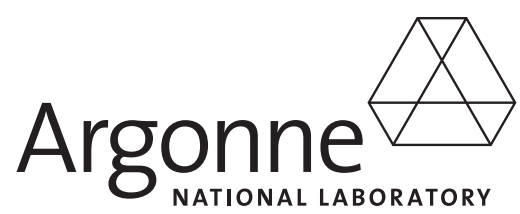

ANL/EVS/AGEM/TR-08-11

\title{
March 2008 Monitoring Results for Barnes, Kansas
}

\section{Environmental Science Division}




\begin{abstract}
About Argonne National Laboratory
Argonne is a U.S. Department of Energy laboratory managed by UChicago Argonne, LLC under contract DE-AC02-06CH11357. The Laboratory's main facility is outside Chicago, at 9700 South Cass Avenue, Argonne, Illinois 60439. For information about Argonne, see www.anl.gov.
\end{abstract}

\title{
Availability of This Report
}

This report is available, at no cost, at http://www.osti.gov/bridge. It is also available on paper to the U.S. Department of Energy and its contractors, for a processing fee, from:

U.S. Department of Energy

Office of Scientific and Technical Information

P.O. Box 62

Oak Ridge, TN 37831-0062

phone (865) 576-8401

fax (865) 576-5728

reports@adonis.osti.gov

\section{Disclaimer}

This report was prepared as an account of work sponsored by an agency of the United States Government. Reference herein to any specific commercial product, process, or service by trade name, trademark, manufacturer, or otherwise, does not necessarily constitute or imply its endorsement, recommendation, or favoring by the United States Government or any agency thereof. The views and opinions of document authors expressed herein do not necessarily state or reflect those of the United States Government or any agency thereof, Argonne National Laboratory, or UChicago Argonne, LLC. 


\section{March 2008 Monitoring Results for Barnes, Kansas}

by

Applied Geosciences and Environmental Management Section

Environmental Science Division, Argonne National Laboratory

August 2008

USDA 


\section{Contents}

Notation

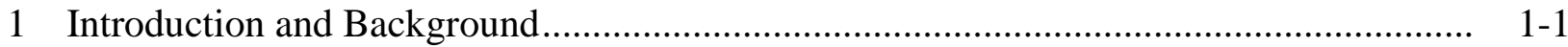

2 Sampling and Analysis Activities ................................................................. 2-1

2.1 Measurement of Groundwater Levels ............................................................... 2-1

2.2 Monitoring Well Sampling and Analyses ......................................................... 2-2

2.3 Handling and Disposal of Investigation-Derived Waste ....................................... 2-3

2.4 Quality Control for Sample Collection, Handling, and Analysis......................... 2-4

3 Results and Discussion....................................................................................

3.1 Groundwater Level Data .......................................................................... 3-1

3.2 Analytical Results for Volatile Organic Compounds in Groundwater Samples and Lateral Distribution of the Contaminants .................................................... 3-2

3.3 Vertical Distribution of Carbon Tetrachloride and Evaluation of Data to Distinguish Aquifer Zones by Depth ........................................................ 3-3

4 Conclusions and Ongoing Tasks .................................................................. $4-1$

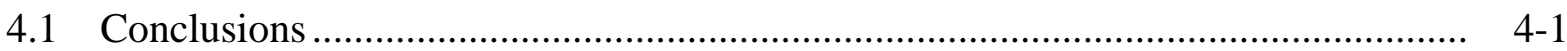

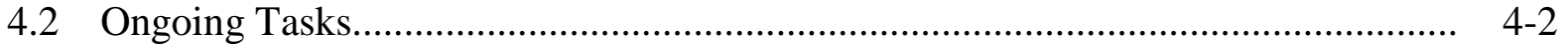

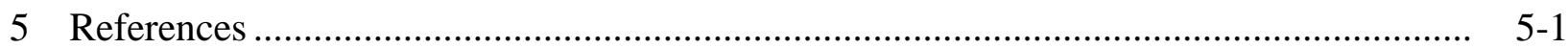

Appendix A: Sampling Activities and Field Measurements at Barnes in March 2008 ........ A-1

Appendix B: Results from the AGEM Laboratory for Dual Analyses of Samples Collected at Barnes in March 2008 and for Quality Control Samples............ B-1

Appendix C: Sample Documentation from Envirosystems, Inc.................................. C-1

Supplement 1: Automatically Measured Water Levels, May 2007 to April 2008................. on CD

\section{Figures}

1.1 Groundwater sampling locations at Barnes in March 2008 .................................. 1-2

2.1 Wells at Barnes equipped with data loggers for water level monitoring, as of April 2008 


\section{Figures (Cont.)}

3.1 Potentiometric surface map depicting the groundwater flow direction at Barnes on December 19, 2007, before pumping of the public water supply wells and during pumping

3.2 Hydrographs summarizing monthly results of long-term water level monitoring in wells at Barnes, January to April 2008

3.3 Analytical results for carbon tetrachloride in groundwater samples collected at Barnes during the 2007 targeted investigation....

3.4 Analytical results for carbon tetrachloride in groundwater samples collected at Barnes in November 2007 and March 2008.

3.5 Analytical results for chloroform in groundwater samples collected at Barnes in March 2008

3.6 Interpreted carbon tetrachloride plume in wells screened in the intermediate aquifer zone (approximately 1250-1254 ft AMSL)....

3.7 Interpreted carbon tetrachloride plume in wells screened in the deep aquifer zone (approximately 1223-1229 ft AMSL)

\section{Tables}

3.1 Hand-measured water levels at Barnes, November 2007, December 2007, and March 2008

3.2 Analytical results from the AGEM Laboratory for volatile organic compounds in groundwater samples collected at Barnes, July 2006 to March 2008

A.1 Sequence of sampling activities at Barnes in March 2008

A.2 Field measurements for groundwater samples collected at Barnes, July 2006 to March 2008

B.1 Analytical results for samples and replicates collected at Barnes in March 2008 and for quality control samples 


\section{Notation}

AGEM Applied Geosciences and Environmental Management

AMSL above mean sea level

BGL below ground level

${ }^{\circ} \mathrm{C} \quad$ degree(s) Celsius

CCC Commodity Credit Corporation

CD compact disc

CLP Contract Laboratory Program

COC chain of custody

DO dissolved oxygen

EPA U.S. Environmental Protection Agency

$\mathrm{ft} \quad$ foot (feet)

gal gallon(s)

in. inch(es)

KDHE Kansas Department of Health and Environment

L liter(s)

$\mu \mathrm{g} / \mathrm{L} \quad$ microgram(s) per liter

$\mu \mathrm{S} / \mathrm{cm} \quad$ microsiemen(s) per centimeter

$\mathrm{mg} / \mathrm{L} \quad$ milligram(s) per liter

$\min \quad$ minute

$\mathrm{mV} \quad$ millivolt(s)

NAPP National Aerial Photography Program

ORP oxidation-reduction potential

RBSL risk-based screening level

TOC top of casing

USDA U.S. Department of Agriculture

VOC volatile organic compound 


\section{March 2008 Monitoring Results for Barnes, Kansas}

\section{Introduction and Background}

The Commodity Credit Corporation of the U.S. Department of Agriculture (CCC/USDA) operated a grain storage facility at Barnes, Kansas, during most of the interval 1949-1974. Carbon tetrachloride contamination was initially detected in 1986 in the town's public water supply wells. In 2006-2007, the CCC/USDA conducted a comprehensive targeted investigation at and near its former property in Barnes to characterize this contamination. Those results were reported previously (Argonne 2007).

In November 2007, the CCC/USDA began quarterly groundwater monitoring at Barnes. The monitoring is being conducted on behalf of the CCC/USDA by Argonne National Laboratory, in accord with the recommendations made in the report for the 2006-2007 targeted investigation (Argonne 2007). The objective is to monitor the carbon tetrachloride contamination identified in the groundwater at Barnes. The sampling is presently conducted in a network of 28 individual monitoring wells (at 19 distinct locations), 2 public water supply wells, and 1 private well (Figure 1.1).

The results of the 2006-2007 targeted investigation (Argonne 2007) and the initial monitoring in November 2007 (Argonne 2008) demonstrated the presence of carbon tetrachloride contamination in groundwater at levels slightly exceeding the Kansas Department of Health and Environment (KDHE) Tier 2 risk-based screening level (RBSL) of $5.0 \mu \mathrm{g} / \mathrm{L}$ for this compound. The contaminant plume appears to extend from the former CCC/USDA property northwestward, toward the Barnes public water supply wells. Information obtained during the 2006-2007 investigation indicates that at least one other potential source might have contributed to the groundwater contaminant plume (Argonne 2007). In particular, the local school district (USD 223) handled, stored, and disposed of chemicals including carbon tetrachloride.

This current report presents the results of the second quarterly monitoring event, conducted in March 2008. During this second monitoring event, low-flow sampling methods were used to purge and sample all wells. This was the initial use of these sampling methods at Barnes. 


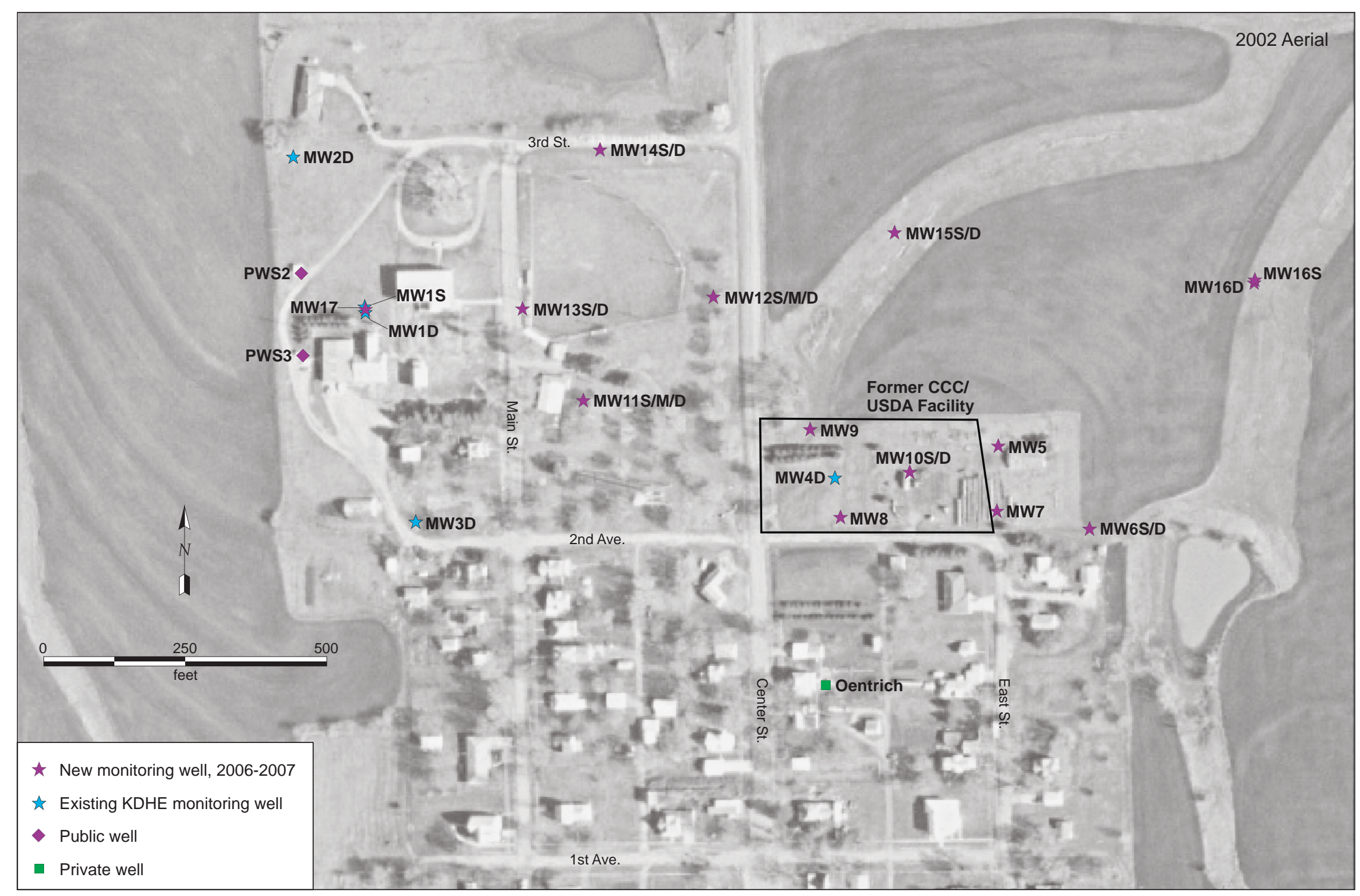

FIGURE 1.1 Groundwater sampling locations at Barnes in March 2008. Source of photograph: NAPP (2002). 


\section{Sampling and Analysis Activities}

\subsection{Measurement of Groundwater Levels}

The March 2008 groundwater sampling event at Barnes involved 28 monitoring wells (MW1S, MW1D, MW2D, MW3D, MW4D, MW5, MW6S, MW6D, MW7, MW8, MW9, MW10S, MW10D, MW11S, MW11M, MW11D, MW12S, MW12M, MW12D, MW13S, MW13D, MW14S, MW14D, MW15S, MW15D, MW16S, MW16D, MW17). All of the well locations are shown in Figure 1.1. A chronological summary of the field activities is in Appendix A, Table A.1.

Before the wells were purged in preparation for the sampling described in Section 2.2, a hand-held water level indicator was used to measure the depth to groundwater and the total depth of each well, to within $0.01 \mathrm{ft}$, from the top of the well casing. Monitoring wells MW1S and MW12S were measured but were found to be dry and consequently could not be sampled. Two public water supply wells (PWS2 and PWS3) and 1 private well (Oentrich) were sampled but not measured. The hand-measured water levels and the volumes purged are in Appendix A, Table A.1. Additional sets of manual water level measurements were made in the wells equipped with data loggers on September 7, 2007, and December 19, 2007, in conjunction with data downloads.

In addition to the manual water level measurements, data recorders have been gathering long-term data on the groundwater elevation and gradient at monitoring wells MW1D, MW2D, MW3D, MW4D, MW7, MW9, MW14D, MW15D, and MW16D and at the Oentrich private well (Figure 2.1). The data loggers record water levels continuously at 30-min intervals. The automatically recorded water levels for May 11, 2007, to April 16, 2008, are in Supplement 1, on the compact disc (CD) inside the back cover of this report.

During the download on December 19, 2007, the data recorder cables for wells MW3D and MW14D were found to be damaged, and the recorders were removed from the wells. After repairs, the recorders were replaced on April 16, 2008. In addition, the recorder batteries for MW7 failed in early March. The batteries were changed during the site visit in April 2008. All ten recorders are now working properly. 
The hand-measured and automatically recorded groundwater level data are presented and discussed in Section 3.1.

\subsection{Monitoring Well Sampling and Analyses}

After measurement of water levels, monitoring wells were purged and sampled by using low-flow groundwater sampling techniques, according to U.S. Environmental Protection Agency (EPA) guidelines (Puls and Barcelona 1996). The Oentrich well and the public water supply wells were sampled at their respective faucets after the existing pumps had been allowed to run for $2-5 \mathrm{~min}$.

The low-flow sampling of monitoring wells involved the use of a bladder pump and field measurement equipment designed to determine when representative formation water was entering the well casing. Stabilization of formation water in the screened area of the well was determined by measuring the static water levels and monitoring the levels of $\mathrm{pH}$, temperature, specific conductivity, oxidation-reduction potential (ORP), and dissolved oxygen (DO) during pumping.

The following procedure was followed for each well sampled:

1. A bladder pump was inserted into the well to a depth midway between the top and bottom of the screen. To minimize disturbance of the solids that are typically present at the bottom of a well, care was taken not to lower the equipment or pump to the bottom of the well screen.

2. The pumping rate for the bladder pump was set to ensure that minimal drawdown occurred in each well during pumping. The rate was monitored by measuring the static water level continuously throughout pumping.

3. Poly tubing was used to connect the bladder pump and to an in-line flow cell. Formation parameters, including $\mathrm{pH}$, temperature, specific conductivity, ORP, and DO, were measured continuously in the in-line flow cell during pumping. Measurements were recorded every 4 min until 3 successive measurements for each parameter were within a range indicating that the formation water was 
stable. The range for formation stabilization varies for each parameter, as follows: $\mathrm{pH}, \pm 0.1$; temperature, $\pm 3 \%$; specific conductivity, $\pm 3 \%$; ORP, $\pm 10 \mathrm{mV} ;$ and DO, $\pm 10 \%$.

4. After formation stabilization occurred, the poly tubing was disconnected from the in-line flow cell, and a representative groundwater sample was transferred from the poly tubing into laboratory-approved containers.

5. Poly tubing for each well was kept and dedicated for reuse at that well. In addition, pumping rate data were recorded for each well as a reference for subsequent sampling events.

The sequence of activities during the March 2008 well sampling event is summarized in Appendix A, Table A.1. The field measurements are in Appendix A, Table A.2.

Groundwater samples designated for analyses for volatile organic compounds (VOCs) were collected in appropriate laboratory containers, labeled, packaged, and chilled to $4^{\circ} \mathrm{C}$ by placement in ice-filled coolers. The samples were shipped via an overnight delivery service to the Applied Geosciences and Environmental Management (AGEM) Laboratory at Argonne for VOCs analyses with EPA Method 524.2 (EPA 1995). Aliquots of selected samples (chosen in the field) were also shipped to Envirosystems, Inc., Columbia, Maryland, for verification VOCs analyses according to EPA Contract Laboratory Program (CLP) protocols.

The analytical results are presented and discussed in Section 3.2.

\subsection{Handling and Disposal of Investigation-Derived Waste}

Purge water generated as potentially contaminated investigation-derived waste was containerized on-site in 55-gal drums. The accumulated purge water was sampled and analyzed for VOCs (including ethylene dibromide) and nitrates. The analytical results indicated carbon tetrachloride at $1.9 \mu \mathrm{g} / \mathrm{L}$ and no detectable concentration of chloroform or ethylene dibromide. Other VOCs were detected, but none were at concentrations exceeding the KDHE RBSL values. Nitrate was present at $4.0 \mathrm{mg} / \mathrm{L}$. With the approval of the KDHE (2008a), the water was discharged on-site. 


\subsection{Quality Control for Sample Collection, Handling, and Analysis}

Quality assurance/quality control procedures followed during the March 2008 monitoring event are described in detail in the Master Work Plan (Argonne 2002). The results are summarized as follows:

- Sample collection and handling activities were monitored by the documentation of samples as they were collected and the use of chain-ofcustody forms and custody seals to ensure sample integrity during handling and shipment.

- Samples designated for VOCs analyses were received with custody seals intact and at the appropriate preservation temperature. All samples sent to the AGEM Laboratory were analyzed within the required holding times.

- Quality control samples collected to monitor sample-handling activities (trip blanks) and method blanks analyzed with the samples to monitor analytical methodologies were all free of carbon tetrachloride and chloroform contamination. Trace detections of carbon tetrachloride in two of three equipment rinsates do not require qualification of groundwater results.

- Groundwater samples were analyzed for VOCs at the AGEM Laboratory with the purge-and-trap method on a gas chromatograph-mass spectrometer system. Calibration checks analyzed with each sample delivery group were required to be within $\pm 20 \%$ of the standard. Surrogate standard determinations performed on samples and blanks were within the specified range of $80-120 \%$ for all samples, in either the initial analysis or a successful reanalysis.

- Results from the AGEM Laboratory for dual analyses of the groundwater samples are in Appendix B, Table B.1. The results of the dual analyses compare well, indicating consistency in the sampling and analytical methodologies. Analytical results for quality control samples are also in Appendix B, Table B.1. 
- In accordance with the procedures defined in the Master Work Plan (Argonne 2002), three groundwater samples were submitted to a second laboratory (Envirosystems) for verification analysis according to the protocols of the EPA's CLP. Documentation is in Appendix C. The results for the sample from location MW12M compare well, with relative percent difference values of $1.4 \%$ for carbon tetrachloride and $2.7 \%$ for chloroform. Carbon tetrachloride concentrations were $18 \mu \mathrm{g} / \mathrm{L}$ (AGEM Laboratory) and $17 \mu \mathrm{g} / \mathrm{L}$ (verification by Envirosystems); chloroform concentrations were $2.6 \mu \mathrm{g} / \mathrm{L}$ (AGEM Laboratory) and $2.9 \mu \mathrm{g} / \mathrm{L}$ (verification by Envirosystems). Agreement is less favorable for two other verification samples; for these samples, the AGEM Laboratory reported lower contaminant concentrations that were not consistently detected by the CLP analysis, with its higher method quantitation limit. Methylene chloride, a frequent laboratory contaminant, was detected in all samples and blanks analyzed by Envirosystems. The AGEM Laboratory results are displayed in the figures in Section 3. 


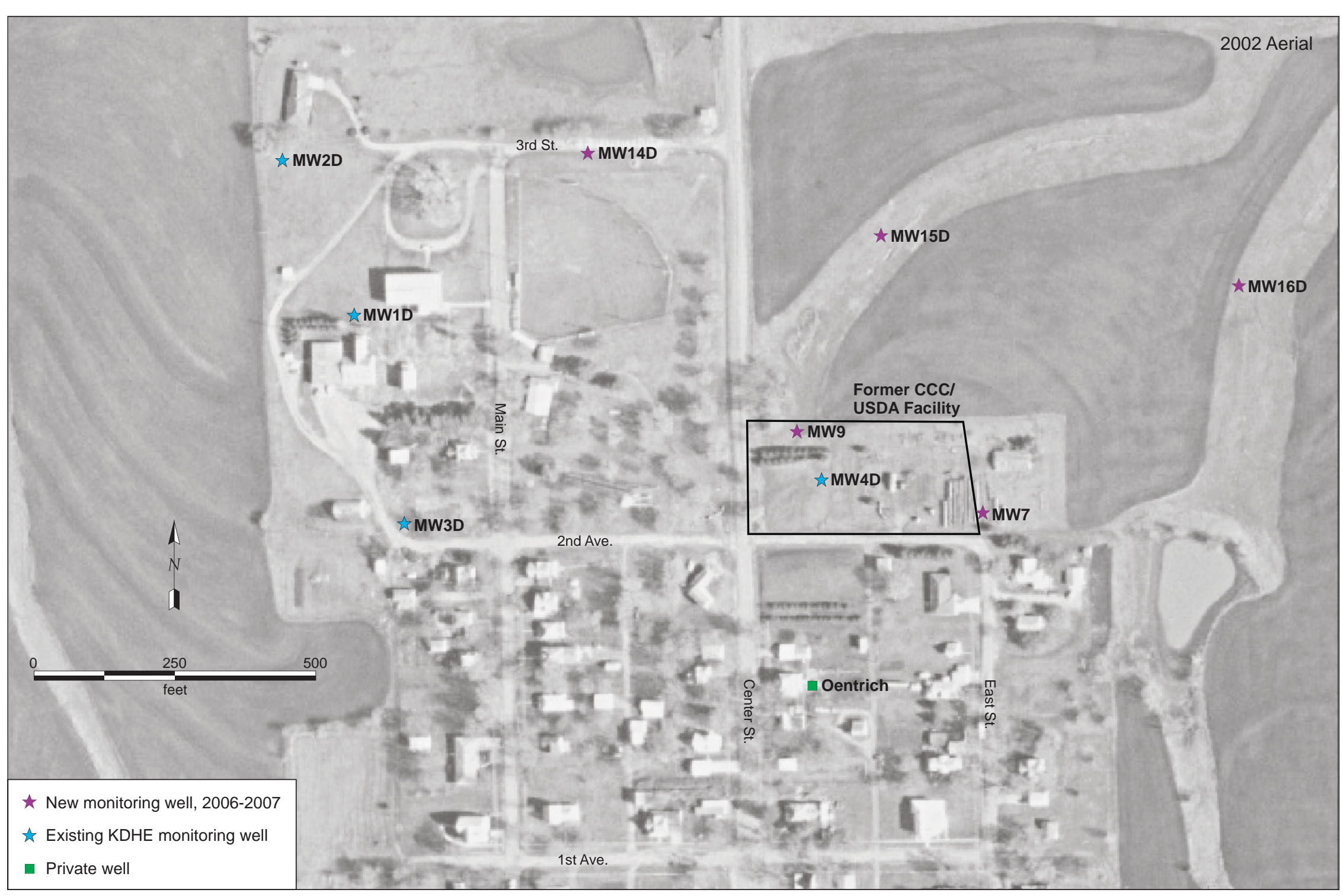

FIGURE 2.1 Wells at Barnes equipped with data loggers for water level monitoring, as of April 2008. Source of photograph: NAPP (2002). 


\section{Results and Discussion}

\subsection{Groundwater Level Data}

The manual water level measurements made during sampling on March 4-10, 2008, are in Table 3.1, together with manual measurements made in November 2007 and December 2007. The manual water level measurements are discussed further in Section 3.3.

The water level contours shown in Figure 3.1 are based on the most recent available data for the complete network of recording transducers installed for long-term water level monitoring at Barnes (see Section 2.1). The complete monitoring network includes wells MW1D, MW2D, MW3D, MW4D, MW7, MW9, MW14D, MW15D, and MW16D, as well as the Oentrich well. The automatically recorded water levels for May 11, 2007, to April 16, 2008, are on the CD, in Supplement 1.

Extensive documentation of the potentiometric surface at Barnes during the targeted investigation (Figure 4.7 in Argonne 2007) indicated that operation of the public water supply wells strongly influences the groundwater flow direction. The more recent contours for the complete network, for December 19, 2007 (Figure 3.1), continue to show that flow directions are significantly different before pumping begins (top) versus during pumping (bottom). The data loggers in monitoring wells MW14D, MW15D, and MW16D, in the northeastern part of the study area, were installed after collection of the data depicted in the earlier report (Figure 4.7 in Argonne 2007). Consequently, Figure 3.1 in the present report reflects finer resolution of the local flow to the northeast.

The hydrographs in Figure 3.2 summarize data for the period January 1, 2008, to April 16, 2008, for the recording transducers. As indicated in Section 2.1, the recorders in wells MW3D and MW14D were removed for repair in December 2007, and thus no traces for those wells appear in Figure 3.2. In addition, the battery in the well MW7 recorder failed in early March 2008, and its trace ends at that point.

The data for well MW15D were displayed only partially in the previous report (Figure 3.1 in Argonne 2008), because a recorder malfunction was suspected. The observed pattern of higher water levels in this deeper well could indicate that vertical gradients are present. 
The patterns in Figure 3.2 of the present report are similar to patterns recorded in 2007 (Figure 3.1 in Argonne 2008). The hydrographs show responses to pumping of the public water supply wells that are characterized by drawdowns of as much as $2 \mathrm{ft}$ during pumping, with almost immediate rebound when pumping stops. The most prominent drawdowns are consistently observed in wells MW1D and MW2D, which are the closest monitoring points to the public wells.

In 2007, water levels at Barnes rose by approximately 7-8 ft. During January 2008, the levels remained relatively stable. The levels have increased slowly since February 2008 and have shown an overall increase of about 1-2 ft since January 2008.

\subsection{Analytical Results for Volatile Organic Compounds in Groundwater Samples and Lateral Distribution of the Contaminants}

The analytical data for VOCs in the groundwater samples collected in March 2008 are in Table 3.2, together with data for the previous sampling events at Barnes. The lateral distribution of carbon tetrachloride in groundwater in the July-August 2006 and March-April 2007 sampling events is illustrated in Figure 3.3, while the November 2007 and March 2008 results are illustrated in Figure 3.4. The overall carbon tetrachloride distribution patterns are similar for the four events. The lateral distribution of chloroform in the March 2008 sampling event is illustrated in Figure 3.5.

Overall, carbon tetrachloride was detected in March 2008 in 23 of the 29 groundwater samples collected (at 16 of the 20 distinct monitoring locations; Figure 3.4 and Table 3.2). Carbon tetrachloride concentrations in the 29 samples were as follows:

- Not detected at an instrument detection limit of $1.0 \mu \mathrm{g} / \mathrm{L}$ in 6 samples.

- Trace levels below the method quantitation limit of $1.0 \mu \mathrm{g} / \mathrm{L}$ in 9 samples.

- Concentrations of 1.0-4.9 $\mu \mathrm{g} / \mathrm{L}$ in 7 samples.

- Concentrations at or above the RBSL value of $5.0 \mu \mathrm{g} / \mathrm{L}$ in 7 samples. 
The maximum carbon tetrachloride concentration detected was $56 \mu \mathrm{g} / \mathrm{L}$ (at MW10S). No carbon tetrachloride was detected in samples from wells MW2D, MW3D, PWS2, and PWS3.

Chloroform concentrations ranging from trace levels to $2.6 \mu \mathrm{g} / \mathrm{L}$ (at $\mathrm{MW} 12 \mathrm{M}$ ) were detected in 12 of the 29 groundwater samples (at 9 of the 20 distinct sampling locations; Table 3.2 and Figure 3.5). Chloroform occurred in association with the more elevated carbon tetrachloride levels identified at MW4D, MW8, MW10S, MW10D, MW12M, MW13S, and MW13D. The results of the November 2007 monitoring event were similar.

The lateral distribution of carbon tetrachloride in groundwater in March 2008 was similar to the distribution observed during the 2007 sampling events, with only a slight increase in contaminant concentrations northwest of the former CCC/USDA property and a decrease in concentrations north of the former CCC/USDA property. The predominant differences noted were fluctuating concentrations in some wells. The most significant changes were observed in wells MW4D, MW10S, MW13D, MW14S, and MW15S. The carbon tetrachloride level increased between the November 2007 and March 2008 events in wells MW4D (1.7 $\mu \mathrm{g} / \mathrm{L}$ to $18 \mu \mathrm{g} / \mathrm{L}), \operatorname{MW} 10 \mathrm{~S}(11 \mu \mathrm{g} / \mathrm{L}$ to $56 \mu \mathrm{g} / \mathrm{L}), \operatorname{MW} 13 \mathrm{D}(5.9 \mu \mathrm{g} / \mathrm{L}$ to $11 \mu \mathrm{g} / \mathrm{L})$, and MW14S $(1.2 \mu \mathrm{g} / \mathrm{L}$ to $4.3 \mu \mathrm{g} / \mathrm{L})$. Conversely, the concentration detected in sentinel well MW15S decreased from $8.7 \mu \mathrm{g} / \mathrm{L}$ in November 2007 to $1.8 \mu \mathrm{g} / \mathrm{L}$ in March 2008 .

In the other sentinel wells, as defined previously (Section 5.1 of Argonne 2007), carbon tetrachloride was detected at trace concentrations below the method quantitation limit of $1.0 \mu \mathrm{g} / \mathrm{L}$ in wells MW1D, MW6S, MW6D, MW14D, MW15D, MW16S, and MW17. No carbon tetrachloride was detected (at an instrument detection limit of $0.1 \mu \mathrm{g} / \mathrm{L}$ ) in sentinel wells MW2D, MW3D, and MW16D.

\subsection{Vertical Distribution of Carbon Tetrachloride and Evaluation of Data to Distinguish Aquifer Zones by Depth}

The designations "S," "M," and " $\mathrm{D}$ " in monitoring well names were assigned at the time of well installation. They indicate shallow, medium, and deep screens (relative depths) in an individual well, rather than the aquifer zone screened. After the March 2008 monitoring, a detailed evaluation of the hand-measured water levels (Table 3.1) and VOCs data was conducted in an attempt to differentiate aquifer zones by depth. The data suggest at least three vertically distinct zones within the aquifer. The aquifer zones are as follows: 
- Shallow Aquifer Zone. The only monitoring wells believed to be completed in the shallow zone are MW1S, MW11S, and MW12S. Wells MW1S and MW12S were dry during the March 2008 sampling event; well MW11S showed a water level elevation of approximately $1309 \mathrm{ft}$ AMSL (above mean sea level).

- Intermediate Aquifer Zone. The intermediate zone is believed to be represented by monitoring wells MW10S, MW11M, MW12M, MW13S, and MW17. Water level elevations measured manually for these wells in March 2008 were approximately 1250-1254 ft AMSL.

- Deep Aquifer Zone. Monitoring wells believed to represent the deep zone include MW1D, MW2D, MW3D, MW4D, MW5, MW6S, MW6D, MW7, MW8, MW9, MW10D, MW11D, MW12D, MW13D, MW14S, MW14D, MW15S, MW15D, MW16S, and MW16D. The water level elevations measured manually in these monitoring wells in March 2008 were approximately 1223-1229 ft AMSL. On the basis of its hand-measured water level in November 2007 (approximately $1220 \mathrm{ft}$ AMSL [Table 3.1] and the automatic measurements [Supplement 1]), the Oentrich well is also considered to be screened in the deep aquifer zone. All of the wells equipped with data loggers for water level monitoring are screened in the deep zone.

The vertical distribution of the carbon tetrachloride in groundwater indicates that the highest concentrations were detected in the intermediate zone, at wells MW10S $(56 \mu \mathrm{g} / \mathrm{L})$, MW12M $(18 \mu / \mathrm{L})$, and MW13S $(17 \mu / \mathrm{L})$. The deep-zone wells at these locations showed lower concentrations $(5.7 \mu / \mathrm{L}$ at MW10D, $1.0 \mu / \mathrm{L}$ at MW12D, and $11 \mu / \mathrm{L}$ at MW13D). Figures 3.6 and 3.7 show the contaminant distribution in the intermediate and deep zones, respectively. The shallow zone well (MW11S) showed no detectable concentrations of carbon tetrachloride. 
TABLE 3.1 Hand-measured water levels at Barnes, November 2007, December 2007, and March 2008.

\begin{tabular}{|c|c|c|c|c|c|c|c|}
\hline \multirow[b]{3}{*}{ Well } & \multirow{3}{*}{$\begin{array}{l}\text { Reference } \\
\text { Elevation } \\
\text { (ft AMSL) }\end{array}$} & \multicolumn{6}{|c|}{ Water Level on Date Indicated } \\
\hline & & \multicolumn{2}{|c|}{$11 / 18-19 / 07$} & \multicolumn{2}{|c|}{$12 / 19 / 07$} & \multicolumn{2}{|c|}{$3 / 4-10 / 08$} \\
\hline & & ft TOCa & $\mathrm{ft}$ AMSL & ft TOCa & ft AMSL & $\mathrm{ft} \mathrm{TOCa}$ & ft AMSL \\
\hline \multicolumn{8}{|c|}{ Shallow aquifer zone } \\
\hline $\begin{array}{l}\text { MW1S } \\
\text { MW11S } \\
\text { MW12S }\end{array}$ & $\begin{array}{l}1351.58 \\
1336.58 \\
1327.46\end{array}$ & $\begin{array}{l}\text { Dry } \\
29.20 \\
\text { Dry }\end{array}$ & $\begin{array}{c}- \\
1307.38 \\
-\end{array}$ & Dry & - & $\begin{array}{c}\text { Dry } \\
27.70 \\
\text { Dry }\end{array}$ & ${ }_{-}^{-}$ \\
\hline \multicolumn{8}{|c|}{ Intermediate aquifer zone } \\
\hline $\begin{array}{l}\text { MW10S } \\
\text { MW11M } \\
\text { MW12M } \\
\text { MW13S } \\
\text { MW17 }\end{array}$ & $\begin{array}{l}1331.33 \\
1336.51 \\
1327.46 \\
1342.36 \\
1351.77\end{array}$ & $\begin{array}{r}77.81 \\
82.33 \\
74.50 \\
92.23 \\
102.68\end{array}$ & $\begin{array}{l}1253.52 \\
1254.18 \\
1252.96 \\
1250.13 \\
1249.09\end{array}$ & & & $\begin{array}{r}77.47 \\
82.65 \\
74.77 \\
92.10 \\
101.75\end{array}$ & $\begin{array}{l}1253.86 \\
1253.86 \\
1252.69 \\
1250.26 \\
1250.02\end{array}$ \\
\hline \multicolumn{8}{|c|}{ Deep aquifer zone } \\
\hline MW1D & 1351.33 & 124.89 & 1226.44 & 125.54 & 1225.79 & 127.66 & 1223.67 \\
\hline MW2D & 1348.85 & 122.56 & 1226.29 & 123.20 & 1225.65 & 125.55 & 1223.30 \\
\hline MW3D & 1345.99 & 126.25 & 1219.74 & 120.09 & 1225.90 & 121.90 & 1224.09 \\
\hline MW4D & 1326.32 & 101.39 & 1224.93 & 100.54 & 1225.78 & 101.74 & 1224.58 \\
\hline MW5 & 1327.20 & 102.78 & 1224.42 & & & 102.00 & 1225.20 \\
\hline MW6S & 1323.13 & 96.10 & 1227.03 & & & 94.50 & 1228.63 \\
\hline MW6D & 1323.15 & 98.50 & 1224.65 & & & 98.50 & 1224.65 \\
\hline MW7 & 1329.91 & 105.50 & 1224.41 & 104.48 & 1225.43 & 105.62 & 1224.29 \\
\hline MW8 & 1330.06 & 105.17 & 1224.89 & & & 104.38 & 1225.68 \\
\hline MW9 & 1321.86 & 97.20 & 1224.66 & 96.25 & 1225.61 & 96.40 & 1225.46 \\
\hline MW10D & 1331.33 & 106.22 & 1225.11 & & & 106.36 & 1224.97 \\
\hline MW11D & 1336.53 & 112.46 & 1224.07 & & & 110.50 & 1226.03 \\
\hline MW12D & 1327.52 & 102.00 & 1225.52 & & & 103.30 & 1224.22 \\
\hline MW13D & 1342.37 & 117.83 & 1224.54 & & & 118.19 & 1224.18 \\
\hline MW14S & 1332.69 & 106.75 & 1225.94 & & & 106.95 & 1225.74 \\
\hline MW14D & 1332.74 & 107.10 & 1225.64 & 107.23 & 1225.51 & 106.95 & 1225.79 \\
\hline MW15S & 1309.34 & 84.33 & 1225.01 & & & 84.66 & 1224.68 \\
\hline MW15D & 1309.29 & 70.20 & 1239.09 & 80.84 & 1228.45 & 80.80 & 1228.49 \\
\hline MW16S & 1299.47 & 75.30 & 1224.17 & & & 75.50 & 1223.97 \\
\hline MW16D & 1299.52 & 74.50 & 1225.02 & 74.62 & 1224.90 & 75.00 & 1224.52 \\
\hline Oentrichb & 1336.93 & $\mathrm{NM}^{\mathrm{C}}$ & - & 116.55 & 1220.38 & NM & NM \\
\hline
\end{tabular}

a TOC, top of casing.

b The Oentrich well water level was measured from the concrete at the top of the well vault. The value shown was corrected by $5.5 \mathrm{ft}$ to give a measured depth from the top of the casing.

c NM, not measured (sampled from faucet). 
TABLE 3.2 Analytical results from the AGEM Laboratory for volatile organic compounds in groundwater samples collected at Barnes, July 2006 to March 2008.

\begin{tabular}{|c|c|c|c|c|c|c|}
\hline \multirow[b]{2}{*}{ Well } & \multirow[b]{2}{*}{$\begin{array}{l}\text { Screen Interval } \\
\quad(\mathrm{ft} B G L)\end{array}$} & \multirow[b]{2}{*}{ Sample } & \multirow[b]{2}{*}{$\begin{array}{l}\text { Sample } \\
\text { Date }\end{array}$} & \multicolumn{3}{|c|}{ Concentration $(\mu \mathrm{g} / \mathrm{L})$} \\
\hline & & & & $\begin{array}{c}\text { Carbon } \\
\text { Tetrachloride }\end{array}$ & Chloroform & $\begin{array}{l}\text { Methylene } \\
\text { Chloride }\end{array}$ \\
\hline \multicolumn{7}{|c|}{ Previously existing KDHE monitoring wells } \\
\hline MW1S & 13.3-23.3 & $\begin{array}{l}\text { Not sampled (well dry) } \\
\text { Not sampled (well dry) } \\
\text { Not sampled (well dry) } \\
\text { Not sampled (well dry) }\end{array}$ & $\begin{array}{r}7 / 19 / 06 \\
4 / 4 / 07 \\
11 / 18 / 07 \\
3 / 4 / 08\end{array}$ & $\begin{array}{l}- \\
- \\
- \\
-\end{array}$ & $\begin{array}{l}- \\
- \\
- \\
-\end{array}$ & $\begin{array}{l}- \\
- \\
- \\
-\end{array}$ \\
\hline MW1D & $139.85-159.4$ & $\begin{array}{l}\text { BAMW1D-W-21688 } \\
\text { BAMW1D-W-22565 } \\
\text { BAMW1D-W-22593 } \\
\text { BAMW1D-W-22627 }\end{array}$ & $\begin{array}{r}7 / 19 / 06 \\
4 / 4 / 07 \\
11 / 18 / 07 \\
3 / 4 / 08\end{array}$ & $\begin{array}{l}1.0 \\
1.2 \\
N D \\
0.2 \mathrm{Jb}^{\mathrm{b}}\end{array}$ & $\begin{array}{l}N D^{a} \\
N D \\
N D \\
N D\end{array}$ & $\begin{array}{l}\text { ND } \\
\text { ND } \\
\text { ND } \\
\text { ND }\end{array}$ \\
\hline MW2D & $133.26-152.93$ & $\begin{array}{l}\text { BAMW2D-W-21687 } \\
\text { BAMW2D-W-22564 } \\
\text { BAMW2D-W-22594 } \\
\text { BAMW2D-W-22628 }\end{array}$ & $\begin{array}{r}7 / 19 / 06 \\
4 / 4 / 07 \\
11 / 18 / 07 \\
3 / 7 / 08\end{array}$ & $\begin{array}{l}\text { ND } \\
\text { ND } \\
\text { ND } \\
\text { ND }\end{array}$ & $\begin{array}{l}\text { ND } \\
\text { ND } \\
\text { ND } \\
\text { ND }\end{array}$ & $\begin{array}{l}\text { ND } \\
\text { ND } \\
\text { ND } \\
\text { ND }\end{array}$ \\
\hline MW3D & $133.02-152.73$ & $\begin{array}{l}\text { BAMW3D-W-21686 } \\
\text { BAMW3D-W-22567 } \\
\text { BAMW3D-W-22595 } \\
\text { BAMW3D-W-22629 }\end{array}$ & $\begin{array}{r}7 / 19 / 06 \\
4 / 4 / 07 \\
11 / 19 / 07 \\
3 / 7 / 08\end{array}$ & $\begin{array}{l}\text { ND } \\
\text { ND } \\
\text { ND } \\
\text { ND }\end{array}$ & $\begin{array}{l}\text { ND } \\
\text { ND } \\
\text { ND } \\
\text { ND }\end{array}$ & $\begin{array}{l}\text { ND } \\
\text { ND } \\
\text { ND } \\
\text { ND }\end{array}$ \\
\hline MW4D & $98.38-118.22$ & $\begin{array}{l}\text { BAMW4D-W-21690 } \\
\text { BAMW4D-W-22583 } \\
\text { BAMW4D-W-22596 } \\
\text { BAMW4D-W-22642 }\end{array}$ & $\begin{array}{r}7 / 20 / 06 \\
4 / 6 / 07 \\
11 / 19 / 07 \\
3 / 9 / 08\end{array}$ & $\begin{array}{r}2.1 \\
3.5 \\
1.7 \\
18\end{array}$ & $\begin{array}{l}\text { ND } \\
0.1 \mathrm{~J} \\
0.4 \mathrm{~J} \\
0.4 \mathrm{~J}\end{array}$ & $\begin{array}{l}\text { ND } \\
\text { ND } \\
\text { ND } \\
\text { ND }\end{array}$ \\
\hline \multicolumn{7}{|c|}{ CCC/USDA wells installed during the 2006-2007 investigation } \\
\hline MW5 & $110-120$ & $\begin{array}{l}\text { BAMW5-W-22589 } \\
\text { BAMW5-W-22597 } \\
\text { BAMW5-W-22637 }\end{array}$ & $\begin{array}{r}4 / 6 / 07 \\
11 / 19 / 07 \\
3 / 8 / 08\end{array}$ & $\begin{array}{l}0.6 \mathrm{~J} \\
0.6 \mathrm{~J} \\
0.7 \mathrm{~J}\end{array}$ & $\begin{array}{l}\text { ND } \\
\text { ND } \\
\text { ND }\end{array}$ & $\begin{array}{l}\text { ND } \\
\text { ND } \\
\text { ND }\end{array}$ \\
\hline MW6S & $90.5-100.5$ & $\begin{array}{l}\text { Not sampled (well dry) } \\
\text { BAMW6S-W-22598 } \\
\text { BAMW6S-W-22635 }\end{array}$ & $\begin{array}{r}4 / 4 / 07 \\
11 / 19 / 07 \\
3 / 8 / 08\end{array}$ & $\begin{array}{l}- \\
0.3 \mathrm{~J} \\
0.4 \mathrm{~J}\end{array}$ & $\begin{array}{l}- \\
\text { ND } \\
\text { ND }\end{array}$ & $\begin{array}{l}- \\
N D \\
N D\end{array}$ \\
\hline MW6D & $105-115$ & $\begin{array}{l}\text { BAMW6D-W-22573 } \\
\text { BAMW6D-W-22599 } \\
\text { BAMW6D-W-22636 }\end{array}$ & $\begin{array}{r}4 / 5 / 07 \\
11 / 19 / 07 \\
3 / 8 / 08\end{array}$ & $\begin{array}{l}\text { ND } \\
0.5 \mathrm{~J} \\
0.8 \mathrm{~J}\end{array}$ & $\begin{array}{l}\text { ND } \\
\text { ND } \\
\text { ND }\end{array}$ & $\begin{array}{l}\text { ND } \\
\text { ND } \\
\text { ND }\end{array}$ \\
\hline MW7 & $116-126$ & $\begin{array}{l}\text { BAMW7-W-22588 } \\
\text { BAMW7-W-22600 } \\
\text { BAMW7-W-22643 }\end{array}$ & $\begin{array}{r}4 / 6 / 07 \\
11 / 19 / 07 \\
3 / 9 / 08\end{array}$ & $\begin{array}{l}1.0 \\
2.6 \\
2.8\end{array}$ & $\begin{array}{l}\text { ND } \\
\text { ND } \\
\text { ND }\end{array}$ & $\begin{array}{l}\text { ND } \\
\text { ND } \\
\text { ND }\end{array}$ \\
\hline MW8 & $110-120$ & $\begin{array}{l}\text { BAMW8-W-22584 } \\
\text { BAMW8-W-22601 } \\
\text { BAMW8-W-22652 }\end{array}$ & $\begin{array}{r}4 / 6 / 07 \\
11 / 19 / 07 \\
3 / 10 / 08\end{array}$ & $\begin{array}{l}14 \\
23 \\
19\end{array}$ & $\begin{array}{l}0.7 \mathrm{~J} \\
0.6 \mathrm{~J} \\
0.6 \mathrm{~J}\end{array}$ & $\begin{array}{l}\text { ND } \\
\text { ND } \\
\text { ND }\end{array}$ \\
\hline
\end{tabular}


TABLE 3.2 (Cont.)

\begin{tabular}{|c|c|c|c|c|c|c|}
\hline \multirow[b]{2}{*}{ Well } & \multirow[b]{2}{*}{$\begin{array}{l}\text { Screen Interval } \\
\quad(\mathrm{ft} B G L)\end{array}$} & \multirow[b]{2}{*}{ Sample } & \multirow[b]{2}{*}{$\begin{array}{l}\text { Sample } \\
\text { Date }\end{array}$} & \multicolumn{3}{|c|}{ Concentration $(\mu \mathrm{g} / \mathrm{L})$} \\
\hline & & & & $\begin{array}{c}\text { Carbon } \\
\text { Tetrachloride }\end{array}$ & Chloroform & $\begin{array}{r}\text { Methyl } \\
\text { Chlori }\end{array}$ \\
\hline \multicolumn{7}{|c|}{ CCC/USDA wells installed during the $2006-2007$ investigation (cont.) } \\
\hline MW9 & $100-110$ & $\begin{array}{l}\text { BAMW9-W-22582 } \\
\text { BAMW9-W-22602 } \\
\text { BAMW9-W-22647 }\end{array}$ & $\begin{array}{r}4 / 5 / 07 \\
11 / 19 / 07 \\
3 / 9 / 08\end{array}$ & $\begin{array}{l}1.0 \\
7.7 \\
3.0\end{array}$ & $\begin{array}{l}\text { ND } \\
0.6 \mathrm{~J} \\
0.3 \mathrm{~J}\end{array}$ & $\begin{array}{l}\text { ND } \\
\text { ND } \\
\text { ND }\end{array}$ \\
\hline MW10S & $93-103$ & $\begin{array}{l}\text { BAMW10S-W-22586 } \\
\text { BAMW10S-W-22603 } \\
\text { BAMW10S-W-22649 }\end{array}$ & $\begin{array}{r}4 / 6 / 07 \\
11 / 19 / 07 \\
3 / 10 / 08\end{array}$ & $\begin{array}{l}20 \\
11 \\
56\end{array}$ & $\begin{array}{l}1.4 \\
0.7 \mathrm{~J} \\
2.0\end{array}$ & $\begin{array}{l}\text { ND } \\
\text { ND } \\
\text { ND }\end{array}$ \\
\hline MW10D & $115-125$ & $\begin{array}{l}\text { BAMW10D-W-22585 } \\
\text { BAMW10D-W-22604 } \\
\text { BAMW10D-W-22646 }\end{array}$ & $\begin{array}{r}4 / 6 / 07 \\
11 / 19 / 07 \\
3 / 9 / 08\end{array}$ & $\begin{array}{l}2.4 \\
6.3 \\
5.7\end{array}$ & $\begin{array}{l}0.2 \mathrm{~J} \\
0.5 \mathrm{~J} \\
0.5 \mathrm{~J}\end{array}$ & $\begin{array}{l}\text { ND } \\
\text { ND } \\
\text { ND }\end{array}$ \\
\hline MW11S & $40-50$ & $\begin{array}{l}\text { BAMW11S-W-22570 } \\
\text { BAMW11S-W-22605 } \\
\text { BAMW11S-W-22630 }\end{array}$ & $\begin{array}{r}4 / 4 / 07 \\
11 / 19 / 07 \\
3 / 5 / 08\end{array}$ & $\begin{array}{l}\text { ND } \\
\text { ND } \\
\text { ND }\end{array}$ & $\begin{array}{l}1.1 \\
0.6 \mathrm{~J} \\
0.6 \mathrm{~J}\end{array}$ & $\begin{array}{l}\text { ND } \\
\text { ND } \\
\text { ND }\end{array}$ \\
\hline MW11M & $90-100$ & $\begin{array}{l}\text { BAMW11M-W-22572 } \\
\text { BAMW11M-W-22606 } \\
\text { BAMW11M-W-22644 }\end{array}$ & $\begin{array}{r}4 / 5 / 07 \\
11 / 19 / 07 \\
3 / 6 / 08\end{array}$ & $\begin{array}{l}\text { ND } \\
3.7 \\
2.4\end{array}$ & $\begin{array}{l}\text { ND } \\
\text { ND } \\
0.5 \mathrm{~J}\end{array}$ & $\begin{array}{l}\text { ND } \\
\text { ND } \\
\text { ND }\end{array}$ \\
\hline MW11D & $125-135$ & $\begin{array}{l}\text { BAMW11D-W-22571 } \\
\text { BAMW11D-W-22607 } \\
\text { BAMW11D-W-22639 }\end{array}$ & $\begin{array}{r}4 / 4 / 07 \\
11 / 19 / 07 \\
3 / 5 / 08\end{array}$ & $\begin{array}{l}1.1 \\
0.8 \mathrm{~J} \\
0.4 \mathrm{~J}\end{array}$ & $\begin{array}{l}\text { ND } \\
\text { ND } \\
\text { ND }\end{array}$ & $\begin{array}{l}\text { ND } \\
\text { ND } \\
\text { ND }\end{array}$ \\
\hline MW12S & $43-50$ & $\begin{array}{l}\text { Not sampled (well dry) } \\
\text { Not sampled (well dry) } \\
\text { Not sampled (well dry) }\end{array}$ & $\begin{array}{r}4 / 5 / 07 \\
11 / 19 / 07 \\
3 / 10 / 08\end{array}$ & $\begin{array}{l}- \\
- \\
-\end{array}$ & $\begin{array}{l}- \\
- \\
-\end{array}$ & $\begin{array}{l}- \\
- \\
-\end{array}$ \\
\hline MW12M & $90-100$ & $\begin{array}{l}\text { BAMW12M-W-22580 } \\
\text { BAMW12M-W-22609 } \\
\text { BAMW12M-W-22651 }\end{array}$ & $\begin{array}{r}4 / 5 / 07 \\
11 / 19 / 07 \\
3 / 10 / 08\end{array}$ & $\begin{array}{l}20 \\
18 \\
18\end{array}$ & $\begin{array}{l}4.2 \\
5.1 \\
2.6\end{array}$ & $\begin{array}{l}\text { ND } \\
\text { ND } \\
\text { ND }\end{array}$ \\
\hline MW12D & $115-125$ & $\begin{array}{l}\text { BAMW12D-W-22576 } \\
\text { BAMW12D-W-22610 } \\
\text { BAMW12D-W-22641 }\end{array}$ & $\begin{array}{r}4 / 5 / 07 \\
11 / 18 / 07 \\
3 / 9 / 08\end{array}$ & $\begin{array}{l}0.6 \mathrm{~J} \\
1.6 \\
1.0\end{array}$ & $\begin{array}{l}\text { ND } \\
\text { ND } \\
\text { ND }\end{array}$ & $\begin{array}{l}\text { ND } \\
\text { ND } \\
\text { ND }\end{array}$ \\
\hline MW13S & $112-122$ & $\begin{array}{l}\text { BAMW13S-W-22575 } \\
\text { BAMW13S-W-22611 } \\
\text { BAMW13S-W-22650 }\end{array}$ & $\begin{array}{r}4 / 5 / 07 \\
11 / 19 / 07 \\
3 / 10 / 08\end{array}$ & $\begin{array}{l}21 \\
17 \\
17\end{array}$ & $\begin{array}{l}1.6 \\
1.8 \\
1.5\end{array}$ & $\begin{array}{l}\text { ND } \\
\text { ND } \\
\text { ND }\end{array}$ \\
\hline MW13D & $127-137$ & $\begin{array}{l}\text { BAMW13D-W-22574 } \\
\text { BAMW13D-W-22612 } \\
\text { BAMW13D-W-22645 }\end{array}$ & $\begin{array}{r}4 / 5 / 07 \\
11 / 19 / 07 \\
3 / 9 / 08\end{array}$ & $\begin{array}{r}3.5 \\
5.9 \\
11\end{array}$ & $\begin{array}{l}0.4 \mathrm{~J} \\
0.2 \mathrm{~J} \\
1.1\end{array}$ & $\begin{array}{l}\text { ND } \\
\text { ND } \\
\text { ND }\end{array}$ \\
\hline MW14S & $108-118$ & $\begin{array}{l}\text { BAMW14S-W-22569 } \\
\text { BAMW14S-W-22613 } \\
\text { BAMW14S-W-22640 }\end{array}$ & $\begin{array}{r}4 / 4 / 07 \\
11 / 18 / 07 \\
3 / 8 / 08\end{array}$ & $\begin{array}{l}0.9 \mathrm{~J} \\
1.2 \\
4.3\end{array}$ & $\begin{array}{l}\text { ND } \\
\text { ND } \\
0.3 \mathrm{~J}\end{array}$ & $\begin{array}{l}\text { ND } \\
\text { ND } \\
\text { ND }\end{array}$ \\
\hline MW14D & $123-133$ & $\begin{array}{l}\text { BAMW14D-W-22568 } \\
\text { BAMW14D-W-22614 } \\
\text { BAMW14D-W-22638 }\end{array}$ & $\begin{array}{r}4 / 4 / 07 \\
11 / 18 / 07 \\
3 / 8 / 08\end{array}$ & $\begin{array}{l}1.2 \\
0.6 \mathrm{~J} \\
0.7 \mathrm{~J}\end{array}$ & $\begin{array}{l}\text { ND } \\
\text { ND } \\
\text { ND }\end{array}$ & $\begin{array}{l}\text { ND } \\
\text { ND } \\
\text { ND }\end{array}$ \\
\hline
\end{tabular}


TABLE 3.2 (Cont.)

\begin{tabular}{|c|c|c|c|c|c|c|}
\hline \multirow[b]{2}{*}{ Well } & \multirow[b]{2}{*}{$\begin{array}{l}\text { Screen Interval } \\
\quad(\mathrm{ft} B G L)\end{array}$} & \multirow[b]{2}{*}{ Sample } & \multirow[b]{2}{*}{$\begin{array}{l}\text { Sample } \\
\text { Date }\end{array}$} & \multicolumn{3}{|c|}{ Concentration $(\mu \mathrm{g} / \mathrm{L})$} \\
\hline & & & & $\begin{array}{c}\text { Carbon } \\
\text { Tetrachloride }\end{array}$ & Chloroform & $\begin{array}{l}\text { Methylene } \\
\text { Chloride }\end{array}$ \\
\hline \multicolumn{7}{|c|}{ CCC/USDA wells installed during the 2006-2007 investigation (cont.) } \\
\hline MW15S & $88-98$ & $\begin{array}{l}\text { BAMW15S-W-22560 } \\
\text { BAMW15S-W-22615 } \\
\text { BAMW15S-W-22648 }\end{array}$ & $\begin{array}{r}4 / 4 / 07 \\
11 / 18 / 07 \\
3 / 10 / 08\end{array}$ & $\begin{array}{l}1.5 \\
8.7 \\
1.8\end{array}$ & $\begin{array}{l}\text { ND } \\
0.4 \mathrm{~J} \\
0.2 \mathrm{~J}\end{array}$ & $\begin{array}{l}\text { ND } \\
\text { ND } \\
\text { ND }\end{array}$ \\
\hline MW15D & $105-115$ & $\begin{array}{l}\text { BAMW15D-W-22561 } \\
\text { BAMW15D-W-22616 } \\
\text { BAMW15D-W-22631 }\end{array}$ & $\begin{array}{r}4 / 4 / 07 \\
11 / 18 / 07 \\
3 / 8 / 08\end{array}$ & $\begin{array}{l}\text { ND } \\
\text { ND } \\
0.2 \mathrm{~J}\end{array}$ & $\begin{array}{l}\text { ND } \\
\text { ND } \\
\text { ND }\end{array}$ & $\begin{array}{l}\text { ND } \\
\text { ND } \\
\text { ND }\end{array}$ \\
\hline MW16S & $76-86$ & $\begin{array}{l}\text { BAMW16S-W-22563 } \\
\text { BAMW16S-W-22617 } \\
\text { BAMW16S-W-22632 }\end{array}$ & $\begin{array}{r}4 / 4 / 07 \\
11 / 19 / 07 \\
3 / 7 / 08\end{array}$ & $\begin{array}{l}\text { ND } \\
\text { ND } \\
0.4 \mathrm{~J}\end{array}$ & $\begin{array}{l}\text { ND } \\
\text { ND } \\
\text { ND }\end{array}$ & $\begin{array}{l}\text { ND } \\
\text { ND } \\
\text { ND }\end{array}$ \\
\hline MW16D & $90-100$ & $\begin{array}{l}\text { BAMW16D-W-22562 } \\
\text { BAMW16D-W-22618 } \\
\text { BAMW16D-W-22633 }\end{array}$ & $\begin{array}{r}4 / 4 / 07 \\
11 / 19 / 07 \\
3 / 7 / 08\end{array}$ & $\begin{array}{l}\text { ND } \\
\text { ND } \\
\text { ND }\end{array}$ & $\begin{array}{l}\text { ND } \\
\text { ND } \\
\text { ND }\end{array}$ & $\begin{array}{l}\text { ND } \\
\text { ND } \\
\text { ND }\end{array}$ \\
\hline MW17 & $120-130$ & $\begin{array}{l}\text { BAMW17D-W-22566 } \\
\text { BAMW17D-W-22619 } \\
\text { BAMW17-W-22634 }\end{array}$ & $\begin{array}{r}4 / 4 / 07 \\
11 / 19 / 07 \\
3 / 5 / 08\end{array}$ & $\begin{array}{l}\text { ND } \\
\text { ND } \\
0.3 \mathrm{~J}\end{array}$ & $\begin{array}{l}\text { ND } \\
\text { ND } \\
\text { ND }\end{array}$ & $\begin{array}{l}\text { ND } \\
\text { ND } \\
\text { ND }\end{array}$ \\
\hline \multicolumn{7}{|c|}{ Private wells } \\
\hline Oentrich & 150 & $\begin{array}{l}\text { BAOENT-W-21693 } \\
\text { BAOENT-W-21713 } \\
\text { BAOENTRICH-W-22579 } \\
\text { BAOENTRICH-W-22622 } \\
\text { BAOENTRICH-W-22654 }\end{array}$ & $\begin{array}{r}7 / 20 / 06 \\
8 / 2 / 06 \\
4 / 5 / 07 \\
11 / 19 / 07 \\
3 / 6 / 08\end{array}$ & $\begin{array}{l}0.3 \mathrm{~J} \\
0.6 \mathrm{~J} \\
0.6 \mathrm{~J} \\
0.8 \mathrm{~J} \\
1.3\end{array}$ & $\begin{array}{l}\text { ND } \\
\text { ND } \\
\text { ND } \\
\text { ND } \\
\text { ND }\end{array}$ & $\begin{array}{l}\text { ND } \\
\text { ND } \\
\text { ND } \\
\text { ND } \\
\text { ND }\end{array}$ \\
\hline Sedivy & 138 & $\begin{array}{l}\text { BACW-W-21849 } \\
\text { BASED2-W-21913 }\end{array}$ & $\begin{array}{l}8 / 22 / 06 \\
9 / 13 / 06\end{array}$ & $\begin{array}{l}\text { ND } \\
\text { ND }\end{array}$ & $\begin{array}{l}\text { ND } \\
\text { ND }\end{array}$ & $\begin{array}{l}\text { ND } \\
\text { ND }\end{array}$ \\
\hline Sedivy1 & 90 & Not sampled (well dry) & 9/13/06 & - & - & - \\
\hline \multicolumn{7}{|c|}{ Public water supply wells } \\
\hline PW2 & 155 & $\begin{array}{l}\text { BAPWS2-W-22510 } \\
\text { BAPW2-W-22578 } \\
\text { BAPW2-W-22620 } \\
\text { BAPWS2-W-22655 }\end{array}$ & $\begin{array}{r}3 / 9 / 07 \\
4 / 5 / 07 \\
11 / 20 / 07 \\
3 / 6 / 08\end{array}$ & $\begin{array}{l}\text { ND } \\
\text { ND } \\
\text { ND } \\
\text { ND }\end{array}$ & $\begin{array}{l}\text { ND } \\
\text { ND } \\
\text { ND } \\
\text { ND }\end{array}$ & $\begin{array}{l}\text { ND } \\
\text { ND } \\
\text { ND } \\
\text { ND }\end{array}$ \\
\hline PWS3 & 160 & $\begin{array}{l}\text { BAPWS3-W-22511 } \\
\text { BAPW3-W-22577 } \\
\text { BAPW3-W-22621 } \\
\text { BAPWS3-W-22656 }\end{array}$ & $\begin{array}{r}3 / 9 / 07 \\
4 / 5 / 07 \\
11 / 20 / 07 \\
3 / 6 / 08\end{array}$ & $\begin{array}{l}0.2 \mathrm{~J} \\
\mathrm{ND} \\
\mathrm{ND} \\
\mathrm{ND}\end{array}$ & $\begin{array}{l}\text { ND } \\
\text { ND } \\
\text { ND } \\
\text { ND }\end{array}$ & $\begin{array}{l}\text { ND } \\
\text { ND } \\
\text { ND } \\
\text { ND }\end{array}$ \\
\hline
\end{tabular}

a ND, contaminant not detected at an instrument detection limit of $0.1 \mu \mathrm{g} / \mathrm{L}$.

b Qualifier $\mathrm{J}$ indicates an estimated concentration below the purge-and-trap method quantitation limit of $1.0 \mu \mathrm{g} / \mathrm{L}$. 

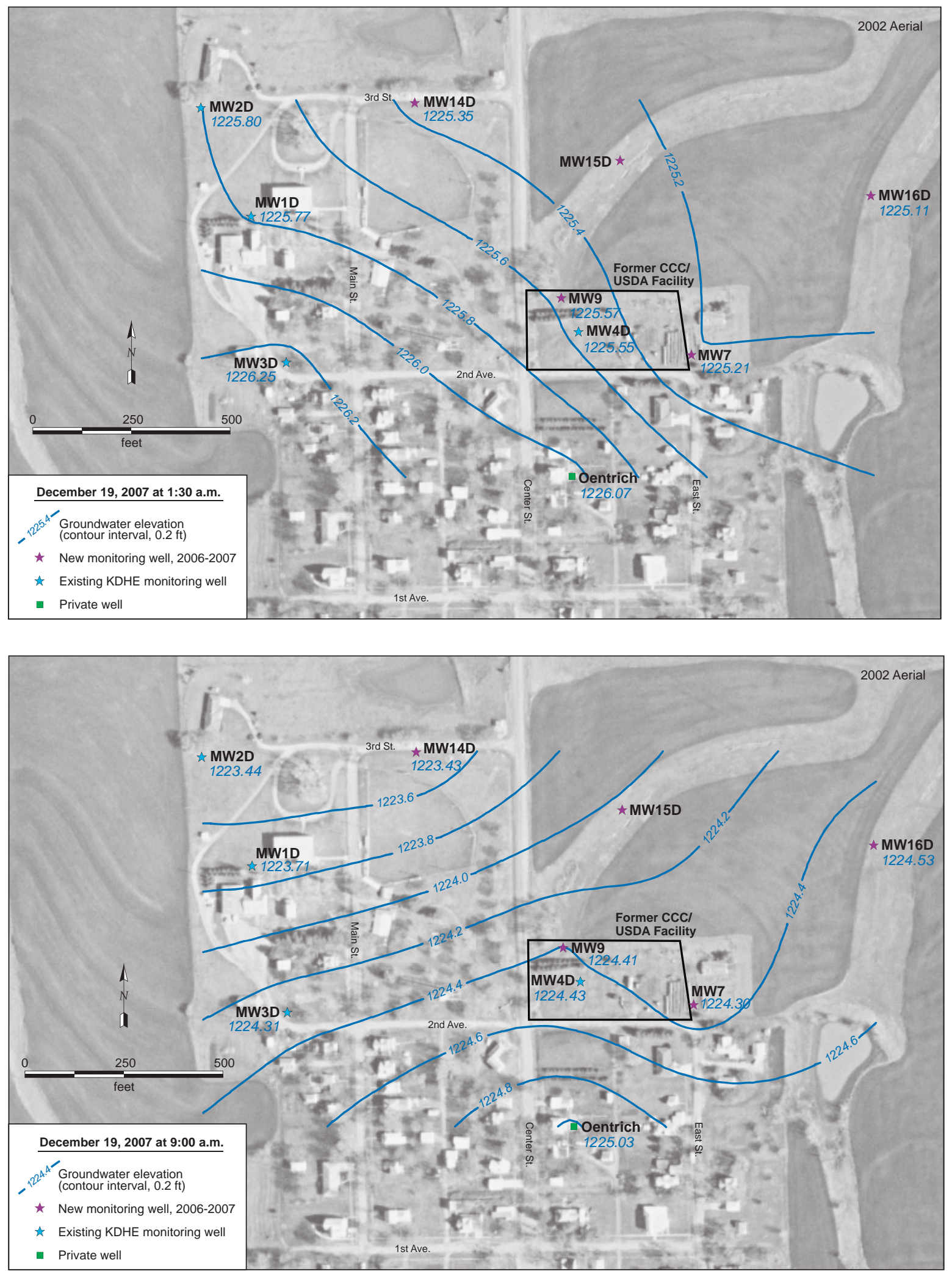

FIGURE 3.1 Potentiometric surface map depicting the groundwater flow direction at Barnes on December 19, 2007, before pumping of the public water supply wells (top) and during pumping (bottom). Source of photograph: NAPP (2002). 
Barnes Monitoring Data
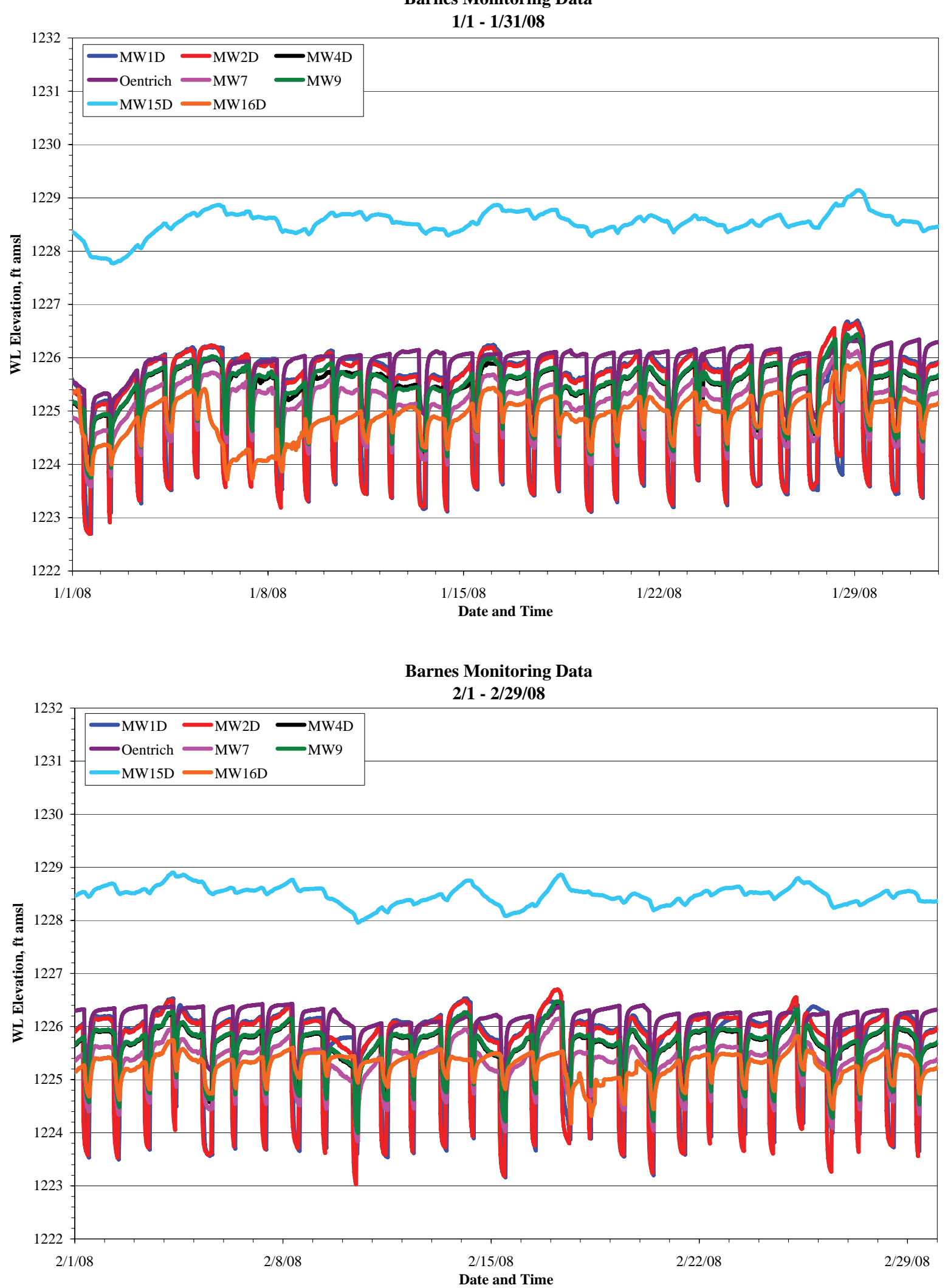

FIGURE 3.2 Hydrographs summarizing monthly results of long-term water level monitoring in wells at Barnes, January to April 2008. 

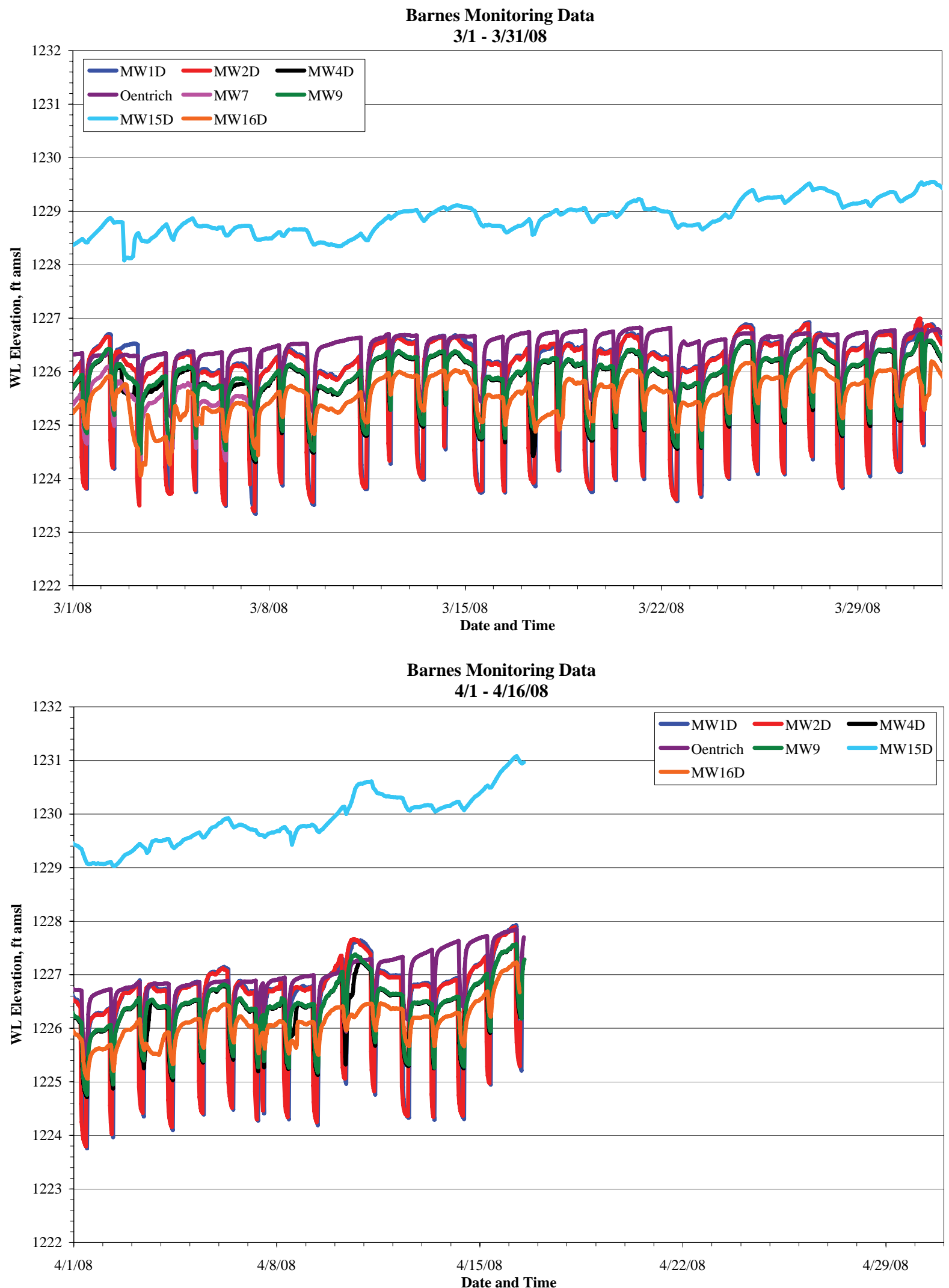

FIGURE 3.2 (Cont.) 


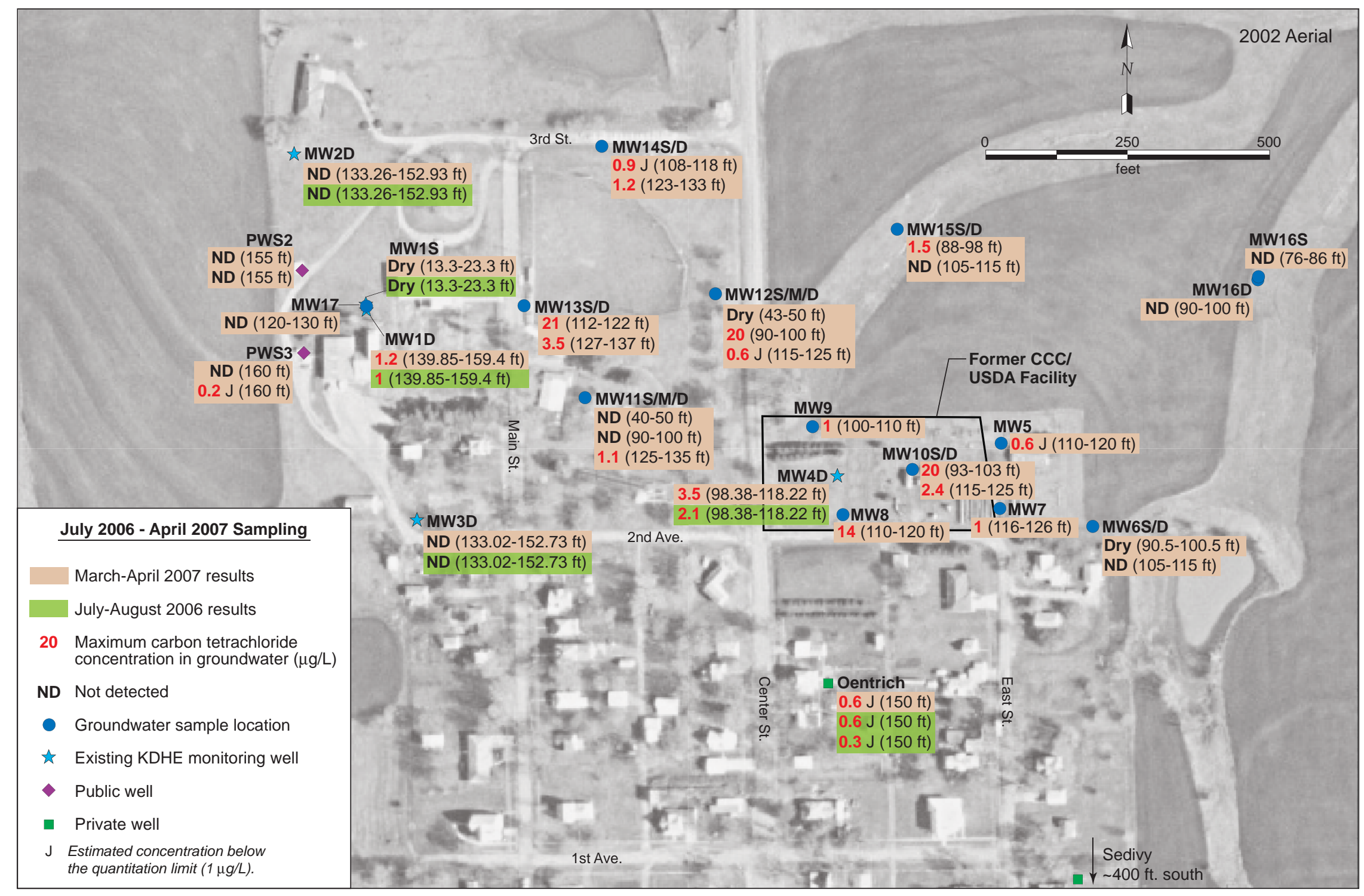

FIGURE 3.3 Analytical results for carbon tetrachloride in groundwater samples collected at Barnes during the 2007 targeted investigation. Source of photograph: NAPP (2002). 


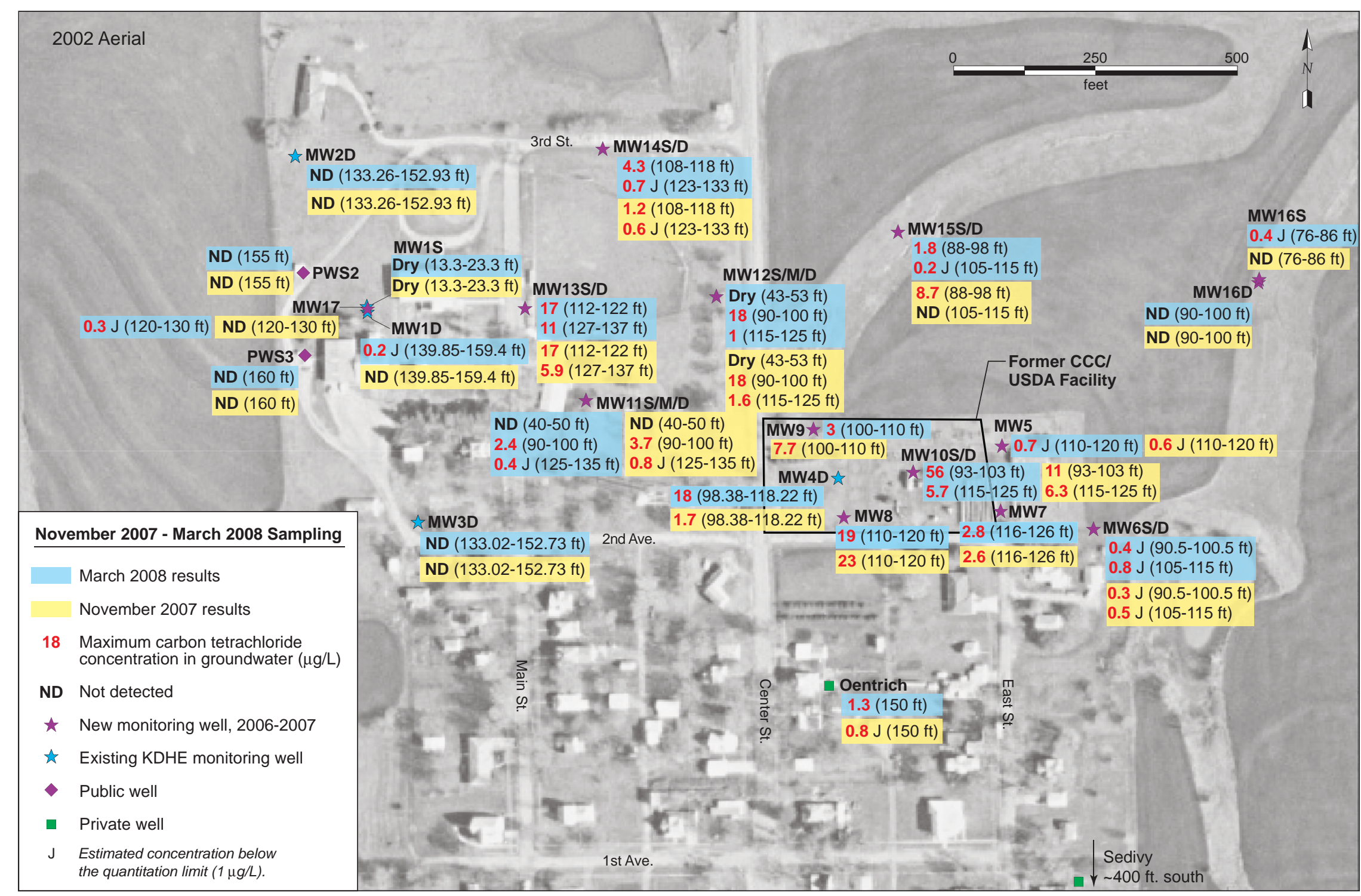

FIGURE 3.4 Analytical results for carbon tetrachloride in groundwater samples collected at Barnes in November 2007 and March 2008 . Source of photograph: NAPP (2002). 


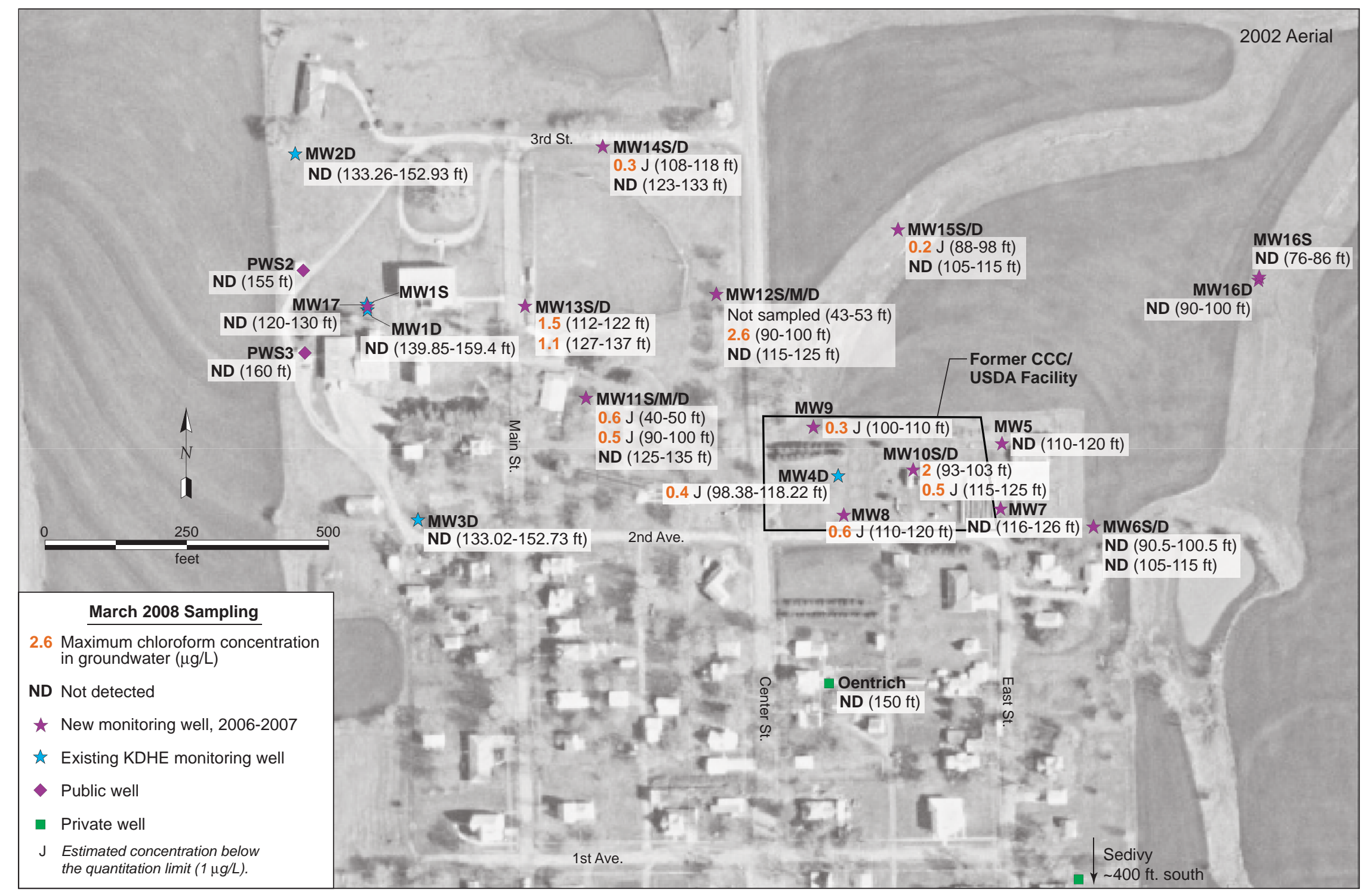

FIGURE 3.5 Analytical results for chloroform in groundwater samples collected at Barnes in March 2008. Source of photograph: NAPP (2002). 


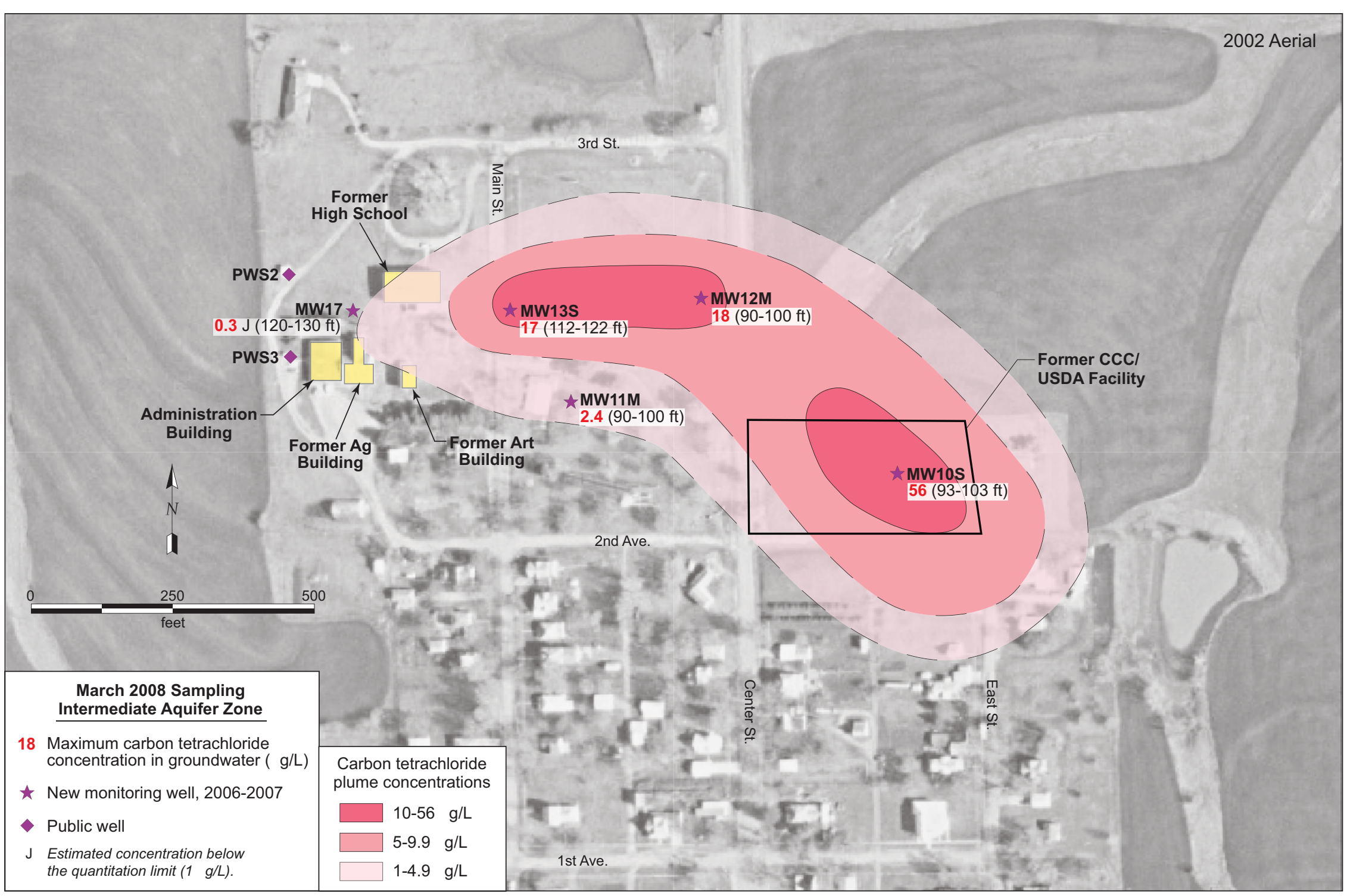

FIGURE 3.6 Interpreted carbon tetrachloride plume in wells screened in the intermediate aquifer zone (approximately 1250-1254 ft AMSL). Source of photograph: NAPP (2002). 


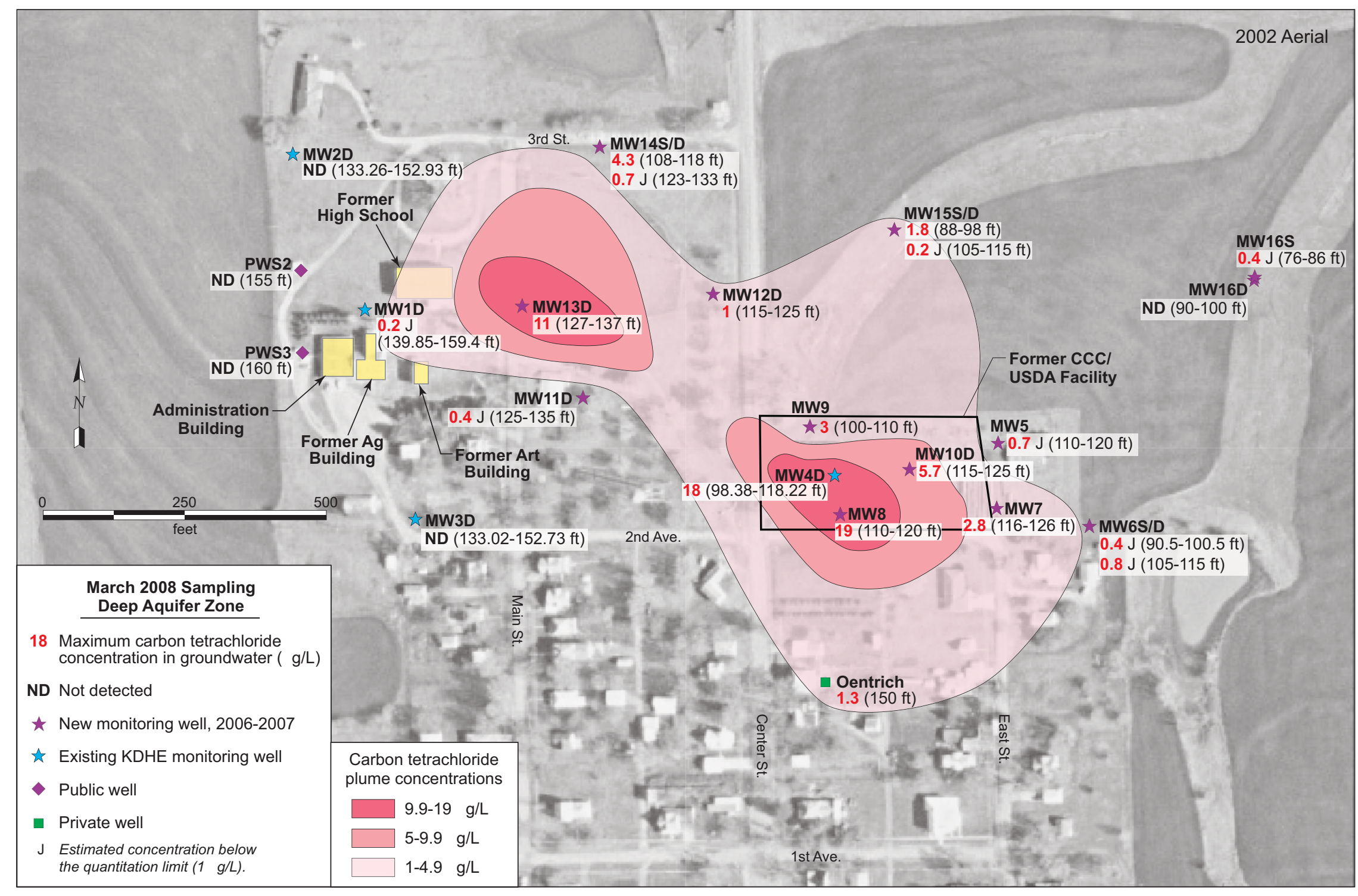

FIGURE 3.7 Interpreted carbon tetrachloride plume in wells screened in the deep aquifer zone (approximately 1223-1229 ft AMSL). Source of photograph: NAPP (2002). 


\section{Conclusions and Ongoing Tasks}

\subsection{Conclusions}

The findings of the March 2008 monitoring event at Barnes support the following conclusions:

- Measurements of groundwater levels obtained manually and through the use of automatic recorders have consistently indicated that the flow direction is strongly influenced by pumping of the public water supply wells. The results continue to indicate

- An apparent groundwater flow direction to the northeast when the public wells are not pumping and

- A northwest trend with an increased gradient when the public wells are pumping.

- Overall, the lateral distribution of carbon tetrachloride in groundwater in March 2008 was similar to the distribution in March-April and November 2007.

- An analysis of manual water level measurements and carbon tetrachloride concentrations suggested that three vertically distinguishable aquifer zones are present at Barnes: a shallow zone with a potentiometric surface at approximately $1309 \mathrm{ft}$ AMSL, an intermediate zone at approximately 1250-1254 ft AMSL, and a deep zone at approximately 1223-1229 ft AMSL. All monitoring wells equipped with data loggers for water level measurements are screened in the deep aquifer zone.

- Compared to the November 2007 monitoring event, the March 2008 data indicate notable increases in carbon tetrachloride concentrations in four monitoring wells. Two of the wells (MW4D and MW10S) are on the former CCC/USDA property, one (MW13D) is adjacent to the high school, and one 
(MW14S) is a downgradient sentinel well. Well MW10S is screened in the intermediate aquifer zone, while wells MW4D, MW13D, and MW14S are screened in the deep aquifer zone.

- The most significant decrease in concentration noted in the March 2008 sampling event occurred at MW15S (north of the former CCC/USDA property), where the carbon tetrachloride concentration dropped below the RBSL value. This well is screened in the deep aquifer zone.

- The vertical distribution of the carbon tetrachloride in groundwater indicates that the highest concentrations occurred in the intermediate aquifer zone. Lower concentrations were detected in the deep aquifer zone, and no carbon tetrachloride was detected in the shallow zone.

\subsection{Ongoing Tasks}

Ongoing tasks related to Barnes are as follows:

- Quarterly monitoring will continue, as recommended in the targeted investigation report (Argonne 2007).

- In an effort to evaluate the effect of pumping of the public water supply wells on the contaminant plume, an attempt will be made to collect the next set of groundwater samples on a schedule coinciding with the well pumping schedule.

- The conceptual contingent interim measure work plan requested by the KDHE (2008b) for protection of the public water supply wells is currently being developed. Cost estimates have been obtained for treatment at the wellhead though use of carbon filtration or air stripping. The CCC/USDA and Argonne have also contacted Washington County Rural Water District \#2 to determine the feasibility of connecting the city of Barnes to that water supply. 


\section{References}

Argonne, 2002, Final Master Work Plan: Environmental Investigations at Former CCC/USDA Facilities in Kansas, 2002 Revision, ANL/ER/TR-02/004, prepared for the Commodity Credit Corporation, U.S. Department of Agriculture, Washington, D.C., by Argonne National Laboratory, Argonne, Illinois, December.

Argonne, 2007, Draft Report: Results of the 2007-2007 Investigation of Potential Contamination at the Former CCC/USDA facility in Barnes, Kansas, ANL/EVS/AGEM/TR-07-12, prepared for the Commodity Credit Corporation, U.S. Department of Agriculture, Washington, D.C., by Argonne National Laboratory, Argonne, Illinois, October.

Argonne, 2008, November 2007 Monitoring Results for Barnes, Kansas, ANL/EVS/AGEM/TR08-04, prepared for the Commodity Credit Corporation, U.S. Department of Agriculture, Washington, D.C., by Argonne National Laboratory, Argonne, Illinois, February.

EPA, 1995, Method 524.2: Measurement of Purgeable Organic Compounds in Water by Capillary Column Gas Chromatography/Mass Spectrometry, Revision 4.1, edited by J.W. Munch, National Exposure Research Laboratory, Office of Research and Development, U.S. Environmental Protection Agency, Cincinnati, Ohio.

KDHE, 2008a, electronic mail message from E. McWilliams (Bureau of Environmental Remediation, Kansas Department of Health and Environment, Topeka, Kansas) to L. Larsen (Larsen and Associates, Inc., Lawrence, Kansas), April 15.

KDHE, 2008b, letter from C. Carey (Bureau of Environmental Remediation, Kansas Department of Health and Environment, Topeka, Kansas) to C. Roe (Commodity Credit Corporation, U.S. Department of Agriculture, Washington, D.C.), regarding the former CCC/USDA facility at Barnes, March 13.

NAPP, 2002, aerial image NAPP 12897 222, National Aerial Photography Program, U.S. Geological Survey (available from Kansas Geospatial Community Commons [http://www.kansasgis.org/]), April 9. 
Puls, R.W., and Barcelona, M.J., 1996, "Low-Flow (Minimal Drawdown) Ground-Water Sampling Procedures," EPA/540/S-95/504, in Ground Water Issue, Superfund Technology Support Center for Ground Water, National Risk Management Research Laboratory, U.S. Environmental Protection Agency, Ada, Oklahoma, April (www.epa.gov/tio/tsp/download/ lwflw2a.pdf). 


\section{Appendix A:}

\section{Sampling Activities and Field Measurements} at Barnes in March 2008 
TABLE A.1 Sequence of sampling activities at Barnes in March 2008.

\begin{tabular}{|c|c|c|c|c|c|c|c|c|c|}
\hline $\begin{array}{l}\text { Sample } \\
\text { Date }\end{array}$ & Time & Location & Sample & Medium & Type & $\begin{array}{l}\text { Depth }^{\mathrm{a}} \\
\text { (ft TOC) }\end{array}$ & $\begin{array}{l}\text { Chain of } \\
\text { Custody }\end{array}$ & $\begin{array}{l}\text { Shipping } \\
\text { Date }\end{array}$ & Sample Description \\
\hline $3 / 4 / 08$ & $N R^{b}$ & MW1S & - & - & - & - & - & - & Well dry. \\
\hline $3 / 4 / 08$ & $19: 16$ & MW1D & BAMW1D-W-22627 & & MW & $139.85-159.4$ & 6068 & $3 / 6 / 08$ & $\begin{array}{l}\text { Depth to water }=127.66 \mathrm{ft} \text { TOC. Depth of } 2 \text {-in. well }= \\
159.40 \mathrm{ft} \mathrm{TOC} \text {. Sample collected by using low-flow } \\
\text { bladder pump after purging of } 5.5 \mathrm{~L} \text {. }\end{array}$ \\
\hline $3 / 5 / 08$ & $10: 26$ & MW17 & BAMW17-W-22634 & Water & MW & $120-130$ & 6069 & $3 / 6 / 08$ & $\begin{array}{l}\text { Depth to water }=101.75 \mathrm{ft} \text { TOC. Depth of } 2 \text {-in. well }= \\
131.15 \mathrm{ft} \text { TOC. Sample collected by using low-flow } \\
\text { bladder pump after purging of } 3.5 \mathrm{~L} \text {. }\end{array}$ \\
\hline $3 / 5 / 08$ & $15: 32$ & MW11S & BAMW11S-W-22630 & Water & MW & $40-50$ & 6069 & $3 / 6 / 08$ & $\begin{array}{l}\text { Depth to water }=27.70 \mathrm{ft} \text { TOC. Depth of } 1 \text {-in. well }= \\
49.65 \mathrm{ft} \mathrm{TOC.} \mathrm{Sample} \mathrm{collected} \mathrm{by} \mathrm{using} \mathrm{low-flow} \\
\text { bladder pump after purging of } 2 \mathrm{~L} \text {. }\end{array}$ \\
\hline $3 / 5 / 08$ & $19: 53$ & MW11D & BAMW11D-W-22639 & Water & MW & $125-135$ & 6069 & $3 / 6 / 08$ & $\begin{array}{l}\text { Depth to water }=110.50 \mathrm{ft} \text { TOC. Depth of } 2 \text {-in. well }= \\
135.00 \mathrm{ft} \mathrm{TOC} \text {. Sample collected by using low-flow } \\
\text { bladder pump after purging of } 3.5 \mathrm{~L} \text {. }\end{array}$ \\
\hline $3 / 6 / 08$ & $10: 46$ & PWS3 & BAPWS3-W-22656 & Water & MW & 160 & 6070 & $3 / 6 / 08$ & $\begin{array}{l}\text { Sample collected from tap in well house. Allowed water } \\
\text { to run from tap for } 2-3 \mathrm{~min} \text {, then sampled. }\end{array}$ \\
\hline $3 / 6 / 08$ & $10: 53$ & PWS2 & BAPWS2-W-22655 & Water & MW & 155 & 6070 & $3 / 6 / 08$ & $\begin{array}{l}\text { Sample collected from tap in well house. Allowed water } \\
\text { to run from tap for } 2-3 \mathrm{~min} \text {, then sampled. }\end{array}$ \\
\hline $3 / 6 / 08$ & $12: 32$ & MW11M & BAMW11M-W-22644 & Water & MW & $90-100$ & 6070 & $3 / 6 / 08$ & $\begin{array}{l}\text { Depth to water }=82.65 \mathrm{ft} \mathrm{TOC} \text {. Depth of } 2 \text {-in. well }= \\
100.00 \mathrm{ft} \mathrm{TOC} \text {. Sample collected by using low-flow } \\
\text { bladder pump after purging of } 5 \mathrm{~L} \text {. }\end{array}$ \\
\hline $3 / 6 / 08$ & $14: 43$ & Oentrich & BAOENTRICH-W-22654 & Water & MW & 150 & 6070 & $3 / 6 / 08$ & $\begin{array}{l}\text { Oentrich private well. Sampled at tap outside house. } \\
\text { Allowed water to run for } 5 \text { min, then sampled. }\end{array}$ \\
\hline $3 / 6 / 08$ & $15: 15$ & QC & BAQCTB-W-22657 & Water & TB & - & 6070 & $3 / 6 / 08$ & $\begin{array}{l}\text { Trip blank sent to the AGEM Laboratory for organic } \\
\text { analyses with water samples listed on chain-of- } \\
\text { custody forms (COCs) 6068, 6069, and } 6070 \text {. }\end{array}$ \\
\hline $3 / 7 / 08$ & $9: 08$ & MW2D & BAMW2D-W-22628 & Water & MW & $133.26-152.93$ & 6072 & $3 / 7 / 08$ & $\begin{array}{l}\text { Depth to water }=125.55 \mathrm{ft} \text {. Depth of } 2 \text {-in. well }=152.93 \\
\mathrm{ft} \text {. Sample collected by using low-flow bladder pump } \\
\text { after purging of } 4.52 \mathrm{~L} \text {. }\end{array}$ \\
\hline $3 / 7 / 08$ & $11: 17$ & MW3D & BAMW3D-W-22629 & Water & MW & $133.02-152.73$ & 6072 & $3 / 7 / 08$ & $\begin{array}{l}\text { Depth to water }=121.90 \mathrm{ft} \mathrm{TOC} \text {. Depth of } 2 \text {-in. well = } \\
152.73 \mathrm{ft} \mathrm{TOC} \text {. Sample collected by using low-flow } \\
\text { bladder pump after purging of } 5.46 \mathrm{~L} \text {. }\end{array}$ \\
\hline $3 / 7 / 08$ & 13:11 & MW16D & BAMW16D-W-22633 & Water & MW & $90-100$ & 6072 & $3 / 7 / 08$ & $\begin{array}{l}\text { Depth to water }=75.00 \mathrm{ft} \text { TOC. Depth of } 2 \text {-in. well }= \\
100.00 \mathrm{ft} \mathrm{TOC} \text {. Sample collected by using low-flow } \\
\text { bladder pump after purging of } 2.78 \mathrm{~L} \text {. }\end{array}$ \\
\hline $3 / 7 / 08$ & $14: 16$ & MW16S & BAMW16S-W-22632 & Water & MW & $76-86$ & 6072 & $3 / 7 / 08$ & $\begin{array}{l}\text { Depth to water }=75.50 \mathrm{ft} \text { TOC. Depth of } 2 \text {-in. well }= \\
86.00 \mathrm{ft} \mathrm{TOC.} \mathrm{Sample} \mathrm{collected} \mathrm{by} \mathrm{using} \mathrm{low-flow} \\
\text { bladder pump after purging of } 2.21 \mathrm{~L} \text {. }\end{array}$ \\
\hline $3 / 7 / 08$ & $15: 10$ & QC & BAQCTB-W-22658 & Water & TB & - & 6072 & $3 / 7 / 08$ & $\begin{array}{l}\text { Trip blank sent to the AGEM Laboratory for organic } \\
\text { analyses with water samples listed on COC } 6072 \text {. }\end{array}$ \\
\hline $3 / 8 / 08$ & $10: 21$ & MW6D & BAMW6D-W-22636 & Water & MW & $105-115$ & 6073 & $3 / 10 / 08$ & $\begin{array}{l}\text { Depth to water }=98.50 \mathrm{ft} \text { TOC. } \text {. Depth of } 2 \text {-in. well }= \\
115.00 \mathrm{ft} \mathrm{TOC} \text {. Sample collected by using low-flow } \\
\text { bladder pump after purging of } 2 \mathrm{~L} \text {. }\end{array}$ \\
\hline
\end{tabular}


TABLE A.1 (Cont.)

\begin{tabular}{|c|c|c|c|c|c|c|c|c|c|}
\hline $\begin{array}{l}\text { Sample } \\
\text { Date }\end{array}$ & Time & Location & Sample & Medium & Type & $\begin{array}{l}\text { Deptha } \\
\text { (ft TOC) }\end{array}$ & $\begin{array}{l}\text { Chain of } \\
\text { Custody }\end{array}$ & $\begin{array}{l}\text { Shipping } \\
\text { Date }\end{array}$ & Sample Description \\
\hline $3 / 8 / 08$ & $12: 14$ & MW5 & BAMW5-W-22637 & Water & MW & $110-120$ & 6073 & $3 / 10 / 08$ & $\begin{array}{l}\text { Depth to water }=102.00 \mathrm{ft} \text { TOC. Depth of } 2 \text {-in. well }= \\
120.00 \mathrm{ft} \mathrm{TOC} \text {. Sample collected by using low-flow } \\
\text { bladder pump after purging of } 2.3 \mathrm{~L} \text {. }\end{array}$ \\
\hline $3 / 8 / 08$ & $14: 01$ & MW14D & BAMW14D-W-22638 & Water & MW & $123-133$ & 6073 & $3 / 10 / 08$ & $\begin{array}{l}\text { Depth to water }=106.95 \mathrm{ft} \text { TOC. } \text {. Depth of } 2 \text {-in. well }= \\
133.00 \mathrm{ft} \mathrm{TOC.} \mathrm{Sample} \mathrm{collected} \mathrm{by} \mathrm{using} \mathrm{low-flow} \\
\text { bladder pump after purging of } 3.5 \mathrm{~L} \text {. }\end{array}$ \\
\hline $3 / 8 / 08$ & $15: 35$ & MW14S & BAMW14S-W-22640 & Water & MW & $108-118$ & 6073 & $3 / 10 / 08$ & $\begin{array}{l}\text { Depth to water }=106.95 \mathrm{ft} \text { TOC. } \text {. Depth of } 2 \text {-in. well }= \\
118.00 \mathrm{ft} \mathrm{TOC} \text {. Sample collected by using low-flow } \\
\text { bladder pump after purging of } 2.62 \mathrm{~L} \text {. }\end{array}$ \\
\hline $3 / 8 / 08$ & $17: 26$ & MW15D & BAMW15D-W-22631 & Water & MW & $105-115$ & 6073 & $3 / 10 / 08$ & $\begin{array}{l}\text { Depth to water }=80.80 \mathrm{ft} \mathrm{TOC} \text {. Depth of } 2 \text {-in. well }= \\
115.00 \mathrm{ft} \mathrm{TOC} \text {. Sample collected by using low-flow } \\
\text { bladder pump after purging of } 2.41 \mathrm{~L} \text {. }\end{array}$ \\
\hline $3 / 8 / 08$ & $17: 55$ & MW6S & BAMW6S-W-22635 & Water & MW & $90.5-100.5$ & 6073 & $3 / 10 / 08$ & $\begin{array}{l}\text { Depth to water }=94.50 \mathrm{ft} \mathrm{TOC} \text {. Depth of } 2 \text {-in. well = } \\
100.50 \mathrm{ft} \mathrm{TOC} \text {. Well went dry with use of low-flow } \\
\text { bladder pump. Continued purging with bailer to } 1.25 \\
\text { gal. Sampled later in the day by using bailer. }\end{array}$ \\
\hline $3 / 9 / 08$ & $10: 05$ & MW12D & BAMW12D-W-22641 & Water & MW & $115-125$ & 6074 & $3 / 10 / 08$ & $\begin{array}{l}\text { Depth to water }=103.30 \mathrm{ft} \text { TOC. Depth of } 2 \text {-in. well }= \\
125.00 \mathrm{ft} \mathrm{TOC} \text {. Sample collected by using low-flow } \\
\text { bladder pump after purging of } 3 \mathrm{~L} \text {. }\end{array}$ \\
\hline $3 / 9 / 08$ & $11: 59$ & MW4D & BAMW4D-W-22642 & Water & MW & $98.38-118.22$ & 6074 & $3 / 10 / 08$ & $\begin{array}{l}\text { Depth to water }=101.74 \mathrm{ft} \mathrm{TOC} \text {. Depth of } 2 \text {-in. well }= \\
118.22 \mathrm{ft} \mathrm{TOC} \text {. Sample collected by using low-flow } \\
\text { bladder pump after purging of } 3 \mathrm{~L} \text {. }\end{array}$ \\
\hline $3 / 9 / 08$ & $13: 33$ & MW7 & BAMW7-W-22643 & Water & MW & $116-126$ & 6074 & $3 / 10 / 08$ & $\begin{array}{l}\text { Depth to water }=105.62 \mathrm{ft} \mathrm{TOC} \text {. Depth of } 2 \text {-in. well }= \\
126.00 \mathrm{ft} \mathrm{TOC} \text {. Sample collected by using low-flow } \\
\text { bladder pump after purging of } 3.2 \mathrm{~L} \text {. }\end{array}$ \\
\hline $3 / 9 / 08$ & $14: 56$ & MW13D & BAMW13D-W-22645 & Water & MW & $127-137$ & 6074 & $3 / 10 / 08$ & $\begin{array}{l}\text { Depth to water }=118.19 \mathrm{ft} \text { TOC. Depth of } 2 \text {-in. well }= \\
137.00 \mathrm{ft} \mathrm{TOC} \text {. Sample collected by using low-flow } \\
\text { bladder pump after purging of } 3.7 \mathrm{~L} \text {. }\end{array}$ \\
\hline $3 / 9 / 08$ & $14: 58$ & MW13D & BAMW13DDUP-W-22667 & Water & MW & 127-137 & 6074 & $3 / 10 / 08$ & Replicate of sample BAMW13D-W-22645. \\
\hline $3 / 9 / 08$ & $16: 19$ & MW10D & BAMW10D-W-22646 & Water & MW & $115-125$ & 6074 & $3 / 10 / 08$ & $\begin{array}{l}\text { Depth to water }=106.36 \mathrm{ft} \mathrm{TOC} \text {. Depth of } 2 \text {-in. well }= \\
125.00 \mathrm{ft} \text { TOC. Sample collected by using low-flow } \\
\text { bladder pump after purging of } 3 \mathrm{~L} \text {. }\end{array}$ \\
\hline $3 / 9 / 08$ & 18:01 & MW9 & BAMW9-W-22647 & Water & MW & $100-110$ & 6074 & $3 / 10 / 08$ & $\begin{array}{l}\text { Depth to water }=96.40 \mathrm{ft} \text { TOC. } \text {. Depth of } 2 \text {-in. well }= \\
110.00 \mathrm{ft} \text {. Sample collected by using low-flow bladder } \\
\text { pump after purging of } 3.94 \mathrm{~L} \text {. }\end{array}$ \\
\hline 3/10/08 & $10: 30$ & MW15S & BAMW15S-W-22648 & Water & MW & $88-98$ & 6075 & $3 / 10 / 08$ & $\begin{array}{l}\text { Depth to water }=84.66 \mathrm{ft} \mathrm{TOC} \text {. Depth of } 2 \text {-in. well }= \\
98.00 \mathrm{ft} \mathrm{TOC.} \mathrm{Sample} \mathrm{collected} \mathrm{by} \mathrm{using} \mathrm{low-flow} \\
\text { bladder pump after purging of } 14 \mathrm{~L} \text {. }\end{array}$ \\
\hline $3 / 10 / 08$ & $10: 31$ & MW15S & BAMW15SDUP-W-22668 & Water & MW & $88-98$ & 6075 & $3 / 10 / 08$ & Replicate of sample BAMW15S-W-22648. \\
\hline 3/10/08 & $11: 15$ & QC & BAQCIR-W-22663 & Water & $\mathrm{RI}$ & - & 6075 & $3 / 10 / 08$ & Rinsate of bladder pump after sampling of well MW15S. \\
\hline $3 / 10 / 08$ & $12: 21$ & MW10S & BAMW10S-W-22649 & Water & MW & $93-103$ & 6075 & $3 / 10 / 08$ & $\begin{array}{l}\text { Depth to water }=77.47 \mathrm{ft} \text { TOC. } \text { Depth of } 2 \text {-in. well }= \\
103.00 \mathrm{ft} \mathrm{TOC} \text {. Sample collected by using low-flow } \\
\text { bladder pump after purging of } 5.6 \mathrm{~L} \text {. }\end{array}$ \\
\hline
\end{tabular}


TABLE A.1 (Cont.)

\begin{tabular}{|c|c|c|c|c|c|c|c|c|c|}
\hline $\begin{array}{l}\text { Sample } \\
\text { Date }\end{array}$ & Time & Location & Sample & Medium & Type & $\begin{array}{l}\text { Depth }^{\mathrm{a}} \\
\text { (ft TOC) }\end{array}$ & $\begin{array}{l}\text { Chain of } \\
\text { Custody }\end{array}$ & $\begin{array}{l}\text { Shipping } \\
\text { Date }\end{array}$ & Sample Description \\
\hline 3/10/08 & $12: 46$ & MW13S & BAMW13S-W-22650 & Water & MW & $112-122$ & 6075 & $3 / 10 / 08$ & $\begin{array}{l}\text { Depth to water }=92.10 \mathrm{ft} \mathrm{TOC} \text {. Depth of } 2 \text {-in. well }= \\
122.00 \mathrm{ft} \mathrm{TOC} \text {. Sample collected by using low-flow } \\
\text { bladder pump after purging of } 2.5 \mathrm{~L} \text {. }\end{array}$ \\
\hline 3/10/08 & NR & MW12S & - & - & - & - & - & - & Well dry. \\
\hline 3/10/08 & $14: 53$ & MW12M & BAMW12M-W-22651 & Water & MW & $90-100$ & 6075 & $3 / 10 / 08$ & $\begin{array}{l}\text { Depth to water }=74.77 \mathrm{ft} \mathrm{TOC} \text {. Depth of } 2 \text {-in. well }= \\
100.00 \mathrm{ft} \mathrm{TOC} \text {. Sample collected by using low-flow } \\
\text { bladder pump after purging of } 2.58 \mathrm{~L} \text {. }\end{array}$ \\
\hline 3/10/08 & $15: 16$ & QC & BAQCIR-W-22664 & Water & RI & - & 6075 & $3 / 10 / 08$ & Rinsate of bladder pump after sampling of well MW12M. \\
\hline 3/10/08 & $15: 55$ & QC & BAQCTB-W-22659 & Water & TB & - & 6075 & $3 / 10 / 08$ & $\begin{array}{l}\text { Trip blank sent to the AGEM Laboratory for organic } \\
\text { analyses with water samples listed on COCs } 6073 \text {, } \\
6074 \text {, and } 6075 \text {. }\end{array}$ \\
\hline 3/10/08 & $16: 15$ & MW8 & BAMW8-W-22652 & Water & MW & $110-120$ & 6075 & $3 / 10 / 08$ & $\begin{array}{l}\text { Depth to water }=104.38 \mathrm{ft} \mathrm{TOC} \text {. Depth of } 2 \text {-in. well }= \\
120.00 \mathrm{ft} \mathrm{TOC} \text {. Sample collected by using low-flow } \\
\text { bladder pump after purging of } 2.2 \mathrm{~L} \text {. }\end{array}$ \\
\hline 3/10/08 & $16: 38$ & QC & BAQCIR-W-22665 & Water & RI & - & 6075 & 3/10/08 & Rinsate of bladder pump after sampling of well MW8. \\
\hline 3/10/08 & $16: 45$ & QC & BAPURGE-W-22666 & Water & BT & - & 6075 & $3 / 10 / 08$ & $\begin{array}{l}\text { Sample of containerized purge water during March } 2008 \\
\text { sampling. }\end{array}$ \\
\hline
\end{tabular}

a Depth is in feet below the top of the well casing.

b NR, not recorded. 
TABLE A.2 Field measurements for groundwater samples collected at Barnes, July 2006 to March 2008.

\begin{tabular}{|c|c|c|c|c|c|c|c|}
\hline Well & $\begin{array}{l}\text { Screen Interval } \\
\quad(\mathrm{ft} B G L)\end{array}$ & $\begin{array}{l}\text { Sample } \\
\text { Date }\end{array}$ & $\begin{array}{c}\text { Temperature } \\
\left({ }^{\circ} \mathrm{C}\right)\end{array}$ & $\mathrm{pH}$ & $\begin{array}{l}\text { Conductivity } \\
(\mu \mathrm{S} / \mathrm{cm})\end{array}$ & $\begin{array}{c}\text { Dissolved } \\
\text { Oxygen } \\
\text { (mg/L) }\end{array}$ & $\begin{array}{c}\text { Oxygen } \\
\text { Reduction } \\
\text { Potential } \\
(\mathrm{mV})\end{array}$ \\
\hline \multicolumn{8}{|c|}{ Previously existing KDHE monitoring wells } \\
\hline \multirow[t]{4}{*}{ MW1S } & $13.3-23.2$ & 7/19/06 & - & - & - & - & - \\
\hline & & $4 / 4 / 07$ & - & - & - & - & - \\
\hline & & $11 / 18 / 07$ & - & - & - & - & - \\
\hline & & $3 / 4 / 08$ & - & - & - & - & - \\
\hline \multirow[t]{4}{*}{ MW1D } & $139.85-159.4$ & 7/19/06 & 22.8 & 7.15 & 945 & - & - \\
\hline & & $4 / 4 / 07$ & 15.7 & 6.30 & 855 & - & - \\
\hline & & $11 / 18 / 07$ & 12.7 & 7.62 & 712 & - & - \\
\hline & & $3 / 4 / 08$ & 5.53 & 7.22 & 1167 & 11.6 & 244.2 \\
\hline \multirow[t]{4}{*}{ MW2D } & $133.26-152.93$ & 7/19/06 & 24.7 & 7.72 & 946 & - & - \\
\hline & & $4 / 4 / 07$ & 15.1 & 6.32 & 887 & - & - \\
\hline & & $11 / 18 / 07$ & 12.1 & 6.96 & 1448 & - & - \\
\hline & & 3/7/08 & 6.5 & 7.22 & 1198 & 4.61 & 196.5 \\
\hline \multirow{4}{*}{ MW3D } & $133.02-152.73$ & 7/19/06 & 23 & 7.06 & 976 & - & - \\
\hline & & $4 / 4 / 07$ & 15.6 & 6.37 & 989 & - & - \\
\hline & & $11 / 19 / 07$ & 10.5 & 7.16 & 1093 & - & - \\
\hline & & 3/7/08 & 8.2 & 7.09 & 1195 & 5.34 & 254.8 \\
\hline \multirow[t]{4}{*}{ MW4D } & $98.38-118.22$ & $7 / 20 / 06$ & 23.5 & 6.26 & 968 & - & - \\
\hline & & $4 / 6 / 07$ & 11.3 & 6.21 & 1018 & - & - \\
\hline & & $11 / 19 / 07$ & 15.7 & 6.98 & 1022 & - & - \\
\hline & & $3 / 9 / 08$ & 11.5 & 7.14 & 859 & 6.57 & 201.2 \\
\hline \multicolumn{8}{|c|}{ CCC/USDA wells installed during the $2006-2007$ investigation } \\
\hline \multirow[t]{3}{*}{ MW5 } & $110-120$ & $4 / 6 / 07$ & 13.9 & 6.17 & 1705 & - & - \\
\hline & & $11 / 19 / 07$ & 15.2 & 6.74 & 3070 & - & - \\
\hline & & $3 / 8 / 08$ & 9.9 & 6.76 & 2770 & 0.66 & 123.2 \\
\hline \multirow[t]{3}{*}{ MW6S } & $90.5-100.5$ & $4 / 4 / 07$ & - & - & - & - & - \\
\hline & & $11 / 19 / 07$ & 12 & 7.60 & 723 & - & - \\
\hline & & $3 / 8 / 08$ & 4.7 & 7.77 & 673 & 6.72 & 272.2 \\
\hline \multirow[t]{3}{*}{ MW6D } & $105-115$ & 4/5/07 & 6.2 & 6.11 & 936 & - & - \\
\hline & & $11 / 19 / 07$ & 13.6 & 7.00 & 1103 & - & - \\
\hline & & $3 / 8 / 08$ & 9.1 & 7.15 & 908 & 5.56 & 241.0 \\
\hline \multirow[t]{3}{*}{ MW7 } & $116-126$ & $4 / 6 / 07$ & 14.1 & 6.30 & 1051 & - & - \\
\hline & & $11 / 19 / 07$ & 14.6 & 7.16 & 890 & - & - \\
\hline & & $3 / 9 / 08$ & 13.1 & 7.10 & 1068 & 4.24 & 186.4 \\
\hline \multirow[t]{3}{*}{ MW8 } & $110-120$ & $4 / 6 / 07$ & 12.1 & 6.23 & 974 & - & - \\
\hline & & $11 / 19 / 07$ & 14.6 & 7.03 & 909 & - & - \\
\hline & & 3/10/08 & 13.1 & 7.09 & 961 & 6.71 & 182.1 \\
\hline \multirow[t]{3}{*}{ MW9 } & $100-110$ & $4 / 5 / 07$ & 12.9 & 6.2 & 976 & - & - \\
\hline & & $11 / 19 / 07$ & 16.5 & 7.21 & 1066 & - & - \\
\hline & & $3 / 9 / 08$ & 11.2 & 7.07 & 928 & 5.80 & 239.0 \\
\hline
\end{tabular}


TABLE A.2 (Cont.)

\begin{tabular}{|c|c|c|c|c|c|c|c|}
\hline Well & $\begin{array}{l}\text { Screen Interval } \\
\quad(\mathrm{ft} B G L)\end{array}$ & $\begin{array}{l}\text { Sample } \\
\text { Date }\end{array}$ & $\begin{array}{l}\text { Temperature } \\
\left({ }^{\circ} \mathrm{C}\right)\end{array}$ & $\mathrm{pH}$ & $\begin{array}{l}\text { Conductivity } \\
(\mu \mathrm{S} / \mathrm{cm})\end{array}$ & $\begin{array}{c}\text { Dissolved } \\
\text { Oxygen } \\
(\mathrm{mg} / \mathrm{L})\end{array}$ & $\begin{array}{l}\text { Oxygen } \\
\text { Reduction } \\
\text { Potential } \\
(\mathrm{mV})\end{array}$ \\
\hline \multicolumn{8}{|c|}{ CCC/USDA wells installed during the 2006-2007 investigation (cont.) } \\
\hline \multirow[t]{3}{*}{ MW10S } & $93-103$ & $4 / 6 / 07$ & 13.2 & 6.36 & 1004 & - & - \\
\hline & & $11 / 19 / 07$ & 14.5 & 7.22 & 942 & - & - \\
\hline & & 3/10/08 & 12.7 & 7.08 & 912 & 5.18 & 176.1 \\
\hline \multirow[t]{3}{*}{ MW10D } & $115-125$ & 4/6/07 & 12.1 & 6.21 & 992 & - & - \\
\hline & & $11 / 19 / 07$ & 14.5 & 7.42 & 1175 & - & - \\
\hline & & $3 / 9 / 08$ & 13.7 & 7.01 & 1024 & 5.07 & 235.9 \\
\hline \multirow[t]{3}{*}{ MW11S } & $40-50$ & $4 / 4 / 07$ & 12.8 & 6.14 & 1027 & - & - \\
\hline & & $11 / 19 / 07$ & 11.2 & 7.15 & 1174 & - & - \\
\hline & & 3/5/08 & 9.4 & 6.81 & 1122 & 2.26 & 240.8 \\
\hline \multirow[t]{3}{*}{ MW11M } & $90-100$ & $4 / 5 / 07$ & 7.5 & 7.60 & 1097 & - & - \\
\hline & & $11 / 19 / 07$ & 11.9 & 7.17 & 1144 & - & - \\
\hline & & 3/6/08 & 10.8 & 7.06 & 997 & 2.65 & 254.1 \\
\hline \multirow[t]{3}{*}{ MW11D } & $125-135$ & $4 / 4 / 07$ & 13.8 & 6.18 & 990 & - & - \\
\hline & & $11 / 19 / 07$ & 13.1 & 7.22 & 987 & - & - \\
\hline & & 3/5/08 & 6.0 & 7.06 & 872 & 6.85 & 252.0 \\
\hline \multirow[t]{3}{*}{ MW12S } & $43-53$ & $4 / 5 / 07$ & - & - & - & - & - \\
\hline & & $11 / 19 / 07$ & - & - & - & - & - \\
\hline & & 3/10/08 & - & - & - & - & - \\
\hline \multirow[t]{3}{*}{ MW12M } & $90-100$ & $4 / 5 / 07$ & 12.6 & 6.42 & 867 & - & - \\
\hline & & $11 / 19 / 07$ & 14.9 & 7.13 & 835 & - & - \\
\hline & & 3/10/08 & 12.6 & 7.13 & 665 & 1.81 & 211.5 \\
\hline \multirow[t]{3}{*}{ MW12D } & $115-125$ & $4 / 5 / 07$ & 14.0 & 6.36 & 930 & - & - \\
\hline & & $11 / 18 / 07$ & 15.6 & 6.95 & 571 & - & - \\
\hline & & $3 / 9 / 08$ & 8.8 & 7.13 & 881 & 5.25 & 237.3 \\
\hline \multirow[t]{3}{*}{ MW13S } & $112-122$ & $4 / 5 / 07$ & 9.8 & 6.42 & 946 & - & - \\
\hline & & $11 / 19 / 07$ & 16.5 & 7.21 & 893 & - & - \\
\hline & & 3/10/08 & 12.2 & 7.13 & 810 & 6.21 & 199.3 \\
\hline \multirow[t]{3}{*}{ MW13D } & $127-137$ & $4 / 5 / 07$ & 14.9 & 6.25 & 397 & - & - \\
\hline & & $11 / 19 / 07$ & 17.0 & 7.00 & 763 & - & - \\
\hline & & $3 / 9 / 08$ & 13.1 & 7.09 & 758 & 5.95 & 212.9 \\
\hline \multirow[t]{3}{*}{ MW14S } & $108-118$ & $4 / 4 / 07$ & 13.4 & 6.50 & 704 & - & - \\
\hline & & $11 / 18 / 07$ & 12.9 & 7.26 & 966 & - & - \\
\hline & & $3 / 8 / 08$ & 13.2 & 7.20 & 729 & 6.59 & 207.8 \\
\hline \multirow[t]{3}{*}{ MW14D } & $123-133$ & $4 / 4 / 07$ & 14.7 & 6.34 & 932 & - & - \\
\hline & & $11 / 18 / 07$ & 13.2 & 7.47 & 739 & - & - \\
\hline & & 3/8/08 & 12.0 & 7.06 & 1424 & 1.95 & 282.4 \\
\hline \multirow[t]{3}{*}{ MW15S } & $88-98$ & $4 / 4 / 07$ & 13.1 & 8.03 & 854 & - & - \\
\hline & & $11 / 18 / 07$ & 13.9 & $\mathrm{NR}^{\mathrm{a}}$ & 1883 & - & - \\
\hline & & 3/10/08 & 12.1 & 8.67 & 697 & 5.49 & 173.2 \\
\hline
\end{tabular}


TABLE A.2 (Cont.)

\begin{tabular}{|c|c|c|c|c|c|c|c|}
\hline Well & $\begin{array}{l}\text { Screen Interval } \\
\quad(\mathrm{ft} B G L)\end{array}$ & $\begin{array}{l}\text { Sample } \\
\text { Date }\end{array}$ & $\begin{array}{c}\text { Temperature } \\
\left({ }^{\circ} \mathrm{C}\right)\end{array}$ & $\mathrm{pH}$ & $\begin{array}{l}\text { Conductivity } \\
(\mu \mathrm{S} / \mathrm{cm})\end{array}$ & $\begin{array}{l}\text { Dissolved } \\
\text { Oxygen } \\
\text { (mg/L) }\end{array}$ & $\begin{array}{l}\text { Oxygen } \\
\text { Reduction } \\
\text { Potential } \\
(\mathrm{mV})\end{array}$ \\
\hline \multicolumn{8}{|c|}{ CCC/USDA wells installed during the 2006-2007 investigation (cont.) } \\
\hline MW15D & $105-115$ & $\begin{array}{r}4 / 4 / 07 \\
11 / 18 / 07 \\
3 / 8 / 08\end{array}$ & $\begin{array}{r}14.8 \\
13.1 \\
9.0\end{array}$ & $\begin{array}{l}6.15 \\
6.85 \\
6.85\end{array}$ & $\begin{array}{l}2980 \\
2190 \\
2912\end{array}$ & $\begin{array}{l}- \\
\overline{0} .57\end{array}$ & $\begin{array}{c}- \\
\overline{-} \\
130.6\end{array}$ \\
\hline MW16S & $76-86$ & $\begin{array}{r}4 / 4 / 07 \\
11 / 19 / 07 \\
3 / 7 / 08\end{array}$ & $\begin{array}{l}12.8 \\
15 \\
7.3\end{array}$ & $\begin{array}{l}6.35 \\
6.94 \\
6.96\end{array}$ & $\begin{array}{l}1708 \\
1616 \\
1968\end{array}$ & $\begin{array}{l}- \\
\overline{3} .45\end{array}$ & $\begin{array}{c}- \\
\overline{-} \\
183.5\end{array}$ \\
\hline MW16D & $90-100$ & $\begin{array}{r}4 / 4 / 07 \\
11 / 19 / 07 \\
3 / 7 / 08\end{array}$ & $\begin{array}{r}14.1 \\
12.5 \\
7.0\end{array}$ & $\begin{array}{l}6.17 \\
6.78 \\
6.86\end{array}$ & $\begin{array}{l}2910 \\
2400 \\
2866\end{array}$ & $\begin{array}{l}- \\
\overline{0} .50\end{array}$ & $\begin{array}{c}- \\
\overline{-} \\
140.0\end{array}$ \\
\hline MW17 & $120-130$ & $\begin{array}{r}4 / 4 / 07 \\
11 / 19 / 07 \\
3 / 5 / 08\end{array}$ & $\begin{array}{l}16 \\
8.3 \\
5.4\end{array}$ & $\begin{array}{l}6.44 \\
7.15 \\
7.12\end{array}$ & $\begin{array}{l}861 \\
610 \\
804\end{array}$ & $\begin{array}{l}- \\
- \\
7.02\end{array}$ & $\begin{array}{c}- \\
- \\
239.4\end{array}$ \\
\hline \multicolumn{8}{|c|}{ Private wells } \\
\hline Oentrich & 150 & $\begin{array}{r}7 / 20 / 06 \\
8 / 2 / 06 \\
4 / 5 / 07 \\
11 / 19 / 07 \\
3 / 6 / 08\end{array}$ & $\begin{array}{c}- \\
- \\
\overline{1} \\
12.1 \\
-\end{array}$ & $\begin{array}{l}- \\
- \\
\overline{8} \\
8.26 \\
-\end{array}$ & $\begin{array}{r}- \\
- \\
- \\
1830 \\
-\end{array}$ & $\begin{array}{l}- \\
- \\
- \\
- \\
-\end{array}$ & $\begin{array}{l}- \\
- \\
- \\
- \\
-\end{array}$ \\
\hline Sedivy & 138 & $\begin{array}{l}\text { 8/22/06 } \\
9 / 13 / 06\end{array}$ & - & $\overline{6} .57$ & $73 \overline{9}$ & - & - \\
\hline
\end{tabular}

a NR, not recroded. 


\section{Appendix B:}

Results from the AGEM Laboratory for Dual Analyses of Samples Collected at Barnes in March 2008 and for Quality Control Samples 
TABLE B.1 Analytical results for samples and replicates collected at Barnes in March 2008 and for quality control samples.

\begin{tabular}{|c|c|c|c|c|c|c|c|c|}
\hline \multirow[b]{2}{*}{ Location } & \multirow[b]{2}{*}{ Sample } & \multirow[b]{2}{*}{$\begin{array}{c}\text { Sample } \\
\text { Date }\end{array}$} & \multirow[b]{2}{*}{$\begin{array}{c}\text { Depth } \\
\text { (ft BGL) }\end{array}$} & \multirow[b]{2}{*}{$\begin{array}{c}\text { Sample } \\
\text { Type }^{a}\end{array}$} & \multicolumn{3}{|c|}{ Concentration $(\mu \mathrm{g} / \mathrm{L})$} & \multirow[b]{2}{*}{$\begin{array}{c}\text { Analysis } \\
\text { Type }\end{array}$} \\
\hline & & & & & $\begin{array}{c}\text { Carbon } \\
\text { Tetrachloride }\end{array}$ & Chloroform & $\begin{array}{l}\text { Methylene } \\
\text { Chloride }\end{array}$ & \\
\hline MW8 & BAMW8-W-22652 & $3 / 10 / 08$ & $110-120$ & MW & 19 & $0.6 \mathrm{Jb}$ & $N^{c}$ & Primary \\
\hline MW8 & BAMW8-W-22652DUP & $3 / 10 / 08$ & $110-120$ & MW & 18 & $0.6 \mathrm{~J}$ & ND & Duplicate \\
\hline MW12D & BAMW12D-W-22641 & $3 / 9 / 08$ & $115-125$ & MW & 1.0 & ND & ND & Primary \\
\hline MW12D & BAMW12D-W-22641DUP & $3 / 9 / 08$ & $115-125$ & MW & 1.1 & ND & ND & Duplicate \\
\hline MW13D & BAMW13D-W-22645 & $3 / 9 / 08$ & $127-137$ & MW & 11 & 1.1 & ND & Primary \\
\hline MW13D & BAMW13DDUP-W-22667 & $3 / 9 / 08$ & $127-137$ & MW & 11 & 1.1 & ND & Replicate \\
\hline MW15S & BAMW15S-W-22648 & $3 / 10 / 08$ & $88-98$ & MW & 1.8 & $0.2 \mathrm{~J}$ & ND & Primary \\
\hline MW15S & BAMW15SDUP-W-22668 & $3 / 10 / 08$ & $88-98$ & MW & 1.6 & ND & ND & Replicate \\
\hline MW16D & BAMW16D-W-22633 & $3 / 7 / 08$ & $90-100$ & MW & ND & ND & ND & Primary \\
\hline MW16D & BAMW16D-W-22633DUP & $3 / 7 / 08$ & $90-100$ & MW & ND & ND & ND & Duplicate \\
\hline PWS2 & BAPWS2-W-22655 & $3 / 6 / 08$ & 155 & MW & ND & ND & ND & Primary \\
\hline PWS2 & BAPWS2-W-22655DUP & $3 / 6 / 08$ & 155 & MW & ND & ND & ND & Duplicate \\
\hline QC & BAQCIR-W-22663 & $3 / 10 / 08$ & - & $\mathrm{RI}$ & ND & ND & ND & Rinsate after MW15S \\
\hline QC & BAQCIR-W-22664 & $3 / 10 / 08$ & - & $\mathrm{RI}$ & 1.5 & ND & ND & Rinsate after MW12M \\
\hline QC & BAQCIR-W-22665 & $3 / 10 / 08$ & - & $\mathrm{RI}$ & 1.9 & ND & ND & Rinsate after MW8 \\
\hline QC & BAQCTB-W-22657 & $3 / 6 / 08$ & - & TB & ND & ND & ND & Trip blank \\
\hline QC & BAQCTB-W-22658 & $3 / 7 / 08$ & - & TB & ND & ND & ND & Trip blank \\
\hline QC & BAQCTB-W-22659 & $3 / 10 / 08$ & - & TB & ND & ND & ND & Trip blank \\
\hline QC & BAPURGE-W-22666 & $3 / 10 / 08$ & - & BT & 2.0 & ND & ND & Waste purge water \\
\hline
\end{tabular}

a Sample types: BT, waste; MW, monitoring well; RI, rinsate; $\mathrm{TB}$, trip blank.

b Qualifier J indicates an estimated concentration below the method quantitation limit of $1.0 \mu \mathrm{g} / \mathrm{L}$.

c ND, contaminant not detected at an instrument detection limit of $0.1 \mu \mathrm{g} / \mathrm{L}$. 


\section{Appendix C:}

Sample Documentation from Envirosystems, Inc. 


\section{ENVIROSYSTEMS, INC.}

9200 Rumsey Road • Suite B102 - Columbia, Maryland 21045-1934

Phone (410) 964-0330 - Fax (410) 740-9306

Email: info@envsystems.com •Webpage: www.envsystems.com/envsys

March 14, 2008

Jorge S. Alvarado, Ph.D

Argonne National Laboratory

Environmental Research Division

Applied Geoscience and Environmental

Management Section

9700 South Cass Avenue, ER-203

Argonne, Illinois 60439

\section{RE: ENVSY Report 080140}

Dear Jorge:

Enclosed are the results of analysis for the samples received on March 11, 2008 for volatile organics analysis by US EPA $8260 \mathrm{~B}$.

Please do not hesitate to call me if you have any questions, comments, or require additional information.

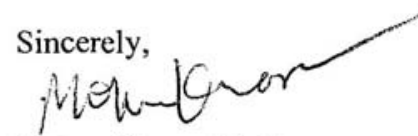

Mohan Khare, Ph.D

President/CEO

$\mathrm{MK} / \mathrm{ncc}$ 


\section{Traffic Reports/Chain of Custody Records}




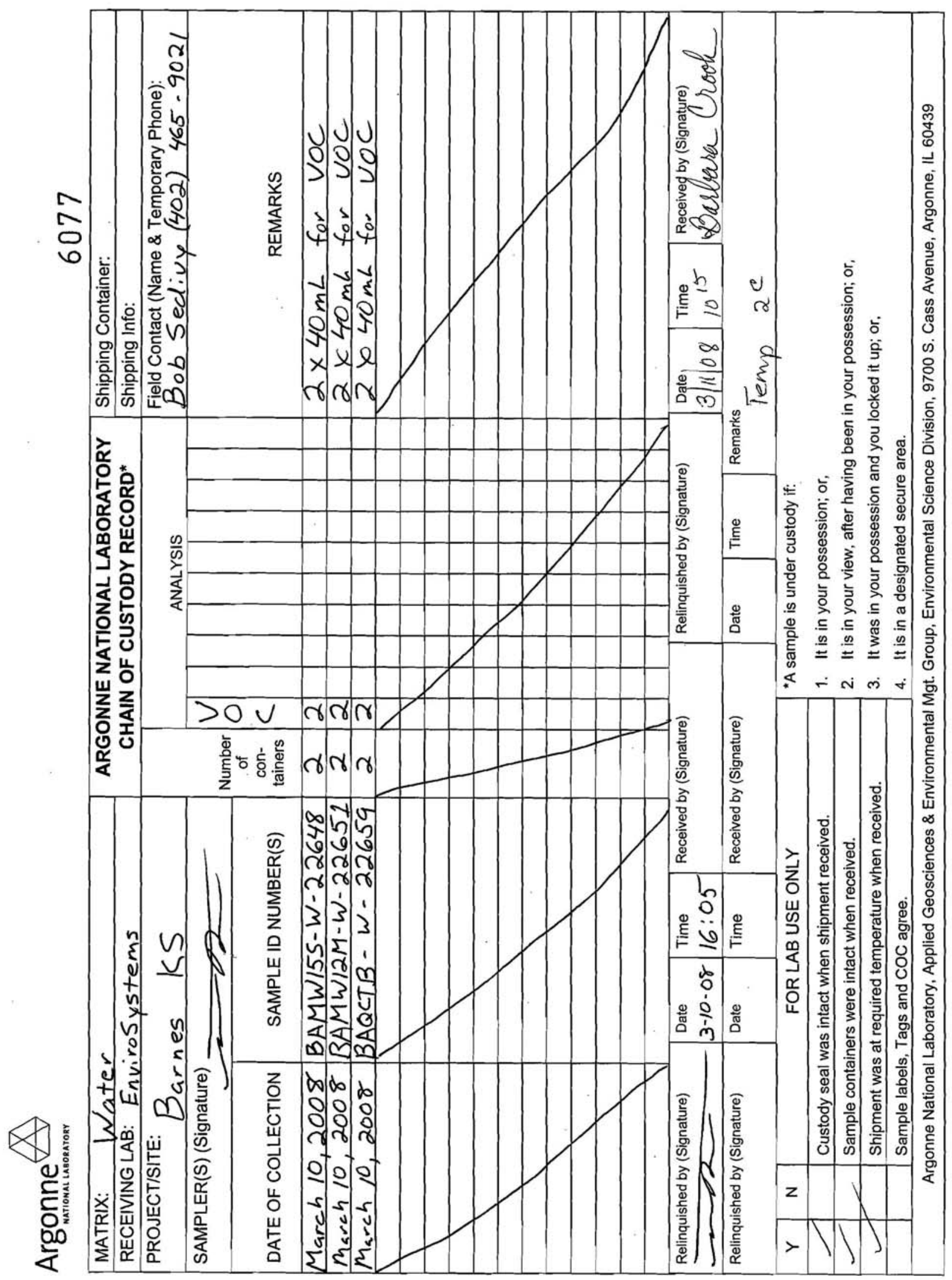




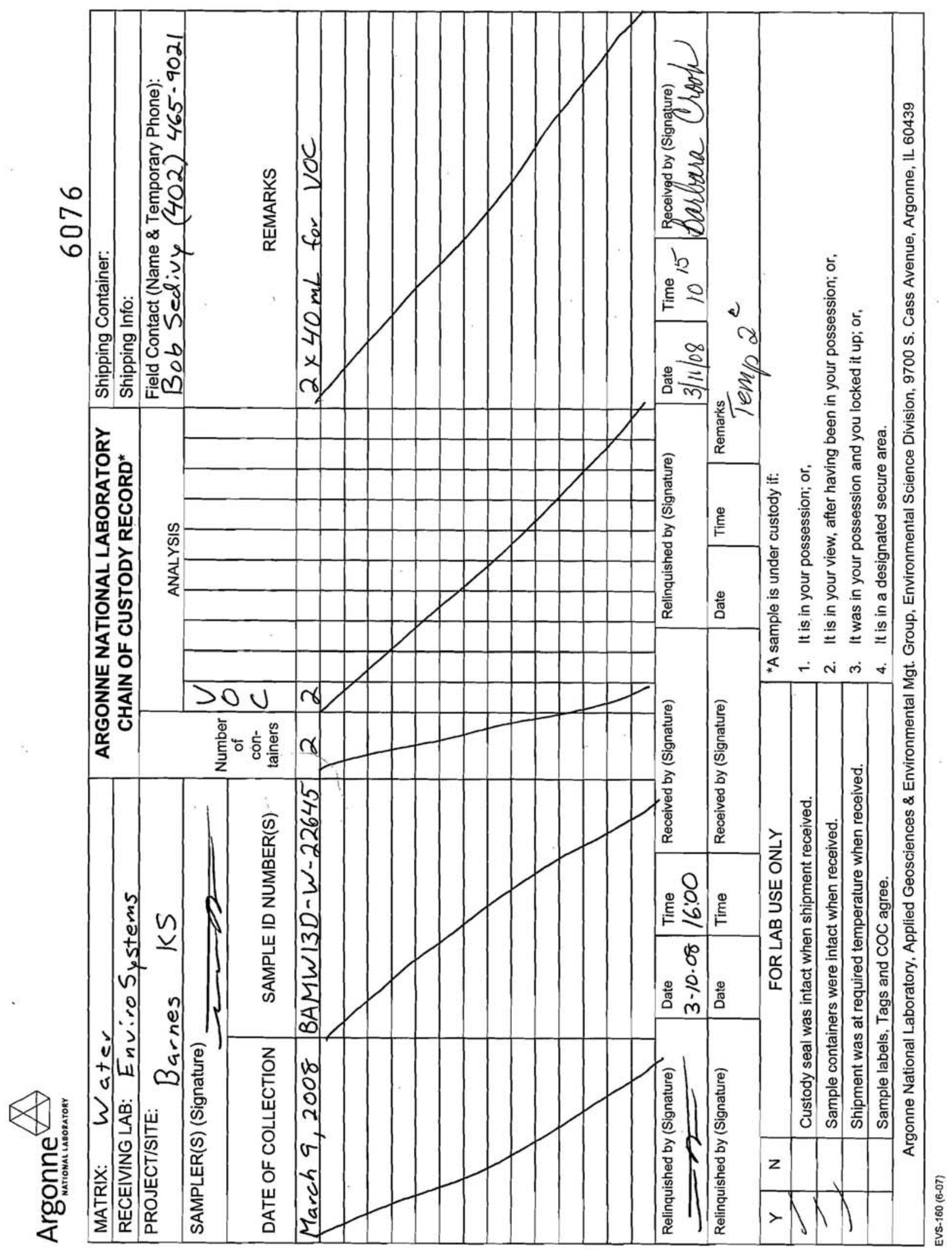




\section{Volatiles Data}


FORM 1

VOLATILE ORGANICS ANALYSIS DATA SHEET

Lab Name: ENVIROSYSTEMS, INC.

Lab Code: ENVSYS Case No.:

Matrix: (soil/water) WATER

Sample wt/vol:

$5.000(\mathrm{~g} / \mathrm{mL}) \mathrm{ML}$

Level: (low/med) LOW

$\%$ Moisture: not dec.

GC Column: RTX-624 ID: 0.18 (mm)

Soil Extract Volume: (uL)
Contract: N/A

SAS NO.: N/A
ARGONNE SAMPLE NO.

BAMW155-W-22648

Lab Sample ID: 0080303-01

Lab File ID: F000435

Date Received: 03/11/08

Date Analyzed: 03/11/08

Dilution Factor: 1.0

Soil Aliquot Volume: (uL)

(ug/L or ug/Kg) UG/L Q

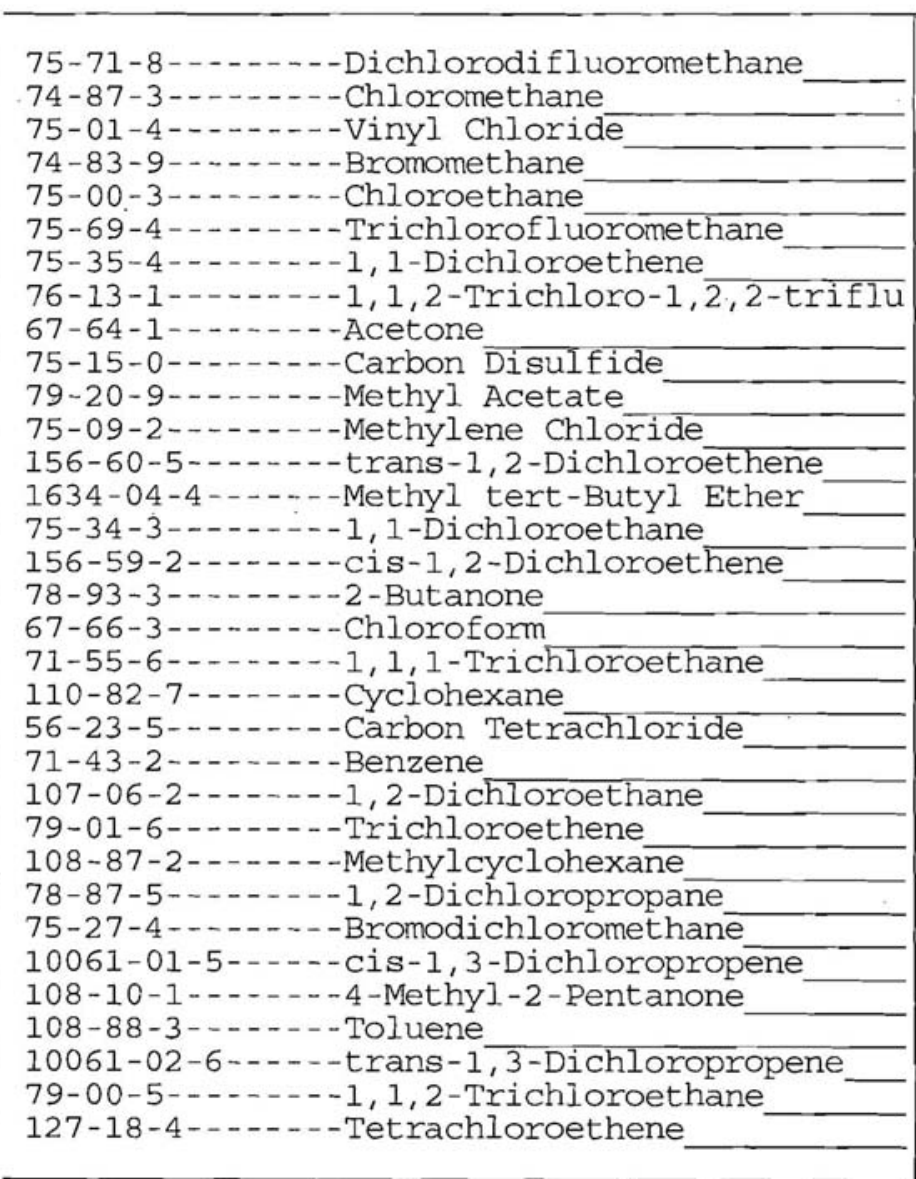

$5.0 \mathrm{U}$

$5.0 \mathrm{U}$

$5.0 \mathrm{U}$

$5.0 \mathrm{U}$

$5.0 \mathrm{U}$

$5.0 \mathrm{U}$

5. $0 \mathrm{U}$

$5.0 \mathrm{U}$

$5.0 \mathrm{U}$

$5.0 \mathrm{U}$

$5.0 \mathrm{U}$

$4.0 \mathrm{~J}$

$5.0 \mathrm{U}$

5. $0 \mathrm{U}$

$5.0 \mathrm{U}$

$5.0 \mathrm{U}$

$5.0 \mathrm{U}$

5. $0 \mathrm{U}$

$5.0 \mathrm{U}$

5. 0 U

$5.0 \mathrm{U}$

$5.0 \mathrm{U}$

$5.0 \mathrm{U}$

$5.0 \mathrm{U}$

$5.0 \mathrm{U}$

$5.0 \mathrm{U}$

5. $0 \mathrm{U}$

$5.0 \mathrm{U}$

5. $0 \mathrm{U}$

$5.0 \mathrm{U}$

5. $0 \mathrm{U}$

$5.0 \mathrm{U}$

5. $0 \mathrm{U}$ 
FORM 1 VOLATILE ORGANICS ANALYSIS DATA SHEET

Lab Name: ENVIROSYSTEMS, INC. Lab Code: ENVSYS Case No.: Matrix: (soil/water) WATER Sample wt/vol: $5.000(\mathrm{~g} / \mathrm{mL}) \mathrm{ML}$

Level: (low/med) LOW

\% Moisture: not dec.

GC Column: RTX-624 ID: 0.18 (mm)

Soil Extract Volume: (uL)
ARGONNE SAMPLE NO.

BAMW155-W-22648

SDG No. : NA

Lab Sample ID: 0080303-01

Lab File ID: F000435

Date Received: 03/11/08

Date Analyzed: 03/11/08

Dilution Factor: 1.0

Soil Aliquot Volume: (uL)

(ug/L or $u g / K g$ ) UG/L

Q

591-78-6-.----2-Hexanone

124-48-1-...--Dibromochloromethane

106-93-4-..--1, 2-Dibromoethane

108-90-7-...--Chlorobenzene

100-41-4-..---Ethylbenzene

1330-20-7-...-Xylene (Total)

100-42-5------Styrene

75-25-2-...--Bromoform

98-82-8--..---Isopropyl benzene

79-34-5 -....-1, 1,2, 2-Tetrachloroethane

541-73-1-...-1, 3-Dichlorobenzene

106-46-7-n..-1,4-Dichlorobenzene

95-50-1-....-1,2-Dichlorobenzene

96-12-8-n--.-1, 2-Dibromo-3-chloropropane

120-82-1-...-1,2,4-Trichlorobenzene

91-20-3-..---Naphthalene

75-65-0--.-.-tert-Butanol

108-20-3-...-Diisopropyl ether

637-92-3-..---Ethyl-tert-butyl ether

994-05-8------tert-Amyl methyl ether

919-94-8-..---tert-Amyl ethyl ether

$5.0 \mathrm{U}$

$5.0 \mathrm{U}$

$5.0 \mathrm{U}$

$5.0 \mathrm{U}$

$5.0 \mathrm{U}$

$5.0 \mathrm{U}$

$5.0 \mathrm{U}$

$5.0 \mathrm{U}$

$5.0 \mathrm{U}$

$5.0 \mathrm{U}$

$5.0 \mathrm{U}$

$5.0 \mathrm{U}$

$5.0 \mathrm{U}$

$5.0 \mathrm{U}$

$5.0 \mathrm{U}$

$10 \mathrm{U}$

$5.0 \mathrm{U}$

$10 \mathrm{U}$

$10 \mathrm{U}$

$10 \mathrm{U}$

$10 \mathrm{U}$

FORM I VOA 
FORM 1

VOLATILE ORGANICS ANALYSIS DATA SHEET

Lab Name: ENVIROSYSTEMS, INC.

Lab Code: ENVSYS Case No.:

Matrix: (soil/water) WATER

Sample wt/vol: $\quad 5.000(\mathrm{~g} / \mathrm{mL}) \mathrm{ML}$

Level: (low/med) LOW

\% Moisture: not dec.

GC Column: RTX-624 ID: 0.18 (mm)

Soil Extract Volume:

(uL)
Contract: $\mathrm{N} / \mathrm{A}$

SAS NO.: N/A
ARGONNE SAMPLE NO.

BAMW12M-W-22651

Lab Sample ID: 0080303-02

Lab File ID: F000436

Date Received: 03/11/08

Date Analyzed: 03/11/08

Dilution Factor: 1.0

Soil Aliquot Volume:

(uL)

CONCENTRATION UNITS:

(ug/L or $u g / \mathrm{Kg}$ ) UG/L

Q

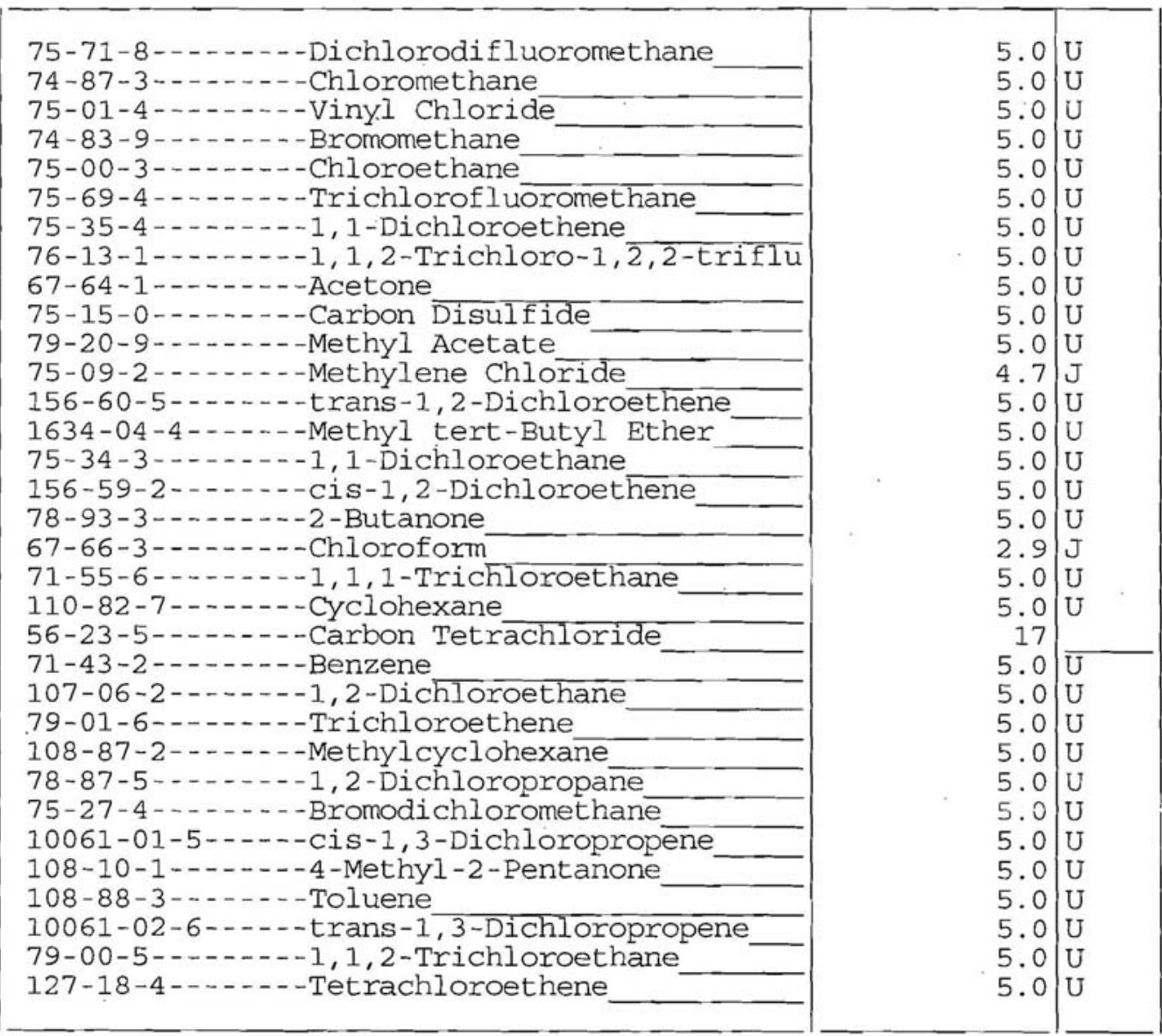

FORM I VOA 
FORM 1

VOLATILE ORGANICS ANALYSIS DATA SHEET

Lab Name: ENVIROSYSTEMS, INC.

Lab Code: ENVSYS Case No. :

Matrix: (soil/water) WATER

Sample wt/vol: $\quad 5.000(\mathrm{~g} / \mathrm{mL}) \mathrm{ML}$

Level: (low/med) LOW

\% Moisture: not dec.

GC Column: RTX-624 ID: 0.18 (mm)

Soil Extract Volume:

(uL)
Contract: N/A

SAS NO.: N/A
ARGONNE SAMPLE NO.

BAMW12M-W-22651

Lab Sample ID: 0080303-02

Lab File ID: F000436

Date Received: 03/11/08

Date Analyzed: 03/11/08

Dilution Factor: 1.0

Soil Aliquot Volume:

(uL)

(ug/L or ug/Kg) UG/L

Q

$5.0 \mathrm{U}$

$5.0 \mathrm{U}$

$5.0 \mathrm{U}$

$5.0 \mathrm{U}$

$5.0 \mathrm{U}$

$5.0 \mathrm{U}$

$5.0 \mathrm{U}$

$5.0 \mathrm{U}$

$5.0 \mathrm{U}$

$5.0 \mathrm{U}$

$5.0 \mathrm{U}$

$5.0 \mathrm{U}$

$5.0 \mathrm{U}$

$5.0 \mathrm{U}$

$5.0 \mathrm{U}$

$10 \mathrm{U}$

$5.0 \mathrm{U}$

$10 \mathrm{U}$

$10 \mathrm{U}$

$10 \mathrm{U}$

$10 \mathrm{U}$ 
FORM 1

VOLATILE ORGANICS ANALYSIS DATA SHEET

Lab Name: ENVIROSYSTEMS, INC.

Lab Code: ENVSYS Case No.:

Matrix: (soil/water) WATER

Sample wt/vol: $\quad 5.000(\mathrm{~g} / \mathrm{mL}) \mathrm{ML}$

Level: (low/med) LOW

$\because$ Moisture: not dec.

GC Column: RTX-624 ID: 0.18 (mm)

Soil Extract Volume: (uL)
Contract: N/A

SAS NO. : N/A
ARGONNE SAMPLE NO.

BAQCTB-W-22659

Lab Sample ID: 0080303-03

Lab File ID: F000437

Date Received: 03/11/08

Date Analyzed: 03/11/08

Dilution Factor: 1.0

Soil Aliquot Volume: (uL)

CAS NO.

\begin{tabular}{|c|}
\hline 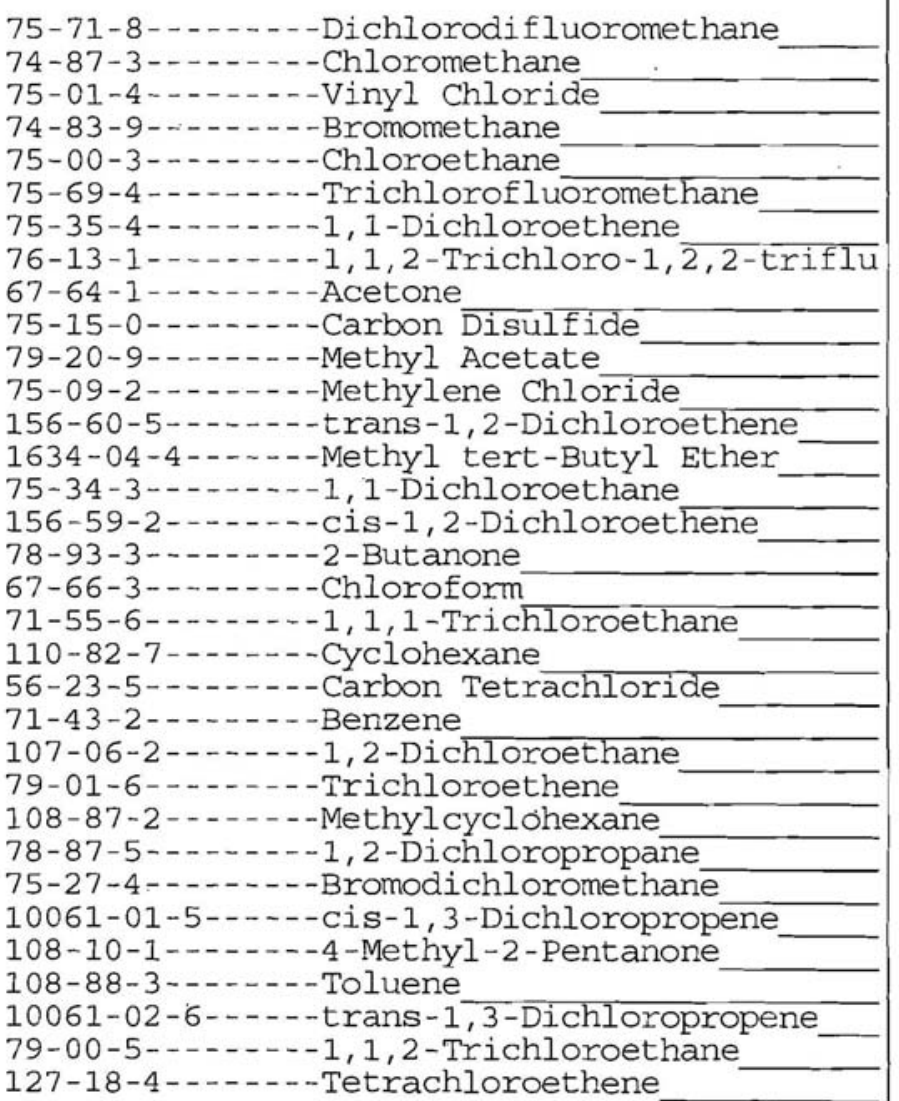 \\
\hline
\end{tabular}

$5.0 \mathrm{U}$

$5.0 \mathrm{U}$

$5.0 \mathrm{U}$

$5.0 \mathrm{U}$

$5.0 \mathrm{U}$

$5.0 \mathrm{U}$

$5.0 \mathrm{U}$

$5.0 \mathrm{U}$

$5.0 \mathrm{U}$

$5.0 \mathrm{U}$

$5.0 \mathrm{U}$

$4.5 \mathrm{~J}$

$5.0 \mathrm{U}$

$5.0 \mathrm{U}$

$5.0 \mathrm{U}$

$5.0 \mathrm{U}$

$5.0 \mathrm{U}$

$5.0 \mathrm{U}$

$5.0 \mathrm{U}$

$5.0 \mathrm{U}$

$5.0 \mathrm{U}$

$5.0 \mathrm{U}$

$5.0 \mathrm{U}$

$5.0 \mathrm{U}$

$5.0 \mathrm{U}$

$5.0 \mathrm{U}$

$5.0 \mathrm{U}$

$5.0 \mathrm{U}$

$5.0 \mathrm{U}$

$5.0 \mathrm{U}$

$5.0 \mathrm{U}$

$5.0 \mathrm{U}$

$5.0 \mathrm{U}$ 
FORM 1

VOLATILE ORGANICS ANALYSIS DATA SHEET

Lab Name: ENVIROSYSTEMS, INC.

Lab Code: ENVSYS Case No.:

Matrix: (soil/water) WATER

Sample wt/vol: $\quad 5.000(\mathrm{~g} / \mathrm{mL}) \mathrm{ML}$

Level: (low/med) LOW

\% Moisture: not dec.

GC Column: RTX-624 ID: 0.18 (mm)

Soil Extract Volume: (uL)
Contract: N/A

SAS NO.: N/A
ARGONNE SAMPLE NO.

BAQCTB-W-22659

Lab Sample ID: 0080303-03

Lab File ID: F000437

Date Received: 03/11/08

Date Analyzed: 03/11/08

Dilution Factor: 1.0

Soil Aliquot Volume: (uL)

CONCENTRATION UNITS:

CAS NO.

COMPOUND

Q

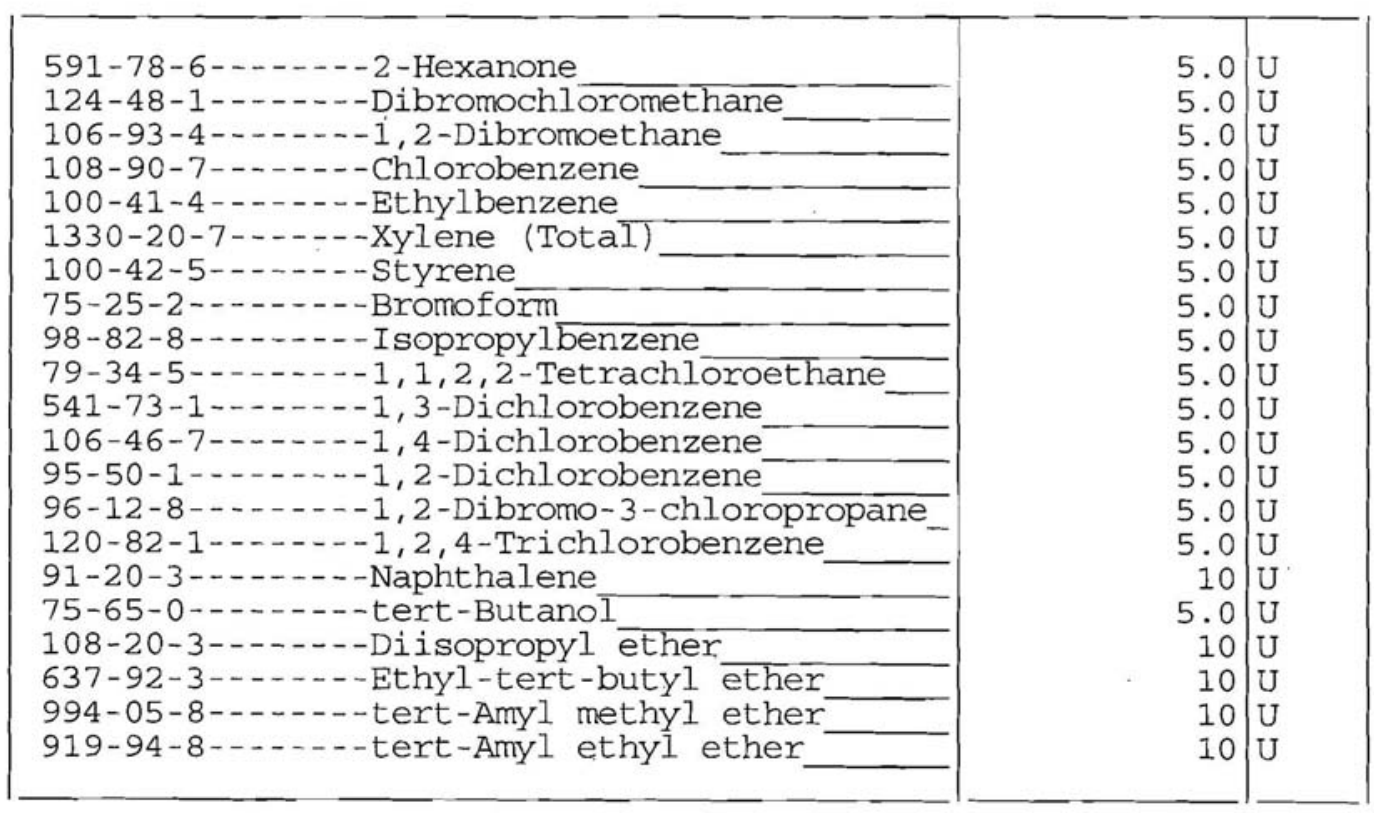


FORM 1 VOLATILE ORGANICS ANALYSIS DATA SHEET

Lab Name: ENVIROSYSTEMS, INC. Lab Code: ENVSYS Case No.: Matrix: (soil/water) WATER Sample wt/vol: $5.000(\mathrm{~g} / \mathrm{mL}) \mathrm{ML}$ Level: (low/med) LOW \% Moisture: not dec. GC Column: RTX-624 ID: 0.18 (mm) Soil Extract Volume: (UL)
Contract: N/A

SAS NO.: N/A
ARGONNE SAMPLE NO. BAMW13D-W-22645
SDG No. : NA

Lab Sample ID: 0080303-04

Lab File ID: F000438

Date Received: 03/11/08

Date Analyzed: 03/11/08

Dilution Factor: 1.0

Soil Aliquot Volume:

(uL)
CAS NO.
COMPOUND
CONCENTRATION UNITS: (ug/L or $\mathrm{ug} / \mathrm{Kg}$ ) UG/L

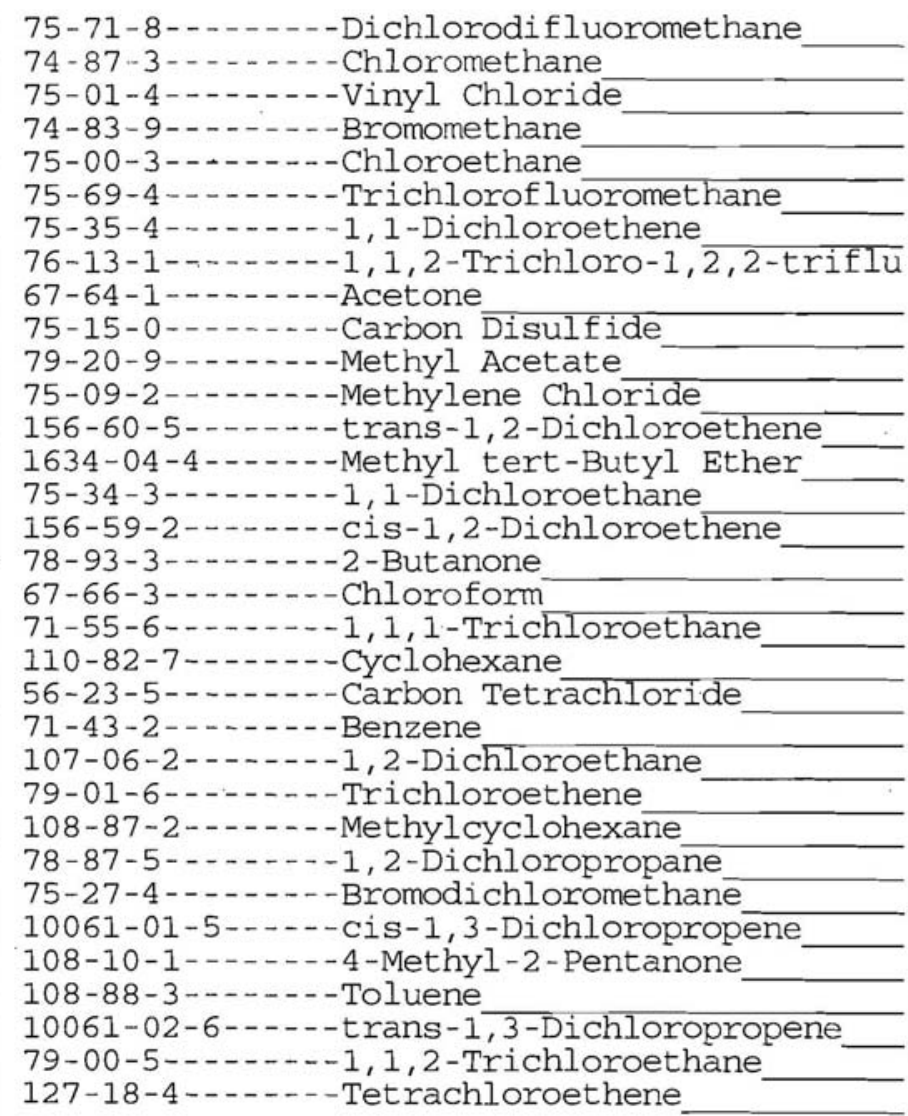


FORM 1

VOLATILE ORGANICS ANALYSIS DATA SHEET

Lab Name: ENVIROSYSTEMS, INC.

Lab Code: ENVSYS Case No. :

Matrix: (soil/water) WATER

Sample wt/vol:

$5.000(\mathrm{~g} / \mathrm{mL}) \mathrm{ML}$

Level: (low/med) Low

\% Moisture: not dec.

GC Column: RTX-624 ID: 0.18 (mm)

Soil Extract Volume: (uL)
Contract: N/A

SAS NO.: N/A
ARGONNE SAMPLE NO.

BAMW13D-W-22645

Lab Sample ID: 0080303-04

Lab File ID: F000438

Date Received: 03/11/08

Date Analyzed: 03/11/08

Dilution Factor: 1.0

Soil Aliquot Volume: (uL)

CAS NO.

COMPOUND

(TS

(ug/L or $u g / K g$ ) UG/L Q

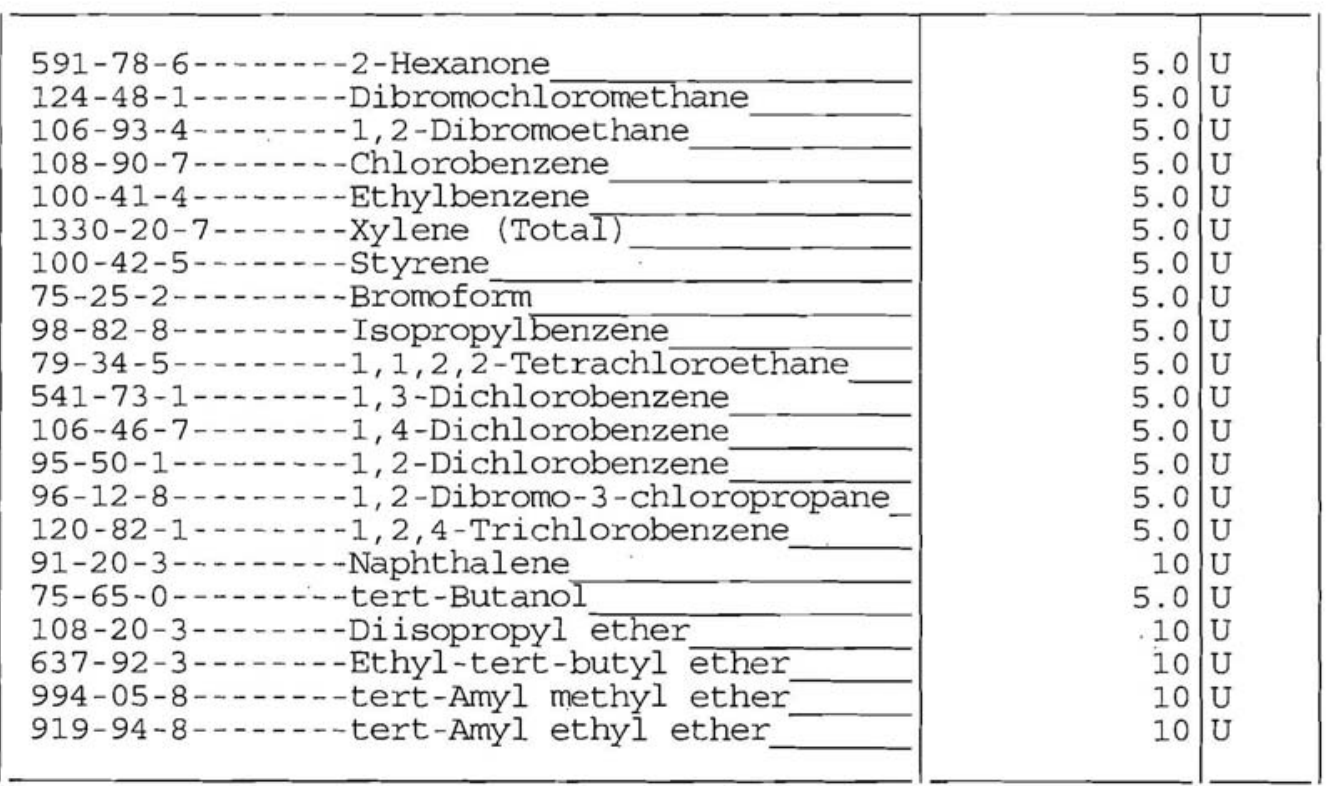


FORM 1

VOLATILE ORGANICS ANALYSIS DATA SHEET

Lab Name: ENVIROSYSTEMS, INC.

Lab Code: ENVSYS Case No.:

Matrix: (soil/water) WATER

Sample wt/vol: $\quad 5.000(\mathrm{~g} / \mathrm{mL}) \mathrm{ML}$

Level: (low/med) Low

$\%$ Moisture: not dec.

GC Column: RTX-624 ID: 0.18 (mm)

Soil Extract Volume: (UL)
Contract: N/A

SAS NO. : N/A
ARGONNE SAMPLE NO.

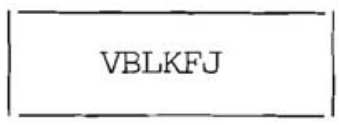

SDG No. : NA

Lab Sample ID: 0C81202-BLKI

Lab File ID: F000434

Date Received:

Date Analyzed: 03/11/08

Dilution Factor: 1.0

Soil Aliquot Volume:

CAS NO.

COMPOUND

CONCENTRATION UNITS:

(ug/L or ug/Kg) UG/L

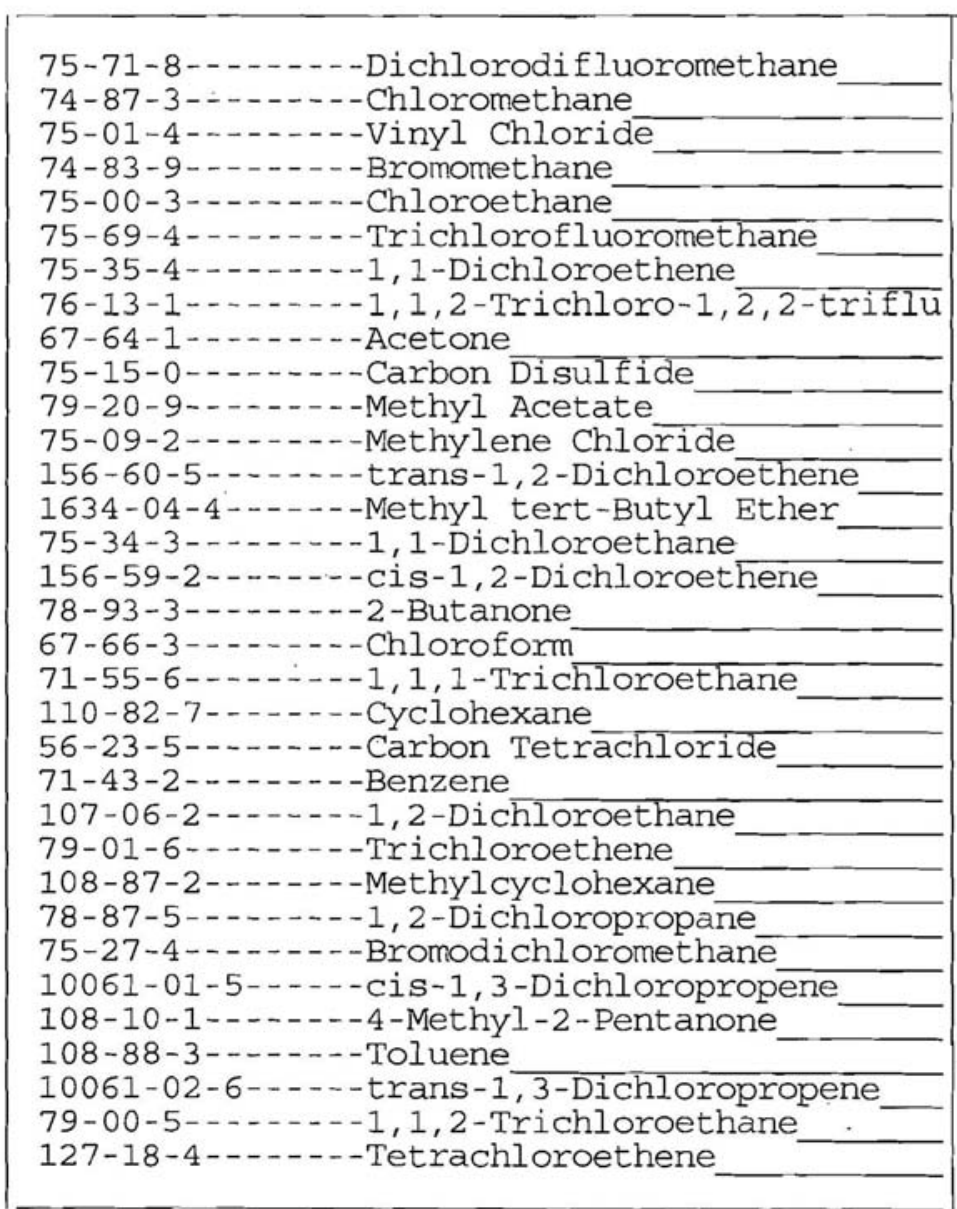

$5.0 \mathrm{U}$

$5.0 \mathrm{U}$

$5.0 \mathrm{U}$

$5.0 \mathrm{U}$

$5.0 \mathrm{U}$

$5.0 \mathrm{U}$

$5.0 \mathrm{U}$

$5.0 \mathrm{U}$

$5.0 \mathrm{U}$

$5.0 \mathrm{U}$

$5.0 \mathrm{U}$

$4.6 \mathrm{~J}$

$5.0 \mathrm{U}$

$5.0 \mathrm{U}$

$5.0 \mathrm{U}$

$5.0 \mathrm{U}$

$5.0 \mathrm{U}$

$5.0 \mathrm{U}$

$5.0 \mathrm{U}$

$5.0 \mathrm{U}$

$5.0 \mathrm{U}$

$5.0 \mathrm{U}$

$5.0 \mathrm{U}$

$5.0 \mathrm{U}$

$5.0 \mathrm{U}$

$5.0 \mathrm{U}$

$5.0 \mathrm{U}$

$5.0 \mathrm{U}$

$5.0 \mathrm{U}$

$5.0 \mathrm{U}$

$5.0 \mathrm{U}$

$5.0 \mathrm{U}$

$5.0 \mathrm{U}$ 
FORM 1 VOLATILE ORGANICS ANALYSIS DATA SHEET

Lab Name: ENVIROSYSTEMS, INC. Contract: N/A Lab Code: ENVSYS Case No.: Matrix: (soil/water) WATER Sample wt/vol: $5.000(\mathrm{~g} / \mathrm{mL}) \mathrm{ML}$

Level: (low/med) LOW

\% Moisture: not dec.

GC Column: RTX-624

ID : $0.18(\mathrm{~mm})$

Soil Extract Volume: (uL)
SAS NO.: N/A SDG NO.: NA Lab Sample ID: 0C81202-BLK1

ARGONNE SAMPLE NO.

VBLKFJ

Lab File ID: F000434

Date Received:

Date Analyzed: 03/11/08

Dilution Factor: 1.0

Soil Aliquot Volume: (uL)

CAS NO.

COMPOUND

CONCENTRATION UNITS:

(ug/L or ug/Kg) UG/L

Q

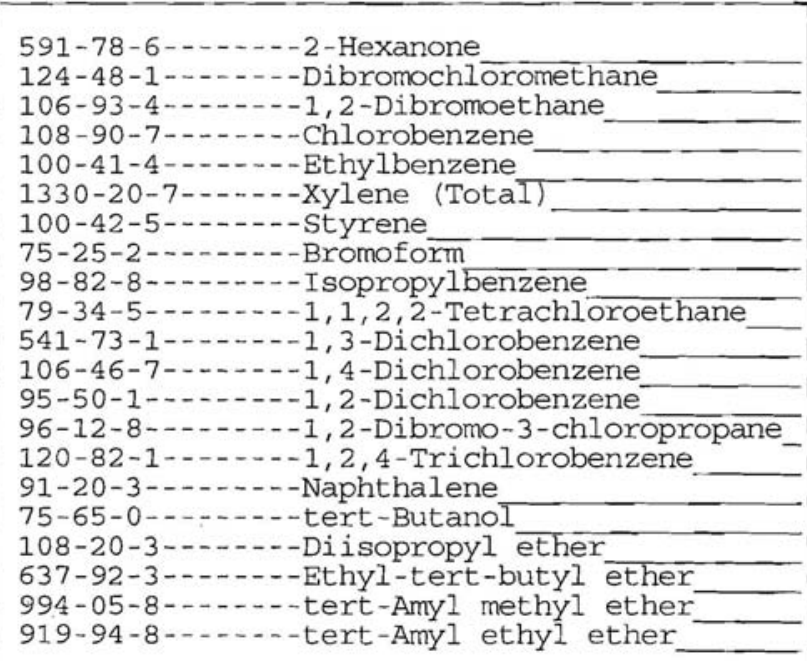

$5.0 \mathrm{U}$

$5.0 \mathrm{U}$

5.0 U

$5.0 \mathrm{U}$

$5.0 \mathrm{U}$

$5.0 \mathrm{U}$

$5.0 \mathrm{U}$

$5.0 \mathrm{U}$

$5.0 \mathrm{U}$

$5.0 \mathrm{U}$

$5.0 \mathrm{U}$

$5.0 \mathrm{U}$

$5.0 \mathrm{U}$

$5.0 \mathrm{U}$

$5.0 \mathrm{U}$

$10 \mathrm{U}$

$5.0 \mathrm{U}$

$10 \mathrm{U}$

$10 \mathrm{U}$

$10 \mathrm{U}$

$10 \mathrm{U}$ 


\section{Supplement 1:}

Automatically Measured Water Levels, May 2007 to April 2008 
TABLE S1.1 Automatically recorded water levels for monitoring wells at Barnes, Kansas,

May 11, 2007, to April 16, 2008.

\begin{tabular}{|c|c|c|c|c|c|c|c|c|c|c|}
\hline \multirow[b]{2}{*}{ Date and Time } & \multicolumn{10}{|c|}{ Depth from Top of Casing (ft) } \\
\hline & MW1D & MW2D & MW3D & MW4D & MW7 & MW9 & Oentrich & MW14D & MW15D & MW16D \\
\hline $5 / 11 / 070: 00$ & 130.452 & 128.066 & 124.711 & 105.285 & 109.216 & 100.96 & 115.745 & 112.239 & 84.06 & 77.46 \\
\hline $5 / 11 / 070: 30$ & 130.452 & 128.062 & 124.709 & 105.285 & 109.22 & 100.958 & 115.746 & 112.239 & 84.054 & 77.465 \\
\hline 5/11/07 1:00 & 130.447 & 128.051 & 124.706 & 105.281 & 109.215 & 100.953 & 115.741 & 112.235 & 84.045 & 77.452 \\
\hline $5 / 11 / 071: 30$ & 130.443 & 128.044 & 124.704 & 105.278 & 109.209 & 100.951 & 115.737 & 112.23 & 84.04 & 77.444 \\
\hline 5/11/07 2:00 & 130.433 & 128.041 & 124.692 & 105.266 & 109.197 & 100.939 & 115.731 & 112.219 & 84.029 & 77.431 \\
\hline $5 / 11 / 072: 30$ & 130.428 & 128.041 & 124.685 & 105.261 & 109.194 & 100.937 & 115.726 & 112.215 & 84.021 & 77.431 \\
\hline 5/11/07 3:00 & 130.421 & 128.039 & 124.681 & 105.257 & 109.188 & 100.932 & 115.718 & 112.208 & 84.014 & 77.427 \\
\hline 5/11/07 3:30 & 130.424 & 128.041 & 124.678 & 105.257 & 109.188 & 100.93 & 115.716 & 112.208 & 84.012 & 77.423 \\
\hline 5/11/07 4:00 & 130.421 & 128.035 & 124.676 & 105.255 & 109.188 & 100.927 & 115.715 & 112.208 & 84.006 & 77.411 \\
\hline 5/11/07 4:30 & 130.421 & 128.039 & 124.679 & 105.255 & 109.188 & 100.93 & 115.715 & 112.208 & 84.001 & 77.419 \\
\hline 5/11/07 5:00 & 130.417 & 128.055 & 124.674 & 105.254 & 109.188 & 100.927 & 115.713 & 112.204 & 83.997 & 77.421 \\
\hline 5/11/07 5:30 & 130.412 & 128.053 & 124.676 & 105.254 & 109.186 & 100.927 & 115.711 & 112.204 & 83.994 & 77.421 \\
\hline 5/11/07 6:00 & 130.435 & 129.838 & 124.69 & 105.273 & 109.205 & 100.946 & 115.726 & 112.222 & 83.997 & 77.427 \\
\hline 5/11/07 6:30 & 130.433 & 130.16 & 124.69 & 105.269 & 109.207 & 100.941 & 115.728 & 112.222 & 83.994 & 77.417 \\
\hline 5/11/07 7:00 & 132.228 & 130.314 & 125.782 & 105.762 & 109.54 & 101.415 & 116.12 & 113.49 & 84.005 & 77.438 \\
\hline 5/11/07 7:30 & 132.583 & 130.41 & 126.129 & 106.074 & 109.851 & 101.731 & 116.38 & 113.869 & 84.042 & 77.525 \\
\hline $5 / 11 / 078: 00$ & 132.748 & 130.471 & 126.291 & 106.229 & 110.009 & 101.887 & 116.513 & 114.037 & 84.071 & 77.597 \\
\hline $5 / 11 / 078: 30$ & 132.845 & 130.514 & 126.395 & 106.331 & 110.111 & 101.986 & 116.594 & 114.141 & 84.101 & 77.674 \\
\hline 5/11/07 9:00 & 132.911 & 128.876 & 126.46 & 106.396 & 110.178 & 102.052 & 116.648 & 114.21 & 84.126 & 77.717 \\
\hline $5 / 11 / 079: 30$ & 132.956 & 128.527 & 126.506 & 106.443 & 110.226 & 102.097 & 116.684 & 114.256 & 84.15 & 77.758 \\
\hline $5 / 11 / 07$ 10:00 & 131.322 & 128.375 & 125.553 & 106.06 & 109.993 & 101.738 & 116.387 & 113.158 & 84.165 & 77.787 \\
\hline $5 / 11 / 07 \quad 10: 30$ & 130.93 & 128.282 & 125.176 & 105.731 & 109.67 & 101.408 & 116.109 & 112.726 & 84.148 & 77.742 \\
\hline 5/11/07 11:00 & 130.767 & 128.221 & 125.016 & 105.579 & 109.521 & 101.257 & 115.981 & 112.56 & 84.134 & 77.682 \\
\hline $5 / 11 / 0711: 30$ & 130.672 & 128.173 & 124.926 & 105.493 & 109.432 & 101.17 & 115.906 & 112.463 & 84. & 77.628 \\
\hline $5 / 11 / 0712: 00$ & 130.608 & 128.128 & 124.863 & 105.431 & 109.369 & 101.109 & 115.855 & 112.399 & 84.104 & 77.583 \\
\hline $5 / 11 / 07$ 12:30 & 130.556 & 128.1 & 124.808 & 105.379 & 109.315 & 101.059 & 115.81 & 112.343 & 84.088 & 77.525 \\
\hline 5/11/07 13:00 & 130.516 & 128.075 & 124.769 & 105.343 & 109.279 & 101.019 & 115.782 & 112.306 & 84.071 & 77.504 \\
\hline 5/11/07 13:30 & 130.485 & 128.053 & 124.739 & 105.312 & 109.247 & 100.989 & 115.758 & 112.272 & 84. & 77.473 \\
\hline $5 / 11 / 0714: 00$ & 130.461 & 128.035 & 124.718 & 105.29 & 109.226 & 100.967 & 115.739 & 112.25 & 84.043 & 77.457 \\
\hline $5 / 11 / 07$ 14:30 & 130.443 & 128.016 & 124.695 & 105.269 & 109.205 & 100.946 & 115.722 & 112.228 & 84.029 & 77.438 \\
\hline $5 / 11 / 07$ 15:00 & 130.421 & 127.998 & 124.674 & 105.248 & 109.184 & 100.925 & 115.705 & 112.206 & 84.014 & 77.413 \\
\hline $5 / 11 / 07$ 15:30 & 130.402 & 127.985 & 124.655 & 105.233 & 109.167 & 100.908 & 115.688 & 112.191 & 84.001 & 77.411 \\
\hline $5 / 11 / 07$ 16:00 & 130.386 & 127.971 & 124.639 & 105.216 & 109.152 & 100.894 & 115.675 & 112.175 & 83.988 & 77.395 \\
\hline 5/11/07 16:30 & 130.369 & 127.964 & 124.625 & 105.202 & 109.136 & 100.88 & 115.664 & 112.157 & 83.977 & 77.384 \\
\hline $5 / 11 / 07$ 17:00 & 130.357 & 127.953 & 124.611 & 105.188 & 109.127 & 100.868 & 115.658 & 112.144 & 83.964 & 77.374 \\
\hline $5 / 11 / 07 \quad 17: 30$ & 130.348 & 127.951 & 124.602 & 105.182 & 109.115 & 100.859 & 115.654 & 112.137 & & 77.364 \\
\hline $5 / 11 / 07$ 18:00 & 130.341 & 127.948 & 124.593 & 105.173 & 109.108 & 100.852 & 115.634 & 112.129 & 83.944 & 77.355 \\
\hline $5 / 11 / 07$ 18:30 & 130.334 & 127.944 & 124.588 & 105.167 & 109.104 & 100.847 & 115.632 & 112.124 & 83.935 & 77.339 \\
\hline 5/11/07 19:00 & 130.331 & 127.946 & 124.586 & 105.167 & 109.102 & 100.842 & 115.63 & 112.12 & & 77.339 \\
\hline $5 / 11 / 07 \quad 19: 30$ & 130.327 & 127.948 & 124.581 & 105.161 & 109.1 & 100.84 & 115.62 & 112.115 & 83.92 & 77.331 \\
\hline $5 / 11 / 0720: 00$ & 130.329 & 127.953 & 124.584 & 105.166 & 109.102 & 100.842 & 115.623 & 112.12 & 83.912 & 77.335 \\
\hline 5/11/07 20:30 & 130.331 & 127.964 & 124.586 & 105.169 & 109.106 & 100.847 & 115.62 & 112.122 & 83.908 & 77.333 \\
\hline $5 / 11 / 0721: 00$ & 1 & 127.962 & 124.593 & 105.176 & 109.114 & 100.854 & 115.634 & 112.129 & 83.905 & 77.339 \\
\hline $5 / 11 / 0721: 30$ & 130.348 & 127.962 & 124.6 & 105.186 & 109.123 & 100.861 & 115.641 & 112.137 & 83.903 & 77.339 \\
\hline 5/11/07 22:00 & 130.346 & 127.962 & 124.6 & 105.186 & 109.123 & 100.864 & 115.641 & 112.137 & & 77.349 \\
\hline 5/11/07 22:30 & 130.348 & 127.958 & 124.604 & 105.19 & 109.127 & 100.866 & 115.65 & 112.142 & 83.896 & 77.347 \\
\hline 5/11/07 23:00 & 130.348 & 127.951 & 124.602 & 105.188 & 109.129 & 100.866 & 115.643 & 112.14 & 83.892 & 77.341 \\
\hline 5/11/07 23:30 & 130.343 & 127.948 & 124.597 & 105.183 & 109.119 & 100.861 & 115.638 & 112.133 & 83.887 & 77.335 \\
\hline $5 / 12 / 07$ 0:00 & 130.336 & 127.946 & 124.593 & 105.178 & 109.115 & 100.854 & 115.63 & 112.129 & 83.877 & 77.335 \\
\hline $5 / 12 / 070: 30$ & 130.334 & 127.944 & 124.591 & 105.176 & 109.115 & 100.854 & 115.62 & 112.126 & 83.876 & 77.324 \\
\hline 5/12/07 1:00 & 130.331 & 127.939 & 124.588 & 105.174 & 109.115 & 100.854 & 115.619 & 112.124 & 83.87 & 77.335 \\
\hline 5/12/07 1:30 & 130.329 & 127.928 & 124.586 & 105.173 & 109.11 & 100.849 & 115.613 & 112.124 & 83.865 & 77.314 \\
\hline 5/12/07 2:00 & 130.327 & 127.919 & 124.581 & 105.168 & 109.108 & 100.842 & 115.613 & 112.117 & 83.859 & 77.31 \\
\hline $5 / 12 / 072: 30$ & 130.324 & 127.914 & 124.57 & 105.157 & 109.095 & 100.831 & 115.596 & 112.106 & 83.852 & 77.308 \\
\hline 5/12/07 3:00 & 130.32 & 127.917 & 124.563 & 105.149 & 109.087 & 100.824 & 115.589 & 112.098 & 83.844 & 77.289 \\
\hline 5/12/07 3:30 & 130.315 & 127.914 & 124.556 & 105.143 & 109.083 & 100.819 & 115.583 & 112.093 & 83.839 & 77.287 \\
\hline 5/12/07 4:00 & 130.312 & 127.912 & 124.558 & 105.145 & 109.083 & 100.819 & 115.585 & 112.093 & 83.835 & 77.297 \\
\hline $5 / 12 / 074: 30$ & 130.31 & 127.91 & 124.556 & 105.145 & 109.083 & 100.819 & 115.581 & 112.093 & 83.831 & 77.304 \\
\hline 5/12/07 5:00 & 130.31 & 127.91 & 124.551 & 105.14 & 109.077 & 100.814 & 115.579 & 112.091 & 83.828 & 77.285 \\
\hline 5/12/07 5:30 & 130.308 & 127.912 & 124.549 & 105.138 & 109.075 & 100.812 & 115.575 & 112.089 & 83.824 & 77.289 \\
\hline & & 129.772 & & 105.138 & 109.077 & 100.814 & 115.628 & 112.091 & 83.82 & 77.281 \\
\hline 5/12/07 6:30 & 130.301 & 130.078 & 124.556 & 105.143 & 109.081 & 100.819 & 115.6 & 112.095 & 83.82 & 77.277 \\
\hline 5/12/07 7:00 & 132.202 & 130.24 & 125.851 & 105.736 & 109.5 & 101.387 & 116.098 & 113.435 & 83.842 & 77.316 \\
\hline $5 / 12 / 07$ 7:30 & 132.533 & 130.342 & 126.18 & 106.034 & 109.801 & 101.687 & 116.336 & 113.785 & 83.887 & 77.395 \\
\hline 5/12/07 8:00 & 132.701 & 130.41 & 126.349 & 106.2 & 109.965 & 101.852 & 116.472 & 113.96 & 83.925 & 77.484 \\
\hline
\end{tabular}


TABLE S1.1 (Cont.)

Depth from Top of Casing (ft)

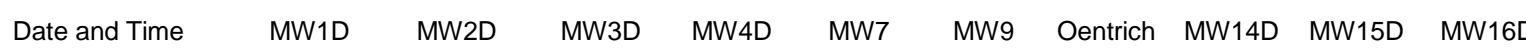

\begin{tabular}{|c|c|c|c|c|c|c|c|c|c|c|}
\hline 5/12/07 8:30 & 132.807 & 130.462 & 126.455 & 106.303 & 110.07 & 101.958 & 116.558 & 114.068 & 83.96 & 77.56 \\
\hline 5/12/07 9:00 & 132.881 & 130.5 & 126.529 & 106.376 & 110.146 & 102.031 & 116.616 & 114.143 & 83.994 & 77.616 \\
\hline 5/12/07 9:30 & 132.935 & 130.525 & 126.58 & 106.429 & 110.199 & 102.085 & 116.656 & 114.199 & 84.023 & 77.661 \\
\hline 5/12/07 10:00 & 132.975 & 130.541 & 126.619 & 106.467 & 110.239 & 102.123 & 116.691 & 114.239 & 84.053 & 77.694 \\
\hline $5 / 12 / 07 \quad 10: 30$ & 132.999 & 130.555 & 126.647 & 106.496 & 110.268 & 102.151 & 116.708 & 114.267 & 84.077 & 77.719 \\
\hline $5 / 12 / 0711: 00$ & 133.02 & 130.557 & 126.668 & 106.517 & 110.287 & 102.17 & 116.725 & 114.287 & 84.101 & 77.742 \\
\hline $5 / 12 / 0711: 30$ & 133.032 & 130.553 & 126.68 & 106.531 & 110.304 & 102.184 & 116.736 & 114.301 & 84.121 & 77.746 \\
\hline $5 / 12 / 07$ 12:00 & 133.03 & 129.223 & 126.684 & 106.531 & 110.304 & 102.186 & 116.736 & 114.303 & 84.137 & 77.757 \\
\hline $5 / 12 / 07 \quad 12: 30$ & 133.03 & 128.554 & 126.682 & 106.531 & 110.304 & 102.186 & 116.757 & 114.303 & 84.156 & 77.773 \\
\hline $5 / 12 / 07$ 13:00 & 132.046 & 128.334 & 126.164 & 106.422 & 110.268 & 102.094 & 116.684 & 114.115 & 84.169 & 77.765 \\
\hline 5/12/07 13:30 & 130.975 & 128.209 & 125.213 & 105.764 & 109.712 & 101.441 & 116.12 & 112.78 & 84.143 & 77.709 \\
\hline $5 / 12 / 07 \quad 14: 00$ & 130.736 & 128.128 & 124.979 & 105.545 & 109.491 & 101.224 & 115.955 & 112.536 & 84.115 & 77.62 \\
\hline $5 / 12 / 07$ 14:30 & 130.61 & 128.064 & 124.854 & 105.426 & 109.373 & 101.104 & 115.838 & 112.408 & 84.091 & 77.568 \\
\hline 5/12/07 15:00 & 130.525 & 128.012 & 124.771 & 105.347 & 109.291 & 101.026 & 115.767 & 112.321 & 84.071 & 77.5 \\
\hline $5 / 12 / 07 \quad 15: 30$ & 130.457 & 127.98 & 124.706 & 105.283 & 109.228 & 100.963 & 115.712 & 112.255 & 84.049 & 77.453 \\
\hline $5 / 12 / 07$ 16:00 & 130.405 & 127.948 & 124.653 & 105.233 & 109.176 & 100.911 & 115.668 & 112.202 & 84.027 & 77.409 \\
\hline $5 / 12 / 07$ 16:30 & 130.367 & 127.93 & 124.616 & 105.201 & 109.144 & 100.88 & 115.642 & 112.166 & 84.008 & 77.382 \\
\hline $5 / 12 / 07$ 17:00 & 130.339 & 127.912 & 124.588 & 105.171 & 109.114 & 100.852 & 115.614 & 112.135 & 83.99 & 77.364 \\
\hline $5 / 12 / 07 \quad 17: 30$ & 130.32 & 127.896 & 124.57 & 105.154 & 109.096 & 100.835 & 115.6 & 112.115 & 83.975 & 77.347 \\
\hline $5 / 12 / 07$ 18:00 & 130.301 & 127.883 & 124.551 & 105.136 & 109.079 & 100.814 & 115.585 & 112.095 & 83.96 & 77.333 \\
\hline $5 / 12 / 07$ 18:30 & 130.286 & 127.871 & 124.535 & 105.123 & 109.064 & 100.798 & 115.572 & 112.084 & 83.947 & 77.31 \\
\hline $5 / 12 / 07 \quad 19: 00$ & 130.272 & 127.862 & 124.521 & 105.107 & 109.051 & 100.786 & 115.553 & 112.067 & 83.933 & 77.297 \\
\hline $5 / 12 / 07$ 19:30 & 130.258 & 127.851 & 124.51 & 105.097 & 109.041 & 100.774 & 115.542 & 112.055 & 83.922 & 77.275 \\
\hline $5 / 12 / 0720: 00$ & 130.249 & 127.853 & 124.5 & 105.088 & 109.03 & 100.765 & 115.534 & 112.047 & 83.911 & 77.277 \\
\hline $5 / 12 / 0720: 30$ & 130.237 & 127.855 & 124.491 & 105.08 & 109.024 & 100.755 & 115.529 & 112.038 & 83.898 & 77.266 \\
\hline $5 / 12 / 0721: 00$ & 130.239 & 127.858 & 124.493 & 105.083 & 109.024 & 100.758 & 115.53 & 112.038 & 83.892 & 77.271 \\
\hline $5 / 12 / 0721: 30$ & 130.242 & 127.858 & 124.493 & 105.087 & 109.028 & 100.76 & 115.532 & 112.042 & 83.887 & 77.269 \\
\hline $5 / 12 / 0722: 00$ & 130.244 & 127.86 & 124.496 & 105.087 & 109.03 & 100.765 & 115.534 & 112.042 & 83.879 & 77.269 \\
\hline $5 / 12 / 0722: 30$ & 130.246 & 127.851 & 124.493 & 105.088 & 109.035 & 100.765 & 115.536 & 112.042 & 83.874 & 77.273 \\
\hline 5/12/07 23:00 & 130.244 & 127.849 & 124.498 & 105.092 & 109.035 & 100.767 & 115.538 & 112.044 & 83.868 & 77.273 \\
\hline 5/12/07 23:30 & 130.239 & 127.846 & 124.491 & 105.083 & 109.028 & 100.762 & 115.532 & 112.038 & 83.861 & 77.275 \\
\hline $5 / 13 / 070: 00$ & 130.234 & 127.842 & 124.486 & 105.082 & 109.026 & 100.758 & 115.532 & 112.033 & 83.853 & 77.269 \\
\hline $5 / 13 / 07$ 0:30 & 130.234 & 127.835 & 124.486 & 105.081 & 109.026 & 100.758 & 115.53 & 112.033 & 83.85 & 77.25 \\
\hline 5/13/07 1:00 & 130.227 & 127.826 & 124.479 & 105.074 & 109.018 & 100.753 & 115.525 & 112.029 & 83.842 & 77.248 \\
\hline 5/13/07 1:30 & 130.218 & 127.821 & 124.473 & 105.07 & 109.013 & 100.748 & 115.521 & 112.02 & 83.835 & 77.244 \\
\hline 5/13/07 2:00 & 130.213 & 127.817 & 124.466 & 105.061 & 109.005 & 100.739 & 115.517 & 112.013 & 83.826 & 77.244 \\
\hline $5 / 13 / 072: 30$ & 130.208 & 127.81 & 124.461 & 105.057 & 109.003 & 100.734 & 115.51 & 112.007 & 83.82 & 77.235 \\
\hline 5/13/07 3:00 & 130.204 & 127.81 & 124.454 & 105.05 & 108.996 & 100.727 & 115.504 & 112.005 & 83.813 & 77.235 \\
\hline 5/13/07 3:30 & 130.199 & 127.799 & 124.452 & 105.047 & 108.992 & 100.724 & 115.502 & 112 & 83.807 & 77.225 \\
\hline 5/13/07 4:00 & 130.199 & 127.799 & 124.449 & 105.047 & 108.99 & 100.722 & 115.506 & 112 & 83.802 & 77.223 \\
\hline 5/13/07 4:30 & 130.187 & 127.801 & 124.441 & 105.038 & 108.982 & 100.713 & 115.499 & 111.989 & 83.794 & 77.2 \\
\hline 5/13/07 5:00 & 130.187 & 127.801 & 124.443 & 105.038 & 108.984 & 100.713 & 115.499 & 111.989 & 83.791 & 77.211 \\
\hline 5/13/07 5:30 & 130.187 & 127.801 & 124.441 & 105.037 & 108.982 & 100.713 & 115.487 & 111.987 & 83.787 & 77.196 \\
\hline 5/13/07 6:00 & 130.187 & 129.237 & 124.441 & 105.038 & 108.982 & 100.715 & 115.487 & 111.989 & 83.783 & 77.202 \\
\hline 5/13/07 6:30 & 130.185 & 129.84 & 124.441 & 105.038 & 108.986 & 100.715 & 115.489 & 111.989 & 83.78 & 77.192 \\
\hline 5/13/07 7:00 & 131.341 & 130.047 & 125.102 & 105.238 & 109.066 & 100.892 & 115.606 & 112.401 & 83.778 & 77.197 \\
\hline $5 / 13 / 077: 30$ & 132.287 & 130.165 & 125.928 & 105.805 & 109.573 & 101.455 & 116.135 & 113.541 & 83.817 & 77.26 \\
\hline 5/13/07 8:00 & 132.505 & 130.24 & 126.145 & 106.008 & 109.778 & 101.661 & 116.307 & 113.767 & 83.853 & 77.347 \\
\hline $5 / 13 / 078: 30$ & 132.632 & 130.299 & 126.275 & 106.133 & 109.906 & 101.785 & 116.421 & 113.9 & 83.89 & 77.418 \\
\hline 5/13/07 9:00 & 132.708 & 130.342 & 126.351 & 106.208 & 109.982 & 101.861 & 116.478 & 113.977 & 83.92 & 77.475 \\
\hline 5/13/07 9:30 & 132.767 & 130.371 & 126.411 & 106.269 & 110.045 & 101.922 & 116.527 & 114.039 & 83.947 & 77.519 \\
\hline $5 / 13 / 07 \quad 10: 00$ & 132.81 & 130.385 & 126.455 & 106.312 & 110.089 & 101.965 & 116.566 & 114.084 & 83.971 & 77.544 \\
\hline $5 / 13 / 07 \quad 10: 30$ & 132.845 & 130.401 & 126.49 & 106.346 & 110.123 & 102.002 & 116.594 & 114.121 & 83.995 & 77.589 \\
\hline $5 / 13 / 07$ 11:00 & 132.859 & 130.401 & 126.506 & 106.363 & 110.142 & 102.017 & 116.613 & 114.137 & 84.014 & 77.606 \\
\hline $5 / 13 / 07 \quad 11: 30$ & 132.869 & 128.479 & 126.518 & 106.376 & 110.155 & 102.031 & 116.616 & 114.148 & 84.034 & 77.618 \\
\hline $5 / 13 / 07 \quad 12: 00$ & 132.876 & 128.23 & 126.522 & 106.381 & 110.159 & 102.036 & 116.62 & 114.154 & 84.049 & 77.639 \\
\hline $5 / 13 / 07$ 12:30 & 130.899 & 128.089 & 125.134 & 105.688 & 109.641 & 101.371 & 116.052 & 112.718 & 84.036 & 77.604 \\
\hline $5 / 13 / 07$ 13:00 & 130.632 & 127.994 & 124.875 & 105.448 & 109.399 & 101.128 & 115.846 & 112.445 & 84.01 & 77.519 \\
\hline $5 / 13 / 07$ 13:30 & 130.483 & 127.923 & 124.727 & 105.307 & 109.26 & 100.989 & 115.73 & 112.292 & 83.984 & 77.442 \\
\hline $5 / 13 / 07 \quad 14: 00$ & 130.386 & 127.874 & 124.633 & 105.217 & 109.167 & 100.899 & 115.653 & 112.193 & 83.962 & 77.395 \\
\hline 5/13/07 14:30 & 130.317 & 127.828 & 124.563 & 105.15 & 109.1 & 100.828 & 115.596 & 112.122 & 83.938 & 77.333 \\
\hline $5 / 13 / 07$ 15:00 & 130.263 & 127.794 & 124.51 & 105.1 & 109.051 & 100.779 & 115.555 & 112.069 & 83.916 & 77.295 \\
\hline $5 / 13 / 07$ 15:30 & 130.218 & 127.762 & 124.466 & 105.055 & 109.007 & 100.734 & 115.521 & 112.022 & 83.894 & 77.254 \\
\hline 5/13/07 16:00 & 130.182 & 127.742 & 124.429 & 105.021 & 108.971 & 100.701 & 115.489 & 111.989 & 83.876 & 77.215 \\
\hline $5 / 13 / 07$ 16:30 & 130.147 & 127.724 & 124.399 & 104.993 & 108.94 & 100.673 & 115.463 & 111.954 & 83.853 & 77.182 \\
\hline $5 / 13 / 07$ 17:00 & 130.126 & 127.713 & 124.375 & 104.971 & 108.921 & 100.649 & 115.442 & 111.929 & 83.839 & 77.173 \\
\hline
\end{tabular}


TABLE S1.1 (Cont.)

Depth from Top of Casing (ft)

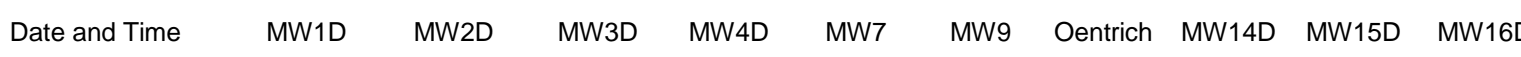

\begin{tabular}{|c|c|c|c|c|c|c|c|c|c|c|}
\hline 5/13/07 17:30 & 130.109 & 127.722 & 124.357 & 104.954 & 108.902 & 100.633 & 115.431 & 111.914 & 83.82 & 77.155 \\
\hline $5 / 13 / 07$ 18:00 & 130.095 & 127.772 & 124.346 & 104.943 & 108.893 & 100.621 & 115.422 & 111.903 & 83.807 & 77.136 \\
\hline $5 / 13 / 07$ 18:30 & 130.09 & 127.744 & 124.343 & 104.961 & 108.889 & 100.63 & 116.09 & 111.894 & 83.793 & 77.124 \\
\hline $5 / 13 / 07 \quad 19: 00$ & 130.161 & 127.713 & 124.434 & 105.069 & 108.98 & 100.743 & 116.881 & 111.954 & 83.826 & 77.13 \\
\hline $5 / 13 / 07$ 19:30 & 130.145 & 127.737 & 124.394 & 104.992 & 108.946 & 100.673 & 115.459 & 111.951 & 83.835 & 77.143 \\
\hline $5 / 13 / 0720: 00$ & 130.102 & 127.708 & 124.35 & 104.95 & 108.9 & 100.63 & 115.604 & 111.905 & 83.811 & 77.134 \\
\hline $5 / 13 / 0720: 30$ & 130.128 & 127.706 & 124.392 & 105.025 & 108.942 & 100.696 & 116.331 & 111.92 & 83.804 & 77.136 \\
\hline $5 / 13 / 0721: 00$ & 130.093 & 127.71 & 124.341 & 104.942 & 108.891 & 100.618 & 115.448 & 111.898 & 83.796 & 77.13 \\
\hline $5 / 13 / 0721: 30$ & 130.088 & 127.715 & 124.339 & 104.937 & 108.889 & 100.614 & 115.303 & 111.896 & 83.789 & 77.132 \\
\hline 5/13/07 22:00 & 130.097 & 127.71 & 124.346 & 104.943 & 108.898 & 100.621 & 115.275 & 111.903 & 83.783 & 77.136 \\
\hline $5 / 13 / 07$ 22:30 & 130.102 & 127.701 & 124.352 & 104.954 & 108.904 & 100.635 & 115.288 & 111.907 & 83.782 & 77.151 \\
\hline 5/13/07 23:00 & 130.095 & 127.692 & 124.341 & 104.943 & 108.896 & 100.621 & 115.215 & 111.901 & 83.776 & 77.173 \\
\hline 5/13/07 23:30 & 130.083 & 127.685 & 124.334 & 104.94 & 108.889 & 100.611 & 115.419 & 111.892 & 83.767 & 77.159 \\
\hline 5/14/07 0:00 & 130.078 & 127.683 & 124.327 & 104.93 & 108.879 & 100.609 & 115.26 & 111.885 & 83.759 & 77.149 \\
\hline 5/14/07 0:30 & 130.067 & 127.669 & 124.318 & 104.923 & 108.874 & 100.6 & 115.232 & 111.876 & 83.75 & 77.151 \\
\hline 5/14/07 1:00 & 130.067 & 127.654 & 124.318 & 104.921 & 108.874 & 100.6 & 115.197 & 111.874 & 83.745 & 77.139 \\
\hline $5 / 14 / 071: 30$ & 130.055 & 127.649 & 124.306 & 104.911 & 108.858 & 100.585 & 115.21 & 111.863 & 83.735 & 77.136 \\
\hline 5/14/07 2:00 & 130.038 & 127.638 & 124.288 & 104.892 & 108.843 & 100.566 & 115.162 & 111.847 & 83.726 & 77.134 \\
\hline $5 / 14 / 072: 30$ & 130.033 & 127.633 & 124.283 & 104.887 & 108.841 & 100.564 & 115.169 & 111.841 & 83.717 & 77.124 \\
\hline 5/14/07 3:00 & 130.029 & 127.624 & 124.272 & 104.875 & 108.828 & 100.552 & 115.152 & 111.832 & 83.71 & 77.122 \\
\hline 5/14/07 3:30 & 130.026 & 127.617 & 124.264 & 104.869 & 108.822 & 100.545 & 115.133 & 111.823 & 83.702 & 77.104 \\
\hline 5/14/07 4:00 & 130.024 & 127.617 & 124.255 & 104.861 & 108.811 & 100.538 & 115.151 & 111.814 & 83.693 & 77.099 \\
\hline 5/14/07 4:30 & 130.026 & 127.61 & 124.251 & 104.856 & 108.807 & 100.531 & 115.142 & 111.808 & 83.686 & 77.093 \\
\hline 5/14/07 5:00 & 130.003 & 127.608 & 124.255 & 104.864 & 108.811 & 100.545 & 115.217 & 111.805 & 83.68 & 77.082 \\
\hline 5/14/07 5:30 & 129.993 & 127.615 & 124.244 & 104.849 & 108.799 & 100.527 & 115.127 & 111.801 & 83.673 & 77.068 \\
\hline 5/14/07 6:00 & 129.989 & 129.502 & 124.239 & 104.844 & 108.797 & 100.524 & 115.094 & 111.799 & 83.667 & 77.066 \\
\hline 5/14/07 6:30 & 129.998 & 129.799 & 124.253 & 104.864 & 108.815 & 100.545 & 115.222 & 111.805 & 83.667 & 77.07 \\
\hline 5/14/07 7:00 & 131.902 & 129.954 & 125.43 & 105.419 & 109.211 & 101.071 & 115.587 & 113.18 & 83.682 & 77.105 \\
\hline 5/14/07 7:30 & 132.226 & 130.049 & 125.754 & 105.726 & 109.508 & 101.38 & 116.073 & 113.521 & 83.723 & 77.19 \\
\hline 5/14/07 8:00 & 132.391 & 130.122 & 125.918 & 105.878 & 109.664 & 101.536 & 116.049 & 113.69 & 83.758 & 77.277 \\
\hline 5/14/07 8:30 & 132.488 & 130.147 & 126.018 & 105.976 & 109.767 & 101.637 & 116.124 & 113.794 & 83.789 & 77.343 \\
\hline 5/14/07 9:00 & 132.557 & 130.176 & 126.085 & 106.05 & 109.833 & 101.703 & 116.501 & 113.864 & 83.817 & 77.399 \\
\hline 5/14/07 9:30 & 132.59 & 130.203 & 126.113 & 106.064 & 109.868 & 101.727 & 116.109 & 113.902 & 83.837 & 77.422 \\
\hline $5 / 14 / 07$ 10:00 & 132.616 & 130.219 & 126.138 & 106.098 & 109.898 & 101.757 & 116.189 & 113.931 & 83.859 & 77.465 \\
\hline $5 / 14 / 07$ 10:30 & 132.642 & 130.23 & 126.173 & 106.129 & 109.929 & 101.786 & 116.213 & 113.96 & 83.881 & 77.486 \\
\hline $5 / 14 / 0711: 00$ & 132.658 & 130.24 & 126.192 & 106.145 & 109.946 & 101.802 & 116.22 & 113.973 & 83.898 & 77.508 \\
\hline 5/14/07 11:30 & 132.67 & 130.249 & 126.203 & 106.16 & 109.963 & 101.816 & 116.192 & 113.993 & 83.92 & 77.533 \\
\hline 5/14/07 12:00 & 132.68 & 130.258 & 126.224 & 106.189 & 109.982 & 101.847 & 116.377 & 113.997 & 83.933 & 77.541 \\
\hline $5 / 14 / 07$ 12:30 & 132.692 & 128.767 & 126.226 & 106.184 & 109.988 & 101.847 & 116.241 & 114.015 & 83.951 & 77.558 \\
\hline 5/14/07 13:00 & 132.701 & 128.243 & 126.238 & 106.193 & 109.999 & 101.856 & 116.204 & 114.024 & 83.966 & 77.575 \\
\hline 5/14/07 13:30 & 131.308 & 128.046 & 125.493 & 105.938 & 109.873 & 101.618 & 116.033 & 113.253 & 83.977 & 77.575 \\
\hline $5 / 14 / 07$ 14:00 & 130.653 & 127.935 & 124.889 & 105.476 & 109.426 & 101.156 & 115.961 & 112.474 & 83.953 & 77.532 \\
\hline $5 / 14 / 07$ 14:30 & 130.443 & 127.858 & 124.681 & 105.267 & 109.23 & 100.951 & 115.438 & 112.259 & 83.929 & 77.459 \\
\hline $5 / 14 / 07$ 15:00 & 130.324 & 127.806 & 124.568 & 105.159 & 109.121 & 100.842 & 115.392 & 112.142 & 83.907 & 77.395 \\
\hline $5 / 14 / 07 \quad 15: 30$ & 130.246 & 127.758 & 124.491 & 105.087 & 109.047 & 100.769 & 115.318 & 112.06 & 83.887 & 77.337 \\
\hline $5 / 14 / 07$ 16:00 & 130.19 & 127.717 & 124.436 & 105.031 & 108.992 & 100.715 & 115.241 & 112.007 & 83.868 & 77.291 \\
\hline 5/14/07 16:30 & 130.145 & 127.683 & 124.387 & 104.985 & 108.948 & 100.67 & 115.183 & 111.958 & 83.848 & 77.25 \\
\hline $5 / 14 / 07 \quad 17: 00$ & 130.107 & 127.642 & 124.355 & 104.957 & 108.914 & 100.642 & 115.277 & 111.918 & 83.826 & 77.211 \\
\hline 5/14/07 17:30 & 130.071 & 127.647 & 124.318 & 104.916 & 108.876 & 100.595 & 115.143 & 111.882 & 83.804 & 77.176 \\
\hline $5 / 14 / 07$ 18:00 & 130.029 & 127.624 & 124.281 & 104.887 & 108.839 & 100.569 & 115.28 & 111.841 & 83.78 & 77.134 \\
\hline $5 / 14 / 07$ 18:30 & 130.029 & 127.622 & 124.279 & 104.88 & 108.837 & 100.56 & 115.111 & 111.839 & 83.768 & 77.134 \\
\hline 5/14/07 19:00 & 130.01 & 127.62 & 124.258 & 104.859 & 108.818 & 100.541 & 115.097 & 111.821 & 83.75 & 77.124 \\
\hline 5/14/07 19:30 & 130.01 & 127.642 & 124.255 & 104.862 & 108.816 & 100.541 & 115.134 & 111.816 & 83.741 & 77.108 \\
\hline $5 / 14 / 0720: 00$ & 130.005 & 127.649 & 124.251 & 104.857 & 108.815 & 100.538 & 115.104 & 111.814 & 83.73 & 77.107 \\
\hline $5 / 14 / 0720: 30$ & 130.022 & 127.683 & 124.269 & 104.878 & 108.834 & 100.555 & 115.143 & 111.83 & 83.73 & 77.124 \\
\hline 5/14/07 21:00 & 130.031 & 127.685 & 124.279 & 104.889 & 108.845 & 100.567 & 115.131 & 111.841 & 83.726 & 77.13 \\
\hline $5 / 14 / 0721: 30$ & 130.071 & 127.697 & 124.318 & 104.928 & 108.885 & 100.609 & 115.168 & 111.878 & 83.735 & 77.165 \\
\hline 5/14/07 22:00 & 130.071 & 127.71 & 124.318 & 104.928 & 108.883 & 100.607 & 115.167 & 111.878 & 83.73 & 77.149 \\
\hline 5/14/07 22:30 & 130.074 & 127.667 & 124.323 & 104.935 & 108.889 & 100.614 & 115.175 & 111.883 & 83.726 & 77.149 \\
\hline 5/14/07 23:00 & 130.102 & 127.665 & 124.353 & 104.964 & 108.917 & 100.64 & 115.204 & 111.912 & 83.735 & 77.18 \\
\hline 5/14/07 23:30 & 130.123 & 127.672 & 124.306 & 104.916 & 108.872 & 100.593 & 115.159 & 111.87 & 83.708 & 77.155 \\
\hline 5/15/07 0:00 & 130.109 & 127.692 & 124.299 & 104.913 & 108.87 & 100.59 & 115.137 & 111.861 & 83.702 & 77.136 \\
\hline 5/15/07 0:30 & 130.097 & 127.685 & 124.306 & 104.919 & 108.874 & 100.6 & 115.149 & 111.863 & 83.702 & 77.114 \\
\hline 5/15/07 1:00 & 130.083 & 127.685 & 124.325 & 104.937 & 108.891 & 100.614 & 115.165 & 111.881 & 83.706 & 77.134 \\
\hline $5 / 15 / 071: 30$ & 130.081 & 127.681 & 124.325 & 104.935 & 108.891 & 100.614 & 115.161 & 111.883 & 83.702 & 77.132 \\
\hline 5/15/07 2:00 & 130.078 & 127.656 & 124.325 & 104.938 & 108.895 & 100.614 & 115.15 & 111.881 & 83.699 & 77.12 \\
\hline
\end{tabular}


TABLE S1.1 (Cont.)

Depth from Top of Casing (ft)

$\begin{array}{lllllllllll}\text { Date and Time } & \text { MW1D } & \text { MW2D } & \text { MW3D } & \text { MW4D } & \text { MW7 } & \text { MW9 } & \text { Oentrich } & \text { MW14D } & \text { MW15D } & \text { MW16 }\end{array}$

\begin{tabular}{|c|c|c|c|c|c|c|c|c|c|c|}
\hline 5/15/07 2:30 & 130.093 & 127.656 & 124.318 & 104.933 & 108.891 & 100.611 & 115.143 & 111.878 & 83.689 & 77.116 \\
\hline 5/15/07 3:00 & 130.102 & 127.674 & 124.292 & 104.906 & 108.862 & 100.585 & 115.124 & 111.856 & 83.676 & 77.114 \\
\hline 5/15/07 3:30 & 130.093 & 127.679 & 124.292 & 104.906 & 108.862 & 100.585 & 115.135 & 111.85 & 83.669 & 77.105 \\
\hline 5/15/07 4:00 & 130.097 & 127.665 & 124.311 & 104.93 & 108.885 & 100.607 & 115.127 & 111.87 & 83.673 & 77.078 \\
\hline $5 / 15 / 074: 30$ & 130.114 & 127.656 & 124.313 & 104.93 & 108.887 & 100.609 & 115.13 & 111.876 & 83.671 & 77.066 \\
\hline 5/15/07 5:00 & 130.116 & 127.66 & 124.306 & 104.923 & 108.879 & 100.602 & 115.108 & 111.872 & 83.669 & 77.066 \\
\hline 5/15/07 5:30 & 130.112 & 127.674 & 124.295 & 104.909 & 108.868 & 100.588 & 115.096 & 111.854 & 83.667 & 77.064 \\
\hline 5/15/07 6:00 & 130.097 & 129.539 & 124.302 & 104.914 & 108.874 & 100.593 & 115.106 & 111.861 & 83.66 & 77.035 \\
\hline 5/15/07 6:30 & 130.09 & 129.838 & 124.311 & 104.93 & 108.885 & 100.607 & 115.113 & 111.872 & 83.658 & 77.023 \\
\hline $5 / 15 / 077: 00$ & 131.715 & 129.958 & 125.477 & 105.485 & 109.279 & 101.139 & 115.581 & 113.227 & 83.689 & 77.016 \\
\hline 5/15/07 7:30 & 132.072 & 130.038 & 125.798 & 105.776 & 109.569 & 101.429 & 115.832 & 113.57 & 83.715 & 77.066 \\
\hline 5/15/07 8:00 & 132.204 & 130.124 & 125.93 & 105.896 & 109.693 & 101.552 & 115.93 & 113.709 & 83.745 & 77.145 \\
\hline 5/15/07 8:30 & 132.289 & 130.206 & 126.011 & 105.976 & 109.778 & 101.635 & 115.997 & 113.794 & 83.772 & 77.203 \\
\hline 5/15/07 9:00 & 132.417 & 130.251 & 126.099 & 106.069 & 109.87 & 101.724 & 116.085 & 113.88 & 83.809 & 77.227 \\
\hline 5/15/07 9:30 & 132.493 & 130.287 & 126.18 & 106.15 & 109.953 & 101.809 & 116.163 & 113.964 & 83.852 & 77.256 \\
\hline $5 / 15 / 07 \quad 10: 00$ & 132.566 & 130.308 & 126.233 & 106.203 & 110.005 & 101.861 & 116.202 & 114.017 & 83.89 & 77.281 \\
\hline $5 / 15 / 07 \quad 10: 30$ & 132.606 & 128.627 & 126.27 & 106.241 & 110.045 & 101.901 & 116.238 & 114.055 & 83.922 & 77.312 \\
\hline 5/15/07 11:00 & 132.635 & 128.268 & 126.293 & 106.265 & 110.068 & 101.922 & 116.245 & 114.079 & 83.949 & 77.445 \\
\hline $5 / 15 / 07 \quad 11: 30$ & 131.258 & 128.109 & 125.301 & 105.84 & 109.801 & 101.522 & 115.896 & 112.934 & 83.966 & 77.662 \\
\hline $5 / 15 / 07$ 12:00 & 130.821 & 128.005 & 124.912 & 105.5 & 109.466 & 101.18 & 115.586 & 112.496 & 83.951 & 77.608 \\
\hline $5 / 15 / 07$ 12:30 & 130.584 & 127.926 & 124.748 & 105.347 & 109.314 & 101.029 & 115.456 & 112.326 & 83.94 & 77.535 \\
\hline 5/15/07 13:00 & 130.417 & 127.869 & 124.648 & 105.248 & 109.211 & 100.925 & 115.37 & 112.217 & 83.929 & 77.477 \\
\hline 5/15/07 13:30 & 130.324 & 127.828 & 124.565 & 105.169 & 109.131 & 100.847 & 115.301 & 112.133 & 83.912 & 77.424 \\
\hline $5 / 15 / 0714: 00$ & 130.263 & 127.792 & 124.51 & 105.114 & 109.075 & 100.791 & 115.255 & 112.075 & 83.899 & 77.376 \\
\hline $5 / 15 / 07 \quad 14: 30$ & 130.225 & 127.762 & 124.47 & 105.076 & 109.037 & 100.755 & 115.225 & 112.038 & 83.885 & 77.354 \\
\hline $5 / 15 / 07$ 15:00 & 130.187 & 127.735 & 124.431 & 105.04 & 108.999 & 100.72 & 115.201 & 111.996 & 83.87 & 77.314 \\
\hline $5 / 15 / 07$ 15:30 & 130.152 & 127.722 & 124.403 & 105.009 & 108.969 & 100.689 & 115.171 & 111.965 & 83.855 & 77.289 \\
\hline 5/15/07 16:00 & 130.128 & 127.701 & 124.375 & 104.987 & 108.944 & 100.668 & 115.148 & 111.94 & 83.84 & 77.275 \\
\hline 5/15/07 16:30 & 130.114 & 127.688 & 124.362 & 104.971 & 108.929 & 100.651 & 115.133 & 111.923 & 83.827 & 77.252 \\
\hline $5 / 15 / 07$ 17:00 & 130.093 & 127.679 & 124.338 & 104.951 & 108.91 & 100.63 & 115.116 & 111.903 & 83.814 & 77.244 \\
\hline $5 / 15 / 07$ 17:30 & 130.078 & 127.665 & 124.325 & 104.94 & 108.896 & 100.618 & 115.105 & 111.887 & 83.802 & 77.235 \\
\hline $5 / 15 / 07$ 18:00 & 130.067 & 127.658 & 124.315 & 104.93 & 108.885 & 100.607 & 115.097 & 111.876 & 83.793 & 77.217 \\
\hline $5 / 15 / 07$ 18:30 & 130.052 & 127.651 & 124.299 & 104.916 & 108.876 & 100.595 & 115.084 & 111.863 & 83.782 & 77.203 \\
\hline 5/15/07 19:00 & 130.045 & 127.647 & 124.294 & 104.909 & 108.868 & 100.588 & 115.08 & 111.858 & 83.774 & 77.205 \\
\hline 5/15/07 19:30 & 130.038 & 127.642 & 124.286 & 104.902 & 108.86 & 100.578 & 115.074 & 111.85 & 83.765 & 77.19 \\
\hline $5 / 15 / 0720: 00$ & 130.033 & 127.638 & 124.283 & 104.899 & 108.856 & 100.576 & 115.072 & 111.847 & 83.759 & 77.192 \\
\hline $5 / 15 / 0720: 30$ & 130.029 & 127.64 & 124.281 & 104.895 & 108.855 & 100.571 & 115.068 & 111.841 & 83.754 & 77.18 \\
\hline $5 / 15 / 0721: 00$ & 130.026 & 127.642 & 124.276 & 104.892 & 108.851 & 100.569 & 115.068 & 111.839 & 83.747 & 77.18 \\
\hline $5 / 15 / 0721: 30$ & 130.026 & 127.64 & 124.276 & 104.894 & 108.853 & 100.571 & 115.07 & 111.839 & 83.743 & 77.178 \\
\hline 5/15/07 22:00 & 130.029 & 127.64 & 124.281 & 104.897 & 108.855 & 100.571 & 115.072 & 111.843 & 83.741 & 77.18 \\
\hline 5/15/07 22:30 & 130.026 & 127.635 & 124.276 & 104.893 & 108.853 & 100.571 & 115.07 & 111.841 & 83.737 & 77.18 \\
\hline 5/15/07 23:00 & 130.024 & 127.635 & 124.276 & 104.893 & 108.853 & 100.569 & 115.07 & 111.839 & 83.734 & 77.18 \\
\hline 5/15/07 23:30 & 130.022 & 127.635 & 124.271 & 104.89 & 108.851 & 100.564 & 115.066 & 111.834 & 83.73 & 77.163 \\
\hline 5/16/07 0:00 & 130.022 & 127.633 & 124.271 & 104.89 & 108.847 & 100.569 & 115.068 & 111.834 & 83.728 & 77.168 \\
\hline 5/16/07 0:30 & 130.024 & 127.626 & 124.274 & 104.893 & 108.853 & 100.569 & 115.07 & 111.836 & 83.726 & 77.176 \\
\hline 5/16/07 1:00 & 130.019 & 127.62 & 124.271 & 104.89 & 108.849 & 100.569 & 115.072 & 111.834 & 83.724 & 77.172 \\
\hline $5 / 16 / 07$ 1:30 & 130.012 & 127.61 & 124.264 & 104.883 & 108.839 & 100.557 & 115.063 & 111.827 & 83.721 & 77.18 \\
\hline 5/16/07 2:00 & 130.005 & 127.599 & 124.258 & 104.876 & 108.834 & 100.552 & 115.057 & 111.821 & 83.717 & 77.157 \\
\hline 5/16/07 2:30 & 129.996 & 127.595 & 124.248 & 104.87 & 108.828 & 100.545 & 115.053 & 111.81 & 83.711 & 77.166 \\
\hline 5/16/07 3:00 & 129.986 & 127.583 & 124.239 & 104.856 & 108.816 & 100.533 & 115.044 & 111.801 & 83.704 & 77.157 \\
\hline 5/16/07 3:30 & 129.981 & 127.579 & 124.23 & 104.852 & 108.811 & 100.527 & 115.04 & 111.794 & 83.7 & 77.147 \\
\hline 5/16/07 4:00 & 129.967 & 127.574 & 124.218 & 104.839 & 108.799 & 100.512 & 115.028 & 111.781 & 83.691 & 77.151 \\
\hline 5/16/07 4:30 & 129.963 & 127.57 & 124.213 & 104.835 & 108.794 & 100.51 & 115.027 & 111.777 & 83.688 & 77.138 \\
\hline 5/16/07 5:00 & 129.96 & 127.565 & 124.214 & 104.832 & 108.794 & 100.508 & 115.025 & 111.774 & 83.682 & 77.144 \\
\hline 5/16/07 5:30 & 129.955 & 127.563 & 124.207 & 104.827 & 108.786 & 100.503 & 115.021 & 111.77 & 83.676 & 77.13 \\
\hline 5/16/07 6:00 & 129.951 & 129.296 & 124.204 & 104.825 & 108.784 & 100.501 & 115.019 & 111.765 & 83.673 & 77.132 \\
\hline $5 / 16 / 076: 30$ & 129.951 & 129.672 & 124.2 & 104.821 & 108.782 & 100.496 & 115.017 & 111.763 & 83.669 & 77.126 \\
\hline 5/16/07 7:00 & 131.703 & 129.849 & 125.356 & 105.307 & 109.093 & 100.953 & 115.434 & 112.928 & 83.676 & 77.126 \\
\hline 5/16/07 7:30 & 132.124 & 129.956 & 125.766 & 105.664 & 109.443 & 101.312 & 115.746 & 113.393 & 83.715 & 77.202 \\
\hline 5/16/07 8:00 & 132.313 & 128.291 & 125.953 & 105.84 & 109.622 & 101.493 & 115.9 & 113.585 & 83.754 & 77.306 \\
\hline 5/16/07 8:30 & 132.427 & 127.971 & 126.064 & 105.947 & 109.733 & 101.597 & 115.993 & 113.701 & 83.787 & 77.388 \\
\hline 5/16/07 9:00 & 130.738 & 127.833 & 124.958 & 105.506 & 109.466 & 101.184 & 115.629 & 112.574 & 83.807 & 77.438 \\
\hline 5/16/07 9:30 & 130.376 & 127.751 & 124.614 & 105.211 & 109.18 & 100.892 & 115.372 & 112.197 & 83.798 & 77.401 \\
\hline $5 / 16 / 07$ 10:00 & 130.23 & 127.697 & 124.473 & 105.078 & 109.047 & 100.76 & 115.259 & 112.049 & 83.789 & 77.339 \\
\hline $5 / 16 / 07 \quad 10: 30$ & 130.14 & 127.658 & 124.387 & 104.995 & 108.963 & 100.673 & 115.186 & 111.958 & 83.778 & 77.306 \\
\hline $5 / 16 / 0711: 00$ & 130.086 & 127.624 & 124.332 & 104.945 & 108.91 & 100.621 & 115.141 & 111.901 & 83.77 & 77.269 \\
\hline
\end{tabular}


TABLE S1.1 (Cont.)

Depth from Top of Casing (ft)

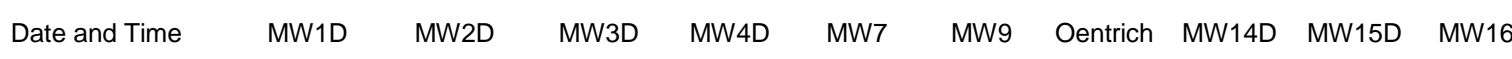

\begin{tabular}{|c|c|c|c|c|c|c|c|c|c|c|}
\hline 5/16/07 11:30 & 130.05 & 127.597 & 124.294 & 104.911 & 108.874 & 100.588 & 115.113 & 111.865 & 83.763 & 77.233 \\
\hline $5 / 16 / 07$ 12:00 & 130.012 & 127.574 & 124.26 & 104.875 & 108.839 & 100.555 & 115.081 & 111.827 & 83.752 & 77.2 \\
\hline 5/16/07 12:30 & 129.986 & 129.244 & 124.232 & 104.849 & 108.815 & 100.527 & 115.058 & 111.801 & 83.743 & 77.178 \\
\hline 5/16/07 13:00 & 129.963 & 129.625 & 124.209 & 104.826 & 108.79 & 100.505 & 115.038 & 111.777 & 83.732 & 77.163 \\
\hline 5/16/07 13:30 & 131.641 & 129.781 & 125.296 & 105.257 & 109.045 & 100.906 & 115.396 & 112.857 & 83.726 & 77.14 \\
\hline $5 / 16 / 07$ 14:00 & 132.077 & 129.877 & 125.712 & 105.616 & 109.397 & 101.264 & 115.712 & 113.349 & 83.754 & 77.207 \\
\hline $5 / 16 / 07$ 14:30 & 132.247 & 128.187 & 125.886 & 105.772 & 109.557 & 101.425 & 115.849 & 113.523 & 83.778 & 77.275 \\
\hline 5/16/07 15:00 & 132.346 & 127.862 & 125.983 & 105.869 & 109.656 & 101.521 & 115.932 & 113.625 & 83.8 & 77.339 \\
\hline 5/16/07 15:30 & 130.625 & 127.715 & 124.85 & 105.399 & 109.363 & 101.081 & 115.537 & 112.465 & 83.806 & 77.374 \\
\hline 5/16/07 16:00 & 130.265 & 127.644 & 124.505 & 105.101 & 109.074 & 100.784 & 115.28 & 112.089 & 83.785 & 77.329 \\
\hline 5/16/07 16:30 & 130.112 & 127.586 & 124.355 & 104.961 & 108.933 & 100.642 & 115.158 & 111.934 & 83.763 & 77.262 \\
\hline 5/16/07 17:00 & 130.031 & 127.542 & 124.271 & 104.883 & 108.851 & 100.562 & 115.088 & 111.85 & 83.744 & 77.207 \\
\hline 5/16/07 17:30 & 129.977 & 127.515 & 124.22 & 104.835 & 108.803 & 100.515 & 115.045 & 111.796 & 83.728 & 77.171 \\
\hline 5/16/07 18:00 & 129.932 & 127.493 & 124.179 & 104.794 & 108.761 & 100.472 & 115.009 & 111.75 & 83.711 & 77.13 \\
\hline 5/16/07 18:30 & 129.903 & 127.474 & 124.149 & 104.764 & 108.733 & 100.444 & 114.987 & 111.719 & 83.696 & 77.101 \\
\hline 5/16/07 19:00 & 129.88 & 127.463 & 124.126 & 104.744 & 108.71 & 100.423 & 114.966 & 111.696 & 83.682 & 77.082 \\
\hline 5/16/07 19:30 & 129.863 & 127.461 & 124.109 & 104.728 & 108.695 & 100.406 & 114.955 & 111.679 & 83.671 & 77.074 \\
\hline 5/16/07 20:00 & 129.851 & 127.463 & 124.096 & 104.714 & 108.683 & 100.394 & 114.943 & 111.665 & 83.658 & 77.066 \\
\hline $5 / 16 / 0720: 30$ & 129.849 & 127.474 & 124.096 & 104.714 & 108.681 & 100.394 & 114.943 & 111.664 & 83.651 & 77.057 \\
\hline 5/16/07 21:00 & 129.849 & 127.481 & 124.098 & 104.72 & 108.685 & 100.399 & 114.947 & 111.666 & 83.645 & 77.063 \\
\hline 5/16/07 21:30 & 129.859 & 127.486 & 124.107 & 104.73 & 108.697 & 100.406 & 114.957 & 111.677 & 83.643 & 77.074 \\
\hline 5/16/07 22:00 & 129.866 & 127.49 & 124.114 & 104.737 & 108.702 & 100.413 & 114.964 & 111.684 & 83.639 & 77.068 \\
\hline 5/16/07 22:30 & 129.87 & 127.495 & 124.119 & 104.742 & 108.708 & 100.42 & 114.97 & 111.688 & 83.637 & 77.078 \\
\hline 5/16/07 23:00 & 129.875 & 127.495 & 124.123 & 104.749 & 108.716 & 100.427 & 114.974 & 111.695 & 83.636 & 77.086 \\
\hline 5/16/07 23:30 & 129.88 & 127.493 & 124.128 & 104.752 & 108.719 & 100.432 & 114.977 & 111.699 & 83.634 & 77.08 \\
\hline 5/17/07 0:00 & 129.88 & 127.493 & 124.128 & 104.754 & 108.719 & 100.43 & 114.975 & 111.699 & 83.63 & 77.074 \\
\hline 5/17/07 0:30 & 129.877 & 127.495 & 124.126 & 104.751 & 108.716 & 100.427 & 114.974 & 111.697 & 83.626 & 77.088 \\
\hline 5/17/07 1:00 & 129.877 & 127.493 & 124.126 & 104.752 & 108.719 & 100.432 & 114.975 & 111.697 & 83.626 & 77.078 \\
\hline 5/17/07 1:30 & 129.882 & 127.49 & 124.128 & 104.756 & 108.721 & 100.434 & 114.981 & 111.701 & 83.625 & 77.088 \\
\hline 5/17/07 2:00 & 129.88 & 127.493 & 124.128 & 104.754 & 108.721 & 100.435 & 114.977 & 111.699 & 83.623 & 77.084 \\
\hline 5/17/07 2:30 & 129.875 & 127.49 & 124.126 & 104.752 & 108.717 & 100.43 & 114.975 & 111.695 & 83.619 & 77.082 \\
\hline 5/17/07 3:00 & 129.877 & 127.488 & 124.126 & 104.756 & 108.721 & 100.432 & 114.977 & 111.697 & 83.617 & 77.082 \\
\hline 5/17/07 3:30 & 129.875 & 127.493 & 124.123 & 104.752 & 108.719 & 100.432 & 114.977 & 111.695 & 83.615 & 77.078 \\
\hline 5/17/07 4:00 & 129.875 & 127.49 & 124.126 & 104.754 & 108.717 & 100.432 & 114.977 & 111.695 & 83.614 & 77.084 \\
\hline 5/17/07 4:30 & 129.877 & 127.49 & 124.126 & 104.756 & 108.721 & 100.432 & 114.977 & 111.697 & 83.612 & 77.084 \\
\hline 5/17/07 5:00 & 129.875 & 127.49 & 124.124 & 104.754 & 108.719 & 100.432 & 114.975 & 111.695 & 83.608 & 77.084 \\
\hline 5/17/07 5:30 & 129.875 & 127.497 & 124.121 & 104.752 & 108.717 & 100.43 & 114.973 & 111.695 & 83.606 & 77.086 \\
\hline 5/17/07 6:00 & 129.877 & 129.341 & 124.126 & 104.756 & 108.721 & 100.432 & 114.979 & 111.697 & 83.608 & 77.101 \\
\hline 5/17/07 6:30 & 129.88 & 129.661 & 124.13 & 104.761 & 108.729 & 100.439 & 114.983 & 111.701 & 83.608 & 77.101 \\
\hline 5/17/07 7:00 & 131.767 & 129.829 & 125.409 & 105.348 & 109.135 & 100.996 & 115.486 & 113.016 & 83.626 & 77.113 \\
\hline 5/17/07 7:30 & 132.114 & 129.938 & 125.752 & 105.655 & 109.439 & 101.309 & 115.755 & 113.388 & 83.669 & 77.216 \\
\hline 5/17/07 8:00 & 132.294 & 130.004 & 125.932 & 105.83 & 109.616 & 101.481 & 115.901 & 113.574 & 83.713 & 77.32 \\
\hline 5/17/07 8:30 & 132.403 & 130.058 & 126.041 & 105.935 & 109.723 & 101.587 & 115.993 & 113.687 & 83.75 & 77.396 \\
\hline 5/17/07 9:00 & 132.474 & 130.092 & 126.113 & 106.002 & 109.793 & 101.656 & 116.055 & 113.76 & 83.783 & 77.458 \\
\hline 5/17/07 9:30 & 132.531 & 130.103 & 126.166 & 106.059 & 109.851 & 101.71 & 116.102 & 113.818 & 83.816 & 77.512 \\
\hline $5 / 17 / 07$ 10:00 & 132.566 & 130.117 & 126.205 & 106.093 & 109.885 & 101.745 & 116.134 & 113.853 & 83.846 & 77.525 \\
\hline $5 / 17 / 07 \quad 10: 30$ & 132.58 & 130.124 & 126.219 & 106.107 & 109.9 & 101.762 & 116.145 & 113.869 & 83.87 & 77.537 \\
\hline 5/17/07 11:00 & 132.595 & 130.165 & 126.233 & 106.122 & 109.915 & 101.779 & 116.159 & 113.884 & 83.892 & 77.576 \\
\hline 5/17/07 11:30 & 132.602 & 128.271 & 126.24 & 106.127 & 109.925 & 101.786 & 116.166 & 113.893 & 83.914 & 77.589 \\
\hline 5/17/07 12:00 & 132.639 & 127.982 & 126.27 & 106.154 & 109.946 & 101.809 & 116.185 & 113.933 & 83.932 & 77.603 \\
\hline $5 / 17 / 07$ 12:30 & 130.693 & 127.828 & 124.921 & 105.493 & 109.468 & 101.177 & 115.628 & 112.529 & 83.927 & 77.601 \\
\hline 5/17/07 13:00 & 130.386 & 127.728 & 124.623 & 105.219 & 109.195 & 100.901 & 115.39 & 112.215 & 83.903 & 77.5 \\
\hline 5/17/07 13:30 & 130.227 & 127.649 & 124.466 & 105.073 & 109.047 & & 115.262 & 112.053 & & 77.415 \\
\hline $5 / 17 / 07$ 14:00 & 130.123 & 127.592 & 124.362 & 104.975 & 108.948 & 100.654 & 115.178 & 111.947 & 83.861 & 77.345 \\
\hline $5 / 17 / 07$ 14:30 & 130.041 & 127.549 & 124.283 & 104.897 & 108.87 & 100.576 & 115.11 & 111.863 & 83.838 & 77.274 \\
\hline 5/17/07 15:00 & 129.981 & 127.508 & 124.225 & 104.842 & 108.811 & 100.522 & 115.062 & 111.801 & 83.816 & 77.221 \\
\hline 5/17/07 15:30 & 129.939 & 127.474 & 124.181 & 104.799 & 108.767 & 100.477 & 115.024 & 111.757 & 83.796 & 77.181 \\
\hline 5/17/07 16:00 & 129.896 & 127.447 & 124.142 & 104.759 & 108.729 & 100.439 & 114.99 & 111.714 & 83.776 & 77.144 \\
\hline 5/17/07 16:30 & 129.863 & 127.427 & 124.107 & 104.728 & 108.697 & 100.406 & 114.962 & 111.681 & 83.755 & 77.121 \\
\hline 5/17/07 17:00 & 129.835 & 127.413 & 124.079 & 104.701 & 108.668 & 100.38 & 114.94 & 111.653 & 83.735 & 77.101 \\
\hline $5 / 17 / 07$ 17:30 & 129.814 & 127.397 & 124.059 & 104.682 & 108.651 & 100.361 & 114.921 & 111.63 & 83.715 & 77.078 \\
\hline 5/17/07 18:00 & 129.799 & 127.381 & 124.042 & 104.668 & 108.636 & 100.345 & 114.909 & 111.615 & 83.7 & 77.068 \\
\hline 5/17/07 18:30 & 129.783 & 127.377 & 124.028 & 104.653 & 108.624 & 100.333 & 114.896 & 111.601 & 83.684 & 77.043 \\
\hline 5/17/07 19:00 & 129.766 & 127.37 & 124.015 & 104.639 & 108.607 & 100.317 & 114.891 & 111.584 & 83.669 & 77.059 \\
\hline 5/17/07 19:30 & 129.762 & 127.372 & 124.008 & 104.634 & 108.603 & 100.312 & 114.876 & 111.577 & 83.656 & 77.045 \\
\hline 5/17/07 20:00 & 129.757 & 127.37 & 124.003 & 104.628 & 108.599 & 100.307 & 114.877 & 111.573 & 83.647 & 77.039 \\
\hline
\end{tabular}


TABLE S1.1 (Cont.)

Depth from Top of Casing (ft)

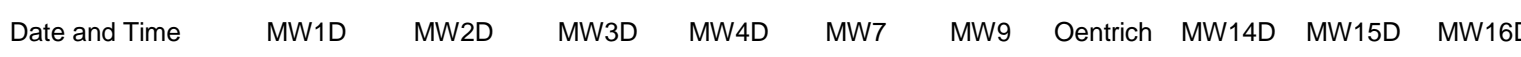

\begin{tabular}{|c|c|c|c|c|c|c|c|c|c|c|}
\hline 5/17/07 20:30 & 129.762 & 127.37 & 124.01 & 104.637 & 108.607 & 100.317 & 114.912 & 111.575 & 83.638 & 77.037 \\
\hline $5 / 17 / 0721: 00$ & 129.752 & 127.375 & 124.001 & 104.628 & 108.598 & 100.307 & 114.883 & 111.568 & 83.63 & 77.024 \\
\hline 5/17/07 21:30 & 129.752 & 127.377 & 124.003 & 104.63 & 108.601 & 100.31 & 114.881 & 111.573 & 83.623 & 77.026 \\
\hline 5/17/07 22:00 & 129.759 & 127.375 & 124.008 & 104.637 & 108.607 & 100.317 & 114.884 & 111.579 & 83.617 & 77.035 \\
\hline 5/17/07 22:30 & 129.762 & 127.37 & 124.01 & 104.638 & 108.607 & 100.317 & 114.886 & 111.582 & 83.612 & 77.024 \\
\hline 5/17/07 23:00 & 129.759 & 127.363 & 124.008 & 104.637 & 108.609 & 100.317 & 114.884 & 111.577 & 83.606 & 77.024 \\
\hline 5/17/07 23:30 & 129.754 & 127.359 & 124.003 & 104.632 & 108.603 & 100.309 & 114.88 & 111.575 & 83.603 & 77.037 \\
\hline 5/18/07 0:00 & 129.75 & 127.347 & 123.994 & 104.625 & 108.594 & 100.305 & 114.874 & 111.568 & 83.597 & 77.028 \\
\hline 5/18/07 0:30 & 129.743 & 127.338 & 123.991 & 104.62 & 108.592 & 100.298 & 114.869 & 111.562 & 83.594 & 77.014 \\
\hline 5/18/07 1:00 & 129.733 & 127.331 & 123.98 & 104.609 & 108.582 & 100.288 & 114.859 & 111.551 & 83.584 & 77.01 \\
\hline 5/18/07 1:30 & 129.724 & 127.325 & 123.971 & 104.599 & 108.569 & 100.279 & 114.851 & 111.54 & 83.577 & 76.993 \\
\hline 5/18/07 2:00 & 129.717 & 127.318 & 123.964 & 104.594 & 108.565 & 100.274 & 114.846 & 111.535 & 83.57 & 76.991 \\
\hline 5/18/07 2:30 & 129.71 & 127.311 & 123.957 & 104.589 & 108.559 & 100.267 & 114.84 & 111.529 & 83.564 & 76.987 \\
\hline 5/18/07 3:00 & 129.702 & 127.307 & 123.95 & 104.582 & 108.552 & 100.26 & 114.833 & 111.52 & 83.557 & 76.985 \\
\hline 5/18/07 3:30 & 129.697 & 127.302 & 123.945 & 104.575 & 108.544 & 100.255 & 114.827 & 111.515 & 83.549 & 76.981 \\
\hline 5/18/07 4:00 & 129.691 & 127.307 & 123.938 & 104.57 & 108.54 & 100.248 & 114.821 & 111.511 & 83.543 & 76.985 \\
\hline 5/18/07 4:30 & 129.686 & 127.302 & 123.936 & 104.568 & 108.538 & 100.248 & 114.82 & 111.509 & 83.536 & 76.965 \\
\hline 5/18/07 5:00 & 129.688 & 127.3 & 123.936 & 104.568 & 108.538 & 100.248 & 114.821 & 111.509 & 83.531 & 76.973 \\
\hline 5/18/07 5:30 & 129.686 & 127.304 & 123.934 & 104.568 & 108.538 & 100.248 & 114.819 & 111.506 & 83.527 & 76.981 \\
\hline 5/18/07 6:00 & 129.684 & 129.205 & 123.931 & 104.566 & 108.537 & 100.246 & 114.817 & 111.504 & 83.521 & 76.971 \\
\hline 5/18/07 6:30 & 129.686 & 129.475 & 123.934 & 104.568 & 108.542 & 100.248 & 114.821 & 111.509 & 83.518 & 76.973 \\
\hline 5/18/07 7:00 & 131.611 & 129.616 & 125.148 & 105.173 & 108.978 & 100.826 & 115.336 & 112.912 & 83.536 & 77.012 \\
\hline 5/18/07 7:30 & 131.899 & 129.709 & 125.432 & 105.447 & 109.239 & 101.088 & 115.632 & 113.215 & 83.573 & 77.091 \\
\hline 5/18/07 8:00 & 132.051 & 129.768 & 125.583 & 105.578 & 109.392 & 101.236 & 115.693 & 113.373 & 83.606 & 77.18 \\
\hline 5/18/07 8:30 & 132.143 & 128.15 & 125.675 & 105.666 & 109.479 & 101.326 & 115.768 & 113.468 & 83.637 & 77.25 \\
\hline 5/18/07 9:00 & 132.204 & 127.783 & 125.738 & 105.73 & 109.544 & 101.389 & 115.824 & 113.535 & 83.663 & 77.303 \\
\hline 5/18/07 9:30 & 130.599 & 127.631 & 124.815 & 105.356 & 109.327 & 101.043 & 115.514 & 112.467 & 83.682 & 77.337 \\
\hline 5/18/07 10:00 & 130.182 & 127.536 & 124.419 & 105.026 & 109.005 & 100.708 & 115.229 & 112.013 & 83.673 & 77.297 \\
\hline $5 / 18 / 07 \quad 10: 30$ & 130.022 & 127.481 & 124.264 & 104.88 & 108.86 & 100.562 & 115.098 & 111.852 & 83.658 & 77.24 \\
\hline 5/18/07 11:00 & 129.925 & 127.433 & 124.165 & 104.789 & 108.765 & 100.47 & 115.02 & 111.752 & 83.647 & 77.186 \\
\hline $5 / 18 / 0711: 30$ & 129.866 & 127.397 & 124.109 & 104.735 & 108.71 & 100.413 & 114.971 & 111.695 & 83.637 & 77.142 \\
\hline $5 / 18 / 07$ 12:00 & 129.816 & 127.368 & 124.061 & 104.688 & 108.662 & 100.369 & 114.932 & 111.641 & 83.627 & 77.105 \\
\hline 5/18/07 12:30 & 129.778 & 127.34 & 124.024 & 104.654 & 108.624 & 100.333 & 114.9 & 111.603 & 83.617 & 77.07 \\
\hline 5/18/07 13:00 & 129.747 & 127.313 & 123.994 & 104.622 & 108.596 & 100.303 & 114.872 & 111.568 & 83.604 & 77.043 \\
\hline 5/18/07 13:30 & 129.719 & 127.295 & 123.968 & 104.596 & 108.567 & 100.281 & 114.849 & 111.544 & 83.59 & 77.018 \\
\hline $5 / 18 / 07$ 14:00 & 129.698 & 127.277 & 123.947 & 104.575 & 108.548 & 100.253 & 114.829 & 111.52 & 83.578 & 77.008 \\
\hline $5 / 18 / 07$ 14:30 & 129.679 & 127.261 & 123.924 & 104.554 & 108.527 & 100.234 & 114.814 & 111.5 & 83.564 & 76.985 \\
\hline $5 / 18 / 07$ 15:00 & 129.66 & 127.245 & 123.908 & 104.537 & 108.51 & 100.215 & 114.795 & 111.48 & 83.555 & 76.975 \\
\hline 5/18/07 15:30 & 129.643 & 127.241 & 123.892 & 104.522 & 108.497 & 100.201 & 114.784 & 111.467 & 83.54 & 76.954 \\
\hline $5 / 18 / 07$ 16:00 & 129.627 & 127.225 & 123.876 & 104.508 & 108.481 & 100.187 & 114.77 & 111.451 & 83.527 & 76.946 \\
\hline 5/18/07 16:30 & 129.62 & 127.22 & 123.869 & 104.499 & 108.472 & 100.178 & 114.763 & 111.444 & 83.516 & 76.925 \\
\hline $5 / 18 / 07$ 17:00 & 129.608 & 127.211 & 123.857 & 104.489 & 108.46 & 100.17 & 114.754 & 111.431 & 83.503 & 76.913 \\
\hline $5 / 18 / 07 \quad 17: 30$ & 129.601 & 127.198 & 123.848 & 104.48 & 108.455 & 100.161 & 114.746 & 111.422 & 83.494 & 76.909 \\
\hline $5 / 18 / 07$ 18:00 & 129.589 & 127.191 & 123.839 & 104.473 & 108.447 & 100.154 & 114.739 & 111.416 & 83.483 & 76.898 \\
\hline $5 / 18 / 07$ 18:30 & 129.577 & 127.186 & 123.825 & 104.459 & 108.434 & 100.137 & 114.72 & 111.402 & 83.47 & 76.894 \\
\hline 5/18/07 19:00 & 129.57 & 127.182 & 123.82 & 104.454 & 108.43 & 100.135 & 114.704 & 111.396 & 83.457 & 76.87 \\
\hline 5/18/07 19:30 & 129.565 & 127.182 & 123.816 & 104.451 & 108.428 & 100.13 & 114.699 & 111.391 & 83.449 & 76.878 \\
\hline $5 / 18 / 0720: 00$ & 129.563 & 127.182 & 123.811 & 104.447 & 108.42 & 100.126 & 114.688 & 111.385 & 83.44 & 76.865 \\
\hline 5/18/07 20:30 & 129.563 & 127.186 & 123.811 & 104.447 & 108.422 & 100.128 & 114.688 & 111.385 & 83.433 & 76.876 \\
\hline $5 / 18 / 0721: 00$ & 129.561 & 127.191 & 123.811 & 104.447 & 108.424 & 100.126 & 114.688 & 111.385 & 83.425 & 76.857 \\
\hline 5/18/07 21:30 & 129.565 & 127.193 & 123.814 & 104.453 & 108.428 & 100.133 & 114.693 & 111.389 & 83.422 & 76.853 \\
\hline 5/18/07 22:00 & 129.57 & 127.195 & 123.818 & 104.458 & 108.432 & 100.137 & 114.699 & 111.393 & 83.418 & 76.865 \\
\hline 5/18/07 22:30 & 129.572 & 127.198 & 123.825 & 104.461 & 108.438 & 100.142 & 114.703 & 111.4 & 83.413 & 76.853 \\
\hline 5/18/07 23:00 & 129.575 & 127.195 & 123.825 & 104.465 & 108.439 & 100.142 & 114.704 & 111.402 & 83.409 & 76.863 \\
\hline 5/18/07 23:30 & 129.575 & 127.193 & 123.825 & 104.465 & 108.439 & 100.144 & 114.704 & 111.402 & 83.405 & 76.865 \\
\hline $5 / 19 / 070: 00$ & 129.575 & 127.193 & 123.825 & 104.463 & 108.436 & 100.142 & 114.703 & 111.4 & 83.401 & 76.859 \\
\hline 5/19/07 0:30 & 129.572 & 127.191 & 123.823 & 104.461 & 108.436 & 100.14 & 114.704 & 111.4 & 83.398 & 76.857 \\
\hline 5/19/07 1:00 & 129.57 & 127.189 & 123.823 & 104.461 & 108.435 & 100.14 & 114.703 & 111.398 & 83.392 & 76.845 \\
\hline $5 / 19 / 071: 30$ & 129.572 & 127.182 & 123.821 & 104.46 & 108.434 & 100.137 & 114.703 & 111.396 & 83.389 & 76.843 \\
\hline 5/19/07 2:00 & 129.568 & 127.179 & 123.813 & 104.453 & 108.428 & 100.133 & 114.699 & 111.393 & 83.383 & 76.838 \\
\hline 5/19/07 2:30 & 129.561 & 127.18 & 123.809 & 104.449 & 108.428 & 100.128 & 114.695 & 111.385 & 83.378 & 76.828 \\
\hline 5/19/07 3:00 & 129.558 & 127.175 & 123.806 & 104.447 & 108.422 & 100.126 & 114.697 & 111.382 & 83.374 & 76.826 \\
\hline 5/19/07 3:30 & 129.558 & 127.168 & 123.809 & 104.447 & 108.424 & 100.13 & 114.697 & 111.385 & 83.372 & 76.824 \\
\hline 5/19/07 4:00 & 129.553 & 127.166 & 123.804 & 104.444 & 108.419 & 100.121 & 114.695 & 111.38 & 83.365 & 76.82 \\
\hline 5/19/07 4:30 & 129.549 & 127.166 & 123.799 & 104.439 & 108.417 & 100.116 & 114.691 & 111.376 & 83.361 & 76.816 \\
\hline 5/19/07 5:00 & 129.546 & 127.164 & 123.797 & 104.437 & 108.415 & 100.118 & 114.691 & 111.374 & 83.355 & 76.816 \\
\hline
\end{tabular}


TABLE S1.1 (Cont.)

Depth from Top of Casing (ft)

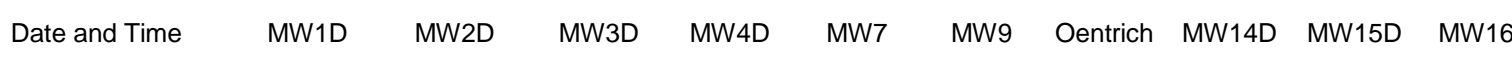

\begin{tabular}{|c|c|c|c|c|c|c|c|c|c|c|}
\hline 5/19/07 5:30 & 129.544 & 127.166 & 123.795 & 104.437 & 108.413 & 100.116 & 114.691 & 111.374 & 83.352 & 76.816 \\
\hline 5/19/07 6:00 & 129.544 & 127.168 & 123.795 & 104.434 & 108.413 & 100.116 & 114.689 & 111.371 & 83.35 & 76.814 \\
\hline 5/19/07 6:30 & 129.544 & 128.788 & 123.795 & 104.435 & 108.409 & 100.116 & 114.689 & 111.369 & 83.344 & 76.816 \\
\hline 5/19/07 7:00 & 129.546 & 129.273 & 123.799 & 104.441 & 108.419 & 100.121 & 114.693 & 111.374 & 83.344 & 76.818 \\
\hline 5/19/07 7:30 & 131.128 & 129.459 & 124.794 & 104.823 & 108.626 & 100.47 & 115.012 & 112.288 & 83.348 & 76.82 \\
\hline 5/19/07 8:00 & 131.72 & 129.575 & 125.354 & 105.266 & 109.056 & 100.918 & 115.409 & 112.992 & 83.387 & 76.892 \\
\hline 5/19/07 8:30 & 131.916 & 129.657 & 125.55 & 105.454 & 109.245 & 101.104 & 115.572 & 113.196 & 83.42 & 76.983 \\
\hline 5/19/07 9:00 & 132.036 & 129.72 & 125.671 & 105.568 & 109.367 & 101.224 & 115.674 & 113.322 & 83.455 & 77.06 \\
\hline 5/19/07 9:30 & 132.119 & 129.761 & 125.754 & 105.649 & 109.445 & 101.304 & 115.743 & 113.406 & 83.484 & 77.147 \\
\hline 5/19/07 10:00 & 132.183 & 129.786 & 125.814 & 105.709 & 109.512 & 101.366 & 115.796 & 113.473 & 83.514 & 77.186 \\
\hline $5 / 19 / 07 \quad 10: 30$ & 132.223 & 129.811 & 125.858 & 105.75 & 109.552 & 101.406 & 115.83 & 113.515 & 83.542 & 77.215 \\
\hline 5/19/07 11:00 & 132.254 & 129.861 & 125.886 & 105.778 & 109.58 & 101.434 & 115.856 & 113.548 & 83.564 & 77.25 \\
\hline $5 / 19 / 07 \quad 11: 30$ & 132.28 & 129.883 & 125.914 & 105.803 & 109.609 & 101.458 & 115.878 & 113.57 & 83.592 & 77.277 \\
\hline $5 / 19 / 07 \quad 12: 00$ & 132.327 & 129.868 & 125.983 & 105.914 & 109.685 & 101.564 & 117.047 & 113.608 & 83.619 & 77.306 \\
\hline $5 / 19 / 07 \quad 12: 30$ & 132.36 & 129.858 & 125.997 & 105.888 & 109.693 & 101.55 & 116.019 & 113.65 & 83.665 & 77.335 \\
\hline 5/19/07 13:00 & 132.337 & 129.861 & 125.971 & 105.857 & 109.668 & 101.517 & 115.914 & 113.632 & 83.673 & 77.343 \\
\hline 5/19/07 13:30 & 132.332 & 129.856 & 125.965 & 105.867 & 109.658 & 101.51 & 115.935 & 113.627 & 83.685 & 77.368 \\
\hline $5 / 19 / 07$ 14:00 & 132.332 & 128.021 & 125.967 & 105.862 & 109.662 & 101.524 & 116.11 & 113.628 & 83.704 & 77.368 \\
\hline $5 / 19 / 07$ 14:30 & 132.332 & 127.708 & 125.967 & 105.854 & 109.664 & 101.514 & 115.962 & 113.628 & 83.719 & 77.374 \\
\hline $5 / 19 / 07$ 15:00 & 130.447 & 127.551 & 124.671 & 105.237 & 109.228 & 100.925 & 115.347 & 112.292 & 83.713 & 77.362 \\
\hline 5/19/07 15:30 & 130.107 & 127.449 & 124.341 & 104.943 & 108.936 & 100.63 & 115.114 & 111.945 & 83.7 & 77.266 \\
\hline $5 / 19 / 07$ 16:00 & 129.941 & 127.375 & 124.176 & 104.792 & 108.786 & 100.477 & 115.008 & 111.774 & 83.678 & 77.178 \\
\hline 5/19/07 16:30 & 129.837 & 127.32 & 124.077 & 104.692 & 108.683 & 100.38 & 114.927 & 111.668 & 83.656 & 77.099 \\
\hline $5 / 19 / 07 \quad 17: 00$ & 129.762 & 127.279 & 124.005 & 104.628 & 108.615 & 100.317 & 114.956 & 111.591 & 83.634 & 77.035 \\
\hline $5 / 19 / 07 \quad 17: 30$ & 129.702 & 127.245 & 123.948 & 104.57 & 108.554 & 100.253 & 114.83 & 111.533 & 83.612 & 77.002 \\
\hline 5/19/07 18:00 & 129.662 & 127.22 & 123.906 & 104.532 & 108.518 & 100.22 & 114.794 & 111.491 & 83.591 & 76.954 \\
\hline $5 / 19 / 07$ 18:30 & 129.629 & 127.202 & 123.874 & 104.504 & 108.485 & 100.189 & 114.797 & 111.455 & 83.575 & 76.923 \\
\hline 5/19/07 19:00 & 129.603 & 127.182 & 123.846 & 104.473 & 108.458 & 100.161 & 114.741 & 111.429 & 83.555 & 76.896 \\
\hline 5/19/07 19:30 & 129.582 & 127.168 & 123.83 & 104.461 & 108.443 & 100.149 & 114.752 & 111.411 & 83.538 & 76.882 \\
\hline $5 / 19 / 0720: 00$ & 129.565 & 127.17 & 123.811 & 104.441 & 108.424 & 100.128 & 114.709 & 111.391 & 83.521 & 76.865 \\
\hline $5 / 19 / 0720: 30$ & 129.551 & 127.182 & 123.797 & 104.43 & 108.415 & 100.114 & 114.708 & 111.38 & 83.507 & 76.861 \\
\hline $5 / 19 / 0721: 00$ & 129.556 & 127.177 & 123.802 & 104.435 & 108.419 & 100.119 & 114.7 & 111.382 & 83.497 & 76.853 \\
\hline $5 / 19 / 0721: 30$ & 129.563 & 127.179 & 123.809 & 104.447 & 108.426 & 100.128 & 114.716 & 111.393 & 83.494 & 76.847 \\
\hline 5/19/07 22:00 & 129.558 & 127.177 & 123.806 & 104.445 & 108.424 & 100.128 & 114.741 & 111.387 & 83.487 & 76.851 \\
\hline 5/19/07 22:30 & 129.561 & 127.175 & 123.809 & 104.447 & 108.426 & 100.13 & 114.726 & 111.389 & 83.477 & 76.849 \\
\hline 5/19/07 23:00 & 129.558 & 127.166 & 123.806 & 104.446 & 108.426 & 100.128 & 114.714 & 111.389 & 83.472 & 76.838 \\
\hline 5/19/07 23:30 & 129.556 & 127.164 & 123.802 & 104.442 & 108.42 & 100.121 & 114.726 & 111.385 & 83.46 & 76.838 \\
\hline $5 / 20 / 070: 00$ & 129.551 & 127.155 & 123.799 & 104.437 & 108.417 & 100.121 & 114.72 & 111.378 & 83.455 & 76.832 \\
\hline $5 / 20 / 07$ 0:30 & 129.542 & 127.145 & 123.79 & 104.432 & 108.409 & 100.114 & 114.712 & 111.371 & 83.446 & 76.822 \\
\hline 5/20/07 1:00 & 129.537 & 127.152 & 123.783 & 104.422 & 108.401 & 100.104 & 114.704 & 111.365 & 83.437 & 76.812 \\
\hline $5 / 20 / 07 \quad 1: 30$ & 129.525 & 127.152 & 123.774 & 104.413 & 108.394 & 100.097 & 114.692 & 111.354 & 83.429 & 76.801 \\
\hline $5 / 20 / 072: 00$ & 129.532 & 127.148 & 123.781 & 104.422 & 108.401 & 100.104 & 114.695 & 111.36 & 83.424 & 76.805 \\
\hline $5 / 20 / 072: 30$ & 129.53 & 127.136 & 123.781 & 104.423 & 108.401 & 100.104 & 114.695 & 111.362 & 83.418 & 76.805 \\
\hline $5 / 20 / 073: 00$ & 129.527 & 127.132 & 123.777 & 104.416 & 108.398 & 100.102 & 114.689 & 111.358 & 83.413 & 76.805 \\
\hline 5/20/07 3:30 & 129.518 & 127.127 & 123.765 & 104.408 & 108.388 & 100.093 & 114.681 & 111.347 & 83.403 & 76.799 \\
\hline 5/20/07 4:00 & 129.511 & 127.121 & 123.758 & 104.401 & 108.382 & 100.083 & 114.673 & 111.34 & 83.396 & 76.797 \\
\hline $5 / 20 / 074: 30$ & 129.509 & 127.111 & 123.758 & 104.399 & 108.38 & 100.081 & 114.671 & 111.338 & 83.39 & 76.799 \\
\hline 5/20/07 5:00 & 129.501 & 127.107 & 123.749 & 104.392 & 108.373 & 100.074 & 114.667 & 111.331 & 83.385 & 76.781 \\
\hline $5 / 20 / 075: 30$ & 129.492 & 127.107 & 123.742 & 104.384 & 108.365 & 100.062 & 114.657 & 111.32 & 83.376 & 76.783 \\
\hline $5 / 20 / 076: 00$ & 129.487 & 127.102 & 123.735 & 104.379 & 108.359 & 100.06 & 114.648 & 111.316 & 83.37 & 76.768 \\
\hline $5 / 20 / 076: 30$ & 129.487 & 127.105 & 123.737 & 104.38 & 108.363 & 100.062 & 114.642 & 111.316 & 83.366 & 76.776 \\
\hline $5 / 20 / 07$ 7:00 & 129.485 & 129.001 & 123.732 & 104.377 & 108.358 & 100.057 & 114.636 & 111.314 & 83.359 & 76.772 \\
\hline $5 / 20 / 077: 30$ & 129.483 & 129.282 & 123.733 & 104.377 & 108.356 & 100.055 & 114.634 & 111.312 & 83.355 & 76.772 \\
\hline 5/20/07 8:00 & 131.429 & 129.43 & 125.065 & 105.011 & 108.803 & 100.658 & 115.165 & 112.691 & 83.372 & 76.805 \\
\hline $5 / 20 / 078: 30$ & 131.736 & 129.52 & 125.368 & 105.281 & 109.077 & 100.932 & 115.411 & 113.016 & 83.407 & 76.886 \\
\hline 5/20/07 9:00 & 131.892 & 129.586 & 125.523 & 105.43 & 109.23 & 101.083 & 115.545 & 113.176 & 83.444 & 76.983 \\
\hline 5/20/07 9:30 & 131.987 & 129.631 & 125.618 & 105.522 & 109.323 & 101.177 & 115.624 & 113.275 & 83.475 & 77.056 \\
\hline $5 / 20 / 07 \quad 10: 00$ & 132.053 & 129.663 & 125.685 & 105.586 & 109.39 & 101.243 & 115.674 & 113.344 & 83.503 & 77.118 \\
\hline $5 / 20 / 07 \quad 10: 30$ & 132.096 & 129.693 & 125.729 & 105.629 & 109.434 & 101.288 & 115.714 & 113.391 & 83.529 & 77.149 \\
\hline $5 / 20 / 0711: 00$ & 132.133 & 129.715 & 125.763 & 105.661 & 109.47 & 101.319 & 115.744 & 113.426 & 83.553 & 77.186 \\
\hline $5 / 20 / 07$ 11:30 & 132.159 & 129.72 & 125.793 & 105.69 & 109.498 & 101.349 & 115.766 & 113.457 & 83.577 & 77.221 \\
\hline $5 / 20 / 07$ 12:00 & 132.183 & 129.731 & 125.816 & 105.714 & 109.521 & 101.371 & 115.783 & 113.481 & 83.599 & 77.246 \\
\hline $5 / 20 / 0712: 30$ & 132.193 & 129.731 & 125.821 & 105.722 & 109.531 & 101.38 & 115.79 & 113.488 & 83.617 & 77.283 \\
\hline 5/20/07 13:00 & 132.2 & 129.736 & 125.833 & 105.731 & 109.538 & 101.387 & 115.798 & 113.497 & 83.636 & 77.291 \\
\hline $5 / 20 / 07$ 13:30 & 132.204 & 129.738 & 125.837 & 105.735 & 109.544 & 101.392 & 115.802 & 113.501 & 83.651 & 77.318 \\
\hline $5 / 20 / 07$ 14:00 & 132.207 & 128.137 & 125.842 & 105.738 & 109.548 & 101.397 & 115.804 & 113.504 & 83.665 & 77.326 \\
\hline
\end{tabular}


TABLE S1.1 (Cont.)

Depth from Top of Casing (ft)

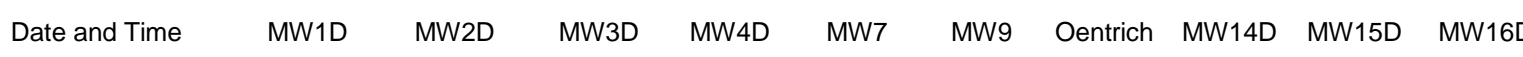

\begin{tabular}{|c|c|c|c|c|c|c|c|c|c|c|}
\hline $5 / 20 / 07$ 14:30 & 132.209 & 127.676 & 125.844 & 105.743 & 109.555 & 101.401 & 115.807 & 113.508 & 83.678 & 77.32 \\
\hline $5 / 20 / 07$ 15:00 & 130.615 & 127.481 & 124.815 & 105.328 & 109.314 & 101.022 & 115.467 & 112.501 & 83.685 & 77.341 \\
\hline 5/20/07 15:30 & 130.083 & 127.365 & 124.313 & 104.918 & 108.913 & 100.604 & 115.1 & 111.923 & 83.66 & 77.269 \\
\hline $5 / 20 / 07$ 16:00 & 129.877 & 127.281 & 124.112 & 104.73 & 108.723 & 100.416 & 114.937 & 111.715 & 83.632 & 77.178 \\
\hline $5 / 20 / 07 \quad 16: 30$ & 129.752 & 127.218 & 123.992 & 104.616 & 108.605 & 100.305 & 114.837 & 111.588 & 83.606 & 77.087 \\
\hline $5 / 20 / 07$ 17:00 & 129.667 & 127.175 & 123.906 & 104.535 & 108.523 & 100.22 & 114.765 & 111.502 & 83.581 & 77.008 \\
\hline $5 / 20 / 07$ 17:30 & 129.603 & 127.136 & 123.843 & 104.475 & 108.462 & 100.161 & 114.708 & 111.436 & 83.558 & 76.963 \\
\hline $5 / 20 / 07$ 18:00 & 129.556 & 127.098 & 123.799 & 104.433 & 108.422 & 100.119 & 114.673 & 111.391 & 83.536 & 76.934 \\
\hline 5/20/07 18:30 & 129.516 & 127.077 & 123.76 & 104.395 & 108.38 & 100.079 & 114.639 & 111.349 & 83.512 & 76.884 \\
\hline 5/20/07 19:00 & 129.48 & 127.066 & 123.725 & 104.361 & 108.344 & 100.046 & 114.607 & 111.314 & 83.49 & 76.851 \\
\hline 5/20/07 19:30 & 129.459 & 127.059 & 123.702 & 104.341 & 108.325 & 100.027 & 114.588 & 111.289 & 83.47 & 76.82 \\
\hline 5/20/07 20:00 & 129.447 & 127.05 & 123.691 & 104.33 & 108.316 & 100.013 & 114.579 & 111.278 & 83.453 & 76.791 \\
\hline 5/20/07 20:30 & 129.44 & 127.048 & 123.684 & 104.325 & 108.308 & 100.008 & 114.571 & 111.272 & 83.44 & 76.799 \\
\hline 5/20/07 21:00 & 129.428 & 127.05 & 123.675 & 104.316 & 108.3 & 99.998 & 114.565 & 111.261 & 83.426 & 76.776 \\
\hline $5 / 20 / 0721: 30$ & 129.428 & 127.055 & 123.672 & 104.316 & 108.3 & 99.998 & 114.568 & 111.261 & 83.415 & 76.776 \\
\hline 5/20/07 22:00 & 129.423 & 127.057 & 123.67 & 104.314 & 108.299 & 99.998 & 114.568 & 111.256 & 83.401 & 76.768 \\
\hline 5/20/07 22:30 & 129.435 & 127.057 & 123.682 & 104.327 & 108.31 & 100.005 & 114.575 & 111.265 & 83.398 & 76.774 \\
\hline 5/20/07 23:00 & 129.438 & 127.048 & 123.684 & 104.329 & 108.312 & 100.013 & 114.577 & 111.27 & 83.389 & 76.768 \\
\hline 5/20/07 23:30 & 129.435 & 127.05 & 123.682 & 104.325 & 108.31 & 100.008 & 114.573 & 111.267 & 83.381 & 76.779 \\
\hline 5/21/07 0:00 & 129.428 & 127.043 & 123.675 & 104.321 & 108.304 & 100.003 & 114.567 & 111.261 & 83.374 & 76.772 \\
\hline 5/21/07 0:30 & 129.428 & 127.039 & 123.675 & 104.321 & 108.304 & 100.003 & 114.567 & 111.261 & 83.366 & 76.76 \\
\hline 5/21/07 1:00 & 129.423 & 127.034 & 123.672 & 104.316 & 108.302 & 100.001 & 114.565 & 111.256 & 83.359 & 76.772 \\
\hline 5/21/07 1:30 & 129.414 & 127.023 & 123.663 & 104.309 & 108.295 & 99.991 & 114.558 & 111.25 & 83.35 & 76.745 \\
\hline 5/21/07 2:00 & 129.411 & 127.018 & 123.659 & 104.306 & 108.291 & 99.989 & 114.554 & 111.245 & 83.343 & 76.741 \\
\hline 5/21/07 2:30 & 129.402 & 127.014 & 123.649 & 104.297 & 108.283 & 99.977 & 114.547 & 111.236 & 83.334 & 76.731 \\
\hline 5/21/07 3:00 & 129.395 & 127.005 & 123.642 & 104.29 & 108.274 & 99.972 & 114.537 & 111.23 & 83.326 & 76.725 \\
\hline 5/21/07 3:30 & 129.39 & 126.998 & 123.638 & 104.285 & 108.27 & 99.97 & 114.531 & 111.223 & 83.319 & 76.719 \\
\hline 5/21/07 4:00 & 129.383 & 126.991 & 123.633 & 104.28 & 108.264 & 99.961 & 114.526 & 111.216 & 83.31 & 76.706 \\
\hline 5/21/07 4:30 & 129.379 & 126.994 & 123.626 & 104.273 & 108.259 & 99.954 & 114.522 & 111.21 & 83.304 & 76.712 \\
\hline 5/21/07 5:00 & 129.369 & 127 & 123.619 & 104.267 & 108.251 & 99.949 & 114.512 & 111.203 & 83.295 & 76.712 \\
\hline 5/21/07 5:30 & 129.371 & 126.996 & 123.617 & 104.266 & 108.251 & 99.946 & 114.514 & 111.203 & 83.289 & 76.7 \\
\hline 5/21/07 6:00 & 129.379 & 128.813 & 123.624 & 104.271 & 108.26 & 99.954 & 114.516 & 111.212 & 83.287 & 76.69 \\
\hline 5/21/07 6:30 & 129.374 & 129.133 & 123.621 & 104.27 & 108.255 & 99.951 & 114.516 & 111.208 & 83.28 & 76.683 \\
\hline 5/21/07 7:00 & 131.206 & 129.294 & 124.736 & 104.785 & 108.599 & 100.437 & 114.946 & 112.501 & 83.287 & 76.69 \\
\hline 5/21/07 7:30 & 131.559 & 129.382 & 125.081 & 105.092 & 108.91 & 100.746 & 115.227 & 112.877 & 83.321 & 76.785 \\
\hline 5/21/07 8:00 & 131.724 & 129.448 & 125.243 & 105.247 & 109.066 & 100.904 & 115.359 & 113.047 & 83.35 & 76.874 \\
\hline 5/21/07 8:30 & 131.821 & 129.493 & 125.345 & 105.344 & 109.167 & 101.005 & 115.448 & 113.151 & 83.38 & 76.944 \\
\hline 5/21/07 9:00 & 131.885 & 129.525 & 125.409 & 105.411 & 109.235 & 101.069 & 115.504 & 113.218 & 83.405 & 77.01 \\
\hline 5/21/07 9:30 & 131.928 & 129.55 & 125.456 & 105.457 & 109.287 & 101.116 & 115.545 & 113.267 & 83.431 & 77.076 \\
\hline $5 / 21 / 07$ 10:00 & 131.965 & 129.559 & 125.493 & 105.488 & 109.317 & 101.149 & 115.573 & 113.302 & 83.453 & 77.103 \\
\hline $5 / 21 / 07$ 10:30 & 131.989 & 127.644 & 125.513 & 105.514 & 109.342 & 101.175 & 115.594 & 113.326 & 83.473 & 77.122 \\
\hline 5/21/07 11:00 & 131.999 & 127.402 & 125.53 & 105.528 & 109.357 & 101.187 & 115.605 & 113.342 & 83.492 & 77.144 \\
\hline 5/21/07 11:30 & 130.052 & 127.275 & 124.281 & 104.883 & 108.881 & 100.574 & 115.059 & 111.903 & 83.483 & 77.12 \\
\hline $5 / 21 / 07$ 12:00 & 129.792 & 127.18 & 124.031 & 104.656 & 108.655 & 100.343 & 114.851 & 111.639 & 83.463 & 77.039 \\
\hline $5 / 21 / 07$ 12:30 & 129.66 & 127.121 & 123.897 & 104.532 & 108.529 & 100.218 & 114.747 & 111.5 & 83.448 & 76.971 \\
\hline $5 / 21 / 07$ 13:00 & 129.567 & 127.075 & 123.807 & 104.444 & 108.436 & 100.128 & 114.67 & 111.407 & 83.429 & 76.911 \\
\hline 5/21/07 13:30 & 129.504 & 127.037 & 123.746 & 104.383 & 108.379 & 100.069 & 114.617 & 111.34 & 83.413 & 76.847 \\
\hline $5 / 21 / 0714: 00$ & 129.457 & 127.012 & 123.702 & 104.341 & 108.331 & 100.024 & 114.578 & 111.296 & 83.394 & 76.807 \\
\hline $5 / 21 / 07$ 14:30 & 129.416 & 126.989 & 123.661 & 104.301 & 108.291 & & 114.544 & 111.254 & & 76.764 \\
\hline $5 / 21 / 07$ 15:00 & 129.39 & 126.971 & 123.633 & 104.279 & 108.268 & 99.961 & 114.522 & 111.227 & 83.365 & 76.748 \\
\hline $5 / 21 / 07$ 15:30 & 129.367 & 126.955 & 123.612 & 104.259 & 108.243 & 99.939 & 114.503 & 111.205 & 83.35 & 76.729 \\
\hline $5 / 21 / 07$ 16:00 & 129.35 & 126.941 & 123.594 & 104.239 & 108.224 & 99.923 & 114.486 & 111.185 & 83.335 & 76.706 \\
\hline 5/21/07 16:30 & 129.334 & 126.925 & 123.58 & 104.221 & 108.211 & 99.909 & 114.474 & 111.17 & 83.322 & 76.692 \\
\hline $5 / 21 / 07$ 17:00 & 129.315 & 126.914 & 123.561 & 104.206 & 108.194 & 99.892 & 114.457 & 111.152 & 83.309 & 76.671 \\
\hline $5 / 21 / 07$ 17:30 & 129.303 & 126.901 & 123.55 & 104.196 & 108.188 & 99.878 & 114.446 & 111.141 & 83.295 & 76.657 \\
\hline $5 / 21 / 0718: 00$ & 129.293 & 126.898 & 123.538 & 104.187 & 108.175 & 99.871 & 114.441 & 111.128 & 83.282 & 76.65 \\
\hline $5 / 21 / 07$ 18:30 & 129.279 & 126.891 & 123.524 & 104.172 & 108.163 & 99.857 & 114.409 & 111.117 & 83.275 & 76.636 \\
\hline 5/21/07 19:00 & 129.274 & 126.891 & 123.52 & 104.173 & 108.16 & 99.855 & 114.4 & 111.112 & 83.262 & 76.626 \\
\hline 5/21/07 19:30 & 129.27 & 126.901 & 123.517 & 104.166 & 108.158 & 99.852 & 114.392 & 111.106 & 83.25 & 76.63 \\
\hline $5 / 21 / 0720: 00$ & 129.267 & 126.907 & 123.513 & 104.165 & 108.154 & 99.847 & 114.385 & 111.103 & 83.241 & 76.619 \\
\hline $5 / 21 / 07$ 20:30 & 129.284 & 126.905 & 123.536 & 104.187 & 108.175 & 99.873 & 114.461 & 111.117 & 83.236 & 76.621 \\
\hline 5/21/07 21:00 & 129.282 & 126.905 & 123.527 & 104.177 & 108.171 & 99.862 & 114.388 & 111.119 & 83.234 & 76.646 \\
\hline 5/21/07 21:30 & 129.282 & 126.901 & 123.527 & 104.18 & 108.169 & 99.864 & 114.426 & 111.117 & 83.228 & 76.632 \\
\hline 5/21/07 22:00 & 129.279 & 126.891 & 123.522 & 104.175 & 108.169 & 99.859 & 114.42 & 111.117 & 83.223 & 76.632 \\
\hline $5 / 21 / 07$ 22:30 & 129.277 & 126.878 & 123.527 & 104.175 & 108.169 & 99.862 & 114.405 & 111.115 & 83.215 & 76.626 \\
\hline 5/21/07 23:00 & 129.265 & 126.887 & 123.51 & 104.168 & 108.156 & 99.85 & 114.435 & 111.103 & 83.206 & 76.613 \\
\hline
\end{tabular}


TABLE S1.1 (Cont.)

Depth from Top of Casing (ft)

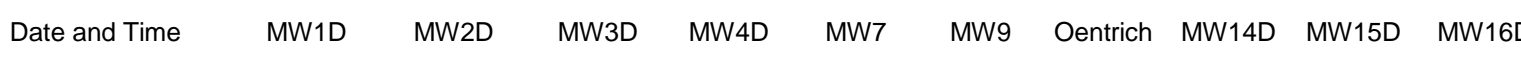

\begin{tabular}{|c|c|c|c|c|c|c|c|c|c|c|}
\hline 5/21/07 23:30 & 129.258 & 126.876 & 123.503 & 104.156 & 108.148 & 99.84 & 114.379 & 111.095 & 83.199 & 76.63 \\
\hline 5/22/07 0:00 & 129.26 & 126.864 & 123.508 & 104.16 & 108.156 & 99.845 & 114.382 & 111.101 & 83.195 & 76.623 \\
\hline 5/22/07 0:30 & 129.251 & 126.857 & 123.494 & 104.161 & 108.137 & 99.833 & 114.481 & 111.09 & 83.188 & 76.609 \\
\hline 5/22/07 1:00 & 129.241 & 126.855 & 123.49 & 104.149 & 108.135 & 99.833 & 114.539 & 111.079 & 83.181 & 76.609 \\
\hline 5/22/07 1:30 & 129.237 & 126.848 & 123.483 & 104.136 & 108.127 & 99.819 & 114.373 & 111.075 & 83.173 & 76.607 \\
\hline 5/22/07 2:00 & 129.23 & 126.839 & 123.478 & 104.144 & 108.125 & 99.819 & 114.571 & 111.068 & 83.169 & 76.617 \\
\hline 5/22/07 2:30 & 129.225 & 126.828 & 123.471 & 104.125 & 108.116 & 99.807 & 114.363 & 111.064 & 83.162 & 76.619 \\
\hline 5/22/07 3:00 & 129.215 & 126.835 & 123.462 & 104.116 & 108.11 & 99.8 & 114.352 & 111.055 & 83.157 & 76.603 \\
\hline 5/22/07 3:30 & 129.204 & 126.835 & 123.453 & 104.106 & 108.097 & 99.789 & 114.356 & 111.041 & 83.147 & 76.592 \\
\hline 5/22/07 4:00 & 129.211 & 126.823 & 123.459 & 104.115 & 108.106 & 99.798 & 114.359 & 111.053 & 83.145 & 76.586 \\
\hline 5/22/07 4:30 & 129.213 & 126.821 & 123.459 & 104.116 & 108.108 & 99.8 & 114.355 & 111.05 & 83.142 & 76.595 \\
\hline 5/22/07 5:00 & 129.199 & 126.839 & 123.448 & 104.104 & 108.095 & 99.786 & 114.341 & 111.037 & 83.136 & 76.584 \\
\hline 5/22/07 5:30 & 129.194 & 126.848 & 123.441 & 104.097 & 108.087 & 99.777 & 114.334 & 111.033 & 83.131 & 76.582 \\
\hline 5/22/07 6:00 & 129.213 & 128.654 & 123.459 & 104.118 & 108.108 & 99.798 & 114.348 & 111.05 & 83.133 & 76.582 \\
\hline 5/22/07 6:30 & 129.225 & 129.005 & 123.471 & 104.128 & 108.12 & 99.81 & 114.355 & 111.064 & 83.133 & 76.595 \\
\hline 5/22/07 7:00 & 131.064 & 129.173 & 124.704 & 104.683 & 108.489 & 100.333 & 114.811 & 112.314 & 83.145 & 76.607 \\
\hline $5 / 22 / 077: 30$ & 131.452 & 129.282 & 125.083 & 105.016 & 108.82 & 100.665 & 115.114 & 112.737 & 83.182 & 76.696 \\
\hline 5/22/07 8:00 & 131.634 & 129.364 & 125.261 & 105.185 & 108.994 & 100.838 & 115.263 & 112.923 & 83.219 & 76.793 \\
\hline 5/22/07 8:30 & 131.743 & 129.409 & 125.375 & 105.293 & 109.106 & 100.951 & 115.361 & 113.039 & 83.254 & 76.884 \\
\hline 5/22/07 9:00 & 131.826 & 127.563 & 125.456 & 105.376 & 109.19 & 101.033 & 115.439 & 113.123 & 83.289 & 76.946 \\
\hline 5/22/07 9:30 & 131.876 & 127.32 & 125.507 & 105.426 & 109.239 & 101.083 & 115.478 & 113.176 & 83.315 & 77.002 \\
\hline 5/22/07 10:00 & 129.974 & 127.186 & 124.207 & 104.811 & 108.816 & 100.503 & 114.95 & 111.834 & 83.321 & 77.018 \\
\hline 5/22/07 10:30 & 129.71 & 127.111 & 123.947 & 104.582 & 108.584 & 100.272 & 114.741 & 111.557 & 83.315 & 76.969 \\
\hline 5/22/07 11:00 & 129.572 & 127.062 & 123.816 & 104.456 & 108.457 & 100.145 & 114.629 & 111.418 & 83.302 & 76.907 \\
\hline 5/22/07 11:30 & 129.494 & 127.023 & 123.735 & 104.383 & 108.38 & 100.069 & 114.567 & 111.338 & 83.293 & 76.843 \\
\hline $5 / 22 / 07$ 12:00 & 129.44 & 126.989 & 123.684 & 104.333 & 108.329 & 100.015 & 114.524 & 111.285 & 83.284 & 76.803 \\
\hline 5/22/07 12:30 & 129.402 & 126.969 & 123.645 & 104.296 & 108.287 & 99.977 & 114.49 & 111.243 & 83.276 & 76.776 \\
\hline 5/22/07 13:00 & 129.366 & 126.948 & 123.61 & 104.261 & 108.253 & 99.944 & 114.458 & 111.208 & 83.267 & 76.752 \\
\hline 5/22/07 13:30 & 129.345 & 126.928 & 123.589 & 104.24 & 108.234 & 99.928 & 114.439 & 111.185 & 83.26 & 76.71 \\
\hline $5 / 22 / 07$ 14:00 & 129.324 & 126.91 & 123.568 & 104.223 & 108.217 & 99.909 & 114.422 & 111.165 & 83.251 & 76.692 \\
\hline $5 / 22 / 07$ 14:30 & 129.31 & 126.898 & 123.552 & 104.208 & 108.2 & 99.89 & 114.409 & 111.148 & 83.243 & 76.686 \\
\hline $5 / 22 / 07$ 15:00 & 129.286 & 126.891 & 123.531 & 104.182 & 108.177 & 99.869 & 114.388 & 111.126 & 83.23 & 76.671 \\
\hline $5 / 22 / 07$ 15:30 & 129.274 & 126.876 & 123.524 & 104.177 & 108.167 & 99.857 & 114.375 & 111.117 & 83.225 & 76.652 \\
\hline 5/22/07 16:00 & 129.27 & 126.86 & 123.515 & 104.173 & 108.161 & 99.855 & 114.363 & 111.11 & 83.221 & 76.644 \\
\hline $5 / 22 / 07$ 16:30 & 129.256 & 126.851 & 123.496 & 104.158 & 108.146 & 99.836 & 114.341 & 111.095 & 83.208 & 76.638 \\
\hline $5 / 22 / 07$ 17:00 & 129.234 & 126.844 & 123.483 & 104.137 & 108.129 & 99.819 & 114.326 & 111.077 & 83.199 & 76.623 \\
\hline 5/22/07 17:30 & 129.232 & 126.826 & 123.476 & 104.132 & 108.121 & 99.812 & 114.32 & 111.07 & 83.192 & 76.619 \\
\hline 5/22/07 18:00 & 129.225 & 126.826 & 123.469 & 104.125 & 108.117 & 99.81 & 114.316 & 111.064 & 83.189 & 76.617 \\
\hline 5/22/07 18:30 & 129.203 & 126.823 & 123.45 & 104.108 & 108.099 & 99.789 & 114.299 & 111.046 & 83.179 & 76.638 \\
\hline 5/22/07 19:00 & 129.203 & 126.837 & 123.45 & 104.109 & 108.102 & 99.789 & 114.297 & 111.044 & 83.171 & 76.619 \\
\hline 5/22/07 19:30 & 129.201 & 126.837 & 123.448 & 104.108 & 108.099 & 99.793 & 114.297 & 111.041 & 83.166 & 76.605 \\
\hline 5/22/07 20:00 & 129.215 & 126.837 & 123.459 & 104.12 & 108.114 & 99.805 & 114.31 & 111.053 & 83.168 & 76.626 \\
\hline $5 / 22 / 07$ 20:30 & 129.215 & 126.86 & 123.462 & 104.121 & 108.114 & 99.807 & 114.312 & 111.057 & 83.162 & 76.615 \\
\hline 5/22/07 21:00 & 129.215 & 126.855 & 123.462 & 104.122 & 108.114 & 99.805 & 114.31 & 111.055 & 83.157 & 76.611 \\
\hline 5/22/07 21:30 & 129.232 & 126.866 & 123.48 & 104.142 & 108.133 & 99.826 & 114.325 & 111.07 & 83.16 & 76.613 \\
\hline 5/22/07 22:00 & 129.232 & 126.857 & 123.478 & 104.14 & 108.135 & 99.824 & 114.325 & 111.072 & 83.158 & 76.623 \\
\hline 5/22/07 22:30 & 129.239 & 126.839 & 123.485 & 104.153 & 108.142 & 99.831 & 114.332 & 111.081 & 83.158 & 76.628 \\
\hline 5/22/07 23:00 & 129.232 & 126.846 & 123.483 & 104.144 & 108.137 & 99.829 & 114.326 & 111.075 & 83.155 & 76.616 \\
\hline 5/22/07 23:30 & 129.222 & 126.842 & 123.469 & 104.127 & 108.12 & 99.812 & 114.311 & 111.061 & 83.147 & 76.605 \\
\hline 5/23/07 0:00 & 129.222 & 126.855 & 123.469 & 104.135 & 108.127 & 99.814 & 114.319 & 111.064 & 83.145 & 76.59 \\
\hline 5/23/07 0:30 & 129.222 & 126.876 & 123.467 & 104.13 & 108.121 & 99.812 & 114.319 & 111.061 & 83.14 & 76.599 \\
\hline 5/23/07 1:00 & 129.227 & 126.878 & 123.473 & 104.139 & 108.133 & 99.819 & 114.324 & 111.064 & 83.142 & 76.601 \\
\hline 5/23/07 1:30 & 129.246 & 126.867 & 123.494 & 104.159 & 108.154 & 99.843 & 114.341 & 111.086 & 83.145 & 76.613 \\
\hline 5/23/07 2:00 & 129.255 & 126.873 & 123.503 & 104.166 & 108.16 & 99.85 & 114.351 & 111.097 & 83.149 & 76.628 \\
\hline 5/23/07 2:30 & 129.246 & 126.887 & 123.494 & 104.154 & 108.15 & 99.838 & 114.341 & 111.086 & 83.144 & 76.611 \\
\hline 5/23/07 3:00 & 129.243 & 126.887 & 123.492 & 104.155 & 108.148 & 99.838 & 114.342 & 111.085 & 83.145 & 76.634 \\
\hline 5/23/07 3:30 & 129.263 & 126.86 & 123.511 & 104.177 & 108.169 & 99.859 & 114.36 & 111.103 & 83.149 & 76.623 \\
\hline 5/23/07 4:00 & 129.265 & 126.853 & 123.508 & 104.175 & 108.165 & 99.857 & 114.356 & 111.101 & 83.145 & 76.632 \\
\hline 5/23/07 4:30 & 129.267 & 126.828 & 123.492 & 104.151 & 108.144 & 99.833 & 114.335 & 111.081 & 83.136 & 76.632 \\
\hline 5/23/07 5:00 & 129.265 & 126.823 & 123.48 & 104.144 & 108.137 & 99.826 & 114.33 & 111.072 & 83.133 & 76.615 \\
\hline 5/23/07 5:30 & 129.267 & 126.837 & 123.457 & 104.12 & 108.112 & 99.805 & 114.309 & 111.053 & 83.125 & 76.626 \\
\hline 5/23/07 6:00 & 129.267 & 128.747 & 123.453 & 104.113 & 108.108 & 99.798 & 114.305 & 111.05 & 83.123 & 76.628 \\
\hline 5/23/07 6:30 & 129.251 & 129.074 & 123.458 & 104.125 & 108.118 & 99.805 & 114.307 & 111.046 & 83.122 & 76.605 \\
\hline 5/23/07 7:00 & 131.026 & 129.232 & 124.671 & 104.725 & 108.546 & 100.378 & 114.825 & 112.452 & 83.151 & 76.617 \\
\hline 5/23/07 7:30 & 131.36 & 129.334 & 125.014 & 105.04 & 108.866 & 100.696 & 115.111 & 112.817 & 83.199 & 76.685 \\
\hline 5/23/07 8:00 & 131.528 & 129.398 & 125.183 & 105.202 & 109.028 & 100.861 & 115.257 & 112.992 & 83.24 & 76.789 \\
\hline
\end{tabular}


TABLE S1.1 (Cont.)

Depth from Top of Casing (ft)

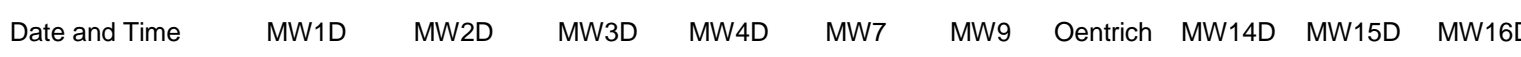

\begin{tabular}{|c|c|c|c|c|c|c|c|c|c|c|}
\hline 5/23/07 8:30 & 131.627 & 129.452 & 125.289 & 105.304 & 109.135 & 100.965 & 115.349 & 113.103 & 83.276 & 76.874 \\
\hline 5/23/07 9:00 & 131.708 & 129.486 & 125.358 & 105.373 & 109.207 & 101.036 & 115.431 & 113.174 & 83.313 & 76.985 \\
\hline 5/23/07 9:30 & 131.762 & 129.505 & 125.412 & 105.429 & 109.264 & 101.09 & 115.459 & 113.225 & 83.348 & 77.073 \\
\hline $5 / 23 / 07$ 10:00 & 131.826 & 127.81 & 125.458 & 105.466 & 109.306 & 101.137 & 115.447 & 113.269 & 83.381 & 77.116 \\
\hline $5 / 23 / 07 \quad 10: 30$ & 131.85 & 127.454 & 125.472 & 105.483 & 109.321 & 101.146 & 115.48 & 113.291 & 83.407 & 77.151 \\
\hline $5 / 23 / 0711: 00$ & 130.386 & 127.302 & 124.477 & 105.045 & 109.055 & 100.748 & 115.159 & 112.135 & 83.426 & 77.17 \\
\hline $5 / 23 / 0711: 30$ & 129.95 & 127.198 & 124.089 & 104.73 & 108.731 & 100.418 & 115.125 & 111.703 & 83.415 & 77.109 \\
\hline $5 / 23 / 07$ 12:00 & 129.773 & 127.13 & 123.924 & 104.57 & 108.573 & 100.258 & 114.726 & 111.535 & 83.404 & 77.037 \\
\hline $5 / 23 / 07$ 12:30 & 129.589 & 127.089 & 123.823 & 104.468 & 108.474 & 100.156 & 114.618 & 111.427 & 83.393 & 77.01 \\
\hline $5 / 23 / 07$ 13:00 & 129.511 & 127.043 & 123.753 & 104.406 & 108.405 & 100.09 & 114.559 & 111.356 & 83.383 & 76.944 \\
\hline $5 / 23 / 07$ 13:30 & 129.468 & 127.016 & 123.716 & 104.366 & 108.365 & 100.057 & 114.575 & 111.309 & 83.378 & 76.898 \\
\hline $5 / 23 / 07 \quad 14: 00$ & 129.435 & 126.991 & 123.672 & 104.321 & 108.321 & 100.008 & 114.509 & 111.272 & 83.369 & 76.958 \\
\hline $5 / 23 / 07 \quad 14: 30$ & 129.392 & 126.975 & 123.64 & 104.29 & 108.289 & 99.977 & 114.462 & 111.234 & 83.361 & 76.932 \\
\hline $5 / 23 / 07$ 15:00 & 129.371 & 126.941 & 123.619 & 104.277 & 108.268 & 99.965 & 114.618 & 111.208 & 83.354 & 76.915 \\
\hline $5 / 23 / 07$ 15:30 & 129.355 & 126.937 & 123.605 & 104.265 & 108.259 & 99.954 & 114.596 & 111.196 & 83.352 & 76.903 \\
\hline $5 / 23 / 07 \quad 16: 00$ & 129.329 & 126.937 & 123.57 & 104.225 & 108.219 & 99.909 & 114.429 & 111.165 & 83.339 & 76.896 \\
\hline $5 / 23 / 07$ 16:30 & 129.319 & 126.914 & 123.566 & 104.22 & 108.217 & 99.906 & 114.454 & 111.156 & 83.337 & 76.889 \\
\hline $5 / 23 / 07 \quad 17: 00$ & 129.317 & 126.905 & 123.566 & 104.225 & 108.22 & 99.911 & 114.505 & 111.154 & 83.335 & 76.88 \\
\hline $5 / 23 / 07 \quad 17: 30$ & 129.298 & 126.88 & 123.543 & 104.199 & 108.194 & 99.883 & 114.429 & 111.139 & 83.328 & 76.882 \\
\hline $5 / 23 / 07$ 18:00 & 129.284 & 126.885 & 123.533 & 104.194 & 108.19 & 99.883 & 114.478 & 111.121 & 83.321 & 76.857 \\
\hline $5 / 23 / 07$ 18:30 & 129.262 & 126.873 & 123.508 & 104.163 & 108.158 & 99.85 & 114.38 & 111.101 & 83.31 & 76.841 \\
\hline 5/23/07 19:00 & 129.265 & 126.878 & 123.517 & 104.175 & 108.169 & 99.864 & 114.478 & 111.103 & 83.308 & 76.834 \\
\hline $5 / 23 / 07$ 19:30 & 129.256 & 126.882 & 123.503 & 104.161 & 108.156 & 99.845 & 114.383 & 111.094 & 83.304 & 76.828 \\
\hline $5 / 23 / 0720: 00$ & 129.256 & 126.891 & 123.506 & 104.161 & 108.16 & 99.845 & 114.362 & 111.096 & 83.302 & 76.82 \\
\hline $5 / 23 / 07 \quad 20: 30$ & 129.258 & 126.898 & 123.508 & 104.173 & 108.163 & 99.857 & 114.561 & 111.096 & 83.3 & 76.826 \\
\hline $5 / 23 / 0721: 00$ & 129.27 & 126.903 & 123.52 & 104.187 & 108.177 & 99.869 & 114.596 & 111.11 & 83.302 & 76.837 \\
\hline $5 / 23 / 0721: 30$ & 129.277 & 126.88 & 123.522 & 104.18 & 108.177 & 99.864 & 114.429 & 111.114 & 83.3 & 76.834 \\
\hline 5/23/07 22:00 & 129.272 & 126.871 & 123.524 & 104.185 & 108.182 & 99.871 & 114.416 & 111.117 & 83.299 & 76.831 \\
\hline 5/23/07 22:30 & 129.274 & 126.839 & 123.508 & 104.166 & 108.165 & 99.855 & 114.394 & 111.099 & 83.291 & 76.829 \\
\hline 5/23/07 23:00 & 129.277 & 126.837 & 123.501 & 104.161 & 108.161 & 99.847 & 114.423 & 111.092 & 83.284 & 76.812 \\
\hline 5/23/07 23:30 & 129.281 & 126.828 & 123.469 & 104.127 & 108.123 & 99.812 & 114.374 & 111.064 & 83.269 & 76.82 \\
\hline 5/24/07 0:00 & 129.274 & 126.839 & 123.464 & 104.127 & 108.121 & 99.812 & 114.404 & 111.055 & 83.26 & 76.808 \\
\hline 5/24/07 0:30 & 129.263 & 126.823 & 123.453 & 104.113 & 108.11 & 99.796 & 114.377 & 111.044 & 83.252 & 76.791 \\
\hline $5 / 24 / 071: 00$ & 129.262 & 126.819 & 123.464 & 104.137 & 108.127 & 99.817 & 114.424 & 111.055 & 83.275 & 76.771 \\
\hline 5/24/07 1:30 & 129.251 & 126.821 & 123.446 & 104.215 & 108.11 & 99.793 & 114.431 & 111.044 & 83.37 & 76.76 \\
\hline $5 / 24 / 072: 00$ & 129.239 & 126.814 & 123.631 & 104.402 & 108.11 & 99.796 & 114.341 & 111.037 & 83.485 & 76.745 \\
\hline 5/24/07 2:30 & 129.232 & 126.787 & 123.443 & 104.49 & 108.112 & 99.8 & 114.497 & 111.035 & 83.535 & 76.737 \\
\hline 5/24/07 3:00 & 129.227 & 126.78 & 123.434 & 104.494 & 108.099 & 99.784 & 114.334 & 111.033 & 83.571 & 76.737 \\
\hline 5/24/07 3:30 & 129.222 & 126.789 & 123.413 & 104.492 & 108.08 & 99.76 & 114.314 & 111.006 & 83.619 & 76.738 \\
\hline 5/24/07 4:00 & 129.213 & 126.758 & 123.404 & 104.47 & 108.068 & 99.753 & 114.313 & 110.999 & 83.662 & 76.743 \\
\hline 5/24/07 4:30 & 129.206 & 126.74 & 123.416 & 104.465 & 108.08 & 99.767 & 114.348 & 111.008 & 83.713 & 76.743 \\
\hline 5/24/07 5:00 & 129.206 & 126.764 & 123.381 & 104.442 & 108.041 & 99.73 & 114.287 & 110.975 & 83.728 & 76.741 \\
\hline 5/24/07 5:30 & 129.203 & 126.798 & 123.367 & 104.46 & 108.032 & 99.713 & 114.267 & 110.962 & 83.724 & 76.743 \\
\hline 5/24/07 6:00 & 129.201 & 128.724 & 123.385 & 104.485 & 108.057 & 99.739 & 114.302 & 111.115 & 83.706 & 76.723 \\
\hline $5 / 24 / 076: 30$ & 129.182 & 129.024 & 123.42 & 104.504 & 108.093 & 99.774 & 114.322 & 111.079 & 83.693 & 76.707 \\
\hline 5/24/07 7:00 & 130.937 & 129.189 & 124.789 & 105.073 & 108.577 & 100.418 & 115.12 & 112.423 & 83.7 & 76.706 \\
\hline 5/24/07 7:30 & 131.287 & 129.294 & 125.102 & 105.295 & 108.86 & 100.698 & 115.148 & 112.764 & 83.724 & 76.775 \\
\hline 5/24/07 8:00 & 131.502 & 129.389 & 125.278 & 105.416 & 109.03 & 100.868 & 115.308 & 112.943 & 83.724 & 76.83 \\
\hline 5/24/07 8:30 & 131.615 & 129.464 & 125.386 & 105.491 & 109.136 & 100.974 & 115.347 & 113.056 & 83.693 & 76.878 \\
\hline 5/24/07 9:00 & 131.717 & 127.715 & 125.483 & 105.536 & 109.235 & 101.071 & 115.47 & 113.156 & 83.665 & 76.924 \\
\hline 5/24/07 9:30 & 131.795 & 127.404 & 125.564 & 105.557 & 109.317 & 101.154 & 115.536 & 113.238 & 83.64 & 76.955 \\
\hline $5 / 24 / 07 \quad 10: 00$ & 130.327 & 127.273 & 124.366 & 105.138 & 108.98 & 100.663 & 115.094 & 112.013 & 83.603 & 76.984 \\
\hline $5 / 24 / 07 \quad 10: 30$ & 129.972 & 127.18 & 124.035 & 104.854 & 108.697 & 100.373 & 114.828 & 111.655 & 83.571 & 76.938 \\
\hline $5 / 24 / 0711: 00$ & 129.83 & 127.127 & 123.901 & 104.676 & 108.563 & 100.244 & 114.713 & 111.513 & 83.52 & 76.886 \\
\hline $5 / 24 / 0711: 30$ & 129.719 & 127.084 & 123.809 & 104.546 & 108.474 & 100.154 & 114.594 & 111.418 & 83.468 & 76.841 \\
\hline $5 / 24 / 07$ 12:00 & 129.655 & 127.062 & 123.751 & 104.458 & 108.415 & 100.097 & 114.595 & 111.356 & 83.431 & 76.812 \\
\hline $5 / 24 / 07 \quad 12: 30$ & 129.487 & 127.039 & 123.712 & 104.38 & 108.379 & 100.06 & 114.563 & 111.314 & 83.409 & 76.803 \\
\hline 5/24/07 13:00 & 129.447 & 127.021 & 123.693 & 104.337 & 108.359 & 100.053 & 114.696 & 111.289 & 83.391 & 76.789 \\
\hline 5/24/07 13:30 & 129.426 & 127 & 123.668 & 104.296 & 108.331 & 100.022 & 114.555 & 111.263 & 83.381 & 76.789 \\
\hline $5 / 24 / 07 \quad 14: 00$ & 129.402 & 126.982 & 123.645 & 104.258 & 108.31 & 99.996 & 114.509 & 111.245 & 83.37 & 76.808 \\
\hline $5 / 24 / 07$ 14:30 & 129.385 & 126.962 & 123.628 & 104.236 & 108.295 & 99.977 & 114.457 & 111.227 & 83.359 & 76.804 \\
\hline 5/24/07 15:00 & 129.367 & 126.955 & 123.612 & 104.218 & 108.278 & 99.963 & 114.453 & 111.208 & 83.35 & 76.804 \\
\hline $5 / 24 / 07 \quad 15: 30$ & 129.345 & 126.948 & 123.589 & 104.22 & 108.255 & 99.939 & 114.452 & 111.188 & 83.341 & 76.793 \\
\hline $5 / 24 / 07$ 16:00 & 129.336 & 126.932 & 123.582 & 104.203 & 108.247 & 99.93 & 114.419 & 111.181 & 83.332 & 76.801 \\
\hline $5 / 24 / 07 \quad 16: 30$ & 129.329 & 126.925 & 123.575 & 104.253 & 108.243 & 99.93 & 114.684 & 111.17 & 83.326 & 76.803 \\
\hline $5 / 24 / 07 \quad 17: 00$ & 129.312 & 126.916 & 123.559 & 104.225 & 108.224 & 99.911 & 114.428 & 111.157 & 83.319 & 76.795 \\
\hline
\end{tabular}


TABLE S1.1 (Cont.)

Depth from Top of Casing (ft)

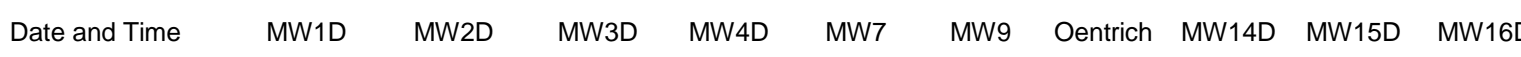

\begin{tabular}{|c|c|c|c|c|c|c|c|c|c|c|}
\hline 5/24/07 17:30 & 129.307 & 126.905 & 123.552 & 104.217 & 108.217 & 99.899 & 114.403 & 111.15 & 83.313 & 76.785 \\
\hline $5 / 24 / 07$ 18:00 & 129.298 & 126.896 & 123.547 & 104.21 & 108.211 & 99.899 & 114.429 & 111.139 & 83.306 & 76.775 \\
\hline $5 / 24 / 07$ 18:30 & 129.286 & 126.891 & 123.533 & 104.21 & 108.201 & 99.888 & 114.66 & 111.128 & 83.297 & 76.76 \\
\hline 5/24/07 19:00 & 129.277 & 126.898 & 123.522 & 104.202 & 108.188 & 99.876 & 114.562 & 111.119 & 83.291 & 76.745 \\
\hline 5/24/07 19:30 & 129.272 & 126.891 & 123.517 & 104.184 & 108.186 & 99.866 & 114.381 & 111.115 & 83.284 & 76.738 \\
\hline $5 / 24 / 0720: 00$ & 129.274 & 126.887 & 123.524 & 104.203 & 108.194 & 99.88 & 114.644 & 111.119 & 83.282 & 76.707 \\
\hline $5 / 24 / 07$ 20:30 & 129.272 & 126.885 & 123.52 & 104.187 & 108.186 & 99.871 & 114.402 & 111.112 & 83.276 & 76.7 \\
\hline $5 / 24 / 0721: 00$ & 129.263 & 126.885 & 123.51 & 104.18 & 108.18 & 99.864 & 114.387 & 111.108 & 83.273 & 76.681 \\
\hline 5/24/07 21:30 & 129.263 & 126.882 & 123.508 & 104.179 & 108.177 & 99.862 & 114.397 & 111.106 & 83.267 & 76.659 \\
\hline 5/24/07 22:00 & 129.262 & 126.878 & 123.51 & 104.182 & 108.182 & 99.864 & 114.406 & 111.103 & 83.265 & 76.634 \\
\hline $5 / 24 / 07$ 22:30 & 129.265 & 126.867 & 123.513 & 104.18 & 108.18 & 99.871 & 114.41 & 111.103 & 83.26 & 76.607 \\
\hline 5/24/07 23:00 & 129.258 & 126.855 & 123.503 & 104.173 & 108.175 & 99.859 & 114.369 & 111.101 & 83.256 & 76.595 \\
\hline 5/24/07 23:30 & 129.251 & 126.846 & 123.496 & 104.163 & 108.169 & 99.847 & 114.358 & 111.092 & 83.249 & 76.574 \\
\hline $5 / 25 / 070: 00$ & 129.239 & 126.835 & 123.485 & 104.155 & 108.156 & 99.84 & 114.375 & 111.079 & 83.238 & 76.554 \\
\hline 5/25/07 0:30 & 129.222 & 126.823 & 123.473 & 104.142 & 108.142 & 99.824 & 114.359 & 111.068 & 83.228 & 76.541 \\
\hline 5/25/07 1:00 & 129.215 & 126.812 & 123.462 & 104.132 & 108.133 & 99.814 & 114.338 & 111.059 & 83.221 & 76.524 \\
\hline $5 / 25 / 071: 30$ & 129.203 & 126.803 & 123.45 & 104.121 & 108.121 & 99.81 & 114.336 & 111.046 & 83.21 & 76.506 \\
\hline 5/25/07 2:00 & 129.194 & 126.796 & 123.441 & 104.109 & 108.11 & 99.791 & 114.339 & 111.037 & 83.201 & 76.502 \\
\hline $5 / 25 / 072: 30$ & 129.18 & 126.787 & 123.437 & 104.096 & 108.1 & 99.781 & 114.36 & 111.024 & 83.19 & 76.481 \\
\hline 5/25/07 3:00 & 129.175 & 126.785 & 123.427 & 104.092 & 108.097 & 99.777 & 114.372 & 111.022 & 83.182 & 76.473 \\
\hline 5/25/07 3:30 & 129.166 & 126.774 & 123.42 & 104.082 & 108.085 & 99.767 & 114.33 & 111.013 & 83.171 & 76.467 \\
\hline $5 / 25 / 074: 00$ & 129.165 & 126.771 & 123.414 & 104.082 & 108.087 & 99.767 & 114.311 & 111.008 & 83.162 & 76.454 \\
\hline $5 / 25 / 074: 30$ & 129.154 & 126.76 & 123.409 & 104.082 & 108.076 & 99.758 & 114.46 & 110.999 & 83.155 & 76.442 \\
\hline $5 / 25 / 075: 00$ & 129.149 & 126.76 & 123.399 & 104.07 & 108.07 & 99.753 & 114.308 & 110.995 & 83.145 & 76.432 \\
\hline 5/25/07 5:30 & 129.14 & 126.762 & 123.395 & 104.056 & 108.061 & 99.741 & 114.273 & 110.986 & 83.136 & 76.436 \\
\hline 5/25/07 6:00 & 129.142 & 128.661 & 123.39 & 104.06 & 108.062 & 99.744 & 114.289 & 110.986 & 83.129 & 76.413 \\
\hline $5 / 25 / 076: 30$ & 129.14 & 128.956 & 123.383 & 104.058 & 108.061 & 99.741 & 114.305 & 110.986 & 83.122 & 76.407 \\
\hline 5/25/07 7:00 & 131.05 & 129.103 & 124.565 & 104.633 & 108.46 & 100.284 & 114.777 & 112.357 & 83.133 & 76.411 \\
\hline 5/25/07 7:30 & 131.381 & 129.185 & 124.884 & 104.926 & 108.757 & 100.581 & 115.035 & 112.708 & 83.164 & 76.501 \\
\hline $5 / 25 / 078: 00$ & 131.532 & 129.25 & 125.046 & 105.068 & 108.904 & 100.727 & 115.15 & 112.868 & 83.19 & 76.601 \\
\hline 5/25/07 8:30 & 131.622 & 129.278 & 125.139 & 105.154 & 108.996 & 100.816 & 115.232 & 112.961 & 83.216 & 76.68 \\
\hline 5/25/07 9:00 & 131.689 & 129.298 & 125.201 & 105.219 & 109.062 & 100.88 & 115.284 & 113.027 & 83.236 & 76.748 \\
\hline 5/25/07 9:30 & 131.72 & 129.309 & 125.236 & 105.252 & 109.095 & 100.915 & 115.304 & 113.063 & 83.256 & 76.8 \\
\hline $5 / 25 / 07 \quad 10: 00$ & 131.741 & 127.685 & 125.257 & 105.274 & 109.119 & 100.939 & 115.33 & 113.085 & 83.273 & 76.855 \\
\hline $5 / 25 / 07 \quad 10: 30$ & 131.753 & 127.245 & 125.273 & 105.287 & 109.133 & 100.953 & 115.335 & 113.096 & 83.284 & 76.899 \\
\hline $5 / 25 / 0711: 00$ & 130.185 & 127.059 & 124.366 & 104.906 & 108.921 & 100.611 & 115.027 & 112.084 & 83.291 & 76.946 \\
\hline 5/25/07 11:30 & 129.65 & 126.944 & 123.869 & 104.515 & 108.538 & 100.211 & 114.622 & 111.506 & 83.265 & 76.92 \\
\hline $5 / 25 / 07$ 12:00 & 129.447 & 126.86 & 123.7 & 104.335 & 108.35 & 100.027 & 114.495 & 111.303 & 83.238 & 76.891 \\
\hline $5 / 25 / 07$ 12:30 & 129.331 & 126.796 & 123.577 & 104.227 & 108.241 & 99.916 & 114.415 & 111.185 & 83.21 & 76.843 \\
\hline $5 / 25 / 07$ 13:00 & 129.246 & 126.744 & 123.483 & 104.144 & 108.154 & 99.833 & 114.341 & 111.097 & 83.181 & 76.83 \\
\hline 5/25/07 13:30 & 129.182 & 126.708 & 123.441 & 104.08 & 108.091 & 99.772 & 114.28 & 111.03 & 83.155 & 76.802 \\
\hline $5 / 25 / 07 \quad 14: 00$ & 129.128 & 126.674 & 123.374 & 104.03 & 108.038 & 99.718 & 114.24 & 110.977 & 83.125 & 76.785 \\
\hline $5 / 25 / 07 \quad 14: 30$ & 129.09 & 126.644 & 123.348 & 103.993 & 108.001 & 99.682 & 114.211 & 110.94 & 83.099 & 76.769 \\
\hline $5 / 25 / 07$ 15:00 & 129.059 & 126.628 & 123.318 & 103.963 & 107.971 & 99.649 & 114.182 & 110.906 & 83.077 & 76.758 \\
\hline $5 / 25 / 07 \quad 15: 30$ & 129.028 & 126.615 & 123.291 & 103.932 & 107.942 & 99.619 & 114.153 & 110.875 & 83.048 & 76.748 \\
\hline $5 / 25 / 07 \quad 16: 00$ & 129.01 & 126.599 & 123.267 & 103.916 & 107.929 & 99.607 & 114.145 & 110.858 & 83.024 & 76.727 \\
\hline $5 / 25 / 07 \quad 16: 30$ & 128.993 & 126.585 & 123.247 & 103.901 & 107.912 & 99.59 & 114.124 & 110.842 & 83.002 & 76.723 \\
\hline $5 / 25 / 07 \quad 17: 00$ & 128.979 & 126.569 & 123.23 & 103.889 & 107.901 & 99.579 & 114.146 & 110.827 & 82.981 & 76.715 \\
\hline $5 / 25 / 07 \quad 17: 30$ & 128.967 & 126.567 & 123.219 & 103.875 & 107.885 & 99.565 & 114.113 & 110.816 & 82.959 & 76.713 \\
\hline $5 / 25 / 07$ 18:00 & 128.953 & 126.551 & 123.2 & 103.86 & 107.872 & 99.546 & 114.085 & 110.8 & 82.939 & 76.696 \\
\hline $5 / 25 / 07$ 18:30 & 128.948 & 126.547 & 123.189 & 103.86 & 107.87 & 99.546 & 114.094 & 110.796 & 82.921 & 76.676 \\
\hline 5/25/07 19:00 & 128.929 & 126.535 & 123.18 & 103.841 & 107.853 & 99.529 & 114.074 & 110.78 & 82.902 & 76.671 \\
\hline 5/25/07 19:30 & 128.925 & 126.535 & 123.168 & 103.837 & 107.849 & 99.524 & 114.081 & 110.774 & 82.886 & 76.663 \\
\hline $5 / 25 / 0720: 00$ & 128.913 & 126.531 & 123.159 & 103.825 & 107.838 & 99.51 & 114.073 & 110.76 & 82.865 & 76.654 \\
\hline $5 / 25 / 0720: 30$ & 128.913 & 126.529 & 123.152 & 103.827 & 107.838 & 99.51 & 114.069 & 110.762 & 82.851 & 76.634 \\
\hline $5 / 25 / 0721: 00$ & 128.908 & 126.526 & 123.147 & 103.823 & 107.836 & 99.508 & 114.063 & 110.758 & 82.834 & 76.638 \\
\hline $5 / 25 / 0721: 30$ & 128.903 & 126.52 & 123.143 & 103.82 & 107.832 & 99.506 & 114.052 & 110.756 & 82.821 & 76.614 \\
\hline 5/25/07 22:00 & 128.903 & 126.517 & 123.143 & 103.82 & 107.832 & 99.503 & 114.057 & 110.754 & 82.806 & 76.611 \\
\hline 5/25/07 22:30 & 128.896 & 126.508 & 123.138 & 103.81 & 107.822 & 99.496 & 114.041 & 110.747 & 82.788 & 76.605 \\
\hline $5 / 25 / 0723: 00$ & 128.891 & 126.488 & 123.131 & 103.808 & 107.822 & 99.491 & 114.022 & 110.743 & 82.773 & 76.597 \\
\hline $5 / 25 / 0723: 30$ & 128.884 & 126.483 & 123.122 & 103.801 & 107.815 & 99.484 & 114.024 & 110.734 & 82.756 & 76.585 \\
\hline 5/26/07 0:00 & 128.865 & 126.47 & 123.113 & 103.784 & 107.796 & 99.47 & 114.005 & 110.718 & 82.736 & 76.572 \\
\hline $5 / 26 / 070: 30$ & 128.865 & 126.463 & 123.103 & 103.779 & 107.794 & 99.466 & 113.999 & 110.712 & 82.721 & 76.57 \\
\hline 5/26/07 1:00 & 128.849 & 126.458 & 123.096 & 103.765 & 107.779 & 99.454 & 113.981 & 110.7 & 82.701 & 76.557 \\
\hline 5/26/07 1:30 & 128.839 & 126.456 & 123.082 & 103.756 & 107.769 & 99.444 & 113.975 & 110.689 & 82.683 & 76.545 \\
\hline $5 / 26 / 072: 00$ & 128.834 & 126.458 & 123.08 & 103.753 & 107.767 & 99.44 & 113.969 & 110.685 & 82.666 & 76.543 \\
\hline
\end{tabular}


TABLE S1.1 (Cont.)

Depth from Top of Casing (ft)

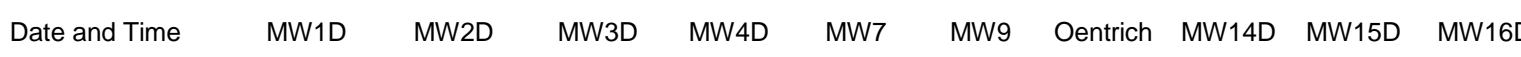

\begin{tabular}{|c|c|c|c|c|c|c|c|c|c|c|}
\hline 5/26/07 2:30 & 128.835 & 126.465 & 123.073 & 103.753 & 107.767 & 99.44 & 113.963 & 110.687 & 82.653 & 76.535 \\
\hline 5/26/07 3:00 & 128.835 & 126.465 & 123.073 & 103.753 & 107.767 & 99.44 & 113.969 & 110.685 & 82.639 & 76.524 \\
\hline 5/26/07 3:30 & 128.844 & 126.463 & 123.082 & 103.763 & 107.779 & 99.449 & 113.974 & 110.696 & 82.629 & 76.514 \\
\hline 5/26/07 4:00 & 128.844 & 126.461 & 123.085 & 103.763 & 107.779 & 99.451 & 113.972 & 110.696 & 82.618 & 76.51 \\
\hline 5/26/07 4:30 & 128.839 & 126.454 & 123.08 & 103.768 & 107.775 & 99.447 & 113.964 & 110.694 & 82.605 & 76.504 \\
\hline 5/26/07 5:00 & 128.832 & 126.445 & 123.073 & 103.753 & 107.769 & 99.44 & 113.967 & 110.687 & 82.59 & 76.499 \\
\hline 5/26/07 5:30 & 128.83 & 126.461 & 123.069 & 103.749 & 107.765 & 99.437 & 113.956 & 110.683 & 82.578 & 76.504 \\
\hline 5/26/07 6:00 & 128.825 & 126.481 & 123.062 & 103.743 & 107.758 & 99.428 & 113.949 & 110.678 & 82.563 & 76.496 \\
\hline 5/26/07 6:30 & 128.834 & 126.476 & 123.073 & 103.758 & 107.773 & 99.442 & 113.965 & 110.687 & 82.557 & 76.481 \\
\hline 5/26/07 7:00 & 128.856 & 126.492 & 123.096 & 103.784 & 107.796 & 99.47 & 114.078 & 110.709 & 82.554 & 76.477 \\
\hline 5/26/07 7:30 & 128.851 & 128.47 & 123.089 & 103.774 & 107.79 & 99.463 & 113.986 & 110.705 & 82.541 & 76.485 \\
\hline 5/26/07 8:00 & 128.856 & 128.717 & 123.108 & 104.01 & 107.813 & 99.482 & 113.99 & 110.723 & 82.865 & 76.458 \\
\hline 5/26/07 8:30 & 130.7 & 128.847 & 124.526 & 104.838 & 108.325 & 100.154 & 114.589 & 112.186 & 82.908 & 76.473 \\
\hline 5/26/07 9:00 & 131.024 & 128.931 & 124.796 & 105.028 & 108.577 & 100.406 & 114.816 & 112.474 & 82.898 & 76.574 \\
\hline 5/26/07 9:30 & 131.131 & 128.987 & 124.926 & 105.076 & 108.7 & 100.529 & 114.961 & 112.611 & 82.843 & 76.681 \\
\hline 5/26/07 10:00 & 131.216 & 129.042 & 125.014 & 105.049 & 108.788 & 100.614 & 115.019 & 112.702 & 82.789 & 76.756 \\
\hline 5/26/07 10:30 & 131.273 & 129.071 & 125.074 & 105.023 & 108.847 & 100.673 & 115.065 & 112.764 & 82.732 & 76.82 \\
\hline 5/26/07 11:00 & 131.374 & 129.087 & 125.127 & 105.006 & 108.904 & 100.727 & 115.107 & 112.819 & 82.698 & 76.859 \\
\hline 5/26/07 11:30 & 131.417 & 129.108 & 125.157 & 105.002 & 108.935 & 100.758 & 115.131 & 112.85 & 82.709 & 76.903 \\
\hline $5 / 26 / 07$ 12:00 & 131.44 & 129.121 & 125.176 & 105.028 & 108.952 & 100.776 & 115.146 & 112.87 & 82.712 & 76.938 \\
\hline $5 / 26 / 07$ 12:30 & 131.469 & 129.126 & 125.194 & 105.09 & 108.975 & 100.798 & 115.162 & 112.89 & 82.723 & 76.969 \\
\hline 5/26/07 13:00 & 131.488 & 127.193 & 125.213 & 105.149 & 108.992 & 100.812 & 115.177 & 112.908 & 82.74 & 76.982 \\
\hline $5 / 26 / 07$ 13:30 & 131.504 & 126.932 & 125.22 & 105.155 & 108.997 & 100.819 & 115.181 & 112.915 & 82.756 & 77.012 \\
\hline $5 / 26 / 0714: 00$ & 129.747 & 126.794 & 123.825 & 104.453 & 108.487 & 100.154 & 114.563 & 111.475 & 82.738 & 77.025 \\
\hline $5 / 26 / 07$ 14:30 & 129.473 & 126.694 & 123.552 & 104.206 & 108.24 & 99.902 & 114.348 & 111.192 & 82.709 & 76.954 \\
\hline $5 / 26 / 07$ 15:00 & 129.298 & 126.628 & 123.411 & 104.072 & 108.104 & 99.765 & 114.229 & 111.041 & 82.681 & 76.899 \\
\hline $5 / 26 / 07$ 15:30 & 129.211 & 126.574 & 123.309 & 103.975 & 108.003 & 99.668 & 114.142 & 110.937 & 82.653 & 76.862 \\
\hline 5/26/07 16:00 & 129.137 & 126.542 & 123.242 & 103.911 & 107.937 & 99.602 & 114.088 & 110.869 & 82.627 & 76.82 \\
\hline 5/26/07 16:30 & 129.064 & 126.513 & 123.189 & 103.858 & 107.883 & 99.548 & 114.043 & 110.813 & 82.605 & 76.793 \\
\hline 5/26/07 17:00 & 129.007 & 126.506 & 123.155 & 103.829 & 107.851 & 99.517 & 114.015 & 110.778 & 82.58 & 76.785 \\
\hline $5 / 26 / 07$ 17:30 & 128.896 & 126.483 & 123.124 & 103.799 & 107.821 & 99.491 & 113.993 & 110.747 & 82.559 & 76.754 \\
\hline $5 / 26 / 07$ 18:00 & 128.884 & 126.497 & 123.117 & 103.794 & 107.815 & 99.484 & 113.994 & 110.738 & 82.541 & 76.729 \\
\hline $5 / 26 / 07$ 18:30 & 128.865 & 126.495 & 123.096 & 103.772 & 107.794 & 99.463 & 113.975 & 110.718 & 82.521 & 76.711 \\
\hline 5/26/07 19:00 & 128.851 & 126.483 & 123.108 & 103.787 & 107.813 & 99.477 & 114.022 & 110.729 & 82.506 & 76.69 \\
\hline $5 / 26 / 07$ 19:30 & 128.839 & 126.47 & 123.101 & 103.982 & 107.805 & 99.473 & 114.017 & 110.723 & 82.639 & 76.657 \\
\hline 5/26/07 20:00 & 128.849 & 126.461 & 123.092 & 104.261 & 107.8 & 99.466 & 113.997 & 110.893 & 82.827 & 76.634 \\
\hline $5 / 26 / 07$ 20:30 & 128.858 & 126.463 & 123.082 & 104.251 & 107.786 & 99.454 & 114.016 & 110.853 & 82.863 & 76.617 \\
\hline $5 / 26 / 0721: 00$ & 128.842 & 126.465 & 123.076 & 104.234 & 107.779 & 99.444 & 113.994 & 110.782 & 82.847 & 76.62 \\
\hline 5/26/07 21:30 & 128.828 & 126.472 & 123.073 & 104.217 & 107.781 & 99.449 & 113.979 & 110.736 & 82.827 & 76.599 \\
\hline 5/26/07 22:00 & 128.82 & 126.472 & 123.076 & 104.199 & 107.781 & 99.449 & 113.971 & 110.718 & 82.803 & 76.592 \\
\hline 5/26/07 22:30 & 128.808 & 126.463 & 123.08 & 104.134 & 107.79 & 99.456 & 113.97 & 110.707 & 82.733 & 76.576 \\
\hline 5/26/07 23:00 & 128.804 & 126.456 & 123.08 & 104.056 & 107.79 & 99.456 & 113.968 & 110.703 & 82.674 & 76.562 \\
\hline 5/26/07 23:30 & 128.799 & 126.449 & 123.076 & 103.986 & 107.788 & 99.454 & 113.962 & 110.698 & 82.609 & 76.549 \\
\hline $5 / 27 / 070: 00$ & 128.792 & 126.442 & 123.069 & 103.918 & 107.777 & 99.444 & 113.952 & 110.689 & 82.556 & 76.537 \\
\hline 5/27/07 0:30 & 128.785 & 126.438 & 123.059 & 103.867 & 107.771 & 99.44 & 113.944 & 110.683 & 82.493 & 76.518 \\
\hline 5/27/07 1:00 & 128.78 & 126.436 & 123.057 & 103.822 & 107.767 & 99.435 & 113.94 & 110.678 & 82.443 & 76.499 \\
\hline 5/27/07 1:30 & 128.773 & 126.431 & 123.05 & 103.784 & 107.763 & 99.428 & 113.932 & 110.674 & 82.414 & 76.483 \\
\hline 5/27/07 2:00 & 128.769 & 126.427 & 123.048 & 103.756 & 107.76 & 99.425 & 113.93 & 110.672 & 82.388 & 76.479 \\
\hline 5/27/07 2:30 & 128.766 & 126.417 & 123.043 & 103.732 & 107.758 & 99.423 & 113.922 & 110.667 & 82.369 & 76.469 \\
\hline 5/27/07 3:00 & 128.764 & 126.411 & 123.039 & 103.711 & 107.754 & 99.421 & 113.918 & 110.663 & 82.351 & 76.458 \\
\hline 5/27/07 3:30 & 128.759 & 126.406 & 123.029 & 103.694 & 107.742 & 99.409 & 113.911 & 110.654 & 82.334 & 76.454 \\
\hline 5/27/07 4:00 & 128.749 & 126.404 & 123.022 & 103.677 & 107.739 & 99.402 & 113.903 & 110.645 & 82.316 & 76.444 \\
\hline 5/27/07 4:30 & 128.745 & 126.411 & 123.022 & 103.665 & 107.735 & 99.402 & 113.899 & 110.645 & 82.299 & 76.432 \\
\hline 5/27/07 5:00 & 128.735 & 126.411 & 123.015 & 103.663 & 107.733 & 99.397 & 113.895 & 110.639 & 82.285 & 76.427 \\
\hline 5/27/07 5:30 & 128.733 & 126.408 & 123.02 & 103.67 & 107.737 & 99.402 & 113.899 & 110.645 & 82.272 & 76.417 \\
\hline 5/27/07 6:00 & 128.728 & 126.408 & 123.025 & 103.67 & 107.737 & 99.404 & 113.899 & 110.647 & 82.262 & 76.421 \\
\hline 5/27/07 6:30 & 128.726 & 126.406 & 123.022 & 103.672 & 107.741 & 99.404 & 113.9 & 110.647 & 82.253 & 76.404 \\
\hline 5/27/07 7:00 & 128.723 & 128.413 & 123.022 & 103.715 & 107.739 & 99.404 & 113.9 & 110.65 & 82.244 & 76.402 \\
\hline 5/27/07 7:30 & 128.721 & 128.645 & 123.015 & 103.71 & 107.735 & 99.4 & 113.895 & 110.641 & 82.237 & 76.434 \\
\hline 5/27/07 8:00 & 130.705 & 128.77 & 124.336 & 104.403 & 108.251 & 100.06 & 114.488 & 112.162 & 82.257 & 76.496 \\
\hline 5/27/07 8:30 & 130.908 & 128.856 & 124.574 & 104.625 & 108.478 & 100.288 & 114.692 & 112.412 & 82.29 & 76.609 \\
\hline 5/27/07 9:00 & 131.19 & 128.908 & 124.708 & 104.752 & 108.611 & 100.418 & 114.806 & 112.556 & 82.316 & 76.688 \\
\hline 5/27/07 9:30 & 131.289 & 128.944 & 124.799 & 104.842 & 108.702 & 100.507 & 114.889 & 112.644 & 82.342 & 76.764 \\
\hline $5 / 27 / 07 \quad 10: 00$ & 131.346 & 127.214 & 124.857 & 104.893 & 108.756 & 100.562 & 114.936 & 112.702 & 82.369 & 76.81 \\
\hline $5 / 27 / 07$ 10:30 & 131.383 & 126.864 & 124.891 & 104.93 & 108.793 & 100.599 & 114.968 & 112.74 & 82.391 & 76.865 \\
\hline 5/27/07 11:00 & 129.672 & 126.715 & 123.86 & 104.463 & 108.506 & 100.173 & 114.576 & 111.555 & 82.406 & 76.896 \\
\hline
\end{tabular}


TABLE S1.1 (Cont.)

Depth from Top of Casing (ft)

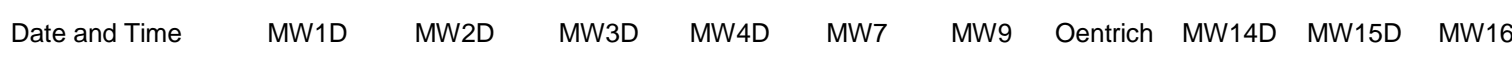

\begin{tabular}{|c|c|c|c|c|c|c|c|c|c|c|}
\hline 5/27/07 11:30 & 129.263 & 126.615 & 123.485 & 104.149 & 108.192 & 99.85 & 114.286 & 111.134 & 82.393 & 76.86 \\
\hline $5 / 27 / 07 \quad 12: 00$ & 129.099 & 126.547 & 123.328 & 104.004 & 108.04 & 99.699 & 114.155 & 110.968 & 82.379 & 76.82 \\
\hline $5 / 27 / 07 \quad 12: 30$ & 129 & 126.49 & 123.23 & 103.91 & 107.944 & 99.602 & 114.072 & 110.867 & 82.358 & 76.791 \\
\hline $5 / 27 / 07$ 13:00 & 128.931 & 126.451 & 123.163 & 103.844 & 107.878 & 99.536 & 114.014 & 110.796 & 82.334 & 76.758 \\
\hline $5 / 27 / 07$ 13:30 & 128.873 & 126.42 & 123.103 & 103.785 & 107.817 & 99.477 & 113.963 & 110.736 & 82.309 & 76.733 \\
\hline $5 / 27 / 07 \quad 14: 00$ & 128.832 & 126.397 & 123.064 & 103.75 & 107.777 & 99.44 & 113.929 & 110.694 & 82.281 & 76.719 \\
\hline $5 / 27 / 07 \quad 14: 30$ & 128.801 & 126.368 & 123.032 & 103.72 & 107.746 & 99.407 & 113.903 & 110.663 & 82.262 & 76.702 \\
\hline $5 / 27 / 07$ 15:00 & 128.773 & 126.347 & 123.008 & 103.692 & 107.722 & 99.383 & 113.883 & 110.636 & 82.242 & 76.694 \\
\hline $5 / 27 / 07$ 15:30 & 128.749 & 126.318 & 122.981 & 103.67 & 107.697 & 99.357 & 113.863 & 110.61 & 82.226 & 76.702 \\
\hline 5/27/07 16:00 & 128.726 & 126.3 & 122.96 & 103.648 & 107.674 & 99.338 & 113.844 & 110.588 & 82.203 & 76.692 \\
\hline 5/27/07 16:30 & 128.698 & 126.284 & 122.93 & 103.619 & 107.647 & 99.308 & 113.82 & 110.557 & 82.179 & 76.706 \\
\hline $5 / 27 / 07 \quad 17: 00$ & 128.676 & 126.265 & 122.911 & 103.598 & 107.628 & 99.286 & 113.801 & 110.537 & 82.159 & 76.721 \\
\hline $5 / 27 / 07 \quad 17: 30$ & 128.66 & 126.254 & 122.893 & 103.582 & 107.609 & 99.27 & 113.788 & 110.523 & 82.141 & 76.723 \\
\hline $5 / 27 / 07$ 18:00 & 128.643 & 126.252 & 122.881 & 103.567 & 107.596 & 99.258 & 113.773 & 110.506 & 82.12 & 76.716 \\
\hline $5 / 27 / 07$ 18:30 & 128.631 & 126.247 & 122.865 & 103.556 & 107.586 & 99.246 & 113.764 & 110.495 & 82.102 & 76.706 \\
\hline 5/27/07 19:00 & 128.626 & 126.245 & 122.86 & 103.553 & 107.581 & 99.242 & 113.76 & 110.488 & 82.087 & 76.685 \\
\hline 5/27/07 19:30 & 128.622 & 126.252 & 122.856 & 103.55 & 107.579 & 99.239 & 113.757 & 110.484 & 82.074 & 76.671 \\
\hline $5 / 27 / 0720: 00$ & 128.62 & 126.259 & 122.856 & 103.549 & 107.577 & 99.239 & 113.755 & 110.484 & 82.061 & 76.657 \\
\hline $5 / 27 / 0720: 30$ & 128.626 & 126.265 & 122.858 & 103.555 & 107.586 & 99.246 & 113.761 & 110.49 & 82.05 & 76.642 \\
\hline $5 / 27 / 0721: 00$ & 128.631 & 126.272 & 122.867 & 103.561 & 107.594 & 99.253 & 113.764 & 110.497 & 82.043 & 76.626 \\
\hline $5 / 27 / 0721: 30$ & 128.641 & 126.275 & 122.874 & 103.57 & 107.602 & 99.263 & 113.77 & 110.506 & 82.037 & 76.607 \\
\hline $5 / 27 / 0722: 00$ & 128.645 & 126.27 & 122.879 & 103.577 & 107.609 & 99.27 & 113.775 & 110.51 & 82.032 & 76.599 \\
\hline 5/27/07 22:30 & 128.648 & 126.27 & 122.881 & 103.579 & 107.611 & 99.27 & 113.777 & 110.512 & 82.023 & 76.592 \\
\hline 5/27/07 23:00 & 128.65 & 126.261 & 122.881 & 103.579 & 107.609 & 99.27 & 113.777 & 110.512 & 82.017 & 76.582 \\
\hline 5/27/07 23:30 & 128.645 & 126.25 & 122.877 & 103.575 & 107.605 & 99.267 & 113.773 & 110.508 & 82.006 & 76.566 \\
\hline $5 / 28 / 070: 00$ & 128.638 & 126.234 & 122.87 & 103.569 & 107.6 & 99.26 & 113.766 & 110.501 & 81.995 & 76.553 \\
\hline $5 / 28 / 070: 30$ & 128.624 & 126.231 & 122.858 & 103.558 & 107.59 & 99.249 & 113.756 & 110.49 & 81.984 & 76.539 \\
\hline 5/28/07 1:00 & 128.615 & 126.227 & 122.847 & 103.546 & 107.577 & 99.237 & 113.745 & 110.479 & 81.971 & 76.541 \\
\hline $5 / 28 / 07 \quad 1: 30$ & 128.607 & 126.213 & 122.84 & 103.541 & 107.571 & 99.232 & 113.74 & 110.472 & 81.96 & 76.534 \\
\hline $5 / 28 / 072: 00$ & 128.603 & 126.204 & 122.835 & 103.536 & 107.569 & 99.227 & 113.736 & 110.468 & 81.951 & 76.52 \\
\hline $5 / 28 / 072: 30$ & 128.591 & 126.197 & 122.826 & 103.524 & 107.558 & 99.218 & 113.724 & 110.457 & 81.94 & 76.505 \\
\hline 5/28/07 3:00 & 128.579 & 126.193 & 122.812 & 103.513 & 107.546 & 99.206 & 113.713 & 110.444 & 81.927 & 76.518 \\
\hline 5/28/07 3:30 & 128.574 & 126.188 & 122.805 & 103.505 & 107.539 & 99.199 & 113.704 & 110.439 & 81.912 & 76.504 \\
\hline 5/28/07 4:00 & 128.567 & 126.184 & 122.8 & 103.499 & 107.533 & 99.192 & 113.696 & 110.433 & 81.899 & 76.499 \\
\hline $5 / 28 / 074: 30$ & 128.563 & 126.184 & 122.798 & 103.498 & 107.531 & 99.192 & 113.696 & 110.43 & 81.892 & 76.491 \\
\hline 5/28/07 5:00 & 128.56 & 126.184 & 122.793 & 103.496 & 107.529 & 99.19 & 113.692 & 110.428 & 81.883 & 76.485 \\
\hline $5 / 28 / 075: 30$ & 128.56 & 126.175 & 122.793 & 103.495 & 107.529 & 99.187 & 113.69 & 110.426 & 81.873 & 76.479 \\
\hline $5 / 28 / 076: 00$ & 128.555 & 126.175 & 122.789 & 103.491 & 107.525 & 99.185 & 113.688 & 110.424 & 81.866 & 76.483 \\
\hline $5 / 28 / 076: 30$ & 128.551 & 126.175 & 122.784 & 103.488 & 107.52 & 99.178 & 113.683 & 110.417 & 81.857 & 76.489 \\
\hline $5 / 28 / 077: 00$ & 128.549 & 126.177 & 122.782 & 103.486 & 107.52 & 99.176 & 113.683 & 110.417 & 81.849 & 76.479 \\
\hline $5 / 28 / 077: 30$ & 128.549 & 126.17 & 122.782 & 103.488 & 107.522 & 99.178 & 113.683 & 110.419 & 81.844 & 76.475 \\
\hline 5/28/07 8:00 & 128.553 & 126.168 & 122.784 & 103.489 & 107.524 & 99.18 & 113.686 & 110.419 & 81.838 & 76.473 \\
\hline $5 / 28 / 078: 30$ & 128.546 & 126.161 & 122.777 & 103.482 & 107.516 & 99.176 & 113.681 & 110.415 & 81.833 & 76.483 \\
\hline $5 / 28 / 07$ 9:00 & 128.544 & 128.15 & 122.777 & 103.48 & 107.516 & 99.173 & 113.679 & 110.413 & 81.824 & 76.489 \\
\hline 5/28/07 9:30 & 128.537 & 128.402 & 122.768 & 103.472 & 107.508 & 99.166 & 113.671 & 110.404 & 81.816 & 76.481 \\
\hline $5 / 28 / 07 \quad 10: 00$ & 130.587 & 128.536 & 124.197 & 104.189 & 108.028 & 99.843 & 114.285 & 111.876 & 81.833 & 76.518 \\
\hline $5 / 28 / 07 \quad 10: 30$ & 130.861 & 128.622 & 124.466 & 104.432 & 108.274 & 100.09 & 114.51 & 112.162 & 81.866 & 76.609 \\
\hline $5 / 28 / 07 \quad 11: 00$ & 131.001 & 128.679 & 124.607 & 104.563 & 108.413 & 100.227 & 114.629 & 112.31 & 81.892 & 76.708 \\
\hline $5 / 28 / 07 \quad 11: 30$ & 131.088 & 128.717 & 124.697 & 104.651 & 108.502 & 100.317 & 114.709 & 112.401 & 81.912 & 76.772 \\
\hline $5 / 28 / 07 \quad 12: 00$ & 131.154 & 128.736 & 124.752 & 104.704 & 108.558 & 100.371 & 114.757 & 112.461 & 81.951 & 76.83 \\
\hline $5 / 28 / 07 \quad 12: 30$ & 131.192 & 128.754 & 124.792 & 104.742 & 108.598 & 100.409 & 114.79 & 112.505 & 81.979 & 76.884 \\
\hline $5 / 28 / 07$ 13:00 & 131.223 & 128.767 & 124.813 & 104.763 & 108.622 & 100.432 & 114.811 & 112.525 & 82.001 & 76.921 \\
\hline 5/28/07 13:30 & 131.244 & 128.781 & 124.831 & 104.783 & 108.641 & 100.451 & 114.826 & 112.543 & 82.019 & 76.987 \\
\hline $5 / 28 / 07 \quad 14: 00$ & 131.263 & 128.792 & 124.845 & 104.799 & 108.657 & 100.468 & 114.839 & 112.56 & 82.03 & 77.01 \\
\hline $5 / 28 / 07 \quad 14: 30$ & 131.272 & 128.795 & 124.859 & 104.813 & 108.67 & 100.479 & 114.852 & 112.576 & 82.037 & 77.039 \\
\hline 5/28/07 15:00 & 131.282 & 128.801 & 124.868 & 104.821 & 108.679 & 100.491 & 114.86 & 112.585 & 82.045 & 77.053 \\
\hline 5/28/07 15:30 & 131.27 & 128.799 & 124.875 & 104.827 & 108.685 & 100.496 & 114.864 & 112.591 & 82.049 & 77.068 \\
\hline $5 / 28 / 07$ 16:00 & 131.275 & 126.914 & 124.88 & 104.831 & 108.693 & 100.501 & 114.867 & 112.596 & 82.056 & 77.082 \\
\hline 5/28/07 16:30 & 131.275 & 126.619 & 124.88 & 104.832 & 108.693 & 100.505 & 114.869 & 112.598 & 82.063 & 77.088 \\
\hline $5 / 28 / 07 \quad 17: 00$ & 129.336 & 126.467 & 123.543 & 104.177 & 108.238 & 99.89 & 114.304 & 111.225 & 82.054 & 77.08 \\
\hline $5 / 28 / 07 \quad 17: 30$ & 129.014 & 126.368 & 123.23 & 103.901 & 107.958 & 99.605 & 114.053 & 110.891 & 82.03 & 77.001 \\
\hline $5 / 28 / 07$ 18:00 & 128.853 & 126.297 & 123.071 & 103.753 & 107.805 & 99.451 & 113.919 & 110.727 & 82 & 76.921 \\
\hline $5 / 28 / 07$ 18:30 & 128.747 & 126.252 & 122.971 & 103.656 & 107.703 & 99.355 & 113.831 & 110.623 & & 76.863 \\
\hline 5/28/07 19:00 & 128.678 & 126.222 & 122.902 & 103.591 & 107.638 & 99.291 & 113.773 & 110.552 & 81.945 & 76.812 \\
\hline $5 / 28 / 07 \quad 19: 30$ & 128.631 & 126.204 & 122.853 & 103.546 & 107.588 & 99.242 & 113.729 & 110.501 & 81.92 & 76.755 \\
\hline 5/28/07 20:00 & 128.601 & 126.191 & 122.823 & 103.519 & 107.56 & 99.213 & 113.707 & 110.47 & 81.897 & 76.719 \\
\hline
\end{tabular}


TABLE S1.1 (Cont.)

Depth from Top of Casing (ft)

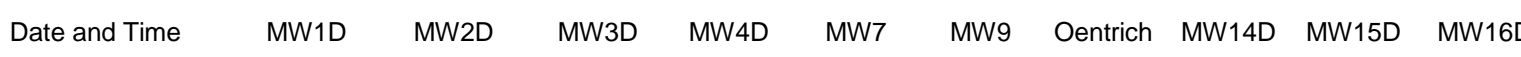

\begin{tabular}{|c|c|c|c|c|c|c|c|c|c|c|}
\hline $5 / 28 / 07$ 20:30 & 128.579 & 126.186 & 122.807 & 103.503 & 107.542 & 99.199 & 113.696 & 110.45 & 81.881 & 76.693 \\
\hline $5 / 28 / 0721: 00$ & 128.567 & 126.182 & 122.793 & 103.493 & 107.531 & 99.185 & 113.685 & 110.439 & 81.864 & 76.667 \\
\hline 5/28/07 21:30 & 128.56 & 126.184 & 122.786 & 103.487 & 107.525 & 99.18 & 113.695 & 110.43 & 81.851 & 76.63 \\
\hline 5/28/07 22:00 & 128.555 & 126.177 & 122.784 & 103.486 & 107.525 & 99.178 & 113.687 & 110.426 & 81.84 & 76.601 \\
\hline 5/28/07 22:30 & 128.558 & 126.177 & 122.784 & 103.489 & 107.527 & 99.183 & 113.683 & 110.43 & 81.827 & 76.588 \\
\hline 5/28/07 23:00 & 128.551 & 126.173 & 122.779 & 103.482 & 107.522 & 99.178 & 113.679 & 110.424 & 81.82 & 76.565 \\
\hline 5/28/07 23:30 & 128.553 & 126.148 & 122.779 & 103.486 & 107.524 & 99.178 & 113.679 & 110.424 & 81.809 & 76.555 \\
\hline $5 / 29 / 070: 00$ & 128.549 & 126.134 & 122.775 & 103.479 & 107.524 & 99.176 & 113.677 & 110.422 & 81.8 & 76.535 \\
\hline 5/29/07 0:30 & 128.525 & 126.118 & 122.752 & 103.458 & 107.497 & 99.15 & 113.656 & 110.397 & 81.789 & 76.52 \\
\hline 5/29/07 1:00 & 128.506 & 126.111 & 122.736 & 103.439 & 107.48 & 99.135 & 113.639 & 110.379 & 81.772 & 76.512 \\
\hline 5/29/07 1:30 & 128.492 & 126.116 & 122.722 & 103.429 & 107.468 & 99.119 & 113.628 & 110.364 & 81.757 & 76.501 \\
\hline 5/29/07 2:00 & 128.485 & 126.109 & 122.715 & 103.422 & 107.461 & 99.114 & 113.622 & 110.357 & 81.744 & 76.495 \\
\hline 5/29/07 2:30 & 128.49 & 126.1 & 122.717 & 103.426 & 107.466 & 99.117 & 113.624 & 110.36 & 81.731 & 76.478 \\
\hline 5/29/07 3:00 & 128.48 & 126.095 & 122.71 & 103.418 & 107.461 & 99.114 & 113.619 & 110.353 & 81.722 & 76.466 \\
\hline 5/29/07 3:30 & 128.471 & 126.091 & 122.701 & 103.41 & 107.453 & 99.107 & 113.609 & 110.346 & 81.709 & 76.456 \\
\hline 5/29/07 4:00 & 128.468 & 126.091 & 122.696 & 103.408 & 107.451 & 99.105 & 113.609 & 110.342 & 81.7 & 76.449 \\
\hline 5/29/07 4:30 & 128.466 & 126.086 & 122.696 & 103.406 & 107.445 & 99.098 & 113.606 & 110.34 & 81.691 & 76.433 \\
\hline 5/29/07 5:00 & 128.464 & 126.089 & 122.694 & 103.403 & 107.447 & 99.098 & 113.604 & 110.337 & 81.68 & 76.427 \\
\hline 5/29/07 5:30 & 128.459 & 126.086 & 122.687 & 103.399 & 107.442 & 99.093 & 113.598 & 110.333 & 81.671 & 76.41 \\
\hline 5/29/07 6:00 & 128.459 & 127.91 & 122.689 & 103.401 & 107.442 & 99.095 & 113.6 & 110.333 & 81.663 & 76.398 \\
\hline 5/29/07 6:30 & 128.459 & 128.255 & 122.687 & 103.401 & 107.444 & 99.095 & 113.6 & 110.333 & 81.656 & 76.387 \\
\hline 5/29/07 7:00 & 130.286 & 126.551 & 123.804 & 103.925 & 107.79 & 99.579 & 113.993 & 111.61 & 81.661 & 76.389 \\
\hline 5/29/07 7:30 & 130.677 & 126.349 & 124.184 & 104.259 & 108.129 & 99.923 & 114.345 & 112.031 & 81.696 & 76.476 \\
\hline 5/29/07 8:00 & 128.958 & 126.263 & 123.177 & 103.842 & 107.895 & 99.546 & 114.006 & 110.855 & 81.715 & 76.555 \\
\hline 5/29/07 8:30 & 128.731 & 126.218 & 122.955 & 103.655 & 107.704 & 99.352 & 113.825 & 110.612 & 81.711 & 76.542 \\
\hline 5/29/07 9:00 & 128.641 & 126.188 & 122.865 & 103.572 & 107.621 & 99.267 & 113.748 & 110.521 & 81.702 & 76.499 \\
\hline 5/29/07 9:30 & 128.594 & 126.161 & 122.821 & 103.53 & 107.579 & 99.225 & 113.711 & 110.47 & 81.696 & 76.47 \\
\hline $5 / 29 / 07$ 10:00 & 128.56 & 126.138 & 122.789 & 103.499 & 107.544 & 99.197 & 113.685 & 110.439 & 81.691 & 76.46 \\
\hline $5 / 29 / 07$ 10:30 & 128.534 & 126.129 & 122.763 & 103.474 & 107.518 & 99.171 & 113.662 & 110.41 & 81.682 & 76.46 \\
\hline 5/29/07 11:00 & 128.513 & 126.12 & 122.74 & 103.455 & 107.499 & 99.152 & 113.648 & 110.391 & 81.674 & 76.449 \\
\hline 5/29/07 11:30 & 128.501 & 126.116 & 122.729 & 103.445 & 107.489 & 99.14 & 113.638 & 110.379 & 81.667 & 76.445 \\
\hline $5 / 29 / 07$ 12:00 & 128.492 & 126.098 & 122.722 & 103.437 & 107.484 & 99.133 & 113.633 & 110.371 & 81.658 & 76.454 \\
\hline $5 / 29 / 07$ 12:30 & 128.487 & 126.082 & 122.717 & 103.43 & 107.478 & 99.128 & 113.629 & 110.364 & 81.649 & 76.443 \\
\hline 5/29/07 13:00 & 128.47 & 126.066 & 122.701 & 103.417 & 107.461 & 99.112 & 113.616 & 110.348 & 81.641 & 76.451 \\
\hline 5/29/07 13:30 & 128.454 & 126.052 & 122.682 & 103.398 & 107.44 & 99.093 & 113.597 & 110.331 & 81.632 & 76.443 \\
\hline $5 / 29 / 07$ 14:00 & 128.44 & 126.041 & 122.671 & 103.383 & 107.432 & 99.081 & 113.588 & 110.317 & 81.625 & 76.456 \\
\hline $5 / 29 / 07 \quad 14: 30$ & 128.421 & 126.034 & 122.653 & 103.369 & 107.413 & 99.065 & 113.573 & 110.3 & 81.615 & 76.478 \\
\hline $5 / 29 / 07$ 15:00 & 128.414 & 126.02 & 122.643 & 103.362 & 107.405 & 99.053 & 113.566 & 110.291 & 81.601 & 76.472 \\
\hline 5/29/07 15:30 & 128.407 & 126.007 & 122.638 & 103.357 & 107.402 & 99.053 & 113.56 & 110.286 & 81.588 & 76.46 \\
\hline 5/29/07 16:00 & 128.395 & 125.998 & 122.627 & 103.345 & 107.39 & 99.039 & 113.549 & 110.271 & 81.577 & 76.474 \\
\hline 5/29/07 16:30 & 128.378 & 125.991 & 122.608 & 103.325 & 107.373 & 99.022 & 113.536 & 110.255 & 81.564 & 76.478 \\
\hline 5/29/07 17:00 & 128.369 & 125.971 & 122.601 & 103.318 & 107.364 & 99.015 & 113.526 & 110.247 & 81.554 & 76.491 \\
\hline $5 / 29 / 07 \quad 17: 30$ & 128.362 & 125.959 & 122.592 & 103.308 & 107.356 & 99.001 & 113.517 & 110.238 & 81.547 & 76.482 \\
\hline 5/29/07 18:00 & 128.345 & 125.948 & 122.574 & 103.295 & 107.341 & 98.992 & 113.506 & 110.224 & 81.534 & 76.47 \\
\hline 5/29/07 18:30 & 128.333 & 125.939 & 122.565 & 103.283 & 107.329 & 98.977 & 113.492 & 110.211 & 81.523 & 76.466 \\
\hline 5/29/07 19:00 & 128.322 & 125.937 & 122.551 & 103.269 & 107.316 & 98.968 & 113.483 & 110.2 & 81.508 & 76.458 \\
\hline 5/29/07 19:30 & 128.312 & 125.927 & 122.546 & 103.263 & 107.31 & 98.961 & 113.477 & 110.191 & 81.497 & 76.462 \\
\hline 5/29/07 20:00 & 128.308 & 125.932 & 122.539 & 103.258 & 107.305 & 98.956 & 113.47 & 110.187 & 81.49 & 76.443 \\
\hline $5 / 29 / 0720: 30$ & 128.3 & 125.927 & 122.532 & 103.251 & 107.299 & 98.947 & 113.466 & 110.178 & 81.481 & 76.447 \\
\hline $5 / 29 / 0721: 00$ & 128.305 & 125.937 & 122.537 & 103.256 & 107.305 & 98.952 & 113.468 & 110.182 & 81.475 & 76.447 \\
\hline 5/29/07 21:30 & 128.298 & 125.943 & 122.532 & 103.251 & 107.299 & 98.947 & 113.464 & 110.178 & 81.468 & 76.441 \\
\hline 5/29/07 22:00 & 128.308 & 125.941 & 122.539 & & 107.308 & 98.959 & 113.472 & 110.187 & 81.466 & 76.431 \\
\hline 5/29/07 22:30 & 128.315 & 125.932 & 122.544 & 103.265 & 107.316 & 98.963 & 113.475 & 110.191 & 81.464 & 76.418 \\
\hline 5/29/07 23:00 & 128.312 & 125.921 & 122.544 & 103.265 & 107.314 & 98.963 & 113.475 & 110.191 & 81.46 & 76.4 \\
\hline 5/29/07 23:30 & 128.305 & 125.916 & 122.537 & 103.256 & 107.305 & 98.956 & 113.47 & 110.187 & 81.453 & 76.379 \\
\hline 5/30/07 0:00 & 128.293 & 125.916 & 122.525 & 103.246 & 107.295 & 98.942 & 113.459 & 110.174 & 81.444 & 76.369 \\
\hline 5/30/07 0:30 & 128.289 & 125.921 & 122.52 & 103.243 & 107.291 & 98.942 & 113.455 & 110.169 & 81.437 & 76.356 \\
\hline 5/30/07 1:00 & 128.286 & 125.93 & 122.52 & 103.243 & 107.293 & 98.94 & 113.453 & 110.167 & 81.431 & 76.381 \\
\hline 5/30/07 1:30 & 128.291 & 125.921 & 122.523 & 103.246 & 107.297 & 98.942 & 113.457 & 110.171 & 81.429 & 76.375 \\
\hline 5/30/07 2:00 & 128.3 & 125.907 & 122.532 & 103.26 & 107.308 & 98.954 & 113.468 & 110.182 & 81.427 & 76.373 \\
\hline 5/30/07 2:30 & 128.296 & 125.894 & 122.527 & 103.252 & 107.301 & 98.947 & 113.462 & 110.176 & 81.424 & 76.365 \\
\hline 5/30/07 3:00 & 128.279 & 125.887 & 122.514 & 103.236 & 107.285 & 98.933 & 113.447 & 110.16 & 81.413 & 76.36 \\
\hline 5/30/07 3:30 & 128.267 & 125.875 & 122.502 & 103.224 & 107.274 & 98.921 & 113.436 & 110.149 & 81.403 & 76.356 \\
\hline 5/30/07 4:00 & 128.258 & 125.857 & 122.493 & 103.215 & 107.266 & 98.914 & 113.429 & 110.14 & 81.394 & 76.354 \\
\hline $0 / 074: 30$ & 128.255 & 125.834 & 122.481 & 103.205 & 107.257 & 98.902 & 113.417 & 110.132 & 81.385 & 76.363 \\
\hline 5/30/07 5:00 & 128.253 & 125.812 & 122.465 & 103.188 & 107.238 & 98.886 & 113.404 & 110.114 & 81.378 & 76.363 \\
\hline
\end{tabular}


TABLE S1.1 (Cont.)

Depth from Top of Casing (ft)

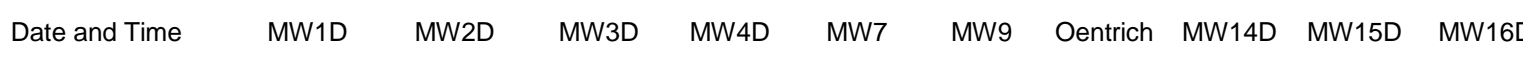

\begin{tabular}{|c|c|c|c|c|c|c|c|c|c|c|}
\hline 5/30/07 5:30 & 128.251 & 125.801 & 122.446 & 103.167 & 107.217 & 98.867 & 113.387 & 110.094 & 81.365 & 76.36 \\
\hline 5/30/07 6:00 & 128.246 & 127.647 & 122.419 & 103.141 & 107.192 & 98.838 & 113.361 & 110.067 & 81.35 & 76.352 \\
\hline 5/30/07 6:30 & 128.241 & 128.032 & 122.407 & 103.131 & 107.182 & 98.831 & 113.353 & 110.061 & 81.339 & 76.354 \\
\hline 5/30/07 7:00 & 129.932 & 128.216 & 123.677 & 103.706 & 107.556 & 99.359 & 113.794 & 111.349 & 81.342 & 76.373 \\
\hline 5/30/07 7:30 & 130.376 & 128.323 & 124.089 & 104.08 & 107.939 & 99.746 & 114.175 & 111.794 & 81.381 & 76.458 \\
\hline 5/30/07 8:00 & 130.584 & 128.391 & 124.285 & 104.266 & 108.127 & 99.932 & 114.346 & 111.994 & 81.429 & 76.532 \\
\hline 5/30/07 8:30 & 130.7 & 128.445 & 124.394 & 104.368 & 108.234 & 100.038 & 114.44 & 112.106 & 81.468 & 76.6 \\
\hline 5/30/07 9:00 & 130.778 & 128.472 & 124.463 & 104.435 & 108.3 & 100.107 & 114.5 & 112.179 & 81.499 & 76.673 \\
\hline 5/30/07 9:30 & 130.828 & 128.518 & 124.514 & 104.485 & 108.352 & 100.154 & 114.546 & 112.237 & 81.531 & 76.741 \\
\hline $5 / 30 / 07$ 10:00 & 130.863 & 128.552 & 124.549 & 104.52 & 108.388 & 100.187 & 114.576 & 112.268 & 81.558 & 76.774 \\
\hline $5 / 30 / 07 \quad 10: 30$ & 130.984 & 128.561 & 124.593 & 104.566 & 108.436 & 100.234 & 114.615 & 112.315 & 81.59 & 76.817 \\
\hline 5/30/07 11:00 & 131.02 & 128.579 & 124.623 & 104.594 & 108.464 & 100.262 & 114.639 & 112.343 & 81.619 & 76.842 \\
\hline $5 / 30 / 0711: 30$ & 131.036 & 127.032 & 124.641 & 104.613 & 108.485 & 100.284 & 114.658 & 112.365 & 81.645 & 76.873 \\
\hline $5 / 30 / 07$ 12:00 & 131.053 & 126.52 & 124.66 & 104.632 & 108.502 & 100.305 & 114.675 & 112.383 & 81.671 & 76.906 \\
\hline $5 / 30 / 07$ 12:30 & 129.572 & 126.334 & 123.73 & 104.265 & 108.331 & 99.996 & 114.395 & 111.533 & 81.691 & 76.921 \\
\hline $5 / 30 / 07$ 13:00 & 128.92 & 126.225 & 123.138 & 103.822 & 107.891 & 99.527 & 113.97 & 110.811 & 81.674 & 76.876 \\
\hline 5/30/07 13:30 & 128.721 & 126.152 & 122.941 & 103.641 & 107.706 & 99.345 & 113.806 & 110.608 & 81.658 & 76.799 \\
\hline $5 / 30 / 07$ 14:00 & 128.61 & 126.1 & 122.833 & 103.539 & 107.602 & 99.242 & 113.711 & 110.492 & 81.639 & 76.739 \\
\hline $5 / 30 / 07 \quad 14: 30$ & 128.537 & 126.054 & 122.759 & 103.47 & 107.529 & 99.171 & 113.649 & 110.419 & 81.625 & 76.691 \\
\hline $5 / 30 / 07$ 15:00 & 128.48 & 126.018 & 122.705 & 103.419 & 107.476 & 99.119 & 113.602 & 110.362 & 81.61 & 76.664 \\
\hline 5/30/07 15:30 & 128.435 & 125.993 & 122.662 & 103.377 & 107.432 & 99.077 & 113.564 & 110.317 & 81.591 & 76.617 \\
\hline $5 / 30 / 07$ 16:00 & 128.399 & 125.968 & 122.627 & 103.344 & 107.398 & 99.041 & 113.534 & 110.28 & 81.577 & 76.582 \\
\hline $5 / 30 / 07 \quad 16: 30$ & 128.373 & 125.959 & 122.601 & 103.318 & 107.375 & 99.018 & 113.514 & 110.253 & 81.56 & 76.553 \\
\hline $5 / 30 / 07$ 17:00 & 128.345 & 125.957 & 122.574 & 103.293 & 107.346 & 98.992 & 113.491 & 110.224 & 81.542 & 76.524 \\
\hline $5 / 30 / 07$ 17:30 & 128.336 & 125.937 & 122.564 & 103.284 & 107.341 & 98.982 & 113.484 & 110.216 & 81.529 & 76.499 \\
\hline 5/30/07 18:00 & 128.336 & 125.921 & 122.565 & 103.287 & 107.343 & 98.985 & 113.486 & 110.213 & 81.519 & 76.451 \\
\hline $5 / 30 / 07$ 18:30 & 128.312 & 125.918 & 122.541 & 103.265 & 107.32 & 98.961 & 113.465 & 110.191 & 81.507 & 76.398 \\
\hline 5/30/07 19:00 & 128.296 & 125.914 & 122.527 & 103.248 & 107.303 & 98.949 & 113.456 & 110.178 & 81.492 & 76.344 \\
\hline 5/30/07 19:30 & 128.296 & 125.909 & 122.525 & 103.25 & 107.305 & 98.947 & 113.454 & 110.176 & 81.483 & 76.294 \\
\hline $5 / 30 / 0720: 00$ & 128.286 & 125.909 & 122.516 & 103.241 & 107.295 & 98.935 & 113.445 & 110.165 & 81.475 & 76.253 \\
\hline 5/30/07 20:30 & 128.284 & 125.921 & 122.516 & 103.241 & 107.293 & 98.937 & 113.445 & 110.167 & 81.466 & 76.212 \\
\hline $5 / 30 / 0721: 00$ & 128.286 & 125.925 & 122.518 & 103.245 & 107.299 & 98.94 & 113.449 & 110.167 & 81.46 & 76.17 \\
\hline 5/30/07 21:30 & 128.293 & 125.925 & 122.525 & 103.253 & 107.306 & 98.949 & 113.456 & 110.176 & 81.457 & 76.129 \\
\hline 5/30/07 22:00 & 128.3 & 125.923 & 122.53 & 103.258 & 107.31 & 98.954 & 113.46 & 110.18 & 81.455 & 76.102 \\
\hline 5/30/07 22:30 & 128.3 & 125.912 & 122.532 & 103.262 & 107.314 & 98.959 & 113.464 & 110.182 & 81.453 & 76.085 \\
\hline 5/30/07 23:00 & 128.3 & 125.909 & 122.53 & 103.258 & 107.312 & 98.956 & 113.462 & 110.18 & 81.451 & 76.069 \\
\hline 5/30/07 23:30 & 128.291 & 125.903 & 122.521 & 103.25 & 107.301 & 98.944 & 113.452 & 110.171 & 81.444 & 76.048 \\
\hline 5/31/07 0:00 & 128.281 & 125.898 & 122.516 & 103.244 & 107.299 & 98.944 & 113.449 & 110.165 & 81.438 & 76.044 \\
\hline 5/31/07 0:30 & 128.277 & 125.889 & 122.509 & 103.238 & 107.291 & 98.935 & 113.441 & 110.158 & 81.433 & 76.032 \\
\hline 5/31/07 1:00 & 128.272 & 125.88 & 122.507 & 103.234 & 107.289 & 98.933 & 113.439 & 110.156 & 81.429 & 76.028 \\
\hline 5/31/07 1:30 & 128.265 & 125.869 & 122.497 & 103.225 & 107.28 & 98.926 & 113.432 & 110.147 & 81.422 & 76.026 \\
\hline 5/31/07 2:00 & 128.255 & 125.857 & 122.49 & 103.22 & 107.274 & 98.916 & 113.425 & 110.14 & 81.414 & 76.017 \\
\hline 5/31/07 2:30 & 128.244 & 125.846 & 122.476 & 103.206 & 107.261 & 98.902 & 113.413 & 110.127 & 81.407 & 76.011 \\
\hline 5/31/07 3:00 & 128.232 & 125.837 & 122.463 & 103.194 & 107.249 & 98.89 & 113.402 & 110.114 & 81.398 & 76.007 \\
\hline $5 / 31 / 07$ 3:30 & 128.22 & 125.85 & 122.454 & 103.184 & 107.24 & 98.881 & 113.393 & 110.103 & 81.389 & 76.003 \\
\hline 5/31/07 4:00 & 128.213 & 125.841 & 122.444 & 103.177 & 107.232 & 98.874 & 113.383 & 110.094 & 81.381 & 75.988 \\
\hline $5 / 31 / 074: 30$ & 128.225 & 125.835 & 122.458 & 103.193 & 107.247 & 98.888 & 113.394 & 110.107 & 81.381 & 75.98 \\
\hline 5/31/07 5:00 & 128.218 & 125.846 & 122.451 & 103.181 & 107.236 & 98.878 & 113.389 & 110.101 & 81.374 & 75.98 \\
\hline 5/31/07 5:30 & 128.208 & 125.841 & 122.444 & 103.177 & 107.23 & 98.874 & 113.383 & 110.092 & 81.366 & 75.978 \\
\hline 5/31/07 6:00 & 128.215 & 127.753 & 122.449 & 103.184 & 107.238 & 98.881 & 113.391 & 110.101 & 81.366 & 75.974 \\
\hline 5/31/07 6:30 & 128.215 & 128.055 & 122.449 & 103.183 & 107.236 & 98.878 & 113.389 & 110.098 & 81.363 & 75.984 \\
\hline 5/31/07 7:00 & 130.149 & 128.198 & 123.656 & 103.77 & 107.64 & 99.425 & 113.852 & 111.484 & 81.372 & 76.011 \\
\hline 5/31/07 7:30 & 130.478 & 128.284 & 123.978 & 104.063 & 107.939 & 99.727 & 114.149 & 111.832 & 81.407 & 76.106 \\
\hline 5/31/07 8:00 & 130.632 & 126.744 & 124.128 & 104.203 & 108.085 & 99.871 & 114.282 & 111.991 & 81.44 & 76.205 \\
\hline 5/31/07 8:30 & 130.722 & 126.281 & 124.22 & 104.29 & 108.175 & 99.96 & 114.363 & 112.086 & 81.468 & 76.284 \\
\hline 5/31/07 9:00 & 129.284 & 127.352 & 123.462 & 104.006 & 108.074 & 99.737 & 114.175 & 111.285 & 81.494 & 76.363 \\
\hline 5/31/07 9:30 & 128.674 & 128.1 & 122.897 & 103.601 & 107.672 & 99.308 & 113.769 & 110.574 & 81.484 & 76.342 \\
\hline $5 / 31 / 07$ 10:00 & 128.998 & 128.252 & 122.904 & 103.565 & 107.525 & 99.168 & 113.632 & 110.404 & 81.475 & 76.282 \\
\hline $5 / 31 / 07$ 10:30 & 130.518 & 128.323 & 124.019 & 104.104 & 107.984 & 99.772 & 114.187 & 111.878 & 81.494 & 76.309 \\
\hline $5 / 31 / 0711: 00$ & 130.684 & 128.361 & 124.181 & 104.252 & 108.139 & 99.921 & 114.326 & 112.047 & 81.516 & 76.375 \\
\hline 5/31/07 11:30 & 130.757 & 128.379 & 124.26 & 104.323 & 108.211 & 99.996 & 114.395 & 112.129 & 81.518 & 76.447 \\
\hline $5 / 31 / 07$ 12:00 & 130.797 & 126.644 & 124.297 & 104.359 & 108.251 & 100.034 & 114.429 & 112.166 & 81.521 & 76.501 \\
\hline $5 / 31 / 07 \quad 12: 30$ & 130.819 & 126.275 & 124.322 & 104.383 & 108.276 & 100.06 & 114.45 & 112.193 & 81.527 & 76.538 \\
\hline $5 / 31 / 07$ 13:00 & 129.09 & 126.102 & 123.298 & 103.925 & 108.003 & 99.647 & 114.081 & 111.03 & 81.529 & 76.571 \\
\hline $5 / 31 / 07$ 13:30 & 128.662 & 125.991 & 122.884 & 103.582 & 107.657 & 99.289 & 113.754 & 110.561 & 81.499 & 76.513 \\
\hline $5 / 31 / 0714: 00$ & 128.487 & 125.914 & 122.712 & 103.422 & 107.491 & 99.126 & 113.606 & 110.384 & 81.47 & 76.415 \\
\hline
\end{tabular}


TABLE S1.1 (Cont.)

Depth from Top of Casing (ft)

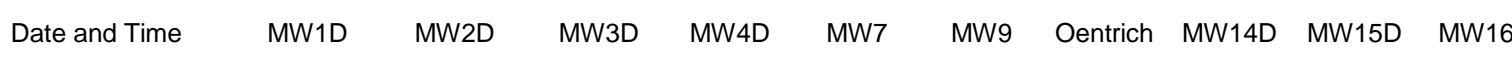

\begin{tabular}{|c|c|c|c|c|c|c|c|c|c|c|}
\hline 5/31/07 14:30 & 128.373 & 125.855 & 122.602 & 103.315 & 107.381 & 99.018 & 113.506 & 110.264 & 81.442 & 76.338 \\
\hline 5/31/07 15:00 & 128.291 & 125.807 & 122.521 & 103.239 & 107.301 & 98.937 & 113.435 & 110.18 & 81.414 & 76.278 \\
\hline 5/31/07 15:30 & 128.232 & 125.762 & 122.463 & 103.182 & 107.244 & 98.881 & 113.384 & 110.12 & 81.387 & 76.231 \\
\hline 5/31/07 16:00 & 128.185 & 125.723 & 122.414 & 103.138 & 107.196 & 98.834 & 113.342 & 110.072 & 81.359 & 76.185 \\
\hline 5/31/07 16:30 & 128.133 & 125.698 & 122.363 & 103.086 & 107.145 & 98.784 & 113.296 & 110.021 & 81.33 & 76.146 \\
\hline $5 / 31 / 07$ 17:00 & 128.099 & 125.683 & 122.329 & 103.053 & 107.112 & 98.754 & 113.266 & 109.983 & 81.304 & 76.107 \\
\hline $5 / 31 / 07$ 17:30 & 128.076 & 125.664 & 122.305 & 103.031 & 107.089 & 98.732 & 113.245 & 109.959 & 81.283 & 76.057 \\
\hline $5 / 31 / 07$ 18:00 & 128.057 & 125.664 & 122.289 & 103.015 & 107.074 & 98.716 & 113.234 & 109.943 & 81.265 & 76.02 \\
\hline $5 / 31 / 07$ 18:30 & 128.04 & 125.678 & 122.271 & 103 & 107.059 & 98.697 & 113.217 & 109.923 & 81.243 & 75.974 \\
\hline 5/31/07 19:00 & 128.036 & 125.696 & 122.269 & 103 & 107.057 & 98.699 & 113.219 & 109.921 & 81.228 & 75.923 \\
\hline 5/31/07 19:30 & 128.05 & 125.685 & 122.283 & 103.016 & 107.072 & 98.713 & 113.234 & 109.934 & 81.219 & 75.902 \\
\hline 5/31/07 20:00 & 128.069 & 125.687 & 122.301 & 103.034 & 107.093 & 98.732 & 113.251 & 109.952 & 81.215 & 75.881 \\
\hline $5 / 31 / 0720: 30$ & 128.062 & 125.671 & 122.291 & 103.024 & 107.087 & 98.725 & 113.246 & 109.946 & 81.204 & 75.869 \\
\hline 5/31/07 21:00 & 128.062 & 125.68 & 122.294 & 103.029 & 107.089 & 98.73 & 113.249 & 109.95 & 81.193 & 75.856 \\
\hline 5/31/07 21:30 & 128.043 & 125.655 & 122.275 & 103.012 & 107.07 & 98.709 & 113.229 & 109.928 & 81.174 & 75.85 \\
\hline 5/31/07 22:00 & 128.052 & 125.66 & 122.287 & 103.022 & 107.082 & 98.721 & 113.242 & 109.939 & 81.171 & 75.84 \\
\hline 5/31/07 22:30 & 128.031 & 125.635 & 122.261 & 102.997 & 107.055 & 98.697 & 113.223 & 109.919 & 81.154 & 75.846 \\
\hline 5/31/07 23:00 & 128.029 & 125.578 & 122.261 & 103 & 107.061 & 98.695 & 113.217 & 109.917 & 81.143 & 75.83 \\
\hline 5/31/07 23:30 & 128.014 & 125.531 & 122.245 & 102.977 & 107.04 & 98.678 & 113.204 & 109.903 & 81.13 & 75.823 \\
\hline 6/1/07 0:00 & 128.019 & 125.51 & 122.185 & 102.917 & 106.975 & 98.617 & 113.149 & 109.844 & 81.103 & 75.811 \\
\hline 6/1/07 0:30 & 128.014 & 125.512 & 122.127 & 102.862 & 106.914 & 98.553 & 113.089 & 109.784 & 81.068 & 75.786 \\
\hline 6/1/07 1:00 & 128 & 125.474 & 122.12 & 102.855 & 106.918 & 98.551 & 113.089 & 109.775 & 81.057 & 75.751 \\
\hline 6/1/07 1:30 & 127.986 & 125.467 & 122.116 & 102.855 & 106.916 & 98.553 & 113.088 & 109.771 & 81.044 & 75.726 \\
\hline $6 / 1 / 072: 00$ & 127.988 & 125.474 & 122.076 & 102.816 & 106.876 & 98.513 & 113.05 & 109.735 & 81.024 & 75.722 \\
\hline $6 / 1 / 072: 30$ & 127.977 & 125.501 & 122.076 & 102.814 & 106.878 & 98.513 & 113.056 & 109.735 & 81.011 & 75.697 \\
\hline 6/1/07 3:00 & 127.962 & 125.506 & 122.079 & 102.819 & 106.882 & 98.515 & 113.054 & 109.733 & 80.996 & 75.683 \\
\hline 6/1/07 3:30 & 127.953 & 125.485 & 122.1 & 102.847 & 106.907 & 98.539 & 113.077 & 109.753 & 80.998 & 75.677 \\
\hline 6/1/07 4:00 & 127.95 & 125.499 & 122.116 & 102.855 & 106.92 & 98.553 & 113.09 & 109.768 & 80.996 & 75.683 \\
\hline $6 / 1 / 074: 30$ & 127.953 & 125.494 & 122.095 & 102.835 & 106.897 & 98.534 & 113.073 & 109.751 & 80.979 & 75.687 \\
\hline $6 / 1 / 075: 00$ & 127.941 & 125.51 & 122.106 & 102.848 & 106.912 & 98.546 & 113.082 & 109.764 & 80.976 & 75.681 \\
\hline 6/1/07 5:30 & 127.938 & 125.517 & 122.1 & 102.84 & 106.905 & 98.537 & 113.075 & 109.755 & 80.963 & 75.681 \\
\hline 6/1/07 6:00 & 127.932 & 127.447 & 122.113 & 102.858 & 106.92 & 98.553 & 113.088 & 109.768 & 80.965 & 75.674 \\
\hline 6/1/07 6:30 & 127.929 & 127.753 & 122.123 & 102.865 & 106.931 & 98.563 & 113.096 & 109.777 & 80.961 & 75.679 \\
\hline 6/1/07 7:00 & 129.752 & 127.917 & 123.488 & 103.524 & 107.385 & 99.178 & 113.625 & 111.174 & 80.976 & 75.705 \\
\hline $6 / 1 / 077: 30$ & 130.1 & 128.007 & 123.816 & 103.818 & 107.687 & 99.482 & 113.92 & 111.524 & 81.011 & 75.821 \\
\hline 6/1/07 8:00 & 130.279 & 128.062 & 123.985 & 103.975 & 107.849 & 99.645 & 114.072 & 111.699 & 81.046 & 75.925 \\
\hline 6/1/07 8:30 & 130.376 & 128.109 & 124.079 & 104.072 & 107.946 & 99.739 & 114.157 & 111.799 & 81.075 & 76.034 \\
\hline 6/1/07 9:00 & 130.445 & 126.186 & 124.139 & 104.128 & 108.007 & 99.798 & 114.211 & 111.86 & 81.105 & 76.094 \\
\hline 6/1/07 9:30 & 130.568 & 125.946 & 124.19 & 104.178 & 108.057 & 99.847 & 114.254 & 111.912 & 81.132 & 76.15 \\
\hline 6/1/07 10:00 & 128.596 & 125.828 & 122.807 & 103.496 & 107.583 & 99.211 & 113.683 & 110.495 & 81.134 & 76.162 \\
\hline 6/1/07 10:30 & 128.334 & 125.748 & 122.555 & 103.27 & 107.35 & 98.98 & 113.463 & 110.231 & 81.119 & 76.098 \\
\hline 6/1/07 11:00 & 128.208 & 125.696 & 122.435 & 103.16 & 107.234 & & 113.357 & 110.103 & 81.108 & 76.045 \\
\hline 6/1/07 11:30 & 128.128 & 125.664 & 122.359 & 103.082 & 107.156 & 98.787 & 113.292 & 110.021 & 81.094 & 75.995 \\
\hline 6/1/07 12:00 & 128.071 & 125.639 & 122.303 & 103.032 & 107.103 & 98.732 & 113.243 & 109.963 & 81.083 & 75.96 \\
\hline $6 / 1 / 0712: 30$ & 128.04 & 125.621 & 122.271 & 103.003 & 107.072 & & 113.215 & & & 75.92 \\
\hline 6/1/07 13:00 & 128.019 & 125.61 & 122.25 & 102.982 & 107.049 & 98.68 & 113.196 & 109.908 & 81.064 & 75.885 \\
\hline 6/1/07 13:30 & 127.998 & 125.596 & 122.234 & 102.965 & 107.032 & 98.664 & 113.183 & 109.888 & 81.055 & 75.863 \\
\hline 6/1/07 14:00 & 127.986 & 125.59 & 122.217 & 102.953 & 107.019 & & 113.176 & 109.875 & 81.046 & 75.842 \\
\hline 6/1/07 14:30 & 127.974 & 125.587 & 122.206 & 102.943 & 107.009 & 98.64 & 113.163 & 109.865 & 81.04 & 75.823 \\
\hline $6 / 1 / 07$ 15:00 & 127.96 & 125.578 & 122.194 & 102.936 & 106.998 & 98.629 & 113.153 & 109.853 & 81.027 & 75.815 \\
\hline $6 / 1 / 0715: 30$ & 127.943 & 125.576 & 122.192 & & & & 113.153 & 109.848 & 81.02 & 75.803 \\
\hline 6/1/07 16:00 & 127.939 & 125.56 & 122.187 & 102.929 & 106.992 & 98.624 & 113.152 & 109.844 & 81.014 & 75.788 \\
\hline 6/1/07 16:30 & 127.932 & 125.546 & 122.185 & 102.926 & 106.99 & 98.621 & 113.148 & 109.841 & 81.009 & 75.772 \\
\hline $6 / 1 / 0717: 00$ & 127.922 & 125.528 & 122.174 & 102.912 & 106.983 & & 113.138 & 109.83 & 81 & 75.755 \\
\hline 6/1/07 17:30 & 127.917 & 125.497 & 122.158 & 102.9 & 106.964 & 98.598 & 113.127 & 109.815 & 80.989 & 75.747 \\
\hline 6/1/07 18:00 & 127.913 & 125.494 & 122.141 & 102.885 & 106.95 & 98.581 & 113.11 & 109.799 & 80.976 & 75.739 \\
\hline $6 / 1 / 0718: 30$ & 127.908 & 125.49 & 122.109 & 102.85 & 106.918 & 98.548 & 113.082 & 109.766 & 80.959 & 75.726 \\
\hline 6/1/07 19:00 & 127.882 & 125.488 & 122.1 & 102.845 & 106.91 & 98.539 & 113.073 & 109.755 & 80.95 & 75.718 \\
\hline 6/1/07 19:30 & 127.873 & 125.476 & 122.095 & 102.843 & 106.908 & 98.539 & 113.071 & 109.755 & 80.942 & 75.703 \\
\hline 6/1/07 20:00 & 127.865 & 125.483 & 122.093 & 102.839 & 106.908 & 98.537 & 113.076 & 109.751 & 80.937 & 75.691 \\
\hline $6 / 1 / 0720: 30$ & 127.851 & 125.49 & 122.081 & 102.827 & 106.891 & 98.52 & 113.06 & 109.737 & 80.926 & 75.683 \\
\hline 6/1/07 21:00 & 127.849 & 125.488 & 122.088 & 102.836 & 106.901 & 98.53 & 113.067 & 109.744 & 80.922 & 75.66 \\
\hline 6/1/07 21:30 & 127.858 & 125.492 & 122.095 & 102.845 & 106.91 & 98.544 & 113.073 & 109.753 & 80.92 & 75.646 \\
\hline 6/1/07 22:00 & 127.858 & 125.481 & 122.095 & 102.843 & 106.91 & 98.541 & 113.073 & 109.753 & 80.917 & 75.643 \\
\hline 6/1/07 22:30 & 127.856 & 125.478 & 122.095 & 102.845 & 106.91 & 98.541 & 113.078 & 109.753 & 80.913 & 75.627 \\
\hline 6/1/07 23:00 & 127.856 & 125.467 & 122.09 & 102.836 & 106.904 & 98.537 & 113.071 & 109.746 & 80.907 & 75.629 \\
\hline
\end{tabular}


TABLE S1.1 (Cont.)

Depth from Top of Casing (ft)

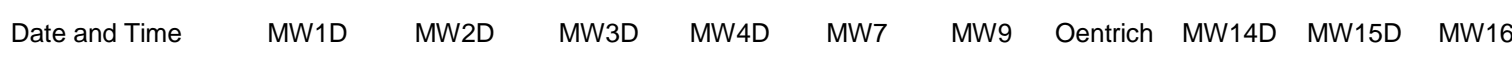

\begin{tabular}{|c|c|c|c|c|c|c|c|c|c|c|}
\hline 6/1/07 23:30 & 127.849 & 125.472 & 122.086 & 102.836 & 106.904 & 98.532 & 113.067 & 109.746 & 80.902 & 75.621 \\
\hline 6/2/07 0:00 & 127.861 & 125.472 & 122.076 & 102.822 & 106.889 & 98.52 & 113.056 & 109.735 & 80.895 & 75.623 \\
\hline 6/2/07 0:30 & 127.856 & 125.476 & 122.076 & 102.826 & 106.893 & 98.522 & 113.056 & 109.735 & 80.895 & 75.606 \\
\hline 6/2/07 1:00 & 127.844 & 125.46 & 122.079 & 102.829 & 106.897 & 98.527 & 113.058 & 109.737 & 80.889 & 75.615 \\
\hline $6 / 2 / 071: 30$ & 127.839 & 125.449 & 122.083 & 102.834 & 106.901 & 98.532 & 113.065 & 109.742 & 80.891 & 75.596 \\
\hline 6/2/07 2:00 & 127.837 & 125.44 & 122.069 & 102.817 & 106.888 & 98.515 & 113.052 & 109.731 & 80.882 & 75.602 \\
\hline 6/2/07 2:30 & 127.832 & 125.433 & 122.056 & 102.803 & 106.872 & 98.503 & 113.039 & 109.715 & 80.872 & 75.6 \\
\hline 6/2/07 3:00 & 127.83 & 125.431 & 122.049 & 102.8 & 106.867 & 98.499 & 113.035 & 109.708 & 80.867 & 75.598 \\
\hline 6/2/07 3:30 & 127.827 & 125.424 & 122.042 & 102.791 & 106.857 & 98.487 & 113.024 & 109.699 & 80.861 & 75.594 \\
\hline 6/2/07 4:00 & 127.823 & 125.419 & 122.037 & 102.786 & 106.855 & 98.485 & 113.02 & 109.695 & 80.854 & 75.586 \\
\hline 6/2/07 4:30 & 127.82 & 125.41 & 122.03 & 102.781 & 106.851 & 98.48 & 113.02 & 109.691 & 80.848 & 75.594 \\
\hline 6/2/07 5:00 & 127.79 & 125.413 & 122.025 & 102.774 & 106.844 & 98.473 & 113.009 & 109.682 & 80.843 & 75.588 \\
\hline 6/2/07 5:30 & 127.783 & 125.415 & 122.016 & 102.767 & 106.838 & 98.466 & 113.005 & 109.675 & 80.836 & 75.59 \\
\hline 6/2/07 6:00 & 127.778 & 125.417 & 122.019 & 102.772 & 106.844 & 98.468 & 113.009 & 109.678 & 80.832 & 75.583 \\
\hline 6/2/07 6:30 & 127.785 & 125.408 & 122.023 & 102.776 & 106.846 & 98.473 & 113.013 & 109.684 & 80.828 & 75.577 \\
\hline 6/2/07 7:00 & 127.787 & 127.347 & 122.023 & 102.776 & 106.846 & 98.473 & 113.011 & 109.684 & 80.826 & 75.581 \\
\hline 6/2/07 7:30 & 127.782 & 127.665 & 122.016 & 102.767 & 106.838 & 98.464 & 113.003 & 109.675 & 80.821 & 75.583 \\
\hline 6/2/07 8:00 & 129.693 & 127.819 & 123.242 & 103.37 & 107.253 & 99.027 & 113.475 & 111.077 & 80.832 & 75.631 \\
\hline 6/2/07 8:30 & 130.078 & 127.912 & 123.58 & 103.67 & 107.56 & 99.34 & 113.777 & 111.444 & 80.869 & 75.741 \\
\hline 6/2/07 9:00 & 130.244 & 127.969 & 123.742 & 103.823 & 107.718 & 99.498 & 113.929 & 111.615 & 80.906 & 75.848 \\
\hline 6/2/07 9:30 & 130.339 & 128.005 & 123.841 & 103.918 & 107.815 & 99.593 & 114.015 & 111.717 & 80.937 & 75.931 \\
\hline 6/2/07 10:00 & 130.407 & 128.037 & 123.901 & 103.975 & 107.876 & 99.652 & 114.072 & 111.777 & 80.963 & 76.005 \\
\hline $6 / 2 / 07 \quad 10: 30$ & 130.447 & 128.062 & 123.941 & 104.013 & 107.916 & 99.689 & 114.104 & 111.816 & 80.989 & 76.063 \\
\hline 6/2/07 11:00 & 130.478 & 128.08 & 123.971 & 104.044 & 107.948 & 99.72 & 114.132 & 111.85 & 81.012 & 76.117 \\
\hline 6/2/07 11:30 & 130.502 & 128.089 & 124.001 & 104.073 & 107.979 & 99.748 & 114.162 & 111.876 & 81.036 & 76.152 \\
\hline 6/2/07 12:00 & 130.523 & 128.098 & 124.019 & 104.092 & 108 & 99.774 & 114.175 & 111.896 & 81.06 & 76.187 \\
\hline 6/2/07 12:30 & 130.535 & 127.966 & 124.033 & 104.106 & 108.011 & 99.784 & 114.187 & 111.909 & 81.081 & 76.214 \\
\hline 6/2/07 13:00 & 130.54 & 126.082 & 124.04 & 104.113 & 108.019 & 99.791 & 114.196 & 111.916 & 81.099 & 76.239 \\
\hline 6/2/07 13:30 & 130.542 & 125.853 & 124.045 & 104.12 & 108.028 & 99.8 & 114.202 & 111.923 & 81.116 & 76.266 \\
\hline $6 / 2 / 07$ 14:00 & 128.49 & 125.766 & 122.708 & 103.405 & 107.497 & 99.121 & 113.623 & 110.399 & 81.107 & 76.231 \\
\hline 6/2/07 14:30 & 128.241 & 125.646 & 122.465 & 103.186 & 107.272 & 98.897 & 113.387 & 110.147 & 81.084 & 76.142 \\
\hline $6 / 2 / 07$ 15:00 & 128.165 & 125.574 & 122.402 & 103.141 & 107.217 & 98.857 & 113.685 & 110.056 & 81.079 & 76.071 \\
\hline 6/2/07 15:30 & 128.026 & 125.522 & 122.254 & 102.986 & 107.067 & 98.69 & 113.232 & 109.923 & 81.053 & 76.016 \\
\hline 6/2/07 16:00 & 127.953 & 125.485 & 122.178 & 102.915 & 106.99 & 98.617 & 113.164 & 109.848 & 81.031 & 75.978 \\
\hline 6/2/07 16:30 & 127.903 & 125.456 & 122.132 & 102.867 & 106.943 & 98.57 & 113.113 & 109.797 & 81.011 & 75.929 \\
\hline 6/2/07 17:00 & 127.865 & 125.429 & 122.095 & 102.831 & 106.905 & 98.532 & 113.077 & 109.757 & 80.99 & 75.881 \\
\hline $6 / 2 / 07$ 17:30 & 127.83 & 125.406 & 122.062 & 102.8 & 106.876 & 98.501 & 113.054 & 109.724 & 80.972 & 75.846 \\
\hline $6 / 2 / 07$ 18:00 & 127.804 & 125.388 & 122.037 & 102.777 & 106.851 & 98.478 & 113.037 & 109.7 & 80.955 & 75.784 \\
\hline $6 / 2 / 07$ 18:30 & 127.78 & 125.376 & 122.012 & 102.753 & 106.829 & 98.454 & 113.018 & 109.673 & 80.937 & 75.73 \\
\hline 6/2/07 19:00 & 127.761 & 125.372 & 121.995 & 102.738 & 106.811 & 98.438 & 113.001 & 109.658 & 80.922 & 75.687 \\
\hline 6/2/07 19:30 & 127.75 & 125.379 & 121.979 & 102.726 & 106.802 & 98.428 & 112.984 & 109.644 & 80.906 & 75.641 \\
\hline 6/2/07 20:00 & 127.747 & 125.365 & 121.977 & 102.727 & 106.8 & 98.428 & 112.982 & 109.642 & 80.895 & 75.608 \\
\hline 6/2/07 20:30 & 127.75 & 125.361 & 121.979 & 102.729 & 106.804 & 98.428 & 112.982 & 109.642 & 80.885 & 75.581 \\
\hline 6/2/07 21:00 & 127.738 & 125.365 & 121.97 & 102.721 & 106.794 & 98.419 & 112.974 & 109.633 & 80.874 & 75.569 \\
\hline $6 / 2 / 0721: 30$ & 127.738 & 125.363 & 121.97 & 102.721 & 106.796 & 98.421 & 112.971 & 109.633 & 80.867 & 75.559 \\
\hline 6/2/07 22:00 & 127.738 & 125.356 & 121.97 & 102.724 & 106.798 & 98.423 & 112.971 & 109.636 & 80.858 & 75.544 \\
\hline 6/2/07 22:30 & 127.733 & 125.351 & 121.968 & 102.719 & 106.792 & 98.419 & 112.965 & 109.629 & 80.852 & 75.544 \\
\hline 6/2/07 23:00 & 127.728 & 125.345 & 121.961 & 102.715 & 106.79 & 98.414 & 112.961 & 109.625 & 80.845 & 75.544 \\
\hline 6/2/07 23:30 & 127.721 & 125.342 & 121.956 & 102.709 & 106.783 & 98.407 & 112.953 & 109.62 & 80.836 & 75.54 \\
\hline $6 / 3 / 070: 00$ & 127.716 & 125.34 & 121.951 & 102.703 & 106.777 & 98.402 & 112.948 & 109.613 & 80.828 & 75.542 \\
\hline $6 / 3 / 070: 30$ & 127.714 & 125.336 & 121.949 & 102.702 & 106.777 & 98.4 & 112.946 & 109.609 & 80.823 & 75.546 \\
\hline $6 / 3 / 071: 00$ & 127.714 & 125.331 & 121.949 & 102.702 & 106.779 & 98.402 & 112.944 & 109.609 & 80.817 & 75.548 \\
\hline $6 / 3 / 07 \quad 1: 30$ & 127.714 & 125.324 & 121.945 & 102.698 & 106.773 & 98.398 & 112.94 & 109.607 & 80.812 & 75.546 \\
\hline $6 / 3 / 072: 00$ & 127.705 & 125.315 & 121.935 & 102.691 & 106.767 & 98.39 & 112.933 & 109.6 & 80.806 & 75.542 \\
\hline $6 / 3 / 072: 30$ & 127.697 & 125.315 & 121.931 & 102.684 & 106.764 & 98.386 & 112.929 & 109.596 & 80.797 & 75.532 \\
\hline 6/3/07 3:00 & 127.688 & 125.304 & 121.921 & 102.677 & 106.75 & 98.376 & 112.918 & 109.585 & 80.789 & 75.526 \\
\hline 6/3/07 3:30 & 127.688 & 125.297 & 121.921 & 102.679 & 106.754 & 98.379 & 112.919 & 109.585 & 80.784 & 75.519 \\
\hline 6/3/07 4:00 & 127.681 & 125.293 & 121.914 & 102.667 & 106.743 & 98.367 & 112.91 & 109.578 & 80.777 & 75.519 \\
\hline 6/3/07 4:30 & 127.669 & 125.288 & 121.905 & 102.662 & 106.737 & 98.365 & 112.904 & 109.569 & 80.769 & 75.509 \\
\hline $6 / 3 / 07$ 5:00 & 127.664 & 125.292 & 121.898 & 102.657 & 106.733 & 98.355 & 112.897 & 109.563 & 80.76 & 75.501 \\
\hline $6 / 3 / 075: 30$ & 127.662 & 125.292 & 121.896 & 102.654 & 106.729 & 98.353 & 112.893 & 109.56 & 80.754 & 75.505 \\
\hline $6 / 3 / 076: 00$ & 127.664 & 125.313 & 121.898 & 102.659 & 106.735 & 98.355 & 112.897 & 109.563 & 80.753 & 75.499 \\
\hline 6/3/07 6:30 & 127.667 & 125.315 & 121.901 & 102.659 & 106.735 & 98.355 & 112.897 & 109.563 & 80.749 & 75.521 \\
\hline 6/3/07 7:00 & 127.681 & 125.313 & 121.917 & 102.679 & 106.754 & 98.379 & 112.946 & 109.578 & 80.753 & 75.519 \\
\hline $6 / 3 / 07 \quad 7: 30$ & 127.686 & 125.311 & 121.919 & 102.679 & 106.754 & 98.379 & 112.922 & 109.585 & 80.751 & 75.54 \\
\hline 6/3/07 8:00 & 127.683 & 125.313 & 121.921 & 102.679 & 106.754 & 98.379 & 112.916 & 109.582 & 80.749 & 75.548 \\
\hline
\end{tabular}


TABLE S1.1 (Cont.)

Depth from Top of Casing (ft)

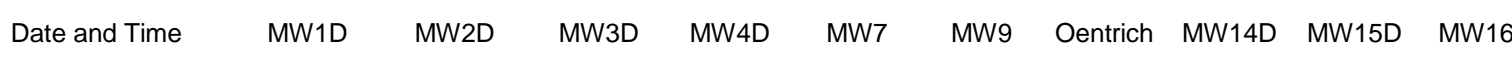

\begin{tabular}{|c|c|c|c|c|c|c|c|c|c|c|}
\hline 6/3/07 8:30 & 127.683 & 125.304 & 121.917 & 102.676 & 106.754 & 98.374 & 112.914 & 109.582 & 80.745 & 75.552 \\
\hline 6/3/07 9:00 & 127.683 & 125.302 & 121.908 & 102.683 & 106.752 & 98.374 & 112.945 & 109.58 & 80.745 & 75.552 \\
\hline 6/3/07 9:30 & 127.676 & 125.292 & 121.912 & 102.669 & 106.749 & 98.369 & 112.951 & 109.574 & 80.743 & 75.557 \\
\hline 6/3/07 10:00 & 127.676 & 125.283 & 121.91 & 102.667 & 106.745 & 98.367 & 112.927 & 109.576 & 80.741 & 75.552 \\
\hline $6 / 3 / 07 \quad 10: 30$ & 127.667 & 125.277 & 121.898 & 102.659 & 106.735 & 98.357 & 112.91 & 109.562 & 80.738 & 75.552 \\
\hline 6/3/07 11:00 & 127.66 & 125.27 & 121.889 & 102.645 & 106.726 & 98.348 & 112.896 & 109.556 & 80.732 & 75.561 \\
\hline 6/3/07 11:30 & 127.65 & 125.261 & 121.882 & 102.641 & 106.722 & 98.343 & 112.886 & 109.547 & 80.729 & 75.557 \\
\hline $6 / 3 / 07 \quad 12: 00$ & 127.646 & 125.245 & 121.877 & 102.634 & 106.712 & 98.336 & 112.878 & 109.54 & 80.725 & 75.561 \\
\hline $6 / 3 / 0712: 30$ & 127.634 & 125.24 & 121.866 & 102.622 & 106.703 & 98.327 & 112.869 & 109.531 & 80.721 & 75.569 \\
\hline 6/3/07 13:00 & 127.62 & 125.227 & 121.85 & 102.61 & 106.69 & 98.31 & 112.857 & 109.518 & 80.714 & 75.567 \\
\hline 6/3/07 13:30 & 127.612 & 125.218 & 121.845 & 102.603 & 106.684 & 98.303 & 112.847 & 109.509 & 80.706 & 75.573 \\
\hline 6/3/07 14:00 & 127.601 & 125.213 & 121.834 & 102.591 & 106.67 & 98.291 & 112.836 & 109.496 & 80.699 & 75.581 \\
\hline 6/3/07 14:30 & 127.591 & 126.894 & 121.824 & 102.583 & 106.663 & 98.284 & 112.828 & 109.489 & 80.694 & 75.577 \\
\hline 6/3/07 15:00 & 127.586 & 127.363 & 121.817 & 102.578 & 106.657 & 98.277 & 112.821 & 109.485 & 80.688 & 75.559 \\
\hline $6 / 3 / 0715: 30$ & 129.26 & 127.547 & 122.886 & 103.01 & 106.88 & 98.65 & 113.07 & 110.488 & 80.688 & 75.555 \\
\hline 6/3/07 16:00 & 129.816 & 127.651 & 123.42 & 103.439 & 107.32 & 99.102 & 113.558 & 111.132 & 80.719 & 75.637 \\
\hline 6/3/07 16:30 & 130.012 & 127.719 & 123.612 & 103.62 & 107.503 & 99.286 & 113.729 & 111.336 & 80.749 & 75.73 \\
\hline 6/3/07 17:00 & 130.116 & 127.76 & 123.721 & 103.72 & 107.609 & 99.392 & 113.824 & 111.444 & 80.775 & 75.805 \\
\hline 6/3/07 17:30 & 130.192 & 127.796 & 123.793 & 103.789 & 107.68 & 99.458 & 113.888 & 111.52 & 80.797 & 75.854 \\
\hline 6/3/07 18:00 & 130.234 & 127.821 & 123.836 & 103.832 & 107.727 & 99.508 & 113.929 & 111.568 & 80.817 & 75.887 \\
\hline $6 / 3 / 07$ 18:30 & 130.27 & 127.844 & 123.873 & 103.866 & 107.762 & 99.541 & 113.96 & 111.604 & 80.834 & 75.91 \\
\hline 6/3/07 19:00 & 130.296 & 127.862 & 123.899 & 103.894 & 107.79 & 99.569 & 113.986 & 111.633 & 80.852 & 75.935 \\
\hline 6/3/07 19:30 & 130.315 & 127.873 & 123.92 & 103.913 & 107.809 & 99.588 & 114.001 & 111.653 & 80.871 & 75.956 \\
\hline 6/3/07 20:00 & 130.339 & 127.892 & 123.941 & 103.934 & 107.832 & 99.612 & 114.022 & 111.677 & 80.895 & 75.993 \\
\hline 6/3/07 20:30 & 130.35 & 127.903 & 123.952 & 103.949 & 107.843 & 99.623 & 114.033 & 111.688 & 80.915 & 76.009 \\
\hline 6/3/07 21:00 & 130.365 & 127.926 & 123.968 & 103.965 & 107.861 & 99.64 & 114.048 & 111.703 & 80.93 & 76.02 \\
\hline $6 / 3 / 0721: 30$ & 130.376 & 126.152 & 123.982 & 103.977 & 107.874 & 99.654 & 114.059 & 111.717 & 80.946 & 76.042 \\
\hline 6/3/07 22:00 & 130.4 & 125.798 & 124.003 & 104.001 & 107.899 & 99.675 & 114.08 & 111.739 & 80.966 & 76.057 \\
\hline $6 / 3 / 0722: 30$ & 128.591 & 125.628 & 122.8 & 103.46 & 107.567 & 99.185 & 113.647 & 110.53 & 80.977 & 76.063 \\
\hline 6/3/07 23:00 & 128.194 & 125.519 & 122.412 & 103.132 & 107.234 & 98.845 & 113.322 & 110.105 & 80.957 & 75.98 \\
\hline 6/3/07 23:30 & 128.014 & 125.453 & 122.236 & 102.969 & 107.065 & 98.676 & 113.17 & 109.923 & 80.933 & 75.879 \\
\hline 6/4/07 0:00 & 127.903 & 125.401 & 122.125 & 102.864 & 106.952 & 98.567 & 113.07 & 109.806 & 80.911 & 75.792 \\
\hline 6/4/07 0:30 & 127.834 & 125.365 & 122.058 & 102.802 & 106.888 & 98.506 & 113.012 & 109.737 & 80.893 & 75.724 \\
\hline $6 / 4 / 071: 00$ & 127.78 & 125.336 & 122.005 & 102.752 & 106.834 & 98.452 & 112.967 & 109.682 & 80.874 & 75.674 \\
\hline 6/4/07 1:30 & 127.74 & 125.304 & 121.968 & 102.714 & 106.798 & 98.416 & 112.933 & 109.64 & 80.858 & 75.639 \\
\hline $6 / 4 / 072: 00$ & 127.709 & 125.288 & 121.938 & 102.688 & 106.771 & 98.39 & 112.911 & 109.611 & 80.841 & 75.608 \\
\hline $6 / 4 / 072: 30$ & 127.681 & 125.274 & 121.91 & 102.659 & 106.743 & 98.362 & 112.884 & 109.582 & 80.826 & 75.588 \\
\hline 6/4/07 3:00 & 127.664 & 125.258 & 121.891 & 102.643 & 106.728 & 98.346 & 112.871 & 109.565 & 80.813 & 75.571 \\
\hline 6/4/07 3:30 & 127.65 & 125.254 & 121.875 & 102.633 & 106.716 & 98.334 & 112.86 & 109.551 & 80.8 & 75.557 \\
\hline 6/4/07 4:00 & 127.633 & 125.247 & 121.864 & 102.617 & 106.699 & 98.317 & 112.849 & 109.536 & 80.788 & 75.54 \\
\hline 6/4/07 4:30 & 127.631 & 125.243 & 121.859 & 102.615 & 106.697 & 98.317 & 112.847 & 109.532 & 80.78 & 75.526 \\
\hline 6/4/07 5:00 & 127.622 & 125.243 & 121.848 & 102.607 & 106.69 & 98.308 & 112.838 & 109.52 & 80.769 & 75.517 \\
\hline $6 / 4 / 075: 30$ & 127.617 & 125.24 & 121.847 & 102.607 & 106.688 & 98.308 & 112.838 & 109.518 & 80.764 & 75.526 \\
\hline 6/4/07 6:00 & 127.617 & 127.055 & 121.845 & 102.607 & 106.69 & 98.308 & 112.836 & 109.518 & 80.756 & 75.521 \\
\hline 6/4/07 6:30 & 127.617 & 127.424 & 121.847 & 102.609 & 106.69 & 98.31 & 112.838 & 109.518 & 80.751 & 75.519 \\
\hline $6 / 4 / 077: 00$ & 129.435 & 127.595 & 122.953 & 103.122 & 107.015 & 98.777 & 113.217 & 110.771 & 80.754 & 75.538 \\
\hline $6 / 4 / 077: 30$ & 129.847 & 127.694 & 123.348 & 103.463 & 107.365 & 99.133 & 113.574 & 111.214 & 80.784 & 75.66 \\
\hline 6/4/07 8:00 & 130.024 & 126.057 & 123.524 & 103.629 & 107.535 & 99.3 & 113.732 & 111.4 & 80.808 & 75.77 \\
\hline 6/4/07 8:30 & 130.13 & 125.68 & 123.633 & 103.732 & 107.642 & 99.406 & 113.828 & 111.509 & 80.832 & 75.861 \\
\hline 6/4/07 9:00 & 128.513 & 125.535 & 122.719 & 103.363 & 107.468 & 99.095 & 113.572 & 110.488 & 80.837 & 75.931 \\
\hline 6/4/07 9:30 & 128.071 & 125.447 & 122.294 & 103.024 & 107.124 & 98.735 & 113.221 & 109.983 & 80.821 & 75.892 \\
\hline $6 / 4 / 07 \quad 10: 00$ & 127.92 & 125.388 & 122.141 & 102.886 & 106.979 & 98.596 & 113.087 & 109.828 & 80.804 & 75.813 \\
\hline $6 / 4 / 07 \quad 10: 30$ & 127.828 & 125.34 & 122.051 & 102.8 & 106.891 & 98.506 & 113.008 & 109.733 & 80.784 & 75.745 \\
\hline 6/4/07 11:00 & 127.766 & 125.306 & 121.993 & 102.745 & 106.834 & 98.449 & 112.958 & 109.671 & 80.769 & 75.699 \\
\hline 6/4/07 11:30 & 127.719 & 125.277 & 121.947 & 102.7 & 106.787 & 98.407 & 112.916 & 109.625 & 80.754 & 75.66 \\
\hline $6 / 4 / 07 \quad 12: 00$ & 127.683 & 125.252 & 121.912 & 102.667 & 106.752 & 98.372 & 112.884 & 109.585 & 80.743 & 75.625 \\
\hline $6 / 4 / 0712: 30$ & 127.653 & 125.227 & 121.882 & 102.638 & 106.722 & 98.343 & 112.86 & 109.556 & 80.732 & 75.612 \\
\hline 6/4/07 13:00 & 127.627 & 125.209 & 121.859 & 102.614 & 106.699 & 98.317 & 112.837 & 109.529 & 80.719 & 75.594 \\
\hline 6/4/07 13:30 & 127.603 & 125.193 & 121.834 & 102.591 & 106.678 & 98.294 & 112.819 & 109.505 & 80.706 & 75.573 \\
\hline $6 / 4 / 07 \quad 14: 00$ & 127.586 & 125.177 & 121.815 & 102.572 & 106.657 & 98.275 & 112.8 & 109.485 & 80.694 & 75.546 \\
\hline 6/4/07 14:30 & 127.57 & 125.161 & 121.799 & 102.557 & 106.642 & 98.261 & 112.787 & 109.47 & 80.681 & 75.521 \\
\hline $6 / 4 / 0715: 00$ & 127.553 & 125.147 & 121.787 & 102.545 & 106.629 & 98.249 & 112.776 & 109.456 & 80.671 & 75.509 \\
\hline $6 / 4 / 0715: 30$ & 127.539 & 125.131 & 121.769 & 102.528 & 106.615 & 98.233 & 112.761 & 109.439 & 80.659 & 75.493 \\
\hline $6 / 4 / 0716: 00$ & 127.523 & 125.118 & 121.755 & 102.514 & 106.598 & 98.216 & 112.746 & 109.423 & 80.646 & 75.484 \\
\hline 6/4/07 16:30 & 127.508 & 125.111 & 121.739 & 102.499 & 106.585 & 98.2 & 112.733 & 109.408 & 80.631 & 75.47 \\
\hline $6 / 4 / 07$ 17:00 & 127.494 & 125.1 & 121.727 & 102.486 & 106.573 & 98.19 & 112.721 & 109.396 & 80.622 & 75.449 \\
\hline
\end{tabular}


TABLE S1.1 (Cont.)

Depth from Top of Casing (ft)

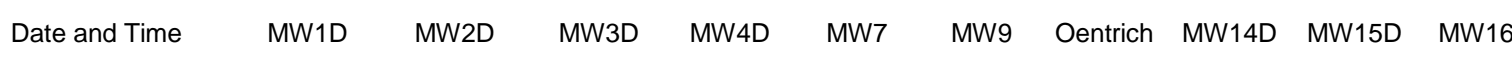

\begin{tabular}{|c|c|c|c|c|c|c|c|c|c|c|}
\hline 6/4/07 17:30 & 127.485 & 125.091 & 121.718 & 102.479 & 106.566 & 98.183 & 112.714 & 109.388 & 80.611 & 75.439 \\
\hline 6/4/07 18:00 & 127.473 & 125.088 & 121.706 & 102.469 & 106.554 & 98.171 & 112.703 & 109.377 & 80.6 & 75.42 \\
\hline $6 / 4 / 07$ 18:30 & 127.464 & 125.088 & 121.697 & 102.462 & 106.547 & 98.164 & 112.695 & 109.368 & 80.59 & 75.414 \\
\hline 6/4/07 19:00 & 127.461 & 125.086 & 121.692 & 102.459 & 106.545 & 98.162 & 112.693 & 109.365 & 80.581 & 75.399 \\
\hline 6/4/07 19:30 & 127.461 & 125.107 & 121.692 & 102.459 & 106.545 & 98.159 & 112.694 & 109.365 & 80.572 & 75.393 \\
\hline 6/4/07 20:00 & 127.459 & 125.118 & 121.69 & 102.459 & 106.543 & 98.159 & 112.692 & 109.361 & 80.565 & 75.387 \\
\hline $6 / 4 / 0720: 30$ & 127.48 & 125.125 & 121.711 & 102.483 & 106.57 & 98.185 & 112.712 & 109.383 & 80.568 & 75.393 \\
\hline 6/4/07 21:00 & 127.487 & 125.122 & 121.72 & 102.49 & 106.577 & 98.192 & 112.718 & 109.392 & 80.566 & 75.412 \\
\hline 6/4/07 21:30 & 127.497 & 125.12 & 121.729 & 102.5 & 106.587 & 98.202 & 112.727 & 109.401 & 80.565 & 75.42 \\
\hline 6/4/07 22:00 & 127.499 & 125.116 & 121.732 & 102.5 & 106.587 & 98.204 & 112.729 & 109.405 & 80.559 & 75.421 \\
\hline 6/4/07 22:30 & 127.492 & 125.116 & 121.725 & 102.493 & 106.579 & 98.197 & 112.72 & 109.396 & 80.553 & 75.416 \\
\hline 6/4/07 23:00 & 127.487 & 125.109 & 121.723 & 102.493 & 106.579 & 98.195 & 112.716 & 109.394 & 80.55 & 75.422 \\
\hline 6/4/07 23:30 & 127.49 & 125.118 & 121.721 & 102.488 & 106.573 & 98.19 & 112.716 & 109.392 & 80.548 & 75.418 \\
\hline 6/5/07 0:00 & 127.485 & 125.125 & 121.713 & 102.485 & 106.571 & 98.188 & 112.709 & 109.387 & 80.542 & 75.41 \\
\hline $6 / 5 / 07 \quad 0: 30$ & 127.473 & 125.12 & 121.725 & 102.495 & 106.579 & 98.197 & 112.718 & 109.396 & 80.546 & 75.416 \\
\hline $6 / 5 / 071: 00$ & 127.466 & 125.12 & 121.727 & 102.5 & 106.587 & 98.202 & 112.722 & 109.401 & 80.546 & 75.42 \\
\hline $6 / 5 / 071: 30$ & 127.466 & 125.116 & 121.727 & 102.497 & 106.581 & 98.2 & 112.72 & 109.399 & 80.544 & 75.421 \\
\hline $6 / 5 / 072: 00$ & 127.461 & 125.116 & 121.723 & 102.493 & 106.581 & 98.195 & 112.716 & 109.396 & 80.542 & 75.416 \\
\hline $6 / 5 / 072: 30$ & 127.454 & 125.113 & 121.725 & 102.493 & 106.579 & 98.197 & 112.716 & 109.396 & 80.541 & 75.416 \\
\hline 6/5/07 3:00 & 127.447 & 125.109 & 121.723 & 102.491 & 106.579 & 98.192 & 112.714 & 109.394 & 80.537 & 75.414 \\
\hline $6 / 5 / 073: 30$ & 127.442 & 125.111 & 121.718 & 102.488 & 106.575 & 98.188 & 112.71 & 109.39 & 80.533 & 75.414 \\
\hline 6/5/07 4:00 & 127.438 & 125.109 & 121.716 & 102.486 & 106.575 & 98.185 & 112.709 & 109.388 & 80.531 & 75.439 \\
\hline 6/5/07 4:30 & 127.433 & 125.116 & 121.716 & 102.488 & 106.575 & 98.188 & 112.707 & 109.388 & 80.529 & 75.439 \\
\hline $6 / 5 / 075: 00$ & 127.435 & 125.12 & 121.711 & 102.485 & 106.573 & 98.185 & 112.705 & 109.385 & 80.526 & 75.443 \\
\hline $6 / 5 / 075: 30$ & 127.466 & 125.122 & 121.72 & 102.495 & 106.579 & 98.195 & 112.712 & 109.394 & 80.526 & 75.445 \\
\hline 6/5/07 6:00 & 127.487 & 127.055 & 121.725 & 102.498 & 106.585 & 98.2 & 112.718 & 109.399 & 80.528 & 75.454 \\
\hline $6 / 5 / 076: 30$ & 127.492 & 127.374 & 121.727 & 102.5 & 106.587 & 98.2 & 112.718 & 109.403 & 80.524 & 75.447 \\
\hline 6/5/07 7:00 & 129.468 & 127.538 & 123.089 & 103.158 & 107.036 & 98.81 & 113.244 & 110.787 & 80.533 & 75.476 \\
\hline 6/5/07 7:30 & 129.825 & 127.635 & 123.434 & 103.467 & 107.356 & 99.133 & 113.559 & 111.159 & 80.577 & 75.625 \\
\hline $6 / 5 / 078: 00$ & 130.003 & 127.708 & 123.612 & 103.632 & 107.525 & 99.303 & 113.75 & 111.34 & 80.622 & 75.743 \\
\hline 6/5/07 8:30 & 130.095 & 127.79 & 123.707 & 103.722 & 107.617 & 99.392 & 113.821 & 111.438 & 80.662 & 75.832 \\
\hline 6/5/07 9:00 & 130.175 & 127.785 & 123.783 & 103.796 & 107.695 & 99.468 & 113.881 & 111.517 & 80.701 & 75.9 \\
\hline 6/5/07 9:30 & 130.246 & 127.81 & 123.841 & 103.844 & 107.741 & 99.517 & 113.918 & 111.571 & 80.732 & 75.96 \\
\hline 6/5/07 10:00 & 130.26 & 127.821 & 123.862 & 103.873 & 107.771 & 99.55 & 113.946 & 111.602 & 80.76 & 75.995 \\
\hline 6/5/07 10:30 & 130.284 & 126.513 & 123.89 & 103.896 & 107.796 & 99.574 & 113.966 & 111.628 & 80.788 & 76.033 \\
\hline 6/5/07 11:00 & 130.296 & 125.757 & 123.901 & 103.91 & 107.811 & 99.588 & 113.977 & 111.641 & 80.812 & 76.047 \\
\hline 6/5/07 11:30 & 129.52 & 125.549 & 123.52 & 103.732 & 107.794 & 99.532 & 113.958 & 111.537 & 80.832 & 76.059 \\
\hline 6/5/07 12:00 & 128.159 & 126.467 & 122.377 & 103.1 & 107.207 & 98.817 & 113.271 & 110.081 & 80.817 & 75.993 \\
\hline 6/5/07 12:30 & 127.936 & 127.402 & 122.157 & 102.898 & 106.998 & 98.61 & 113.076 & 109.85 & & 75.881 \\
\hline 6/5/07 13:00 & 127.806 & 127.563 & 122.03 & 102.783 & 106.872 & 98.487 & 112.965 & 109.717 & 80.782 & 75.784 \\
\hline 6/5/07 13:30 & 129.859 & 127.649 & 123.466 & 103.498 & 107.386 & 99.164 & 113.58 & 111.188 & 80.797 & 75.784 \\
\hline 6/5/07 14:00 & 130.029 & 125.694 & 123.638 & 103.653 & 107.546 & 99.324 & 113.732 & 111.367 & & 75.834 \\
\hline $6 / 5 / 07 \quad 14: 30$ & 130.119 & 125.469 & 123.728 & 103.739 & 107.636 & 99.411 & 113.813 & 111.46 & 80.837 & 75.904 \\
\hline 6/5/07 15:00 & 128.099 & 125.342 & 122.317 & 103.031 & 107.137 & 98.751 & 113.207 & 110.019 & 80.83 & 75.894 \\
\hline 6/5/07 15:30 & 127.856 & 125.261 & 122.079 & 102.815 & 106.918 & 98.53 & 113.001 & 109.768 & & 75.801 \\
\hline 6/5/07 16:00 & 127.726 & 125.195 & 121.951 & 102.696 & 106.794 & 98.405 & 112.89 & 109.638 & 80.788 & 75.699 \\
\hline 6/5/07 16:30 & 127.638 & 125.147 & 121.866 & 102.615 & 106.709 & 98.324 & 112.813 & 109.549 & 80.765 & 75.623 \\
\hline 6/5/07 17:00 & 127.575 & 125.107 & 121.801 & 102.555 & 106.648 & 98.261 & 112.755 & 109.481 & 80.743 & 75.567 \\
\hline 6/5/07 17:30 & 127.523 & 125.068 & 121.753 & 102.507 & 106.598 & 98.214 & 112.712 & 109.43 & 80.721 & 75.519 \\
\hline 6/5/07 18:00 & 127.482 & 125.041 & 121.711 & 102.469 & 106.56 & 98.171 & 112.678 & 109.39 & 80.701 & 75.483 \\
\hline 6/5/07 18:30 & 127.442 & 125.014 & 121.672 & 102.429 & 106.522 & 98.134 & 112.642 & 109.348 & & 75.443 \\
\hline 6/5/07 19:00 & 127.414 & 124.995 & 121.644 & 102.405 & 106.495 & 98.11 & 112.62 & 109.321 & 80.657 & 75.408 \\
\hline 6/5/07 19:30 & 127.388 & 124.982 & 121.621 & 102.381 & 106.471 & 98.086 & 112.595 & 109.295 & 80.636 & 75.406 \\
\hline 6/5/07 20:00 & 127.369 & 124.968 & 121.6 & 102.364 & 106.455 & 98.07 & 112.619 & 109.277 & & 75.387 \\
\hline $6 / 5 / 07 \quad 20: 30$ & 127.355 & 124.948 & 121.588 & 102.352 & 106.442 & 98.058 & 112.598 & 109.263 & 80.601 & 75.365 \\
\hline 6/5/07 21:00 & 127.341 & 124.943 & 121.575 & 102.338 & 106.429 & 98.042 & 112.583 & 109.246 & 80.585 & 75.344 \\
\hline 6/5/07 21:30 & 127.324 & 124.932 & 121.556 & 102.321 & 106.412 & 98.027 & 112.563 & 109.23 & 80.568 & 75.333 \\
\hline $6 / 5 / 07$ 22:00 & 127.315 & 124.921 & 121.549 & 102.316 & 106.406 & 98.018 & 112.556 & 109.222 & 80.555 & 75.323 \\
\hline 6/5/07 22:30 & 127.305 & 124.909 & 121.535 & 102.305 & 106.398 & 98.011 & 112.546 & 109.213 & 80.541 & 75.315 \\
\hline 6/5/07 23:00 & 127.293 & 124.893 & 121.528 & 102.297 & 106.389 & 98.001 & 112.531 & 109.201 & 80.526 & 75.301 \\
\hline 6/5/07 23:30 & 127.279 & 124.873 & 121.514 & 102.281 & 106.375 & 97.985 & 112.515 & 109.188 & 80.511 & 75.284 \\
\hline 6/6/07 0:00 & 127.263 & 124.848 & 121.498 & 102.264 & 106.356 & 97.968 & 112.5 & 109.173 & 80.498 & 75.278 \\
\hline $6 / 6 / 070: 30$ & 127.244 & 124.83 & 121.478 & 102.245 & 106.335 & 97.947 & 112.477 & 109.151 & 80.482 & 75.249 \\
\hline 6/6/07 1:00 & 127.22 & 124.814 & 121.452 & 102.219 & 106.311 & 97.921 & 112.455 & 109.126 & 80.465 & 75.232 \\
\hline 6/6/07 1:30 & 127.201 & 124.796 & 121.436 & 102.204 & 106.295 & 97.91 & 112.437 & 109.111 & 80.448 & 75.209 \\
\hline 6/6/07 2:00 & 127.185 & 124.78 & 121.42 & 102.186 & 106.278 & 97.891 & 112.421 & 109.091 & 80.434 & 75.195 \\
\hline
\end{tabular}


TABLE S1.1 (Cont.)

Depth from Top of Casing (ft)

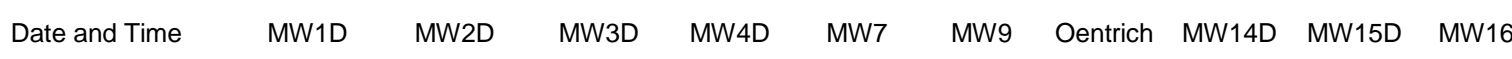

\begin{tabular}{|c|c|c|c|c|c|c|c|c|c|c|}
\hline 6/6/07 2:30 & 127.166 & 124.769 & 121.399 & 102.169 & 106.259 & 97.874 & 112.403 & 109.075 & 80.419 & 75.182 \\
\hline 6/6/07 3:00 & 127.149 & 124.748 & 121.385 & 102.152 & 106.246 & 97.855 & 112.386 & 109.058 & 80.404 & 75.168 \\
\hline 6/6/07 3:30 & 127.137 & 124.735 & 121.373 & 102.142 & 106.236 & 97.844 & 112.375 & 109.049 & 80.391 & 75.154 \\
\hline $6 / 6 / 074: 00$ & 127.114 & 124.723 & 121.348 & 102.123 & 106.212 & 97.825 & 112.354 & 109.029 & 80.375 & 75.139 \\
\hline $6 / 6 / 074: 30$ & 127.104 & 124.71 & 121.341 & 102.109 & 106.202 & 97.811 & 112.343 & 109.016 & 80.36 & 75.135 \\
\hline 6/6/07 5:00 & 127.095 & 124.692 & 121.327 & 102.097 & 106.193 & 97.803 & 112.33 & 109.002 & 80.345 & 75.123 \\
\hline 6/6/07 5:30 & 127.081 & 124.682 & 121.315 & 102.085 & 106.179 & 97.787 & 112.318 & 108.989 & 80.332 & 75.108 \\
\hline 6/6/07 6:00 & 127.059 & 126.64 & 121.295 & 102.066 & 106.158 & 97.768 & 112.3 & 108.971 & 80.316 & 75.091 \\
\hline 6/6/07 6:30 & 127.052 & 126.93 & 121.288 & 102.061 & 106.154 & 97.761 & 112.292 & 108.962 & 80.305 & 75.09 \\
\hline $6 / 6 / 077: 00$ & 129.036 & 127.066 & 122.539 & 102.683 & 106.585 & 98.343 & 112.802 & 110.39 & 80.31 & 75.114 \\
\hline $6 / 6 / 077: 30$ & 129.345 & 127.145 & 122.844 & 102.955 & 106.868 & 98.624 & 113.071 & 110.72 & 80.336 & 75.239 \\
\hline 6/6/07 8:00 & 129.492 & 127.189 & 122.99 & 103.091 & 107.011 & 98.765 & 113.201 & 110.871 & 80.356 & 75.34 \\
\hline 6/6/07 8:30 & 129.577 & 125.249 & 123.073 & 103.167 & 107.091 & 98.848 & 113.272 & 110.959 & 80.376 & 75.412 \\
\hline 6/6/07 9:00 & 129.62 & 124.993 & 123.117 & 103.22 & 107.141 & 98.895 & 113.317 & 111.008 & 80.393 & 75.474 \\
\hline 6/6/07 9:30 & 127.648 & 124.859 & 121.873 & 102.583 & 106.703 & 98.306 & 112.781 & 109.582 & 80.388 & 75.472 \\
\hline 6/6/07 10:00 & 127.376 & 124.766 & 121.602 & 102.343 & 106.455 & 98.053 & 112.548 & 109.299 & 80.364 & 75.365 \\
\hline $6 / 6 / 07 \quad 10: 30$ & 127.232 & 124.701 & 121.459 & 102.213 & 106.316 & 97.919 & 112.422 & 109.153 & 80.345 & 75.274 \\
\hline 6/6/07 11:00 & 127.14 & 124.658 & 121.369 & 102.126 & 106.231 & 97.834 & 112.342 & 109.056 & 80.317 & 75.201 \\
\hline 6/6/07 11:30 & 127.069 & 124.614 & 121.304 & 102.064 & 106.164 & 97.77 & 112.283 & 108.987 & 80.297 & 75.139 \\
\hline $6 / 6 / 07 \quad 12: 00$ & 127.019 & 124.576 & 121.248 & 102.012 & 106.113 & 97.716 & 112.24 & 108.936 & 80.273 & 75.086 \\
\hline $6 / 6 / 0712: 30$ & 126.981 & 124.544 & 121.214 & 101.98 & 106.078 & 97.683 & 112.208 & 108.896 & 80.255 & 75.057 \\
\hline 6/6/07 13:00 & 126.941 & 124.526 & 121.177 & 101.943 & 106.036 & 97.648 & 112.171 & 108.858 & 80.233 & 75.017 \\
\hline 6/6/07 13:30 & 126.91 & 124.501 & 121.147 & 101.908 & 106.01 & 97.62 & 112.145 & 108.829 & 80.212 & 74.98 \\
\hline $6 / 6 / 0714: 00$ & 126.884 & 124.485 & 121.121 & 101.881 & 105.985 & 97.594 & 112.126 & 108.803 & 80.194 & 74.953 \\
\hline 6/6/07 14:30 & 126.861 & 124.465 & 121.096 & 101.862 & 105.964 & 97.568 & 112.096 & 108.77 & 80.172 & 74.935 \\
\hline $6 / 6 / 0715: 00$ & 126.849 & 124.449 & 121.084 & 101.847 & 105.945 & 97.554 & 112.089 & 108.763 & 80.164 & 74.92 \\
\hline 6/6/07 15:30 & 126.83 & 124.426 & 121.066 & 101.833 & 105.932 & 97.539 & 112.07 & 108.743 & 80.139 & 74.906 \\
\hline 6/6/07 16:00 & 126.816 & 124.413 & 121.052 & 101.816 & 105.924 & 97.525 & 112.059 & 108.73 & 80.126 & 74.893 \\
\hline 6/6/07 16:30 & 126.797 & 124.397 & 121.033 & 101.797 & 105.899 & 97.506 & 112.042 & 108.712 & 80.107 & 74.876 \\
\hline $6 / 6 / 0717: 00$ & 126.778 & 124.381 & 121.006 & 101.78 & 105.878 & 97.488 & 112.019 & 108.692 & 80.091 & 74.852 \\
\hline 6/6/07 17:30 & 126.759 & 124.358 & 120.996 & 101.764 & 105.865 & 97.466 & 112.004 & 108.677 & 80.074 & 74.842 \\
\hline $6 / 6 / 07$ 18:00 & 126.74 & 124.338 & 120.978 & 101.747 & 105.848 & 97.452 & 111.989 & 108.657 & 80.054 & 74.821 \\
\hline 6/6/07 18:30 & 126.719 & 124.34 & 120.957 & 101.721 & 105.823 & 97.431 & 111.968 & 108.635 & 80.034 & 74.8 \\
\hline 6/6/07 19:00 & 126.702 & 124.349 & 120.941 & 101.707 & 105.808 & 97.412 & 111.951 & 108.617 & 80.017 & 74.782 \\
\hline 6/6/07 19:30 & 126.705 & 124.34 & 120.945 & 101.716 & 105.816 & 97.417 & 111.986 & 108.622 & 80.01 & 74.778 \\
\hline 6/6/07 20:00 & 126.714 & 124.331 & 120.955 & 101.721 & 105.829 & 97.44 & 112.104 & 108.626 & 79.995 & 74.776 \\
\hline $6 / 6 / 0720: 30$ & 126.707 & 124.329 & 120.941 & 101.713 & 105.817 & 97.419 & 112.001 & 108.622 & 79.984 & 74.776 \\
\hline 6/6/07 21:00 & 126.695 & 124.324 & 120.929 & 101.702 & 105.806 & 97.407 & 112.023 & 108.61 & 79.967 & 74.761 \\
\hline 6/6/07 21:30 & 126.683 & 124.322 & 120.925 & 101.697 & 105.798 & 97.4 & 112.011 & 108.602 & 79.952 & 74.747 \\
\hline 6/6/07 22:00 & 126.688 & 124.311 & 120.922 & 101.695 & 105.804 & 97.4 & 111.978 & 108.602 & 79.941 & 74.749 \\
\hline 6/6/07 22:30 & 126.688 & 124.29 & 120.922 & 101.702 & 105.804 & 97.398 & 111.962 & 108.602 & 79.929 & 74.74 \\
\hline 6/6/07 23:00 & 126.669 & 124.286 & 120.908 & 101.68 & 105.783 & 97.386 & 111.958 & 108.588 & 79.914 & 74.73 \\
\hline 6/6/07 23:30 & 126.655 & 124.265 & 120.892 & 101.664 & 105.764 & 97.37 & 111.952 & 108.573 & 79.897 & 74.728 \\
\hline 6/7/07 0:00 & 126.648 & 124.252 & 120.883 & 101.656 & 105.762 & 97.36 & 111.946 & 108.562 & 79.89 & 74.716 \\
\hline 6/7/07 0:30 & 126.627 & 124.249 & & 101.635 & 105.745 & & & & & 74.701 \\
\hline 6/7/07 1:00 & 126.617 & 124.24 & 120.853 & 101.625 & 105.732 & 97.332 & 111.942 & 108.537 & 79.857 & 74.693 \\
\hline 6/7/07 1:30 & 126.613 & 124.231 & 120.846 & 101.621 & 105.728 & 97.33 & 111.938 & 108.528 & 79.849 & 74.688 \\
\hline 6/7/07 2:00 & 126.603 & 124.245 & 120.839 & 101.613 & 105.718 & 97.318 & & 108.522 & & 74.674 \\
\hline 6/7/07 2:30 & 126.594 & 124.27 & 120.832 & 101.606 & 105.711 & 97.311 & 111.93 & 108.513 & 79.822 & 74.678 \\
\hline 6/7/07 3:00 & 126.601 & 124.279 & 120.837 & 101.614 & 105.715 & 97.32 & 111.924 & 108.518 & 79.816 & 74.689 \\
\hline 6/7/07 3:30 & & 124.274 & & 101.645 & & & & 108.549 & & 74.718 \\
\hline 6/7/07 4:00 & 126.643 & 124.27 & 120.874 & 101.654 & 105.762 & 97.365 & 111.92 & 108.562 & 79.818 & 74.721 \\
\hline 6/7/07 4:30 & 126.636 & 124.279 & 120.869 & 101.647 & 105.751 & 97.351 & 111.922 & 108.555 & 79.809 & 74.716 \\
\hline $6 / 7 / 075: 00$ & 126.631 & 124.292 & & 101.644 & 105.751 & & 111.918 & 108.548 & 79.803 & 74.705 \\
\hline 6/7/07 5:30 & 126.641 & 124.295 & 120.876 & 101.656 & 105.764 & 97.358 & 111.916 & 108.557 & 79.801 & 74.718 \\
\hline 6/7/07 6:00 & 126.655 & 126.225 & 120.888 & 101.668 & 105.774 & 97.372 & 111.914 & 108.571 & 79.803 & 74.73 \\
\hline 6/7/07 6:30 & 126.66 & 126.576 & 120.895 & 101.673 & 105.781 & 97.379 & 111.914 & 108.575 & 79.799 & 74.73 \\
\hline 6/7/07 7:00 & 128.643 & 126.755 & 122.25 & 102.323 & 106.215 & 97.973 & 112.431 & 109.947 & 79.811 & 74.755 \\
\hline 6/7/07 7:30 & 129.029 & 126.873 & 122.629 & 102.66 & 106.562 & 98.324 & 112.751 & 110.355 & 79.855 & 74.9 \\
\hline 6/7/07 8:00 & 129.213 & 126.95 & 122.814 & 102.834 & 106.743 & 98.506 & 112.917 & 110.55 & 79.895 & 75.034 \\
\hline 6/7/07 8:30 & 129.336 & 127.012 & 122.937 & 102.953 & 106.865 & 98.626 & 113.022 & 110.674 & 79.936 & 75.139 \\
\hline 6/7/07 9:00 & 129.414 & 127.062 & 123.015 & 103.031 & 106.945 & 98.709 & 113.091 & 110.758 & 79.971 & 75.21 \\
\hline 6/7/07 9:30 & 129.478 & 127.093 & 123.08 & 103.093 & 107.009 & 98.768 & 113.14 & 110.822 & 80.004 & 75.286 \\
\hline 6/7/07 10:00 & 129.527 & 127.127 & 123.131 & 103.143 & 107.059 & 98.82 & 113.178 & 110.875 & 80.037 & 75.34 \\
\hline 6/7/07 10:30 & 129.563 & 127.152 & 123.166 & 103.179 & 107.095 & 98.857 & 113.206 & 110.913 & 80.069 & 75.375 \\
\hline 6/7/07 11:00 & 129.598 & 127.177 & 123.203 & 103.212 & 107.129 & 98.892 & 113.229 & 110.946 & 80.1 & 75.406 \\
\hline
\end{tabular}


TABLE S1.1 (Cont.)

Depth from Top of Casing (ft)

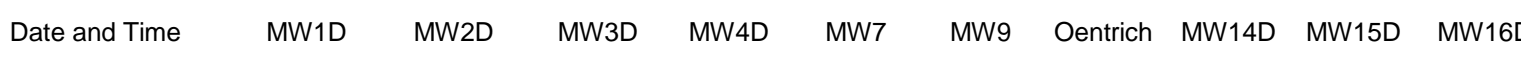

\begin{tabular}{|c|c|c|c|c|c|c|c|c|c|c|}
\hline 6/7/07 11:30 & 129.62 & 127.186 & 123.228 & 103.236 & 107.154 & 98.919 & 113.253 & 110.972 & 80.128 & 75.443 \\
\hline 6/7/07 12:00 & 129.646 & 125.501 & 123.251 & 103.262 & 107.181 & 98.942 & 113.278 & 110.995 & 80.155 & 75.462 \\
\hline 6/7/07 12:30 & 129.653 & 125.068 & 123.263 & 103.275 & 107.192 & 98.954 & 113.289 & 111.01 & 80.177 & 75.483 \\
\hline 6/7/07 13:00 & 127.955 & 124.889 & 122.169 & 102.805 & 106.939 & 98.546 & 112.943 & 109.941 & 80.198 & 75.499 \\
\hline 6/7/07 13:30 & 127.459 & 124.78 & 121.681 & 102.412 & 106.537 & 98.131 & 112.526 & 109.385 & 80.185 & 75.396 \\
\hline 6/7/07 14:00 & 127.267 & 124.707 & 121.494 & 102.24 & 106.354 & 97.954 & 112.359 & 109.188 & 80.168 & 75.28 \\
\hline 6/7/07 14:30 & 127.161 & 124.66 & 121.387 & 102.14 & 106.255 & 97.855 & 112.263 & 109.08 & 80.159 & 75.197 \\
\hline 6/7/07 15:00 & 127.08 & 124.619 & 121.313 & 102.069 & 106.179 & 97.78 & 112.197 & 109 & 80.144 & 75.133 \\
\hline $6 / 7 / 07$ 15:30 & 127.026 & 124.585 & 121.258 & 102.023 & 106.132 & 97.733 & 112.152 & 108.949 & 80.129 & 75.084 \\
\hline 6/7/07 16:00 & 126.986 & 124.567 & 121.221 & 101.985 & 106.094 & 97.69 & 112.118 & 108.907 & 80.12 & 75.044 \\
\hline 6/7/07 16:30 & 126.955 & 124.551 & 121.188 & 101.956 & 106.063 & 97.662 & 112.092 & 108.872 & 80.107 & 75.011 \\
\hline 6/7/07 17:00 & 126.934 & 124.542 & 121.167 & 101.937 & 106.044 & 97.643 & 112.073 & 108.854 & 80.098 & 74.993 \\
\hline 6/7/07 17:30 & 126.917 & 124.542 & 121.151 & 101.923 & 106.031 & 97.631 & 112.06 & 108.836 & 80.085 & 74.986 \\
\hline 6/7/07 18:00 & 126.908 & 124.544 & 121.147 & 101.916 & 106.021 & 97.624 & 112.056 & 108.827 & 80.08 & 74.974 \\
\hline 6/7/07 18:30 & 126.908 & 124.551 & 121.142 & 101.914 & 106.021 & 97.622 & 112.047 & 108.823 & 80.074 & 74.97 \\
\hline 6/7/07 19:00 & 126.91 & 124.589 & 121.147 & 101.923 & 106.027 & 97.627 & 112.053 & 108.827 & 80.07 & 74.968 \\
\hline 6/7/07 19:30 & 126.922 & 124.624 & 121.156 & 101.932 & 106.036 & 97.636 & 112.086 & 108.836 & 80.069 & 74.978 \\
\hline 6/7/07 20:00 & 126.957 & 124.651 & 121.191 & 101.973 & 106.076 & 97.674 & 112.125 & 108.867 & 80.082 & 75.001 \\
\hline 6/7/07 20:30 & 126.995 & 124.692 & 121.23 & 102.009 & 106.116 & 97.716 & 112.17 & 108.909 & 80.089 & 75.034 \\
\hline 6/7/07 21:00 & 127.019 & 124.726 & 121.255 & 102.039 & 106.141 & 97.742 & 112.193 & 108.931 & 80.098 & 75.059 \\
\hline 6/7/07 21:30 & 127.057 & 124.75 & 121.292 & 102.078 & 106.185 & 97.784 & 112.226 & 108.971 & 80.115 & 75.098 \\
\hline 6/7/07 22:00 & 127.095 & 124.78 & 121.329 & 102.113 & 106.215 & 97.815 & 112.237 & 109.005 & 80.124 & 75.131 \\
\hline 6/7/07 22:30 & 127.118 & 124.798 & 121.355 & 102.142 & 106.244 & 97.844 & 112.255 & 109.033 & 80.137 & 75.15 \\
\hline 6/7/07 23:00 & 127.149 & 124.805 & 121.385 & 102.171 & 106.273 & 97.874 & 112.281 & 109.062 & 80.148 & 75.178 \\
\hline 6/7/07 23:30 & 127.165 & 124.83 & 121.401 & 102.188 & 106.293 & 97.893 & 112.3 & 109.08 & 80.161 & 75.199 \\
\hline 6/8/07 0:00 & 127.175 & 124.85 & 121.413 & 102.193 & 106.297 & 97.9 & 112.301 & 109.089 & 80.166 & 75.195 \\
\hline 6/8/07 0:30 & 127.196 & 124.859 & 121.433 & 102.224 & 106.324 & 97.926 & 112.32 & 109.113 & 80.177 & 75.224 \\
\hline 6/8/07 1:00 & 127.22 & 124.866 & 121.454 & 102.243 & 106.345 & 97.947 & 112.337 & 109.133 & 80.19 & 75.241 \\
\hline 6/8/07 1:30 & 127.229 & 124.875 & 121.468 & 102.252 & 106.354 & 97.957 & 112.354 & 109.144 & 80.201 & 75.251 \\
\hline 6/8/07 2:00 & 127.236 & 124.891 & 121.473 & 102.262 & 106.364 & 97.971 & 112.359 & 109.153 & 80.209 & 75.255 \\
\hline 6/8/07 2:30 & 127.246 & 124.909 & 121.484 & 102.273 & 106.372 & 97.973 & 112.373 & 109.162 & 80.218 & 75.278 \\
\hline 6/8/07 3:00 & 127.263 & 124.916 & 121.498 & 102.288 & 106.387 & 97.99 & 112.374 & 109.175 & 80.229 & 75.29 \\
\hline 6/8/07 3:30 & 127.277 & 124.921 & 121.517 & 102.309 & 106.408 & 98.009 & 112.389 & 109.195 & 80.242 & 75.307 \\
\hline 6/8/07 4:00 & 127.284 & 124.934 & 121.524 & 102.314 & 106.412 & 98.013 & 112.391 & 109.202 & 80.251 & 75.33 \\
\hline 6/8/07 4:30 & 127.291 & 124.943 & 121.528 & 102.319 & 106.417 & 98.02 & 112.404 & 109.208 & 80.26 & 75.33 \\
\hline 6/8/07 5:00 & 127.305 & 124.957 & 121.544 & 102.335 & 106.434 & 98.034 & 112.408 & 109.222 & 80.271 & 75.34 \\
\hline 6/8/07 5:30 & 127.312 & 124.968 & 121.551 & 102.341 & 106.44 & 98.046 & 112.423 & 109.23 & 80.281 & 75.348 \\
\hline 6/8/07 6:00 & 127.326 & 126.98 & 121.565 & 102.357 & 106.455 & 98.06 & 112.436 & 109.244 & 80.292 & 75.352 \\
\hline 6/8/07 6:30 & 127.336 & 127.275 & 121.577 & 102.367 & 106.463 & 98.067 & 112.438 & 109.253 & 80.301 & 75.371 \\
\hline 6/8/07 7:00 & 129.412 & 127.427 & 123.027 & 103.101 & 106.987 & 98.753 & 113.052 & 110.733 & 80.336 & 75.425 \\
\hline 6/8/07 7:30 & 129.736 & 127.529 & 123.344 & 103.386 & 107.284 & 99.05 & 113.334 & 111.068 & 80.386 & 75.567 \\
\hline 6/8/07 8:00 & 129.892 & 127.599 & 123.501 & 103.537 & 107.436 & 99.206 & 113.484 & 111.234 & 80.434 & 75.704 \\
\hline 6/8/07 8:30 & 129.993 & 127.656 & 123.605 & 103.634 & 107.539 & 99.305 & 113.58 & 111.338 & 80.478 & 75.805 \\
\hline 6/8/07 9:00 & 130.069 & 127.694 & 123.682 & 103.708 & 107.613 & 99.38 & 113.667 & 111.413 & 80.524 & 75.877 \\
\hline 6/8/07 9:30 & 130.128 & 127.722 & 123.739 & 103.765 & 107.67 & 99.439 & 113.707 & 111.473 & 80.572 & 75.937 \\
\hline 6/8/07 10:00 & 130.166 & 127.733 & 123.779 & 103.804 & 107.712 & 99.482 & 113.736 & 111.515 & 80.611 & 75.989 \\
\hline 6/8/07 10:30 & 130.194 & 127.749 & 123.804 & 103.829 & 107.731 & 99.503 & 113.757 & 111.542 & 80.646 & 76.02 \\
\hline 6/8/07 11:00 & 130.208 & 127.762 & 123.82 & 103.846 & 107.752 & 99.52 & 113.77 & 111.56 & 80.681 & 76.039 \\
\hline 6/8/07 11:30 & 130.225 & 127.767 & 123.836 & 103.86 & 107.769 & 99.539 & 113.777 & 111.575 & 80.714 & 76.059 \\
\hline 6/8/07 12:00 & 130.239 & 125.821 & 123.853 & 103.875 & 107.784 & 99.55 & 113.8 & 111.591 & 80.743 & 76.07 \\
\hline $6 / 8 / 0712: 30$ & 130.244 & 125.533 & 123.86 & 103.879 & 107.788 & 99.555 & 113.807 & 111.597 & 80.771 & 76.09 \\
\hline 6/8/07 13:00 & 128.239 & 125.374 & 122.46 & 103.174 & 107.293 & 98.897 & 113.207 & 110.171 & 80.769 & 76.049 \\
\hline 6/8/07 13:30 & 127.925 & 125.277 & 122.153 & 102.9 & 107.011 & 98.617 & 112.944 & 109.848 & 80.749 & 75.908 \\
\hline 6/8/07 14:00 & 127.759 & 125.268 & 121.988 & 102.748 & 106.855 & 98.456 & 112.807 & 109.678 & 80.732 & 75.789 \\
\hline 6/8/07 14:30 & 127.66 & 125.175 & 121.889 & 102.653 & 106.756 & 98.36 & 112.719 & 109.574 & 80.719 & 75.714 \\
\hline 6/8/07 15:00 & 127.65 & 125.116 & 121.901 & 102.719 & 106.773 & 98.412 & 113.671 & 109.549 & 80.718 & 75.679 \\
\hline 6/8/07 15:30 & 127.558 & 125.064 & 121.79 & 102.559 & 106.657 & 98.266 & 112.702 & 109.472 & 80.71 & 75.636 \\
\hline 6/8/07 16:00 & 127.492 & 125.029 & 121.727 & 102.497 & 106.596 & 98.204 & 112.677 & 109.408 & 80.692 & 75.584 \\
\hline 6/8/07 16:30 & 127.442 & 124.995 & 121.679 & 102.452 & 106.549 & 98.157 & 112.642 & 109.354 & 80.677 & 75.534 \\
\hline 6/8/07 17:00 & 127.404 & 124.977 & 121.644 & 102.419 & 106.516 & 98.124 & 112.613 & 109.319 & 80.664 & 75.509 \\
\hline 6/8/07 17:30 & 127.374 & 124.946 & 121.612 & 102.388 & 106.484 & 98.093 & 112.585 & 109.288 & 80.649 & 75.476 \\
\hline 6/8/07 18:00 & 127.348 & 124.93 & 121.586 & 102.39 & 106.461 & 98.067 & 112.665 & 109.261 & 80.638 & 75.454 \\
\hline 6/8/07 18:30 & 127.324 & 124.912 & 121.563 & 102.34 & 106.438 & 98.044 & 112.56 & 109.237 & 80.627 & 75.429 \\
\hline 6/8/07 19:00 & 127.305 & 124.896 & 121.549 & 102.324 & 106.423 & 98.034 & 112.576 & 109.219 & 80.616 & 75.412 \\
\hline 6/8/07 19:30 & 127.286 & 124.889 & 121.526 & 102.316 & 106.402 & 98.009 & 112.551 & 109.199 & 80.605 & 75.406 \\
\hline 6/8/07 20:00 & 127.267 & 124.877 & 121.51 & 102.297 & 106.389 & 97.997 & 112.523 & 109.184 & 80.598 & 75.387 \\
\hline
\end{tabular}


TABLE S1.1 (Cont.)

Depth from Top of Casing (ft)

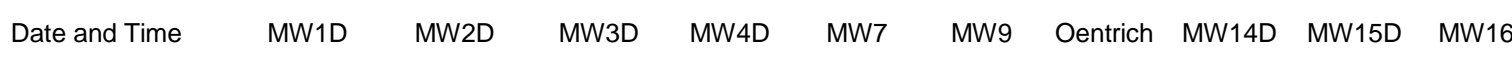

\begin{tabular}{|c|c|c|c|c|c|c|c|c|c|c|}
\hline 6/8/07 20:30 & 127.26 & 124.889 & 121.503 & 102.288 & 106.385 & 97.992 & 112.583 & 109.175 & 80.588 & 75.387 \\
\hline 6/8/07 21:00 & 127.251 & 124.877 & 121.494 & 102.278 & 106.373 & 97.983 & 112.57 & 109.166 & 80.579 & 75.375 \\
\hline $6 / 8 / 0721: 30$ & 127.258 & 124.873 & 121.505 & 102.316 & 106.389 & 98.004 & 112.711 & 109.166 & 80.577 & 75.369 \\
\hline 6/8/07 22:00 & 127.253 & 124.862 & 121.494 & 102.279 & 106.377 & 97.985 & 112.478 & 109.166 & 80.574 & 75.375 \\
\hline 6/8/07 22:30 & 127.246 & 124.862 & 121.487 & 102.273 & 106.372 & 97.978 & 112.505 & 109.157 & 80.568 & 75.371 \\
\hline 6/8/07 23:00 & 127.237 & 124.859 & 121.48 & 102.263 & 106.36 & 97.966 & 112.494 & 109.151 & 80.565 & 75.367 \\
\hline 6/8/07 23:30 & 127.236 & 124.855 & 121.477 & 102.264 & 106.362 & 97.968 & 112.507 & 109.151 & 80.563 & 75.365 \\
\hline 6/9/07 0:00 & 127.232 & 124.848 & 121.475 & 102.266 & 106.36 & 97.966 & 112.569 & 109.146 & 80.559 & 75.363 \\
\hline 6/9/07 0:30 & 127.229 & 124.841 & 121.473 & 102.259 & 106.356 & 97.961 & 112.48 & 109.146 & 80.557 & 75.363 \\
\hline 6/9/07 1:00 & 127.222 & 124.834 & 121.466 & 102.252 & 106.349 & 97.957 & 112.477 & 109.137 & 80.552 & 75.354 \\
\hline 6/9/07 1:30 & 127.215 & 124.83 & 121.457 & 102.243 & 106.343 & 97.947 & 112.47 & 109.128 & 80.546 & 75.346 \\
\hline 6/9/07 2:00 & 127.211 & 124.821 & 121.452 & 102.24 & 106.339 & 97.94 & 112.467 & 109.124 & 80.544 & 75.344 \\
\hline 6/9/07 2:30 & 127.203 & 124.816 & 121.447 & 102.235 & 106.333 & 97.943 & 112.481 & 109.118 & 80.541 & 75.342 \\
\hline 6/9/07 3:00 & 127.199 & 124.809 & 121.438 & 102.228 & 106.324 & 97.928 & 112.503 & 109.111 & 80.537 & 75.332 \\
\hline 6/9/07 3:30 & 127.192 & 124.8 & 121.433 & 102.219 & 106.318 & 97.921 & 112.587 & 109.104 & 80.531 & 75.33 \\
\hline 6/9/07 4:00 & 127.187 & 124.789 & 121.422 & 102.216 & 106.309 & 97.91 & 112.574 & 109.095 & 80.529 & 75.317 \\
\hline 6/9/07 4:30 & 127.182 & 124.785 & 121.413 & 102.207 & 106.301 & 97.9 & 112.464 & 109.084 & 80.528 & 75.307 \\
\hline 6/9/07 5:00 & 127.177 & 124.787 & 121.406 & 102.197 & 106.293 & 97.9 & 112.549 & 109.078 & 80.524 & 75.305 \\
\hline 6/9/07 5:30 & 127.173 & 124.787 & 121.401 & 102.186 & 106.284 & 97.886 & 112.46 & 109.071 & 80.52 & 75.307 \\
\hline 6/9/07 6:00 & 127.166 & 124.785 & 121.406 & 102.195 & 106.293 & 97.895 & 112.375 & 109.078 & 80.522 & 75.307 \\
\hline $6 / 9 / 076: 30$ & 127.161 & 124.785 & 121.403 & 102.195 & 106.292 & 97.893 & 112.365 & 109.075 & 80.52 & 75.313 \\
\hline 6/9/07 7:00 & 127.154 & 126.547 & 121.401 & 102.192 & 106.29 & 97.893 & 112.378 & 109.073 & 80.517 & 75.311 \\
\hline 6/9/07 7:30 & 127.149 & 126.987 & 121.399 & 102.192 & 106.288 & 97.891 & 112.346 & 109.071 & 80.513 & 75.313 \\
\hline 6/9/07 8:00 & 128.899 & 127.159 & 122.428 & 102.645 & 106.541 & 98.282 & 112.62 & 110.206 & 80.517 & 75.315 \\
\hline 6/9/07 8:30 & 129.407 & 127.259 & 122.916 & 103.053 & 106.962 & 98.716 & 113.105 & 110.78 & 80.552 & 75.441 \\
\hline 6/9/07 9:00 & 129.591 & 127.32 & 123.099 & 103.213 & 107.133 & 98.888 & 113.318 & 110.972 & 80.579 & 75.565 \\
\hline 6/9/07 9:30 & 129.695 & 127.352 & 123.205 & 103.315 & 107.238 & 98.996 & 113.419 & 111.079 & 80.605 & 75.648 \\
\hline 6/9/07 10:00 & 129.757 & 127.386 & 123.263 & 103.362 & 107.301 & 99.053 & 113.449 & 111.143 & 80.633 & 75.704 \\
\hline 6/9/07 10:30 & 129.788 & 127.397 & 123.295 & 103.379 & 107.331 & 99.081 & 113.496 & 111.179 & 80.657 & 75.749 \\
\hline 6/9/07 11:00 & 129.83 & 127.515 & 123.346 & 103.437 & 107.388 & 99.138 & 113.575 & 111.338 & 80.675 & 75.782 \\
\hline 6/9/07 11:30 & 129.835 & 125.678 & 123.441 & 103.444 & 107.381 & 99.124 & 113.512 & 111.216 & 80.697 & 75.803 \\
\hline 6/9/07 12:00 & 129.844 & 125.24 & 123.349 & 103.443 & 107.392 & 99.131 & 113.531 & 111.225 & 80.714 & 75.818 \\
\hline 6/9/07 12:30 & 128.142 & 125.05 & 122.359 & 102.996 & 107.143 & 98.749 & 113.202 & 110.127 & 80.721 & 75.826 \\
\hline 6/9/07 13:00 & 127.631 & 124.925 & 121.864 & 102.595 & 106.731 & 98.32 & 112.783 & 109.549 & 80.695 & 75.702 \\
\hline 6/9/07 13:30 & 127.426 & 124.834 & 121.66 & 102.416 & 106.537 & 98.129 & 112.707 & 109.341 & 80.671 & 75.582 \\
\hline 6/9/07 14:00 & 127.3 & 124.764 & 121.54 & 102.295 & 106.419 & 98.016 & 112.558 & 109.208 & 80.647 & 75.472 \\
\hline 6/9/07 14:30 & 127.208 & 124.714 & 121.447 & 102.209 & 106.33 & 97.926 & 112.481 & 109.115 & 80.624 & 75.387 \\
\hline 6/9/07 15:00 & 127.135 & 124.673 & 121.373 & 102.149 & 106.259 & 97.855 & 112.386 & 109.042 & 80.6 & 75.34 \\
\hline 6/9/07 15:30 & 127.085 & 124.639 & 121.325 & 102.09 & 106.208 & 97.803 & 112.359 & 108.991 & 80.579 & 75.288 \\
\hline 6/9/07 16:00 & 127.043 & 124.612 & 121.285 & 102.054 & 106.172 & 97.77 & 112.355 & 108.949 & 80.559 & 75.251 \\
\hline 6/9/07 16:30 & 127.01 & 124.589 & 121.253 & 102.019 & 106.137 & 97.733 & 112.313 & 108.916 & 80.541 & 75.218 \\
\hline 6/9/07 17:00 & 126.981 & 124.569 & 121.223 & 102.002 & 106.109 & 97.704 & 112.317 & 108.887 & 80.524 & 75.189 \\
\hline 6/9/07 17:30 & 126.958 & 124.551 & 121.202 & 101.974 & 106.092 & 97.686 & 112.262 & 108.863 & 80.506 & 75.168 \\
\hline 6/9/07 18:00 & 126.939 & 124.535 & 121.181 & 101.954 & 106.069 & 97.667 & 112.238 & 108.84 & 80.489 & 75.141 \\
\hline 6/9/07 18:30 & 126.915 & & 121.159 & 101.943 & 106.046 & 97.641 & & & & 75.131 \\
\hline 6/9/07 19:00 & 126.903 & 124.515 & 121.149 & 101.921 & 106.036 & 97.631 & 112.213 & 108.809 & 80.461 & 75.119 \\
\hline 6/9/07 19:30 & 126.891 & 124.51 & 121.137 & 101.911 & 106.027 & 97.622 & 112.213 & 108.796 & 80.448 & 75.115 \\
\hline 6/9/07 20:00 & 126.884 & 124.515 & 121.128 & 101.907 & 106.019 & 97.615 & 112.212 & 108.787 & 80.437 & 75.102 \\
\hline 6/9/07 20:30 & 126.877 & 124.51 & 121.124 & 101.902 & 106.015 & 97.613 & 112.187 & 108.781 & 80.426 & 75.106 \\
\hline 6/9/07 21:00 & 126.884 & 124.508 & 121.131 & 101.904 & 106.023 & 97.62 & 112.149 & 108.788 & 80.417 & 75.102 \\
\hline 6/9/07 21:30 & 126.877 & & 121.121 & 101.9 & & 97.61 & 112.143 & 108.783 & 80.412 & 75.102 \\
\hline 6/9/07 22:00 & 126.873 & 124.501 & 121.119 & 101.9 & 106.017 & 97.61 & 112.167 & 108.778 & 80.404 & 75.102 \\
\hline 6/9/07 22:30 & 126.87 & 124.496 & 121.117 & 101.897 & 106.014 & 97.605 & 112.14 & 108.776 & 80.397 & 75.1 \\
\hline 6/9/07 23:00 & 126.868 & 124.481 & 121.11 & 101.895 & 106.01 & 97.603 & 112.136 & 108.772 & & 75.091 \\
\hline 6/9/07 23:30 & 126.861 & 124.462 & 121.105 & 101.887 & 106.002 & 97.596 & 112.12 & 108.765 & 80.382 & 75.087 \\
\hline 6/10/07 0:00 & 126.849 & 124.451 & 121.093 & 101.875 & 105.991 & 97.586 & 112.111 & 108.756 & 80.371 & 75.075 \\
\hline 6/10/07 0:30 & 126.83 & 124.433 & 121.075 & 101.856 & 105.972 & 97.568 & 112.108 & 108.736 & 80.358 & 75.067 \\
\hline 6/10/07 1:00 & 126.816 & 124.41 & 121.061 & 101.842 & 105.962 & 97.554 & 112.091 & 108.725 & 80.347 & 75.05 \\
\hline 6/10/07 1:30 & 126.802 & 124.415 & 121.047 & 101.831 & 105.947 & 97.539 & 112.175 & 108.705 & 80.334 & 75.036 \\
\hline $6 / 10 / 072: 00$ & 126.778 & 124.413 & 121.022 & 101.804 & 105.922 & 97.513 & 112.114 & 108.684 & 80.321 & 75.013 \\
\hline $6 / 10 / 072: 30$ & 126.783 & 124.397 & 121.029 & 101.814 & 105.93 & 97.518 & 112.088 & 108.69 & 80.316 & 75.025 \\
\hline 6/10/07 3:00 & 126.785 & 124.383 & 121.029 & 101.811 & 105.93 & 97.52 & 112.062 & 108.69 & 80.308 & 75.027 \\
\hline 6/10/07 3:30 & 126.766 & 124.383 & 121.013 & 101.794 & 105.913 & 97.504 & 112.046 & 108.674 & 80.297 & 75.005 \\
\hline 6/10/07 4:00 & 126.752 & 124.381 & 120.996 & 101.78 & 105.899 & 97.49 & 112.028 & 108.659 & 80.286 & 74.993 \\
\hline $6 / 10 / 074: 30$ & 126.754 & 124.399 & 120.999 & 101.783 & 105.901 & 97.49 & 112.014 & 108.659 & 80.279 & 74.992 \\
\hline 6/10/07 5:00 & 126.745 & 124.394 & 120.992 & 101.778 & 105.896 & 97.485 & 112.002 & 108.653 & 80.271 & 74.984 \\
\hline
\end{tabular}


TABLE S1.1 (Cont.)

Depth from Top of Casing (ft)

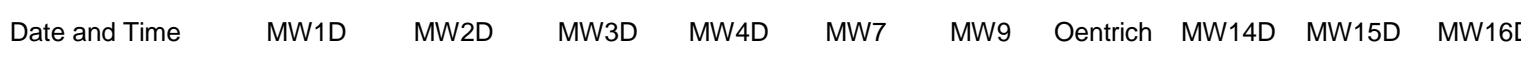

\begin{tabular}{|c|c|c|c|c|c|c|c|c|c|c|}
\hline 6/10/07 5:30 & 126.766 & 124.392 & 121.01 & 101.799 & 105.915 & 97.504 & 111.983 & 108.672 & 80.273 & 75.003 \\
\hline 6/10/07 6:00 & 126.764 & 124.392 & 121.008 & 101.795 & 105.911 & 97.499 & 111.981 & 108.668 & 80.27 & 75.001 \\
\hline 6/10/07 6:30 & 126.759 & 124.394 & 121.003 & 101.792 & 105.909 & 97.497 & 111.981 & 108.663 & 80.264 & 75 \\
\hline 6/10/07 7:00 & 126.756 & 124.385 & 121.003 & 101.792 & 105.907 & 97.497 & 111.977 & 108.666 & 80.26 & 74.998 \\
\hline 6/10/07 7:30 & 126.761 & 124.376 & 121.008 & 101.795 & 105.911 & 97.502 & 111.971 & 108.668 & 80.258 & 74.998 \\
\hline 6/10/07 8:00 & 126.756 & 126.204 & 121.001 & 101.785 & 105.903 & 97.492 & 111.965 & 108.664 & 80.253 & 75.013 \\
\hline 6/10/07 8:30 & 126.752 & 126.606 & 120.992 & 101.776 & 105.896 & 97.485 & 111.955 & 108.652 & 80.246 & 74.998 \\
\hline 6/10/07 9:00 & 128.601 & 126.789 & 122.227 & 102.345 & 106.233 & 97.982 & 112.484 & 109.866 & 80.253 & 75.005 \\
\hline 6/10/07 9:30 & 129.059 & 126.896 & 122.678 & 102.715 & 106.629 & 98.386 & 112.852 & 110.379 & 80.29 & 75.146 \\
\hline 6/10/07 10:00 & 129.248 & 126.964 & 122.863 & 102.898 & 106.808 & 98.562 & 112.989 & 110.574 & 80.323 & 75.28 \\
\hline $6 / 10 / 07$ 10:30 & 129.362 & 127.075 & 122.976 & 102.996 & 106.92 & 98.673 & 113.089 & 110.694 & 80.356 & 75.381 \\
\hline 6/10/07 11:00 & 129.431 & 127.152 & 123.043 & 103.06 & 106.987 & 98.739 & 113.147 & 110.767 & 80.376 & 75.447 \\
\hline 6/10/07 11:30 & 129.549 & 127.198 & 123.198 & 103.258 & 107.145 & 98.928 & 114.159 & 110.873 & 80.419 & 75.515 \\
\hline 6/10/07 12:00 & 129.627 & 127.227 & 123.277 & 103.339 & 107.226 & 99.01 & 114.204 & 110.955 & 80.461 & 75.579 \\
\hline 6/10/07 12:30 & 129.676 & 127.247 & 123.33 & 103.387 & 107.28 & 99.062 & 114.239 & 111.008 & 80.5 & 75.629 \\
\hline 6/10/07 13:00 & 129.705 & 127.247 & 123.355 & 103.413 & 107.305 & 99.088 & 114.256 & 111.037 & 80.531 & 75.668 \\
\hline 6/10/07 13:30 & 129.726 & 127.257 & 123.379 & 103.434 & 107.325 & 99.11 & 114.278 & 111.057 & 80.561 & 75.693 \\
\hline $6 / 10 / 07$ 14:00 & 129.728 & 127.254 & 123.381 & 103.436 & 107.327 & 99.11 & 114.278 & 111.061 & 80.579 & 75.71 \\
\hline 6/10/07 14:30 & 129.738 & 125.794 & 123.39 & 103.444 & 107.339 & 99.121 & 114.286 & 111.07 & 80.601 & 75.724 \\
\hline $6 / 10 / 07$ 15:00 & 129.738 & 125.075 & 123.388 & 103.443 & 107.337 & 99.119 & 114.293 & 111.07 & 80.62 & 75.734 \\
\hline 6/10/07 15:30 & 128.558 & 124.83 & 122.712 & 103.039 & 107.177 & 98.86 & 113.325 & 110.749 & 80.614 & 75.726 \\
\hline $6 / 10 / 07$ 16:00 & 127.473 & 124.73 & 121.704 & 102.429 & 106.573 & 98.159 & 112.56 & 109.396 & 80.577 & 75.612 \\
\hline $6 / 10 / 07$ 16:30 & 127.217 & 124.676 & 121.452 & 102.198 & 106.333 & 97.919 & 112.348 & 109.135 & 80.541 & 75.459 \\
\hline 6/10/07 17:00 & 127.111 & 124.628 & 121.362 & 102.166 & 106.253 & 97.869 & 113.078 & 109.009 & 80.511 & 75.344 \\
\hline $6 / 10 / 07$ 17:30 & 127.059 & 124.585 & 121.318 & 102.13 & 106.21 & 97.832 & 113.164 & 108.956 & 80.504 & 75.269 \\
\hline $6 / 10 / 07$ 18:00 & 127.007 & 124.558 & 121.269 & 102.083 & 106.166 & 97.787 & 113.119 & 108.905 & 80.493 & 75.215 \\
\hline 6/10/07 18:30 & 126.967 & 124.531 & 121.228 & 102.045 & 106.126 & 97.749 & 113.076 & 108.863 & 80.476 & 75.174 \\
\hline $6 / 10 / 07$ 19:00 & 126.936 & 124.512 & 121.198 & 102.019 & 106.097 & 97.721 & 113.046 & 108.832 & 80.463 & 75.151 \\
\hline $6 / 10 / 07$ 19:30 & 126.912 & 124.428 & 121.174 & 101.995 & 106.076 & 97.7 & 113.021 & 108.809 & 80.448 & 75.131 \\
\hline 6/10/07 20:00 & 126.891 & 124.394 & 121.156 & 101.976 & 106.055 & 97.681 & 113.005 & 108.787 & 80.437 & 75.114 \\
\hline $6 / 10 / 0720: 30$ & 126.801 & 124.376 & 121.047 & 101.821 & 105.949 & 97.535 & 112.07 & 108.714 & 80.412 & 75.089 \\
\hline $6 / 10 / 0721: 00$ & 126.766 & 124.367 & 121.015 & 101.79 & 105.915 & 97.506 & 112.085 & 108.674 & 80.386 & 75.069 \\
\hline 6/10/07 21:30 & 126.747 & 124.36 & 120.992 & 101.769 & 105.896 & 97.483 & 112.024 & 108.657 & 80.365 & 75.046 \\
\hline 6/10/07 22:00 & 126.735 & 124.36 & 120.982 & 101.764 & 105.884 & 97.471 & 112.032 & 108.643 & 80.349 & 75.034 \\
\hline 6/10/07 22:30 & 126.731 & 124.358 & 120.976 & 101.759 & 105.88 & 97.466 & 112.02 & 108.639 & 80.336 & 75.023 \\
\hline 6/10/07 23:00 & 126.73 & 124.358 & 120.976 & 101.759 & 105.88 & 97.466 & 111.998 & 108.639 & 80.323 & 75.025 \\
\hline $6 / 10 / 07$ 23:30 & 126.73 & 124.349 & 120.974 & 101.759 & 105.88 & 97.471 & 111.939 & 108.639 & 80.31 & 75.023 \\
\hline 6/11/07 0:00 & 126.723 & 124.351 & 120.971 & 101.769 & 105.876 & 97.469 & 111.984 & 108.632 & 80.301 & 75.017 \\
\hline 6/11/07 0:30 & 126.721 & 124.342 & 120.969 & 101.749 & 105.875 & 97.473 & 112.038 & 108.626 & 80.288 & 75.009 \\
\hline 6/11/07 1:00 & 126.719 & 124.331 & 120.967 & 101.754 & 105.876 & 97.464 & 111.954 & 108.63 & 80.279 & 75.017 \\
\hline 6/11/07 1:30 & 126.714 & 124.322 & 120.959 & 101.745 & 105.867 & 97.457 & 111.971 & 108.623 & 80.268 & 75 \\
\hline 6/11/07 2:00 & 126.7 & 124.308 & 120.948 & 101.735 & 105.854 & 97.443 & 111.961 & 108.61 & 80.255 & 74.986 \\
\hline 6/11/07 2:30 & 126.69 & 124.301 & 120.936 & 101.725 & 105.844 & 97.433 & 111.952 & 108.599 & 80.244 & 74.969 \\
\hline 6/11/07 3:00 & 126.676 & 124.292 & 120.925 & 101.711 & 105.833 & 97.424 & 111.938 & 108.586 & 80.231 & 74.968 \\
\hline 6/11/07 3:30 & 126.671 & 124.29 & 120.918 & 101.706 & 105.825 & 97.415 & 111.928 & 108.579 & 80.222 & 74.957 \\
\hline 6/11/07 4:00 & 126.662 & 124.283 & 120.908 & 101.697 & 105.819 & 97.405 & 111.924 & 108.57 & 80.211 & 74.945 \\
\hline 6/11/07 4:30 & 126.657 & 124.288 & 120.904 & 101.694 & 105.814 & 97.403 & 111.914 & 108.568 & 80.201 & 74.941 \\
\hline 6/11/07 5:00 & 126.652 & 124.288 & 120.902 & 101.69 & 105.812 & 97.398 & 111.915 & 108.564 & 80.192 & 74.941 \\
\hline 6/11/07 5:30 & 126.645 & 124.295 & 120.902 & 101.692 & 105.812 & 97.4 & 111.907 & 108.564 & 80.187 & 74.936 \\
\hline 6/11/07 6:00 & 126.655 & 126.302 & 120.902 & 101.694 & 105.814 & 97.4 & 111.907 & 108.564 & 80.181 & 74.936 \\
\hline 6/11/07 6:30 & 126.662 & 126.597 & 120.911 & 101.701 & 105.821 & 97.405 & 111.914 & 108.573 & 80.177 & 74.947 \\
\hline 6/11/07 7:00 & 128.695 & 126.76 & 122.211 & 102.369 & 106.292 & 98.027 & 112.427 & 110.047 & 80.196 & 74.996 \\
\hline 6/11/07 7:30 & 129.022 & 126.855 & 122.53 & 102.648 & 106.589 & 98.327 & 112.713 & 110.39 & 80.231 & 75.137 \\
\hline 6/11/07 8:00 & 129.192 & 126.928 & 122.699 & 102.81 & 106.754 & 98.492 & 112.899 & 110.563 & 80.268 & 75.28 \\
\hline 6/11/07 8:30 & 129.293 & 126.968 & 122.798 & 102.905 & 106.853 & 98.588 & 113.009 & 110.669 & 80.299 & 75.371 \\
\hline 6/11/07 9:00 & 129.364 & 127 & 122.87 & 102.974 & 106.924 & 98.657 & 113.045 & 110.742 & 80.33 & 75.437 \\
\hline 6/11/07 9:30 & 129.409 & 127.018 & 122.918 & 103.019 & 106.971 & 98.704 & 113.116 & 110.791 & 80.358 & 75.484 \\
\hline 6/11/07 10:00 & 129.437 & 124.986 & 122.951 & 103.053 & 107.004 & 98.739 & 113.179 & 110.822 & 80.38 & 75.515 \\
\hline $6 / 11 / 07$ 10:30 & 129.457 & 124.753 & 122.969 & 103.077 & 107.025 & 98.756 & 113.164 & 110.842 & 80.402 & 75.548 \\
\hline $6 / 11 / 0711: 00$ & 127.388 & 124.626 & 121.621 & 102.352 & 106.499 & 98.082 & 112.507 & 109.314 & 80.397 & 75.503 \\
\hline 6/11/07 11:30 & 127.135 & 124.553 & 121.373 & 102.138 & 106.271 & 97.853 & 112.294 & 109.056 & 80.38 & 75.379 \\
\hline 6/11/07 12:00 & 127.005 & 124.492 & 121.246 & 102.018 & 106.149 & 97.733 & 112.288 & 108.92 & 80.362 & 75.282 \\
\hline $6 / 11 / 07$ 12:30 & 126.929 & 124.442 & 121.17 & 101.947 & 106.074 & 97.662 & 112.191 & 108.843 & 80.347 & 75.211 \\
\hline 6/11/07 13:00 & 126.865 & 124.406 & 121.11 & 101.888 & 106.014 & 97.601 & 112.079 & 108.778 & 80.33 & 75.158 \\
\hline 6/11/07 13:30 & 126.816 & 124.374 & 121.063 & 101.842 & 105.97 & 97.558 & 112.071 & 108.728 & 80.312 & 75.11 \\
\hline $6 / 11 / 0714: 00$ & 126.775 & 124.347 & 121.019 & 101.811 & 105.922 & 97.511 & 112.018 & 108.685 & 80.294 & 75.065 \\
\hline
\end{tabular}


TABLE S1.1 (Cont.)

Depth from Top of Casing (ft)

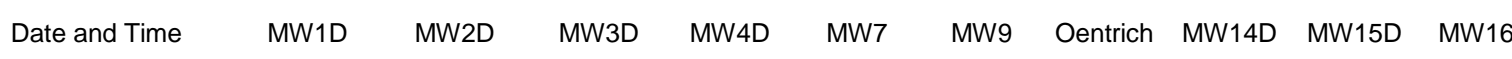

\begin{tabular}{|c|c|c|c|c|c|c|c|c|c|c|}
\hline 6/11/07 14:30 & 126.742 & 124.333 & 120.987 & 101.78 & 105.894 & 97.481 & 111.968 & 108.652 & 80.277 & 75.031 \\
\hline 6/11/07 15:00 & 126.719 & 124.313 & 120.962 & 101.747 & 105.871 & 97.457 & 111.942 & 108.63 & 80.26 & 75.011 \\
\hline $6 / 11 / 0715: 30$ & 126.697 & 124.301 & 120.945 & 101.737 & 105.856 & 97.445 & 112.074 & 108.608 & 80.246 & 74.997 \\
\hline $6 / 11 / 0716: 00$ & 126.683 & 124.29 & 120.929 & 101.718 & 105.84 & 97.426 & 111.921 & 108.592 & 80.233 & 74.978 \\
\hline 6/11/07 16:30 & 126.669 & 124.283 & 120.916 & 101.706 & 105.827 & 97.415 & 111.909 & 108.579 & 80.22 & 74.959 \\
\hline 6/11/07 17:00 & 126.657 & 124.27 & 120.906 & 101.695 & 105.816 & 97.403 & 111.903 & 108.57 & 80.207 & 74.957 \\
\hline $6 / 11 / 07$ 17:30 & 126.65 & 124.258 & 120.899 & 101.688 & 105.812 & 97.398 & 111.875 & 108.561 & 80.2 & 74.947 \\
\hline $6 / 11 / 07$ 18:00 & 126.639 & 124.256 & 120.888 & 101.678 & 105.8 & 97.386 & 111.855 & 108.553 & 80.187 & 74.936 \\
\hline $6 / 11 / 0718: 30$ & 126.626 & 124.256 & 120.876 & 101.67 & 105.789 & 97.377 & 111.837 & 108.542 & 80.176 & 74.928 \\
\hline 6/11/07 19:00 & 126.624 & 124.261 & 120.871 & 101.664 & 105.787 & 97.374 & 111.819 & 108.535 & 80.166 & 74.92 \\
\hline 6/11/07 19:30 & 126.624 & 124.261 & 120.874 & 101.668 & 105.787 & 97.377 & 111.803 & 108.535 & 80.161 & 74.926 \\
\hline $6 / 11 / 0720: 00$ & 126.629 & 124.256 & 120.876 & 101.67 & 105.795 & 97.379 & 111.787 & 108.539 & 80.155 & 74.926 \\
\hline $6 / 11 / 0720: 30$ & 126.629 & 124.258 & 120.878 & 101.671 & 105.793 & 97.379 & 111.785 & 108.542 & 80.152 & 74.922 \\
\hline 6/11/07 21:00 & 126.624 & 124.272 & 120.874 & 101.668 & 105.787 & 97.374 & 111.774 & 108.535 & 80.142 & 74.918 \\
\hline $6 / 11 / 0721: 30$ & 126.626 & 124.286 & 120.878 & 101.671 & 105.793 & 97.377 & 111.766 & 108.539 & 80.139 & 74.922 \\
\hline 6/11/07 22:00 & 126.639 & 124.288 & 120.888 & 101.683 & 105.804 & 97.389 & 111.758 & 108.548 & 80.139 & 74.926 \\
\hline 6/11/07 22:30 & 126.655 & 124.288 & 120.906 & 101.702 & 105.823 & 97.407 & 111.754 & 108.566 & 80.141 & 74.943 \\
\hline 6/11/07 23:00 & 126.657 & 124.283 & 120.907 & 101.701 & 105.823 & 97.407 & 111.757 & 108.568 & 80.137 & 74.945 \\
\hline $6 / 11 / 0723: 30$ & 126.652 & 124.27 & 120.904 & 101.701 & 105.819 & 97.403 & 111.755 & 108.564 & 80.133 & 74.936 \\
\hline 6/12/07 0:00 & 126.65 & 124.258 & 120.902 & 101.699 & 105.819 & 97.405 & 111.755 & 108.561 & 80.129 & 74.934 \\
\hline 6/12/07 0:30 & 126.634 & 124.254 & 120.885 & 101.68 & 105.8 & 97.389 & 111.751 & 108.546 & 80.12 & 74.916 \\
\hline 6/12/07 1:00 & 126.624 & 124.24 & 120.876 & 101.67 & 105.793 & 97.379 & 111.745 & 108.537 & 80.113 & 74.91 \\
\hline 6/12/07 1:30 & 126.619 & 124.233 & 120.871 & 101.668 & 105.789 & 97.374 & 111.739 & 108.533 & 80.105 & 74.901 \\
\hline 6/12/07 2:00 & 126.61 & 124.231 & 120.858 & 101.654 & 105.777 & 97.363 & 111.736 & 108.522 & 80.098 & 74.887 \\
\hline $6 / 12 / 072: 30$ & 126.603 & 124.218 & 120.853 & 101.649 & 105.772 & 97.356 & 111.726 & 108.513 & 80.093 & 74.876 \\
\hline 6/12/07 3:00 & 126.596 & 124.22 & 120.848 & 101.645 & 105.766 & 97.351 & 111.722 & 108.508 & 80.087 & 74.885 \\
\hline 6/12/07 3:30 & 126.589 & 124.218 & 120.839 & 101.633 & 105.758 & 97.344 & 111.718 & 108.499 & 80.078 & 74.876 \\
\hline 6/12/07 4:00 & 126.586 & 124.211 & 120.839 & 101.635 & 105.757 & 97.341 & 111.711 & 108.499 & 80.074 & 74.883 \\
\hline 6/12/07 4:30 & 126.584 & 124.208 & 120.837 & 101.635 & 105.757 & 97.341 & 111.705 & 108.497 & 80.072 & 74.876 \\
\hline 6/12/07 5:00 & 126.579 & 124.208 & 120.83 & 101.628 & 105.749 & 97.334 & 111.701 & 108.493 & 80.067 & 74.866 \\
\hline 6/12/07 5:30 & 126.577 & 124.188 & 120.83 & 101.626 & 105.749 & 97.332 & 111.695 & 108.488 & 80.061 & 74.864 \\
\hline 6/12/07 6:00 & 126.582 & 126.297 & 120.834 & 101.628 & 105.757 & 97.341 & 111.69 & 108.497 & 80.059 & 74.87 \\
\hline 6/12/07 6:30 & 126.636 & 126.54 & 121.14 & 101.625 & 105.736 & 97.318 & 111.689 & 108.471 & 80.163 & 74.86 \\
\hline 6/12/07 7:00 & 128.693 & 126.678 & 122.361 & 102.429 & 106.339 & 98.089 & 112.391 & 110.054 & 80.03 & 74.943 \\
\hline $6 / 12 / 077: 30$ & 128.958 & 126.771 & 122.622 & 102.662 & 106.587 & 98.336 & 112.635 & 110.328 & 80.07 & 75.065 \\
\hline 6/12/07 8:00 & 129.104 & 126.835 & 122.763 & 102.795 & 106.72 & 98.47 & 112.766 & 110.477 & 80.109 & 75.168 \\
\hline 6/12/07 8:30 & 129.218 & 124.921 & 122.863 & 102.891 & 106.821 & 98.567 & 112.872 & 110.576 & 80.148 & 75.234 \\
\hline 6/12/07 9:00 & 129.267 & 124.651 & 122.927 & 102.951 & 106.882 & 98.628 & 112.923 & 110.643 & 80.181 & 75.29 \\
\hline 6/12/07 9:30 & 127.393 & 124.517 & 121.565 & 102.288 & 106.438 & 98.016 & 112.32 & 109.263 & 80.188 & 75.296 \\
\hline $6 / 12 / 07$ 10:00 & 127.107 & 124.433 & 121.274 & 102.04 & 106.179 & 97.759 & 112.068 & 108.956 & 80.177 & 75.211 \\
\hline $6 / 12 / 07 \quad 10: 30$ & 126.969 & 124.374 & 121.142 & 101.918 & 106.055 & 97.631 & 111.946 & 108.816 & 80.168 & 75.133 \\
\hline 6/12/07 11:00 & 126.882 & 124.333 & 121.054 & 101.838 & & 97.551 & 111.873 & 108.728 & 80.159 & 75.091 \\
\hline 6/12/07 11:30 & 126.82 & 124.292 & 120.994 & 101.781 & 105.911 & 97.49 & 111.829 & 108.663 & 80.146 & 75.063 \\
\hline 6/12/07 12:00 & 126.766 & 124.252 & 120.957 & 101.742 & 105.871 & 97.455 & 111.795 & 108.623 & 80.137 & 75.025 \\
\hline $6 / 12 / 0712: 30$ & 126.714 & 124.213 & & 101.702 & & & & & & 75.015 \\
\hline 6/12/07 13:00 & 126.624 & 124.19 & 120.874 & 101.663 & 105.791 & 97.372 & 111.753 & 108.539 & 80.105 & 75.027 \\
\hline 6/12/07 13:30 & 126.587 & 124.179 & 120.837 & 101.625 & 105.753 & 97.334 & 111.725 & 108.499 & 80.089 & 75.06 \\
\hline $6 / 12 / 0714: 00$ & 126.561 & 124.172 & 120.811 & 101.604 & 105.732 & 97.315 & 111.712 & 108.477 & 80.074 & 75.046 \\
\hline $6 / 12 / 07$ 14:30 & 126.551 & 124.159 & 120.802 & 101.595 & 105.72 & 97.306 & 111.7 & 108.464 & 80.067 & 74.996 \\
\hline $6 / 12 / 07$ 15:00 & 126.544 & 124.136 & 120.795 & 101.589 & 105.715 & 97.299 & 111.691 & 108.46 & 80.058 & 74.924 \\
\hline $6 / 12 / 0715: 30$ & & & & 101.578 & & & & 108.446 & & 74.874 \\
\hline 6/12/07 16:00 & 126.506 & 124.127 & 120.76 & 101.554 & 105.678 & 97.261 & 111.695 & 108.422 & 80.034 & 74.862 \\
\hline $6 / 12 / 0716: 30$ & 126.506 & 124.111 & 120.758 & 101.556 & 105.682 & 97.263 & 111.677 & 108.422 & 80.026 & 74.877 \\
\hline 6/12/07 17:00 & 126.499 & 124.095 & & 101.545 & & & & 108.413 & 80.019 & 74.854 \\
\hline 6/12/07 17:30 & 126.48 & 124.088 & 120.735 & 101.53 & 105.656 & 97.238 & 111.652 & 108.398 & 80.004 & 74.835 \\
\hline 6/12/07 18:00 & 126.466 & 124.093 & 120.716 & 101.513 & 105.64 & 97.219 & 111.644 & 108.38 & 79.991 & 74.81 \\
\hline 6/12/07 18:30 & 126.461 & 124.086 & 120.712 & 101.509 & 105.633 & 97.216 & 111.637 & 108.373 & 79.984 & 74.792 \\
\hline 6/12/07 19:00 & 126.461 & 124.086 & 120.714 & 101.514 & 105.64 & 97.219 & 111.633 & 108.378 & 79.978 & 74.773 \\
\hline 6/12/07 19:30 & 126.456 & 124.082 & 120.71 & 101.508 & 105.635 & 97.216 & 111.628 & 108.371 & 79.971 & 74.761 \\
\hline $6 / 12 / 0720: 00$ & 126.456 & 124.066 & 120.71 & 101.508 & 105.635 & 97.214 & 111.624 & 108.371 & 79.965 & 74.757 \\
\hline 6/12/07 20:30 & 126.447 & 124.061 & 120.703 & 101.502 & 105.627 & 97.209 & 111.618 & 108.364 & 79.958 & 74.753 \\
\hline 6/12/07 21:00 & 126.437 & 124.07 & 120.693 & 101.489 & 105.616 & 97.195 & 111.652 & 108.353 & 79.951 & 74.752 \\
\hline $6 / 12 / 0721: 30$ & 126.433 & 124.075 & 120.686 & 101.485 & 105.612 & 97.193 & 111.629 & 108.349 & 79.945 & 74.746 \\
\hline 6/12/07 22:00 & 126.437 & 124.079 & 120.691 & 101.492 & 105.619 & 97.198 & 111.61 & 108.353 & 79.941 & 74.746 \\
\hline 6/12/07 22:30 & 126.444 & 124.077 & 120.698 & 101.499 & 105.625 & 97.205 & 111.602 & 108.36 & 79.94 & 74.748 \\
\hline 6/12/07 23:00 & 126.447 & 124.072 & 120.698 & 101.501 & 105.627 & 97.205 & 111.596 & 108.36 & 79.934 & 74.75 \\
\hline
\end{tabular}


TABLE S1.1 (Cont.)

Depth from Top of Casing (ft)

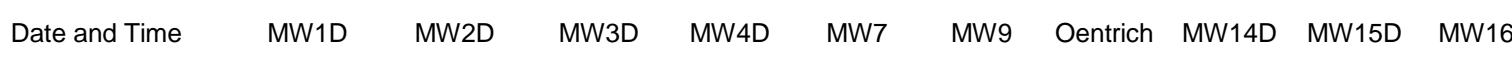

\begin{tabular}{|c|c|c|c|c|c|c|c|c|c|c|}
\hline 6/12/07 23:30 & 126.444 & 124.061 & 120.698 & 101.499 & 105.625 & 97.205 & 111.592 & 108.36 & 79.932 & 74.753 \\
\hline 6/13/07 0:00 & 126.44 & 124.057 & 120.693 & 101.495 & 105.621 & 97.202 & 111.586 & 108.356 & 79.927 & 74.759 \\
\hline 6/13/07 0:30 & 126.43 & 124.045 & 120.684 & 101.483 & 105.612 & 97.19 & 111.583 & 108.349 & 79.921 & 74.754 \\
\hline 6/13/07 1:00 & 126.426 & 124.032 & 120.679 & 101.478 & 105.608 & 97.188 & 111.573 & 108.342 & 79.916 & 74.745 \\
\hline 6/13/07 1:30 & 126.414 & 124.025 & 120.668 & 101.47 & 105.597 & 97.176 & 111.571 & 108.333 & 79.91 & 74.746 \\
\hline 6/13/07 2:00 & 126.402 & 124.02 & 120.654 & 101.458 & 105.585 & 97.164 & 111.564 & 108.318 & 79.899 & 74.736 \\
\hline 6/13/07 2:30 & 126.395 & 124.009 & 120.649 & 101.449 & 105.578 & 97.157 & 111.558 & 108.311 & 79.892 & 74.726 \\
\hline 6/13/07 3:00 & 126.388 & 124.007 & 120.64 & 101.444 & 105.574 & 97.15 & 111.552 & 108.302 & 79.882 & 74.717 \\
\hline 6/13/07 3:30 & 126.378 & 124 & 120.633 & 101.435 & 105.564 & 97.141 & 111.547 & 108.296 & 79.877 & 74.709 \\
\hline 6/13/07 4:00 & 126.374 & 124 & 120.629 & 101.43 & 105.56 & 97.139 & 111.543 & 108.289 & 79.871 & 74.703 \\
\hline 6/13/07 4:30 & 126.369 & 124 & 120.624 & 101.428 & 105.556 & 97.134 & 111.537 & 108.287 & 79.866 & 74.697 \\
\hline 6/13/07 5:00 & 126.367 & 124 & 120.622 & 101.427 & 105.555 & 97.134 & 111.535 & 108.285 & 79.86 & 74.701 \\
\hline 6/13/07 5:30 & 126.369 & 123.998 & 120.624 & 101.427 & 105.555 & 97.136 & 111.531 & 108.287 & 79.857 & 74.69 \\
\hline 6/13/07 6:00 & 126.366 & 125.762 & 120.622 & 101.425 & 105.553 & 97.132 & 111.528 & 108.285 & 79.851 & 74.69 \\
\hline 6/13/07 6:30 & 126.366 & 126.22 & 120.62 & 101.423 & 105.553 & 97.132 & 111.528 & 108.285 & 79.847 & 74.692 \\
\hline 6/13/07 7:00 & 128.109 & 126.404 & 121.642 & 101.878 & 105.798 & 97.516 & 111.879 & 109.381 & 79.847 & 74.697 \\
\hline 6/13/07 7:30 & 128.636 & 126.515 & 122.148 & 102.283 & 106.221 & 97.954 & 112.29 & 110.003 & 79.881 & 74.825 \\
\hline 6/13/07 8:00 & 128.828 & 126.592 & 122.34 & 102.462 & 106.408 & 98.138 & 112.466 & 110.206 & 79.912 & 74.951 \\
\hline 6/13/07 8:30 & 128.943 & 124.633 & 122.456 & 102.572 & 106.524 & 98.251 & 112.567 & 110.324 & 79.941 & 75.056 \\
\hline 6/13/07 9:00 & 129.024 & 124.381 & 122.537 & 102.646 & 106.6 & 98.329 & 112.633 & 110.406 & 79.969 & 75.137 \\
\hline 6/13/07 9:30 & 127.036 & 124.254 & 121.274 & 102.009 & 106.164 & 97.742 & 112.074 & 108.975 & 79.976 & 75.156 \\
\hline $6 / 13 / 07$ 10:00 & 126.759 & 124.184 & 121.006 & 101.778 & 105.924 & 97.497 & 111.842 & 108.69 & 79.962 & 75.067 \\
\hline 6/13/07 10:30 & 126.627 & 124.145 & 120.878 & 101.661 & 105.8 & 97.372 & 111.73 & 108.555 & 79.949 & 74.976 \\
\hline 6/13/07 11:00 & 126.553 & 124.113 & 120.807 & 101.595 & 105.734 & 97.308 & 111.667 & 108.477 & 79.941 & 74.907 \\
\hline $6 / 13 / 0711: 30$ & 126.516 & 124.088 & 120.77 & 101.563 & 105.698 & 97.275 & 111.626 & 108.437 & 79.932 & 74.847 \\
\hline 6/13/07 12:00 & 126.483 & 124.059 & 120.74 & 101.533 & 105.667 & 97.245 & 111.598 & 108.406 & 79.923 & 74.808 \\
\hline 6/13/07 12:30 & 126.463 & 124.043 & 120.714 & 101.511 & 105.642 & 97.221 & 111.573 & 108.378 & 79.912 & 74.788 \\
\hline $6 / 13 / 07$ 13:00 & 126.447 & 124.016 & 120.68 & 101.482 & 105.612 & 97.191 & 111.555 & 108.347 & 79.897 & 74.775 \\
\hline 6/13/07 13:30 & 126.435 & 124.007 & 120.668 & 101.466 & 105.597 & 97.179 & 111.544 & 108.333 & 79.888 & 74.769 \\
\hline 6/13/07 14:00 & 126.426 & 123.998 & 120.645 & 101.442 & 105.574 & 97.155 & 111.529 & 108.309 & 79.871 & 74.759 \\
\hline $6 / 13 / 07$ 14:30 & 126.418 & 123.998 & 120.631 & 101.432 & 105.562 & 97.141 & 111.519 & 108.296 & 79.86 & 74.757 \\
\hline 6/13/07 15:00 & 126.409 & 123.989 & 120.624 & 101.425 & 105.555 & 97.132 & 111.51 & 108.285 & 79.849 & 74.752 \\
\hline 6/13/07 15:30 & 126.364 & 123.977 & 120.62 & 101.423 & 105.555 & 97.132 & 111.5 & 108.283 & 79.84 & 74.754 \\
\hline $6 / 13 / 07$ 16:00 & 126.357 & 123.97 & 120.612 & 101.416 & 105.549 & 97.124 & 111.495 & 108.276 & 79.833 & 74.744 \\
\hline $6 / 13 / 07$ 16:30 & 126.345 & 123.948 & 120.604 & 101.406 & 105.538 & 97.113 & 111.487 & 108.265 & 79.822 & 74.732 \\
\hline 6/13/07 17:00 & 126.343 & 123.918 & 120.603 & 101.402 & 105.534 & 97.113 & 111.484 & 108.263 & 79.814 & 74.723 \\
\hline $6 / 13 / 07$ 17:30 & 126.319 & 123.911 & 120.575 & 101.378 & 105.509 & 97.087 & 111.476 & 108.238 & 79.798 & 74.709 \\
\hline 6/13/07 18:00 & 126.288 & 123.905 & 120.545 & 101.346 & 105.48 & 97.056 & 111.467 & 108.209 & 79.781 & 74.705 \\
\hline 6/13/07 18:30 & 126.279 & 123.9 & 120.536 & 101.34 & 105.473 & 97.049 & 111.456 & 108.198 & 79.77 & 74.692 \\
\hline $6 / 13 / 07$ 19:00 & 126.272 & 123.898 & 120.529 & 101.334 & 105.465 & 97.042 & 111.45 & 108.19 & 79.761 & 74.682 \\
\hline $6 / 13 / 07$ 19:30 & 126.272 & 123.889 & 120.529 & 101.332 & 105.465 & 97.042 & 111.471 & 108.192 & 79.755 & 74.674 \\
\hline 6/13/07 20:00 & 126.263 & 123.9 & 120.52 & 101.349 & 105.458 & 97.032 & 111.483 & 108.181 & 79.746 & 74.661 \\
\hline 6/13/07 20:30 & 126.256 & 123.907 & 120.513 & 101.316 & 105.45 & 97.025 & 111.449 & 108.174 & 79.737 & 74.641 \\
\hline 6/13/07 21:00 & 126.267 & 123.907 & 120.525 & 101.332 & 105.465 & 97.037 & 111.476 & 108.185 & 79.735 & 74.62 \\
\hline $6 / 13 / 0721: 30$ & 126.277 & 123.902 & 120.534 & 101.339 & 105.471 & 97.049 & 111.447 & 108.196 & 79.733 & 74.608 \\
\hline 6/13/07 22:00 & 126.277 & 123.914 & 120.534 & 101.339 & 105.469 & 97.047 & 111.429 & 108.194 & 79.729 & 74.593 \\
\hline 6/13/07 22:30 & 126.269 & 123.902 & 120.527 & 101.332 & 105.465 & 97.04 & 111.421 & 108.187 & 79.722 & 74.581 \\
\hline 6/13/07 23:00 & 126.284 & 123.905 & 120.541 & 101.346 & 105.479 & 97.056 & 111.412 & 108.203 & 79.724 & 74.575 \\
\hline $6 / 13 / 0723: 30$ & 126.272 & 123.902 & 120.529 & 101.335 & 105.465 & 97.042 & 111.408 & 108.192 & 79.717 & 74.571 \\
\hline 6/14/07 0:00 & 126.272 & 123.893 & 120.529 & 101.337 & 105.469 & 97.044 & 111.4 & 108.192 & 79.713 & 74.564 \\
\hline 6/14/07 0:30 & 126.267 & 123.88 & 120.525 & 101.334 & 105.465 & 97.04 & 111.394 & 108.188 & 79.707 & 74.552 \\
\hline 6/14/07 1:00 & 126.258 & 123.868 & 120.515 & 101.323 & 105.456 & 97.032 & 111.392 & 108.181 & 79.702 & 74.542 \\
\hline 6/14/07 1:30 & 126.248 & 123.866 & 120.506 & 101.313 & 105.444 & 97.021 & 111.388 & 108.168 & 79.694 & 74.534 \\
\hline 6/14/07 2:00 & 126.239 & 123.848 & 120.497 & 101.304 & 105.437 & 97.011 & 111.385 & 108.159 & 79.685 & 74.529 \\
\hline 6/14/07 2:30 & 126.234 & 123.85 & 120.492 & 101.299 & 105.433 & 97.007 & 111.381 & 108.156 & 79.678 & 74.531 \\
\hline 6/14/07 3:00 & 126.23 & 123.846 & 120.474 & 101.28 & 105.414 & 96.988 & 111.377 & 108.136 & 79.667 & 74.535 \\
\hline 6/14/07 3:30 & 126.225 & 123.841 & 120.476 & 101.284 & 105.416 & 96.99 & 111.369 & 108.139 & 79.663 & 74.535 \\
\hline 6/14/07 4:00 & 126.222 & 123.825 & 120.471 & 101.28 & 105.414 & 96.99 & 111.366 & 108.135 & 79.656 & 74.533 \\
\hline 6/14/07 4:30 & 126.22 & 123.823 & 120.464 & 101.271 & 105.406 & 96.981 & 111.362 & 108.128 & 79.648 & 74.531 \\
\hline 6/14/07 5:00 & 126.22 & 123.823 & 120.453 & 101.261 & 105.393 & 96.969 & 111.36 & 108.116 & 79.639 & 74.532 \\
\hline 6/14/07 5:30 & 126.215 & 123.821 & 120.448 & 101.256 & 105.391 & 96.964 & 111.356 & 108.11 & 79.632 & 74.519 \\
\hline 6/14/07 6:00 & 126.211 & 123.818 & 120.448 & 101.256 & 105.393 & 96.967 & 111.352 & 108.112 & 79.63 & 74.519 \\
\hline 6/14/07 6:30 & 126.208 & 123.816 & 120.446 & 101.254 & 105.389 & 96.964 & 111.348 & 108.108 & 79.624 & 74.521 \\
\hline 6/14/07 7:00 & 126.203 & 125.841 & 120.444 & 101.254 & 105.391 & 96.962 & 111.343 & 108.108 & 79.621 & 74.515 \\
\hline 6/14/07 7:30 & 126.203 & 126.191 & 120.441 & 101.249 & 105.387 & 96.96 & 111.339 & 108.104 & 79.615 & 74.527 \\
\hline $6 / 14 / 078: 00$ & 128.26 & 126.363 & 121.873 & 101.943 & 105.846 & 97.594 & 111.928 & 109.542 & 79.628 & 74.56 \\
\hline
\end{tabular}


TABLE S1.1 (Cont.)

Depth from Top of Casing (ft)

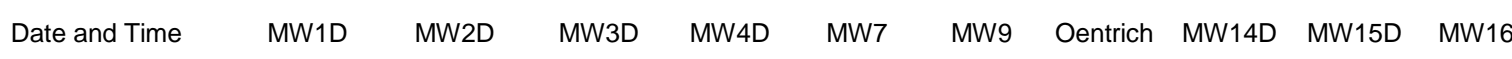

\begin{tabular}{|c|c|c|c|c|c|c|c|c|c|c|}
\hline 6/14/07 8:30 & 128.641 & 126.476 & 122.248 & 102.269 & 106.189 & 97.935 & 112.255 & 109.954 & 79.667 & 74.709 \\
\hline 6/14/07 9:00 & 128.825 & 126.556 & 122.428 & 102.438 & 106.362 & 98.107 & 112.417 & 110.144 & 79.698 & 74.821 \\
\hline $6 / 14 / 079: 30$ & 128.946 & 126.617 & 122.549 & 102.548 & 106.476 & 98.221 & 112.516 & 110.266 & 79.731 & 74.914 \\
\hline $6 / 14 / 07$ 10:00 & 129.029 & 126.656 & 122.627 & 102.621 & 106.552 & 98.298 & 112.586 & 110.35 & 79.761 & 74.988 \\
\hline $6 / 14 / 07 \quad 10: 30$ & 129.09 & 126.685 & 122.687 & 102.679 & 106.611 & 98.357 & 112.64 & 110.412 & 79.794 & 75.038 \\
\hline $6 / 14 / 0711: 00$ & 129.13 & 126.694 & 122.726 & 102.717 & 106.65 & 98.395 & 112.674 & 110.453 & 79.818 & 75.087 \\
\hline $6 / 14 / 0711: 30$ & 129.163 & 126.717 & 122.759 & 102.745 & 106.682 & 98.426 & 112.702 & 110.485 & 79.844 & 75.149 \\
\hline $6 / 14 / 07 \quad 12: 00$ & 129.175 & 126.789 & 122.773 & 102.758 & 106.695 & 98.437 & 112.723 & 110.496 & 79.866 & 75.213 \\
\hline $6 / 14 / 07$ 12:30 & 129.192 & 126.814 & 122.791 & 102.779 & 106.714 & 98.459 & 112.738 & 110.521 & 79.888 & 75.244 \\
\hline $6 / 14 / 07$ 13:00 & 129.239 & 126.764 & 122.83 & 102.802 & 106.731 & 98.482 & 112.758 & 110.565 & 79.908 & 75.271 \\
\hline $6 / 14 / 07$ 13:30 & 129.289 & 126.687 & 122.863 & 102.819 & 106.749 & 98.499 & 112.773 & 110.588 & 79.927 & 75.266 \\
\hline $6 / 14 / 07 \quad 14: 00$ & 129.248 & 126.671 & 122.844 & 102.824 & 106.762 & 98.506 & 112.785 & 110.576 & 79.945 & 75.268 \\
\hline $6 / 14 / 07 \quad 14: 30$ & 129.166 & 126.667 & 122.786 & 102.795 & 106.735 & 98.478 & 112.764 & 110.51 & 79.956 & 75.288 \\
\hline $6 / 14 / 07$ 15:00 & 129.149 & 126.66 & 122.766 & 102.777 & 106.72 & 98.461 & 112.758 & 110.492 & 79.969 & 75.275 \\
\hline $6 / 14 / 07 \quad 15: 30$ & 129.142 & 126.656 & 122.766 & 102.776 & 106.718 & 98.461 & 112.753 & 110.485 & 79.978 & 75.271 \\
\hline $6 / 14 / 07$ 16:00 & 129.137 & 126.653 & 122.759 & 102.772 & 106.716 & 98.456 & 112.749 & 110.483 & 79.989 & 75.271 \\
\hline $6 / 14 / 0716: 30$ & 129.13 & 126.649 & 122.754 & 102.769 & 106.71 & 98.452 & 112.751 & 110.477 & 79.997 & 75.271 \\
\hline $6 / 14 / 07$ 17:00 & 129.13 & 126.646 & 122.754 & 102.767 & 106.712 & 98.452 & 112.753 & 110.477 & 80.006 & 75.274 \\
\hline $6 / 14 / 07$ 17:30 & 129.123 & 126.644 & 122.747 & 102.764 & 106.707 & 98.449 & 112.753 & 110.47 & 80.013 & 75.267 \\
\hline $6 / 14 / 07$ 18:00 & 129.123 & 126.646 & 122.747 & 102.762 & 106.705 & 98.447 & 112.753 & 110.47 & 80.023 & 75.271 \\
\hline $6 / 14 / 0718: 30$ & 129.121 & 126.646 & 122.747 & 102.762 & 106.705 & 98.447 & 112.753 & 110.465 & 80.03 & 75.267 \\
\hline $6 / 14 / 07$ 19:00 & 129.123 & 126.649 & 122.747 & 102.764 & 106.709 & 98.449 & 112.755 & 110.47 & 80.037 & 75.271 \\
\hline $6 / 14 / 07$ 19:30 & 129.121 & 126.644 & 122.747 & 102.765 & 106.709 & 98.452 & 112.755 & 110.468 & 80.045 & 75.28 \\
\hline $6 / 14 / 0720: 00$ & 129.126 & 126.653 & 122.752 & 102.771 & 106.714 & 98.454 & 112.753 & 110.47 & 80.052 & 75.282 \\
\hline $6 / 14 / 0720: 30$ & 129.121 & 126.66 & 122.747 & 102.765 & 106.71 & 98.449 & 112.747 & 110.468 & 80.059 & 75.282 \\
\hline 6/14/07 21:00 & 129.128 & 126.674 & 122.756 & 102.776 & 106.72 & 98.461 & 112.753 & 110.479 & 80.069 & 75.288 \\
\hline $6 / 14 / 0721: 30$ & 129.132 & 124.676 & 122.761 & 102.784 & 106.729 & 98.466 & 112.755 & 110.483 & 80.076 & 75.309 \\
\hline $6 / 14 / 0722: 00$ & 129.147 & 124.399 & 122.777 & 102.8 & 106.743 & 98.48 & 112.756 & 110.499 & 80.087 & 75.317 \\
\hline $6 / 14 / 0722: 30$ & 127.083 & 124.247 & 121.32 & 102.047 & 106.212 & 97.782 & 112.086 & 109.024 & 80.072 & 75.269 \\
\hline 6/14/07 23:00 & 126.785 & 124.152 & 121.029 & 101.79 & 105.943 & 97.514 & 111.829 & 108.717 & 80.043 & 75.131 \\
\hline $6 / 14 / 0723: 30$ & 126.626 & 124.082 & 120.874 & 101.647 & 105.797 & 97.365 & 111.69 & 108.555 & 80.015 & 75.011 \\
\hline $6 / 15 / 070: 00$ & 126.527 & 124.032 & 120.777 & 101.558 & 105.701 & 97.271 & 111.608 & 108.455 & 79.989 & 74.926 \\
\hline 6/15/07 0:30 & 126.456 & 123.989 & 120.707 & 101.492 & 105.633 & 97.209 & 111.55 & 108.38 & 79.965 & 74.858 \\
\hline 6/15/07 1:00 & 126.402 & 123.957 & 120.654 & 101.446 & 105.585 & 97.158 & 111.507 & 108.327 & 79.943 & 74.804 \\
\hline 6/15/07 1:30 & 126.362 & 123.932 & 120.615 & 101.408 & 105.547 & 97.12 & 111.473 & 108.285 & 79.919 & 74.763 \\
\hline 6/15/07 2:00 & 126.329 & 123.911 & 120.582 & 101.378 & 105.517 & 97.091 & 111.449 & 108.252 & 79.899 & 74.73 \\
\hline 6/15/07 2:30 & 126.303 & 123.893 & 120.557 & 101.356 & 105.492 & 97.066 & 111.426 & 108.227 & 79.879 & 74.707 \\
\hline 6/15/07 3:00 & 126.279 & 123.88 & 120.536 & 101.335 & 105.473 & 97.047 & 111.41 & 108.203 & 79.858 & 74.69 \\
\hline 6/15/07 3:30 & 126.262 & 123.868 & 120.52 & 101.32 & 105.458 & 97.03 & 111.396 & 108.187 & 79.84 & 74.674 \\
\hline 6/15/07 4:00 & 126.248 & 123.868 & 120.504 & 101.308 & 105.444 & 97.016 & 111.383 & 108.172 & 79.823 & 74.659 \\
\hline 6/15/07 4:30 & 126.236 & 123.859 & 120.495 & 101.299 & 105.435 & 97.009 & 111.372 & 108.161 & 79.807 & 74.647 \\
\hline 6/15/07 5:00 & 126.234 & 123.857 & 120.493 & 101.296 & 105.435 & 97.004 & 111.361 & 108.159 & 79.794 & 74.643 \\
\hline 6/15/07 5:30 & 126.227 & 123.859 & 120.483 & 101.289 & 105.425 & 96.997 & 111.351 & 108.15 & 79.781 & 74.635 \\
\hline 6/15/07 6:00 & 126.222 & 125.694 & 120.481 & 101.289 & 105.425 & 96.997 & 111.34 & 108.147 & 79.77 & 74.626 \\
\hline 6/15/07 6:30 & 126.222 & 126.089 & 120.481 & 101.29 & 105.425 & 96.997 & 111.333 & 108.147 & 79.761 & 74.624 \\
\hline 6/15/07 7:00 & 128.066 & 126.261 & 121.605 & 101.823 & 105.753 & 97.469 & 111.776 & 109.387 & 79.759 & 74.635 \\
\hline 6/15/07 7:30 & 128.508 & 126.365 & 122.032 & 102.18 & 106.128 & 97.853 & 112.114 & 109.888 & 79.788 & 74.773 \\
\hline 6/15/07 8:00 & 128.688 & 126.433 & 122.213 & 102.345 & 106.301 & 98.023 & 112.277 & 110.076 & 79.814 & 74.905 \\
\hline 6/15/07 8:30 & 128.797 & 124.451 & 122.319 & 102.45 & 106.408 & 98.129 & 112.371 & 110.184 & 79.838 & 75.005 \\
\hline 6/15/07 9:00 & 128.87 & 124.206 & 122.393 & 102.521 & 106.482 & 98.204 & 112.433 & 110.264 & 79.864 & 75.083 \\
\hline 6/15/07 9:30 & 126.849 & 124.086 & 121.093 & 101.842 & 106.004 & 97.572 & 111.844 & 108.794 & 79.857 & 75.075 \\
\hline $6 / 15 / 07 \quad 10: 00$ & 126.586 & 124.007 & 120.837 & 101.616 & 105.77 & 97.337 & 111.628 & 108.522 & 79.838 & 74.965 \\
\hline $6 / 15 / 07 \quad 10: 30$ & 126.461 & 123.959 & 120.71 & 101.504 & 105.65 & 97.219 & 111.525 & 108.391 & 79.822 & 74.866 \\
\hline $6 / 15 / 0711: 00$ & 126.378 & 123.923 & 120.633 & 101.43 & 105.574 & 97.146 & 111.46 & 108.309 & 79.805 & 74.796 \\
\hline $6 / 15 / 0711: 30$ & 126.329 & 123.893 & 120.582 & 101.385 & 105.526 & 97.099 & 111.415 & 108.258 & 79.792 & 74.746 \\
\hline $6 / 15 / 07 \quad 12: 00$ & 126.288 & 123.866 & 120.546 & 101.351 & 105.488 & 97.061 & 111.383 & 108.218 & 79.776 & 74.707 \\
\hline $6 / 15 / 07 \quad 12: 30$ & 126.262 & 123.846 & 120.518 & 101.325 & 105.463 & 97.035 & 111.36 & 108.187 & 79.764 & 74.678 \\
\hline $6 / 15 / 0713: 00$ & 126.234 & 123.825 & 120.492 & 101.297 & 105.435 & 97.009 & 111.342 & 108.161 & 79.748 & 74.643 \\
\hline 6/15/07 13:30 & 126.213 & 123.812 & 120.471 & 101.278 & 105.416 & 96.988 & 111.327 & 108.141 & 79.733 & 74.624 \\
\hline $6 / 15 / 07 \quad 14: 00$ & 126.194 & 123.8 & 120.455 & 101.261 & 105.402 & 96.974 & 111.314 & 108.123 & 79.72 & 74.606 \\
\hline $6 / 15 / 0714: 30$ & 126.178 & 123.787 & 120.437 & 101.246 & 105.383 & 96.957 & 111.302 & 108.105 & 79.707 & 74.585 \\
\hline $6 / 15 / 07$ 15:00 & 126.165 & 123.778 & 120.425 & 101.235 & 105.376 & 96.948 & 111.293 & 108.094 & 79.693 & 74.573 \\
\hline $6 / 15 / 07$ 15:30 & 126.154 & 123.766 & 120.414 & 101.225 & 105.366 & 96.934 & 111.286 & 108.081 & 79.68 & 74.568 \\
\hline $6 / 15 / 0716: 00$ & 126.139 & 123.757 & 120.4 & 101.213 & 105.349 & 96.922 & 111.274 & 108.068 & 79.665 & 74.554 \\
\hline $6 / 15 / 0716: 30$ & 126.13 & 123.746 & 120.393 & 101.206 & 105.343 & 96.917 & 111.271 & 108.059 & 79.654 & 74.546 \\
\hline $6 / 15 / 07$ 17:00 & 126.123 & 123.735 & 120.386 & 101.197 & 105.336 & 96.91 & 111.261 & 108.052 & 79.641 & 74.533 \\
\hline
\end{tabular}


TABLE S1.1 (Cont.)

Depth from Top of Casing (ft)

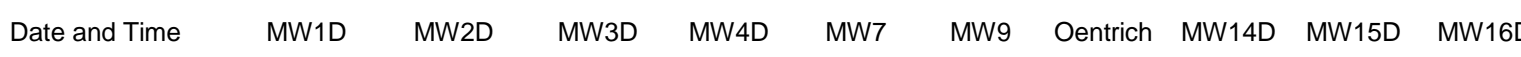

\begin{tabular}{|c|c|c|c|c|c|c|c|c|c|c|}
\hline 6/15/07 17:30 & 126.114 & 123.728 & 120.374 & 101.189 & 105.326 & 96.898 & 111.256 & 108.039 & 79.626 & 74.523 \\
\hline $6 / 15 / 0718: 00$ & 126.102 & 123.728 & 120.363 & 101.175 & 105.315 & 96.889 & 111.248 & 108.028 & 79.613 & 74.521 \\
\hline 6/15/07 18:30 & 126.095 & 123.728 & 120.356 & 101.17 & 105.309 & 96.879 & 111.242 & 108.021 & 79.602 & 74.513 \\
\hline 6/15/07 19:00 & 126.095 & 123.728 & 120.358 & 101.172 & 105.313 & 96.879 & 111.237 & 108.023 & 79.597 & 74.515 \\
\hline 6/15/07 19:30 & 126.095 & 123.728 & 120.356 & 101.172 & 105.311 & 96.882 & 111.231 & 108.021 & 79.587 & 74.506 \\
\hline 6/15/07 20:00 & 126.095 & 123.725 & 120.356 & 101.173 & 105.313 & 96.882 & 111.225 & 108.021 & 79.578 & 74.511 \\
\hline $6 / 15 / 0720: 30$ & 126.095 & 123.728 & 120.356 & 101.173 & 105.311 & 96.879 & 111.222 & 108.021 & 79.571 & 74.508 \\
\hline $6 / 15 / 0721: 00$ & 126.095 & 123.732 & 120.356 & 101.172 & 105.313 & 96.884 & 111.216 & 108.021 & 79.564 & 74.504 \\
\hline 6/15/07 21:30 & 126.095 & 123.735 & 120.356 & 101.175 & 105.315 & 96.882 & 111.209 & 108.021 & 79.556 & 74.508 \\
\hline 6/15/07 22:00 & 126.097 & 123.737 & 120.36 & 101.178 & 105.319 & 96.886 & 111.205 & 108.026 & 79.552 & 74.506 \\
\hline 6/15/07 22:30 & 126.102 & 123.739 & 120.363 & 101.182 & 105.322 & 96.891 & 111.199 & 108.03 & 79.549 & 74.511 \\
\hline 6/15/07 23:00 & 126.104 & 123.741 & 120.367 & 101.185 & 105.324 & 96.894 & 111.194 & 108.032 & 79.545 & 74.517 \\
\hline 6/15/07 23:30 & 126.104 & 123.739 & 120.367 & 101.187 & 105.328 & 96.896 & 111.194 & 108.035 & 79.541 & 74.517 \\
\hline 6/16/07 0:00 & 126.106 & 123.746 & 120.367 & 101.189 & 105.326 & 96.896 & 111.188 & 108.035 & 79.538 & 74.517 \\
\hline 6/16/07 0:30 & 126.107 & 123.739 & 120.367 & 101.189 & 105.328 & 96.896 & 111.186 & 108.035 & 79.534 & 74.519 \\
\hline 6/16/07 1:00 & 126.111 & 123.741 & 120.375 & 101.194 & 105.334 & 96.9 & 111.182 & 108.041 & 79.53 & 74.523 \\
\hline 6/16/07 1:30 & 126.104 & 123.735 & 120.37 & 101.189 & 105.328 & 96.896 & 111.179 & 108.035 & 79.528 & 74.519 \\
\hline 6/16/07 2:00 & 126.107 & 123.732 & 120.367 & 101.192 & 105.33 & 96.898 & 111.175 & 108.037 & 79.525 & 74.517 \\
\hline 6/16/07 2:30 & 126.104 & 123.73 & 120.367 & 101.185 & 105.328 & 96.896 & 111.173 & 108.032 & 79.521 & 74.521 \\
\hline 6/16/07 3:00 & 126.1 & 123.73 & 120.36 & 101.182 & 105.321 & 96.889 & 111.169 & 108.028 & 79.514 & 74.511 \\
\hline 6/16/07 3:30 & 126.095 & 123.723 & 120.36 & 101.18 & 105.321 & 96.886 & 111.164 & 108.026 & 79.51 & 74.513 \\
\hline 6/16/07 4:00 & 126.097 & 123.721 & 120.36 & 101.182 & 105.324 & 96.891 & 111.162 & 108.028 & 79.508 & 74.508 \\
\hline 6/16/07 4:30 & 126.092 & 123.721 & 120.356 & 101.177 & 105.319 & 96.886 & 111.158 & 108.021 & 79.503 & 74.506 \\
\hline 6/16/07 5:00 & 126.085 & 123.725 & 120.353 & 101.175 & 105.317 & 96.882 & 111.152 & 108.019 & 79.499 & 74.504 \\
\hline $6 / 16 / 075: 30$ & 126.085 & 123.735 & 120.344 & 101.175 & 105.313 & 96.884 & 111.15 & 108.017 & 79.493 & 74.504 \\
\hline 6/16/07 6:00 & 126.092 & 123.725 & 120.358 & 101.182 & 105.322 & 96.891 & 111.15 & 108.024 & 79.493 & 74.513 \\
\hline 6/16/07 6:30 & 126.099 & 123.737 & 120.363 & 101.187 & 105.328 & 96.896 & 111.15 & 108.03 & 79.493 & 74.515 \\
\hline 6/16/07 7:00 & 126.095 & 123.732 & 120.358 & 101.182 & 105.321 & 96.891 & 111.148 & 108.024 & 79.488 & 74.515 \\
\hline 6/16/07 7:30 & 126.1 & 125.882 & 120.363 & 101.187 & 105.33 & 96.896 & 111.148 & 108.03 & 79.486 & 74.519 \\
\hline 6/16/07 8:00 & 126.102 & 126.136 & 120.365 & 101.187 & 105.33 & 96.898 & 111.169 & 108.032 & 79.486 & 74.515 \\
\hline 6/16/07 8:30 & 128.288 & 126.265 & 121.813 & 101.974 & 105.915 & 97.636 & 111.847 & 109.66 & 79.514 & 74.601 \\
\hline 6/16/07 9:00 & 128.558 & 126.345 & 122.076 & 102.216 & 106.166 & 97.888 & 112.069 & 109.939 & 79.554 & 74.767 \\
\hline 6/16/07 9:30 & 128.695 & 126.399 & 122.213 & 102.343 & 106.299 & 98.023 & 112.206 & 110.08 & 79.59 & 74.883 \\
\hline $6 / 16 / 07$ 10:00 & 128.783 & 126.44 & 122.298 & 102.424 & 106.383 & 98.105 & 112.281 & 110.171 & 79.625 & 74.969 \\
\hline $6 / 16 / 07$ 10:30 & 128.834 & 126.465 & 122.354 & 102.476 & 106.44 & 98.157 & 112.344 & 110.226 & 79.652 & 75.027 \\
\hline 6/16/07 11:00 & 128.875 & 126.488 & 122.398 & 102.517 & 106.484 & 98.202 & 112.371 & 110.271 & 79.676 & 75.069 \\
\hline $6 / 16 / 0711: 30$ & 128.903 & 126.504 & 122.428 & 102.547 & 106.516 & 98.232 & 112.396 & 110.302 & 79.704 & 75.106 \\
\hline 6/16/07 12:00 & 128.927 & 126.506 & 122.449 & 102.569 & 106.535 & 98.256 & 112.433 & 110.322 & 79.726 & 75.133 \\
\hline 6/16/07 12:30 & 128.939 & 125.687 & 122.465 & 102.584 & 106.552 & 98.27 & 112.434 & 110.34 & 79.75 & 75.162 \\
\hline $6 / 16 / 07$ 13:00 & 128.943 & 124.41 & 122.472 & 102.59 & 106.558 & 98.277 & 112.451 & 110.346 & 79.766 & 75.168 \\
\hline $6 / 16 / 07$ 13:30 & 128.951 & 124.177 & 122.479 & 102.593 & 106.566 & 98.284 & 112.467 & 110.353 & 79.785 & 75.176 \\
\hline $6 / 16 / 07$ 14:00 & 126.804 & 124.047 & 121.057 & 101.814 & 105.985 & 97.549 & 111.756 & 108.757 & 79.772 & 75.091 \\
\hline 6/16/07 14:30 & 126.556 & 123.957 & 120.814 & 101.595 & 105.757 & 97.32 & 111.552 & 108.5 & 79.75 & 74.951 \\
\hline 6/16/07 15:00 & 126.421 & 123.889 & 120.679 & 101.475 & 105.627 & 97.195 & 111.449 & 108.36 & 79.731 & 74.847 \\
\hline $6 / 16 / 07$ 15:30 & 126.329 & 123.841 & 120.589 & 101.39 & 105.539 & 97.106 & 111.39 & 108.267 & 79.709 & 74.771 \\
\hline 6/16/07 16:00 & 126.26 & 123.805 & 120.523 & 101.33 & 105.475 & 97.044 & 111.35 & 108.198 & 79.693 & 74.715 \\
\hline $6 / 16 / 07$ 16:30 & 126.213 & 123.766 & 120.476 & 101.284 & 105.429 & 96.997 & 111.292 & 108.147 & 79.674 & 74.67 \\
\hline $6 / 16 / 07$ 17:00 & 126.173 & 123.741 & 120.437 & 101.247 & 105.389 & 96.96 & 111.252 & 108.108 & 79.656 & 74.624 \\
\hline $6 / 16 / 07$ 17:30 & 126.135 & 123.716 & 120.4 & 101.211 & 105.355 & 96.922 & 111.24 & 108.068 & 79.637 & 74.589 \\
\hline $6 / 16 / 07$ 18:00 & 126.109 & 123.696 & 120.372 & 101.185 & 105.33 & 96.896 & 111.222 & 108.043 & 79.621 & 74.564 \\
\hline 6/16/07 18:30 & 126.085 & 123.68 & 120.349 & 101.165 & 105.309 & 96.875 & 111.227 & 108.019 & 79.602 & 74.535 \\
\hline $6 / 16 / 07$ 19:00 & 126.066 & 123.671 & 120.333 & 101.147 & 105.292 & 96.856 & 111.211 & 108.001 & 79.587 & 74.517 \\
\hline 6/16/07 19:30 & 126.045 & 123.664 & 120.312 & 101.128 & 105.271 & 96.839 & 111.176 & 107.979 & 79.573 & 74.506 \\
\hline 6/16/07 20:00 & 126.033 & 123.662 & 120.3 & 101.122 & 105.263 & 96.83 & 111.18 & 107.968 & 79.56 & 74.492 \\
\hline 6/16/07 20:30 & 126.031 & 123.667 & 120.298 & 101.118 & 105.26 & 96.825 & 111.16 & 107.964 & 79.549 & 74.492 \\
\hline $6 / 16 / 0721: 00$ & 126.028 & 123.673 & 120.296 & 101.116 & 105.262 & 96.825 & 111.156 & 107.961 & 79.538 & 74.484 \\
\hline 6/16/07 21:30 & 126.033 & 123.676 & 120.298 & 101.12 & 105.262 & 96.827 & 111.159 & 107.964 & 79.532 & 74.484 \\
\hline 6/16/07 22:00 & 126.04 & 123.664 & 120.305 & 101.13 & 105.273 & 96.837 & 111.128 & 107.975 & 79.527 & 74.49 \\
\hline $6 / 16 / 07$ 22:30 & 126.043 & 123.657 & 120.309 & 101.128 & 105.277 & 96.839 & 111.128 & 107.977 & 79.521 & 74.492 \\
\hline 6/16/07 23:00 & 126.031 & 123.653 & 120.298 & 101.122 & 105.263 & 96.83 & 111.16 & 107.964 & 79.512 & 74.483 \\
\hline 6/16/07 23:30 & 126.024 & 123.642 & 120.291 & 101.113 & 105.258 & 96.825 & 111.135 & 107.957 & 79.505 & 74.475 \\
\hline $6 / 17 / 07$ 0:00 & 126.017 & 123.626 & 120.284 & 101.111 & 105.252 & 96.816 & 111.105 & 107.95 & 79.495 & 74.471 \\
\hline 6/17/07 0:30 & 126.009 & 123.626 & 120.277 & 101.101 & 105.246 & 96.811 & 111.093 & 107.944 & 79.488 & 74.465 \\
\hline 6/17/07 1:00 & 125.991 & 123.61 & 120.259 & 101.084 & 105.225 & 96.792 & 111.079 & 107.926 & 79.477 & 74.444 \\
\hline 6/17/07 1:30 & 125.993 & 123.603 & 120.259 & 101.085 & 105.227 & 96.792 & 111.073 & 107.928 & 79.469 & 74.446 \\
\hline $6 / 17 / 072: 00$ & 125.976 & 123.599 & 120.243 & 101.068 & 105.212 & 96.778 & 111.065 & 107.911 & 79.458 & 74.434 \\
\hline
\end{tabular}


TABLE S1.1 (Cont.)

Depth from Top of Casing (ft)

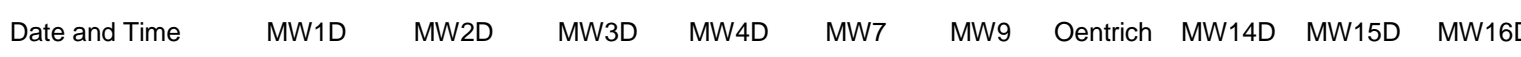

\begin{tabular}{|c|c|c|c|c|c|c|c|c|c|c|}
\hline 6/17/07 2:30 & 125.967 & 123.589 & 120.238 & 101.063 & 105.206 & 96.773 & 111.06 & 107.904 & 79.451 & 74.432 \\
\hline 6/17/07 3:00 & 125.967 & 123.576 & 120.233 & 101.058 & 105.202 & 96.768 & 111.052 & 107.9 & 79.444 & 74.427 \\
\hline 6/17/07 3:30 & 125.953 & 123.565 & 120.222 & 101.047 & 105.193 & 96.759 & 111.048 & 107.889 & 79.434 & 74.415 \\
\hline 6/17/07 4:00 & 125.941 & 123.558 & 120.212 & 101.037 & 105.183 & 96.747 & 111.041 & 107.88 & 79.424 & 74.405 \\
\hline 6/17/07 4:30 & 125.931 & 123.544 & 120.201 & 101.025 & 105.17 & 96.733 & 111.033 & 107.869 & 79.414 & 74.394 \\
\hline 6/17/07 5:00 & 125.922 & 123.54 & 120.189 & 101.018 & 105.162 & 96.724 & 111.025 & 107.858 & 79.405 & 74.386 \\
\hline 6/17/07 5:30 & 125.915 & 123.537 & 120.178 & 101.004 & 105.151 & 96.712 & 111.018 & 107.847 & 79.396 & 74.374 \\
\hline 6/17/07 6:00 & 125.91 & 123.535 & 120.173 & 101.001 & 105.147 & 96.707 & 111.012 & 107.84 & 79.387 & 74.372 \\
\hline 6/17/07 6:30 & 125.908 & 123.524 & 120.173 & 100.999 & 105.145 & 96.707 & 111.008 & 107.84 & 79.381 & 74.37 \\
\hline 6/17/07 7:00 & 125.906 & 123.517 & 120.168 & 100.994 & 105.142 & 96.705 & 111.004 & 107.838 & 79.374 & 74.363 \\
\hline 6/17/07 7:30 & 125.901 & 125.578 & 120.159 & 100.987 & 105.132 & 96.695 & 111.029 & 107.826 & 79.364 & 74.357 \\
\hline 6/17/07 8:00 & 125.896 & 125.857 & 120.155 & 100.98 & 105.126 & 96.691 & 111.045 & 107.82 & 79.357 & 74.349 \\
\hline 6/17/07 8:30 & 128.012 & 125.991 & 121.662 & 101.759 & 105.686 & 97.426 & 111.825 & 109.339 & 79.379 & 74.415 \\
\hline 6/17/07 9:00 & 128.307 & 126.068 & 121.951 & 102.03 & 105.955 & 97.688 & 111.968 & 109.655 & 79.41 & 74.564 \\
\hline 6/17/07 9:30 & 128.451 & 126.125 & 122.093 & 102.147 & 106.09 & 97.822 & 112.125 & 109.803 & 79.437 & 74.68 \\
\hline $6 / 17 / 07$ 10:00 & 128.537 & 126.161 & 122.178 & 102.226 & 106.174 & 97.905 & 112.171 & 109.892 & 79.462 & 74.76 \\
\hline $6 / 17 / 07$ 10:30 & 128.591 & 126.188 & 122.234 & 102.279 & 106.231 & 97.959 & 112.218 & 109.95 & 79.482 & 74.822 \\
\hline $6 / 17 / 0711: 00$ & 128.626 & 126.204 & 122.273 & 102.314 & 106.265 & 97.997 & 112.259 & 109.988 & 79.501 & 74.857 \\
\hline 6/17/07 11:30 & 128.653 & 126.222 & 122.298 & 102.338 & 106.293 & 98.02 & 112.284 & 110.016 & 79.521 & 74.895 \\
\hline 6/17/07 12:00 & 128.669 & 126.229 & 122.312 & 102.357 & 106.311 & 98.037 & 112.297 & 110.036 & 79.538 & 74.917 \\
\hline $6 / 17 / 07$ 12:30 & 128.688 & 126.222 & 122.333 & 102.374 & 106.328 & 98.058 & 112.31 & 110.051 & 79.555 & 74.938 \\
\hline 6/17/07 13:00 & 128.7 & 126.218 & 122.345 & 102.385 & 106.341 & 98.067 & 112.323 & 110.065 & 79.573 & 74.955 \\
\hline 6/17/07 13:30 & 128.695 & 125.95 & 122.342 & 102.381 & 106.337 & 98.065 & 112.328 & 110.061 & 79.586 & 74.95 \\
\hline $6 / 17 / 0714: 00$ & 128.688 & 124.131 & 122.338 & 102.378 & 106.332 & 98.06 & 112.332 & 110.056 & 79.591 & 74.946 \\
\hline $6 / 17 / 07$ 14:30 & 128.679 & 123.875 & 122.328 & 102.364 & 106.322 & 98.051 & 112.319 & 110.049 & 79.602 & 74.946 \\
\hline $6 / 17 / 07$ 15:00 & 126.53 & 123.73 & 120.781 & 101.533 & 105.711 & 97.266 & 111.568 & 108.484 & 79.578 & 74.859 \\
\hline $6 / 17 / 07$ 15:30 & 126.26 & 123.628 & 120.518 & 101.299 & 105.463 & 97.023 & 111.349 & 108.207 & 79.549 & 74.709 \\
\hline $6 / 17 / 07$ 16:00 & 126.109 & 123.555 & 120.365 & 101.163 & 105.322 & 96.882 & 111.229 & 108.05 & 79.517 & 74.589 \\
\hline $6 / 17 / 07$ 16:30 & 126 & 123.501 & 120.263 & 101.065 & 105.22 & 96.785 & 111.148 & 107.944 & 79.49 & 74.5 \\
\hline 6/17/07 17:00 & 125.929 & 123.46 & 120.192 & 101.001 & 105.151 & 96.714 & 111.092 & 107.871 & 79.464 & 74.432 \\
\hline $6 / 17 / 07$ 17:30 & 125.87 & 123.474 & 120.136 & 100.944 & 105.098 & 96.66 & 111.051 & 107.811 & 79.442 & 74.38 \\
\hline 6/17/07 18:00 & 125.825 & 123.406 & 120.09 & 100.903 & 105.058 & 96.618 & 111.017 & 107.765 & 79.412 & 74.332 \\
\hline 6/17/07 18:30 & 125.844 & 123.385 & 120.129 & 100.992 & 105.098 & 96.693 & 111.927 & 107.769 & 79.396 & 74.299 \\
\hline 6/17/07 19:00 & 125.775 & 123.442 & 120.05 & 100.856 & 105.016 & 96.594 & 111.034 & 107.714 & 79.375 & 74.272 \\
\hline 6/17/07 19:30 & 125.747 & 123.456 & 120.016 & 100.834 & 104.991 & 96.556 & 111.418 & 107.687 & 79.353 & 74.254 \\
\hline 6/17/07 20:00 & 125.811 & 123.447 & 120.097 & 100.967 & 105.071 & 96.667 & 111.899 & 107.733 & 79.353 & 74.245 \\
\hline $6 / 17 / 0720: 30$ & 125.825 & 123.372 & 120.111 & 100.984 & 105.09 & 96.684 & 111.912 & 107.749 & 79.353 & 74.252 \\
\hline 6/17/07 21:00 & 125.823 & 123.363 & 120.108 & 100.973 & 105.088 & 96.684 & 111.932 & 107.749 & 79.346 & 74.245 \\
\hline 6/17/07 21:30 & 125.738 & 123.356 & 120.004 & 100.836 & 104.98 & 96.54 & 110.951 & 107.678 & 79.324 & 74.237 \\
\hline 6/17/07 22:00 & 125.724 & 123.347 & 119.991 & 100.82 & 104.97 & 96.526 & 110.927 & 107.665 & 79.313 & 74.233 \\
\hline 6/17/07 22:30 & 125.719 & 123.333 & 119.99 & 100.806 & 104.974 & 96.537 & 111.383 & 107.661 & 79.298 & 74.223 \\
\hline 6/17/07 23:00 & 125.71 & 123.322 & 119.976 & 100.818 & 104.953 & 96.512 & 110.909 & 107.649 & 79.283 & 74.208 \\
\hline 6/17/07 23:30 & 125.695 & 123.306 & 119.965 & 100.792 & 104.944 & 96.502 & 110.932 & 107.638 & 79.272 & 74.196 \\
\hline 6/18/07 0:00 & 125.684 & 123.29 & 119.953 & 100.782 & 104.93 & 96.493 & 110.958 & 107.625 & 79.259 & 74.179 \\
\hline 6/18/07 0:30 & 125.671 & 123.283 & 119.939 & 100.767 & 104.919 & 96.476 & 110.887 & 107.616 & 79.245 & 74.169 \\
\hline 6/18/07 1:00 & 125.657 & 123.27 & 119.926 & 100.755 & 104.905 & 96.464 & & & 79.232 & 74.148 \\
\hline 6/18/07 1:30 & 125.645 & 123.252 & 119.914 & 100.744 & 104.894 & 96.455 & 110.881 & 107.587 & 79.221 & 74.148 \\
\hline 6/18/07 2:00 & 125.629 & 123.245 & 119.902 & 100.732 & 104.885 & 96.441 & 110.858 & 107.572 & 79.204 & 74.132 \\
\hline 6/18/07 2:30 & 125.617 & 123.261 & 119.884 & 100.715 & 104.865 & 96.427 & 110.858 & 107.557 & 79.193 & 74.119 \\
\hline 6/18/07 3:00 & 125.61 & 123.258 & 119.879 & 100.71 & 104.862 & 96.422 & 110.834 & 107.552 & 79.18 & 74.113 \\
\hline 6/18/07 3:30 & 125.619 & 123.265 & 119.891 & 100.724 & 104.875 & 96.436 & 110.826 & 107.563 & 79.177 & 74.121 \\
\hline 6/18/07 4:00 & 125.617 & 123.258 & 119.889 & 100.724 & 104.873 & 96.431 & 110.818 & 107.559 & 79.167 & 74.115 \\
\hline 6/18/07 4:30 & 125.629 & 123.261 & 119.9 & 100.732 & 104.888 & 96.446 & 110.815 & 107.572 & 79.163 & 74.126 \\
\hline 6/18/07 5:00 & 125.617 & 123.254 & 119.889 & 100.722 & 104.875 & 96.434 & 110.808 & 107.561 & 79.152 & 74.111 \\
\hline 6/18/07 5:30 & 125.622 & 123.256 & 119.896 & 100.73 & 104.883 & 96.441 & 110.811 & 107.566 & 79.147 & 74.109 \\
\hline 6/18/07 6:00 & 125.619 & 125.358 & 119.889 & 100.725 & 104.879 & 96.436 & 110.804 & 107.563 & 79.14 & 74.115 \\
\hline 6/18/07 6:30 & 125.619 & 125.637 & 119.891 & 100.725 & 104.879 & 96.438 & 110.798 & 107.563 & 79.132 & 74.115 \\
\hline 6/18/07 7:00 & 127.792 & 125.778 & 121.445 & 101.551 & 105.479 & 97.205 & 111.551 & 109.135 & 79.154 & 74.192 \\
\hline 6/18/07 7:30 & 128.09 & 125.853 & 121.739 & 101.818 & 105.76 & 97.487 & 111.802 & 109.443 & 79.197 & 74.37 \\
\hline 6/18/07 8:00 & 128.241 & 125.9 & 121.887 & 101.952 & 105.905 & 97.629 & 111.94 & 109.6 & 79.23 & 74.498 \\
\hline 6/18/07 8:30 & 128.317 & 125.948 & 121.965 & 102.021 & 105.977 & 97.704 & 112.034 & 109.68 & 79.254 & 74.57 \\
\hline 6/18/07 9:00 & 128.369 & 125.975 & 122.014 & 102.071 & 106.027 & 97.751 & 112.09 & 109.735 & 79.274 & 74.624 \\
\hline 6/18/07 9:30 & 128.419 & 126 & 122.067 & 102.116 & 106.08 & 97.808 & 112.164 & 109.784 & 79.3 & 74.665 \\
\hline 6/18/07 10:00 & 128.442 & 126.039 & 122.09 & 102.14 & 106.103 & 97.827 & 112.197 & 109.81 & 79.322 & 74.7 \\
\hline 6/18/07 10:30 & 128.468 & 126.068 & 122.118 & 102.173 & 106.13 & 97.85 & 112.222 & 109.837 & 79.344 & 74.723 \\
\hline $6 / 18 / 0711: 00$ & 128.506 & 124.408 & 122.155 & 102.211 & 106.166 & 97.888 & 112.252 & 109.874 & 79.368 & 74.762 \\
\hline
\end{tabular}


TABLE S1.1 (Cont.)

Depth from Top of Casing (ft)

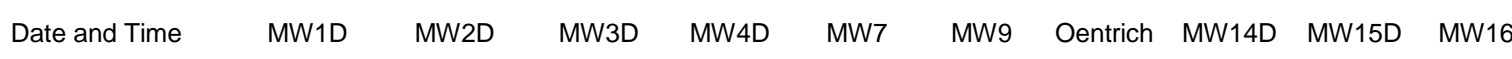

\begin{tabular}{|c|c|c|c|c|c|c|c|c|c|c|}
\hline 6/18/07 11:30 & 128.542 & 123.868 & 122.19 & 102.24 & 106.202 & 97.924 & 112.281 & 109.912 & 79.392 & 74.795 \\
\hline $6 / 18 / 07$ 12:00 & 127.031 & 123.676 & 121.14 & 101.75 & 105.964 & 97.544 & 112.033 & 108.943 & 79.381 & 74.742 \\
\hline 6/18/07 12:30 & 126.395 & 123.558 & 120.515 & 101.309 & 105.467 & 97.021 & 111.331 & 108.212 & 79.329 & 74.624 \\
\hline 6/18/07 13:00 & 126.184 & 123.483 & 120.319 & 101.12 & 105.286 & 96.844 & 111.179 & 108.008 & 79.313 & 74.529 \\
\hline 6/18/07 13:30 & 126.071 & 123.422 & 120.198 & 100.996 & 105.174 & 96.743 & 111.127 & 107.878 & 79.293 & 74.45 \\
\hline $6 / 18 / 07$ 14:00 & 125.984 & 123.397 & 120.116 & 100.929 & 105.092 & 96.644 & 111.025 & 107.798 & 79.276 & 74.419 \\
\hline 6/18/07 14:30 & 125.925 & 123.351 & 120.057 & 100.882 & 105.037 & 96.589 & 110.972 & 107.742 & 79.257 & 74.382 \\
\hline $6 / 18 / 07$ 15:00 & 125.882 & 123.333 & 120.03 & 100.861 & 105.008 & 96.568 & 110.911 & 107.702 & 79.241 & 74.343 \\
\hline 6/18/07 15:30 & 125.846 & 123.317 & 119.991 & 100.83 & 104.97 & 96.53 & 110.887 & 107.663 & 79.222 & 74.324 \\
\hline 6/18/07 16:00 & 125.823 & 123.315 & 119.965 & 100.82 & 104.947 & 96.5 & 110.888 & 107.641 & 79.211 & 74.305 \\
\hline 6/18/07 16:30 & 125.794 & 123.304 & 119.946 & 100.777 & 104.936 & 96.486 & 110.859 & 107.621 & 79.193 & 74.281 \\
\hline 6/18/07 17:00 & 125.78 & 123.299 & 119.947 & 100.775 & 104.936 & 96.488 & 110.849 & 107.623 & 79.186 & 74.293 \\
\hline 6/18/07 17:30 & 125.768 & 123.301 & 119.935 & 100.772 & 104.923 & 96.474 & 110.85 & 107.612 & 79.176 & 74.27 \\
\hline 6/18/07 18:00 & 125.761 & 123.297 & 119.938 & 100.761 & 104.926 & 96.488 & 110.846 & 107.605 & 79.167 & 74.254 \\
\hline $6 / 18 / 07$ 18:30 & 125.747 & 123.297 & 119.933 & 100.765 & 104.925 & 96.479 & 110.855 & 107.612 & 79.164 & 74.237 \\
\hline 6/18/07 19:00 & 125.74 & 123.299 & 119.926 & 100.765 & 104.919 & 96.474 & 110.848 & 107.605 & 79.156 & 74.223 \\
\hline 6/18/07 19:30 & 125.733 & 123.299 & 119.933 & 100.763 & 104.925 & 96.481 & 110.804 & 107.605 & 79.151 & 74.2 \\
\hline 6/18/07 20:00 & 125.723 & 123.306 & 119.931 & 100.792 & 104.925 & 96.476 & 110.808 & 107.607 & 79.145 & 74.177 \\
\hline 6/18/07 20:30 & 125.714 & 123.317 & 119.933 & 100.791 & 104.926 & 96.481 & 110.798 & 107.609 & 79.142 & 74.161 \\
\hline 6/18/07 21:00 & 125.705 & 123.326 & 119.937 & 100.799 & 104.932 & 96.488 & 110.821 & 107.614 & 79.138 & 74.14 \\
\hline $6 / 18 / 0721: 30$ & 125.7 & 123.34 & 119.954 & 100.786 & 104.947 & 96.507 & 110.807 & 107.625 & 79.14 & 74.121 \\
\hline 6/18/07 22:00 & 125.69 & 123.338 & 119.963 & 100.825 & 104.959 & 96.514 & 110.8 & 107.636 & 79.14 & 74.099 \\
\hline 6/18/07 22:30 & 125.683 & 123.351 & 119.97 & 100.827 & 104.968 & 96.521 & 110.814 & 107.643 & 79.138 & 74.082 \\
\hline 6/18/07 23:00 & 125.679 & 123.356 & 119.97 & 100.813 & 104.968 & 96.526 & 110.863 & 107.647 & 79.136 & 74.068 \\
\hline 6/18/07 23:30 & 125.674 & 123.358 & 119.984 & 100.829 & 104.984 & 96.54 & 110.887 & 107.66 & 79.138 & 74.051 \\
\hline 6/19/07 0:00 & 125.707 & 123.365 & 119.991 & 100.832 & 104.987 & 96.545 & 110.871 & 107.665 & 79.138 & 74.041 \\
\hline 6/19/07 0:30 & 125.702 & 123.365 & 119.991 & 100.836 & 104.989 & 96.547 & 110.856 & 107.667 & 79.138 & 74.028 \\
\hline 6/19/07 1:00 & 125.695 & 123.36 & 119.995 & 100.842 & 104.997 & 96.554 & 110.849 & 107.674 & 79.136 & 74.022 \\
\hline 6/19/07 1:30 & 125.726 & 123.36 & 120 & 100.842 & 105.001 & 96.554 & 110.841 & 107.676 & 79.136 & 74.014 \\
\hline 6/19/07 2:00 & 125.723 & 123.365 & 119.998 & 100.839 & 104.995 & 96.552 & 110.834 & 107.669 & 79.136 & 74.006 \\
\hline 6/19/07 2:30 & 125.726 & 123.372 & 119.997 & 100.841 & 104.995 & 96.552 & 110.824 & 107.671 & 79.132 & 73.997 \\
\hline 6/19/07 3:00 & 125.731 & 123.379 & 120 & 100.846 & 105.003 & 96.559 & 110.818 & 107.676 & 79.134 & 73.993 \\
\hline 6/19/07 3:30 & 125.736 & 123.385 & 120.005 & 100.855 & 105.008 & 96.563 & 110.809 & 107.683 & 79.134 & 73.991 \\
\hline 6/19/07 4:00 & 125.74 & 123.394 & 120.014 & 100.861 & 105.014 & 96.571 & 110.805 & 107.689 & 79.136 & 73.983 \\
\hline 6/19/07 4:30 & 125.75 & 123.399 & 120.016 & 100.867 & 105.02 & 96.578 & 110.801 & 107.696 & 79.136 & 73.975 \\
\hline 6/19/07 5:00 & 125.757 & 123.408 & 120.023 & 100.875 & 105.029 & 96.585 & 110.797 & 107.705 & 79.138 & 73.966 \\
\hline 6/19/07 5:30 & 125.761 & 123.404 & 120.032 & 100.88 & 105.035 & 96.589 & 110.793 & 107.709 & 79.138 & 73.96 \\
\hline 6/19/07 6:00 & 125.771 & 125.503 & 120.041 & 100.891 & 105.046 & 96.601 & 110.787 & 107.718 & 79.14 & 73.96 \\
\hline 6/19/07 6:30 & 125.768 & 125.76 & 120.039 & 100.886 & 105.039 & 96.597 & 110.785 & 107.716 & 79.138 & 73.962 \\
\hline 6/19/07 7:00 & 127.906 & 125.903 & 121.454 & 101.652 & 105.606 & 97.315 & 111.454 & 109.294 & 79.167 & 74.045 \\
\hline 6/19/07 7:30 & 128.18 & 125.993 & 121.725 & 101.895 & 105.857 & 97.57 & 111.675 & 109.584 & 79.206 & 74.196 \\
\hline 6/19/07 8:00 & 128.329 & 126.07 & 121.875 & 102.033 & 106.002 & 97.714 & 111.796 & 109.737 & 79.245 & 74.328 \\
\hline 6/19/07 8:30 & 128.423 & 124.211 & 121.968 & 102.128 & 106.101 & 97.813 & 111.873 & 109.837 & 79.278 & 74.44 \\
\hline 6/19/07 9:00 & 128.499 & 123.898 & 122.042 & 102.2 & 106.177 & 97.884 & 111.923 & 109.914 & 79.315 & 74.516 \\
\hline 6/19/07 9:30 & 126.624 & 123.755 & 120.878 & 101.628 & 105.817 & 97.374 & 111.414 & 108.606 & 79.331 & 74.566 \\
\hline $6 / 19 / 07$ 10:00 & 126.272 & 123.669 & 120.539 & 101.349 & 105.522 & 97.075 & & & 79.322 & 74.496 \\
\hline 6/19/07 10:30 & 126.126 & 123.619 & 120.391 & 101.216 & 105.381 & 96.938 & 111.027 & 108.083 & 79.315 & 74.463 \\
\hline 6/19/07 11:00 & 126.036 & 123.576 & 120.307 & 101.135 & 105.3 & 96.856 & 110.952 & 107.991 & 79.305 & 74.446 \\
\hline 6/19/07 11:30 & 125.986 & 123.551 & 120.254 & 101.092 & 105.252 & 96.809 & 110.902 & 107.939 & 79.3 & 74.432 \\
\hline 6/19/07 12:00 & 125.946 & 123.528 & 120.215 & 101.051 & 105.21 & 96.769 & 110.868 & 107.897 & 79.295 & 74.438 \\
\hline 6/19/07 12:30 & 125.917 & 123.517 & 120.187 & 101.029 & 105.183 & 96.743 & 110.842 & 107.867 & 79.287 & 74.44 \\
\hline 6/19/07 13:00 & 125.896 & 123.499 & 120.166 & 101.01 & 105.164 & 96.724 & 110.821 & 107.846 & 79.284 & 74.419 \\
\hline 6/19/07 13:30 & 125.882 & 123.487 & 120.155 & 100.998 & 105.153 & 96.71 & 110.806 & 107.831 & 79.28 & 74.398 \\
\hline 6/19/07 14:00 & 125.866 & 123.472 & 120.136 & 100.98 & 105.134 & 96.693 & 110.795 & 107.813 & 79.272 & 74.382 \\
\hline 6/19/07 14:30 & 125.854 & 123.46 & 120.127 & 100.97 & 105.126 & 96.684 & 110.785 & 107.802 & 79.269 & 74.365 \\
\hline $6 / 19 / 07$ 15:00 & 125.839 & 123.44 & 120.111 & 100.954 & 105.111 & 96.669 & 110.78 & 107.787 & 79.261 & 74.351 \\
\hline 6/19/07 15:30 & 125.825 & 123.426 & 120.097 & 100.944 & 105.1 & 96.658 & 110.774 & 107.776 & 79.254 & 74.336 \\
\hline 6/19/07 16:00 & 125.806 & 123.438 & 120.081 & 100.925 & 105.079 & 96.641 & 110.767 & 107.756 & 79.245 & 74.322 \\
\hline $6 / 19 / 07$ 16:30 & 125.792 & 123.428 & 120.067 & 100.91 & 105.065 & 96.625 & 110.761 & 107.743 & 79.237 & 74.314 \\
\hline 6/19/07 17:00 & 125.804 & 123.413 & 120.072 & 100.925 & 105.079 & 96.639 & 110.75 & 107.752 & 79.237 & 74.322 \\
\hline 6/19/07 17:30 & 125.794 & 123.41 & 120.069 & 100.915 & 105.069 & 96.629 & 110.744 & 107.745 & 79.232 & 74.316 \\
\hline 6/19/07 18:00 & 125.778 & 123.406 & 120.053 & 100.899 & 105.054 & 96.613 & 110.74 & 107.729 & 79.222 & 74.299 \\
\hline 6/19/07 18:30 & 125.771 & 123.392 & 120.046 & 100.898 & 105.05 & 96.608 & 110.728 & 107.718 & 79.213 & 74.287 \\
\hline 6/19/07 19:00 & 125.769 & 123.383 & 120.044 & 100.896 & 105.048 & 96.608 & 110.727 & 107.716 & 79.21 & 74.289 \\
\hline 6/19/07 19:30 & 125.759 & 123.369 & 120.032 & 100.88 & 105.039 & 96.594 & 110.723 & 107.707 & 79.202 & 74.276 \\
\hline 6/19/07 20:00 & 125.745 & 123.374 & 120.02 & 100.872 & 105.025 & 96.585 & 110.718 & 107.694 & 79.193 & 74.262 \\
\hline
\end{tabular}


TABLE S1.1 (Cont.)

Depth from Top of Casing (ft)

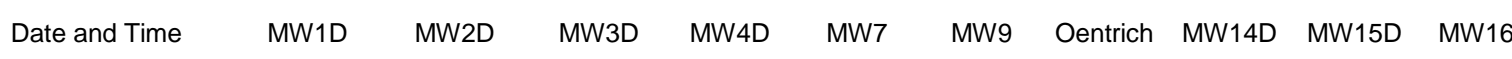

\begin{tabular}{|c|c|c|c|c|c|c|c|c|c|c|}
\hline 6/19/07 20:30 & 125.736 & 123.385 & 120.004 & 100.858 & 105.016 & 96.57 & 110.714 & 107.685 & 79.184 & 74.258 \\
\hline 6/19/07 21:00 & 125.736 & 123.383 & 120.011 & 100.865 & 105.02 & 96.575 & 110.708 & 107.687 & 79.182 & 74.256 \\
\hline 6/19/07 21:30 & 125.747 & 123.358 & 120.023 & 100.873 & 105.025 & 96.582 & 110.706 & 107.7 & 79.178 & 74.264 \\
\hline 6/19/07 22:00 & 125.747 & 123.36 & 120.023 & 100.873 & 105.027 & 96.585 & 110.7 & 107.7 & 79.184 & 74.264 \\
\hline 6/19/07 22:30 & 125.723 & 123.36 & 120 & 100.848 & 105.003 & 96.559 & 110.696 & 107.671 & 79.18 & 74.243 \\
\hline 6/19/07 23:00 & 125.724 & 123.363 & 120 & 100.853 & 105.006 & 96.563 & 110.689 & 107.674 & 79.18 & 74.243 \\
\hline 6/19/07 23:30 & 125.723 & 123.358 & 120 & 100.853 & 105.008 & 96.561 & 110.685 & 107.676 & 79.178 & 74.254 \\
\hline 6/20/07 0:00 & 125.728 & 123.363 & 120.004 & 100.856 & 105.012 & 96.566 & 110.68 & 107.676 & 79.178 & 74.258 \\
\hline 6/20/07 0:30 & 125.721 & 123.376 & 119.997 & 100.849 & 105.006 & 96.559 & 110.678 & 107.671 & 79.175 & 74.248 \\
\hline 6/20/07 1:00 & 125.726 & 123.39 & 120.002 & 100.856 & 105.01 & 96.563 & 110.672 & 107.676 & 79.175 & 74.252 \\
\hline 6/20/07 1:30 & 125.74 & 123.379 & 120.018 & 100.87 & 105.025 & 96.58 & 110.666 & 107.692 & 79.18 & 74.27 \\
\hline 6/20/07 2:00 & 125.752 & 123.356 & 120.03 & 100.882 & 105.037 & 96.589 & 110.662 & 107.703 & 79.182 & 74.274 \\
\hline 6/20/07 2:30 & 125.747 & 123.333 & 120.023 & 100.873 & 105.029 & 96.585 & 110.661 & 107.696 & 79.18 & 74.268 \\
\hline 6/20/07 3:00 & 125.719 & 123.335 & 119.997 & 100.849 & 105.004 & 96.556 & 110.659 & 107.672 & 79.169 & 74.248 \\
\hline 6/20/07 3:30 & 125.695 & 123.335 & 119.975 & 100.824 & 104.978 & 96.533 & 110.657 & 107.647 & 79.162 & 74.231 \\
\hline 6/20/07 4:00 & 125.698 & 123.326 & 119.974 & 100.827 & 104.984 & 96.537 & 110.651 & 107.647 & 79.158 & 74.231 \\
\hline 6/20/07 4:30 & 125.7 & 123.335 & 119.979 & 100.829 & 104.984 & 96.537 & 110.647 & 107.65 & 79.156 & 74.237 \\
\hline 6/20/07 5:00 & 125.697 & 123.331 & 119.97 & 100.822 & 104.974 & 96.53 & 110.642 & 107.641 & 79.152 & 74.225 \\
\hline 6/20/07 5:30 & 125.69 & 123.338 & 119.976 & 100.83 & 104.985 & 96.542 & 110.636 & 107.649 & 79.153 & 74.231 \\
\hline 6/20/07 6:00 & 125.688 & 125.347 & 119.974 & 100.827 & 104.982 & 96.537 & 110.632 & 107.647 & 79.149 & 74.233 \\
\hline 6/20/07 6:30 & 125.7 & 125.669 & 119.979 & 100.834 & 104.987 & 96.542 & 110.628 & 107.649 & 79.147 & 74.235 \\
\hline 6/20/07 7:00 & 127.773 & 125.823 & 121.443 & 101.578 & 105.507 & 97.228 & 111.276 & 109.115 & 79.171 & 74.285 \\
\hline 6/20/07 7:30 & 128.118 & 125.927 & 121.783 & 101.878 & 105.819 & 97.544 & 111.546 & 109.48 & 79.214 & 74.456 \\
\hline 6/20/07 8:00 & 128.284 & 125.989 & 121.947 & 102.024 & 105.977 & 97.702 & 111.693 & 109.653 & 79.247 & 74.593 \\
\hline 6/20/07 8:30 & 128.388 & 126.034 & 122.049 & 102.123 & 106.076 & 97.799 & 111.782 & 109.759 & 79.276 & 74.686 \\
\hline 6/20/07 9:00 & 128.452 & 126.07 & 122.113 & 102.185 & 106.139 & 97.865 & 111.844 & 109.823 & 79.302 & 74.75 \\
\hline 6/20/07 9:30 & 128.501 & 126.093 & 122.162 & 102.229 & 106.187 & 97.912 & 111.888 & 109.874 & 79.33 & 74.812 \\
\hline 6/20/07 10:00 & 128.539 & 126.093 & 122.201 & 102.266 & 106.225 & 97.95 & 111.92 & 109.914 & 79.355 & 74.849 \\
\hline 6/20/07 10:30 & 128.56 & 126.093 & 122.224 & 102.288 & 106.252 & 97.971 & 111.942 & 109.937 & 79.377 & 74.882 \\
\hline $6 / 20 / 0711: 00$ & 128.568 & 126.095 & 122.229 & 102.292 & 106.253 & 97.978 & 111.963 & 109.945 & 79.394 & 74.895 \\
\hline 6/20/07 11:30 & 128.568 & 124.025 & 122.229 & 102.293 & 106.255 & 97.98 & 111.978 & 109.945 & 79.409 & 74.901 \\
\hline 6/20/07 12:00 & 128.565 & 123.775 & 122.229 & 102.292 & 106.253 & 97.975 & 111.989 & 109.943 & 79.426 & 74.903 \\
\hline 6/20/07 12:30 & 126.421 & 123.639 & 120.687 & 101.459 & 105.642 & 97.198 & 111.24 & 108.389 & 79.407 & 74.818 \\
\hline 6/20/07 13:00 & 126.154 & 123.549 & 120.425 & 101.232 & 105.404 & 96.957 & 111.026 & 108.115 & 79.383 & 74.671 \\
\hline 6/20/07 13:30 & 126.012 & 123.481 & 120.284 & 101.106 & 105.273 & 96.825 & 110.91 & 107.968 & 79.361 & 74.56 \\
\hline $6 / 20 / 07$ 14:00 & 125.917 & 123.428 & 120.194 & 101.022 & 105.185 & 96.74 & 110.836 & 107.873 & 79.339 & 74.477 \\
\hline $6 / 20 / 07$ 14:30 & 125.851 & 123.388 & 120.127 & 100.96 & 105.119 & 96.677 & 110.785 & 107.805 & 79.32 & 74.415 \\
\hline 6/20/07 15:00 & 125.799 & 123.349 & 120.074 & 100.91 & 105.069 & 96.627 & 110.749 & 107.751 & 79.298 & 74.363 \\
\hline $6 / 20 / 07$ 15:30 & 125.754 & 123.317 & 120.032 & 100.868 & 105.027 & 96.585 & 110.723 & 107.707 & 79.28 & 74.318 \\
\hline 6/20/07 16:00 & 125.717 & 123.297 & 119.995 & 100.832 & 104.993 & 96.549 & 110.701 & 107.669 & 79.263 & 74.281 \\
\hline 6/20/07 16:30 & 125.686 & 123.283 & 119.965 & 100.806 & 104.962 & 96.521 & 110.684 & 107.636 & 79.245 & 74.248 \\
\hline $6 / 20 / 07$ 17:00 & 125.667 & 123.267 & 119.944 & 100.784 & 104.944 & 96.502 & 110.669 & 107.616 & 79.23 & 74.225 \\
\hline $6 / 20 / 07$ 17:30 & 125.648 & 123.247 & 119.928 & 100.772 & 104.926 & 96.486 & 110.656 & 107.601 & 79.215 & 74.208 \\
\hline 6/20/07 18:00 & 125.629 & 123.236 & 119.91 & 100.756 & 104.909 & 96.469 & 110.646 & 107.583 & 79.199 & 74.188 \\
\hline $6 / 20 / 07$ 18:30 & 125.61 & 123.224 & 119.893 & 100.737 & 104.896 & 96.453 & & & 79.182 & 74.171 \\
\hline 6/20/07 19:00 & 125.598 & 123.213 & 119.88 & 100.725 & 104.886 & 96.441 & 110.628 & 107.552 & 79.167 & 74.157 \\
\hline 6/20/07 19:30 & 125.589 & 123.209 & 119.87 & 100.717 & 104.873 & 96.431 & 110.622 & 107.541 & 79.156 & 74.15 \\
\hline 6/20/07 20:00 & 125.575 & 123.209 & 119.859 & 100.705 & 104.862 & 96.42 & & 107.528 & 79.142 & 74.14 \\
\hline 6/20/07 20:30 & 125.572 & 123.206 & 119.854 & 100.705 & 104.86 & 96.417 & 110.605 & 107.525 & 79.132 & 74.138 \\
\hline 6/20/07 21:00 & 125.575 & 123.206 & 119.859 & 100.705 & 104.864 & 96.422 & 110.605 & 107.526 & 79.12 & 74.132 \\
\hline $6 / 20 / 0721: 30$ & 125.57 & 123.204 & 119.852 & 100.703 & 104.862 & 96.417 & 110.592 & 107.523 & 79.11 & 74.13 \\
\hline 6/20/07 22:00 & 125.57 & 123.202 & 119.852 & 100.705 & 104.864 & 96.42 & 110.588 & 107.523 & 79.105 & 74.13 \\
\hline 6/20/07 22:30 & 125.567 & 123.193 & 119.85 & 100.701 & 104.861 & 96.417 & 110.584 & 107.521 & 79.096 & 74.124 \\
\hline $6 / 20 / 07$ 23:00 & 125.565 & 123.199 & 119.847 & 100.699 & 104.86 & 96.413 & 110.581 & 107.516 & 79.084 & 74.117 \\
\hline 6/20/07 23:30 & 125.554 & 123.199 & 119.838 & 100.691 & 104.846 & 96.403 & 110.577 & 107.508 & 79.077 & 74.117 \\
\hline 6/21/07 0:00 & 125.558 & 123.197 & 119.843 & 100.698 & 104.856 & 96.41 & 110.569 & 107.515 & 79.072 & 74.119 \\
\hline 6/21/07 0:30 & 125.563 & 123.193 & 119.845 & 100.699 & 104.856 & 96.413 & 110.562 & 107.516 & 79.064 & 74.121 \\
\hline 6/21/07 1:00 & 125.558 & 123.188 & 119.843 & 100.696 & 104.854 & 96.408 & 110.56 & 107.514 & 79.059 & 74.117 \\
\hline 6/21/07 1:30 & 125.556 & 123.193 & 119.838 & 100.691 & 104.852 & 96.405 & 110.556 & 107.51 & 79.051 & 74.117 \\
\hline 6/21/07 2:00 & 125.551 & 123.193 & 119.833 & 100.689 & 104.846 & 96.401 & 110.551 & 107.503 & 79.046 & 74.109 \\
\hline 6/21/07 2:30 & 125.553 & 123.188 & 119.838 & 100.693 & 104.852 & 96.403 & 110.547 & 107.508 & 79.04 & 74.109 \\
\hline 6/21/07 3:00 & 125.553 & 123.177 & 119.838 & 100.693 & 104.85 & 96.403 & 110.543 & 107.51 & 79.038 & 74.109 \\
\hline 6/21/07 3:30 & 125.551 & 123.165 & 119.831 & 100.689 & 104.846 & 96.401 & 110.539 & 107.505 & 79.029 & 74.103 \\
\hline $6 / 21 / 074: 00$ & 125.539 & 123.165 & 119.822 & 100.677 & 104.837 & 96.391 & 110.536 & 107.494 & 79.022 & 74.09 \\
\hline 6/21/07 4:30 & 125.528 & 123.159 & 119.81 & 100.665 & 104.822 & 96.377 & 110.536 & 107.481 & 79.011 & 74.086 \\
\hline 6/21/07 5:00 & 125.52 & 123.156 & 119.808 & 100.668 & 104.824 & 96.377 & 110.528 & 107.479 & 79.007 & 74.08 \\
\hline
\end{tabular}


TABLE S1.1 (Cont.)

Depth from Top of Casing (ft)

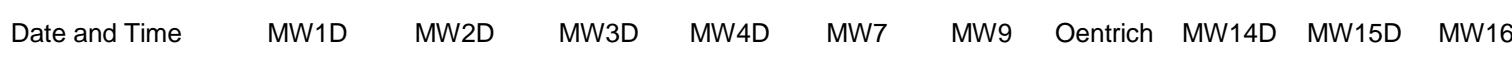

\begin{tabular}{|c|c|c|c|c|c|c|c|c|c|c|}
\hline 6/21/07 5:30 & 125.52 & 125.068 & 119.803 & 100.66 & 104.818 & 96.37 & 110.524 & 107.475 & 79 & 74.082 \\
\hline 6/21/07 6:00 & 125.516 & 125.429 & 119.801 & 100.658 & 104.818 & 96.37 & 110.519 & 107.47 & 78.994 & 74.074 \\
\hline 6/21/07 6:30 & 127.485 & 125.601 & 121.167 & 101.325 & 105.252 & 96.971 & 111.103 & 108.823 & 79.005 & 74.097 \\
\hline 6/21/07 7:00 & 127.88 & 125.696 & 121.549 & 101.651 & 105.598 & 97.32 & 111.422 & 109.243 & 79.042 & 74.268 \\
\hline 6/21/07 7:30 & 128.055 & 125.76 & 121.723 & 101.809 & 105.762 & 97.485 & 111.572 & 109.425 & 79.077 & 74.398 \\
\hline 6/21/07 8:00 & 128.156 & 125.803 & 121.825 & 101.906 & 105.861 & 97.584 & 111.664 & 109.534 & 79.108 & 74.492 \\
\hline 6/21/07 8:30 & 128.222 & 123.755 & 121.891 & 101.966 & 105.928 & 97.648 & 111.726 & 109.6 & 79.136 & 74.564 \\
\hline 6/21/07 9:00 & 128.265 & 123.526 & 121.933 & 102.009 & 105.972 & 97.69 & 111.769 & 109.649 & 79.162 & 74.611 \\
\hline 6/21/07 9:30 & 126.144 & 123.404 & 120.416 & 101.206 & 105.393 & 96.943 & 111.043 & 108.116 & 79.156 & 74.558 \\
\hline $6 / 21 / 07$ 10:00 & 125.899 & 123.326 & 120.176 & 100.998 & 105.174 & 96.724 & 110.846 & 107.864 & 79.14 & 74.432 \\
\hline $6 / 21 / 07 \quad 10: 30$ & 125.771 & 123.27 & 120.051 & 100.884 & 105.052 & 96.603 & 110.741 & 107.734 & 79.125 & 74.339 \\
\hline $6 / 21 / 0711: 00$ & 125.695 & 123.243 & 119.974 & 100.813 & 104.98 & 96.533 & 110.674 & 107.654 & 79.112 & 74.266 \\
\hline $6 / 21 / 0711: 30$ & 125.639 & 123.227 & 119.919 & 100.763 & 104.928 & 96.481 & 110.629 & 107.597 & 79.099 & 74.212 \\
\hline $6 / 21 / 07$ 12:00 & 125.603 & 123.188 & 119.887 & 100.734 & 104.898 & 96.45 & 110.595 & 107.561 & 79.088 & 74.188 \\
\hline $6 / 21 / 0712: 30$ & 125.591 & 123.159 & 119.877 & 100.743 & 104.885 & 96.436 & 110.56 & 107.55 & 79.083 & 74.161 \\
\hline 6/21/07 13:00 & 125.556 & 123.138 & 119.838 & 100.684 & 104.848 & 96.398 & 110.607 & 107.512 & 79.068 & 74.136 \\
\hline 6/21/07 13:30 & 125.523 & 123.116 & 119.808 & 100.656 & 104.818 & 96.372 & 110.559 & 107.479 & 79.055 & 74.109 \\
\hline $6 / 21 / 0714: 00$ & 125.501 & 123.095 & 119.785 & 100.637 & 104.795 & 96.349 & 110.537 & 107.457 & 79.042 & 74.082 \\
\hline $6 / 21 / 0714: 30$ & 125.48 & 123.072 & 119.766 & 100.615 & 104.774 & 96.33 & 110.523 & 107.437 & 79.029 & 74.064 \\
\hline $6 / 21 / 0715: 00$ & 125.457 & 123.066 & 119.741 & 100.593 & 104.755 & 96.306 & 110.514 & 107.413 & 79.014 & 74.047 \\
\hline 6/21/07 15:30 & 125.438 & 123.057 & 119.72 & 100.57 & 104.73 & 96.285 & 110.506 & 107.393 & 79.002 & 74.028 \\
\hline $6 / 21 / 07$ 16:00 & 125.426 & 123.045 & 119.71 & 100.563 & 104.725 & 96.278 & 110.496 & 107.384 & 78.992 & 74.02 \\
\hline 6/21/07 16:30 & 125.419 & 123.032 & 119.704 & 100.556 & 104.717 & 96.269 & 110.489 & 107.375 & 78.983 & 74.012 \\
\hline 6/21/07 17:00 & 125.407 & 123.023 & 119.692 & 100.544 & 104.706 & 96.259 & 110.481 & 107.364 & 78.972 & 73.999 \\
\hline $6 / 21 / 0717: 30$ & 125.388 & 123.004 & 119.674 & 100.529 & 104.687 & 96.24 & 110.473 & 107.346 & 78.963 & 73.981 \\
\hline $6 / 21 / 07$ 18:00 & 125.383 & 123.004 & 119.672 & 100.527 & 104.685 & 96.241 & 110.47 & 107.34 & 78.952 & 73.977 \\
\hline 6/21/07 18:30 & 125.367 & 122.993 & 119.651 & 100.506 & 104.669 & 96.222 & 110.458 & 107.322 & 78.937 & 73.958 \\
\hline 6/21/07 19:00 & 125.364 & 122.984 & 119.651 & 100.505 & 104.667 & 96.219 & 110.453 & 107.32 & 78.928 & 73.956 \\
\hline $6 / 21 / 0719: 30$ & 125.355 & 122.977 & 119.641 & 100.494 & 104.656 & 96.21 & 110.447 & 107.309 & 78.918 & 73.95 \\
\hline $6 / 21 / 0720: 00$ & 125.348 & 123.011 & 119.635 & 100.489 & 104.652 & 96.205 & 110.436 & 107.304 & 78.909 & 73.944 \\
\hline $6 / 21 / 0720: 30$ & 125.341 & 123.016 & 119.628 & 100.486 & 104.645 & 96.198 & 110.455 & 107.298 & 78.9 & 73.933 \\
\hline $6 / 21 / 0721: 00$ & 125.376 & 123.023 & 119.662 & 100.52 & 104.683 & 96.233 & 110.478 & 107.335 & 78.908 & 73.952 \\
\hline 6/21/07 21:30 & 125.374 & 123.023 & 119.66 & 100.522 & 104.683 & 96.231 & 110.494 & 107.331 & 78.904 & 73.96 \\
\hline 6/21/07 22:00 & 125.388 & 123.011 & 119.676 & 100.527 & 104.698 & 96.269 & 110.514 & 107.34 & 78.902 & 73.973 \\
\hline 6/21/07 22:30 & 125.383 & 123.011 & 119.669 & 100.525 & 104.696 & 96.25 & 110.961 & 107.34 & 78.896 & 73.968 \\
\hline 6/21/07 23:00 & 125.376 & 123.02 & 119.66 & 100.522 & 104.683 & 96.231 & 110.467 & 107.335 & 78.891 & 73.966 \\
\hline 6/21/07 23:30 & 125.376 & 123.018 & 119.667 & 100.515 & 104.688 & 96.257 & 110.534 & 107.329 & 78.885 & 73.962 \\
\hline 6/22/07 0:00 & 125.381 & 123.036 & 119.667 & 100.531 & 104.688 & 96.236 & 110.463 & 107.339 & 78.885 & 73.971 \\
\hline 6/22/07 0:30 & 125.378 & 123.02 & 119.664 & 100.524 & 104.687 & 96.238 & 110.477 & 107.338 & & 73.962 \\
\hline 6/22/07 1:00 & 125.397 & 123 & 119.683 & 100.543 & 104.706 & 96.255 & 110.437 & 107.353 & 78.884 & 73.975 \\
\hline 6/22/07 1:30 & 125.383 & 122.979 & 119.669 & 100.527 & 104.69 & 96.238 & 110.462 & 107.342 & 78.876 & 73.964 \\
\hline 6/22/07 2:00 & 125.359 & 122.977 & 119.648 & 100.506 & 104.671 & 96.219 & 110.462 & 107.32 & & 73.946 \\
\hline $6 / 22 / 072: 30$ & 125.341 & 122.989 & 119.628 & 100.484 & 104.65 & 96.196 & 110.432 & 107.3 & 78.856 & 73.929 \\
\hline 6/22/07 3:00 & 125.338 & 122.998 & 119.625 & 100.481 & 104.652 & 96.203 & 110.898 & 107.298 & 78.854 & 73.931 \\
\hline 6/22/07 3:30 & & 122.998 & & & & & & & & 73.944 \\
\hline 6/22/07 4:00 & 125.357 & 122.97 & 119.644 & 100.532 & 104.667 & 96.219 & 110.448 & 107.315 & 78.854 & 73.95 \\
\hline 6/22/07 4:30 & 125.364 & 122.941 & 119.65 & 100.508 & 104.677 & 96.224 & 110.424 & 107.32 & 78.85 & 73.948 \\
\hline 6/22/07 5:00 & 125.333 & 122.932 & 119.618 & 100.482 & 104.641 & 96.191 & 110.449 & 107.293 & & 73.927 \\
\hline 6/22/07 5:30 & 125.3 & 122.925 & 119.586 & 100.448 & 104.604 & 96.156 & 110.423 & 107.26 & 78.825 & 73.896 \\
\hline 6/22/07 6:00 & 125.296 & 124.943 & 119.581 & 100.439 & 104.606 & 96.153 & 110.406 & 107.256 & 78.819 & 73.892 \\
\hline 6/22/07 6:30 & & 125.252 & & 100.432 & & 96.144 & & 107.246 & & 73.89 \\
\hline 6/22/07 7:00 & 127.336 & 125.408 & 120.899 & 101.12 & 105.071 & 96.773 & 111.008 & 108.719 & 78.828 & 73.933 \\
\hline 6/22/07 7:30 & 127.667 & 125.503 & 121.218 & 101.401 & 105.364 & 97.07 & 111.274 & 109.071 & 78.861 & 74.088 \\
\hline 6/22/07 8:00 & 127.832 & 125.565 & & 101.551 & & 97.228 & 111.416 & 109.244 & 78.893 & 74.231 \\
\hline 6/22/07 8:30 & 127.932 & 125.617 & 121.484 & 101.644 & 105.625 & 97.329 & 111.495 & 109.347 & 78.92 & 74.322 \\
\hline 6/22/07 9:00 & 127.993 & 125.653 & 121.547 & 101.702 & 105.686 & 97.388 & 111.553 & 109.412 & 78.948 & 74.39 \\
\hline 6/22/07 9:30 & 128.043 & 124.701 & 121.6 & 101.756 & 105.739 & 97.44 & 111.602 & 109.465 & 78.976 & 74.452 \\
\hline 6/22/07 10:00 & 128.085 & 125.644 & 121.637 & 101.792 & 105.777 & 97.48 & 111.63 & 109.505 & 79.002 & 74.492 \\
\hline 6/22/07 10:30 & 128.107 & 125.676 & 121.663 & 101.745 & 105.802 & 97.504 & 111.646 & 109.529 & 79.02 & 74.525 \\
\hline 6/22/07 11:00 & 128.071 & 125.692 & 121.63 & 101.79 & 105.772 & 97.476 & 111.614 & 109.492 & 79.037 & 74.518 \\
\hline $6 / 22 / 0711: 30$ & 128.109 & 125.694 & 121.669 & 101.823 & 105.808 & 97.511 & 111.648 & 109.534 & 79.053 & 74.537 \\
\hline 6/22/07 12:00 & 128.128 & 123.605 & 121.686 & 101.837 & 105.825 & 97.528 & 111.666 & 109.549 & 79.072 & 74.551 \\
\hline 6/22/07 12:30 & 128.128 & 123.363 & 121.69 & 101.844 & 105.833 & 97.532 & 111.679 & 109.556 & 79.086 & 74.566 \\
\hline 6/22/07 13:00 & 125.996 & 123.227 & 120.272 & 101.06 & 105.254 & 96.799 & 110.981 & 107.977 & 79.068 & 74.481 \\
\hline 6/22/07 13:30 & 125.738 & 123.131 & 120.018 & 100.836 & 105.018 & 96.566 & 110.769 & 107.707 & 79.045 & 74.332 \\
\hline 6/22/07 14:00 & 125.596 & 123.057 & 119.877 & 100.712 & 104.885 & 96.434 & 110.65 & 107.563 & 79.02 & 74.223 \\
\hline
\end{tabular}


TABLE S1.1 (Cont.)

Depth from Top of Casing (ft)

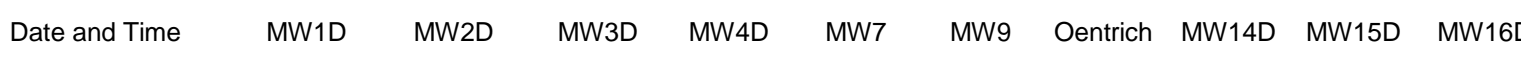

\begin{tabular}{|c|c|c|c|c|c|c|c|c|c|c|}
\hline $6 / 22 / 07$ 14:30 & 125.499 & 123.007 & 119.782 & 100.618 & 104.791 & 96.339 & 110.572 & 107.461 & 78.996 & 74.132 \\
\hline $6 / 22 / 07$ 15:00 & 125.426 & 122.966 & 119.708 & 100.55 & 104.719 & 96.266 & 110.511 & 107.386 & 78.974 & 74.064 \\
\hline 6/22/07 15:30 & 125.374 & 122.927 & 119.657 & 100.501 & 104.669 & 96.219 & 110.473 & 107.333 & 78.952 & 74.012 \\
\hline 6/22/07 16:00 & 125.331 & 122.898 & 119.618 & 100.463 & 104.631 & 96.182 & 110.441 & 107.293 & 78.933 & 73.977 \\
\hline 6/22/07 16:30 & 125.294 & 122.873 & 119.579 & 100.427 & 104.593 & 96.144 & 110.413 & 107.253 & 78.915 & 73.937 \\
\hline 6/22/07 17:00 & 125.263 & 122.85 & 119.546 & 100.398 & 104.563 & 96.111 & 110.389 & 107.222 & 78.893 & 73.902 \\
\hline $6 / 22 / 07$ 17:30 & 125.234 & 122.828 & 119.519 & 100.372 & 104.538 & 96.087 & 110.372 & 107.195 & 78.874 & 73.88 \\
\hline 6/22/07 18:00 & 125.213 & 122.818 & 119.498 & 100.351 & 104.515 & 96.066 & 110.35 & 107.171 & 78.856 & 73.855 \\
\hline 6/22/07 18:30 & 125.19 & 122.807 & 119.479 & 100.332 & 104.496 & 96.047 & 110.313 & 107.152 & 78.839 & 73.834 \\
\hline 6/22/07 19:00 & 125.175 & 122.805 & 119.461 & 100.32 & 104.485 & 96.031 & 110.3 & 107.136 & 78.823 & 73.818 \\
\hline 6/22/07 19:30 & 125.168 & 122.803 & 119.456 & 100.314 & 104.479 & 96.026 & 110.292 & 107.129 & 78.81 & 73.812 \\
\hline $6 / 22 / 07$ 20:00 & 125.166 & 122.846 & 119.456 & 100.314 & 104.479 & 96.028 & 110.285 & 107.127 & 78.799 & 73.809 \\
\hline 6/22/07 20:30 & 125.164 & 122.882 & 119.454 & 100.312 & 104.477 & 96.026 & 110.279 & 107.123 & 78.79 & 73.805 \\
\hline 6/22/07 21:00 & 125.206 & 122.834 & 119.509 & 100.427 & 104.542 & 96.125 & 111.233 & 107.149 & 78.782 & 73.795 \\
\hline 6/22/07 21:30 & 125.246 & 122.839 & 119.553 & 100.465 & 104.586 & 96.167 & 111.287 & 107.193 & 78.788 & 73.809 \\
\hline 6/22/07 22:00 & 125.194 & 122.887 & 119.482 & 100.348 & 104.513 & 96.059 & 110.352 & 107.156 & 78.784 & 73.83 \\
\hline 6/22/07 22:30 & 125.194 & 122.866 & 119.486 & 100.353 & 104.523 & 96.083 & 110.478 & 107.153 & 78.775 & 73.828 \\
\hline 6/22/07 23:00 & 125.248 & 122.837 & 119.537 & 100.405 & 104.574 & 96.125 & 110.336 & 107.211 & 78.793 & 73.886 \\
\hline 6/22/07 23:30 & 125.298 & 122.8 & 119.523 & 100.384 & 104.557 & 96.104 & 110.352 & 107.198 & 78.767 & 73.811 \\
\hline 6/23/07 0:00 & 125.274 & 122.821 & 119.486 & 100.351 & 104.517 & 96.066 & 110.314 & 107.162 & 78.804 & 73.836 \\
\hline 6/23/07 0:30 & 125.26 & 122.812 & 119.447 & 100.31 & 104.479 & 96.026 & 110.296 & 107.122 & 78.745 & 73.832 \\
\hline 6/23/07 1:00 & 125.239 & 122.791 & 119.475 & 100.336 & 104.511 & 96.059 & 110.278 & 107.152 & 78.704 & 73.811 \\
\hline 6/23/07 1:30 & 125.227 & 122.794 & 119.463 & 100.329 & 104.496 & 96.045 & 110.266 & 107.136 & 78.701 & 73.801 \\
\hline 6/23/07 2:00 & 125.22 & 122.764 & 119.445 & 100.308 & 104.475 & 96.024 & 110.256 & 107.121 & 78.688 & 73.791 \\
\hline 6/23/07 2:30 & 125.213 & 122.76 & 119.445 & 100.308 & 104.481 & 96.024 & 110.256 & 107.121 & 78.681 & 73.78 \\
\hline 6/23/07 3:00 & 125.199 & 122.757 & 119.419 & 100.281 & 104.447 & 95.995 & 110.243 & 107.091 & 78.668 & 73.766 \\
\hline 6/23/07 3:30 & 125.19 & 122.75 & 119.41 & 100.274 & 104.443 & 95.986 & 110.239 & 107.08 & 78.66 & 73.752 \\
\hline 6/23/07 4:00 & 125.178 & 122.76 & 119.408 & 100.272 & 104.441 & 95.988 & 110.231 & 107.083 & 78.655 & 73.735 \\
\hline 6/23/07 4:30 & 125.17 & 122.76 & 119.399 & 100.265 & 104.431 & 95.979 & 110.229 & 107.072 & 78.644 & 73.72 \\
\hline 6/23/07 5:00 & 125.159 & 122.757 & 119.403 & 100.279 & 104.447 & 95.991 & 110.229 & 107.083 & 78.644 & 73.706 \\
\hline 6/23/07 5:30 & 125.159 & 122.755 & 119.408 & 100.276 & 104.445 & 95.993 & 110.221 & 107.081 & 78.64 & 73.704 \\
\hline 6/23/07 6:00 & 125.152 & 122.762 & 119.41 & 100.276 & 104.441 & 95.988 & 110.22 & 107.08 & 78.634 & 73.713 \\
\hline 6/23/07 6:30 & 125.145 & 122.757 & 119.401 & 100.27 & 104.439 & 95.986 & 110.217 & 107.078 & 78.629 & 73.706 \\
\hline 6/23/07 7:00 & 125.142 & 124.75 & 119.408 & 100.279 & 104.449 & 95.993 & 110.217 & 107.085 & 78.627 & 73.708 \\
\hline 6/23/07 7:30 & 125.14 & 125.088 & 119.408 & 100.277 & 104.445 & 95.988 & 110.216 & 107.081 & 78.625 & 73.723 \\
\hline 6/23/07 8:00 & 127.111 & 125.247 & 120.693 & 100.929 & 104.879 & 96.575 & 110.784 & 108.511 & 78.637 & 73.774 \\
\hline 6/23/07 8:30 & 127.506 & 125.342 & 121.056 & 101.235 & 105.204 & 96.908 & 111.094 & 108.909 & 78.67 & 73.94 \\
\hline 6/23/07 9:00 & 127.672 & 125.408 & 121.223 & 101.389 & 105.366 & 97.068 & 111.239 & 109.082 & 78.703 & 74.066 \\
\hline 6/23/07 9:30 & 127.773 & 125.453 & 121.322 & 101.48 & 105.461 & 97.162 & 111.327 & 109.186 & 78.731 & 74.169 \\
\hline $6 / 23 / 07$ 10:00 & 127.844 & 125.481 & 121.392 & 101.547 & 105.536 & 97.235 & 111.391 & 109.259 & 78.76 & 74.256 \\
\hline $6 / 23 / 07$ 10:30 & 127.887 & 125.499 & 121.438 & 101.594 & 105.581 & 97.282 & 111.432 & 109.305 & 78.784 & 74.308 \\
\hline 6/23/07 11:00 & 127.917 & 125.522 & 121.47 & 101.623 & 105.614 & 97.313 & 111.466 & 109.339 & 78.808 & 74.384 \\
\hline 6/23/07 11:30 & 127.934 & 125.528 & 121.487 & 101.642 & 105.631 & 97.332 & 111.482 & 109.357 & 78.83 & 74.442 \\
\hline $6 / 23 / 07$ 12:00 & 127.96 & 125.535 & 121.51 & 101.663 & 105.656 & 97.353 & 111.501 & 109.381 & 78.849 & 74.487 \\
\hline 6/23/07 12:30 & 127.964 & 125.537 & 121.521 & 101.675 & 105.667 & 97.365 & 111.512 & 109.392 & 78.865 & 74.523 \\
\hline 6/23/07 13:00 & 127.972 & 125.537 & 121.531 & 101.682 & 105.675 & 97.374 & 111.524 & 109.401 & & 74.547 \\
\hline 6/23/07 13:30 & 127.972 & 125.537 & 121.533 & 101.685 & 105.677 & 97.374 & 111.531 & 109.401 & 78.895 & 74.537 \\
\hline 6/23/07 14:00 & 127.974 & 123.63 & 121.533 & 101.688 & 105.682 & 97.379 & 111.537 & 109.403 & 78.906 & 74.481 \\
\hline $6 / 23 / 07$ 14:30 & 127.974 & 123.277 & 121.535 & 101.688 & 105.684 & 97.379 & 111.542 & 109.403 & 78.915 & 74.452 \\
\hline 6/23/07 15:00 & 126.048 & 123.104 & 120.316 & 101.061 & 105.275 & 96.82 & 111.003 & 108.05 & 78.909 & 74.429 \\
\hline 6/23/07 15:30 & 125.66 & 122.995 & 119.935 & 100.748 & 104.94 & 96.481 & 110.694 & 107.634 & 78.88 & 74.275 \\
\hline $6 / 23 / 07$ 16:00 & 125.478 & 122.925 & 119.759 & 100.591 & 104.774 & 96.318 & 110.545 & 107.45 & 78.854 & 74.14 \\
\hline 6/23/07 16:30 & 125.367 & 122.877 & 119.65 & 100.488 & 104.667 & 96.212 & 110.451 & 107.335 & 78.828 & 74.041 \\
\hline 6/23/07 17:00 & 125.296 & 122.841 & 119.579 & 100.425 & 104.601 & 96.146 & 110.386 & 107.26 & 78.806 & 73.975 \\
\hline 6/23/07 17:30 & 125.244 & 122.807 & 119.528 & 100.377 & 104.551 & 96.097 & 110.344 & 107.209 & 78.786 & 73.921 \\
\hline $6 / 23 / 07$ 18:00 & 125.208 & 122.787 & 119.493 & 100.346 & 104.521 & 96.066 & 110.312 & 107.174 & 78.767 & 73.882 \\
\hline 6/23/07 18:30 & 125.173 & 122.775 & 119.458 & 100.315 & 104.489 & 96.033 & 110.292 & 107.138 & 78.747 & 73.849 \\
\hline $6 / 23 / 07$ 19:00 & 125.154 & 122.766 & 119.44 & 100.296 & 104.469 & 96.017 & 110.271 & 107.118 & 78.732 & 73.826 \\
\hline 6/23/07 19:30 & 125.14 & 122.757 & 119.424 & 100.286 & 104.456 & 96.002 & 110.256 & 107.103 & 78.718 & 73.808 \\
\hline 6/23/07 20:00 & 125.135 & 122.753 & 119.428 & 100.276 & 104.46 & 96.028 & 110.384 & 107.094 & 78.703 & 73.801 \\
\hline 6/23/07 20:30 & 125.118 & 122.75 & 119.408 & 100.284 & 104.441 & 95.986 & 110.241 & 107.085 & 78.69 & 73.789 \\
\hline 6/23/07 21:00 & 125.116 & 122.753 & 119.405 & 100.269 & 104.439 & 95.986 & 110.26 & 107.081 & 78.679 & 73.785 \\
\hline 6/23/07 21:30 & 125.114 & 122.755 & 119.403 & 100.269 & 104.439 & 95.984 & 110.233 & 107.078 & 78.668 & 73.779 \\
\hline 6/23/07 22:00 & 125.112 & 122.757 & 119.401 & 100.296 & 104.441 & 95.984 & 110.233 & 107.076 & 78.66 & 73.781 \\
\hline 6/23/07 22:30 & 125.118 & 122.75 & 119.412 & 100.27 & 104.447 & 95.993 & 110.223 & 107.08 & 78.653 & 73.783 \\
\hline 6/23/07 23:00 & 125.118 & 122.748 & 119.41 & 100.279 & 104.449 & 95.995 & 110.24 & 107.085 & 78.648 & 73.789 \\
\hline
\end{tabular}


TABLE S1.1 (Cont.)

Depth from Top of Casing (ft)

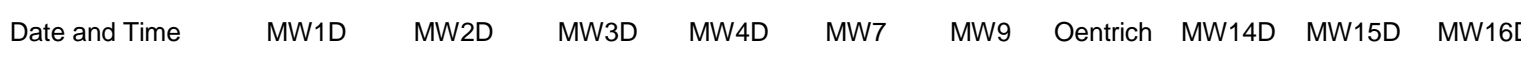

\begin{tabular}{|c|c|c|c|c|c|c|c|c|c|c|}
\hline 6/23/07 23:30 & 125.111 & 122.744 & 119.403 & 100.27 & 104.441 & 95.986 & 110.239 & 107.078 & 78.638 & 73.779 \\
\hline 6/24/07 0:00 & 125.109 & 122.732 & 119.398 & 100.265 & 104.437 & 95.979 & 110.187 & 107.076 & 78.629 & 73.775 \\
\hline 6/24/07 0:30 & 125.102 & 122.726 & 119.391 & 100.264 & 104.431 & 95.976 & 110.255 & 107.067 & 78.62 & 73.772 \\
\hline 6/24/07 1:00 & 125.092 & 122.714 & 119.384 & 100.255 & 104.426 & 95.967 & 110.238 & 107.058 & 78.611 & 73.758 \\
\hline 6/24/07 1:30 & 125.083 & 122.705 & 119.375 & 100.248 & 104.414 & 95.96 & 110.209 & 107.049 & 78.6 & 73.748 \\
\hline 6/24/07 2:00 & 125.076 & 122.696 & 119.366 & 100.236 & 104.409 & 95.951 & 110.206 & 107.043 & 78.59 & 73.744 \\
\hline 6/24/07 2:30 & 125.069 & 122.694 & 119.359 & 100.229 & 104.397 & 95.941 & 110.182 & 107.034 & 78.579 & 73.733 \\
\hline 6/24/07 3:00 & 125.06 & 122.685 & 119.35 & 100.22 & 104.391 & 95.934 & 110.17 & 107.023 & 78.568 & 73.721 \\
\hline 6/24/07 3:30 & 125.055 & 122.687 & 119.347 & 100.217 & 104.388 & 95.932 & 110.185 & 107.021 & 78.563 & 73.725 \\
\hline 6/24/07 4:00 & 125.05 & 122.692 & 119.341 & 100.21 & 104.382 & 95.925 & 110.17 & 107.014 & 78.554 & 73.717 \\
\hline 6/24/07 4:30 & 125.047 & 122.696 & 119.338 & 100.207 & 104.384 & 95.925 & 110.494 & 107.012 & 78.548 & 73.713 \\
\hline 6/24/07 5:00 & 125.05 & 122.698 & 119.343 & 100.219 & 104.388 & 95.932 & 110.168 & 107.018 & 78.544 & 73.715 \\
\hline 6/24/07 5:30 & 125.05 & 122.701 & 119.345 & 100.222 & 104.391 & 95.936 & 110.16 & 107.021 & 78.539 & 73.719 \\
\hline 6/24/07 6:00 & 125.06 & 122.701 & 119.35 & 100.224 & 104.393 & 95.941 & 110.175 & 107.025 & 78.537 & 73.721 \\
\hline 6/24/07 6:30 & 125.059 & 122.701 & 119.352 & 100.226 & 104.395 & 95.939 & 110.16 & 107.027 & 78.533 & 73.723 \\
\hline 6/24/07 7:00 & 125.062 & 122.705 & 119.352 & 100.227 & 104.397 & 95.939 & 110.149 & 107.027 & 78.528 & 73.723 \\
\hline 6/24/07 7:30 & 125.062 & 124.58 & 119.354 & 100.231 & 104.401 & 95.943 & 110.162 & 107.029 & 78.528 & 73.727 \\
\hline 6/24/07 8:00 & 125.064 & 124.957 & 119.359 & 100.229 & 104.405 & 95.948 & 110.124 & 107.032 & 78.522 & 73.727 \\
\hline 6/24/07 8:30 & 126.988 & 125.134 & 120.686 & 100.873 & 104.805 & 96.512 & 110.714 & 108.331 & 78.535 & 73.741 \\
\hline 6/24/07 9:00 & 127.404 & 125.236 & 121.091 & 101.209 & 105.164 & 96.879 & 111.047 & 108.781 & 78.572 & 73.904 \\
\hline 6/24/07 9:30 & 127.589 & 125.302 & 121.274 & 101.377 & 105.338 & 97.051 & 111.205 & 108.973 & 78.605 & 74.052 \\
\hline 6/24/07 10:00 & 127.7 & 124.283 & 121.38 & 101.475 & 105.44 & 97.155 & 111.302 & 109.084 & 78.637 & 74.149 \\
\hline 6/24/07 10:30 & 127.763 & 123.277 & 121.445 & 101.537 & 105.505 & 97.219 & 111.362 & 109.15 & 78.662 & 74.217 \\
\hline 6/24/07 11:00 & 127.813 & 123.066 & 121.494 & 101.42 & 105.553 & 97.266 & 111.409 & 109.204 & 78.686 & 74.273 \\
\hline 6/24/07 11:30 & 125.662 & 122.95 & 119.944 & 100.753 & 104.951 & 96.493 & 110.657 & 107.645 & 78.679 & 74.205 \\
\hline 6/24/07 12:00 & 125.437 & 122.871 & 119.722 & 100.563 & 104.747 & 96.29 & 110.469 & 107.412 & 78.664 & 74.087 \\
\hline 6/24/07 12:30 & 125.319 & 122.821 & 119.607 & 100.457 & 104.639 & 96.182 & 110.365 & 107.291 & 78.651 & 73.992 \\
\hline 6/24/07 13:00 & 125.237 & 122.782 & 119.528 & 100.386 & 104.563 & 96.106 & 110.299 & 107.209 & 78.637 & 73.919 \\
\hline 6/24/07 13:30 & 125.187 & 122.75 & 119.477 & 100.336 & 104.511 & 96.054 & 110.252 & 107.153 & 78.622 & 73.87 \\
\hline 6/24/07 14:00 & 125.147 & 122.726 & 119.438 & 100.3 & 104.473 & 96.019 & 110.22 & 107.114 & 78.609 & 73.83 \\
\hline $6 / 24 / 07$ 14:30 & 125.116 & 122.71 & 119.408 & 100.272 & 104.443 & 95.991 & 110.196 & 107.083 & 78.596 & 73.797 \\
\hline 6/24/07 15:00 & 125.09 & 122.696 & 119.384 & 100.248 & 104.422 & 95.969 & 110.171 & 107.058 & 78.583 & 73.772 \\
\hline 6/24/07 15:30 & 125.071 & 122.678 & 119.366 & 100.233 & 104.405 & 95.951 & 110.156 & 107.036 & 78.57 & 73.752 \\
\hline 6/24/07 16:00 & 125.059 & 122.667 & 119.352 & 100.219 & 104.393 & 95.939 & 110.147 & 107.025 & 78.561 & 73.737 \\
\hline 6/24/07 16:30 & 125.038 & 122.653 & 119.331 & 100.202 & 104.374 & 95.918 & 110.135 & 107.005 & 78.548 & 73.717 \\
\hline 6/24/07 17:00 & 125.026 & 122.646 & 119.32 & 100.191 & 104.363 & 95.906 & 110.122 & 106.994 & 78.535 & 73.704 \\
\hline $6 / 24 / 07$ 17:30 & 125.014 & 122.637 & 119.308 & 100.179 & 104.351 & 95.896 & 110.115 & 106.983 & 78.526 & 73.688 \\
\hline 6/24/07 18:00 & 125.005 & 122.635 & 119.301 & 100.174 & 104.344 & 95.889 & 110.107 & 106.974 & 78.517 & 73.686 \\
\hline 6/24/07 18:30 & 125 & 122.63 & 119.294 & 100.167 & 104.336 & 95.882 & 110.102 & 106.968 & 78.507 & 73.677 \\
\hline 6/24/07 19:00 & 124.998 & 122.635 & 119.292 & 100.165 & 104.336 & 95.882 & 110.1 & 106.963 & 78.496 & 73.671 \\
\hline 6/24/07 19:30 & 124.991 & 122.635 & 119.287 & 100.162 & 104.33 & 95.877 & 110.094 & 106.961 & 78.489 & 73.667 \\
\hline 6/24/07 20:00 & 124.995 & 122.63 & 119.29 & 100.165 & 104.336 & 95.882 & 110.092 & 106.961 & 78.485 & 73.673 \\
\hline 6/24/07 20:30 & 124.995 & 122.633 & 119.292 & 100.167 & 104.336 & 95.882 & 110.092 & & 78.478 & 73.671 \\
\hline 6/24/07 21:00 & 124.991 & 122.642 & 119.288 & 100.164 & 104.336 & 95.877 & 110.089 & 106.959 & 78.472 & 73.671 \\
\hline 6/24/07 21:30 & 124.993 & 122.646 & 119.287 & 100.165 & 104.334 & 95.877 & 110.087 & 106.959 & 78.467 & 73.669 \\
\hline 6/24/07 22:00 & 125 & 122.646 & 119.297 & 100.176 & 104.344 & 95.889 & 110.088 & & 78.463 & 73.675 \\
\hline 6/24/07 22:30 & 125.007 & 122.648 & 119.303 & 100.181 & 104.35 & 95.896 & 110.086 & 106.974 & 78.461 & 73.679 \\
\hline 6/24/07 23:00 & 125.01 & 122.653 & 119.306 & 100.183 & 104.353 & 95.896 & 110.085 & 106.977 & 78.458 & 73.682 \\
\hline 6/24/07 23:30 & 125.01 & 122.653 & 119.306 & 100.184 & 104.353 & 95.899 & 110.083 & 106.977 & 78.454 & 73.684 \\
\hline 6/25/07 0:00 & 125.012 & 122.653 & 119.31 & 100.188 & 104.357 & 95.901 & 110.079 & 106.981 & 78.452 & 73.688 \\
\hline 6/25/07 0:30 & 125.012 & 122.646 & 119.31 & 100.188 & 104.357 & 95.906 & 110.079 & 106.981 & 78.448 & 73.688 \\
\hline 6/25/07 1:00 & 125.014 & 122.651 & 119.31 & 100.189 & 104.359 & 95.91 & 110.075 & 106.981 & 78.445 & 73.686 \\
\hline 6/25/07 1:30 & 125.007 & 122.648 & 119.306 & 100.184 & 104.355 & 95.906 & 110.071 & 106.974 & 78.437 & 73.677 \\
\hline 6/25/07 2:00 & 125.012 & 122.646 & 119.306 & 100.188 & 104.355 & 95.899 & 110.071 & 106.977 & 78.436 & 73.682 \\
\hline 6/25/07 2:30 & 125.01 & 122.653 & 119.306 & 100.184 & 104.357 & 95.901 & 110.069 & 106.979 & 78.432 & 73.679 \\
\hline 6/25/07 3:00 & 125.007 & 122.648 & 119.306 & 100.183 & 104.355 & 95.899 & 110.066 & 106.974 & 78.428 & 73.679 \\
\hline 6/25/07 3:30 & 125.014 & 122.644 & 119.313 & 100.191 & 104.363 & 95.906 & 110.069 & 106.981 & 78.426 & 73.682 \\
\hline 6/25/07 4:00 & 125.01 & 122.642 & 119.308 & 100.186 & 104.357 & 95.901 & 110.066 & 106.979 & 78.423 & 73.675 \\
\hline 6/25/07 4:30 & 125.003 & 122.644 & 119.299 & 100.181 & 104.35 & 95.896 & 110.062 & 106.972 & 78.417 & 73.673 \\
\hline 6/25/07 5:00 & 125 & 122.644 & 119.301 & 100.179 & 104.35 & 95.894 & 110.06 & 106.972 & 78.415 & 73.675 \\
\hline 6/25/07 5:30 & 125.003 & 122.644 & 119.299 & 100.181 & 104.351 & 95.894 & 110.062 & 106.972 & 78.412 & 73.673 \\
\hline 6/25/07 6:00 & 124.998 & 124.791 & 119.301 & 100.184 & 104.353 & 95.899 & 110.055 & 106.974 & 78.41 & 73.675 \\
\hline 6/25/07 6:30 & 124.993 & 125.027 & 119.303 & 100.184 & 104.355 & 95.899 & 110.09 & 106.974 & 78.41 & 73.677 \\
\hline 6/25/07 7:00 & 127.194 & 125.15 & 120.772 & 100.985 & 104.953 & 96.648 & 110.802 & 108.604 & 78.441 & 73.772 \\
\hline 6/25/07 7:30 & 127.437 & 125.234 & 121.017 & 101.208 & 105.183 & 96.882 & 111.056 & 108.863 & 78.476 & 73.936 \\
\hline 6/25/07 8:00 & 127.57 & 125.295 & 121.147 & 101.325 & 105.309 & 97.007 & 111.15 & 109.002 & 78.511 & 74.054 \\
\hline
\end{tabular}


TABLE S1.1 (Cont.)

Depth from Top of Casing (ft)

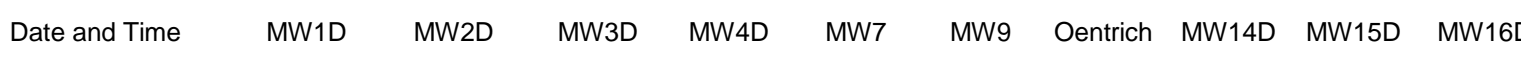

\begin{tabular}{|c|c|c|c|c|c|c|c|c|c|c|}
\hline 6/25/07 8:30 & 127.66 & 125.331 & 121.235 & 101.409 & 105.397 & 97.096 & 111.238 & 109.091 & 78.541 & 74.143 \\
\hline 6/25/07 9:00 & 127.726 & 124.097 & 121.297 & 101.471 & 105.465 & 97.155 & 111.276 & 109.155 & 78.568 & 74.205 \\
\hline 6/25/07 9:30 & 127.759 & 123.256 & 121.336 & 101.509 & 105.501 & 97.197 & 111.301 & 109.192 & 78.592 & 74.252 \\
\hline $6 / 25 / 07$ 10:00 & 127.376 & 123.048 & 121.315 & 101.328 & 105.539 & 97.233 & 111.324 & 109.232 & 78.62 & 74.291 \\
\hline 6/25/07 10:30 & 125.636 & 122.934 & 119.926 & 100.742 & 104.944 & 96.481 & 110.604 & 107.625 & 78.607 & 74.205 \\
\hline 6/25/07 11:00 & 125.419 & 122.859 & 119.71 & 100.558 & 104.743 & 96.283 & 110.412 & 107.402 & 78.592 & 74.08 \\
\hline 6/25/07 11:30 & 125.298 & 122.807 & 119.593 & 100.45 & 104.633 & 96.172 & 110.308 & 107.275 & 78.579 & 73.983 \\
\hline 6/25/07 12:00 & 125.222 & 122.775 & 119.519 & 100.384 & 104.559 & 96.101 & 110.24 & 107.195 & 78.566 & 73.917 \\
\hline 6/25/07 12:30 & 125.17 & 122.739 & 119.465 & 100.334 & 104.509 & 96.052 & 110.198 & 107.142 & 78.554 & 73.863 \\
\hline 6/25/07 13:00 & 125.14 & 122.712 & 119.433 & 100.305 & 104.477 & 96.021 & 110.166 & 107.109 & 78.544 & 73.83 \\
\hline $6 / 25 / 07$ 13:30 & 125.102 & 122.689 & 119.401 & 100.272 & 104.443 & 95.988 & 110.142 & 107.072 & 78.53 & 73.795 \\
\hline $6 / 25 / 0714: 00$ & 125.071 & 122.671 & 119.371 & 100.244 & 104.416 & 95.96 & 110.125 & 107.043 & 78.517 & 73.766 \\
\hline 6/25/07 14:30 & 125.05 & 122.651 & 119.35 & 100.224 & 104.393 & 95.941 & 110.108 & 107.021 & 78.504 & 73.744 \\
\hline 6/25/07 15:00 & 125.031 & 122.635 & 119.334 & 100.208 & 104.378 & 95.925 & 110.096 & 107.001 & 78.493 & 73.725 \\
\hline 6/25/07 15:30 & 125.012 & 122.628 & 119.313 & 100.187 & 104.363 & 95.906 & 110.087 & 106.986 & 78.48 & 73.706 \\
\hline 6/25/07 16:00 & 124.998 & 122.621 & 119.299 & 100.175 & 104.346 & 95.889 & 110.077 & 106.97 & 78.472 & 73.696 \\
\hline 6/25/07 16:30 & 124.988 & 122.61 & 119.287 & 100.167 & 104.338 & 95.882 & 110.07 & 106.959 & 78.46 & 73.684 \\
\hline 6/25/07 17:00 & 124.981 & 122.599 & 119.28 & 100.158 & 104.332 & 95.875 & 110.064 & 106.95 & 78.45 & 73.671 \\
\hline 6/25/07 17:30 & 124.97 & 122.592 & 119.271 & 100.15 & 104.321 & 95.863 & 110.06 & 106.941 & 78.439 & 73.665 \\
\hline 6/25/07 18:00 & 124.958 & 122.589 & 119.257 & 100.139 & 104.313 & 95.856 & 110.055 & 106.93 & 78.43 & 73.653 \\
\hline 6/25/07 18:30 & 124.951 & 122.589 & 119.253 & 100.134 & 104.304 & 95.849 & 110.045 & 106.921 & 78.423 & 73.646 \\
\hline 6/25/07 19:00 & 124.948 & 124.676 & 119.25 & 100.133 & 104.306 & 95.847 & 110.041 & 106.921 & 78.417 & 73.644 \\
\hline 6/25/07 19:30 & 124.946 & 124.943 & 119.25 & 100.132 & 104.304 & 95.847 & 110.038 & 106.919 & 78.412 & 73.644 \\
\hline 6/25/07 20:00 & 127.109 & 125.086 & 120.809 & 100.958 & 104.898 & 96.615 & 110.774 & 108.48 & 78.437 & 73.727 \\
\hline $6 / 25 / 0720: 30$ & 127.392 & 125.179 & 121.089 & 101.204 & 105.161 & 96.875 & 111.01 & 108.778 & 78.471 & 73.899 \\
\hline 6/25/07 21:00 & 127.544 & 125.245 & 121.235 & 101.34 & 105.307 & 97.016 & 111.138 & 108.931 & 78.502 & 74.023 \\
\hline 6/25/07 21:30 & 127.641 & 125.288 & 121.329 & 101.43 & 105.397 & 97.106 & 111.218 & 109.031 & 78.526 & 74.116 \\
\hline 6/25/07 22:00 & 127.707 & 125.333 & 121.399 & 101.494 & 105.465 & 97.174 & 111.275 & 109.099 & 78.55 & 74.184 \\
\hline 6/25/07 22:30 & 127.756 & 124.159 & 121.445 & 101.54 & 105.511 & 97.221 & 111.314 & 109.15 & 78.576 & 74.231 \\
\hline 6/25/07 23:00 & 127.797 & 123.263 & 121.487 & 101.582 & 105.555 & 97.263 & 111.344 & 109.192 & 78.601 & 74.273 \\
\hline 6/25/07 23:30 & 127.607 & 123.045 & 121.507 & 101.373 & 105.579 & 97.289 & 111.369 & 109.221 & 78.622 & 74.302 \\
\hline 6/26/07 0:00 & 125.653 & 122.93 & 119.939 & 100.754 & 104.953 & 96.493 & 110.588 & 107.639 & 78.609 & 74.213 \\
\hline 6/26/07 0:30 & 125.423 & 122.855 & 119.713 & 100.558 & 104.747 & 96.285 & 110.392 & 107.402 & 78.59 & 74.089 \\
\hline 6/26/07 1:00 & 125.301 & 122.812 & 119.591 & 100.45 & 104.633 & 96.17 & 110.283 & 107.273 & 78.578 & 73.992 \\
\hline 6/26/07 1:30 & 125.222 & 122.778 & 119.517 & 100.384 & 104.563 & 96.101 & 110.214 & 107.195 & 78.565 & 73.921 \\
\hline 6/26/07 2:00 & 125.18 & 122.748 & 119.477 & 100.346 & 104.521 & 96.061 & 110.165 & 107.152 & 78.555 & 73.88 \\
\hline 6/26/07 2:30 & 125.144 & 122.735 & 119.442 & 100.315 & 104.489 & 96.031 & 110.131 & 107.116 & 78.543 & 73.845 \\
\hline 6/26/07 3:00 & 125.123 & 122.723 & 119.412 & 100.288 & 104.46 & 96.002 & 110.107 & 107.085 & 78.531 & 73.818 \\
\hline 6/26/07 3:30 & 125.102 & 122.716 & 119.398 & 100.279 & 104.449 & 95.991 & 110.088 & 107.072 & 78.522 & 73.799 \\
\hline 6/26/07 4:00 & 125.088 & 122.712 & 119.389 & 100.269 & 104.437 & 95.981 & 110.073 & 107.061 & 78.517 & 73.789 \\
\hline 6/26/07 4:30 & 125.076 & 122.71 & 119.382 & 100.262 & 104.433 & 95.972 & 110.058 & 107.052 & 78.509 & 73.777 \\
\hline 6/26/07 5:00 & 125.067 & 122.705 & 119.375 & 100.258 & 104.428 & 95.969 & 110.049 & 107.045 & 78.502 & 73.768 \\
\hline 6/26/07 5:30 & 125.069 & 122.698 & 119.373 & 100.257 & 104.427 & 95.967 & 110.041 & 107.043 & 78.496 & 73.764 \\
\hline 6/26/07 6:00 & 125.062 & 124.544 & 119.371 & 100.253 & 104.424 & 95.962 & 110.034 & 107.038 & 78.493 & 73.762 \\
\hline 6/26/07 6:30 & 125.057 & 124.932 & 119.364 & 100.248 & 104.418 & 95.96 & 110.028 & 107.034 & 78.489 & 73.762 \\
\hline 6/26/07 7:00 & 126.948 & 125.107 & 120.663 & 100.87 & 104.799 & 96.502 & 110.559 & 108.294 & 78.495 & 73.772 \\
\hline 6/26/07 7:30 & 127.381 & 125.213 & 121.084 & 101.215 & 105.168 & 96.879 & 110.912 & 108.765 & 78.526 & 73.934 \\
\hline 6/26/07 8:00 & 127.563 & 123.778 & 121.26 & 101.375 & 105.336 & 97.047 & 111.076 & 108.953 & 78.555 & 74.066 \\
\hline 6/26/07 8:30 & 127.672 & 123.184 & 121.369 & 101.475 & 105.44 & 97.15 & 111.171 & 109.067 & 78.587 & 74.165 \\
\hline 6/26/07 9:00 & 126.371 & 122.989 & 120.619 & 101.144 & 105.378 & 96.966 & 110.976 & 108.518 & 78.613 & 74.242 \\
\hline 6/26/07 9:30 & 125.568 & 122.891 & 119.859 & 100.684 & 104.883 & 96.422 & 110.454 & 107.552 & 78.601 & 74.163 \\
\hline $6 / 26 / 07$ 10:00 & 125.367 & 122.83 & 119.662 & 100.512 & 104.7 & 96.238 & 110.291 & 107.349 & 78.589 & 74.047 \\
\hline 6/26/07 10:30 & 125.258 & 122.8 & 119.556 & 100.42 & 104.601 & 96.139 & 110.199 & 107.235 & 78.574 & 73.961 \\
\hline 6/26/07 11:00 & 125.199 & 122.764 & 119.496 & 100.369 & 104.545 & 96.085 & 110.137 & 107.171 & 78.565 & 73.905 \\
\hline $6 / 26 / 0711: 30$ & 125.168 & 122.753 & 119.468 & 100.345 & 104.521 & 96.059 & 110.095 & 107.14 & 78.557 & 73.88 \\
\hline $6 / 26 / 07$ 12:00 & 125.133 & 122.741 & 119.433 & 100.312 & 104.487 & 96.028 & 110.067 & 107.105 & 78.548 & 73.847 \\
\hline 6/26/07 12:30 & 125.118 & 122.719 & 119.421 & 100.303 & 104.475 & 96.017 & 110.047 & 107.091 & 78.541 & 73.83 \\
\hline 6/26/07 13:00 & 125.107 & 122.701 & 119.408 & 100.291 & 104.462 & 96.002 & 110.032 & 107.078 & 78.533 & 73.816 \\
\hline 6/26/07 13:30 & 125.086 & 122.685 & 119.387 & 100.27 & 104.441 & 95.981 & 110.018 & 107.056 & 78.522 & 73.797 \\
\hline $6 / 26 / 07$ 14:00 & 125.069 & 122.669 & 119.371 & 100.251 & 104.424 & 95.965 & 110.007 & 107.038 & 78.511 & 73.779 \\
\hline 6/26/07 14:30 & 125.047 & 122.664 & 119.352 & 100.238 & 104.409 & 95.948 & 110 & 107.019 & 78.498 & 73.762 \\
\hline 6/26/07 15:00 & 125.034 & 122.658 & 119.338 & 100.222 & 104.397 & 95.936 & 109.992 & 107.005 & 78.489 & 73.748 \\
\hline 6/26/07 15:30 & 125.026 & 122.642 & 119.331 & 100.218 & 104.39 & 95.929 & 109.985 & 106.998 & 78.48 & 73.744 \\
\hline 6/26/07 16:00 & 125.024 & 122.639 & 119.327 & 100.214 & 104.384 & 95.925 & 109.977 & 106.994 & 78.474 & 73.744 \\
\hline 6/26/07 16:30 & 125.005 & 122.628 & 119.31 & 100.196 & 104.369 & 95.91 & 109.975 & 106.976 & 78.461 & 73.729 \\
\hline $6 / 26 / 07$ 17:00 & 125.003 & 122.614 & 119.306 & 100.195 & 104.365 & 95.906 & 109.97 & 106.974 & 78.454 & 73.727 \\
\hline
\end{tabular}


TABLE S1.1 (Cont.)

Depth from Top of Casing (ft)

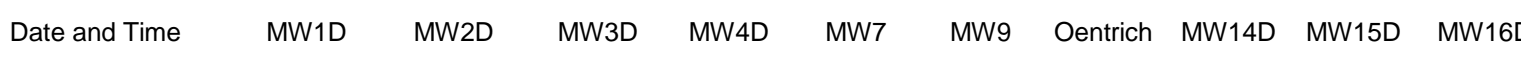

\begin{tabular}{|c|c|c|c|c|c|c|c|c|c|c|}
\hline 6/26/07 17:30 & 124.991 & 124.029 & 119.297 & 100.186 & 104.355 & 95.896 & 109.964 & 106.963 & 78.447 & 73.715 \\
\hline $6 / 26 / 07$ 18:00 & 124.979 & 124.757 & 119.285 & 100.174 & 104.344 & 95.885 & 109.958 & 106.952 & 78.436 & 73.702 \\
\hline 6/26/07 18:30 & 125.894 & 124.957 & 119.678 & 100.439 & 104.359 & 95.941 & 110.02 & 107.039 & 78.426 & 73.688 \\
\hline 6/26/07 19:00 & 127.17 & 123.41 & 120.765 & 100.99 & 104.961 & 96.655 & 110.711 & 108.586 & 78.454 & 73.804 \\
\hline 6/26/07 19:30 & 127.381 & 122.955 & 120.973 & 101.177 & 105.159 & 96.851 & 110.904 & 108.81 & 78.484 & 73.942 \\
\hline 6/26/07 20:00 & 125.908 & 122.796 & 120.185 & 100.842 & 105.071 & 96.634 & 110.687 & 108.015 & 78.504 & 74.041 \\
\hline 6/26/07 20:30 & 125.333 & 122.723 & 119.63 & 100.475 & 104.669 & 96.203 & 110.292 & 107.318 & 78.491 & 73.969 \\
\hline $6 / 26 / 0721: 00$ & 125.168 & 122.694 & 119.47 & 100.332 & 104.521 & 96.057 & 110.159 & 107.147 & 78.474 & 73.876 \\
\hline 6/26/07 21:30 & 125.09 & 122.68 & 119.396 & 100.27 & 104.452 & 95.986 & 110.086 & 107.067 & 78.467 & 73.816 \\
\hline 6/26/07 22:00 & 125.057 & 122.669 & 119.361 & 100.244 & 104.42 & 95.958 & 110.037 & 107.034 & 78.463 & 73.787 \\
\hline 6/26/07 22:30 & 125.045 & 122.667 & 119.35 & 100.236 & 104.412 & 95.948 & 110.003 & 107.019 & 78.463 & 73.772 \\
\hline 6/26/07 23:00 & 125.031 & 122.658 & 119.338 & 100.226 & 104.395 & 95.934 & 109.981 & 107.005 & 78.456 & 73.762 \\
\hline 6/26/07 23:30 & 125.029 & 122.642 & 119.336 & 100.224 & 104.395 & 95.934 & 109.964 & 107.003 & 78.456 & 73.758 \\
\hline 6/27/07 0:00 & 125.019 & 122.635 & 119.327 & 100.215 & 104.386 & 95.927 & 109.952 & 106.994 & 78.45 & 73.746 \\
\hline 6/27/07 0:30 & 125.01 & 122.619 & 119.315 & 100.202 & 104.374 & 95.915 & 109.943 & 106.981 & 78.443 & 73.733 \\
\hline 6/27/07 1:00 & 124.998 & 122.605 & 119.306 & 100.195 & 104.368 & 95.908 & 109.934 & 106.97 & 78.434 & 73.723 \\
\hline 6/27/07 1:30 & 124.986 & 122.592 & 119.292 & 100.179 & 104.351 & 95.892 & 109.928 & 106.959 & 78.425 & 73.708 \\
\hline 6/27/07 2:00 & 124.972 & 122.585 & 119.278 & 100.167 & 104.338 & 95.88 & 109.921 & 106.943 & 78.415 & 73.698 \\
\hline 6/27/07 2:30 & 124.96 & 122.571 & 119.264 & 100.155 & 104.328 & 95.868 & 109.915 & 106.93 & 78.41 & 73.688 \\
\hline 6/27/07 3:00 & 124.948 & 122.558 & 119.255 & 100.146 & 104.319 & 95.859 & 109.909 & 106.924 & 78.402 & 73.682 \\
\hline 6/27/07 3:30 & 124.934 & 122.546 & 119.241 & 100.131 & 104.302 & 95.842 & 109.908 & 106.906 & 78.39 & 73.665 \\
\hline 6/27/07 4:00 & 124.92 & 122.535 & 119.229 & 100.121 & 104.294 & 95.833 & 109.9 & 106.895 & 78.382 & 73.653 \\
\hline 6/27/07 4:30 & 124.918 & 122.526 & 119.216 & 100.11 & 104.279 & 95.821 & 109.896 & 106.881 & 78.373 & 73.642 \\
\hline 6/27/07 5:00 & 124.917 & 122.526 & 119.206 & 100.098 & 104.271 & 95.811 & 109.894 & 106.872 & 78.367 & 73.632 \\
\hline 6/27/07 5:30 & 124.913 & 122.535 & 119.197 & 100.089 & 104.264 & 95.8 & 109.889 & 106.862 & 78.358 & 73.624 \\
\hline 6/27/07 6:00 & 124.906 & 124.478 & 119.199 & 100.094 & 104.266 & 95.804 & 109.883 & 106.862 & 78.354 & 73.626 \\
\hline 6/27/07 6:30 & 124.904 & 124.825 & 119.209 & 100.103 & 104.275 & 95.814 & 109.883 & 106.873 & 78.353 & 73.636 \\
\hline 6/27/07 7:00 & 126.865 & 124.993 & 120.474 & 100.747 & 104.702 & 96.389 & 110.427 & 108.265 & 78.366 & 73.671 \\
\hline 6/27/07 7:30 & 127.239 & 125.084 & 120.842 & 101.058 & 105.033 & 96.726 & 110.734 & 108.668 & 78.402 & 73.837 \\
\hline 6/27/07 8:00 & 127.414 & 125.147 & 121.015 & 101.218 & 105.2 & 96.893 & 110.88 & 108.851 & 78.436 & 73.994 \\
\hline 6/27/07 8:30 & 127.511 & 123.104 & 121.11 & 101.307 & 105.296 & 96.988 & 110.97 & 108.951 & 78.465 & 74.078 \\
\hline 6/27/07 9:00 & 127.577 & 122.896 & 121.177 & 101.368 & 105.361 & 97.049 & 111.028 & 109.02 & 78.495 & 74.147 \\
\hline 6/27/07 9:30 & 125.492 & 122.784 & 119.792 & 100.62 & 104.822 & 96.356 & 110.353 & 107.486 & 78.491 & 74.097 \\
\hline $6 / 27 / 07$ 10:00 & 125.27 & 122.723 & 119.572 & 100.436 & 104.624 & 96.158 & 110.168 & 107.255 & 78.478 & 73.981 \\
\hline $6 / 27 / 07$ 10:30 & 125.151 & 122.673 & 119.459 & 100.332 & 104.515 & 96.052 & 110.071 & 107.134 & 78.463 & 73.894 \\
\hline 6/27/07 11:00 & 125.09 & 122.658 & 119.396 & 100.277 & 104.456 & 95.991 & 110.009 & 107.067 & 78.452 & 73.841 \\
\hline $6 / 27 / 0711: 30$ & 125.038 & 122.626 & 119.348 & 100.232 & 104.407 & 95.943 & 109.969 & 107.017 & 78.439 & 73.789 \\
\hline 6/27/07 12:00 & 125.019 & 122.605 & 119.329 & 100.222 & 104.391 & 95.929 & 109.936 & 106.992 & 78.432 & 73.762 \\
\hline 6/27/07 12:30 & 125.043 & 122.578 & 119.6 & 100.225 & 104.374 & 95.91 & 110.046 & 106.968 & 78.631 & 73.692 \\
\hline $6 / 27 / 07$ 13:00 & 125.005 & 122.555 & 119.278 & 100.172 & 104.347 & 95.885 & 110.024 & 106.946 & 78.742 & 73.694 \\
\hline 6/27/07 13:30 & 124.984 & 122.555 & 119.253 & 100.146 & 104.321 & 95.859 & 110.001 & 106.919 & 78.778 & 73.688 \\
\hline $6 / 27 / 07$ 14:00 & 124.967 & 122.551 & 119.23 & 100.124 & 104.3 & 95.837 & 109.981 & 106.895 & 78.607 & 73.675 \\
\hline 6/27/07 14:30 & 124.956 & 122.515 & 119.23 & 100.129 & 104.302 & 95.84 & 109.979 & 106.894 & 78.478 & 73.651 \\
\hline 6/27/07 15:00 & 124.956 & 122.49 & 119.225 & 100.12 & 104.296 & 95.833 & 109.973 & 106.89 & 78.601 & 73.636 \\
\hline $6 / 27 / 07$ 15:30 & 124.956 & 122.483 & 119.193 & 100.086 & 104.262 & 95.8 & 109.945 & 106.857 & 78.817 & 73.636 \\
\hline $6 / 27 / 07$ 16:00 & 124.941 & 122.472 & 119.165 & 100.096 & 104.235 & 95.771 & & 106.831 & 78.845 & 73.644 \\
\hline 6/27/07 16:30 & 124.927 & 122.449 & 119.158 & 100.057 & 104.23 & 95.769 & 109.915 & 106.824 & 78.81 & 73.655 \\
\hline 6/27/07 17:00 & 124.915 & 122.44 & 119.147 & 100.045 & 104.224 & 95.76 & 109.911 & 106.815 & 78.773 & 73.642 \\
\hline 6/27/07 17:30 & 124.913 & 122.438 & 119.128 & 100.025 & 104.201 & 95.736 & 109.892 & 106.795 & 78.729 & 73.634 \\
\hline 6/27/07 18:00 & 124.908 & 122.435 & 119.114 & 100.012 & 104.19 & 95.724 & 109.879 & 106.782 & 78.679 & 73.622 \\
\hline 6/27/07 18:30 & 124.896 & 124.145 & 119.112 & 100.012 & 104.19 & 95.724 & 109.877 & 106.78 & 78.616 & 73.605 \\
\hline 6/27/07 19:00 & 124.889 & 124.633 & 119.11 & 100.01 & 104.184 & 95.72 & 109.872 & 106.775 & 78.517 & 73.595 \\
\hline 6/27/07 19:30 & 126.395 & 124.832 & 120.236 & 100.52 & 104.439 & 96.127 & 110.277 & 107.776 & 78.425 & 73.586 \\
\hline 6/27/07 20:00 & 127.007 & 122.966 & 120.795 & 100.939 & 104.896 & 96.603 & 110.726 & 108.467 & 78.391 & 73.704 \\
\hline 6/27/07 20:30 & 127.232 & 122.732 & 121.001 & 101.126 & 105.092 & 96.799 & 110.904 & 108.684 & 78.382 & 73.816 \\
\hline $6 / 27 / 0721: 00$ & 125.454 & 122.63 & 119.66 & 100.484 & 104.687 & 96.224 & 110.339 & 107.357 & 78.366 & 73.839 \\
\hline 6/27/07 21:30 & 125.218 & 122.576 & 119.413 & 100.282 & 104.473 & 96.007 & 110.135 & 107.092 & 78.334 & 73.756 \\
\hline 6/27/07 22:00 & 125.097 & 122.531 & 119.306 & 100.189 & 104.374 & 95.908 & 110.044 & 106.983 & 78.316 & 73.69 \\
\hline 6/27/07 22:30 & 125.04 & 122.499 & 119.258 & 100.141 & 104.323 & 95.859 & 109.996 & 106.926 & 78.294 & 73.636 \\
\hline 6/27/07 23:00 & 125.003 & 122.478 & 119.214 & 100.098 & 104.277 & 95.816 & 109.954 & 106.877 & 78.275 & 73.599 \\
\hline 6/27/07 23:30 & 124.977 & 122.469 & 119.179 & 100.07 & 104.249 & 95.788 & 109.924 & 106.846 & 78.257 & 73.572 \\
\hline 6/28/07 0:00 & 124.951 & 122.46 & 119.154 & 100.05 & 104.228 & 95.767 & 109.904 & 106.822 & 78.242 & 73.547 \\
\hline 6/28/07 0:30 & 124.934 & 122.447 & 119.144 & 100.041 & 104.218 & 95.757 & 109.894 & 106.811 & 78.235 & 73.529 \\
\hline 6/28/07 1:00 & 124.917 & 122.438 & 119.135 & 100.034 & 104.212 & 95.753 & 109.887 & 106.804 & 78.229 & 73.51 \\
\hline 6/28/07 1:30 & 124.906 & 122.428 & 119.126 & 100.022 & 104.201 & 95.738 & 109.874 & 106.791 & 78.222 & \\
\hline 6/28/07 2:00 & 124.894 & 122.417 & 119.117 & 100.012 & 104.19 & 95.729 & 109.866 & 106.782 & 78.214 & 73.487 \\
\hline
\end{tabular}


TABLE S1.1 (Cont.)

Depth from Top of Casing (ft)

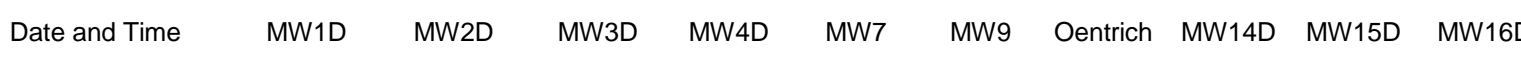

\begin{tabular}{|c|c|c|c|c|c|c|c|c|c|c|}
\hline 6/28/07 2:30 & 124.884 & 122.406 & 119.107 & 100.007 & 104.184 & 95.722 & 109.859 & 106.773 & 78.205 & 73.473 \\
\hline 6/28/07 3:00 & 124.875 & 122.397 & 119.096 & 99.991 & 104.167 & 95.703 & 109.845 & 106.757 & 78.196 & 73.462 \\
\hline 6/28/07 3:30 & 124.865 & 122.385 & 119.084 & 99.984 & 104.159 & 95.696 & 109.836 & 106.749 & 78.19 & 73.452 \\
\hline 6/28/07 4:00 & 124.856 & 122.381 & 119.075 & 99.974 & 104.152 & 95.689 & 109.83 & 106.74 & 78.183 & 73.444 \\
\hline 6/28/07 4:30 & 124.847 & 122.379 & 119.065 & 99.963 & 104.142 & 95.677 & 109.821 & 106.731 & 78.172 & 73.429 \\
\hline 6/28/07 5:00 & 124.839 & 122.383 & 119.059 & 99.958 & 104.134 & 95.668 & 109.813 & 106.722 & 78.166 & 73.417 \\
\hline 6/28/07 5:30 & 124.832 & 122.385 & 119.054 & 99.957 & 104.134 & 95.672 & 109.812 & 106.722 & 78.161 & 73.405 \\
\hline 6/28/07 6:00 & 124.806 & 124.399 & 119.056 & 99.963 & 104.14 & 95.677 & 109.815 & 106.726 & 78.155 & 73.394 \\
\hline 6/28/07 6:30 & 124.766 & 124.705 & 119.061 & 99.967 & 104.142 & 95.679 & 109.815 & 106.728 & 78.152 & 73.384 \\
\hline 6/28/07 7:00 & 126.759 & 124.859 & 120.55 & 100.732 & 104.675 & 96.38 & 110.499 & 108.205 & 78.17 & 73.425 \\
\hline 6/28/07 7:30 & 127.154 & 124.968 & 120.872 & 101.01 & 104.97 & 96.679 & 110.775 & 108.55 & 78.207 & 73.564 \\
\hline 6/28/07 8:00 & 127.319 & 123.036 & 121.034 & 101.158 & 105.126 & 96.832 & 110.919 & 108.719 & 78.246 & 73.698 \\
\hline 6/28/07 8:30 & 127.43 & 122.789 & 121.142 & 101.259 & 105.233 & 96.938 & 111.011 & 108.834 & 78.281 & 73.795 \\
\hline 6/28/07 9:00 & 125.431 & 122.671 & 119.734 & 100.554 & 104.765 & 96.297 & 110.375 & 107.432 & 78.29 & 73.797 \\
\hline 6/28/07 9:30 & 125.161 & 122.596 & 119.47 & 100.337 & 104.532 & 96.064 & 110.153 & 107.154 & 78.292 & 73.721 \\
\hline 6/28/07 10:00 & 125.043 & 122.549 & 119.35 & 100.231 & 104.418 & 95.951 & 110.048 & 107.03 & 78.29 & 73.673 \\
\hline $6 / 28 / 07$ 10:30 & 124.962 & 122.51 & 119.274 & 100.163 & 104.344 & 95.88 & 109.982 & 106.948 & 78.283 & 73.63 \\
\hline 6/28/07 11:00 & 124.915 & 122.485 & 119.225 & 100.12 & 104.3 & 95.837 & 109.939 & 106.897 & 78.277 & 73.605 \\
\hline 6/28/07 11:30 & 124.877 & 122.458 & 119.191 & 100.084 & 104.264 & 95.802 & 109.911 & 106.859 & 78.27 & 73.597 \\
\hline 6/28/07 12:00 & 124.849 & 122.444 & 119.163 & 100.06 & 104.239 & 95.776 & 109.888 & 106.833 & 78.262 & 73.599 \\
\hline 6/28/07 12:30 & 124.825 & 122.428 & 119.14 & 100.038 & 104.216 & 95.755 & 109.868 & 106.808 & 78.257 & 73.599 \\
\hline 6/28/07 13:00 & 124.806 & 122.413 & 119.121 & 100.022 & 104.199 & 95.738 & 109.855 & 106.788 & 78.246 & 73.603 \\
\hline 6/28/07 13:30 & 124.795 & 122.394 & 119.107 & 100.01 & 104.186 & 95.727 & 109.843 & 106.777 & 78.236 & 73.603 \\
\hline 6/28/07 14:00 & 124.776 & 122.381 & 119.093 & 99.994 & 104.172 & 95.71 & 109.832 & 106.762 & 78.227 & 73.586 \\
\hline $6 / 28 / 07$ 14:30 & 124.759 & 122.37 & 119.075 & 99.977 & 104.155 & 95.694 & 109.819 & 106.742 & 78.214 & 73.56 \\
\hline 6/28/07 15:00 & 124.745 & 122.356 & 119.059 & 99.964 & 104.138 & 95.677 & 109.806 & 106.726 & 78.205 & 73.545 \\
\hline 6/28/07 15:30 & 124.735 & 122.34 & 119.051 & 99.955 & 104.134 & 95.67 & 109.796 & 106.717 & 78.194 & 73.529 \\
\hline 6/28/07 16:00 & 124.721 & 122.336 & 119.04 & 99.939 & 104.119 & 95.658 & 109.787 & 106.704 & 78.187 & 73.518 \\
\hline 6/28/07 16:30 & 124.702 & 122.333 & 119.022 & 99.926 & 104.102 & 95.639 & 109.77 & 106.686 & 78.178 & 73.508 \\
\hline 6/28/07 17:00 & 124.695 & 122.324 & 119.015 & 99.92 & 104.098 & 95.632 & 109.764 & 106.68 & 78.166 & 73.5 \\
\hline 6/28/07 17:30 & 124.698 & 122.322 & 119.012 & 99.92 & 104.096 & 95.63 & 109.763 & 106.678 & 78.155 & 73.491 \\
\hline 6/28/07 18:00 & 124.688 & 122.317 & 119.006 & 99.91 & 104.089 & 95.625 & 109.755 & 106.671 & 78.15 & 73.487 \\
\hline 6/28/07 18:30 & 124.683 & 122.311 & 119.001 & 99.908 & 104.087 & 95.623 & 109.751 & 106.667 & 78.141 & 73.485 \\
\hline 6/28/07 19:00 & 124.679 & 122.311 & 118.996 & 99.905 & 104.081 & 95.618 & 109.746 & 106.662 & 78.133 & 73.479 \\
\hline 6/28/07 19:30 & 124.674 & 122.311 & 118.989 & 99.9 & 104.075 & 95.611 & 109.742 & 106.655 & 78.131 & 73.473 \\
\hline 6/28/07 20:00 & 124.669 & 122.304 & 118.991 & 99.898 & 104.075 & 95.613 & 109.738 & 106.655 & 78.124 & 73.473 \\
\hline 6/28/07 20:30 & 124.672 & 122.302 & 118.991 & 99.9 & 104.075 & 95.611 & 109.74 & 106.655 & 78.12 & 73.473 \\
\hline 6/28/07 21:00 & 124.667 & 122.302 & 118.985 & 99.893 & 104.068 & 95.606 & 109.734 & 106.649 & 78.115 & 73.467 \\
\hline 6/28/07 21:30 & 124.662 & 122.304 & 118.98 & 99.891 & 104.068 & 95.602 & 109.731 & 106.646 & 78.109 & 73.462 \\
\hline 6/28/07 22:00 & 124.66 & 122.306 & 118.98 & 99.891 & 104.066 & 95.602 & 109.729 & 106.644 & 78.104 & 73.467 \\
\hline 6/28/07 22:30 & 124.662 & 122.306 & 118.982 & 99.895 & 104.07 & 95.604 & 109.731 & 106.646 & 78.102 & 73.464 \\
\hline 6/28/07 23:00 & 124.667 & 122.304 & 118.987 & 99.898 & 104.073 & 95.609 & 109.736 & 106.651 & 78.1 & 73.469 \\
\hline 6/28/07 23:30 & 124.667 & 122.299 & 118.987 & 99.898 & 104.073 & 95.609 & 109.734 & 106.651 & 78.095 & 73.467 \\
\hline 6/29/07 0:00 & 124.665 & 122.292 & 118.987 & 99.896 & 104.073 & 95.609 & 109.732 & 106.651 & 78.093 & 73.469 \\
\hline 6/29/07 0:30 & 124.658 & 122.277 & 118.98 & 99.891 & 104.07 & 95.604 & 109.727 & 106.644 & 78.085 & 73.46 \\
\hline 6/29/07 1:00 & 124.655 & 122.27 & 118.975 & 99.886 & 104.062 & 95.597 & 109.723 & & 78.082 & 73.458 \\
\hline 6/29/07 1:30 & 124.655 & 122.261 & 118.959 & 99.87 & 104.047 & 95.58 & 109.708 & 106.624 & 78.074 & 73.448 \\
\hline 6/29/07 2:00 & 124.65 & 122.252 & 118.952 & 99.86 & 104.039 & 95.571 & 109.702 & 106.615 & 78.067 & 73.44 \\
\hline 6/29/07 2:30 & 124.648 & 122.236 & 118.941 & 99.853 & 104.03 & 95.562 & 109.693 & 106.607 & 78.061 & 73.431 \\
\hline 6/29/07 3:00 & 124.644 & 122.229 & 118.936 & 99.846 & 104.024 & 95.557 & 109.686 & 106.598 & 78.054 & 73.423 \\
\hline 6/29/07 3:30 & 124.613 & 122.222 & 118.927 & 99.831 & 104.009 & 95.543 & 109.674 & 106.587 & 78.047 & 73.415 \\
\hline 6/29/07 4:00 & 124.606 & 122.213 & 118.913 & 99.822 & 103.999 & 95.533 & 109.665 & 106.576 & 78.039 & 73.407 \\
\hline 6/29/07 4:30 & 124.603 & 122.215 & 118.906 & 99.815 & 103.995 & 95.526 & 109.657 & 106.567 & 78.034 & 73.4 \\
\hline 6/29/07 5:00 & 124.601 & 122.211 & 118.899 & 99.808 & 103.986 & 95.519 & 109.648 & 106.56 & 78.025 & 73.394 \\
\hline 6/29/07 5:30 & 124.596 & 122.222 & 118.894 & 99.81 & 103.99 & 95.519 & 109.65 & 106.558 & 78.019 & 73.392 \\
\hline 6/29/07 6:00 & 124.591 & 124.14 & 118.892 & 99.808 & 103.986 & 95.519 & 109.646 & 106.558 & 78.015 & 73.39 \\
\hline 6/29/07 6:30 & 124.594 & 124.508 & 118.899 & 99.815 & 103.992 & 95.524 & 109.654 & 106.569 & 78.012 & 73.392 \\
\hline 6/29/07 7:00 & 126.52 & 124.68 & 120.141 & 100.434 & 104.391 & 96.068 & 110.191 & 107.919 & 78.021 & 73.419 \\
\hline 6/29/07 7:30 & 126.92 & 124.787 & 120.536 & 100.761 & 104.74 & 96.427 & 110.523 & 108.358 & 78.058 & 73.593 \\
\hline 6/29/07 8:00 & 127.099 & 124.85 & 120.71 & 100.921 & 104.911 & 96.599 & 110.681 & 108.544 & 78.091 & 73.737 \\
\hline 6/29/07 8:30 & 127.213 & 124.902 & 120.823 & 101.027 & 105.02 & 96.707 & 110.778 & 108.659 & 78.124 & 73.841 \\
\hline 6/29/07 9:00 & 127.277 & 124.934 & 120.89 & 101.092 & 105.088 & 96.776 & 110.84 & 108.73 & 78.154 & 73.919 \\
\hline 6/29/07 9:30 & 127.333 & 123.281 & 120.943 & 101.139 & 105.138 & 96.823 & 110.883 & 108.785 & 78.181 & 73.973 \\
\hline 6/29/07 10:00 & 127.366 & 122.787 & 120.976 & 101.171 & 105.174 & 96.86 & 110.915 & 108.818 & 78.207 & 74.008 \\
\hline 6/29/07 10:30 & 125.766 & 122.608 & 120.058 & 100.735 & 104.978 & 96.53 & 110.585 & 107.877 & 78.225 & 74.037 \\
\hline 6/29/07 11:00 & 125.168 & 122.499 & 119.482 & 100.336 & 104.542 & 96.066 & 110.149 & 107.171 & 78.211 & 73.917 \\
\hline
\end{tabular}


TABLE S1.1 (Cont.)

Depth from Top of Casing (ft)

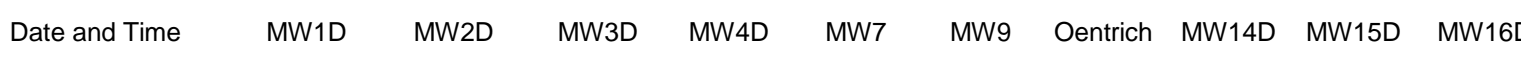

\begin{tabular}{|c|c|c|c|c|c|c|c|c|c|c|}
\hline 6/29/07 11:30 & 124.979 & 122.424 & 119.295 & 100.172 & 104.367 & 95.896 & 109.988 & 106.977 & 78.196 & 73.797 \\
\hline 6/29/07 12:00 & 124.868 & 122.372 & 119.188 & 100.075 & 104.264 & 95.793 & 109.894 & 106.861 & 78.181 & 73.702 \\
\hline 6/29/07 12:30 & 124.792 & 122.333 & 119.11 & 100.003 & 104.19 & 95.72 & 109.828 & 106.781 & 78.166 & 73.632 \\
\hline 6/29/07 13:00 & 124.738 & 122.299 & 119.056 & 99.955 & 104.138 & 95.672 & 109.783 & 106.726 & 78.152 & 73.58 \\
\hline 6/29/07 13:30 & 124.7 & 122.265 & 119.019 & 99.92 & 104.102 & 95.637 & 109.749 & 106.689 & 78.137 & 73.537 \\
\hline 6/29/07 14:00 & 124.667 & 122.245 & 118.984 & 99.888 & 104.068 & 95.602 & 109.719 & 106.651 & 78.122 & 73.504 \\
\hline 6/29/07 14:30 & 124.634 & 122.224 & 118.952 & 99.857 & 104.037 & 95.573 & 109.691 & 106.618 & 78.107 & 73.475 \\
\hline 6/29/07 15:00 & 124.61 & 122.213 & 118.932 & 99.836 & 104.016 & 95.552 & 109.672 & 106.596 & 78.093 & 73.454 \\
\hline 6/29/07 15:30 & 124.587 & 122.202 & 118.911 & 99.815 & 103.999 & 95.529 & 109.654 & 106.576 & 78.08 & 73.431 \\
\hline 6/29/07 16:00 & 124.575 & 122.186 & 118.897 & 99.807 & 103.988 & 95.522 & 109.644 & 106.562 & 78.067 & 73.421 \\
\hline 6/29/07 16:30 & 124.568 & 122.17 & 118.888 & 99.796 & 103.976 & 95.51 & 109.633 & 106.553 & 78.056 & 73.408 \\
\hline 6/29/07 17:00 & 124.554 & 122.159 & 118.876 & 99.784 & 103.967 & 95.498 & 109.624 & 106.538 & 78.043 & 73.398 \\
\hline 6/29/07 17:30 & 124.532 & 122.147 & 118.855 & 99.765 & 103.948 & 95.479 & 109.605 & 106.518 & 78.026 & 73.382 \\
\hline 6/29/07 18:00 & 124.52 & 122.145 & 118.843 & 99.757 & 103.934 & 95.472 & 109.595 & 106.507 & 78.015 & 73.369 \\
\hline 6/29/07 18:30 & 124.511 & 122.147 & 118.832 & 99.746 & 103.925 & 95.46 & 109.586 & 106.496 & 78.002 & 73.359 \\
\hline 6/29/07 19:00 & 124.509 & 122.147 & 118.832 & 99.745 & 103.925 & 95.456 & 109.584 & 106.494 & 77.995 & 73.355 \\
\hline 6/29/07 19:30 & 124.509 & 122.141 & 118.834 & 99.748 & 103.927 & 95.463 & 109.586 & 106.496 & 77.988 & 73.357 \\
\hline 6/29/07 20:00 & 124.509 & 122.138 & 118.834 & 99.747 & 103.929 & 95.46 & 109.584 & 106.496 & 77.98 & 73.359 \\
\hline 6/29/07 20:30 & 124.504 & 122.141 & 118.83 & 99.741 & 103.925 & 95.458 & 109.584 & 106.491 & 77.971 & 73.347 \\
\hline 6/29/07 21:00 & 124.502 & 122.141 & 118.823 & 99.738 & 103.919 & 95.451 & 109.577 & 106.487 & 77.967 & 73.345 \\
\hline 6/29/07 21:30 & 124.499 & 122.147 & 118.825 & 99.74 & 103.921 & 95.453 & 109.577 & 106.487 & 77.962 & 73.345 \\
\hline 6/29/07 22:00 & 124.499 & 122.15 & 118.827 & 99.743 & 103.921 & 95.456 & 109.575 & 106.487 & 77.956 & 73.347 \\
\hline 6/29/07 22:30 & 124.506 & 122.147 & 118.832 & 99.748 & 103.931 & 95.463 & 109.58 & 106.494 & 77.953 & 73.351 \\
\hline 6/29/07 23:00 & 124.509 & 122.15 & 118.834 & 99.75 & 103.931 & 95.463 & 109.579 & 106.496 & 77.947 & 73.351 \\
\hline 6/29/07 23:30 & 124.509 & 122.143 & 118.832 & 99.748 & 103.929 & 95.463 & 109.577 & 106.496 & 77.943 & 73.349 \\
\hline 6/30/07 0:00 & 124.509 & 122.131 & 118.832 & 99.752 & 103.931 & 95.467 & 109.573 & 106.496 & 77.942 & 73.355 \\
\hline 6/30/07 0:30 & 124.502 & 122.12 & 118.827 & 99.743 & 103.925 & 95.458 & 109.569 & 106.49 & 77.934 & 73.349 \\
\hline 6/30/07 1:00 & 124.492 & 122.109 & 118.818 & 99.735 & 103.917 & 95.448 & 109.565 & 106.48 & 77.927 & 73.338 \\
\hline 6/30/07 1:30 & 124.48 & 122.095 & 118.807 & 99.721 & 103.904 & 95.432 & 109.556 & 106.467 & 77.918 & 73.324 \\
\hline 6/30/07 2:00 & 124.476 & 122.084 & 118.797 & 99.712 & 103.896 & 95.425 & 109.55 & 106.458 & 77.91 & 73.318 \\
\hline 6/30/07 2:30 & 124.473 & 122.075 & 118.781 & 99.696 & 103.879 & 95.408 & 109.533 & 106.443 & 77.899 & 73.303 \\
\hline 6/30/07 3:00 & 124.466 & 122.063 & 118.772 & 99.69 & 103.872 & 95.401 & 109.526 & 106.434 & 77.89 & 73.297 \\
\hline 6/30/07 3:30 & 124.435 & 122.057 & 118.763 & 99.683 & 103.862 & 95.392 & 109.517 & 106.425 & 77.881 & 73.289 \\
\hline 6/30/07 4:00 & 124.424 & 122.052 & 118.755 & 99.679 & 103.851 & 95.38 & 109.505 & 106.414 & 77.871 & 73.276 \\
\hline 6/30/07 4:30 & 124.416 & 122.048 & 118.744 & 99.678 & 103.845 & 95.373 & 109.498 & 106.405 & 77.862 & 73.272 \\
\hline 6/30/07 5:00 & 124.414 & 122.041 & 118.74 & 99.676 & 103.841 & 95.371 & 109.496 & 106.401 & 77.855 & 73.268 \\
\hline 6/30/07 5:30 & 124.409 & 122.036 & 118.732 & 99.674 & 103.835 & 95.364 & 109.487 & 106.394 & 77.848 & 73.26 \\
\hline 6/30/07 6:00 & 124.405 & 122.034 & 118.728 & 99.672 & 103.832 & 95.361 & 109.481 & 106.388 & 77.84 & 73.258 \\
\hline 6/30/07 6:30 & 124.402 & 123.013 & 118.721 & 99.671 & 103.826 & 95.357 & 109.479 & 106.387 & 77.833 & 73.254 \\
\hline 6/30/07 7:00 & 124.398 & 124.208 & 118.719 & 99.64 & 103.824 & 95.352 & 109.477 & 106.383 & 77.827 & 73.252 \\
\hline 6/30/07 7:30 & 124.395 & 124.444 & 118.721 & 99.757 & 103.826 & 95.354 & 109.479 & 106.385 & 77.824 & 73.254 \\
\hline 6/30/07 8:00 & 126.612 & 124.567 & 120.229 & 100.475 & 104.45 & 96.134 & 110.232 & 108.043 & 77.853 & 73.365 \\
\hline 6/30/07 8:30 & 126.86 & 124.653 & 120.476 & 100.692 & 104.681 & 96.368 & 110.45 & 108.305 & 77.888 & 73.535 \\
\hline 6/30/07 9:00 & 126.99 & 124.71 & 120.605 & 100.813 & 104.808 & 96.493 & 110.564 & 108.44 & 77.919 & 73.648 \\
\hline 6/30/07 9:30 & 127.078 & 124.744 & 120.693 & 100.894 & 104.894 & 96.575 & 110.641 & 108.53 & 77.951 & 73.739 \\
\hline $6 / 30 / 07$ 10:00 & 127.137 & 124.778 & 120.749 & 100.947 & 104.951 & 96.634 & 110.694 & 108.592 & 77.977 & 73.799 \\
\hline $6 / 30 / 07$ 10:30 & 127.175 & 123.018 & 120.784 & 100.982 & 104.985 & 96.669 & 110.726 & 108.626 & 78.001 & 73.837 \\
\hline 6/30/07 11:00 & 127.208 & 122.58 & 120.823 & 101.018 & 105.024 & 96.705 & 110.756 & 108.663 & 78.021 & 73.876 \\
\hline $6 / 30 / 07$ 11:30 & 125.454 & 122.404 & 119.761 & 100.504 & 104.746 & 96.283 & 110.335 & 107.526 & 78.032 & 73.894 \\
\hline 6/30/07 12:00 & 124.96 & 122.292 & 119.278 & 100.143 & 104.351 & 95.877 & 109.956 & 106.967 & 78.01 & 73.758 \\
\hline 6/30/07 12:30 & 124.773 & 122.215 & 119.096 & 99.979 & 104.178 & 95.705 & 109.796 & 106.777 & 77.991 & 73.63 \\
\hline $6 / 30 / 07$ 13:00 & 124.66 & 122.159 & 118.984 & 99.877 & 104.073 & 95.602 & 109.7 & 106.66 & 77.975 & 73.539 \\
\hline 6/30/07 13:30 & 124.582 & 122.122 & 118.906 & 99.805 & 103.995 & 95.526 & 109.633 & 106.578 & 77.953 & 73.462 \\
\hline 6/30/07 14:00 & 124.523 & 122.093 & 118.851 & 99.755 & 103.942 & 95.47 & 109.582 & 106.518 & 77.934 & 73.405 \\
\hline 6/30/07 14:30 & 124.487 & 122.066 & 118.814 & 99.722 & 103.908 & 95.439 & 109.552 & 106.48 & 77.919 & 73.365 \\
\hline $6 / 30 / 07$ 15:00 & 124.457 & 122.048 & 118.784 & 99.693 & 103.881 & 95.411 & 109.526 & 106.449 & 77.907 & 73.334 \\
\hline 6/30/07 15:30 & 124.428 & 122.027 & 118.755 & 99.667 & 103.855 & 95.385 & 109.501 & 106.421 & 77.888 & 73.307 \\
\hline 6/30/07 16:00 & 124.407 & 122.014 & 118.733 & 99.648 & 103.832 & 95.361 & 109.483 & 106.398 & 77.871 & 73.287 \\
\hline 6/30/07 16:30 & 124.39 & 122 & 118.716 & 99.633 & 103.818 & 95.349 & 109.472 & 106.383 & 77.859 & 73.274 \\
\hline 6/30/07 17:00 & 124.376 & 121.984 & 118.705 & 99.621 & 103.805 & 95.333 & 109.46 & 106.37 & 77.846 & 73.258 \\
\hline 6/30/07 17:30 & 124.362 & 121.977 & 118.691 & 99.607 & 103.792 & 95.324 & 109.447 & 106.354 & 77.829 & 73.243 \\
\hline 6/30/07 18:00 & 124.346 & 121.968 & 118.672 & 99.593 & 103.776 & 95.307 & 109.437 & 106.339 & 77.816 & 73.229 \\
\hline 6/30/07 18:30 & 124.336 & 121.964 & 118.665 & 99.584 & 103.771 & 95.298 & 109.428 & 106.33 & 77.801 & 73.218 \\
\hline 6/30/07 19:00 & 124.331 & 121.955 & 118.659 & 99.579 & 103.763 & 95.293 & 109.421 & 106.321 & 77.79 & 73.21 \\
\hline 6/30/07 19:30 & 124.327 & 121.957 & 118.654 & 99.576 & 103.759 & 95.288 & 109.413 & 106.319 & 77.777 & 73.202 \\
\hline $6 / 30 / 0720: 00$ & 124.315 & 121.959 & 118.645 & 99.567 & 103.752 & 95.279 & 109.411 & 106.308 & 77.766 & 73.196 \\
\hline
\end{tabular}


TABLE S1.1 (Cont.)

\begin{tabular}{|c|c|c|c|c|c|c|c|c|c|c|}
\hline \multirow[b]{2}{*}{ Date and Time } & \multicolumn{10}{|c|}{ Depth from Top of Casing (ft) } \\
\hline & MW1D & MW2D & MW3D & MW4D & MW7 & MW9 & Oentrich & MW14D & MW15D & MW16D \\
\hline 6/30/07 20:30 & 124.315 & 121.959 & 118.645 & 99.567 & 103.752 & 95.279 & 109.406 & 106.308 & 77.759 & 73.196 \\
\hline 6/30/07 21:00 & 124.317 & 121.966 & 118.649 & 99.572 & 103.759 & 95.283 & 109.409 & 106.312 & 77.754 & 73.198 \\
\hline 6/30/07 21:30 & 124.317 & 121.973 & 118.647 & 99.572 & 103.757 & 95.283 & 109.407 & 106.312 & 77.746 & 73.198 \\
\hline 6/30/07 22:00 & 124.327 & 121.975 & 118.654 & 99.583 & 103.767 & 95.295 & 109.411 & 106.319 & 77.742 & 73.204 \\
\hline 6/30/07 22:30 & 124.331 & 121.982 & 118.661 & 99.59 & 103.773 & 95.3 & 109.411 & 106.328 & 77.739 & 73.21 \\
\hline 6/30/07 23:00 & 124.334 & 121.982 & 118.663 & 99.59 & 103.775 & 95.302 & 109.411 & 106.328 & 77.735 & 73.208 \\
\hline 6/30/07 23:30 & 124.341 & 121.982 & 118.67 & 99.598 & 103.78 & 95.309 & 109.409 & 106.334 & 77.733 & 73.216 \\
\hline 7/1/07 0:00 & 124.343 & 121.98 & 118.673 & 99.596 & 103.782 & 95.309 & 109.407 & 106.337 & 77.731 & 73.218 \\
\hline 7/1/07 0:30 & 124.343 & 121.973 & 118.672 & 99.598 & 103.784 & 95.314 & 109.406 & 106.339 & 77.724 & 73.214 \\
\hline 7/1/07 1:00 & 124.346 & 121.961 & 118.673 & 99.598 & 103.784 & 95.312 & 109.404 & 106.339 & 77.72 & 73.216 \\
\hline 7/1/07 1:30 & 124.334 & 121.952 & 118.661 & 99.586 & 103.773 & 95.3 & 109.402 & 106.325 & 77.711 & 73.204 \\
\hline 7/1/07 2:00 & 124.322 & 121.943 & 118.654 & 99.578 & 103.763 & 95.291 & 109.396 & 106.314 & 77.7 & 73.196 \\
\hline $7 / 1 / 072: 30$ & 124.312 & 121.934 & 118.645 & 99.571 & 103.756 & 95.283 & 109.389 & 106.308 & 77.695 & 73.185 \\
\hline 7/1/07 3:00 & 124.305 & 121.927 & 118.633 & 99.559 & 103.744 & 95.272 & 109.385 & 106.299 & 77.683 & 73.181 \\
\hline 7/1/07 3:30 & 124.294 & 121.93 & 118.624 & 99.55 & 103.737 & 95.265 & 109.379 & 106.29 & 77.676 & 73.169 \\
\hline $7 / 1 / 074: 00$ & 124.286 & 121.927 & 118.619 & 99.545 & 103.731 & 95.258 & 109.374 & 106.281 & 77.669 & 73.165 \\
\hline $7 / 1 / 074: 30$ & 124.282 & 121.93 & 118.619 & 99.547 & 103.733 & 95.262 & 109.372 & 106.284 & 77.665 & 73.169 \\
\hline 7/1/07 5:00 & 124.284 & 121.925 & 118.617 & 99.547 & 103.733 & 95.26 & 109.368 & 106.281 & 77.658 & 73.169 \\
\hline 7/1/07 5:30 & 124.289 & 121.927 & 118.619 & 99.55 & 103.735 & 95.265 & 109.366 & 106.284 & 77.654 & 73.171 \\
\hline 7/1/07 6:00 & 124.286 & 121.925 & 118.617 & 99.547 & 103.731 & 95.26 & 109.362 & 106.279 & 77.647 & 73.165 \\
\hline 7/1/07 6:30 & 124.282 & 121.93 & 118.619 & 99.547 & 103.734 & 95.262 & 109.36 & 106.281 & 77.645 & 73.169 \\
\hline 7/1/07 7:00 & 124.279 & 122.968 & 118.617 & 99.547 & 103.733 & 95.26 & 109.359 & 106.281 & 77.639 & 73.167 \\
\hline 7/1/07 7:30 & 124.289 & 124.093 & 118.622 & 99.553 & 103.737 & 95.265 & 109.357 & 106.283 & 77.636 & 73.171 \\
\hline 7/1/07 8:00 & 124.286 & 124.329 & 118.619 & 99.719 & 103.733 & 95.262 & 109.355 & 106.283 & 77.63 & 73.167 \\
\hline 7/1/07 8:30 & 126.53 & 124.465 & 120.273 & 100.444 & 104.405 & 96.104 & 110.16 & 107.93 & 77.667 & 73.293 \\
\hline 7/1/07 9:00 & 126.78 & 124.558 & 120.518 & 100.665 & 104.635 & 96.335 & 110.374 & 108.19 & 77.7 & 73.462 \\
\hline 7/1/07 9:30 & 126.922 & 124.61 & 120.654 & 100.79 & 104.768 & 96.467 & 110.495 & 108.333 & 77.735 & 73.591 \\
\hline $7 / 1 / 07$ 10:00 & 127.017 & 124.653 & 120.749 & 100.878 & 104.858 & 96.559 & 110.572 & 108.433 & 77.765 & 73.682 \\
\hline 7/1/07 10:30 & 127.073 & 124.685 & 120.804 & 100.928 & 104.915 & 96.613 & 110.624 & 108.491 & 77.792 & 73.739 \\
\hline 7/1/07 11:00 & 127.116 & 124.707 & 120.849 & 100.97 & 104.955 & 96.651 & 110.662 & 108.535 & 77.822 & 73.785 \\
\hline 7/1/07 11:30 & 127.149 & 124.728 & 120.883 & 100.999 & 104.989 & 96.688 & 110.69 & 108.57 & 77.851 & 73.816 \\
\hline 7/1/07 12:00 & 127.175 & 122.973 & 120.904 & 101.023 & 105.008 & 96.71 & 110.711 & 108.591 & 77.87 & 73.843 \\
\hline 7/1/07 12:30 & 127.194 & 122.533 & 120.925 & 101.042 & 105.029 & 96.726 & 110.726 & 108.612 & 77.894 & 73.863 \\
\hline 7/1/07 13:00 & 125.412 & 122.347 & 119.722 & 100.465 & 104.706 & 96.245 & 110.245 & 107.472 & 77.91 & 73.876 \\
\hline 7/1/07 13:30 & 124.913 & 122.236 & 119.237 & 100.1 & 104.311 & 95.84 & 109.858 & 106.923 & 77.89 & 73.731 \\
\hline 7/1/07 14:00 & 124.719 & 122.159 & 119.043 & 99.932 & 104.134 & 95.661 & 109.693 & 106.722 & 77.873 & 73.599 \\
\hline 7/1/07 14:30 & 124.603 & 122.102 & 118.927 & 99.829 & 104.024 & 95.552 & 109.597 & 106.605 & 77.853 & 73.5 \\
\hline 7/1/07 15:00 & 124.523 & 122.061 & 118.853 & 99.757 & 103.95 & 95.479 & 109.535 & 106.523 & 77.838 & 73.425 \\
\hline $7 / 1 / 07$ 15:30 & 124.464 & 122.034 & 118.793 & 99.705 & 103.895 & 95.425 & 109.492 & 106.463 & 77.822 & 73.367 \\
\hline 7/1/07 16:00 & 124.421 & 122.007 & 118.751 & 99.669 & 103.856 & 95.387 & 109.458 & 106.421 & 77.801 & 73.326 \\
\hline 7/1/07 16:30 & 124.398 & 121.982 & 118.728 & 99.645 & 103.832 & 95.361 & 109.436 & 106.394 & 77.789 & 73.301 \\
\hline $7 / 1 / 0717: 00$ & 124.369 & 121.966 & 118.7 & 99.619 & 103.807 & 95.338 & 109.417 & 106.368 & 77.774 & 73.272 \\
\hline 7/1/07 17:30 & 124.343 & 121.95 & 118.677 & 99.596 & 103.784 & 95.316 & 109.404 & 106.341 & 77.761 & 73.249 \\
\hline 7/1/07 18:00 & 124.324 & 121.934 & 118.659 & 99.581 & 103.767 & 95.298 & 109.387 & 106.323 & 77.744 & 73.235 \\
\hline $7 / 1 / 07$ 18:30 & 124.31 & 121.925 & 118.645 & 99.567 & 103.754 & 95.283 & 109.376 & 106.308 & 77.733 & 73.218 \\
\hline 7/1/07 19:00 & 124.296 & 121.921 & 118.631 & 99.553 & 103.738 & 95.267 & 109.362 & 106.292 & 77.722 & 73.204 \\
\hline 7/1/07 19:30 & 124.284 & 121.925 & 118.619 & 99.543 & 103.731 & 95.26 & 109.353 & 106.281 & 77.707 & 73.192 \\
\hline $7 / 1 / 0720: 00$ & 124.279 & 121.923 & 118.614 & 99.541 & 103.727 & 95.258 & 109.349 & 106.279 & 77.698 & 73.19 \\
\hline $7 / 1 / 0720: 30$ & 124.282 & 121.921 & 118.617 & 99.545 & 103.733 & 95.262 & 109.344 & 106.281 & 77.693 & 73.192 \\
\hline 7/1/07 21:00 & 124.282 & 121.93 & 118.617 & 99.545 & 103.733 & 95.262 & 109.338 & 106.281 & 77.685 & 73.19 \\
\hline $7 / 1 / 0721: 30$ & 124.279 & 121.934 & 118.612 & 99.543 & 103.731 & 95.26 & 109.332 & 106.277 & 77.676 & 73.187 \\
\hline 7/1/07 22:00 & 124.286 & 121.932 & 118.619 & 99.553 & 103.738 & 95.267 & 109.327 & 106.284 & 77.672 & 73.192 \\
\hline 7/1/07 22:30 & 124.296 & 121.939 & 118.629 & 99.559 & 103.744 & 95.274 & 109.323 & 106.293 & 77.671 & 73.196 \\
\hline 7/1/07 23:00 & 124.291 & 121.939 & 118.626 & 99.557 & 103.744 & 95.274 & 109.319 & 106.29 & 77.667 & 73.194 \\
\hline 7/1/07 23:30 & 124.296 & 121.927 & 118.633 & 99.564 & 103.75 & 95.276 & 109.315 & 106.294 & 77.661 & 73.2 \\
\hline 7/2/07 0:00 & 124.301 & 121.941 & 118.638 & 99.565 & 103.752 & 95.283 & 109.312 & 106.299 & 77.659 & 73.204 \\
\hline 7/2/07 0:30 & 124.286 & 121.923 & 118.619 & 99.552 & 103.738 & 95.267 & 109.31 & 106.281 & 77.652 & 73.187 \\
\hline 7/2/07 1:00 & 124.296 & 121.923 & 118.636 & 99.567 & 103.754 & 95.281 & 109.302 & 106.297 & 77.654 & 73.2 \\
\hline 7/2/07 1:30 & 124.282 & 121.918 & 118.619 & 99.545 & 103.736 & 95.265 & 109.302 & 106.281 & 77.643 & 73.185 \\
\hline $7 / 2 / 072: 00$ & 124.282 & 121.914 & 118.617 & 99.55 & 103.738 & 95.265 & 109.297 & 106.279 & 77.637 & 73.185 \\
\hline $7 / 2 / 072: 30$ & 124.279 & 121.912 & 118.614 & 99.547 & 103.737 & 95.265 & 109.295 & 106.277 & 77.634 & 73.183 \\
\hline 7/2/07 3:00 & 124.275 & 121.912 & 118.61 & 99.543 & 103.729 & 95.258 & 109.289 & 106.273 & 77.628 & 73.179 \\
\hline 7/2/07 3:30 & 124.27 & 121.905 & 118.605 & 99.541 & 103.727 & 95.255 & 109.285 & 106.268 & 77.623 & 73.175 \\
\hline $7 / 2 / 074: 00$ & 124.268 & 121.896 & 118.608 & 99.541 & 103.729 & 95.253 & 109.282 & 106.268 & 77.621 & 73.175 \\
\hline 7/2/07 4:30 & 124.263 & 121.887 & 118.601 & 99.534 & 103.721 & 95.253 & 109.276 & 106.264 & 77.617 & 73.175 \\
\hline 7/2/07 5:00 & 124.26 & 121.884 & 118.591 & 99.524 & 103.712 & 95.241 & 109.274 & 106.253 & 77.608 & 73.165 \\
\hline
\end{tabular}


TABLE S1.1 (Cont.)

Depth from Top of Casing (ft)

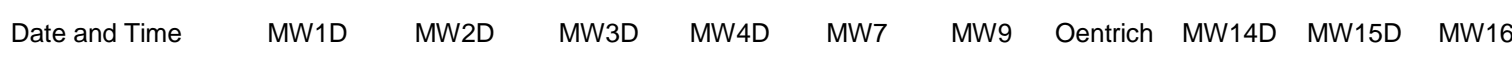

\begin{tabular}{|c|c|c|c|c|c|c|c|c|c|c|}
\hline 7/2/07 5:30 & 124.258 & 121.878 & 118.587 & 99.515 & 103.702 & 95.232 & 109.272 & 106.244 & 77.602 & 73.159 \\
\hline 7/2/07 6:00 & 124.26 & 123.846 & 118.578 & 99.512 & 103.697 & 95.225 & 109.27 & 106.239 & 77.593 & 73.15 \\
\hline 7/2/07 6:30 & 124.253 & 124.188 & 118.575 & 99.51 & 103.696 & 95.225 & 109.265 & 106.235 & 77.589 & 73.148 \\
\hline 7/2/07 7:00 & 126.222 & 124.358 & 119.859 & 100.156 & 104.117 & 95.795 & 109.822 & 107.634 & 77.599 & 73.179 \\
\hline 7/2/07 7:30 & 126.601 & 124.453 & 120.224 & 100.456 & 104.443 & 96.127 & 110.147 & 108.043 & 77.628 & 73.34 \\
\hline 7/2/07 8:00 & 126.778 & 124.517 & 120.402 & 100.618 & 104.614 & 96.299 & 110.301 & 108.232 & 77.663 & 73.487 \\
\hline 7/2/07 8:30 & 126.877 & 124.558 & 120.504 & 100.713 & 104.713 & 96.398 & 110.389 & 108.333 & 77.695 & 73.589 \\
\hline 7/2/07 9:00 & 126.939 & 124.587 & 120.566 & 100.773 & 104.776 & 96.46 & 110.45 & 108.4 & 77.722 & 73.653 \\
\hline 7/2/07 9:30 & 126.983 & 124.608 & 120.608 & 100.815 & 104.82 & 96.5 & 110.491 & 108.444 & 77.742 & 73.696 \\
\hline 7/2/07 10:00 & 127.017 & 124.628 & 120.638 & 100.844 & 104.848 & 96.53 & 110.521 & 108.475 & 77.763 & 73.731 \\
\hline $7 / 2 / 07 \quad 10: 30$ & 127.038 & 123.703 & 120.664 & 100.866 & 104.875 & 96.556 & 110.543 & 108.499 & 77.785 & 73.758 \\
\hline 7/2/07 11:00 & 127.059 & 122.521 & 120.686 & 100.889 & 104.898 & 96.578 & 110.558 & 108.524 & 77.809 & 73.785 \\
\hline 7/2/07 11:30 & 127.071 & 122.297 & 120.696 & 100.772 & 104.911 & 96.589 & 110.572 & 108.533 & 77.827 & 73.808 \\
\hline $7 / 2 / 07$ 12:00 & 124.903 & 122.172 & 119.234 & 100.091 & 104.311 & 95.835 & 109.83 & 106.928 & 77.816 & 73.715 \\
\hline $7 / 2 / 0712: 30$ & 124.667 & 122.088 & 118.996 & 99.888 & 104.094 & 95.618 & 109.629 & 106.68 & 77.796 & 73.576 \\
\hline 7/2/07 13:00 & 124.539 & 122.029 & 118.871 & 99.776 & 103.974 & 95.5 & 109.518 & 106.547 & 77.781 & 73.471 \\
\hline 7/2/07 13:30 & 124.454 & 121.982 & 118.788 & 99.698 & 103.895 & 95.42 & 109.451 & 106.459 & 77.765 & 73.39 \\
\hline $7 / 2 / 0714: 00$ & 124.39 & 121.943 & 118.728 & 99.641 & 103.835 & 95.361 & 109.404 & 106.394 & 77.748 & 73.328 \\
\hline $7 / 2 / 0714: 30$ & 124.343 & 121.912 & 118.677 & 99.598 & 103.788 & 95.319 & 109.37 & 106.346 & 77.733 & 73.283 \\
\hline $7 / 2 / 07$ 15:00 & 124.306 & 121.887 & 118.64 & 99.564 & 103.754 & 95.281 & 109.344 & 106.306 & 77.717 & 73.243 \\
\hline $7 / 2 / 07$ 15:30 & 124.272 & 121.859 & 118.61 & 99.531 & 103.721 & 95.248 & 109.327 & 106.273 & 77.7 & 73.216 \\
\hline $7 / 2 / 0716: 00$ & 124.247 & 121.834 & 118.582 & 99.507 & 103.698 & 95.225 & 109.31 & 106.246 & 77.685 & 73.187 \\
\hline $7 / 2 / 07$ 16:30 & 124.22 & 121.821 & 118.557 & 99.483 & 103.674 & 95.201 & 109.293 & 106.219 & 77.667 & 73.163 \\
\hline $7 / 2 / 0717: 00$ & 124.199 & 121.805 & 118.534 & 99.46 & 103.651 & 95.177 & 109.276 & 106.195 & 77.654 & 73.142 \\
\hline $7 / 2 / 07$ 17:30 & 124.185 & 121.785 & 118.522 & 99.448 & 103.639 & 95.168 & 109.265 & 106.184 & 77.639 & 73.128 \\
\hline $7 / 2 / 07$ 18:00 & 124.164 & 121.771 & 118.504 & 99.433 & 103.622 & 95.151 & 109.252 & 106.164 & 77.623 & 73.111 \\
\hline $7 / 2 / 07$ 18:30 & 124.145 & 121.762 & 118.485 & 99.414 & 103.603 & 95.13 & 109.238 & 106.146 & 77.606 & 73.092 \\
\hline $7 / 2 / 07$ 19:00 & 124.128 & 121.762 & 118.469 & 99.4 & 103.59 & 95.116 & 109.227 & 106.131 & 77.593 & 73.08 \\
\hline $7 / 2 / 07$ 19:30 & 124.119 & 121.76 & 118.457 & 99.393 & 103.584 & 95.109 & 109.218 & 106.12 & 77.58 & 73.07 \\
\hline $7 / 2 / 0720: 00$ & 124.121 & 121.755 & 118.46 & 99.397 & 103.584 & 95.109 & 109.218 & 106.122 & 77.571 & 73.072 \\
\hline $7 / 2 / 0720: 30$ & 124.116 & 121.748 & 118.455 & 99.393 & 103.584 & 95.109 & 109.212 & 106.118 & 77.56 & 73.066 \\
\hline $7 / 2 / 0721: 00$ & 124.116 & 121.751 & 118.457 & 99.391 & 103.58 & 95.109 & 109.21 & 106.117 & 77.553 & 73.068 \\
\hline $7 / 2 / 0721: 30$ & 124.104 & 121.762 & 118.446 & 99.383 & 103.573 & 95.097 & 109.203 & 106.107 & 77.542 & 73.055 \\
\hline $7 / 2 / 07$ 22:00 & 124.107 & 121.764 & 118.448 & 99.388 & 103.575 & 95.102 & 109.203 & 106.111 & 77.536 & 73.059 \\
\hline $7 / 2 / 07$ 22:30 & 124.119 & 121.769 & 118.46 & 99.4 & 103.588 & 95.116 & 109.203 & 106.122 & 77.532 & 73.07 \\
\hline $7 / 2 / 07$ 23:00 & 124.123 & 121.766 & 118.467 & 99.402 & 103.594 & 95.118 & 109.199 & 106.126 & 77.529 & 73.072 \\
\hline $7 / 2 / 0723: 30$ & 124.128 & 121.769 & 118.469 & 99.409 & 103.599 & 95.126 & 109.195 & 106.131 & 77.523 & 73.074 \\
\hline $7 / 3 / 07$ 0:00 & 124.123 & 121.769 & 118.469 & 99.405 & 103.598 & 95.126 & 109.192 & 106.128 & 77.518 & 73.072 \\
\hline $7 / 3 / 070: 30$ & 124.126 & 121.755 & 118.466 & 99.407 & 103.596 & 95.126 & 109.186 & 106.128 & 77.514 & 73.07 \\
\hline 7/3/07 1:00 & 124.128 & 121.744 & 118.469 & 99.405 & 103.595 & 95.123 & 109.184 & 106.128 & 77.51 & 73.07 \\
\hline $7 / 3 / 071: 30$ & 124.114 & 121.742 & 118.455 & 99.393 & 103.582 & 95.111 & 109.182 & 106.115 & 77.501 & 73.059 \\
\hline $7 / 3 / 072: 00$ & 124.097 & 121.732 & 118.439 & 99.381 & 103.567 & 95.093 & 109.178 & 106.1 & 77.492 & 73.043 \\
\hline $7 / 3 / 072: 30$ & 124.102 & 121.723 & 118.446 & 99.383 & 103.571 & 95.097 & 109.173 & 106.102 & 77.488 & 73.045 \\
\hline $7 / 3 / 073: 00$ & 124.088 & 121.705 & 118.432 & 99.371 & 103.559 & 95.088 & 109.171 & 106.091 & 77.481 & 73.037 \\
\hline 7/3/07 3:30 & 124.083 & 121.694 & 118.427 & 99.364 & 103.556 & 95.078 & 109.169 & 106.086 & 77.475 & 73.035 \\
\hline $7 / 3 / 074: 00$ & 124.081 & 121.683 & 118.407 & 99.345 & 103.535 & 95.06 & 109.158 & 106.066 & 77.464 & 73.016 \\
\hline $7 / 3 / 074: 30$ & 124.076 & 121.673 & 118.395 & 99.335 & 103.525 & 95.05 & 109.15 & 106.055 & 77.455 & 73.006 \\
\hline $7 / 3 / 07$ 5:00 & 124.041 & 121.662 & 118.383 & 99.323 & 103.512 & 95.038 & 109.143 & 106.042 & 77.446 & 72.995 \\
\hline 7/3/07 5:30 & 124.034 & 121.664 & 118.374 & 99.316 & 103.506 & 95.034 & 109.137 & 106.034 & 77.44 & 72.989 \\
\hline $7 / 3 / 076: 00$ & 124.019 & 123.621 & 118.362 & 99.304 & 103.493 & 95.022 & 109.128 & 106.022 & 77.429 & 72.977 \\
\hline $7 / 3 / 076: 30$ & 124.029 & 123.959 & 118.363 & 99.3 & 103.491 & 95.015 & 109.124 & 106.027 & 77.422 & 72.97 \\
\hline 7/3/07 7:00 & 126.038 & 124.118 & 119.801 & 100.022 & 103.971 & 95.665 & 109.762 & 107.428 & 77.431 & 73.003 \\
\hline $7 / 3 / 077: 30$ & 126.402 & 124.224 & 120.157 & 100.322 & 104.292 & 95.99 & 110.07 & 107.815 & 77.464 & 73.187 \\
\hline 7/3/07 8:00 & 126.572 & 124.29 & 120.321 & 100.47 & 104.45 & 96.148 & 110.217 & 107.988 & 77.495 & 73.33 \\
\hline 7/3/07 8:30 & 126.679 & 124.329 & 120.427 & 100.57 & 104.553 & 96.25 & 110.312 & 108.101 & 77.523 & 73.427 \\
\hline $7 / 3 / 07$ 9:00 & 126.747 & 122.41 & 120.499 & 100.634 & 104.624 & 96.32 & 110.374 & 108.174 & 77.551 & 73.498 \\
\hline 7/3/07 9:30 & 126.79 & 122.104 & 120.541 & 100.673 & 104.664 & 96.358 & 110.414 & 108.214 & 77.573 & 73.545 \\
\hline $7 / 3 / 07$ 10:00 & 124.811 & 121.959 & 119.142 & 99.972 & 104.209 & 95.731 & 109.792 & 106.844 & 77.578 & 73.533 \\
\hline $7 / 3 / 07 \quad 10: 30$ & 124.476 & 121.88 & 118.813 & 99.705 & 103.917 & 95.441 & 109.516 & 106.494 & 77.564 & 73.396 \\
\hline 7/3/07 11:00 & 124.324 & 121.821 & 118.663 & 99.574 & 103.778 & 95.302 & 109.385 & 106.335 & 77.551 & 73.278 \\
\hline $7 / 3 / 0711: 30$ & 124.242 & 121.78 & 118.582 & 99.502 & 103.704 & 95.227 & 109.312 & 106.251 & 77.54 & 73.21 \\
\hline $7 / 3 / 07 \quad 12: 00$ & 124.178 & 121.744 & 118.522 & 99.447 & 103.643 & 95.168 & 109.259 & 106.186 & 77.529 & 73.15 \\
\hline $7 / 3 / 07 \quad 12: 30$ & 124.14 & 121.723 & 118.485 & 99.412 & 103.605 & 95.13 & 109.225 & 106.146 & 77.519 & 73.109 \\
\hline 7/3/07 13:00 & 124.107 & 121.71 & 118.448 & 99.379 & 103.573 & 95.097 & 109.197 & 106.113 & 77.506 & 73.074 \\
\hline 7/3/07 13:30 & 124.081 & 121.696 & 118.425 & 99.357 & 103.55 & 95.074 & 109.176 & 106.084 & 77.495 & 73.049 \\
\hline $7 / 3 / 07$ 14:00 & 124.067 & 121.683 & 118.413 & 99.35 & 103.538 & 95.064 & 109.163 & 106.073 & 77.488 & 73.037 \\
\hline
\end{tabular}


TABLE S1.1 (Cont.)

Depth from Top of Casing (ft)

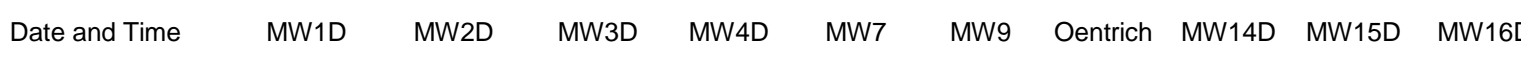

\begin{tabular}{|c|c|c|c|c|c|c|c|c|c|c|}
\hline 7/3/07 14:30 & 124.057 & 121.664 & 118.402 & 99.335 & 103.529 & 95.055 & 109.152 & 106.062 & 77.483 & 73.024 \\
\hline 7/3/07 15:00 & 124.038 & 121.653 & 118.383 & 99.321 & 103.512 & 95.038 & 109.139 & 106.042 & 77.471 & 73.008 \\
\hline 7/3/07 15:30 & 124.024 & 121.63 & 118.37 & 99.305 & 103.502 & 95.024 & 109.128 & 106.029 & 77.46 & 72.997 \\
\hline 7/3/07 16:00 & 124.01 & 121.626 & 118.356 & 99.295 & 103.485 & 95.012 & 109.116 & 106.015 & 77.451 & 72.983 \\
\hline 7/3/07 16:30 & 123.989 & 121.603 & 118.335 & 99.273 & 103.466 & 94.989 & 109.1 & 105.993 & 77.436 & 72.964 \\
\hline $7 / 3 / 07$ 17:00 & 123.982 & 121.583 & 118.328 & 99.269 & 103.46 & 94.986 & 109.094 & 105.987 & 77.429 & 72.958 \\
\hline $7 / 3 / 07$ 17:30 & 123.963 & 121.56 & 118.309 & 99.247 & 103.441 & 94.968 & 109.077 & 105.969 & 77.416 & 72.941 \\
\hline $7 / 3 / 07$ 18:00 & 123.941 & 121.551 & 118.286 & 99.226 & 103.419 & 94.946 & 109.062 & 105.947 & 77.4 & 72.917 \\
\hline 7/3/07 18:30 & 123.918 & 121.535 & 118.265 & 99.205 & 103.4 & 94.925 & 109.049 & 105.925 & 77.387 & 72.898 \\
\hline 7/3/07 19:00 & 123.908 & 121.533 & 118.254 & 99.193 & 103.39 & 94.913 & 109.043 & 105.912 & 77.374 & 72.888 \\
\hline 7/3/07 19:30 & 123.892 & 121.56 & 118.238 & 99.18 & 103.375 & 94.897 & 109.038 & 105.898 & 77.359 & 72.875 \\
\hline $7 / 3 / 0720: 00$ & 123.885 & 121.569 & 118.233 & 99.181 & 103.371 & 94.892 & 109.032 & 105.892 & 77.348 & 72.869 \\
\hline $7 / 3 / 0720: 30$ & 123.918 & 121.565 & 118.263 & 99.212 & 103.403 & 94.925 & 109.039 & 105.923 & 77.35 & 72.896 \\
\hline 7/3/07 21:00 & 123.925 & 121.562 & 118.272 & 99.221 & 103.413 & 94.939 & 109.045 & 105.934 & 77.348 & 72.904 \\
\hline 7/3/07 21:30 & 123.922 & 121.567 & 118.27 & 99.214 & 103.409 & 94.932 & 109.043 & 105.929 & 77.341 & 72.9 \\
\hline $7 / 3 / 0722: 00$ & 123.918 & 121.565 & 118.266 & 99.214 & 103.405 & 94.93 & 109.038 & 105.923 & 77.335 & 72.896 \\
\hline 7/3/07 22:30 & 123.922 & 121.56 & 118.272 & 99.216 & 103.411 & 94.935 & 109.038 & 105.927 & 77.333 & 72.902 \\
\hline 7/3/07 23:00 & 123.92 & 121.56 & 118.268 & 99.216 & 103.407 & 94.932 & 109.036 & 105.925 & 77.326 & 72.898 \\
\hline 7/3/07 23:30 & 123.915 & 121.565 & 118.265 & 99.212 & 103.405 & 94.93 & 109.032 & 105.923 & 77.32 & 72.894 \\
\hline 7/4/07 0:00 & 123.916 & 121.567 & 118.263 & 99.212 & 103.403 & 94.928 & 109.028 & 105.923 & 77.315 & 72.894 \\
\hline $7 / 4 / 070: 30$ & 123.92 & 121.56 & 118.27 & 99.216 & 103.407 & 94.93 & 109.028 & 105.925 & 77.313 & 72.896 \\
\hline $7 / 4 / 071: 00$ & 123.922 & 121.547 & 118.272 & 99.221 & 103.413 & 94.935 & 109.026 & 105.929 & 77.309 & 72.904 \\
\hline $7 / 4 / 071: 30$ & 123.918 & 121.535 & 118.267 & 99.212 & 103.407 & 94.93 & 109.024 & 105.925 & 77.306 & 72.896 \\
\hline $7 / 4 / 072: 00$ & 123.901 & 121.526 & 118.251 & 99.198 & 103.39 & 94.916 & 109.017 & 105.909 & 77.296 & 72.882 \\
\hline $7 / 4 / 072: 30$ & 123.889 & 121.528 & 118.24 & 99.188 & 103.379 & 94.902 & 109.011 & 105.898 & 77.289 & 72.869 \\
\hline $7 / 4 / 07$ 3:00 & 123.882 & 121.513 & 118.23 & 99.18 & 103.373 & 94.897 & 109.004 & 105.89 & 77.283 & 72.865 \\
\hline $7 / 4 / 07$ 3:30 & 123.882 & 121.51 & 118.226 & 99.183 & 103.375 & 94.895 & 109.004 & 105.89 & 77.28 & 72.865 \\
\hline $7 / 4 / 074: 00$ & 123.87 & 121.519 & 118.217 & 99.166 & 103.361 & 94.883 & 108.998 & 105.878 & 77.272 & 72.855 \\
\hline $7 / 4 / 074: 30$ & 123.866 & 121.517 & 118.212 & 99.166 & 103.358 & 94.88 & 108.994 & 105.874 & 77.267 & 72.851 \\
\hline $7 / 4 / 075: 00$ & 123.873 & 121.519 & 118.219 & 99.174 & 103.369 & 94.892 & 108.996 & 105.883 & 77.265 & 72.861 \\
\hline $7 / 4 / 07$ 5:30 & 123.873 & 121.515 & 118.219 & 99.174 & 103.367 & 94.89 & 108.992 & 105.88 & 77.261 & 72.859 \\
\hline $7 / 4 / 076: 00$ & 123.875 & 121.513 & 118.224 & 99.178 & 103.369 & 94.892 & 108.992 & 105.883 & 77.258 & 72.861 \\
\hline $7 / 4 / 076: 30$ & 123.87 & 121.522 & 118.217 & 99.171 & 103.365 & 94.888 & 108.989 & 105.878 & 77.254 & 72.857 \\
\hline $7 / 4 / 07$ 7:00 & 123.87 & 123.58 & 118.217 & 99.173 & 103.365 & 94.885 & 108.987 & 105.876 & 77.248 & 72.853 \\
\hline $7 / 4 / 07$ 7:30 & 123.875 & 123.882 & 118.222 & 99.178 & 103.369 & 94.892 & 108.985 & 105.88 & 77.247 & 72.861 \\
\hline $7 / 4 / 078: 00$ & 125.967 & 124.025 & 119.611 & 99.901 & 103.872 & 95.55 & 109.631 & 107.397 & 77.263 & 72.91 \\
\hline $7 / 4 / 078: 30$ & 126.296 & 124.113 & 119.931 & 100.167 & 104.159 & 95.84 & 109.907 & 107.742 & 77.296 & 73.084 \\
\hline $7 / 4 / 079: 00$ & 126.444 & 124.17 & 120.081 & 100.303 & 104.308 & 95.983 & 110.044 & 107.9 & 77.328 & 73.216 \\
\hline 7/4/07 9:30 & 126.539 & 124.215 & 120.168 & 100.389 & 104.395 & 96.073 & 110.128 & 107.997 & 77.355 & 73.305 \\
\hline $7 / 4 / 07$ 10:00 & 126.596 & 124.258 & 120.227 & 100.444 & 104.454 & 96.132 & 110.187 & 108.059 & 77.38 & 73.369 \\
\hline $7 / 4 / 0710: 30$ & 126.643 & 124.292 & 120.272 & 100.489 & 104.498 & 96.177 & 110.226 & 108.105 & 77.405 & 73.419 \\
\hline 7/4/07 11:00 & 126.686 & 124.313 & 120.321 & 100.532 & 104.542 & 96.221 & 110.258 & 108.152 & 77.435 & 73.467 \\
\hline $7 / 4 / 0711: 30$ & 126.721 & 124.326 & 120.356 & 100.563 & 104.576 & 96.257 & 110.281 & 108.185 & 77.459 & 73.504 \\
\hline $7 / 4 / 0712: 00$ & 126.743 & 124.342 & 120.377 & 100.584 & 104.597 & 96.276 & 110.297 & 108.207 & 77.481 & 73.529 \\
\hline 7/4/07 12:30 & 126.756 & 124.354 & 120.391 & 100.598 & 104.612 & 96.287 & 110.312 & 108.221 & 77.501 & 73.543 \\
\hline 7/4/07 13:00 & 126.773 & 124.34 & 120.409 & 100.618 & 104.631 & 96.306 & 110.322 & 108.24 & 77.521 & 73.564 \\
\hline $7 / 4 / 0713: 30$ & 126.787 & 124.349 & 120.423 & 100.629 & 104.645 & 96.323 & 110.331 & 108.256 & 77.543 & 73.58 \\
\hline $7 / 4 / 0714: 00$ & 126.773 & 122.973 & 120.414 & 100.618 & 104.635 & 96.311 & 110.341 & 108.243 & 77.554 & 73.578 \\
\hline $7 / 4 / 0714: 30$ & 126.778 & 122.186 & 120.419 & 100.627 & 104.643 & 96.318 & 110.342 & 108.247 & 77.571 & 73.586 \\
\hline $7 / 4 / 0715: 00$ & 126.022 & 121.973 & 120.18 & 100.33 & 104.62 & 96.278 & 110.307 & 108.212 & 77.584 & 73.584 \\
\hline $7 / 4 / 07$ 15:30 & 124.57 & 121.848 & 118.908 & 99.776 & 104.003 & 95.524 & 109.576 & 106.598 & 77.567 & 73.469 \\
\hline $7 / 4 / 07$ 16:00 & 124.338 & 121.773 & 118.681 & 99.584 & 103.796 & 95.316 & 109.379 & 106.359 & 77.545 & 73.326 \\
\hline $7 / 4 / 0716: 30$ & 124.213 & 121.703 & 118.557 & 99.472 & 103.677 & 95.199 & 109.27 & 106.228 & 77.527 & 73.218 \\
\hline 7/4/07 17:00 & 124.135 & 121.658 & 118.481 & 99.405 & 103.605 & 95.13 & 109.199 & 106.146 & 77.512 & 73.144 \\
\hline $7 / 4 / 0717: 30$ & 124.062 & 121.63 & 118.409 & 99.336 & 103.539 & 95.062 & 109.15 & 106.073 & 77.49 & 73.074 \\
\hline $7 / 4 / 07$ 18:00 & 124.017 & 121.612 & 118.363 & 99.297 & 103.495 & 95.017 & 109.114 & 106.024 & 77.473 & 73.026 \\
\hline 7/4/07 18:30 & 123.989 & 121.594 & 118.337 & 99.274 & 103.47 & 94.994 & 109.088 & 105.998 & 77.459 & 72.999 \\
\hline $7 / 4 / 07$ 19:00 & 123.967 & 121.592 & 118.316 & 99.257 & 103.449 & 94.975 & 109.068 & 105.976 & 77.444 & 72.979 \\
\hline 7/4/07 19:30 & 123.951 & 121.592 & 118.303 & 99.243 & 103.44 & 94.958 & 109.052 & 105.96 & 77.433 & 72.964 \\
\hline $7 / 4 / 0720: 00$ & 123.944 & 121.578 & 118.293 & 99.24 & 103.432 & 94.953 & 109.041 & 105.951 & 77.42 & 72.952 \\
\hline $7 / 4 / 0720: 30$ & 123.948 & 121.576 & 118.298 & 99.245 & 103.441 & 94.961 & 109.032 & 105.958 & 77.412 & 72.956 \\
\hline $7 / 4 / 0721: 00$ & 123.939 & 121.569 & 118.289 & 99.235 & 103.428 & 94.953 & 109.022 & 105.947 & 77.403 & 72.946 \\
\hline 7/4/07 21:30 & 123.932 & 121.569 & 118.284 & 99.231 & 103.426 & 94.949 & 109.015 & 105.94 & 77.392 & 72.939 \\
\hline $7 / 4 / 0722: 00$ & 123.925 & 121.562 & 118.277 & 99.226 & 103.419 & 94.944 & 109.005 & 105.936 & 77.385 & 72.939 \\
\hline $7 / 4 / 0722: 30$ & 123.925 & 121.558 & 118.274 & 99.224 & 103.417 & 94.942 & 109 & 105.931 & 77.377 & 72.933 \\
\hline 7/4/07 23:00 & 123.92 & 121.556 & 118.27 & 99.221 & 103.415 & 94.935 & 108.992 & 105.927 & 77.372 & 72.931 \\
\hline
\end{tabular}


TABLE S1.1 (Cont.)

\begin{tabular}{|c|c|c|c|c|c|c|c|c|c|c|}
\hline \multirow[b]{2}{*}{ Date and Time } & \multicolumn{10}{|c|}{ Depth from Top of Casing (ft) } \\
\hline & MW1D & MW2D & MW3D & MW4D & MW7 & MW9 & Oentrich & MW14D & MW15D & MW16D \\
\hline 7/4/07 23:30 & 123.913 & 121.556 & 118.265 & 99.216 & 103.409 & 94.93 & 108.988 & 105.923 & 77.363 & 72.925 \\
\hline $7 / 5 / 07$ 0:00 & 123.913 & 121.551 & 118.265 & 99.217 & 103.407 & 94.932 & 108.981 & 105.92 & 77.359 & 72.921 \\
\hline 7/5/07 0:30 & 123.911 & 121.547 & 118.263 & 99.216 & 103.409 & 94.93 & 108.976 & 105.921 & 77.353 & 72.921 \\
\hline 7/5/07 1:00 & 123.908 & 121.542 & 118.261 & 99.212 & 103.407 & 94.93 & 108.97 & 105.918 & 77.348 & 72.919 \\
\hline 7/5/07 1:30 & 123.901 & 121.54 & 118.254 & 99.205 & 103.398 & 94.92 & 108.968 & 105.912 & 77.342 & 72.91 \\
\hline $7 / 5 / 072: 00$ & 123.899 & 121.535 & 118.249 & 99.202 & 103.398 & 94.918 & 108.962 & 105.907 & 77.335 & 72.904 \\
\hline $7 / 5 / 072: 30$ & 123.896 & 121.522 & 118.249 & 99.202 & 103.394 & 94.918 & 108.959 & 105.905 & 77.331 & 72.904 \\
\hline 7/5/07 3:00 & 123.892 & 121.515 & 118.245 & 99.197 & 103.39 & 94.911 & 108.955 & 105.9 & 77.326 & 72.9 \\
\hline 7/5/07 3:30 & 123.89 & 121.517 & 118.233 & 99.185 & 103.377 & 94.899 & 108.953 & 105.89 & 77.317 & 72.888 \\
\hline $7 / 5 / 074: 00$ & 123.887 & 121.517 & 118.226 & 99.18 & 103.371 & 94.897 & 108.947 & 105.88 & 77.309 & 72.882 \\
\hline $7 / 5 / 074: 30$ & 123.88 & 121.522 & 118.228 & 99.181 & 103.375 & 94.899 & 108.943 & 105.883 & 77.306 & 72.886 \\
\hline 7/5/07 5:00 & 123.875 & 121.522 & 118.23 & 99.185 & 103.379 & 94.901 & 108.938 & 105.887 & 77.302 & 72.89 \\
\hline 7/5/07 5:30 & 123.871 & 121.519 & 118.233 & 99.188 & 103.38 & 94.904 & 108.934 & 105.887 & 77.296 & 72.89 \\
\hline 7/5/07 6:00 & 123.875 & 123.442 & 118.233 & 99.188 & 103.38 & 94.904 & 108.93 & 105.887 & 77.295 & 72.892 \\
\hline 7/5/07 6:30 & 123.873 & 123.803 & 118.23 & 99.186 & 103.377 & 94.902 & 108.927 & 105.883 & 77.289 & 72.89 \\
\hline 7/5/07 7:00 & 125.851 & 123.977 & 119.639 & 99.882 & 103.828 & 95.517 & 109.539 & 107.246 & 77.298 & 72.91 \\
\hline $7 / 5 / 077: 30$ & 126.246 & 124.086 & 120.018 & 100.196 & 104.171 & 95.861 & 109.856 & 107.667 & 77.331 & 73.097 \\
\hline 7/5/07 8:00 & 126.428 & 122.644 & 120.199 & 100.36 & 104.34 & 96.033 & 110.01 & 107.855 & 77.366 & 73.247 \\
\hline 7/5/07 8:30 & 126.544 & 122.039 & 120.309 & 100.461 & 104.449 & 96.141 & 110.102 & 107.973 & 77.4 & 73.355 \\
\hline 7/5/07 9:00 & 125.242 & 121.862 & 119.56 & 100.112 & 104.391 & 95.969 & 109.914 & 107.47 & 77.429 & 73.427 \\
\hline 7/5/07 9:30 & 124.419 & 121.762 & 118.763 & 99.657 & 103.879 & 95.394 & 109.362 & 106.443 & 77.422 & 73.34 \\
\hline $7 / 5 / 07$ 10:00 & 124.232 & 121.701 & 118.577 & 99.497 & 103.706 & 95.225 & 109.197 & 106.248 & 77.412 & 73.227 \\
\hline 7/5/07 10:30 & 124.123 & 121.66 & 118.474 & 99.407 & 103.613 & 95.133 & 109.111 & 106.138 & 77.403 & 73.14 \\
\hline 7/5/07 11:00 & 124.064 & 121.63 & 118.413 & 99.355 & 103.552 & 95.074 & 109.054 & 106.077 & 77.394 & 73.08 \\
\hline 7/5/07 11:30 & 124.02 & 121.608 & 118.374 & 99.317 & 103.514 & 95.034 & 109.017 & 106.033 & 77.389 & 73.041 \\
\hline 7/5/07 12:00 & 123.989 & 121.592 & 118.342 & 99.288 & 103.487 & 95.008 & 108.987 & 106 & 77.383 & 73.012 \\
\hline $7 / 5 / 07$ 12:30 & 123.967 & 121.571 & 118.321 & 99.271 & 103.464 & 94.989 & 108.97 & 105.978 & 77.376 & 72.987 \\
\hline 7/5/07 13:00 & 123.948 & 121.56 & 118.307 & 99.255 & 103.451 & 94.972 & 108.953 & 105.96 & 77.368 & 72.97 \\
\hline 7/5/07 13:30 & 123.93 & 121.542 & 118.286 & 99.235 & 103.428 & 94.951 & 108.943 & 105.941 & 77.359 & 72.952 \\
\hline $7 / 5 / 0714: 00$ & 123.92 & 121.528 & 118.275 & 99.224 & 103.419 & 94.942 & 108.934 & 105.929 & 77.353 & 72.941 \\
\hline 7/5/07 14:30 & 123.901 & 121.517 & 118.256 & 99.209 & 103.403 & 94.928 & 108.927 & 105.911 & 77.344 & 72.923 \\
\hline $7 / 5 / 07 \quad 15: 00$ & 123.89 & 121.501 & 118.245 & 99.193 & 103.39 & 94.911 & 108.921 & 105.896 & 77.337 & 72.91 \\
\hline 7/5/07 15:30 & 123.878 & 121.483 & 118.233 & 99.183 & 103.379 & 94.899 & 108.916 & 105.887 & 77.33 & 72.9 \\
\hline $7 / 5 / 07$ 16:00 & 123.861 & 121.465 & 118.217 & 99.171 & 103.363 & 94.89 & 108.91 & 105.87 & 77.32 & 72.888 \\
\hline 7/5/07 16:30 & 123.842 & 121.451 & 118.198 & 99.152 & 103.344 & 94.869 & 108.906 & 105.854 & 77.311 & 72.871 \\
\hline 7/5/07 17:00 & 123.823 & 121.438 & 118.18 & 99.131 & 103.325 & 94.85 & 108.9 & 105.834 & 77.298 & 72.853 \\
\hline 7/5/07 17:30 & 123.809 & 121.433 & 118.166 & 99.119 & 103.312 & 94.836 & 108.895 & 105.817 & 77.289 & 72.838 \\
\hline 7/5/07 18:00 & 123.795 & 121.433 & 118.152 & 99.107 & 103.301 & 94.824 & 108.891 & 105.806 & 77.28 & 72.828 \\
\hline $7 / 5 / 0718: 30$ & 123.788 & 121.433 & 118.145 & 99.104 & 103.297 & 94.824 & 108.883 & 105.799 & 77.272 & 72.824 \\
\hline 7/5/07 19:00 & 123.793 & 121.433 & 118.15 & 99.107 & 103.301 & 94.826 & 108.878 & 105.803 & 77.269 & 72.828 \\
\hline 7/5/07 19:30 & 123.79 & 121.433 & 118.15 & 99.107 & 103.302 & 94.826 & 108.872 & 105.803 & 77.263 & 72.83 \\
\hline $7 / 5 / 0720: 00$ & 123.79 & 121.435 & 118.15 & 99.107 & 103.302 & 94.826 & 108.868 & 105.801 & 77.256 & 72.826 \\
\hline $7 / 5 / 0720: 30$ & 123.793 & 121.442 & 118.15 & 99.107 & 103.301 & 94.824 & 108.866 & 105.801 & 77.25 & 72.826 \\
\hline 7/5/07 21:00 & 123.793 & 121.454 & 118.152 & 99.111 & 103.304 & 94.824 & 108.859 & 105.801 & 77.245 & 72.828 \\
\hline $7 / 5 / 0721: 30$ & 123.8 & 121.456 & 118.159 & 99.121 & 103.312 & 94.836 & 108.855 & 105.81 & 77.243 & 72.834 \\
\hline 7/5/07 22:00 & 123.809 & 121.46 & 118.168 & 99.13 & 103.323 & 94.847 & 108.85 & 105.821 & 77.241 & 72.842 \\
\hline 7/5/07 22:30 & 123.814 & 121.46 & 118.173 & 99.135 & 103.327 & 94.847 & 108.846 & 105.823 & 77.239 & 72.844 \\
\hline 7/5/07 23:00 & 123.816 & 121.454 & 118.178 & 99.14 & 103.329 & 94.852 & 108.842 & 105.828 & 77.237 & 72.848 \\
\hline 7/5/07 23:30 & 123.818 & 121.451 & 118.178 & 99.138 & 103.333 & 94.852 & 108.84 & 105.83 & 77.234 & 72.848 \\
\hline 7/6/07 0:00 & 123.811 & 121.449 & 118.171 & 99.131 & 103.325 & 94.845 & 108.84 & 105.823 & 77.228 & 72.84 \\
\hline $7 / 6 / 070: 30$ & 123.811 & 121.442 & 118.171 & 99.131 & 103.325 & 94.845 & 108.835 & 105.821 & 77.226 & 72.842 \\
\hline 7/6/07 1:00 & 123.807 & 121.433 & 118.168 & 99.126 & 103.32 & 94.84 & 108.833 & 105.816 & 77.221 & 72.838 \\
\hline 7/6/07 1:30 & 123.8 & 121.433 & 118.159 & 99.119 & 103.312 & 94.833 & 108.831 & 105.81 & 77.217 & 72.832 \\
\hline $7 / 6 / 072: 00$ & 123.795 & 121.426 & 118.154 & 99.112 & 103.306 & 94.824 & 108.827 & 105.803 & 77.212 & 72.824 \\
\hline 7/6/07 2:30 & 123.79 & 121.424 & 118.15 & 99.111 & 103.304 & 94.824 & 108.825 & 105.801 & 77.208 & 72.824 \\
\hline 7/6/07 3:00 & 123.788 & 121.422 & 118.145 & 99.104 & 103.297 & 94.817 & 108.823 & 105.794 & 77.202 & 72.82 \\
\hline 7/6/07 3:30 & 123.785 & 121.42 & 118.141 & 99.102 & 103.293 & 94.814 & 108.82 & 105.79 & 77.197 & 72.815 \\
\hline 7/6/07 4:00 & 123.783 & 121.415 & 118.138 & 99.1 & 103.293 & 94.817 & 108.816 & 105.79 & 77.197 & 72.817 \\
\hline $7 / 6 / 074: 30$ & 123.778 & 121.42 & 118.136 & 99.099 & 103.293 & 94.812 & 108.81 & 105.788 & 77.193 & 72.813 \\
\hline $7 / 6 / 07$ 5:00 & 123.776 & 121.422 & 118.133 & 99.095 & 103.287 & 94.807 & 108.808 & 105.781 & 77.188 & 72.811 \\
\hline $7 / 6 / 075: 30$ & 123.771 & 121.417 & 118.138 & 99.102 & 103.295 & 94.814 & 108.804 & 105.788 & 77.188 & 72.817 \\
\hline $7 / 6 / 076: 00$ & 123.776 & 123.072 & 118.143 & 99.105 & 103.299 & 94.822 & 108.799 & 105.792 & 77.189 & 72.822 \\
\hline $7 / 6 / 076: 30$ & 123.778 & 123.682 & 118.136 & 99.099 & 103.291 & 94.814 & 108.797 & 105.788 & 77.186 & 72.815 \\
\hline 7/6/07 7:00 & 125.3 & 123.893 & 119.005 & 99.514 & 103.434 & 95.076 & 109.064 & 106.536 & 77.182 & 72.815 \\
\hline 7/6/07 7:30 & 126.09 & 124.011 & 119.738 & 99.998 & 103.978 & 95.656 & 109.61 & 107.532 & 77.217 & 72.964 \\
\hline 7/6/07 8:00 & 126.31 & 124.093 & 119.956 & 100.191 & 104.186 & 95.866 & 109.8 & 107.765 & 77.248 & 73.128 \\
\hline
\end{tabular}


TABLE S1.1 (Cont.)

Depth from Top of Casing (ft)

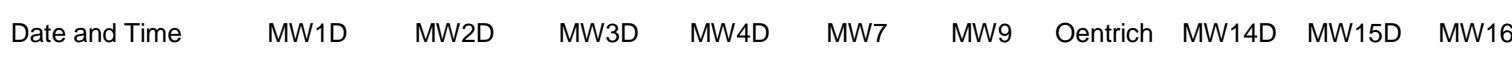

\begin{tabular}{|c|c|c|c|c|c|c|c|c|c|c|}
\hline 7/6/07 8:30 & 126.421 & 124.145 & 120.078 & 100.305 & 104.304 & 95.983 & 109.909 & 107.891 & 77.28 & 73.245 \\
\hline 7/6/07 9:00 & 126.518 & 124.172 & 120.161 & 100.382 & 104.386 & 96.064 & 109.978 & 107.98 & 77.313 & 73.332 \\
\hline 7/6/07 9:30 & 126.57 & 124.199 & 120.217 & 100.434 & 104.443 & 96.12 & 110.025 & 108.037 & 77.342 & 73.392 \\
\hline 7/6/07 10:00 & 126.6 & 124.211 & 120.247 & 100.461 & 104.473 & 96.151 & 110.059 & 108.068 & 77.365 & 73.425 \\
\hline $7 / 6 / 07 \quad 10: 30$ & 126.629 & 124.215 & 120.275 & 100.489 & 104.498 & 96.177 & 110.085 & 108.097 & 77.385 & 73.452 \\
\hline 7/6/07 11:00 & 126.641 & 124.222 & 120.291 & 100.501 & 104.515 & 96.191 & 110.104 & 108.112 & 77.405 & 73.473 \\
\hline 7/6/07 11:30 & 126.648 & 124.222 & 120.298 & 100.508 & 104.521 & 96.198 & 110.115 & 108.116 & 77.429 & 73.489 \\
\hline $7 / 6 / 07 \quad 12: 00$ & 126.653 & 124.222 & 120.305 & 100.515 & 104.53 & 96.205 & 110.126 & 108.125 & 77.446 & 73.498 \\
\hline $7 / 6 / 07$ 12:30 & 126.655 & 124.22 & 120.305 & 100.518 & 104.532 & 96.207 & 110.134 & 108.125 & 77.462 & 73.508 \\
\hline 7/6/07 13:00 & 126.655 & 124.213 & 120.305 & 100.518 & 104.532 & 96.207 & 110.141 & 108.125 & 77.477 & 73.512 \\
\hline 7/6/07 13:30 & 126.652 & 124.206 & 120.305 & 100.517 & 104.534 & 96.207 & 110.149 & 108.128 & 77.494 & 73.514 \\
\hline 7/6/07 14:00 & 126.645 & 122.481 & 120.301 & 100.513 & 104.525 & 96.203 & 110.156 & 108.119 & 77.505 & 73.512 \\
\hline 7/6/07 14:30 & 126.641 & 121.984 & 120.296 & 100.508 & 104.523 & 96.2 & 110.16 & 108.114 & 77.518 & 73.514 \\
\hline 7/6/07 15:00 & 124.946 & 121.785 & 119.283 & 100.008 & 104.273 & 95.809 & 109.783 & 107.072 & 77.525 & 73.506 \\
\hline 7/6/07 15:30 & 124.36 & 121.664 & 118.712 & 99.598 & 103.822 & 95.338 & 109.34 & 106.392 & 77.497 & 73.353 \\
\hline 7/6/07 16:00 & 124.152 & 121.581 & 118.506 & 99.417 & 103.632 & 95.149 & 109.171 & 106.173 & 77.471 & 73.208 \\
\hline $7 / 6 / 07 \quad 16: 30$ & 124.027 & 121.524 & 118.385 & 99.309 & 103.514 & 95.036 & 109.071 & 106.047 & 77.451 & 73.101 \\
\hline 7/6/07 17:00 & 123.942 & 121.474 & 118.303 & 99.236 & 103.436 & 94.958 & 109.005 & 105.96 & 77.433 & 73.018 \\
\hline 7/6/07 17:30 & 123.883 & 121.44 & 118.244 & 99.18 & 103.382 & 94.902 & 108.962 & 105.898 & 77.411 & 72.958 \\
\hline 7/6/07 18:00 & 123.833 & 121.408 & 118.194 & 99.135 & 103.333 & 94.854 & 108.93 & 105.847 & 77.39 & 72.91 \\
\hline $7 / 6 / 07$ 18:30 & 123.797 & 121.386 & 118.159 & 99.105 & 103.302 & 94.824 & 108.906 & 105.812 & 77.374 & 72.873 \\
\hline 7/6/07 19:00 & 123.767 & 121.365 & 118.129 & 99.078 & 103.276 & 94.798 & 108.885 & 105.783 & 77.355 & 72.848 \\
\hline 7/6/07 19:30 & 123.745 & 121.352 & 118.108 & 99.057 & 103.253 & 94.774 & 108.868 & 105.759 & 77.339 & 72.826 \\
\hline $7 / 6 / 0720: 00$ & 123.724 & 121.336 & 118.087 & 99.038 & 103.236 & 94.756 & 108.855 & 105.737 & 77.322 & 72.805 \\
\hline $7 / 6 / 0720: 30$ & 123.705 & 121.327 & 118.069 & 99.023 & 103.219 & 94.739 & 108.844 & 105.721 & 77.309 & 72.789 \\
\hline 7/6/07 21:00 & 123.696 & 121.315 & 118.06 & 99.011 & 103.209 & 94.727 & 108.847 & 105.71 & 77.298 & 72.776 \\
\hline $7 / 6 / 0721: 30$ & 123.681 & 121.313 & 118.046 & 99.002 & 103.198 & 94.718 & 108.825 & 105.697 & 77.285 & 72.768 \\
\hline $7 / 6 / 07$ 22:00 & 123.672 & 121.306 & 118.039 & 98.995 & 103.192 & 94.711 & 108.813 & 105.688 & 77.272 & 72.758 \\
\hline $7 / 6 / 07$ 22:30 & 123.667 & 121.302 & 118.032 & 98.99 & 103.186 & 94.704 & 108.805 & 105.681 & 77.263 & 72.753 \\
\hline 7/6/07 23:00 & 123.663 & 121.302 & 118.027 & 98.987 & 103.183 & 94.704 & 108.799 & 105.677 & 77.25 & 72.749 \\
\hline $7 / 6 / 0723: 30$ & 123.658 & 121.295 & 118.023 & 98.985 & 103.179 & 94.699 & 108.794 & 105.675 & 77.243 & 72.745 \\
\hline 7/7/07 0:00 & 123.658 & 121.286 & 118.023 & 98.983 & 103.179 & 94.699 & 108.79 & 105.672 & 77.234 & 72.743 \\
\hline 7/7/07 0:30 & 123.651 & 121.281 & 118.016 & 98.976 & 103.173 & 94.697 & 108.784 & 105.666 & 77.223 & 72.735 \\
\hline 7/7/07 1:00 & 123.641 & 121.277 & 118.009 & 98.968 & 103.165 & 94.682 & 108.78 & 105.657 & 77.212 & 72.724 \\
\hline 7/7/07 1:30 & 123.637 & 121.263 & 118.004 & 98.964 & 103.16 & 94.678 & 108.775 & 105.652 & 77.204 & 72.72 \\
\hline $7 / 7 / 072: 00$ & 123.629 & 121.252 & 117.995 & 98.956 & 103.15 & 94.671 & 108.771 & 105.646 & 77.195 & 72.712 \\
\hline $7 / 7 / 072: 30$ & 123.627 & 121.247 & 117.986 & 98.947 & 103.143 & 94.661 & 108.767 & 105.635 & 77.186 & 72.704 \\
\hline 7/7/07 3:00 & 123.622 & 121.243 & 117.974 & 98.935 & 103.133 & 94.649 & 108.762 & 105.624 & 77.175 & 72.693 \\
\hline 7/7/07 3:30 & 123.62 & 121.236 & 117.967 & 98.931 & 103.127 & 94.645 & 108.758 & 105.617 & 77.167 & 72.687 \\
\hline 7/7/07 4:00 & 123.615 & 121.229 & 117.963 & 98.926 & 103.122 & 94.64 & 108.752 & 105.613 & 77.16 & 72.683 \\
\hline 7/7/07 4:30 & 123.613 & 121.227 & 117.956 & 98.919 & 103.116 & 94.635 & 108.747 & 105.606 & 77.151 & 72.677 \\
\hline 7/7/07 5:00 & 123.611 & 121.227 & 117.951 & 98.914 & 103.11 & 94.628 & 108.743 & 105.602 & 77.143 & 72.671 \\
\hline 7/7/07 5:30 & 123.603 & 121.225 & 117.948 & 98.914 & 103.11 & 94.628 & 108.739 & 105.597 & 77.136 & 72.671 \\
\hline 7/7/07 6:00 & 123.599 & 122.506 & 117.949 & 98.914 & 103.11 & 94.628 & 108.735 & 105.597 & 77.13 & 72.669 \\
\hline 7/7/07 6:30 & 123.596 & 123.376 & 117.944 & 98.909 & 103.104 & 94.626 & 108.733 & 105.593 & 77.125 & 72.664 \\
\hline 7/7/07 7:00 & 123.596 & 123.605 & 117.942 & 99.197 & 103.101 & 94.623 & 108.728 & 105.588 & 77.116 & 72.662 \\
\hline 7/7/07 7:30 & 125.806 & 123.73 & 119.6 & 99.807 & 103.771 & 95.46 & 109.546 & 107.229 & 77.151 & 72.789 \\
\hline 7/7/07 8:00 & 126.055 & 123.803 & 119.84 & 100.015 & 103.995 & 95.686 & 109.751 & 107.483 & 77.18 & 72.96 \\
\hline 7/7/07 8:30 & 126.18 & 123.859 & 119.965 & 100.127 & 104.113 & 95.807 & 109.871 & 107.614 & 77.208 & 73.072 \\
\hline 7/7/07 9:00 & 126.26 & 123.886 & 120.044 & 100.2 & 104.192 & 95.88 & 109.943 & 107.698 & 77.234 & 73.152 \\
\hline 7/7/07 9:30 & 126.315 & 123.902 & 120.099 & 100.249 & 104.245 & 95.936 & 109.993 & 107.756 & 77.254 & 73.21 \\
\hline 7/7/07 10:00 & 126.345 & 123.911 & 120.127 & 100.277 & 104.271 & 95.962 & 110.025 & 107.787 & 77.271 & 73.241 \\
\hline 7/7/07 10:30 & 126.362 & 123.918 & 120.146 & 100.293 & 104.29 & 95.981 & 110.046 & 107.802 & 77.289 & 73.266 \\
\hline 7/7/07 11:00 & 126.371 & 123.927 & 120.153 & 100.298 & 104.298 & 95.988 & 110.061 & 107.814 & 77.3 & 73.276 \\
\hline 7/7/07 11:30 & 126.374 & 123.934 & 120.16 & 100.306 & 104.304 & 95.993 & 110.07 & 107.818 & 77.315 & 73.287 \\
\hline 7/7/07 12:00 & 126.388 & 122.138 & 120.169 & 100.313 & 104.311 & 96 & 110.076 & 107.826 & 77.326 & 73.293 \\
\hline 7/7/07 12:30 & 126.392 & 121.714 & 120.178 & 100.322 & 104.321 & 96.012 & 110.089 & 107.838 & 77.342 & 73.305 \\
\hline 7/7/07 13:00 & 124.566 & 121.524 & 118.915 & 99.698 & 103.957 & 95.484 & 109.571 & 106.642 & 77.344 & 73.291 \\
\hline 7/7/07 13:30 & 124.091 & 121.413 & 118.448 & 99.336 & 103.565 & 95.081 & 109.193 & 106.12 & 77.317 & 73.132 \\
\hline 7/7/07 14:00 & 123.892 & 121.333 & 118.249 & 99.169 & 103.384 & 94.901 & 109.032 & 105.916 & 77.293 & 72.989 \\
\hline 7/7/07 14:30 & 123.774 & 121.277 & 118.136 & 99.066 & 103.276 & 94.795 & 108.936 & 105.796 & 77.271 & 72.884 \\
\hline 7/7/07 15:00 & 123.693 & 121.243 & 118.057 & 98.995 & 103.199 & 94.718 & 108.872 & 105.712 & 77.248 & 72.807 \\
\hline 7/7/07 15:30 & 123.637 & 121.209 & 117.997 & 98.943 & 103.148 & 94.666 & 108.818 & 105.653 & 77.228 & 72.749 \\
\hline 7/7/07 16:00 & 123.599 & 121.177 & 117.965 & 98.912 & 103.112 & 94.633 & 108.789 & 105.617 & 77.21 & 72.71 \\
\hline 7/7/07 16:30 & 123.563 & 121.147 & 117.93 & 98.88 & 103.08 & 94.598 & 108.759 & 105.582 & 77.193 & 72.675 \\
\hline 7/7/07 17:00 & 123.533 & 121.129 & 117.898 & 98.85 & 103.049 & 94.569 & 108.735 & 105.551 & 77.177 & 72.646 \\
\hline
\end{tabular}


TABLE S1.1 (Cont.)

\begin{tabular}{|c|c|c|c|c|c|c|c|c|c|c|}
\hline \multirow[b]{2}{*}{ Date and Time } & \multicolumn{10}{|c|}{ Depth from Top of Casing (ft) } \\
\hline & MW1D & MW2D & MW3D & MW4D & MW7 & MW9 & Oentrich & MW14D & MW15D & MW16D \\
\hline 7/7/07 17:30 & 123.504 & 121.109 & 117.872 & 98.826 & 103.024 & 94.546 & 108.711 & 105.52 & 77.162 & 72.617 \\
\hline 7/7/07 18:00 & 123.485 & 121.154 & 117.854 & 98.809 & 103.009 & 94.529 & 108.696 & 105.502 & 77.145 & 72.6 \\
\hline 7/7/07 18:30 & 123.464 & 121.161 & 117.833 & 98.79 & 102.988 & 94.51 & 108.682 & 105.482 & 77.129 & 72.58 \\
\hline 7/7/07 19:00 & 123.523 & 121.172 & 117.907 & 98.837 & 103.074 & 94.628 & 109.673 & 105.527 & 77.129 & 72.571 \\
\hline 7/7/07 19:30 & 123.523 & 121.179 & 117.905 & 98.921 & 103.076 & 94.628 & 109.69 & 105.522 & 77.123 & 72.569 \\
\hline 7/7/07 20:00 & 123.537 & 121.179 & 117.919 & 98.933 & 103.091 & 94.642 & 109.701 & 105.538 & 77.123 & 72.574 \\
\hline 7/7/07 20:30 & 123.542 & 121.181 & 117.926 & 98.942 & 103.099 & 94.649 & 109.713 & 105.544 & 77.119 & 72.582 \\
\hline 7/7/07 21:00 & 123.542 & 121.122 & 117.928 & 98.942 & 103.097 & 94.649 & 109.709 & 105.544 & 77.112 & 72.582 \\
\hline 7/7/07 21:30 & 123.544 & 121.093 & 117.93 & 98.945 & 103.101 & 94.652 & 109.705 & 105.547 & 77.11 & 72.586 \\
\hline 7/7/07 22:00 & 123.483 & 121.075 & 117.852 & 98.811 & 103.017 & 94.531 & 108.704 & 105.502 & 77.099 & 72.588 \\
\hline 7/7/07 22:30 & 123.447 & 121.066 & 117.817 & 98.782 & 102.981 & 94.498 & 108.664 & 105.467 & 77.081 & 72.569 \\
\hline 7/7/07 23:00 & 123.431 & 121.064 & 117.801 & 98.763 & 102.964 & 94.48 & 108.633 & 105.449 & 77.066 & 72.553 \\
\hline 7/7/07 23:30 & 123.419 & 121.055 & 117.789 & 98.756 & 102.956 & 94.47 & 108.621 & 105.438 & 77.051 & 72.543 \\
\hline $7 / 8 / 070: 00$ & 123.414 & 121.043 & 117.785 & 98.752 & 102.948 & 94.465 & 108.61 & 105.431 & 77.04 & 72.534 \\
\hline 7/8/07 0:30 & 123.41 & 121.039 & 117.782 & 98.745 & 102.946 & 94.461 & 108.603 & 105.427 & 77.033 & 72.53 \\
\hline 7/8/07 1:00 & 123.398 & 121.03 & 117.771 & 98.733 & 102.933 & 94.449 & 108.597 & 105.413 & 77.022 & 72.516 \\
\hline 7/8/07 1:30 & 123.391 & 121.027 & 117.761 & 98.728 & 102.927 & 94.444 & 108.589 & 105.409 & 77.012 & 72.509 \\
\hline $7 / 8 / 072: 00$ & 123.386 & 121.023 & 117.755 & 98.721 & 102.92 & 94.437 & 108.587 & 105.4 & 77.001 & 72.501 \\
\hline $7 / 8 / 072: 30$ & 123.381 & 121.011 & 117.755 & 98.721 & 102.922 & 94.44 & 108.584 & 105.4 & 76.992 & 72.499 \\
\hline $7 / 8 / 073: 00$ & 123.379 & 121 & 117.75 & 98.714 & 102.914 & 94.432 & 108.58 & 105.396 & 76.985 & 72.495 \\
\hline 7/8/07 3:30 & 123.379 & 120.996 & 117.741 & 98.706 & 102.905 & 94.423 & 108.578 & 105.387 & 76.974 & 72.483 \\
\hline $7 / 8 / 074: 00$ & 123.377 & 120.991 & 117.727 & 98.694 & 102.893 & 94.411 & 108.574 & 105.373 & 76.963 & 72.47 \\
\hline 7/8/07 4:30 & 123.372 & 120.989 & 117.722 & 98.689 & 102.887 & 94.404 & 108.57 & 105.367 & 76.953 & 72.466 \\
\hline 7/8/07 5:00 & 123.369 & 120.984 & 117.715 & 98.682 & 102.88 & 94.399 & 108.568 & 105.36 & 76.944 & 72.46 \\
\hline 7/8/07 5:30 & 123.343 & 120.984 & 117.715 & 98.685 & 102.884 & 94.402 & 108.565 & 105.362 & 76.942 & 72.462 \\
\hline 7/8/07 6:00 & 123.339 & 120.989 & 117.711 & 98.68 & 102.88 & 94.397 & 108.559 & 105.356 & 76.933 & 72.456 \\
\hline 7/8/07 6:30 & 123.336 & 120.982 & 117.708 & 98.68 & 102.876 & 94.395 & 108.557 & 105.354 & 76.924 & 72.454 \\
\hline 7/8/07 7:00 & 123.332 & 123.12 & 117.715 & 98.685 & 102.884 & 94.402 & 108.553 & 105.36 & 76.92 & 72.458 \\
\hline 7/8/07 7:30 & 123.329 & 123.385 & 117.706 & 98.676 & 102.874 & 94.393 & 108.55 & 105.351 & 76.913 & 72.452 \\
\hline 7/8/07 8:00 & 125.515 & 123.517 & 119.193 & 99.481 & 103.464 & 95.135 & 109.274 & 106.965 & 76.935 & 72.545 \\
\hline $7 / 8 / 078: 30$ & 125.799 & 123.594 & 119.466 & 99.717 & 103.714 & 95.389 & 109.503 & 107.26 & 76.979 & 72.726 \\
\hline $7 / 8 / 079: 00$ & 125.936 & 123.651 & 119.602 & 99.839 & 103.845 & 95.519 & 109.629 & 107.404 & 77.014 & 72.851 \\
\hline 7/8/07 9:30 & 126.017 & 123.678 & 119.681 & 99.907 & 103.919 & 95.597 & 109.71 & 107.486 & 77.039 & 72.931 \\
\hline $7 / 8 / 07$ 10:00 & 126.074 & 123.705 & 119.738 & 99.965 & 103.976 & 95.653 & 109.76 & 107.548 & 77.06 & 72.989 \\
\hline 7/8/07 10:30 & 126.102 & 123.716 & 119.768 & 99.991 & 104.007 & 95.679 & 109.798 & 107.576 & 77.075 & 73.024 \\
\hline 7/8/07 11:00 & 126.128 & 123.728 & 119.794 & 100.017 & 104.037 & 95.708 & 109.824 & 107.607 & 77.094 & 73.055 \\
\hline 7/8/07 11:30 & 126.142 & 123.735 & 119.808 & 100.031 & 104.045 & 95.719 & 109.847 & 107.621 & 77.108 & 73.072 \\
\hline 7/8/07 12:00 & 126.158 & 123.737 & 119.826 & 100.044 & 104.06 & 95.736 & 109.862 & 107.639 & 77.123 & 73.092 \\
\hline $7 / 8 / 0712: 30$ & 126.163 & 123.794 & 119.831 & 100.048 & 104.068 & 95.661 & 109.875 & 107.64 & 77.136 & 73.099 \\
\hline 7/8/07 13:00 & 126.163 & 122.199 & 119.833 & 100.051 & 104.073 & 95.663 & 109.871 & 107.64 & 77.143 & 73.105 \\
\hline 7/8/07 13:30 & 126.225 & 121.646 & 119.926 & 100.189 & 104.163 & 95.788 & 110.892 & 107.69 & 77.164 & 73.115 \\
\hline $7 / 8 / 0714: 00$ & 124.728 & 121.449 & 119.093 & 99.784 & 104.03 & 95.533 & 110.65 & 106.915 & 77.188 & 73.136 \\
\hline 7/8/07 14:30 & 124.031 & 121.34 & 118.411 & 99.354 & 103.54 & 95.01 & 110.15 & 106.056 & 77.169 & 73.008 \\
\hline 7/8/07 15:00 & 123.821 & 121.211 & 118.203 & 99.18 & 103.352 & 94.822 & 109.97 & 105.836 & 77.153 & 72.863 \\
\hline 7/8/07 15:30 & 123.71 & 121.123 & 118.094 & 99.083 & 103.253 & 94.718 & 109.872 & 105.721 & 77.136 & 72.77 \\
\hline 7/8/07 16:00 & 123.575 & 121.066 & 117.944 & 98.894 & 103.095 & 94.529 & 108.802 & 105.602 & 77.118 & 72.695 \\
\hline 7/8/07 16:30 & 123.483 & 121.025 & 117.852 & 98.799 & 103.007 & 94.444 & 108.721 & 105.504 & 77.088 & 72.625 \\
\hline 7/8/07 17:00 & 123.424 & 120.993 & 117.794 & 98.744 & 102.952 & 94.388 & 108.659 & 105.444 & 77.064 & 72.567 \\
\hline 7/8/07 17:30 & 123.384 & 120.971 & 117.755 & 98.707 & 102.912 & 94.35 & 108.62 & 105.405 & 77.042 & 72.524 \\
\hline 7/8/07 18:00 & 123.351 & 120.957 & 117.722 & 98.678 & 102.88 & 94.317 & 108.59 & 105.369 & 77.022 & 72.491 \\
\hline $7 / 8 / 0718: 30$ & 123.327 & 120.937 & 117.701 & 98.657 & 102.861 & 94.298 & 108.569 & 105.347 & 77.005 & 72.47 \\
\hline 7/8/07 19:00 & 123.313 & 120.923 & 117.683 & 98.644 & 102.847 & 94.282 & 108.554 & 105.331 & 76.987 & 72.45 \\
\hline 7/8/07 19:30 & 123.294 & 120.909 & 117.666 & 98.628 & 102.828 & 94.267 & 108.541 & 105.312 & 76.972 & 72.435 \\
\hline $7 / 8 / 0720: 00$ & 123.28 & 120.907 & 117.652 & 98.618 & 102.819 & 94.256 & 108.529 & 105.299 & 76.957 & 72.423 \\
\hline $7 / 8 / 0720: 30$ & 123.268 & 120.909 & 117.639 & 98.604 & 102.806 & 94.242 & 108.52 & 105.285 & 76.942 & 72.408 \\
\hline 7/8/07 21:00 & 123.261 & 120.918 & 117.634 & 98.602 & 102.804 & 94.234 & 108.513 & 105.278 & 76.931 & 72.402 \\
\hline $7 / 8 / 0721: 30$ & 123.263 & 120.925 & 117.636 & 98.606 & 102.806 & 94.242 & 108.505 & 105.28 & 76.92 & 72.406 \\
\hline 7/8/07 22:00 & 123.27 & 120.923 & 117.645 & 98.616 & 102.815 & 94.251 & 108.495 & 105.29 & 76.913 & 72.414 \\
\hline 7/8/07 22:30 & 123.28 & 120.912 & 117.655 & 98.625 & 102.826 & 94.26 & 108.49 & 105.299 & 76.906 & 72.421 \\
\hline 7/8/07 23:00 & 123.28 & 120.894 & 117.653 & 98.625 & 102.825 & 94.26 & 108.484 & 105.299 & 76.9 & 72.416 \\
\hline 7/8/07 23:30 & 123.265 & 120.889 & 117.641 & 98.611 & 102.811 & 94.246 & 108.482 & 105.287 & 76.887 & 72.404 \\
\hline 7/9/07 0:00 & 123.244 & 120.889 & 117.62 & 98.59 & 102.79 & 94.227 & 108.479 & 105.268 & 76.872 & 72.385 \\
\hline $7 / 9 / 070: 30$ & 123.242 & 120.887 & 117.618 & 98.589 & 102.79 & 94.225 & 108.475 & 105.263 & 76.863 & 72.381 \\
\hline $7 / 9 / 071: 00$ & 123.239 & 120.882 & 117.616 & 98.59 & 102.788 & 94.225 & 108.469 & 105.261 & 76.856 & 72.381 \\
\hline 7/9/07 1:30 & 123.242 & 120.88 & 117.616 & 98.589 & 102.788 & 94.225 & 108.467 & 105.261 & 76.847 & 72.379 \\
\hline $7 / 9 / 072: 00$ & 123.232 & 120.878 & 117.606 & 98.585 & 102.783 & 94.218 & 108.461 & 105.252 & 76.837 & 72.371 \\
\hline
\end{tabular}


TABLE S1.1 (Cont.)

Depth from Top of Casing (ft)

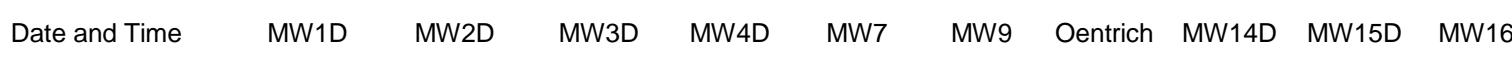

\begin{tabular}{|c|c|c|c|c|c|c|c|c|c|c|}
\hline 7/9/07 2:30 & 123.232 & 120.871 & 117.609 & 98.582 & 102.785 & 94.218 & 108.46 & 105.252 & 76.834 & 72.371 \\
\hline 7/9/07 3:00 & 123.232 & 120.869 & 117.609 & 98.582 & 102.781 & 94.216 & 108.456 & 105.252 & 76.824 & 72.373 \\
\hline 7/9/07 3:30 & 123.232 & 120.878 & 117.599 & 98.57 & 102.771 & 94.206 & 108.454 & 105.243 & 76.819 & 72.361 \\
\hline 7/9/07 4:00 & 123.228 & 120.896 & 117.597 & 98.57 & 102.769 & 94.206 & 108.452 & 105.241 & 76.808 & 72.361 \\
\hline $7 / 9 / 074: 30$ & 123.223 & 120.903 & 117.604 & 98.583 & 102.781 & 94.216 & 108.446 & 105.247 & 76.806 & 72.367 \\
\hline $7 / 9 / 075: 00$ & 123.247 & 120.907 & 117.625 & 98.602 & 102.802 & 94.234 & 108.452 & 105.267 & 76.808 & 72.385 \\
\hline 7/9/07 5:30 & 123.249 & 120.916 & 117.632 & 98.609 & 102.807 & 94.244 & 108.461 & 105.276 & 76.806 & 72.39 \\
\hline 7/9/07 6:00 & 123.261 & 122.97 & 117.639 & 98.613 & 102.811 & 94.249 & 108.463 & 105.278 & 76.804 & 72.394 \\
\hline 7/9/07 6:30 & 123.275 & 123.249 & 117.65 & 98.623 & 102.826 & 94.263 & 108.469 & 105.294 & 76.804 & 72.406 \\
\hline 7/9/07 7:00 & 125.397 & 123.401 & 119.197 & 99.424 & 103.384 & 94.993 & 109.192 & 106.808 & 76.823 & 72.468 \\
\hline 7/9/07 7:30 & 125.695 & 123.515 & 119.491 & 99.672 & 103.651 & 95.26 & 109.455 & 107.122 & 76.856 & 72.646 \\
\hline $7 / 9 / 078: 00$ & 125.856 & 123.587 & 119.646 & 99.814 & 103.801 & 95.408 & 109.59 & 107.286 & 76.887 & 72.782 \\
\hline 7/9/07 8:30 & 125.969 & 123.633 & 119.759 & 99.926 & 103.914 & 95.519 & 109.672 & 107.402 & 76.926 & 72.892 \\
\hline 7/9/07 9:00 & 126.043 & 123.678 & 119.833 & 99.991 & 103.984 & 95.59 & 109.732 & 107.479 & 76.959 & 72.967 \\
\hline 7/9/07 9:30 & 126.087 & 123.698 & 119.877 & 100.034 & 104.028 & 95.635 & 109.774 & 107.528 & 76.988 & 73.012 \\
\hline 7/9/07 10:00 & 126.135 & 123.714 & 119.926 & 100.077 & 104.073 & 95.679 & 109.8 & 107.576 & 77.016 & 73.063 \\
\hline 7/9/07 10:30 & 126.156 & 123.728 & 119.946 & 100.094 & 104.096 & 95.701 & 109.824 & 107.599 & 77.036 & 73.086 \\
\hline 7/9/07 11:00 & 126.173 & 123.753 & 119.963 & 100.11 & 104.112 & 95.715 & 109.841 & 107.614 & 77.057 & 73.105 \\
\hline 7/9/07 11:30 & 126.187 & 123.769 & 119.979 & 100.129 & 104.125 & 95.734 & 109.856 & 107.63 & 77.079 & 73.128 \\
\hline $7 / 9 / 07 \quad 12: 00$ & 126.215 & 123.771 & 120.004 & 100.151 & 104.153 & 95.759 & 109.869 & 107.656 & 77.099 & 73.15 \\
\hline 7/9/07 12:30 & 126.229 & 123.771 & 120.02 & 100.168 & 104.169 & 95.774 & 109.875 & 107.674 & 77.118 & 73.171 \\
\hline 7/9/07 13:00 & 126.229 & 123.764 & 120.023 & 100.17 & 104.171 & 95.778 & 109.882 & 107.676 & 77.136 & 73.177 \\
\hline 7/9/07 13:30 & 126.234 & 121.719 & 120.03 & 100.172 & 104.172 & 95.781 & 109.892 & 107.678 & 77.149 & 73.183 \\
\hline 7/9/07 14:00 & 126.229 & 121.431 & 120.02 & 100.162 & 104.167 & 95.774 & 109.897 & 107.672 & 77.162 & 73.177 \\
\hline 7/9/07 14:30 & 124.109 & 121.281 & 118.469 & 99.335 & 103.578 & 95.012 & 109.158 & 106.151 & 77.147 & 73.092 \\
\hline $7 / 9 / 07 \quad 15: 00$ & 123.804 & 121.197 & 118.166 & 99.08 & 103.302 & 94.739 & 108.921 & 105.832 & 77.119 & 72.919 \\
\hline 7/9/07 15:30 & 123.646 & 121.134 & 118.013 & 98.949 & 103.165 & 94.6 & 108.795 & 105.673 & 77.097 & 72.791 \\
\hline $7 / 9 / 07 \quad 16: 00$ & 123.556 & 121.093 & 117.928 & 98.875 & 103.082 & 94.52 & 108.716 & 105.579 & 77.081 & 72.708 \\
\hline 7/9/07 16:30 & 123.492 & 121.059 & 117.866 & 98.814 & 103.021 & 94.458 & 108.662 & 105.513 & 77.06 & 72.642 \\
\hline 7/9/07 17:00 & 123.455 & 121.025 & 117.828 & 98.785 & 102.988 & 94.425 & 108.626 & 105.475 & 77.046 & 72.605 \\
\hline 7/9/07 17:30 & 123.417 & 120.995 & 117.791 & 98.747 & 102.95 & 94.388 & 108.6 & 105.438 & 77.029 & 72.567 \\
\hline 7/9/07 18:00 & 123.379 & 120.986 & 117.756 & 98.714 & 102.916 & 94.352 & 108.579 & 105.4 & 77.012 & 72.532 \\
\hline 7/9/07 18:30 & 123.355 & 120.977 & 117.731 & 98.692 & 102.895 & 94.331 & 108.563 & 105.373 & 76.998 & 72.507 \\
\hline 7/9/07 19:00 & 123.341 & 121.458 & 117.717 & 98.683 & 102.882 & 94.319 & 108.549 & 105.36 & 76.983 & 72.493 \\
\hline 7/9/07 19:30 & 123.334 & 123.131 & 117.71 & 98.675 & 102.876 & 94.312 & 108.544 & 105.356 & 76.972 & 72.483 \\
\hline $7 / 9 / 0720: 00$ & 123.31 & 123.374 & 117.687 & 98.735 & 102.853 & 94.291 & 108.532 & 105.329 & 76.955 & 72.462 \\
\hline $7 / 9 / 0720: 30$ & 125.532 & 123.508 & 119.209 & 99.488 & 103.47 & 95.066 & 109.287 & 106.985 & 76.974 & 72.569 \\
\hline 7/9/07 21:00 & 125.79 & 123.589 & 119.456 & 99.702 & 103.698 & 95.297 & 109.498 & 107.249 & 77 & 72.739 \\
\hline $7 / 9 / 0721: 30$ & 125.929 & 123.648 & 119.595 & 99.827 & 103.834 & 95.427 & 109.614 & 107.393 & 77.024 & 72.861 \\
\hline $7 / 9 / 0722: 00$ & 126.014 & 123.685 & 119.678 & 99.905 & 103.917 & 95.512 & 109.685 & 107.481 & 77.046 & 72.946 \\
\hline 7/9/07 22:30 & 126.069 & 123.71 & 119.736 & 99.96 & 103.971 & 95.564 & 109.734 & 107.539 & 77.066 & 73.003 \\
\hline 7/9/07 23:00 & 126.111 & 123.732 & 119.778 & 99.998 & 104.014 & 95.606 & 109.766 & 107.583 & 77.086 & 73.049 \\
\hline 7/9/07 23:30 & 126.135 & 123.753 & 119.805 & 100.025 & 104.041 & 95.632 & 109.789 & 107.612 & 77.105 & 73.078 \\
\hline $7 / 10 / 07$ 0:00 & 126.159 & 123.76 & 119.831 & 100.051 & 104.072 & 95.66 & 109.804 & 107.636 & 77.123 & 73.107 \\
\hline $7 / 10 / 07$ 0:30 & 126.177 & 123.757 & 119.849 & 100.072 & 104.087 & 95.682 & 109.815 & 107.654 & 77.138 & 73.13 \\
\hline 7/10/07 1:00 & 126.187 & 121.76 & 119.861 & 100.081 & 104.1 & 95.691 & 109.826 & 107.667 & 77.147 & 73.146 \\
\hline 7/10/07 1:30 & 126.184 & 121.438 & 119.859 & 100.081 & 104.098 & 95.691 & 109.833 & 107.667 & 77.154 & 73.15 \\
\hline $7 / 10 / 072: 00$ & 124.154 & 121.293 & 118.515 & 99.367 & 103.615 & 95.052 & 109.207 & 106.208 & 77.153 & 73.099 \\
\hline 7/10/07 2:30 & 123.807 & 121.206 & 118.173 & 99.092 & 103.312 & 94.746 & 108.932 & 105.841 & 77.13 & 72.929 \\
\hline 7/10/07 3:00 & 123.658 & 121.177 & 118.027 & 98.961 & 103.177 & 94.612 & 108.795 & 105.688 & 77.114 & 72.815 \\
\hline 7/10/07 3:30 & 123.566 & 121.136 & 117.939 & 98.888 & 103.095 & 94.534 & 108.711 & 105.593 & 77.099 & 72.735 \\
\hline 7/10/07 4:00 & 123.535 & 121.107 & 117.907 & 98.868 & 103.068 & 94.506 & 108.655 & 105.557 & 77.094 & 72.702 \\
\hline 7/10/07 4:30 & 123.497 & 121.079 & 117.87 & 98.828 & 103.034 & 94.468 & 108.617 & 105.517 & 77.079 & 72.656 \\
\hline 7/10/07 5:00 & 123.473 & 121.048 & 117.842 & 98.802 & 103.004 & 94.44 & 108.59 & 105.489 & 77.066 & 72.625 \\
\hline 7/10/07 5:30 & 123.457 & 121.036 & 117.815 & 98.776 & 102.979 & 94.414 & 108.568 & 105.46 & 77.053 & 72.596 \\
\hline $7 / 10 / 076: 00$ & 123.419 & 123.091 & 117.782 & 98.745 & 102.948 & 94.383 & 108.555 & 105.427 & 77.035 & 72.563 \\
\hline $7 / 10 / 076: 30$ & 123.405 & 123.363 & 117.77 & 98.735 & 102.935 & 94.374 & 108.544 & 105.416 & 77.024 & 72.553 \\
\hline 7/10/07 7:00 & 125.485 & 123.51 & 119.186 & 99.49 & 103.47 & 95.062 & 109.216 & 106.948 & 77.036 & 72.615 \\
\hline $7 / 10 / 07$ 7:30 & 125.778 & 123.592 & 119.468 & 99.736 & 103.737 & 95.326 & 109.447 & 107.253 & 77.066 & 72.797 \\
\hline $7 / 10 / 078: 00$ & 125.927 & 121.658 & 119.616 & 99.87 & 103.879 & 95.47 & 109.569 & 107.408 & 77.094 & 72.927 \\
\hline $7 / 10 / 078: 30$ & 126.012 & 121.399 & 119.697 & 99.95 & 103.961 & 95.547 & 109.642 & 107.497 & 77.116 & 73.012 \\
\hline 7/10/07 9:00 & 124.043 & 121.279 & 118.443 & 99.29 & 103.525 & 94.958 & 109.045 & 106.093 & 77.123 & 73.016 \\
\hline 7/10/07 9:30 & 123.767 & 121.211 & 118.161 & 99.095 & 103.287 & 94.72 & 108.812 & 105.799 & 77.11 & 72.894 \\
\hline $7 / 10 / 07 \quad 10: 00$ & 123.641 & 121.168 & 118.027 & 98.962 & 103.173 & 94.607 & 108.703 & 105.666 & 77.097 & 72.797 \\
\hline $7 / 10 / 07 \quad 10: 30$ & 123.57 & 121.143 & 117.953 & 98.902 & 103.11 & 94.543 & 108.636 & 105.595 & 77.084 & 72.733 \\
\hline 7/10/07 11:00 & 123.53 & 121.127 & 117.904 & 98.868 & 103.068 & 94.503 & 108.591 & 105.547 & 77.077 & 72.689 \\
\hline
\end{tabular}


TABLE S1.1 (Cont.)

\begin{tabular}{|c|c|c|c|c|c|c|c|c|c|c|}
\hline \multirow[b]{2}{*}{ Date and Time } & \multicolumn{10}{|c|}{ Depth from Top of Casing (ft) } \\
\hline & MW1D & MW2D & MW3D & MW4D & MW7 & MW9 & Oentrich & MW14D & MW15D & MW16D \\
\hline 7/10/07 11:30 & 123.502 & 121.113 & 117.877 & 98.847 & 103.045 & 94.48 & 108.563 & 105.52 & 77.07 & 72.66 \\
\hline $7 / 10 / 07$ 12:00 & 123.483 & 121.104 & 117.861 & 98.828 & 103.028 & 94.465 & 108.538 & 105.504 & 77.06 & 72.644 \\
\hline 7/10/07 12:30 & 123.473 & 121.095 & 117.844 & 98.819 & 103.019 & 94.454 & 108.523 & 105.489 & 77.053 & 72.629 \\
\hline 7/10/07 13:00 & 123.462 & 121.091 & 117.837 & 98.813 & 103.009 & 94.442 & 108.51 & 105.478 & 77.049 & 72.619 \\
\hline $7 / 10 / 07$ 13:30 & 123.455 & 121.086 & 117.829 & 98.802 & 103.004 & 94.44 & 108.497 & 105.471 & 77.038 & 72.611 \\
\hline 7/10/07 14:00 & 123.447 & 121.086 & 117.823 & 98.8 & 103.002 & 94.432 & 108.487 & 105.466 & 77.031 & 72.607 \\
\hline $7 / 10 / 0714: 30$ & 123.443 & 121.084 & 117.817 & 98.797 & 102.998 & 94.43 & 108.476 & 105.458 & 77.027 & 72.6 \\
\hline 7/10/07 15:00 & 123.443 & 121.082 & 117.814 & 98.799 & 102.998 & 94.432 & 108.47 & 105.46 & 77.022 & 72.6 \\
\hline 7/10/07 15:30 & 123.438 & 121.077 & 117.814 & 98.797 & 102.996 & 94.428 & 108.468 & 105.455 & 77.014 & 72.598 \\
\hline 7/10/07 16:00 & 123.443 & 121.073 & 117.817 & 98.797 & 102.996 & 94.432 & 108.467 & 105.46 & 77.012 & 72.596 \\
\hline 7/10/07 16:30 & 123.436 & 121.075 & 117.812 & 98.795 & 102.99 & 94.428 & 108.461 & 105.453 & 77.003 & 72.59 \\
\hline 7/10/07 17:00 & 123.429 & 121.075 & 117.812 & 98.788 & 102.986 & 94.423 & 108.455 & 105.449 & 77.001 & 72.588 \\
\hline 7/10/07 17:30 & 123.431 & 121.066 & 117.812 & 98.792 & 102.99 & 94.428 & 108.452 & 105.453 & 76.998 & 72.586 \\
\hline 7/10/07 18:00 & 123.429 & 121.066 & 117.81 & 98.79 & 102.986 & 94.423 & 108.448 & 105.451 & 76.992 & 72.584 \\
\hline 7/10/07 18:30 & 123.421 & 121.066 & 117.805 & 98.782 & 102.981 & 94.416 & 108.444 & 105.442 & 76.988 & 72.578 \\
\hline 7/10/07 19:00 & 123.421 & 121.061 & 117.803 & 98.783 & 102.981 & 94.418 & 108.438 & 105.44 & 76.985 & 72.578 \\
\hline 7/10/07 19:30 & 123.421 & 121.068 & 117.803 & 98.782 & 102.979 & 94.416 & 108.437 & 105.44 & 76.981 & 72.578 \\
\hline 7/10/07 20:00 & 123.417 & 121.064 & 117.798 & 98.778 & 102.975 & 94.414 & 108.433 & 105.435 & 76.976 & 72.574 \\
\hline $7 / 10 / 0720: 30$ & 123.421 & 121.064 & 117.803 & 98.785 & 102.981 & 94.418 & 108.433 & 105.444 & 76.972 & 72.576 \\
\hline 7/10/07 21:00 & 123.419 & 121.073 & 117.8 & 98.782 & 102.977 & 94.416 & 108.447 & 105.44 & 76.97 & 72.576 \\
\hline 7/10/07 21:30 & 123.414 & 121.086 & 117.798 & 98.782 & 102.975 & 94.414 & 108.468 & 105.438 & 76.97 & 72.574 \\
\hline 7/10/07 22:00 & 123.424 & 121.098 & 117.807 & 98.794 & 102.988 & 94.428 & 108.455 & 105.449 & 76.97 & 72.584 \\
\hline 7/10/07 22:30 & 123.438 & 121.102 & 117.821 & 98.807 & 103.002 & 94.44 & 108.439 & 105.464 & 76.97 & 72.594 \\
\hline 7/10/07 23:00 & 123.45 & 121.109 & 117.833 & 98.818 & 103.011 & 94.449 & 108.428 & 105.473 & 76.974 & 72.605 \\
\hline 7/10/07 23:30 & 123.457 & 121.111 & 117.84 & 98.825 & 103.021 & 94.456 & 108.42 & 105.48 & 76.974 & 72.611 \\
\hline 7/11/07 0:00 & 123.462 & 121.111 & 117.847 & 98.83 & 103.024 & 94.465 & 108.413 & 105.484 & 76.976 & 72.619 \\
\hline 7/11/07 0:30 & 123.469 & 121.109 & 117.849 & 98.835 & 103.028 & 94.463 & 108.409 & 105.489 & 76.977 & 72.621 \\
\hline 7/11/07 1:00 & 123.464 & 121.109 & 117.849 & 98.833 & 103.028 & 94.465 & 108.405 & 105.489 & 76.977 & 72.621 \\
\hline 7/11/07 1:30 & 123.462 & 121.1 & 117.849 & 98.833 & 103.028 & 94.465 & 108.399 & 105.489 & 76.976 & 72.621 \\
\hline 7/11/07 2:00 & 123.457 & 121.102 & 117.847 & 98.832 & 103.026 & 94.465 & 108.396 & 105.486 & 76.974 & 72.619 \\
\hline 7/11/07 2:30 & 123.457 & 121.104 & 117.84 & 98.823 & 103.019 & 94.456 & 108.394 & 105.478 & 76.972 & 72.613 \\
\hline 7/11/07 3:00 & 123.452 & 121.104 & 117.838 & 98.83 & 103.023 & 94.461 & 108.388 & 105.48 & 76.97 & 72.615 \\
\hline 7/11/07 3:30 & 123.462 & 121.104 & 117.844 & 98.83 & 103.026 & 94.463 & 108.384 & 105.484 & 76.968 & 72.619 \\
\hline 7/11/07 4:00 & 123.459 & 121.102 & 117.844 & 98.832 & 103.026 & 94.463 & 108.381 & 105.482 & 76.966 & 72.617 \\
\hline 7/11/07 4:30 & 123.457 & 121.1 & 117.844 & 98.83 & 103.024 & 94.461 & 108.379 & 105.482 & 76.965 & 72.615 \\
\hline 7/11/07 5:00 & 123.452 & 121.109 & 117.842 & 98.828 & 103.024 & 94.461 & 108.375 & 105.482 & 76.961 & 72.617 \\
\hline 7/11/07 5:30 & 123.45 & 121.116 & 117.84 & 98.826 & 103.021 & 94.458 & 108.371 & 105.478 & 76.961 & 72.615 \\
\hline 7/11/07 6:00 & 123.445 & 123.138 & 117.84 & 98.838 & 103.026 & 94.463 & 108.367 & 105.482 & 76.961 & 72.617 \\
\hline 7/11/07 6:30 & 123.471 & 123.456 & 117.854 & 98.84 & 103.036 & 94.473 & 108.364 & 105.493 & 76.966 & 72.627 \\
\hline 7/11/07 7:00 & 125.556 & 123.617 & 119.376 & 99.624 & 103.573 & 95.182 & 109.047 & 106.97 & 76.988 & 72.691 \\
\hline 7/11/07 7:30 & 125.896 & 123.719 & 119.708 & 99.908 & 103.879 & 95.488 & 109.323 & 107.331 & 77.031 & 72.888 \\
\hline 7/11/07 8:00 & 126.069 & 123.791 & 119.877 & 100.055 & 104.037 & 95.649 & 109.483 & 107.508 & 77.07 & 73.035 \\
\hline 7/11/07 8:30 & 126.173 & 123.841 & 119.976 & 100.148 & 104.134 & 95.743 & 109.571 & 107.612 & 77.106 & 73.134 \\
\hline 7/11/07 9:00 & 126.243 & 123.877 & 120.05 & 100.218 & 104.205 & 95.814 & 109.622 & 107.69 & 77.14 & 73.206 \\
\hline 7/11/07 9:30 & 126.3 & 123.902 & 120.104 & 100.267 & 104.258 & 95.866 & 109.659 & 107.745 & 77.169 & 73.262 \\
\hline 7/11/07 10:00 & 126.338 & 123.923 & 120.143 & 100.301 & 104.296 & 95.901 & 109.684 & 107.783 & 77.197 & 73.301 \\
\hline 7/11/07 10:30 & 126.362 & 123.934 & 120.168 & 100.325 & 104.319 & 95.927 & 109.704 & 107.809 & 77.223 & 73.33 \\
\hline 7/11/07 11:00 & 126.381 & 123.952 & 120.187 & 100.343 & 104.34 & 95.946 & 109.721 & 107.831 & 77.243 & 73.351 \\
\hline $7 / 11 / 0711: 30$ & 126.392 & 123.955 & 120.199 & 100.356 & 104.35 & 95.957 & 109.73 & 107.84 & 77.261 & 73.365 \\
\hline $7 / 11 / 07$ 12:00 & 126.409 & 123.939 & 120.215 & 100.37 & 104.367 & 95.974 & 109.74 & 107.858 & 77.282 & 73.386 \\
\hline $7 / 11 / 07$ 12:30 & 126.414 & 122.025 & 120.222 & 100.375 & 104.37 & 95.979 & 109.751 & 107.864 & 77.3 & 73.396 \\
\hline 7/11/07 13:00 & 126.4 & 121.66 & 120.208 & 100.36 & 104.355 & 95.967 & 109.76 & 107.851 & 77.306 & 73.384 \\
\hline 7/11/07 13:30 & 124.433 & 121.49 & 118.802 & 99.638 & 103.885 & 95.326 & 109.145 & 106.494 & 77.304 & 73.336 \\
\hline $7 / 11 / 0714: 00$ & 124.036 & 121.383 & 118.411 & 99.323 & 103.542 & 94.979 & 108.837 & 106.071 & 77.274 & 73.159 \\
\hline 7/11/07 14:30 & 123.856 & 121.311 & 118.233 & 99.173 & 103.38 & 94.822 & 108.69 & 105.885 & 77.254 & 73.022 \\
\hline 7/11/07 15:00 & 123.745 & 121.252 & 118.126 & 99.078 & 103.281 & 94.72 & 108.604 & 105.772 & 77.232 & 72.925 \\
\hline $7 / 11 / 07$ 15:30 & 123.672 & 121.209 & 118.052 & 99.011 & 103.209 & 94.649 & 108.546 & 105.697 & 77.213 & 72.853 \\
\hline 7/11/07 16:00 & 123.613 & 121.172 & 117.997 & 98.959 & 103.156 & 94.595 & 108.506 & 105.637 & 77.195 & 72.793 \\
\hline 7/11/07 16:30 & 123.568 & 121.145 & 117.953 & 98.919 & 103.114 & 94.555 & 108.474 & 105.591 & 77.177 & 72.753 \\
\hline 7/11/07 17:00 & 123.53 & 121.125 & 117.916 & 98.885 & 103.08 & 94.52 & 108.454 & 105.555 & 77.158 & 72.718 \\
\hline 7/11/07 17:30 & 123.502 & 121.107 & 117.886 & 98.859 & 103.051 & 94.491 & 108.435 & 105.524 & 77.142 & 72.687 \\
\hline 7/11/07 18:00 & 123.481 & 121.091 & 117.867 & 98.838 & 103.032 & 94.473 & 108.422 & 105.504 & 77.127 & 72.664 \\
\hline $7 / 11 / 07$ 18:30 & 123.464 & 121.082 & 117.849 & 98.823 & 103.017 & 94.456 & 108.409 & 105.486 & 77.116 & 72.65 \\
\hline 7/11/07 19:00 & 123.445 & 121.079 & 117.83 & 98.809 & 103 & 94.442 & 108.399 & 105.466 & 77.101 & 72.631 \\
\hline 7/11/07 19:30 & 123.438 & 121.073 & 117.826 & 98.802 & 102.996 & 94.437 & 108.392 & 105.462 & 77.092 & 72.627 \\
\hline $7 / 11 / 0720: 00$ & 123.431 & 121.066 & 117.817 & 98.799 & 102.99 & 94.428 & 108.382 & 105.453 & 77.083 & 72.619 \\
\hline
\end{tabular}


TABLE S1.1 (Cont.)

Depth from Top of Casing (ft)

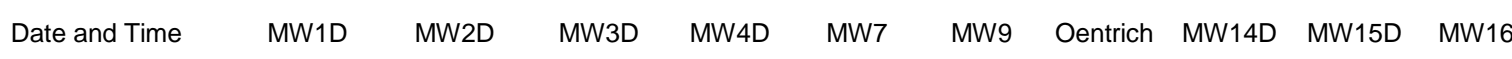

\begin{tabular}{|c|c|c|c|c|c|c|c|c|c|c|}
\hline 7/11/07 20:30 & 123.426 & 121.07 & 117.815 & 98.794 & 102.99 & 94.425 & 108.376 & 105.451 & 77.075 & 72.619 \\
\hline $7 / 11 / 0721: 00$ & 123.419 & 121.073 & 117.81 & 98.79 & 102.981 & 94.421 & 108.369 & 105.442 & 77.066 & 72.609 \\
\hline $7 / 11 / 0721: 30$ & 123.424 & 121.084 & 117.812 & 98.794 & 102.986 & 94.425 & 108.365 & 105.447 & 77.059 & 72.613 \\
\hline 7/11/07 22:00 & 123.426 & 121.084 & 117.817 & 98.8 & 102.992 & 94.432 & 108.358 & 105.451 & 77.055 & 72.619 \\
\hline $7 / 11 / 0722: 30$ & 123.436 & 121.077 & 117.826 & 98.809 & 103.002 & 94.444 & 108.35 & 105.462 & 77.053 & 72.625 \\
\hline 7/11/07 23:00 & 123.436 & 121.07 & 117.826 & 98.809 & 103.002 & 94.444 & 108.345 & 105.46 & 77.049 & 72.627 \\
\hline 7/11/07 23:30 & 123.429 & 121.061 & 117.821 & 98.802 & 102.998 & 94.437 & 108.339 & 105.456 & 77.042 & 72.621 \\
\hline $7 / 12 / 070: 00$ & 123.426 & 121.048 & 117.814 & 98.797 & 102.99 & 94.432 & 108.335 & 105.449 & 77.035 & 72.615 \\
\hline 7/12/07 0:30 & 123.424 & 121.032 & 117.805 & 98.787 & 102.981 & 94.423 & 108.333 & 105.442 & 77.027 & 72.607 \\
\hline 7/12/07 1:00 & 123.421 & 121.016 & 117.793 & 98.775 & 102.971 & 94.409 & 108.329 & 105.427 & 77.018 & 72.594 \\
\hline 7/12/07 1:30 & 123.386 & 120.996 & 117.773 & 98.757 & 102.95 & 94.39 & 108.326 & 105.409 & 77.009 & 72.576 \\
\hline 7/12/07 2:00 & 123.381 & 120.996 & 117.759 & 98.738 & 102.935 & 94.374 & 108.324 & 105.391 & 76.998 & 72.561 \\
\hline 7/12/07 2:30 & 123.351 & 120.989 & 117.738 & 98.721 & 102.916 & 94.355 & 108.32 & 105.374 & 76.987 & 72.545 \\
\hline 7/12/07 3:00 & 123.348 & 120.984 & 117.738 & 98.726 & 102.92 & 94.357 & 108.313 & 105.371 & 76.979 & 72.547 \\
\hline 7/12/07 3:30 & 123.351 & 120.982 & 117.736 & 98.718 & 102.908 & 94.348 & 108.311 & 105.365 & 76.972 & 72.536 \\
\hline 7/12/07 4:00 & 123.346 & 120.977 & 117.731 & 98.714 & 102.912 & 94.35 & 108.307 & 105.365 & 76.968 & 72.54 \\
\hline 7/12/07 4:30 & 123.341 & 120.968 & 117.722 & 98.713 & 102.912 & 94.35 & 108.301 & 105.363 & 76.961 & 72.54 \\
\hline 7/12/07 5:00 & 123.339 & 120.964 & 117.72 & 98.707 & 102.901 & 94.338 & 108.299 & 105.356 & 76.953 & 72.53 \\
\hline 7/12/07 5:30 & 123.339 & 120.964 & 117.718 & 98.699 & 102.893 & 94.333 & 108.296 & 105.347 & 76.948 & 72.522 \\
\hline 7/12/07 6:00 & 123.334 & 123.018 & 117.708 & 98.695 & 102.887 & 94.326 & 108.292 & 105.341 & 76.942 & 72.516 \\
\hline 7/12/07 6:30 & 123.329 & 123.333 & 117.706 & 98.695 & 102.889 & 94.329 & 108.288 & 105.341 & 76.939 & 72.52 \\
\hline 7/12/07 7:00 & 125.404 & 123.499 & 119.1 & 99.419 & 103.384 & 94.975 & 108.928 & 106.843 & 76.953 & 72.569 \\
\hline 7/12/07 7:30 & 125.747 & 123.599 & 119.431 & 99.698 & 103.691 & 95.283 & 109.206 & 107.209 & 76.987 & 72.764 \\
\hline 7/12/07 8:00 & 125.915 & 123.651 & 119.6 & 99.848 & 103.849 & 95.444 & 109.347 & 107.386 & 77.022 & 72.91 \\
\hline 7/12/07 8:30 & 126.019 & 123.689 & 119.699 & 99.939 & 103.944 & 95.54 & 109.433 & 107.49 & 77.051 & 73.006 \\
\hline 7/12/07 9:00 & 126.076 & 123.714 & 119.759 & 99.993 & 104.001 & 95.597 & 109.49 & 107.552 & 77.077 & 73.068 \\
\hline 7/12/07 9:30 & 126.113 & 123.739 & 119.799 & 100.031 & 104.037 & 95.632 & 109.529 & 107.59 & 77.099 & 73.109 \\
\hline $7 / 12 / 07$ 10:00 & 126.142 & 123.748 & 119.831 & 100.06 & 104.07 & 95.663 & 109.556 & 107.623 & 77.123 & 73.144 \\
\hline $7 / 12 / 07 \quad 10: 30$ & 126.163 & 123.755 & 119.852 & 100.079 & 104.093 & 95.686 & 109.582 & 107.645 & 77.145 & 73.169 \\
\hline 7/12/07 11:00 & 126.175 & 123.764 & 119.861 & 100.088 & 104.1 & 95.696 & 109.597 & 107.656 & 77.16 & 73.181 \\
\hline $7 / 12 / 0711: 30$ & 126.182 & 123.762 & 119.87 & 100.098 & 104.11 & 95.705 & 109.608 & 107.663 & 77.177 & 73.194 \\
\hline $7 / 12 / 07$ 12:00 & 126.191 & 123.76 & 119.879 & 100.106 & 104.121 & 95.715 & 109.617 & 107.674 & 77.195 & 73.208 \\
\hline $7 / 12 / 07$ 12:30 & 126.187 & 123.75 & 119.88 & 100.106 & 104.123 & 95.715 & 109.625 & 107.671 & 77.208 & 73.21 \\
\hline 7/12/07 13:00 & 126.187 & 123.737 & 119.879 & 100.108 & 104.121 & 95.717 & 109.631 & 107.672 & 77.221 & 73.214 \\
\hline 7/12/07 13:30 & 126.18 & 123.725 & 119.872 & 100.1 & 104.117 & 95.708 & 109.64 & 107.667 & 77.23 & 73.212 \\
\hline $7 / 12 / 0714: 00$ & 126.168 & 121.787 & 119.861 & 100.086 & 104.104 & 95.696 & 109.644 & 107.654 & 77.236 & 73.204 \\
\hline $7 / 12 / 0714: 30$ & 126.154 & 121.435 & 119.849 & 100.075 & 104.093 & 95.686 & 109.649 & 107.64 & 77.241 & 73.198 \\
\hline 7/12/07 15:00 & 124.185 & 121.265 & 118.559 & 99.398 & 103.649 & 95.09 & 109.069 & 106.253 & 77.232 & 73.154 \\
\hline $7 / 12 / 07$ 15:30 & 123.804 & 121.168 & 118.187 & 99.104 & 103.325 & 94.76 & 108.767 & 105.845 & 77.202 & 72.985 \\
\hline $7 / 12 / 0716: 00$ & 123.627 & 121.109 & 118.011 & 98.956 & 103.165 & 94.602 & 108.622 & 105.662 & 77.177 & 72.848 \\
\hline $7 / 12 / 07$ 16:30 & 123.53 & 121.057 & 117.916 & 98.869 & 103.078 & 94.51 & 108.534 & 105.559 & 77.156 & 72.76 \\
\hline 7/12/07 17:00 & 123.469 & 121.009 & 117.856 & 98.818 & 103.021 & 94.458 & 108.478 & 105.496 & 77.138 & 72.695 \\
\hline 7/12/07 17:30 & 123.414 & 120.98 & 117.8 & 98.769 & 102.967 & 94.407 & 108.438 & 105.44 & 77.116 & 72.638 \\
\hline $7 / 12 / 07$ 18:00 & 123.367 & 120.986 & 117.754 & 98.721 & 102.924 & 94.359 & 108.412 & 105.393 & 77.097 & 72.592 \\
\hline $7 / 12 / 07$ 18:30 & 123.334 & 121.016 & 117.722 & 98.699 & 102.893 & 94.329 & 108.391 & 105.358 & 77.075 & 72.557 \\
\hline 7/12/07 19:00 & 123.348 & 121.002 & 117.736 & 98.706 & 102.91 & 94.35 & 108.367 & 105.369 & 77.075 & 72.576 \\
\hline 7/12/07 19:30 & 123.372 & 120.971 & 117.759 & 98.751 & 102.941 & 94.378 & 108.346 & 105.394 & 77.07 & 72.594 \\
\hline 7/12/07 20:00 & 123.362 & 120.955 & 117.752 & 98.728 & 102.929 & 94.369 & 108.339 & 105.387 & 77.059 & 72.582 \\
\hline $7 / 12 / 0720: 30$ & 123.325 & 120.957 & 117.713 & 98.694 & 102.886 & 94.326 & 108.335 & 105.347 & 77.038 & 72.536 \\
\hline $7 / 12 / 0721: 00$ & 123.308 & 120.977 & 117.696 & 98.678 & 102.87 & 94.31 & 108.328 & 105.329 & 77.024 & 72.522 \\
\hline $7 / 12 / 0721: 30$ & 123.31 & 120.975 & 117.701 & 98.687 & 102.88 & 94.317 & 108.316 & 105.336 & 77.018 & 72.532 \\
\hline 7/12/07 22:00 & 123.332 & 120.971 & 117.722 & 98.709 & 102.901 & 94.338 & 108.307 & 105.354 & 77.018 & 72.553 \\
\hline $7 / 12 / 0722: 30$ & 123.332 & 120.957 & 117.722 & 98.707 & 102.901 & 94.341 & 108.303 & 105.356 & 77.011 & 72.549 \\
\hline 7/12/07 23:00 & 123.325 & 120.966 & 117.719 & 98.702 & 102.899 & 94.338 & 108.298 & 105.354 & 77.007 & 72.545 \\
\hline 7/12/07 23:30 & 123.325 & 120.966 & 117.704 & 98.687 & 102.884 & 94.322 & 108.294 & 105.338 & 76.998 & 72.53 \\
\hline $7 / 13 / 07$ 0:00 & 123.317 & 120.968 & 117.71 & 98.699 & 102.891 & 94.329 & 108.288 & 105.343 & 76.994 & 72.536 \\
\hline 7/13/07 0:30 & 123.322 & 120.968 & 117.713 & 98.699 & 102.893 & 94.333 & 108.283 & 105.347 & 76.99 & 72.54 \\
\hline 7/13/07 1:00 & 123.325 & 120.977 & 117.717 & 98.702 & 102.899 & 94.336 & 108.277 & 105.349 & 76.985 & 72.543 \\
\hline 7/13/07 1:30 & 123.322 & 120.966 & 117.711 & 98.702 & 102.895 & 94.331 & 108.271 & 105.345 & 76.977 & 72.536 \\
\hline 7/13/07 2:00 & 123.325 & 120.948 & 117.724 & 98.713 & 102.906 & 94.343 & 108.268 & 105.356 & 76.977 & 72.551 \\
\hline $7 / 13 / 072: 30$ & 123.322 & 120.93 & 117.715 & 98.702 & 102.899 & 94.336 & 108.264 & 105.347 & 76.972 & 72.54 \\
\hline 7/13/07 3:00 & 123.325 & 120.914 & 117.694 & 98.683 & 102.878 & 94.317 & 108.262 & 105.329 & 76.961 & 72.522 \\
\hline 7/13/07 3:30 & 123.299 & & 117.678 & 98.668 & 102.861 & 94.298 & 108.26 & 105.311 & 76.948 & 72.505 \\
\hline 7/13/07 4:00 & 123.275 & 120.9 & 117.662 & 98.668 & 102.842 & 94.282 & 108.258 & 105.294 & 76.939 & 72.491 \\
\hline 7/13/07 4:30 & 123.268 & 120.907 & 117.653 & 98.663 & 102.838 & 94.277 & 108.253 & 105.285 & 76.931 & 72.485 \\
\hline 7/13/07 5:00 & 123.265 & 120.918 & 117.655 & 98.661 & 102.836 & 94.275 & 108.247 & 105.285 & 76.928 & 72.487 \\
\hline
\end{tabular}


TABLE S1.1 (Cont.)

\begin{tabular}{|c|c|c|c|c|c|c|c|c|c|c|}
\hline \multirow[b]{2}{*}{ Date and Time } & \multicolumn{10}{|c|}{ Depth from Top of Casing (ft) } \\
\hline & MW1D & MW2D & MW3D & MW4D & MW7 & MW9 & Oentrich & MW14D & MW15D & MW16D \\
\hline 7/13/07 5:30 & 123.258 & 120.93 & 117.65 & 98.657 & 102.84 & 94.272 & 108.245 & 105.283 & 76.922 & 72.485 \\
\hline 7/13/07 6:00 & 123.273 & 122.941 & 117.667 & 98.656 & 102.851 & 94.289 & 108.239 & 105.299 & 76.922 & 72.495 \\
\hline 7/13/07 6:30 & 123.284 & 123.245 & 117.678 & 98.654 & 102.863 & 94.303 & 108.234 & 105.31 & 76.924 & 72.509 \\
\hline 7/13/07 7:00 & 125.343 & 123.401 & 119.176 & 99.447 & 103.39 & 94.993 & 108.898 & 106.762 & 76.946 & 72.571 \\
\hline 7/13/07 7:30 & 125.686 & 123.503 & 119.514 & 99.724 & 103.7 & 95.305 & 109.184 & 107.129 & 76.981 & 72.77 \\
\hline 7/13/07 8:00 & 125.851 & 123.565 & 119.678 & 99.87 & 103.853 & 95.462 & 109.323 & 107.302 & 77.016 & 72.915 \\
\hline 7/13/07 8:30 & 125.955 & 123.614 & 119.782 & 99.963 & 103.952 & 95.561 & 109.407 & 107.408 & 77.049 & 73.016 \\
\hline 7/13/07 9:00 & 126.024 & 123.648 & 119.845 & 100.02 & 104.011 & 95.618 & 109.467 & 107.473 & 77.079 & 73.078 \\
\hline 7/13/07 9:30 & 126.071 & 123.667 & 119.893 & 100.069 & 104.062 & 95.672 & 109.507 & 107.526 & 77.108 & 73.132 \\
\hline 7/13/07 10:00 & 126.106 & 123.682 & 119.93 & 100.1 & 104.096 & 95.703 & 109.535 & 107.565 & 77.134 & 73.165 \\
\hline $7 / 13 / 07$ 10:30 & 126.123 & 123.696 & 119.949 & 100.119 & 104.115 & 95.722 & 109.556 & 107.581 & 77.158 & 73.19 \\
\hline 7/13/07 11:00 & 126.139 & 121.696 & 119.967 & 100.134 & 104.131 & 95.736 & 109.571 & 107.601 & 77.18 & 73.21 \\
\hline 7/13/07 11:30 & 126.154 & 121.426 & 119.979 & 100.148 & 104.144 & 95.755 & 109.584 & 107.614 & 77.2 & 73.227 \\
\hline 7/13/07 12:00 & 124.083 & 121.283 & 118.462 & 99.343 & 103.578 & 95.017 & 108.861 & 106.131 & 77.193 & 73.154 \\
\hline 7/13/07 12:30 & 123.797 & 121.193 & 118.182 & 99.112 & 103.329 & 94.767 & 108.628 & 105.832 & 77.177 & 72.999 \\
\hline 7/13/07 13:00 & 123.653 & 121.125 & 118.039 & 98.985 & 103.194 & 94.633 & 108.508 & 105.682 & 77.16 & 72.875 \\
\hline 7/13/07 13:30 & 123.556 & 121.086 & 117.944 & 98.904 & 103.106 & 94.546 & 108.435 & 105.584 & 77.143 & 72.789 \\
\hline 7/13/07 14:00 & 123.485 & 121.045 & 117.877 & 98.842 & 103.042 & 94.48 & 108.386 & 105.513 & 77.127 & 72.72 \\
\hline 7/13/07 14:30 & 123.443 & 121.011 & 117.835 & 98.804 & 103.002 & 94.44 & 108.354 & 105.469 & 77.11 & 72.675 \\
\hline 7/13/07 15:00 & 123.4 & 120.984 & 117.793 & 98.768 & 102.965 & 94.404 & 108.328 & 105.425 & 77.095 & 72.638 \\
\hline 7/13/07 15:30 & 123.367 & 120.957 & 117.759 & 98.735 & 102.931 & 94.371 & 108.307 & 105.394 & 77.081 & 72.605 \\
\hline 7/13/07 16:00 & 123.339 & 120.934 & 117.733 & 98.713 & 102.906 & 94.345 & 108.292 & 105.365 & 77.064 & 72.58 \\
\hline 7/13/07 16:30 & 123.315 & 120.916 & 117.71 & 98.689 & 102.884 & 94.324 & 108.281 & 105.341 & 77.051 & 72.557 \\
\hline 7/13/07 17:00 & 123.291 & 120.896 & 117.687 & 98.666 & 102.863 & 94.3 & 108.269 & 105.316 & 77.036 & 72.534 \\
\hline 7/13/07 17:30 & 123.273 & 120.878 & 117.667 & 98.649 & 102.846 & 94.284 & 108.262 & 105.296 & 77.024 & 72.516 \\
\hline 7/13/07 18:00 & 123.251 & 120.875 & 117.645 & 98.626 & 102.823 & 94.263 & 108.254 & 105.276 & 77.009 & 72.495 \\
\hline 7/13/07 18:30 & 123.23 & 120.859 & 117.625 & 98.685 & 102.804 & 94.242 & 108.249 & 105.256 & 76.992 & 72.474 \\
\hline 7/13/07 19:00 & 123.23 & 120.853 & 117.63 & 98.609 & 102.807 & 94.244 & 108.309 & 105.259 & 76.99 & 72.472 \\
\hline 7/13/07 19:30 & 123.216 & 120.835 & 117.611 & 98.595 & 102.794 & 94.23 & 108.283 & 105.243 & 76.979 & 72.464 \\
\hline 7/13/07 20:00 & 123.206 & 120.853 & 117.604 & 98.587 & 102.787 & 94.22 & 108.262 & 105.232 & 76.968 & 72.456 \\
\hline 7/13/07 20:30 & 123.19 & 120.83 & 117.588 & 98.571 & 102.767 & 94.204 & 108.248 & 105.216 & 76.955 & 72.439 \\
\hline 7/13/07 21:00 & 123.211 & 120.816 & 117.611 & 98.589 & 102.792 & 94.227 & 108.284 & 105.239 & 76.959 & 72.439 \\
\hline 7/13/07 21:30 & 123.185 & 120.81 & 117.585 & 98.563 & 102.764 & 94.209 & 108.253 & 105.21 & 76.944 & 72.431 \\
\hline 7/13/07 22:00 & 123.171 & 120.812 & 117.567 & 98.552 & 102.747 & 94.185 & 108.275 & 105.195 & 76.935 & 72.421 \\
\hline 7/13/07 22:30 & 123.164 & 120.801 & 117.562 & 98.549 & 102.745 & 94.18 & 108.248 & 105.19 & 76.928 & 72.418 \\
\hline 7/13/07 23:00 & 123.164 & 120.798 & 117.562 & 98.551 & 102.747 & 94.183 & 108.234 & 105.19 & 76.922 & 72.416 \\
\hline 7/13/07 23:30 & 123.157 & 120.794 & 117.553 & 98.539 & 102.735 & 94.171 & 108.222 & 105.181 & 76.913 & 72.408 \\
\hline 7/14/07 0:00 & 123.15 & 120.794 & 117.548 & 98.537 & 102.733 & 94.166 & 108.215 & 105.177 & 76.904 & 72.404 \\
\hline 7/14/07 0:30 & 123.145 & 120.785 & 117.544 & 98.532 & 102.726 & 94.166 & 108.207 & 105.172 & 76.896 & 72.4 \\
\hline 7/14/07 1:00 & 123.147 & 120.773 & 117.544 & 98.533 & 102.728 & 94.164 & 108.203 & 105.172 & 76.893 & 72.4 \\
\hline 7/14/07 1:30 & 123.14 & 120.762 & 117.537 & 98.525 & 102.72 & 94.157 & 108.199 & 105.166 & 76.883 & 72.394 \\
\hline 7/14/07 2:00 & 123.128 & 120.755 & 117.528 & 98.513 & 102.71 & 94.147 & 108.193 & 105.155 & 76.874 & 72.383 \\
\hline 7/14/07 2:30 & 123.117 & 120.744 & 117.514 & 98.501 & 102.695 & 94.133 & 108.19 & 105.141 & 76.863 & 72.369 \\
\hline 7/14/07 3:00 & 123.11 & 120.73 & 117.502 & 98.492 & 102.688 & 94.124 & 108.186 & 105.133 & 76.852 & 72.363 \\
\hline 7/14/07 3:30 & 123.11 & 120.724 & 117.493 & 98.485 & 102.68 & 94.114 & 108.184 & 105.121 & 76.845 & 72.354 \\
\hline 7/14/07 4:00 & 123.107 & 120.714 & 117.481 & 98.47 & 102.667 & 94.103 & 108.18 & 105.11 & 76.834 & 72.344 \\
\hline 7/14/07 4:30 & 123.102 & 120.712 & 117.479 & 98.465 & 102.661 & 94.098 & 108.176 & 105.104 & 76.824 & 72.338 \\
\hline 7/14/07 5:00 & 123.072 & 120.705 & 117.472 & 98.456 & 102.655 & 94.088 & 108.176 & 105.095 & 76.815 & 72.328 \\
\hline 7/14/07 5:30 & 123.067 & 120.708 & 117.463 & 98.454 & 102.653 & 94.084 & 108.17 & 105.09 & 76.808 & 72.325 \\
\hline 7/14/07 6:00 & 123.067 & 120.708 & 117.459 & 98.442 & 102.642 & 94.074 & 108.19 & 105.083 & 76.799 & 72.319 \\
\hline 7/14/07 6:30 & 123.062 & 122.75 & 117.456 & 98.444 & 102.644 & 94.077 & 108.226 & 105.083 & 76.795 & 72.317 \\
\hline 7/14/07 7:00 & 123.06 & 123.02 & 117.456 & 98.444 & 102.649 & 94.084 & 108.223 & 105.088 & 76.791 & 72.323 \\
\hline 7/14/07 7:30 & 125.177 & 123.159 & 119.021 & 99.274 & 103.222 & 94.824 & 108.899 & 106.607 & 76.813 & 72.402 \\
\hline 7/14/07 8:00 & 125.463 & 123.249 & 119.297 & 99.5 & 103.478 & 95.081 & 109.199 & 106.908 & 76.843 & 72.584 \\
\hline 7/14/07 8:30 & 125.603 & 123.304 & 119.435 & 99.624 & 103.613 & 95.215 & 109.326 & 107.056 & 76.872 & 72.712 \\
\hline 7/14/07 9:00 & 125.695 & 123.338 & 119.528 & 99.708 & 103.702 & 95.302 & 109.407 & 107.152 & 76.906 & 72.803 \\
\hline 7/14/07 9:30 & 125.757 & 123.363 & 119.59 & 99.765 & 103.761 & 95.361 & 109.464 & 107.216 & 76.933 & 72.867 \\
\hline 7/14/07 10:00 & 125.792 & 123.383 & 119.623 & 99.795 & 103.792 & 95.394 & 109.492 & 107.251 & 76.953 & 72.902 \\
\hline $7 / 14 / 07$ 10:30 & 125.816 & 123.467 & 119.648 & 99.817 & 103.816 & 95.418 & 109.532 & 107.278 & 76.974 & 72.931 \\
\hline $7 / 14 / 0711: 00$ & 125.839 & 123.492 & 119.673 & 99.903 & 103.839 & 95.439 & 109.541 & 107.298 & 76.992 & 72.952 \\
\hline 7/14/07 11:30 & 125.927 & 122.073 & 119.796 & 100.003 & 103.961 & 95.597 & 110.565 & 107.375 & 77.029 & 72.985 \\
\hline 7/14/07 12:00 & 125.955 & 121.333 & 119.824 & 100.029 & 103.988 & 95.625 & 110.598 & 107.404 & 77.06 & 73.012 \\
\hline $7 / 14 / 0712: 30$ & 124.717 & 121.1 & 119.102 & 99.607 & 103.904 & 95.444 & 110.43 & 106.99 & 77.084 & 73.032 \\
\hline 7/14/07 13:00 & 123.715 & 120.968 & 118.099 & 98.993 & 103.232 & 94.668 & 108.807 & 105.754 & 77.06 & 72.892 \\
\hline 7/14/07 13:30 & 123.469 & 120.88 & 117.859 & 98.79 & 103.011 & 94.444 & 108.596 & 105.502 & 77.035 & 72.729 \\
\hline 7/14/07 14:00 & 123.332 & 120.821 & 117.724 & 98.671 & 102.886 & 94.317 & 108.498 & 105.363 & 77.014 & 72.611 \\
\hline
\end{tabular}


TABLE S1.1 (Cont.)

Depth from Top of Casing (ft)

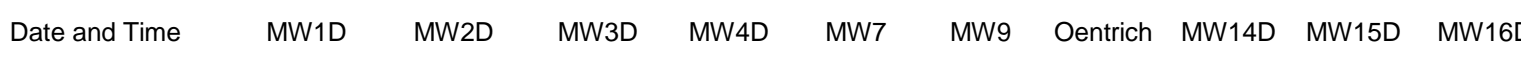

\begin{tabular}{|c|c|c|c|c|c|c|c|c|c|c|}
\hline 7/14/07 14:30 & 123.247 & 120.776 & 117.643 & 98.592 & 102.809 & 94.265 & 108.449 & 105.268 & 76.992 & 72.522 \\
\hline 7/14/07 15:00 & 123.18 & 120.742 & 117.574 & 98.542 & 102.745 & 94.176 & 108.403 & 105.208 & 76.977 & 72.462 \\
\hline 7/14/07 15:30 & 123.136 & 120.71 & 117.53 & 98.511 & 102.699 & 94.136 & 108.358 & 105.161 & 76.959 & 72.416 \\
\hline 7/14/07 16:00 & 123.098 & 120.687 & 117.496 & 98.466 & 102.67 & 94.105 & 108.337 & 105.124 & 76.941 & 72.381 \\
\hline $7 / 14 / 07$ 16:30 & 123.067 & 120.669 & 117.463 & 98.447 & 102.638 & 94.072 & 108.327 & 105.09 & 76.922 & 72.348 \\
\hline 7/14/07 17:00 & 123.091 & 120.649 & 117.44 & 98.43 & 102.617 & 94.053 & 108.329 & 105.068 & 76.896 & 72.338 \\
\hline 7/14/07 17:30 & 123.072 & 120.646 & 117.424 & 98.403 & 102.602 & 94.037 & 108.292 & 105.05 & 76.88 & 72.305 \\
\hline 7/14/07 18:00 & 123.003 & 120.64 & 117.405 & 98.382 & 102.587 & 94.022 & 108.289 & 105.028 & 76.867 & 72.284 \\
\hline 7/14/07 18:30 & 122.998 & 120.644 & 117.398 & 98.384 & 102.581 & 94.015 & 108.314 & 105.026 & 76.854 & 72.282 \\
\hline 7/14/07 19:00 & 122.996 & 120.646 & 117.396 & 98.375 & 102.579 & 94.015 & 108.269 & 105.02 & 76.847 & 72.276 \\
\hline 7/14/07 19:30 & 122.994 & 120.653 & 117.394 & 98.387 & 102.581 & 94.013 & 108.269 & 105.022 & 76.841 & 72.278 \\
\hline $7 / 14 / 07$ 20:00 & 122.998 & 120.649 & 117.4 & 98.408 & 102.585 & 94.018 & 108.261 & 105.026 & 76.834 & 72.282 \\
\hline $7 / 14 / 0720: 30$ & 123.006 & 120.642 & 117.405 & 98.394 & 102.592 & 94.027 & 108.283 & 105.03 & 76.828 & 72.288 \\
\hline 7/14/07 21:00 & 123.001 & 120.653 & 117.398 & 98.384 & 102.583 & 94.018 & 108.262 & 105.026 & 76.821 & 72.28 \\
\hline 7/14/07 21:30 & 122.998 & 120.66 & 117.398 & 98.38 & 102.585 & 94.025 & 108.254 & 105.02 & 76.813 & 72.276 \\
\hline 7/14/07 22:00 & 123.003 & 120.662 & 117.401 & 98.39 & 102.592 & 94.022 & 108.274 & 105.028 & 76.81 & 72.284 \\
\hline 7/14/07 22:30 & 123.013 & 120.66 & 117.415 & 98.404 & 102.602 & 94.034 & 108.297 & 105.039 & 76.81 & 72.294 \\
\hline 7/14/07 23:00 & 123.015 & 120.66 & 117.414 & 98.406 & 102.602 & 94.037 & 108.293 & 105.042 & 76.806 & 72.297 \\
\hline 7/14/07 23:30 & 123.013 & 120.658 & 117.412 & 98.406 & 102.6 & 94.034 & 108.295 & 105.037 & 76.8 & 72.29 \\
\hline 7/15/07 0:00 & 123.015 & 120.658 & 117.414 & 98.406 & 102.602 & 94.037 & 108.289 & 105.037 & 76.797 & 72.292 \\
\hline 7/15/07 0:30 & 123.01 & 120.658 & 117.41 & 98.403 & 102.598 & 94.034 & 108.275 & 105.035 & 76.793 & 72.29 \\
\hline 7/15/07 1:00 & 123.01 & 120.656 & 117.412 & 98.404 & 102.6 & 94.039 & 108.276 & 105.037 & 76.788 & 72.292 \\
\hline 7/15/07 1:30 & 123.01 & 120.644 & 117.412 & 98.404 & 102.6 & 94.037 & 108.274 & 105.037 & 76.782 & 72.292 \\
\hline 7/15/07 2:00 & 123.008 & 120.633 & 117.41 & 98.401 & 102.598 & 94.034 & 108.272 & 105.035 & 76.776 & 72.29 \\
\hline 7/15/07 2:30 & 122.994 & 120.635 & 117.396 & 98.387 & 102.585 & 94.02 & 108.268 & 105.022 & 76.769 & 72.278 \\
\hline 7/15/07 3:00 & 122.987 & 120.631 & 117.389 & 98.378 & 102.575 & 94.013 & 108.262 & 105.013 & 76.764 & 72.27 \\
\hline 7/15/07 3:30 & 122.984 & 120.633 & 117.386 & 98.382 & 102.577 & 94.013 & 108.26 & 105.011 & 76.756 & 72.27 \\
\hline 7/15/07 4:00 & 122.982 & 120.633 & 117.384 & 98.38 & 102.577 & 94.008 & 108.26 & 105.008 & 76.751 & 72.268 \\
\hline 7/15/07 4:30 & 122.982 & 120.64 & 117.384 & 98.38 & 102.577 & 94.013 & 108.258 & 105.009 & 76.747 & 72.27 \\
\hline 7/15/07 5:00 & 122.98 & 120.637 & 117.384 & 98.382 & 102.579 & 94.013 & 108.259 & 105.011 & 76.743 & 72.272 \\
\hline $7 / 15 / 075: 30$ & 122.991 & 120.637 & 117.386 & 98.389 & 102.585 & 94.018 & 108.261 & 105.017 & 76.74 & 72.274 \\
\hline 7/15/07 6:00 & 122.987 & 120.642 & 117.389 & 98.389 & 102.587 & 94.02 & 108.261 & 105.017 & 76.74 & 72.276 \\
\hline 7/15/07 6:30 & 122.984 & 120.64 & 117.391 & 98.389 & 102.585 & 94.02 & 108.261 & 105.017 & 76.732 & 72.278 \\
\hline 7/15/07 7:00 & 122.982 & 120.644 & 117.396 & 98.392 & 102.589 & 94.022 & 108.263 & 105.02 & 76.732 & 72.278 \\
\hline 7/15/07 7:30 & 122.994 & 122.612 & 117.393 & 98.389 & 102.585 & 94.02 & 108.262 & 105.017 & 76.729 & 72.278 \\
\hline 7/15/07 8:00 & 122.991 & 122.97 & 117.396 & 98.392 & 102.589 & 94.02 & 108.264 & 105.02 & 76.727 & 72.28 \\
\hline 7/15/07 8:30 & 124.984 & 123.143 & 118.705 & 99.059 & 103.015 & 94.602 & 108.833 & 106.414 & 76.74 & 72.311 \\
\hline 7/15/07 9:00 & 125.378 & 123.24 & 119.086 & 99.367 & 103.358 & 94.949 & 109.156 & 106.848 & 76.773 & 72.507 \\
\hline 7/15/07 9:30 & 125.56 & 123.304 & 119.266 & 99.526 & 103.527 & 95.116 & 109.315 & 107.036 & 76.81 & 72.66 \\
\hline 7/15/07 10:00 & 125.662 & 123.351 & 119.366 & 99.619 & 103.626 & 95.215 & 109.407 & 107.14 & 76.839 & 72.764 \\
\hline $7 / 15 / 07$ 10:30 & 125.723 & 123.383 & 119.431 & 99.677 & 103.687 & 95.279 & 109.468 & 107.207 & 76.865 & 72.828 \\
\hline 7/15/07 11:00 & 125.773 & 123.404 & 119.48 & 99.722 & 103.735 & 95.323 & 109.514 & 107.258 & 76.891 & 72.877 \\
\hline 7/15/07 11:30 & 125.802 & 123.422 & 119.512 & 99.753 & 103.767 & 95.356 & 109.546 & 107.291 & 76.913 & 72.915 \\
\hline $7 / 15 / 07$ 12:00 & 125.828 & 123.426 & 119.537 & 99.776 & 103.792 & 95.382 & 109.567 & 107.318 & 76.937 & 72.944 \\
\hline $7 / 15 / 07$ 12:30 & 125.842 & 123.426 & 119.551 & 99.791 & 103.807 & 95.394 & 109.582 & 107.333 & 76.959 & 72.96 \\
\hline $7 / 15 / 07$ 13:00 & 125.851 & 123.428 & 119.56 & 99.795 & 103.813 & 95.404 & 109.591 & 107.342 & 76.974 & 72.972 \\
\hline $7 / 15 / 07$ 13:30 & 125.851 & 123.424 & 119.562 & 99.796 & 103.815 & 95.406 & 109.595 & 107.344 & 76.988 & 72.977 \\
\hline 7/15/07 14:00 & 125.851 & 123.426 & 119.565 & 99.802 & 103.82 & 95.408 & 109.599 & 107.346 & 77.001 & 72.985 \\
\hline 7/15/07 14:30 & 125.849 & 121.499 & 119.562 & 99.802 & 103.816 & 95.408 & 109.598 & 107.34 & 77.016 & 72.985 \\
\hline $7 / 15 / 07$ 15:00 & 125.851 & 121.15 & 119.565 & 99.803 & 103.822 & 95.413 & 109.6 & 107.344 & 77.025 & 72.991 \\
\hline 7/15/07 15:30 & 123.901 & 120.984 & 118.286 & 99.13 & 103.386 & 94.824 & 109.02 & 105.969 & 77.022 & 72.95 \\
\hline 7/15/07 16:00 & 123.516 & 120.88 & 117.911 & 98.835 & 103.061 & 94.494 & 108.709 & 105.559 & 77 & 72.778 \\
\hline 7/15/07 16:30 & 123.346 & 120.803 & 117.74 & 98.689 & 102.905 & 94.338 & 108.566 & 105.38 & 76.979 & 72.644 \\
\hline 7/15/07 17:00 & 123.237 & 120.755 & 117.634 & 98.595 & 102.802 & 94.237 & 108.474 & 105.268 & 76.957 & 72.54 \\
\hline 7/15/07 17:30 & 123.162 & 120.719 & 117.558 & 98.527 & 102.729 & 94.166 & 108.408 & 105.19 & 76.935 & 72.466 \\
\hline 7/15/07 18:00 & 123.112 & 120.705 & 117.512 & 98.48 & 102.684 & 94.121 & 108.368 & 105.141 & 76.92 & 72.414 \\
\hline 7/15/07 18:30 & 123.074 & 120.674 & 117.474 & 98.452 & 102.651 & 94.091 & 108.339 & 105.102 & 76.904 & 72.381 \\
\hline 7/15/07 19:00 & 123.06 & 120.653 & 117.46 & 98.44 & 102.642 & 94.077 & 108.324 & 105.086 & 76.893 & 72.367 \\
\hline 7/15/07 19:30 & 123.029 & 120.642 & 117.433 & 98.415 & 102.613 & 94.051 & 108.299 & 105.057 & 76.876 & 72.338 \\
\hline 7/15/07 20:00 & 123.008 & 120.637 & 117.41 & 98.392 & 102.59 & 94.029 & 108.284 & 105.035 & 76.861 & 72.315 \\
\hline $7 / 15 / 0720: 30$ & 122.996 & 120.644 & 117.398 & 98.387 & 102.583 & 94.018 & 108.275 & 105.024 & 76.85 & 72.303 \\
\hline 7/15/07 21:00 & 122.994 & 120.642 & 117.393 & 98.385 & 102.583 & 94.015 & 108.271 & 105.022 & 76.841 & 72.301 \\
\hline 7/15/07 21:30 & 122.994 & 120.66 & 117.396 & 98.392 & 102.585 & 94.02 & 108.271 & 105.02 & 76.835 & 72.303 \\
\hline $7 / 15 / 0722: 00$ & 122.996 & 120.714 & 117.4 & 98.39 & 102.587 & 94.022 & 108.273 & 105.026 & 76.83 & 72.305 \\
\hline 7/15/07 22:30 & 123.01 & 120.671 & 117.412 & 98.408 & 102.602 & 94.034 & 108.282 & 105.037 & 76.828 & 72.315 \\
\hline 7/15/07 23:00 & 123.069 & 120.662 & 117.472 & 98.466 & 102.665 & 94.098 & 108.29 & 105.095 & 76.845 & 72.375 \\
\hline
\end{tabular}


TABLE S1.1 (Cont.)

Depth from Top of Casing (ft)

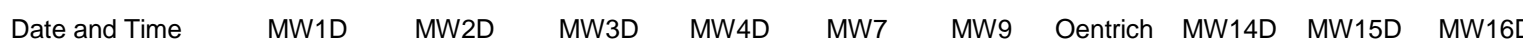

\begin{tabular}{|c|c|c|c|c|c|c|c|c|c|c|}
\hline 7/15/07 23:30 & 123.029 & 120.66 & 117.433 & 98.418 & 102.617 & 94.055 & 108.292 & 105.055 & 76.826 & 72.328 \\
\hline 7/16/07 0:00 & 123.013 & 120.64 & 117.417 & 98.411 & 102.604 & 94.041 & 108.284 & 105.04 & 76.815 & 72.313 \\
\hline 7/16/07 0:30 & 123.01 & 120.601 & 117.414 & 98.411 & 102.606 & 94.041 & 108.284 & 105.039 & 76.812 & 72.313 \\
\hline 7/16/07 1:00 & 122.996 & 120.61 & 117.398 & 98.385 & 102.587 & 94.022 & 108.269 & 105.024 & 76.799 & 72.297 \\
\hline 7/16/07 1:30 & 122.954 & 120.612 & 117.356 & 98.349 & 102.545 & 93.982 & 108.235 & 104.982 & 76.78 & 72.257 \\
\hline 7/16/07 2:00 & 122.961 & 120.642 & 117.363 & 98.359 & 102.554 & 93.989 & 108.241 & 104.988 & 76.778 & 72.268 \\
\hline 7/16/07 2:30 & 122.963 & 120.635 & 117.368 & 98.365 & 102.56 & 93.994 & 108.244 & 104.991 & 76.775 & 72.272 \\
\hline 7/16/07 3:00 & 122.998 & 120.617 & 117.4 & 98.399 & 102.596 & 94.029 & 108.274 & 105.026 & 76.78 & 72.309 \\
\hline 7/16/07 3:30 & 122.987 & 120.61 & 117.393 & 98.387 & 102.585 & 94.02 & 108.265 & 105.017 & 76.775 & 72.294 \\
\hline 7/16/07 4:00 & 122.968 & 120.608 & 117.373 & 98.366 & 102.566 & 93.999 & 108.248 & 104.995 & 76.762 & 72.276 \\
\hline 7/16/07 4:30 & 122.963 & 120.601 & 117.368 & 98.361 & 102.558 & 93.994 & 108.244 & 104.991 & 76.756 & 72.27 \\
\hline 7/16/07 5:00 & 122.958 & 120.594 & 117.363 & 98.365 & 102.556 & 93.992 & 108.241 & 104.984 & 76.749 & 72.266 \\
\hline 7/16/07 5:30 & 122.956 & 120.597 & 117.356 & 98.356 & 102.55 & 93.982 & 108.233 & 104.979 & 76.741 & 72.261 \\
\hline 7/16/07 6:00 & 122.954 & 122.596 & 117.352 & 98.351 & 102.545 & 93.98 & 108.229 & 104.973 & 76.738 & 72.255 \\
\hline 7/16/07 6:30 & 122.954 & 122.891 & 117.354 & 98.353 & 102.549 & 93.985 & 108.233 & 104.978 & 76.73 & 72.259 \\
\hline 7/16/07 7:00 & 125.012 & 123.048 & 118.873 & 99.138 & 103.095 & 94.694 & 108.931 & 106.448 & 76.751 & 72.33 \\
\hline 7/16/07 7:30 & 125.329 & 123.131 & 119.176 & 99.393 & 103.371 & 94.972 & 109.194 & 106.777 & 76.78 & 72.52 \\
\hline 7/16/07 8:00 & 125.492 & 123.19 & 119.336 & 99.536 & 103.523 & 95.125 & 109.335 & 106.948 & 76.812 & 72.66 \\
\hline 7/16/07 8:30 & 125.582 & 123.238 & 119.429 & 99.617 & 103.615 & 95.213 & 109.418 & 107.041 & 76.841 & 72.753 \\
\hline 7/16/07 9:00 & 125.641 & 123.272 & 119.486 & 99.672 & 103.67 & 95.269 & 109.47 & 107.103 & 76.863 & 72.815 \\
\hline 7/16/07 9:30 & 125.686 & 123.29 & 119.533 & 99.714 & 103.712 & 95.314 & 109.51 & 107.149 & 76.887 & 72.859 \\
\hline 7/16/07 10:00 & 125.723 & 123.308 & 119.569 & 99.75 & 103.748 & 95.349 & 109.544 & 107.187 & 76.913 & 72.896 \\
\hline 7/16/07 10:30 & 125.742 & 121.32 & 119.591 & 99.767 & 103.769 & 95.371 & 109.564 & 107.209 & 76.933 & 72.923 \\
\hline 7/16/07 11:00 & 125.761 & 121.068 & 119.609 & 99.788 & 103.786 & 95.392 & 109.583 & 107.227 & 76.952 & 72.941 \\
\hline 7/16/07 11:30 & 123.705 & 120.934 & 118.097 & 98.985 & 103.224 & 94.661 & 108.866 & 105.755 & 76.948 & 72.867 \\
\hline 7/16/07 12:00 & 123.433 & 120.841 & 117.828 & 98.769 & 102.986 & 94.421 & 108.64 & 105.467 & 76.933 & 72.716 \\
\hline 7/16/07 12:30 & 123.294 & 120.78 & 117.692 & 98.651 & 102.859 & 94.298 & 108.522 & 105.325 & 76.92 & 72.602 \\
\hline 7/16/07 13:00 & 123.199 & 120.739 & 117.6 & 98.566 & 102.775 & 94.209 & 108.443 & 105.232 & 76.906 & 72.514 \\
\hline 7/16/07 13:30 & 123.138 & 120.705 & 117.541 & 98.513 & 102.714 & 94.15 & 108.391 & 105.165 & 76.894 & 72.454 \\
\hline $7 / 16 / 07$ 14:00 & 123.095 & 120.68 & 117.497 & 98.475 & 102.676 & 94.11 & 108.351 & 105.121 & 76.88 & 72.408 \\
\hline $7 / 16 / 07$ 14:30 & 123.062 & 120.662 & 117.465 & 98.447 & 102.644 & 94.084 & 108.325 & 105.088 & 76.869 & 72.377 \\
\hline 7/16/07 15:00 & 123.039 & 120.644 & 117.442 & 98.425 & 102.621 & 94.06 & 108.306 & 105.064 & 76.858 & 72.354 \\
\hline 7/16/07 15:30 & 123.015 & 120.628 & 117.421 & 98.406 & 102.602 & 94.039 & 108.287 & 105.044 & 76.847 & 72.332 \\
\hline 7/16/07 16:00 & 122.998 & 120.612 & 117.403 & 98.389 & 102.585 & 94.022 & 108.274 & 105.024 & 76.837 & 72.315 \\
\hline $7 / 16 / 07$ 16:30 & 122.984 & 120.603 & 117.386 & 98.377 & 102.575 & 94.011 & 108.261 & 105.013 & 76.824 & 72.303 \\
\hline 7/16/07 17:00 & 122.968 & 120.597 & 117.371 & 98.359 & 102.556 & 93.992 & 108.246 & 104.993 & 76.812 & 72.286 \\
\hline 7/16/07 17:30 & 122.956 & 120.585 & 117.361 & 98.351 & 102.549 & 93.985 & 108.237 & 104.982 & 76.799 & 72.276 \\
\hline $7 / 16 / 07$ 18:00 & 122.949 & 120.578 & 117.356 & 98.349 & 102.545 & 93.982 & 108.233 & 104.978 & 76.789 & 72.274 \\
\hline 7/16/07 18:30 & 122.939 & 120.576 & 117.345 & 98.339 & 102.535 & 93.968 & 108.223 & 104.966 & 76.778 & 72.261 \\
\hline 7/16/07 19:00 & 122.932 & 120.574 & 117.338 & 98.33 & 102.53 & 93.963 & 108.219 & 104.958 & 76.767 & 72.253 \\
\hline 7/16/07 19:30 & 122.93 & 120.576 & 117.336 & 98.33 & 102.528 & 93.963 & 108.216 & 104.955 & 76.762 & 72.253 \\
\hline 7/16/07 20:00 & 122.93 & 120.572 & 117.333 & 98.33 & 102.526 & 93.963 & 108.216 & 104.955 & 76.754 & 72.251 \\
\hline $7 / 16 / 07$ 20:30 & 122.93 & 120.576 & 117.336 & 98.33 & 102.528 & 93.961 & 108.216 & 104.953 & 76.747 & 72.249 \\
\hline $7 / 16 / 0721: 00$ & 122.925 & 120.583 & 117.331 & 98.327 & 102.524 & 93.959 & 108.212 & 104.951 & 76.738 & 72.247 \\
\hline $7 / 16 / 0721: 30$ & 122.93 & 120.587 & 117.336 & 98.334 & 102.531 & 93.963 & 108.216 & 104.955 & 76.734 & 72.247 \\
\hline 7/16/07 22:00 & 122.937 & 120.587 & 117.343 & 98.342 & 102.537 & 93.971 & 108.221 & 104.962 & 76.732 & 72.257 \\
\hline 7/16/07 22:30 & 122.939 & 120.587 & 117.348 & 98.346 & 102.543 & 93.975 & 108.227 & 104.969 & 76.729 & 72.261 \\
\hline 7/16/07 23:00 & 122.939 & 120.583 & 117.348 & 98.344 & 102.541 & 93.975 & 108.223 & 104.966 & 76.723 & 72.259 \\
\hline 7/16/07 23:30 & 122.937 & 120.585 & 117.345 & 98.342 & 102.537 & 93.973 & 108.221 & 104.964 & 76.719 & 72.255 \\
\hline 7/17/07 0:00 & 122.935 & 120.583 & 117.34 & 98.337 & 102.533 & 93.968 & 108.219 & 104.96 & 76.716 & 72.253 \\
\hline 7/17/07 0:30 & 122.937 & 120.581 & 117.343 & 98.34 & 102.535 & 93.971 & 108.219 & 104.962 & 76.714 & 72.253 \\
\hline 7/17/07 1:00 & 122.932 & 120.576 & 117.34 & 98.339 & 102.533 & 93.971 & 108.218 & 104.96 & 76.708 & 72.253 \\
\hline 7/17/07 1:30 & 122.93 & 120.574 & 117.338 & 98.337 & 102.535 & 93.966 & 108.218 & 104.957 & 76.708 & 72.251 \\
\hline 7/17/07 2:00 & 122.928 & 120.572 & 117.334 & 98.332 & 102.53 & 93.961 & 108.212 & 104.953 & 76.701 & 72.247 \\
\hline 7/17/07 2:30 & 122.925 & 120.565 & 117.331 & 98.33 & 102.528 & 93.961 & 108.21 & 104.953 & 76.699 & 72.245 \\
\hline 7/17/07 3:00 & 122.925 & 120.558 & 117.331 & 98.328 & 102.524 & 93.956 & 108.21 & 104.951 & 76.695 & 72.243 \\
\hline 7/17/07 3:30 & 122.925 & 120.553 & 117.324 & 98.322 & 102.516 & 93.952 & 108.203 & 104.942 & 76.69 & 72.237 \\
\hline 7/17/07 4:00 & 122.92 & 120.556 & 117.32 & 98.315 & 102.512 & 93.949 & 108.199 & 104.937 & 76.684 & 72.232 \\
\hline 7/17/07 4:30 & 122.918 & 120.551 & 117.312 & 98.311 & 102.507 & 93.942 & 108.193 & 104.933 & & 72.226 \\
\hline $7 / 17 / 07$ 5:00 & 122.916 & 120.553 & 117.315 & 98.315 & 102.509 & 93.942 & 108.193 & 104.933 & 76.677 & 72.23 \\
\hline 7/17/07 5:30 & 122.913 & 120.553 & 117.315 & 98.309 & 102.51 & 93.942 & 108.193 & 104.933 & 76.671 & 72.226 \\
\hline 7/17/07 6:00 & 122.911 & 122.551 & 117.312 & 98.311 & 102.51 & 93.942 & 108.191 & 104.931 & & 72.228 \\
\hline $7 / 17 / 076: 30$ & 122.909 & & 117.313 & 98.313 & 102.509 & 93.942 & 108.191 & 104.933 & 76.664 & 72.228 \\
\hline 7/17/07 7:00 & 124.929 & 123.057 & 118.658 & 99.002 & 102.965 & 94.548 & 108.79 & 106.374 & 76.675 & 72.268 \\
\hline 7/17/07 7:30 & 125.293 & 123.204 & 119.012 & 99.293 & 103.281 & 94.875 & 109.091 & 106.766 & 76.705 & 72.466 \\
\hline & & & & & & & & & & \\
\hline
\end{tabular}


TABLE S1.1 (Cont.)

Depth from Top of Casing (ft)

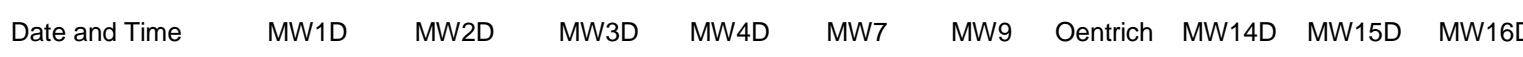

\begin{tabular}{|c|c|c|c|c|c|c|c|c|c|c|}
\hline 7/17/07 8:30 & 125.622 & 123.297 & 119.364 & 99.664 & 103.622 & 95.241 & 110.335 & 107.087 & 76.784 & 72.72 \\
\hline 7/17/07 9:00 & 125.712 & 123.313 & 119.433 & 99.665 & 103.693 & 95.281 & 109.443 & 107.202 & 76.828 & 72.817 \\
\hline 7/17/07 9:30 & 125.714 & 123.326 & 119.433 & 99.676 & 103.693 & 95.279 & 109.48 & 107.205 & 76.845 & 72.867 \\
\hline $7 / 17 / 07 \quad 10: 00$ & 125.733 & 123.333 & 119.454 & 99.693 & 103.71 & 95.3 & 109.495 & 107.224 & 76.861 & 72.892 \\
\hline $7 / 17 / 07$ 10:30 & 125.747 & 123.347 & 119.468 & 99.707 & 103.723 & 95.312 & 109.507 & 107.24 & 76.88 & 72.91 \\
\hline 7/17/07 11:00 & 125.754 & 123.356 & 119.472 & 99.712 & 103.729 & 95.319 & 109.512 & 107.249 & 76.896 & 72.919 \\
\hline 7/17/07 11:30 & 125.764 & 123.363 & 119.484 & 99.727 & 103.742 & 95.333 & 109.523 & 107.258 & 76.915 & 72.935 \\
\hline $7 / 17 / 07$ 12:00 & 125.778 & 123.358 & 119.5 & 99.738 & 103.754 & 95.347 & 109.534 & 107.273 & 76.931 & 72.95 \\
\hline $7 / 17 / 07$ 12:30 & 125.78 & 121.268 & 119.505 & 99.746 & 103.761 & 95.352 & 109.544 & 107.277 & 76.946 & 72.96 \\
\hline 7/17/07 13:00 & 125.78 & 121.025 & 119.505 & 99.746 & 103.761 & 95.354 & 109.544 & 107.278 & 76.959 & 72.962 \\
\hline 7/17/07 13:30 & 123.646 & 120.887 & 118.043 & 98.935 & 103.175 & 94.609 & 108.817 & 105.699 & 76.946 & 72.861 \\
\hline $7 / 17 / 07$ 14:00 & 123.386 & 120.801 & 117.787 & 98.723 & 102.945 & 94.381 & 108.599 & 105.427 & 76.926 & 72.7 \\
\hline $7 / 17 / 07$ 14:30 & 123.244 & 120.744 & 117.648 & 98.604 & 102.811 & 94.246 & 108.477 & 105.279 & 76.907 & 72.576 \\
\hline $7 / 17 / 07$ 15:00 & 123.157 & 120.696 & 117.56 & 98.527 & 102.733 & 94.169 & 108.404 & 105.188 & 76.889 & 72.491 \\
\hline $7 / 17 / 07$ 15:30 & 123.098 & 120.662 & 117.507 & 98.477 & 102.678 & 94.112 & 108.355 & 105.13 & 76.874 & 72.435 \\
\hline 7/17/07 16:00 & 123.053 & 120.633 & 117.459 & 98.434 & 102.63 & 94.072 & 108.315 & 105.079 & 76.858 & 72.385 \\
\hline 7/17/07 16:30 & 123.017 & 120.612 & 117.423 & 98.404 & 102.6 & 94.039 & 108.285 & 105.044 & 76.845 & 72.352 \\
\hline 7/17/07 17:00 & 122.989 & 120.592 & 117.396 & 98.378 & 102.577 & 94.013 & 108.261 & 105.017 & 76.832 & 72.323 \\
\hline 7/17/07 17:30 & 122.963 & 120.572 & 117.373 & 98.358 & 102.556 & 93.992 & 108.242 & 104.991 & 76.815 & 72.301 \\
\hline $7 / 17 / 07$ 18:00 & 122.944 & 120.558 & 117.352 & 98.339 & 102.535 & 93.971 & 108.223 & 104.971 & 76.802 & 72.282 \\
\hline 7/17/07 18:30 & 122.923 & 120.549 & 117.333 & 98.32 & 102.514 & 93.952 & 108.208 & 104.951 & 76.789 & 72.263 \\
\hline 7/17/07 19:00 & 122.909 & 120.544 & 117.317 & 98.308 & 102.505 & 93.942 & 108.197 & 104.938 & 76.775 & 72.249 \\
\hline 7/17/07 19:30 & 122.902 & 120.544 & 117.31 & 98.301 & 102.497 & 93.933 & 108.189 & 104.929 & 76.764 & 72.243 \\
\hline $7 / 17 / 0720: 00$ & 122.892 & 120.54 & 117.301 & 98.296 & 102.491 & 93.926 & 108.184 & 104.92 & 76.754 & 72.235 \\
\hline $7 / 17 / 0720: 30$ & 122.894 & 120.551 & 117.303 & 98.297 & 102.493 & 93.928 & 108.184 & 104.922 & 76.745 & 72.235 \\
\hline $7 / 17 / 0721: 00$ & 122.892 & 120.556 & 117.301 & 98.296 & 102.491 & 93.93 & 108.184 & 104.92 & 76.738 & 72.232 \\
\hline 7/17/07 21:30 & 122.899 & 120.56 & 117.31 & 98.306 & 102.501 & 93.935 & 108.189 & 104.929 & 76.732 & 72.239 \\
\hline 7/17/07 22:00 & 122.904 & 120.565 & 117.312 & 98.313 & 102.507 & 93.942 & 108.191 & 104.931 & 76.729 & 72.243 \\
\hline 7/17/07 22:30 & 122.911 & 120.562 & 117.322 & 98.316 & 102.512 & 93.947 & 108.197 & 104.938 & 76.727 & 72.249 \\
\hline 7/17/07 23:00 & 122.916 & 120.56 & 117.324 & 98.32 & 102.516 & 93.952 & 108.199 & 104.942 & 76.721 & 72.253 \\
\hline 7/17/07 23:30 & 122.913 & 120.558 & 117.322 & 98.32 & 102.514 & 93.952 & 108.199 & 104.94 & 76.718 & 72.249 \\
\hline 7/18/07 0:00 & 122.911 & 120.558 & 117.322 & 98.316 & 102.51 & 93.949 & 108.197 & 104.938 & 76.712 & 72.247 \\
\hline 7/18/07 0:30 & 122.909 & 120.558 & 117.319 & 98.315 & 102.512 & 93.947 & 108.197 & 104.938 & 76.706 & 72.245 \\
\hline 7/18/07 1:00 & 122.909 & 120.549 & 117.319 & 98.318 & 102.512 & 93.952 & 108.197 & 104.937 & 76.705 & 72.245 \\
\hline 7/18/07 1:30 & 122.909 & 120.544 & 117.319 & 98.316 & 102.512 & 93.949 & 108.197 & 104.935 & 76.699 & 72.245 \\
\hline 7/18/07 2:00 & 122.899 & 120.544 & 117.31 & 98.309 & 102.503 & 93.94 & 108.189 & 104.929 & 76.694 & 72.237 \\
\hline 7/18/07 2:30 & 122.894 & 120.542 & 117.306 & 98.304 & 102.501 & 93.935 & 108.186 & 104.922 & 76.688 & 72.232 \\
\hline 7/18/07 3:00 & 122.892 & 120.535 & 117.303 & 98.304 & 102.497 & 93.935 & 108.184 & 104.922 & 76.688 & 72.232 \\
\hline 7/18/07 3:30 & 122.892 & 120.528 & 117.301 & 98.301 & 102.495 & 93.93 & 108.182 & 104.918 & 76.682 & 72.228 \\
\hline 7/18/07 4:00 & 122.885 & 120.526 & 117.299 & 98.294 & 102.491 & 93.926 & 108.178 & 104.915 & 76.677 & 72.226 \\
\hline $7 / 18 / 074: 30$ & 122.88 & 120.517 & 117.289 & 98.289 & 102.484 & 93.921 & 108.172 & 104.909 & 76.671 & 72.22 \\
\hline 7/18/07 5:00 & 122.876 & 120.517 & 117.287 & 98.285 & 102.48 & 93.916 & 108.169 & 104.902 & 76.668 & 72.216 \\
\hline 7/18/07 5:30 & 122.868 & 120.517 & 117.28 & 98.28 & 102.476 & 93.909 & 108.163 & 104.898 & 76.66 & 72.21 \\
\hline $7 / 18 / 076: 00$ & 122.868 & 122.558 & 117.28 & 98.28 & 102.476 & 93.912 & 108.165 & 104.898 & 76.657 & 72.21 \\
\hline $7 / 18 / 076: 30$ & 122.868 & 122.821 & 117.28 & 98.278 & 102.476 & 93.912 & 108.163 & 104.898 & 76.649 & 72.212 \\
\hline 7/18/07 7:00 & 124.977 & 122.968 & 118.841 & 99.099 & 103.061 & 94.659 & 108.899 & 106.418 & 76.673 & 72.301 \\
\hline 7/18/07 7:30 & 125.26 & 123.054 & 119.114 & 99.328 & 103.308 & 94.913 & 109.135 & 106.711 & 76.701 & 72.483 \\
\hline 7/18/07 8:00 & 125.411 & 123.113 & 119.262 & 99.464 & 103.449 & 95.057 & 109.269 & 106.868 & 76.73 & 72.621 \\
\hline $7 / 18 / 078: 30$ & 125.501 & 123.159 & 119.354 & 99.547 & 103.54 & 95.144 & 109.349 & 106.966 & 76.756 & 72.71 \\
\hline 7/18/07 9:00 & 125.56 & 123.188 & 119.412 & 99.601 & 103.598 & 95.201 & 109.404 & 107.025 & 76.78 & 72.774 \\
\hline 7/18/07 9:30 & 125.605 & 123.206 & 119.458 & 99.643 & 103.639 & 95.243 & 109.443 & 107.07 & 76.804 & 72.817 \\
\hline 7/18/07 10:00 & 125.638 & 123.22 & 119.491 & 99.669 & 103.67 & 95.272 & 109.472 & 107.103 & 76.823 & 72.848 \\
\hline $7 / 18 / 07 \quad 10: 30$ & 125.657 & 123.227 & 119.509 & 99.684 & 103.685 & 95.288 & 109.487 & 107.12 & 76.837 & 72.869 \\
\hline 7/18/07 11:00 & 125.671 & 121.245 & 119.523 & 99.7 & 103.698 & 95.3 & 109.502 & 107.136 & 76.856 & 72.886 \\
\hline $7 / 18 / 0711: 30$ & 125.679 & 120.966 & 119.532 & 99.705 & 103.708 & 95.309 & 109.509 & 107.145 & 76.872 & 72.894 \\
\hline $7 / 18 / 07 \quad 12: 00$ & 123.627 & 120.823 & 118.025 & 98.906 & 103.148 & 94.586 & 108.794 & 105.677 & 76.865 & 72.815 \\
\hline $7 / 18 / 07$ 12:30 & 123.329 & 120.726 & 117.733 & 98.671 & 102.889 & 94.326 & 108.55 & 105.369 & 76.847 & 72.65 \\
\hline 7/18/07 13:00 & 123.183 & 120.664 & 117.585 & 98.542 & 102.754 & 94.19 & 108.426 & 105.214 & 76.83 & 72.528 \\
\hline 7/18/07 13:30 & 123.079 & 120.617 & 117.484 & 98.452 & 102.657 & 94.093 & 108.336 & 105.108 & 76.813 & 72.429 \\
\hline $7 / 18 / 07 \quad 14: 00$ & 123.015 & 120.585 & 117.426 & 98.399 & 102.598 & 94.037 & 108.283 & 105.046 & 76.797 & 72.367 \\
\hline $7 / 18 / 07$ 14:30 & 122.972 & 120.558 & 117.38 & 98.358 & 102.558 & 93.994 & 108.245 & 105 & 76.786 & 72.325 \\
\hline 7/18/07 15:00 & 122.937 & 120.538 & 117.347 & 98.33 & 102.531 & 93.966 & 108.217 & 104.968 & 76.773 & 72.29 \\
\hline $7 / 18 / 07$ 15:30 & 122.906 & 120.519 & 117.317 & 98.303 & 102.499 & 93.935 & 108.197 & 104.935 & 76.758 & 72.259 \\
\hline 7/18/07 16:00 & 122.887 & 120.501 & 117.296 & 98.285 & 102.48 & 93.919 & 108.178 & 104.913 & 76.745 & 72.241 \\
\hline $7 / 18 / 07$ 16:30 & 122.868 & 120.488 & 117.28 & 98.27 & 102.465 & 93.902 & 108.165 & 104.898 & 76.734 & 72.224 \\
\hline 7/18/07 17:00 & 122.852 & 120.483 & 117.264 & 98.254 & 102.451 & 93.888 & 108.152 & 104.88 & 76.725 & 72.212 \\
\hline
\end{tabular}


TABLE S1.1 (Cont.)

Depth from Top of Casing (ft)

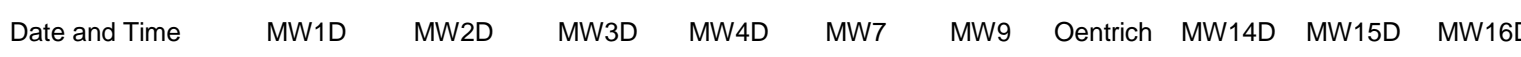

\begin{tabular}{|c|c|c|c|c|c|c|c|c|c|c|}
\hline 7/18/07 17:30 & 122.838 & 120.549 & 117.25 & 98.244 & 102.438 & 93.879 & 108.14 & 104.869 & 76.714 & 72.195 \\
\hline 7/18/07 18:00 & 122.833 & 120.56 & 117.245 & 98.306 & 102.434 & 93.872 & 108.135 & 104.86 & 76.705 & 72.191 \\
\hline 7/18/07 18:30 & 122.902 & 120.497 & 117.331 & 98.382 & 102.537 & 94.004 & 109.185 & 104.918 & 76.712 & 72.191 \\
\hline 7/18/07 19:00 & 122.916 & 120.472 & 117.343 & 98.395 & 102.547 & 94.015 & 109.2 & 104.931 & 76.714 & 72.199 \\
\hline 7/18/07 19:30 & 122.85 & 120.456 & 117.264 & 98.256 & 102.459 & 93.89 & 108.196 & 104.88 & 76.701 & 72.204 \\
\hline $7 / 18 / 07$ 20:00 & 122.824 & 120.454 & 117.238 & 98.23 & 102.429 & 93.867 & 108.159 & 104.853 & 76.69 & 72.187 \\
\hline $7 / 18 / 0720: 30$ & 122.805 & 120.46 & 117.22 & 98.218 & 102.413 & 93.848 & 108.134 & 104.836 & 76.679 & 72.17 \\
\hline 7/18/07 21:00 & 122.805 & 120.46 & 117.218 & 98.216 & 102.413 & 93.85 & 108.116 & 104.833 & 76.668 & 72.168 \\
\hline 7/18/07 21:30 & 122.809 & 120.46 & 117.225 & 98.223 & 102.419 & 93.853 & 108.109 & 104.84 & 76.666 & 72.17 \\
\hline 7/18/07 22:00 & 122.812 & 120.445 & 117.225 & 98.223 & 102.421 & 93.855 & 108.111 & 104.84 & 76.659 & 72.173 \\
\hline 7/18/07 22:30 & 122.805 & 120.417 & 117.22 & 98.218 & 102.415 & 93.85 & 108.115 & 104.836 & 76.651 & 72.166 \\
\hline 7/18/07 23:00 & 122.798 & 120.413 & 117.213 & 98.208 & 102.408 & 93.843 & 108.107 & 104.827 & 76.642 & 72.158 \\
\hline 7/18/07 23:30 & 122.769 & 120.431 & 117.181 & 98.177 & 102.372 & 93.81 & 108.101 & 104.796 & 76.629 & 72.127 \\
\hline 7/19/07 0:00 & 122.762 & 120.426 & 117.178 & 98.175 & 102.373 & 93.808 & 108.096 & 104.791 & 76.623 & 72.127 \\
\hline 7/19/07 0:30 & 122.779 & 120.415 & 117.192 & 98.196 & 102.391 & 93.824 & 108.086 & 104.807 & 76.62 & 72.144 \\
\hline 7/19/07 1:00 & 122.776 & 120.408 & 117.19 & 98.191 & 102.387 & 93.824 & 108.09 & 104.809 & 76.618 & 72.139 \\
\hline 7/19/07 1:30 & 122.762 & 120.406 & 117.178 & 98.179 & 102.373 & 93.808 & 108.086 & 104.793 & 76.607 & 72.127 \\
\hline 7/19/07 2:00 & 122.755 & 120.408 & 117.169 & 98.166 & 102.366 & 93.801 & 108.082 & 104.787 & 76.601 & 72.119 \\
\hline 7/19/07 2:30 & 122.755 & 120.406 & 117.169 & 98.168 & 102.364 & 93.801 & 108.079 & 104.785 & 76.596 & 72.117 \\
\hline 7/19/07 3:00 & 122.755 & 120.399 & 117.169 & 98.17 & 102.37 & 93.801 & 108.077 & 104.783 & 76.59 & 72.117 \\
\hline 7/19/07 3:30 & 122.755 & 120.472 & 117.169 & 98.17 & 102.368 & 93.801 & 108.073 & 104.785 & 76.587 & 72.119 \\
\hline 7/19/07 4:00 & 122.743 & 120.463 & 117.16 & 98.168 & 102.358 & 93.791 & 108.071 & 104.774 & 76.577 & 72.106 \\
\hline 7/19/07 4:30 & 122.826 & 120.463 & 117.238 & 98.242 & 102.442 & 93.876 & 108.124 & 104.851 & 76.601 & 72.189 \\
\hline 7/19/07 5:00 & 122.816 & 120.479 & 117.232 & 98.232 & 102.432 & 93.867 & 108.12 & 104.845 & 76.594 & 72.177 \\
\hline 7/19/07 5:30 & 122.814 & 120.49 & 117.23 & 98.234 & 102.429 & 93.862 & 108.118 & 104.844 & 76.594 & 72.173 \\
\hline 7/19/07 6:00 & 122.828 & 122.574 & 117.243 & 98.251 & 102.442 & 93.879 & 108.127 & 104.858 & 76.596 & 72.185 \\
\hline 7/19/07 6:30 & 122.842 & 122.816 & 117.255 & 98.263 & 102.461 & 93.895 & 108.135 & 104.876 & 76.598 & 72.204 \\
\hline 7/19/07 7:00 & 124.998 & 122.936 & 118.859 & 99.116 & 103.074 & 94.677 & 108.905 & 106.436 & 76.625 & 72.307 \\
\hline 7/19/07 7:30 & 125.26 & 123.052 & 119.114 & 99.319 & 103.299 & 94.906 & 109.126 & 106.709 & 76.653 & 72.481 \\
\hline 7/19/07 8:00 & 125.385 & 123.102 & 119.236 & 99.431 & 103.419 & 95.024 & 109.234 & 106.839 & 76.677 & 72.596 \\
\hline 7/19/07 8:30 & 125.499 & 123.129 & 119.352 & 99.541 & 103.535 & 95.137 & 109.344 & 106.957 & 76.712 & 72.712 \\
\hline 7/19/07 9:00 & 125.556 & 123.199 & 119.408 & 99.588 & 103.584 & 95.187 & 109.391 & 107.012 & 76.736 & 72.764 \\
\hline 7/19/07 9:30 & 125.582 & 123.22 & 119.433 & 99.612 & 103.611 & 95.215 & 109.415 & 107.043 & 76.754 & 72.793 \\
\hline 7/19/07 10:00 & 125.653 & 123.245 & 119.505 & 99.686 & 103.683 & 95.286 & 109.462 & 107.114 & 76.788 & 72.867 \\
\hline $7 / 19 / 07$ 10:30 & 125.674 & 123.263 & 119.528 & 99.705 & 103.704 & 95.307 & 109.481 & 107.136 & 76.812 & 72.892 \\
\hline 7/19/07 11:00 & 125.702 & 123.283 & 119.553 & 99.729 & 103.729 & 95.33 & 109.5 & 107.165 & 76.834 & 72.917 \\
\hline 7/19/07 11:30 & 125.716 & 123.292 & 119.567 & 99.745 & 103.742 & 95.347 & 109.513 & 107.18 & 76.852 & 72.929 \\
\hline $7 / 19 / 07$ 12:00 & 125.738 & 123.313 & 119.59 & 99.769 & 103.765 & 95.371 & 109.526 & 107.202 & 76.876 & 72.956 \\
\hline 7/19/07 12:30 & 125.747 & 123.317 & 119.599 & 99.777 & 103.775 & 95.38 & 109.537 & 107.211 & 76.896 & 72.966 \\
\hline 7/19/07 13:00 & 125.766 & 123.322 & 119.618 & 99.796 & 103.792 & 95.396 & 109.545 & 107.231 & 76.917 & 72.987 \\
\hline 7/19/07 13:30 & 125.775 & 121.284 & 119.625 & 99.803 & 103.801 & 95.406 & 109.552 & 107.238 & 76.933 & 72.997 \\
\hline 7/19/07 14:00 & 125.775 & 121.03 & 119.627 & 99.805 & 103.801 & 95.406 & 109.562 & 107.242 & 76.948 & 73.001 \\
\hline 7/19/07 14:30 & 123.667 & 120.898 & 118.066 & 98.957 & 103.196 & 94.633 & 108.797 & 105.715 & 76.935 & 72.902 \\
\hline $7 / 19 / 07$ 15:00 & 123.398 & 120.816 & 117.798 & 98.74 & 102.954 & 94.392 & 108.582 & 105.434 & 76.918 & 72.733 \\
\hline 7/19/07 15:30 & 123.263 & 120.76 & 117.666 & 98.628 & 102.834 & 94.27 & 108.467 & 105.294 & 76.904 & 72.619 \\
\hline 7/19/07 16:00 & 123.176 & 120.721 & 117.583 & 98.554 & 102.752 & 94.194 & 108.396 & 105.205 & 76.887 & 72.534 \\
\hline $7 / 19 / 07$ 16:30 & 123.119 & 120.701 & 117.528 & 98.502 & 102.703 & 94.14 & 108.349 & 105.145 & 76.874 & 72.478 \\
\hline 7/19/07 17:00 & 123.079 & 120.671 & 117.488 & 98.468 & 102.667 & 94.105 & 108.315 & 105.108 & 76.867 & 72.439 \\
\hline 7/19/07 17:30 & 123.06 & 120.646 & 117.467 & 98.449 & 102.644 & 94.084 & 108.298 & 105.09 & 76.852 & 72.408 \\
\hline 7/19/07 18:00 & 123.024 & 120.635 & 117.435 & 98.425 & 102.619 & 94.058 & 108.274 & 105.052 & 76.841 & 72.385 \\
\hline 7/19/07 18:30 & 123.003 & 120.626 & 117.417 & 98.406 & 102.6 & 94.039 & 108.259 & 105.03 & 76.83 & 72.365 \\
\hline 7/19/07 19:00 & 122.989 & 120.619 & 117.405 & 98.394 & 102.59 & 94.027 & 108.245 & 105.017 & 76.821 & 72.354 \\
\hline 7/19/07 19:30 & 122.98 & 120.619 & 117.391 & 98.42 & 102.579 & 94.02 & 108.247 & 105.009 & 76.812 & 72.344 \\
\hline 7/19/07 20:00 & 122.975 & 120.608 & 117.389 & 98.382 & 102.579 & 94.018 & 108.241 & 105.004 & 76.806 & 72.338 \\
\hline $7 / 19 / 07$ 20:30 & 122.97 & 120.61 & 117.384 & 98.378 & 102.573 & 94.011 & 108.239 & 104.997 & 76.8 & 72.334 \\
\hline 7/19/07 21:00 & 122.961 & 120.612 & 117.375 & 98.371 & 102.566 & 94.001 & 108.233 & 104.988 & 76.791 & 72.325 \\
\hline 7/19/07 21:30 & 122.958 & 120.621 & 117.375 & 98.373 & 102.566 & 94.004 & 108.223 & 104.986 & 76.786 & 72.325 \\
\hline 7/19/07 22:00 & 122.963 & 120.612 & 117.38 & 98.378 & 102.571 & 94.011 & 108.217 & 104.993 & 76.784 & 72.332 \\
\hline 7/19/07 22:30 & 122.972 & 120.615 & 117.389 & 98.389 & 102.581 & 94.02 & 108.209 & 104.999 & 76.78 & 72.34 \\
\hline 7/19/07 23:00 & 122.965 & 120.619 & 117.382 & 98.382 & 102.573 & 94.013 & 108.205 & 104.995 & 76.775 & 72.332 \\
\hline 7/19/07 23:30 & 122.968 & 120.624 & 117.382 & 98.382 & 102.575 & 94.013 & 108.198 & 104.995 & 76.773 & 72.332 \\
\hline 7/20/07 0:00 & 122.97 & 120.635 & 117.386 & 98.387 & 102.581 & 94.02 & 108.194 & 104.999 & 76.771 & 72.336 \\
\hline 7/20/07 0:30 & 122.977 & 120.63 & 117.391 & 98.396 & 102.589 & 94.025 & 108.19 & 105.004 & 76.767 & 72.34 \\
\hline 7/20/07 1:00 & 122.987 & 120.64 & 117.4 & 98.404 & 102.594 & 94.034 & 108.184 & 105.013 & 76.769 & 72.35 \\
\hline 7/20/07 1:30 & 122.987 & 120.612 & 117.403 & 98.401 & 102.594 & 94.037 & 108.182 & 105.015 & 76.767 & 72.348 \\
\hline 7/20/07 2:00 & 122.989 & 120.603 & 117.407 & 98.413 & 102.604 & 94.039 & 108.177 & 105.017 & 76.76 & 72.35 \\
\hline
\end{tabular}


TABLE S1.1 (Cont.)

Depth from Top of Casing (ft)

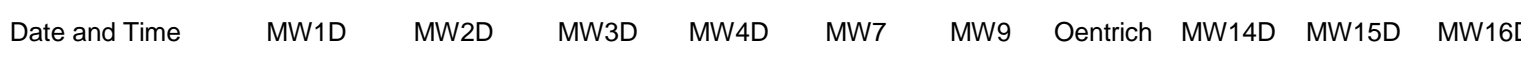

\begin{tabular}{|c|c|c|c|c|c|c|c|c|c|c|}
\hline 7/20/07 2:30 & 123.032 & 120.594 & 117.382 & 98.38 & 102.575 & 94.015 & 108.252 & 104.995 & 76.723 & 72.328 \\
\hline 7/20/07 3:00 & 123.02 & 120.601 & 117.37 & 98.371 & 102.564 & 94.004 & 108.242 & 104.984 & 76.719 & 72.315 \\
\hline 7/20/07 3:30 & 123.01 & 120.601 & 117.363 & 98.365 & 102.558 & 93.996 & 108.234 & 104.975 & 76.718 & 72.307 \\
\hline 7/20/07 4:00 & 122.998 & 120.599 & 117.368 & 98.371 & 102.562 & 94.001 & 108.238 & 104.977 & 76.714 & 72.311 \\
\hline 7/20/07 4:30 & 122.994 & 120.603 & 117.368 & 98.373 & 102.564 & 94.001 & 108.24 & 104.979 & 76.714 & 72.315 \\
\hline 7/20/07 5:00 & 122.987 & 120.599 & 117.366 & 98.368 & 102.56 & 93.999 & 108.238 & 104.977 & 76.71 & 72.311 \\
\hline 7/20/07 5:30 & 122.98 & 120.599 & 117.37 & 98.375 & 102.566 & 94.004 & 108.242 & 104.982 & 76.71 & 72.317 \\
\hline 7/20/07 6:00 & 122.972 & 122.462 & 117.368 & 98.37 & 102.564 & 94.004 & 108.24 & 104.977 & 76.706 & 72.313 \\
\hline 7/20/07 6:30 & 122.965 & 122.902 & 117.366 & 98.37 & 102.562 & 94.001 & 108.238 & 104.977 & 76.703 & 72.311 \\
\hline 7/20/07 7:00 & 124.761 & 123.082 & 118.557 & 98.959 & 102.895 & 94.477 & 108.715 & 106.213 & 76.714 & 72.325 \\
\hline 7/20/07 7:30 & 125.307 & 123.19 & 119.033 & 99.323 & 103.306 & 94.901 & 109.111 & 106.777 & 76.751 & 72.526 \\
\hline 7/20/07 8:00 & 125.497 & 123.258 & 119.218 & 99.483 & 103.479 & 95.074 & 109.275 & 106.974 & 76.776 & 72.683 \\
\hline 7/20/07 8:30 & 125.61 & 123.306 & 119.329 & 99.586 & 103.588 & 95.182 & 109.376 & 107.089 & 76.806 & 72.795 \\
\hline 7/20/07 9:00 & 125.684 & 123.342 & 119.396 & 99.655 & 103.66 & 95.253 & 109.441 & 107.164 & 76.834 & 72.873 \\
\hline 7/20/07 9:30 & 125.728 & 123.363 & 119.447 & 99.7 & 103.708 & 95.305 & 109.487 & 107.218 & 76.858 & 72.927 \\
\hline 7/20/07 10:00 & 125.766 & 123.374 & 119.484 & 99.734 & 103.746 & 95.342 & 109.52 & 107.255 & 76.883 & 72.964 \\
\hline 7/20/07 10:30 & 125.783 & 123.385 & 119.507 & 99.752 & 103.765 & 95.359 & 109.541 & 107.276 & 76.902 & 72.987 \\
\hline 7/20/07 11:00 & 125.797 & 123.381 & 119.523 & 99.767 & 103.78 & 95.373 & 109.554 & 107.291 & 76.92 & 73.003 \\
\hline 7/20/07 11:30 & 125.801 & 123.392 & 119.532 & 99.774 & 103.786 & 95.382 & 109.562 & 107.297 & 76.937 & 73.016 \\
\hline $7 / 20 / 07$ 12:00 & 125.804 & 123.394 & 119.539 & 99.774 & 103.788 & 95.382 & 109.564 & 107.3 & 76.95 & 73.02 \\
\hline $7 / 20 / 07$ 12:30 & 125.818 & 123.394 & 119.544 & 99.784 & 103.796 & 95.389 & 109.573 & 107.308 & 76.966 & 73.026 \\
\hline $7 / 20 / 07$ 13:00 & 125.818 & 123.392 & 119.546 & 99.791 & 103.805 & 95.396 & 109.58 & 107.315 & 76.981 & 73.039 \\
\hline $7 / 20 / 07$ 13:30 & 125.816 & 121.873 & 119.551 & 99.795 & 103.809 & 95.401 & 109.582 & 107.315 & 76.998 & 73.049 \\
\hline $7 / 20 / 07$ 14:00 & 125.816 & 121.227 & 119.553 & 99.796 & 103.809 & 95.406 & 109.584 & 107.315 & 77.011 & 73.053 \\
\hline $7 / 20 / 07$ 14:30 & 124.48 & 121.016 & 118.862 & 99.385 & 103.702 & 95.203 & 109.368 & 106.784 & 77.022 & 73.049 \\
\hline $7 / 20 / 07$ 15:00 & 123.596 & 120.891 & 118.006 & 98.918 & 103.146 & 94.586 & 108.786 & 105.648 & 77 & 72.9 \\
\hline $7 / 20 / 07$ 15:30 & 123.379 & 120.814 & 117.784 & 98.732 & 102.945 & 94.383 & 108.598 & 105.416 & 76.977 & 72.745 \\
\hline $7 / 20 / 07$ 16:00 & 123.251 & 120.764 & 117.662 & 98.625 & 102.828 & 94.267 & 108.49 & 105.285 & 76.961 & 72.631 \\
\hline 7/20/07 16:30 & 123.173 & 120.721 & 117.585 & 98.556 & 102.758 & 94.197 & 108.424 & 105.205 & 76.946 & 72.553 \\
\hline 7/20/07 17:00 & 123.117 & 120.692 & 117.532 & 98.511 & 102.708 & 94.147 & 108.377 & 105.148 & 76.928 & 72.499 \\
\hline $7 / 20 / 07$ 17:30 & 123.076 & 120.667 & 117.491 & 98.473 & 102.668 & 94.107 & 108.341 & 105.106 & 76.917 & 72.458 \\
\hline $7 / 20 / 07$ 18:00 & 123.046 & 120.655 & 117.46 & 98.446 & 102.642 & 94.081 & 108.315 & 105.075 & 76.902 & 72.427 \\
\hline $7 / 20 / 07$ 18:30 & 123.02 & 120.64 & 117.437 & 98.425 & 102.617 & 94.06 & 108.296 & 105.048 & 76.887 & 72.402 \\
\hline 7/20/07 19:00 & 123.008 & 120.637 & 117.423 & 98.415 & 102.608 & 94.051 & 108.287 & 105.035 & 76.88 & 72.392 \\
\hline 7/20/07 19:30 & 122.994 & 120.633 & 117.41 & 98.403 & 102.594 & 94.037 & 108.274 & 105.022 & 76.871 & 72.377 \\
\hline $7 / 20 / 0720: 00$ & 122.987 & 120.624 & 117.405 & 98.401 & 102.596 & 94.034 & 108.27 & 105.02 & 76.861 & 72.375 \\
\hline $7 / 20 / 07$ 20:30 & 122.984 & 120.628 & 117.403 & 98.397 & 102.59 & 94.029 & 108.261 & 105.013 & 76.852 & 72.367 \\
\hline $7 / 20 / 0721: 00$ & 122.98 & 120.637 & 117.396 & 98.392 & 102.583 & 94.022 & 108.259 & 105.006 & 76.845 & 72.359 \\
\hline 7/20/07 21:30 & 122.982 & 120.646 & 117.4 & 98.399 & 102.589 & 94.027 & 108.268 & 105.009 & 76.839 & 72.363 \\
\hline 7/20/07 22:00 & 122.989 & 120.651 & 117.407 & 98.408 & 102.598 & 94.034 & 108.266 & 105.017 & 76.837 & 72.371 \\
\hline $7 / 20 / 07$ 22:30 & 122.996 & 120.649 & 117.415 & 98.416 & 102.608 & 94.048 & 108.263 & 105.024 & 76.834 & 72.377 \\
\hline $7 / 20 / 07$ 23:00 & 123.003 & 120.653 & 117.419 & 98.42 & 102.611 & 94.051 & 108.259 & 105.031 & 76.828 & 72.379 \\
\hline 7/20/07 23:30 & 123.001 & 120.651 & 117.421 & 98.421 & 102.611 & 94.051 & 108.253 & 105.028 & 76.826 & 72.381 \\
\hline $7 / 21 / 070: 00$ & 123.006 & 120.655 & 117.423 & 98.423 & 102.613 & 94.055 & 108.247 & 105.033 & 76.824 & 72.383 \\
\hline 7/21/07 0:30 & 123.006 & 120.651 & 117.421 & 98.423 & 102.611 & 94.053 & 108.245 & 105.033 & 76.819 & 72.381 \\
\hline 7/21/07 1:00 & 123.006 & 120.646 & 117.423 & 98.425 & 102.615 & 94.055 & 108.247 & 105.035 & 76.815 & 72.379 \\
\hline $7 / 21 / 07$ 1:30 & 123.006 & 120.642 & 117.423 & 98.421 & 102.615 & 94.055 & 108.245 & 105.035 & 76.813 & 72.381 \\
\hline 7/21/07 2:00 & 122.998 & 120.64 & 117.417 & 98.418 & 102.609 & 94.051 & 108.242 & 105.026 & 76.808 & 72.375 \\
\hline 7/21/07 2:30 & 122.998 & 120.635 & 117.414 & 98.415 & 102.604 & 94.046 & 108.238 & 105.022 & 76.804 & 72.371 \\
\hline 7/21/07 3:00 & 122.998 & 120.637 & 117.412 & 98.411 & 102.602 & 94.041 & 108.238 & 105.02 & 76.8 & 72.369 \\
\hline 7/21/07 3:30 & 122.996 & 120.64 & 117.407 & 98.409 & 102.598 & 94.041 & 108.234 & 105.015 & 76.797 & 72.365 \\
\hline 7/21/07 4:00 & 122.994 & 120.635 & 117.41 & 98.409 & 102.6 & 94.041 & 108.232 & 105.017 & 76.791 & 72.367 \\
\hline $7 / 21 / 074: 30$ & 122.989 & 120.64 & 117.41 & 98.413 & 102.604 & 94.046 & 108.23 & 105.02 & 76.789 & 72.371 \\
\hline 7/21/07 5:00 & 122.982 & 120.642 & 117.41 & 98.411 & 102.6 & 94.041 & 108.23 & 105.015 & 76.788 & 72.369 \\
\hline 7/21/07 5:30 & 122.991 & 120.642 & 117.412 & 98.413 & 102.604 & 94.044 & 108.227 & 105.02 & 76.786 & 72.371 \\
\hline 7/21/07 6:00 & 122.989 & 120.642 & 117.414 & 98.416 & 102.604 & 94.044 & 108.227 & 105.021 & 76.784 & 72.369 \\
\hline 7/21/07 6:30 & 122.984 & 120.646 & 117.412 & 98.418 & 102.606 & 94.046 & 108.225 & 105.022 & 76.778 & 72.371 \\
\hline 7/21/07 7:00 & 122.994 & 122.712 & 117.414 & 98.416 & 102.607 & 94.048 & 108.223 & 105.019 & 76.78 & 72.371 \\
\hline 7/21/07 7:30 & 122.998 & 122.968 & 117.419 & 98.423 & 102.611 & 94.053 & 108.223 & 105.026 & 76.776 & 72.377 \\
\hline 7/21/07 8:00 & 125.135 & 123.102 & 119.014 & 99.25 & 103.224 & 94.831 & 108.985 & 106.58 & 76.8 & 72.487 \\
\hline 7/21/07 8:30 & 125.402 & 123.184 & 119.273 & 99.491 & 103.464 & 95.071 & 109.21 & 106.857 & 76.834 & 72.671 \\
\hline 7/21/07 9:00 & 125.544 & 123.236 & 119.41 & 99.615 & 103.594 & 95.201 & 109.332 & 107.003 & 76.863 & 72.799 \\
\hline 7/21/07 9:30 & 125.629 & 123.272 & 119.493 & 99.691 & 103.676 & 95.283 & 109.407 & 107.089 & 76.891 & 72.886 \\
\hline $7 / 21 / 07$ 10:00 & 125.686 & 123.297 & 119.551 & 99.743 & 103.733 & 95.34 & 109.462 & 107.149 & 76.915 & 72.946 \\
\hline $7 / 21 / 07$ 10:30 & 125.728 & 121.671 & 119.593 & 99.776 & 103.771 & 95.378 & 109.498 & 107.193 & 76.937 & 72.985 \\
\hline 7/21/07 11:00 & 125.749 & 121.179 & 119.616 & 99.798 & 103.794 & 95.401 & 109.529 & 107.218 & 76.957 & 73.012 \\
\hline
\end{tabular}


TABLE S1.1 (Cont.)

Depth from Top of Casing (ft)

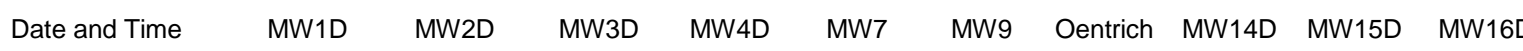

\begin{tabular}{|c|c|c|c|c|c|c|c|c|c|c|}
\hline 7/21/07 11:30 & 124.126 & 121 & 118.526 & 99.267 & 103.542 & 95.005 & 109.128 & 106.261 & 76.972 & 73.026 \\
\hline $7 / 21 / 07$ 12:00 & 123.554 & 120.893 & 117.965 & 98.888 & 103.11 & 94.55 & 108.701 & 105.595 & 76.953 & 72.875 \\
\hline 7/21/07 12:30 & 123.362 & 120.828 & 117.775 & 98.73 & 102.937 & 94.376 & 108.542 & 105.396 & 76.939 & 72.739 \\
\hline 7/21/07 13:00 & 123.254 & 120.78 & 117.671 & 98.639 & 102.842 & 94.282 & 108.454 & 105.287 & 76.928 & 72.64 \\
\hline 7/21/07 13:30 & 123.183 & 120.744 & 117.602 & 98.578 & 102.775 & 94.218 & 108.399 & 105.214 & 76.92 & 72.569 \\
\hline $7 / 21 / 07$ 14:00 & 123.14 & 120.712 & 117.556 & 98.539 & 102.733 & 94.176 & 108.36 & 105.166 & 76.907 & 72.524 \\
\hline $7 / 21 / 07$ 14:30 & 123.1 & 120.694 & 117.518 & 98.504 & 102.697 & 94.14 & 108.337 & 105.13 & 76.898 & 72.485 \\
\hline $7 / 21 / 07$ 15:00 & 123.069 & 120.669 & 117.488 & 98.476 & 102.668 & 94.114 & 108.318 & 105.097 & 76.889 & 72.456 \\
\hline 7/21/07 15:30 & 123.048 & 120.653 & 117.467 & 98.457 & 102.648 & 94.093 & 108.305 & 105.077 & 76.878 & 72.433 \\
\hline 7/21/07 16:00 & 123.027 & 120.633 & 117.447 & 98.437 & 102.629 & 94.072 & 108.294 & 105.053 & 76.867 & 72.414 \\
\hline 7/21/07 16:30 & 123.008 & 120.635 & 117.428 & 98.421 & 102.613 & 94.055 & 108.285 & 105.035 & 76.858 & 72.394 \\
\hline $7 / 21 / 07$ 17:00 & 122.991 & 120.621 & 117.412 & 98.404 & 102.594 & 94.037 & 108.273 & 105.017 & 76.848 & 72.379 \\
\hline 7/21/07 17:30 & 122.984 & 120.615 & 117.407 & 98.406 & 102.592 & 94.037 & 108.272 & 105.013 & 76.837 & 72.371 \\
\hline $7 / 21 / 07$ 18:00 & 122.977 & 120.606 & 117.398 & 98.392 & 102.583 & 94.027 & 108.264 & 105.004 & 76.835 & 72.365 \\
\hline 7/21/07 18:30 & 122.968 & 120.599 & 117.386 & 98.387 & 102.575 & 94.018 & 108.257 & 104.995 & 76.824 & 72.354 \\
\hline 7/21/07 19:00 & 122.956 & 120.603 & 117.38 & 98.378 & 102.568 & 94.011 & 108.249 & 104.984 & 76.817 & 72.348 \\
\hline 7/21/07 19:30 & 122.951 & 120.606 & 117.373 & 98.375 & 102.562 & 94.006 & 108.243 & 104.98 & 76.812 & 72.344 \\
\hline $7 / 21 / 07$ 20:00 & 122.951 & 122.53 & 117.373 & 98.377 & 102.564 & 94.008 & 108.245 & 104.98 & 76.804 & 72.344 \\
\hline $7 / 21 / 07$ 20:30 & 122.958 & 122.875 & 117.382 & 98.382 & 102.569 & 94.013 & 108.247 & 104.986 & 76.804 & 72.352 \\
\hline $7 / 21 / 0721: 00$ & 124.939 & 123.043 & 118.825 & 99.112 & 103.055 & 94.659 & 108.852 & 106.355 & 76.819 & 72.385 \\
\hline 7/21/07 21:30 & 125.31 & 123.154 & 119.181 & 99.402 & 103.369 & 94.979 & 109.183 & 106.76 & 76.85 & 72.594 \\
\hline $7 / 21 / 07$ 22:00 & 125.489 & 123.224 & 119.354 & 99.557 & 103.539 & 95.146 & 109.327 & 106.945 & 76.885 & 72.751 \\
\hline 7/21/07 22:30 & 125.601 & 122.165 & 119.463 & 99.659 & 103.639 & 95.25 & 109.415 & 107.056 & 76.913 & 72.855 \\
\hline 7/21/07 23:00 & 125.676 & 121.202 & 119.539 & 99.726 & 103.714 & 95.326 & 109.474 & 107.136 & 76.942 & 72.931 \\
\hline 7/21/07 23:30 & 125.716 & 120.998 & 119.579 & 99.464 & 103.752 & 95.363 & 109.511 & 107.178 & 76.963 & 72.975 \\
\hline 7/22/07 0:00 & 123.577 & 120.88 & 117.99 & 98.904 & 103.135 & 94.574 & 108.736 & 105.624 & 76.952 & 72.877 \\
\hline 7/22/07 0:30 & 123.36 & 120.814 & 117.775 & 98.732 & 102.939 & 94.381 & 108.548 & 105.396 & 76.942 & 72.739 \\
\hline 7/22/07 1:00 & 123.242 & 120.769 & 117.655 & 98.628 & 102.828 & 94.27 & 108.454 & 105.272 & 76.933 & 72.633 \\
\hline 7/22/07 1:30 & 123.171 & 120.733 & 117.59 & 98.571 & 102.766 & 94.209 & 108.396 & 105.203 & 76.92 & 72.567 \\
\hline 7/22/07 2:00 & 123.126 & 120.712 & 117.541 & 98.526 & 102.72 & 94.161 & 108.358 & 105.152 & 76.913 & 72.518 \\
\hline 7/22/07 2:30 & 123.1 & 120.699 & 117.509 & 98.499 & 102.691 & 94.133 & 108.33 & 105.117 & 76.902 & 72.483 \\
\hline 7/22/07 3:00 & 123.081 & 120.683 & 117.486 & 98.48 & 102.668 & 94.11 & 108.311 & 105.095 & 76.896 & 72.458 \\
\hline 7/22/07 3:30 & 123.069 & 120.669 & 117.474 & 98.466 & 102.657 & 94.1 & 108.298 & 105.079 & 76.891 & 72.445 \\
\hline 7/22/07 4:00 & 123.062 & 120.662 & 117.456 & 98.451 & 102.64 & 94.084 & 108.289 & 105.061 & 76.883 & 72.427 \\
\hline 7/22/07 4:30 & 123.022 & 120.658 & 117.442 & 98.44 & 102.629 & 94.072 & 108.279 & 105.048 & 76.874 & 72.412 \\
\hline 7/22/07 5:00 & 123.015 & 120.658 & 117.437 & 98.435 & 102.623 & 94.067 & 108.274 & 105.044 & 76.872 & 72.408 \\
\hline 7/22/07 5:30 & 123.01 & 120.658 & 117.43 & 98.432 & 102.617 & 94.06 & 108.27 & 105.037 & 76.867 & 72.402 \\
\hline 7/22/07 6:00 & 123.013 & 120.651 & 117.435 & 98.434 & 102.623 & 94.062 & 108.262 & 105.04 & 76.863 & 72.406 \\
\hline 7/22/07 6:30 & 123.01 & 120.649 & 117.433 & 98.435 & 102.623 & 94.062 & 108.258 & 105.037 & 76.859 & 72.406 \\
\hline 7/22/07 7:00 & 123.006 & 122.478 & 117.428 & 98.428 & 102.617 & 94.058 & 108.255 & 105.033 & 76.856 & 72.4 \\
\hline 7/22/07 7:30 & 123.001 & 122.884 & 117.421 & 98.423 & 102.611 & 94.051 & 108.253 & 105.028 & 76.85 & 72.396 \\
\hline 7/22/07 8:00 & 124.865 & 123.066 & 118.751 & 99.078 & 102.996 & 94.593 & 108.792 & 106.263 & 76.858 & 72.41 \\
\hline 7/22/07 8:30 & 125.319 & 123.177 & 119.19 & 99.412 & 103.377 & 94.986 & 109.15 & 106.766 & 76.891 & 72.611 \\
\hline 7/22/07 9:00 & 125.508 & 123.236 & 119.376 & 99.576 & 103.554 & 95.161 & 109.312 & 106.961 & 76.92 & 72.768 \\
\hline 7/22/07 9:30 & 125.617 & 121.685 & 119.482 & 99.674 & 103.655 & 95.264 & 109.406 & 107.074 & 76.946 & 72.875 \\
\hline $7 / 22 / 07$ 10:00 & 125.688 & 121.147 & 119.551 & 99.737 & 103.723 & 95.333 & 109.469 & 107.147 & 76.97 & 72.948 \\
\hline $7 / 22 / 07$ 10:30 & 124.182 & 120.968 & 118.582 & 99.261 & 103.55 & 95.024 & 109.154 & 106.359 & 76.99 & 72.989 \\
\hline 7/22/07 11:00 & 123.521 & 120.864 & 117.934 & 98.861 & 103.084 & 94.522 & 108.686 & 105.562 & 76.972 & 72.853 \\
\hline 7/22/07 11:30 & 123.329 & 120.801 & 117.747 & 98.702 & 102.912 & 94.352 & 108.557 & 105.365 & 76.963 & 72.722 \\
\hline $7 / 22 / 07$ 12:00 & 123.221 & 120.753 & 117.641 & 98.611 & 102.811 & 94.251 & 108.485 & 105.254 & 76.948 & 72.621 \\
\hline $7 / 22 / 07$ 12:30 & 123.159 & 120.721 & 117.581 & 98.558 & 102.754 & 94.199 & 108.402 & 105.188 & 76.942 & 72.557 \\
\hline 7/22/07 13:00 & 123.11 & 120.696 & 117.528 & 98.516 & 102.707 & 94.15 & 108.381 & 105.139 & 76.931 & 72.512 \\
\hline 7/22/07 13:30 & 123.074 & 120.669 & 117.497 & 98.487 & 102.678 & 94.121 & 108.359 & 105.104 & 76.922 & 72.478 \\
\hline 7/22/07 14:00 & 123.05 & 120.651 & 117.47 & 98.459 & 102.651 & 94.093 & 108.333 & 105.077 & 76.911 & 72.45 \\
\hline $7 / 22 / 07$ 14:30 & 123.024 & 120.633 & 117.444 & 98.437 & 102.627 & 94.072 & 108.307 & 105.051 & 76.904 & 72.426 \\
\hline $7 / 22 / 07$ 15:00 & 123.003 & 120.621 & 117.428 & 98.419 & 102.613 & 94.053 & 108.292 & 105.033 & 76.894 & 72.408 \\
\hline 7/22/07 15:30 & 122.987 & 120.608 & 117.41 & 98.406 & 102.594 & 94.039 & 108.276 & 105.015 & 76.885 & 72.39 \\
\hline 7/22/07 16:00 & 122.975 & 120.599 & 117.398 & 98.396 & 102.583 & 94.025 & 108.267 & 105.002 & 76.876 & 72.377 \\
\hline 7/22/07 16:30 & 122.961 & 120.581 & 117.384 & 98.38 & 102.568 & 94.013 & 108.256 & 104.986 & 76.869 & 72.364 \\
\hline 7/22/07 17:00 & 122.954 & 120.574 & 117.375 & 98.371 & 102.562 & 94.001 & 108.222 & 104.982 & 76.861 & 72.354 \\
\hline 7/22/07 17:30 & 122.935 & 120.569 & 117.359 & 98.356 & 102.545 & 93.985 & 108.233 & 104.962 & 76.85 & 72.34 \\
\hline 7/22/07 18:00 & 122.925 & 120.567 & 117.349 & 98.349 & 102.537 & 93.978 & 108.224 & 104.951 & 76.845 & 72.331 \\
\hline 7/22/07 18:30 & 122.923 & 120.558 & 117.345 & 98.347 & 102.535 & 93.975 & 108.222 & 104.949 & 76.839 & 72.33 \\
\hline 7/22/07 19:00 & 122.923 & 120.553 & 117.347 & 98.344 & 102.537 & 93.978 & 108.224 & 104.951 & 76.834 & 72.331 \\
\hline 7/22/07 19:30 & 122.911 & 120.551 & 117.336 & 98.337 & 102.524 & 93.966 & 108.212 & 104.937 & 76.824 & 72.317 \\
\hline $7 / 22 / 07$ 20:00 & 122.906 & 120.549 & 117.331 & 98.33 & 102.52 & 93.961 & 108.208 & 104.935 & 76.819 & 72.315 \\
\hline
\end{tabular}


TABLE S1.1 (Cont.)

Depth from Top of Casing (ft)

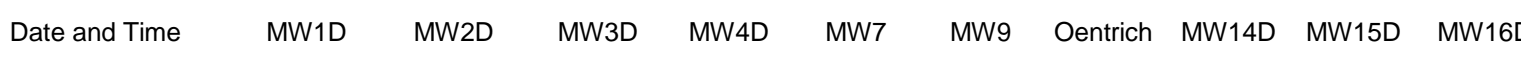

\begin{tabular}{|c|c|c|c|c|c|c|c|c|c|c|}
\hline 7/22/07 20:30 & 122.906 & 120.628 & 117.329 & 98.33 & 102.518 & 93.961 & 108.207 & 104.935 & 76.812 & 72.309 \\
\hline $7 / 22 / 07$ 21:00 & 122.899 & 120.606 & 117.324 & 98.332 & 102.518 & 93.954 & 108.203 & 104.927 & 76.806 & 72.309 \\
\hline $7 / 22 / 07$ 21:30 & 122.987 & 120.576 & 117.428 & 98.489 & 102.632 & 94.105 & 109.259 & 104.999 & 76.819 & 72.328 \\
\hline 7/22/07 22:00 & 122.965 & 120.562 & 117.391 & 98.39 & 102.583 & 94.022 & 108.252 & 104.997 & 76.823 & 72.346 \\
\hline 7/22/07 22:30 & 122.935 & 120.551 & 117.361 & 98.351 & 102.552 & 94.011 & 108.199 & 104.957 & 76.817 & 72.338 \\
\hline 7/22/07 23:00 & 122.918 & 120.553 & 117.343 & 98.337 & 102.533 & 93.975 & 108.17 & 104.944 & 76.806 & 72.327 \\
\hline 7/22/07 23:30 & 122.899 & 120.553 & 117.322 & 98.325 & 102.514 & 93.952 & 108.182 & 104.929 & 76.799 & 72.309 \\
\hline 7/23/07 0:00 & 122.911 & 120.556 & 117.336 & 98.33 & 102.524 & 93.966 & 108.194 & 104.933 & 76.797 & 72.317 \\
\hline 7/23/07 0:30 & 122.904 & 120.547 & 117.331 & 98.33 & 102.531 & 93.973 & 108.78 & 104.931 & 76.793 & 72.315 \\
\hline 7/23/07 1:00 & 122.904 & 120.54 & 117.329 & 98.33 & 102.522 & 93.959 & 108.206 & 104.931 & 76.789 & 72.313 \\
\hline 7/23/07 1:30 & 122.899 & 120.542 & 117.326 & 98.342 & 102.516 & 93.954 & 108.179 & 104.929 & 76.786 & 72.306 \\
\hline 7/23/07 2:00 & 122.894 & 120.538 & 117.317 & 98.323 & 102.51 & 93.952 & 108.191 & 104.922 & 76.782 & 72.304 \\
\hline 7/23/07 2:30 & 122.894 & 120.535 & 117.324 & 98.318 & 102.514 & 93.966 & 108.187 & 104.917 & 76.776 & 72.3 \\
\hline 7/23/07 3:00 & 122.89 & 120.529 & 117.315 & 98.332 & 102.51 & 93.947 & 108.185 & 104.917 & 76.776 & 72.298 \\
\hline 7/23/07 3:30 & 122.885 & 120.524 & 117.312 & 98.318 & 102.503 & 93.94 & 108.174 & 104.915 & 76.773 & 72.294 \\
\hline 7/23/07 4:00 & 122.88 & 120.51 & 117.306 & 98.306 & 102.501 & 93.938 & 108.687 & 104.906 & 76.765 & 72.29 \\
\hline 7/23/07 4:30 & 122.876 & 120.506 & 117.299 & 98.299 & 102.49 & 93.926 & 108.168 & 104.902 & 76.764 & 72.284 \\
\hline 7/23/07 5:00 & 122.866 & 120.504 & 117.289 & 98.287 & 102.48 & 93.921 & 108.151 & 104.893 & 76.756 & 72.273 \\
\hline 7/23/07 5:30 & 122.859 & 120.504 & 117.282 & 98.289 & 102.474 & 93.914 & 108.153 & 104.887 & 76.753 & 72.269 \\
\hline 7/23/07 6:00 & 122.854 & 122.642 & 117.28 & 98.284 & 102.474 & 93.912 & 108.172 & 104.88 & 76.747 & 72.263 \\
\hline 7/23/07 6:30 & 122.857 & 122.909 & 117.282 & 98.285 & 102.476 & 93.914 & 108.17 & 104.885 & 76.747 & 72.265 \\
\hline 7/23/07 7:00 & 125.033 & 123.041 & 118.772 & 99.095 & 103.057 & 94.647 & 108.883 & 106.489 & 76.767 & 72.368 \\
\hline 7/23/07 7:30 & 125.317 & 123.127 & 119.051 & 99.328 & 103.316 & 94.909 & 109.128 & 106.788 & 76.8 & 72.569 \\
\hline 7/23/07 8:00 & 125.454 & 123.168 & 119.188 & 99.446 & 103.443 & 95.038 & 109.248 & 106.934 & 76.824 & 72.693 \\
\hline 7/23/07 8:30 & 125.541 & 123.202 & 119.278 & 99.526 & 103.529 & 95.123 & 109.326 & 107.025 & 76.85 & 72.784 \\
\hline 7/23/07 9:00 & 125.586 & 123.229 & 119.32 & 99.562 & 103.569 & 95.165 & 109.365 & 107.072 & 76.871 & 72.832 \\
\hline 7/23/07 9:30 & 125.617 & 123.267 & 119.354 & 99.596 & 103.603 & 95.198 & 109.397 & 107.105 & 76.885 & 72.869 \\
\hline $7 / 23 / 07$ 10:00 & 125.645 & 123.283 & 119.382 & 99.622 & 103.628 & 95.224 & 109.421 & 107.133 & 76.906 & 72.898 \\
\hline $7 / 23 / 07$ 10:30 & 125.683 & 123.317 & 119.419 & 99.662 & 103.668 & 95.262 & 109.457 & 107.173 & 76.924 & 72.937 \\
\hline 7/23/07 11:00 & 125.702 & 123.308 & 119.438 & 99.674 & 103.683 & 95.281 & 109.474 & 107.191 & 76.942 & 72.958 \\
\hline $7 / 23 / 07$ 11:30 & 125.735 & 123.315 & 119.472 & 99.71 & 103.719 & 95.316 & 109.502 & 107.225 & 76.968 & 72.995 \\
\hline 7/23/07 12:00 & 125.728 & 123.331 & 119.465 & 99.703 & 103.714 & 95.307 & 109.498 & 107.218 & 76.974 & 72.987 \\
\hline $7 / 23 / 07$ 12:30 & 125.735 & 123.333 & 119.475 & 99.712 & 103.721 & 95.316 & 109.507 & 107.229 & 76.988 & 72.999 \\
\hline 7/23/07 13:00 & 125.752 & 123.333 & 119.491 & 99.729 & 103.742 & 95.335 & 109.524 & 107.245 & 77.005 & 73.022 \\
\hline $7 / 23 / 07$ 13:30 & 125.759 & 122.329 & 119.498 & 99.734 & 103.744 & 95.34 & 109.527 & 107.251 & 77.018 & 73.026 \\
\hline $7 / 23 / 07$ 14:00 & 125.754 & 121.197 & 119.495 & 99.741 & 103.748 & 95.34 & 109.531 & 107.249 & 77.031 & 73.032 \\
\hline $7 / 23 / 07$ 14:30 & 125.749 & 120.975 & 119.488 & 99.488 & 103.738 & 95.333 & 109.524 & 107.242 & 77.038 & 73.026 \\
\hline $7 / 23 / 07$ 15:00 & 123.587 & 120.841 & 117.99 & 98.888 & 103.12 & 94.56 & 108.771 & 105.626 & 77.018 & 72.906 \\
\hline 7/23/07 15:30 & 123.343 & 120.753 & 117.756 & 98.697 & 102.91 & 94.35 & 108.572 & 105.376 & 77.001 & 72.751 \\
\hline $7 / 23 / 07$ 16:00 & 123.204 & 120.689 & 117.62 & 98.583 & 102.787 & 94.225 & 108.455 & 105.234 & 76.985 & 72.625 \\
\hline $7 / 23 / 07$ 16:30 & 123.117 & 120.642 & 117.532 & 98.502 & 102.701 & 94.14 & 108.376 & 105.141 & 76.966 & 72.536 \\
\hline $7 / 23 / 07$ 17:00 & 123.048 & 120.612 & 117.467 & 98.442 & 102.638 & 94.081 & 108.318 & 105.075 & 76.948 & 72.472 \\
\hline $7 / 23 / 07$ 17:30 & 122.998 & 120.585 & 117.421 & 98.403 & 102.596 & 94.037 & 108.275 & 105.026 & 76.933 & 72.422 \\
\hline $7 / 23 / 07$ 18:00 & 122.968 & 120.56 & 117.391 & 98.375 & 102.566 & 94.006 & 108.252 & 104.995 & 76.918 & 72.389 \\
\hline 7/23/07 18:30 & 122.939 & 120.544 & 117.361 & 98.347 & 102.539 & 93.98 & 108.23 & 104.966 & 76.891 & 72.362 \\
\hline 7/23/07 19:00 & 122.913 & 120.535 & 117.338 & 98.325 & 102.516 & 93.956 & 108.205 & 104.94 & 76.869 & 72.335 \\
\hline $7 / 23 / 07$ 19:30 & 122.899 & 120.526 & 117.324 & 98.316 & 102.505 & 93.945 & 108.196 & 104.927 & 76.85 & 72.323 \\
\hline 7/23/07 20:00 & 122.892 & 120.526 & 117.315 & 98.308 & 102.499 & 93.938 & 108.19 & 104.918 & 76.839 & 72.317 \\
\hline $7 / 23 / 07$ 20:30 & 122.883 & 120.522 & 117.308 & 98.303 & 102.49 & 93.93 & 108.181 & 104.906 & 76.826 & 72.306 \\
\hline $7 / 23 / 0721: 00$ & 122.878 & 120.522 & 117.303 & 98.301 & 102.491 & 93.928 & 108.181 & 104.904 & 76.812 & 72.302 \\
\hline $7 / 23 / 0721: 30$ & 122.873 & 120.517 & 117.299 & 98.296 & 102.486 & 93.923 & 108.175 & 104.9 & 76.802 & 72.296 \\
\hline 7/23/07 22:00 & 122.871 & 120.515 & 117.296 & 98.297 & 102.484 & 93.923 & 108.175 & 104.898 & 76.791 & 72.296 \\
\hline $7 / 23 / 07$ 22:30 & 122.866 & 120.513 & 117.292 & 98.292 & 102.48 & 93.921 & 108.171 & 104.893 & 76.782 & 72.29 \\
\hline 7/23/07 23:00 & 122.866 & 120.51 & 117.292 & 98.292 & 102.482 & 93.921 & 108.171 & 104.895 & 76.775 & 72.29 \\
\hline 7/23/07 23:30 & 122.864 & 120.513 & 117.287 & 98.289 & 102.48 & 93.921 & 108.169 & 104.891 & 76.769 & 72.286 \\
\hline 7/24/07 0:00 & 122.864 & 120.506 & 117.287 & 98.289 & 102.478 & 93.921 & 108.169 & 104.891 & 76.76 & 72.286 \\
\hline 7/24/07 0:30 & 122.864 & 120.504 & 117.287 & 98.29 & 102.478 & 93.919 & 108.167 & 104.891 & 76.753 & 72.284 \\
\hline 7/24/07 1:00 & 122.859 & 120.501 & 117.285 & 98.285 & 102.472 & 93.914 & 108.165 & 104.884 & 76.745 & 72.28 \\
\hline 7/24/07 1:30 & 122.854 & 120.492 & 117.28 & 98.285 & 102.471 & 93.909 & 108.162 & 104.882 & 76.738 & 72.275 \\
\hline 7/24/07 2:00 & 122.852 & 120.488 & 117.28 & 98.28 & 102.471 & 93.907 & 108.16 & 104.88 & 76.73 & 72.273 \\
\hline 7/24/07 2:30 & 122.84 & 120.481 & 117.271 & 98.268 & 102.453 & 93.895 & 108.147 & 104.864 & 76.723 & 72.259 \\
\hline 7/24/07 3:00 & 122.842 & 120.474 & 117.266 & 98.268 & 102.457 & 93.897 & 108.149 & 104.867 & 76.718 & 72.261 \\
\hline 7/24/07 3:30 & 122.833 & 120.479 & 117.259 & 98.261 & 102.45 & 93.888 & 108.143 & 104.86 & 76.712 & 72.253 \\
\hline $7 / 24 / 074: 00$ & 122.826 & 120.474 & 117.257 & 98.254 & 102.444 & 93.881 & 108.135 & 104.851 & 76.705 & 72.244 \\
\hline 7/24/07 4:30 & 122.828 & 120.47 & 117.253 & 98.261 & 102.444 & 93.883 & 108.137 & 104.854 & 76.701 & 72.247 \\
\hline 7/24/07 5:00 & 122.828 & 120.472 & 117.255 & 98.256 & 102.446 & 93.886 & 108.137 & 104.853 & 76.695 & 72.247 \\
\hline
\end{tabular}


TABLE S1.1 (Cont.)

Depth from Top of Casing (ft)

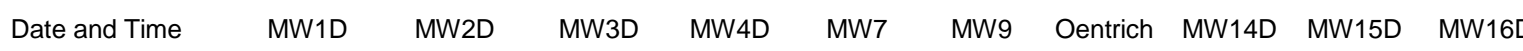

\begin{tabular}{|c|c|c|c|c|c|c|c|c|c|c|}
\hline 7/24/07 5:30 & 122.821 & 120.476 & 117.25 & 98.253 & 102.44 & 93.879 & 108.131 & 104.847 & 76.69 & 72.24 \\
\hline 7/24/07 6:00 & 122.821 & 122.521 & 117.25 & 98.251 & 102.442 & 93.881 & 108.131 & 104.849 & 76.688 & 72.242 \\
\hline 7/24/07 6:30 & 122.824 & 122.809 & 117.25 & 98.256 & 102.444 & 93.886 & 108.135 & 104.851 & 76.684 & 72.244 \\
\hline $7 / 24 / 077: 00$ & 124.939 & 122.954 & 118.816 & 99.079 & 103.022 & 94.63 & 108.866 & 106.37 & 76.706 & 72.333 \\
\hline $7 / 24 / 07$ 7:30 & 125.251 & 123.043 & 119.116 & 99.327 & 103.295 & 94.906 & 109.127 & 106.693 & 76.738 & 72.534 \\
\hline 7/24/07 8:00 & 125.402 & 123.109 & 119.264 & 99.46 & 103.436 & 95.048 & 109.256 & 106.848 & 76.764 & 72.67 \\
\hline 7/24/07 8:30 & 125.494 & 123.145 & 119.357 & 99.541 & 103.525 & 95.137 & 109.341 & 106.946 & 76.788 & 72.763 \\
\hline 7/24/07 9:00 & 125.56 & 123.168 & 119.424 & 99.601 & 103.588 & 95.196 & 109.399 & 107.012 & 76.813 & 72.829 \\
\hline $7 / 24 / 07$ 9:30 & 125.596 & 123.188 & 119.461 & 99.636 & 103.624 & 95.231 & 109.433 & 107.052 & 76.832 & 72.869 \\
\hline $7 / 24 / 07$ 10:00 & 125.619 & 123.202 & 119.484 & 99.656 & 103.647 & 95.255 & 109.453 & 107.076 & 76.85 & 72.896 \\
\hline $7 / 24 / 07$ 10:30 & 125.641 & 123.211 & 119.507 & 99.677 & 103.668 & 95.276 & 109.474 & 107.1 & 76.869 & 72.918 \\
\hline $7 / 24 / 0711: 00$ & 125.657 & 123.217 & 119.521 & 99.691 & 103.683 & 95.29 & 109.485 & 107.114 & 76.885 & 72.937 \\
\hline $7 / 24 / 07$ 11:30 & 125.667 & 121.358 & 119.53 & 99.701 & 103.695 & 95.3 & 109.494 & 107.125 & 76.898 & 72.949 \\
\hline $7 / 24 / 07$ 12:00 & 125.674 & 120.991 & 119.539 & 99.706 & 103.698 & 95.309 & 109.506 & 107.131 & 76.913 & 72.958 \\
\hline $7 / 24 / 07$ 12:30 & 123.767 & 120.821 & 118.175 & 99.007 & 103.261 & 94.711 & 108.909 & 105.834 & 76.911 & 72.925 \\
\hline $7 / 24 / 07$ 13:00 & 123.362 & 120.723 & 117.78 & 98.705 & 102.925 & 94.364 & 108.584 & 105.398 & 76.889 & 72.745 \\
\hline $7 / 24 / 07$ 13:30 & 123.185 & 120.646 & 117.604 & 98.556 & 102.764 & 94.206 & 108.433 & 105.214 & 76.871 & 72.604 \\
\hline $7 / 24 / 07$ 14:00 & 123.076 & 120.594 & 117.497 & 98.466 & 102.665 & 94.105 & 108.343 & 105.106 & 76.85 & 72.503 \\
\hline $7 / 24 / 07$ 14:30 & 123.006 & 120.56 & 117.428 & 98.401 & 102.598 & 94.039 & 108.28 & 105.033 & 76.832 & 72.433 \\
\hline $7 / 24 / 07$ 15:00 & 122.954 & 120.531 & 117.375 & 98.356 & 102.552 & 93.989 & 108.236 & 104.975 & 76.81 & 72.377 \\
\hline $7 / 24 / 07$ 15:30 & 122.916 & 120.506 & 117.34 & 98.323 & 102.516 & 93.956 & 108.206 & 104.942 & 76.793 & 72.34 \\
\hline $7 / 24 / 07$ 16:00 & 122.887 & 120.485 & 117.312 & 98.299 & 102.491 & 93.933 & 108.182 & 104.913 & 76.776 & 72.313 \\
\hline $7 / 24 / 07$ 16:30 & 122.861 & 120.467 & 117.287 & 98.277 & 102.471 & 93.907 & 108.161 & 104.887 & 76.764 & 72.288 \\
\hline $7 / 24 / 07 \quad 17: 00$ & 122.84 & 120.483 & 117.266 & 98.256 & 102.446 & 93.886 & 108.141 & 104.865 & 76.747 & 72.263 \\
\hline 7/24/07 17:30 & 122.821 & 120.458 & 117.248 & 98.24 & 102.431 & 93.872 & 108.124 & 104.847 & 76.734 & 72.247 \\
\hline $7 / 24 / 07$ 18:00 & 122.826 & 120.447 & 117.25 & 98.245 & 102.431 & 93.872 & 108.124 & 104.855 & 76.721 & 72.238 \\
\hline $7 / 24 / 07$ 18:30 & 122.812 & 120.44 & 117.238 & 98.233 & 102.425 & 93.862 & 108.118 & 104.838 & 76.714 & 72.236 \\
\hline $7 / 24 / 07$ 19:00 & 122.798 & 120.436 & 117.227 & 98.223 & 102.411 & 93.85 & 108.107 & 104.824 & 76.701 & 72.228 \\
\hline $7 / 24 / 07 \quad 19: 30$ & 122.79 & 120.433 & 117.22 & 98.218 & 102.408 & 93.846 & 108.101 & 104.818 & 76.69 & 72.222 \\
\hline $7 / 24 / 0720: 00$ & 122.788 & 120.429 & 117.215 & 98.214 & 102.406 & 93.843 & 108.097 & 104.814 & 76.682 & 72.215 \\
\hline $7 / 24 / 0720: 30$ & 122.783 & 120.424 & 117.211 & 98.213 & 102.402 & 93.839 & 108.096 & 104.811 & 76.673 & 72.211 \\
\hline $7 / 24 / 0721: 00$ & 122.779 & 120.429 & 117.206 & 98.209 & 102.398 & 93.836 & 108.094 & 104.807 & 76.668 & 72.207 \\
\hline $7 / 24 / 07$ 21:30 & 122.779 & 120.433 & 117.206 & 98.208 & 102.396 & 93.836 & 108.092 & 104.805 & 76.659 & 72.203 \\
\hline $7 / 24 / 07$ 22:00 & 122.779 & 120.431 & 117.206 & 98.211 & 102.4 & 93.836 & 108.092 & 104.805 & 76.653 & 72.207 \\
\hline $7 / 24 / 0722: 30$ & 122.783 & 120.433 & 117.211 & 98.216 & 102.404 & 93.843 & 108.097 & 104.811 & 76.647 & 72.209 \\
\hline $7 / 24 / 07$ 23:00 & 122.783 & 120.433 & 117.211 & 98.215 & 102.402 & 93.843 & 108.097 & 104.809 & 76.642 & 72.209 \\
\hline $7 / 24 / 07$ 23:30 & 122.786 & 120.433 & 117.213 & 98.218 & 102.406 & 93.846 & 108.099 & 104.811 & 76.64 & 72.209 \\
\hline $7 / 25 / 07$ 0:00 & 122.788 & 120.436 & 117.213 & 98.218 & 102.406 & 93.848 & 108.101 & 104.811 & 76.631 & 72.211 \\
\hline 7/25/07 0:30 & 122.783 & 120.436 & 117.213 & 98.216 & 102.406 & 93.846 & 108.099 & 104.812 & 76.627 & 72.209 \\
\hline $7 / 25 / 07$ 1:00 & 122.786 & 120.436 & 117.215 & 98.22 & 102.411 & 93.845 & 108.101 & 104.813 & 76.618 & 72.211 \\
\hline $7 / 25 / 07$ 1:30 & 122.783 & 120.433 & 117.215 & 98.221 & 102.41 & 93.848 & 108.103 & 104.813 & 76.612 & 72.211 \\
\hline $7 / 25 / 072: 00$ & 122.786 & 120.429 & 117.215 & 98.221 & 102.41 & 93.85 & 108.103 & 104.813 & 76.605 & 72.211 \\
\hline 7/25/07 2:30 & 122.786 & 120.426 & 117.215 & 98.218 & 102.408 & 93.848 & 108.101 & 104.813 & 76.592 & 72.207 \\
\hline 7/25/07 3:00 & 122.779 & 120.426 & 117.208 & 98.215 & 102.402 & 93.839 & 108.095 & 104.807 & 76.583 & 72.201 \\
\hline $7 / 25 / 07$ 3:30 & 122.781 & 120.429 & 117.208 & 98.211 & 102.398 & 93.839 & 108.094 & 104.807 & 76.576 & 72.199 \\
\hline 7/25/07 4:00 & 122.779 & 120.426 & 117.208 & 98.213 & 102.402 & 93.841 & 108.094 & 104.805 & 76.572 & 72.201 \\
\hline $7 / 25 / 074: 30$ & 122.779 & 120.426 & 117.208 & 98.216 & 102.402 & 93.841 & 108.095 & 104.805 & 76.566 & 72.201 \\
\hline 7/25/07 5:00 & 122.779 & 120.426 & 117.208 & 98.213 & 102.4 & 93.841 & 108.093 & 104.805 & 76.563 & 72.199 \\
\hline 7/25/07 5:30 & 122.779 & 120.426 & 117.208 & 98.213 & 102.4 & 93.843 & 108.095 & 104.807 & 76.557 & 72.199 \\
\hline $7 / 25 / 076: 00$ & 122.779 & 122.54 & 117.206 & 98.214 & 102.4 & 93.841 & 108.095 & 104.805 & 76.553 & 72.199 \\
\hline $7 / 25 / 076: 30$ & 122.779 & 122.798 & 117.208 & 98.214 & 102.4 & 93.841 & 108.095 & 104.805 & 76.548 & 72.195 \\
\hline 7/25/07 7:00 & 124.934 & 122.941 & 118.688 & 99.014 & 102.979 & 94.569 & 108.809 & 106.394 & 76.572 & 72.298 \\
\hline $7 / 25 / 077: 30$ & 125.211 & 123.025 & 118.959 & 99.24 & 103.228 & 94.819 & 109.043 & 106.686 & 76.596 & 72.49 \\
\hline 7/25/07 8:00 & 125.355 & 123.088 & 119.1 & 99.369 & 103.363 & 94.956 & 109.169 & 106.837 & 76.62 & 72.623 \\
\hline $7 / 25 / 078: 30$ & 125.447 & 123.12 & 119.19 & 99.45 & 103.451 & 95.045 & 109.252 & 106.93 & 76.642 & 72.716 \\
\hline 7/25/07 9:00 & 125.508 & 123.147 & 119.25 & 99.507 & 103.512 & 95.104 & 109.308 & 106.996 & 76.666 & 72.78 \\
\hline 7/25/07 9:30 & 125.541 & 123.165 & 119.287 & 99.539 & 103.546 & 95.14 & 109.34 & 107.032 & 76.684 & 72.819 \\
\hline $7 / 25 / 07$ 10:00 & 125.563 & 123.181 & 119.315 & 99.565 & 103.575 & 95.165 & 109.364 & 107.058 & 76.699 & 72.848 \\
\hline $7 / 25 / 07 \quad 10: 30$ & 125.582 & 121.256 & 119.334 & 99.584 & 103.59 & 95.187 & 109.383 & 107.078 & 76.716 & 72.871 \\
\hline $7 / 25 / 07 \quad 11: 00$ & 125.598 & 120.927 & 119.352 & 99.6 & 103.609 & 95.203 & 109.398 & 107.096 & 76.734 & 72.889 \\
\hline $7 / 25 / 07$ 11:30 & 123.653 & 120.767 & 118.071 & 98.926 & 103.175 & 94.619 & 108.822 & 105.721 & 76.734 & 72.854 \\
\hline $7 / 25 / 07$ 12:00 & 123.294 & 120.667 & 117.715 & 98.652 & 102.868 & 94.305 & 108.529 & 105.334 & 76.718 & 72.683 \\
\hline $7 / 25 / 07 \quad 12: 30$ & 123.126 & 120.601 & 117.548 & 98.511 & 102.716 & 94.152 & 108.386 & 105.161 & 76.697 & 72.546 \\
\hline $7 / 25 / 07$ 13:00 & 123.022 & 120.56 & 117.449 & 98.421 & 102.619 & 94.058 & 108.298 & 105.053 & 76.682 & 72.449 \\
\hline $7 / 25 / 07$ 13:30 & 122.961 & 120.528 & 117.386 & 98.366 & 102.562 & 94.001 & 108.245 & 104.986 & 76.67 & 72.385 \\
\hline $7 / 25 / 07$ 14:00 & 122.918 & 120.501 & 117.343 & 98.328 & 102.52 & 93.963 & 108.208 & 104.942 & 76.66 & 72.342 \\
\hline
\end{tabular}


TABLE S1.1 (Cont.)

Depth from Top of Casing (ft)

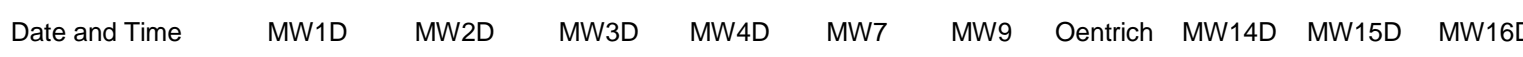

\begin{tabular}{|c|c|c|c|c|c|c|c|c|c|c|}
\hline $7 / 25 / 07$ 14:30 & 122.885 & 120.479 & 117.312 & 98.301 & 102.491 & 93.935 & 108.182 & 104.911 & 76.647 & 72.309 \\
\hline $7 / 25 / 07$ 15:00 & 122.857 & 120.456 & 117.285 & 98.275 & 102.467 & 93.907 & 108.157 & 104.882 & 76.635 & 72.28 \\
\hline $7 / 25 / 07$ 15:30 & 122.831 & 120.442 & 117.259 & 98.254 & 102.444 & 93.883 & 108.135 & 104.858 & 76.622 & 72.255 \\
\hline $7 / 25 / 07$ 16:00 & 122.809 & 120.431 & 117.241 & 98.235 & 102.423 & 93.862 & 108.116 & 104.836 & 76.612 & 72.234 \\
\hline $7 / 25 / 07$ 16:30 & 122.795 & 120.438 & 117.225 & 98.221 & 102.411 & 93.848 & 108.103 & 104.822 & 76.6 & 72.22 \\
\hline $7 / 25 / 07$ 17:00 & 122.783 & 120.492 & 117.216 & 98.211 & 102.4 & 93.839 & 108.093 & 104.811 & 76.588 & 72.209 \\
\hline $7 / 25 / 07$ 17:30 & 122.774 & 120.504 & 117.204 & 98.299 & 102.392 & 93.829 & 108.086 & 104.802 & 76.581 & 72.199 \\
\hline $7 / 25 / 07$ 18:00 & 122.852 & 120.515 & 117.296 & 98.351 & 102.491 & 93.966 & 109.142 & 104.862 & 76.588 & 72.199 \\
\hline $7 / 25 / 07$ 18:30 & 122.864 & 120.513 & 117.31 & 98.364 & 102.507 & 93.98 & 109.163 & 104.878 & 76.592 & 72.211 \\
\hline 7/25/07 19:00 & 122.876 & 120.438 & 117.319 & 98.375 & 102.52 & 93.992 & 109.172 & 104.889 & 76.594 & 72.226 \\
\hline $7 / 25 / 07$ 19:30 & 122.873 & 120.422 & 117.319 & 98.375 & 102.518 & 93.992 & 109.168 & 104.886 & 76.594 & 72.228 \\
\hline $7 / 25 / 0720: 00$ & 122.79 & 120.411 & 117.222 & 98.214 & 102.415 & 93.848 & 108.567 & 104.82 & 76.579 & 72.218 \\
\hline $7 / 25 / 0720: 30$ & 122.772 & 120.406 & 117.201 & 98.199 & 102.392 & 93.827 & 108.073 & 104.8 & 76.57 & 72.203 \\
\hline $7 / 25 / 0721: 00$ & 122.76 & 120.408 & 117.188 & 98.189 & 102.379 & 93.815 & 108.063 & 104.787 & 76.563 & 72.191 \\
\hline $7 / 25 / 0721: 30$ & 122.762 & 120.413 & 117.195 & 98.194 & 102.389 & 93.846 & 108.078 & 104.785 & 76.559 & 72.187 \\
\hline $7 / 25 / 07$ 22:00 & 122.765 & 120.415 & 117.199 & 98.209 & 102.394 & 93.855 & 108.071 & 104.787 & 76.557 & 72.191 \\
\hline 7/25/07 22:30 & 122.767 & 120.413 & 117.199 & 98.204 & 102.391 & 93.829 & 108.052 & 104.791 & 76.553 & 72.193 \\
\hline 7/25/07 23:00 & 122.762 & 120.408 & 117.192 & 98.206 & 102.387 & 93.824 & 108.065 & 104.791 & 76.552 & 72.193 \\
\hline 7/25/07 23:30 & 122.765 & 120.399 & 117.192 & 98.194 & 102.383 & 93.82 & 108.059 & 104.791 & 76.548 & 72.187 \\
\hline 7/26/07 0:00 & 122.76 & 120.397 & 117.19 & 98.187 & 102.379 & 93.813 & 108.045 & 104.785 & 76.541 & 72.182 \\
\hline 7/26/07 0:30 & 122.755 & 120.397 & 117.188 & 98.18 & 102.383 & 93.841 & 108.066 & 104.778 & 76.537 & 72.176 \\
\hline 7/26/07 1:00 & 122.748 & 120.397 & 117.178 & 98.187 & 102.37 & 93.806 & 108.041 & 104.776 & 76.533 & 72.174 \\
\hline 7/26/07 1:30 & 122.746 & 120.388 & 117.176 & 98.194 & 102.368 & 93.803 & 108.038 & 104.772 & 76.529 & 72.172 \\
\hline 7/26/07 2:00 & 122.748 & 120.383 & 117.183 & 98.177 & 102.372 & 93.824 & 108.017 & 104.774 & 76.526 & 72.17 \\
\hline 7/26/07 2:30 & 122.739 & 120.377 & 117.171 & 98.171 & 102.372 & 93.803 & 108.65 & 104.765 & 76.52 & 72.166 \\
\hline 7/26/07 3:00 & 122.731 & 120.377 & 117.162 & 98.171 & 102.354 & 93.791 & 108.029 & 104.761 & 76.517 & 72.16 \\
\hline 7/26/07 3:30 & 122.729 & 120.379 & 117.158 & 98.168 & 102.349 & 93.782 & 108.022 & 104.754 & 76.509 & 72.151 \\
\hline 7/26/07 4:00 & 122.729 & 120.374 & 117.158 & 98.19 & 102.35 & 93.782 & 108.016 & 104.754 & 76.507 & 72.151 \\
\hline $7 / 26 / 074: 30$ & 122.729 & 120.381 & 117.16 & 98.19 & 102.352 & 93.787 & 108.026 & 104.756 & 76.504 & 72.158 \\
\hline 7/26/07 5:00 & 122.727 & 120.386 & 117.155 & 98.197 & 102.349 & 93.784 & 108.02 & 104.751 & 76.5 & 72.153 \\
\hline 7/26/07 5:30 & 122.731 & 120.395 & 117.162 & 98.189 & 102.356 & 93.791 & 108.023 & 104.761 & 76.496 & 72.16 \\
\hline 7/26/07 6:00 & 122.736 & 122.329 & 117.167 & 98.171 & 102.36 & 93.796 & 108.048 & 104.763 & 76.498 & 72.162 \\
\hline $7 / 26 / 076: 30$ & 122.741 & 122.712 & 117.174 & 98.182 & 102.368 & 93.803 & 108.058 & 104.769 & 76.498 & 72.168 \\
\hline 7/26/07 7:00 & 124.691 & 122.884 & 118.452 & 98.828 & 102.769 & 94.35 & 108.598 & 106.12 & 76.511 & 72.195 \\
\hline $7 / 26 / 077: 30$ & 125.121 & 122.984 & 118.869 & 99.145 & 103.129 & 94.727 & 108.937 & 106.584 & 76.541 & 72.395 \\
\hline 7/26/07 8:00 & 125.296 & 123.045 & 119.031 & 99.302 & 103.295 & 94.887 & 109.11 & 106.773 & 76.574 & 72.555 \\
\hline 7/26/07 8:30 & 125.4 & 123.09 & 119.13 & 99.395 & 103.394 & 94.986 & 109.192 & 106.879 & 76.6 & 72.66 \\
\hline 7/26/07 9:00 & 125.461 & 123.12 & 119.199 & 99.453 & 103.459 & 95.05 & 109.258 & 106.948 & 76.623 & 72.726 \\
\hline 7/26/07 9:30 & 125.506 & 123.152 & 119.243 & 99.496 & 103.512 & 95.095 & 109.746 & 106.992 & 76.647 & 72.776 \\
\hline 7/26/07 10:00 & 125.539 & 123.152 & 119.278 & 99.529 & 103.535 & 95.125 & 109.328 & 107.027 & 76.666 & 72.811 \\
\hline $7 / 26 / 07 \quad 10: 30$ & 125.57 & 123.165 & 119.313 & 99.553 & 103.573 & 95.161 & 109.785 & 107.058 & 76.688 & 72.84 \\
\hline $7 / 26 / 0711: 00$ & 125.572 & 123.17 & 119.322 & 99.584 & 103.569 & 95.161 & 109.358 & 107.065 & 76.703 & 72.852 \\
\hline $7 / 26 / 07$ 11:30 & 125.584 & 123.165 & 119.331 & 99.572 & 103.582 & 95.177 & 109.382 & 107.076 & 76.721 & 72.873 \\
\hline $7 / 26 / 07$ 12:00 & 125.587 & 123.163 & 119.343 & 99.576 & 103.584 & 95.182 & 109.388 & 107.08 & 76.734 & 72.875 \\
\hline $7 / 26 / 07$ 12:30 & 125.584 & 123.161 & 119.343 & 99.577 & 103.586 & 95.182 & 109.376 & 107.08 & 76.747 & 72.879 \\
\hline $7 / 26 / 07$ 13:00 & 125.582 & 123.168 & 119.34 & 99.577 & 103.586 & 95.18 & 109.387 & 107.078 & 76.754 & 72.885 \\
\hline $7 / 26 / 07$ 13:30 & 125.582 & 123.168 & 119.34 & 99.579 & 103.588 & 95.182 & 109.392 & 107.078 & 76.767 & 72.885 \\
\hline $7 / 26 / 07$ 14:00 & 125.586 & 121.209 & 119.34 & 99.586 & 103.596 & 95.189 & 109.399 & 107.083 & 76.771 & 72.898 \\
\hline $7 / 26 / 07$ 14:30 & 125.589 & 120.893 & 119.343 & 99.588 & 103.596 & 95.189 & 109.399 & 107.085 & 76.778 & 72.896 \\
\hline $7 / 26 / 07$ 15:00 & 123.599 & 120.737 & 118.018 & 98.878 & 103.125 & 94.567 & 108.783 & 105.661 & 76.769 & 72.844 \\
\hline $7 / 26 / 07$ 15:30 & 123.258 & 120.646 & 117.682 & 98.614 & 102.826 & 94.265 & 108.502 & 105.296 & 76.747 & 72.664 \\
\hline $7 / 26 / 07$ 16:00 & 123.095 & 120.578 & 117.521 & 98.478 & 102.682 & 94.119 & 108.361 & 105.128 & 76.725 & 72.53 \\
\hline $7 / 26 / 07$ 16:30 & 123.003 & 120.535 & 117.43 & 98.401 & 102.598 & 94.037 & 108.283 & 105.035 & 76.708 & 72.439 \\
\hline $7 / 26 / 07$ 17:00 & 122.935 & 120.519 & 117.363 & 98.34 & 102.535 & 93.973 & 108.222 & 104.964 & 76.692 & 72.371 \\
\hline $7 / 26 / 07$ 17:30 & 122.887 & 120.476 & 117.317 & 98.301 & 102.491 & 93.93 & 108.183 & 104.915 & 76.677 & 72.323 \\
\hline 7/26/07 18:00 & 122.88 & 120.449 & 117.303 & 98.285 & 102.48 & 93.919 & 108.171 & 104.911 & 76.664 & 72.294 \\
\hline $7 / 26 / 07$ 18:30 & 122.835 & 120.426 & 117.264 & 98.247 & 102.442 & 93.881 & 108.137 & 104.86 & 76.651 & 72.265 \\
\hline 7/26/07 19:00 & 122.802 & 120.408 & 117.232 & 98.223 & 102.411 & 93.853 & 108.109 & 104.829 & 76.635 & 72.236 \\
\hline 7/26/07 19:30 & 122.781 & 120.395 & 117.211 & 98.202 & 102.392 & 93.829 & 108.092 & 104.807 & 76.622 & 72.213 \\
\hline $7 / 26 / 07$ 20:00 & 122.762 & 120.39 & 117.192 & 98.189 & 102.373 & 93.815 & 108.075 & 104.787 & 76.611 & 72.197 \\
\hline $7 / 26 / 0720: 30$ & 122.748 & 120.399 & 117.181 & 98.173 & 102.36 & 93.803 & 108.064 & 104.774 & 76.6 & 72.184 \\
\hline $7 / 26 / 0721: 00$ & 122.741 & 120.413 & 117.174 & 98.168 & 102.356 & 93.796 & 108.056 & 104.767 & 76.592 & 72.176 \\
\hline $7 / 26 / 0721: 30$ & 122.75 & 120.424 & 117.181 & 98.182 & 102.37 & 93.806 & 108.065 & 104.774 & 76.59 & 72.187 \\
\hline $7 / 26 / 0722: 00$ & 122.762 & 120.429 & 117.195 & 98.197 & 102.383 & 93.822 & 108.08 & 104.789 & 76.588 & 72.201 \\
\hline 7/26/07 22:30 & 122.774 & 120.424 & 117.211 & 98.211 & 102.398 & 93.836 & 108.088 & 104.802 & 76.588 & 72.213 \\
\hline 7/26/07 23:00 & 122.776 & 120.424 & 117.208 & 98.213 & 102.4 & 93.836 & 108.086 & 104.805 & 76.581 & 72.211 \\
\hline
\end{tabular}


TABLE S1.1 (Cont.)

Depth from Top of Casing (ft)

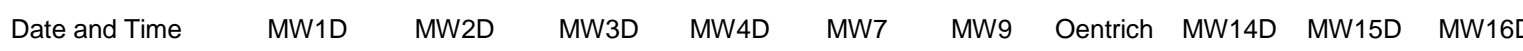

\begin{tabular}{|c|c|c|c|c|c|c|c|c|c|c|}
\hline 7/26/07 23:30 & 122.774 & 120.42 & 117.208 & 98.206 & 102.394 & 93.834 & 108.084 & 104.8 & 76.577 & 72.205 \\
\hline 7/27/07 0:00 & 122.772 & 120.411 & 117.206 & 98.209 & 102.396 & 93.834 & 108.086 & 104.8 & 76.574 & 72.207 \\
\hline 7/27/07 0:30 & 122.767 & 120.408 & 117.202 & 98.204 & 102.391 & 93.829 & 108.08 & 104.793 & 76.568 & 72.199 \\
\hline 7/27/07 1:00 & 122.76 & 120.402 & 117.195 & 98.192 & 102.379 & 93.822 & 108.076 & 104.787 & 76.561 & 72.193 \\
\hline 7/27/07 1:30 & 122.755 & 120.404 & 117.19 & 98.195 & 102.381 & 93.817 & 108.072 & 104.783 & 76.555 & 72.189 \\
\hline 7/27/07 2:00 & 122.75 & 120.404 & 117.185 & 98.189 & 102.375 & 93.815 & 108.068 & 104.778 & 76.548 & 72.187 \\
\hline $7 / 27 / 072: 30$ & 122.75 & 120.413 & 117.185 & 98.191 & 102.375 & 93.815 & 108.07 & 104.778 & 76.546 & 72.187 \\
\hline 7/27/07 3:00 & 122.75 & 120.411 & 117.185 & 98.191 & 102.375 & 93.813 & 108.072 & 104.778 & 76.542 & 72.184 \\
\hline 7/27/07 3:30 & 122.76 & 120.402 & 117.197 & 98.201 & 102.387 & 93.827 & 108.078 & 104.787 & 76.541 & 72.195 \\
\hline 7/27/07 4:00 & 122.762 & 120.392 & 117.195 & 98.199 & 102.387 & 93.829 & 108.079 & 104.787 & 76.539 & 72.195 \\
\hline 7/27/07 4:30 & 122.76 & 120.392 & 117.188 & 98.189 & 102.377 & 93.815 & 108.072 & 104.781 & 76.533 & 72.187 \\
\hline $7 / 27 / 075: 00$ & 122.757 & 120.392 & 117.178 & 98.183 & 102.369 & 93.808 & 108.064 & 104.771 & 76.528 & 72.178 \\
\hline 7/27/07 5:30 & 122.757 & 120.39 & 117.178 & 98.182 & 102.368 & 93.806 & 108.064 & 104.769 & 76.522 & 72.174 \\
\hline 7/27/07 6:00 & 122.755 & 122.356 & 117.176 & 98.18 & 102.368 & 93.806 & 108.064 & 104.769 & 76.518 & 72.176 \\
\hline 7/27/07 6:30 & 122.755 & 122.671 & 117.174 & 98.18 & 102.368 & 93.803 & 108.06 & 104.765 & 76.518 & 72.17 \\
\hline 7/27/07 7:00 & 124.766 & 122.832 & 118.656 & 98.936 & 102.87 & 94.472 & 108.723 & 106.188 & 76.531 & 72.226 \\
\hline 7/27/07 7:30 & 125.104 & 122.925 & 118.984 & 99.203 & 103.165 & 94.769 & 109.003 & 106.549 & 76.557 & 72.42 \\
\hline 7/27/07 8:00 & 125.274 & 122.991 & 119.149 & 99.352 & 103.325 & 94.93 & 109.151 & 106.724 & 76.587 & 72.573 \\
\hline 7/27/07 8:30 & 125.371 & 123.038 & 119.246 & 99.438 & 103.415 & 95.022 & 109.237 & 106.821 & 76.611 & 72.668 \\
\hline 7/27/07 9:00 & 125.442 & 123.068 & 119.317 & 99.5 & 103.485 & 95.09 & 109.301 & 106.897 & 76.636 & 72.743 \\
\hline 7/27/07 9:30 & 125.487 & 123.095 & 119.364 & 99.545 & 103.529 & 95.135 & 109.342 & 106.945 & 76.658 & 72.788 \\
\hline $7 / 27 / 07$ 10:00 & 125.52 & 121.739 & 119.396 & 99.574 & 103.561 & 95.168 & 109.374 & 106.979 & 76.681 & 72.823 \\
\hline $7 / 27 / 07$ 10:30 & 125.546 & 121.021 & 119.421 & 99.598 & 103.588 & 95.194 & 109.397 & 107.005 & 76.701 & 72.852 \\
\hline $7 / 27 / 0711: 00$ & 124.485 & 120.814 & 118.885 & 99.212 & 103.552 & 95.099 & 109.275 & 106.85 & 76.721 & 72.879 \\
\hline 7/27/07 11:30 & 123.398 & 120.696 & 117.819 & 98.73 & 102.956 & 94.397 & 108.612 & 105.438 & 76.71 & 72.749 \\
\hline $7 / 27 / 07 \quad 12: 00$ & 123.178 & 120.626 & 117.602 & 98.556 & 102.762 & 94.201 & 108.43 & 105.212 & 76.697 & 72.6 \\
\hline $7 / 27 / 07$ 12:30 & 123.058 & 120.581 & 117.484 & 98.454 & 102.653 & 94.095 & 108.328 & 105.088 & 76.686 & 72.492 \\
\hline 7/27/07 13:00 & 122.984 & 120.547 & 117.412 & 98.394 & 102.587 & 94.029 & 108.266 & 105.013 & 76.677 & 72.42 \\
\hline $7 / 27 / 07$ 13:30 & 122.937 & 120.522 & 117.368 & 98.351 & 102.543 & 93.985 & 108.229 & 104.964 & 76.668 & 72.373 \\
\hline $7 / 27 / 07$ 14:00 & 122.902 & 120.501 & 117.333 & 98.321 & 102.51 & 93.956 & 108.197 & 104.929 & 76.66 & 72.337 \\
\hline 7/27/07 14:30 & 122.873 & 120.483 & 117.306 & 98.297 & 102.488 & 93.93 & 108.174 & 104.9 & 76.653 & 72.311 \\
\hline $7 / 27 / 07$ 15:00 & 122.854 & 120.465 & 117.285 & 98.28 & 102.467 & 93.912 & 108.155 & 104.88 & 76.644 & 72.288 \\
\hline $7 / 27 / 07$ 15:30 & 122.838 & 120.451 & 117.269 & 98.266 & 102.451 & 93.893 & 108.14 & 104.862 & 76.635 & 72.271 \\
\hline $7 / 27 / 07$ 16:00 & 122.824 & 120.436 & 117.255 & 98.249 & 102.438 & 93.879 & 108.127 & 104.849 & 76.623 & 72.253 \\
\hline $7 / 27 / 07$ 16:30 & 122.807 & 120.431 & 117.241 & 98.239 & 102.423 & 93.864 & 108.112 & 104.831 & 76.616 & 72.24 \\
\hline 7/27/07 17:00 & 122.79 & 120.42 & 117.225 & 98.223 & 102.409 & 93.853 & 108.105 & 104.816 & 76.607 & 72.228 \\
\hline 7/27/07 17:30 & 122.783 & 120.413 & 117.218 & 98.216 & 102.402 & 93.846 & 108.095 & 104.809 & 76.6 & 72.22 \\
\hline $7 / 27 / 07$ 18:00 & 122.772 & 120.402 & 117.206 & 98.209 & 102.392 & 93.834 & 108.088 & 104.798 & 76.594 & 72.211 \\
\hline $7 / 27 / 07$ 18:30 & 122.762 & 120.397 & 117.197 & 98.199 & 102.387 & 93.827 & 108.078 & 104.789 & 76.587 & 72.201 \\
\hline $7 / 27 / 07$ 19:00 & 122.753 & 120.399 & 117.19 & 98.19 & 102.375 & 93.817 & 108.069 & 104.778 & 76.579 & 72.193 \\
\hline $7 / 27 / 07 \quad 19: 30$ & 122.748 & 120.408 & 117.183 & 98.187 & 102.372 & 93.815 & 108.067 & 104.774 & 76.574 & 72.187 \\
\hline $7 / 27 / 0720: 00$ & 122.75 & 120.408 & 117.185 & 98.19 & 102.373 & 93.815 & 108.069 & 104.776 & 76.572 & 72.191 \\
\hline $7 / 27 / 0720: 30$ & 122.755 & 120.411 & 117.192 & 98.197 & 102.381 & 93.822 & 108.074 & 104.782 & 76.568 & 72.195 \\
\hline $7 / 27 / 0721: 00$ & 122.757 & 120.42 & 117.195 & 98.199 & 102.385 & 93.822 & 108.076 & 104.785 & 76.566 & 72.195 \\
\hline $7 / 27 / 0721: 30$ & 122.76 & 120.426 & 117.195 & 98.202 & 102.387 & 93.824 & 108.08 & 104.785 & 76.565 & 72.199 \\
\hline $7 / 27 / 07$ 22:00 & 122.769 & 120.431 & 117.206 & 98.214 & 102.394 & 93.836 & 108.086 & 104.796 & 76.566 & 72.205 \\
\hline $7 / 27 / 0722: 30$ & 122.776 & 120.424 & 117.213 & 98.221 & 102.406 & 93.846 & 108.093 & 104.803 & 76.57 & 72.215 \\
\hline $7 / 27 / 07$ 23:00 & 122.783 & 120.424 & 117.22 & 98.225 & 102.411 & 93.853 & 108.099 & 104.809 & 76.568 & 72.22 \\
\hline 7/27/07 23:30 & 122.774 & 120.415 & 117.211 & 98.216 & 102.402 & 93.843 & 108.091 & 104.8 & 76.565 & 72.211 \\
\hline $7 / 28 / 07$ 0:00 & 122.772 & 120.408 & 117.208 & 98.216 & 102.402 & 93.841 & 108.089 & 104.798 & 76.563 & 72.209 \\
\hline 7/28/07 0:30 & 122.767 & 120.404 & 117.204 & 98.211 & 102.392 & 93.836 & 108.085 & 104.794 & 76.561 & 72.203 \\
\hline 7/28/07 1:00 & 122.76 & 120.395 & 117.197 & 98.201 & 102.387 & 93.829 & 108.078 & 104.787 & 76.553 & 72.199 \\
\hline 7/28/07 1:30 & 122.755 & 120.392 & 117.19 & 98.195 & 102.381 & 93.822 & 108.074 & 104.78 & 76.55 & 72.193 \\
\hline $7 / 28 / 07$ 2:00 & 122.75 & 120.399 & 117.183 & 98.19 & 102.375 & 93.817 & 108.067 & 104.774 & 76.546 & 72.187 \\
\hline $7 / 28 / 07$ 2:30 & 122.746 & 120.402 & 117.183 & 98.189 & 102.375 & 93.815 & 108.063 & 104.769 & 76.542 & 72.184 \\
\hline 7/28/07 3:00 & 122.75 & 120.402 & 117.188 & 98.195 & 102.383 & 93.822 & 108.07 & 104.776 & 76.542 & 72.191 \\
\hline 7/28/07 3:30 & 122.75 & 120.392 & 117.188 & 98.197 & 102.381 & 93.82 & 108.072 & 104.778 & 76.541 & 72.191 \\
\hline 7/28/07 4:00 & 122.755 & 120.395 & 117.19 & 98.197 & 102.383 & 93.824 & 108.072 & 104.781 & 76.541 & 72.193 \\
\hline $7 / 28 / 074: 30$ & 122.748 & 120.395 & 117.183 & 98.19 & 102.375 & 93.817 & 108.067 & 104.774 & 76.535 & 72.187 \\
\hline $7 / 28 / 075: 00$ & 122.746 & 120.39 & 117.181 & 98.19 & 102.373 & 93.815 & 108.063 & 104.772 & 76.533 & 72.182 \\
\hline $7 / 28 / 07$ 5:30 & 122.746 & 120.397 & 117.183 & 98.189 & 102.375 & 93.817 & 108.065 & 104.771 & 76.529 & 72.187 \\
\hline 7/28/07 6:00 & 122.741 & 120.395 & 117.178 & 98.187 & 102.372 & 93.813 & 108.061 & 104.767 & 76.528 & 72.18 \\
\hline $7 / 28 / 076: 30$ & 122.748 & 122.499 & 117.185 & 98.192 & 102.377 & 93.817 & 108.068 & 104.776 & 76.531 & 72.189 \\
\hline $7 / 28 / 07$ 7:00 & 122.748 & 122.778 & 117.183 & 98.19 & 102.375 & 93.817 & 108.063 & 104.771 & 76.526 & 72.182 \\
\hline 7/28/07 7:30 & 124.887 & 122.927 & 118.647 & 98.976 & 102.929 & 94.524 & 108.757 & 106.343 & 76.548 & 72.273 \\
\hline 7/28/07 8:00 & 125.187 & 123.018 & 118.936 & 99.217 & 103.196 & 94.795 & 109.011 & 106.658 & 76.577 & 72.476 \\
\hline
\end{tabular}


TABLE S1.1 (Cont.)

Depth from Top of Casing (ft)

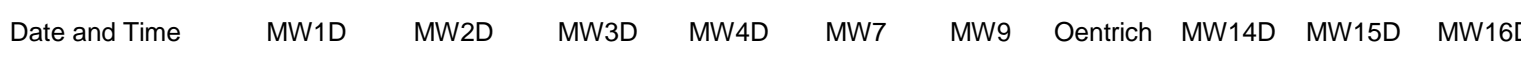

\begin{tabular}{|c|c|c|c|c|c|c|c|c|c|c|}
\hline 7/28/07 8:30 & 125.341 & 123.086 & 119.086 & 99.352 & 103.341 & 94.937 & 109.142 & 106.815 & 76.609 & 72.614 \\
\hline 7/28/07 9:00 & 125.435 & 123.122 & 119.181 & 99.439 & 103.434 & 95.031 & 109.23 & 106.917 & 76.635 & 72.712 \\
\hline 7/28/07 9:30 & 125.506 & 123.156 & 119.253 & 99.501 & 103.504 & 95.097 & 109.292 & 106.992 & 76.664 & 72.782 \\
\hline 7/28/07 10:00 & 125.544 & 123.181 & 119.29 & 99.538 & 103.54 & 95.135 & 109.328 & 107.029 & 76.688 & 72.827 \\
\hline $7 / 28 / 07$ 10:30 & 125.577 & 123.195 & 119.324 & 99.57 & 103.573 & 95.17 & 109.36 & 107.065 & 76.708 & 72.86 \\
\hline $7 / 28 / 07$ 11:00 & 125.601 & 123.197 & 119.347 & 99.593 & 103.596 & 95.196 & 109.382 & 107.089 & 76.73 & 72.891 \\
\hline $7 / 28 / 07$ 11:30 & 125.619 & 123.206 & 119.366 & 99.608 & 103.615 & 95.213 & 109.397 & 107.107 & 76.749 & 72.908 \\
\hline $7 / 28 / 07$ 12:00 & 125.622 & 123.204 & 119.371 & 99.608 & 103.618 & 95.215 & 109.405 & 107.114 & 76.765 & 72.914 \\
\hline $7 / 28 / 07$ 12:30 & 125.627 & 123.199 & 119.377 & 99.619 & 103.624 & 95.222 & 109.413 & 107.118 & 76.78 & 72.927 \\
\hline 7/28/07 13:00 & 125.627 & 123.202 & 119.377 & 99.62 & 103.628 & 95.222 & 109.412 & 107.118 & 76.795 & 72.931 \\
\hline 7/28/07 13:30 & 125.622 & 123.217 & 119.375 & 99.619 & 103.626 & 95.222 & 109.414 & 107.116 & 76.808 & 72.935 \\
\hline $7 / 28 / 07$ 14:00 & 125.624 & 121.195 & 119.38 & 99.624 & 103.63 & 95.229 & 109.416 & 107.118 & 76.821 & 72.943 \\
\hline 7/28/07 14:30 & 125.624 & 120.891 & 119.384 & 99.717 & 103.641 & 95.243 & 109.981 & 107.116 & 76.832 & 72.943 \\
\hline $7 / 28 / 07$ 15:00 & 123.587 & 120.739 & 118.027 & 98.876 & 103.158 & 94.633 & 109.717 & 105.622 & 76.834 & 72.848 \\
\hline 7/28/07 15:30 & 123.261 & 120.649 & 117.687 & 98.633 & 102.844 & 94.293 & 108.613 & 105.294 & 76.812 & 72.679 \\
\hline $7 / 28 / 07$ 16:00 & 123.098 & 120.587 & 117.525 & 98.483 & 102.686 & 94.126 & 108.348 & 105.133 & 76.791 & 72.546 \\
\hline $7 / 28 / 07$ 16:30 & 123.003 & 120.544 & 117.433 & 98.406 & 102.6 & 94.039 & 108.269 & 105.033 & 76.775 & 72.455 \\
\hline 7/28/07 17:00 & 122.944 & 120.51 & 117.375 & 98.356 & 102.549 & 93.992 & 108.233 & 104.973 & 76.762 & 72.395 \\
\hline 7/28/07 17:30 & 122.899 & 120.485 & 117.331 & 98.311 & 102.507 & 93.949 & 108.194 & 104.924 & 76.749 & 72.35 \\
\hline $7 / 28 / 07$ 18:00 & 122.866 & 120.472 & 117.296 & 98.28 & 102.472 & 93.914 & 108.135 & 104.891 & 76.738 & 72.317 \\
\hline 7/28/07 18:30 & 122.84 & 120.463 & 117.271 & 98.259 & 102.45 & 93.893 & 108.114 & 104.864 & 76.729 & 72.292 \\
\hline 7/28/07 19:00 & 122.831 & 120.456 & 117.266 & 98.251 & 102.451 & 93.912 & 108.141 & 104.851 & 76.721 & 72.28 \\
\hline $7 / 28 / 07$ 19:30 & 122.816 & 120.454 & 117.248 & 98.242 & 102.432 & 93.874 & 108.101 & 104.843 & 76.712 & 72.267 \\
\hline $7 / 28 / 07$ 20:00 & 122.807 & 120.449 & 117.241 & 98.235 & 102.425 & 93.864 & 108.102 & 104.833 & 76.706 & 72.261 \\
\hline $7 / 28 / 07$ 20:30 & 122.805 & 120.458 & 117.236 & 98.233 & 102.423 & 93.862 & 108.095 & 104.831 & 76.701 & 72.255 \\
\hline $7 / 28 / 0721: 00$ & 122.802 & 120.465 & 117.234 & 98.268 & 102.425 & 93.86 & 108.668 & 104.827 & 76.695 & 72.255 \\
\hline $7 / 28 / 0721: 30$ & 122.809 & 120.472 & 117.243 & 98.245 & 102.431 & 93.872 & 108.128 & 104.833 & 76.697 & 72.263 \\
\hline 7/28/07 22:00 & 122.814 & 120.465 & 117.25 & 98.252 & 102.436 & 93.879 & 108.132 & 104.84 & 76.697 & 72.267 \\
\hline 7/28/07 22:30 & 122.824 & 120.458 & 117.259 & 98.259 & 102.448 & 93.888 & 108.114 & 104.849 & 76.695 & 72.273 \\
\hline 7/28/07 23:00 & 122.821 & 120.447 & 117.259 & 98.252 & 102.446 & 93.909 & 108.116 & 104.842 & 76.69 & 72.267 \\
\hline 7/28/07 23:30 & 122.809 & 120.451 & 117.245 & 98.249 & 102.432 & 93.874 & 108.105 & 104.836 & 76.684 & 72.259 \\
\hline 7/29/07 0:00 & 122.8 & 120.451 & 117.234 & 98.239 & 102.423 & 93.864 & 108.107 & 104.824 & 76.68 & 72.251 \\
\hline 7/29/07 0:30 & 122.805 & 120.422 & 117.238 & 98.245 & 102.427 & 93.869 & 108.121 & 104.827 & 76.677 & 72.251 \\
\hline 7/29/07 1:00 & 122.814 & 120.415 & 117.243 & 98.244 & 102.431 & 93.874 & 108.125 & 104.831 & 76.671 & 72.259 \\
\hline 7/29/07 1:30 & 122.826 & 120.415 & 117.208 & 98.213 & 102.4 & 93.841 & 108.1 & 104.8 & 76.64 & 72.228 \\
\hline 7/29/07 2:00 & 122.814 & 120.408 & 117.204 & 98.208 & 102.396 & 93.836 & 108.065 & 104.791 & 76.631 & 72.218 \\
\hline 7/29/07 2:30 & 122.795 & 120.406 & 117.201 & 98.245 & 102.394 & 93.834 & 108.089 & 104.791 & 76.629 & 72.22 \\
\hline 7/29/07 3:00 & 122.788 & 120.402 & 117.197 & 98.206 & 102.392 & 93.831 & 108.085 & 104.787 & 76.625 & 72.215 \\
\hline 7/29/07 3:30 & 122.781 & 120.399 & 117.192 & 98.204 & 102.389 & 93.827 & 108.066 & 104.785 & 76.62 & 72.213 \\
\hline 7/29/07 4:00 & 122.776 & 120.392 & 117.19 & 98.197 & 102.383 & 93.824 & 108.074 & 104.781 & 76.62 & 72.207 \\
\hline 7/29/07 4:30 & 122.769 & 120.381 & 117.188 & 98.195 & 102.381 & 93.82 & 108.063 & 104.778 & 76.612 & 72.205 \\
\hline 7/29/07 5:00 & 122.769 & 120.386 & 117.178 & 98.183 & 102.372 & 93.815 & 108.07 & 104.769 & 76.609 & 72.199 \\
\hline 7/29/07 5:30 & 122.765 & 120.397 & 117.171 & 98.18 & 102.366 & 93.808 & 108.063 & 104.761 & 76.603 & 72.191 \\
\hline $7 / 29 / 076: 00$ & 122.757 & 120.413 & 117.171 & 98.185 & 102.37 & 93.808 & 108.065 & 104.762 & 76.601 & 72.191 \\
\hline 7/29/07 6:30 & 122.76 & 120.404 & 117.185 & 98.195 & 102.381 & 93.82 & 108.071 & 104.776 & 76.605 & 72.207 \\
\hline 7/29/07 7:00 & 122.743 & 120.415 & 117.19 & 98.211 & 102.387 & 93.829 & 108.077 & 104.78 & 76.601 & 72.209 \\
\hline 7/29/07 7:30 & 122.776 & 121.93 & 117.192 & 98.201 & 102.391 & 93.836 & 108.084 & 104.787 & 76.587 & 72.218 \\
\hline 7/29/07 8:00 & 122.743 & 122.58 & 117.201 & 98.209 & 102.398 & 93.838 & 108.09 & 104.794 & 76.575 & 72.22 \\
\hline 7/29/07 8:30 & 124.012 & 122.796 & 118.057 & 98.669 & 102.514 & 94.055 & 108.326 & 105.274 & 76.575 & 72.215 \\
\hline 7/29/07 9:00 & 124.91 & 122.911 & 118.892 & 99.138 & 103.087 & 94.694 & 108.92 & 106.448 & 76.603 & 72.377 \\
\hline 7/29/07 9:30 & 125.135 & 122.986 & 119.118 & 99.326 & 103.297 & 94.906 & 109.114 & 106.689 & 76.635 & 72.561 \\
\hline 7/29/07 10:00 & 125.274 & 123.045 & 119.236 & 99.433 & 103.409 & 95.017 & 109.217 & 106.812 & 76.657 & 72.674 \\
\hline $7 / 29 / 07$ 10:30 & 125.334 & 123.088 & 119.31 & 99.503 & 103.479 & 95.088 & 109.282 & 106.89 & 76.679 & 72.751 \\
\hline 7/29/07 11:00 & 125.404 & 123.109 & 119.373 & 99.557 & 103.54 & 95.149 & 109.333 & 106.954 & 76.697 & 72.815 \\
\hline 7/29/07 11:30 & 125.44 & 123.136 & 119.412 & 99.594 & 103.578 & 95.184 & 109.364 & 106.994 & 76.712 & 72.854 \\
\hline $7 / 29 / 07$ 12:00 & 125.494 & 123.159 & 119.433 & 99.613 & 103.599 & 95.208 & 109.392 & 107.021 & 76.729 & 72.883 \\
\hline 7/29/07 12:30 & 125.506 & 123.154 & 119.461 & 99.641 & 103.63 & 95.236 & 109.419 & 107.047 & 76.749 & 72.91 \\
\hline $7 / 29 / 07$ 13:00 & 125.537 & 123.165 & 119.488 & 99.662 & 103.653 & 95.262 & 109.437 & 107.074 & 76.769 & 72.939 \\
\hline 7/29/07 13:30 & 125.539 & 121.181 & 119.488 & 99.658 & 103.645 & 95.255 & 109.441 & 107.067 & 76.778 & 72.933 \\
\hline $7 / 29 / 07$ 14:00 & 125.553 & 120.903 & 119.495 & 99.67 & 103.66 & 95.269 & 109.454 & 107.083 & 76.791 & 72.951 \\
\hline 7/29/07 14:30 & 123.677 & 120.755 & 117.988 & 98.871 & 103.11 & 94.55 & 108.746 & 105.62 & 76.784 & 72.877 \\
\hline 7/29/07 15:00 & 123.398 & 120.664 & 117.696 & 98.637 & 102.853 & 94.289 & 108.498 & 105.307 & 76.769 & 72.705 \\
\hline $7 / 29 / 07$ 15:30 & 123.232 & 120.606 & 117.544 & 98.506 & 102.708 & 94.147 & 108.369 & 105.148 & 76.753 & 72.573 \\
\hline $7 / 29 / 07$ 16:00 & 123.138 & 120.565 & 117.451 & 98.43 & 102.625 & 94.065 & 108.292 & 105.053 & 76.738 & 72.482 \\
\hline $7 / 29 / 07$ 16:30 & 123.072 & 120.533 & 117.391 & 98.378 & 102.569 & 94.01 & 108.241 & 104.991 & 76.729 & 72.424 \\
\hline 7/29/07 17:00 & 123.024 & 120.506 & 117.352 & 98.342 & 102.531 & 93.971 & 108.203 & 104.949 & 76.716 & 72.379 \\
\hline
\end{tabular}


TABLE S1.1 (Cont.)

\begin{tabular}{|c|c|c|c|c|c|c|c|c|c|c|}
\hline \multirow[b]{2}{*}{ Date and Time } & \multicolumn{10}{|c|}{ Depth from Top of Casing (ft) } \\
\hline & MW1D & MW2D & MW3D & MW4D & MW7 & MW9 & Oentrich & MW14D & MW15D & MW16D \\
\hline 7/29/07 17:30 & 122.991 & 120.485 & & 98.311 & 102.499 & 93.94 & 108.175 & 104.916 & 76.705 & 72.346 \\
\hline $7 / 29 / 07$ 18:00 & 122.965 & 120.488 & & 98.289 & 102.478 & 93.916 & 108.154 & 104.889 & 76.694 & 72.319 \\
\hline 7/29/07 18:30 & 122.939 & 120.474 & & 98.271 & 102.461 & 93.9 & 108.137 & 104.867 & 76.684 & 72.301 \\
\hline 7/29/07 19:00 & 122.939 & 120.463 & & 98.266 & 102.469 & 93.897 & 108.688 & 104.869 & 76.68 & 72.29 \\
\hline 7/29/07 19:30 & 122.911 & 120.46 & & 98.254 & 102.446 & 93.883 & 108.113 & 104.851 & 76.673 & 72.286 \\
\hline 7/29/07 20:00 & 122.899 & 120.458 & & 98.257 & 102.44 & 93.878 & 108.102 & 104.844 & 76.67 & 72.28 \\
\hline $7 / 29 / 0720: 30$ & 122.89 & 120.456 & & 98.251 & 102.436 & 93.874 & 108.095 & 104.84 & 76.666 & 72.273 \\
\hline $7 / 29 / 0721: 00$ & 122.878 & 120.46 & & 98.244 & 102.434 & 93.869 & 108.097 & 104.836 & 76.662 & 72.269 \\
\hline 7/29/07 21:30 & 122.871 & 120.467 & & 98.249 & 102.436 & 93.876 & 108.09 & 104.838 & 76.659 & 72.269 \\
\hline $7 / 29 / 0722: 00$ & 122.861 & 120.467 & & 98.247 & 102.438 & 93.874 & 108.453 & 104.838 & 76.659 & 72.271 \\
\hline $7 / 29 / 0722: 30$ & 122.854 & 120.463 & & 98.259 & 102.444 & 93.883 & 108.131 & 104.842 & 76.66 & 72.273 \\
\hline 7/29/07 23:00 & 122.852 & 120.456 & & 98.259 & 102.444 & 93.886 & 108.129 & 104.844 & 76.659 & 72.278 \\
\hline 7/29/07 23:30 & 122.847 & 120.449 & & 98.256 & 102.44 & 93.883 & 108.126 & 104.84 & 76.657 & 72.273 \\
\hline $7 / 30 / 07$ 0:00 & 122.809 & 120.442 & & 98.249 & 102.436 & 93.876 & 108.114 & 104.836 & 76.651 & 72.267 \\
\hline 7/30/07 0:30 & 122.8 & 120.442 & & 98.242 & 102.427 & 93.869 & 108.104 & 104.827 & 76.647 & 72.259 \\
\hline 7/30/07 1:00 & 122.798 & 120.44 & & 98.239 & 102.425 & 93.864 & 108.102 & 104.822 & 76.644 & 72.255 \\
\hline 7/30/07 1:30 & 122.793 & 120.438 & & 98.239 & 102.423 & 93.864 & 108.102 & 104.82 & 76.642 & 72.255 \\
\hline 7/30/07 2:00 & 122.793 & 120.433 & & 98.237 & 102.423 & 93.864 & 108.098 & 104.818 & 76.636 & 72.251 \\
\hline 7/30/07 2:30 & 122.788 & 120.429 & & 98.233 & 102.417 & 93.857 & 108.092 & 104.813 & 76.631 & 72.247 \\
\hline 7/30/07 3:00 & 122.788 & 120.422 & & 98.228 & 102.413 & 93.855 & 108.09 & 104.809 & 76.629 & 72.244 \\
\hline 7/30/07 3:30 & 122.784 & 120.424 & & 98.225 & 102.411 & 93.853 & 108.088 & 104.809 & 76.625 & 72.242 \\
\hline 7/30/07 4:00 & 122.781 & 120.42 & & 98.218 & 102.402 & 93.843 & 108.078 & 104.798 & 76.618 & 72.232 \\
\hline 7/30/07 4:30 & 122.777 & 120.422 & & 98.221 & 102.406 & 93.846 & 108.083 & 104.8 & 76.614 & 72.236 \\
\hline 7/30/07 5:00 & 122.777 & 120.42 & & 98.216 & 102.402 & 93.843 & 108.078 & 104.798 & 76.612 & 72.232 \\
\hline $7 / 30 / 07$ 5:30 & 122.772 & 120.413 & & 98.221 & 102.406 & 93.846 & 108.081 & 104.798 & 76.611 & 72.237 \\
\hline 7/30/07 6:00 & 122.77 & 122.333 & & 98.22 & 102.404 & 93.845 & 108.079 & 104.798 & 76.605 & 72.234 \\
\hline 7/30/07 6:30 & 122.767 & 122.712 & & 98.214 & 102.398 & 93.841 & 108.076 & 104.793 & 76.601 & 72.228 \\
\hline 7/30/07 7:00 & 124.7 & 122.882 & & 98.867 & 102.806 & 94.39 & 108.618 & 106.137 & 76.614 & 72.261 \\
\hline 7/30/07 7:30 & 125.112 & 122.984 & & 99.179 & 103.154 & 94.748 & 108.952 & 106.589 & 76.644 & 72.468 \\
\hline $7 / 30 / 078: 00$ & 125.294 & 123.047 & & 99.334 & 103.323 & 94.92 & 109.108 & 106.777 & 76.675 & 72.627 \\
\hline 7/30/07 8:30 & 125.402 & 123.095 & & 99.431 & 103.428 & 95.022 & 109.202 & 106.89 & 76.697 & 72.734 \\
\hline 7/30/07 9:00 & 125.464 & 123.131 & & 99.489 & 103.491 & 95.085 & 109.26 & 106.959 & 76.719 & 72.803 \\
\hline 7/30/07 9:30 & 125.511 & 121.136 & & 99.534 & 103.54 & 95.132 & 109.301 & 107.008 & 76.738 & 72.854 \\
\hline $7 / 30 / 07$ 10:00 & 125.546 & 120.859 & & 99.567 & 103.571 & 95.163 & 109.333 & 107.043 & 76.764 & 72.891 \\
\hline 7/30/07 10:30 & 123.512 & 120.742 & & 98.916 & 103.087 & 94.557 & 109.56 & 105.544 & 76.76 & 72.823 \\
\hline 7/30/07 11:00 & 123.226 & 120.662 & & 98.604 & 102.817 & 94.251 & 108.43 & 105.261 & 76.75 & 72.668 \\
\hline 7/30/07 11:30 & 123.103 & 120.615 & & 98.499 & 102.701 & 94.138 & 108.355 & 105.13 & 76.743 & 72.563 \\
\hline 7/30/07 12:00 & 123.051 & 120.572 & & 98.43 & 102.627 & 94.067 & 108.265 & 105.051 & 76.736 & 72.484 \\
\hline 7/30/07 12:30 & 123.013 & 120.54 & & 98.39 & 102.581 & 94.018 & 108.255 & 104.997 & 76.732 & 72.433 \\
\hline 7/30/07 13:00 & 122.992 & 120.517 & & 98.351 & 102.543 & 93.98 & 108.219 & 104.953 & 76.721 & 72.389 \\
\hline 7/30/07 13:30 & 122.895 & 120.501 & & 98.323 & 102.512 & 93.952 & 108.185 & 104.92 & 76.714 & 72.354 \\
\hline $7 / 30 / 07$ 14:00 & 122.871 & 120.485 & & 98.304 & 102.49 & 93.933 & 108.158 & 104.898 & 76.706 & 72.332 \\
\hline 7/30/07 14:30 & 122.855 & 120.472 & & 98.289 & 102.474 & 93.916 & 108.142 & 104.88 & 76.699 & 72.317 \\
\hline 7/30/07 15:00 & 122.838 & 120.458 & & 98.273 & 102.457 & 93.902 & 108.126 & 104.862 & 76.69 & 72.301 \\
\hline $7 / 30 / 07$ 15:30 & 122.824 & 120.449 & & 98.261 & 102.448 & 93.89 & 108.118 & 104.849 & 76.681 & 72.286 \\
\hline 7/30/07 16:00 & 122.812 & 120.436 & & 98.249 & 102.434 & 93.878 & 108.109 & 104.833 & 76.675 & 72.276 \\
\hline 7/30/07 16:30 & 122.803 & 120.429 & & 98.242 & 102.427 & 93.872 & 108.101 & 104.829 & 76.668 & 72.27 \\
\hline 7/30/07 17:00 & 122.788 & 120.426 & & 98.23 & 102.415 & 93.857 & 108.089 & 104.814 & 76.659 & 72.255 \\
\hline 7/30/07 17:30 & 122.784 & 120.415 & & 98.225 & 102.411 & 93.853 & 108.086 & 104.809 & 76.651 & 72.249 \\
\hline 7/30/07 18:00 & 122.777 & 120.408 & & 98.221 & 102.406 & 93.848 & 108.082 & 104.8 & 76.642 & 72.244 \\
\hline 7/30/07 18:30 & 122.767 & 120.406 & & 98.211 & 102.396 & 93.839 & 108.076 & 104.793 & 76.636 & 72.234 \\
\hline 7/30/07 19:00 & 122.76 & 120.424 & & 98.204 & 102.389 & 93.834 & 108.07 & 104.785 & 76.631 & 72.228 \\
\hline 7/30/07 19:30 & 122.758 & 120.429 & & 98.206 & 102.391 & 93.829 & 108.068 & 104.783 & 76.622 & 72.226 \\
\hline 7/30/07 20:00 & 122.774 & 120.429 & & 98.22 & 102.4 & 93.843 & 108.078 & 104.796 & 76.622 & 72.234 \\
\hline 7/30/07 20:30 & 122.781 & 120.431 & & 98.226 & 102.408 & 93.85 & 108.083 & 104.805 & 76.62 & 72.242 \\
\hline 7/30/07 21:00 & 122.781 & 120.44 & & 98.228 & 102.413 & 93.855 & 108.085 & 104.805 & 76.618 & 72.244 \\
\hline $7 / 30 / 0721: 30$ & 122.784 & 120.449 & & 98.232 & 102.413 & 93.855 & 108.086 & 104.807 & 76.614 & 72.249 \\
\hline 7/30/07 22:00 & 122.793 & 120.456 & & 98.244 & 102.423 & 93.867 & 108.088 & 104.816 & 76.616 & 72.257 \\
\hline 7/30/07 22:30 & 122.8 & 120.451 & & 98.249 & 102.432 & 93.874 & 108.092 & 104.825 & 76.618 & 72.265 \\
\hline 7/30/07 23:00 & 122.807 & 120.451 & & 98.254 & 102.436 & 93.881 & 108.096 & 104.831 & 76.62 & 72.267 \\
\hline 7/30/07 23:30 & 122.807 & 120.449 & & 98.252 & 102.434 & 93.879 & 108.094 & 104.829 & 76.614 & 72.267 \\
\hline 7/31/07 0:00 & 122.805 & 120.449 & & 98.251 & 102.434 & 93.876 & 108.094 & 104.829 & 76.611 & 72.265 \\
\hline 7/31/07 0:30 & 122.8 & 120.449 & & 98.249 & 102.431 & 93.874 & 108.094 & 104.827 & 76.607 & 72.261 \\
\hline 7/31/07 1:00 & 122.803 & 120.451 & & 98.249 & 102.432 & 93.876 & 108.094 & 104.827 & 76.607 & 72.265 \\
\hline 7/31/07 1:30 & 122.803 & 120.445 & & 98.251 & 102.432 & 93.876 & 108.094 & 104.827 & 76.603 & 72.266 \\
\hline $7 / 31 / 072: 00$ & 122.803 & 120.44 & & 98.251 & 102.434 & 93.876 & 108.094 & 104.829 & 76.601 & 72.266 \\
\hline
\end{tabular}


TABLE S1.1 (Cont.)

\begin{tabular}{|c|c|c|c|c|c|c|c|c|c|c|}
\hline \multirow[b]{2}{*}{ Date and Time } & \multicolumn{10}{|c|}{ Depth from Top of Casing (ft) } \\
\hline & MW1D & MW2D & MW3D & MW4D & MW7 & MW9 & Oentrich & MW14D & MW15D & MW16D \\
\hline 7/31/07 2:30 & 122.8 & 120.431 & & 98.244 & 102.429 & 93.872 & 108.092 & 104.823 & 76.598 & 72.259 \\
\hline 7/31/07 3:00 & 122.791 & 120.429 & & 98.239 & 102.425 & 93.867 & 108.098 & 104.816 & 76.594 & 72.253 \\
\hline 7/31/07 3:30 & 122.798 & 120.422 & & 98.232 & 102.415 & 93.857 & 108.09 & 104.809 & 76.588 & 72.247 \\
\hline 7/31/07 4:00 & 122.798 & 120.411 & & 98.23 & 102.413 & 93.855 & 108.088 & 104.807 & 76.587 & 72.247 \\
\hline 7/31/07 4:30 & 122.795 & 120.408 & & 98.221 & 102.406 & 93.85 & 108.08 & 104.8 & 76.581 & 72.238 \\
\hline 7/31/07 5:00 & 122.793 & 120.408 & & 98.213 & 102.396 & 93.838 & 108.073 & 104.789 & 76.573 & 72.228 \\
\hline 7/31/07 5:30 & 122.788 & 120.406 & & 98.209 & 102.394 & 93.836 & 108.069 & 104.787 & 76.572 & 72.228 \\
\hline 7/31/07 6:00 & 122.786 & 122.335 & & 98.209 & 102.394 & 93.836 & 108.069 & 104.787 & 76.566 & 72.224 \\
\hline 7/31/07 6:30 & 122.781 & 122.689 & & 98.208 & 102.391 & 93.834 & 108.067 & 104.782 & 76.566 & 72.224 \\
\hline 7/31/07 7:00 & 124.729 & 122.859 & & 98.935 & 102.851 & 94.456 & 108.685 & 106.139 & 76.577 & 72.261 \\
\hline 7/31/07 7:30 & 125.128 & 122.959 & & 99.236 & 103.194 & 94.805 & 109.009 & 106.569 & 76.607 & 72.478 \\
\hline 7/31/07 8:00 & 125.303 & 123.027 & & 99.388 & 103.358 & 94.97 & 109.16 & 106.75 & 76.64 & 72.635 \\
\hline 7/31/07 8:30 & 125.405 & 123.081 & & 99.479 & 103.455 & 95.066 & 109.252 & 106.859 & 76.664 & 72.734 \\
\hline 7/31/07 9:00 & 125.478 & 123.118 & & 99.545 & 103.523 & 95.135 & 109.313 & 106.932 & 76.69 & 72.807 \\
\hline 7/31/07 9:30 & 125.535 & 123.152 & & 99.594 & 103.58 & 95.189 & 109.358 & 106.99 & 76.719 & 72.867 \\
\hline $7 / 31 / 07$ 10:00 & 125.57 & 123.168 & & 99.631 & 103.615 & 95.222 & 109.389 & 107.028 & 76.736 & 72.902 \\
\hline $7 / 31 / 07$ 10:30 & 125.598 & 123.183 & & 99.66 & 103.645 & 95.257 & 109.413 & 107.061 & 76.758 & 72.935 \\
\hline 7/31/07 11:00 & 125.62 & 123.186 & & 99.677 & 103.664 & 95.274 & 109.432 & 107.083 & 76.775 & 72.958 \\
\hline 7/31/07 11:30 & 125.634 & 121.444 & & 99.691 & 103.679 & 95.288 & 109.447 & 107.094 & 76.789 & 72.974 \\
\hline $7 / 31 / 07$ 12:00 & 125.65 & 121.009 & & 99.694 & 103.685 & 95.297 & 109.458 & 107.103 & 76.808 & 72.984 \\
\hline 7/31/07 12:30 & 123.97 & 120.832 & & 99.072 & 103.342 & 94.8 & 108.966 & 105.976 & 76.813 & 72.968 \\
\hline 7/31/07 13:00 & 123.474 & 120.723 & & 98.73 & 102.948 & 94.39 & 108.583 & 105.418 & 76.799 & 72.794 \\
\hline 7/31/07 13:30 & 123.292 & 120.653 & & 98.581 & 102.788 & 94.23 & 108.427 & 105.23 & 76.788 & 72.658 \\
\hline $7 / 31 / 07$ 14:00 & 123.181 & 120.601 & & 98.481 & 102.68 & 94.124 & 108.316 & 105.113 & 76.773 & 72.55 \\
\hline $7 / 31 / 07$ 14:30 & 123.013 & 120.558 & & 98.423 & 102.613 & 94.06 & 108.278 & 105.037 & 76.758 & 72.476 \\
\hline 7/31/07 15:00 & 122.963 & 120.522 & & 98.38 & 102.568 & 94.013 & 108.236 & 104.986 & 76.751 & 72.428 \\
\hline 7/31/07 15:30 & 122.914 & 120.508 & & 98.335 & 102.522 & 93.966 & 108.193 & 104.935 & 76.736 & 72.379 \\
\hline $7 / 31 / 07$ 16:00 & 122.878 & 120.485 & & 98.306 & 102.493 & 93.935 & 108.161 & 104.902 & 76.723 & 72.344 \\
\hline 7/31/07 16:30 & 122.859 & 120.476 & & 98.292 & 102.476 & 93.921 & 108.148 & 104.885 & 76.715 & 72.33 \\
\hline 7/31/07 17:00 & 122.843 & 120.465 & & 98.276 & 102.461 & 93.904 & 108.135 & 104.867 & 76.705 & 72.313 \\
\hline 7/31/07 17:30 & 122.831 & 120.456 & & 98.268 & 102.453 & 93.895 & 108.127 & 104.856 & 76.695 & 72.303 \\
\hline $7 / 31 / 07$ 18:00 & 122.821 & 120.44 & & 98.256 & 102.44 & 93.883 & 108.114 & 104.843 & 76.686 & 72.29 \\
\hline 7/31/07 18:30 & 122.81 & 120.429 & & 98.249 & 102.432 & 93.876 & 108.106 & 104.834 & 76.677 & 72.278 \\
\hline 7/31/07 19:00 & 122.793 & 120.417 & & 98.232 & 102.419 & 93.862 & 108.093 & 104.818 & 76.668 & 72.266 \\
\hline 7/31/07 19:30 & 122.781 & 120.406 & & 98.223 & 102.41 & 93.853 & 108.084 & 104.807 & 76.657 & 72.255 \\
\hline $7 / 31 / 0720: 00$ & 122.77 & 120.404 & & 98.211 & 102.396 & 93.838 & 108.072 & 104.794 & 76.646 & 72.243 \\
\hline $7 / 31 / 0720: 30$ & 122.758 & 120.411 & & 98.204 & 102.387 & 93.829 & 108.063 & 104.785 & 76.64 & 72.232 \\
\hline 7/31/07 21:00 & 122.755 & 120.417 & & 98.202 & 102.385 & 93.827 & 108.061 & 104.781 & 76.631 & 72.23 \\
\hline $7 / 31 / 0721: 30$ & 122.765 & 120.415 & & 98.211 & 102.392 & 93.838 & 108.069 & 104.787 & 76.629 & 72.239 \\
\hline 7/31/07 22:00 & 122.77 & 120.411 & & 98.216 & 102.398 & 93.841 & 108.074 & 104.794 & 76.627 & 72.243 \\
\hline 7/31/07 22:30 & 122.77 & 120.413 & & 98.214 & 102.396 & 93.839 & 108.072 & 104.792 & 76.622 & 72.241 \\
\hline 7/31/07 23:00 & 122.765 & 120.413 & & 98.211 & 102.392 & 93.836 & 108.068 & 104.787 & 76.618 & 72.235 \\
\hline 7/31/07 23:30 & 122.767 & 120.402 & & 98.213 & 102.396 & 93.838 & 108.07 & 104.789 & 76.614 & 72.237 \\
\hline 8/1/07 0:00 & 122.765 & 120.399 & & 98.211 & 102.394 & 93.838 & 108.068 & 104.787 & 76.612 & 72.237 \\
\hline 8/1/07 0:30 & 122.76 & 120.397 & & 98.202 & 102.383 & 93.824 & 108.059 & 104.778 & 76.605 & 72.226 \\
\hline 8/1/07 1:00 & 122.76 & 120.392 & & 98.197 & 102.383 & 93.822 & 108.055 & 104.776 & 76.598 & 72.222 \\
\hline 8/1/07 1:30 & 122.758 & 120.39 & & 98.195 & 102.379 & 93.822 & 108.055 & 104.774 & 76.596 & 72.222 \\
\hline 8/1/07 2:00 & 122.76 & 120.386 & & 98.192 & 102.375 & 93.817 & 108.05 & 104.769 & 76.59 & 72.216 \\
\hline 8/1/07 2:30 & 122.758 & 120.381 & & 98.192 & 102.377 & 93.817 & 108.049 & 104.769 & 76.587 & 72.218 \\
\hline 8/1/07 3:00 & 122.758 & 120.377 & & 98.185 & 102.368 & 93.81 & 108.042 & 104.762 & 76.585 & 72.211 \\
\hline 8/1/07 3:30 & 122.753 & 120.368 & & 98.182 & 102.364 & 93.808 & 108.038 & 104.758 & 76.579 & 72.205 \\
\hline 8/1/07 4:00 & 122.751 & 120.368 & & 98.178 & 102.362 & 93.803 & 108.038 & 104.754 & 76.574 & 72.203 \\
\hline 8/1/07 4:30 & 122.748 & 120.363 & & 98.168 & 102.352 & 93.794 & 108.029 & 104.745 & 76.568 & 72.193 \\
\hline $8 / 1 / 075: 00$ & 122.746 & 120.361 & & 98.168 & 102.352 & 93.796 & 108.031 & 104.745 & 76.565 & 72.195 \\
\hline 8/1/07 5:30 & 122.744 & 120.361 & & 98.164 & 102.351 & 93.794 & 108.029 & 104.743 & 76.561 & 72.193 \\
\hline 8/1/07 6:00 & 122.741 & 122.32 & & 98.161 & 102.347 & 93.787 & 108.023 & 104.736 & 76.559 & 72.187 \\
\hline 8/1/07 6:30 & 122.736 & 122.673 & & 98.161 & 102.345 & 93.787 & 108.023 & 104.736 & 76.552 & 72.187 \\
\hline 8/1/07 7:00 & 124.627 & 122.846 & & 98.842 & 102.783 & 94.371 & 108.599 & 106.138 & 76.559 & 72.222 \\
\hline 8/1/07 7:30 & 125.024 & 122.948 & & 99.138 & 103.114 & 94.708 & 108.917 & 106.552 & 76.592 & 72.435 \\
\hline 8/1/07 8:00 & 125.199 & 123.016 & & 99.296 & 103.283 & 94.878 & 109.074 & 106.738 & 76.622 & 72.598 \\
\hline 8/1/07 8:30 & 125.303 & 123.056 & & 99.393 & 103.388 & 94.981 & 109.168 & 106.848 & 76.647 & 72.705 \\
\hline 8/1/07 9:00 & 125.379 & 123.088 & & 99.455 & 103.455 & 95.05 & 109.234 & 106.924 & 76.671 & 72.78 \\
\hline 8/1/07 9:30 & 125.419 & 123.104 & & 99.491 & 103.495 & 95.09 & 109.27 & 106.967 & 76.694 & 72.821 \\
\hline $8 / 1 / 07$ 10:00 & 125.502 & 123.118 & & 99.519 & 103.523 & 95.116 & 109.298 & 106.996 & 76.71 & 72.852 \\
\hline $8 / 1 / 07$ 10:30 & 125.525 & 123.122 & & 99.538 & 103.546 & 95.142 & 109.318 & 107.021 & 76.73 & 72.879 \\
\hline 8/1/07 11:00 & 125.537 & 123.131 & & 99.553 & 103.559 & 95.154 & 109.332 & 107.034 & 76.747 & 72.896 \\
\hline
\end{tabular}


TABLE S1.1 (Cont.)

\begin{tabular}{|c|c|c|c|c|c|c|c|c|c|c|}
\hline \multirow[b]{2}{*}{ Date and Time } & \multicolumn{10}{|c|}{ Depth from Top of Casing (ft) } \\
\hline & MW1D & MW2D & MW3D & MW4D & MW7 & MW9 & Oentrich & MW14D & MW15D & MW16D \\
\hline 8/1/07 11:30 & 125.542 & 121.519 & & 99.558 & 103.565 & 95.163 & 109.339 & 107.039 & 76.758 & 72.906 \\
\hline $8 / 1 / 07 \quad 12: 00$ & 125.551 & 120.977 & & 99.567 & 103.573 & 95.17 & 109.347 & 107.05 & 76.771 & 72.918 \\
\hline 8/1/07 12:30 & 124.008 & 120.789 & & 99.091 & 103.388 & 94.864 & 109.029 & 106.204 & 76.789 & 72.922 \\
\hline 8/1/07 13:00 & 123.348 & 120.678 & & 98.697 & 102.918 & 94.359 & 108.556 & 105.392 & 76.771 & 72.77 \\
\hline $8 / 1 / 0713: 30$ & 123.152 & 120.601 & & 98.535 & 102.741 & 94.185 & 108.391 & 105.183 & 76.758 & 72.621 \\
\hline $8 / 1 / 0714: 00$ & 123.039 & 120.549 & & 98.44 & 102.638 & 94.084 & 108.295 & 105.068 & 76.75 & 72.518 \\
\hline 8/1/07 14:30 & 122.961 & 120.501 & & 98.371 & 102.564 & 94.011 & 108.226 & 104.986 & 76.732 & 72.437 \\
\hline $8 / 1 / 07 \quad 15: 00$ & 122.907 & 120.46 & & 98.323 & 102.512 & 93.959 & 108.179 & 104.929 & 76.717 & 72.381 \\
\hline 8/1/07 15:30 & 122.86 & 120.431 & & 98.28 & 102.471 & 93.916 & 108.143 & 104.885 & 76.708 & 72.338 \\
\hline $8 / 1 / 0716: 00$ & 122.817 & 120.411 & & 98.24 & 102.429 & 93.874 & 108.104 & 104.84 & 76.695 & 72.292 \\
\hline 8/1/07 16:30 & 122.786 & 120.39 & & 98.213 & 102.398 & 93.846 & 108.075 & 104.805 & 76.677 & 72.261 \\
\hline $8 / 1 / 07 \quad 17: 00$ & 122.767 & 120.399 & & 98.195 & 102.385 & 93.834 & 108.064 & 104.789 & 76.668 & 72.247 \\
\hline $8 / 1 / 0717: 30$ & 122.746 & 120.39 & & 98.18 & 102.366 & 93.81 & 108.043 & 104.769 & 76.655 & 72.226 \\
\hline 8/1/07 18:00 & 122.751 & 120.392 & & 98.194 & 102.375 & 93.82 & 108.051 & 104.774 & 76.651 & 72.235 \\
\hline $8 / 1 / 07$ 18:30 & 122.744 & 120.395 & & 98.185 & 102.37 & 93.813 & 108.047 & 104.767 & 76.642 & 72.226 \\
\hline $8 / 1 / 0719: 00$ & 122.746 & 120.377 & & 98.187 & 102.373 & 93.815 & 108.047 & 104.767 & 76.638 & 72.228 \\
\hline $8 / 1 / 07$ 19:30 & 122.748 & 120.381 & & 98.19 & 102.377 & 93.82 & 108.051 & 104.774 & 76.633 & 72.232 \\
\hline 8/1/07 20:00 & 122.732 & 120.388 & & 98.173 & 102.356 & 93.801 & 108.034 & 104.752 & 76.622 & 72.21 \\
\hline $8 / 1 / 0720: 30$ & 122.734 & 120.39 & & 98.18 & 102.364 & 93.805 & 108.04 & 104.756 & 76.616 & 72.216 \\
\hline $8 / 1 / 0721: 00$ & 122.741 & 120.411 & & 98.183 & 102.37 & 93.815 & 108.045 & 104.765 & 76.616 & 72.224 \\
\hline $8 / 1 / 0721: 30$ & 122.741 & 120.395 & & 98.19 & 102.373 & 93.815 & 108.043 & 104.758 & 76.605 & 72.22 \\
\hline 8/1/07 22:00 & 122.765 & 120.379 & & 98.209 & 102.396 & 93.841 & 108.062 & 104.787 & 76.612 & 72.247 \\
\hline $8 / 1 / 0722: 30$ & 122.748 & 120.402 & & 98.192 & 102.375 & 93.82 & 108.051 & 104.772 & 76.601 & 72.224 \\
\hline 8/1/07 23:00 & 122.736 & 120.433 & & 98.173 & 102.364 & 93.808 & 108.04 & 104.761 & 76.59 & 72.211 \\
\hline 8/1/07 23:30 & 122.727 & 120.442 & & 98.206 & 102.385 & 93.827 & 108.055 & 104.774 & 76.59 & 72.232 \\
\hline $8 / 2 / 070: 00$ & 122.72 & 120.436 & & 98.239 & 102.421 & 93.864 & 108.066 & 104.812 & 76.6 & 72.266 \\
\hline 8/2/07 0:30 & 122.718 & 120.426 & & 98.244 & 102.427 & 93.869 & 108.068 & 104.818 & 76.601 & 72.269 \\
\hline 8/2/07 1:00 & 122.725 & 120.422 & & 98.24 & 102.423 & 93.867 & 108.072 & 104.814 & 76.594 & 72.265 \\
\hline 8/2/07 1:30 & 122.727 & 120.413 & & 98.228 & 102.41 & 93.855 & 108.07 & 104.805 & 76.592 & 72.253 \\
\hline 8/2/07 2:00 & 122.722 & 120.408 & & 98.221 & 102.406 & 93.85 & 108.069 & 104.798 & 76.587 & 72.249 \\
\hline 8/2/07 2:30 & 122.72 & 120.406 & & 98.214 & 102.396 & 93.841 & 108.064 & 104.789 & 76.581 & 72.241 \\
\hline 8/2/07 3:00 & 122.718 & 120.399 & & 98.208 & 102.394 & 93.834 & 108.06 & 104.785 & 76.574 & 72.234 \\
\hline 8/2/07 3:30 & 122.713 & 120.406 & & 98.206 & 102.389 & 93.831 & 108.058 & 104.778 & 76.576 & 72.23 \\
\hline 8/2/07 4:00 & 122.71 & 120.406 & & 98.201 & 102.383 & 93.829 & 108.055 & 104.776 & 76.568 & 72.228 \\
\hline $8 / 2 / 074: 30$ & 122.706 & 120.411 & & 98.209 & 102.392 & 93.836 & 108.06 & 104.783 & 76.568 & 72.237 \\
\hline 8/2/07 5:00 & 122.703 & 120.415 & & 98.211 & 102.391 & 93.838 & 108.06 & 104.783 & 76.565 & 72.235 \\
\hline 8/2/07 5:30 & 122.701 & 121.71 & & 98.214 & 102.396 & 93.841 & 108.064 & 104.787 & 76.568 & 72.239 \\
\hline 8/2/07 6:00 & 122.727 & 122.569 & & 98.221 & 102.402 & 93.845 & 108.069 & 104.794 & 76.563 & 72.247 \\
\hline 8/2/07 6:30 & 122.994 & 122.793 & & 98.599 & 102.408 & 93.85 & 108.071 & 104.796 & 76.561 & 72.249 \\
\hline 8/2/07 7:00 & 125 & 122.918 & & 99.138 & 103.085 & 94.696 & 108.899 & 106.441 & 76.592 & 72.4 \\
\hline 8/2/07 7:30 & 125.237 & 123.007 & & 99.334 & 103.301 & 94.913 & 109.1 & 106.682 & 76.621 & 72.59 \\
\hline 8/2/07 8:00 & 125.367 & 123.056 & & 99.45 & 103.424 & 95.036 & 109.217 & 106.819 & 76.651 & 72.716 \\
\hline 8/2/07 8:30 & 125.457 & 123.095 & & 99.529 & 103.508 & 95.121 & 109.298 & 106.91 & 76.677 & 72.803 \\
\hline 8/2/07 9:00 & 125.509 & 121.143 & & 99.579 & 103.563 & 95.175 & 109.35 & 106.968 & 76.703 & 72.863 \\
\hline 8/2/07 9:30 & 125.549 & 120.875 & & 99.615 & 103.599 & 95.212 & 109.382 & 107.008 & 76.727 & 72.904 \\
\hline $8 / 2 / 07 \quad 10: 00$ & 123.533 & 120.748 & & 98.842 & 103.082 & 94.527 & 108.702 & 105.58 & 76.727 & 72.853 \\
\hline $8 / 2 / 07 \quad 10: 30$ & 123.24 & 120.674 & & 98.621 & 102.828 & 94.275 & 108.464 & 105.274 & 76.716 & 72.693 \\
\hline $8 / 2 / 0711: 00$ & 123.11 & 120.626 & & 98.514 & 102.712 & 94.154 & 108.351 & 105.137 & 76.708 & 72.584 \\
\hline $8 / 2 / 0711: 30$ & 123.032 & 120.592 & & 98.45 & 102.642 & 94.088 & 108.286 & 105.059 & 76.703 & 72.509 \\
\hline $8 / 2 / 07 \quad 12: 00$ & 122.985 & 120.565 & & 98.409 & 102.596 & 94.046 & 108.242 & 105.009 & 76.699 & 72.457 \\
\hline $8 / 2 / 0712: 30$ & 122.949 & 120.54 & & 98.378 & 102.562 & 94.01 & 108.214 & 104.973 & 76.695 & 72.42 \\
\hline 8/2/07 13:00 & 122.923 & 120.519 & & 98.352 & 102.541 & 93.987 & 108.192 & 104.944 & 76.69 & 72.394 \\
\hline $8 / 2 / 07$ 13:30 & 122.899 & 120.504 & & 98.33 & 102.516 & 93.966 & 108.175 & 104.92 & 76.682 & 72.368 \\
\hline $8 / 2 / 0714: 00$ & 122.876 & 120.492 & & 98.309 & 102.497 & 93.942 & 108.158 & 104.898 & 76.675 & 72.346 \\
\hline $8 / 2 / 07 \quad 14: 30$ & 122.86 & 120.481 & & 98.299 & 102.482 & 93.926 & 108.145 & 104.882 & 76.673 & 72.331 \\
\hline $8 / 2 / 07$ 15:00 & 122.85 & 120.47 & & 98.289 & 102.472 & 93.919 & 108.135 & 104.871 & 76.666 & 72.321 \\
\hline 8/2/07 15:30 & 122.836 & 120.458 & & 98.276 & 102.459 & 93.907 & 108.124 & 104.86 & 76.662 & 72.309 \\
\hline 8/2/07 16:00 & 122.826 & 120.451 & & 98.266 & 102.45 & 93.897 & 108.115 & 104.849 & 76.655 & 72.301 \\
\hline 8/2/07 16:30 & 122.814 & 120.442 & & 98.256 & 102.442 & 93.883 & 108.105 & 104.836 & 76.647 & 72.29 \\
\hline $8 / 2 / 07 \quad 17: 00$ & 122.808 & 120.433 & & 98.249 & 102.432 & 93.876 & 108.101 & 104.829 & 76.642 & 72.284 \\
\hline $8 / 2 / 07 \quad 17: 30$ & 122.8 & 120.424 & & 98.242 & 102.427 & 93.874 & 108.094 & 104.82 & 76.635 & 72.276 \\
\hline $8 / 2 / 07$ 18:00 & 122.791 & 120.422 & & 98.233 & 102.417 & 93.862 & 108.086 & 104.812 & 76.629 & 72.267 \\
\hline $8 / 2 / 07$ 18:30 & 122.781 & 120.42 & & 98.225 & 102.41 & 93.853 & 108.079 & 104.803 & 76.623 & 72.259 \\
\hline $8 / 2 / 07$ 19:00 & 122.779 & 120.426 & & 98.223 & 102.408 & 93.848 & 108.075 & 104.798 & 76.62 & 72.257 \\
\hline $8 / 2 / 07$ 19:30 & 122.775 & 120.426 & & 98.221 & 102.404 & 93.845 & 108.071 & 104.796 & 76.611 & 72.253 \\
\hline 8/2/07 20:00 & 122.782 & 120.429 & & 98.228 & 102.41 & 93.853 & 108.079 & 104.803 & 76.609 & 72.259 \\
\hline
\end{tabular}


TABLE S1.1 (Cont.)

\begin{tabular}{|c|c|c|c|c|c|c|c|c|c|c|}
\hline \multirow[b]{2}{*}{ Date and Time } & \multicolumn{10}{|c|}{ Depth from Top of Casing (ft) } \\
\hline & MW1D & MW2D & MW3D & MW4D & MW7 & MW9 & Oentrich & MW14D & MW15D & MW16D \\
\hline $8 / 2 / 07$ 20:30 & 122.781 & 120.431 & & 98.228 & 102.411 & 93.853 & 108.079 & 104.803 & 76.605 & 72.259 \\
\hline 8/2/07 21:00 & 122.786 & 120.438 & & 98.232 & 102.413 & 93.857 & 108.081 & 104.807 & 76.603 & 72.263 \\
\hline 8/2/07 21:30 & 122.786 & 120.436 & & 98.235 & 102.417 & 93.86 & 108.083 & 104.807 & 76.601 & 72.263 \\
\hline 8/2/07 22:00 & 122.791 & 120.436 & & 98.24 & 102.421 & 93.867 & 108.085 & 104.812 & 76.598 & 72.267 \\
\hline $8 / 2 / 0722: 30$ & 122.793 & 120.438 & & 98.239 & 102.423 & 93.867 & 108.086 & 104.816 & 76.594 & 72.269 \\
\hline 8/2/07 23:00 & 122.791 & 120.436 & & 98.239 & 102.421 & 93.864 & 108.085 & 104.812 & 76.592 & 72.268 \\
\hline 8/2/07 23:30 & 122.795 & 120.44 & & 98.244 & 102.423 & 93.869 & 108.086 & 104.814 & 76.592 & 72.269 \\
\hline $8 / 3 / 07$ 0:00 & 122.793 & 120.445 & & 98.24 & 102.421 & 93.867 & 108.086 & 104.814 & 76.59 & 72.267 \\
\hline 8/3/07 0:30 & 122.796 & 120.449 & & 98.244 & 102.423 & 93.869 & 108.086 & 104.814 & 76.592 & 72.269 \\
\hline 8/3/07 1:00 & 122.8 & 120.449 & & 98.247 & 102.431 & 93.874 & 108.092 & 104.82 & 76.588 & 72.273 \\
\hline $8 / 3 / 07$ 1:30 & 122.803 & 120.451 & & 98.254 & 102.432 & 93.876 & 108.092 & 104.825 & 76.588 & 72.276 \\
\hline 8/3/07 2:00 & 122.805 & 120.449 & & 98.252 & 102.436 & 93.881 & 108.096 & 104.825 & 76.587 & 72.28 \\
\hline 8/3/07 2:30 & 122.805 & 120.449 & & 98.254 & 102.434 & 93.881 & 108.096 & 104.825 & 76.588 & 72.28 \\
\hline 8/3/07 3:00 & 122.803 & 120.451 & & 98.252 & 102.434 & 93.876 & 108.094 & 104.825 & 76.586 & 72.278 \\
\hline 8/3/07 3:30 & 122.803 & 120.447 & & 98.254 & 102.434 & 93.876 & 108.094 & 104.825 & 76.583 & 72.275 \\
\hline 8/3/07 4:00 & 122.807 & 120.445 & & 98.256 & 102.436 & 93.881 & 108.094 & 104.827 & 76.583 & 72.282 \\
\hline $8 / 3 / 074: 30$ & 122.8 & 120.442 & & 98.251 & 102.431 & 93.876 & 108.09 & 104.823 & 76.583 & 72.275 \\
\hline 8/3/07 5:00 & 122.8 & 120.449 & & 98.247 & 102.431 & 93.874 & 108.09 & 104.823 & 76.585 & 72.273 \\
\hline 8/3/07 5:30 & 122.798 & 120.451 & & 98.247 & 102.431 & 93.871 & 108.088 & 104.818 & 76.579 & 72.274 \\
\hline 8/3/07 6:00 & 122.8 & 122.485 & & 98.256 & 102.434 & 93.876 & 108.09 & 104.823 & 76.572 & 72.275 \\
\hline 8/3/07 6:30 & 122.807 & 122.787 & & 98.257 & 102.44 & 93.883 & 108.094 & 104.829 & 76.577 & 72.284 \\
\hline 8/3/07 7:00 & 124.87 & 122.943 & & 98.993 & 102.939 & 94.527 & 108.73 & 106.319 & 76.594 & 72.344 \\
\hline $8 / 3 / 077: 30$ & 125.197 & 123.032 & & 99.248 & 103.224 & 94.819 & 109.006 & 106.669 & 76.622 & 72.546 \\
\hline 8/3/07 8:00 & 125.357 & 123.102 & & 99.389 & 103.377 & 94.972 & 109.143 & 106.835 & 76.651 & 72.695 \\
\hline 8/3/07 8:30 & 125.452 & 123.138 & & 99.476 & 103.468 & 95.066 & 109.23 & 106.937 & 76.677 & 72.794 \\
\hline 8/3/07 9:00 & 125.52 & 123.165 & & 99.536 & 103.535 & 95.13 & 109.291 & 107.005 & 76.699 & 72.86 \\
\hline 8/3/07 9:30 & 125.556 & 123.188 & & 99.57 & 103.573 & 95.168 & 109.341 & 107.045 & 76.717 & 72.904 \\
\hline $8 / 3 / 07$ 10:00 & 125.584 & 123.208 & & 99.6 & 103.599 & 95.198 & 109.366 & 107.074 & 76.745 & 72.935 \\
\hline $8 / 3 / 07$ 10:30 & 125.608 & 123.213 & & 99.622 & 103.628 & 95.224 & 109.386 & 107.101 & 76.762 & 72.966 \\
\hline 8/3/07 11:00 & 125.632 & 123.22 & & 99.639 & 103.641 & 95.238 & 109.399 & 107.118 & 76.78 & 72.983 \\
\hline $8 / 3 / 07$ 11:30 & 125.634 & 123.222 & & 99.646 & 103.653 & 95.248 & 109.408 & 107.129 & 76.797 & 72.997 \\
\hline $8 / 3 / 07 \quad 12: 00$ & 125.641 & 123.222 & & 99.651 & 103.657 & 95.253 & 109.416 & 107.134 & 76.811 & 73.001 \\
\hline $8 / 3 / 07$ 12:30 & 125.643 & 123.217 & & 99.656 & 103.662 & 95.26 & 109.421 & 107.138 & 76.821 & 73.011 \\
\hline 8/3/07 13:00 & 125.644 & 123.22 & & 99.66 & 103.668 & 95.262 & 109.425 & 107.14 & 76.839 & 73.018 \\
\hline 8/3/07 13:30 & 125.644 & 121.136 & & 99.66 & 103.668 & 95.262 & 109.427 & 107.138 & 76.852 & 73.02 \\
\hline $8 / 3 / 0714: 00$ & 125.643 & 120.893 & & 99.565 & 103.668 & 95.262 & 109.427 & 107.14 & 76.861 & 73.022 \\
\hline $8 / 3 / 07 \quad 14: 30$ & 123.514 & 120.76 & & 98.836 & 103.068 & 94.515 & 108.695 & 105.56 & 76.847 & 72.9 \\
\hline $8 / 3 / 07$ 15:00 & 123.261 & 120.674 & & 98.633 & 102.84 & 94.284 & 108.481 & 105.292 & 76.828 & 72.731 \\
\hline $8 / 3 / 07$ 15:30 & 123.124 & 120.612 & & 98.516 & 102.716 & 94.159 & 108.362 & 105.15 & 76.813 & 72.604 \\
\hline $8 / 3 / 07$ 16:00 & 123.037 & 120.565 & & 98.44 & 102.632 & 94.076 & 108.285 & 105.059 & 76.798 & 72.519 \\
\hline 8/3/07 16:30 & 122.973 & 120.526 & & 98.387 & 102.579 & 94.02 & 108.231 & 104.995 & 76.784 & 72.454 \\
\hline $8 / 3 / 07$ 17:00 & 122.923 & 120.497 & & 98.342 & 102.531 & 93.975 & 108.192 & 104.944 & 76.769 & 72.408 \\
\hline $8 / 3 / 07$ 17:30 & 122.888 & 120.47 & & 98.311 & 102.497 & 93.942 & 108.16 & 104.909 & 76.756 & 72.371 \\
\hline 8/3/07 18:00 & 122.852 & 120.447 & & 98.28 & 102.467 & 93.911 & 108.129 & 104.874 & 76.743 & 72.338 \\
\hline 8/3/07 18:30 & 122.826 & 120.442 & & 98.257 & 102.442 & 93.886 & 108.107 & 104.847 & 76.725 & 72.311 \\
\hline 8/3/07 19:00 & 122.803 & 120.436 & & 98.237 & 102.421 & 93.864 & 108.088 & 104.825 & 76.714 & 72.29 \\
\hline 8/3/07 19:30 & 122.8 & 120.433 & & 98.233 & 102.421 & 93.864 & 108.088 & 104.823 & 76.708 & 72.29 \\
\hline $8 / 3 / 0720: 00$ & 122.793 & 120.431 & & 98.232 & 102.415 & 93.86 & 108.081 & 104.814 & 76.697 & 72.284 \\
\hline $8 / 3 / 0720: 30$ & 122.788 & 120.431 & & 98.23 & 102.415 & 93.855 & 108.079 & 104.812 & 76.691 & 72.278 \\
\hline 8/3/07 21:00 & 122.788 & 120.433 & & 98.23 & 102.413 & 93.855 & 108.077 & 104.809 & 76.682 & 72.276 \\
\hline $8 / 3 / 0721: 30$ & 122.786 & 120.426 & & 98.23 & 102.41 & 93.855 & 108.077 & 104.807 & 76.686 & 72.273 \\
\hline $8 / 3 / 07$ 22:00 & 122.788 & 120.42 & & 98.23 & 102.415 & 93.855 & 108.075 & 104.807 & 76.675 & 72.276 \\
\hline 8/3/07 22:30 & 122.781 & 120.408 & & 98.223 & 102.406 & 93.848 & 108.071 & 104.803 & 76.671 & 72.268 \\
\hline $8 / 3 / 0723: 00$ & 122.774 & 120.406 & & 98.216 & 102.4 & 93.843 & 108.065 & 104.796 & 76.662 & 72.261 \\
\hline 8/3/07 23:30 & 122.762 & 120.404 & & 98.204 & 102.387 & 93.831 & 108.056 & 104.783 & 76.653 & 72.249 \\
\hline 8/4/07 0:00 & 122.76 & 120.395 & & 98.202 & 102.385 & 93.829 & 108.052 & 104.781 & 76.645 & 72.245 \\
\hline 8/4/07 0:30 & 122.76 & 120.392 & & 98.201 & 102.383 & 93.827 & 108.05 & 104.778 & 76.642 & 72.244 \\
\hline 8/4/07 1:00 & 122.751 & 120.374 & & 98.192 & 102.375 & 93.82 & 108.045 & 104.772 & 76.636 & 72.237 \\
\hline 8/4/07 1:30 & 122.746 & 120.365 & & 98.189 & 102.37 & 93.815 & 108.039 & 104.765 & 76.633 & 72.232 \\
\hline $8 / 4 / 072: 00$ & 122.729 & 120.358 & & 98.171 & 102.356 & 93.801 & 108.026 & 104.752 & 76.625 & 72.218 \\
\hline 8/4/07 2:30 & 122.718 & 120.352 & & 98.161 & 102.343 & 93.787 & 108.013 & 104.736 & 76.614 & 72.203 \\
\hline 8/4/07 3:00 & 122.71 & 120.343 & & 98.154 & 102.337 & 93.779 & 108.007 & 104.73 & 76.607 & 72.197 \\
\hline 8/4/07 3:30 & 122.706 & 120.329 & & 98.149 & 102.332 & 93.772 & 108.002 & 104.725 & 76.605 & 72.193 \\
\hline $8 / 4 / 074: 00$ & 122.696 & 120.322 & & 98.139 & 102.322 & 93.765 & 107.994 & 104.714 & 76.596 & 72.184 \\
\hline 8/4/07 4:30 & 122.682 & 120.318 & & 98.125 & 102.311 & 93.751 & 107.983 & 104.703 & 76.585 & 72.17 \\
\hline 8/4/07 5:00 & 122.677 & 120.315 & & 98.121 & 102.305 & 93.749 & 107.979 & 104.696 & 76.581 & 72.166 \\
\hline
\end{tabular}


TABLE S1.1 (Cont.)

\begin{tabular}{|c|c|c|c|c|c|c|c|c|c|c|}
\hline \multirow[b]{2}{*}{ Date and Time } & \multicolumn{10}{|c|}{ Depth from Top of Casing (ft) } \\
\hline & MW1D & MW2D & MW3D & MW4D & MW7 & MW9 & Oentrich & MW14D & MW15D & MW16D \\
\hline 8/4/07 5:30 & 122.67 & 120.313 & & 98.116 & 102.299 & 93.742 & 107.974 & 104.692 & 76.574 & 72.162 \\
\hline $8 / 4 / 076: 00$ & 122.668 & 120.313 & & 98.116 & 102.297 & 93.739 & 107.972 & 104.69 & 76.568 & 72.158 \\
\hline 8/4/07 6:30 & 122.666 & 120.315 & & 98.115 & 102.297 & 93.739 & 107.97 & 104.688 & 76.563 & 72.158 \\
\hline 8/4/07 7:00 & 122.666 & 122.05 & & 98.115 & 102.295 & 93.737 & 107.968 & 104.685 & 76.559 & 72.156 \\
\hline 8/4/07 7:30 & 122.668 & 122.526 & & 98.12 & 102.303 & 93.742 & 107.974 & 104.689 & 76.555 & 72.162 \\
\hline 8/4/07 8:00 & 124.412 & 122.741 & & 98.716 & 102.602 & 94.197 & 108.432 & 105.766 & 76.557 & 72.168 \\
\hline 8/4/07 8:30 & 124.958 & 122.834 & & 99.088 & 103.044 & 94.652 & 108.858 & 106.398 & 76.587 & 72.366 \\
\hline 8/4/07 9:00 & 125.175 & 122.895 & & 99.267 & 103.232 & 94.84 & 109.036 & 106.616 & 76.611 & 72.536 \\
\hline 8/4/07 9:30 & 125.286 & 122.934 & & 99.365 & 103.342 & 94.951 & 109.139 & 106.74 & 76.635 & 72.654 \\
\hline $8 / 4 / 07$ 10:00 & 125.346 & 122.964 & & 99.417 & 103.4 & 95.01 & 109.194 & 106.8 & 76.653 & 72.718 \\
\hline $8 / 4 / 07$ 10:30 & 125.383 & 122.973 & & 99.451 & 103.434 & 95.045 & 109.228 & 106.839 & 76.673 & 72.759 \\
\hline 8/4/07 11:00 & 125.416 & 122.984 & & 99.477 & 103.462 & 95.071 & 109.258 & 106.873 & 76.684 & 72.79 \\
\hline $8 / 4 / 0711: 30$ & 125.424 & 122.991 & & 99.486 & 103.474 & 95.083 & 109.269 & 106.881 & 76.703 & 72.805 \\
\hline 8/4/07 12:00 & 125.438 & 121.592 & & 99.496 & 103.485 & 95.095 & 109.282 & 106.895 & 76.712 & 72.817 \\
\hline $8 / 4 / 07$ 12:30 & 125.445 & 120.893 & & 99.503 & 103.493 & 95.102 & 109.295 & 106.908 & 76.729 & 72.827 \\
\hline 8/4/07 13:00 & 124.263 & 120.68 & & 99.071 & 103.415 & 94.944 & 109.117 & 106.603 & 76.745 & 72.836 \\
\hline 8/4/07 13:30 & 123.268 & 120.558 & & 98.602 & 102.826 & 94.272 & 108.482 & 105.303 & 76.718 & 72.689 \\
\hline $8 / 4 / 0714: 00$ & 123.042 & 120.481 & & 98.421 & 102.63 & 94.067 & 108.291 & 105.071 & 76.706 & 72.528 \\
\hline 8/4/07 14:30 & 122.914 & 120.424 & & 98.318 & 102.514 & 93.954 & 108.186 & 104.94 & 76.69 & 72.412 \\
\hline $8 / 4 / 07$ 15:00 & 122.836 & 120.381 & & 98.249 & 102.444 & 93.886 & 108.116 & 104.862 & 76.677 & 72.335 \\
\hline $8 / 4 / 07$ 15:30 & 122.779 & 120.347 & & 98.199 & 102.389 & 93.831 & 108.071 & 104.803 & 76.659 & 72.276 \\
\hline $8 / 4 / 0716: 00$ & 122.734 & 120.327 & & 98.159 & 102.351 & 93.794 & 108.035 & 104.761 & 76.647 & 72.235 \\
\hline 8/4/07 16:30 & 122.701 & 120.304 & & 98.133 & 102.318 & 93.761 & 108.004 & 104.723 & 76.636 & 72.201 \\
\hline $8 / 4 / 07$ 17:00 & 122.677 & 120.288 & & 98.111 & 102.297 & 93.739 & 107.987 & 104.705 & 76.625 & 72.179 \\
\hline $8 / 4 / 07$ 17:30 & 122.656 & 120.272 & & 98.092 & 102.28 & 93.723 & 107.964 & 104.681 & 76.614 & 72.156 \\
\hline 8/4/07 18:00 & 122.642 & 120.259 & & 98.078 & 102.265 & 93.709 & 107.951 & 104.663 & 76.601 & 72.142 \\
\hline $8 / 4 / 0718: 30$ & 122.625 & 120.252 & & 98.063 & 102.25 & 93.692 & 107.94 & 104.645 & 76.592 & 72.125 \\
\hline $8 / 4 / 07$ 19:00 & 122.611 & 120.243 & & 98.052 & 102.234 & 93.68 & 107.928 & 104.634 & 76.585 & 72.112 \\
\hline 8/4/07 19:30 & 122.606 & 120.243 & & 98.047 & 102.233 & 93.676 & 107.925 & 104.628 & 76.576 & 72.108 \\
\hline $8 / 4 / 0720: 00$ & 122.597 & 120.238 & & 98.042 & 102.223 & 93.664 & 107.917 & 104.617 & 76.566 & 72.098 \\
\hline $8 / 4 / 0720: 30$ & 122.597 & 120.247 & & 98.037 & 102.225 & 93.669 & 107.917 & 104.619 & 76.559 & 72.098 \\
\hline 8/4/07 21:00 & 122.592 & 120.247 & & 98.034 & 102.219 & 93.664 & 107.913 & 104.614 & 76.552 & 72.092 \\
\hline $8 / 4 / 0721: 30$ & 122.599 & 120.254 & & 98.047 & 102.231 & 93.671 & 107.923 & 104.623 & 76.542 & 72.096 \\
\hline 8/4/07 22:00 & 122.599 & 120.254 & & 98.049 & 102.233 & 93.676 & 107.924 & 104.621 & 76.539 & 72.102 \\
\hline 8/4/07 22:30 & 122.606 & 120.241 & & 98.056 & 102.238 & 93.683 & 107.926 & 104.628 & 76.535 & 72.108 \\
\hline 8/4/07 23:00 & 122.609 & 120.238 & & 98.056 & 102.24 & 93.685 & 107.926 & 104.632 & 76.529 & 72.11 \\
\hline 8/4/07 23:30 & 122.595 & 120.236 & & 98.04 & 102.227 & 93.669 & 107.915 & 104.619 & 76.522 & 72.094 \\
\hline 8/5/07 0:00 & 122.59 & 120.231 & & 98.037 & 102.225 & 93.666 & 107.911 & 104.614 & 76.513 & 72.091 \\
\hline 8/5/07 0:30 & 122.588 & 120.229 & & 98.039 & 102.221 & 93.664 & 107.908 & 104.612 & 76.511 & 72.087 \\
\hline 8/5/07 1:00 & 122.58 & 120.225 & & 98.032 & 102.217 & 93.659 & 107.906 & 104.606 & 76.502 & 72.081 \\
\hline 8/5/07 1:30 & 122.58 & 120.216 & & 98.028 & 102.214 & 93.655 & 107.904 & 104.601 & 76.498 & 72.079 \\
\hline 8/5/07 2:00 & 122.573 & 120.207 & & 98.023 & 102.206 & 93.65 & 107.898 & 104.597 & 76.493 & 72.071 \\
\hline 8/5/07 2:30 & 122.566 & 120.207 & & 98.013 & 102.2 & 93.643 & 107.894 & 104.59 & 76.487 & 72.065 \\
\hline 8/5/07 3:00 & 122.557 & 120.2 & & 98.006 & 102.191 & 93.631 & 107.885 & 104.581 & 76.478 & 72.054 \\
\hline 8/5/07 3:30 & 122.557 & 120.195 & & 98.006 & 102.191 & 93.633 & 107.879 & 104.579 & 76.474 & 72.054 \\
\hline 8/5/07 4:00 & 122.552 & 120.188 & & 98.001 & 102.187 & 93.626 & 107.878 & 104.574 & 76.467 & 72.052 \\
\hline 8/5/07 4:30 & 122.545 & 120.179 & & 97.996 & 102.177 & 93.619 & 107.868 & 104.568 & 76.461 & 72.044 \\
\hline 8/5/07 5:00 & 122.538 & 120.179 & & 97.989 & 102.172 & 93.612 & 107.862 & 104.561 & 76.454 & 72.038 \\
\hline 8/5/07 5:30 & 122.528 & 120.175 & & 97.982 & 102.164 & 93.605 & 107.851 & 104.552 & 76.448 & 72.029 \\
\hline 8/5/07 6:00 & 122.531 & 120.173 & & 97.98 & 102.166 & 93.607 & 107.853 & 104.552 & 76.443 & 72.029 \\
\hline 8/5/07 6:30 & 122.524 & 122.306 & & 97.977 & 102.162 & 93.603 & 107.847 & 104.548 & 76.435 & 72.024 \\
\hline 8/5/07 7:00 & 122.526 & 122.558 & & 98.047 & 102.164 & 93.603 & 107.849 & 104.548 & 76.43 & 72.025 \\
\hline 8/5/07 7:30 & 124.703 & 122.694 & & 98.807 & 102.767 & 94.359 & 108.591 & 106.168 & 76.455 & 72.154 \\
\hline 8/5/07 8:00 & 124.965 & 122.771 & & 99.022 & 103.007 & 94.597 & 108.809 & 106.441 & 76.485 & 72.35 \\
\hline 8/5/07 8:30 & 125.107 & 122.821 & & 99.145 & 103.141 & 94.732 & 108.933 & 106.589 & 76.509 & 72.482 \\
\hline 8/5/07 9:00 & 125.192 & 122.868 & & 99.217 & 103.217 & 94.812 & 109.006 & 106.676 & 76.531 & 72.567 \\
\hline 8/5/07 9:30 & 125.239 & 120.991 & & 99.265 & 103.274 & 94.871 & 109.017 & 106.729 & 76.555 & 72.625 \\
\hline 8/5/07 10:00 & 125.282 & 120.653 & & 99.308 & 103.31 & 94.901 & 109.096 & 106.773 & 76.572 & 72.664 \\
\hline $8 / 5 / 07$ 10:30 & 123.391 & 120.513 & & 98.666 & 102.922 & 94.369 & 108.561 & 105.46 & 76.579 & 72.656 \\
\hline 8/5/07 11:00 & 123.02 & 120.424 & & 98.399 & 102.613 & 94.053 & 108.265 & 105.057 & 76.565 & 72.493 \\
\hline 8/5/07 11:30 & 122.873 & 120.377 & & 98.278 & 102.48 & 93.921 & 108.135 & 104.902 & 76.553 & 72.373 \\
\hline $8 / 5 / 0712: 00$ & 122.781 & 120.343 & & 98.199 & 102.394 & 93.836 & 108.06 & 104.807 & 76.544 & 72.282 \\
\hline $8 / 5 / 07 \quad 12: 30$ & 122.732 & 120.318 & & 98.158 & 102.349 & 93.791 & 108.017 & 104.756 & 76.537 & 72.23 \\
\hline 8/5/07 13:00 & 122.696 & 120.299 & & 98.13 & 102.318 & 93.761 & 107.992 & 104.719 & 76.531 & 72.195 \\
\hline 8/5/07 13:30 & 122.67 & 120.284 & & 98.106 & 102.295 & 93.737 & 107.974 & 104.692 & 76.524 & 72.166 \\
\hline 8/5/07 14:00 & 122.651 & 120.265 & & 98.094 & 102.28 & 93.723 & 107.956 & 104.674 & 76.518 & 72.147 \\
\hline
\end{tabular}


TABLE S1.1 (Cont.)

\begin{tabular}{|c|c|c|c|c|c|c|c|c|c|c|}
\hline \multirow[b]{2}{*}{ Date and Time } & \multicolumn{10}{|c|}{ Depth from Top of Casing (ft) } \\
\hline & MW1D & MW2D & MW3D & MW4D & MW7 & MW9 & Oentrich & MW14D & MW15D & MW16D \\
\hline 8/5/07 14:30 & 122.635 & 120.25 & & 98.077 & 102.261 & 93.709 & 107.943 & 104.659 & 76.513 & 72.131 \\
\hline $8 / 5 / 07 \quad 15: 00$ & 122.618 & 120.243 & & 98.061 & 102.246 & 93.69 & 107.93 & 104.641 & 76.507 & 72.112 \\
\hline 8/5/07 15:30 & 122.604 & 120.229 & & 98.046 & 102.231 & 93.673 & 107.917 & 104.623 & 76.496 & 72.098 \\
\hline 8/5/07 16:00 & 122.595 & 120.227 & & 98.04 & 102.223 & 93.666 & 107.908 & 104.614 & 76.487 & 72.087 \\
\hline 8/5/07 16:30 & 122.583 & 120.218 & & 98.032 & 102.215 & 93.657 & 107.898 & 104.606 & 76.482 & 72.077 \\
\hline $8 / 5 / 07$ 17:00 & 122.58 & 120.211 & & 98.027 & 102.212 & 93.655 & 107.897 & 104.603 & 76.481 & 72.075 \\
\hline $8 / 5 / 07 \quad 17: 30$ & 122.573 & 120.197 & & 98.018 & 102.202 & 93.645 & 107.887 & 104.592 & 76.472 & 72.067 \\
\hline 8/5/07 18:00 & 122.566 & 120.193 & & 98.011 & 102.2 & 93.64 & 107.883 & 104.585 & 76.467 & 72.06 \\
\hline 8/5/07 18:30 & 122.55 & 120.188 & & 97.999 & 102.183 & 93.626 & 107.868 & 104.572 & 76.455 & 72.046 \\
\hline 8/5/07 19:00 & 122.545 & 120.186 & & 97.992 & 102.177 & 93.619 & 107.866 & 104.566 & 76.45 & 72.04 \\
\hline $8 / 5 / 07 \quad 19: 30$ & 122.54 & 120.186 & & 97.989 & 102.172 & 93.612 & 107.859 & 104.559 & 76.441 & 72.034 \\
\hline $8 / 5 / 0720: 00$ & 122.54 & 120.193 & & 97.989 & 102.174 & 93.617 & 107.859 & 104.561 & 76.439 & 72.036 \\
\hline $8 / 5 / 0720: 30$ & 122.538 & 120.195 & & 97.99 & 102.174 & 93.614 & 107.861 & 104.559 & 76.434 & 72.036 \\
\hline 8/5/07 21:00 & 122.545 & 120.2 & & 97.997 & 102.183 & 93.622 & 107.868 & 104.568 & 76.432 & 72.042 \\
\hline 8/5/07 21:30 & 122.547 & 120.211 & & 98.001 & 102.183 & 93.624 & 107.87 & 104.568 & 76.428 & 72.044 \\
\hline 8/5/07 22:00 & 122.552 & 120.197 & & 98.006 & 102.189 & 93.631 & 107.874 & 104.572 & 76.426 & 72.048 \\
\hline 8/5/07 22:30 & 122.566 & 120.207 & & 98.018 & 102.2 & 93.643 & 107.883 & 104.586 & 76.424 & 72.059 \\
\hline 8/5/07 23:00 & 122.554 & 120.227 & & 97.999 & 102.187 & 93.631 & 107.876 & 104.575 & 76.42 & 72.048 \\
\hline 8/5/07 23:30 & 122.557 & 120.225 & & 98.015 & 102.193 & 93.633 & 107.878 & 104.577 & 76.419 & 72.05 \\
\hline $8 / 6 / 070: 00$ & 122.58 & 120.216 & & 98.03 & 102.215 & 93.659 & 107.889 & 104.603 & 76.423 & 72.077 \\
\hline 8/6/07 0:30 & 122.578 & 120.204 & & 98.028 & 102.212 & 93.657 & 107.889 & 104.601 & 76.417 & 72.067 \\
\hline 8/6/07 1:00 & 122.571 & 120.202 & & 98.018 & 102.208 & 93.647 & 107.885 & 104.592 & 76.411 & 72.063 \\
\hline 8/6/07 1:30 & 122.559 & 120.202 & & 98.008 & 102.193 & 93.633 & 107.878 & 104.579 & 76.408 & 72.048 \\
\hline 8/6/07 2:00 & 122.557 & 120.195 & & 98.008 & 102.191 & 93.633 & 107.876 & 104.577 & 76.402 & 72.046 \\
\hline 8/6/07 2:30 & 122.557 & 120.193 & & 98.007 & 102.191 & 93.636 & 107.876 & 104.577 & 76.404 & 72.044 \\
\hline 8/6/07 3:00 & 122.547 & 120.195 & & 97.999 & 102.181 & 93.624 & 107.868 & 104.568 & 76.396 & 72.036 \\
\hline 8/6/07 3:30 & 122.545 & 120.193 & & 97.999 & 102.181 & 93.624 & 107.866 & 104.568 & 76.395 & 72.036 \\
\hline 8/6/07 4:00 & 122.55 & 120.197 & & 98.003 & 102.185 & 93.626 & 107.87 & 104.568 & 76.393 & 72.04 \\
\hline 8/6/07 4:30 & 122.55 & 120.195 & & 97.999 & 102.181 & 93.624 & 107.868 & 104.568 & 76.393 & 72.038 \\
\hline 8/6/07 5:00 & 122.55 & 120.188 & & 98.003 & 102.183 & 93.626 & 107.868 & 104.568 & 76.391 & 72.038 \\
\hline 8/6/07 5:30 & 122.547 & 120.191 & & 97.997 & 102.179 & 93.622 & 107.866 & 104.566 & 76.389 & 72.036 \\
\hline 8/6/07 6:00 & 122.54 & 122.095 & & 97.992 & 102.177 & 93.617 & 107.862 & 104.561 & 76.384 & 72.029 \\
\hline 8/6/07 6:30 & 122.547 & 122.467 & & 97.997 & 102.183 & 93.626 & 107.866 & 104.566 & 76.385 & 72.036 \\
\hline 8/6/07 7:00 & 124.497 & 122.628 & & 98.717 & 102.632 & 94.237 & 108.473 & 105.907 & 76.398 & 72.071 \\
\hline 8/6/07 7:30 & 124.904 & 122.728 & & 99.019 & 102.975 & 94.588 & 108.799 & 106.343 & 76.434 & 72.292 \\
\hline 8/6/07 8:00 & 125.071 & 122.793 & & 99.164 & 103.129 & 94.739 & 108.948 & 106.516 & 76.461 & 72.437 \\
\hline 8/6/07 8:30 & 125.176 & 122.841 & & 99.253 & 103.228 & 94.838 & 109.038 & 106.625 & 76.485 & 72.54 \\
\hline 8/6/07 9:00 & 125.242 & 122.877 & & 99.315 & 103.293 & 94.901 & 109.098 & 106.693 & 76.509 & 72.608 \\
\hline 8/6/07 9:30 & 125.294 & 122.907 & & 99.36 & 103.344 & 94.953 & 109.145 & 106.749 & 76.533 & 72.66 \\
\hline $8 / 6 / 07$ 10:00 & 125.329 & 122.911 & & 99.391 & 103.377 & 94.989 & 109.177 & 106.784 & 76.552 & 72.697 \\
\hline 8/6/07 10:30 & 125.36 & 122.93 & & 99.418 & 103.403 & 95.017 & 109.201 & 106.813 & 76.576 & 72.728 \\
\hline 8/6/07 11:00 & 125.362 & 122.941 & & 99.424 & 103.409 & 95.019 & 109.207 & 106.815 & 76.587 & 72.732 \\
\hline $8 / 6 / 0711: 30$ & 125.383 & 122.957 & & 99.448 & 103.432 & 95.04 & 109.229 & 106.839 & 76.597 & 72.759 \\
\hline $8 / 6 / 07$ 12:00 & 125.395 & 122.959 & & 99.451 & 103.44 & 95.047 & 109.237 & 106.85 & 76.605 & 72.765 \\
\hline $8 / 6 / 0712: 30$ & 125.409 & 122.952 & & 99.472 & 103.455 & 95.066 & 109.248 & 106.864 & 76.62 & 72.782 \\
\hline 8/6/07 13:00 & 125.416 & 121.542 & & 99.469 & 103.462 & 95.073 & 109.256 & 106.873 & 76.631 & 72.792 \\
\hline 8/6/07 13:30 & 125.403 & 120.848 & & 99.463 & 103.447 & 95.059 & 109.244 & 106.859 & 76.636 & 72.782 \\
\hline $8 / 6 / 0714: 00$ & 124.202 & 120.628 & & 99.022 & 103.367 & 94.892 & 109.057 & 106.523 & 76.644 & 72.796 \\
\hline $8 / 6 / 07 \quad 14: 30$ & 123.226 & 120.504 & & 98.556 & 102.785 & 94.227 & 108.426 & 105.261 & 76.622 & 72.639 \\
\hline $8 / 6 / 07$ 15:00 & 122.994 & 120.417 & & 98.369 & 102.575 & 94.018 & 108.236 & 105.017 & 76.599 & 72.472 \\
\hline $8 / 6 / 07 \quad 15: 30$ & 122.864 & 120.361 & & 98.261 & 102.459 & 93.9 & 108.123 & 104.887 & 76.585 & 72.356 \\
\hline $8 / 6 / 0716: 00$ & 122.777 & 120.324 & & 98.185 & 102.379 & 93.822 & 108.05 & 104.798 & 76.564 & 72.269 \\
\hline 8/6/07 16:30 & 122.718 & 120.29 & & 98.133 & 102.326 & 93.768 & 108.001 & 104.738 & 76.553 & 72.211 \\
\hline $8 / 6 / 07 \quad 17: 00$ & 122.68 & 120.263 & & 98.106 & 102.29 & 93.732 & 107.966 & 104.698 & 76.538 & 72.168 \\
\hline $8 / 6 / 07$ 17:30 & 122.647 & 120.247 & & 98.073 & 102.259 & 93.704 & 107.938 & 104.665 & 76.526 & 72.137 \\
\hline 8/6/07 18:00 & 122.618 & 120.231 & & 98.051 & 102.234 & 93.68 & 107.913 & 104.639 & 76.513 & 72.11 \\
\hline 8/6/07 18:30 & 122.602 & 120.22 & & 98.035 & 102.219 & 93.664 & 107.9 & 104.621 & 76.503 & 72.094 \\
\hline $8 / 6 / 07$ 19:00 & 122.588 & 120.216 & & 98.023 & 102.21 & 93.65 & 107.887 & 104.606 & 76.49 & 72.079 \\
\hline $8 / 6 / 07$ 19:30 & 122.576 & 120.209 & & 98.015 & 102.196 & 93.64 & 107.878 & 104.592 & 76.483 & 72.069 \\
\hline $8 / 6 / 0720: 00$ & 122.571 & 120.207 & & 98.011 & 102.193 & 93.638 & 107.872 & 104.588 & 76.476 & 72.063 \\
\hline $8 / 6 / 0720: 30$ & 122.564 & 120.202 & & 98.004 & 102.189 & 93.631 & 107.868 & 104.581 & 76.468 & 72.058 \\
\hline $8 / 6 / 0721: 00$ & 122.557 & 120.204 & & 97.999 & 102.185 & 93.629 & 107.864 & 104.577 & 76.465 & 72.052 \\
\hline $8 / 6 / 0721: 30$ & 122.554 & 120.209 & & 97.997 & 102.181 & 93.624 & 107.861 & 104.572 & 76.454 & 72.048 \\
\hline 8/6/07 22:00 & 122.557 & 120.209 & & 98.001 & 102.183 & 93.629 & 107.864 & 104.574 & 76.447 & 72.052 \\
\hline 8/6/07 22:30 & 122.562 & 120.193 & & 98.006 & 102.193 & 93.633 & 107.87 & 104.579 & 76.443 & 72.054 \\
\hline 8/6/07 23:00 & 122.559 & 120.188 & & 98.006 & 102.189 & 93.633 & 107.87 & 104.579 & 76.437 & 72.054 \\
\hline
\end{tabular}


TABLE S1.1 (Cont.)

\begin{tabular}{|c|c|c|c|c|c|c|c|c|c|c|}
\hline \multirow[b]{2}{*}{ Date and Time } & \multicolumn{10}{|c|}{ Depth from Top of Casing (ft) } \\
\hline & MW1D & MW2D & MW3D & MW4D & MW7 & MW9 & Oentrich & MW14D & MW15D & MW16D \\
\hline 8/6/07 23:30 & 122.547 & 120.2 & & 97.99 & 102.174 & 93.619 & 107.857 & 104.568 & 76.426 & 72.042 \\
\hline $8 / 7 / 070: 00$ & 122.538 & 120.197 & & 97.99 & 102.17 & 93.612 & 107.849 & 104.559 & 76.421 & 72.034 \\
\hline 8/7/07 0:30 & 122.552 & 120.209 & & 98.001 & 102.185 & 93.629 & 107.864 & 104.572 & 76.419 & 72.048 \\
\hline 8/7/07 1:00 & 122.55 & 120.211 & & 98.001 & 102.181 & 93.624 & 107.862 & 104.57 & 76.415 & 72.044 \\
\hline 8/7/07 1:30 & 122.562 & 120.213 & & 98.013 & 102.193 & 93.633 & 107.87 & 104.579 & 76.413 & 72.052 \\
\hline 8/7/07 2:00 & 122.564 & 120.213 & & 98.011 & 102.196 & 93.638 & 107.872 & 104.586 & 76.413 & 72.056 \\
\hline 8/7/07 2:30 & 122.562 & 120.209 & & 98.016 & 102.194 & 93.638 & 107.872 & 104.581 & 76.41 & 72.052 \\
\hline 8/7/07 3:00 & 122.564 & 120.216 & & 98.013 & 102.196 & 93.638 & 107.874 & 104.583 & 76.408 & 72.054 \\
\hline 8/7/07 3:30 & 122.559 & 120.259 & & 98.006 & 102.191 & 93.636 & 107.87 & 104.579 & 76.402 & 72.052 \\
\hline 8/7/07 4:00 & 122.564 & 120.256 & & 98.027 & 102.196 & 93.638 & 107.872 & 104.581 & 76.402 & 72.052 \\
\hline 8/7/07 4:30 & 122.606 & 120.247 & & 98.068 & 102.242 & 93.685 & 107.889 & 104.623 & 76.413 & 72.096 \\
\hline 8/7/07 5:00 & 122.609 & 120.207 & & 98.063 & 102.24 & 93.685 & 107.894 & 104.628 & 76.413 & 72.096 \\
\hline 8/7/07 5:30 & 122.602 & 120.227 & & 98.051 & 102.236 & 93.678 & 107.891 & 104.621 & 76.411 & 72.087 \\
\hline 8/7/07 6:00 & 122.566 & 122.29 & & 98.003 & 102.191 & 93.64 & 107.874 & 104.586 & 76.396 & 72.048 \\
\hline 8/7/07 6:30 & 122.583 & 122.583 & & 98.035 & 102.215 & 93.662 & 107.885 & 104.601 & 76.4 & 72.073 \\
\hline 8/7/07 7:00 & 124.679 & 122.739 & & 98.792 & 102.737 & 94.333 & 108.538 & 106.124 & 76.42 & 72.151 \\
\hline 8/7/07 7:30 & 124.991 & 122.846 & & 99.036 & 103.009 & 94.609 & 108.807 & 106.454 & 76.443 & 72.34 \\
\hline 8/7/07 8:00 & 125.159 & 122.907 & & 99.174 & 103.162 & 94.762 & 108.944 & 106.627 & 76.472 & 72.49 \\
\hline 8/7/07 8:30 & 125.265 & 122.95 & & 99.274 & 103.268 & 94.866 & 109.03 & 106.735 & 76.498 & 72.6 \\
\hline 8/7/07 9:00 & 125.334 & 122.975 & & 99.334 & 103.335 & 94.932 & 109.088 & 106.808 & 76.522 & 72.67 \\
\hline 8/7/07 9:30 & 125.374 & 122.986 & & 99.374 & 103.375 & 94.972 & 109.126 & 106.853 & 76.546 & 72.716 \\
\hline 8/7/07 10:00 & 125.398 & 123.002 & & 99.396 & 103.398 & 94.996 & 109.152 & 106.877 & 76.557 & 72.741 \\
\hline $8 / 7 / 07$ 10:30 & 125.409 & 123.004 & & 99.412 & 103.413 & 95.017 & 109.175 & 106.897 & 76.573 & 72.763 \\
\hline 8/7/07 11:00 & 125.426 & 123.013 & & 99.426 & 103.434 & 95.031 & 109.19 & 106.912 & 76.59 & 72.784 \\
\hline 8/7/07 11:30 & 125.428 & 123.025 & & 99.431 & 103.434 & 95.036 & 109.201 & 106.917 & 76.598 & 72.788 \\
\hline $8 / 7 / 07$ 12:00 & 125.435 & 123.029 & & 99.438 & 103.441 & 95.04 & 109.209 & 106.921 & 76.608 & 72.798 \\
\hline $8 / 7 / 07$ 12:30 & 125.447 & 123.034 & & 99.451 & 103.457 & 95.055 & 109.222 & 106.935 & 76.623 & 72.813 \\
\hline 8/7/07 13:00 & 125.454 & 123.043 & & 99.46 & 103.466 & 95.062 & 109.229 & 106.943 & 76.638 & 72.825 \\
\hline 8/7/07 13:30 & 125.457 & 123.047 & & 99.465 & 103.472 & 95.066 & 109.235 & 106.948 & 76.649 & 72.832 \\
\hline 8/7/07 14:00 & 125.466 & 123.052 & & 99.476 & 103.481 & 95.076 & 109.242 & 106.957 & 76.66 & 72.842 \\
\hline 8/7/07 14:30 & 125.471 & 121.408 & & 99.479 & 103.485 & 95.083 & 109.248 & 106.959 & 76.671 & 72.848 \\
\hline $8 / 7 / 07$ 15:00 & 125.476 & 120.887 & & 99.486 & 103.493 & 95.09 & 109.253 & 106.966 & 76.682 & 72.859 \\
\hline $8 / 7 / 07$ 15:30 & 123.875 & 120.694 & & 98.985 & 103.28 & 94.751 & 108.903 & 106.042 & 76.691 & 72.865 \\
\hline 8/7/07 16:00 & 123.259 & 120.594 & & 98.6 & 102.826 & 94.27 & 108.45 & 105.292 & 76.671 & 72.698 \\
\hline 8/7/07 16:30 & 123.058 & 120.524 & & 98.44 & 102.646 & 94.088 & 108.283 & 105.084 & 76.653 & 72.544 \\
\hline $8 / 7 / 07$ 17:00 & 122.954 & 120.467 & & 98.354 & 102.55 & 93.996 & 108.191 & 104.975 & 76.64 & 72.449 \\
\hline 8/7/07 17:30 & 122.885 & 120.424 & & 98.294 & 102.488 & 93.933 & 108.131 & 104.904 & 76.629 & 72.377 \\
\hline 8/7/07 18:00 & 122.826 & 120.39 & & 98.244 & 102.436 & 93.881 & 108.09 & 104.845 & 76.612 & 72.317 \\
\hline 8/7/07 18:30 & 122.781 & 120.363 & & 98.206 & 102.394 & 93.841 & 108.058 & 104.803 & 76.597 & 72.271 \\
\hline $8 / 7 / 07$ 19:00 & 122.748 & 120.354 & & 98.171 & 102.358 & 93.805 & 108.029 & 104.767 & 76.583 & 72.236 \\
\hline 8/7/07 19:30 & 122.718 & 120.343 & & 98.151 & 102.333 & 93.779 & 108.005 & 104.736 & 76.57 & 72.208 \\
\hline 8/7/07 20:00 & 122.71 & 120.352 & & 98.146 & 102.328 & 93.775 & 107.999 & 104.73 & 76.562 & 72.203 \\
\hline 8/7/07 20:30 & 122.699 & 120.352 & & 98.135 & 102.318 & 93.763 & 107.988 & 104.716 & 76.553 & 72.189 \\
\hline 8/7/07 21:00 & 122.701 & 120.361 & & 98.144 & 102.322 & 93.77 & 107.988 & 104.721 & 76.549 & 72.193 \\
\hline 8/7/07 21:30 & 122.706 & 120.361 & & 98.149 & 102.328 & 93.777 & 107.99 & 104.725 & 76.548 & 72.197 \\
\hline 8/7/07 22:00 & 122.715 & 120.354 & & 98.159 & 102.339 & 93.787 & 107.986 & 104.732 & 76.544 & 72.205 \\
\hline 8/7/07 22:30 & 122.715 & 120.329 & & 98.158 & 102.339 & 93.787 & 107.982 & 104.734 & 76.538 & 72.203 \\
\hline 8/7/07 23:00 & 122.708 & 120.295 & & 98.15 & 102.33 & 93.777 & 107.979 & 104.725 & 76.531 & 72.195 \\
\hline 8/7/07 23:30 & 122.687 & 120.284 & & 98.121 & 102.309 & 93.756 & 107.973 & 104.705 & 76.52 & 72.174 \\
\hline 8/8/07 0:00 & 122.651 & 120.288 & & 98.089 & 102.273 & 93.718 & 107.947 & 104.668 & 76.502 & 72.135 \\
\hline 8/8/07 0:30 & 122.637 & 120.293 & & 98.078 & 102.263 & 93.709 & 107.935 & 104.654 & 76.494 & 72.125 \\
\hline 8/8/07 1:00 & 122.642 & 120.304 & & 98.089 & 102.269 & 93.711 & 107.941 & 104.659 & 76.49 & 72.133 \\
\hline 8/8/07 1:30 & 122.649 & 120.318 & & 98.092 & 102.276 & 93.723 & 107.947 & 104.668 & 76.489 & 72.141 \\
\hline 8/8/07 2:00 & 122.656 & 120.299 & & 98.108 & 102.286 & 93.73 & 107.95 & 104.674 & 76.485 & 72.149 \\
\hline 8/8/07 2:30 & 122.67 & 120.288 & & 98.121 & 102.303 & 93.746 & 107.95 & 104.69 & 76.489 & 72.164 \\
\hline 8/8/07 3:00 & 122.654 & 120.284 & & 98.1 & 102.282 & 93.728 & 107.947 & 104.674 & 76.476 & 72.143 \\
\hline 8/8/07 3:30 & 122.642 & 120.268 & & 98.088 & 102.273 & 93.721 & 107.941 & 104.663 & 76.468 & 72.133 \\
\hline 8/8/07 4:00 & 122.637 & 120.256 & & 98.085 & 102.267 & 93.713 & 107.937 & 104.657 & 76.465 & 72.129 \\
\hline 8/8/07 4:30 & 122.623 & 120.256 & & 98.071 & 102.253 & 93.695 & 107.926 & 104.645 & 76.455 & 72.114 \\
\hline 8/8/07 5:00 & 122.611 & 120.263 & & 98.056 & 102.238 & 93.678 & 107.915 & 104.63 & 76.448 & 72.098 \\
\hline 8/8/07 5:30 & 122.611 & 120.263 & & 98.058 & 102.24 & 93.68 & 107.913 & 104.628 & 76.445 & 72.1 \\
\hline 8/8/07 6:00 & 122.614 & 122.184 & & 98.069 & 102.244 & 93.685 & 107.919 & 104.63 & 76.443 & 72.104 \\
\hline 8/8/07 6:30 & 122.616 & 122.533 & & 98.064 & 102.246 & 93.688 & 107.92 & 104.637 & 76.439 & 72.104 \\
\hline 8/8/07 7:00 & 124.589 & 122.707 & & 98.805 & 102.724 & 94.326 & 108.549 & 106.007 & 76.455 & 72.156 \\
\hline 8/8/07 7:30 & 124.97 & 122.816 & & 99.095 & 103.051 & 94.659 & 108.859 & 106.414 & 76.487 & 72.373 \\
\hline 8/8/07 8:00 & 125.156 & 122.891 & & 99.253 & 103.221 & 94.828 & 109.011 & 106.603 & 76.518 & 72.534 \\
\hline
\end{tabular}


TABLE S1.1 (Cont.)

\begin{tabular}{|c|c|c|c|c|c|c|c|c|c|c|}
\hline \multirow[b]{2}{*}{ Date and Time } & \multicolumn{10}{|c|}{ Depth from Top of Casing (ft) } \\
\hline & MW1D & MW2D & MW3D & MW4D & MW7 & MW9 & Oentrich & MW14D & MW15D & MW16D \\
\hline 8/8/07 8:30 & 125.263 & 122.934 & & 99.353 & 103.325 & 94.932 & 109.101 & 106.715 & 76.55 & 72.643 \\
\hline 8/8/07 9:00 & 125.343 & 122.966 & & 99.42 & 103.4 & 95.005 & 109.16 & 106.793 & 76.572 & 72.72 \\
\hline 8/8/07 9:30 & 125.39 & 121.066 & & 99.465 & 103.447 & 95.055 & 109.201 & 106.846 & 76.601 & 72.774 \\
\hline $8 / 8 / 07$ 10:00 & 125.419 & 120.76 & & 99.491 & 103.478 & 95.083 & 109.229 & 106.875 & 76.622 & 72.805 \\
\hline $8 / 8 / 07 \quad 10: 30$ & 123.46 & 120.615 & & 98.748 & 102.994 & 94.437 & 108.594 & 105.509 & 76.625 & 72.759 \\
\hline $8 / 8 / 0711: 00$ & 123.129 & 120.519 & & 98.504 & 102.712 & 94.149 & 108.328 & 105.159 & 76.61 & 72.594 \\
\hline 8/8/07 11:30 & 122.975 & 120.454 & & 98.373 & 102.577 & 94.015 & 108.204 & 105.002 & 76.601 & 72.47 \\
\hline $8 / 8 / 07 \quad 12: 00$ & 122.881 & 120.408 & & 98.294 & 102.484 & 93.928 & 108.129 & 104.902 & 76.59 & 72.373 \\
\hline 8/8/07 12:30 & 122.81 & 120.377 & & 98.233 & 102.421 & 93.862 & 108.078 & 104.834 & 76.577 & 72.302 \\
\hline 8/8/07 13:00 & 122.765 & 120.345 & & 98.197 & 102.379 & 93.822 & 108.041 & 104.787 & 76.565 & 72.257 \\
\hline 8/8/07 13:30 & 122.736 & 120.322 & & 98.166 & 102.352 & 93.794 & 108.018 & 104.754 & 76.561 & 72.224 \\
\hline 8/8/07 14:00 & 122.701 & 120.306 & & 98.137 & 102.32 & 93.763 & 107.99 & 104.721 & 76.548 & 72.193 \\
\hline $8 / 8 / 07 \quad 14: 30$ & 122.68 & 120.29 & & 98.114 & 102.299 & 93.739 & 107.969 & 104.696 & 76.537 & 72.17 \\
\hline $8 / 8 / 07$ 15:00 & 122.658 & 120.272 & & 98.101 & 102.282 & 93.723 & 107.954 & 104.676 & 76.527 & 72.152 \\
\hline 8/8/07 15:30 & 122.642 & 120.259 & & 98.082 & 102.267 & 93.709 & 107.939 & 104.661 & 76.518 & 72.135 \\
\hline 8/8/07 16:00 & 122.625 & 120.245 & & 98.07 & 102.25 & 93.692 & 107.926 & 104.645 & 76.513 & 72.118 \\
\hline 8/8/07 16:30 & 122.614 & 120.234 & & 98.058 & 102.238 & 93.683 & 107.917 & 104.632 & 76.502 & 72.106 \\
\hline $8 / 8 / 07$ 17:00 & 122.599 & 120.227 & & 98.044 & 102.227 & 93.669 & 107.902 & 104.617 & 76.491 & 72.094 \\
\hline $8 / 8 / 07 \quad 17: 30$ & 122.588 & 120.211 & & 98.032 & 102.214 & 93.657 & 107.893 & 104.606 & 76.483 & 72.079 \\
\hline 8/8/07 18:00 & 122.58 & 120.218 & & 98.025 & 102.21 & 93.65 & 107.885 & 104.599 & 76.474 & 72.073 \\
\hline $8 / 8 / 07$ 18:30 & 122.566 & 120.225 & & 98.011 & 102.193 & 93.633 & 107.874 & 104.583 & 76.467 & 72.058 \\
\hline 8/8/07 19:00 & 122.571 & 120.225 & & 98.021 & 102.202 & 93.638 & 107.877 & 104.588 & 76.463 & 72.065 \\
\hline $8 / 8 / 07$ 19:30 & 122.58 & 120.247 & & 98.03 & 102.21 & 93.65 & 107.885 & 104.597 & 76.459 & 72.073 \\
\hline $8 / 8 / 0720: 00$ & 122.578 & 120.263 & & 98.028 & 102.21 & 93.647 & 107.883 & 104.592 & 76.455 & 72.069 \\
\hline $8 / 8 / 07 \quad 20: 30$ & 122.599 & 120.268 & & 98.056 & 102.233 & 93.671 & 107.9 & 104.614 & 76.459 & 72.094 \\
\hline $8 / 8 / 0721: 00$ & 122.618 & 120.286 & & 98.068 & 102.253 & 93.69 & 107.905 & 104.634 & 76.463 & 72.112 \\
\hline $8 / 8 / 0721: 30$ & 122.625 & 120.288 & & 98.075 & 102.257 & 93.699 & 107.907 & 104.641 & 76.465 & 72.118 \\
\hline 8/8/07 22:00 & 122.637 & 120.315 & & 98.095 & 102.273 & 93.713 & 107.905 & 104.657 & 76.467 & 72.131 \\
\hline 8/8/07 22:30 & 122.642 & 120.329 & & 98.097 & 102.274 & 93.716 & 107.905 & 104.659 & 76.467 & 72.131 \\
\hline 8/8/07 23:00 & 122.668 & 120.358 & & 98.126 & 102.303 & 93.746 & 107.9 & 104.688 & 76.478 & 72.16 \\
\hline 8/8/07 23:30 & 122.682 & 120.358 & & 98.14 & 102.318 & 93.758 & 107.902 & 104.701 & 76.474 & 72.17 \\
\hline $8 / 9 / 07 \quad 0: 00$ & 122.708 & 120.372 & & 98.173 & 102.345 & 93.787 & 107.896 & 104.725 & 76.485 & 72.197 \\
\hline 8/9/07 0:30 & 122.715 & 120.363 & & 98.17 & 102.347 & 93.791 & 107.898 & 104.732 & 76.487 & 72.199 \\
\hline 8/9/07 1:00 & 122.727 & 120.358 & & 98.182 & 102.362 & 93.803 & 107.898 & 104.745 & 76.489 & 72.209 \\
\hline 8/9/07 1:30 & 122.722 & 120.358 & & 98.173 & 102.354 & 93.801 & 107.898 & 104.743 & 76.487 & 72.205 \\
\hline 8/9/07 2:00 & 122.715 & 120.361 & & 98.164 & 102.345 & 93.789 & 107.898 & 104.732 & 76.485 & 72.197 \\
\hline 8/9/07 2:30 & 122.715 & 120.356 & & 98.166 & 102.347 & 93.791 & 107.898 & 104.732 & 76.485 & 72.197 \\
\hline 8/9/07 3:00 & 122.713 & 120.358 & & 98.17 & 102.347 & 93.791 & 107.896 & 104.732 & 76.485 & 72.199 \\
\hline 8/9/07 3:30 & 122.715 & 120.372 & & 98.164 & 102.343 & 93.787 & 107.896 & 104.727 & 76.483 & 72.193 \\
\hline 8/9/07 4:00 & 122.713 & 120.381 & & 98.169 & 102.345 & 93.787 & 107.894 & 104.73 & 76.483 & 72.195 \\
\hline 8/9/07 4:30 & 122.727 & 120.39 & & 98.183 & 102.362 & 93.805 & 107.894 & 104.745 & 76.489 & 72.211 \\
\hline 8/9/07 5:00 & 122.732 & 120.408 & & 98.19 & 102.368 & 93.81 & 107.89 & 104.75 & 76.487 & 72.215 \\
\hline 8/9/07 5:30 & 122.744 & 120.415 & & 98.202 & 102.377 & 93.82 & 107.89 & 104.761 & 76.493 & 72.226 \\
\hline 8/9/07 6:00 & 122.755 & 122.44 & & 98.221 & 102.4 & 93.843 & 107.889 & 104.783 & 76.502 & 72.249 \\
\hline 8/9/07 6:30 & 122.77 & 122.755 & & 98.228 & 102.404 & 93.848 & 107.887 & 104.789 & 76.507 & 72.253 \\
\hline 8/9/07 7:00 & 124.828 & 122.932 & & 98.962 & 102.899 & 94.487 & 108.576 & 106.272 & 76.526 & 72.315 \\
\hline 8/9/07 7:30 & 125.166 & 123.02 & & 99.229 & 103.198 & 94.793 & 108.855 & 106.636 & 76.559 & 72.526 \\
\hline ৪/9/07 8:00 & 125.338 & 123.093 & & 99.379 & 103.361 & 94.956 & 108.994 & 106.815 & 76.59 & 72.683 \\
\hline 8/9/07 8:30 & 125.44 & 123.134 & & 99.464 & 103.455 & 95.052 & 109.079 & 106.921 & 76.621 & 72.784 \\
\hline 8/9/07 9:00 & 125.509 & 123.172 & & 99.529 & 103.521 & 95.118 & 109.135 & 106.992 & 76.655 & 72.854 \\
\hline 8/9/07 9:30 & 125.554 & 123.195 & & 99.574 & 103.567 & 95.163 & 109.175 & 107.039 & 76.677 & 72.906 \\
\hline 8/9/07 10:00 & 125.584 & 123.215 & & 99.608 & 103.603 & 95.198 & 109.201 & 107.072 & 76.704 & 72.943 \\
\hline 8/9/07 10:30 & 125.615 & 123.231 & & 99.632 & 103.634 & 95.231 & 109.223 & 107.107 & 76.728 & 72.974 \\
\hline 8/9/07 11:00 & 125.637 & 123.247 & & 99.651 & 103.651 & 95.253 & 109.242 & 107.127 & 76.747 & 72.997 \\
\hline $8 / 9 / 0711: 30$ & 125.653 & 123.254 & & 99.672 & 103.67 & 95.269 & 109.255 & 107.143 & 76.767 & 73.018 \\
\hline 8/9/07 12:00 & 125.667 & 123.254 & & 99.684 & 103.683 & 95.283 & 109.267 & 107.158 & 76.787 & 73.032 \\
\hline 8/9/07 12:30 & 125.676 & 123.254 & & 99.691 & 103.693 & 95.293 & 109.274 & 107.167 & 76.804 & 73.046 \\
\hline 8/9/07 13:00 & 125.674 & 121.367 & & 99.694 & 103.697 & 95.293 & 109.282 & 107.167 & 76.819 & 73.049 \\
\hline $8 / 9 / 0713: 30$ & 125.674 & 121.016 & & 99.696 & 103.698 & 95.295 & 109.289 & 107.167 & 76.833 & 73.057 \\
\hline 8/9/07 14:00 & 123.767 & 120.855 & & 99.026 & 103.28 & 94.732 & 108.735 & 105.832 & 76.835 & 73.015 \\
\hline 8/9/07 14:30 & 123.384 & 120.757 & & 98.748 & 102.96 & 94.404 & 108.429 & 105.416 & 76.817 & 72.84 \\
\hline $8 / 9 / 07$ 15:00 & 123.216 & 120.696 & & 98.609 & 102.806 & 94.251 & 108.29 & 105.243 & 76.804 & 72.699 \\
\hline 8/9/07 15:30 & 123.117 & 120.653 & & 98.526 & 102.716 & 94.161 & 108.211 & 105.139 & 76.793 & 72.602 \\
\hline 8/9/07 16:00 & 123.053 & 120.619 & & 98.471 & 102.657 & 94.102 & 108.159 & 105.072 & 76.784 & 72.538 \\
\hline 8/9/07 16:30 & 123.011 & 120.596 & & 98.435 & 102.617 & 94.062 & 108.123 & 105.028 & 76.773 & 72.492 \\
\hline 8/9/07 17:00 & 122.975 & 120.583 & & 98.404 & 102.587 & 94.032 & 108.099 & 104.993 & 76.763 & 72.459 \\
\hline
\end{tabular}


TABLE S1.1 (Cont.)

\begin{tabular}{|c|c|c|c|c|c|c|c|c|c|c|}
\hline \multirow[b]{2}{*}{ Date and Time } & \multicolumn{10}{|c|}{ Depth from Top of Casing (ft) } \\
\hline & MW1D & MW2D & MW3D & MW4D & MW7 & MW9 & Oentrich & MW14D & MW15D & MW16D \\
\hline 8/9/07 17:30 & 122.954 & 120.565 & & 98.386 & 102.568 & 94.013 & 108.078 & 104.973 & 76.758 & 72.435 \\
\hline $8 / 9 / 07$ 18:00 & 122.935 & 120.549 & & 98.371 & 102.55 & 93.994 & 108.063 & 104.953 & 76.75 & 72.418 \\
\hline 8/9/07 18:30 & 122.918 & 120.544 & & 98.356 & 102.533 & 93.98 & 108.054 & 104.935 & 76.743 & 72.399 \\
\hline 8/9/07 19:00 & 122.904 & 120.542 & & 98.343 & 102.52 & 93.966 & 108.044 & 104.92 & 76.738 & 72.385 \\
\hline 8/9/07 19:30 & 122.899 & 120.542 & & 98.34 & 102.516 & 93.963 & 108.039 & 104.916 & 76.734 & 72.383 \\
\hline $8 / 9 / 0720: 00$ & 122.897 & 120.54 & & 98.342 & 102.516 & 93.961 & 108.029 & 104.913 & 76.729 & 72.381 \\
\hline $8 / 9 / 0720: 30$ & 122.897 & 120.544 & & 98.34 & 102.52 & 93.963 & 108.024 & 104.913 & 76.729 & 72.381 \\
\hline $8 / 9 / 0721: 00$ & 122.895 & 120.551 & & 98.338 & 102.514 & 93.961 & 108.02 & 104.911 & 76.725 & 72.377 \\
\hline 8/9/07 21:30 & 122.897 & 120.56 & & 98.347 & 102.52 & 93.966 & 108.014 & 104.913 & 76.726 & 72.383 \\
\hline 8/9/07 22:00 & 122.907 & 120.558 & & 98.354 & 102.53 & 93.973 & 108.009 & 104.922 & 76.726 & 72.387 \\
\hline 8/9/07 22:30 & 122.914 & 120.556 & & 98.361 & 102.537 & 93.982 & 108.007 & 104.931 & 76.725 & 72.393 \\
\hline 8/9/07 23:00 & 122.914 & 120.553 & & 98.362 & 102.537 & 93.982 & 108.003 & 104.931 & 76.726 & 72.395 \\
\hline 8/9/07 23:30 & 122.911 & 120.547 & & 98.361 & 102.537 & 93.98 & 107.999 & 104.927 & 76.723 & 72.393 \\
\hline 8/10/07 0:00 & 122.907 & 120.54 & & 98.355 & 102.531 & 93.977 & 107.997 & 104.924 & 76.721 & 72.389 \\
\hline 8/10/07 0:30 & 122.899 & 120.538 & & 98.345 & 102.526 & 93.97 & 107.996 & 104.918 & 76.719 & 72.383 \\
\hline 8/10/07 1:00 & 122.892 & 120.544 & & 98.342 & 102.518 & 93.966 & 107.994 & 104.911 & 76.714 & 72.377 \\
\hline $8 / 10 / 07$ 1:30 & 122.89 & 120.547 & & 98.338 & 102.514 & 93.961 & 107.992 & 104.907 & 76.71 & 72.375 \\
\hline $8 / 10 / 072: 00$ & 122.895 & 120.572 & & 98.349 & 102.522 & 93.968 & 107.986 & 104.911 & 76.712 & 72.379 \\
\hline 8/10/07 2:30 & 122.897 & 120.596 & & 98.354 & 102.528 & 93.973 & 107.984 & 104.916 & 76.71 & 72.383 \\
\hline $8 / 10 / 07$ 3:00 & 122.921 & 120.601 & & 98.381 & 102.554 & 93.999 & 107.982 & 104.938 & 76.717 & 72.41 \\
\hline 8/10/07 3:30 & 122.956 & 120.599 & & 98.412 & 102.587 & 94.039 & 107.977 & 104.973 & 76.726 & 72.445 \\
\hline 8/10/07 4:00 & 122.956 & 120.59 & & 98.414 & 102.585 & 94.036 & 107.977 & 104.975 & 76.728 & 72.443 \\
\hline $8 / 10 / 074: 30$ & 122.956 & 120.581 & & 98.404 & 102.581 & 94.032 & 107.975 & 104.971 & 76.726 & 72.435 \\
\hline 8/10/07 5:00 & 122.959 & 120.562 & & 98.392 & 102.569 & 94.02 & 107.977 & 104.96 & 76.725 & 72.424 \\
\hline 8/10/07 5:30 & 122.959 & 120.56 & & 98.383 & 102.56 & 94.01 & 107.977 & 104.953 & 76.725 & 72.414 \\
\hline 8/10/07 6:00 & 122.916 & 122.546 & & 98.366 & 102.543 & 93.992 & 107.977 & 104.933 & 76.717 & 72.395 \\
\hline 8/10/07 6:30 & 122.911 & 122.855 & & 98.433 & 102.537 & 93.987 & 107.973 & 104.929 & 76.715 & 72.394 \\
\hline 8/10/07 7:00 & 124.963 & 122.998 & & 99.151 & 103.072 & 94.685 & 108.71 & 106.388 & 76.734 & 72.462 \\
\hline 8/10/07 7:30 & 125.294 & 123.077 & & 99.415 & 103.367 & 94.981 & 108.992 & 106.735 & 76.771 & 72.683 \\
\hline 8/10/07 8:00 & 125.447 & 123.138 & & 99.543 & 103.506 & 95.123 & 109.112 & 106.893 & 76.8 & 72.82 \\
\hline 8/10/07 8:30 & 125.528 & 123.179 & & 99.613 & 103.584 & 95.198 & 109.183 & 106.979 & 76.82 & 72.902 \\
\hline 8/10/07 9:00 & 125.589 & 123.217 & & 99.67 & 103.645 & 95.26 & 109.226 & 107.043 & 76.844 & 72.968 \\
\hline 8/10/07 9:30 & 125.632 & 123.22 & & 99.708 & 103.685 & 95.302 & 109.256 & 107.087 & 76.867 & 73.013 \\
\hline 8/10/07 10:00 & 125.672 & 123.238 & & 99.737 & 103.725 & 95.354 & 109.269 & 107.118 & 76.889 & 73.042 \\
\hline 8/10/07 10:30 & 125.674 & 123.245 & & 99.743 & 103.721 & 95.337 & 109.328 & 107.129 & 76.905 & 73.055 \\
\hline 8/10/07 11:00 & 125.691 & 121.229 & & 99.763 & 103.742 & 95.354 & 109.333 & 107.149 & 76.926 & 73.075 \\
\hline 8/10/07 11:30 & 125.703 & 120.971 & & 99.768 & 103.75 & 95.37 & 109.383 & 107.158 & 76.944 & 73.09 \\
\hline 8/10/07 12:00 & 123.616 & 120.832 & & 98.919 & 103.156 & 94.609 & 108.639 & 105.653 & 76.933 & 72.98 \\
\hline 8/10/07 12:30 & 123.337 & 120.751 & & 98.709 & 102.912 & 94.362 & 108.426 & 105.361 & 76.92 & 72.805 \\
\hline 8/10/07 13:00 & 123.197 & 120.694 & & 98.593 & 102.785 & 94.234 & 108.3 & 105.217 & 76.903 & 72.685 \\
\hline 8/10/07 13:30 & 123.11 & 120.651 & & 98.523 & 102.708 & 94.159 & 108.217 & 105.13 & 76.894 & 72.6 \\
\hline 8/10/07 14:00 & 123.051 & 120.619 & & 98.473 & 102.653 & 94.107 & 108.162 & 105.068 & 76.883 & 72.542 \\
\hline 8/10/07 14:30 & 123.011 & 120.594 & & 98.435 & 102.613 & 94.065 & 108.126 & 105.024 & 76.874 & 72.499 \\
\hline 8/10/07 15:00 & 122.975 & 120.567 & & 98.405 & 102.585 & 94.034 & 108.099 & 104.991 & 76.865 & 72.466 \\
\hline 8/10/07 15:30 & 122.951 & 120.551 & & 98.383 & 102.562 & 94.013 & 108.079 & 104.966 & 76.85 & 72.443 \\
\hline 8/10/07 16:00 & 122.925 & 120.538 & & 98.363 & 102.539 & 93.989 & 108.064 & 104.942 & 76.841 & 72.418 \\
\hline 8/10/07 16:30 & 122.911 & 120.526 & & 98.344 & 102.528 & 94.001 & 108.046 & 104.922 & 76.832 & 72.397 \\
\hline 8/10/07 17:00 & 122.89 & 120.515 & & 98.332 & 102.507 & 93.959 & 108.073 & 104.907 & 76.824 & 72.383 \\
\hline 8/10/07 17:30 & 122.883 & 120.508 & & 98.325 & 102.499 & 93.954 & 108.057 & 104.898 & 76.817 & 72.377 \\
\hline 8/10/07 18:00 & 122.871 & 120.499 & & 98.314 & 102.49 & 93.942 & 108.044 & 104.887 & 76.808 & 72.364 \\
\hline 8/10/07 18:30 & 122.862 & 120.494 & & 98.307 & 102.482 & 93.933 & 108.034 & 104.878 & 76.804 & 72.356 \\
\hline 8/10/07 19:00 & 122.855 & 120.492 & & 98.299 & 102.474 & 93.923 & 108.027 & 104.871 & 76.797 & 72.348 \\
\hline 8/10/07 19:30 & 122.85 & 120.492 & & 98.295 & 102.471 & 93.921 & 108.019 & 104.865 & 76.791 & 72.344 \\
\hline 8/10/07 20:00 & 122.847 & 120.492 & & 98.294 & 102.469 & 93.916 & 108.015 & 104.86 & 76.785 & 72.34 \\
\hline 8/10/07 20:30 & 122.845 & 120.492 & & 98.29 & 102.467 & 93.916 & 107.986 & 104.86 & 76.784 & 72.337 \\
\hline 8/10/07 21:00 & 122.843 & 120.494 & & 98.289 & 102.465 & 93.914 & 108.058 & 104.858 & 76.78 & 72.338 \\
\hline 8/10/07 21:30 & 122.845 & 120.497 & & 98.29 & 102.469 & 93.919 & 108.067 & 104.86 & 76.778 & 72.34 \\
\hline 8/10/07 22:00 & 122.847 & 120.492 & & 98.297 & 102.472 & 93.921 & 108.074 & 104.862 & 76.776 & 72.344 \\
\hline 8/10/07 22:30 & 122.852 & 120.494 & & 98.299 & 102.476 & 93.923 & 108.049 & 104.867 & 76.774 & 72.344 \\
\hline 8/10/07 23:00 & 122.843 & 120.494 & & 98.295 & 102.469 & 93.916 & 108.031 & 104.86 & 76.771 & 72.337 \\
\hline 8/10/07 23:30 & 122.845 & 120.49 & & 98.295 & 102.469 & 93.921 & 108.016 & 104.86 & 76.769 & 72.337 \\
\hline 8/11/07 0:00 & 122.847 & 120.494 & & 98.299 & 102.472 & 93.921 & 108.007 & 104.865 & 76.765 & 72.342 \\
\hline $8 / 11 / 07$ 0:30 & 122.845 & 120.488 & & 98.295 & 102.471 & 93.919 & 107.999 & 104.862 & 76.763 & 72.34 \\
\hline 8/11/07 1:00 & 122.847 & 120.485 & & 98.299 & 102.474 & 93.921 & 107.993 & 104.865 & 76.761 & 72.342 \\
\hline 8/11/07 1:30 & 122.84 & 120.481 & & 98.292 & 102.465 & 93.914 & 107.991 & 104.858 & 76.758 & 72.335 \\
\hline 8/11/07 2:00 & 122.838 & 120.472 & & 98.287 & 102.465 & 93.916 & 107.988 & 104.856 & 76.754 & 72.333 \\
\hline
\end{tabular}


TABLE S1.1 (Cont.)

\begin{tabular}{|c|c|c|c|c|c|c|c|c|c|c|}
\hline \multirow[b]{2}{*}{ Date and Time } & \multicolumn{10}{|c|}{ Depth from Top of Casing (ft) } \\
\hline & MW1D & MW2D & MW3D & MW4D & MW7 & MW9 & Oentrich & MW14D & MW15D & MW16D \\
\hline 8/11/07 2:30 & 122.831 & 120.467 & & 98.281 & 102.457 & 93.907 & 107.986 & 104.847 & 76.749 & 72.325 \\
\hline 8/11/07 3:00 & 122.826 & 120.474 & & 98.278 & 102.453 & 93.902 & 107.984 & 104.843 & 76.747 & 72.321 \\
\hline 8/11/07 3:30 & 122.821 & 120.472 & & 98.275 & 102.451 & 93.897 & 107.98 & 104.838 & 76.739 & 72.317 \\
\hline 8/11/07 4:00 & 122.826 & 120.47 & & 98.282 & 102.457 & 93.904 & 107.978 & 104.845 & 76.739 & 72.323 \\
\hline $8 / 11 / 074: 30$ & 122.826 & 120.463 & & 98.28 & 102.453 & 93.902 & 107.976 & 104.843 & 76.739 & 72.321 \\
\hline $8 / 11 / 075: 00$ & 122.824 & 120.467 & & 98.275 & 102.453 & 93.897 & 107.974 & 104.838 & 76.732 & 72.317 \\
\hline 8/11/07 5:30 & 122.824 & 120.465 & & 98.268 & 102.44 & 93.888 & 107.972 & 104.831 & 76.728 & 72.309 \\
\hline 8/11/07 6:00 & 122.819 & 120.465 & & 98.276 & 102.451 & 93.897 & 107.97 & 104.836 & 76.73 & 72.315 \\
\hline 8/11/07 6:30 & 122.819 & 122.331 & & 98.271 & 102.446 & 93.895 & 107.968 & 104.836 & 76.725 & 72.313 \\
\hline 8/11/07 7:00 & 122.819 & 122.723 & & 98.273 & 102.448 & 93.895 & 107.966 & 104.836 & 76.726 & 72.313 \\
\hline 8/11/07 7:30 & 124.724 & 122.893 & & 98.969 & 102.868 & 94.472 & 108.541 & 106.12 & 76.734 & 72.34 \\
\hline 8/11/07 8:00 & 125.159 & 122.995 & & 99.286 & 103.23 & 94.845 & 108.884 & 106.598 & 76.769 & 72.559 \\
\hline 8/11/07 8:30 & 125.334 & 123.063 & & 99.434 & 103.394 & 95.01 & 109.04 & 106.777 & 76.796 & 72.714 \\
\hline 8/11/07 9:00 & 125.442 & 123.12 & & 99.529 & 103.495 & 95.113 & 109.13 & 106.888 & 76.826 & 72.819 \\
\hline 8/11/07 9:30 & 125.513 & 123.156 & & 99.591 & 103.563 & 95.179 & 109.192 & 106.961 & 76.852 & 72.892 \\
\hline $8 / 11 / 0710: 00$ & 125.568 & 123.12 & & 99.643 & 103.617 & 95.229 & 109.234 & 107.019 & 76.879 & 72.947 \\
\hline $8 / 11 / 07 \quad 10: 30$ & 125.606 & 123.134 & & 99.675 & 103.649 & 95.267 & 109.265 & 107.056 & 76.898 & 72.985 \\
\hline 8/11/07 11:00 & 125.566 & 123.131 & & 99.674 & 103.658 & 95.271 & 109.269 & 107.039 & 76.918 & 73.008 \\
\hline 8/11/07 11:30 & 125.58 & 123.134 & & 99.682 & 103.67 & 95.283 & 109.282 & 107.05 & 76.937 & 73.022 \\
\hline $8 / 11 / 0712: 00$ & 125.582 & 123.136 & & 99.687 & 103.672 & 95.286 & 109.291 & 107.054 & 76.949 & 73.03 \\
\hline 8/11/07 12:30 & 125.584 & 123.138 & & 99.688 & 103.672 & 95.288 & 109.303 & 107.056 & 76.961 & 73.032 \\
\hline 8/11/07 13:00 & 125.585 & 121.578 & & 99.687 & 103.676 & 95.288 & 109.312 & 107.059 & 76.972 & 73.037 \\
\hline 8/11/07 13:30 & 125.587 & 121.073 & & 99.693 & 103.679 & 95.293 & 109.318 & 107.063 & 76.985 & 73.043 \\
\hline $8 / 11 / 0714: 00$ & 124.041 & 120.884 & & 99.155 & 103.445 & 94.923 & 108.94 & 106.18 & 76.994 & 73.045 \\
\hline $8 / 11 / 0714: 30$ & 123.443 & 120.78 & & 98.788 & 103.004 & 94.454 & 108.497 & 105.473 & 76.977 & 72.884 \\
\hline 8/11/07 15:00 & 123.247 & 120.71 & & 98.635 & 102.83 & 94.277 & 108.332 & 105.27 & 76.962 & 72.736 \\
\hline 8/11/07 15:30 & 123.138 & 120.667 & & 98.543 & 102.729 & 94.178 & 108.244 & 105.159 & 76.949 & 72.633 \\
\hline 8/11/07 16:00 & 123.063 & 120.63 & & 98.479 & 102.665 & 94.114 & 108.184 & 105.082 & 76.935 & 72.561 \\
\hline 8/11/07 16:30 & 123.022 & 120.606 & & 98.445 & 102.623 & 94.076 & 108.144 & 105.04 & 76.926 & 72.518 \\
\hline 8/11/07 17:00 & 122.982 & 120.59 & & 98.411 & 102.59 & 94.041 & 108.114 & 105 & 76.916 & 72.481 \\
\hline 8/11/07 17:30 & 122.961 & 120.578 & & 98.392 & 102.569 & 94.022 & 108.096 & 104.975 & 76.909 & 72.456 \\
\hline 8/11/07 18:00 & 122.944 & 120.569 & & 98.38 & 102.556 & 94.008 & 108.081 & 104.958 & 76.9 & 72.439 \\
\hline 8/11/07 18:30 & 122.933 & 120.567 & & 98.369 & 102.545 & 93.996 & 108.067 & 104.947 & 76.894 & 72.426 \\
\hline 8/11/07 19:00 & 122.923 & 120.572 & & 98.364 & 102.537 & 93.989 & 108.058 & 104.938 & 76.885 & 72.418 \\
\hline 8/11/07 19:30 & 122.921 & 120.572 & & 98.364 & 102.539 & 93.989 & 108.048 & 104.935 & 76.885 & 72.418 \\
\hline 8/11/07 20:00 & 122.925 & 120.576 & & 98.368 & 102.543 & 93.994 & 108.043 & 104.94 & 76.881 & 72.421 \\
\hline 8/11/07 20:30 & 122.928 & 120.581 & & 98.373 & 102.547 & 93.996 & 108.037 & 104.94 & 76.879 & 72.421 \\
\hline 8/11/07 21:00 & 122.93 & 120.587 & & 98.375 & 102.549 & 94.001 & 108.031 & 104.942 & 76.879 & 72.422 \\
\hline 8/11/07 21:30 & 122.933 & 120.596 & & 98.383 & 102.554 & 94.006 & 108.028 & 104.947 & 76.878 & 72.427 \\
\hline 8/11/07 22:00 & 122.942 & 120.601 & & 98.392 & 102.564 & 94.018 & 108.022 & 104.955 & 76.878 & 72.437 \\
\hline 8/11/07 22:30 & 122.951 & 120.599 & & 98.397 & 102.571 & 94.02 & 108.02 & 104.964 & 76.879 & 72.443 \\
\hline 8/11/07 23:00 & 122.954 & 120.601 & & 98.402 & 102.573 & 94.025 & 108.016 & 104.966 & 76.879 & 72.445 \\
\hline 8/11/07 23:30 & 122.951 & 120.603 & & 98.402 & 102.571 & 94.025 & 108.015 & 104.966 & 76.876 & 72.443 \\
\hline 8/12/07 0:00 & 122.954 & 120.601 & & 98.402 & 102.575 & 94.029 & 108.011 & 104.969 & 76.878 & 72.445 \\
\hline 8/12/07 0:30 & 122.956 & 120.603 & & 98.406 & 102.577 & 94.029 & 108.009 & 104.971 & 76.876 & 72.447 \\
\hline $8 / 12 / 071: 00$ & 122.956 & 120.601 & & 98.407 & 102.579 & 94.032 & 108.005 & 104.971 & 76.874 & 72.45 \\
\hline 8/12/07 1:30 & 122.956 & 120.603 & & 98.407 & 102.581 & 94.032 & 108.003 & 104.971 & 76.872 & 72.447 \\
\hline 8/12/07 2:00 & 122.956 & 120.606 & & 98.409 & 102.581 & 94.032 & 108.001 & 104.971 & 76.872 & 72.449 \\
\hline 8/12/07 2:30 & 122.956 & 120.606 & & 98.406 & 102.579 & 94.032 & 107.998 & 104.971 & 76.867 & 72.447 \\
\hline 8/12/07 3:00 & 122.956 & 120.606 & & 98.413 & 102.585 & 94.034 & 107.998 & 104.973 & 76.87 & 72.451 \\
\hline 8/12/07 3:30 & 122.959 & 120.608 & & 98.411 & 102.583 & 94.034 & 107.994 & 104.973 & 76.868 & 72.449 \\
\hline 8/12/07 4:00 & 122.959 & 120.608 & & 98.411 & 102.585 & 94.036 & 107.994 & 104.973 & 76.865 & 72.449 \\
\hline 8/12/07 4:30 & 122.959 & 120.61 & & 98.412 & 102.587 & 94.036 & 107.99 & 104.973 & 76.865 & 72.452 \\
\hline 8/12/07 5:00 & 122.961 & 120.619 & & 98.414 & 102.589 & 94.039 & 107.988 & 104.975 & 76.861 & 72.453 \\
\hline 8/12/07 5:30 & 122.966 & 120.624 & & 98.419 & 102.592 & 94.043 & 107.985 & 104.98 & 76.863 & 72.457 \\
\hline 8/12/07 6:00 & 122.97 & 120.628 & & 98.426 & 102.598 & 94.048 & 107.985 & 104.986 & 76.861 & 72.461 \\
\hline 8/12/07 6:30 & 122.973 & 122.462 & & 98.431 & 102.6 & 94.051 & 107.983 & 104.989 & 76.865 & 72.466 \\
\hline 8/12/07 7:00 & 122.98 & 122.911 & & 98.435 & 102.604 & 94.055 & 107.983 & 104.993 & 76.865 & 72.468 \\
\hline $8 / 12 / 077: 30$ & 124.807 & 123.102 & & 99.029 & 102.922 & 94.51 & 108.435 & 106.184 & 76.87 & 72.483 \\
\hline 8/12/07 8:00 & 125.315 & 123.206 & & 99.384 & 103.337 & 94.944 & 108.841 & 106.775 & 76.907 & 72.691 \\
\hline 8/12/07 8:30 & 125.513 & 123.281 & & 99.549 & 103.521 & 95.128 & 109.023 & 106.981 & 76.938 & 72.861 \\
\hline 8/12/07 9:00 & 125.627 & 123.331 & & 99.646 & 103.628 & 95.231 & 109.145 & 107.098 & 76.966 & 72.972 \\
\hline 8/12/07 9:30 & 125.698 & 123.378 & & 99.713 & 103.698 & 95.307 & 109.222 & 107.174 & 76.994 & 73.049 \\
\hline 8/12/07 10:00 & 125.752 & 123.397 & & 99.761 & 103.752 & 95.354 & 109.263 & 107.229 & 77.014 & 73.105 \\
\hline 8/12/07 10:30 & 125.792 & 123.417 & & 99.801 & 103.79 & 95.396 & 109.295 & 107.271 & 77.04 & 73.146 \\
\hline 8/12/07 11:00 & 125.818 & 123.426 & & 99.825 & 103.815 & 95.422 & 109.319 & 107.298 & 77.064 & 73.173 \\
\hline
\end{tabular}


TABLE S1.1 (Cont.)

\begin{tabular}{|c|c|c|c|c|c|c|c|c|c|c|}
\hline \multirow[b]{2}{*}{ Date and Time } & \multicolumn{10}{|c|}{ Depth from Top of Casing (ft) } \\
\hline & MW1D & MW2D & MW3D & MW4D & MW7 & MW9 & Oentrich & MW14D & MW15D & MW16D \\
\hline 8/12/07 11:30 & 125.84 & 123.428 & & 99.842 & 103.832 & 95.441 & 109.34 & 107.32 & 77.08 & 73.194 \\
\hline 8/12/07 12:00 & 125.847 & 123.433 & & 99.851 & 103.845 & 95.453 & 109.355 & 107.329 & 77.099 & 73.208 \\
\hline 8/12/07 12:30 & 125.851 & 123.431 & & 99.856 & 103.851 & 95.458 & 109.368 & 107.333 & 77.117 & 73.216 \\
\hline 8/12/07 13:00 & 125.856 & 123.435 & & 99.858 & 103.853 & 95.46 & 109.377 & 107.335 & 77.13 & 73.222 \\
\hline 8/12/07 13:30 & 125.852 & 123.431 & & 99.856 & 103.853 & 95.46 & 109.389 & 107.335 & 77.145 & 73.225 \\
\hline 8/12/07 14:00 & 125.858 & 123.426 & & 99.863 & 103.856 & 95.465 & 109.396 & 107.342 & 77.161 & 73.23 \\
\hline 8/12/07 14:30 & 125.854 & 123.431 & & 99.86 & 103.855 & 95.462 & 109.406 & 107.338 & 77.171 & 73.229 \\
\hline 8/12/07 15:00 & 125.851 & 123.431 & & 99.854 & 103.851 & 95.458 & 109.411 & 107.335 & 77.182 & 73.231 \\
\hline 8/12/07 15:30 & 125.849 & 123.428 & & 99.86 & 103.851 & 95.46 & 109.415 & 107.333 & 77.193 & 73.235 \\
\hline 8/12/07 16:00 & 125.854 & 121.626 & & 99.86 & 103.86 & 95.465 & 109.421 & 107.34 & 77.2 & 73.241 \\
\hline 8/12/07 16:30 & 125.849 & 121.224 & & 99.858 & 103.855 & 95.462 & 109.428 & 107.338 & 77.209 & 73.241 \\
\hline 8/12/07 17:00 & 124.041 & 121.048 & & 99.25 & 103.516 & 94.979 & 108.946 & 106.127 & 77.208 & 73.221 \\
\hline 8/12/07 17:30 & 123.592 & 120.946 & & 98.946 & 103.154 & 94.609 & 108.583 & 105.622 & 77.193 & 73.055 \\
\hline 8/12/07 18:00 & 123.41 & 120.882 & & 98.791 & 102.986 & 94.442 & 108.435 & 105.434 & 77.176 & 72.904 \\
\hline 8/12/07 18:30 & 123.306 & 120.832 & & 98.71 & 102.893 & 94.35 & 108.347 & 105.323 & 77.16 & 72.807 \\
\hline 8/12/07 19:00 & 123.24 & 120.805 & & 98.652 & 102.832 & 94.289 & 108.29 & 105.254 & 77.145 & 72.736 \\
\hline 8/12/07 19:30 & 123.19 & 120.782 & & 98.609 & 102.787 & 94.241 & 108.253 & 105.203 & 77.132 & 72.687 \\
\hline 8/12/07 20:00 & 123.162 & 120.769 & & 98.585 & 102.762 & 94.22 & 108.225 & 105.175 & 77.125 & 72.658 \\
\hline 8/12/07 20:30 & 123.136 & 120.769 & & 98.564 & 102.739 & 94.197 & 108.206 & 105.15 & 77.115 & 72.631 \\
\hline 8/12/07 21:00 & 123.122 & 120.773 & & 98.557 & 102.728 & 94.182 & 108.189 & 105.135 & 77.108 & 72.619 \\
\hline 8/12/07 21:30 & 123.122 & 120.778 & & 98.559 & 102.729 & 94.185 & 108.174 & 105.135 & 77.106 & 72.619 \\
\hline 8/12/07 22:00 & 123.129 & 120.773 & & 98.564 & 102.735 & 94.192 & 108.163 & 105.141 & 77.104 & 72.623 \\
\hline 8/12/07 22:30 & 123.126 & 120.769 & & 98.571 & 102.741 & 94.194 & 108.153 & 105.141 & 77.104 & 72.623 \\
\hline 8/12/07 23:00 & 123.126 & 120.764 & & 98.567 & 102.737 & 94.194 & 108.148 & 105.137 & 77.097 & 72.621 \\
\hline 8/12/07 23:30 & 123.122 & 120.755 & & 98.564 & 102.731 & 94.19 & 108.142 & 105.135 & 77.095 & 72.617 \\
\hline 8/13/07 0:00 & 123.117 & 120.757 & & 98.56 & 102.729 & 94.19 & 108.136 & 105.133 & 77.093 & 72.613 \\
\hline 8/13/07 0:30 & 123.11 & 120.746 & & 98.55 & 102.72 & 94.178 & 108.134 & 105.124 & 77.088 & 72.605 \\
\hline 8/13/07 1:00 & 123.112 & 120.737 & & 98.554 & 102.724 & 94.18 & 108.129 & 105.126 & 77.084 & 72.607 \\
\hline 8/13/07 1:30 & 123.098 & 120.721 & & 98.541 & 102.714 & 94.166 & 108.125 & 105.113 & 77.079 & 72.592 \\
\hline 8/13/07 2:00 & 123.089 & 120.712 & & 98.531 & 102.707 & 94.159 & 108.123 & 105.102 & 77.069 & 72.584 \\
\hline 8/13/07 2:30 & 123.086 & 120.701 & & 98.519 & 102.689 & 94.147 & 108.119 & 105.09 & 77.062 & 72.571 \\
\hline $8 / 13 / 07$ 3:00 & 123.084 & 120.692 & & 98.509 & 102.68 & 94.133 & 108.118 & 105.077 & 77.055 & 72.561 \\
\hline 8/13/07 3:30 & 123.081 & 120.692 & & 98.498 & 102.67 & 94.126 & 108.112 & 105.068 & 77.049 & 72.553 \\
\hline 8/13/07 4:00 & 123.044 & 120.687 & & 98.492 & 102.663 & 94.116 & 108.11 & 105.059 & 77.042 & 72.543 \\
\hline 8/13/07 4:30 & 123.044 & 120.685 & & 98.49 & 102.661 & 94.116 & 108.106 & 105.057 & 77.036 & 72.54 \\
\hline 8/13/07 5:00 & 123.039 & 120.685 & & 98.488 & 102.659 & 94.112 & 108.103 & 105.053 & 77.034 & 72.538 \\
\hline 8/13/07 5:30 & 123.039 & 120.685 & & 98.486 & 102.657 & 94.107 & 108.099 & 105.051 & 77.027 & 72.536 \\
\hline 8/13/07 6:00 & 123.039 & 122.648 & & 98.486 & 102.659 & 94.114 & 108.095 & 105.053 & 77.025 & 72.538 \\
\hline 8/13/07 6:30 & 123.037 & 122.95 & & 98.485 & 102.655 & 94.109 & 108.095 & 105.048 & 77.018 & 72.534 \\
\hline 8/13/07 7:00 & 125.06 & 123.1 & & 99.267 & 103.179 & 94.793 & 108.767 & 106.481 & 77.034 & 72.602 \\
\hline 8/13/07 7:30 & 125.388 & 123.193 & & 99.574 & 103.472 & 95.09 & 109.038 & 106.833 & 77.064 & 72.811 \\
\hline 8/13/07 8:00 & 125.549 & 123.254 & & 99.649 & 103.617 & 95.241 & 109.182 & 106.994 & 77.093 & 72.954 \\
\hline 8/13/07 8:30 & 125.639 & 123.288 & & 99.734 & 103.7 & 95.319 & 109.294 & 107.09 & 77.119 & 73.047 \\
\hline 8/13/07 9:00 & 125.7 & 123.32 & & 99.813 & 103.761 & 95.377 & 109.367 & 107.156 & 77.143 & 73.113 \\
\hline 8/13/07 9:30 & 125.741 & 123.338 & & 99.823 & 103.805 & 95.425 & 109.729 & 107.194 & 77.163 & 73.152 \\
\hline 8/13/07 10:00 & 125.771 & 123.36 & & 99.849 & 103.832 & 95.462 & 109.429 & 107.227 & 77.18 & 73.179 \\
\hline 8/13/07 10:30 & 125.788 & 123.369 & & 99.87 & 103.849 & 95.465 & 109.647 & 107.247 & 77.2 & 73.206 \\
\hline 8/13/07 11:00 & 125.809 & 123.381 & & 99.891 & 103.866 & 95.488 & 109.491 & 107.269 & 77.219 & 73.226 \\
\hline 8/13/07 11:30 & 125.821 & 123.383 & & 99.901 & 103.879 & 95.498 & 109.483 & 107.28 & 77.235 & 73.241 \\
\hline 8/13/07 12:00 & 125.83 & 123.385 & & 99.909 & 103.887 & 95.507 & 109.482 & 107.291 & 77.252 & 73.249 \\
\hline 8/13/07 12:30 & 125.83 & 121.587 & & 99.911 & 103.891 & 95.509 & 109.479 & 107.295 & 77.263 & 73.256 \\
\hline 8/13/07 13:00 & 125.832 & 121.211 & & 99.913 & 103.893 & 95.514 & 109.478 & 107.293 & 77.276 & 73.26 \\
\hline 8/13/07 13:30 & 123.994 & 121.039 & & 99.229 & 103.479 & 94.948 & 108.92 & 106.053 & 77.276 & 73.224 \\
\hline 8/13/07 14:00 & 123.578 & 120.934 & & 98.927 & 103.135 & 94.593 & 108.604 & 105.602 & 77.257 & 73.042 \\
\hline $8 / 13 / 07$ 14:30 & 123.398 & 120.862 & & 98.784 & 102.977 & 94.435 & 108.459 & 105.42 & 77.243 & 72.896 \\
\hline 8/13/07 15:00 & 123.292 & 120.81 & & 98.691 & 102.878 & 94.333 & 108.37 & 105.307 & 77.222 & 72.794 \\
\hline 8/13/07 15:30 & 123.219 & 120.773 & & 98.628 & 102.809 & 94.267 & 108.311 & 105.23 & 77.209 & 72.722 \\
\hline 8/13/07 16:00 & 123.164 & 120.746 & & 98.583 & 102.756 & 94.215 & 108.271 & 105.177 & 77.193 & 72.664 \\
\hline 8/13/07 16:30 & 123.126 & 120.717 & & 98.552 & 102.728 & 94.182 & 108.243 & 105.141 & 77.182 & 72.629 \\
\hline 8/13/07 17:00 & 123.098 & 120.692 & & 98.526 & 102.699 & 94.159 & 108.222 & 105.113 & 77.169 & 72.6 \\
\hline 8/13/07 17:30 & 123.072 & 120.658 & & 98.499 & 102.672 & 94.131 & 108.205 & 105.082 & 77.152 & 72.571 \\
\hline 8/13/07 18:00 & 123.044 & 120.642 & & 98.476 & 102.648 & 94.105 & 108.192 & 105.057 & 77.141 & 72.546 \\
\hline 8/13/07 18:30 & 123.011 & 120.646 & & 98.445 & 102.617 & 94.074 & 108.183 & 105.024 & 77.121 & 72.516 \\
\hline 8/13/07 19:00 & 122.994 & 120.655 & & 98.432 & 102.602 & 94.06 & 108.175 & 105.004 & 77.11 & 72.499 \\
\hline 8/13/07 19:30 & 122.996 & 120.658 & & 98.442 & 102.608 & 94.065 & 108.164 & 105.009 & 77.106 & 72.503 \\
\hline 8/13/07 20:00 & 123.008 & 120.649 & & 98.452 & 102.619 & 94.076 & 108.154 & 105.02 & 77.103 & 72.513 \\
\hline
\end{tabular}


TABLE S1.1 (Cont.)

\begin{tabular}{|c|c|c|c|c|c|c|c|c|c|c|}
\hline \multirow[b]{2}{*}{ Date and Time } & \multicolumn{10}{|c|}{ Depth from Top of Casing (ft) } \\
\hline & MW1D & MW2D & MW3D & MW4D & MW7 & MW9 & Oentrich & MW14D & MW15D & MW16D \\
\hline 8/13/07 20:30 & 123.008 & 120.642 & & 98.452 & 102.623 & 94.076 & 108.149 & 105.02 & 77.097 & 72.515 \\
\hline 8/13/07 21:00 & 122.999 & 120.644 & & 98.44 & 102.611 & 94.067 & 108.15 & 105.013 & 77.09 & 72.501 \\
\hline 8/13/07 21:30 & 122.992 & 120.637 & & 98.435 & 102.608 & 94.06 & 108.145 & 105.004 & 77.082 & 72.497 \\
\hline 8/13/07 22:00 & 122.994 & 120.63 & & 98.44 & 102.608 & 94.062 & 108.139 & 105.006 & 77.077 & 72.497 \\
\hline 8/13/07 22:30 & 122.989 & 120.626 & & 98.435 & 102.606 & 94.06 & 108.137 & 105.002 & 77.071 & 72.492 \\
\hline 8/13/07 23:00 & 122.982 & 120.621 & & 98.426 & 102.598 & 94.053 & 108.133 & 104.993 & 77.064 & 72.485 \\
\hline 8/13/07 23:30 & 122.977 & 120.617 & & 98.425 & 102.594 & 94.048 & 108.13 & 104.991 & 77.062 & 72.483 \\
\hline 8/14/07 0:00 & 122.973 & 120.612 & & 98.419 & 102.589 & 94.043 & 108.126 & 104.984 & 77.056 & 72.476 \\
\hline 8/14/07 0:30 & 122.971 & 120.606 & & 98.414 & 102.587 & 94.041 & 108.126 & 104.982 & 77.049 & 72.472 \\
\hline 8/14/07 1:00 & 122.966 & 120.606 & & 98.409 & 102.581 & 94.034 & 108.122 & 104.978 & 77.045 & 72.468 \\
\hline $8 / 14 / 071: 30$ & 122.959 & 120.603 & & 98.404 & 102.573 & 94.027 & 108.12 & 104.969 & 77.038 & 72.461 \\
\hline 8/14/07 2:00 & 122.956 & 120.599 & & 98.404 & 102.575 & 94.027 & 108.118 & 104.971 & 77.032 & 72.46 \\
\hline 8/14/07 2:30 & 122.954 & 120.592 & & 98.399 & 102.571 & 94.025 & 108.116 & 104.964 & 77.027 & 72.458 \\
\hline 8/14/07 3:00 & 122.949 & 120.59 & & 98.398 & 102.568 & 94.022 & 108.112 & 104.962 & 77.027 & 72.455 \\
\hline 8/14/07 3:30 & 122.949 & 120.59 & & 98.392 & 102.56 & 94.015 & 108.111 & 104.955 & 77.016 & 72.447 \\
\hline 8/14/07 4:00 & 122.94 & 120.587 & & 98.388 & 102.558 & 94.01 & 108.109 & 104.951 & 77.01 & 72.443 \\
\hline 8/14/07 4:30 & 122.94 & 120.59 & & 98.388 & 102.558 & 94.01 & 108.107 & 104.951 & 77.005 & 72.443 \\
\hline 8/14/07 5:00 & 122.937 & 120.59 & & 98.388 & 102.558 & 94.013 & 108.103 & 104.951 & 77.003 & 72.443 \\
\hline 8/14/07 5:30 & 122.937 & 120.59 & & 98.392 & 102.558 & 94.013 & 108.101 & 104.949 & 76.997 & 72.443 \\
\hline 8/14/07 6:00 & 122.94 & 122.682 & & 98.39 & 102.558 & 94.013 & 108.099 & 104.951 & 76.992 & 72.443 \\
\hline 8/14/07 6:30 & 122.94 & 122.977 & & 98.394 & 102.56 & 94.015 & 108.099 & 104.953 & 76.99 & 72.443 \\
\hline 8/14/07 7:00 & 125.069 & 123.143 & & 99.184 & 103.116 & 94.72 & 108.786 & 106.516 & 77.007 & 72.54 \\
\hline 8/14/07 7:30 & 125.383 & 123.193 & & 99.431 & 103.419 & 95.05 & 109.969 & 106.831 & 77.036 & 72.737 \\
\hline 8/14/07 8:00 & 125.559 & 123.24 & & 99.579 & 103.594 & 95.227 & 110.184 & 107.014 & 77.069 & 72.884 \\
\hline 8/14/07 8:30 & 125.608 & 123.274 & & 99.625 & 103.611 & 95.215 & 109.281 & 107.083 & 77.09 & 72.979 \\
\hline 8/14/07 9:00 & 125.66 & 123.301 & & 99.67 & 103.662 & 95.262 & 109.325 & 107.136 & 77.108 & 73.034 \\
\hline 8/14/07 9:30 & 125.689 & 123.322 & & 99.7 & 103.691 & 95.293 & 109.374 & 107.169 & 77.121 & 73.07 \\
\hline 8/14/07 10:00 & 125.717 & 123.331 & & 99.723 & 103.721 & 95.33 & 109.363 & 107.198 & 77.134 & 73.096 \\
\hline 8/14/07 10:30 & 125.736 & 123.34 & & 99.746 & 103.74 & 95.342 & 109.487 & 107.22 & 77.15 & 73.123 \\
\hline 8/14/07 11:00 & 125.748 & 123.344 & & 99.753 & 103.748 & 95.352 & 109.454 & 107.231 & 77.163 & 73.135 \\
\hline 8/14/07 11:30 & 125.759 & 123.342 & & 99.762 & 103.761 & 95.375 & 109.448 & 107.242 & 77.178 & 73.148 \\
\hline 8/14/07 12:00 & 125.764 & 123.344 & & 99.796 & 103.763 & 95.37 & 109.49 & 107.247 & 77.191 & 73.154 \\
\hline 8/14/07 12:30 & 125.762 & 123.34 & & 99.789 & 103.767 & 95.373 & 109.512 & 107.249 & 77.202 & 73.159 \\
\hline 8/14/07 13:00 & 125.762 & 123.333 & & 99.784 & 103.769 & 95.373 & 109.515 & 107.249 & 77.211 & 73.163 \\
\hline 8/14/07 13:30 & 125.762 & 123.32 & & 99.789 & 103.773 & 95.38 & 109.513 & 107.249 & 77.222 & 73.164 \\
\hline 8/14/07 14:00 & 125.75 & 123.317 & & 99.767 & 103.765 & 95.37 & 109.535 & 107.238 & 77.23 & 73.165 \\
\hline 8/14/07 14:30 & 125.743 & 123.315 & & 99.758 & 103.757 & 95.363 & 109.534 & 107.231 & 77.239 & 73.158 \\
\hline 8/14/07 15:00 & 125.738 & 123.317 & & 99.758 & 103.756 & 95.361 & 109.535 & 107.229 & 77.244 & 73.161 \\
\hline 8/14/07 15:30 & 125.736 & 121.825 & & 99.755 & 103.754 & 95.356 & 109.533 & 107.227 & 77.252 & 73.16 \\
\hline 8/14/07 16:00 & 125.733 & 121.186 & & 99.753 & 103.752 & 95.359 & 109.515 & 107.227 & 77.256 & 73.159 \\
\hline 8/14/07 16:30 & 124.417 & 120.982 & & 99.322 & 103.653 & 95.168 & 109.288 & 106.726 & 77.261 & 73.165 \\
\hline 8/14/07 17:00 & 123.556 & 120.864 & & 98.892 & 103.106 & 94.564 & 108.751 & 105.589 & 77.237 & 73.003 \\
\hline 8/14/07 17:30 & 123.341 & 120.787 & & 98.719 & 102.914 & 94.369 & 108.552 & 105.363 & 77.215 & 72.844 \\
\hline 8/14/07 18:00 & 123.223 & 120.739 & & 98.619 & 102.804 & 94.258 & 108.436 & 105.239 & 77.197 & 72.733 \\
\hline $8 / 14 / 0718: 30$ & 123.143 & 120.705 & & 98.55 & 102.731 & 94.187 & 108.359 & 105.157 & 77.18 & 72.652 \\
\hline 8/14/07 19:00 & 123.091 & 120.676 & & 98.512 & 102.686 & 94.14 & 108.306 & 105.104 & 77.165 & 72.6 \\
\hline 8/14/07 19:30 & 123.06 & 120.658 & & 98.481 & 102.655 & 94.109 & 108.245 & 105.073 & 77.15 & 72.567 \\
\hline 8/14/07 20:00 & 123.029 & 120.649 & & 98.457 & 102.629 & 94.083 & 108.262 & 105.042 & 77.136 & 72.536 \\
\hline 8/14/07 20:30 & 123.011 & 120.644 & & 98.44 & 102.611 & 94.067 & 108.238 & 105.024 & 77.126 & 72.517 \\
\hline 8/14/07 21:00 & 123.001 & 120.635 & & 98.435 & 102.604 & 94.058 & 108.185 & 105.013 & 77.115 & 72.507 \\
\hline 8/14/07 21:30 & 122.994 & 120.626 & & 98.431 & 102.602 & 94.058 & 108.22 & 105.009 & 77.106 & 72.501 \\
\hline 8/14/07 22:00 & 122.987 & 120.626 & & 98.424 & 102.594 & 94.051 & 108.204 & 105 & 77.101 & 72.492 \\
\hline 8/14/07 22:30 & 122.982 & 120.615 & & 98.418 & 102.589 & 94.046 & 108.19 & 104.991 & 77.091 & 72.484 \\
\hline 8/14/07 23:00 & 122.977 & 120.61 & & 98.416 & 102.587 & 94.039 & 108.196 & 104.989 & 77.086 & 72.48 \\
\hline 8/14/07 23:30 & 122.966 & 120.606 & & 98.404 & 102.575 & 94.029 & 108.192 & 104.978 & 77.075 & 72.47 \\
\hline 8/15/07 0:00 & 122.961 & 120.601 & & 98.404 & 102.573 & 94.027 & 108.194 & 104.973 & 77.069 & 72.466 \\
\hline 8/15/07 0:30 & 122.956 & 120.594 & & 98.399 & 102.566 & 94.022 & 108.182 & 104.969 & 77.064 & 72.46 \\
\hline 8/15/07 1:00 & 122.951 & 120.576 & & 98.395 & 102.564 & 94.02 & 108.17 & 104.964 & 77.058 & 72.458 \\
\hline $8 / 15 / 071: 30$ & 122.949 & 120.578 & & 98.383 & 102.556 & 94.013 & 108.173 & 104.958 & 77.049 & 72.451 \\
\hline 8/15/07 2:00 & 122.947 & 120.576 & & 98.369 & 102.541 & 93.994 & 108.17 & 104.94 & 77.042 & 72.431 \\
\hline 8/15/07 2:30 & 122.942 & 120.572 & & 98.375 & 102.541 & 93.996 & 108.159 & 104.94 & 77.034 & 72.433 \\
\hline 8/15/07 3:00 & 122.937 & 120.567 & & 98.375 & 102.543 & 93.996 & 108.147 & 104.94 & 77.029 & 72.435 \\
\hline 8/15/07 3:30 & 122.935 & 120.565 & & 98.364 & 102.537 & 93.989 & 108.143 & 104.931 & 77.025 & 72.425 \\
\hline 8/15/07 4:00 & 122.935 & 120.567 & & 98.363 & 102.531 & 93.987 & 108.151 & 104.929 & 77.014 & 72.422 \\
\hline 8/15/07 4:30 & 122.93 & 120.562 & & 98.361 & 102.531 & 93.989 & 108.151 & 104.927 & 77.01 & 72.42 \\
\hline $8 / 15 / 075: 00$ & 122.928 & 120.558 & & 98.364 & 102.533 & 93.989 & 108.15 & 104.927 & 77.003 & 72.421 \\
\hline
\end{tabular}


TABLE S1.1 (Cont.)

\begin{tabular}{|c|c|c|c|c|c|c|c|c|c|c|}
\hline \multirow[b]{2}{*}{ Date and Time } & \multicolumn{10}{|c|}{ Depth from Top of Casing (ft) } \\
\hline & MW1D & MW2D & MW3D & MW4D & MW7 & MW9 & Oentrich & MW14D & MW15D & MW16D \\
\hline 8/15/07 5:30 & 122.925 & 120.565 & & 98.361 & 102.53 & 93.985 & 108.151 & 104.924 & 76.999 & 72.418 \\
\hline 8/15/07 6:00 & 122.925 & 122.605 & & 98.357 & 102.526 & 93.982 & 108.141 & 104.92 & 76.992 & 72.414 \\
\hline 8/15/07 6:30 & 122.918 & 122.861 & & 98.363 & 102.539 & 93.992 & 108.128 & 104.929 & 76.99 & 72.425 \\
\hline 8/15/07 7:00 & 125.024 & 122.995 & & 99.239 & 103.135 & 94.751 & 108.867 & 106.454 & 77.01 & 72.534 \\
\hline $8 / 15 / 07$ 7:30 & 125.296 & 123.079 & & 99.429 & 103.379 & 94.996 & 109.112 & 106.74 & 77.036 & 72.726 \\
\hline 8/15/07 8:00 & 125.44 & 123.134 & & 99.551 & 103.514 & 95.132 & 109.231 & 106.888 & 77.062 & 72.861 \\
\hline 8/15/07 8:30 & 125.525 & 123.17 & & 99.623 & 103.594 & 95.212 & 109.303 & 106.977 & 77.079 & 72.948 \\
\hline 8/15/07 9:00 & 125.582 & 123.197 & & 99.675 & 103.645 & 95.267 & 109.367 & 107.034 & 77.099 & 73.003 \\
\hline 8/15/07 9:30 & 125.62 & 123.224 & & 99.71 & 103.683 & 95.307 & 109.399 & 107.076 & 77.117 & 73.045 \\
\hline 8/15/07 10:00 & 125.648 & 123.24 & & 99.735 & 103.71 & 95.333 & 109.431 & 107.105 & 77.132 & 73.074 \\
\hline $8 / 15 / 07 \quad 10: 30$ & 125.672 & 123.245 & & 99.758 & 103.735 & 95.356 & 109.452 & 107.129 & 77.149 & 73.097 \\
\hline 8/15/07 11:00 & 125.688 & 123.254 & & 99.773 & 103.75 & 95.37 & 109.459 & 107.145 & 77.163 & 73.115 \\
\hline 8/15/07 11:30 & 125.696 & 123.256 & & 99.779 & 103.761 & 95.38 & 109.483 & 107.156 & 77.178 & 73.128 \\
\hline $8 / 15 / 07$ 12:00 & 125.702 & 123.265 & & 99.787 & 103.763 & 95.385 & 109.493 & 107.163 & 77.189 & 73.136 \\
\hline 8/15/07 12:30 & 125.7 & 121.63 & & 99.785 & 103.763 & 95.382 & 109.496 & 107.16 & 77.198 & 73.134 \\
\hline 8/15/07 13:00 & 125.712 & 121.166 & & 99.799 & 103.776 & 95.396 & 109.517 & 107.171 & 77.213 & 73.15 \\
\hline 8/15/07 13:30 & 124.067 & 120.977 & & 99.224 & 103.499 & 94.974 & 109.077 & 106.175 & 77.222 & 73.152 \\
\hline 8/15/07 14:00 & 123.533 & 120.871 & & 98.872 & 103.085 & 94.541 & 108.66 & 105.555 & 77.204 & 72.983 \\
\hline 8/15/07 14:30 & 123.337 & 120.796 & & 98.721 & 102.91 & 94.366 & 108.511 & 105.356 & 77.187 & 72.832 \\
\hline $8 / 15 / 07$ 15:00 & 123.228 & 120.744 & & 98.628 & 102.809 & 94.265 & 108.417 & 105.241 & 77.174 & 72.726 \\
\hline 8/15/07 15:30 & 123.152 & 120.71 & & 98.564 & 102.741 & 94.201 & 108.365 & 105.166 & 77.161 & 72.652 \\
\hline 8/15/07 16:00 & 123.098 & 120.683 & & 98.516 & 102.689 & 94.147 & 108.323 & 105.108 & 77.147 & 72.596 \\
\hline 8/15/07 16:30 & 123.063 & 120.664 & & 98.486 & 102.661 & 94.119 & 108.289 & 105.073 & 77.136 & 72.561 \\
\hline 8/15/07 17:00 & 123.037 & 120.653 & & 98.466 & 102.636 & 94.095 & 108.278 & 105.048 & 77.126 & 72.536 \\
\hline $8 / 15 / 07$ 17:30 & 123.015 & 120.637 & & 98.45 & 102.619 & 94.076 & 108.259 & 105.026 & 77.117 & 72.518 \\
\hline 8/15/07 18:00 & 123.006 & 120.624 & & 98.44 & 102.611 & 94.069 & 108.252 & 105.015 & 77.108 & 72.505 \\
\hline 8/15/07 18:30 & 122.987 & 120.619 & & 98.424 & 102.594 & 94.053 & 108.24 & 104.997 & 77.099 & 72.488 \\
\hline 8/15/07 19:00 & 122.973 & 120.61 & & 98.414 & 102.583 & 94.039 & 108.239 & 104.986 & 77.09 & 72.476 \\
\hline 8/15/07 19:30 & 122.973 & 120.658 & & 98.409 & 102.577 & 94.036 & 108.221 & 104.982 & 77.082 & 72.472 \\
\hline 8/15/07 20:00 & 122.966 & 120.628 & & 98.406 & 102.571 & 94.029 & 108.195 & 104.973 & 77.073 & 72.466 \\
\hline 8/15/07 20:30 & 123.013 & 120.633 & & 98.535 & 102.648 & 94.133 & 109.21 & 105.004 & 77.071 & 72.464 \\
\hline 8/15/07 21:00 & 122.982 & 120.633 & & 98.421 & 102.592 & 94.048 & 108.22 & 104.991 & 77.073 & 72.478 \\
\hline 8/15/07 21:30 & 122.982 & 120.637 & & 98.425 & 102.594 & 94.051 & 108.233 & 104.993 & 77.069 & 72.485 \\
\hline 8/15/07 22:00 & 122.985 & 120.635 & & 98.433 & 102.598 & 94.055 & 108.212 & 104.995 & 77.067 & 72.484 \\
\hline 8/15/07 22:30 & 122.987 & 120.635 & & 98.435 & 102.6 & 94.058 & 108.192 & 104.997 & 77.066 & 72.488 \\
\hline 8/15/07 23:00 & 122.987 & 120.63 & & 98.433 & 102.6 & 94.055 & 108.181 & 104.995 & 77.062 & 72.484 \\
\hline 8/15/07 23:30 & 122.985 & 120.63 & & 98.43 & 102.6 & 94.053 & 108.171 & 104.995 & 77.06 & 72.48 \\
\hline 8/16/07 0:00 & 122.982 & 120.637 & & 98.43 & 102.596 & 94.051 & 108.163 & 104.993 & 77.056 & 72.481 \\
\hline 8/16/07 0:30 & 122.982 & 120.64 & & 98.43 & 102.596 & 94.051 & 108.159 & 104.993 & 77.053 & 72.478 \\
\hline 8/16/07 1:00 & 122.987 & 120.649 & & 98.435 & 102.6 & 94.055 & 108.154 & 104.997 & 77.053 & 72.484 \\
\hline 8/16/07 1:30 & 122.989 & 120.649 & & 98.438 & 102.602 & 94.058 & 108.152 & 104.997 & 77.049 & 72.484 \\
\hline 8/16/07 2:00 & 122.996 & 120.651 & & 98.447 & 102.613 & 94.067 & 108.153 & 105.006 & 77.051 & 72.493 \\
\hline 8/16/07 2:30 & 123.001 & 120.658 & & 98.452 & 102.615 & 94.072 & 108.165 & 105.011 & 77.049 & 72.497 \\
\hline 8/16/07 3:00 & 123.003 & 120.66 & & 98.454 & 102.619 & 94.074 & 108.176 & 105.013 & 77.049 & 72.501 \\
\hline 8/16/07 3:30 & 123.006 & 120.658 & & 98.459 & 102.625 & 94.079 & 108.176 & 105.017 & 77.049 & 72.501 \\
\hline 8/16/07 4:00 & 123.008 & 120.653 & & 98.461 & 102.625 & 94.081 & 108.174 & 105.02 & 77.049 & 72.505 \\
\hline 8/16/07 4:30 & 123.011 & 120.655 & & 98.461 & 102.627 & 94.083 & 108.172 & 105.02 & 77.049 & 72.503 \\
\hline 8/16/07 5:00 & 123.008 & 120.651 & & 98.455 & 102.621 & 94.076 & 108.17 & 105.015 & 77.044 & 72.499 \\
\hline 8/16/07 5:30 & 123.008 & 120.66 & & 98.457 & 102.625 & 94.079 & 108.17 & 105.017 & 77.04 & 72.503 \\
\hline 8/16/07 6:00 & 123.001 & 122.666 & & 98.456 & 102.619 & 94.074 & 108.168 & 105.011 & 77.036 & 72.495 \\
\hline 8/16/07 6:30 & 123.003 & 122.982 & & 98.464 & 102.627 & 94.081 & 108.168 & 105.02 & 77.036 & 72.497 \\
\hline 8/16/07 7:00 & 125.045 & 123.183 & & 99.203 & 103.114 & 94.717 & 108.782 & 106.487 & 77.055 & 72.569 \\
\hline 8/16/07 7:30 & 125.388 & 123.245 & & 99.456 & 103.415 & 95.026 & 109.062 & 106.855 & 77.086 & 72.778 \\
\hline 8/16/07 8:00 & 125.596 & 123.315 & & 99.641 & 103.641 & 95.278 & 110.336 & 107.047 & 77.115 & 72.939 \\
\hline 8/16/07 8:30 & 125.665 & 123.358 & & 99.704 & 103.677 & 95.286 & 109.304 & 107.14 & 77.141 & 73.043 \\
\hline 8/16/07 9:00 & 125.733 & 123.39 & & 99.784 & 103.742 & 95.349 & 109.369 & 107.209 & 77.165 & 73.111 \\
\hline 8/16/07 9:30 & 125.778 & 123.419 & & 99.803 & 103.786 & 95.392 & 109.42 & 107.258 & 77.185 & 73.158 \\
\hline $8 / 16 / 07$ 10:00 & 125.806 & 123.44 & & 99.832 & 103.816 & 95.425 & 109.476 & 107.287 & 77.2 & 73.189 \\
\hline 8/16/07 10:30 & 125.845 & 123.462 & & 99.863 & 103.853 & 95.462 & 109.486 & 107.326 & 77.222 & 73.228 \\
\hline 8/16/07 11:00 & 125.856 & 123.49 & & 99.884 & 103.868 & 95.479 & 109.489 & 107.34 & 77.239 & 73.247 \\
\hline 8/16/07 11:30 & 125.882 & 123.512 & & 99.909 & 103.896 & 95.507 & 109.518 & 107.369 & 77.259 & 73.276 \\
\hline 8/16/07 12:00 & 125.908 & 123.524 & & 99.934 & 103.919 & 95.526 & 109.525 & 107.391 & 77.279 & 73.301 \\
\hline 8/16/07 12:30 & 125.932 & 123.53 & & 99.958 & 103.946 & 95.554 & 109.543 & 107.417 & 77.302 & 73.33 \\
\hline 8/16/07 13:00 & 125.946 & 123.53 & & 99.972 & 103.959 & 95.568 & 109.537 & 107.43 & 77.32 & 73.342 \\
\hline 8/16/07 13:30 & 125.953 & 123.528 & & 99.977 & 103.969 & 95.578 & 109.532 & 107.439 & 77.335 & 73.354 \\
\hline 8/16/07 14:00 & 125.951 & 121.764 & & 99.975 & 103.967 & 95.578 & 109.54 & 107.439 & 77.346 & 73.357 \\
\hline
\end{tabular}


TABLE S1.1 (Cont.)

\begin{tabular}{|c|c|c|c|c|c|c|c|c|c|c|}
\hline \multirow[b]{2}{*}{ Date and Time } & \multicolumn{10}{|c|}{ Depth from Top of Casing (ft) } \\
\hline & MW1D & MW2D & MW3D & MW4D & MW7 & MW9 & Oentrich & MW14D & MW15D & MW16D \\
\hline 8/16/07 14:30 & 125.951 & 121.345 & & 99.982 & 103.971 & 95.58 & 109.577 & 107.442 & 77.361 & 73.361 \\
\hline 8/16/07 15:00 & 124.183 & 121.17 & & 99.386 & 103.647 & 95.116 & 109.115 & 106.27 & 77.362 & 73.342 \\
\hline 8/16/07 15:30 & 123.713 & 121.066 & & 99.065 & 103.268 & 94.725 & 108.751 & 105.739 & 77.346 & 73.164 \\
\hline 8/16/07 16:00 & 123.533 & 120.995 & & 98.919 & 103.106 & 94.562 & 108.587 & 105.551 & 77.329 & 73.02 \\
\hline 8/16/07 16:30 & 123.426 & 120.943 & & 98.827 & 103.007 & 94.465 & 108.492 & 105.438 & 77.318 & 72.918 \\
\hline 8/16/07 17:00 & 123.353 & 120.914 & & 98.765 & 102.941 & 94.397 & 108.429 & 105.365 & 77.303 & 72.844 \\
\hline 8/16/07 17:30 & 123.299 & 120.887 & & 98.721 & 102.891 & 94.345 & 108.385 & 105.31 & 77.291 & 72.788 \\
\hline 8/16/07 18:00 & 123.27 & 120.871 & & 98.695 & 102.863 & 94.319 & 108.36 & 105.279 & 77.283 & 72.759 \\
\hline 8/16/07 18:30 & 123.247 & 120.864 & & 98.673 & 102.84 & 94.296 & 108.339 & 105.254 & 77.27 & 72.732 \\
\hline 8/16/07 19:00 & 123.226 & 120.857 & & 98.659 & 102.823 & 94.281 & 108.32 & 105.234 & 77.263 & 72.714 \\
\hline 8/16/07 19:30 & 123.219 & 120.893 & & 98.657 & 102.817 & 94.274 & 108.303 & 105.228 & 77.257 & 72.708 \\
\hline 8/16/07 20:00 & 123.211 & 120.941 & & 98.652 & 102.815 & 94.272 & 108.289 & 105.219 & 77.252 & 72.701 \\
\hline 8/16/07 20:30 & 123.249 & 120.889 & & 98.771 & 102.874 & 94.364 & 109.269 & 105.239 & 77.25 & 72.699 \\
\hline 8/16/07 21:00 & 123.304 & 120.875 & & 98.817 & 102.937 & 94.428 & 109.387 & 105.296 & 77.267 & 72.722 \\
\hline 8/16/07 21:30 & 123.247 & 120.873 & & 98.683 & 102.855 & 94.307 & 108.298 & 105.259 & 77.259 & 72.732 \\
\hline 8/16/07 22:00 & 123.233 & 120.871 & & 98.669 & 102.838 & 94.296 & 108.292 & 105.239 & 77.256 & 72.72 \\
\hline 8/16/07 22:30 & 123.228 & 120.871 & & 98.669 & 102.832 & 94.289 & 108.329 & 105.237 & 77.248 & 72.716 \\
\hline 8/16/07 23:00 & 123.221 & 120.878 & & 98.669 & 102.828 & 94.284 & 108.304 & 105.232 & 77.248 & 72.712 \\
\hline 8/16/07 23:30 & 123.228 & 120.875 & & 98.673 & 102.832 & 94.289 & 108.302 & 105.237 & 77.248 & 72.716 \\
\hline 8/17/07 0:00 & 123.233 & 120.869 & & 98.674 & 102.84 & 94.293 & 108.287 & 105.241 & 77.248 & 72.72 \\
\hline $8 / 17 / 07$ 0:30 & 123.228 & 120.862 & & 98.674 & 102.834 & 94.289 & 108.274 & 105.237 & 77.246 & 72.712 \\
\hline 8/17/07 1:00 & 123.223 & 120.859 & & 98.667 & 102.83 & 94.286 & 108.264 & 105.232 & 77.241 & 72.707 \\
\hline 8/17/07 1:30 & 123.219 & 120.855 & & 98.662 & 102.825 & 94.279 & 108.254 & 105.225 & 77.239 & 72.703 \\
\hline 8/17/07 2:00 & 123.211 & 120.857 & & 98.659 & 102.821 & 94.274 & 108.247 & 105.221 & 77.235 & 72.698 \\
\hline $8 / 17 / 072: 30$ & 123.209 & 120.853 & & 98.655 & 102.815 & 94.272 & 108.243 & 105.217 & 77.232 & 72.695 \\
\hline $8 / 17 / 07$ 3:00 & 123.207 & 120.844 & & 98.653 & 102.817 & 94.272 & 108.237 & 105.217 & 77.23 & 72.695 \\
\hline 8/17/07 3:30 & 123.202 & 120.841 & & 98.65 & 102.811 & 94.267 & 108.245 & 105.212 & 77.226 & 72.689 \\
\hline 8/17/07 4:00 & 123.2 & 120.835 & & 98.645 & 102.806 & 94.26 & 108.252 & 105.208 & 77.219 & 72.685 \\
\hline $8 / 17 / 074: 30$ & 123.197 & 120.83 & & 98.64 & 102.804 & 94.256 & 108.246 & 105.201 & 77.215 & 72.679 \\
\hline 8/17/07 5:00 & 123.188 & 120.83 & & 98.633 & 102.796 & 94.251 & 108.238 & 105.197 & 77.211 & 72.674 \\
\hline 8/17/07 5:30 & 123.181 & 120.835 & & 98.629 & 102.79 & 94.244 & 108.233 & 105.19 & 77.206 & 72.669 \\
\hline 8/17/07 6:00 & 123.183 & 122.771 & & 98.633 & 102.796 & 94.246 & 108.225 & 105.19 & 77.206 & 72.67 \\
\hline 8/17/07 6:30 & 123.185 & 123.088 & & 98.638 & 102.8 & 94.251 & 108.218 & 105.195 & 77.204 & 72.674 \\
\hline 8/17/07 7:00 & 125.182 & 123.24 & & 99.405 & 103.302 & 94.911 & 108.908 & 106.598 & 77.22 & 72.736 \\
\hline $8 / 17 / 07$ 7:30 & 125.525 & 123.331 & & 99.665 & 103.603 & 95.217 & 109.185 & 106.963 & 77.246 & 72.947 \\
\hline 8/17/07 8:00 & 125.684 & 123.385 & & 99.796 & 103.75 & 95.363 & 109.306 & 107.129 & 77.274 & 73.094 \\
\hline 8/17/07 8:30 & 125.778 & 123.428 & & 99.882 & 103.843 & 95.455 & 109.433 & 107.227 & 77.296 & 73.187 \\
\hline 8/17/07 9:00 & 125.835 & 121.721 & & 99.934 & 103.898 & 95.512 & 109.473 & 107.289 & 77.318 & 73.249 \\
\hline 8/17/07 9:30 & 125.878 & 121.317 & & 99.972 & 103.936 & 95.552 & 109.497 & 107.331 & 77.335 & 73.291 \\
\hline $8 / 17 / 0710: 00$ & 124.138 & 121.159 & & 99.355 & 103.609 & 95.073 & 109.042 & 106.217 & 77.346 & 73.297 \\
\hline $8 / 17 / 07 \quad 10: 30$ & 123.684 & 121.066 & & 99.05 & 103.247 & 94.699 & 108.688 & 105.708 & 77.331 & 73.134 \\
\hline 8/17/07 11:00 & 123.519 & 121.009 & & 98.919 & 103.095 & 94.548 & 108.532 & 105.533 & 77.324 & 73.003 \\
\hline 8/17/07 11:30 & 123.422 & 120.966 & & 98.836 & 103.009 & 94.461 & 108.44 & 105.434 & 77.314 & 72.912 \\
\hline $8 / 17 / 07$ 12:00 & 123.365 & 120.932 & & 98.783 & 102.954 & 94.411 & 108.381 & 105.374 & 77.309 & 72.852 \\
\hline $8 / 17 / 07$ 12:30 & 123.32 & 120.907 & & 98.748 & 102.914 & 94.369 & 108.371 & 105.327 & 77.302 & 72.807 \\
\hline 8/17/07 13:00 & 123.285 & 120.887 & & 98.714 & 102.88 & 94.336 & 108.343 & 105.294 & 77.294 & 72.767 \\
\hline 8/17/07 13:30 & 123.261 & 120.873 & & 98.693 & 102.857 & 94.312 & 108.318 & 105.268 & 77.289 & 72.743 \\
\hline 8/17/07 14:00 & 123.24 & 120.862 & & 98.674 & 102.838 & 94.293 & 108.3 & 105.25 & 77.283 & 72.724 \\
\hline $8 / 17 / 0714: 30$ & 123.228 & 120.844 & & 98.666 & 102.826 & 94.284 & 108.323 & 105.237 & 77.276 & 72.712 \\
\hline $8 / 17 / 07$ 15:00 & 123.214 & 120.83 & & 98.65 & 102.813 & 94.267 & 108.311 & 105.221 & 77.27 & 72.697 \\
\hline 8/17/07 15:30 & 123.195 & 120.819 & & 98.635 & 102.798 & 94.251 & 108.289 & 105.203 & 77.259 & 72.681 \\
\hline 8/17/07 16:00 & 123.183 & 120.81 & & 98.629 & 102.785 & 94.239 & 108.282 & 105.192 & 77.257 & 72.668 \\
\hline 8/17/07 16:30 & 123.174 & 120.798 & & 98.614 & 102.777 & 94.23 & 108.291 & 105.181 & 77.248 & 72.66 \\
\hline 8/17/07 17:00 & 123.162 & 120.785 & & 98.606 & 102.771 & 94.225 & 108.302 & 105.17 & 77.241 & 72.65 \\
\hline 8/17/07 17:30 & 123.15 & 120.778 & & 98.591 & 102.756 & 94.208 & 108.281 & 105.159 & 77.233 & 72.635 \\
\hline 8/17/07 18:00 & 123.136 & 120.78 & & 98.578 & 102.741 & 94.197 & 108.281 & 105.144 & 77.226 & 72.623 \\
\hline 8/17/07 18:30 & 123.129 & 120.771 & & 98.578 & 102.735 & 94.192 & 108.281 & 105.137 & 77.217 & 72.617 \\
\hline 8/17/07 19:00 & 123.133 & 120.771 & & 98.581 & 102.741 & 94.197 & 108.264 & 105.141 & 77.219 & 72.623 \\
\hline 8/17/07 19:30 & 123.124 & 120.767 & & 98.569 & 102.733 & 94.187 & 108.252 & 105.133 & 77.209 & 72.612 \\
\hline 8/17/07 20:00 & 123.126 & 120.76 & & 98.573 & 102.733 & 94.185 & 108.247 & 105.133 & 77.204 & 72.612 \\
\hline 8/17/07 20:30 & 123.122 & 120.771 & & 98.568 & 102.728 & 94.182 & 108.235 & 105.13 & 77.2 & 72.61 \\
\hline 8/17/07 21:00 & 123.112 & 120.78 & & 98.562 & 102.722 & 94.175 & 108.229 & 105.121 & 77.193 & 72.6 \\
\hline 8/17/07 21:30 & 123.122 & 120.782 & & 98.576 & 102.733 & 94.185 & 108.223 & 105.133 & 77.193 & 72.612 \\
\hline 8/17/07 22:00 & 123.133 & 120.789 & & 98.585 & 102.745 & 94.197 & 108.216 & 105.141 & 77.193 & 72.621 \\
\hline 8/17/07 22:30 & 123.136 & 120.796 & & 98.587 & 102.745 & 94.201 & 108.214 & 105.144 & 77.189 & 72.621 \\
\hline 8/17/07 23:00 & 123.143 & 120.794 & & 98.592 & 102.75 & 94.204 & 108.21 & 105.148 & 77.191 & 72.625 \\
\hline
\end{tabular}


TABLE S1.1 (Cont.)

\begin{tabular}{|c|c|c|c|c|c|c|c|c|c|c|}
\hline \multirow[b]{2}{*}{ Date and Time } & \multicolumn{10}{|c|}{ Depth from Top of Casing (ft) } \\
\hline & MW1D & MW2D & MW3D & MW4D & MW7 & MW9 & Oentrich & MW14D & MW15D & MW16D \\
\hline 8/17/07 23:30 & 123.146 & 120.789 & & 98.595 & 102.756 & 94.211 & 108.21 & 105.152 & 77.187 & 72.629 \\
\hline 8/18/07 0:00 & 123.143 & 120.785 & & 98.595 & 102.756 & 94.208 & 108.206 & 105.152 & 77.187 & 72.629 \\
\hline 8/18/07 0:30 & 123.143 & 120.785 & & 98.592 & 102.752 & 94.206 & 108.206 & 105.15 & 77.182 & 72.625 \\
\hline 8/18/07 1:00 & 123.138 & 120.782 & & 98.587 & 102.748 & 94.201 & 108.204 & 105.144 & 77.18 & 72.621 \\
\hline 8/18/07 1:30 & 123.136 & 120.776 & & 98.585 & 102.747 & 94.199 & 108.2 & 105.146 & 77.178 & 72.619 \\
\hline 8/18/07 2:00 & 123.134 & 120.769 & & 98.583 & 102.743 & 94.197 & 108.2 & 105.141 & 77.176 & 72.617 \\
\hline 8/18/07 2:30 & 123.131 & 120.755 & & 98.578 & 102.739 & 94.194 & 108.198 & 105.137 & 77.174 & 72.612 \\
\hline 8/18/07 3:00 & 123.124 & 120.744 & & 98.573 & 102.731 & 94.187 & 108.198 & 105.133 & 77.169 & 72.602 \\
\hline 8/18/07 3:30 & 123.11 & 120.737 & & 98.554 & 102.716 & 94.171 & 108.198 & 105.117 & 77.163 & 72.59 \\
\hline 8/18/07 4:00 & 123.096 & 120.739 & & 98.542 & 102.705 & 94.157 & 108.2 & 105.104 & 77.154 & 72.577 \\
\hline 8/18/07 4:30 & 123.096 & 120.742 & & 98.54 & 102.697 & 94.152 & 108.196 & 105.097 & 77.152 & 72.571 \\
\hline 8/18/07 5:00 & 123.093 & 120.742 & & 98.542 & 102.701 & 94.157 & 108.192 & 105.099 & 77.149 & 72.576 \\
\hline 8/18/07 5:30 & 123.093 & 120.746 & & 98.543 & 102.703 & 94.154 & 108.188 & 105.099 & 77.147 & 72.578 \\
\hline 8/18/07 6:00 & 123.096 & 120.751 & & 98.545 & 102.705 & 94.157 & 108.187 & 105.102 & 77.145 & 72.578 \\
\hline 8/18/07 6:30 & 123.098 & 120.753 & & 98.55 & 102.708 & 94.164 & 108.185 & 105.106 & 77.143 & 72.584 \\
\hline 8/18/07 7:00 & 123.105 & 122.728 & & 98.557 & 102.716 & 94.166 & 108.181 & 105.11 & 77.141 & 72.588 \\
\hline $8 / 18 / 07$ 7:30 & 123.107 & 123.072 & & 98.557 & 102.718 & 94.171 & 108.183 & 105.115 & 77.139 & 72.59 \\
\hline 8/18/07 8:00 & 125.107 & 123.236 & & 99.26 & 103.162 & 94.758 & 108.775 & 106.534 & 77.149 & 72.631 \\
\hline 8/18/07 8:30 & 125.48 & 123.331 & & 99.536 & 103.487 & 95.09 & 109.114 & 106.935 & 77.182 & 72.842 \\
\hline 8/18/07 9:00 & 125.648 & 123.383 & & 99.679 & 103.645 & 95.248 & 109.257 & 107.112 & 77.206 & 72.995 \\
\hline 8/18/07 9:30 & 125.743 & 123.424 & & 99.765 & 103.735 & 95.34 & 109.336 & 107.214 & 77.228 & 73.092 \\
\hline 8/18/07 10:00 & 125.8 & 123.465 & & 99.813 & 103.794 & 95.394 & 109.366 & 107.273 & 77.246 & 73.154 \\
\hline $8 / 18 / 07$ 10:30 & 125.84 & 123.483 & & 99.853 & 103.83 & 95.434 & 109.471 & 107.313 & 77.268 & 73.197 \\
\hline 8/18/07 11:00 & 125.882 & 123.487 & & 99.891 & 103.872 & 95.476 & 109.493 & 107.357 & 77.287 & 73.241 \\
\hline 8/18/07 11:30 & 125.901 & 123.49 & & 99.91 & 103.893 & 95.498 & 109.503 & 107.377 & 77.305 & 73.261 \\
\hline 8/18/07 12:00 & 125.908 & 123.501 & & 99.91 & 103.898 & 95.509 & 109.505 & 107.384 & 77.316 & 73.268 \\
\hline 8/18/07 12:30 & 125.906 & 123.499 & & 99.913 & 103.896 & 95.502 & 109.565 & 107.386 & 77.333 & 73.276 \\
\hline 8/18/07 13:00 & 125.918 & 123.512 & & 99.922 & 103.904 & 95.514 & 109.572 & 107.393 & 77.344 & 73.287 \\
\hline 8/18/07 13:30 & 125.918 & 123.505 & & 99.925 & 103.906 & 95.512 & 109.579 & 107.395 & 77.355 & 73.29 \\
\hline 8/18/07 14:00 & 125.934 & 123.503 & & 99.937 & 103.923 & 95.531 & 109.577 & 107.411 & 77.364 & 73.305 \\
\hline 8/18/07 14:30 & 125.927 & 123.501 & & 99.932 & 103.915 & 95.524 & 109.575 & 107.406 & 77.375 & 73.301 \\
\hline 8/18/07 15:00 & 125.927 & 123.494 & & 99.93 & 103.917 & 95.524 & 109.596 & 107.404 & 77.383 & 73.303 \\
\hline 8/18/07 15:30 & 125.923 & 123.492 & & 99.927 & 103.912 & 95.517 & 109.596 & 107.402 & 77.39 & 73.301 \\
\hline 8/18/07 16:00 & 125.915 & 123.283 & & 99.925 & 103.914 & 95.519 & 109.614 & 107.397 & 77.399 & 73.303 \\
\hline 8/18/07 16:30 & 125.913 & 121.397 & & 99.927 & 103.912 & 95.514 & 109.601 & 107.395 & 77.407 & 73.307 \\
\hline 8/18/07 17:00 & 125.913 & 121.168 & & 99.67 & 103.914 & 95.519 & 109.596 & 107.393 & 77.414 & 73.307 \\
\hline 8/18/07 17:30 & 123.772 & 121.041 & & 99.095 & 103.308 & 94.762 & 108.879 & 105.803 & 77.392 & 73.177 \\
\hline 8/18/07 18:00 & 123.528 & 120.95 & & 98.9 & 103.093 & 94.543 & 108.674 & 105.544 & 77.377 & 73.012 \\
\hline 8/18/07 18:30 & 123.398 & 120.9 & & 98.791 & 102.969 & 94.425 & 108.569 & 105.411 & 77.359 & 72.89 \\
\hline 8/18/07 19:00 & 123.306 & 120.873 & & 98.71 & 102.882 & 94.336 & 108.497 & 105.316 & 77.34 & 72.796 \\
\hline 8/18/07 19:30 & 123.259 & 120.85 & & 98.676 & 102.844 & 94.303 & 108.389 & 105.263 & 77.327 & 72.749 \\
\hline 8/18/07 20:00 & 123.228 & 120.837 & & 98.655 & 102.817 & 94.272 & 108.419 & 105.237 & 77.322 & 72.722 \\
\hline 8/18/07 20:30 & 123.207 & 120.823 & & 98.633 & 102.794 & 94.248 & 108.391 & 105.212 & 77.311 & 72.698 \\
\hline 8/18/07 21:00 & 123.19 & 120.819 & & 98.623 & 102.787 & 94.237 & 108.375 & 105.199 & 77.302 & 72.683 \\
\hline 8/18/07 21:30 & 123.176 & 120.81 & & 98.61 & 102.771 & 94.225 & 108.364 & 105.186 & 77.294 & 72.669 \\
\hline 8/18/07 22:00 & 123.167 & 120.798 & & 98.607 & 102.766 & 94.215 & 108.345 & 105.175 & 77.289 & 72.658 \\
\hline 8/18/07 22:30 & 123.164 & 120.796 & & 98.6 & 102.76 & 94.213 & 108.349 & 105.17 & 77.279 & 72.65 \\
\hline 8/18/07 23:00 & 123.15 & 120.801 & & 98.587 & 102.75 & 94.201 & 108.35 & 105.159 & 77.272 & 72.638 \\
\hline 8/18/07 23:30 & 123.152 & 120.796 & & 98.586 & 102.75 & 94.204 & 108.283 & 105.155 & 77.267 & 72.633 \\
\hline 8/19/07 0:00 & 123.15 & 120.794 & & 98.591 & 102.748 & 94.204 & 108.336 & 105.157 & 77.263 & 72.635 \\
\hline 8/19/07 0:30 & 123.145 & 120.787 & & 98.588 & 102.747 & 94.199 & 108.323 & 105.152 & 77.259 & 72.633 \\
\hline 8/19/07 1:00 & 123.145 & 120.778 & & 98.585 & 102.745 & 94.199 & 108.327 & 105.15 & 77.252 & 72.629 \\
\hline 8/19/07 1:30 & 123.141 & 120.769 & & 98.578 & 102.739 & 94.19 & 108.324 & 105.146 & 77.248 & 72.621 \\
\hline 8/19/07 2:00 & 123.131 & 120.762 & & 98.573 & 102.733 & 94.187 & 108.323 & 105.139 & 77.244 & 72.614 \\
\hline 8/19/07 2:30 & 123.119 & 120.753 & & 98.562 & 102.724 & 94.173 & 108.318 & 105.126 & 77.235 & 72.605 \\
\hline 8/19/07 3:00 & 123.115 & 120.753 & & 98.557 & 102.716 & 94.168 & 108.313 & 105.119 & 77.23 & 72.598 \\
\hline 8/19/07 3:30 & 123.105 & 120.753 & & 98.547 & 102.707 & 94.161 & 108.315 & 105.11 & 77.222 & 72.59 \\
\hline 8/19/07 4:00 & 123.103 & 120.748 & & 98.548 & 102.705 & 94.159 & 108.308 & 105.108 & 77.219 & 72.588 \\
\hline 8/19/07 4:30 & 123.105 & 120.751 & & 98.55 & 102.708 & 94.164 & 108.31 & 105.113 & 77.215 & 72.592 \\
\hline 8/19/07 5:00 & 123.103 & 120.744 & & 98.549 & 102.707 & 94.159 & 108.242 & 105.11 & 77.211 & 72.588 \\
\hline 8/19/07 5:30 & 123.103 & 120.742 & & 98.548 & 102.708 & 94.159 & 108.304 & 105.108 & 77.206 & 72.59 \\
\hline 8/19/07 6:00 & 123.098 & 120.744 & & 98.545 & 102.703 & 94.159 & 108.305 & 105.106 & 77.202 & 72.584 \\
\hline 8/19/07 6:30 & 123.093 & 120.742 & & 98.54 & 102.701 & 94.152 & 108.299 & 105.102 & 77.197 & 72.579 \\
\hline 8/19/07 7:00 & 123.096 & 122.721 & & 98.541 & 102.701 & 94.154 & 108.299 & 105.102 & 77.193 & 72.579 \\
\hline $8 / 19 / 077: 30$ & 123.096 & 123.016 & & 98.538 & 102.701 & 94.154 & 108.291 & 105.102 & 77.187 & 72.579 \\
\hline 8/19/07 8:00 & 125.138 & 123.156 & & 99.337 & 103.24 & 94.852 & 108.98 & 106.556 & 77.202 & 72.656 \\
\hline
\end{tabular}


TABLE S1.1 (Cont.)

\begin{tabular}{|c|c|c|c|c|c|c|c|c|c|c|}
\hline \multirow[b]{2}{*}{ Date and Time } & \multicolumn{10}{|c|}{ Depth from Top of Casing (ft) } \\
\hline & MW1D & MW2D & MW3D & MW4D & MW7 & MW9 & Oentrich & MW14D & MW15D & MW16D \\
\hline 8/19/07 8:30 & 125.452 & 123.238 & & 99.584 & 103.521 & 95.137 & 109.243 & 106.888 & 77.235 & 72.867 \\
\hline 8/19/07 9:00 & 125.601 & 123.295 & & 99.708 & 103.658 & 95.276 & 109.374 & 107.043 & 77.254 & 73.001 \\
\hline 8/19/07 9:30 & 125.684 & 123.338 & & 99.784 & 103.737 & 95.352 & 109.463 & 107.127 & 77.276 & 73.086 \\
\hline 8/19/07 10:00 & 125.743 & 123.367 & & 99.832 & 103.792 & 95.406 & 109.507 & 107.189 & 77.294 & 73.148 \\
\hline $8 / 19 / 07 \quad 10: 30$ & 125.788 & 123.385 & & 99.873 & 103.837 & 95.455 & 109.547 & 107.238 & 77.313 & 73.196 \\
\hline 8/19/07 11:00 & 125.821 & 123.392 & & 99.903 & 103.868 & 95.484 & 109.58 & 107.269 & 77.329 & 73.227 \\
\hline 8/19/07 11:30 & 125.837 & 123.39 & & 99.915 & 103.883 & 95.5 & 109.609 & 107.287 & 77.342 & 73.241 \\
\hline 8/19/07 12:00 & 125.844 & 123.39 & & 99.92 & 103.887 & 95.507 & 109.624 & 107.295 & 77.357 & 73.252 \\
\hline 8/19/07 12:30 & 125.847 & 123.388 & & 99.92 & 103.891 & 95.507 & 109.635 & 107.295 & 77.366 & 73.253 \\
\hline 8/19/07 13:00 & 125.84 & 123.385 & & 99.92 & 103.883 & 95.502 & 109.651 & 107.291 & 77.375 & 73.253 \\
\hline 8/19/07 13:30 & 125.844 & 123.39 & & 99.916 & 103.887 & 95.505 & 109.656 & 107.295 & 77.385 & 73.259 \\
\hline 8/19/07 14:00 & 125.84 & 121.433 & & 99.911 & 103.885 & 95.502 & 109.663 & 107.291 & 77.392 & 73.258 \\
\hline 8/19/07 14:30 & 125.837 & 121.15 & & 99.984 & 103.881 & 95.498 & 109.673 & 107.287 & 77.397 & 73.256 \\
\hline 8/19/07 15:00 & 123.821 & 121.009 & & 99.109 & 103.329 & 94.786 & 108.949 & 105.845 & 77.386 & 73.162 \\
\hline 8/19/07 15:30 & 123.521 & 120.921 & & 98.879 & 103.072 & 94.527 & 108.707 & 105.531 & 77.368 & 72.991 \\
\hline 8/19/07 16:00 & 123.374 & 120.864 & & 98.759 & 102.939 & 94.39 & 108.578 & 105.378 & 77.35 & 72.857 \\
\hline 8/19/07 16:30 & 123.285 & 120.823 & & 98.681 & 102.851 & 94.305 & 108.5 & 105.285 & 77.337 & 72.767 \\
\hline 8/19/07 17:00 & 123.223 & 120.789 & & 98.635 & 102.798 & 94.253 & 108.447 & 105.225 & 77.322 & 72.71 \\
\hline 8/19/07 17:30 & 123.181 & 120.762 & & 98.597 & 102.76 & 94.215 & 108.409 & 105.183 & 77.307 & 72.664 \\
\hline 8/19/07 18:00 & 123.148 & 120.737 & & 98.567 & 102.729 & 94.185 & 108.386 & 105.148 & 77.296 & 72.629 \\
\hline 8/19/07 18:30 & 123.119 & 120.726 & & 98.543 & 102.705 & 94.161 & 108.361 & 105.121 & 77.285 & 72.602 \\
\hline 8/19/07 19:00 & 123.096 & 120.717 & & 98.521 & 102.684 & 94.138 & 108.344 & 105.097 & 77.27 & 72.579 \\
\hline 8/19/07 19:30 & 123.081 & 120.726 & & 98.512 & 102.672 & 94.126 & 108.33 & 105.082 & 77.263 & 72.567 \\
\hline 8/19/07 20:00 & 123.07 & 120.721 & & 98.507 & 102.663 & 94.116 & 108.32 & 105.073 & 77.254 & 72.557 \\
\hline 8/19/07 20:30 & 123.089 & 120.726 & & 98.514 & 102.686 & 94.149 & 108.283 & 105.082 & 77.25 & 72.565 \\
\hline 8/19/07 21:00 & 123.077 & 120.723 & & 98.512 & 102.67 & 94.124 & 108.338 & 105.077 & 77.244 & 72.563 \\
\hline 8/19/07 21:30 & 123.081 & 120.723 & & 98.512 & 102.676 & 94.131 & 108.342 & 105.082 & 77.243 & 72.567 \\
\hline 8/19/07 22:00 & 123.074 & 120.723 & & 98.545 & 102.67 & 94.121 & 108.337 & 105.077 & 77.235 & 72.561 \\
\hline 8/19/07 22:30 & 123.079 & 120.721 & & 98.533 & 102.676 & 94.131 & 108.353 & 105.079 & 77.232 & 72.563 \\
\hline 8/19/07 23:00 & 123.079 & 120.701 & & 98.517 & 102.676 & 94.131 & 108.358 & 105.079 & 77.226 & 72.563 \\
\hline 8/19/07 23:30 & 123.079 & 120.701 & & 98.516 & 102.676 & 94.131 & 108.31 & 105.079 & 77.22 & 72.561 \\
\hline 8/20/07 0:00 & 123.06 & 120.696 & & 98.526 & 102.657 & 94.116 & 108.307 & 105.059 & 77.211 & 72.538 \\
\hline 8/20/07 0:30 & 123.058 & 120.685 & & 98.494 & 102.657 & 94.109 & 108.326 & 105.057 & 77.208 & 72.54 \\
\hline 8/20/07 1:00 & 123.055 & 120.68 & & 98.49 & 102.661 & 94.124 & 108.485 & 105.053 & 77.202 & 72.536 \\
\hline 8/20/07 1:30 & 123.041 & 120.669 & & 98.478 & 102.638 & 94.093 & 108.316 & 105.042 & 77.197 & 72.521 \\
\hline 8/20/07 2:00 & 123.032 & 120.664 & & 98.468 & 102.63 & 94.081 & 108.27 & 105.033 & 77.189 & 72.515 \\
\hline $8 / 20 / 072: 30$ & 123.025 & 120.655 & & 98.459 & 102.623 & 94.074 & 108.288 & 105.026 & 77.184 & 72.507 \\
\hline 8/20/07 3:00 & 123.018 & 120.653 & & 98.459 & 102.617 & 94.069 & 108.3 & 105.02 & 77.176 & 72.499 \\
\hline 8/20/07 3:30 & 123.011 & 120.646 & & 98.45 & 102.611 & 94.062 & 108.282 & 105.013 & 77.171 & 72.495 \\
\hline 8/20/07 4:00 & 123.008 & 120.64 & & 98.452 & 102.609 & 94.06 & 108.262 & 105.011 & 77.165 & 72.492 \\
\hline 8/20/07 4:30 & 123.003 & 120.637 & & 98.445 & 102.608 & 94.058 & 108.25 & 105.004 & 77.161 & 72.486 \\
\hline 8/20/07 5:00 & 122.994 & 120.64 & & 98.436 & 102.594 & 94.048 & 108.246 & 104.995 & 77.152 & 72.478 \\
\hline 8/20/07 5:30 & 122.994 & 122.082 & & 98.435 & 102.594 & 94.048 & 108.24 & 104.993 & 77.149 & 72.476 \\
\hline 8/20/07 6:00 & 122.994 & 122.773 & & 98.44 & 102.596 & 94.048 & 108.236 & 104.995 & 77.145 & 72.478 \\
\hline 8/20/07 6:30 & 124.176 & 122.986 & & 98.908 & 102.674 & 94.192 & 108.401 & 105.237 & 77.143 & 72.484 \\
\hline 8/20/07 7:00 & 125.206 & 123.109 & & 99.363 & 103.287 & 94.899 & 109.055 & 106.631 & 77.174 & 72.654 \\
\hline 8/20/07 7:30 & 125.433 & 123.179 & & 99.549 & 103.493 & 95.106 & 109.247 & 106.868 & 77.198 & 72.838 \\
\hline 8/20/07 8:00 & 125.558 & 123.229 & & 99.673 & 103.609 & 95.224 & 109.356 & 106.997 & 77.219 & 72.958 \\
\hline 8/20/07 8:30 & 125.636 & 123.258 & & 99.723 & 103.681 & 95.297 & 109.431 & 107.078 & 77.241 & 73.036 \\
\hline 8/20/07 9:00 & 125.684 & 123.283 & & 99.766 & 103.725 & 95.34 & 109.495 & 107.127 & 77.256 & 73.086 \\
\hline 8/20/07 9:30 & 125.712 & 123.295 & & 99.797 & 103.757 & 95.37 & 109.538 & 107.158 & 77.272 & 73.119 \\
\hline 8/20/07 10:00 & 125.736 & 123.304 & & 99.81 & 103.776 & 95.392 & 109.551 & 107.178 & 77.287 & 73.142 \\
\hline 8/20/07 10:30 & 125.754 & 123.326 & & 99.839 & 103.796 & 95.41 & 109.566 & 107.198 & 77.3 & 73.16 \\
\hline 8/20/07 11:00 & 125.759 & 123.342 & & 99.837 & 103.803 & 95.415 & 109.576 & 107.207 & 77.313 & 73.168 \\
\hline 8/20/07 11:30 & 125.778 & 123.344 & & 99.854 & 103.826 & 95.439 & 109.922 & 107.225 & 77.326 & 73.189 \\
\hline 8/20/07 12:00 & 125.797 & 123.351 & & 99.87 & 103.839 & 95.458 & 109.623 & 107.247 & 77.342 & 73.208 \\
\hline 8/20/07 12:30 & 125.799 & 123.356 & & 99.877 & 103.841 & 95.46 & 109.614 & 107.249 & 77.351 & 73.216 \\
\hline 8/20/07 13:00 & 125.807 & 121.809 & & 99.889 & 103.856 & 95.476 & 109.614 & 107.258 & 77.362 & 73.227 \\
\hline 8/20/07 13:30 & 125.809 & 121.268 & & 99.909 & 103.862 & 95.469 & 109.925 & 107.26 & 77.372 & 73.23 \\
\hline 8/20/07 14:00 & 124.306 & 121.07 & & 99.353 & 103.653 & 95.139 & 109.283 & 106.465 & 77.379 & 73.233 \\
\hline $8 / 20 / 0714: 30$ & 123.651 & 120.943 & & 98.972 & 103.183 & 94.64 & 108.794 & 105.659 & 77.359 & 73.069 \\
\hline 8/20/07 15:00 & 123.438 & 120.878 & & 98.834 & 102.986 & 94.439 & 108.62 & 105.445 & 77.338 & 72.908 \\
\hline $8 / 20 / 0715: 30$ & 123.306 & 120.83 & & 98.695 & 102.868 & 94.324 & 108.536 & 105.312 & 77.318 & 72.784 \\
\hline 8/20/07 16:00 & 123.235 & 120.801 & & 98.64 & 102.806 & 94.263 & 108.452 & 105.237 & 77.303 & 72.714 \\
\hline 8/20/07 16:30 & 123.19 & 120.769 & & 98.606 & 102.764 & 94.22 & 108.396 & 105.188 & 77.291 & 72.668 \\
\hline 8/20/07 17:00 & 123.162 & 120.764 & & 98.576 & 102.741 & 94.199 & 108.354 & 105.161 & 77.283 & 72.641 \\
\hline
\end{tabular}


TABLE S1.1 (Cont.)

\begin{tabular}{|c|c|c|c|c|c|c|c|c|c|c|}
\hline \multirow[b]{2}{*}{ Date and Time } & \multicolumn{10}{|c|}{ Depth from Top of Casing (ft) } \\
\hline & MW1D & MW2D & MW3D & MW4D & MW7 & MW9 & Oentrich & MW14D & MW15D & MW16D \\
\hline 8/20/07 17:30 & 123.124 & 120.787 & & 98.552 & 102.705 & 94.161 & 108.338 & 105.121 & 77.268 & 72.6 \\
\hline 8/20/07 18:00 & 123.122 & 120.803 & & 98.543 & 102.707 & 94.164 & 108.317 & 105.119 & 77.263 & 72.602 \\
\hline 8/20/07 18:30 & 123.136 & 120.753 & & 98.579 & 102.726 & 94.182 & 108.29 & 105.133 & 77.265 & 72.617 \\
\hline 8/20/07 19:00 & 123.162 & 120.746 & & 98.59 & 102.754 & 94.211 & 108.313 & 105.161 & 77.272 & 72.643 \\
\hline 8/20/07 19:30 & 123.107 & 120.746 & & 98.545 & 102.693 & 94.152 & 108.313 & 105.106 & 77.246 & 72.577 \\
\hline 8/20/07 20:00 & 123.1 & 120.73 & & 98.523 & 102.691 & 94.147 & 108.317 & 105.097 & 77.239 & 72.577 \\
\hline 8/20/07 20:30 & 123.103 & 120.728 & & 98.536 & 102.697 & 94.154 & 108.313 & 105.104 & 77.237 & 72.586 \\
\hline 8/20/07 21:00 & 123.091 & 120.76 & & 98.525 & 102.682 & 94.138 & 108.302 & 105.09 & 77.228 & 72.565 \\
\hline 8/20/07 21:30 & 123.081 & 120.776 & & 98.525 & 102.674 & 94.128 & 108.293 & 105.077 & 77.22 & 72.557 \\
\hline 8/20/07 22:00 & 123.11 & 120.778 & & 98.55 & 102.708 & 94.164 & 108.283 & 105.11 & 77.226 & 72.592 \\
\hline 8/20/07 22:30 & 123.131 & 120.796 & & 98.575 & 102.728 & 94.182 & 108.293 & 105.13 & 77.226 & 72.608 \\
\hline 8/20/07 23:00 & 123.129 & 120.778 & & 98.571 & 102.728 & 94.185 & 108.287 & 105.13 & 77.224 & 72.608 \\
\hline 8/20/07 23:30 & 123.148 & 120.769 & & 98.59 & 102.75 & 94.206 & 108.275 & 105.152 & 77.228 & 72.629 \\
\hline 8/21/07 0:00 & 123.133 & 120.771 & & 98.562 & 102.726 & 94.182 & 108.275 & 105.135 & 77.22 & 72.606 \\
\hline 8/21/07 0:30 & 123.119 & 120.778 & & 98.564 & 102.718 & 94.173 & 108.27 & 105.119 & 77.215 & 72.592 \\
\hline 8/21/07 1:00 & 123.124 & 120.773 & & 98.569 & 102.722 & 94.178 & 108.264 & 105.124 & 77.211 & 72.596 \\
\hline $8 / 21 / 071: 30$ & 123.131 & 120.757 & & 98.574 & 102.731 & 94.185 & 108.258 & 105.13 & 77.213 & 72.606 \\
\hline $8 / 21 / 072: 00$ & 123.129 & 120.757 & & 98.569 & 102.728 & 94.182 & 108.279 & 105.128 & 77.208 & 72.598 \\
\hline $8 / 21 / 072: 30$ & 123.115 & 120.767 & & 98.552 & 102.707 & 94.166 & 108.277 & 105.113 & 77.202 & 72.583 \\
\hline $8 / 21 / 073: 00$ & 123.11 & 120.785 & & 98.554 & 102.705 & 94.161 & 108.267 & 105.106 & 77.197 & 72.579 \\
\hline 8/21/07 3:30 & 123.122 & 120.785 & & 98.564 & 102.718 & 94.178 & 108.26 & 105.119 & 77.198 & 72.592 \\
\hline 8/21/07 4:00 & 123.138 & 120.782 & & 98.585 & 102.737 & 94.197 & 108.252 & 105.137 & 77.202 & 72.61 \\
\hline $8 / 21 / 074: 30$ & 123.141 & 120.789 & & 98.581 & 102.737 & 94.197 & 108.266 & 105.139 & 77.202 & 72.61 \\
\hline 8/21/07 5:00 & 123.136 & 120.798 & & 98.58 & 102.737 & 94.194 & 108.269 & 105.135 & 77.2 & 72.608 \\
\hline $8 / 21 / 075: 30$ & 123.143 & 122.39 & & 98.588 & 102.741 & 94.199 & 108.261 & 105.141 & 77.202 & 72.612 \\
\hline 8/21/07 6:00 & 123.155 & 122.991 & & 98.6 & 102.754 & 94.211 & 108.252 & 105.152 & 77.204 & 72.625 \\
\hline 8/21/07 6:30 & 124.603 & 123.193 & & 99.077 & 102.891 & 94.446 & 108.498 & 105.81 & 77.208 & 72.633 \\
\hline $8 / 21 / 07$ 7:00 & 125.395 & 123.32 & & 99.496 & 103.432 & 95.036 & 109.053 & 106.846 & 77.239 & 72.811 \\
\hline 8/21/07 7:30 & 125.608 & 123.399 & & 99.672 & 103.63 & 95.234 & 109.228 & 107.07 & 77.267 & 72.987 \\
\hline $8 / 21 / 078: 00$ & 125.74 & 123.456 & & 99.789 & 103.754 & 95.356 & 109.325 & 107.205 & 77.298 & 73.117 \\
\hline $8 / 21 / 078: 30$ & 125.821 & 123.49 & & 99.861 & 103.834 & 95.441 & 109.404 & 107.291 & 77.326 & 73.204 \\
\hline 8/21/07 9:00 & 125.877 & 123.521 & & 99.911 & 103.887 & 95.493 & 109.452 & 107.349 & 77.346 & 73.259 \\
\hline 8/21/07 9:30 & 125.91 & 123.537 & & 99.94 & 103.919 & 95.526 & 109.474 & 107.384 & 77.366 & 73.297 \\
\hline $8 / 21 / 07 \quad 10: 00$ & 125.944 & 121.59 & & 99.975 & 103.954 & 95.559 & 109.514 & 107.419 & 77.388 & 73.33 \\
\hline $8 / 21 / 07$ 10:30 & 125.96 & 121.308 & & 99.994 & 103.976 & 95.58 & 109.522 & 107.439 & 77.409 & 73.355 \\
\hline 8/21/07 11:00 & 123.975 & 121.168 & & 99.269 & 103.491 & 94.953 & 108.893 & 106.007 & 77.405 & 73.295 \\
\hline $8 / 21 / 0711: 30$ & 123.677 & 121.084 & & 99.041 & 103.23 & 94.689 & 108.651 & 105.686 & 77.392 & 73.125 \\
\hline 8/21/07 12:00 & 123.533 & 121.025 & & 98.924 & 103.101 & 94.557 & 108.53 & 105.535 & 77.385 & 72.999 \\
\hline $8 / 21 / 0712: 30$ & 123.445 & 120.982 & & 98.852 & 103.019 & 94.475 & 108.455 & 105.447 & 77.373 & 72.913 \\
\hline 8/21/07 13:00 & 123.386 & 120.95 & & 98.802 & 102.96 & 94.421 & 108.405 & 105.387 & 77.366 & 72.85 \\
\hline 8/21/07 13:30 & 123.341 & 120.923 & & 98.76 & 102.922 & 94.38 & 108.372 & 105.343 & 77.357 & 72.807 \\
\hline $8 / 21 / 0714: 00$ & 123.308 & 120.903 & & 98.731 & 102.889 & 94.352 & 108.349 & 105.31 & 77.35 & 72.774 \\
\hline $8 / 21 / 07$ 14:30 & 123.285 & 120.884 & & 98.71 & 102.868 & 94.326 & 108.33 & 105.279 & 77.342 & 72.745 \\
\hline 8/21/07 15:00 & 123.259 & 120.859 & & 98.686 & 102.846 & 94.3 & 108.319 & 105.261 & 77.333 & 72.72 \\
\hline $8 / 21 / 0715: 30$ & 123.24 & 120.846 & & 98.669 & 102.826 & 94.284 & 108.311 & 105.239 & 77.326 & 72.704 \\
\hline 8/21/07 16:00 & 123.219 & 120.832 & & 98.649 & 102.804 & 94.263 & 108.3 & 105.217 & 77.316 & 72.683 \\
\hline 8/21/07 16:30 & 123.202 & 120.825 & & 98.633 & 102.788 & 94.246 & 108.292 & 105.199 & 77.309 & 72.666 \\
\hline 8/21/07 17:00 & 123.195 & 120.814 & & 98.623 & 102.788 & 94.258 & 108.322 & 105.188 & 77.3 & 72.654 \\
\hline 8/21/07 17:30 & 123.181 & 120.803 & & 98.614 & 102.769 & 94.23 & 108.308 & 105.177 & 77.296 & 72.648 \\
\hline 8/21/07 18:00 & 123.174 & 120.776 & & 98.609 & 102.764 & 94.225 & 108.3 & 105.17 & 77.291 & 72.642 \\
\hline 8/21/07 18:30 & 123.162 & 120.755 & & 98.595 & 102.748 & 94.211 & 108.294 & 105.159 & 77.283 & 72.627 \\
\hline 8/21/07 19:00 & 123.136 & 120.791 & & 98.562 & 102.724 & 94.182 & 108.29 & 105.133 & 77.27 & 72.598 \\
\hline 8/21/07 19:30 & 123.107 & 120.873 & & 98.541 & 102.699 & 94.157 & 108.285 & 105.106 & 77.259 & 72.578 \\
\hline 8/21/07 20:00 & 123.145 & 120.859 & & 98.595 & 102.745 & 94.201 & 108.294 & 105.141 & 77.267 & 72.621 \\
\hline 8/21/07 20:30 & 123.233 & 120.855 & & 98.745 & 102.851 & 94.34 & 109.192 & 105.217 & 77.281 & 72.646 \\
\hline 8/21/07 21:00 & 123.223 & 120.857 & & 98.652 & 102.819 & 94.274 & 108.318 & 105.225 & 77.285 & 72.662 \\
\hline $8 / 21 / 0721: 30$ & 123.209 & 120.853 & & 98.647 & 102.807 & 94.26 & 108.303 & 105.208 & 77.285 & 72.679 \\
\hline 8/21/07 22:00 & 123.214 & 120.859 & & 98.652 & 102.807 & 94.263 & 108.288 & 105.212 & 77.285 & 72.685 \\
\hline 8/21/07 22:30 & 123.209 & 120.871 & & 98.664 & 102.802 & 94.258 & 108.308 & 105.208 & 77.283 & 72.676 \\
\hline 8/21/07 23:00 & 123.216 & 120.864 & & 98.657 & 102.809 & 94.267 & 108.284 & 105.212 & 77.287 & 72.683 \\
\hline 8/21/07 23:30 & 123.226 & 120.864 & & 98.667 & 102.821 & 94.279 & 108.323 & 105.223 & 77.285 & 72.691 \\
\hline 8/22/07 0:00 & 123.219 & 120.857 & & 98.659 & 102.813 & 94.272 & 108.299 & 105.217 & 77.283 & 72.683 \\
\hline 8/22/07 0:30 & 123.223 & 120.844 & & 98.657 & 102.817 & 94.277 & 108.28 & 105.221 & 77.283 & 72.687 \\
\hline 8/22/07 1:00 & 123.211 & 120.841 & & 98.652 & 102.806 & 94.263 & 108.269 & 105.208 & 77.279 & 72.672 \\
\hline 8/22/07 1:30 & 123.197 & 120.841 & & 98.636 & 102.787 & 94.248 & 108.265 & 105.197 & 77.274 & 72.658 \\
\hline 8/22/07 2:00 & 123.195 & 120.835 & & 98.638 & 102.787 & 94.246 & 108.261 & 105.19 & 77.27 & 72.658 \\
\hline
\end{tabular}


TABLE S1.1 (Cont.)

Depth from Top of Casing (ft)

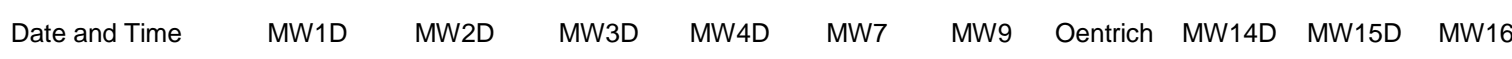

\begin{tabular}{|c|c|c|c|c|c|c|c|c|c|}
\hline 8/22/07 2:30 & 123.195 & 120.828 & 98.631 & 102.788 & 94.246 & 108.253 & 105.192 & 77.27 & 72.662 \\
\hline 8/22/07 3:00 & 123.188 & 120.825 & 98.631 & 102.781 & 94.241 & 108.254 & 105.186 & 77.268 & 72.652 \\
\hline 8/22/07 3:30 & 123.183 & 120.832 & 98.624 & 102.777 & 94.239 & 108.248 & 105.181 & 77.263 & 72.648 \\
\hline 8/22/07 4:00 & 123.181 & 120.837 & 98.624 & 102.773 & 94.234 & 108.252 & 105.179 & 77.261 & 72.642 \\
\hline 8/22/07 4:30 & 123.185 & 120.848 & 98.628 & 102.779 & 94.237 & 108.248 & 105.181 & 77.261 & 72.648 \\
\hline 8/22/07 5:00 & 123.19 & 120.857 & 98.631 & 102.787 & 94.244 & 108.246 & 105.186 & 77.261 & 72.652 \\
\hline 8/22/07 5:30 & 123.2 & 122.22 & 98.647 & 102.796 & 94.256 & 108.24 & 105.197 & 77.267 & 72.664 \\
\hline 8/22/07 6:00 & 123.211 & 122.97 & 98.655 & 102.807 & 94.265 & 108.24 & 105.208 & 77.268 & 72.673 \\
\hline 8/22/07 6:30 & 124.083 & 123.188 & 99.095 & 102.834 & 94.307 & 108.31 & 105.212 & 77.27 & 72.677 \\
\hline 8/22/07 7:00 & 125.405 & 123.315 & 99.563 & 103.476 & 95.099 & 109.051 & 106.824 & 77.3 & 72.836 \\
\hline 8/22/07 7:30 & 125.634 & 123.39 & 99.754 & 103.691 & 95.311 & 109.241 & 107.067 & 77.329 & 73.024 \\
\hline 8/22/07 8:00 & 125.764 & 123.44 & 99.865 & 103.811 & 95.432 & 109.348 & 107.2 & 77.357 & 73.15 \\
\hline 8/22/07 8:30 & 125.84 & 123.476 & 99.93 & 103.883 & 95.507 & 109.421 & 107.28 & 77.379 & 73.227 \\
\hline 8/22/07 9:00 & 125.892 & 123.496 & 99.98 & 103.933 & 95.559 & 109.468 & 107.333 & 77.401 & 73.282 \\
\hline 8/22/07 9:30 & 125.927 & 123.51 & 100.013 & 103.969 & 95.594 & 109.5 & 107.375 & 77.421 & 73.324 \\
\hline 8/22/07 10:00 & 125.948 & 123.515 & 100.027 & 103.988 & 95.611 & 109.524 & 107.393 & 77.434 & 73.344 \\
\hline 8/22/07 10:30 & 125.96 & 123.533 & 100.04 & 104.001 & 95.625 & 109.545 & 107.408 & 77.451 & 73.354 \\
\hline 8/22/07 11:00 & 125.967 & 123.555 & 100.049 & 104.011 & 95.632 & 109.563 & 107.415 & 77.464 & 73.369 \\
\hline 8/22/07 11:30 & 125.984 & 123.571 & 100.066 & 104.028 & 95.651 & 109.573 & 107.43 & 77.479 & 73.388 \\
\hline 8/22/07 12:00 & 126.01 & 123.573 & 100.087 & 104.047 & 95.672 & 109.58 & 107.453 & 77.497 & 73.409 \\
\hline 8/22/07 12:30 & 126.024 & 123.578 & 100.106 & 104.068 & 95.689 & 109.595 & 107.473 & 77.515 & 73.429 \\
\hline 8/22/07 13:00 & 126.029 & 121.599 & 100.113 & 104.07 & 95.696 & 109.601 & 107.475 & 77.528 & 73.436 \\
\hline 8/22/07 13:30 & 126.033 & 121.347 & 100.113 & 104.075 & 95.7 & 109.608 & 107.481 & 77.541 & 73.442 \\
\hline $8 / 22 / 07$ 14:00 & 123.987 & 121.206 & 99.282 & 103.491 & 94.958 & 108.886 & 105.998 & 77.526 & 73.33 \\
\hline $8 / 22 / 07$ 14:30 & 123.712 & 121.107 & 99.07 & 103.253 & 94.713 & 108.664 & 105.715 & 77.51 & 73.159 \\
\hline 8/22/07 15:00 & 123.571 & 121.03 & 98.95 & 103.122 & 94.581 & 108.551 & 105.569 & 77.493 & 73.028 \\
\hline 8/22/07 15:30 & 123.471 & 120.986 & 98.86 & 103.026 & 94.489 & 108.483 & 105.467 & 77.479 & 72.933 \\
\hline 8/22/07 16:00 & 123.391 & 120.955 & 98.791 & 102.952 & 94.418 & 108.442 & 105.385 & 77.456 & 72.851 \\
\hline 8/22/07 16:30 & 123.341 & 120.921 & 98.753 & 102.912 & 94.376 & 108.41 & 105.338 & 77.442 & 72.803 \\
\hline 8/22/07 17:00 & 123.315 & 120.912 & 98.731 & 102.889 & 94.347 & 108.386 & 105.31 & 77.434 & 72.776 \\
\hline $8 / 22 / 07 \quad 17: 30$ & 123.282 & 120.9 & 98.695 & 102.855 & 94.319 & 108.371 & 105.279 & 77.423 & 72.745 \\
\hline 8/22/07 18:00 & 123.266 & 120.884 & 98.691 & 102.846 & 94.307 & 108.356 & 105.263 & 77.414 & 72.73 \\
\hline 8/22/07 18:30 & 123.256 & 120.873 & 98.684 & 102.836 & 94.296 & 108.345 & 105.252 & 77.407 & 72.722 \\
\hline 8/22/07 19:00 & 123.244 & 120.884 & 98.666 & 102.825 & 94.284 & 108.337 & 105.239 & 77.399 & 72.708 \\
\hline 8/22/07 19:30 & 123.233 & 120.925 & 98.662 & 102.813 & 94.274 & 108.331 & 105.225 & 77.39 & 72.695 \\
\hline 8/22/07 20:00 & 123.244 & 120.948 & 98.676 & 102.826 & 94.289 & 108.32 & 105.237 & 77.39 & 72.708 \\
\hline $8 / 22 / 0720: 30$ & 123.282 & 120.971 & 98.721 & 102.87 & 94.326 & 108.309 & 105.265 & 77.397 & 72.741 \\
\hline 8/22/07 21:00 & 123.308 & 121.032 & 98.745 & 102.899 & 94.359 & 108.307 & 105.307 & 77.407 & 72.78 \\
\hline $8 / 22 / 0721: 30$ & 123.325 & 121.018 & 98.771 & 102.914 & 94.373 & 108.299 & 105.321 & 77.409 & 72.79 \\
\hline 8/22/07 22:00 & 123.391 & 120.998 & 98.84 & 102.984 & 94.442 & 108.288 & 105.38 & 77.429 & 72.858 \\
\hline 8/22/07 22:30 & 123.374 & 120.982 & 98.815 & 102.96 & 94.423 & 108.294 & 105.367 & 77.421 & 72.825 \\
\hline 8/22/07 23:00 & 123.384 & 120.968 & 98.793 & 102.943 & 94.404 & 108.331 & 105.349 & 77.407 & 72.811 \\
\hline 8/22/07 23:30 & 123.382 & 120.948 & 98.778 & 102.927 & 94.395 & 108.329 & 105.338 & 77.401 & 72.798 \\
\hline $8 / 23 / 070: 00$ & 123.379 & 120.93 & 98.759 & 102.914 & 94.376 & 108.333 & 105.323 & 77.397 & 72.782 \\
\hline $8 / 23 / 07$ 0:30 & 123.374 & 120.905 & 98.741 & 102.893 & 94.355 & 108.333 & 105.303 & 77.388 & 72.763 \\
\hline 8/23/07 1:00 & 123.372 & 120.889 & 98.719 & 102.886 & 94.338 & 108.934 & 105.281 & 77.381 & 72.743 \\
\hline 8/23/07 1:30 & 123.365 & 120.871 & 98.695 & 102.851 & 94.312 & 108.369 & 105.259 & 77.372 & 72.72 \\
\hline $8 / 23 / 07$ 2:00 & 123.358 & 120.869 & 98.679 & 102.83 & 94.293 & 108.361 & 105.241 & 77.364 & 72.703 \\
\hline $8 / 23 / 072: 30$ & 123.344 & 120.862 & 98.664 & 102.813 & 94.274 & 108.354 & 105.223 & 77.357 & 72.687 \\
\hline 8/23/07 3:00 & 123.334 & 120.841 & 98.666 & 102.815 & 94.277 & 108.342 & 105.223 & 77.353 & 72.689 \\
\hline 8/23/07 3:30 & 123.327 & 120.821 & 98.654 & 102.809 & 94.267 & 108.341 & 105.214 & 77.35 & 72.681 \\
\hline 8/23/07 4:00 & 123.306 & 120.823 & 98.633 & 102.788 & 94.246 & 108.338 & 105.197 & 77.338 & 72.662 \\
\hline 8/23/07 4:30 & 123.28 & 120.81 & 98.614 & 102.766 & 94.223 & 108.337 & 105.175 & 77.327 & 72.64 \\
\hline 8/23/07 5:00 & 123.268 & 120.798 & 98.619 & 102.771 & 94.23 & 108.333 & 105.177 & 77.326 & 72.645 \\
\hline 8/23/07 5:30 & 123.261 & 120.767 & 98.598 & 102.758 & 94.215 & 108.331 & 105.166 & 77.32 & 72.631 \\
\hline 8/23/07 6:00 & 123.214 & 122.871 & 98.598 & 102.743 & 94.199 & 108.36 & 105.148 & 77.309 & 72.614 \\
\hline 8/23/07 6:30 & 123.219 & 123.127 & 98.588 & 102.699 & 94.157 & 108.354 & 105.11 & 77.291 & 72.573 \\
\hline 8/23/07 7:00 & 125.204 & 123.279 & 99.382 & 103.302 & 94.908 & 109.046 & 106.711 & 77.316 & 72.718 \\
\hline $8 / 23 / 077: 30$ & 125.483 & 123.367 & 99.61 & 103.556 & 95.163 & 109.27 & 107.001 & 77.346 & 72.92 \\
\hline 8/23/07 8:00 & 125.636 & 123.422 & 99.741 & 103.698 & 95.309 & 109.384 & 107.158 & 77.372 & 73.065 \\
\hline 8/23/07 8:30 & 125.745 & 123.46 & 99.823 & 103.788 & 95.394 & 109.457 & 107.251 & 77.399 & 73.161 \\
\hline 8/23/07 9:00 & 125.809 & 123.478 & 99.873 & 103.847 & 95.455 & 109.503 & 107.315 & 77.418 & 73.224 \\
\hline 8/23/07 9:30 & 125.884 & 123.483 & 99.904 & 103.885 & 95.495 & 109.551 & 107.355 & 77.438 & 73.268 \\
\hline $8 / 23 / 07$ 10:00 & 125.896 & 123.487 & 99.918 & 103.895 & 95.5 & 109.603 & 107.366 & 77.451 & 73.274 \\
\hline $8 / 23 / 07 \quad 10: 30$ & 125.903 & 123.496 & 99.947 & 103.91 & 95.514 & 109.924 & 107.375 & 77.462 & 73.292 \\
\hline 8/23/07 11:00 & 125.908 & 123.508 & 99.939 & 103.912 & 95.517 & 109.646 & 107.38 & 77.471 & 73.299 \\
\hline
\end{tabular}


TABLE S1.1 (Cont.)

\begin{tabular}{|c|c|c|c|c|c|c|c|c|c|c|}
\hline \multirow[b]{2}{*}{ Date and Time } & \multicolumn{10}{|c|}{ Depth from Top of Casing (ft) } \\
\hline & MW1D & MW2D & MW3D & MW4D & MW7 & MW9 & Oentrich & MW14D & MW15D & MW16D \\
\hline 8/23/07 11:30 & 125.92 & 123.515 & & 99.942 & 103.921 & 95.528 & 109.649 & 107.391 & 77.486 & 73.313 \\
\hline $8 / 23 / 07$ 12:00 & 125.929 & 123.517 & & 99.954 & 103.933 & 95.54 & 109.654 & 107.406 & 77.499 & 73.328 \\
\hline 8/23/07 12:30 & 125.937 & 123.512 & & 99.961 & 103.946 & 95.55 & 109.653 & 107.419 & 77.512 & 73.34 \\
\hline 8/23/07 13:00 & 125.941 & 123.521 & & 99.97 & 103.95 & 95.559 & 109.657 & 107.419 & 77.519 & 73.348 \\
\hline $8 / 23 / 07$ 13:30 & 125.936 & 121.571 & & 99.968 & 103.948 & 95.557 & 109.659 & 107.417 & 77.526 & 73.348 \\
\hline 8/23/07 14:00 & 125.944 & 121.29 & & 99.975 & 103.957 & 95.561 & 109.662 & 107.422 & 77.538 & 73.354 \\
\hline $8 / 23 / 0714: 30$ & 123.953 & 121.154 & & 99.238 & 103.455 & 94.92 & 109.026 & 105.976 & 77.53 & 73.284 \\
\hline 8/23/07 15:00 & 123.658 & 121.066 & & 99.016 & 103.2 & 94.661 & 108.803 & 105.666 & 77.512 & 73.111 \\
\hline 8/23/07 15:30 & 123.516 & 121.007 & & 98.895 & 103.068 & 94.529 & 108.677 & 105.516 & 77.495 & 72.983 \\
\hline 8/23/07 16:00 & 123.426 & 120.964 & & 98.824 & 102.986 & 94.446 & 108.6 & 105.423 & 77.482 & 72.896 \\
\hline 8/23/07 16:30 & 123.365 & 120.93 & & 98.765 & 102.927 & 94.392 & 108.551 & 105.361 & 77.469 & 72.832 \\
\hline 8/23/07 17:00 & 123.325 & 120.905 & & 98.733 & 102.889 & 94.355 & 108.527 & 105.318 & 77.458 & 72.788 \\
\hline 8/23/07 17:30 & 123.289 & 120.878 & & 98.702 & 102.857 & 94.322 & 108.498 & 105.285 & 77.445 & 72.751 \\
\hline 8/23/07 18:00 & 123.263 & 120.871 & & 98.679 & 102.836 & 94.298 & 108.474 & 105.256 & 77.434 & 72.726 \\
\hline 8/23/07 18:30 & 123.24 & 120.866 & & 98.657 & 102.809 & 94.274 & 108.455 & 105.23 & 77.425 & 72.699 \\
\hline 8/23/07 19:00 & 123.23 & 120.857 & & 98.653 & 102.804 & 94.265 & 108.442 & 105.219 & 77.416 & 72.691 \\
\hline 8/23/07 19:30 & 123.221 & 120.859 & & 98.652 & 102.798 & 94.258 & 108.434 & 105.212 & 77.41 & 72.685 \\
\hline 8/23/07 20:00 & 123.219 & 120.85 & & 98.642 & 102.796 & 94.256 & 108.427 & 105.208 & 77.403 & 72.679 \\
\hline 8/23/07 20:30 & 123.219 & 120.848 & & 98.647 & 102.798 & 94.26 & 108.423 & 105.21 & 77.394 & 72.683 \\
\hline 8/23/07 21:00 & 123.207 & 120.828 & & 98.638 & 102.787 & 94.248 & 108.418 & 105.199 & 77.386 & 72.668 \\
\hline 8/23/07 21:30 & 123.204 & 120.853 & & 98.635 & 102.787 & 94.248 & 108.414 & 105.195 & 77.379 & 72.666 \\
\hline 8/23/07 22:00 & 123.188 & 120.887 & & 98.607 & 102.767 & 94.23 & 108.406 & 105.181 & 77.366 & 72.648 \\
\hline 8/23/07 22:30 & 123.207 & 120.866 & & 98.647 & 102.788 & 94.246 & 108.404 & 105.192 & 77.366 & 72.666 \\
\hline 8/23/07 23:00 & 123.237 & 120.857 & & 98.676 & 102.828 & 94.284 & 108.397 & 105.223 & 77.368 & 72.703 \\
\hline 8/23/07 23:30 & 123.259 & 120.896 & & 98.661 & 102.813 & 94.274 & 108.427 & 105.221 & 77.353 & 72.689 \\
\hline 8/24/07 0:00 & 123.249 & 120.85 & & 98.653 & 102.802 & 94.26 & 108.444 & 105.208 & 77.342 & 72.675 \\
\hline $8 / 24 / 07$ 0:30 & 123.237 & 120.878 & & 98.681 & 102.838 & 94.298 & 108.459 & 105.237 & 77.348 & 72.71 \\
\hline $8 / 24 / 07$ 1:00 & 123.24 & 120.88 & & 98.641 & 102.792 & 94.253 & 108.447 & 105.199 & 77.324 & 72.662 \\
\hline 8/24/07 1:30 & 123.235 & 120.844 & & 98.674 & 102.817 & 94.279 & 108.459 & 105.221 & 77.324 & 72.689 \\
\hline 8/24/07 2:00 & 123.228 & 120.93 & & 98.66 & 102.832 & 94.296 & 108.472 & 105.241 & 77.324 & 72.705 \\
\hline $8 / 24 / 072: 30$ & 123.23 & 120.946 & & 98.635 & 102.792 & 94.253 & 108.453 & 105.192 & 77.302 & 72.662 \\
\hline $8 / 24 / 07$ 3:00 & 123.209 & 120.9 & & 98.731 & 102.887 & 94.347 & 108.474 & 105.279 & 77.324 & 72.759 \\
\hline 8/24/07 3:30 & 123.221 & 120.823 & & 98.74 & 102.893 & 94.357 & 108.474 & 105.294 & 77.322 & 72.763 \\
\hline 8/24/07 4:00 & 123.226 & 120.789 & & 98.681 & 102.851 & 94.312 & 108.479 & 105.256 & 77.303 & 72.718 \\
\hline $8 / 24 / 074: 30$ & 123.235 & 120.803 & & 98.602 & 102.764 & 94.225 & 108.43 & 105.177 & 77.274 & 72.631 \\
\hline 8/24/07 5:00 & 123.223 & 120.853 & & 98.573 & 102.733 & 94.194 & 108.395 & 105.144 & 77.259 & 72.604 \\
\hline $8 / 24 / 075: 30$ & 123.209 & 122.487 & & 98.605 & 102.747 & 94.206 & 108.381 & 105.15 & 77.259 & 72.621 \\
\hline 8/24/07 6:00 & 123.2 & 123.032 & & 98.66 & 102.798 & 94.258 & 108.408 & 105.197 & 77.274 & 72.67 \\
\hline 8/24/07 6:30 & 124.769 & 123.236 & & 99.222 & 103.047 & 94.64 & 108.789 & 106.142 & 77.279 & 72.685 \\
\hline $8 / 24 / 077: 00$ & 125.405 & 123.351 & & 99.632 & 103.548 & 95.168 & 109.245 & 106.89 & 77.32 & 72.904 \\
\hline 8/24/07 7:30 & 125.622 & 123.431 & & 99.811 & 103.75 & 95.373 & 109.418 & 107.121 & 77.351 & 73.092 \\
\hline 8/24/07 8:00 & 125.743 & 123.49 & & 99.918 & 103.86 & 95.484 & 109.521 & 107.24 & 77.375 & 73.204 \\
\hline 8/24/07 8:30 & 125.835 & 121.605 & & 99.987 & 103.942 & 95.564 & 109.585 & 107.329 & 77.405 & 73.291 \\
\hline 8/24/07 9:00 & 125.941 & 121.345 & & 100.042 & 103.995 & 95.618 & 109.668 & 107.386 & 77.427 & 73.348 \\
\hline 8/24/07 9:30 & 123.998 & 121.211 & & 99.291 & 103.506 & 94.972 & 109.022 & 106.011 & 77.429 & 73.305 \\
\hline 8/24/07 10:00 & 123.715 & 121.143 & & 99.084 & 103.264 & 94.725 & 108.784 & 105.717 & 77.421 & 73.148 \\
\hline 8/24/07 10:30 & 123.578 & 121.086 & & 98.967 & 103.137 & 94.597 & 108.669 & 105.573 & 77.41 & 73.028 \\
\hline 8/24/07 11:00 & 123.504 & 121.048 & & 98.914 & 103.072 & 94.534 & 108.599 & 105.498 & 77.405 & 72.958 \\
\hline $8 / 24 / 0711: 30$ & 123.45 & 121.018 & & 98.859 & 103.017 & 94.479 & 108.567 & 105.442 & 77.396 & 72.896 \\
\hline 8/24/07 12:00 & 123.408 & 121.002 & & 98.824 & 102.979 & 94.442 & 108.554 & 105.4 & 77.388 & 72.856 \\
\hline $8 / 24 / 0712: 30$ & 123.377 & 120.998 & & 98.798 & 102.95 & 94.411 & 108.534 & 105.369 & 77.383 & 72.823 \\
\hline 8/24/07 13:00 & 123.363 & 120.991 & & 98.79 & 102.939 & 94.399 & 108.515 & 105.352 & 77.377 & 72.811 \\
\hline 8/24/07 13:30 & 123.356 & 120.982 & & 98.791 & 102.939 & 94.399 & 108.498 & 105.347 & 77.375 & 72.807 \\
\hline $8 / 24 / 0714: 00$ & 123.351 & 120.957 & & 98.781 & 102.929 & 94.395 & 108.491 & 105.343 & 77.372 & 72.798 \\
\hline $8 / 24 / 0714: 30$ & 123.341 & 120.948 & & 98.769 & 102.92 & 94.383 & 108.481 & 105.332 & 77.37 & 72.788 \\
\hline 8/24/07 15:00 & 123.339 & 120.948 & & 98.74 & 102.901 & 94.366 & 108.477 & 105.316 & 77.359 & 72.772 \\
\hline $8 / 24 / 07$ 15:30 & 123.337 & 120.946 & & 98.738 & 102.891 & 94.357 & 108.472 & 105.303 & 77.351 & 72.761 \\
\hline $8 / 24 / 07$ 16:00 & 123.334 & 120.946 & & 98.741 & 102.886 & 94.35 & 108.468 & 105.296 & 77.35 & 72.755 \\
\hline 8/24/07 16:30 & 123.327 & 120.939 & & 98.74 & 102.886 & 94.35 & 108.464 & 105.296 & 77.34 & 72.755 \\
\hline 8/24/07 17:00 & 123.32 & 120.948 & & 98.738 & 102.884 & 94.352 & 108.462 & 105.292 & 77.338 & 72.751 \\
\hline 8/24/07 17:30 & 123.32 & 120.955 & & 98.734 & 102.882 & 94.345 & 108.46 & 105.29 & 77.335 & 72.747 \\
\hline 8/24/07 18:00 & 123.315 & 120.961 & & 98.745 & 102.891 & 94.357 & 108.458 & 105.299 & 77.337 & 72.757 \\
\hline 8/24/07 18:30 & 123.315 & 120.968 & & 98.752 & 102.899 & 94.362 & 108.455 & 105.307 & 77.338 & 72.763 \\
\hline 8/24/07 19:00 & 123.318 & 120.98 & & 98.755 & 102.901 & 94.364 & 108.456 & 105.305 & 77.338 & 72.763 \\
\hline 8/24/07 19:30 & 123.325 & 120.993 & & 98.767 & 102.912 & 94.376 & 108.455 & 105.314 & 77.337 & 72.772 \\
\hline 8/24/07 20:00 & 123.337 & 121.002 & & 98.772 & 102.922 & 94.385 & 108.455 & 105.327 & 77.342 & 72.784 \\
\hline
\end{tabular}


TABLE S1.1 (Cont.)

\begin{tabular}{|c|c|c|c|c|c|c|c|c|c|c|}
\hline \multirow[b]{2}{*}{ Date and Time } & \multicolumn{10}{|c|}{ Depth from Top of Casing (ft) } \\
\hline & MW1D & MW2D & MW3D & MW4D & MW7 & MW9 & Oentrich & MW14D & MW15D & MW16D \\
\hline 8/24/07 20:30 & 123.353 & 121.007 & & 98.802 & 102.937 & 94.399 & 108.465 & 105.341 & 77.346 & 72.799 \\
\hline 8/24/07 21:00 & 123.36 & 121.016 & & 98.819 & 102.943 & 94.406 & 108.497 & 105.349 & 77.35 & 72.803 \\
\hline 8/24/07 21:30 & 123.363 & 121.005 & & 98.802 & 102.948 & 94.409 & 108.528 & 105.356 & 77.353 & 72.809 \\
\hline 8/24/07 22:00 & 123.374 & 121.005 & & 98.803 & 102.964 & 94.416 & 109.156 & 105.365 & 77.357 & 72.817 \\
\hline $8 / 24 / 07$ 22:30 & 123.368 & 121.007 & & 98.795 & 102.956 & 94.437 & 108.562 & 105.356 & 77.353 & 72.807 \\
\hline 8/24/07 23:00 & 123.363 & 121.009 & & 98.798 & 102.948 & 94.413 & 108.572 & 105.356 & 77.351 & 72.805 \\
\hline 8/24/07 23:30 & 123.36 & 121.011 & & 98.805 & 102.948 & 94.411 & 108.546 & 105.354 & 77.35 & 72.803 \\
\hline 8/25/07 0:00 & 123.365 & 121.02 & & 98.809 & 102.948 & 94.413 & 108.524 & 105.356 & 77.346 & 72.807 \\
\hline 8/25/07 0:30 & 123.37 & 121.025 & & 98.809 & 102.952 & 94.418 & 108.5 & 105.358 & 77.348 & 72.809 \\
\hline 8/25/07 1:00 & 123.382 & 121.02 & & 98.817 & 102.965 & 94.43 & 108.489 & 105.372 & 77.35 & 72.821 \\
\hline 8/25/07 1:30 & 123.384 & 121.02 & & 98.821 & 102.967 & 94.432 & 108.482 & 105.374 & 77.348 & 72.823 \\
\hline 8/25/07 2:00 & 123.379 & 121.025 & & 98.815 & 102.96 & 94.425 & 108.48 & 105.367 & 77.348 & 72.815 \\
\hline $8 / 25 / 072: 30$ & 123.374 & 121.025 & & 98.817 & 102.964 & 94.43 & 108.48 & 105.369 & 77.348 & 72.819 \\
\hline $8 / 25 / 073: 00$ & 123.386 & 121.041 & & 98.822 & 102.969 & 94.435 & 108.478 & 105.376 & 77.348 & 72.825 \\
\hline 8/25/07 3:30 & 123.384 & 121.05 & & 98.826 & 102.967 & 94.432 & 108.476 & 105.374 & 77.348 & 72.823 \\
\hline 8/25/07 4:00 & 123.398 & 121.052 & & 98.843 & 102.985 & 94.449 & 108.47 & 105.389 & 77.351 & 72.84 \\
\hline 8/25/07 4:30 & 123.41 & 121.057 & & 98.848 & 102.994 & 94.461 & 108.472 & 105.4 & 77.357 & 72.848 \\
\hline 8/25/07 5:00 & 123.412 & 121.064 & & 98.85 & 102.998 & 94.461 & 108.472 & 105.403 & 77.357 & 72.848 \\
\hline 8/25/07 5:30 & 123.415 & 121.07 & & 98.857 & 102.998 & 94.465 & 108.471 & 105.405 & 77.357 & 72.852 \\
\hline 8/25/07 6:00 & 123.426 & 121.075 & & 98.865 & 103.009 & 94.475 & 108.488 & 105.414 & 77.362 & 72.863 \\
\hline 8/25/07 6:30 & 123.429 & 123.045 & & 98.872 & 103.013 & 94.479 & 108.493 & 105.418 & 77.366 & 72.865 \\
\hline 8/25/07 7:00 & 123.434 & 123.365 & & 98.876 & 103.019 & 94.484 & 108.503 & 105.423 & 77.37 & 72.869 \\
\hline 8/25/07 7:30 & 125.435 & 123.524 & & 99.596 & 103.493 & 95.099 & 109.12 & 106.866 & 77.388 & 72.931 \\
\hline 8/25/07 8:00 & 125.776 & 123.617 & & 99.854 & 103.796 & 95.408 & 109.404 & 107.231 & 77.42 & 73.142 \\
\hline 8/25/07 8:30 & 125.939 & 123.68 & & 99.994 & 103.946 & 95.561 & 109.543 & 107.4 & 77.449 & 73.295 \\
\hline 8/25/07 9:00 & 126.038 & 123.728 & & 100.08 & 104.041 & 95.656 & 109.629 & 107.504 & 77.481 & 73.396 \\
\hline 8/25/07 9:30 & 126.104 & 123.759 & & 100.139 & 104.104 & 95.717 & 109.685 & 107.57 & 77.506 & 73.462 \\
\hline 8/25/07 10:00 & 126.152 & 121.796 & & 100.183 & 104.152 & 95.764 & 109.726 & 107.619 & 77.53 & 73.514 \\
\hline 8/25/07 10:30 & 126.185 & 121.533 & & 100.214 & 104.184 & 95.797 & 109.76 & 107.656 & 77.556 & 73.549 \\
\hline 8/25/07 11:00 & 124.183 & 121.406 & & 99.482 & 103.689 & 95.161 & 109.128 & 106.2 & 77.558 & 73.489 \\
\hline 8/25/07 11:30 & 123.904 & 121.32 & & 99.272 & 103.447 & 94.913 & 108.897 & 105.905 & 77.552 & 73.326 \\
\hline 8/25/07 12:00 & 123.774 & 121.263 & & 99.158 & 103.325 & 94.795 & 108.788 & 105.768 & 77.543 & 73.21 \\
\hline 8/25/07 12:30 & 123.686 & 121.224 & & 99.088 & 103.243 & 94.71 & 108.728 & 105.679 & 77.538 & 73.123 \\
\hline 8/25/07 13:00 & 123.63 & 121.19 & & 99.038 & 103.19 & 94.659 & 108.731 & 105.62 & 77.532 & 73.065 \\
\hline 8/25/07 13:30 & 123.59 & 121.166 & & 99.002 & 103.15 & 94.621 & 108.706 & 105.577 & 77.525 & 73.018 \\
\hline 8/25/07 14:00 & 123.554 & 121.145 & & 98.974 & 103.12 & 94.588 & 108.675 & 105.542 & 77.517 & 72.987 \\
\hline 8/25/07 14:30 & 123.53 & 121.122 & & 98.95 & 103.099 & 94.569 & 108.656 & 105.52 & 77.512 & 72.964 \\
\hline 8/25/07 15:00 & 123.507 & 121.102 & & 98.931 & 103.078 & 94.548 & 108.636 & 105.496 & 77.506 & 72.943 \\
\hline $8 / 25 / 07$ 15:30 & 123.486 & 121.084 & & 98.907 & 103.053 & 94.524 & 108.622 & 105.473 & 77.497 & 72.918 \\
\hline 8/25/07 16:00 & 123.469 & 121.075 & & 98.893 & 103.038 & 94.508 & 108.617 & 105.454 & 77.49 & 72.9 \\
\hline 8/25/07 16:30 & 123.448 & 121.07 & & 98.872 & 103.019 & 94.487 & 108.615 & 105.434 & 77.484 & 72.881 \\
\hline 8/25/07 17:00 & 123.438 & 121.064 & & 98.865 & 103.011 & 94.482 & 108.598 & 105.427 & 77.48 & 72.877 \\
\hline 8/25/07 17:30 & 123.434 & 121.057 & & 98.862 & 103.007 & 94.479 & 108.594 & 105.418 & 77.475 & 72.869 \\
\hline 8/25/07 18:00 & 123.426 & 121.05 & & 98.855 & 103 & 94.47 & 108.594 & 105.414 & 77.468 & 72.863 \\
\hline $8 / 25 / 07$ 18:30 & 123.422 & 121.045 & & 98.85 & 102.996 & 94.463 & 108.6 & 105.407 & 77.464 & 72.856 \\
\hline 8/25/07 19:00 & 123.417 & 121.045 & & 98.843 & 102.99 & 94.456 & 108.598 & 105.4 & 77.458 & 72.85 \\
\hline 8/25/07 19:30 & 123.41 & 121.045 & & 98.84 & 102.983 & 94.449 & 108.588 & 105.394 & 77.456 & 72.846 \\
\hline 8/25/07 20:00 & 123.408 & 121.054 & & 98.838 & 102.983 & 94.449 & 108.581 & 105.394 & 77.451 & 72.844 \\
\hline 8/25/07 20:30 & 123.405 & 121.061 & & 98.84 & 102.983 & 94.449 & 108.585 & 105.392 & 77.451 & 72.844 \\
\hline 8/25/07 21:00 & 123.417 & 121.066 & & 98.852 & 102.99 & 94.458 & 108.579 & 105.4 & 77.451 & 72.853 \\
\hline 8/25/07 21:30 & 123.422 & 121.064 & & 98.857 & 102.998 & 94.465 & 108.575 & 105.407 & 77.453 & 72.858 \\
\hline 8/25/07 22:00 & 123.429 & 121.066 & & 98.86 & 103.007 & 94.475 & 108.583 & 105.416 & 77.453 & 72.867 \\
\hline 8/25/07 22:30 & 123.429 & 121.061 & & 98.859 & 103.004 & 94.47 & 108.573 & 105.414 & 77.453 & 72.863 \\
\hline 8/25/07 23:00 & 123.426 & 121.054 & & 98.86 & 103.004 & 94.47 & 108.571 & 105.414 & 77.451 & 72.863 \\
\hline 8/25/07 23:30 & 123.424 & 121.045 & & 98.853 & 102.998 & 94.465 & 108.577 & 105.407 & 77.449 & 72.858 \\
\hline 8/26/07 0:00 & 123.417 & 121.045 & & 98.845 & 102.99 & 94.458 & 108.579 & 105.403 & 77.445 & 72.85 \\
\hline 8/26/07 0:30 & 123.408 & 121.041 & & 98.84 & 102.983 & 94.451 & 108.586 & 105.394 & 77.444 & 72.844 \\
\hline 8/26/07 1:00 & 123.41 & 121.039 & & 98.84 & 102.985 & 94.451 & 108.583 & 105.394 & 77.44 & 72.842 \\
\hline $8 / 26 / 071: 30$ & 123.405 & 121.036 & & 98.838 & 102.981 & 94.446 & 108.577 & 105.392 & 77.438 & 72.842 \\
\hline 8/26/07 2:00 & 123.403 & 121.032 & & 98.833 & 102.975 & 94.442 & 108.571 & 105.385 & 77.434 & 72.836 \\
\hline 8/26/07 2:30 & 123.405 & 121.03 & & 98.829 & 102.973 & 94.439 & 108.569 & 105.385 & 77.432 & 72.834 \\
\hline 8/26/07 3:00 & 123.403 & 121.032 & & 98.826 & 102.971 & 94.437 & 108.566 & 105.38 & 77.429 & 72.829 \\
\hline 8/26/07 3:30 & 123.4 & 121.025 & & 98.822 & 102.965 & 94.432 & 108.564 & 105.374 & 77.425 & 72.825 \\
\hline 8/26/07 4:00 & 123.4 & 121.02 & & 98.827 & 102.965 & 94.435 & 108.56 & 105.376 & 77.425 & 72.825 \\
\hline 8/26/07 4:30 & 123.403 & 121.016 & & 98.819 & 102.964 & 94.43 & 108.558 & 105.374 & 77.421 & 72.823 \\
\hline 8/26/07 5:00 & 123.403 & 121.02 & & 98.815 & 102.96 & 94.428 & 108.558 & 105.369 & 77.42 & 72.817 \\
\hline
\end{tabular}


TABLE S1.1 (Cont.)

\begin{tabular}{|c|c|c|c|c|c|c|c|c|c|c|}
\hline \multirow[b]{2}{*}{ Date and Time } & \multicolumn{10}{|c|}{ Depth from Top of Casing (ft) } \\
\hline & MW1D & MW2D & MW3D & MW4D & MW7 & MW9 & Oentrich & MW14D & MW15D & MW16D \\
\hline 8/26/07 5:30 & 123.403 & 121.02 & & 98.814 & 102.956 & 94.423 & 108.56 & 105.365 & 77.416 & 72.815 \\
\hline 8/26/07 6:00 & 123.4 & 121.014 & & 98.815 & 102.96 & 94.425 & 108.558 & 105.367 & 77.414 & 72.817 \\
\hline 8/26/07 6:30 & 123.4 & 121.023 & & 98.814 & 102.96 & 94.428 & 108.556 & 105.367 & 77.414 & 72.817 \\
\hline 8/26/07 7:00 & 123.405 & 122.603 & & 98.81 & 102.954 & 94.423 & 108.558 & 105.365 & 77.41 & 72.811 \\
\hline $8 / 26 / 07$ 7:30 & 123.403 & 123.147 & & 98.824 & 102.954 & 94.423 & 108.556 & 105.365 & 77.412 & 72.811 \\
\hline 8/26/07 8:00 & 124.828 & 123.344 & & 99.358 & 103.162 & 94.751 & 108.868 & 106.146 & 77.416 & 72.84 \\
\hline 8/26/07 8:30 & 125.504 & 123.46 & & 99.744 & 103.645 & 95.271 & 109.401 & 107.003 & 77.425 & 72.993 \\
\hline 8/26/07 9:00 & 125.717 & 123.519 & & 99.911 & 103.841 & 95.469 & 109.589 & 107.22 & 77.451 & 73.169 \\
\hline 8/26/07 9:30 & 125.854 & 123.564 & & 100.013 & 103.95 & 95.578 & 109.699 & 107.342 & 77.477 & 73.282 \\
\hline 8/26/07 10:00 & 125.92 & 123.596 & & 100.065 & 104.009 & 95.637 & 109.769 & 107.408 & 77.497 & 73.346 \\
\hline 8/26/07 10:30 & 125.975 & 123.612 & & 100.104 & 104.054 & 95.679 & 109.806 & 107.455 & 77.515 & 73.394 \\
\hline 8/26/07 11:00 & 126.055 & 123.626 & & 100.132 & 104.081 & 95.71 & 109.838 & 107.486 & 77.534 & 73.427 \\
\hline 8/26/07 11:30 & 126.074 & 123.632 & & 100.147 & 104.102 & 95.729 & 109.865 & 107.506 & 77.549 & 73.45 \\
\hline 8/26/07 12:00 & 126.086 & 123.632 & & 100.161 & 104.115 & 95.743 & 109.879 & 107.521 & 77.56 & 73.464 \\
\hline 8/26/07 12:30 & 126.092 & 123.635 & & 100.164 & 104.121 & 95.75 & 109.897 & 107.528 & 77.574 & 73.474 \\
\hline 8/26/07 13:00 & 126.095 & 123.632 & & 100.166 & 104.123 & 95.755 & 109.906 & 107.53 & 77.585 & 73.479 \\
\hline $8 / 26 / 07$ 13:30 & 126.097 & 122.15 & & 100.17 & 104.125 & 95.752 & 109.915 & 107.532 & 77.597 & 73.485 \\
\hline 8/26/07 14:00 & 126.095 & 121.558 & & 100.171 & 104.123 & 95.75 & 109.924 & 107.535 & 77.604 & 73.485 \\
\hline 8/26/07 14:30 & 124.7 & 121.345 & & 99.656 & 103.965 & 95.484 & 109.658 & 106.906 & 77.611 & 73.481 \\
\hline 8/26/07 15:00 & 123.937 & 121.222 & & 99.253 & 103.445 & 94.915 & 109.119 & 105.941 & 77.589 & 73.315 \\
\hline 8/26/07 15:30 & 123.717 & 121.154 & & 99.077 & 103.249 & 94.715 & 108.928 & 105.708 & 77.571 & 73.15 \\
\hline 8/26/07 16:00 & 123.592 & 121.095 & & 98.972 & 103.131 & 94.6 & 108.76 & 105.582 & 77.554 & 73.032 \\
\hline 8/26/07 16:30 & 123.516 & 121.057 & & 98.91 & 103.061 & 94.527 & 108.695 & 105.502 & 77.539 & 72.956 \\
\hline 8/26/07 17:00 & 123.457 & 121.03 & & 98.86 & 103.011 & 94.479 & 108.65 & 105.445 & 77.526 & 72.9 \\
\hline 8/26/07 17:30 & 123.419 & 121.009 & & 98.826 & 102.973 & 94.442 & 108.614 & 105.405 & 77.514 & 72.86 \\
\hline 8/26/07 18:00 & 123.393 & 120.991 & & 98.805 & 102.952 & 94.421 & 108.593 & 105.38 & 77.503 & 72.836 \\
\hline 8/26/07 18:30 & 123.372 & 120.982 & & 98.784 & 102.929 & 94.399 & 108.575 & 105.356 & 77.493 & 72.811 \\
\hline 8/26/07 19:00 & 123.351 & 120.973 & & 98.769 & 102.914 & 94.38 & 108.56 & 105.336 & 77.482 & 72.793 \\
\hline 8/26/07 19:30 & 123.344 & 120.973 & & 98.762 & 102.908 & 94.373 & 108.556 & 105.33 & 77.477 & 72.784 \\
\hline 8/26/07 20:00 & 123.337 & 120.982 & & 98.757 & 102.899 & 94.366 & 108.548 & 105.318 & 77.469 & 72.774 \\
\hline 8/26/07 20:30 & 123.334 & 120.989 & & 98.759 & 102.901 & 94.369 & 108.544 & 105.316 & 77.462 & 72.774 \\
\hline 8/26/07 21:00 & 123.339 & 120.993 & & 98.769 & 102.908 & 94.376 & 108.554 & 105.325 & 77.462 & 72.782 \\
\hline 8/26/07 21:30 & 123.348 & 120.998 & & 98.776 & 102.918 & 94.385 & 108.554 & 105.334 & 77.46 & 72.79 \\
\hline 8/26/07 22:00 & 123.356 & 120.989 & & 98.783 & 102.924 & 94.392 & 108.558 & 105.338 & 77.458 & 72.792 \\
\hline 8/26/07 22:30 & 123.36 & 120.98 & & 98.783 & 102.925 & 94.392 & 108.56 & 105.343 & 77.456 & 72.794 \\
\hline 8/26/07 23:00 & 123.351 & 120.977 & & 98.772 & 102.918 & 94.385 & 108.56 & 105.336 & 77.451 & 72.786 \\
\hline 8/26/07 23:30 & 123.341 & 120.975 & & 98.769 & 102.908 & 94.373 & 108.552 & 105.325 & 77.445 & 72.776 \\
\hline 8/27/07 0:00 & 123.339 & 120.975 & & 98.762 & 102.905 & 94.373 & 108.546 & 105.321 & 77.44 & 72.776 \\
\hline $8 / 27 / 07$ 0:30 & 123.334 & 120.968 & & 98.762 & 102.905 & 94.369 & 108.546 & 105.318 & 77.438 & 72.769 \\
\hline 8/27/07 1:00 & 123.337 & 120.966 & & 98.762 & 102.908 & 94.373 & 108.546 & 105.323 & 77.436 & 72.776 \\
\hline 8/27/07 1:30 & 123.332 & 120.961 & & 98.759 & 102.901 & 94.366 & 108.543 & 105.316 & 77.431 & 72.769 \\
\hline 8/27/07 2:00 & 123.325 & 120.961 & & 98.755 & 102.893 & 94.362 & 108.537 & 105.307 & 77.425 & 72.761 \\
\hline $8 / 27 / 072: 30$ & 123.322 & 120.966 & & 98.752 & 102.895 & 94.362 & 108.535 & 105.307 & 77.42 & 72.759 \\
\hline 8/27/07 3:00 & 123.325 & 120.966 & & 98.753 & 102.895 & 94.364 & 108.535 & 105.31 & 77.414 & 72.762 \\
\hline 8/27/07 3:30 & 123.325 & 120.961 & & 98.753 & 102.897 & 94.364 & 108.531 & 105.31 & 77.412 & 72.763 \\
\hline 8/27/07 4:00 & 123.325 & 120.957 & & 98.755 & 102.899 & 94.364 & 108.531 & 105.312 & 77.409 & 72.763 \\
\hline 8/27/07 4:30 & 123.32 & 120.957 & & 98.748 & 102.897 & 94.359 & 108.531 & 105.305 & 77.405 & 72.759 \\
\hline 8/27/07 5:00 & 123.315 & 120.957 & & 98.745 & 102.887 & 94.355 & 108.529 & 105.301 & 77.399 & 72.753 \\
\hline 8/27/07 5:30 & 123.318 & 122.347 & & 98.747 & 102.891 & 94.355 & 108.533 & 105.303 & 77.396 & 72.753 \\
\hline 8/27/07 6:00 & 123.315 & 123.093 & & 98.747 & 102.889 & 94.357 & 108.529 & 105.301 & 77.396 & 72.753 \\
\hline 8/27/07 6:30 & 124.173 & 123.299 & & 99.153 & 102.906 & 94.373 & 108.572 & 105.303 & 77.388 & 72.753 \\
\hline 8/27/07 7:00 & 125.502 & 123.424 & & 99.605 & 103.527 & 95.137 & 109.297 & 106.943 & 77.418 & 72.91 \\
\hline 8/27/07 7:30 & 125.721 & 123.494 & & 99.78 & 103.729 & 95.342 & 109.483 & 107.171 & 77.442 & 73.088 \\
\hline 8/27/07 8:00 & 125.84 & 123.549 & & 99.889 & 103.845 & 95.462 & 109.59 & 107.3 & 77.466 & 73.21 \\
\hline 8/27/07 8:30 & 125.918 & 123.587 & & 99.959 & 103.919 & 95.531 & 109.66 & 107.377 & 77.484 & 73.291 \\
\hline 8/27/07 9:00 & 125.974 & 121.741 & & 100.011 & 103.974 & 95.59 & 109.71 & 107.439 & 77.508 & 73.349 \\
\hline 8/27/07 9:30 & 126.01 & 121.426 & & 100.047 & 104.014 & 95.625 & 109.744 & 107.479 & 77.53 & 73.39 \\
\hline 8/27/07 10:00 & 124.14 & 121.288 & & 99.399 & 103.618 & 95.092 & 109.211 & 106.169 & 77.534 & 73.374 \\
\hline 8/27/07 10:30 & 123.8 & 121.209 & & 99.151 & 103.333 & 94.795 & 108.933 & 105.794 & 77.523 & 73.212 \\
\hline 8/27/07 11:00 & 123.656 & 121.159 & & 99.038 & 103.198 & 94.666 & 108.811 & 105.646 & 77.521 & 73.092 \\
\hline 8/27/07 11:30 & 123.573 & 121.125 & & 98.972 & 103.125 & 94.593 & 108.74 & 105.562 & 77.512 & 73.009 \\
\hline 8/27/07 12:00 & 123.521 & 121.095 & & 98.924 & 103.072 & 94.548 & 108.694 & 105.511 & 77.506 & 72.953 \\
\hline 8/27/07 12:30 & 123.488 & 121.073 & & 98.895 & 103.044 & 94.51 & 108.663 & 105.471 & 77.504 & 72.918 \\
\hline 8/27/07 13:00 & 123.455 & 121.061 & & 98.871 & 103.013 & 94.487 & 108.64 & 105.44 & 77.497 & 72.887 \\
\hline 8/27/07 13:30 & 123.436 & 121.043 & & 98.855 & 102.998 & 94.468 & 108.623 & 105.42 & 77.491 & 72.869 \\
\hline 8/27/07 14:00 & 123.419 & 121.032 & & 98.84 & 102.981 & 94.451 & 108.61 & 105.405 & 77.486 & 72.85 \\
\hline
\end{tabular}


TABLE S1.1 (Cont.)

\begin{tabular}{|c|c|c|c|c|c|c|c|c|c|c|}
\hline \multirow[b]{2}{*}{ Date and Time } & \multicolumn{10}{|c|}{ Depth from Top of Casing (ft) } \\
\hline & MW1D & MW2D & MW3D & MW4D & MW7 & MW9 & Oentrich & MW14D & MW15D & MW16D \\
\hline 8/27/07 14:30 & 123.408 & 121.018 & & 98.827 & 102.969 & 94.437 & 108.602 & 105.389 & 77.48 & 72.838 \\
\hline $8 / 27 / 07$ 15:00 & 123.389 & 121.007 & & 98.812 & 102.956 & 94.425 & 108.589 & 105.376 & 77.475 & 72.821 \\
\hline 8/27/07 15:30 & 123.377 & 121.002 & & 98.8 & 102.941 & 94.411 & 108.582 & 105.361 & 77.468 & 72.807 \\
\hline 8/27/07 16:00 & 123.367 & 120.995 & & 98.791 & 102.933 & 94.402 & 108.571 & 105.352 & 77.462 & 72.798 \\
\hline $8 / 27 / 0716: 30$ & 123.363 & 120.989 & & 98.786 & 102.927 & 94.397 & 108.567 & 105.345 & 77.455 & 72.795 \\
\hline 8/27/07 17:00 & 123.356 & 120.982 & & 98.781 & 102.922 & 94.395 & 108.561 & 105.338 & 77.447 & 72.786 \\
\hline 8/27/07 17:30 & 123.348 & 120.977 & & 98.774 & 102.916 & 94.388 & 108.559 & 105.332 & 77.44 & 72.782 \\
\hline 8/27/07 18:00 & 123.344 & 120.973 & & 98.769 & 102.91 & 94.383 & 108.552 & 105.325 & 77.436 & 72.776 \\
\hline 8/27/07 18:30 & 123.339 & 120.977 & & 98.765 & 102.908 & 94.378 & 108.548 & 105.321 & 77.431 & 72.769 \\
\hline 8/27/07 19:00 & 123.334 & 120.975 & & 98.76 & 102.901 & 94.373 & 108.54 & 105.316 & 77.425 & 72.763 \\
\hline 8/27/07 19:30 & 123.341 & 120.975 & & 98.765 & 102.908 & 94.378 & 108.55 & 105.323 & 77.42 & 72.765 \\
\hline 8/27/07 20:00 & 123.337 & 120.986 & & 98.764 & 102.905 & 94.373 & 108.542 & 105.318 & 77.418 & 72.765 \\
\hline 8/27/07 20:30 & 123.339 & 121.002 & & 98.769 & 102.906 & 94.376 & 108.544 & 105.321 & 77.416 & 72.767 \\
\hline 8/27/07 21:00 & 123.348 & 121.014 & & 98.779 & 102.92 & 94.385 & 108.554 & 105.327 & 77.418 & 72.778 \\
\hline 8/27/07 21:30 & 123.365 & 121.023 & & 98.796 & 102.935 & 94.402 & 108.565 & 105.345 & 77.418 & 72.794 \\
\hline 8/27/07 22:00 & 123.377 & 121.027 & & 98.807 & 102.946 & 94.418 & 108.567 & 105.356 & 77.421 & 72.803 \\
\hline 8/27/07 22:30 & 123.384 & 121.032 & & 98.815 & 102.954 & 94.423 & 108.569 & 105.365 & 77.425 & 72.811 \\
\hline 8/27/07 23:00 & 123.389 & 121.034 & & 98.821 & 102.958 & 94.428 & 108.571 & 105.372 & 77.425 & 72.815 \\
\hline 8/27/07 23:30 & 123.391 & 121.034 & & 98.822 & 102.962 & 94.43 & 108.571 & 105.374 & 77.425 & 72.819 \\
\hline 8/28/07 0:00 & 123.396 & 121.03 & & 98.826 & 102.964 & 94.432 & 108.572 & 105.376 & 77.425 & 72.82 \\
\hline 8/28/07 0:30 & 123.398 & 121.03 & & 98.824 & 102.965 & 94.435 & 108.569 & 105.378 & 77.425 & 72.822 \\
\hline 8/28/07 1:00 & 123.393 & 121.03 & & 98.822 & 102.964 & 94.43 & 108.569 & 105.374 & 77.423 & 72.815 \\
\hline 8/28/07 1:30 & 123.393 & 121.023 & & 98.824 & 102.964 & 94.43 & 108.569 & 105.374 & 77.421 & 72.817 \\
\hline 8/28/07 2:00 & 123.393 & 121.023 & & 98.817 & 102.96 & 94.43 & 108.567 & 105.374 & 77.418 & 72.815 \\
\hline 8/28/07 2:30 & 123.384 & 121.02 & & 98.814 & 102.954 & 94.425 & 108.567 & 105.367 & 77.418 & 72.809 \\
\hline 8/28/07 3:00 & 123.384 & 121.014 & & 98.812 & 102.952 & 94.425 & 108.565 & 105.365 & 77.416 & 72.809 \\
\hline 8/28/07 3:30 & 123.382 & 121.014 & & 98.81 & 102.95 & 94.418 & 108.565 & 105.361 & 77.412 & 72.803 \\
\hline 8/28/07 4:00 & 123.374 & 121.011 & & 98.805 & 102.946 & 94.413 & 108.561 & 105.356 & 77.407 & 72.798 \\
\hline 8/28/07 4:30 & 123.374 & 121.009 & & 98.805 & 102.943 & 94.413 & 108.563 & 105.356 & 77.403 & 72.798 \\
\hline 8/28/07 5:00 & 123.374 & 121.014 & & 98.805 & 102.943 & 94.413 & 108.561 & 105.354 & 77.401 & 72.799 \\
\hline $8 / 28 / 075: 30$ & 123.37 & 122.564 & & 98.802 & 102.939 & 94.409 & 108.561 & 105.349 & 77.397 & 72.795 \\
\hline 8/28/07 6:00 & 123.367 & 123.147 & & 98.803 & 102.943 & 94.413 & 108.559 & 105.354 & 77.396 & 72.799 \\
\hline 8/28/07 6:30 & 124.849 & 123.351 & & 99.331 & 103.11 & 94.696 & 108.86 & 106.06 & 77.397 & 72.801 \\
\hline 8/28/07 7:00 & 125.584 & 123.469 & & 99.739 & 103.643 & 95.271 & 109.391 & 106.999 & 77.431 & 72.989 \\
\hline 8/28/07 7:30 & 125.799 & 123.546 & & 99.915 & 103.841 & 95.472 & 109.571 & 107.22 & 77.458 & 73.168 \\
\hline 8/28/07 8:00 & 125.92 & 123.601 & & 100.02 & 103.954 & 95.585 & 109.678 & 107.344 & 77.482 & 73.285 \\
\hline 8/28/07 8:30 & 126 & 123.642 & & 100.094 & 104.033 & 95.66 & 109.742 & 107.43 & 77.508 & 73.369 \\
\hline 8/28/07 9:00 & 126.057 & 123.673 & & 100.142 & 104.083 & 95.715 & 109.791 & 107.486 & 77.534 & 73.425 \\
\hline 8/28/07 9:30 & 126.1 & 123.696 & & 100.18 & 104.125 & 95.755 & 109.825 & 107.53 & 77.554 & 73.466 \\
\hline 8/28/07 10:00 & 126.133 & 123.716 & & 100.209 & 104.157 & 95.79 & 109.849 & 107.563 & 77.574 & 73.502 \\
\hline 8/28/07 10:30 & 126.154 & 123.728 & & 100.232 & 104.178 & 95.809 & 109.868 & 107.585 & 77.595 & 73.524 \\
\hline 8/28/07 11:00 & 126.178 & 123.746 & & 100.249 & 104.199 & 95.83 & 109.889 & 107.608 & 77.613 & 73.545 \\
\hline 8/28/07 11:30 & 126.185 & 121.975 & & 100.261 & 104.209 & 95.842 & 109.9 & 107.619 & 77.632 & 73.558 \\
\hline 8/28/07 12:00 & 126.204 & 121.605 & & 100.275 & 104.224 & 95.854 & 109.915 & 107.636 & 77.646 & 73.572 \\
\hline 8/28/07 12:30 & 124.395 & 121.433 & & 99.599 & 103.834 & 95.316 & 109.383 & 106.43 & 77.652 & 73.547 \\
\hline 8/28/07 13:00 & 123.982 & 121.338 & & 99.31 & 103.491 & 94.96 & 109.053 & 105.974 & 77.635 & 73.365 \\
\hline 8/28/07 13:30 & 123.807 & 121.272 & & 99.169 & 103.335 & 94.802 & 108.91 & 105.792 & 77.624 & 73.223 \\
\hline 8/28/07 14:00 & 123.705 & 121.224 & & 99.088 & 103.24 & 94.71 & 108.828 & 105.688 & 77.615 & 73.127 \\
\hline 8/28/07 14:30 & 123.639 & 121.193 & & 99.031 & 103.179 & 94.654 & 108.775 & 105.62 & 77.606 & 73.061 \\
\hline 8/28/07 15:00 & 123.587 & 121.168 & & 98.989 & 103.133 & 94.607 & 108.737 & 105.569 & 77.597 & 73.009 \\
\hline 8/28/07 15:30 & 123.559 & 121.147 & & 98.96 & 103.104 & 94.578 & 108.719 & 105.538 & 77.585 & 72.979 \\
\hline 8/28/07 16:00 & 123.53 & 121.122 & & 98.939 & 103.078 & 94.553 & 108.706 & 105.509 & 77.578 & 72.95 \\
\hline 8/28/07 16:30 & 123.507 & 121.091 & & 98.919 & 103.061 & 94.534 & 108.687 & 105.489 & 77.569 & 72.929 \\
\hline 8/28/07 17:00 & 123.486 & 121.07 & & 98.896 & 103.04 & 94.51 & 108.672 & 105.465 & 77.558 & 72.906 \\
\hline 8/28/07 17:30 & 123.455 & 121.059 & & 98.865 & 103.011 & 94.482 & 108.646 & 105.434 & 77.545 & 72.875 \\
\hline 8/28/07 18:00 & 123.434 & 121.068 & & 98.848 & 102.99 & 94.461 & 108.632 & 105.414 & 77.534 & 72.857 \\
\hline 8/28/07 18:30 & 123.422 & 121.073 & & 98.841 & 102.979 & 94.449 & 108.625 & 105.4 & 77.525 & 72.844 \\
\hline 8/28/07 19:00 & 123.431 & 121.077 & & 98.855 & 102.994 & 94.463 & 108.617 & 105.409 & 77.525 & 72.856 \\
\hline 8/28/07 19:30 & 123.436 & 121.077 & & 98.857 & 102.996 & 94.465 & 108.614 & 105.411 & 77.523 & 72.859 \\
\hline 8/28/07 20:00 & 123.441 & 121.079 & & 98.86 & 103.002 & 94.47 & 108.614 & 105.418 & 77.521 & 72.865 \\
\hline 8/28/07 20:30 & 123.441 & 121.1 & & 98.864 & 103.004 & 94.47 & 108.612 & 105.418 & 77.519 & 72.865 \\
\hline 8/28/07 21:00 & 123.445 & 121.12 & & 98.867 & 103.004 & 94.475 & 108.614 & 105.423 & 77.515 & 72.865 \\
\hline 8/28/07 21:30 & 123.464 & 121.152 & & 98.891 & 103.026 & 94.496 & 108.619 & 105.44 & 77.519 & 72.885 \\
\hline 8/28/07 22:00 & 123.483 & 121.188 & & 98.915 & 103.045 & 94.515 & 108.621 & 105.46 & 77.525 & 72.904 \\
\hline 8/28/07 22:30 & 123.516 & 121.22 & & 98.945 & 103.082 & 94.55 & 108.615 & 105.491 & 77.534 & 72.933 \\
\hline 8/28/07 23:00 & 123.556 & 121.24 & & 98.988 & 103.12 & 94.59 & 108.61 & 105.529 & 77.55 & 72.974 \\
\hline
\end{tabular}


TABLE S1.1 (Cont.)

\begin{tabular}{|c|c|c|c|c|c|c|c|c|c|c|}
\hline \multirow[b]{2}{*}{ Date and Time } & \multicolumn{10}{|c|}{ Depth from Top of Casing (ft) } \\
\hline & MW1D & MW2D & MW3D & MW4D & MW7 & MW9 & Oentrich & MW14D & MW15D & MW16D \\
\hline 8/28/07 23:30 & 123.585 & 121.249 & & 99.017 & 103.146 & 94.619 & 108.61 & 105.56 & 77.558 & 73.001 \\
\hline 8/29/07 0:00 & 123.608 & 121.27 & & 99.029 & 103.169 & 94.64 & 108.606 & 105.584 & 77.567 & 73.018 \\
\hline 8/29/07 0:30 & 123.616 & 121.238 & & 99.041 & 103.179 & 94.649 & 108.606 & 105.595 & 77.573 & 73.028 \\
\hline 8/29/07 1:00 & 123.632 & 121.22 & & 99.062 & 103.196 & 94.666 & 108.602 & 105.608 & 77.58 & 73.044 \\
\hline 8/29/07 1:30 & 123.658 & 121.204 & & 99.026 & 103.162 & 94.637 & 108.752 & 105.584 & 77.556 & 73.016 \\
\hline $8 / 29 / 072: 00$ & 123.66 & 121.204 & & 99.007 & 103.152 & 94.626 & 108.745 & 105.571 & 77.55 & 73.003 \\
\hline $8 / 29 / 072: 30$ & 123.658 & 121.202 & & 98.993 & 103.129 & 94.6 & 108.732 & 105.547 & 77.543 & 72.976 \\
\hline 8/29/07 3:00 & 123.646 & 121.204 & & 98.998 & 103.131 & 94.604 & 108.726 & 105.547 & 77.543 & 72.98 \\
\hline 8/29/07 3:30 & 123.642 & 121.213 & & 98.995 & 103.133 & 94.604 & 108.726 & 105.547 & 77.543 & 72.982 \\
\hline $8 / 29 / 074: 00$ & 123.637 & 121.22 & & 98.996 & 103.131 & 94.604 & 108.726 & 105.547 & 77.545 & 72.983 \\
\hline 8/29/07 4:30 & 123.632 & 121.224 & & 99.008 & 103.143 & 94.616 & 108.732 & 105.558 & 77.547 & 72.995 \\
\hline 8/29/07 5:00 & 123.627 & 122.644 & & 99.014 & 103.148 & 94.621 & 108.734 & 105.562 & 77.549 & 72.999 \\
\hline $8 / 29 / 075: 30$ & 123.623 & 123.34 & & 99.017 & 103.152 & 94.626 & 108.741 & 105.569 & 77.55 & 73.003 \\
\hline 8/29/07 6:00 & 124.603 & 123.567 & & 99.498 & 103.221 & 94.741 & 108.888 & 105.713 & 77.552 & 73.014 \\
\hline 8/29/07 6:30 & 125.754 & 123.696 & & 99.946 & 103.843 & 95.476 & 109.567 & 107.196 & 77.589 & 73.193 \\
\hline $8 / 29 / 07$ 7:00 & 126.003 & 123.775 & & 100.142 & 104.06 & 95.691 & 109.768 & 107.442 & 77.624 & 73.386 \\
\hline $8 / 29 / 07$ 7:30 & 126.135 & 123.839 & & 100.258 & 104.188 & 95.82 & 109.883 & 107.579 & 77.654 & 73.514 \\
\hline 8/29/07 8:00 & 126.22 & 122.313 & & 100.33 & 104.268 & 95.901 & 109.956 & 107.663 & 77.681 & 73.603 \\
\hline 8/29/07 8:30 & 126.282 & 121.818 & & 100.387 & 104.327 & 95.96 & 110.01 & 107.729 & 77.707 & 73.663 \\
\hline 8/29/07 9:00 & 124.894 & 121.658 & & 99.875 & 104.15 & 95.653 & 109.689 & 106.939 & 77.729 & 73.704 \\
\hline 8/29/07 9:30 & 124.306 & 121.574 & & 99.537 & 103.714 & 95.187 & 109.239 & 106.193 & 77.722 & 73.564 \\
\hline 8/29/07 10:00 & 124.133 & 121.517 & & 99.406 & 103.563 & 95.036 & 109.087 & 106.016 & 77.716 & 73.438 \\
\hline $8 / 29 / 07$ 10:30 & 124.046 & 121.485 & & 99.336 & 103.483 & 94.956 & 109.006 & 105.925 & 77.718 & 73.357 \\
\hline 8/29/07 11:00 & 123.989 & 121.46 & & 99.289 & 103.43 & 94.904 & 108.959 & 105.868 & 77.715 & 73.297 \\
\hline 8/29/07 11:30 & 123.956 & 121.449 & & 99.26 & 103.401 & 94.875 & 108.929 & 105.834 & 77.716 & 73.264 \\
\hline $8 / 29 / 0712: 00$ & 123.942 & 121.433 & & 99.241 & 103.375 & 94.849 & 108.912 & 105.808 & 77.715 & 73.233 \\
\hline 8/29/07 12:30 & 123.925 & 121.424 & & 99.232 & 103.365 & 94.84 & 108.897 & 105.794 & 77.716 & 73.221 \\
\hline 8/29/07 13:00 & 123.911 & 121.408 & & 99.217 & 103.352 & 94.826 & 108.891 & 105.779 & 77.716 & 73.204 \\
\hline 8/29/07 13:30 & 123.901 & 121.397 & & 99.208 & 103.344 & 94.819 & 108.886 & 105.77 & 77.718 & 73.198 \\
\hline 8/29/07 14:00 & 123.802 & 121.383 & & 99.194 & 103.329 & 94.802 & 108.882 & 105.755 & 77.716 & 73.183 \\
\hline 8/29/07 14:30 & 123.767 & 121.372 & & 99.182 & 103.318 & 94.791 & 108.882 & 105.744 & 77.711 & 73.173 \\
\hline 8/29/07 15:00 & 123.75 & 121.358 & & 99.169 & 103.304 & 94.781 & 108.873 & 105.728 & 77.709 & 73.159 \\
\hline 8/29/07 15:30 & 123.741 & 121.349 & & 99.157 & 103.293 & 94.767 & 108.869 & 105.717 & 77.709 & 73.146 \\
\hline 8/29/07 16:00 & 123.727 & 121.345 & & 99.145 & 103.283 & 94.758 & 108.861 & 105.706 & 77.703 & 73.135 \\
\hline 8/29/07 16:30 & 123.717 & 121.34 & & 99.139 & 103.274 & 94.751 & 108.854 & 105.695 & 77.698 & 73.129 \\
\hline 8/29/07 17:00 & 123.712 & 121.333 & & 99.132 & 103.27 & 94.741 & 108.848 & 105.69 & 77.696 & 73.123 \\
\hline 8/29/07 17:30 & 123.708 & 121.333 & & 99.129 & 103.266 & 94.739 & 108.844 & 105.684 & 77.691 & 73.119 \\
\hline 8/29/07 18:00 & 123.701 & 121.336 & & 99.124 & 103.259 & 94.734 & 108.839 & 105.677 & 77.689 & 73.113 \\
\hline 8/29/07 18:30 & 123.698 & 121.34 & & 99.124 & 103.257 & 94.732 & 108.837 & 105.675 & 77.685 & 73.111 \\
\hline 8/29/07 19:00 & 123.701 & 121.347 & & 99.126 & 103.261 & 94.736 & 108.839 & 105.679 & 77.685 & 73.115 \\
\hline 8/29/07 19:30 & 123.705 & 121.354 & & 99.132 & 103.266 & 94.739 & 108.843 & 105.682 & 77.683 & 73.119 \\
\hline 8/29/07 20:00 & 123.712 & 121.367 & & 99.138 & 103.27 & 94.743 & 108.848 & 105.688 & 77.685 & 73.123 \\
\hline 8/29/07 20:30 & 123.72 & 121.376 & & 99.148 & 103.28 & 94.753 & 108.854 & 105.697 & 77.687 & 73.132 \\
\hline 8/29/07 21:00 & 123.729 & 121.385 & & 99.158 & 103.291 & 94.762 & 108.859 & 105.706 & 77.689 & 73.14 \\
\hline 8/29/07 21:30 & 123.741 & 121.392 & & 99.17 & 103.302 & 94.776 & 108.863 & 105.719 & 77.694 & 73.152 \\
\hline 8/29/07 22:00 & 123.748 & 121.395 & & 99.179 & 103.31 & 94.786 & 108.865 & 105.728 & 77.698 & 73.161 \\
\hline 8/29/07 22:30 & 123.757 & 121.395 & & 99.184 & 103.318 & 94.791 & 108.865 & 105.735 & 77.702 & 73.167 \\
\hline 8/29/07 23:00 & 123.76 & 121.397 & & 99.186 & 103.32 & 94.795 & 108.865 & 105.737 & 77.703 & 73.169 \\
\hline 8/29/07 23:30 & 123.762 & 121.401 & & 99.188 & 103.321 & 94.793 & 108.863 & 105.739 & 77.703 & 73.171 \\
\hline 8/30/07 0:00 & 123.762 & 121.404 & & 99.189 & 103.321 & 94.795 & 108.865 & 105.741 & 77.705 & 73.171 \\
\hline $8 / 30 / 07$ 0:30 & 123.76 & 121.406 & & 99.189 & 103.325 & 94.798 & 108.865 & 105.744 & 77.707 & 73.173 \\
\hline 8/30/07 1:00 & 123.76 & 121.408 & & 99.194 & 103.325 & 94.8 & 108.863 & 105.748 & 77.709 & 73.175 \\
\hline 8/30/07 1:30 & 123.757 & 121.404 & & 99.196 & 103.329 & 94.8 & 108.863 & 105.748 & 77.711 & 73.177 \\
\hline 8/30/07 2:00 & 123.757 & 121.399 & & 99.198 & 103.331 & 94.805 & 108.863 & 105.75 & 77.713 & 73.179 \\
\hline 8/30/07 2:30 & 123.757 & 121.395 & & 99.191 & 103.327 & 94.802 & 108.861 & 105.746 & 77.711 & 73.177 \\
\hline 8/30/07 3:00 & 123.755 & 121.39 & & 99.191 & 103.323 & 94.8 & 108.859 & 105.744 & 77.711 & 73.173 \\
\hline 8/30/07 3:30 & 123.753 & 121.385 & & 99.184 & 103.32 & 94.795 & 108.858 & 105.739 & 77.711 & 73.169 \\
\hline 8/30/07 4:00 & 123.753 & 121.383 & & 99.179 & 103.314 & 94.79 & 108.858 & 105.735 & 77.707 & 73.163 \\
\hline 8/30/07 4:30 & 123.748 & 121.383 & & 99.179 & 103.31 & 94.783 & 108.856 & 105.728 & 77.705 & 73.16 \\
\hline 8/30/07 5:00 & 123.746 & 121.39 & & 99.177 & 103.308 & 94.781 & 108.854 & 105.726 & 77.703 & 73.159 \\
\hline 8/30/07 5:30 & 123.746 & 123.113 & & 99.177 & 103.308 & 94.781 & 108.852 & 105.726 & 77.702 & 73.159 \\
\hline 8/30/07 6:00 & 123.743 & 123.601 & & 99.184 & 103.318 & 94.791 & 108.85 & 105.732 & 77.702 & 73.167 \\
\hline 8/30/07 6:30 & 125.447 & 123.787 & & 99.735 & 103.559 & 95.163 & 109.229 & 106.786 & 77.703 & 73.169 \\
\hline 8/30/07 7:00 & 126.01 & 123.562 & & 100.092 & 104.009 & 95.63 & 109.661 & 107.45 & 77.735 & 73.369 \\
\hline 8/30/07 7:30 & 126.208 & 123.891 & & 100.259 & 104.197 & 95.816 & 109.83 & 107.659 & 77.759 & 73.541 \\
\hline 8/30/07 8:00 & 125.738 & 123.977 & & 100.175 & 104.026 & 95.58 & 109.605 & 106.921 & 77.774 & 73.624 \\
\hline
\end{tabular}


TABLE S1.1 (Cont.)

\begin{tabular}{|c|c|c|c|c|c|c|c|c|c|c|}
\hline \multirow[b]{2}{*}{ Date and Time } & \multicolumn{10}{|c|}{ Depth from Top of Casing (ft) } \\
\hline & MW1D & MW2D & MW3D & MW4D & MW7 & MW9 & Oentrich & MW14D & MW15D & MW16D \\
\hline 8/30/07 8:30 & 126.312 & 124.022 & & 100.356 & 104.298 & 95.92 & 109.928 & 107.765 & 77.794 & 73.665 \\
\hline 8/30/07 9:00 & 126.404 & 124.059 & & 100.428 & 104.378 & 96 & 110.005 & 107.858 & 77.814 & 73.737 \\
\hline 8/30/07 9:30 & 126.452 & 124.088 & & 100.473 & 104.424 & 96.047 & 110.05 & 107.904 & 77.832 & 73.785 \\
\hline 8/30/07 10:00 & 126.49 & 124.1 & & 100.507 & 104.464 & 96.089 & 110.078 & 107.946 & 77.855 & 73.828 \\
\hline $8 / 30 / 07$ 10:30 & 126.516 & 124.104 & & 100.531 & 104.489 & 96.113 & 110.102 & 107.973 & 77.871 & 73.855 \\
\hline 8/30/07 11:00 & 126.532 & 124.109 & & 100.547 & 104.508 & 96.129 & 110.119 & 107.991 & 77.886 & 73.876 \\
\hline 8/30/07 11:30 & 126.537 & 122.066 & & 100.552 & 104.513 & 96.136 & 110.132 & 107.995 & 77.901 & 73.884 \\
\hline 8/30/07 12:00 & 126.542 & 121.825 & & 100.466 & 104.517 & 96.143 & 110.146 & 108.002 & 77.914 & 73.892 \\
\hline 8/30/07 12:30 & 124.455 & 121.694 & & 99.748 & 103.942 & 95.42 & 109.449 & 106.454 & 77.903 & 73.775 \\
\hline 8/30/07 13:00 & 124.202 & 121.614 & & 99.551 & 103.714 & 95.187 & 109.242 & 106.186 & 77.888 & 73.599 \\
\hline 8/30/07 13:30 & 124.069 & 121.555 & & 99.439 & 103.592 & 95.066 & 109.135 & 106.051 & 77.875 & 73.479 \\
\hline 8/30/07 14:00 & 123.984 & 121.515 & & 99.368 & 103.512 & 94.989 & 109.068 & 105.965 & 77.868 & 73.396 \\
\hline 8/30/07 14:30 & 123.927 & 121.474 & & 99.319 & 103.46 & 94.934 & 109.023 & 105.903 & 77.856 & 73.334 \\
\hline 8/30/07 15:00 & 123.885 & 121.442 & & 99.279 & 103.419 & 94.894 & 108.991 & 105.863 & 77.845 & 73.291 \\
\hline 8/30/07 15:30 & 123.845 & 121.417 & & 99.244 & 103.38 & 94.857 & 108.957 & 105.821 & 77.834 & 73.252 \\
\hline 8/30/07 16:00 & 123.812 & 121.395 & & 99.213 & 103.352 & 94.831 & 108.927 & 105.788 & 77.823 & 73.218 \\
\hline 8/30/07 16:30 & 123.786 & 121.381 & & 99.193 & 103.327 & 94.805 & 108.906 & 105.761 & 77.812 & 73.194 \\
\hline 8/30/07 17:00 & 123.762 & 121.367 & & 99.172 & 103.308 & 94.783 & 108.886 & 105.739 & 77.801 & 73.173 \\
\hline 8/30/07 17:30 & 123.748 & 121.354 & & 99.157 & 103.293 & 94.769 & 108.876 & 105.726 & 77.792 & 73.156 \\
\hline 8/30/07 18:00 & 123.738 & 121.345 & & 99.148 & 103.285 & 94.762 & 108.865 & 105.713 & 77.783 & 73.148 \\
\hline 8/30/07 18:30 & 123.724 & 121.34 & & 99.136 & 103.27 & 94.748 & 108.854 & 105.697 & 77.772 & 73.136 \\
\hline 8/30/07 19:00 & 123.715 & 121.336 & & 99.127 & 103.261 & 94.736 & 108.844 & 105.688 & 77.764 & 73.125 \\
\hline 8/30/07 19:30 & 123.708 & 121.336 & & 99.122 & 103.257 & 94.732 & 108.839 & 105.682 & 77.755 & 73.121 \\
\hline 8/30/07 20:00 & 123.703 & 121.34 & & 99.119 & 103.253 & 94.727 & 108.837 & 105.677 & 77.75 & 73.117 \\
\hline 8/30/07 20:30 & 123.701 & 121.342 & & 99.12 & 103.255 & 94.727 & 108.833 & 105.677 & 77.742 & 73.115 \\
\hline 8/30/07 21:00 & 123.705 & 121.34 & & 99.126 & 103.255 & 94.729 & 108.839 & 105.679 & 77.737 & 73.119 \\
\hline 8/30/07 21:30 & 123.708 & 121.342 & & 99.129 & 103.261 & 94.731 & 108.841 & 105.684 & 77.731 & 73.119 \\
\hline 8/30/07 22:00 & 123.708 & 121.34 & & 99.126 & 103.257 & 94.731 & 108.839 & 105.682 & 77.727 & 73.117 \\
\hline 8/30/07 22:30 & 123.708 & 121.34 & & 99.127 & 103.259 & 94.732 & 108.841 & 105.682 & 77.724 & 73.117 \\
\hline 8/30/07 23:00 & 123.705 & 121.34 & & 99.126 & 103.259 & 94.729 & 108.839 & 105.682 & 77.722 & 73.115 \\
\hline 8/30/07 23:30 & 123.708 & 121.34 & & 99.125 & 103.259 & 94.729 & 108.839 & 105.682 & 77.718 & 73.117 \\
\hline 8/31/07 0:00 & 123.705 & 121.34 & & 99.126 & 103.257 & 94.732 & 108.839 & 105.679 & 77.713 & 73.115 \\
\hline $8 / 31 / 07$ 0:30 & 123.705 & 121.338 & & 99.126 & 103.259 & 94.732 & 108.839 & 105.682 & 77.711 & 73.115 \\
\hline 8/31/07 1:00 & 123.705 & 121.336 & & 99.126 & 103.259 & 94.732 & 108.839 & 105.679 & 77.709 & 73.115 \\
\hline $8 / 31 / 071: 30$ & 123.705 & 121.333 & & 99.122 & 103.257 & 94.729 & 108.839 & 105.679 & 77.707 & 73.111 \\
\hline $8 / 31 / 072: 00$ & 123.701 & 121.327 & & 99.122 & 103.255 & 94.729 & 108.837 & 105.677 & 77.703 & 73.111 \\
\hline $8 / 31 / 072: 30$ & 123.701 & 121.324 & & 99.115 & 103.249 & 94.722 & 108.831 & 105.673 & 77.7 & 73.105 \\
\hline 8/31/07 3:00 & 123.698 & 121.324 & & 99.113 & 103.245 & 94.72 & 108.825 & 105.668 & 77.692 & 73.101 \\
\hline 8/31/07 3:30 & 123.698 & 121.32 & & 99.112 & 103.245 & 94.717 & 108.825 & 105.666 & 77.691 & 73.099 \\
\hline 8/31/07 4:00 & 123.696 & 121.313 & & 99.11 & 103.242 & 94.715 & 108.824 & 105.662 & 77.687 & 73.099 \\
\hline 8/31/07 4:30 & 123.694 & 121.311 & & 99.107 & 103.238 & 94.713 & 108.822 & 105.659 & 77.683 & 73.094 \\
\hline $8 / 31 / 075: 00$ & 123.694 & 121.313 & & 99.101 & 103.232 & 94.706 & 108.816 & 105.653 & 77.678 & 73.086 \\
\hline $8 / 31 / 075: 30$ & 123.691 & 122.036 & & 99.098 & 103.232 & 94.703 & 108.812 & 105.651 & 77.672 & 73.086 \\
\hline 8/31/07 6:00 & 123.689 & 123.428 & & 99.101 & 103.234 & 94.706 & 108.814 & 105.653 & 77.667 & 73.088 \\
\hline 8/31/07 6:30 & 123.686 & 123.66 & & 99.413 & 103.23 & 94.703 & 108.812 & 105.651 & 77.663 & 73.084 \\
\hline $8 / 31 / 07$ 7:00 & 125.84 & 123.78 & & 99.94 & 103.847 & 95.465 & 109.554 & 107.273 & 77.689 & 73.231 \\
\hline $8 / 31 / 077: 30$ & 126.083 & 123.859 & & 100.137 & 104.072 & 95.688 & 109.764 & 107.526 & 77.713 & 73.423 \\
\hline 8/31/07 8:00 & 126.206 & 123.911 & & 100.247 & 104.192 & 95.811 & 109.877 & 107.656 & 77.738 & 73.549 \\
\hline $8 / 31 / 078: 30$ & 126.284 & 123.943 & & 100.321 & 104.271 & 95.889 & 109.95 & 107.74 & 77.761 & 73.632 \\
\hline 8/31/07 9:00 & 126.336 & 123.97 & & 100.366 & 104.319 & 95.941 & 109.995 & 107.794 & 77.779 & 73.686 \\
\hline 8/31/07 9:30 & 126.374 & 123.995 & & 100.402 & 104.357 & 95.976 & 110.027 & 107.829 & 77.796 & 73.727 \\
\hline 8/31/07 10:00 & 126.4 & 122.02 & & 100.423 & 104.382 & 96.002 & 110.051 & 107.858 & 77.814 & 73.756 \\
\hline 8/31/07 10:30 & 126.421 & 121.769 & & 100.449 & 104.407 & 96.028 & 110.072 & 107.882 & 77.831 & 73.781 \\
\hline 8/31/07 11:00 & 124.414 & 121.642 & & 99.698 & 103.896 & 95.375 & 109.426 & 106.412 & 77.827 & 73.706 \\
\hline 8/31/07 11:30 & 124.145 & 121.555 & & 99.494 & 103.655 & 95.132 & 109.201 & 106.129 & 77.818 & 73.539 \\
\hline 8/31/07 12:00 & 124.013 & 121.499 & & 99.382 & 103.533 & 95.012 & 109.088 & 105.992 & 77.807 & 73.419 \\
\hline $8 / 31 / 07$ 12:30 & 123.927 & 121.458 & & 99.308 & 103.457 & 94.932 & 109.021 & 105.903 & 77.799 & 73.332 \\
\hline 8/31/07 13:00 & 123.871 & 121.426 & & 99.26 & 103.401 & 94.878 & 108.972 & 105.845 & 77.788 & 73.272 \\
\hline 8/31/07 13:30 & 123.826 & 121.399 & & 99.222 & 103.36 & 94.835 & 108.936 & 105.799 & 77.779 & 73.227 \\
\hline $8 / 31 / 0714: 00$ & 123.795 & 121.379 & & 99.194 & 103.333 & 94.807 & 108.908 & 105.768 & 77.772 & 73.194 \\
\hline $8 / 31 / 0714: 30$ & 123.769 & 121.363 & & 99.172 & 103.306 & 94.783 & 108.887 & 105.744 & 77.761 & 73.169 \\
\hline 8/31/07 15:00 & 123.75 & 121.342 & & 99.155 & 103.287 & 94.762 & 108.869 & 105.721 & 77.757 & 73.148 \\
\hline $8 / 31 / 0715: 30$ & 123.731 & 121.327 & & 99.136 & 103.27 & 94.746 & 108.854 & 105.701 & 77.748 & 73.132 \\
\hline 8/31/07 16:00 & 123.71 & 121.315 & & 99.117 & 103.253 & 94.727 & 108.835 & 105.682 & 77.742 & 73.113 \\
\hline 8/31/07 16:30 & 123.698 & 121.306 & & 99.103 & 103.24 & 94.713 & 108.824 & 105.668 & 77.733 & 73.099 \\
\hline 8/31/07 17:00 & 123.684 & 121.295 & & 99.095 & 103.226 & 94.703 & 108.81 & 105.653 & 77.726 & 73.088 \\
\hline
\end{tabular}


TABLE S1.1 (Cont.)

\begin{tabular}{|c|c|c|c|c|c|c|c|c|c|c|}
\hline \multirow[b]{2}{*}{ Date and Time } & \multicolumn{10}{|c|}{ Depth from Top of Casing (ft) } \\
\hline & MW1D & MW2D & MW3D & MW4D & MW7 & MW9 & Oentrich & MW14D & MW15D & MW16D \\
\hline 8/31/07 17:30 & 123.675 & 121.29 & & 99.086 & 103.221 & 94.694 & 108.805 & 105.644 & 77.716 & 73.08 \\
\hline 8/31/07 18:00 & 123.663 & 121.281 & & 99.074 & 103.207 & 94.685 & 108.793 & 105.633 & 77.709 & 73.066 \\
\hline 8/31/07 18:30 & 123.658 & 121.274 & & 99.069 & 103.2 & 94.677 & 108.788 & 105.626 & 77.702 & 73.059 \\
\hline 8/31/07 19:00 & 123.649 & 121.272 & & 99.064 & 103.196 & 94.673 & 108.782 & 105.617 & 77.696 & 73.055 \\
\hline 8/31/07 19:30 & 123.642 & 121.274 & & 99.055 & 103.188 & 94.666 & 108.777 & 105.613 & 77.689 & 73.047 \\
\hline 8/31/07 20:00 & 123.637 & 121.281 & & 99.055 & 103.186 & 94.661 & 108.773 & 105.608 & 77.681 & 73.045 \\
\hline 8/31/07 20:30 & 123.642 & 121.283 & & 99.06 & 103.19 & 94.666 & 108.777 & 105.613 & 77.678 & 73.049 \\
\hline 8/31/07 21:00 & 123.646 & 121.29 & & 99.065 & 103.196 & 94.67 & 108.782 & 105.617 & 77.676 & 73.053 \\
\hline 8/31/07 21:30 & 123.653 & 121.295 & & 99.07 & 103.202 & 94.677 & 108.788 & 105.624 & 77.674 & 73.057 \\
\hline 8/31/07 22:00 & 123.658 & 121.299 & & 99.075 & 103.207 & 94.682 & 108.79 & 105.628 & 77.67 & 73.061 \\
\hline 8/31/07 22:30 & 123.663 & 121.295 & & 99.081 & 103.211 & 94.687 & 108.795 & 105.633 & 77.67 & 73.068 \\
\hline 8/31/07 23:00 & 123.665 & 121.299 & & 99.082 & 103.213 & 94.687 & 108.797 & 105.635 & 77.667 & 73.068 \\
\hline 8/31/07 23:30 & 123.663 & 121.302 & & 99.082 & 103.213 & 94.689 & 108.797 & 105.635 & 77.665 & 73.066 \\
\hline 9/1/07 0:00 & 123.665 & 121.304 & & 99.086 & 103.213 & 94.689 & 108.799 & 105.637 & 77.663 & 73.066 \\
\hline 9/1/07 0:30 & 123.668 & 121.304 & & 99.087 & 103.217 & 94.692 & 108.799 & 105.637 & 77.661 & 73.068 \\
\hline 9/1/07 1:00 & 123.67 & 121.308 & & 99.089 & 103.219 & 94.696 & 108.801 & 105.639 & 77.663 & 73.07 \\
\hline $9 / 1 / 071: 30$ & 123.67 & 121.308 & & 99.093 & 103.221 & 94.696 & 108.803 & 105.644 & 77.659 & 73.072 \\
\hline 9/1/07 2:00 & 123.672 & 121.308 & & 99.095 & 103.224 & 94.698 & 108.803 & 105.646 & 77.661 & 73.074 \\
\hline $9 / 1 / 072: 30$ & 123.675 & 121.308 & & 99.095 & 103.226 & 94.701 & 108.803 & 105.646 & 77.657 & 73.076 \\
\hline $9 / 1 / 073: 00$ & 123.677 & 121.306 & & 99.095 & 103.224 & 94.701 & 108.805 & 105.646 & 77.657 & 73.076 \\
\hline 9/1/07 3:30 & 123.677 & 121.302 & & 99.093 & 103.222 & 94.699 & 108.803 & 105.646 & 77.656 & 73.074 \\
\hline $9 / 1 / 074: 00$ & 123.675 & 121.297 & & 99.091 & 103.222 & 94.699 & 108.799 & 105.644 & 77.656 & 73.074 \\
\hline $9 / 1 / 074: 30$ & 123.67 & 121.297 & & 99.086 & 103.222 & 94.694 & 108.801 & 105.642 & 77.65 & 73.07 \\
\hline 9/1/07 5:00 & 123.663 & 121.297 & & 99.084 & 103.213 & 94.687 & 108.795 & 105.635 & 77.646 & 73.063 \\
\hline $9 / 1 / 075: 30$ & 123.665 & 121.299 & & 99.084 & 103.213 & 94.689 & 108.793 & 105.635 & 77.644 & 73.066 \\
\hline 9/1/07 6:00 & 123.665 & 123.111 & & 99.084 & 103.217 & 94.689 & 108.796 & 105.637 & 77.641 & 73.066 \\
\hline 9/1/07 6:30 & 123.663 & 123.526 & & 99.084 & 103.213 & 94.689 & 108.794 & 105.635 & 77.639 & 73.063 \\
\hline 9/1/07 7:00 & 125.476 & 123.696 & & 99.698 & 103.544 & 95.151 & 109.257 & 106.864 & 77.644 & 73.08 \\
\hline 9/1/07 7:30 & 125.941 & 123.803 & & 100.016 & 103.933 & 95.554 & 109.629 & 107.377 & 77.676 & 73.293 \\
\hline 9/1/07 8:00 & 126.121 & 123.871 & & 100.171 & 104.104 & 95.726 & 109.789 & 107.568 & 77.7 & 73.456 \\
\hline $9 / 1 / 078: 30$ & 126.23 & 123.911 & & 100.262 & 104.209 & 95.83 & 109.884 & 107.678 & 77.722 & 73.562 \\
\hline $9 / 1 / 079: 00$ & 126.298 & 123.95 & & 100.326 & 104.275 & 95.896 & 109.946 & 107.749 & 77.746 & 73.636 \\
\hline 9/1/07 9:30 & 126.343 & 123.973 & & 100.366 & 104.321 & 95.941 & 109.986 & 107.798 & 77.766 & 73.682 \\
\hline 9/1/07 10:00 & 126.381 & 123.984 & & 100.399 & 104.355 & 95.976 & 110.018 & 107.833 & 77.785 & 73.721 \\
\hline 9/1/07 10:30 & 126.404 & 123.993 & & 100.423 & 104.38 & 96.002 & 110.042 & 107.858 & 77.805 & 73.748 \\
\hline 9/1/07 11:00 & 126.416 & 124.002 & & 100.435 & 104.393 & 96.014 & 110.057 & 107.873 & 77.82 & 73.764 \\
\hline 9/1/07 11:30 & 126.428 & 124 & & 100.448 & 104.407 & 96.03 & 110.068 & 107.884 & 77.838 & 73.781 \\
\hline 9/1/07 12:00 & 126.435 & 122.254 & & 100.456 & 104.416 & 96.037 & 110.08 & 107.893 & 77.855 & 73.791 \\
\hline 9/1/07 12:30 & 126.435 & 121.853 & & 100.454 & 104.418 & 96.04 & 110.089 & 107.893 & 77.864 & 73.795 \\
\hline 9/1/07 13:00 & 124.688 & 121.68 & & 99.858 & 104.1 & 95.59 & 109.642 & 106.726 & 77.869 & 73.777 \\
\hline 9/1/07 13:30 & 124.232 & 121.576 & & 99.553 & 103.729 & 95.205 & 109.28 & 106.217 & 77.851 & 73.598 \\
\hline 9/1/07 14:00 & 124.057 & 121.506 & & 99.41 & 103.563 & 95.043 & 109.128 & 106.034 & 77.84 & 73.45 \\
\hline 9/1/07 14:30 & 123.949 & 121.456 & & 99.32 & 103.464 & 94.944 & 109.038 & 105.923 & 77.827 & 73.349 \\
\hline 9/1/07 15:00 & 123.878 & 121.415 & & 99.26 & 103.401 & 94.878 & 108.977 & 105.85 & 77.82 & 73.276 \\
\hline 9/1/07 15:30 & 123.826 & 121.383 & & 99.213 & 103.354 & 94.831 & 108.932 & 105.799 & 77.807 & 73.225 \\
\hline 9/1/07 16:00 & 123.786 & 121.363 & & 99.179 & 103.316 & 94.795 & 108.897 & 105.757 & 77.797 & 73.181 \\
\hline 9/1/07 16:30 & 123.755 & 121.342 & & 99.15 & 103.283 & 94.762 & 108.869 & 105.721 & 77.786 & 73.15 \\
\hline 9/1/07 17:00 & 123.731 & 121.324 & & 99.129 & 103.262 & 94.741 & 108.848 & 105.699 & 77.777 & 73.128 \\
\hline 9/1/07 17:30 & 123.71 & 121.317 & & 99.112 & 103.245 & 94.722 & 108.833 & 105.679 & 77.77 & 73.109 \\
\hline 9/1/07 18:00 & 123.694 & 121.299 & & 99.098 & 103.23 & 94.703 & 108.818 & 105.662 & 77.757 & 73.092 \\
\hline 9/1/07 18:30 & 123.686 & 121.29 & & 99.091 & 103.224 & 94.701 & 108.812 & 105.655 & 77.751 & 73.086 \\
\hline 9/1/07 19:00 & 123.672 & 121.288 & & 99.076 & 103.209 & 94.685 & 108.799 & 105.639 & 77.744 & 73.07 \\
\hline 9/1/07 19:30 & 123.658 & 121.286 & & 99.067 & 103.198 & 94.673 & 108.788 & 105.626 & 77.733 & 73.059 \\
\hline 9/1/07 20:00 & 123.656 & 121.295 & & 99.067 & 103.196 & 94.673 & 108.786 & 105.624 & 77.729 & 73.057 \\
\hline 9/1/07 20:30 & 123.653 & 121.295 & & 99.067 & 103.194 & 94.67 & 108.786 & 105.622 & 77.726 & 73.055 \\
\hline 9/1/07 21:00 & 123.658 & 121.299 & & 99.074 & 103.202 & 94.68 & 108.792 & 105.629 & 77.722 & 73.061 \\
\hline 9/1/07 21:30 & 123.663 & 121.304 & & 99.076 & 103.205 & 94.682 & 108.793 & 105.631 & 77.72 & 73.063 \\
\hline 9/1/07 22:00 & 123.668 & 121.306 & & 99.084 & 103.211 & 94.689 & 108.799 & 105.637 & 77.718 & 73.07 \\
\hline 9/1/07 22:30 & 123.672 & 121.304 & & 99.086 & 103.217 & 94.694 & 108.803 & 105.642 & 77.716 & 73.072 \\
\hline 9/1/07 23:00 & 123.672 & 121.306 & & 99.089 & 103.215 & 94.694 & 108.803 & 105.642 & 77.715 & 73.072 \\
\hline 9/1/07 23:30 & 123.675 & 121.313 & & 99.088 & 103.217 & 94.694 & 108.803 & 105.642 & 77.713 & 73.071 \\
\hline 9/2/07 0:00 & 123.675 & 121.313 & & 99.091 & 103.219 & 94.696 & 108.805 & 105.644 & 77.713 & 73.076 \\
\hline 9/2/07 0:30 & 123.679 & 121.313 & & 99.094 & 103.224 & 94.701 & 108.805 & 105.648 & 77.711 & 73.078 \\
\hline 9/2/07 1:00 & 123.679 & 121.308 & & 99.096 & 103.226 & 94.701 & 108.807 & 105.648 & 77.709 & 73.078 \\
\hline 9/2/07 1:30 & 123.682 & 121.306 & & 99.093 & 103.222 & 94.701 & 108.807 & 105.648 & 77.711 & 73.078 \\
\hline 9/2/07 2:00 & 123.682 & 121.306 & & 99.091 & 103.219 & 94.696 & 108.803 & 105.644 & 77.707 & 73.072 \\
\hline
\end{tabular}


TABLE S1.1 (Cont.)

\begin{tabular}{|c|c|c|c|c|c|c|c|c|c|c|}
\hline \multirow[b]{2}{*}{ Date and Time } & \multicolumn{10}{|c|}{ Depth from Top of Casing (ft) } \\
\hline & MW1D & MW2D & MW3D & MW4D & MW7 & MW9 & Oentrich & MW14D & MW15D & MW16D \\
\hline 9/2/07 2:30 & 123.682 & 121.304 & & 99.089 & 103.219 & 94.696 & 108.803 & 105.642 & 77.705 & 73.07 \\
\hline 9/2/07 3:00 & 123.679 & 121.306 & & 99.088 & 103.219 & 94.696 & 108.799 & 105.644 & 77.703 & 73.072 \\
\hline 9/2/07 3:30 & 123.677 & 121.306 & & 99.088 & 103.215 & 94.691 & 108.799 & 105.642 & 77.702 & 73.07 \\
\hline 9/2/07 4:00 & 123.675 & 121.306 & & 99.089 & 103.217 & 94.696 & 108.799 & 105.642 & 77.702 & 73.07 \\
\hline 9/2/07 4:30 & 123.672 & 121.306 & & 99.088 & 103.221 & 94.696 & 108.797 & 105.642 & 77.702 & 73.072 \\
\hline 9/2/07 5:00 & 123.672 & 121.302 & & 99.091 & 103.217 & 94.698 & 108.797 & 105.642 & 77.698 & 73.07 \\
\hline 9/2/07 5:30 & 123.675 & 121.304 & & 99.089 & 103.219 & 94.698 & 108.795 & 105.642 & 77.698 & 73.07 \\
\hline 9/2/07 6:00 & 123.67 & 122.83 & & 99.086 & 103.215 & 94.691 & 108.795 & 105.637 & 77.692 & 73.068 \\
\hline 9/2/07 6:30 & 123.672 & 123.458 & & 99.089 & 103.217 & 94.694 & 108.793 & 105.639 & 77.692 & 73.07 \\
\hline 9/2/07 7:00 & 125.029 & 123.671 & & 99.553 & 103.323 & 94.882 & 108.998 & 106.138 & 77.692 & 73.076 \\
\hline 9/2/07 7:30 & 125.873 & 123.787 & & 99.971 & 103.875 & 95.495 & 109.567 & 107.304 & 77.722 & 73.249 \\
\hline 9/2/07 8:00 & 126.097 & 123.855 & & 100.151 & 104.083 & 95.705 & 109.753 & 107.537 & 77.75 & 73.436 \\
\hline 9/2/07 8:30 & 126.216 & 123.907 & & 100.258 & 104.199 & 95.823 & 109.857 & 107.663 & 77.774 & 73.558 \\
\hline 9/2/07 9:00 & 126.284 & 123.945 & & 100.32 & 104.268 & 95.891 & 109.937 & 107.736 & 77.797 & 73.632 \\
\hline 9/2/07 9:30 & 126.336 & 123.973 & & 100.371 & 104.319 & 95.943 & 109.975 & 107.789 & 77.816 & 73.688 \\
\hline 9/2/07 10:00 & 126.374 & 122.36 & & 100.406 & 104.359 & 95.983 & 110.005 & 107.831 & 77.836 & 73.729 \\
\hline $9 / 2 / 07 \quad 10: 30$ & 126.404 & 121.88 & & 100.435 & 104.388 & 96.009 & 110.033 & 107.86 & 77.855 & 73.76 \\
\hline 9/2/07 11:00 & 124.837 & 121.701 & & 99.92 & 104.192 & 95.693 & 109.702 & 106.957 & 77.868 & 73.779 \\
\hline 9/2/07 11:30 & 124.258 & 121.594 & & 99.582 & 103.754 & 95.231 & 109.263 & 106.244 & 77.855 & 73.613 \\
\hline $9 / 2 / 07 \quad 12: 00$ & 124.074 & 121.537 & & 99.432 & 103.586 & 95.064 & 109.113 & 106.052 & 77.845 & 73.469 \\
\hline 9/2/07 12:30 & 123.968 & 121.483 & & 99.348 & 103.489 & 94.967 & 109.025 & 105.945 & 77.838 & 73.369 \\
\hline 9/2/07 13:00 & 123.909 & 121.447 & & 99.294 & 103.434 & 94.911 & 108.972 & 105.879 & 77.831 & 73.303 \\
\hline 9/2/07 13:30 & 123.854 & 121.419 & & 99.246 & 103.38 & 94.861 & 108.942 & 105.823 & 77.82 & 73.247 \\
\hline 9/2/07 14:00 & 123.816 & 121.397 & & 99.21 & 103.342 & 94.823 & 108.912 & 105.783 & 77.814 & 73.206 \\
\hline 9/2/07 14:30 & 123.788 & 121.374 & & 99.187 & 103.321 & 94.8 & 108.895 & 105.759 & 77.807 & 73.181 \\
\hline 9/2/07 15:00 & 123.767 & 121.358 & & 99.165 & 103.297 & 94.779 & 108.878 & 105.735 & 77.799 & 73.156 \\
\hline 9/2/07 15:30 & 123.746 & 121.345 & & 99.144 & 103.278 & 94.755 & 108.863 & 105.71 & 77.792 & 73.136 \\
\hline 9/2/07 16:00 & 123.727 & 121.338 & & 99.13 & 103.261 & 94.739 & 108.85 & 105.693 & 77.786 & 73.117 \\
\hline 9/2/07 16:30 & 123.712 & 121.322 & & 99.118 & 103.247 & 94.727 & 108.839 & 105.679 & 77.781 & 73.105 \\
\hline 9/2/07 17:00 & 123.705 & 121.315 & & 99.106 & 103.24 & 94.722 & 108.831 & 105.673 & 77.775 & 73.099 \\
\hline 9/2/07 17:30 & 123.689 & 121.315 & & 99.096 & 103.224 & 94.706 & 108.816 & 105.657 & 77.77 & 73.082 \\
\hline 9/2/07 18:00 & 123.684 & 121.311 & & 99.093 & 103.219 & 94.698 & 108.812 & 105.648 & 77.764 & 73.078 \\
\hline 9/2/07 18:30 & 123.682 & 121.306 & & 99.093 & 103.221 & 94.701 & 108.812 & 105.648 & 77.762 & 73.078 \\
\hline 9/2/07 19:00 & 123.679 & 121.306 & & 99.089 & 103.217 & 94.699 & 108.81 & 105.646 & 77.757 & 73.074 \\
\hline 9/2/07 19:30 & 123.675 & 121.34 & & 99.086 & 103.213 & 94.694 & 108.807 & 105.642 & 77.751 & 73.07 \\
\hline 9/2/07 20:00 & 123.675 & 121.327 & & 99.086 & 103.213 & 94.691 & 108.805 & 105.639 & 77.75 & 73.07 \\
\hline 9/2/07 20:30 & 123.705 & 121.329 & & 99.196 & 103.264 & 94.774 & 109.712 & 105.653 & 77.748 & 73.074 \\
\hline 9/2/07 21:00 & 123.696 & 121.336 & & 99.105 & 103.24 & 94.715 & 108.779 & 105.664 & 77.75 & 73.086 \\
\hline 9/2/07 21:30 & 123.698 & 121.34 & & 99.111 & 103.24 & 94.713 & 108.825 & 105.666 & 77.75 & 73.092 \\
\hline 9/2/07 22:00 & 123.703 & 121.345 & & 99.119 & 103.243 & 94.722 & 108.834 & 105.668 & 77.748 & 73.099 \\
\hline 9/2/07 22:30 & 123.71 & 121.34 & & 99.125 & 103.251 & 94.729 & 108.827 & 105.675 & 77.75 & 73.103 \\
\hline 9/2/07 23:00 & 123.71 & 121.336 & & 99.126 & 103.251 & 94.732 & 108.824 & 105.677 & 77.75 & 73.103 \\
\hline 9/2/07 23:30 & 123.71 & 121.338 & & 99.12 & 103.249 & 94.729 & 108.816 & 105.675 & 77.748 & 73.101 \\
\hline 9/3/07 0:00 & 123.705 & 121.336 & & 99.117 & 103.243 & 94.722 & 108.81 & 105.67 & 77.746 & 73.094 \\
\hline 9/3/07 0:30 & 123.705 & 121.34 & & 99.119 & 103.245 & 94.722 & 108.802 & 105.673 & 77.744 & 73.099 \\
\hline 9/3/07 1:00 & 123.705 & 121.336 & & 99.118 & 103.243 & 94.724 & 108.804 & 105.67 & 77.744 & 73.097 \\
\hline 9/3/07 1:30 & 123.708 & 121.333 & & 99.119 & 103.247 & 94.724 & 108.794 & 105.675 & 77.742 & 73.094 \\
\hline 9/3/07 2:00 & 123.705 & 121.333 & & 99.115 & 103.245 & 94.72 & 108.791 & 105.67 & 77.742 & 73.094 \\
\hline 9/3/07 2:30 & 123.698 & 121.333 & & 99.113 & 103.24 & 94.717 & 108.789 & 105.666 & 77.74 & 73.09 \\
\hline 9/3/07 3:00 & 123.701 & 121.331 & & 99.113 & 103.24 & 94.717 & 108.792 & 105.666 & 77.74 & 73.09 \\
\hline 9/3/07 3:30 & 123.701 & 121.329 & & 99.113 & 103.243 & 94.72 & 108.787 & 105.668 & 77.74 & 73.092 \\
\hline 9/3/07 4:00 & 123.698 & 121.331 & & 99.112 & 103.242 & 94.717 & 108.785 & 105.666 & 77.738 & 73.09 \\
\hline 9/3/07 4:30 & 123.696 & 121.329 & & 99.108 & 103.236 & 94.715 & 108.789 & 105.664 & 77.738 & 73.086 \\
\hline $9 / 3 / 075: 00$ & 123.698 & 121.331 & & 99.112 & 103.236 & 94.717 & 108.785 & 105.664 & 77.738 & 73.084 \\
\hline 9/3/07 5:30 & 123.698 & 122.936 & & 99.111 & 103.238 & 94.715 & 108.785 & 105.664 & 77.737 & 73.088 \\
\hline 9/3/07 6:00 & 123.701 & 123.481 & & 99.115 & 103.24 & 94.717 & 108.783 & 105.666 & 77.737 & 73.09 \\
\hline 9/3/07 6:30 & 125.26 & 123.685 & & 99.668 & 103.441 & 95.045 & 109.123 & 106.492 & 77.738 & 73.092 \\
\hline 9/3/07 7:00 & 125.925 & 123.796 & & 100.059 & 103.946 & 95.58 & 109.614 & 107.322 & 77.772 & 73.287 \\
\hline 9/3/07 7:30 & 126.14 & 123.871 & & 100.235 & 104.15 & 95.783 & 109.797 & 107.546 & 77.801 & 73.473 \\
\hline 9/3/07 8:00 & 126.253 & 123.916 & & 100.336 & 104.258 & 95.898 & 109.892 & 107.665 & 77.823 & 73.589 \\
\hline 9/3/07 8:30 & 126.329 & 123.954 & & 100.404 & 104.33 & 95.969 & 109.962 & 107.743 & 77.845 & 73.665 \\
\hline 9/3/07 9:00 & 126.381 & 122.061 & & 100.445 & 104.378 & 96.018 & 110.007 & 107.796 & 77.868 & 73.717 \\
\hline 9/3/07 9:30 & 126.419 & 121.784 & & 100.48 & 104.412 & 96.054 & 110.039 & 107.833 & 77.884 & 73.754 \\
\hline 9/3/07 10:00 & 124.464 & 121.653 & & 99.721 & 103.925 & 95.406 & 109.398 & 106.454 & 77.884 & 73.702 \\
\hline 9/3/07 10:30 & 124.164 & 121.574 & & 99.504 & 103.664 & 95.142 & 109.15 & 106.14 & 77.873 & 73.539 \\
\hline 9/3/07 11:00 & 124.027 & 121.521 & & 99.394 & 103.539 & 95.017 & 109.036 & 105.998 & 77.864 & 73.417 \\
\hline
\end{tabular}


TABLE S1.1 (Cont.)

\begin{tabular}{|c|c|c|c|c|c|c|c|c|c|c|}
\hline \multirow[b]{2}{*}{ Date and Time } & \multicolumn{10}{|c|}{ Depth from Top of Casing (ft) } \\
\hline & MW1D & MW2D & MW3D & MW4D & MW7 & MW9 & Oentrich & MW14D & MW15D & MW16D \\
\hline 9/3/07 11:30 & 123.946 & 121.476 & & 99.325 & 103.464 & 94.944 & 108.966 & 105.914 & 77.855 & 73.336 \\
\hline 9/3/07 12:00 & 123.887 & 121.444 & & 99.277 & 103.411 & 94.894 & 108.925 & 105.856 & 77.847 & 73.278 \\
\hline 9/3/07 12:30 & 123.847 & 121.417 & & 99.239 & 103.371 & 94.852 & 108.899 & 105.812 & 77.842 & 73.233 \\
\hline 9/3/07 13:00 & 123.814 & 121.397 & & 99.211 & 103.341 & 94.821 & 108.88 & 105.781 & 77.836 & 73.199 \\
\hline 9/3/07 13:30 & 123.786 & 121.379 & & 99.186 & 103.316 & 94.795 & 108.867 & 105.753 & 77.827 & 73.171 \\
\hline 9/3/07 14:00 & 123.764 & 121.358 & & 99.165 & 103.293 & 94.776 & 108.858 & 105.73 & 77.823 & 73.15 \\
\hline 9/3/07 14:30 & 123.748 & 121.336 & & 99.15 & 103.278 & 94.76 & 108.846 & 105.713 & 77.812 & 73.134 \\
\hline 9/3/07 15:00 & 123.724 & 121.322 & & 99.127 & 103.257 & 94.736 & 108.835 & 105.69 & 77.805 & 73.111 \\
\hline 9/3/07 15:30 & 123.705 & 121.308 & & 99.108 & 103.236 & 94.72 & 108.824 & 105.67 & 77.797 & 73.092 \\
\hline 9/3/07 16:00 & 123.691 & 121.297 & & 99.093 & 103.222 & 94.703 & 108.818 & 105.655 & 77.79 & 73.077 \\
\hline 9/3/07 16:30 & 123.677 & 121.29 & & 99.082 & 103.207 & 94.691 & 108.805 & 105.642 & 77.783 & 73.066 \\
\hline 9/3/07 17:00 & 123.668 & 121.283 & & 99.072 & 103.198 & 94.682 & 108.795 & 105.633 & 77.775 & 73.053 \\
\hline 9/3/07 17:30 & 123.66 & 121.272 & & 99.065 & 103.194 & 94.677 & 108.788 & 105.624 & 77.77 & 73.049 \\
\hline 9/3/07 18:00 & 123.653 & 121.268 & & 99.06 & 103.186 & 94.668 & 108.786 & 105.617 & 77.764 & 73.042 \\
\hline 9/3/07 18:30 & 123.644 & 121.261 & & 99.051 & 103.177 & 94.658 & 108.775 & 105.606 & 77.761 & 73.032 \\
\hline 9/3/07 19:00 & 123.634 & 121.256 & & 99.044 & 103.171 & 94.654 & 108.769 & 105.6 & 77.753 & 73.026 \\
\hline 9/3/07 19:30 & 123.63 & 121.258 & & 99.037 & 103.165 & 94.647 & 108.765 & 105.593 & 77.748 & 73.02 \\
\hline 9/3/07 20:00 & 123.625 & 121.27 & & 99.034 & 103.162 & 94.642 & 108.763 & 105.591 & 77.744 & 73.018 \\
\hline 9/3/07 20:30 & 123.627 & 121.272 & & 99.037 & 103.163 & 94.642 & 108.765 & 105.591 & 77.74 & 73.016 \\
\hline 9/3/07 21:00 & 123.637 & 121.272 & & 99.049 & 103.175 & 94.654 & 108.773 & 105.6 & 77.738 & 73.024 \\
\hline 9/3/07 21:30 & 123.639 & 121.27 & & 99.051 & 103.179 & 94.659 & 108.775 & 105.604 & 77.737 & 73.03 \\
\hline 9/3/07 22:00 & 123.642 & 121.27 & & 99.053 & 103.179 & 94.658 & 108.775 & 105.607 & 77.737 & 73.03 \\
\hline 9/3/07 22:30 & 123.637 & 121.268 & & 99.05 & 103.177 & 94.656 & 108.771 & 105.604 & 77.735 & 73.028 \\
\hline 9/3/07 23:00 & 123.637 & 121.263 & & 99.046 & 103.175 & 94.654 & 108.771 & 105.602 & 77.731 & 73.026 \\
\hline 9/3/07 23:30 & 123.637 & 121.261 & & 99.046 & 103.173 & 94.654 & 108.767 & 105.6 & 77.727 & 73.026 \\
\hline 9/4/07 0:00 & 123.632 & 121.261 & & 99.043 & 103.169 & 94.651 & 108.765 & 105.595 & 77.727 & 73.022 \\
\hline 9/4/07 0:30 & 123.63 & 121.254 & & 99.039 & 103.165 & 94.647 & 108.764 & 105.593 & 77.724 & 73.018 \\
\hline 9/4/07 1:00 & 123.625 & 121.247 & & 99.037 & 103.163 & 94.644 & 108.762 & 105.589 & 77.718 & 73.014 \\
\hline 9/4/07 1:30 & 123.623 & 121.243 & & 99.031 & 103.158 & 94.642 & 108.758 & 105.586 & 77.718 & 73.01 \\
\hline $9 / 4 / 072: 00$ & 123.616 & 121.236 & & 99.025 & 103.154 & 94.633 & 108.752 & 105.577 & 77.711 & 73.003 \\
\hline 9/4/07 2:30 & 123.611 & 121.227 & & 99.017 & 103.148 & 94.628 & 108.75 & 105.573 & 77.709 & 72.997 \\
\hline 9/4/07 3:00 & 123.606 & 121.224 & & 99.013 & 103.141 & 94.623 & 108.747 & 105.569 & 77.705 & 72.991 \\
\hline 9/4/07 3:30 & 123.597 & 121.222 & & 99.005 & 103.131 & 94.611 & 108.741 & 105.56 & 77.702 & 72.982 \\
\hline $9 / 4 / 074: 00$ & 123.592 & 121.218 & & 99.001 & 103.127 & 94.609 & 108.739 & 105.555 & 77.698 & 72.979 \\
\hline 9/4/07 4:30 & 123.59 & 121.213 & & 98.998 & 103.125 & 94.604 & 108.735 & 105.553 & 77.7 & 72.976 \\
\hline 9/4/07 5:00 & 123.587 & 121.213 & & 98.998 & 103.122 & 94.602 & 108.733 & 105.549 & 77.692 & 72.972 \\
\hline 9/4/07 5:30 & 123.583 & 122.347 & & 98.995 & 103.12 & 94.597 & 108.732 & 105.547 & 77.691 & 72.97 \\
\hline 9/4/07 6:00 & 123.585 & 123.315 & & 98.995 & 103.122 & 94.602 & 108.73 & 105.549 & 77.689 & 72.972 \\
\hline 9/4/07 6:30 & 123.587 & 123.544 & & 99.408 & 103.123 & 94.604 & 108.73 & 105.549 & 77.689 & 72.975 \\
\hline 9/4/07 7:00 & 125.757 & 123.671 & & 99.899 & 103.78 & 95.417 & 109.528 & 107.152 & 77.718 & 73.134 \\
\hline 9/4/07 7:30 & 126 & 123.75 & & 100.094 & 104.003 & 95.639 & 109.734 & 107.402 & 77.746 & 73.326 \\
\hline 9/4/07 8:00 & 126.133 & 123.803 & & 100.206 & 104.127 & 95.766 & 109.849 & 107.539 & 77.772 & 73.454 \\
\hline 9/4/07 8:30 & 126.215 & 123.839 & & 100.278 & 104.205 & 95.846 & 109.924 & 107.623 & 77.794 & 73.537 \\
\hline 9/4/07 9:00 & 126.268 & 123.866 & & 100.326 & 104.256 & 95.896 & 109.973 & 107.676 & 77.812 & 73.593 \\
\hline 9/4/07 9:30 & 126.305 & 123.884 & & 100.357 & 104.291 & 95.931 & 110.006 & 107.714 & 77.829 & 73.629 \\
\hline 9/4/07 10:00 & 126.334 & 123.9 & & 100.383 & 104.319 & 95.957 & 110.033 & 107.743 & 77.844 & 73.659 \\
\hline 9/4/07 10:30 & 126.35 & 123.909 & & 100.402 & 104.34 & 95.976 & 110.05 & 107.76 & 77.862 & 73.682 \\
\hline 9/4/07 11:00 & 126.365 & 123.911 & & 100.416 & 104.351 & 95.995 & 110.065 & 107.78 & 77.877 & 73.698 \\
\hline 9/4/07 11:30 & 126.376 & 123.916 & & 100.424 & 104.361 & 96.002 & 110.076 & 107.787 & 77.888 & 73.706 \\
\hline 9/4/07 12:00 & 126.379 & 123.911 & & 100.426 & 104.365 & 96.004 & 110.085 & 107.791 & 77.901 & 73.713 \\
\hline 9/4/07 12:30 & 126.381 & 122.533 & & 100.428 & 104.367 & 96.009 & 110.091 & 107.796 & 77.908 & 73.717 \\
\hline 9/4/07 13:00 & 126.379 & 121.841 & & 100.428 & 104.365 & 96.009 & 110.097 & 107.796 & 77.921 & 73.717 \\
\hline 9/4/07 13:30 & 125.239 & 121.63 & & 99.944 & 104.285 & 95.865 & 109.937 & 107.55 & 77.928 & 73.717 \\
\hline 9/4/07 14:00 & 124.23 & 121.51 & & 99.518 & 103.698 & 95.179 & 109.295 & 106.206 & 77.904 & 73.553 \\
\hline 9/4/07 14:30 & 124.008 & 121.431 & & 99.342 & 103.497 & 94.981 & 109.109 & 105.978 & 77.89 & 73.388 \\
\hline 9/4/07 15:00 & 123.885 & 121.376 & & 99.244 & 103.384 & 94.866 & 109.006 & 105.852 & 77.873 & 73.276 \\
\hline 9/4/07 15:30 & 123.807 & 121.333 & & 99.177 & 103.312 & 94.793 & 108.944 & 105.77 & 77.858 & 73.193 \\
\hline 9/4/07 16:00 & 123.748 & 121.302 & & 99.125 & 103.261 & 94.741 & 108.902 & 105.713 & 77.845 & 73.138 \\
\hline 9/4/07 16:30 & 123.703 & 121.279 & & 99.086 & 103.217 & 94.701 & 108.865 & 105.666 & 77.832 & 73.09 \\
\hline 9/4/07 17:00 & 123.67 & 121.256 & & 99.058 & 103.19 & 94.673 & 108.84 & 105.635 & 77.825 & 73.057 \\
\hline 9/4/07 17:30 & 123.646 & 121.243 & & 99.037 & 103.167 & 94.652 & 108.825 & 105.611 & 77.81 & 73.035 \\
\hline 9/4/07 18:00 & 123.627 & 121.231 & & 99.02 & 103.148 & 94.63 & 108.81 & 105.589 & 77.803 & 73.016 \\
\hline 9/4/07 18:30 & 123.613 & 121.22 & & 99.01 & 103.135 & 94.616 & 108.799 & 105.575 & 77.792 & 73.001 \\
\hline 9/4/07 19:00 & 123.604 & 121.218 & & 98.998 & 103.129 & 94.609 & 108.79 & 105.566 & 77.785 & 72.991 \\
\hline 9/4/07 19:30 & 123.59 & 121.218 & & 98.989 & 103.116 & 94.597 & 108.78 & 105.551 & 77.777 & 72.979 \\
\hline 9/4/07 20:00 & 123.587 & 121.218 & & 98.987 & 103.114 & 94.595 & 108.775 & 105.549 & 77.772 & 72.977 \\
\hline
\end{tabular}


TABLE S1.1 (Cont.)

\begin{tabular}{|c|c|c|c|c|c|c|c|c|c|c|}
\hline \multirow[b]{2}{*}{ Date and Time } & \multicolumn{10}{|c|}{ Depth from Top of Casing (ft) } \\
\hline & MW1D & MW2D & MW3D & MW4D & MW7 & MW9 & Oentrich & MW14D & MW15D & MW16D \\
\hline 9/4/07 20:30 & 123.59 & 121.218 & & 98.991 & 103.116 & 94.6 & 108.773 & 105.551 & 77.766 & 72.976 \\
\hline 9/4/07 21:00 & 123.585 & 121.213 & & 98.989 & 103.116 & 94.597 & 108.767 & 105.549 & 77.764 & 72.977 \\
\hline 9/4/07 21:30 & 123.587 & 121.209 & & 98.993 & 103.12 & 94.597 & 108.765 & 105.549 & 77.761 & 72.979 \\
\hline 9/4/07 22:00 & 123.582 & 121.213 & & 98.987 & 103.112 & 94.593 & 108.762 & 105.544 & 77.755 & 72.97 \\
\hline 9/4/07 22:30 & 123.58 & 121.209 & & 98.981 & 103.11 & 94.593 & 108.76 & 105.54 & 77.751 & 72.968 \\
\hline 9/4/07 23:00 & 123.582 & 121.211 & & 98.987 & 103.112 & 94.595 & 108.758 & 105.544 & 77.748 & 72.972 \\
\hline 9/4/07 23:30 & 123.578 & 121.211 & & 98.982 & 103.108 & 94.59 & 108.756 & 105.54 & 77.742 & 72.966 \\
\hline 9/5/07 0:00 & 123.578 & 121.206 & & 98.982 & 103.108 & 94.59 & 108.756 & 105.54 & 77.74 & 72.968 \\
\hline 9/5/07 0:30 & 123.58 & 121.2 & & 98.984 & 103.114 & 94.595 & 108.756 & 105.542 & 77.735 & 72.968 \\
\hline 9/5/07 1:00 & 123.575 & 121.202 & & 98.979 & 103.104 & 94.588 & 108.754 & 105.538 & 77.731 & 72.96 \\
\hline 9/5/07 1:30 & 123.571 & 121.202 & & 98.977 & 103.101 & 94.581 & 108.752 & 105.531 & 77.729 & 72.958 \\
\hline 9/5/07 2:00 & 123.568 & 121.195 & & 98.975 & 103.103 & 94.583 & 108.75 & 105.531 & 77.726 & 72.958 \\
\hline $9 / 5 / 072: 30$ & 123.568 & 121.19 & & 98.975 & 103.101 & 94.583 & 108.748 & 105.529 & 77.72 & 72.956 \\
\hline 9/5/07 3:00 & 123.564 & 121.186 & & 98.97 & 103.095 & 94.578 & 108.75 & 105.527 & 77.718 & 72.95 \\
\hline 9/5/07 3:30 & 123.559 & 121.181 & & 98.963 & 103.091 & 94.574 & 108.747 & 105.52 & 77.713 & 72.944 \\
\hline 9/5/07 4:00 & 123.552 & 121.177 & & 98.958 & 103.084 & 94.569 & 108.747 & 105.516 & 77.709 & 72.939 \\
\hline $9 / 5 / 074: 30$ & 123.547 & 121.177 & & 98.955 & 103.078 & 94.56 & 108.744 & 105.509 & 77.705 & 72.931 \\
\hline 9/5/07 5:00 & 123.547 & 121.17 & & 98.953 & 103.078 & 94.56 & 108.743 & 105.509 & 77.703 & 72.931 \\
\hline 9/5/07 5:30 & 123.545 & 123.249 & & 98.953 & 103.078 & 94.559 & 108.743 & 105.507 & 77.702 & 72.931 \\
\hline 9/5/07 6:00 & 123.538 & 123.501 & & 99.072 & 103.07 & 94.553 & 108.743 & 105.5 & 77.696 & 72.922 \\
\hline 9/5/07 6:30 & 125.66 & 123.637 & & 99.758 & 103.649 & 95.274 & 109.451 & 107.081 & 77.718 & 73.043 \\
\hline 9/5/07 7:00 & 125.925 & 123.716 & & 99.968 & 103.889 & 95.514 & 109.674 & 107.353 & 77.746 & 73.239 \\
\hline $9 / 5 / 077: 30$ & 126.066 & 123.768 & & 100.088 & 104.024 & 95.651 & 109.79 & 107.501 & 77.768 & 73.38 \\
\hline 9/5/07 8:00 & 126.152 & 123.807 & & 100.161 & 104.104 & 95.733 & 109.866 & 107.585 & 77.79 & 73.467 \\
\hline 9/5/07 8:30 & 126.204 & 123.832 & & 100.212 & 104.159 & 95.785 & 109.914 & 107.643 & 77.81 & 73.524 \\
\hline 9/5/07 9:00 & 126.241 & 123.855 & & 100.245 & 104.196 & 95.825 & 109.95 & 107.681 & 77.829 & 73.566 \\
\hline 9/5/07 9:30 & 126.267 & 123.866 & & 100.273 & 104.224 & 95.851 & 109.974 & 107.709 & 77.847 & 73.595 \\
\hline 9/5/07 10:00 & 126.291 & 123.88 & & 100.292 & 104.245 & 95.875 & 109.997 & 107.734 & 77.862 & 73.617 \\
\hline 9/5/07 10:30 & 126.303 & 123.882 & & 100.307 & 104.26 & 95.889 & 110.01 & 107.747 & 77.879 & 73.638 \\
\hline 9/5/07 11:00 & 126.315 & 123.893 & & 100.319 & 104.273 & 95.903 & 110.023 & 107.76 & 77.893 & 73.653 \\
\hline 9/5/07 11:30 & 126.322 & 123.891 & & 100.321 & 104.277 & 95.905 & 110.035 & 107.767 & 77.903 & 73.659 \\
\hline $9 / 5 / 07$ 12:00 & 126.326 & 122.034 & & 100.328 & 104.283 & 95.91 & 110.044 & 107.771 & 77.912 & 73.663 \\
\hline 9/5/07 12:30 & 126.326 & 121.703 & & 100.333 & 104.291 & 95.919 & 110.05 & 107.776 & 77.925 & 73.675 \\
\hline 9/5/07 13:00 & 124.45 & 121.544 & & 99.661 & 103.877 & 95.368 & 109.509 & 106.446 & 77.919 & 73.632 \\
\hline 9/5/07 13:30 & 124.086 & 121.451 & & 99.397 & 103.567 & 95.05 & 109.212 & 106.06 & 77.903 & 73.448 \\
\hline 9/5/07 14:00 & 123.92 & 121.385 & & 99.267 & 103.417 & 94.901 & 109.075 & 105.89 & 77.886 & 73.309 \\
\hline 9/5/07 14:30 & 123.823 & 121.333 & & 99.186 & 103.327 & 94.809 & 108.945 & 105.788 & 77.871 & 73.214 \\
\hline 9/5/07 15:00 & 123.757 & 121.304 & & 99.129 & 103.264 & 94.751 & 108.893 & 105.721 & 77.86 & 73.146 \\
\hline 9/5/07 15:30 & 123.708 & 121.281 & & 99.087 & 103.221 & 94.703 & 108.851 & 105.67 & 77.845 & 73.092 \\
\hline 9/5/07 16:00 & 123.675 & 121.256 & & 99.06 & 103.186 & 94.675 & 108.822 & 105.637 & 77.836 & 73.059 \\
\hline 9/5/07 16:30 & 123.649 & 121.245 & & 99.039 & 103.169 & 94.652 & 108.802 & 105.613 & 77.825 & 73.035 \\
\hline 9/5/07 17:00 & 123.63 & 121.236 & & 99.02 & 103.146 & 94.632 & 108.783 & 105.591 & 77.816 & 73.014 \\
\hline 9/5/07 17:30 & 123.613 & 121.227 & & 99.008 & 103.135 & 94.618 & 108.77 & 105.577 & 77.809 & 73.001 \\
\hline 9/5/07 18:00 & 123.604 & 121.229 & & 98.998 & 103.123 & 94.609 & 108.76 & 105.565 & 77.801 & 72.989 \\
\hline 9/5/07 18:30 & 123.594 & 121.211 & & 98.993 & 103.123 & 94.602 & 108.757 & 105.558 & 77.796 & 72.981 \\
\hline 9/5/07 19:00 & 123.604 & 121.213 & & 98.993 & 103.127 & 94.609 & 108.755 & 105.565 & 77.792 & 72.975 \\
\hline 9/5/07 19:30 & 123.58 & 121.215 & & 98.979 & 103.104 & 94.585 & 108.74 & 105.54 & 77.783 & 72.964 \\
\hline 9/5/07 20:00 & 123.578 & 121.227 & & 98.982 & 103.106 & 94.588 & 108.741 & 105.54 & 77.779 & 72.966 \\
\hline 9/5/07 20:30 & 123.585 & 121.236 & & 98.991 & 103.112 & 94.595 & 108.727 & 105.547 & 77.777 & 72.97 \\
\hline 9/5/07 21:00 & 123.597 & 121.243 & & 98.999 & 103.122 & 94.607 & 108.759 & 105.558 & 77.777 & 72.981 \\
\hline 9/5/07 21:30 & 123.601 & 121.247 & & 99.008 & 103.131 & 94.616 & 108.767 & 105.562 & 77.775 & 72.989 \\
\hline 9/5/07 22:00 & 123.611 & 121.24 & & 99.013 & 103.141 & 94.623 & 108.772 & 105.571 & 77.774 & 72.995 \\
\hline 9/5/07 22:30 & 123.613 & 121.24 & & 99.02 & 103.144 & 94.628 & 108.775 & 105.575 & 77.774 & 72.999 \\
\hline 9/5/07 23:00 & 123.611 & 121.24 & & 99.013 & 103.137 & 94.621 & 108.768 & 105.569 & 77.77 & 72.991 \\
\hline 9/5/07 23:30 & 123.608 & 121.238 & & 99.012 & 103.135 & 94.621 & 108.768 & 105.569 & 77.768 & 72.989 \\
\hline 9/6/07 0:00 & 123.606 & 121.234 & & 99.012 & 103.137 & 94.618 & 108.766 & 105.566 & 77.766 & 72.989 \\
\hline 9/6/07 0:30 & 123.606 & 121.238 & & 99.012 & 103.133 & 94.616 & 108.764 & 105.564 & 77.762 & 72.987 \\
\hline 9/6/07 1:00 & 123.601 & 121.234 & & 99.006 & 103.131 & 94.614 & 108.762 & 105.562 & 77.759 & 72.985 \\
\hline 9/6/07 1:30 & 123.606 & 121.218 & & 99.01 & 103.135 & 94.619 & 108.762 & 105.567 & 77.757 & 72.987 \\
\hline $9 / 6 / 072: 00$ & 123.601 & 121.211 & & 99.005 & 103.131 & 94.614 & 108.758 & 105.562 & 77.753 & 72.983 \\
\hline 9/6/07 2:30 & 123.599 & 121.211 & & 98.991 & 103.116 & 94.6 & 108.749 & 105.549 & 77.748 & 72.966 \\
\hline 9/6/07 3:00 & 123.582 & 121.211 & & 98.984 & 103.11 & 94.595 & 108.745 & 105.542 & 77.744 & 72.96 \\
\hline 9/6/07 3:30 & 123.58 & 121.209 & & 98.989 & 103.112 & 94.593 & 108.743 & 105.538 & 77.74 & 72.96 \\
\hline $9 / 6 / 074: 00$ & 123.58 & 121.204 & & 98.984 & 103.11 & 94.592 & 108.743 & 105.54 & 77.738 & 72.96 \\
\hline 9/6/07 4:30 & 123.58 & 121.204 & & 98.984 & 103.106 & 94.588 & 108.741 & 105.538 & 77.735 & 72.956 \\
\hline 9/6/07 5:00 & 123.578 & 121.206 & & 98.982 & 103.104 & 94.588 & 108.739 & 105.535 & 77.735 & 72.956 \\
\hline
\end{tabular}


TABLE S1.1 (Cont.)

\begin{tabular}{|c|c|c|c|c|c|c|c|c|c|c|}
\hline \multirow[b]{2}{*}{ Date and Time } & \multicolumn{10}{|c|}{ Depth from Top of Casing (ft) } \\
\hline & MW1D & MW2D & MW3D & MW4D & MW7 & MW9 & Oentrich & MW14D & MW15D & MW16D \\
\hline 9/6/07 5:30 & 123.578 & 121.211 & & 98.981 & 103.104 & 94.588 & 108.737 & 105.533 & 77.731 & 72.954 \\
\hline 9/6/07 6:00 & 123.575 & 123.233 & & 98.982 & 103.104 & 94.588 & 108.737 & 105.533 & 77.729 & 72.954 \\
\hline 9/6/07 6:30 & 123.58 & 123.487 & & 98.989 & 103.108 & 94.595 & 108.741 & 105.538 & 77.729 & 72.958 \\
\hline 9/6/07 7:00 & 125.674 & 123.614 & & 99.828 & 103.7 & 95.342 & 109.477 & 107.061 & 77.753 & 73.07 \\
\hline $9 / 6 / 077: 30$ & 125.941 & 123.705 & & 100.042 & 103.946 & 95.589 & 109.712 & 107.342 & 77.781 & 73.27 \\
\hline 9/6/07 8:00 & 126.078 & 123.753 & & 100.145 & 104.081 & 95.714 & 110.221 & 107.482 & 77.805 & 73.4 \\
\hline 9/6/07 8:30 & 126.166 & 123.789 & & 100.231 & 104.157 & 95.799 & 109.904 & 107.57 & 77.829 & 73.491 \\
\hline 9/6/07 9:00 & 126.218 & 123.812 & & 100.278 & 104.207 & 95.851 & 109.956 & 107.626 & 77.849 & 73.545 \\
\hline 9/6/07 9:30 & 126.253 & 123.843 & & 100.312 & 104.241 & 95.889 & 109.993 & 107.663 & 77.866 & 73.584 \\
\hline 9/6/07 10:00 & 126.277 & 123.857 & & 100.336 & 104.264 & 95.91 & 110.015 & 107.69 & 77.88 & 73.609 \\
\hline 9/6/07 10:30 & 126.308 & 123.866 & & 100.361 & 104.294 & 95.941 & 110.037 & 107.719 & 77.897 & 73.642 \\
\hline 9/6/07 11:00 & 126.324 & 123.873 & & 100.374 & 104.311 & 95.955 & 110.049 & 107.736 & 77.914 & 73.659 \\
\hline 9/6/07 11:30 & 126.334 & 123.88 & & 100.386 & 104.319 & 95.964 & 110.056 & 107.745 & 77.93 & 73.671 \\
\hline 9/6/07 12:00 & 126.338 & 123.875 & & 100.392 & 104.327 & 95.971 & 110.061 & 107.752 & 77.939 & 73.677 \\
\hline 9/6/07 12:30 & 126.345 & 121.893 & & 100.395 & 104.332 & 95.976 & 110.068 & 107.76 & 77.95 & 73.685 \\
\hline 9/6/07 13:00 & 126.345 & 121.635 & & 100.318 & 104.332 & 95.978 & 110.074 & 107.758 & 77.963 & 73.687 \\
\hline 9/6/07 13:30 & 124.294 & 121.49 & & 99.553 & 103.744 & 95.231 & 109.342 & 106.269 & 77.945 & 73.572 \\
\hline 9/6/07 14:00 & 124.02 & 121.404 & & 99.337 & 103.499 & 94.984 & 109.113 & 105.985 & 77.923 & 73.383 \\
\hline 9/6/07 14:30 & 123.871 & 121.34 & & 99.217 & 103.36 & 94.842 & 108.983 & 105.832 & 77.903 & 73.247 \\
\hline 9/6/07 15:00 & 123.779 & 121.293 & & 99.142 & 103.278 & 94.762 & 108.906 & 105.739 & 77.886 & 73.159 \\
\hline $9 / 6 / 07$ 15:30 & 123.712 & 121.254 & & 99.084 & 103.217 & 94.703 & 108.847 & 105.673 & 77.873 & 73.097 \\
\hline 9/6/07 16:00 & 123.663 & 121.231 & & 99.041 & 103.171 & 94.656 & 108.806 & 105.624 & 77.856 & 73.045 \\
\hline 9/6/07 16:30 & 123.625 & 121.209 & & 99.008 & 103.139 & 94.621 & 108.774 & 105.584 & 77.844 & 73.008 \\
\hline 9/6/07 17:00 & 123.604 & 121.184 & & 98.987 & 103.112 & 94.597 & 108.751 & 105.558 & 77.832 & 72.982 \\
\hline 9/6/07 17:30 & 123.578 & 121.163 & & 98.967 & 103.093 & 94.576 & 108.734 & 105.535 & 77.818 & 72.96 \\
\hline 9/6/07 18:00 & 123.556 & 121.17 & & 98.941 & 103.072 & 94.555 & 108.712 & 105.513 & 77.809 & 72.935 \\
\hline 9/6/07 18:30 & 123.535 & 121.15 & & 98.925 & 103.051 & 94.536 & 108.691 & 105.491 & 77.792 & 72.915 \\
\hline 9/6/07 19:00 & 123.54 & 121.141 & & 98.936 & 103.059 & 94.543 & 108.698 & 105.498 & 77.792 & 72.923 \\
\hline 9/6/07 19:30 & 123.521 & 121.145 & & 98.915 & 103.04 & 94.524 & 108.68 & 105.478 & 77.781 & 72.904 \\
\hline 9/6/07 20:00 & 123.512 & 121.138 & & 98.908 & 103.034 & 94.515 & 108.672 & 105.469 & 77.772 & 72.894 \\
\hline 9/6/07 20:30 & 123.514 & 121.12 & & 98.913 & 103.038 & 94.52 & 108.676 & 105.471 & 77.768 & 72.9 \\
\hline 9/6/07 21:00 & 123.504 & 121.19 & & 98.903 & 103.028 & 94.512 & 108.668 & 105.462 & 77.759 & 72.888 \\
\hline 9/6/07 21:30 & 123.49 & 121.195 & & 98.894 & 103.011 & 94.498 & 108.655 & 105.451 & 77.75 & 72.873 \\
\hline 9/6/07 22:00 & 123.613 & 121.222 & & 98.967 & 103.106 & 94.588 & 108.679 & 105.522 & 77.755 & 72.966 \\
\hline 9/6/07 22:30 & 123.623 & 121.202 & & 98.974 & 103.084 & 94.564 & 108.691 & 105.509 & 77.729 & 72.939 \\
\hline 9/6/07 23:00 & 123.653 & 121.2 & & 99.015 & 103.137 & 94.621 & 108.691 & 105.56 & 77.72 & 72.993 \\
\hline 9/6/07 23:30 & 123.575 & 121.245 & & 98.965 & 103.11 & 94.6 & 108.745 & 105.547 & 77.687 & 73.591 \\
\hline 9/7/07 0:00 & 123.568 & 121.243 & & 98.989 & 103.084 & 94.567 & 108.715 & 105.514 & 77.678 & 73.295 \\
\hline 9/7/07 0:30 & 123.549 & 121.231 & & 99.029 & 103.144 & 94.625 & 108.758 & 105.56 & 77.692 & 73.167 \\
\hline 9/7/07 1:00 & 123.561 & 121.245 & & 99.015 & 103.137 & 94.621 & 108.76 & 105.567 & 77.692 & 73.082 \\
\hline 9/7/07 1:30 & 123.556 & 121.247 & & 99.006 & 103.129 & 94.614 & 108.756 & 105.56 & 77.691 & 73.022 \\
\hline 9/7/07 2:00 & 123.564 & 121.258 & & 99.025 & 103.146 & 94.63 & 108.766 & 105.573 & 77.694 & 72.977 \\
\hline 9/7/07 2:30 & 123.552 & 121.281 & & 99.024 & 103.146 & 94.63 & 108.766 & 105.573 & 77.696 & 72.946 \\
\hline 9/7/07 3:00 & 123.573 & 121.274 & & 99.034 & 103.156 & 94.642 & 108.769 & 105.587 & 77.702 & 72.925 \\
\hline 9/7/07 3:30 & 123.599 & 121.281 & & 99.058 & 103.183 & 94.665 & 108.773 & 105.609 & 77.709 & 72.919 \\
\hline 9/7/07 4:00 & 123.646 & 121.286 & & 99.051 & 103.175 & 94.661 & 108.777 & 105.607 & 77.709 & 72.917 \\
\hline 9/7/07 4:30 & 123.646 & 121.293 & & 99.062 & 103.183 & 94.665 & 108.781 & 105.613 & 77.715 & 72.917 \\
\hline 9/7/07 5:00 & 123.658 & 121.295 & & 99.063 & 103.188 & 94.67 & 108.782 & 105.618 & 77.716 & 72.921 \\
\hline 9/7/07 5:30 & 123.663 & 122.716 & & 99.068 & 103.19 & 94.673 & 108.784 & 105.62 & 77.718 & 72.977 \\
\hline 9/7/07 6:00 & 123.665 & 123.426 & & 99.074 & 103.196 & 94.675 & 108.788 & 105.624 & 77.722 & 73.03 \\
\hline 9/7/07 6:30 & 124.714 & 123.646 & & 99.565 & 103.266 & 94.795 & 108.933 & 105.757 & 77.729 & 73.051 \\
\hline 9/7/07 7:00 & 125.87 & 123.782 & & 100.014 & 103.895 & 95.533 & 109.595 & 107.264 & 77.77 & 73.235 \\
\hline 9/7/07 7:30 & 126.104 & 123.861 & & 100.206 & 104.113 & 95.752 & 109.787 & 107.506 & 77.805 & 73.427 \\
\hline 9/7/07 8:00 & 126.241 & 122.274 & & 100.328 & 104.249 & 95.889 & 109.89 & 107.65 & 77.84 & 73.568 \\
\hline 9/7/07 8:30 & 126.324 & 121.853 & & 100.402 & 104.327 & 95.964 & 109.965 & 107.732 & 77.868 & 73.651 \\
\hline 9/7/07 9:00 & 124.736 & 121.701 & & & 104.102 & 95.608 & 109.594 & 106.782 & 77.888 & 73.698 \\
\hline 9/7/07 9:30 & 124.239 & 121.624 & & 99.554 & 103.557 & 95.208 & 109.203 & 106.209 & 77.886 & 73.564 \\
\hline 9/7/07 10:00 & 124.081 & 121.576 & & 99.432 & 103.415 & 95.066 & 109.064 & 106.042 & 77.886 & 73.444 \\
\hline 9/7/07 10:30 & 124.001 & 121.58 & & 99.368 & 103.344 & 94.993 & 108.987 & 105.961 & & 73.367 \\
\hline 9/7/07 11:00 & 123.953 & 121.562 & & 99.334 & 103.3 & 94.948 & 108.944 & & 77.877 & 73.318 \\
\hline 9/7/07 11:30 & 126.251 & 121.555 & & 99.317 & 103.28 & 94.927 & 108.918 & 105.877 & 77.886 & 73.288 \\
\hline 9/7/07 12:00 & 123.911 & 121.553 & & 99.301 & 103.266 & 94.915 & 108.897 & 105.859 & 77.891 & 73.272 \\
\hline 9/7/07 12:30 & 123.899 & 121.542 & 118.1 & 99.299 & 103.257 & 94.906 & & 105.848 & 77.886 & 73.262 \\
\hline 9/7/07 13:00 & 123.904 & 121.531 & 118.077 & 99.294 & 103.259 & 94.908 & 108.92 & 105.85 & & 73.261 \\
\hline 9/7/07 13:30 & 123.887 & 121.524 & 118.06 & 99.285 & 103.247 & 94.894 & 108.903 & 105.837 & & 73.247 \\
\hline 9/7/07 14:00 & 123.88 & 121.51 & 118.055 & 99.273 & 103.234 & 94.887 & 108.895 & 105.826 & & 73.237 \\
\hline
\end{tabular}


TABLE S1.1 (Cont.)

\begin{tabular}{|c|c|c|c|c|c|c|c|c|c|c|}
\hline \multirow[b]{2}{*}{ Date and Time } & \multicolumn{10}{|c|}{ Depth from Top of Casing (ft) } \\
\hline & MW1D & MW2D & MW3D & MW4D & MW7 & MW9 & Oentrich & MW14D & MW15D & MW16D \\
\hline 9/7/07 14:30 & 123.871 & 121.503 & 118.045 & 99.267 & 103.23 & 94.88 & 108.89 & 105.819 & & 73.231 \\
\hline 9/7/07 15:00 & 123.861 & 121.494 & 118.034 & 99.253 & 103.215 & 94.866 & 108.884 & 105.806 & & 73.216 \\
\hline 9/7/07 15:30 & 123.852 & 121.487 & 118.024 & 99.248 & 103.209 & 94.859 & 108.88 & 105.799 & & 73.21 \\
\hline 9/7/07 16:00 & 123.842 & 121.481 & 118.017 & 99.239 & 103.201 & 94.849 & 108.879 & 105.788 & & 73.202 \\
\hline 9/7/07 16:30 & 123.833 & 121.481 & 118.014 & 99.23 & 103.192 & 94.84 & 108.877 & 105.779 & & 73.194 \\
\hline 9/7/07 17:00 & 123.826 & 121.483 & 118.014 & 99.227 & 103.188 & 94.838 & 108.873 & 105.775 & & 73.19 \\
\hline 9/7/07 17:30 & 123.826 & 121.487 & 118.017 & 99.23 & 103.186 & 94.835 & 108.871 & 105.772 & & 73.187 \\
\hline 9/7/07 18:00 & 123.828 & 121.49 & 118.019 & 99.232 & 103.19 & 94.84 & 108.871 & 105.775 & & 73.19 \\
\hline 9/7/07 18:30 & 123.828 & 121.494 & 117.896 & 99.236 & 103.194 & 94.842 & 108.869 & 105.779 & & 73.196 \\
\hline 9/7/07 19:00 & 123.835 & 121.508 & 117.909 & 99.239 & 103.198 & 94.847 & 108.865 & 105.783 & & 73.2 \\
\hline 9/7/07 19:30 & 123.838 & 121.515 & 117.916 & 99.244 & 103.201 & 94.849 & 108.864 & 105.786 & & 73.202 \\
\hline 9/7/07 20:00 & 123.849 & 121.526 & 117.929 & 99.256 & 103.213 & 94.861 & 108.86 & 105.799 & & 73.214 \\
\hline 9/7/07 20:30 & 123.857 & 121.533 & 117.936 & 99.263 & 103.22 & 94.868 & 108.856 & 105.806 & & 73.221 \\
\hline 9/7/07 21:00 & 123.868 & 121.54 & 117.939 & 99.277 & 103.232 & 94.88 & 108.856 & 105.817 & & 73.233 \\
\hline 9/7/07 21:30 & 123.875 & 121.533 & 117.936 & 99.284 & 103.24 & 94.887 & 108.858 & 105.825 & & 73.239 \\
\hline 9/7/07 22:00 & 123.885 & 121.542 & 117.939 & 99.287 & 103.245 & 94.892 & 108.858 & 105.834 & & 73.243 \\
\hline 9/7/07 22:30 & 123.878 & 121.54 & 117.941 & 99.282 & 103.24 & 94.889 & 108.858 & 105.825 & & 73.237 \\
\hline 9/7/07 23:00 & 123.885 & 121.537 & 117.931 & 99.289 & 103.249 & 94.896 & 108.854 & 105.834 & & 73.247 \\
\hline 9/7/07 23:30 & 123.885 & 121.531 & 117.934 & 99.287 & 103.245 & 94.896 & 108.856 & 105.834 & & 73.243 \\
\hline $9 / 8 / 070: 00$ & 123.883 & 121.526 & 117.929 & 99.282 & 103.241 & 94.892 & 108.854 & 105.83 & & 73.241 \\
\hline 9/8/07 0:30 & 123.878 & 121.521 & 117.921 & 99.279 & 103.236 & 94.887 & 108.854 & 105.825 & & 73.237 \\
\hline 9/8/07 1:00 & 123.875 & 121.524 & 117.921 & 99.275 & 103.236 & 94.882 & 108.85 & 105.823 & & 73.235 \\
\hline 9/8/07 1:30 & 123.868 & 121.521 & 117.916 & 99.268 & 103.226 & 94.878 & 108.85 & 105.814 & & 73.227 \\
\hline 9/8/07 2:00 & 123.866 & 121.517 & 117.916 & 99.272 & 103.228 & 94.88 & 108.85 & 105.815 & & 73.229 \\
\hline 9/8/07 2:30 & 123.864 & 121.508 & 117.909 & 99.268 & 103.226 & 94.875 & 108.847 & 105.814 & & 73.227 \\
\hline 9/8/07 3:00 & 123.861 & 121.503 & 117.901 & 99.263 & 103.219 & 94.871 & 108.847 & 105.808 & & 73.221 \\
\hline 9/8/07 3:30 & 123.857 & 121.494 & 117.893 & 99.256 & 103.217 & 94.868 & 108.847 & 105.804 & & 73.218 \\
\hline 9/8/07 4:00 & 123.854 & 121.483 & 117.883 & 99.251 & 103.209 & 94.861 & 108.845 & 105.797 & & 73.212 \\
\hline 9/8/07 4:30 & 123.852 & 121.483 & 117.876 & 99.239 & 103.2 & 94.852 & 108.845 & 105.788 & & 73.204 \\
\hline 9/8/07 5:00 & 123.849 & 121.483 & 117.881 & 99.232 & 103.19 & 94.838 & 108.845 & 105.777 & & 73.192 \\
\hline 9/8/07 5:30 & 123.845 & 121.485 & 117.881 & 99.232 & 103.188 & 94.838 & 108.843 & 105.777 & & 73.194 \\
\hline $9 / 8 / 076: 00$ & 123.84 & 122.714 & 117.886 & 99.232 & 103.192 & 94.84 & 108.841 & 105.777 & & 73.194 \\
\hline 9/8/07 6:30 & 123.835 & 123.614 & 119.337 & 99.236 & 103.192 & 94.842 & 108.841 & 105.777 & & 73.194 \\
\hline $9 / 8 / 077: 00$ & 123.835 & 123.834 & 119.587 & 99.618 & 103.192 & 94.845 & 108.841 & 105.782 & & 73.196 \\
\hline 9/8/07 7:30 & 125.606 & 123.936 & 119.713 & 100.083 & 103.814 & 95.608 & 109.592 & 107.413 & & 73.347 \\
\hline 9/8/07 8:00 & 125.802 & 124.004 & 119.783 & 100.268 & 104.024 & 95.82 & 109.789 & 107.647 & & 73.529 \\
\hline 9/8/07 8:30 & 125.92 & 124.047 & 119.834 & 100.361 & 104.134 & 95.929 & 109.9 & 107.769 & & 73.642 \\
\hline 9/8/07 9:00 & 125.996 & 124.084 & 119.871 & 100.426 & 104.203 & 95.997 & 109.968 & 107.84 & & 73.719 \\
\hline 9/8/07 9:30 & 126.05 & 124.104 & 119.892 & 100.467 & 104.25 & 96.044 & 110.011 & 107.889 & & 73.772 \\
\hline 9/8/07 10:00 & 126.092 & 124.122 & 119.914 & 100.5 & 104.285 & 96.08 & 110.043 & 107.926 & & 73.81 \\
\hline 9/8/07 10:30 & 126.131 & 124.127 & 119.922 & 100.519 & 104.304 & 96.101 & 110.067 & 107.949 & & 73.835 \\
\hline 9/8/07 11:00 & 126.178 & 124.136 & 119.932 & 100.535 & 104.327 & 96.12 & 110.084 & 107.968 & & 73.855 \\
\hline 9/8/07 11:30 & 126.365 & 124.14 & 119.937 & 100.54 & 104.332 & 96.127 & 110.101 & 107.973 & & 73.863 \\
\hline 9/8/07 12:00 & 126.53 & 122.936 & 119.944 & 100.55 & 104.34 & 96.132 & 110.112 & 107.984 & & 73.874 \\
\hline 9/8/07 12:30 & 126.554 & 122.084 & 118.533 & 100.555 & 104.348 & 96.141 & 110.121 & 107.988 & & 73.886 \\
\hline 9/8/07 13:00 & 126.371 & 121.873 & 118.289 & 100.175 & 104.359 & 96.155 & 110.131 & 107.999 & & 73.899 \\
\hline 9/8/07 13:30 & 124.502 & 121.753 & 118.158 & 99.73 & 103.744 & 95.396 & 109.394 & 106.412 & & 73.754 \\
\hline 9/8/07 14:00 & 124.242 & 121.667 & 118.067 & 99.553 & 103.542 & 95.193 & 109.214 & 106.18 & & 73.586 \\
\hline 9/8/07 14:30 & 124.112 & 121.612 & 118.014 & 99.449 & 103.426 & 95.076 & 109.119 & 106.051 & & 73.469 \\
\hline 9/8/07 15:00 & 124.024 & 121.576 & 117.974 & 99.377 & 103.348 & 94.996 & 109.06 & 105.965 & & 73.384 \\
\hline 9/8/07 15:30 & 123.97 & 121.553 & 117.949 & 99.33 & 103.297 & 94.948 & 109.019 & 105.91 & & 73.328 \\
\hline 9/8/07 16:00 & 123.932 & 121.535 & 117.926 & 99.299 & 103.26 & 94.913 & 108.993 & 105.87 & & 73.291 \\
\hline 9/8/07 16:30 & 123.901 & 121.521 & 117.914 & 99.282 & 103.243 & 94.894 & 108.971 & 105.848 & & 73.27 \\
\hline 9/8/07 17:00 & 123.885 & 121.515 & 117.906 & 99.265 & 103.226 & 94.878 & 108.954 & 105.828 & & 73.249 \\
\hline 9/8/07 17:30 & 123.866 & 121.515 & 117.906 & 99.254 & 103.215 & 94.863 & 108.942 & 105.814 & & 73.235 \\
\hline 9/8/07 18:00 & 123.861 & 121.519 & 117.906 & 99.249 & 103.209 & 94.859 & 108.935 & 105.808 & & 73.229 \\
\hline 9/8/07 18:30 & 123.859 & 121.517 & 117.903 & 99.253 & 103.209 & 94.859 & 108.929 & 105.806 & & 73.225 \\
\hline 9/8/07 19:00 & 123.861 & 121.519 & 117.909 & 99.254 & 103.211 & 94.861 & 108.92 & 105.808 & & 73.227 \\
\hline 9/8/07 19:30 & 123.861 & 121.531 & 117.919 & 99.254 & 103.211 & 94.864 & 108.914 & 105.808 & & 73.229 \\
\hline 9/8/07 20:00 & 123.861 & 121.54 & 117.931 & 99.258 & 103.213 & 94.864 & 108.91 & 105.808 & & 73.229 \\
\hline 9/8/07 20:30 & 123.873 & 121.546 & 117.934 & 99.275 & 103.226 & 94.878 & 108.903 & 105.821 & & 73.241 \\
\hline 9/8/07 21:00 & 123.885 & 121.549 & 117.936 & 99.279 & 103.236 & 94.887 & 108.901 & 105.832 & & 73.249 \\
\hline 9/8/07 21:30 & 123.89 & 121.558 & 117.941 & 99.289 & 103.243 & 94.892 & 108.897 & 105.837 & & 73.252 \\
\hline 9/8/07 22:00 & 123.892 & 121.56 & 117.944 & 99.291 & 103.243 & 94.892 & 108.893 & 105.834 & & 73.252 \\
\hline 9/8/07 22:30 & 123.897 & 121.562 & 117.951 & 99.298 & 103.253 & 94.904 & 108.891 & 105.843 & & 73.261 \\
\hline 9/8/07 23:00 & 123.904 & 121.565 & 117.951 & 99.299 & 103.255 & 94.904 & 108.89 & 105.845 & & 73.264 \\
\hline
\end{tabular}


TABLE S1.1 (Cont.)

\begin{tabular}{|c|c|c|c|c|c|c|c|c|c|c|}
\hline \multirow[b]{2}{*}{ Date and Time } & \multicolumn{10}{|c|}{ Depth from Top of Casing (ft) } \\
\hline & MW1D & MW2D & MW3D & MW4D & MW7 & MW9 & Oentrich & MW14D & MW15D & MW16D \\
\hline 9/8/07 23:30 & 123.906 & 121.567 & 117.956 & 99.303 & 103.259 & 94.908 & 108.888 & 105.85 & & 73.268 \\
\hline 9/9/07 0:00 & 123.909 & 121.587 & 117.977 & 99.304 & 103.26 & 94.911 & 108.886 & 105.855 & & 73.268 \\
\hline 9/9/07 0:30 & 123.913 & 121.599 & 117.987 & 99.308 & 103.266 & 94.913 & 108.884 & 105.859 & & 73.272 \\
\hline 9/9/07 1:00 & 123.925 & 121.599 & 117.989 & 99.335 & 103.287 & 94.937 & 108.876 & 105.874 & & 73.297 \\
\hline 9/9/07 1:30 & 123.942 & 121.601 & 117.992 & 99.344 & 103.299 & 94.951 & 108.876 & 105.888 & & 73.303 \\
\hline 9/9/07 2:00 & 123.947 & 121.601 & 117.989 & 99.342 & 103.297 & 94.948 & 108.876 & 105.89 & & 73.303 \\
\hline 9/9/07 2:30 & 123.944 & 121.605 & 117.994 & 99.346 & 103.3 & 94.956 & 108.875 & 105.892 & & 73.307 \\
\hline 9/9/07 3:00 & 123.942 & 121.612 & 117.997 & 99.342 & 103.299 & 94.951 & 108.875 & 105.89 & & 73.303 \\
\hline 9/9/07 3:30 & 123.937 & 121.614 & 118.004 & 99.351 & 103.304 & 94.955 & 108.873 & 105.894 & & 73.307 \\
\hline 9/9/07 4:00 & 123.932 & 121.626 & 118.014 & 99.354 & 103.312 & 94.96 & 108.871 & 105.899 & & 73.316 \\
\hline 9/9/07 4:30 & 123.93 & 121.626 & 118.014 & 99.36 & 103.316 & 94.967 & 108.871 & 105.905 & & 73.32 \\
\hline 9/9/07 5:00 & 123.925 & 121.628 & 118.017 & 99.372 & 103.327 & 94.977 & 108.873 & 105.916 & & 73.33 \\
\hline 9/9/07 5:30 & 123.927 & 121.644 & 118.027 & 99.368 & 103.327 & 94.979 & 108.876 & 105.919 & & 73.33 \\
\hline 9/9/07 6:00 & 123.925 & 121.66 & 118.042 & 99.375 & 103.329 & 94.979 & 108.878 & 105.919 & & 73.332 \\
\hline 9/9/07 6:30 & 123.925 & 123.12 & 118.06 & 99.394 & 103.346 & 94.993 & 108.88 & 105.934 & & 73.345 \\
\hline 9/9/07 7:00 & 123.923 & 123.805 & 119.529 & 99.408 & 103.359 & 95.01 & 108.882 & 105.947 & & 73.361 \\
\hline $9 / 9 / 077: 30$ & 124.868 & 124.013 & 119.776 & 99.859 & 103.428 & 95.125 & 109.017 & 106.166 & & 73.374 \\
\hline 9/9/07 8:00 & 125.702 & 124.134 & 119.909 & 100.285 & 104.014 & 95.809 & 109.648 & 107.61 & & 73.541 \\
\hline 9/9/07 8:30 & 125.892 & 124.211 & 119.99 & 100.462 & 104.222 & 96.014 & 109.838 & 107.838 & & 73.725 \\
\hline 9/9/07 9:00 & 126.005 & 124.263 & 120.048 & 100.573 & 104.34 & 96.136 & 109.945 & 107.971 & & 73.849 \\
\hline 9/9/07 9:30 & 126.081 & 124.299 & 120.083 & 100.641 & 104.414 & 96.209 & 110.01 & 108.048 & & 73.932 \\
\hline 9/9/07 10:00 & 126.14 & 122.333 & 118.795 & 100.688 & 104.469 & 96.266 & 110.06 & 108.106 & & 73.987 \\
\hline 9/9/07 10:30 & 126.192 & 122.095 & 118.511 & 100.722 & 104.504 & 96.299 & 109.279 & 108.143 & & 74.027 \\
\hline 9/9/07 11:00 & 124.573 & 121.977 & 118.377 & 99.971 & 103.993 & 95.646 & 109.342 & 106.673 & & 73.959 \\
\hline 9/9/07 11:30 & 124.384 & 121.905 & 118.299 & 99.78 & 103.767 & 95.42 & 109.176 & 106.406 & & 73.797 \\
\hline 9/9/07 12:00 & 124.317 & 121.855 & 118.246 & 99.682 & 103.658 & 95.307 & 109.092 & 106.279 & & 73.69 \\
\hline 9/9/07 12:30 & 124.258 & 121.818 & 118.208 & 99.62 & 103.586 & 95.238 & 109.035 & 106.202 & & 73.613 \\
\hline 9/9/07 13:00 & 124.206 & 121.791 & 118.188 & 99.572 & 103.537 & 95.189 & 109.002 & 106.146 & & 73.558 \\
\hline 9/9/07 13:30 & 124.171 & 121.771 & 118.16 & 99.547 & 103.5 & 95.153 & 108.975 & 106.109 & & 73.518 \\
\hline 9/9/07 14:00 & 124.147 & 121.741 & 118.133 & 99.52 & 103.479 & 95.135 & 108.957 & 106.084 & & 73.493 \\
\hline 9/9/07 14:30 & 124.124 & 121.716 & 118.11 & 99.503 & 103.462 & 95.113 & 108.943 & 106.062 & & 73.473 \\
\hline 9/9/07 15:00 & 124.1 & 121.696 & 118.085 & 99.475 & 103.434 & 95.085 & 108.932 & 106.036 & & 73.444 \\
\hline 9/9/07 15:30 & 124.072 & 121.685 & 118.075 & 99.451 & 103.409 & 95.062 & 108.924 & 106.011 & & 73.421 \\
\hline 9/9/07 16:00 & 124.048 & 121.676 & 118.06 & 99.43 & 103.386 & 95.04 & 108.919 & 105.987 & & 73.4 \\
\hline 9/9/07 16:30 & 124.036 & 121.669 & 118.055 & 99.422 & 103.379 & 95.031 & 108.913 & 105.978 & & 73.392 \\
\hline 9/9/07 17:00 & 124.022 & 121.66 & 118.047 & 99.413 & 103.367 & 95.022 & 108.906 & 105.965 & & 73.381 \\
\hline 9/9/07 17:30 & 124.017 & 121.655 & 118.045 & 99.41 & 103.361 & 95.014 & 108.902 & 105.96 & & 73.374 \\
\hline 9/9/07 18:00 & 124.01 & 121.651 & 118.04 & 99.396 & 103.356 & 95.007 & 108.898 & 105.952 & & 73.363 \\
\hline 9/9/07 18:30 & 124.003 & 121.648 & 118.034 & 99.396 & 103.35 & 95.003 & 108.894 & 105.948 & & 73.361 \\
\hline 9/9/07 19:00 & 123.999 & 121.646 & 118.037 & 99.389 & 103.346 & 94.998 & 108.891 & 105.941 & & 73.355 \\
\hline 9/9/07 19:30 & 123.991 & 121.648 & 118.037 & 99.385 & 103.342 & 94.993 & 108.889 & 105.936 & & 73.349 \\
\hline 9/9/07 20:00 & 123.991 & 121.651 & 118.04 & 99.387 & 103.342 & 94.996 & 108.885 & 105.938 & & 73.353 \\
\hline 9/9/07 20:30 & 123.994 & 121.664 & 118.055 & 99.391 & 103.342 & 94.995 & 108.883 & 105.939 & & 73.353 \\
\hline 9/9/07 21:00 & 123.996 & 121.671 & 118.057 & 99.396 & 103.346 & 94.998 & 108.879 & 105.941 & & 73.355 \\
\hline 9/9/07 21:30 & 124.008 & 121.669 & 118.057 & 99.406 & 103.361 & 95.014 & 108.879 & 105.956 & & 73.369 \\
\hline 9/9/07 22:00 & 124.015 & 121.671 & 118.06 & 99.413 & 103.367 & 95.017 & 108.879 & 105.96 & & 73.373 \\
\hline 9/9/07 22:30 & 124.015 & 121.671 & 118.055 & 99.41 & 103.363 & 95.017 & 108.878 & 105.959 & & 73.374 \\
\hline 9/9/07 23:00 & 124.017 & 121.671 & 118.062 & 99.411 & 103.365 & 95.019 & 108.878 & 105.963 & & 73.371 \\
\hline 9/9/07 23:30 & 124.017 & 121.658 & 118.047 & 99.41 & 103.363 & 95.017 & 108.878 & 105.958 & & 73.369 \\
\hline 9/10/07 0:00 & 124.015 & 121.644 & 118.032 & 99.408 & 103.369 & 95.019 & 108.874 & 105.963 & & 73.376 \\
\hline 9/10/07 0:30 & 124.013 & 121.637 & 118.027 & 99.394 & 103.354 & 95.005 & 108.872 & 105.947 & & 73.359 \\
\hline 9/10/07 1:00 & 124.008 & 121.644 & 118.034 & 99.382 & 103.339 & 94.993 & 108.872 & 105.934 & & 73.347 \\
\hline 9/10/07 1:30 & 124.005 & 121.651 & 118.04 & 99.379 & 103.333 & 94.986 & 108.868 & 105.927 & & 73.34 \\
\hline 9/10/07 2:00 & 123.998 & 121.651 & 118.034 & 99.387 & 103.342 & 94.995 & 108.864 & 105.934 & & 73.351 \\
\hline 9/10/07 2:30 & 123.994 & 121.653 & 118.037 & 99.394 & 103.348 & 95 & 108.863 & 105.941 & & 73.357 \\
\hline 9/10/07 3:00 & 123.994 & 121.646 & 118.032 & 99.394 & 103.346 & 95 & 108.861 & 105.941 & & 73.355 \\
\hline 9/10/07 3:30 & 123.989 & 121.644 & 118.034 & 99.394 & 103.348 & 95.003 & 108.859 & 105.941 & & 73.357 \\
\hline 9/10/07 4:00 & 123.987 & 121.639 & 118.027 & 99.391 & 103.344 & 94.998 & 108.857 & 105.936 & & 73.353 \\
\hline 9/10/07 4:30 & 123.989 & 121.642 & 118.019 & 99.384 & 103.34 & 94.993 & 108.857 & 105.934 & & 73.347 \\
\hline 9/10/07 5:00 & 123.987 & 121.644 & 118.027 & 99.38 & 103.337 & 94.988 & 108.857 & 105.927 & & 73.34 \\
\hline 9/10/07 5:30 & 123.982 & 121.635 & 118.022 & 99.385 & 103.339 & 94.991 & 108.855 & 105.929 & & 73.345 \\
\hline 9/10/07 6:00 & 123.982 & 123.49 & 119.294 & 99.384 & 103.342 & 94.995 & 108.853 & 105.934 & & 73.349 \\
\hline 9/10/07 6:30 & 123.982 & 123.837 & 119.728 & 99.372 & 103.329 & 94.988 & 108.855 & 105.923 & & 73.336 \\
\hline 9/10/07 7:00 & 125.532 & 123.988 & 119.899 & 100.094 & 103.769 & 95.573 & 109.445 & 107.249 & & 73.371 \\
\hline 9/10/07 7:30 & 125.849 & 124.077 & 120.002 & 100.368 & 104.096 & 95.905 & 109.758 & 107.647 & & 73.584 \\
\hline 9/10/07 8:00 & 126.003 & 124.156 & 120.081 & 100.505 & 104.247 & 96.056 & 109.905 & 107.811 & & 73.731 \\
\hline
\end{tabular}


TABLE S1.1 (Cont.)

Depth from Top of Casing (ft)

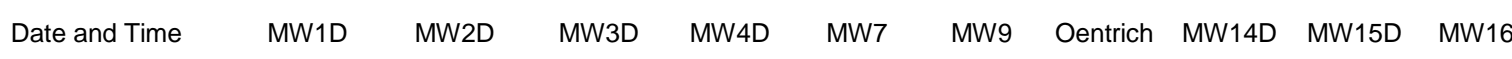

\begin{tabular}{|c|c|c|c|c|c|c|c|c|c|}
\hline 9/10/07 8:30 & 126.104 & 124.204 & 120.131 & 100.585 & 104.336 & 96.148 & 109.993 & 107.913 & 73.828 \\
\hline 9/10/07 9:00 & 126.17 & 124.247 & 120.176 & 100.654 & 104.414 & 96.226 & 110.047 & 107.991 & 73.911 \\
\hline 9/10/07 9:30 & 126.222 & 124.279 & 120.212 & 100.705 & 104.46 & 96.273 & 110.085 & 108.039 & 73.963 \\
\hline 9/10/07 10:00 & 126.267 & 124.301 & 120.234 & 100.743 & 104.504 & 96.315 & 110.115 & 108.086 & 74.006 \\
\hline 9/10/07 10:30 & 126.3 & 124.306 & 120.239 & 100.774 & 104.538 & 96.348 & 110.135 & 108.117 & 74.045 \\
\hline $9 / 10 / 0711: 00$ & 126.324 & 124.315 & 120.249 & 100.793 & 104.559 & 96.372 & 110.156 & 108.143 & 74.066 \\
\hline 9/10/07 11:30 & 126.338 & 124.344 & 120.275 & 100.8 & 104.563 & 96.372 & 110.175 & 108.148 & 74.07 \\
\hline $9 / 10 / 07$ 12:00 & 126.345 & 122.431 & 118.901 & 100.809 & 104.572 & 96.384 & 110.184 & 108.157 & 74.082 \\
\hline $9 / 10 / 07$ 12:30 & 126.357 & 122.172 & 118.586 & 100.834 & 104.605 & 96.412 & 110.199 & 108.188 & 74.112 \\
\hline 9/10/07 13:00 & 124.733 & 122.038 & 118.433 & 100.045 & 104.077 & 95.733 & 109.51 & 106.771 & 74.045 \\
\hline 9/10/07 13:30 & 124.507 & 121.95 & 118.347 & 99.844 & 103.832 & 95.484 & 109.281 & 106.479 & 73.872 \\
\hline $9 / 10 / 07$ 14:00 & 124.4 & 121.884 & 118.281 & 99.728 & 103.704 & 95.359 & 109.169 & 106.339 & 73.748 \\
\hline $9 / 10 / 0714: 30$ & 124.336 & 121.848 & 118.241 & 99.654 & 103.62 & 95.276 & 109.103 & 106.246 & 73.656 \\
\hline $9 / 10 / 07$ 15:00 & 124.296 & 121.823 & 118.213 & 99.594 & 103.559 & 95.212 & 109.062 & 106.18 & 73.589 \\
\hline $9 / 10 / 07$ 15:30 & 124.268 & 121.807 & 118.196 & 99.563 & 103.523 & 95.179 & 109.03 & 106.14 & 73.551 \\
\hline 9/10/07 16:00 & 124.242 & 121.798 & 118.183 & 99.544 & 103.504 & 95.158 & 109.005 & 106.114 & 73.526 \\
\hline $9 / 10 / 0716: 30$ & 124.223 & 121.78 & 118.168 & 99.534 & 103.489 & 95.142 & 108.99 & 106.096 & 73.508 \\
\hline $9 / 10 / 07$ 17:00 & 124.206 & 121.766 & 118.155 & 99.527 & 103.479 & 95.135 & 108.977 & 106.087 & 73.5 \\
\hline 9/10/07 17:30 & 124.202 & 121.755 & 118.14 & 99.509 & 103.462 & 95.116 & 108.97 & 106.069 & 73.481 \\
\hline $9 / 10 / 07$ 18:00 & 124.192 & 121.748 & 118.138 & 99.496 & 103.451 & 95.106 & 108.962 & 106.058 & 73.469 \\
\hline $9 / 10 / 0718: 30$ & 124.187 & 121.739 & 118.128 & 99.487 & 103.439 & 95.094 & 108.96 & 106.045 & 73.454 \\
\hline 9/10/07 19:00 & 124.178 & 121.737 & 118.123 & 99.48 & 103.436 & 95.09 & 108.953 & 106.038 & 73.452 \\
\hline 9/10/07 19:30 & 124.171 & 121.739 & 118.125 & 99.473 & 103.426 & 95.08 & 108.949 & 106.027 & 73.442 \\
\hline 9/10/07 20:00 & 124.166 & 121.744 & 118.13 & 99.473 & 103.424 & 95.078 & 108.945 & 106.027 & 73.438 \\
\hline $9 / 10 / 0720: 30$ & 124.157 & 121.746 & 118.128 & 99.475 & 103.426 & 95.08 & 108.941 & 106.027 & 73.442 \\
\hline 9/10/07 21:00 & 124.152 & 121.746 & 118.133 & 99.48 & 103.43 & 95.085 & 108.939 & 106.034 & 73.446 \\
\hline $9 / 10 / 0721: 30$ & 124.147 & 121.744 & 118.128 & 99.48 & 103.432 & 95.087 & 108.938 & 106.034 & 73.446 \\
\hline 9/10/07 22:00 & 124.145 & 121.739 & 118.125 & 99.48 & 103.436 & 95.087 & 108.938 & 106.034 & 73.444 \\
\hline $9 / 10 / 0722: 30$ & 124.138 & 121.739 & 118.125 & 99.477 & 103.428 & 95.085 & 108.936 & 106.032 & 73.441 \\
\hline 9/10/07 23:00 & 124.131 & 121.739 & 118.12 & 99.475 & 103.426 & 95.083 & 108.936 & 106.027 & 73.439 \\
\hline 9/10/07 23:30 & 124.126 & 121.728 & 118.115 & 99.473 & 103.426 & 95.083 & 108.936 & 106.029 & 73.44 \\
\hline $9 / 11 / 070: 00$ & 124.121 & 121.725 & 118.11 & 99.473 & 103.426 & 95.078 & 108.934 & 106.025 & 73.438 \\
\hline 9/11/07 0:30 & 124.117 & 121.719 & 118.103 & 99.463 & 103.415 & 95.069 & 108.934 & 106.016 & 73.427 \\
\hline 9/11/07 1:00 & 124.112 & 121.705 & 118.09 & 99.461 & 103.413 & 95.066 & 108.932 & 106.012 & 73.425 \\
\hline $9 / 11 / 071: 30$ & 124.112 & 121.698 & 118.082 & 99.453 & 103.405 & 95.059 & 108.932 & 106.007 & 73.417 \\
\hline $9 / 11 / 072: 00$ & 124.102 & 121.689 & 118.075 & 99.441 & 103.394 & 95.047 & 108.932 & 105.996 & 73.407 \\
\hline 9/11/07 2:30 & 124.098 & 121.68 & 118.065 & 99.432 & 103.386 & 95.04 & 108.93 & 105.987 & 73.398 \\
\hline 9/11/07 3:00 & 124.093 & 121.676 & 118.062 & 99.427 & 103.379 & 95.031 & 108.928 & 105.976 & 73.392 \\
\hline 9/11/07 3:30 & 124.088 & 121.669 & 118.052 & 99.416 & 103.371 & 95.024 & 108.928 & 105.967 & 73.382 \\
\hline 9/11/07 4:00 & 124.083 & 121.662 & 118.05 & 99.413 & 103.365 & 95.019 & 108.926 & 105.963 & 73.378 \\
\hline 9/11/07 4:30 & 124.079 & 121.655 & 118.042 & 99.404 & 103.358 & 95.012 & 108.926 & 105.956 & 73.371 \\
\hline 9/11/07 5:00 & 124.074 & 121.651 & 118.034 & 99.401 & 103.354 & 95.01 & 108.924 & 105.952 & 73.365 \\
\hline $9 / 11 / 075: 30$ & 124.074 & 121.637 & 118.024 & 99.394 & 103.35 & 95.003 & 108.924 & 105.945 & 73.361 \\
\hline 9/11/07 $6: 00$ & 124.072 & 123.639 & 119.504 & 99.385 & 103.337 & 94.991 & 108.924 & 105.936 & 73.351 \\
\hline 9/11/07 $6: 30$ & 124.067 & 123.873 & 119.783 & 99.54 & 103.329 & 94.981 & 108.924 & 105.925 & 73.34 \\
\hline $9 / 11 / 077: 00$ & 125.75 & 124.002 & 119.924 & 100.216 & 103.919 & 95.724 & 109.635 & 107.435 & 73.458 \\
\hline $9 / 11 / 077: 30$ & 125.96 & 124.07 & 120.002 & 100.416 & 104.15 & 95.96 & 109.849 & 107.698 & 73.648 \\
\hline 9/11/07 8:00 & 126.069 & 124.122 & 120.055 & 100.523 & 104.275 & 96.084 & 109.965 & 107.834 & 73.776 \\
\hline 9/11/07 8:30 & 126.149 & 124.159 & 120.093 & 100.588 & 104.346 & 96.155 & 110.036 & 107.911 & 73.851 \\
\hline 9/11/07 9:00 & 126.204 & 124.188 & 120.126 & 100.635 & 104.397 & 96.205 & 110.085 & 107.964 & 73.907 \\
\hline 9/11/07 9:30 & 126.251 & 124.215 & 120.154 & 100.667 & 104.431 & 96.24 & 110.12 & 107.999 & 73.944 \\
\hline $9 / 11 / 07$ 10:00 & 126.282 & 124.231 & 120.171 & 100.698 & 104.464 & 96.273 & 110.145 & 108.033 & 73.979 \\
\hline $9 / 11 / 07$ 10:30 & 126.312 & 124.242 & 120.189 & 100.721 & 104.487 & 96.297 & 110.164 & 108.059 & 74.004 \\
\hline 9/11/07 11:00 & 126.35 & 122.335 & 118.806 & 100.74 & 104.504 & 96.315 & 110.179 & 108.077 & 74.024 \\
\hline $9 / 11 / 0711: 30$ & 126.4 & 122.07 & 118.483 & 100.748 & 104.519 & 96.33 & 110.194 & 108.093 & 74.039 \\
\hline $9 / 11 / 0712: 00$ & 124.818 & 121.939 & 118.339 & 99.951 & 103.978 & 95.637 & 109.518 & 106.676 & 73.948 \\
\hline 9/11/07 12:30 & 124.518 & 121.855 & 118.251 & 99.737 & 103.727 & 95.38 & 109.291 & 106.377 & 73.77 \\
\hline 9/11/07 13:00 & 124.339 & 121.803 & 118.191 & 99.628 & 103.601 & 95.257 & 109.176 & 106.235 & 73.648 \\
\hline 9/11/07 13:30 & 124.225 & 121.757 & 118.148 & 99.558 & 103.523 & 95.179 & 109.11 & 106.149 & 73.563 \\
\hline $9 / 11 / 0714: 00$ & 124.161 & 121.728 & 118.113 & 99.509 & 103.47 & 95.127 & 109.069 & 106.091 & 73.506 \\
\hline 9/11/07 14:30 & 124.121 & 121.705 & 118.09 & 99.472 & 103.432 & 95.087 & 109.041 & 106.049 & 73.462 \\
\hline $9 / 11 / 07$ 15:00 & 124.083 & 121.685 & 118.07 & 99.444 & 103.401 & 95.059 & 109.018 & 106.014 & 73.427 \\
\hline 9/11/07 15:30 & 124.06 & 121.669 & 118.057 & 99.423 & 103.382 & 95.038 & 109.001 & 105.991 & 73.407 \\
\hline $9 / 11 / 0716: 00$ & 124.043 & 121.662 & 118.047 & 99.408 & 103.363 & 95.019 & 108.99 & 105.972 & 73.386 \\
\hline $9 / 11 / 0716: 30$ & 124.022 & 121.655 & 118.04 & 99.396 & 103.35 & 95.005 & 108.981 & 105.959 & 73.371 \\
\hline $9 / 11 / 07$ 17:00 & 124.01 & 121.648 & 118.034 & 99.387 & 103.342 & 94.998 & 108.973 & 105.948 & 73.363 \\
\hline
\end{tabular}


TABLE S1.1 (Cont.)

\begin{tabular}{|c|c|c|c|c|c|c|c|c|c|c|}
\hline \multirow[b]{2}{*}{ Date and Time } & \multicolumn{10}{|c|}{ Depth from Top of Casing (ft) } \\
\hline & MW1D & MW2D & MW3D & MW4D & MW7 & MW9 & Oentrich & MW14D & MW15D & MW16D \\
\hline 9/11/07 17:30 & 124.003 & 121.644 & 118.027 & 99.382 & 103.337 & 94.991 & 108.966 & 105.941 & & 73.357 \\
\hline 9/11/07 18:00 & 123.998 & 121.644 & 118.027 & 99.379 & 103.333 & 94.988 & 108.96 & 105.936 & & 73.353 \\
\hline 9/11/07 18:30 & 123.994 & 121.642 & 118.024 & 99.375 & 103.329 & 94.981 & 108.955 & 105.93 & & 73.345 \\
\hline 9/11/07 19:00 & 123.994 & 121.642 & 118.024 & 99.375 & 103.327 & 94.981 & 108.953 & 105.93 & & 73.346 \\
\hline 9/11/07 19:30 & 123.989 & 121.648 & 118.029 & 99.373 & 103.325 & 94.979 & 108.947 & 105.927 & & 73.343 \\
\hline 9/11/07 20:00 & 123.989 & 121.653 & 118.037 & 99.375 & 103.327 & 94.981 & 108.945 & 105.93 & & 73.342 \\
\hline 9/11/07 20:30 & 123.994 & 121.662 & 118.042 & 99.384 & 103.333 & 94.988 & 108.941 & 105.934 & & 73.349 \\
\hline 9/11/07 21:00 & 124.001 & 121.667 & 118.05 & 99.389 & 103.34 & 94.995 & 108.939 & 105.941 & & 73.352 \\
\hline 9/11/07 21:30 & 124.005 & 121.669 & 118.047 & 99.396 & 103.346 & 95 & 108.938 & 105.948 & & 73.359 \\
\hline 9/11/07 22:00 & 124.013 & 121.671 & 118.052 & 99.403 & 103.352 & 95.007 & 108.936 & 105.952 & & 73.365 \\
\hline 9/11/07 22:30 & 124.017 & 121.669 & 118.05 & 99.403 & 103.352 & 95.007 & 108.936 & 105.956 & & 73.365 \\
\hline 9/11/07 23:00 & 124.017 & 121.669 & 118.052 & 99.406 & 103.356 & 95.01 & 108.932 & 105.954 & & 73.367 \\
\hline 9/11/07 23:30 & 124.02 & 121.664 & 118.047 & 99.404 & 103.356 & 95.012 & 108.932 & 105.959 & & 73.367 \\
\hline 9/12/07 0:00 & 124.017 & 121.667 & 118.05 & 99.403 & 103.352 & 95.007 & 108.932 & 105.954 & & 73.363 \\
\hline 9/12/07 0:30 & 124.013 & 121.664 & 118.05 & 99.399 & 103.352 & 95.005 & 108.93 & 105.95 & & 73.361 \\
\hline 9/12/07 1:00 & 124.01 & 121.664 & 118.047 & 99.401 & 103.352 & 95.007 & 108.928 & 105.952 & & 73.361 \\
\hline 9/12/07 1:30 & 124.008 & 121.66 & 118.04 & 99.399 & 103.35 & 95.005 & 108.928 & 105.952 & & 73.363 \\
\hline 9/12/07 2:00 & 124.003 & 121.662 & 118.045 & 99.399 & 103.352 & 95.007 & 108.924 & 105.952 & & 73.361 \\
\hline 9/12/07 2:30 & 124.001 & 121.658 & 118.042 & 99.396 & 103.346 & 95 & 108.924 & 105.945 & & 73.357 \\
\hline 9/12/07 3:00 & 123.998 & 121.644 & 118.032 & 99.396 & 103.348 & 95.005 & 108.923 & 105.947 & & 73.359 \\
\hline 9/12/07 3:30 & 123.994 & 121.637 & 118.019 & 99.394 & 103.344 & 95.003 & 108.923 & 105.943 & & 73.354 \\
\hline 9/12/07 4:00 & 123.994 & 121.639 & 118.017 & 99.382 & 103.335 & 94.988 & 108.923 & 105.934 & & 73.344 \\
\hline 9/12/07 4:30 & 123.989 & 121.635 & 118.014 & 99.375 & 103.325 & 94.979 & 108.921 & 105.923 & & 73.334 \\
\hline 9/12/07 5:00 & 123.987 & 121.628 & 118.014 & 99.375 & 103.327 & 94.981 & 108.921 & 105.925 & & 73.336 \\
\hline 9/12/07 5:30 & 123.984 & 123.165 & 118.007 & 99.372 & 103.325 & 94.977 & 108.917 & 105.923 & & 73.334 \\
\hline 9/12/07 6:00 & 123.979 & 123.766 & 119.486 & 99.366 & 103.319 & 94.972 & 108.917 & 105.918 & & 73.328 \\
\hline 9/12/07 6:30 & 125.128 & 123.954 & 119.703 & 99.845 & 103.441 & 95.189 & 109.157 & 106.559 & & 73.323 \\
\hline 9/12/07 7:00 & 125.759 & 124.059 & 119.834 & 100.24 & 103.969 & 95.762 & 109.687 & 107.566 & & 73.503 \\
\hline 9/12/07 7:30 & 125.927 & 124.131 & 119.907 & 100.411 & 104.161 & 95.96 & 109.871 & 107.783 & & 73.679 \\
\hline 9/12/07 8:00 & 126.034 & 122.465 & 119.151 & 100.502 & 104.268 & 96.066 & 109.97 & 107.902 & & 73.791 \\
\hline 9/12/07 8:30 & 126.114 & 122.061 & 118.483 & 100.56 & 104.334 & 96.134 & 110.036 & 107.971 & & 73.861 \\
\hline 9/12/07 9:00 & 124.797 & 121.918 & 118.309 & 100.013 & 104.094 & 95.762 & 109.664 & 106.913 & & 73.892 \\
\hline 9/12/07 9:30 & 124.419 & 121.834 & 118.216 & 99.73 & 103.727 & 95.38 & 109.298 & 106.377 & & 73.746 \\
\hline 9/12/07 10:00 & 124.291 & 121.775 & 118.166 & 99.609 & 103.588 & 95.241 & 109.165 & 106.215 & & 73.623 \\
\hline 9/12/07 10:30 & 124.225 & 121.739 & 118.128 & 99.54 & 103.504 & 95.158 & 109.092 & 106.125 & & 73.536 \\
\hline 9/12/07 11:00 & 124.197 & 121.714 & 118.097 & 99.491 & 103.451 & 95.104 & 109.048 & 106.069 & & 73.476 \\
\hline 9/12/07 11:30 & 124.152 & 121.687 & 118.072 & 99.461 & 103.415 & 95.073 & 109.02 & 106.029 & & 73.438 \\
\hline 9/12/07 12:00 & 124.076 & 121.669 & 118.055 & 99.434 & 103.392 & 95.047 & 109.003 & 106.003 & & 73.407 \\
\hline 9/12/07 12:30 & 124.041 & 121.651 & 118.034 & 99.408 & 103.363 & 95.021 & 108.99 & 105.974 & & 73.38 \\
\hline 9/12/07 13:00 & 124.022 & 121.628 & 118.012 & 99.392 & 103.346 & 95.005 & 108.979 & 105.954 & & 73.361 \\
\hline 9/12/07 13:30 & 124.003 & 121.608 & 117.994 & 99.375 & 103.329 & 94.989 & 108.971 & 105.936 & & 73.343 \\
\hline 9/12/07 14:00 & 123.984 & 121.585 & 117.974 & 99.354 & 103.308 & 94.965 & 108.97 & 105.914 & & 73.318 \\
\hline 9/12/07 14:30 & 123.963 & 121.569 & 117.951 & 99.334 & 103.287 & 94.948 & 108.962 & 105.894 & & 73.301 \\
\hline 9/12/07 15:00 & 123.942 & 121.551 & 117.936 & 99.311 & 103.27 & 94.927 & 108.96 & 105.872 & & 73.28 \\
\hline 9/12/07 15:30 & 123.925 & 121.533 & 117.921 & 99.294 & 103.247 & 94.906 & 108.956 & 105.855 & & 73.262 \\
\hline 9/12/07 16:00 & 123.909 & 121.521 & 117.906 & 99.277 & 103.232 & 94.89 & 108.955 & 105.837 & & 73.245 \\
\hline 9/12/07 16:30 & 123.89 & 121.515 & 117.896 & 99.263 & 103.219 & 94.875 & 108.949 & 105.821 & & 73.231 \\
\hline 9/12/07 17:00 & 123.873 & 121.508 & 117.893 & 99.251 & 103.205 & 94.863 & 108.947 & 105.806 & & 73.218 \\
\hline 9/12/07 17:30 & 123.864 & 121.499 & 117.881 & 99.246 & 103.2 & 94.854 & 108.943 & 105.799 & & 73.212 \\
\hline 9/12/07 18:00 & 123.859 & 121.492 & 117.873 & 99.241 & 103.194 & 94.849 & 108.941 & 105.793 & & 73.206 \\
\hline 9/12/07 18:30 & 123.849 & 121.485 & 117.868 & 99.23 & 103.184 & 94.84 & 108.939 & 105.786 & & 73.195 \\
\hline 9/12/07 19:00 & 123.842 & 121.485 & 117.868 & 99.223 & 103.177 & 94.83 & 108.936 & 105.777 & & 73.19 \\
\hline 9/12/07 19:30 & 123.835 & 121.483 & 117.863 & 99.213 & 103.169 & 94.826 & 108.934 & 105.77 & & 73.183 \\
\hline 9/12/07 20:00 & 123.833 & 121.496 & 117.873 & 99.249 & 103.171 & 94.826 & 108.932 & 105.773 & & 73.183 \\
\hline 9/12/07 20:30 & 123.833 & 121.501 & 117.878 & 99.222 & 103.171 & 94.828 & 108.932 & 105.77 & & 73.183 \\
\hline 9/12/07 21:00 & 123.842 & 121.503 & 117.888 & 99.234 & 103.184 & 94.835 & 108.918 & 105.782 & & 73.194 \\
\hline 9/12/07 21:30 & 123.849 & 121.508 & 117.891 & 99.236 & 103.188 & 94.842 & 108.912 & 105.786 & & 73.196 \\
\hline 9/12/07 22:00 & 123.854 & 121.508 & 117.888 & 99.239 & 103.192 & 94.845 & 108.908 & 105.788 & & 73.2 \\
\hline 9/12/07 22:30 & 123.857 & 121.51 & 117.891 & 99.239 & 103.192 & 94.849 & 108.906 & 105.79 & & 73.2 \\
\hline 9/12/07 23:00 & 123.854 & 121.508 & 117.888 & 99.242 & 103.192 & 94.847 & 108.904 & 105.793 & & 73.2 \\
\hline 9/12/07 23:30 & 123.859 & 121.503 & 117.886 & 99.242 & 103.194 & 94.849 & 108.904 & 105.794 & & 73.202 \\
\hline 9/13/07 0:00 & 123.854 & 121.499 & 117.883 & 99.239 & 103.188 & 94.845 & 108.9 & 105.79 & & 73.196 \\
\hline 9/13/07 0:30 & 123.852 & 121.496 & 117.873 & 99.236 & 103.188 & 94.84 & 108.9 & 105.786 & & 73.194 \\
\hline 9/13/07 1:00 & 123.849 & 121.494 & 117.876 & 99.232 & 103.182 & 94.838 & 108.9 & 105.782 & & 73.19 \\
\hline 9/13/07 1:30 & 123.847 & 121.494 & 117.871 & 99.227 & 103.179 & 94.833 & 108.9 & 105.779 & & 73.185 \\
\hline 9/13/07 2:00 & 123.842 & 121.492 & 117.871 & 99.227 & 103.179 & 94.833 & 108.898 & 105.777 & & 73.185 \\
\hline
\end{tabular}


TABLE S1.1 (Cont.)

Depth from Top of Casing (ft)

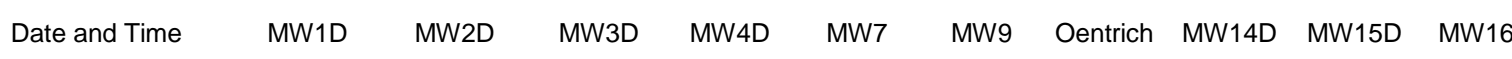

\begin{tabular}{|c|c|c|c|c|c|c|c|c|c|}
\hline 9/13/07 2:30 & 123.842 & 121.49 & 117.871 & 99.229 & 103.179 & 94.83 & 108.894 & 105.775 & 73.185 \\
\hline 9/13/07 3:00 & 123.84 & 121.487 & 117.868 & 99.225 & 103.175 & 94.83 & 108.894 & 105.775 & 73.181 \\
\hline 9/13/07 3:30 & 123.835 & 121.485 & 117.868 & 99.223 & 103.173 & 94.828 & 108.893 & 105.772 & 73.179 \\
\hline 9/13/07 4:00 & 123.833 & 121.485 & 117.871 & 99.22 & 103.175 & 94.828 & 108.893 & 105.77 & 73.177 \\
\hline 9/13/07 4:30 & 123.831 & 121.487 & 117.873 & 99.218 & 103.173 & 94.826 & 108.891 & 105.768 & 73.177 \\
\hline 9/13/07 5:00 & 123.828 & 121.483 & 117.868 & 99.22 & 103.171 & 94.826 & 108.889 & 105.768 & 73.177 \\
\hline 9/13/07 5:30 & 123.826 & 123.483 & 119.342 & 99.222 & 103.175 & 94.83 & 108.889 & 105.772 & 73.179 \\
\hline 9/13/07 6:00 & 123.826 & 123.73 & 119.629 & 99.284 & 103.171 & 94.828 & 108.885 & 105.768 & 73.177 \\
\hline $9 / 13 / 076: 30$ & 125.518 & 123.857 & 119.768 & 100.054 & 103.754 & 95.564 & 109.615 & 107.273 & 73.287 \\
\hline 9/13/07 7:00 & 125.747 & 123.939 & 119.856 & 100.256 & 103.99 & 95.802 & 109.843 & 107.544 & 73.475 \\
\hline 9/13/07 7:30 & 125.866 & 123.993 & 119.919 & 100.369 & 104.117 & 95.929 & 109.963 & 107.683 & 73.605 \\
\hline 9/13/07 8:00 & 125.946 & 124.027 & 119.957 & 100.445 & 104.197 & 96.009 & 110.036 & 107.77 & 73.694 \\
\hline 9/13/07 8:30 & 126.007 & 124.056 & 119.987 & 100.493 & 104.254 & 96.063 & 110.085 & 107.825 & 73.752 \\
\hline 9/13/07 9:00 & 126.06 & 124.084 & 120.007 & 100.524 & 104.287 & 96.099 & 110.12 & 107.864 & 73.789 \\
\hline 9/13/07 9:30 & 126.1 & 124.106 & 120.04 & 100.548 & 104.309 & 96.125 & 110.147 & 107.889 & 73.813 \\
\hline 9/13/07 10:00 & 126.13 & 124.12 & 120.055 & 100.576 & 104.34 & 96.15 & 110.169 & 107.918 & 73.845 \\
\hline 9/13/07 10:30 & 126.166 & 124.122 & 120.055 & 100.598 & 104.365 & 96.179 & 110.184 & 107.946 & 73.871 \\
\hline 9/13/07 11:00 & 126.284 & 124.131 & 120.068 & 100.609 & 104.378 & 96.188 & 110.199 & 107.957 & 73.884 \\
\hline $9 / 13 / 0711: 30$ & 126.542 & 124.127 & 120.065 & 100.61 & 104.378 & 96.193 & 110.211 & 107.964 & 73.888 \\
\hline $9 / 13 / 07$ 12:00 & 126.577 & 123.424 & 120.065 & 100.616 & 104.389 & 96.202 & 110.22 & 107.971 & 73.899 \\
\hline $9 / 13 / 0712: 30$ & 126.572 & 122.136 & 118.581 & 100.617 & 104.384 & 96.195 & 110.229 & 107.967 & 73.896 \\
\hline 9/13/07 13:00 & 126.577 & 121.923 & 118.332 & 100.247 & 104.389 & 96.2 & 110.239 & 107.971 & 73.903 \\
\hline 9/13/07 13:30 & 124.552 & 121.805 & 118.201 & 99.764 & 103.778 & 95.434 & 109.48 & 106.459 & 73.772 \\
\hline $9 / 13 / 0714: 00$ & 124.296 & 121.728 & 118.11 & 99.59 & 103.577 & 95.231 & 109.287 & 106.226 & 73.613 \\
\hline $9 / 13 / 0714: 30$ & 124.169 & 121.671 & 118.062 & 99.489 & 103.46 & 95.116 & 109.187 & 106.098 & 73.498 \\
\hline $9 / 13 / 07$ 15:00 & 124.083 & 121.635 & 118.022 & 99.422 & 103.384 & 95.043 & 109.125 & 106.014 & 73.417 \\
\hline $9 / 13 / 0715: 30$ & 124.031 & 121.608 & 117.994 & 99.377 & 103.339 & 94.995 & 109.082 & 105.958 & 73.363 \\
\hline $9 / 13 / 07$ 16:00 & 123.994 & 121.605 & 117.989 & 99.344 & 103.3 & 94.958 & 109.052 & 105.919 & 73.324 \\
\hline $9 / 13 / 0716: 30$ & 123.961 & 121.601 & 117.987 & 99.325 & 103.276 & 94.934 & 109.03 & 105.892 & 73.297 \\
\hline 9/13/07 17:00 & 123.956 & 121.601 & 117.979 & 99.327 & 103.28 & 94.939 & 109.011 & 105.887 & 73.297 \\
\hline $9 / 13 / 07$ 17:30 & 123.951 & 121.598 & 117.984 & 99.323 & 103.276 & 94.934 & 108.998 & 105.883 & 73.287 \\
\hline $9 / 13 / 07$ 18:00 & 123.949 & 121.605 & 117.989 & 99.325 & 103.274 & 94.929 & 108.99 & 105.881 & 73.285 \\
\hline $9 / 13 / 07$ 18:30 & 123.953 & 121.608 & 117.992 & 99.329 & 103.278 & 94.932 & 108.981 & 105.883 & 73.286 \\
\hline 9/13/07 19:00 & 123.956 & 121.619 & 117.994 & 99.334 & 103.287 & 94.939 & 108.97 & 105.89 & 73.295 \\
\hline 9/13/07 19:30 & 123.958 & 121.628 & 118.011 & 99.339 & 103.289 & 94.944 & 108.964 & 105.89 & 73.295 \\
\hline $9 / 13 / 0720: 00$ & 123.965 & 121.642 & 118.022 & 99.351 & 103.299 & 94.953 & 108.96 & 105.901 & 73.305 \\
\hline $9 / 13 / 0720: 30$ & 123.977 & 121.657 & 118.037 & 99.363 & 103.312 & 94.965 & 108.955 & 105.912 & 73.316 \\
\hline 9/13/07 21:00 & 123.989 & 121.664 & 118.045 & 99.377 & 103.323 & 94.979 & 108.949 & 105.925 & 73.326 \\
\hline $9 / 13 / 0721: 30$ & 124.003 & 121.676 & 118.057 & 99.391 & 103.339 & 94.993 & 108.947 & 105.938 & 73.338 \\
\hline $9 / 13 / 0722: 00$ & 124.013 & 121.682 & 118.065 & 99.396 & 103.348 & 95.005 & 108.943 & 105.949 & 73.347 \\
\hline 9/13/07 22:30 & 124.024 & 121.696 & 118.08 & 99.411 & 103.359 & 95.017 & 108.943 & 105.961 & 73.359 \\
\hline 9/13/07 23:00 & 124.031 & 121.712 & 118.097 & 99.418 & 103.363 & 95.021 & 108.941 & 105.965 & 73.365 \\
\hline 9/13/07 23:30 & 124.041 & 121.725 & 118.11 & 99.435 & 103.38 & 95.038 & 108.938 & 105.981 & 73.38 \\
\hline $9 / 14 / 070: 00$ & 124.062 & 121.739 & 118.123 & 99.447 & 103.396 & 95.054 & 108.936 & 105.996 & 73.394 \\
\hline 9/14/07 0:30 & 124.072 & 121.753 & 118.135 & 99.463 & 103.409 & 95.069 & 108.934 & 106.012 & 73.407 \\
\hline 9/14/07 1:00 & 124.081 & 121.762 & 118.14 & 99.477 & 103.422 & 95.083 & 108.932 & 106.023 & 73.419 \\
\hline 9/14/07 1:30 & 124.083 & 121.764 & 118.14 & 99.494 & 103.436 & 95.097 & 108.932 & 106.038 & 73.431 \\
\hline $9 / 14 / 072: 00$ & 124.081 & 121.759 & 118.143 & 99.499 & 103.441 & 95.102 & 108.932 & 106.045 & 73.438 \\
\hline $9 / 14 / 072: 30$ & 124.079 & 121.769 & 118.15 & 99.496 & 103.447 & 95.106 & 108.932 & 106.047 & 73.442 \\
\hline 9/14/07 3:00 & 124.081 & 121.78 & 118.157 & 99.503 & 103.432 & 95.092 & 108.934 & 106.036 & 73.427 \\
\hline 9/14/07 3:30 & 124.074 & 121.78 & 118.16 & 99.506 & 103.451 & 95.109 & 108.93 & 106.049 & 73.442 \\
\hline $9 / 14 / 074: 00$ & 124.072 & 121.791 & 118.173 & 99.516 & 103.464 & 95.123 & 108.928 & 106.063 & 73.458 \\
\hline 9/14/07 4:30 & 124.069 & 121.798 & 118.181 & 99.522 & 103.466 & 95.125 & 108.928 & 106.065 & 73.458 \\
\hline 9/14/07 5:00 & 124.069 & 121.805 & 118.188 & 99.527 & 103.476 & 95.137 & 108.928 & 106.076 & 73.469 \\
\hline 9/14/07 5:30 & 124.065 & 123.392 & 118.206 & 99.532 & 103.479 & 95.139 & 108.928 & 106.08 & 73.473 \\
\hline 9/14/07 6:00 & 124.062 & 123.929 & 119.813 & 99.549 & 103.489 & 95.149 & 108.926 & 106.089 & 73.483 \\
\hline 9/14/07 6:30 & 125.303 & 124.131 & 120.043 & 100.119 & 103.702 & 95.483 & 109.253 & 106.912 & 73.504 \\
\hline $9 / 14 / 07$ 7:00 & 125.844 & 124.256 & 120.181 & 100.492 & 104.199 & 96.011 & 109.758 & 107.734 & 73.688 \\
\hline $9 / 14 / 077: 30$ & 126.026 & 124.342 & 120.265 & 100.667 & 104.397 & 96.212 & 109.94 & 107.951 & 73.87 \\
\hline 9/14/07 8:00 & 126.131 & 124.394 & 120.325 & 100.781 & 104.528 & 96.344 & 110.051 & 108.095 & 74.008 \\
\hline 9/14/07 8:30 & 126.204 & 124.444 & 120.353 & 100.857 & 104.61 & 96.426 & 110.126 & 108.177 & 74.095 \\
\hline 9/14/07 9:00 & 126.267 & 124.474 & 120.413 & 100.905 & 104.662 & 96.478 & 110.179 & 108.234 & 74.151 \\
\hline 9/14/07 9:30 & 126.315 & 124.514 & 120.451 & 100.957 & 104.713 & 96.528 & 110.216 & 108.283 & 74.202 \\
\hline 9/14/07 10:00 & 126.362 & 124.537 & 120.474 & 100.984 & 104.738 & 96.556 & 110.254 & 108.314 & 74.229 \\
\hline $9 / 14 / 07 \quad 10: 30$ & 126.4 & 124.542 & 120.484 & 101.024 & 104.784 & 96.598 & 110.282 & 108.36 & 74.273 \\
\hline $9 / 14 / 0711: 00$ & 126.442 & 122.827 & 119.387 & 101.039 & 104.803 & 96.615 & 110.312 & 108.381 & 74.293 \\
\hline
\end{tabular}


TABLE S1.1 (Cont.)

\begin{tabular}{|c|c|c|c|c|c|c|c|c|c|c|}
\hline \multirow[b]{2}{*}{ Date and Time } & \multicolumn{10}{|c|}{ Depth from Top of Casing (ft) } \\
\hline & MW1D & MW2D & MW3D & MW4D & MW7 & MW9 & Oentrich & MW14D & MW15D & MW16D \\
\hline 9/14/07 11:30 & 126.549 & 122.451 & 118.874 & 101.045 & 104.806 & 96.62 & 110.331 & 108.385 & & 74.302 \\
\hline $9 / 14 / 07$ 12:00 & 125.329 & 122.295 & 118.697 & 100.378 & 104.441 & 96.113 & 109.82 & 107.214 & & 74.285 \\
\hline 9/14/07 12:30 & 124.847 & 122.206 & 118.598 & 100.107 & 104.094 & 95.757 & 109.491 & 106.762 & & 74.105 \\
\hline 9/14/07 13:00 & 124.667 & 122.138 & 118.531 & 99.975 & 103.948 & 95.606 & 109.353 & 106.594 & & 73.973 \\
\hline $9 / 14 / 07$ 13:30 & 124.566 & 122.088 & 118.48 & 99.899 & 103.858 & 95.521 & 109.276 & 106.497 & & 73.88 \\
\hline 9/14/07 14:00 & 124.502 & 122.052 & 118.43 & 99.839 & 103.797 & 95.462 & 109.23 & 106.428 & & 73.814 \\
\hline $9 / 14 / 0714: 30$ & 124.455 & 122.025 & 118.41 & 99.795 & 103.75 & 95.413 & 109.199 & 106.377 & & 73.762 \\
\hline 9/14/07 15:00 & 124.417 & 122.002 & 118.39 & 99.764 & 103.715 & 95.377 & 109.176 & 106.339 & & 73.723 \\
\hline 9/14/07 15:30 & 124.381 & 121.986 & 118.369 & 99.742 & 103.689 & 95.351 & 109.159 & 106.311 & & 73.696 \\
\hline 9/14/07 16:00 & 124.36 & 121.975 & 118.36 & 99.723 & 103.668 & 95.33 & 109.146 & 106.288 & & 73.675 \\
\hline 9/14/07 16:30 & 124.341 & 121.961 & 118.349 & 99.706 & 103.655 & 95.314 & 109.135 & 106.27 & & 73.658 \\
\hline 9/14/07 17:00 & 124.327 & 121.954 & 118.342 & 99.701 & 103.647 & 95.309 & 109.127 & 106.26 & & 73.648 \\
\hline 9/14/07 17:30 & 124.32 & 121.952 & 118.337 & 99.685 & 103.632 & 95.295 & 109.12 & 106.249 & & 73.638 \\
\hline 9/14/07 18:00 & 124.306 & 121.948 & 118.331 & 99.682 & 103.628 & 95.288 & 109.112 & 106.24 & & 73.632 \\
\hline 9/14/07 18:30 & 124.303 & 121.941 & 118.324 & 99.677 & 103.626 & 95.285 & 109.11 & 106.238 & & 73.628 \\
\hline 9/14/07 19:00 & 124.299 & 121.939 & 118.322 & 99.677 & 103.62 & 95.281 & 109.105 & 106.233 & & 73.624 \\
\hline 9/14/07 19:30 & 124.294 & 121.945 & 118.327 & 99.67 & 103.615 & 95.274 & 109.101 & 106.226 & & 73.615 \\
\hline 9/14/07 20:00 & 124.289 & 121.952 & 118.329 & 99.668 & 103.611 & 95.271 & 109.097 & 106.222 & & 73.613 \\
\hline 9/14/07 20:30 & 124.291 & 121.959 & 118.324 & 99.677 & 103.618 & 95.278 & 109.093 & 106.229 & & 73.624 \\
\hline 9/14/07 21:00 & 124.294 & 121.964 & 118.329 & 99.685 & 103.63 & 95.288 & 109.092 & 106.235 & & 73.629 \\
\hline 9/14/07 21:30 & 124.289 & 121.97 & 118.337 & 99.692 & 103.632 & 95.292 & 109.088 & 106.242 & & 73.634 \\
\hline 9/14/07 22:00 & 124.287 & 121.97 & 118.339 & 99.692 & 103.637 & 95.297 & 109.088 & 106.246 & & 73.636 \\
\hline 9/14/07 22:30 & 124.28 & 121.968 & 118.352 & 99.699 & 103.645 & 95.304 & 109.086 & 106.253 & & 73.644 \\
\hline 9/14/07 23:00 & 124.28 & 121.975 & 118.354 & 99.699 & 103.645 & 95.304 & 109.088 & 106.255 & & 73.644 \\
\hline 9/14/07 23:30 & 124.277 & 121.973 & 118.354 & 99.699 & 103.643 & 95.302 & 109.088 & 106.251 & & 73.64 \\
\hline 9/15/07 0:00 & 124.273 & 121.968 & 118.352 & 99.706 & 103.647 & 95.309 & 109.086 & 106.255 & & 73.648 \\
\hline 9/15/07 0:30 & 124.275 & 121.964 & 118.344 & 99.701 & 103.649 & 95.307 & 109.088 & 106.257 & & 73.644 \\
\hline 9/15/07 1:00 & 124.27 & 121.966 & 118.344 & 99.696 & 103.643 & 95.304 & 109.088 & 106.251 & & 73.642 \\
\hline 9/15/07 1:30 & 124.27 & 121.964 & 118.341 & 99.697 & 103.639 & 95.3 & 109.088 & 106.246 & & 73.638 \\
\hline 9/15/07 2:00 & 124.268 & 121.968 & 118.354 & 99.694 & 103.639 & 95.3 & 109.088 & 106.251 & & 73.64 \\
\hline 9/15/07 2:30 & 124.265 & 121.957 & 118.344 & 99.696 & 103.636 & 95.292 & 109.088 & 106.244 & & 73.634 \\
\hline 9/15/07 3:00 & 124.261 & 121.954 & 118.334 & 99.701 & 103.643 & 95.304 & 109.088 & 106.253 & & 73.644 \\
\hline 9/15/07 3:30 & 124.263 & 121.948 & 118.334 & 99.694 & 103.634 & 95.3 & 109.09 & 106.244 & & 73.636 \\
\hline 9/15/07 4:00 & 124.261 & 121.934 & 118.314 & 99.689 & 103.628 & 95.29 & 109.09 & 106.238 & & 73.629 \\
\hline 9/15/07 4:30 & 124.261 & 121.925 & 118.304 & 99.668 & 103.624 & 95.285 & 109.09 & 106.233 & & 73.624 \\
\hline 9/15/07 5:00 & 124.258 & 121.93 & 118.332 & 99.661 & 103.605 & 95.267 & 109.088 & 106.213 & & 73.607 \\
\hline 9/15/07 5:30 & 124.254 & 121.916 & 118.297 & 99.671 & 103.599 & 95.257 & 109.088 & 106.204 & & 73.598 \\
\hline 9/15/07 6:00 & 124.251 & 121.93 & 118.309 & 99.644 & 103.616 & 95.278 & 109.084 & 106.226 & & 73.622 \\
\hline 9/15/07 6:30 & 124.254 & 123.682 & 119.138 & 99.652 & 103.59 & 95.25 & 109.088 & 106.198 & & 73.595 \\
\hline 9/15/07 7:00 & 124.247 & 124.131 & 119.861 & 99.661 & 103.607 & 95.267 & 109.08 & 106.211 & & 73.607 \\
\hline 9/15/07 7:30 & 125.624 & 124.333 & 120.083 & 100.274 & 103.906 & 95.686 & 109.471 & 107.338 & & 73.648 \\
\hline 9/15/07 8:00 & 126.062 & 124.403 & 120.159 & 100.595 & 104.325 & 96.125 & 109.891 & 107.935 & & 73.839 \\
\hline 9/15/07 8:30 & 126.22 & 124.467 & 120.212 & 100.772 & 104.527 & 96.327 & 110.053 & 108.153 & & 74.031 \\
\hline 9/15/07 9:00 & 126.324 & 124.524 & 120.302 & 100.824 & 104.591 & 96.391 & 110.16 & 108.23 & & 74.099 \\
\hline 9/15/07 9:30 & 126.391 & 124.537 & 120.322 & 100.893 & 104.66 & 96.457 & 110.218 & 108.296 & & 74.173 \\
\hline 9/15/07 10:00 & 126.452 & 122.762 & 119.312 & 100.941 & 104.713 & 96.516 & 110.263 & 108.363 & & 74.236 \\
\hline 9/15/07 10:30 & 126.501 & 122.392 & 118.808 & 100.948 & 104.728 & 96.532 & 110.297 & 108.381 & & 74.254 \\
\hline 9/15/07 11:00 & 125.069 & 122.222 & 118.624 & 100.323 & 104.378 & 96.047 & 109.818 & 107.145 & & 74.239 \\
\hline 9/15/07 11:30 & 124.752 & 122.122 & 118.516 & 100.049 & 104.033 & 95.691 & 109.503 & 106.698 & & 74.055 \\
\hline 9/15/07 12:00 & 124.627 & 122.057 & 118.448 & 99.906 & 103.875 & 95.533 & 109.373 & 106.523 & & 73.913 \\
\hline 9/15/07 12:30 & 124.559 & 121.998 & 118.387 & 99.816 & 103.773 & 95.434 & 109.302 & 106.412 & & 73.806 \\
\hline 9/15/07 13:00 & 124.514 & 121.957 & 118.344 & 99.754 & 103.714 & 95.373 & 109.257 & 106.346 & & 73.739 \\
\hline 9/15/07 13:30 & 124.485 & 121.918 & 118.302 & 99.706 & 103.656 & 95.316 & 109.227 & 106.284 & & 73.679 \\
\hline 9/15/07 14:00 & 124.457 & 121.889 & 118.269 & 99.663 & 103.62 & 95.281 & 109.206 & 106.242 & & 73.64 \\
\hline 9/15/07 14:30 & 124.44 & 121.88 & 118.261 & 99.632 & 103.577 & 95.238 & 109.191 & 106.2 & & 73.597 \\
\hline 9/15/07 15:00 & 124.421 & 121.873 & 118.249 & 99.606 & 103.55 & 95.212 & 109.18 & 106.171 & & 73.567 \\
\hline 9/15/07 15:30 & 124.405 & 121.866 & 118.233 & 99.603 & 103.548 & 95.21 & 109.169 & 106.169 & & 73.568 \\
\hline 9/15/07 16:00 & 124.391 & 121.861 & 118.211 & 99.587 & 103.531 & 95.191 & 109.161 & 106.147 & & 73.547 \\
\hline 9/15/07 16:30 & 124.377 & 121.828 & 118.187 & 99.575 & 103.521 & 95.182 & 109.152 & 106.138 & & 73.537 \\
\hline 9/15/07 17:00 & 124.362 & 121.825 & 118.176 & 99.563 & 103.514 & 95.172 & 109.146 & 106.125 & & 73.529 \\
\hline 9/15/07 17:30 & 124.358 & 121.823 & 118.178 & 99.542 & 103.493 & 95.151 & 109.144 & 106.105 & & 73.506 \\
\hline 9/15/07 18:00 & 124.346 & 121.787 & 118.166 & 99.53 & 103.478 & 95.137 & 109.14 & 106.089 & & 73.493 \\
\hline 9/15/07 18:30 & 124.334 & 121.782 & 118.153 & 99.515 & 103.464 & 95.125 & 109.137 & 106.078 & & 73.479 \\
\hline 9/15/07 19:00 & 124.325 & 121.78 & 118.143 & 99.501 & 103.449 & 95.109 & 109.135 & 106.06 & & 73.464 \\
\hline 9/15/07 19:30 & 124.315 & 121.778 & 118.135 & 99.492 & 103.438 & 95.097 & 109.131 & 106.049 & & 73.454 \\
\hline 9/15/07 20:00 & 124.301 & 121.773 & 118.135 & 99.484 & 103.43 & 95.09 & 109.127 & 106.043 & & 73.446 \\
\hline
\end{tabular}


TABLE S1.1 (Cont.)

\begin{tabular}{|c|c|c|c|c|c|c|c|c|c|c|}
\hline \multirow[b]{2}{*}{ Date and Time } & \multicolumn{10}{|c|}{ Depth from Top of Casing (ft) } \\
\hline & MW1D & MW2D & MW3D & MW4D & MW7 & MW9 & Oentrich & MW14D & MW15D & MW16D \\
\hline 9/15/07 20:30 & 124.289 & 121.771 & 118.135 & 99.478 & 103.424 & 95.085 & 109.123 & 106.036 & & 73.439 \\
\hline 9/15/07 21:00 & 124.277 & 121.771 & 118.135 & 99.48 & 103.428 & 95.087 & 109.122 & 106.036 & & 73.442 \\
\hline 9/15/07 21:30 & 124.268 & 121.769 & 118.137 & 99.482 & 103.428 & 95.085 & 109.118 & 106.036 & & 73.44 \\
\hline 9/15/07 22:00 & 124.258 & 121.746 & 118.125 & 99.482 & 103.42 & 95.08 & 109.118 & 106.032 & & 73.436 \\
\hline 9/15/07 22:30 & 124.251 & 121.748 & 118.12 & 99.478 & 103.424 & 95.083 & 109.114 & 106.034 & & 73.438 \\
\hline 9/15/07 23:00 & 124.244 & 121.746 & 118.107 & 99.475 & 103.417 & 95.076 & 109.114 & 106.025 & & 73.427 \\
\hline 9/15/07 23:30 & 124.239 & 121.712 & 118.1 & 99.456 & 103.403 & 95.061 & 109.114 & 106.014 & & 73.413 \\
\hline 9/16/07 0:00 & 124.232 & 121.71 & 118.09 & 99.451 & 103.396 & 95.057 & 109.112 & 106.007 & & 73.408 \\
\hline 9/16/07 0:30 & 124.228 & 121.712 & 118.082 & 99.437 & 103.386 & 95.045 & 109.11 & 105.996 & & 73.396 \\
\hline 9/16/07 1:00 & 124.216 & 121.71 & 118.079 & 99.435 & 103.38 & 95.04 & 109.108 & 105.99 & & 73.392 \\
\hline 9/16/07 1:30 & 124.209 & 121.71 & 118.07 & 99.427 & 103.373 & 95.031 & 109.108 & 105.981 & & 73.384 \\
\hline 9/16/07 2:00 & 124.202 & 121.678 & 118.06 & 99.423 & 103.367 & 95.026 & 109.105 & 105.976 & & 73.378 \\
\hline 9/16/07 2:30 & 124.197 & 121.667 & 118.05 & 99.413 & 103.359 & 95.019 & 109.103 & 105.969 & & 73.371 \\
\hline 9/16/07 3:00 & 124.19 & 121.664 & 118.037 & 99.399 & 103.35 & 95.01 & 109.103 & 105.961 & & 73.361 \\
\hline 9/16/07 3:30 & 124.18 & 121.664 & 118.019 & 99.389 & 103.339 & 94.998 & 109.101 & 105.948 & & 73.348 \\
\hline 9/16/07 4:00 & 124.176 & 121.662 & 118.029 & 99.382 & 103.329 & 94.988 & 109.099 & 105.934 & & 73.34 \\
\hline 9/16/07 4:30 & 124.166 & 121.66 & 118.024 & 99.38 & 103.325 & 94.984 & 109.097 & 105.932 & & 73.338 \\
\hline 9/16/07 5:00 & 124.159 & 121.66 & 118.019 & 99.373 & 103.318 & 94.974 & 109.097 & 105.925 & & 73.33 \\
\hline 9/16/07 5:30 & 124.152 & 121.655 & 118.014 & 99.365 & 103.312 & 94.97 & 109.093 & 105.919 & & 73.324 \\
\hline 9/16/07 6:00 & 124.147 & 121.657 & 118.017 & 99.361 & 103.306 & 94.962 & 109.092 & 105.912 & & 73.317 \\
\hline 9/16/07 6:30 & 124.138 & 123.564 & 119.221 & 99.366 & 103.312 & 94.97 & 109.088 & 105.916 & & 73.322 \\
\hline 9/16/07 7:00 & 124.131 & 123.868 & 119.607 & 99.365 & 103.312 & 94.967 & 109.084 & 105.917 & & 73.321 \\
\hline $9 / 16 / 077: 30$ & 125.717 & 124.007 & 119.766 & 100.069 & 103.757 & 95.552 & 109.662 & 107.32 & & 73.367 \\
\hline 9/16/07 8:00 & 126.005 & 124.09 & 119.856 & 100.311 & 104.051 & 95.853 & 109.951 & 107.676 & & 73.568 \\
\hline 9/16/07 8:30 & 126.142 & 124.138 & 119.919 & 100.433 & 104.191 & 95.993 & 110.09 & 107.829 & & 73.706 \\
\hline 9/16/07 9:00 & 126.227 & 124.19 & 119.965 & 100.511 & 104.279 & 96.077 & 110.171 & 107.922 & & 73.799 \\
\hline 9/16/07 9:30 & 126.287 & 124.22 & 119.995 & 100.559 & 104.327 & 96.129 & 110.224 & 107.973 & & 73.855 \\
\hline 9/16/07 10:00 & 126.336 & 124.231 & 120.02 & 100.605 & 104.38 & 96.176 & 110.259 & 108.024 & & 73.906 \\
\hline 9/16/07 10:30 & 126.369 & 124.247 & 120.03 & 100.636 & 104.41 & 96.209 & 110.284 & 108.055 & & 73.942 \\
\hline 9/16/07 11:00 & 126.412 & 124.247 & 120.038 & 100.64 & 104.422 & 96.224 & 110.306 & 108.073 & & 73.956 \\
\hline 9/16/07 11:30 & 126.542 & 122.687 & 119.755 & 100.654 & 104.435 & 96.233 & 110.323 & 108.084 & & 73.971 \\
\hline 9/16/07 12:00 & 126.632 & 122.179 & 118.614 & 100.667 & 104.439 & 96.24 & 110.338 & 108.091 & & 73.977 \\
\hline 9/16/07 12:30 & 125.365 & 122.029 & 118.412 & 100.154 & 104.264 & 95.948 & 110.015 & 107.227 & & 73.999 \\
\hline 9/16/07 13:00 & 124.622 & 121.909 & 118.316 & 99.809 & 103.799 & 95.462 & 109.576 & 106.481 & & 73.822 \\
\hline 9/16/07 13:30 & 124.374 & 121.821 & 118.208 & 99.692 & 103.681 & 95.337 & 109.403 & 106.322 & & 73.723 \\
\hline 9/16/07 14:00 & 124.306 & 121.741 & 118.133 & 99.592 & 103.569 & 95.229 & 109.322 & 106.207 & & 73.605 \\
\hline $9 / 16 / 07$ 14:30 & 124.263 & 121.687 & 118.085 & 99.509 & 103.481 & 95.139 & 109.272 & 106.114 & & 73.512 \\
\hline 9/16/07 15:00 & 124.242 & 121.669 & 118.052 & 99.439 & 103.396 & 95.059 & 109.24 & 106.029 & & 73.423 \\
\hline 9/16/07 15:30 & 124.221 & 121.635 & 118.017 & 99.394 & 103.35 & 95.012 & 109.214 & 105.979 & & 73.375 \\
\hline 9/16/07 16:00 & 124.204 & 121.617 & 118.001 & 99.37 & 103.329 & 94.986 & 109.193 & 105.95 & & 73.349 \\
\hline 9/16/07 16:30 & 124.19 & 121.596 & 117.981 & 99.348 & 103.295 & 94.955 & 109.178 & 105.914 & & 73.316 \\
\hline 9/16/07 17:00 & 124.18 & 121.571 & 117.959 & 99.33 & 103.28 & 94.941 & 109.167 & 105.897 & & 73.297 \\
\hline 9/16/07 17:30 & 124.171 & 121.553 & 117.941 & 99.308 & 103.26 & 94.92 & 109.161 & 105.877 & & 73.274 \\
\hline 9/16/07 18:00 & 124.161 & 121.542 & 117.924 & 99.287 & 103.238 & 94.896 & 109.15 & 105.852 & & 73.254 \\
\hline 9/16/07 18:30 & 124.15 & 121.53 & 117.916 & 99.272 & 103.22 & 94.88 & 109.146 & 105.835 & & 73.237 \\
\hline 9/16/07 19:00 & 124.069 & 121.521 & 117.905 & 99.26 & 103.209 & 94.868 & 109.138 & 105.821 & & 73.225 \\
\hline 9/16/07 19:30 & 123.994 & 121.526 & 117.905 & 99.248 & 103.196 & 94.852 & 109.127 & 105.81 & & 73.21 \\
\hline 9/16/07 20:00 & 123.935 & 121.526 & 117.908 & 99.244 & 103.188 & 94.847 & 109.152 & 105.799 & & 73.202 \\
\hline 9/16/07 20:30 & 123.897 & 121.526 & 117.909 & 99.248 & 103.194 & 94.852 & 109.132 & 105.804 & & 73.206 \\
\hline 9/16/07 21:00 & 123.885 & 121.526 & 117.908 & 99.249 & 103.194 & 94.854 & 109.113 & 105.804 & & 73.208 \\
\hline 9/16/07 21:30 & 123.878 & 121.517 & 117.9 & 99.249 & 103.196 & 94.854 & 109.104 & 105.804 & & 73.204 \\
\hline 9/16/07 22:00 & 123.88 & 121.519 & 117.9 & 99.248 & 103.194 & 94.854 & 109.086 & 105.804 & & 73.204 \\
\hline 9/16/07 22:30 & 123.878 & 121.524 & 117.903 & 99.241 & 103.186 & 94.847 & 109.077 & 105.795 & & 73.195 \\
\hline 9/16/07 23:00 & 123.871 & 121.521 & 117.903 & 99.242 & 103.19 & 94.847 & 109.069 & 105.797 & & 73.198 \\
\hline 9/16/07 23:30 & 123.873 & 121.526 & 117.906 & 99.248 & 103.19 & 94.847 & 109.066 & 105.797 & & 73.197 \\
\hline 9/17/07 0:00 & 123.873 & 121.524 & 117.905 & 99.244 & 103.19 & 94.849 & 109.062 & 105.799 & & 73.199 \\
\hline 9/17/07 0:30 & 123.875 & 121.528 & 117.908 & 99.248 & 103.194 & 94.852 & 109.056 & 105.804 & & 73.202 \\
\hline 9/17/07 1:00 & 123.875 & 121.54 & 117.918 & 99.249 & 103.196 & 94.854 & 109.054 & 105.804 & & 73.202 \\
\hline 9/17/07 1:30 & 123.878 & 121.551 & 117.936 & 99.253 & 103.198 & 94.854 & 109.052 & 105.804 & & 73.202 \\
\hline 9/17/07 2:00 & 123.88 & 121.558 & 117.941 & 99.265 & 103.209 & 94.868 & 109.047 & 105.817 & & 73.214 \\
\hline 9/17/07 2:30 & 123.894 & 121.564 & 117.946 & 99.273 & 103.222 & 94.88 & 109.045 & 105.828 & & 73.224 \\
\hline 9/17/07 3:00 & 123.901 & 121.562 & 117.946 & 99.284 & 103.228 & 94.887 & 109.041 & 105.835 & & 73.23 \\
\hline 9/17/07 3:30 & 123.911 & 121.565 & 117.951 & 99.287 & 103.236 & 94.894 & 109.039 & 105.841 & & 73.237 \\
\hline 9/17/07 4:00 & 123.913 & 121.562 & 117.946 & 99.289 & 103.234 & 94.894 & 109.041 & 105.841 & & 73.233 \\
\hline 9/17/07 4:30 & 123.916 & 121.565 & 117.943 & 99.291 & 103.238 & 94.896 & 109.037 & 105.841 & & 73.235 \\
\hline 9/17/07 5:00 & 123.916 & 121.567 & 117.931 & 99.287 & 103.234 & 94.896 & 109.037 & 105.843 & & 73.235 \\
\hline
\end{tabular}


TABLE S1.1 (Cont.)

\begin{tabular}{|c|c|c|c|c|c|c|c|c|c|c|}
\hline \multirow[b]{2}{*}{ Date and Time } & \multicolumn{10}{|c|}{ Depth from Top of Casing (ft) } \\
\hline & MW1D & MW2D & MW3D & MW4D & MW7 & MW9 & Oentrich & MW14D & MW15D & MW16D \\
\hline 9/17/07 5:30 & 123.916 & 123.177 & 118.671 & 99.291 & 103.234 & 94.896 & 109.033 & 105.841 & & 73.233 \\
\hline 9/17/07 6:00 & 123.916 & 123.648 & 119.534 & 99.294 & 103.24 & 94.899 & 109.031 & 105.843 & & 73.235 \\
\hline 9/17/07 6:30 & 125.286 & 123.818 & 119.74 & 99.873 & 103.483 & 95.276 & 109.422 & 106.802 & & 73.237 \\
\hline 9/17/07 7:00 & 125.974 & 123.923 & 119.851 & 100.212 & 103.927 & 95.738 & 109.852 & 107.457 & & 73.427 \\
\hline 9/17/07 7:30 & 126.225 & 123.995 & 119.924 & 100.362 & 104.104 & 95.912 & 110.019 & 107.646 & & 73.59 \\
\hline 9/17/07 8:00 & 126.348 & 124.031 & 119.97 & 100.455 & 104.207 & 96.016 & 110.111 & 107.754 & & 73.7 \\
\hline 9/17/07 8:30 & 126.423 & 124.066 & 120.007 & 100.516 & 104.269 & 96.084 & 110.171 & 107.827 & & 73.77 \\
\hline 9/17/07 9:00 & 126.473 & 124.095 & 120.035 & 100.554 & 104.311 & 96.125 & 110.212 & 107.871 & & 73.815 \\
\hline 9/17/07 9:30 & 126.506 & 124.111 & 120.057 & 100.585 & 104.346 & 96.16 & 110.241 & 107.905 & & 73.851 \\
\hline 9/17/07 10:00 & 126.532 & 124.125 & 120.067 & 100.609 & 104.372 & 96.186 & 110.263 & 107.938 & & 73.882 \\
\hline 9/17/07 10:30 & 126.558 & 122.546 & 119.253 & 100.629 & 104.391 & 96.205 & 110.282 & 107.956 & & 73.9 \\
\hline 9/17/07 11:00 & 126.57 & 122.1 & 118.528 & 100.643 & 104.405 & 96.219 & 110.297 & 107.971 & & 73.917 \\
\hline 9/17/07 11:30 & 125.171 & 121.93 & 118.326 & 100.054 & 104.14 & 95.813 & 109.888 & 106.986 & & 73.915 \\
\hline 9/17/07 12:00 & 124.497 & 121.832 & 118.225 & 99.742 & 103.733 & 95.394 & 109.501 & 106.408 & & 73.744 \\
\hline 9/17/07 12:30 & 124.296 & 121.764 & 118.15 & 99.596 & 103.571 & 95.229 & 109.349 & 106.22 & & 73.599 \\
\hline 9/17/07 13:00 & 124.197 & 121.714 & 118.094 & 99.52 & 103.478 & 95.142 & 109.266 & 106.118 & & 73.504 \\
\hline 9/17/07 13:30 & 124.124 & 121.673 & 118.051 & 99.454 & 103.413 & 95.073 & 109.217 & 106.043 & & 73.431 \\
\hline 9/17/07 14:00 & 124.069 & 121.644 & 118.026 & 99.415 & 103.359 & 95.024 & 109.18 & 105.99 & & 73.371 \\
\hline 9/17/07 14:30 & 124.031 & 121.617 & 118.007 & 99.373 & 103.325 & 94.988 & 109.155 & 105.95 & & 73.334 \\
\hline 9/17/07 15:00 & 123.998 & 121.596 & 117.978 & 99.351 & 103.3 & 94.955 & 109.138 & 105.921 & & 73.307 \\
\hline 9/17/07 15:30 & 123.975 & 121.571 & 117.956 & 99.325 & 103.274 & 94.937 & 109.125 & 105.897 & & 73.283 \\
\hline 9/17/07 16:00 & 123.951 & 121.558 & 117.936 & 99.301 & 103.253 & 94.913 & 109.116 & 105.872 & & 73.258 \\
\hline 9/17/07 16:30 & 123.925 & 121.551 & 117.931 & 99.287 & 103.234 & 94.894 & 109.107 & 105.852 & & 73.237 \\
\hline 9/17/07 17:00 & 123.911 & 121.54 & 117.92 & 99.273 & 103.22 & 94.882 & 109.099 & 105.839 & & 73.227 \\
\hline 9/17/07 17:30 & 123.904 & 121.53 & 117.913 & 99.263 & 103.215 & 94.878 & 109.09 & 105.828 & & 73.221 \\
\hline 9/17/07 18:00 & 123.894 & 121.528 & 117.91 & 99.256 & 103.205 & 94.863 & 109.088 & 105.821 & & 73.204 \\
\hline 9/17/07 18:30 & 123.883 & 121.54 & 117.92 & 99.249 & 103.196 & 94.856 & 109.08 & 105.808 & & 73.194 \\
\hline 9/17/07 19:00 & 123.88 & 121.558 & 117.943 & 99.249 & 103.192 & 94.854 & 109.077 & 105.808 & & 73.195 \\
\hline 9/17/07 19:30 & 123.887 & 121.571 & 117.948 & 99.268 & 103.211 & 94.871 & 109.073 & 105.819 & & 73.21 \\
\hline 9/17/07 20:00 & 123.911 & 121.571 & 117.948 & 99.28 & 103.228 & 94.889 & 109.067 & 105.837 & & 73.227 \\
\hline 9/17/07 20:30 & 123.916 & 121.571 & 117.953 & 99.296 & 103.238 & 94.896 & 109.06 & 105.843 & & 73.235 \\
\hline 9/17/07 21:00 & 123.92 & 121.578 & 117.956 & 99.291 & 103.238 & 94.896 & 109.058 & 105.846 & & 73.233 \\
\hline 9/17/07 21:30 & 123.923 & 121.574 & 117.956 & 99.299 & 103.24 & 94.901 & 109.056 & 105.85 & & 73.235 \\
\hline 9/17/07 22:00 & 123.927 & 121.578 & 117.953 & 99.301 & 103.241 & 94.906 & 109.052 & 105.852 & & 73.239 \\
\hline 9/17/07 22:30 & 123.927 & 121.635 & 118.002 & 99.296 & 103.24 & 94.904 & 109.05 & 105.85 & & 73.233 \\
\hline 9/17/07 23:00 & 123.925 & 121.66 & 118.05 & 99.306 & 103.243 & 94.904 & 109.05 & 105.855 & & 73.235 \\
\hline 9/17/07 23:30 & 123.965 & 121.662 & 118.044 & 99.368 & 103.304 & 94.96 & 109.037 & 105.903 & & 73.295 \\
\hline 9/18/07 0:00 & 124.015 & 121.639 & 118.026 & 99.385 & 103.331 & 94.993 & 109.037 & 105.939 & & 73.322 \\
\hline 9/18/07 0:30 & 124.015 & 121.63 & 118.017 & 99.389 & 103.331 & 94.993 & 109.037 & 105.939 & & 73.318 \\
\hline 9/18/07 1:00 & 124.013 & 121.633 & 118.016 & 99.36 & 103.308 & 94.967 & 109.039 & 105.917 & & 73.293 \\
\hline 9/18/07 1:30 & 124.013 & 121.626 & 118.014 & 99.351 & 103.299 & 94.962 & 109.039 & 105.908 & & 73.287 \\
\hline 9/18/07 2:00 & 124.008 & 121.623 & 118.006 & 99.353 & 103.303 & 94.965 & 109.039 & 105.91 & & 73.288 \\
\hline 9/18/07 2:30 & 124.008 & 121.635 & 118.011 & 99.349 & 103.295 & 94.958 & 109.037 & 105.905 & & 73.283 \\
\hline 9/18/07 3:00 & 124.003 & 121.626 & 118.014 & 99.349 & 103.293 & 94.955 & 109.037 & 105.901 & & 73.28 \\
\hline 9/18/07 3:30 & 123.998 & 121.608 & 117.993 & 99.36 & 103.302 & 94.962 & 109.031 & 105.91 & & 73.289 \\
\hline 9/18/07 4:00 & 123.998 & 121.61 & 117.986 & 99.348 & 103.297 & 94.958 & 109.03 & 105.903 & & 73.284 \\
\hline 9/18/07 4:30 & 123.998 & 121.601 & 117.983 & 99.334 & 103.276 & 94.937 & 109.03 & 105.886 & & 73.262 \\
\hline 9/18/07 5:00 & 123.996 & 121.626 & 117.999 & 99.329 & 103.276 & 94.934 & 109.028 & 105.883 & & 73.262 \\
\hline 9/18/07 5:30 & 123.996 & 123.299 & 118.583 & 99.327 & 103.268 & 94.927 & 109.028 & 105.874 & & 73.256 \\
\hline 9/18/07 6:00 & 123.984 & 123.789 & 119.52 & 99.36 & 103.297 & 94.953 & 109.02 & 105.899 & & 73.282 \\
\hline 9/18/07 6:30 & 125.298 & 123.961 & 119.725 & 99.909 & 103.531 & 95.311 & 109.364 & 106.893 & & 73.303 \\
\hline 9/18/07 7:00 & 125.771 & 124.068 & 119.833 & 100.252 & 103.978 & 95.783 & 109.807 & 107.586 & & 73.487 \\
\hline 9/18/07 7:30 & 125.929 & 124.143 & 119.914 & 100.402 & 104.148 & 95.952 & 109.98 & 107.781 & & 73.642 \\
\hline 9/18/07 8:00 & 126.012 & 124.17 & 119.951 & 100.497 & 104.258 & 96.061 & 110.073 & 107.891 & & 73.756 \\
\hline 9/18/07 8:30 & 126.062 & 124.217 & 119.989 & 100.567 & 104.329 & 96.132 & 110.13 & 107.969 & & 73.837 \\
\hline 9/18/07 9:00 & 126.102 & 124.24 & 120.035 & 100.588 & 104.359 & 96.165 & 110.171 & 108.004 & & 73.87 \\
\hline 9/18/07 9:30 & 126.128 & 124.249 & 120.043 & 100.647 & 104.407 & 96.207 & 110.197 & 108.046 & & 73.917 \\
\hline 9/18/07 10:00 & 126.152 & 122.476 & 119.022 & 100.657 & 104.435 & 96.24 & 110.22 & 108.081 & & 73.948 \\
\hline 9/18/07 10:30 & 126.166 & 122.113 & 118.528 & 100.666 & 104.447 & 96.247 & 110.237 & 108.088 & & 73.961 \\
\hline 9/18/07 11:00 & 124.712 & 121.975 & 118.364 & 100.033 & 104.094 & 95.762 & 109.745 & 106.853 & & 73.948 \\
\hline 9/18/07 11:30 & 124.403 & 121.884 & 118.278 & 99.759 & 103.755 & 95.413 & 109.435 & 106.419 & & 73.762 \\
\hline 9/18/07 12:00 & 124.275 & 121.832 & 118.22 & 99.658 & 103.622 & 95.281 & 109.3 & 106.262 & & 73.64 \\
\hline $9 / 18 / 07$ 12:30 & 124.206 & 121.793 & 118.181 & 99.577 & 103.531 & 95.193 & 109.227 & 106.171 & & 73.547 \\
\hline 9/18/07 13:00 & 124.166 & 121.762 & 118.145 & 99.518 & 103.481 & 95.144 & 109.178 & 106.116 & & 73.489 \\
\hline 9/18/07 13:30 & 124.133 & 121.735 & 118.122 & 99.499 & 103.451 & 95.111 & 109.142 & 106.074 & & 73.452 \\
\hline 9/18/07 14:00 & 124.119 & 121.712 & 118.094 & 99.468 & 103.418 & 95.08 & 109.122 & 106.041 & & 73.414 \\
\hline
\end{tabular}


TABLE S1.1 (Cont.)

\begin{tabular}{|c|c|c|c|c|c|c|c|c|c|c|}
\hline \multirow[b]{2}{*}{ Date and Time } & \multicolumn{10}{|c|}{ Depth from Top of Casing (ft) } \\
\hline & MW1D & MW2D & MW3D & MW4D & MW7 & MW9 & Oentrich & MW14D & MW15D & MW16D \\
\hline 9/18/07 14:30 & 124.109 & 121.698 & 118.077 & 99.446 & 103.394 & 95.057 & 109.107 & 106.014 & & 73.39 \\
\hline 9/18/07 15:00 & 124.1 & 121.689 & 118.067 & 99.422 & 103.367 & 95.031 & 109.095 & 105.987 & & 73.359 \\
\hline 9/18/07 15:30 & 124.098 & 121.678 & 118.059 & 99.411 & 103.358 & 95.019 & 109.086 & 105.976 & & 73.351 \\
\hline 9/18/07 16:00 & 124.093 & 121.662 & 118.044 & 99.401 & 103.348 & 95.01 & 109.077 & 105.963 & & 73.338 \\
\hline 9/18/07 16:30 & 124.093 & 121.671 & 118.049 & 99.392 & 103.339 & 95 & 109.071 & 105.954 & & 73.33 \\
\hline 9/18/07 17:00 & 124.091 & 121.671 & 118.046 & 99.373 & 103.318 & 94.981 & 109.065 & 105.934 & & 73.309 \\
\hline 9/18/07 17:30 & 124.081 & 121.671 & 118.049 & 99.389 & 103.333 & 94.995 & 109.06 & 105.945 & & 73.322 \\
\hline 9/18/07 18:00 & 124.076 & 121.669 & 118.049 & 99.389 & 103.331 & 94.993 & 109.054 & 105.945 & & 73.32 \\
\hline 9/18/07 18:30 & 124.074 & 121.671 & 118.054 & 99.391 & 103.333 & 94.995 & 109.05 & 105.945 & & 73.32 \\
\hline 9/18/07 19:00 & 124.067 & 121.673 & 118.054 & 99.391 & 103.335 & 94.995 & 109.048 & 105.941 & & 73.32 \\
\hline 9/18/07 19:30 & 124.062 & 121.696 & 118.072 & 99.392 & 103.337 & 95 & 109.043 & 105.948 & & 73.324 \\
\hline 9/18/07 20:00 & 124.057 & 121.703 & 118.087 & 99.397 & 103.34 & 95.003 & 109.041 & 105.948 & & 73.326 \\
\hline 9/18/07 20:30 & 124.05 & 121.741 & 118.107 & 99.425 & 103.361 & 95.024 & 109.035 & 105.97 & & 73.347 \\
\hline 9/18/07 21:00 & 124.048 & 121.771 & 118.17 & 99.423 & 103.371 & 95.033 & 109.031 & 105.981 & & 73.353 \\
\hline 9/18/07 21:30 & 124.039 & 121.784 & 118.157 & 99.482 & 103.411 & 95.076 & 109.024 & 106.014 & & 73.394 \\
\hline 9/18/07 22:00 & 124.029 & 121.807 & 118.187 & 99.506 & 103.453 & 95.116 & 109.018 & 106.056 & & 73.433 \\
\hline 9/18/07 22:30 & 124.024 & 121.812 & 118.198 & 99.513 & 103.449 & 95.113 & 109.016 & 106.054 & & 74.759 \\
\hline 9/18/07 23:00 & 124.022 & 121.832 & 118.21 & 99.53 & 103.476 & 95.139 & 109.015 & 106.083 & & 74.449 \\
\hline 9/18/07 23:30 & 124.022 & 121.834 & 118.215 & 99.547 & 103.481 & 95.146 & 109.015 & 106.089 & & 74.225 \\
\hline 9/19/07 0:00 & 124.02 & 121.841 & 118.225 & 99.561 & 103.498 & 95.16 & 109.015 & 106.105 & & 74.035 \\
\hline 9/19/07 0:30 & 124.017 & 121.837 & 118.223 & 99.566 & 103.504 & 95.168 & 109.015 & 106.111 & & 73.843 \\
\hline 9/19/07 1:00 & 124.015 & 121.841 & 118.225 & 99.565 & 103.506 & 95.17 & 109.015 & 106.118 & & 73.673 \\
\hline 9/19/07 1:30 & 124.015 & 121.841 & 118.225 & 99.565 & 103.506 & 95.17 & 109.015 & 106.114 & & 73.526 \\
\hline 9/19/07 2:00 & 124.01 & 121.839 & 118.22 & 99.568 & 103.51 & 95.175 & 109.011 & 106.118 & & 73.483 \\
\hline 9/19/07 2:30 & 124.01 & 121.837 & 118.22 & 99.565 & 103.508 & 95.172 & 109.013 & 106.118 & & 73.477 \\
\hline 9/19/07 3:00 & 124.003 & 121.841 & 118.218 & 99.563 & 103.506 & 95.175 & 109.013 & 106.116 & & 73.469 \\
\hline 9/19/07 3:30 & 124.003 & 121.85 & 118.23 & 99.563 & 103.502 & 95.17 & 109.013 & 106.111 & & 73.467 \\
\hline 9/19/07 4:00 & 123.996 & 121.857 & 118.24 & 99.57 & 103.512 & 95.175 & 109.011 & 106.118 & & 73.469 \\
\hline 9/19/07 4:30 & 123.996 & 121.868 & 118.25 & 99.577 & 103.517 & 95.184 & 109.011 & 106.127 & & 73.477 \\
\hline 9/19/07 5:00 & 123.989 & 121.875 & 118.25 & 99.587 & 103.525 & 95.191 & 109.009 & 106.134 & & 73.487 \\
\hline 9/19/07 5:30 & 123.987 & 123.304 & 118.258 & 99.597 & 103.535 & 95.201 & 109.005 & 106.142 & & 73.498 \\
\hline 9/19/07 6:00 & 123.984 & 123.923 & 119.81 & 99.606 & 103.542 & 95.208 & 109.005 & 106.151 & & 73.504 \\
\hline 9/19/07 6:30 & 125.012 & 124.122 & 120.042 & 100.104 & 103.645 & 95.391 & 109.206 & 106.541 & & 73.512 \\
\hline 9/19/07 7:00 & 125.693 & 124.236 & 120.166 & 100.504 & 104.207 & 96.021 & 109.794 & 107.727 & & 73.671 \\
\hline 9/19/07 7:30 & 125.858 & 124.301 & 120.244 & 100.672 & 104.405 & 96.221 & 109.974 & 107.949 & & 73.855 \\
\hline 9/19/07 8:00 & 125.948 & 124.353 & 120.292 & 100.776 & 104.519 & 96.337 & 110.072 & 108.07 & & 73.977 \\
\hline 9/19/07 8:30 & 126.008 & 122.467 & 118.916 & 100.838 & 104.589 & 96.405 & 110.135 & 108.143 & & 74.058 \\
\hline 9/19/07 9:00 & 126.053 & 122.261 & 118.664 & 100.721 & 104.639 & 96.455 & 110.177 & 108.194 & & 74.112 \\
\hline 9/19/07 9:30 & 124.464 & 122.156 & 118.553 & 100.097 & 104.102 & 95.771 & 109.495 & 106.787 & & 74.045 \\
\hline 9/19/07 10:00 & 124.282 & 122.093 & 118.48 & 99.937 & 103.908 & 95.573 & 109.304 & 106.554 & & 73.904 \\
\hline 9/19/07 10:30 & 124.202 & 122.061 & 118.447 & 99.849 & 103.807 & 95.474 & 109.208 & 106.443 & & 73.812 \\
\hline 9/19/07 11:00 & 124.169 & 122.038 & 118.424 & 99.795 & 103.748 & 95.415 & 109.155 & 106.379 & & 73.741 \\
\hline 9/19/07 11:30 & 124.15 & 122.011 & 118.397 & 99.771 & 103.717 & 95.384 & 109.122 & 106.339 & & 73.702 \\
\hline 9/19/07 12:00 & 124.14 & 121.993 & 118.371 & 99.747 & 103.693 & 95.363 & 109.099 & 106.317 & & 73.677 \\
\hline 9/19/07 12:30 & 124.133 & 121.977 & 118.364 & 99.723 & 103.668 & 95.335 & 109.084 & 106.288 & & 73.651 \\
\hline 9/19/07 13:00 & 124.133 & 121.964 & 118.346 & 99.709 & 103.649 & 95.316 & 109.073 & 106.269 & & 73.629 \\
\hline 9/19/07 13:30 & 124.131 & 121.945 & 118.331 & 99.694 & 103.639 & 95.304 & 109.065 & 106.257 & & 73.617 \\
\hline 9/19/07 14:00 & 124.128 & 121.936 & 118.319 & 99.678 & 103.62 & 95.288 & 109.058 & 106.238 & & 73.599 \\
\hline 9/19/07 14:30 & 124.126 & 121.925 & 118.311 & 99.663 & 103.603 & 95.271 & 109.054 & 106.222 & & 73.584 \\
\hline 9/19/07 15:00 & 124.126 & 121.909 & 118.293 & 99.656 & 103.601 & 95.267 & 109.048 & 106.213 & & 73.576 \\
\hline 9/19/07 15:30 & 124.135 & 121.893 & 118.276 & 99.644 & 103.588 & 95.255 & 109.047 & 106.202 & & 73.566 \\
\hline 9/19/07 16:00 & 124.15 & 121.882 & 118.266 & 99.63 & 103.571 & 95.241 & 109.047 & 106.187 & & 73.549 \\
\hline 9/19/07 16:30 & 124.152 & 121.868 & 118.25 & 99.615 & 103.556 & 95.222 & 109.043 & 106.169 & & 73.535 \\
\hline 9/19/07 17:00 & 124.154 & 121.877 & 118.253 & 99.601 & 103.542 & 95.212 & 109.043 & 106.158 & & 73.522 \\
\hline 9/19/07 17:30 & 124.161 & 121.896 & 118.293 & 99.59 & 103.529 & 95.198 & 109.039 & 106.145 & & 73.51 \\
\hline 9/19/07 18:00 & 124.166 & 121.88 & 118.266 & 99.599 & 103.537 & 95.203 & 109.046 & 106.149 & & 73.512 \\
\hline 9/19/07 18:30 & 124.199 & 121.871 & 118.256 & 99.639 & 103.571 & 95.264 & 109.003 & 106.173 & & 73.518 \\
\hline 9/19/07 19:00 & 124.199 & 121.868 & 118.25 & 99.594 & 103.54 & 95.21 & 109.003 & 106.156 & & 73.516 \\
\hline 9/19/07 19:30 & 124.202 & 121.875 & 118.256 & 99.584 & 103.54 & 95.198 & 109.41 & 106.149 & & 73.512 \\
\hline 9/19/07 20:00 & 124.206 & 121.871 & 118.248 & 99.596 & 103.533 & 95.222 & 109.028 & 106.142 & & 73.506 \\
\hline 9/19/07 20:30 & 124.204 & 121.873 & 118.248 & 99.587 & 103.538 & 95.201 & 109.035 & 106.153 & & 73.516 \\
\hline 9/19/07 21:00 & 124.211 & 121.882 & 118.258 & 99.59 & 103.533 & 95.198 & 109.044 & 106.145 & & 73.508 \\
\hline 9/19/07 21:30 & 124.211 & 121.886 & 118.268 & 99.594 & 103.535 & 95.201 & 109.023 & 106.147 & & 73.512 \\
\hline 9/19/07 22:00 & 124.211 & 121.875 & 118.253 & 99.606 & 103.542 & 95.208 & 109.03 & 106.153 & & 73.518 \\
\hline 9/19/07 22:30 & 124.216 & 121.871 & 118.248 & 99.606 & 103.546 & 95.212 & 109.012 & 106.162 & & 73.526 \\
\hline 9/19/07 23:00 & 124.218 & 121.864 & 118.243 & 99.597 & 103.535 & 95.203 & 109.007 & 106.149 & & 73.512 \\
\hline
\end{tabular}


TABLE S1.1 (Cont.)

\begin{tabular}{|c|c|c|c|c|c|c|c|c|c|c|}
\hline \multirow[b]{2}{*}{ Date and Time } & \multicolumn{10}{|c|}{ Depth from Top of Casing (ft) } \\
\hline & MW1D & MW2D & MW3D & MW4D & MW7 & MW9 & Oentrich & MW14D & MW15D & MW16D \\
\hline 9/19/07 23:30 & 124.218 & 121.859 & 118.238 & 99.592 & 103.531 & 95.196 & 108.998 & 106.142 & & 73.508 \\
\hline 9/20/07 0:00 & 124.216 & 121.859 & 118.24 & 99.582 & 103.525 & 95.189 & 108.961 & 106.138 & & 73.502 \\
\hline 9/20/07 0:30 & 124.213 & 121.859 & 118.238 & 99.58 & 103.519 & 95.184 & 109.011 & 106.134 & & 73.5 \\
\hline 9/20/07 1:00 & 124.211 & 121.859 & 118.24 & 99.582 & 103.521 & 95.186 & 109.005 & 106.134 & & 73.498 \\
\hline 9/20/07 1:30 & 124.209 & 121.855 & 118.235 & 99.584 & 103.521 & 95.186 & 108.989 & 106.134 & & 73.498 \\
\hline 9/20/07 2:00 & 124.209 & 121.855 & 118.235 & 99.584 & 103.523 & 95.189 & 108.979 & 106.134 & & 73.498 \\
\hline 9/20/07 2:30 & 124.209 & 121.85 & 118.233 & 99.575 & 103.516 & 95.184 & 108.974 & 106.129 & & 73.491 \\
\hline 9/20/07 3:00 & 124.204 & 121.846 & 118.225 & 99.58 & 103.517 & 95.186 & 108.966 & 106.129 & & 73.491 \\
\hline 9/20/07 3:30 & 124.202 & 121.841 & 118.22 & 99.572 & 103.514 & 95.179 & 108.964 & 106.127 & & 73.489 \\
\hline 9/20/07 4:00 & 124.202 & 121.837 & 118.215 & 99.566 & 103.508 & 95.175 & 108.961 & 106.12 & & 73.483 \\
\hline 9/20/07 4:30 & 124.202 & 121.837 & 118.218 & 99.561 & 103.5 & 95.17 & 108.955 & 106.116 & & 73.477 \\
\hline 9/20/07 5:00 & 124.202 & 121.837 & 118.213 & 99.556 & 103.497 & 95.165 & 108.953 & 106.109 & & 73.473 \\
\hline 9/20/07 5:30 & 124.197 & 123.319 & 118.215 & 99.558 & 103.5 & 95.168 & 108.951 & 106.111 & & 73.477 \\
\hline 9/20/07 6:00 & 124.199 & 123.968 & 119.687 & 99.556 & 103.497 & 95.165 & 108.949 & 106.111 & & 73.475 \\
\hline 9/20/07 6:30 & 125.232 & 124.165 & 119.914 & 100.028 & 103.584 & 95.316 & 109.126 & 106.519 & & 73.471 \\
\hline 9/20/07 7:00 & 125.974 & 124.27 & 120.024 & 100.424 & 104.14 & 95.948 & 109.719 & 107.758 & & 73.634 \\
\hline 9/20/07 7:30 & 126.154 & 124.335 & 120.103 & 100.593 & 104.334 & 96.143 & 109.903 & 107.978 & & 73.81 \\
\hline 9/20/07 8:00 & 126.256 & 124.381 & 120.148 & 100.683 & 104.441 & 96.249 & 110.004 & 108.088 & & 73.923 \\
\hline 9/20/07 8:30 & 126.326 & 124.41 & 120.186 & 100.741 & 104.506 & 96.311 & 110.068 & 108.159 & & 73.995 \\
\hline 9/20/07 9:00 & 126.381 & 124.431 & 120.206 & 100.784 & 104.549 & 96.358 & 110.113 & 108.206 & & 74.045 \\
\hline 9/20/07 9:30 & 126.421 & 124.444 & 120.221 & 100.814 & 104.582 & 96.389 & 110.143 & 108.241 & & 74.081 \\
\hline 9/20/07 10:00 & 126.457 & 124.453 & 120.231 & 100.829 & 104.601 & 96.41 & 110.168 & 108.261 & & 74.103 \\
\hline 9/20/07 10:30 & 126.49 & 124.458 & 120.236 & 100.843 & 104.618 & 96.424 & 110.186 & 108.277 & & 74.12 \\
\hline 9/20/07 11:00 & 126.572 & 124.453 & 120.236 & 100.85 & 104.624 & 96.433 & 110.203 & 108.285 & & 74.13 \\
\hline 9/20/07 11:30 & 126.778 & 124.447 & 120.229 & 100.853 & 104.629 & 96.438 & 110.216 & 108.29 & & 74.138 \\
\hline 9/20/07 12:00 & 126.868 & 124.437 & 120.221 & 100.853 & 104.629 & 96.44 & 110.227 & 108.29 & & 74.143 \\
\hline 9/20/07 12:30 & 126.87 & 124.431 & 120.216 & 100.845 & 104.624 & 96.431 & 110.237 & 108.281 & & 74.14 \\
\hline 9/20/07 13:00 & 126.858 & 122.526 & 119.004 & 100.84 & 104.618 & 96.426 & 110.242 & 108.274 & & 74.136 \\
\hline 9/20/07 13:30 & 126.851 & 122.229 & 118.636 & 100.834 & 104.612 & 96.419 & 110.248 & 108.268 & & 74.134 \\
\hline 9/20/07 14:00 & 125.062 & 122.077 & 118.47 & 100.097 & 104.129 & 95.802 & 109.655 & 106.86 & & 74.058 \\
\hline 9/20/07 14:30 & 124.634 & 121.982 & 118.371 & 99.866 & 103.853 & 95.516 & 109.403 & 106.525 & & 73.87 \\
\hline 9/20/07 15:00 & 124.455 & 121.914 & 118.301 & 99.74 & 103.71 & 95.375 & 109.285 & 106.364 & & 73.733 \\
\hline 9/20/07 15:30 & 124.353 & 121.866 & 118.248 & 99.659 & 103.613 & 95.283 & 109.212 & 106.264 & & 73.634 \\
\hline 9/20/07 16:00 & 124.282 & 121.83 & 118.21 & 99.601 & 103.552 & 95.217 & 109.167 & 106.193 & & 73.562 \\
\hline 9/20/07 16:30 & 124.228 & 121.803 & 118.18 & 99.554 & 103.506 & 95.17 & 109.137 & 106.142 & & 73.512 \\
\hline 9/20/07 17:00 & 124.185 & 121.782 & 118.16 & 99.523 & 103.47 & 95.135 & 109.114 & 106.107 & & 73.471 \\
\hline 9/20/07 17:30 & 124.157 & 121.764 & 118.147 & 99.497 & 103.447 & 95.111 & 109.099 & 106.076 & & 73.442 \\
\hline 9/20/07 18:00 & 124.135 & 121.757 & 118.137 & 99.485 & 103.428 & 95.094 & 109.086 & 106.056 & & 73.423 \\
\hline 9/20/07 18:30 & 124.121 & 121.741 & 118.122 & 99.472 & 103.417 & 95.08 & 109.075 & 106.041 & & 73.408 \\
\hline 9/20/07 19:00 & 124.112 & 121.732 & 118.109 & 99.463 & 103.405 & 95.071 & 109.067 & 106.029 & & 73.398 \\
\hline 9/20/07 19:30 & 124.098 & 121.723 & 118.109 & 99.444 & 103.394 & 95.057 & 109.039 & 106.018 & & 73.381 \\
\hline 9/20/07 20:00 & 124.086 & 121.723 & 118.094 & 99.439 & 103.39 & 95.047 & 109.522 & 106.005 & & 73.371 \\
\hline 9/20/07 20:30 & 124.079 & 121.725 & 118.104 & 99.432 & 103.377 & 95.061 & 109.07 & 105.996 & & 73.365 \\
\hline 9/20/07 21:00 & 124.074 & 121.723 & 118.099 & 99.43 & 103.382 & 95.054 & 109.059 & 105.996 & & 73.365 \\
\hline 9/20/07 21:30 & 124.076 & 121.721 & 118.099 & 99.441 & 103.38 & 95.045 & 109.096 & 105.998 & & 73.367 \\
\hline 9/20/07 22:00 & 124.076 & 121.723 & 118.099 & 99.435 & 103.377 & 95.038 & 109.076 & 105.996 & & 73.363 \\
\hline 9/20/07 22:30 & 124.072 & 121.721 & 118.099 & 99.435 & 103.375 & 95.04 & 109.07 & 105.992 & & 73.359 \\
\hline 9/20/07 23:00 & 124.074 & 121.716 & 118.092 & 99.435 & 103.377 & 95.043 & 109.056 & 105.994 & & 73.361 \\
\hline 9/20/07 23:30 & 124.072 & 121.712 & 118.089 & 99.434 & 103.375 & 95.04 & 109.045 & 105.99 & & 73.359 \\
\hline 9/21/07 0:00 & 124.067 & 121.707 & 118.084 & 99.429 & 103.367 & 95.036 & 109.038 & 105.987 & & 73.351 \\
\hline 9/21/07 0:30 & 124.062 & 121.707 & 118.079 & 99.427 & 103.367 & 95.036 & 109.03 & 105.983 & & 73.351 \\
\hline 9/21/07 1:00 & 124.062 & 121.703 & 118.079 & 99.425 & 103.363 & 95.031 & 109.026 & 105.979 & & 73.347 \\
\hline 9/21/07 1:30 & 124.06 & 121.694 & 118.074 & 99.42 & 103.361 & 95.031 & 109.02 & 105.979 & & 73.345 \\
\hline 9/21/07 2:00 & 124.053 & 121.689 & 118.059 & 99.416 & 103.356 & 95.021 & 109.019 & 105.972 & & 73.336 \\
\hline 9/21/07 2:30 & 124.048 & 121.678 & 118.054 & 99.406 & 103.35 & 95.014 & 109.017 & 105.965 & & 73.33 \\
\hline 9/21/07 3:00 & 124.043 & 121.669 & 118.049 & 99.403 & 103.342 & 95.007 & 109.015 & 105.959 & & 73.324 \\
\hline 9/21/07 3:30 & 124.036 & 121.667 & 118.044 & 99.392 & 103.335 & 95 & 109.015 & 105.952 & & 73.316 \\
\hline 9/21/07 4:00 & 124.024 & 121.671 & 118.044 & 99.384 & 103.325 & 94.991 & 109.013 & 105.943 & & 73.307 \\
\hline 9/21/07 4:30 & 124.02 & 121.673 & 118.051 & 99.382 & 103.319 & 94.984 & 109.013 & 105.936 & & 73.301 \\
\hline 9/21/07 5:00 & 124.02 & 121.671 & 118.044 & 99.389 & 103.329 & 94.993 & 109.009 & 105.943 & & 73.307 \\
\hline 9/21/07 5:30 & 124.024 & 121.671 & 118.044 & 99.392 & 103.329 & 94.995 & 109.009 & 105.943 & & 73.307 \\
\hline 9/21/07 6:00 & 124.027 & 123.623 & 119.485 & 99.385 & 103.327 & 94.995 & 109.009 & 105.943 & & 73.305 \\
\hline 9/21/07 6:30 & 124.022 & 123.861 & 119.767 & 99.499 & 103.321 & 94.988 & 109.009 & 105.941 & & 73.301 \\
\hline 9/21/07 7:00 & 125.962 & 123.986 & 119.909 & 100.207 & 103.896 & 95.71 & 109.723 & 107.413 & & 73.407 \\
\hline 9/21/07 7:30 & 126.277 & 124.061 & 119.992 & 100.402 & 104.132 & 95.95 & 109.944 & 107.679 & & 73.599 \\
\hline 9/21/07 8:00 & 126.416 & 123.1 & 120.037 & 100.516 & 104.26 & 96.077 & 110.057 & 107.814 & & 73.731 \\
\hline
\end{tabular}


TABLE S1.1 (Cont.)

\begin{tabular}{|c|c|c|c|c|c|c|c|c|c|c|}
\hline \multirow[b]{2}{*}{ Date and Time } & \multicolumn{10}{|c|}{ Depth from Top of Casing (ft) } \\
\hline & MW1D & MW2D & MW3D & MW4D & MW7 & MW9 & Oentrich & MW14D & MW15D & MW16D \\
\hline 9/21/07 8:30 & 126.504 & 122.172 & 118.603 & 100.579 & 104.33 & 96.148 & 110.13 & 107.893 & & 73.812 \\
\hline 9/21/07 9:00 & 126.544 & 121.986 & 118.382 & 100.209 & 104.376 & 96.193 & 110.177 & 107.938 & & 73.859 \\
\hline 9/21/07 9:30 & 124.62 & 121.889 & 118.273 & 99.801 & 103.801 & 95.467 & 109.452 & 106.479 & & 73.764 \\
\hline $9 / 21 / 0710: 00$ & 124.369 & 121.825 & 118.208 & 99.649 & 103.624 & 95.29 & 109.285 & 106.273 & & 73.628 \\
\hline $9 / 21 / 07 \quad 10: 30$ & 124.254 & 121.782 & 118.162 & 99.57 & 103.529 & 95.191 & 109.202 & 106.167 & & 73.529 \\
\hline 9/21/07 11:00 & 124.195 & 121.75 & 118.132 & 99.515 & 103.468 & 95.132 & 109.155 & 106.1 & & 73.467 \\
\hline 9/21/07 11:30 & 124.147 & 121.725 & 118.104 & 99.478 & 103.426 & 95.092 & 109.123 & 106.056 & & 73.419 \\
\hline $9 / 21 / 07$ 12:00 & 124.112 & 121.712 & 118.092 & 99.451 & 103.398 & 95.064 & 109.101 & 106.023 & & 73.384 \\
\hline 9/21/07 12:30 & 124.083 & 121.71 & 118.084 & 99.429 & 103.373 & 95.04 & 109.086 & 105.996 & & 73.357 \\
\hline 9/21/07 13:00 & 124.067 & 121.712 & 118.087 & 99.416 & 103.363 & 95.031 & 109.077 & 105.985 & & 73.343 \\
\hline 9/21/07 13:30 & 124.06 & 121.707 & 118.087 & 99.416 & 103.358 & 95.026 & 109.069 & 105.981 & & 73.334 \\
\hline 9/21/07 14:00 & 124.06 & 121.707 & 118.082 & 99.423 & 103.363 & 95.031 & 109.06 & 105.981 & & 73.338 \\
\hline $9 / 21 / 0714: 30$ & 124.06 & 121.71 & 118.069 & 99.422 & 103.361 & 95.028 & 109.054 & 105.976 & & 73.338 \\
\hline 9/21/07 15:00 & 124.062 & 121.712 & 118.087 & 99.422 & 103.361 & 95.031 & 109.05 & 105.976 & & 73.336 \\
\hline 9/21/07 15:30 & 124.06 & 121.707 & 118.069 & 99.425 & 103.361 & 95.028 & 109.048 & 105.976 & & 73.334 \\
\hline $9 / 21 / 0716: 00$ & 124.065 & 121.714 & 118.074 & 99.427 & 103.371 & 95.036 & 109.045 & 105.983 & & 73.338 \\
\hline $9 / 21 / 0716: 30$ & 124.065 & 121.73 & 118.104 & 99.422 & 103.359 & 95.028 & 109.045 & 105.976 & & 73.33 \\
\hline 9/21/07 17:00 & 124.062 & 121.746 & 118.102 & 99.43 & 103.369 & 95.036 & 109.041 & 105.983 & & 73.338 \\
\hline $9 / 21 / 0717: 30$ & 124.079 & 121.759 & 118.132 & 99.451 & 103.39 & 95.057 & 109.037 & 106.001 & & 73.357 \\
\hline 9/21/07 18:00 & 124.093 & 121.766 & 118.145 & 99.468 & 103.401 & 95.066 & 109.035 & 106.01 & & 73.365 \\
\hline 9/21/07 18:30 & 124.112 & 121.778 & 118.15 & 99.48 & 103.418 & 95.083 & 109.033 & 106.029 & & 73.384 \\
\hline 9/21/07 19:00 & 124.119 & 121.791 & 118.165 & 99.487 & 103.424 & 95.094 & 109.033 & 106.038 & & 73.388 \\
\hline 9/21/07 19:30 & 124.124 & 121.796 & 118.172 & 99.499 & 103.434 & 95.102 & 109.031 & 106.047 & & 73.396 \\
\hline 9/21/07 20:00 & 124.138 & 121.807 & 118.185 & 99.513 & 103.447 & 95.113 & 109.031 & 106.058 & & 73.409 \\
\hline $9 / 21 / 0720: 30$ & 124.15 & 121.823 & 118.193 & 99.516 & 103.457 & 95.123 & 109.031 & 106.069 & & 73.415 \\
\hline 9/21/07 21:00 & 124.159 & 121.839 & 118.213 & 99.53 & 103.464 & 95.135 & 109.03 & 106.078 & & 73.425 \\
\hline 9/21/07 21:30 & 124.169 & 121.85 & 118.223 & 99.546 & 103.48 & 95.149 & 109.026 & 106.094 & & 73.44 \\
\hline 9/21/07 22:00 & 124.187 & 121.861 & 118.235 & 99.565 & 103.5 & 95.17 & 109.024 & 106.111 & & 73.458 \\
\hline 9/21/07 22:30 & 124.202 & 121.875 & 118.253 & 99.573 & 103.508 & 95.177 & 109.024 & 106.12 & & 73.464 \\
\hline 9/21/07 23:00 & 124.211 & 121.889 & 118.268 & 99.585 & 103.519 & 95.186 & 109.022 & 106.131 & & 73.475 \\
\hline 9/21/07 23:30 & 124.225 & 121.9 & 118.281 & 99.604 & 103.537 & 95.205 & 109.022 & 106.149 & & 73.493 \\
\hline 9/22/07 0:00 & 124.239 & 121.909 & 118.291 & 99.615 & 103.552 & 95.219 & 109.02 & 106.162 & & 73.506 \\
\hline 9/22/07 0:30 & 124.251 & 121.914 & 118.293 & 99.627 & 103.561 & 95.229 & 109.018 & 106.173 & & 73.516 \\
\hline 9/22/07 1:00 & 124.261 & 121.911 & 118.296 & 99.635 & 103.569 & 95.238 & 109.018 & 106.182 & & 73.524 \\
\hline 9/22/07 1:30 & 124.268 & 121.911 & 118.291 & 99.639 & 103.573 & 95.241 & 109.018 & 106.187 & & 73.526 \\
\hline 9/22/07 2:00 & 124.268 & 121.914 & 118.291 & 99.634 & 103.571 & 95.238 & 109.018 & 106.187 & & 73.526 \\
\hline 9/22/07 2:30 & 124.261 & 121.914 & 118.296 & 99.635 & 103.571 & 95.238 & 109.016 & 106.182 & & 73.524 \\
\hline 9/22/07 3:00 & 124.258 & 121.918 & 118.301 & 99.635 & 103.571 & 95.238 & 109.016 & 106.184 & & 73.522 \\
\hline 9/22/07 3:30 & 124.256 & 121.925 & 118.306 & 99.639 & 103.575 & 95.241 & 109.015 & 106.187 & & 73.529 \\
\hline 9/22/07 4:00 & 124.254 & 121.925 & 118.306 & 99.644 & 103.578 & 95.245 & 109.015 & 106.193 & & 73.531 \\
\hline 9/22/07 4:30 & 124.251 & 121.927 & 118.311 & 99.649 & 103.584 & 95.252 & 109.015 & 106.198 & & 73.535 \\
\hline 9/22/07 5:00 & 124.249 & 121.943 & 118.316 & 99.651 & 103.586 & 95.25 & 109.013 & 106.2 & & 73.535 \\
\hline 9/22/07 5:30 & 124.249 & 121.945 & 118.326 & 99.654 & 103.584 & 95.252 & 109.015 & 106.202 & & 73.541 \\
\hline 9/22/07 6:00 & 124.244 & 121.95 & 118.331 & 99.665 & 103.597 & 95.264 & 109.013 & 106.211 & & 73.549 \\
\hline 9/22/07 6:30 & 124.242 & 123.773 & 119.349 & 99.67 & 103.605 & 95.274 & 109.011 & 106.22 & & 73.558 \\
\hline 9/22/07 7:00 & 124.242 & 124.163 & 119.893 & 99.675 & 103.609 & 95.278 & 109.009 & 106.222 & & 73.56 \\
\hline 9/22/07 7:30 & 125.719 & 124.331 & 120.082 & 100.319 & 103.961 & 95.759 & 109.48 & 107.477 & & 73.589 \\
\hline 9/22/07 8:00 & 126.076 & 124.428 & 120.196 & 100.616 & 104.342 & 96.15 & 109.835 & 107.962 & & 73.801 \\
\hline 9/22/07 8:30 & 126.239 & 124.492 & 120.264 & 100.76 & 104.506 & 96.315 & 109.989 & 108.15 & & 73.963 \\
\hline 9/22/07 9:00 & 126.345 & 124.537 & 120.309 & 100.852 & 104.606 & 96.417 & 110.077 & 108.254 & & 74.068 \\
\hline 9/22/07 9:30 & 126.416 & 124.56 & 120.332 & 100.905 & 104.666 & 96.478 & 110.139 & 108.321 & & 74.136 \\
\hline 9/22/07 10:00 & 126.473 & 124.58 & 120.36 & 100.943 & 104.705 & 96.521 & 110.182 & 108.365 & & 74.178 \\
\hline 9/22/07 10:30 & 126.516 & 124.587 & 120.365 & 100.967 & 104.734 & 96.546 & 110.211 & 108.392 & & 74.208 \\
\hline 9/22/07 11:00 & 126.558 & 124.594 & 120.377 & 100.986 & 104.757 & 96.57 & 110.229 & 108.414 & & 74.231 \\
\hline 9/22/07 11:30 & 126.601 & 124.596 & 120.38 & 100.995 & 104.761 & 96.572 & 110.246 & 108.418 & & 74.24 \\
\hline 9/22/07 12:00 & 126.858 & 124.598 & 120.385 & 100.998 & 104.768 & 96.584 & 110.261 & 108.429 & & 74.25 \\
\hline 9/22/07 12:30 & 127 & 122.605 & 119.059 & 101 & 104.772 & 96.587 & 110.273 & 108.434 & & 74.256 \\
\hline 9/22/07 13:00 & 127.024 & 122.358 & 118.765 & 100.888 & 104.778 & 96.591 & 110.282 & 108.436 & & 74.262 \\
\hline 9/22/07 13:30 & 125.112 & 122.227 & 118.618 & 100.204 & 104.222 & 95.894 & 109.608 & 106.928 & & 74.153 \\
\hline 9/22/07 14:00 & 124.764 & 122.143 & 118.533 & 100.004 & 103.982 & 95.653 & 109.399 & 106.649 & & 73.971 \\
\hline 9/22/07 14:30 & 124.608 & 122.086 & 118.475 & 99.895 & 103.858 & 95.528 & 109.294 & 106.51 & & 73.847 \\
\hline 9/22/07 15:00 & 124.518 & 122.045 & 118.429 & 99.825 & 103.778 & 95.45 & 109.23 & 106.424 & & 73.762 \\
\hline 9/22/07 15:30 & 124.464 & 122.011 & 118.394 & 99.777 & 103.725 & 95.396 & 109.189 & 106.364 & & 73.702 \\
\hline 9/22/07 16:00 & 124.417 & 121.991 & 118.374 & 99.738 & 103.685 & 95.358 & 109.161 & 106.322 & & 73.661 \\
\hline 9/22/07 16:30 & 124.377 & 121.97 & 118.349 & 99.713 & 103.653 & 95.323 & 109.142 & 106.288 & & 73.624 \\
\hline 9/22/07 17:00 & 124.351 & 121.957 & 118.334 & 99.692 & 103.634 & 95.304 & 109.125 & 106.266 & & 73.603 \\
\hline
\end{tabular}


TABLE S1.1 (Cont.)

\begin{tabular}{|c|c|c|c|c|c|c|c|c|c|c|}
\hline \multirow[b]{2}{*}{ Date and Time } & \multicolumn{10}{|c|}{ Depth from Top of Casing (ft) } \\
\hline & MW1D & MW2D & MW3D & MW4D & MW7 & MW9 & Oentrich & MW14D & MW15D & MW16D \\
\hline 9/22/07 17:30 & 124.332 & 121.945 & 118.326 & 99.675 & 103.613 & 95.283 & 109.114 & 106.244 & & 73.582 \\
\hline 9/22/07 18:00 & 124.315 & 121.934 & 118.316 & 99.661 & 103.601 & 95.271 & 109.105 & 106.229 & & 73.568 \\
\hline 9/22/07 18:30 & 124.306 & 121.925 & 118.306 & 99.652 & 103.594 & 95.262 & 109.097 & 106.22 & & 73.558 \\
\hline 9/22/07 19:00 & 124.294 & 121.925 & 118.303 & 99.64 & 103.58 & 95.25 & 109.092 & 106.207 & & 73.545 \\
\hline 9/22/07 19:30 & 124.284 & 121.925 & 118.306 & 99.635 & 103.573 & 95.243 & 109.086 & 106.198 & & 73.537 \\
\hline 9/22/07 20:00 & 124.277 & 121.927 & 118.303 & 99.634 & 103.571 & 95.243 & 109.08 & 106.196 & & 73.535 \\
\hline 9/22/07 20:30 & 124.28 & 121.927 & 118.306 & 99.637 & 103.575 & 95.243 & 109.077 & 106.198 & & 73.537 \\
\hline 9/22/07 21:00 & 124.28 & 121.923 & 118.303 & 99.639 & 103.573 & 95.245 & 109.073 & 106.196 & & 73.535 \\
\hline 9/22/07 21:30 & 124.28 & 121.918 & 118.293 & 99.64 & 103.575 & 95.245 & 109.069 & 106.198 & & 73.537 \\
\hline 9/22/07 22:00 & 124.28 & 121.918 & 118.283 & 99.635 & 103.573 & 95.243 & 109.067 & 106.193 & & 73.535 \\
\hline 9/22/07 22:30 & 124.275 & 121.911 & 118.273 & 99.632 & 103.569 & 95.238 & 109.063 & 106.191 & & 73.531 \\
\hline 9/22/07 23:00 & 124.273 & 121.905 & 118.271 & 99.632 & 103.569 & 95.238 & 109.062 & 106.191 & & 73.531 \\
\hline 9/22/07 23:30 & 124.273 & 121.905 & 118.266 & 99.625 & 103.563 & 95.234 & 109.06 & 106.184 & & 73.524 \\
\hline 9/23/07 0:00 & 124.265 & 121.898 & 118.261 & 99.62 & 103.556 & 95.226 & 109.058 & 106.178 & & 73.518 \\
\hline 9/23/07 0:30 & 124.261 & 121.893 & 118.258 & 99.615 & 103.554 & 95.224 & 109.056 & 106.176 & & 73.516 \\
\hline 9/23/07 1:00 & 124.256 & 121.893 & 118.256 & 99.611 & 103.546 & 95.217 & 109.056 & 106.169 & & 73.508 \\
\hline 9/23/07 1:30 & 124.254 & 121.882 & 118.245 & 99.609 & 103.544 & 95.215 & 109.054 & 106.167 & & 73.506 \\
\hline 9/23/07 2:00 & 124.249 & 121.88 & 118.258 & 99.603 & 103.544 & 95.212 & 109.052 & 106.162 & & 73.504 \\
\hline 9/23/07 2:30 & 124.244 & 121.875 & 118.258 & 99.596 & 103.535 & 95.203 & 109.05 & 106.153 & & 73.493 \\
\hline 9/23/07 3:00 & 124.239 & 121.873 & 118.253 & 99.594 & 103.531 & 95.201 & 109.05 & 106.151 & & 73.489 \\
\hline 9/23/07 3:30 & 124.237 & 121.868 & 118.248 & 99.589 & 103.527 & 95.198 & 109.047 & 106.147 & & 73.487 \\
\hline 9/23/07 4:00 & 124.235 & 121.868 & 118.245 & 99.584 & 103.521 & 95.191 & 109.047 & 106.14 & & 73.481 \\
\hline 9/23/07 4:30 & 124.23 & 121.868 & 118.248 & 99.582 & 103.517 & 95.186 & 109.045 & 106.138 & & 73.477 \\
\hline 9/23/07 5:00 & 124.225 & 121.868 & 118.243 & 99.582 & 103.519 & 95.191 & 109.045 & 106.138 & & 73.479 \\
\hline 9/23/07 5:30 & 124.225 & 121.871 & 118.248 & 99.582 & 103.521 & 95.191 & 109.043 & 106.138 & & 73.479 \\
\hline 9/23/07 6:00 & 124.223 & 121.871 & 118.253 & 99.584 & 103.519 & 95.191 & 109.043 & 106.138 & & 73.477 \\
\hline 9/23/07 6:30 & 124.223 & 123.793 & 119.457 & 99.584 & 103.521 & 95.191 & 109.041 & 106.14 & & 73.479 \\
\hline 9/23/07 7:00 & 124.223 & 124.095 & 119.835 & 99.587 & 103.523 & 95.193 & 109.039 & 106.145 & & 73.483 \\
\hline 9/23/07 7:30 & 125.799 & 124.24 & 119.999 & 100.302 & 103.98 & 95.783 & 109.617 & 107.548 & & 73.537 \\
\hline 9/23/07 8:00 & 126.078 & 124.331 & 120.098 & 100.545 & 104.273 & 96.084 & 109.901 & 107.9 & & 73.741 \\
\hline 9/23/07 8:30 & 126.215 & 124.39 & 120.163 & 100.671 & 104.418 & 96.231 & 110.034 & 108.057 & & 73.884 \\
\hline 9/23/07 9:00 & 126.308 & 124.426 & 120.206 & 100.753 & 104.507 & 96.318 & 110.115 & 108.153 & & 73.979 \\
\hline 9/23/07 9:30 & 126.499 & 124.453 & 120.236 & 100.805 & 104.568 & 96.377 & 110.169 & 108.217 & & 74.043 \\
\hline 9/23/07 10:00 & 126.705 & 122.594 & 119.079 & 100.841 & 104.608 & 96.417 & 110.207 & 108.259 & & 74.087 \\
\hline 9/23/07 10:30 & 126.823 & 122.299 & 118.709 & 100.862 & 104.631 & 96.443 & 110.235 & 108.285 & & 74.116 \\
\hline 9/23/07 11:00 & 125.171 & 122.159 & 118.553 & 100.168 & 104.197 & 95.872 & 109.67 & 106.931 & & 74.078 \\
\hline 9/23/07 11:30 & 124.726 & 122.075 & 118.46 & 99.942 & 103.917 & 95.589 & 109.414 & 106.59 & & 73.907 \\
\hline 9/23/07 12:00 & 124.537 & 122.011 & 118.402 & 99.828 & 103.788 & 95.457 & 109.296 & 106.443 & & 73.781 \\
\hline 9/23/07 12:30 & 124.445 & 121.966 & 118.351 & 99.752 & 103.704 & 95.375 & 109.229 & 106.35 & & 73.692 \\
\hline 9/23/07 13:00 & 124.381 & 121.925 & 118.311 & 99.699 & 103.649 & 95.321 & 109.189 & 106.291 & & 73.63 \\
\hline 9/23/07 13:30 & 124.334 & 121.895 & 118.278 & 99.656 & 103.603 & 95.274 & 109.163 & 106.24 & & 73.58 \\
\hline 9/23/07 14:00 & 124.291 & 121.873 & 118.256 & 99.62 & 103.563 & 95.236 & 109.142 & 106.2 & & 73.539 \\
\hline 9/23/07 14:30 & 124.258 & 121.855 & 118.233 & 99.596 & 103.538 & 95.208 & 109.129 & 106.169 & & 73.506 \\
\hline 9/23/07 15:00 & 124.235 & 121.832 & 118.213 & 99.575 & 103.512 & 95.184 & 109.118 & 106.142 & & 73.483 \\
\hline 9/23/07 15:30 & 124.216 & 121.818 & 118.198 & 99.553 & 103.497 & 95.168 & 109.11 & 106.122 & & 73.464 \\
\hline 9/23/07 16:00 & 124.192 & 121.809 & 118.185 & 99.535 & 103.476 & 95.149 & 109.105 & 106.103 & & 73.446 \\
\hline $9 / 23 / 0716: 30$ & 124.178 & 121.8 & 118.18 & 99.523 & 103.46 & 95.137 & 109.099 & 106.089 & & 73.431 \\
\hline 9/23/07 17:00 & 124.164 & 121.794 & 118.175 & 99.515 & 103.457 & 95.127 & 109.093 & 106.078 & & 73.421 \\
\hline 9/23/07 17:30 & 124.157 & 121.789 & 118.17 & 99.508 & 103.447 & 95.116 & 109.09 & 106.067 & & 73.413 \\
\hline 9/23/07 18:00 & 124.15 & 121.787 & 118.167 & 99.503 & 103.439 & 95.111 & 109.088 & 106.063 & & 73.405 \\
\hline 9/23/07 18:30 & 124.145 & 121.784 & 118.165 & 99.499 & 103.436 & 95.104 & 109.084 & 106.058 & & 73.4 \\
\hline 9/23/07 19:00 & 124.143 & 121.78 & 118.16 & 99.497 & 103.434 & 95.104 & 109.082 & 106.054 & & 73.396 \\
\hline 9/23/07 19:30 & 124.14 & 121.784 & 118.165 & 99.494 & 103.434 & 95.104 & 109.08 & 106.056 & & 73.396 \\
\hline 9/23/07 20:00 & 124.135 & 121.791 & 118.165 & 99.492 & 103.428 & 95.099 & 109.078 & 106.049 & & 73.39 \\
\hline 9/23/07 20:30 & 124.138 & 121.789 & 118.17 & 99.497 & 103.434 & 95.104 & 109.077 & 106.052 & & 73.394 \\
\hline 9/23/07 21:00 & 124.143 & 121.782 & 118.155 & 99.503 & 103.439 & 95.111 & 109.077 & 106.06 & & 73.4 \\
\hline 9/23/07 21:30 & 124.145 & 121.78 & 118.155 & 99.499 & 103.439 & 95.106 & 109.077 & 106.058 & & 73.398 \\
\hline 9/23/07 22:00 & 124.138 & 121.778 & 118.157 & 99.494 & 103.43 & 95.099 & 109.077 & 106.049 & & 73.388 \\
\hline 9/23/07 22:30 & 124.135 & 121.769 & 118.15 & 99.491 & 103.426 & 95.099 & 109.075 & 106.049 & & 73.388 \\
\hline 9/23/07 23:00 & 124.131 & 121.766 & 118.145 & 99.489 & 103.424 & 95.094 & 109.073 & 106.045 & & 73.384 \\
\hline 9/23/07 23:30 & 124.128 & 121.762 & 118.14 & 99.48 & 103.418 & 95.09 & 109.075 & 106.038 & & 73.376 \\
\hline 9/24/07 0:00 & 124.124 & 121.762 & 118.142 & 99.477 & 103.413 & 95.085 & 109.071 & 106.036 & & 73.376 \\
\hline 9/24/07 0:30 & 124.119 & 121.757 & 118.137 & 99.473 & 103.411 & 95.08 & 109.071 & 106.032 & & 73.369 \\
\hline 9/24/07 1:00 & 124.119 & 121.759 & 118.137 & 99.472 & 103.411 & 95.083 & 109.071 & 106.032 & & 73.371 \\
\hline 9/24/07 1:30 & 124.114 & 121.762 & 118.14 & 99.47 & 103.405 & 95.076 & 109.069 & 106.025 & & 73.367 \\
\hline 9/24/07 2:00 & 124.109 & 121.76 & 118.13 & 99.473 & 103.411 & 95.08 & 109.067 & 106.027 & & 73.367 \\
\hline
\end{tabular}


TABLE S1.1 (Cont.)

Depth from Top of Casing (ft)

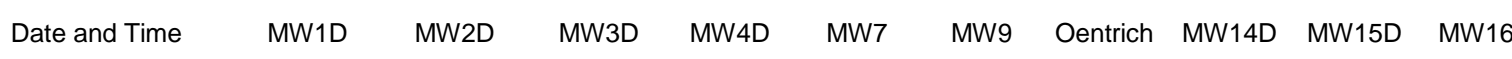

\begin{tabular}{|c|c|c|c|c|c|c|c|c|c|}
\hline 9/24/07 2:30 & 124.117 & 121.748 & 118.122 & 99.473 & 103.411 & 95.085 & 109.065 & 106.029 & 73.371 \\
\hline 9/24/07 3:00 & 124.117 & 121.741 & 118.117 & 99.473 & 103.409 & 95.08 & 109.065 & 106.027 & 73.365 \\
\hline 9/24/07 3:30 & 124.109 & 121.741 & 118.122 & 99.46 & 103.398 & 95.071 & 109.067 & 106.018 & 73.355 \\
\hline 9/24/07 4:00 & 124.1 & 121.744 & 118.117 & 99.454 & 103.392 & 95.064 & 109.067 & 106.01 & 73.349 \\
\hline 9/24/07 4:30 & 124.095 & 121.744 & 118.119 & 99.456 & 103.392 & 95.061 & 109.067 & 106.012 & 73.349 \\
\hline 9/24/07 5:00 & 124.1 & 121.735 & 118.117 & 99.456 & 103.394 & 95.059 & 109.065 & 106.01 & 73.349 \\
\hline 9/24/07 5:30 & 124.098 & 123.494 & 119.268 & 99.454 & 103.392 & 95.061 & 109.065 & 106.012 & 73.349 \\
\hline 9/24/07 6:00 & 124.093 & 123.839 & 119.737 & 99.451 & 103.384 & 95.054 & 109.065 & 106.003 & 73.338 \\
\hline 9/24/07 6:30 & 125.714 & 123.993 & 119.909 & 100.125 & 103.769 & 95.58 & 109.593 & 107.227 & 73.359 \\
\hline 9/24/07 7:00 & 126.232 & 124.086 & 120.012 & 100.392 & 104.106 & 95.927 & 109.925 & 107.646 & 73.56 \\
\hline 9/24/07 7:30 & 126.419 & 124.145 & 120.08 & 100.524 & 104.256 & 96.08 & 110.073 & 107.811 & 73.702 \\
\hline 9/24/07 8:00 & 126.52 & 124.183 & 120.118 & 100.609 & 104.351 & 96.172 & 110.162 & 107.913 & 73.804 \\
\hline 9/24/07 8:30 & 126.584 & 124.222 & 120.161 & 100.662 & 104.41 & 96.233 & 110.218 & 107.975 & 73.87 \\
\hline 9/24/07 9:00 & 126.627 & 124.251 & 120.193 & 100.698 & 104.448 & 96.271 & 110.257 & 108.015 & 73.911 \\
\hline 9/24/07 9:30 & 126.66 & 124.274 & 120.211 & 100.736 & 104.49 & 96.311 & 110.284 & 108.057 & 73.954 \\
\hline 9/24/07 10:00 & 126.698 & 124.286 & 120.229 & 100.769 & 104.521 & 96.339 & 110.304 & 108.09 & 73.987 \\
\hline 9/24/07 10:30 & 126.717 & 124.292 & 120.236 & 100.786 & 104.546 & 96.363 & 110.321 & 108.113 & 74.01 \\
\hline 9/24/07 11:00 & 126.733 & 122.843 & 120.163 & 100.795 & 104.555 & 96.374 & 110.334 & 108.124 & 74.025 \\
\hline 9/24/07 11:30 & 126.74 & 122.313 & 118.737 & 100.802 & 104.561 & 96.384 & 110.349 & 108.133 & 74.035 \\
\hline 9/24/07 12:00 & 125.544 & 122.12 & 118.52 & 100.274 & 104.391 & 96.099 & 110.049 & 107.429 & 74.041 \\
\hline 9/24/07 12:30 & 124.736 & 122.016 & 118.402 & 99.93 & 103.923 & 95.594 & 109.58 & 106.614 & 73.884 \\
\hline 9/24/07 13:00 & 124.507 & 121.948 & 118.331 & 99.773 & 103.742 & 95.408 & 109.42 & 106.406 & 73.729 \\
\hline 9/24/07 13:30 & 124.386 & 121.9 & 118.283 & 99.685 & 103.639 & 95.309 & 109.334 & 106.293 & 73.623 \\
\hline 9/24/07 14:00 & 124.31 & 121.861 & 118.243 & 99.621 & 103.575 & 95.243 & 109.281 & 106.22 & 73.553 \\
\hline 9/24/07 14:30 & 124.263 & 121.837 & 118.22 & 99.58 & 103.529 & 95.198 & 109.244 & 106.169 & 73.502 \\
\hline 9/24/07 15:00 & 124.221 & 121.814 & 118.195 & 99.553 & 103.495 & 95.16 & 109.221 & 106.129 & 73.46 \\
\hline 9/24/07 15:30 & 124.199 & 121.8 & 118.18 & 99.53 & 103.472 & 95.142 & 109.206 & 106.109 & 73.436 \\
\hline 9/24/07 16:00 & 124.173 & 121.791 & 118.17 & 99.511 & 103.449 & 95.123 & 109.191 & 106.085 & 73.417 \\
\hline 9/24/07 16:30 & 124.159 & 121.787 & 118.165 & 99.501 & 103.438 & 95.109 & 109.18 & 106.069 & 73.398 \\
\hline 9/24/07 17:00 & 124.145 & 121.782 & 118.16 & 99.492 & 103.432 & 95.099 & 109.172 & 106.056 & 73.39 \\
\hline 9/24/07 17:30 & 124.143 & 121.78 & 118.16 & 99.492 & 103.426 & 95.094 & 109.165 & 106.056 & 73.386 \\
\hline 9/24/07 18:00 & 124.135 & 121.78 & 118.157 & 99.489 & 103.422 & 95.092 & 109.157 & 106.049 & 73.38 \\
\hline 9/24/07 18:30 & 124.135 & 121.775 & 118.157 & 99.489 & 103.422 & 95.092 & 109.154 & 106.047 & 73.378 \\
\hline 9/24/07 19:00 & 124.133 & 121.782 & 118.155 & 99.487 & 103.42 & 95.09 & 109.15 & 106.045 & 73.374 \\
\hline 9/24/07 19:30 & 124.131 & 121.784 & 118.16 & 99.485 & 103.418 & 95.09 & 109.148 & 106.045 & 73.374 \\
\hline 9/24/07 20:00 & 124.133 & 121.794 & 118.172 & 99.492 & 103.426 & 95.099 & 109.144 & 106.049 & 73.378 \\
\hline 9/24/07 20:30 & 124.138 & 121.798 & 118.175 & 99.494 & 103.43 & 95.102 & 109.142 & 106.054 & 73.38 \\
\hline 9/24/07 21:00 & 124.145 & 121.816 & 118.185 & 99.497 & 103.438 & 95.109 & 109.14 & 106.06 & 73.388 \\
\hline 9/24/07 21:30 & 124.147 & 121.848 & 118.213 & 99.506 & 103.441 & 95.111 & 109.138 & 106.063 & 73.39 \\
\hline 9/24/07 22:00 & 124.157 & 121.88 & 118.258 & 99.532 & 103.462 & 95.127 & 109.133 & 106.08 & 73.405 \\
\hline 9/24/07 22:30 & 124.187 & 121.895 & 118.278 & 99.578 & 103.493 & 95.163 & 109.129 & 106.111 & 73.438 \\
\hline 9/24/07 23:00 & 124.228 & 121.905 & 118.283 & 99.599 & 103.527 & 95.196 & 109.125 & 106.142 & 73.471 \\
\hline 9/24/07 23:30 & 124.249 & 121.914 & 118.293 & 99.611 & 103.546 & 95.215 & 109.125 & 106.165 & 73.491 \\
\hline 9/25/07 0:00 & 124.258 & 121.925 & 118.311 & 99.623 & 103.557 & 95.226 & 109.123 & 106.173 & 73.498 \\
\hline 9/25/07 0:30 & 124.265 & 121.905 & 118.281 & 99.635 & 103.565 & 95.234 & 109.123 & 106.182 & 73.502 \\
\hline 9/25/07 1:00 & 124.287 & 121.92 & 118.303 & 99.635 & 103.58 & 95.248 & 109.12 & 106.198 & 73.518 \\
\hline 9/25/07 1:30 & 124.27 & 121.927 & 118.301 & 99.625 & 103.554 & 95.224 & 109.123 & 106.176 & 73.491 \\
\hline 9/25/07 2:00 & 124.277 & 121.932 & 118.311 & 99.637 & 103.573 & 95.243 & 109.118 & 106.191 & 73.512 \\
\hline 9/25/07 2:30 & 124.277 & 121.945 & 118.321 & 99.646 & 103.573 & 95.243 & 109.116 & 106.189 & 73.508 \\
\hline 9/25/07 3:00 & 124.287 & 121.943 & 118.321 & 99.652 & 103.578 & 95.25 & 109.114 & 106.196 & 73.514 \\
\hline 9/25/07 3:30 & 124.299 & 121.941 & 118.324 & 99.656 & 103.596 & 95.267 & 109.114 & 106.215 & 73.537 \\
\hline 9/25/07 4:00 & 124.299 & 121.945 & 118.321 & 99.663 & 103.592 & 95.264 & 109.114 & 106.209 & 73.524 \\
\hline 9/25/07 4:30 & 124.294 & 121.964 & 118.346 & 99.665 & 103.594 & 95.264 & 109.11 & 106.211 & 73.514 \\
\hline 9/25/07 5:00 & 124.289 & 121.966 & 118.351 & 99.659 & 103.596 & 95.264 & 109.108 & 106.209 & 73.506 \\
\hline 9/25/07 5:30 & 124.282 & 123.292 & 118.349 & 99.68 & 103.618 & 95.29 & 109.105 & 106.235 & 73.493 \\
\hline 9/25/07 6:00 & 124.28 & 123.975 & 119.868 & 99.677 & 103.62 & 95.29 & 109.105 & 106.238 & 73.485 \\
\hline 9/25/07 6:30 & 125.076 & 124.195 & 120.125 & 100.161 & 103.666 & 95.356 & 109.206 & 106.277 & 73.481 \\
\hline 9/25/07 7:00 & 125.953 & 124.324 & 120.251 & 100.574 & 104.269 & 96.087 & 109.873 & 107.781 & 73.615 \\
\hline 9/25/07 7:30 & 126.123 & 124.399 & 120.334 & 100.748 & 104.483 & 96.304 & 110.055 & 108.024 & 73.793 \\
\hline 9/25/07 8:00 & 126.216 & 124.476 & 120.41 & 100.864 & 104.605 & 96.419 & 110.154 & 108.15 & 73.969 \\
\hline 9/25/07 8:30 & 126.279 & 124.517 & 120.443 & 100.936 & 104.675 & 96.497 & 110.222 & 108.234 & 74.082 \\
\hline 9/25/07 9:00 & 126.317 & 124.553 & 120.483 & 100.993 & 104.755 & 96.577 & 110.263 & 108.314 & 74.161 \\
\hline 9/25/07 9:30 & 126.362 & 124.569 & 120.521 & 101.043 & 104.793 & 96.61 & 110.295 & 108.354 & 74.219 \\
\hline 9/25/07 10:00 & 126.395 & 124.576 & 120.528 & 101.074 & 104.829 & 96.648 & 110.318 & 108.394 & 74.262 \\
\hline 9/25/07 10:30 & 126.435 & 124.598 & 120.528 & 101.088 & 104.846 & 96.667 & 110.338 & 108.412 & 74.293 \\
\hline 9/25/07 11:00 & 126.478 & 122.725 & 119.178 & 101.096 & 104.854 & 96.676 & 110.355 & 108.423 & 74.306 \\
\hline
\end{tabular}


TABLE S1.1 (Cont.)

Depth from Top of Casing (ft)

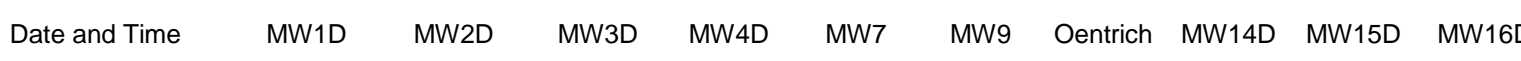

\begin{tabular}{|c|c|c|c|c|c|c|c|c|c|}
\hline 9/25/07 11:30 & 126.511 & 122.496 & 118.906 & 100.95 & 104.875 & 96.695 & 110.365 & 108.436 & 74.308 \\
\hline $9 / 25 / 07$ 12:00 & 124.948 & 122.381 & 118.777 & 100.326 & 104.33 & 96.004 & 109.679 & 107.041 & 74.236 \\
\hline $9 / 25 / 07$ 12:30 & 124.757 & 122.304 & 118.694 & 100.144 & 104.113 & 95.785 & 109.473 & 106.784 & 74.083 \\
\hline 9/25/07 13:00 & 124.677 & 122.252 & 118.641 & 100.049 & 104.005 & 95.674 & 109.368 & 106.658 & 73.967 \\
\hline 9/25/07 13:30 & 124.648 & 122.213 & 118.598 & 99.985 & 103.931 & 95.601 & 109.306 & 106.576 & 73.888 \\
\hline $9 / 25 / 0714: 00$ & 124.615 & 122.186 & 118.568 & 99.94 & 103.883 & 95.556 & 109.266 & 106.528 & 73.837 \\
\hline 9/25/07 14:30 & 124.582 & 122.154 & 118.54 & 99.907 & 103.847 & 95.519 & 109.238 & 106.486 & 73.797 \\
\hline $9 / 25 / 07$ 15:00 & 124.556 & 122.129 & 118.51 & 99.878 & 103.822 & 95.493 & 109.217 & 106.459 & 73.768 \\
\hline $9 / 25 / 0715: 30$ & 124.535 & 122.12 & 118.505 & 99.847 & 103.79 & 95.465 & 109.204 & 106.426 & 73.735 \\
\hline 9/25/07 16:00 & 124.504 & 122.113 & 118.497 & 99.83 & 103.767 & 95.439 & 109.191 & 106.399 & 73.71 \\
\hline $9 / 25 / 07 \quad 16: 30$ & 124.481 & 122.109 & 118.49 & 99.823 & 103.759 & 95.429 & 109.182 & 106.388 & 73.7 \\
\hline $9 / 25 / 07$ 17:00 & 124.471 & 122.102 & 118.482 & 99.818 & 103.754 & 95.427 & 109.174 & 106.384 & 73.696 \\
\hline 9/25/07 17:30 & 124.466 & 122.1 & 118.485 & 99.811 & 103.75 & 95.42 & 109.165 & 106.377 & 73.688 \\
\hline 9/25/07 18:00 & 124.459 & 122.097 & 118.477 & 99.811 & 103.746 & 95.415 & 109.159 & 106.37 & 73.684 \\
\hline $9 / 25 / 0718: 30$ & 124.459 & 122.091 & 118.47 & 99.809 & 103.742 & 95.415 & 109.154 & 106.37 & 73.684 \\
\hline 9/25/07 19:00 & 124.455 & 122.086 & 118.467 & 99.802 & 103.742 & 95.413 & 109.15 & 106.368 & 73.677 \\
\hline 9/25/07 19:30 & 124.452 & 122.095 & 118.472 & 99.795 & 103.735 & 95.403 & 109.148 & 106.364 & 73.669 \\
\hline $9 / 25 / 0720: 00$ & 124.44 & 122.104 & 118.482 & 99.799 & 103.731 & 95.403 & 109.14 & 106.357 & 73.671 \\
\hline 9/25/07 20:30 & 124.431 & 122.111 & 118.487 & 99.806 & 103.738 & 95.406 & 109.135 & 106.362 & 73.677 \\
\hline 9/25/07 21:00 & 124.429 & 122.118 & 118.497 & 99.813 & 103.744 & 95.415 & 109.135 & 106.373 & 73.686 \\
\hline $9 / 25 / 0721: 30$ & 124.421 & 122.122 & 118.497 & 99.821 & 103.754 & 95.424 & 109.129 & 106.379 & 73.694 \\
\hline 9/25/07 22:00 & 124.421 & 122.129 & 118.505 & 99.828 & 103.759 & 95.429 & 109.127 & 106.384 & 73.696 \\
\hline 9/25/07 22:30 & 124.419 & 122.131 & 118.505 & 99.833 & 103.765 & 95.439 & 109.127 & 106.39 & 73.7 \\
\hline 9/25/07 23:00 & 124.419 & 122.131 & 118.513 & 99.839 & 103.773 & 95.443 & 109.125 & 106.397 & 73.708 \\
\hline $9 / 25 / 0723: 30$ & 124.417 & 122.129 & 118.51 & 99.84 & 103.773 & 95.446 & 109.123 & 106.399 & 73.708 \\
\hline 9/26/07 0:00 & 124.417 & 122.127 & 118.508 & 99.842 & 103.774 & 95.448 & 109.123 & 106.401 & 73.71 \\
\hline $9 / 26 / 070: 30$ & 124.414 & 122.118 & 118.502 & 99.839 & 103.773 & 95.446 & 109.12 & 106.397 & 73.708 \\
\hline 9/26/07 1:00 & 124.414 & 122.115 & 118.495 & 99.835 & 103.769 & 95.441 & 109.118 & 106.395 & 73.704 \\
\hline 9/26/07 1:30 & 124.412 & 122.109 & 118.49 & 99.828 & 103.761 & 95.436 & 109.118 & 106.388 & 73.7 \\
\hline 9/26/07 2:00 & 124.407 & 122.097 & 118.482 & 99.825 & 103.757 & 95.432 & 109.114 & 106.384 & 73.696 \\
\hline 9/26/07 2:30 & 124.41 & 122.086 & 118.472 & 99.818 & 103.752 & 95.427 & 109.114 & 106.379 & 73.69 \\
\hline 9/26/07 3:00 & 124.405 & 122.079 & 118.465 & 99.809 & 103.742 & 95.415 & 109.112 & 106.368 & 73.684 \\
\hline 9/26/07 3:30 & 124.405 & 122.068 & 118.447 & 99.797 & 103.733 & 95.406 & 109.11 & 106.357 & 73.673 \\
\hline 9/26/07 4:00 & 124.403 & 122.059 & 118.437 & 99.789 & 103.721 & 95.396 & 109.108 & 106.348 & 73.661 \\
\hline 9/26/07 4:30 & 124.4 & 122.052 & 118.437 & 99.777 & 103.712 & 95.384 & 109.107 & 106.337 & 73.653 \\
\hline 9/26/07 5:00 & 124.4 & 122.047 & 118.429 & 99.768 & 103.704 & 95.375 & 109.105 & 106.326 & 73.642 \\
\hline 9/26/07 5:30 & 124.395 & 122.05 & 118.432 & 99.763 & 103.696 & 95.37 & 109.105 & 106.322 & 73.64 \\
\hline 9/26/07 6:00 & 124.391 & 122.052 & 118.429 & 99.761 & 103.695 & 95.368 & 109.101 & 106.317 & 73.634 \\
\hline $9 / 26 / 076: 30$ & 124.388 & 123.932 & 119.581 & 99.761 & 103.696 & 95.368 & 109.099 & 106.319 & 73.638 \\
\hline 9/26/07 7:00 & 124.386 & 124.254 & 119.999 & 99.768 & 103.698 & 95.368 & 109.099 & 106.322 & 73.638 \\
\hline 9/26/07 7:30 & 125.927 & 124.406 & 120.171 & 100.455 & 104.119 & 95.922 & 109.64 & 107.677 & 73.682 \\
\hline 9/26/07 8:00 & 126.225 & 124.49 & 120.264 & 100.71 & 104.431 & 96.242 & 109.942 & 108.057 & 73.88 \\
\hline $9 / 26 / 078: 30$ & 126.371 & 124.546 & 120.327 & 100.838 & 104.582 & 96.393 & 110.083 & 108.223 & 74.029 \\
\hline 9/26/07 9:00 & 126.468 & 124.594 & 120.375 & 100.912 & 104.669 & 96.48 & 110.167 & 108.314 & 74.122 \\
\hline 9/26/07 9:30 & 126.53 & 122.995 & 119.851 & 100.967 & 104.726 & 96.537 & 110.222 & 108.374 & 74.184 \\
\hline 9/26/07 10:00 & 126.579 & 122.535 & 118.959 & 101.008 & 104.774 & 96.587 & 110.259 & 108.425 & 74.233 \\
\hline $9 / 26 / 07 \quad 10: 30$ & 125.332 & 122.365 & 118.762 & 100.474 & 104.561 & 96.257 & 109.927 & 107.484 & 74.254 \\
\hline 9/26/07 11:00 & 124.904 & 122.267 & 118.659 & 100.161 & 104.15 & 95.82 & 109.521 & 106.833 & 74.097 \\
\hline 9/26/07 11:30 & 124.785 & 122.211 & 118.598 & 100.025 & 103.986 & 95.658 & 109.379 & 106.649 & 73.956 \\
\hline $9 / 26 / 07$ 12:00 & 124.679 & 122.161 & 118.548 & 99.942 & 103.894 & 95.568 & 109.298 & 106.543 & 73.861 \\
\hline $9 / 26 / 07$ 12:30 & 124.601 & 122.113 & 118.5 & 99.89 & 103.837 & 95.509 & 109.249 & 106.483 & 73.799 \\
\hline 9/26/07 13:00 & 124.544 & 122.084 & 118.465 & 99.844 & 103.79 & 95.465 & 109.217 & 106.43 & 73.748 \\
\hline 9/26/07 13:30 & 124.495 & 122.052 & 118.439 & 99.802 & 103.746 & 95.42 & 109.199 & 106.384 & 73.698 \\
\hline 9/26/07 14:00 & 124.457 & 122.036 & 118.419 & 99.775 & 103.715 & 95.391 & 109.184 & 106.355 & 73.667 \\
\hline $9 / 26 / 07$ 14:30 & 124.426 & 122.013 & 118.397 & 99.749 & 103.687 & 95.363 & 109.17 & 106.324 & 73.64 \\
\hline $9 / 26 / 0715: 00$ & 124.405 & 121.993 & 118.379 & 99.733 & 103.672 & 95.344 & 109.161 & 106.304 & 73.626 \\
\hline 9/26/07 15:30 & 124.388 & 121.984 & 118.369 & 99.711 & 103.649 & 95.323 & 109.154 & 106.282 & 73.601 \\
\hline 9/26/07 16:00 & 124.365 & 121.975 & 118.354 & 99.694 & 103.634 & 95.304 & 109.148 & 106.264 & 73.584 \\
\hline $9 / 26 / 0716: 30$ & 124.346 & 121.968 & 118.349 & 99.687 & 103.624 & 95.295 & 109.144 & 106.253 & 73.57 \\
\hline 9/26/07 17:00 & 124.334 & 121.97 & 118.349 & 99.68 & 103.615 & 95.288 & 109.138 & 106.242 & 73.564 \\
\hline 9/26/07 17:30 & 124.327 & 121.964 & 118.344 & 99.675 & 103.607 & 95.278 & 109.135 & 106.233 & 73.558 \\
\hline $9 / 26 / 07$ 18:00 & 124.325 & 121.959 & 118.344 & 99.675 & 103.611 & 95.283 & 109.131 & 106.238 & 73.558 \\
\hline 9/26/07 18:30 & 124.322 & 121.952 & 118.334 & 99.67 & 103.603 & 95.276 & 109.129 & 106.229 & 73.549 \\
\hline 9/26/07 19:00 & 124.322 & 121.959 & 118.341 & 99.663 & 103.601 & 95.274 & 109.127 & 106.229 & 73.549 \\
\hline 9/26/07 19:30 & 124.313 & 121.968 & 118.346 & 99.659 & 103.592 & 95.264 & 109.127 & 106.22 & 73.539 \\
\hline $9 / 26 / 0720: 00$ & 124.313 & 121.975 & 118.356 & 99.668 & 103.605 & 95.271 & 109.123 & 106.226 & 73.545 \\
\hline
\end{tabular}


TABLE S1.1 (Cont.)

\begin{tabular}{|c|c|c|c|c|c|c|c|c|c|c|}
\hline \multirow[b]{2}{*}{ Date and Time } & \multicolumn{10}{|c|}{ Depth from Top of Casing (ft) } \\
\hline & MW1D & MW2D & MW3D & MW4D & MW7 & MW9 & Oentrich & MW14D & MW15D & MW16D \\
\hline 9/26/07 20:30 & 124.317 & 121.975 & 118.356 & 99.678 & 103.611 & 95.281 & 109.12 & 106.235 & & 73.553 \\
\hline 9/26/07 21:00 & 124.327 & 121.975 & 118.354 & 99.683 & 103.615 & 95.285 & 109.118 & 106.24 & & 73.559 \\
\hline 9/26/07 21:30 & 124.329 & 121.977 & 118.359 & 99.682 & 103.616 & 95.285 & 109.12 & 106.242 & & 73.558 \\
\hline 9/26/07 22:00 & 124.327 & 121.975 & 118.356 & 99.68 & 103.615 & 95.288 & 109.118 & 106.242 & & 73.558 \\
\hline 9/26/07 22:30 & 124.327 & 121.973 & 118.356 & 99.687 & 103.618 & 95.29 & 109.118 & 106.244 & & 73.558 \\
\hline 9/26/07 23:00 & 124.325 & 121.973 & 118.356 & 99.683 & 103.616 & 95.29 & 109.116 & 106.242 & & 73.558 \\
\hline 9/26/07 23:30 & 124.322 & 121.968 & 118.351 & 99.68 & 103.615 & 95.285 & 109.116 & 106.24 & & 73.555 \\
\hline 9/27/07 0:00 & 124.322 & 121.964 & 118.346 & 99.682 & 103.616 & 95.288 & 109.116 & 106.242 & & 73.555 \\
\hline 9/27/07 0:30 & 124.32 & 121.964 & 118.346 & 99.677 & 103.611 & 95.281 & 109.116 & 106.235 & & 73.551 \\
\hline 9/27/07 1:00 & 124.315 & 121.97 & 118.351 & 99.671 & 103.607 & 95.276 & 109.116 & 106.231 & & 73.545 \\
\hline 9/27/07 1:30 & 124.313 & 121.97 & 118.354 & 99.673 & 103.605 & 95.278 & 109.114 & 106.231 & & 73.545 \\
\hline 9/27/07 2:00 & 124.31 & 121.964 & 118.346 & 99.68 & 103.613 & 95.283 & 109.11 & 106.235 & & 73.551 \\
\hline 9/27/07 2:30 & 124.31 & 121.966 & 118.346 & 99.677 & 103.613 & 95.285 & 109.108 & 106.235 & & 73.551 \\
\hline 9/27/07 3:00 & 124.308 & 121.964 & 118.346 & 99.673 & 103.607 & 95.278 & 109.108 & 106.231 & & 73.547 \\
\hline 9/27/07 3:30 & 124.306 & 121.959 & 118.344 & 99.675 & 103.611 & 95.281 & 109.107 & 106.231 & & 73.549 \\
\hline 9/27/07 4:00 & 124.303 & 121.973 & 118.349 & 99.675 & 103.611 & 95.278 & 109.107 & 106.231 & & 73.547 \\
\hline 9/27/07 4:30 & 124.301 & 121.977 & 118.359 & 99.673 & 103.607 & 95.276 & 109.105 & 106.229 & & 73.547 \\
\hline 9/27/07 5:00 & 124.296 & 121.975 & 118.356 & 99.683 & 103.62 & 95.288 & 109.101 & 106.238 & & 73.553 \\
\hline 9/27/07 5:30 & 124.296 & 121.979 & 118.361 & 99.69 & 103.624 & 95.292 & 109.101 & 106.244 & & 73.559 \\
\hline 9/27/07 6:00 & 124.296 & 123.875 & 119.72 & 99.689 & 103.618 & 95.288 & 109.101 & 106.24 & & 73.555 \\
\hline 9/27/07 6:30 & 124.299 & 124.149 & 120.06 & 99.692 & 103.624 & 95.295 & 109.101 & 106.246 & & 73.558 \\
\hline 9/27/07 7:00 & 125.887 & 124.299 & 120.216 & 100.478 & 104.146 & 95.962 & 109.756 & 107.652 & & 73.64 \\
\hline 9/27/07 7:30 & 126.135 & 124.392 & 120.322 & 100.7 & 104.416 & 96.235 & 110.006 & 107.96 & & 73.841 \\
\hline 9/27/07 8:00 & 126.267 & 124.451 & 120.385 & 100.828 & 104.559 & 96.381 & 110.132 & 108.119 & & 73.983 \\
\hline 9/27/07 8:30 & 126.357 & 124.496 & 120.43 & 100.908 & 104.65 & 96.471 & 110.209 & 108.215 & & 74.076 \\
\hline 9/27/07 9:00 & 126.426 & 124.533 & 120.476 & 100.962 & 104.707 & 96.528 & 110.261 & 108.279 & & 74.14 \\
\hline 9/27/07 9:30 & 126.475 & 124.56 & 120.501 & 101.003 & 104.749 & 96.572 & 110.299 & 108.325 & & 74.184 \\
\hline 9/27/07 10:00 & 126.516 & 124.589 & 120.528 & 101.037 & 104.789 & 96.612 & 110.325 & 108.367 & & 74.225 \\
\hline 9/27/07 10:30 & 126.556 & 124.603 & 120.549 & 101.067 & 104.816 & 96.641 & 110.346 & 108.396 & & 74.256 \\
\hline 9/27/07 11:00 & 126.601 & 124.612 & 120.556 & 101.089 & 104.843 & 96.667 & 110.366 & 108.425 & & 74.279 \\
\hline 9/27/07 11:30 & 126.752 & 124.621 & 120.566 & 101.105 & 104.86 & 96.683 & 110.381 & 108.438 & & 74.297 \\
\hline 9/27/07 12:00 & 126.991 & 124.628 & 120.571 & 101.115 & 104.869 & 96.693 & 110.391 & 108.449 & & 74.31 \\
\hline 9/27/07 12:30 & 127.066 & 123.347 & 120.571 & 101.124 & 104.879 & 96.704 & 110.402 & 108.46 & & 74.322 \\
\hline 9/27/07 13:00 & 127.081 & 122.655 & 119.087 & 101.127 & 104.884 & 96.707 & 110.411 & 108.467 & & 74.328 \\
\hline 9/27/07 13:30 & 126.258 & 122.453 & 118.855 & 100.648 & 104.824 & 96.601 & 110.28 & 108.288 & & 74.333 \\
\hline 9/27/07 14:00 & 125.135 & 122.34 & 118.732 & 100.257 & 104.252 & 95.927 & 109.657 & 106.955 & & 74.183 \\
\hline 9/27/07 14:30 & 124.859 & 122.27 & 118.659 & 100.094 & 104.06 & 95.733 & 109.486 & 106.736 & & 74.026 \\
\hline 9/27/07 15:00 & 124.724 & 122.224 & 118.611 & 100.001 & 103.952 & 95.625 & 109.39 & 106.612 & & 73.917 \\
\hline 9/27/07 15:30 & 124.646 & 122.188 & 118.573 & 99.94 & 103.887 & 95.561 & 109.33 & 106.541 & & 73.847 \\
\hline 9/27/07 16:00 & 124.594 & 122.161 & 118.545 & 99.902 & 103.845 & 95.516 & 109.292 & 106.492 & & 73.799 \\
\hline 9/27/07 16:30 & 124.556 & 122.145 & 118.528 & 99.87 & 103.811 & 95.483 & 109.264 & 106.455 & & 73.762 \\
\hline 9/27/07 17:00 & 124.525 & 122.134 & 118.513 & 99.849 & 103.788 & 95.462 & 109.244 & 106.428 & & 73.735 \\
\hline 9/27/07 17:30 & 124.504 & 122.129 & 118.51 & 99.837 & 103.773 & 95.446 & 109.227 & 106.41 & & 73.718 \\
\hline 9/27/07 18:00 & 124.492 & 122.129 & 118.508 & 99.828 & 103.763 & 95.434 & 109.214 & 106.397 & & 73.706 \\
\hline 9/27/07 18:30 & 124.485 & 122.129 & 118.51 & 99.828 & 103.759 & 95.432 & 109.204 & 106.395 & & 73.702 \\
\hline 9/27/07 19:00 & 124.483 & 122.134 & 118.508 & 99.828 & 103.757 & 95.432 & 109.197 & 106.393 & & 73.698 \\
\hline 9/27/07 19:30 & 124.483 & 122.14 & 118.518 & 99.83 & 103.761 & 95.432 & 109.191 & 106.393 & & 73.698 \\
\hline 9/27/07 20:00 & 124.485 & 122.149 & 118.528 & 99.837 & 103.767 & 95.436 & 109.184 & 106.397 & & 73.702 \\
\hline 9/27/07 20:30 & 124.495 & 122.154 & 118.533 & 99.845 & 103.773 & 95.446 & 109.18 & 106.406 & & 73.712 \\
\hline 9/27/07 21:00 & 124.502 & 122.165 & 118.545 & 99.854 & 103.782 & 95.453 & 109.176 & 106.412 & & 73.718 \\
\hline 9/27/07 21:30 & 124.509 & 122.168 & 118.55 & 99.858 & 103.788 & 95.46 & 109.172 & 106.419 & & 73.722 \\
\hline 9/27/07 22:00 & 124.516 & 122.177 & 118.55 & 99.868 & 103.799 & 95.472 & 109.169 & 106.428 & & 73.733 \\
\hline 9/27/07 22:30 & 124.523 & 122.186 & 118.56 & 99.875 & 103.803 & 95.476 & 109.167 & 106.435 & & 73.737 \\
\hline 9/27/07 23:00 & 124.523 & 122.188 & 118.568 & 99.88 & 103.809 & 95.483 & 109.165 & 106.439 & & 73.741 \\
\hline 9/27/07 23:30 & 124.521 & 122.195 & 118.57 & 99.892 & 103.822 & 95.493 & 109.161 & 106.45 & & 73.751 \\
\hline 9/28/07 0:00 & 124.516 & 122.193 & 118.57 & 99.897 & 103.824 & 95.498 & 109.159 & 106.455 & & 73.756 \\
\hline 9/28/07 0:30 & 124.514 & 122.195 & 118.568 & 99.897 & 103.83 & 95.502 & 109.155 & 106.461 & & 73.76 \\
\hline 9/28/07 1:00 & 124.511 & 122.193 & 118.573 & 99.894 & 103.824 & 95.5 & 109.155 & 106.457 & & 73.758 \\
\hline 9/28/07 1:30 & 124.509 & 122.193 & 118.576 & 99.899 & 103.826 & 95.5 & 109.154 & 106.457 & & 73.758 \\
\hline 9/28/07 2:00 & 124.507 & 122.186 & 118.568 & 99.897 & 103.828 & 95.502 & 109.154 & 106.459 & & 73.76 \\
\hline 9/28/07 2:30 & 124.502 & 122.186 & 118.565 & 99.895 & 103.826 & 95.5 & 109.152 & 106.457 & & 73.758 \\
\hline 9/28/07 3:00 & 124.502 & 122.181 & 118.563 & 99.892 & 103.824 & 95.498 & 109.15 & 106.452 & & 73.756 \\
\hline 9/28/07 3:30 & 124.497 & 122.177 & 118.555 & 99.892 & 103.822 & 95.495 & 109.148 & 106.452 & & 73.754 \\
\hline 9/28/07 4:00 & 124.495 & 122.186 & 118.563 & 99.889 & 103.818 & 95.49 & 109.148 & 106.448 & & 73.749 \\
\hline 9/28/07 4:30 & 124.495 & 122.188 & 118.565 & 99.883 & 103.814 & 95.488 & 109.146 & 106.441 & & 73.743 \\
\hline 9/28/07 5:00 & 124.488 & 122.181 & 118.563 & 99.895 & 103.822 & 95.495 & 109.142 & 106.45 & & 73.753 \\
\hline
\end{tabular}


TABLE S1.1 (Cont.)

Depth from Top of Casing (ft)

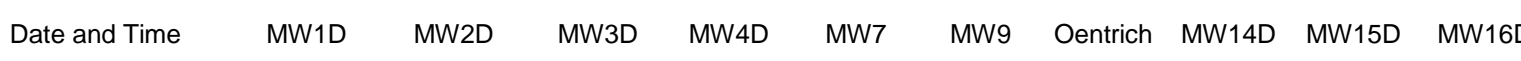

\begin{tabular}{|c|c|c|c|c|c|c|c|c|c|}
\hline 9/28/07 5:30 & 124.485 & 122.19 & 118.57 & 99.895 & 103.83 & 95.5 & 109.142 & 106.457 & 73.758 \\
\hline 9/28/07 6:00 & 124.485 & 124.134 & 119.823 & 99.894 & 103.82 & 95.493 & 109.142 & 106.448 & 73.751 \\
\hline 9/28/07 6:30 & 124.483 & 124.422 & 120.166 & 99.899 & 103.83 & 95.5 & 109.138 & 106.455 & 73.76 \\
\hline 9/28/07 7:00 & 126.088 & 124.555 & 120.324 & 100.64 & 104.317 & 96.125 & 109.749 & 107.898 & 73.835 \\
\hline 9/28/07 7:30 & 126.35 & 124.641 & 120.415 & 100.867 & 104.591 & 96.405 & 110.012 & 108.223 & 74.033 \\
\hline 9/28/07 8:00 & 126.483 & 124.7 & 120.481 & 100.986 & 104.728 & 96.542 & 110.143 & 108.372 & 74.167 \\
\hline 9/28/07 8:30 & 126.575 & 124.737 & 120.526 & 101.062 & 104.814 & 96.627 & 110.222 & 108.465 & 74.256 \\
\hline 9/28/07 9:00 & 126.641 & 122.886 & 119.367 & 101.117 & 104.873 & 96.688 & 110.278 & 108.527 & 74.322 \\
\hline 9/28/07 9:30 & 126.688 & 122.603 & 119.014 & 101.151 & 104.911 & 96.726 & 110.318 & 108.567 & 74.362 \\
\hline 9/28/07 10:00 & 125.171 & 122.474 & 118.868 & 100.459 & 104.479 & 96.158 & 109.754 & 107.214 & 74.328 \\
\hline $9 / 28 / 07 \quad 10: 30$ & 124.937 & 122.388 & 118.782 & 100.245 & 104.212 & 95.886 & 109.508 & 106.888 & 74.163 \\
\hline 9/28/07 11:00 & 124.842 & 122.331 & 118.719 & 100.137 & 104.091 & 95.764 & 109.398 & 106.751 & 74.047 \\
\hline 9/28/07 11:30 & 124.785 & 122.29 & 118.669 & 100.061 & 104.009 & 95.686 & 109.336 & 106.663 & 73.962 \\
\hline $9 / 28 / 07$ 12:00 & 124.719 & 122.247 & 118.636 & 100.013 & 103.952 & 95.627 & 109.294 & 106.603 & 73.898 \\
\hline $9 / 28 / 0712: 30$ & 124.665 & 122.217 & 118.603 & 99.973 & 103.91 & 95.587 & 109.268 & 106.559 & 73.857 \\
\hline 9/28/07 13:00 & 124.622 & 122.19 & 118.57 & 99.935 & 103.868 & 95.545 & 109.251 & 106.512 & 73.811 \\
\hline 9/28/07 13:30 & 124.589 & 122.163 & 118.54 & 99.905 & 103.843 & 95.519 & 109.238 & 106.486 & 73.784 \\
\hline $9 / 28 / 07 \quad 14: 00$ & 124.561 & 122.134 & 118.51 & 99.876 & 103.814 & 95.49 & 109.229 & 106.457 & 73.756 \\
\hline 9/28/07 14:30 & 124.533 & 122.104 & 118.487 & 99.854 & 103.79 & 95.465 & 109.221 & 106.43 & 73.731 \\
\hline $9 / 28 / 07$ 15:00 & 124.504 & 122.079 & 118.465 & 99.823 & 103.759 & 95.436 & 109.214 & 106.399 & 73.702 \\
\hline 9/28/07 15:30 & 124.478 & 122.063 & 118.445 & 99.799 & 103.735 & 95.408 & 109.208 & 106.373 & 73.677 \\
\hline 9/28/07 16:00 & 124.452 & 122.05 & 118.427 & 99.775 & 103.708 & 95.387 & 109.204 & 106.346 & 73.653 \\
\hline 9/28/07 16:30 & 124.429 & 122.036 & 118.419 & 99.761 & 103.693 & 95.368 & 109.2 & 106.328 & 73.638 \\
\hline 9/28/07 17:00 & 124.417 & 122.029 & 118.407 & 99.746 & 103.681 & 95.354 & 109.199 & 106.315 & 73.623 \\
\hline $9 / 28 / 07 \quad 17: 30$ & 124.4 & 122.018 & 118.394 & 99.735 & 103.668 & 95.34 & 109.2 & 106.302 & 73.613 \\
\hline 9/28/07 18:00 & 124.388 & 122.009 & 118.389 & 99.723 & 103.66 & 95.335 & 109.191 & 106.291 & 73.605 \\
\hline 9/28/07 18:30 & 124.379 & 122.018 & 118.382 & 99.719 & 103.651 & 95.323 & 109.187 & 106.282 & 73.594 \\
\hline 9/28/07 19:00 & 124.37 & 122.018 & 118.374 & 99.713 & 103.643 & 95.318 & 109.185 & 106.275 & 73.586 \\
\hline 9/28/07 19:30 & 124.372 & 122.022 & 118.392 & 99.721 & 103.653 & 95.323 & 109.184 & 106.282 & 73.594 \\
\hline 9/28/07 20:00 & 124.369 & 122.027 & 118.407 & 99.721 & 103.651 & 95.323 & 109.18 & 106.28 & 73.594 \\
\hline $9 / 28 / 0720: 30$ & 124.372 & 122.025 & 118.402 & 99.727 & 103.653 & 95.323 & 109.178 & 106.28 & 73.593 \\
\hline 9/28/07 21:00 & 124.381 & 122.02 & 118.394 & 99.728 & 103.662 & 95.333 & 109.174 & 106.291 & 73.603 \\
\hline 9/28/07 21:30 & 124.381 & 122.043 & 118.414 & 99.725 & 103.656 & 95.33 & 109.176 & 106.286 & 73.596 \\
\hline 9/28/07 22:00 & 124.374 & 122.038 & 118.422 & 99.727 & 103.653 & 95.323 & 109.176 & 106.282 & 73.59 \\
\hline 9/28/07 22:30 & 124.388 & 122.038 & 118.419 & 99.746 & 103.674 & 95.347 & 109.172 & 106.302 & 73.613 \\
\hline 9/28/07 23:00 & 124.398 & 122.059 & 118.434 & 99.74 & 103.675 & 95.347 & 109.174 & 106.304 & 73.611 \\
\hline 9/28/07 23:30 & 124.398 & 122.045 & 118.427 & 99.74 & 103.674 & 95.344 & 109.172 & 106.304 & 73.609 \\
\hline 9/29/07 0:00 & 124.407 & 122.047 & 118.419 & 99.761 & 103.689 & 95.363 & 109.17 & 106.319 & 73.625 \\
\hline 9/29/07 0:30 & 124.407 & 122.054 & 118.432 & 99.747 & 103.679 & 95.351 & 109.172 & 106.308 & 73.613 \\
\hline 9/29/07 1:00 & 124.398 & 122.056 & 118.432 & 99.752 & 103.679 & 95.351 & 109.17 & 106.306 & 73.613 \\
\hline 9/29/07 1:30 & 124.407 & 122.054 & 118.439 & 99.754 & 103.685 & 95.361 & 109.169 & 106.315 & 73.619 \\
\hline 9/29/07 2:00 & 124.407 & 122.05 & 118.427 & 99.761 & 103.695 & 95.368 & 109.167 & 106.322 & 73.629 \\
\hline 9/29/07 2:30 & 124.417 & 122.038 & 118.422 & 99.754 & 103.693 & 95.366 & 109.165 & 106.322 & 73.626 \\
\hline 9/29/07 3:00 & 124.407 & 122.025 & 118.407 & 99.754 & 103.685 & 95.356 & 109.167 & 106.313 & 73.617 \\
\hline 9/29/07 3:30 & 124.403 & 122.013 & 118.394 & 99.74 & 103.677 & 95.347 & 109.167 & 106.302 & 73.607 \\
\hline 9/29/07 4:00 & 124.391 & 122.004 & 118.389 & 99.727 & 103.66 & 95.335 & 109.169 & 106.288 & 73.592 \\
\hline 9/29/07 4:30 & 124.381 & 121.993 & 118.371 & 99.718 & 103.649 & 95.323 & 109.169 & 106.277 & 73.582 \\
\hline 9/29/07 5:00 & 124.372 & 121.991 & 118.374 & 99.706 & 103.643 & 95.316 & 109.165 & 106.271 & 73.576 \\
\hline 9/29/07 5:30 & 124.362 & 122 & 118.376 & 99.699 & 103.63 & 95.304 & 109.167 & 106.257 & 73.563 \\
\hline 9/29/07 6:00 & 124.353 & 123.789 & 119.563 & 99.699 & 103.626 & 95.302 & 109.165 & 106.255 & 73.563 \\
\hline 9/29/07 6:30 & 124.348 & 124.14 & 120.029 & 99.708 & 103.634 & 95.309 & 109.161 & 106.26 & 73.57 \\
\hline 9/29/07 7:00 & 125.908 & 124.306 & 120.218 & 100.405 & 104.041 & 95.856 & 109.704 & 107.519 & 73.598 \\
\hline 9/29/07 7:30 & 126.386 & 124.41 & 120.329 & 100.678 & 104.386 & 96.207 & 110.064 & 107.94 & 73.807 \\
\hline 9/29/07 8:00 & 126.629 & 124.474 & 120.397 & 100.819 & 104.544 & 96.37 & 110.202 & 108.119 & 73.96 \\
\hline 9/29/07 8:30 & 126.783 & 124.521 & 120.448 & 100.912 & 104.643 & 96.469 & 110.28 & 108.221 & 74.064 \\
\hline 9/29/07 9:00 & 126.882 & 124.555 & 120.483 & 100.967 & 104.709 & 96.537 & 110.335 & 108.296 & 74.136 \\
\hline 9/29/07 9:30 & 126.951 & 124.567 & 120.506 & 101.012 & 104.753 & 96.582 & 110.368 & 108.343 & 74.181 \\
\hline 9/29/07 10:00 & 126.998 & 124.585 & 120.516 & 101.041 & 104.787 & 96.617 & 110.395 & 108.381 & 74.217 \\
\hline 9/29/07 10:30 & 127.022 & 124.592 & 120.528 & 101.056 & 104.808 & 96.631 & 110.419 & 108.401 & 74.24 \\
\hline 9/29/07 11:00 & 127.036 & 124.598 & 120.533 & 101.068 & 104.818 & 96.641 & 110.434 & 108.409 & 74.252 \\
\hline 9/29/07 11:30 & 127.043 & 124.608 & 120.539 & 101.076 & 104.825 & 96.653 & 110.447 & 108.42 & 74.262 \\
\hline $9 / 29 / 0712: 00$ & 127.05 & 124.412 & 120.554 & 101.086 & 104.833 & 96.66 & 110.458 & 108.425 & 74.272 \\
\hline $9 / 29 / 0712: 30$ & 127.057 & 122.66 & 119.1 & 101.089 & 104.843 & 96.664 & 110.475 & 108.434 & 74.283 \\
\hline 9/29/07 13:00 & 127.064 & 122.428 & 118.83 & 100.729 & 104.852 & 96.676 & 110.481 & 108.443 & 74.289 \\
\hline 9/29/07 13:30 & 125.128 & 122.304 & 118.699 & 100.243 & 104.249 & 95.922 & 109.751 & 106.964 & 74.159 \\
\hline 9/29/07 14:00 & 124.823 & 122.224 & 118.606 & 100.059 & 104.026 & 95.703 & 109.557 & 106.705 & 73.987 \\
\hline
\end{tabular}


TABLE S1.1 (Cont.)

\begin{tabular}{|c|c|c|c|c|c|c|c|c|c|c|}
\hline \multirow[b]{2}{*}{ Date and Time } & \multicolumn{10}{|c|}{ Depth from Top of Casing (ft) } \\
\hline & MW1D & MW2D & MW3D & MW4D & MW7 & MW9 & Oentrich & MW14D & MW15D & MW16D \\
\hline 9/29/07 14:30 & 124.682 & 122.17 & 118.558 & 99.954 & 103.906 & 95.582 & 109.458 & 106.574 & & 73.867 \\
\hline 9/29/07 15:00 & 124.592 & 122.127 & 118.51 & 99.883 & 103.826 & 95.502 & 109.399 & 106.488 & & 73.787 \\
\hline 9/29/07 15:30 & 124.533 & 122.106 & 118.482 & 99.842 & 103.788 & 95.457 & 109.356 & 106.437 & & 73.737 \\
\hline 9/29/07 16:00 & 124.495 & 122.079 & 118.46 & 99.808 & 103.742 & 95.417 & 109.334 & 106.393 & & 73.692 \\
\hline 9/29/07 16:30 & 124.464 & 122.056 & 118.434 & 99.785 & 103.721 & 95.396 & 109.311 & 106.366 & & 73.667 \\
\hline 9/29/07 17:00 & 124.44 & 122.047 & 118.412 & 99.761 & 103.695 & 95.373 & 109.296 & 106.339 & & 73.642 \\
\hline 9/29/07 17:30 & 124.417 & 122.036 & 118.397 & 99.744 & 103.677 & 95.351 & 109.285 & 106.322 & & 73.624 \\
\hline 9/29/07 18:00 & 124.405 & 122.025 & 118.392 & 99.735 & 103.664 & 95.34 & 109.277 & 106.311 & & 73.609 \\
\hline 9/29/07 18:30 & 124.391 & 122.013 & 118.379 & 99.728 & 103.655 & 95.33 & 109.268 & 106.297 & & 73.599 \\
\hline 9/29/07 19:00 & 124.386 & 122.013 & 118.382 & 99.72 & 103.651 & 95.321 & 109.264 & 106.288 & & 73.589 \\
\hline 9/29/07 19:30 & 124.374 & 122.009 & 118.376 & 99.711 & 103.639 & 95.314 & 109.259 & 106.275 & & 73.578 \\
\hline 9/29/07 20:00 & 124.372 & 122 & 118.379 & 99.709 & 103.641 & 95.314 & 109.255 & 106.277 & & 73.578 \\
\hline 9/29/07 20:30 & 124.369 & 121.995 & 118.374 & 99.702 & 103.636 & 95.311 & 109.251 & 106.271 & & 73.576 \\
\hline 9/29/07 21:00 & 124.36 & 121.998 & 118.376 & 99.69 & 103.626 & 95.295 & 109.249 & 106.262 & & 73.562 \\
\hline 9/29/07 21:30 & 124.351 & 121.986 & 118.366 & 99.694 & 103.62 & 95.292 & 109.246 & 106.255 & & 73.558 \\
\hline 9/29/07 22:00 & 124.355 & 121.998 & 118.376 & 99.692 & 103.622 & 95.297 & 109.246 & 106.257 & & 73.56 \\
\hline 9/29/07 22:30 & 124.346 & 122.002 & 118.379 & 99.685 & 103.613 & 95.283 & 109.246 & 106.246 & & 73.549 \\
\hline 9/29/07 23:00 & 124.348 & 122.013 & 118.389 & 99.697 & 103.618 & 95.292 & 109.24 & 106.253 & & 73.555 \\
\hline 9/29/07 23:30 & 124.355 & 122.018 & 118.399 & 99.696 & 103.63 & 95.302 & 109.242 & 106.262 & & 73.566 \\
\hline 9/30/07 0:00 & 124.36 & 122.002 & 118.384 & 99.711 & 103.639 & 95.311 & 109.236 & 106.275 & & 73.572 \\
\hline 9/30/07 0:30 & 124.374 & 121.998 & 118.369 & 99.715 & 103.645 & 95.316 & 109.232 & 106.28 & & 73.578 \\
\hline 9/30/07 1:00 & 124.367 & 122.011 & 118.387 & 99.697 & 103.628 & 95.3 & 109.236 & 106.262 & & 73.56 \\
\hline 9/30/07 1:30 & 124.355 & 122.002 & 118.382 & 99.696 & 103.626 & 95.295 & 109.234 & 106.257 & & 73.555 \\
\hline 9/30/07 2:00 & 124.36 & 121.991 & 118.371 & 99.706 & 103.636 & 95.307 & 109.232 & 106.271 & & 73.568 \\
\hline 9/30/07 2:30 & 124.358 & 121.977 & 118.356 & 99.697 & 103.624 & 95.3 & 109.234 & 106.26 & & 73.558 \\
\hline 9/30/07 3:00 & 124.351 & 121.968 & 118.349 & 99.687 & 103.616 & 95.29 & 109.234 & 106.253 & & 73.547 \\
\hline 9/30/07 3:30 & 124.336 & 121.95 & 118.336 & 99.675 & 103.603 & 95.276 & 109.236 & 106.235 & & 73.531 \\
\hline 9/30/07 4:00 & 124.327 & 121.939 & 118.321 & 99.666 & 103.597 & 95.269 & 109.232 & 106.229 & & 73.529 \\
\hline 9/30/07 4:30 & 124.317 & 121.932 & 118.313 & 99.646 & 103.58 & 95.252 & 109.236 & 106.213 & & 73.51 \\
\hline 9/30/07 5:00 & 124.299 & 121.925 & 118.306 & 99.639 & 103.565 & 95.234 & 109.234 & 106.196 & & 73.495 \\
\hline 9/30/07 5:30 & 124.291 & 121.918 & 118.298 & 99.632 & 103.561 & 95.234 & 109.232 & 106.191 & & 73.493 \\
\hline 9/30/07 6:00 & 124.284 & 121.921 & 118.303 & 99.62 & 103.552 & 95.226 & 109.232 & 106.182 & & 73.487 \\
\hline 9/30/07 6:30 & 124.275 & 121.92 & 118.303 & 99.621 & 103.548 & 95.226 & 109.23 & 106.182 & & 73.483 \\
\hline 9/30/07 7:00 & 124.277 & 123.902 & 119.591 & 99.621 & 103.55 & 95.222 & 109.229 & 106.182 & & 73.481 \\
\hline 9/30/07 7:30 & 124.277 & 124.165 & 119.914 & 99.618 & 103.548 & 95.222 & 109.229 & 106.182 & & 73.479 \\
\hline 9/30/07 8:00 & 126.189 & 124.294 & 120.06 & 100.39 & 104.068 & 95.877 & 109.882 & 107.67 & & 73.56 \\
\hline 9/30/07 8:30 & 126.551 & 124.367 & 120.14 & 100.6 & 104.329 & 96.141 & 110.122 & 107.962 & & 73.764 \\
\hline 9/30/07 9:00 & 126.705 & 124.419 & 120.198 & 100.712 & 104.452 & 96.266 & 110.242 & 108.102 & & 73.888 \\
\hline 9/30/07 9:30 & 126.788 & 124.458 & 120.229 & 100.778 & 104.53 & 96.339 & 110.323 & 108.188 & & 73.969 \\
\hline 9/30/07 10:00 & 126.835 & 124.483 & 120.266 & 100.826 & 104.582 & 96.393 & 110.37 & 108.241 & & 74.028 \\
\hline 9/30/07 10:30 & 126.877 & 124.501 & 120.287 & 100.857 & 104.614 & 96.429 & 110.41 & 108.279 & & 74.068 \\
\hline 9/30/07 11:00 & 126.903 & 124.53 & 120.309 & 100.884 & 104.645 & 96.457 & 110.434 & 108.305 & & 74.097 \\
\hline 9/30/07 11:30 & 126.922 & 124.535 & 120.324 & 100.903 & 104.671 & 96.473 & 110.453 & 108.332 & & 74.128 \\
\hline 9/30/07 12:00 & 126.939 & 124.537 & 120.329 & 100.929 & 104.69 & 96.504 & 110.468 & 108.354 & & 74.153 \\
\hline 9/30/07 12:30 & 126.953 & 122.571 & 119.016 & 100.933 & 104.702 & 96.516 & 110.486 & 108.365 & & 74.163 \\
\hline 9/30/07 13:00 & 126.958 & 122.353 & 118.752 & 100.743 & 104.705 & 96.521 & 110.489 & 108.365 & & 74.167 \\
\hline 9/30/07 13:30 & 125.026 & 122.258 & 118.651 & 100.149 & 104.153 & 95.828 & 109.817 & 106.871 & & 74.053 \\
\hline 9/30/07 14:00 & 124.724 & 122.229 & 118.618 & 99.985 & 103.95 & 95.618 & 109.61 & 106.625 & & 73.909 \\
\hline 9/30/07 14:30 & 124.613 & 122.206 & 118.593 & 99.926 & 103.866 & 95.531 & 109.508 & 106.523 & & 73.824 \\
\hline 9/30/07 15:00 & 124.575 & 122.202 & 118.586 & 99.904 & 103.845 & 95.512 & 109.446 & 106.494 & & 73.797 \\
\hline 9/30/07 15:30 & 124.559 & 122.186 & 118.578 & 99.889 & 103.824 & 95.498 & 109.394 & 106.466 & & 73.768 \\
\hline 9/30/07 16:00 & 124.551 & 122.181 & 118.563 & 99.892 & 103.822 & 95.493 & 109.369 & 106.463 & & 73.76 \\
\hline 9/30/07 16:30 & 124.549 & 122.188 & 118.57 & 99.868 & 103.803 & 95.476 & 109.349 & 106.446 & & 73.739 \\
\hline 9/30/07 17:00 & 124.53 & 122.188 & 118.563 & 99.874 & 103.801 & 95.472 & 109.334 & 106.437 & & 73.733 \\
\hline 9/30/07 17:30 & 124.54 & 122.204 & 118.578 & 99.881 & 103.809 & 95.481 & 109.322 & 106.446 & & 73.735 \\
\hline 9/30/07 18:00 & 124.54 & 122.206 & 118.591 & 99.883 & 103.811 & 95.483 & 109.309 & 106.448 & & 73.737 \\
\hline 9/30/07 18:30 & 124.551 & 122.22 & 118.601 & 99.906 & 103.82 & 95.49 & 109.306 & 106.455 & & 73.739 \\
\hline 9/30/07 19:00 & 124.561 & 122.229 & 118.608 & 99.902 & 103.837 & 95.507 & 109.302 & 106.47 & & 73.754 \\
\hline 9/30/07 19:30 & 124.57 & 122.24 & 118.613 & 99.921 & 103.843 & 95.516 & 109.294 & 106.477 & & 73.762 \\
\hline 9/30/07 20:00 & 124.582 & 122.251 & 118.633 & 99.928 & 103.856 & 95.528 & 109.292 & 106.488 & & 73.768 \\
\hline 9/30/07 20:30 & 124.587 & 122.263 & 118.641 & 99.945 & 103.868 & 95.54 & 109.289 & 106.499 & & 73.783 \\
\hline 9/30/07 21:00 & 124.599 & 122.261 & 118.646 & 99.956 & 103.877 & 95.547 & 109.281 & 106.51 & & 73.787 \\
\hline 9/30/07 21:30 & 124.599 & 122.265 & 118.649 & 99.964 & 103.889 & 95.561 & 109.281 & 106.521 & & 73.801 \\
\hline 9/30/07 22:00 & 124.599 & 122.265 & 118.646 & 99.966 & 103.887 & 95.559 & 109.279 & 106.521 & & 73.799 \\
\hline 9/30/07 22:30 & 124.594 & 122.267 & 118.649 & 99.964 & 103.893 & 95.566 & 109.277 & 106.528 & & 73.805 \\
\hline 9/30/07 23:00 & 124.589 & 122.276 & 118.659 & 99.966 & 103.891 & 95.561 & 109.277 & 106.525 & & 73.803 \\
\hline
\end{tabular}


TABLE S1.1 (Cont.)

Depth from Top of Casing (ft)

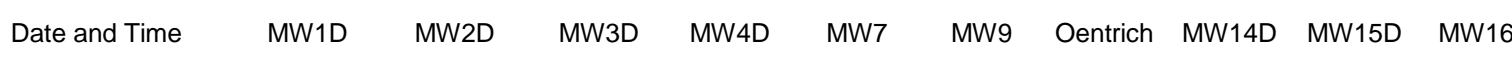

\begin{tabular}{|c|c|c|c|c|c|c|c|c|c|}
\hline 9/30/07 23:30 & 124.589 & 122.288 & 118.666 & 99.971 & 103.893 & 95.566 & 109.277 & 106.525 & 73.804 \\
\hline $10 / 1 / 070: 00$ & 124.585 & 122.29 & 118.674 & 99.982 & 103.902 & 95.575 & 109.276 & 106.534 & 73.812 \\
\hline 10/1/07 0:30 & 124.582 & 122.306 & 118.684 & 99.995 & 103.915 & 95.587 & 109.277 & 106.548 & 73.826 \\
\hline 10/1/07 1:00 & 124.582 & 122.315 & 118.699 & 99.994 & 103.917 & 95.589 & 109.291 & 106.552 & 73.828 \\
\hline 10/1/07 1:30 & 124.58 & 122.326 & 118.709 & 100.009 & 103.931 & 95.606 & 109.287 & 106.565 & 73.841 \\
\hline $10 / 1 / 072: 00$ & 124.575 & 122.319 & 118.704 & 100.021 & 103.946 & 95.618 & 109.292 & 106.576 & 73.853 \\
\hline $10 / 1 / 072: 30$ & 124.577 & 122.322 & 118.699 & 100.028 & 103.952 & 95.627 & 109.292 & 106.585 & 73.861 \\
\hline 10/1/07 3:00 & 124.58 & 122.317 & 118.699 & 100.025 & 103.944 & 95.62 & 109.294 & 106.581 & 73.853 \\
\hline 10/1/07 3:30 & 124.58 & 122.322 & 118.702 & 100.025 & 103.948 & 95.622 & 109.296 & 106.583 & 73.855 \\
\hline 10/1/07 4:00 & 124.58 & 122.324 & 118.709 & 100.021 & 103.944 & 95.618 & 109.294 & 106.579 & 73.851 \\
\hline 10/1/07 4:30 & 124.577 & 122.324 & 118.704 & 100.025 & 103.948 & 95.622 & 109.292 & 106.581 & 73.859 \\
\hline $10 / 1 / 075: 00$ & 124.575 & 122.326 & 118.702 & 100.026 & 103.952 & 95.627 & 109.291 & 106.585 & 73.863 \\
\hline 10/1/07 5:30 & 124.577 & 122.331 & 118.717 & 100.026 & 103.95 & 95.622 & 109.292 & 106.581 & 73.859 \\
\hline 10/1/07 6:00 & 124.575 & 124.172 & 119.803 & 100.035 & 103.953 & 95.625 & 109.289 & 106.585 & 73.861 \\
\hline 10/1/07 6:30 & 124.575 & 124.528 & 120.261 & 100.037 & 103.957 & 95.634 & 109.289 & 106.592 & 73.868 \\
\hline 10/1/07 7:00 & 126.088 & 124.682 & 120.443 & 100.705 & 104.349 & 96.155 & 109.805 & 107.9 & 73.898 \\
\hline $10 / 1 / 077: 30$ & 126.409 & 124.768 & 120.544 & 100.972 & 104.686 & 96.499 & 110.126 & 108.319 & 74.105 \\
\hline $10 / 1 / 078: 00$ & 126.561 & 124.83 & 120.609 & 101.107 & 104.839 & 96.653 & 110.286 & 108.489 & 74.254 \\
\hline 10/1/07 8:30 & 126.66 & 124.884 & 120.659 & 101.182 & 104.93 & 96.744 & 110.376 & 108.589 & 74.351 \\
\hline 10/1/07 9:00 & 126.728 & 124.904 & 120.687 & 101.245 & 104.991 & 96.803 & 110.432 & 108.651 & 74.415 \\
\hline 10/1/07 9:30 & 126.785 & 124.934 & 120.705 & 101.286 & 105.044 & 96.86 & 110.473 & 108.708 & 74.471 \\
\hline $10 / 1 / 07 \quad 10: 00$ & 126.832 & 124.95 & 120.74 & 101.303 & 105.062 & 96.879 & 110.503 & 108.728 & 74.492 \\
\hline $10 / 1 / 07 \quad 10: 30$ & 126.873 & 124.957 & 120.748 & 101.341 & 105.094 & 96.907 & 110.52 & 108.755 & 74.523 \\
\hline 10/1/07 11:00 & 126.958 & 123.258 & 119.888 & 101.351 & 105.113 & 96.928 & 110.539 & 108.779 & 74.546 \\
\hline $10 / 1 / 0711: 30$ & 127.227 & 122.85 & 119.271 & 101.362 & 105.121 & 96.935 & 110.552 & 108.788 & 74.558 \\
\hline $10 / 1 / 07 \quad 12: 00$ & 125.875 & 122.669 & 119.069 & 100.75 & 104.816 & 96.504 & 110.132 & 107.666 & 74.539 \\
\hline $10 / 1 / 07 \quad 12: 30$ & 125.277 & 122.562 & 118.959 & 100.455 & 104.439 & 96.113 & 109.775 & 107.139 & 74.362 \\
\hline $10 / 1 / 0713: 00$ & 125.062 & 122.483 & 118.87 & 100.312 & 104.271 & 95.945 & 109.632 & 106.946 & 74.214 \\
\hline 10/1/07 13:30 & 124.941 & 122.428 & 118.817 & 100.219 & 104.165 & 95.842 & 109.552 & 106.833 & 74.107 \\
\hline $10 / 1 / 07 \quad 14: 00$ & 124.859 & 122.39 & 118.771 & 100.15 & 104.091 & 95.764 & 109.503 & 106.749 & 74.027 \\
\hline $10 / 1 / 07 \quad 14: 30$ & 124.802 & 122.353 & 118.734 & 100.104 & 104.039 & 95.714 & 109.471 & 106.694 & 73.971 \\
\hline $10 / 1 / 07$ 15:00 & 124.759 & 122.317 & 118.704 & 100.068 & 104.001 & 95.674 & 109.446 & 106.652 & 73.931 \\
\hline $10 / 1 / 07 \quad 15: 30$ & 124.724 & 122.295 & 118.681 & 100.032 & 103.963 & 95.641 & 109.428 & 106.616 & 73.892 \\
\hline 10/1/07 16:00 & 124.691 & 122.263 & 118.649 & 99.999 & 103.931 & 95.606 & 109.414 & 106.581 & 73.861 \\
\hline 10/1/07 16:30 & 124.662 & 122.238 & 118.618 & 99.975 & 103.91 & 95.585 & 109.403 & 106.556 & 73.841 \\
\hline $10 / 1 / 07 \quad 17: 00$ & 124.632 & 122.231 & 118.611 & 99.951 & 103.879 & 95.556 & 109.396 & 106.523 & 73.806 \\
\hline $10 / 1 / 07 \quad 17: 30$ & 124.606 & 122.213 & 118.598 & 99.925 & 103.853 & 95.531 & 109.388 & 106.499 & 73.783 \\
\hline 10/1/07 18:00 & 124.589 & 122.195 & 118.576 & 99.92 & 103.849 & 95.523 & 109.383 & 106.492 & 73.777 \\
\hline $10 / 1 / 07$ 18:30 & 124.58 & 122.177 & 118.558 & 99.902 & 103.828 & 95.505 & 109.379 & 106.474 & 73.758 \\
\hline $10 / 1 / 07$ 19:00 & 124.561 & 122.165 & 118.548 & 99.883 & 103.813 & 95.488 & 109.377 & 106.455 & 73.741 \\
\hline 10/1/07 19:30 & 124.544 & 122.159 & 118.538 & 99.868 & 103.801 & 95.474 & 109.375 & 106.441 & 73.727 \\
\hline $10 / 1 / 0720: 00$ & 124.528 & 122.152 & 118.535 & 99.858 & 103.784 & 95.46 & 109.369 & 106.424 & 73.713 \\
\hline $10 / 1 / 0720: 30$ & 124.521 & 122.143 & 118.525 & 99.85 & 103.776 & 95.453 & 109.364 & 106.417 & 73.706 \\
\hline 10/1/07 21:00 & 124.514 & 122.138 & 118.52 & 99.845 & 103.774 & 95.446 & 109.362 & 106.41 & 73.7 \\
\hline 10/1/07 21:30 & 124.504 & 122.124 & 118.5 & 99.837 & 103.767 & 95.441 & 109.356 & 106.404 & 73.694 \\
\hline $10 / 1 / 0722: 00$ & 124.497 & 122.116 & 118.495 & 99.832 & 103.759 & 95.434 & 109.354 & 106.399 & 73.688 \\
\hline 10/1/07 22:30 & 124.49 & 122.1 & 118.48 & 99.816 & 103.746 & 95.42 & 109.356 & 106.384 & 73.671 \\
\hline $10 / 1 / 07$ 23:00 & 124.476 & 122.09 & 118.475 & 99.808 & 103.733 & 95.408 & 109.353 & 106.373 & 73.659 \\
\hline 10/1/07 23:30 & 124.464 & 122.075 & 118.477 & 99.792 & 103.719 & 95.394 & 109.353 & 106.357 & 73.644 \\
\hline $10 / 2 / 070: 00$ & 124.455 & 122.059 & 118.465 & 99.783 & 103.712 & 95.382 & 109.354 & 106.348 & 73.636 \\
\hline $10 / 2 / 070: 30$ & 124.44 & 122.05 & 118.45 & 99.763 & 103.695 & 95.368 & 109.353 & 106.331 & 73.617 \\
\hline 10/2/07 1:00 & 124.426 & 122.05 & 118.447 & 99.747 & 103.679 & 95.354 & 109.356 & 106.319 & 73.605 \\
\hline 10/2/07 1:30 & 124.41 & 122.034 & 118.437 & 99.742 & 103.672 & 95.344 & 109.349 & 106.308 & 73.599 \\
\hline $10 / 2 / 072: 00$ & 124.405 & 122.023 & 118.417 & 99.74 & 103.668 & 95.342 & 109.349 & 106.304 & 73.593 \\
\hline $10 / 2 / 072: 30$ & 124.398 & 122.016 & 118.414 & 99.723 & 103.655 & 95.328 & 109.349 & 106.291 & 73.58 \\
\hline $10 / 2 / 073: 00$ & 124.384 & 122 & 118.397 & 99.715 & 103.641 & 95.316 & 109.347 & 106.282 & 73.57 \\
\hline 10/2/07 3:30 & 124.374 & 121.984 & 118.382 & 99.704 & 103.636 & 95.307 & 109.349 & 106.271 & 73.56 \\
\hline $10 / 2 / 074: 00$ & 124.362 & 121.959 & 118.364 & 99.687 & 103.626 & 95.295 & 109.349 & 106.26 & 73.549 \\
\hline $10 / 2 / 074: 30$ & 124.348 & 121.948 & 118.344 & 99.67 & 103.603 & 95.276 & 109.351 & 106.242 & 73.529 \\
\hline 10/2/07 5:00 & 124.327 & 121.934 & 118.331 & 99.646 & 103.582 & 95.252 & 109.351 & 106.218 & 73.506 \\
\hline $10 / 2 / 075: 30$ & 124.308 & 121.92 & 118.324 & 99.639 & 103.565 & 95.238 & 109.353 & 106.202 & 73.491 \\
\hline $10 / 2 / 076: 00$ & 124.296 & 123.777 & 119.636 & 99.62 & 103.554 & 95.224 & 109.353 & 106.191 & 73.481 \\
\hline $10 / 2 / 076: 30$ & 124.282 & 124.059 & 119.987 & 99.615 & 103.542 & 95.215 & 109.353 & 106.176 & 73.467 \\
\hline $10 / 2 / 07$ 7:00 & 126.074 & 124.186 & 120.128 & 100.355 & 104.016 & 95.837 & 109.985 & 107.539 & 73.516 \\
\hline $10 / 2 / 07$ 7:30 & 126.473 & 124.281 & 120.211 & 100.581 & 104.292 & 96.115 & 110.254 & 107.856 & 73.706 \\
\hline 10/2/07 8:00 & 126.629 & 124.333 & 120.266 & 100.688 & 104.42 & 96.242 & 110.389 & 107.998 & 73.828 \\
\hline
\end{tabular}


TABLE S1.1 (Cont.)

Depth from Top of Casing (ft)

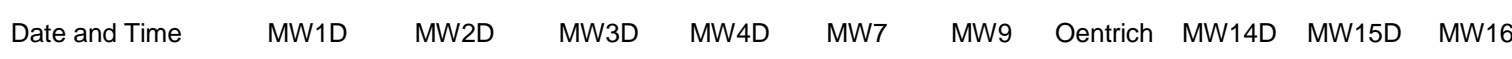

\begin{tabular}{|c|c|c|c|c|c|c|c|c|c|}
\hline 10/2/07 8:30 & 126.707 & 124.369 & 120.307 & 100.783 & 104.508 & 96.33 & 110.466 & 108.084 & 73.919 \\
\hline 10/2/07 9:00 & 126.787 & 124.383 & 120.319 & 100.831 & 104.563 & 96.386 & 110.518 & 108.153 & 73.979 \\
\hline 10/2/07 9:30 & 126.811 & 124.39 & 120.337 & 100.845 & 104.597 & 96.417 & 110.547 & 108.186 & 74.016 \\
\hline $10 / 2 / 07$ 10:00 & 126.839 & 124.403 & 120.337 & 100.857 & 104.612 & 96.436 & 110.575 & 108.201 & 74.054 \\
\hline $10 / 2 / 07 \quad 10: 30$ & 126.868 & 124.417 & 120.357 & 100.865 & 104.614 & 96.438 & 110.603 & 108.21 & 75.091 \\
\hline $10 / 2 / 0711: 00$ & 126.889 & 124.428 & 120.365 & 100.876 & 104.627 & 96.452 & 110.618 & 108.223 & 74.732 \\
\hline 10/2/07 11:30 & 126.908 & 122.796 & 119.394 & 100.894 & 104.646 & 96.471 & 110.633 & 108.239 & 74.413 \\
\hline $10 / 2 / 07 \quad 12: 00$ & 126.927 & 122.41 & 118.845 & 100.909 & 104.658 & 96.483 & 110.642 & 108.257 & 74.223 \\
\hline $10 / 2 / 0712: 30$ & 125.561 & 122.279 & 118.694 & 100.29 & 104.351 & 96.035 & 110.175 & 107.178 & 74.113 \\
\hline $10 / 2 / 07$ 13:00 & 125.199 & 122.208 & 118.618 & 100.026 & 104.001 & 95.672 & 109.822 & 106.689 & 73.934 \\
\hline $10 / 2 / 07$ 13:30 & 125.05 & 122.172 & 118.578 & 99.93 & 103.879 & 95.547 & 109.675 & 106.543 & 73.82 \\
\hline $10 / 2 / 07 \quad 14: 00$ & 124.96 & 122.147 & 118.553 & 99.875 & 103.816 & 95.488 & 109.589 & 106.474 & 73.754 \\
\hline $10 / 2 / 07$ 14:30 & 124.899 & 122.131 & 118.533 & 99.844 & 103.784 & 95.453 & 109.542 & 106.43 & 73.71 \\
\hline $10 / 2 / 07$ 15:00 & 124.866 & 122.124 & 118.528 & 99.825 & 103.763 & 95.432 & 109.506 & 106.408 & 73.679 \\
\hline $10 / 2 / 07 \quad 15: 30$ & 124.833 & 122.113 & 118.513 & 99.813 & 103.746 & 95.42 & 109.488 & 106.388 & 73.657 \\
\hline $10 / 2 / 07$ 16:00 & 124.733 & 122.109 & 118.508 & 99.811 & 103.742 & 95.417 & 109.471 & 106.384 & 73.653 \\
\hline $10 / 2 / 07 \quad 16: 30$ & 124.585 & 122.115 & 118.509 & 99.799 & 103.729 & 95.403 & 109.46 & 106.37 & 73.638 \\
\hline $10 / 2 / 07 \quad 17: 00$ & 124.499 & 122.122 & 118.518 & 99.802 & 103.729 & 95.401 & 109.446 & 106.366 & 73.634 \\
\hline $10 / 2 / 07$ 17:30 & 124.473 & 122.138 & 118.525 & 99.804 & 103.735 & 95.408 & 109.439 & 106.37 & 73.638 \\
\hline $10 / 2 / 07$ 18:00 & 124.471 & 122.143 & 118.538 & 99.818 & 103.74 & 95.413 & 109.43 & 106.377 & 73.642 \\
\hline $10 / 2 / 07$ 18:30 & 124.488 & 122.149 & 118.545 & 99.833 & 103.755 & 95.429 & 109.424 & 106.39 & 73.656 \\
\hline $10 / 2 / 07 \quad 19: 00$ & 124.495 & 122.159 & 118.555 & 99.84 & 103.761 & 95.439 & 109.42 & 106.399 & 73.663 \\
\hline 10/2/07 19:30 & 124.504 & 122.168 & 118.56 & 99.845 & 103.769 & 95.443 & 109.418 & 106.406 & 73.669 \\
\hline $10 / 2 / 0720: 00$ & 124.507 & 122.181 & 118.576 & 99.856 & 103.782 & 95.455 & 109.414 & 106.415 & 73.675 \\
\hline $10 / 2 / 0720: 30$ & 124.509 & 122.19 & 118.588 & 99.864 & 103.788 & 95.465 & 109.409 & 106.421 & 73.684 \\
\hline 10/2/07 21:00 & 124.509 & 122.197 & 118.596 & 99.878 & 103.801 & 95.476 & 109.407 & 106.437 & 73.696 \\
\hline $10 / 2 / 0721: 30$ & 124.502 & 122.199 & 118.601 & 99.889 & 103.809 & 95.486 & 109.405 & 106.446 & 73.704 \\
\hline $10 / 2 / 0722: 00$ & 124.499 & 122.204 & 118.603 & 99.893 & 103.816 & 95.49 & 109.405 & 106.452 & 73.71 \\
\hline $10 / 2 / 07$ 22:30 & 124.499 & 122.208 & 118.606 & 99.897 & 103.82 & 95.498 & 109.405 & 106.457 & 73.714 \\
\hline $10 / 2 / 0723: 00$ & 124.497 & 122.208 & 118.608 & 99.899 & 103.826 & 95.498 & 109.401 & 106.459 & 73.718 \\
\hline $10 / 2 / 0723: 30$ & 124.495 & 122.217 & 118.611 & 99.906 & 103.828 & 95.505 & 109.398 & 106.463 & 73.721 \\
\hline $10 / 3 / 070: 00$ & 124.492 & 122.22 & 118.621 & 99.907 & 103.83 & 95.507 & 109.398 & 106.466 & 73.721 \\
\hline $10 / 3 / 07$ 0:30 & 124.492 & 122.22 & 118.621 & 99.918 & 103.841 & 95.516 & 109.394 & 106.474 & 73.731 \\
\hline $10 / 3 / 07$ 1:00 & 124.492 & 122.22 & 118.621 & 99.918 & 103.839 & 95.514 & 109.394 & 106.474 & 73.733 \\
\hline 10/3/07 1:30 & 124.492 & 122.215 & 118.618 & 99.92 & 103.843 & 95.521 & 109.392 & 106.479 & 73.733 \\
\hline $10 / 3 / 072: 00$ & 124.492 & 122.199 & 118.603 & 99.916 & 103.843 & 95.519 & 109.39 & 106.477 & 73.733 \\
\hline $10 / 3 / 072: 30$ & 124.495 & 122.202 & 118.593 & 99.909 & 103.839 & 95.514 & 109.398 & 106.472 & 73.729 \\
\hline 10/3/07 3:00 & 124.495 & 122.193 & 118.576 & 99.895 & 103.822 & 95.5 & 109.396 & 106.459 & 73.716 \\
\hline 10/3/07 3:30 & 124.492 & 122.197 & 118.581 & 99.897 & 103.82 & 95.498 & 109.392 & 106.455 & 73.715 \\
\hline $10 / 3 / 074: 00$ & 124.492 & 122.202 & 118.583 & 99.89 & 103.814 & 95.49 & 109.388 & 106.452 & 73.708 \\
\hline $10 / 3 / 074: 30$ & 124.49 & 122.206 & 118.59 & 99.892 & 103.818 & 95.495 & 109.386 & 106.455 & 73.712 \\
\hline $10 / 3 / 07$ 5:00 & 124.485 & 122.211 & 118.593 & 99.901 & 103.826 & 95.5 & 109.383 & 106.457 & 73.715 \\
\hline $10 / 3 / 075: 30$ & 124.485 & 122.217 & 118.603 & 99.909 & 103.83 & 95.505 & 109.383 & 106.463 & 73.721 \\
\hline $10 / 3 / 076: 00$ & 124.485 & 124.102 & 119.788 & 99.911 & 103.834 & 95.509 & 109.381 & 106.468 & 73.727 \\
\hline $10 / 3 / 076: 30$ & 124.485 & 124.421 & 120.186 & 99.918 & 103.841 & 95.514 & 109.377 & 106.472 & 73.731 \\
\hline $10 / 3 / 07$ 7:00 & 126.043 & 124.564 & 120.344 & 100.621 & 104.277 & 96.084 & 109.944 & 107.849 & 73.779 \\
\hline $10 / 3 / 07$ 7:30 & 126.329 & 124.651 & 120.443 & 100.867 & 104.582 & 96.4 & 110.227 & 108.215 & 73.985 \\
\hline $10 / 3 / 078: 00$ & 126.471 & 124.71 & 120.506 & 100.991 & 104.726 & 96.542 & 110.363 & 108.374 & 74.121 \\
\hline $10 / 3 / 078: 30$ & 126.563 & 124.635 & 120.551 & 101.065 & 104.814 & 96.627 & 110.443 & 108.465 & 74.215 \\
\hline 10/3/07 9:00 & 126.624 & 122.771 & 119.228 & 101.124 & 104.875 & 96.693 & 110.494 & 108.529 & 74.285 \\
\hline 10/3/07 9:30 & 126.672 & 122.567 & 118.984 & 100.815 & 104.917 & 96.733 & 110.532 & 108.573 & 74.331 \\
\hline $10 / 3 / 07 \quad 10: 00$ & 125.043 & 122.465 & 118.873 & 100.369 & 104.361 & 96.04 & 109.848 & 107.068 & 74.231 \\
\hline $10 / 3 / 07 \quad 10: 30$ & 124.887 & 122.397 & 118.807 & 100.211 & 104.169 & 95.842 & 109.67 & 106.84 & 74.083 \\
\hline 10/3/07 11:00 & 124.816 & 122.351 & 118.759 & 100.128 & 104.07 & 95.747 & 109.58 & 106.731 & 73.987 \\
\hline $10 / 3 / 07 \quad 11: 30$ & 124.776 & 122.315 & 118.719 & 100.064 & 104.005 & 95.681 & 109.529 & 106.66 & 73.919 \\
\hline $10 / 3 / 07$ 12:00 & 124.726 & 122.281 & 118.684 & 100.026 & 103.963 & 95.641 & 109.495 & 106.616 & 73.87 \\
\hline $10 / 3 / 07$ 12:30 & 124.686 & 122.251 & 118.656 & 99.99 & 103.925 & 95.604 & 109.471 & 106.576 & 73.83 \\
\hline $10 / 3 / 07$ 13:00 & 124.651 & 122.22 & 118.623 & 99.963 & 103.896 & 95.571 & 109.452 & 106.541 & 73.797 \\
\hline $10 / 3 / 07$ 13:30 & 124.622 & 122.195 & 118.596 & 99.933 & 103.864 & 95.542 & 109.445 & 106.512 & 73.768 \\
\hline $10 / 3 / 0714: 00$ & 124.592 & 122.181 & 118.581 & 99.902 & 103.83 & 95.509 & 109.437 & 106.477 & 73.733 \\
\hline $10 / 3 / 07 \quad 14: 30$ & 124.563 & 122.17 & 118.565 & 99.883 & 103.809 & 95.488 & 109.428 & 106.455 & 73.713 \\
\hline $10 / 3 / 07$ 15:00 & 124.547 & 122.145 & 118.548 & 99.868 & 103.797 & 95.474 & 109.424 & 106.437 & 73.696 \\
\hline $10 / 3 / 07 \quad 15: 30$ & 124.535 & 122.134 & 118.538 & 99.852 & 103.78 & 95.457 & 109.42 & 106.424 & 73.679 \\
\hline $10 / 3 / 07$ 16:00 & 124.514 & 122.124 & 118.523 & 99.833 & 103.761 & 95.439 & 109.418 & 106.401 & 73.663 \\
\hline $10 / 3 / 07 \quad 16: 30$ & 124.499 & 122.115 & 118.5 & 99.823 & 103.75 & 95.429 & 109.413 & 106.393 & 73.653 \\
\hline $10 / 3 / 07$ 17:00 & 124.488 & 122.109 & 118.489 & 99.816 & 103.744 & 95.42 & 109.409 & 106.381 & 73.642 \\
\hline
\end{tabular}


TABLE S1.1 (Cont.)

\begin{tabular}{|c|c|c|c|c|c|c|c|c|c|c|}
\hline \multirow[b]{2}{*}{ Date and Time } & \multicolumn{10}{|c|}{ Depth from Top of Casing (ft) } \\
\hline & MW1D & MW2D & MW3D & MW4D & MW7 & MW9 & Oentrich & MW14D & MW15D & MW16D \\
\hline 10/3/07 17:30 & 124.476 & 122.097 & 118.479 & 99.804 & 103.733 & 95.41 & 109.407 & 106.373 & & 73.634 \\
\hline 10/3/07 18:00 & 124.471 & 122.09 & 118.477 & 99.795 & 103.723 & 95.401 & 109.405 & 106.362 & & 73.624 \\
\hline 10/3/07 18:30 & 124.457 & 122.088 & 118.477 & 99.79 & 103.714 & 95.391 & 109.403 & 106.35 & & 73.615 \\
\hline 10/3/07 19:00 & 124.455 & 122.086 & 118.487 & 99.783 & 103.714 & 95.389 & 109.401 & 106.348 & & 73.611 \\
\hline 10/3/07 19:30 & 124.45 & 122.088 & 118.487 & 99.783 & 103.708 & 95.384 & 109.399 & 106.346 & & 73.609 \\
\hline 10/3/07 20:00 & 124.447 & 122.09 & 118.492 & 99.782 & 103.708 & 95.382 & 109.398 & 106.344 & & 73.607 \\
\hline 10/3/07 20:30 & 124.443 & 122.097 & 118.497 & 99.785 & 103.71 & 95.384 & 109.394 & 106.342 & & 73.605 \\
\hline 10/3/07 21:00 & 124.45 & 122.1 & 118.5 & 99.789 & 103.714 & 95.389 & 109.392 & 106.348 & & 73.611 \\
\hline 10/3/07 21:30 & 124.452 & 122.104 & 118.502 & 99.795 & 103.715 & 95.394 & 109.39 & 106.353 & & 73.613 \\
\hline 10/3/07 22:00 & 124.457 & 122.104 & 118.508 & 99.797 & 103.723 & 95.399 & 109.392 & 106.357 & & 73.62 \\
\hline 10/3/07 22:30 & 124.459 & 122.106 & 118.502 & 99.801 & 103.725 & 95.401 & 109.39 & 106.359 & & 73.62 \\
\hline 10/3/07 23:00 & 124.462 & 122.104 & 118.499 & 99.802 & 103.729 & 95.403 & 109.39 & 106.364 & & 73.624 \\
\hline 10/3/07 23:30 & 124.459 & 122.097 & 118.5 & 99.804 & 103.727 & 95.403 & 109.388 & 106.362 & & 73.622 \\
\hline $10 / 4 / 070: 00$ & 124.457 & 122.095 & 118.49 & 99.799 & 103.721 & 95.399 & 109.386 & 106.357 & & 73.615 \\
\hline 10/4/07 0:30 & 124.457 & 122.093 & 118.495 & 99.79 & 103.717 & 95.394 & 109.388 & 106.355 & & 73.611 \\
\hline 10/4/07 1:00 & 124.455 & 122.09 & 118.492 & 99.79 & 103.715 & 95.394 & 109.386 & 106.35 & & 73.609 \\
\hline 10/4/07 1:30 & 124.455 & 122.088 & 118.482 & 99.79 & 103.717 & 95.391 & 109.386 & 106.348 & & 73.609 \\
\hline 10/4/07 2:00 & 124.452 & 122.084 & 118.48 & 99.787 & 103.714 & 95.387 & 109.386 & 106.348 & & 73.607 \\
\hline 10/4/07 2:30 & 124.452 & 122.075 & 118.477 & 99.785 & 103.712 & 95.387 & 109.386 & 106.346 & & 73.603 \\
\hline 10/4/07 3:00 & 124.45 & 122.07 & 118.469 & 99.777 & 103.704 & 95.38 & 109.388 & 106.339 & & 73.596 \\
\hline 10/4/07 3:30 & 124.447 & 122.066 & 118.462 & 99.77 & 103.696 & 95.373 & 109.388 & 106.331 & & 73.588 \\
\hline 10/4/07 4:00 & 124.45 & 122.059 & 118.445 & 99.763 & 103.689 & 95.366 & 109.388 & 106.322 & & 73.58 \\
\hline 10/4/07 4:30 & 124.447 & 122.052 & 118.437 & 99.759 & 103.685 & 95.361 & 109.388 & 106.319 & & 73.576 \\
\hline 10/4/07 5:00 & 124.445 & 122.052 & 118.436 & 99.754 & 103.681 & 95.356 & 109.39 & 106.315 & & 73.572 \\
\hline $10 / 4 / 075: 30$ & 124.443 & 122.056 & 118.455 & 99.746 & 103.674 & 95.349 & 109.388 & 106.308 & & 73.566 \\
\hline 10/4/07 6:00 & 124.44 & 123.993 & 119.86 & 99.75 & 103.672 & 95.347 & 109.39 & 106.306 & & 73.566 \\
\hline 10/4/07 6:30 & 124.44 & 124.265 & 120.177 & 99.75 & 103.679 & 95.347 & 109.39 & 106.311 & & 73.572 \\
\hline 10/4/07 7:00 & 126.078 & 124.397 & 120.329 & 100.56 & 104.222 & 96.04 & 110.075 & 107.756 & & 73.657 \\
\hline 10/4/07 7:30 & 126.322 & 124.483 & 120.425 & 100.776 & 104.481 & 96.304 & 110.318 & 108.057 & & 73.853 \\
\hline 10/4/07 8:00 & 126.447 & 124.544 & 120.488 & 100.893 & 104.614 & 96.436 & 110.445 & 108.199 & & 73.985 \\
\hline 10/4/07 8:30 & 126.525 & 124.587 & 120.535 & 100.972 & 104.704 & 96.525 & 110.517 & 108.294 & & 74.081 \\
\hline 10/4/07 9:00 & 126.579 & 124.61 & 120.559 & 101.027 & 104.763 & 96.584 & 110.565 & 108.358 & & 74.145 \\
\hline 10/4/07 9:30 & 126.627 & 124.628 & 120.583 & 101.064 & 104.804 & 96.627 & 110.603 & 108.405 & & 74.19 \\
\hline 10/4/07 10:00 & 126.667 & 124.646 & 120.598 & 101.082 & 104.826 & 96.645 & 110.631 & 108.427 & & 74.21 \\
\hline 10/4/07 10:30 & 126.7 & 124.655 & 120.612 & 101.105 & 104.846 & 96.667 & 110.652 & 108.451 & & 74.233 \\
\hline 10/4/07 11:00 & 126.726 & 123.732 & 120.618 & 101.119 & 104.865 & 96.683 & 110.669 & 108.467 & & 74.252 \\
\hline 10/4/07 11:30 & 126.828 & 122.689 & 119.147 & 101.129 & 104.873 & 96.693 & 110.688 & 108.476 & & 74.264 \\
\hline 10/4/07 12:00 & 126.977 & 122.467 & 118.892 & 100.705 & 104.881 & 96.7 & 110.697 & 108.487 & & 74.273 \\
\hline $10 / 4 / 0712: 30$ & 125.199 & 122.347 & 118.757 & 100.269 & 104.268 & 95.938 & 109.942 & 106.984 & & 74.143 \\
\hline 10/4/07 13:00 & 124.906 & 122.258 & 118.671 & 100.095 & 104.06 & 95.726 & 109.758 & 106.742 & & 73.979 \\
\hline 10/4/07 13:30 & 124.745 & 122.208 & 118.61 & 99.992 & 103.938 & 95.606 & 109.664 & 106.609 & & 73.861 \\
\hline $10 / 4 / 0714: 00$ & 124.651 & 122.168 & 118.572 & 99.923 & 103.858 & 95.526 & 109.606 & 106.523 & & 73.775 \\
\hline $10 / 4 / 0714: 30$ & 124.585 & 122.138 & 118.543 & 99.873 & 103.811 & 95.476 & 109.567 & 106.468 & & 73.721 \\
\hline 10/4/07 15:00 & 124.542 & 122.109 & 118.513 & 99.842 & 103.771 & 95.439 & 109.54 & 106.428 & & 73.679 \\
\hline $10 / 4 / 0715: 30$ & 124.509 & 122.093 & 118.494 & 99.811 & 103.74 & 95.408 & 109.521 & 106.393 & & 73.646 \\
\hline 10/4/07 16:00 & 124.478 & 122.077 & 118.477 & 99.787 & 103.714 & 95.382 & 109.508 & 106.366 & & 73.617 \\
\hline 10/4/07 16:30 & 124.457 & 122.075 & 118.472 & 99.773 & 103.7 & 95.363 & 109.497 & 106.35 & & 73.601 \\
\hline 10/4/07 17:00 & 124.44 & 122.072 & 118.469 & 99.763 & 103.687 & 95.351 & 109.488 & 106.333 & & 73.586 \\
\hline 10/4/07 17:30 & 124.431 & 122.072 & 118.457 & 99.761 & 103.685 & 95.349 & 109.48 & 106.326 & & 73.582 \\
\hline 10/4/07 18:00 & 124.431 & 122.079 & 118.48 & 99.759 & 103.685 & 95.347 & 109.476 & 106.328 & & 73.582 \\
\hline 10/4/07 18:30 & 124.431 & 122.079 & 118.477 & 99.764 & 103.687 & 95.351 & 109.471 & 106.331 & & 73.584 \\
\hline 10/4/07 19:00 & 124.436 & 122.075 & 118.474 & 99.77 & 103.693 & 95.358 & 109.465 & 106.333 & & 73.586 \\
\hline 10/4/07 19:30 & 124.436 & 122.079 & 118.477 & 99.768 & 103.689 & 95.356 & 109.461 & 106.333 & & 73.584 \\
\hline 10/4/07 20:00 & 124.436 & 122.088 & 118.485 & 99.763 & 103.687 & 95.351 & 109.461 & 106.331 & & 73.58 \\
\hline $10 / 4 / 0720: 30$ & 124.436 & 122.102 & 118.49 & 99.773 & 103.695 & 95.356 & 109.456 & 106.333 & & 73.586 \\
\hline 10/4/07 21:00 & 124.443 & 122.102 & 118.499 & 99.783 & 103.702 & 95.368 & 109.454 & 106.342 & & 73.595 \\
\hline 10/4/07 21:30 & 124.45 & 122.111 & 118.513 & 99.792 & 103.71 & 95.375 & 109.454 & 106.348 & & 73.598 \\
\hline 10/4/07 22:00 & 124.459 & 122.111 & 118.505 & 99.795 & 103.715 & 95.382 & 109.45 & 106.355 & & 73.605 \\
\hline 10/4/07 22:30 & 124.469 & 122.115 & 118.515 & 99.801 & 103.729 & 95.391 & 109.446 & 106.366 & & 73.617 \\
\hline 10/4/07 23:00 & 124.464 & 122.115 & 118.508 & 99.802 & 103.725 & 95.391 & 109.448 & 106.364 & & 73.613 \\
\hline 10/4/07 23:30 & 124.469 & 122.113 & 118.507 & 99.806 & 103.727 & 95.396 & 109.446 & 106.368 & & 73.617 \\
\hline 10/5/07 0:00 & 124.473 & 122.104 & 118.502 & 99.806 & 103.729 & 95.394 & 109.446 & 106.368 & & 73.615 \\
\hline 10/5/07 0:30 & 124.469 & 122.1 & 118.49 & 99.801 & 103.725 & 95.389 & 109.446 & 106.364 & & 73.611 \\
\hline 10/5/07 1:00 & 124.464 & 122.097 & 118.482 & 99.795 & 103.717 & 95.382 & 109.445 & 106.357 & & 73.605 \\
\hline 10/5/07 1:30 & 124.464 & 122.097 & 118.482 & 99.792 & 103.718 & 95.38 & 109.445 & 106.355 & & 73.605 \\
\hline $10 / 5 / 072: 00$ & 124.459 & 122.093 & 118.475 & 99.792 & 103.714 & 95.38 & 109.446 & 106.355 & & 73.599 \\
\hline
\end{tabular}


TABLE S1.1 (Cont.)

\begin{tabular}{|c|c|c|c|c|c|c|c|c|c|c|}
\hline \multirow[b]{2}{*}{ Date and Time } & \multicolumn{10}{|c|}{ Depth from Top of Casing (ft) } \\
\hline & MW1D & MW2D & MW3D & MW4D & MW7 & MW9 & Oentrich & MW14D & MW15D & MW16D \\
\hline 10/5/07 2:30 & 124.457 & 122.086 & 118.477 & 99.787 & 103.715 & 95.38 & 109.445 & 106.353 & & 73.598 \\
\hline 10/5/07 3:00 & 124.45 & 122.081 & 118.469 & 99.787 & 103.71 & 95.375 & 109.445 & 106.348 & & 73.594 \\
\hline 10/5/07 3:30 & 124.447 & 122.081 & 118.466 & 99.783 & 103.706 & 95.373 & 109.445 & 106.344 & & 73.59 \\
\hline 10/5/07 4:00 & 124.445 & 122.081 & 118.461 & 99.778 & 103.702 & 95.368 & 109.443 & 106.339 & & 73.586 \\
\hline 10/5/07 4:30 & 124.445 & 122.088 & 118.474 & 99.778 & 103.7 & 95.363 & 109.445 & 106.335 & & 73.582 \\
\hline 10/5/07 5:00 & 124.443 & 122.093 & 118.477 & 99.782 & 103.7 & 95.366 & 109.443 & 106.337 & & 73.582 \\
\hline 10/5/07 5:30 & 124.438 & 123.891 & 119.51 & 99.785 & 103.708 & 95.373 & 109.441 & 106.342 & & 73.589 \\
\hline 10/5/07 6:00 & 124.44 & 124.258 & 120.004 & 99.785 & 103.712 & 95.375 & 109.441 & 106.346 & & 73.591 \\
\hline 10/5/07 6:30 & 125.913 & 124.412 & 120.183 & 100.433 & 104.07 & 95.863 & 109.931 & 107.606 & & 73.609 \\
\hline 10/5/07 7:00 & 126.244 & 124.505 & 120.286 & 100.707 & 104.416 & 96.221 & 110.263 & 108.049 & & 73.812 \\
\hline 10/5/07 7:30 & 126.386 & 124.571 & 120.355 & 100.843 & 104.578 & 96.381 & 110.411 & 108.226 & & 73.963 \\
\hline 10/5/07 8:00 & 126.473 & 123.864 & 120.409 & 100.926 & 104.667 & 96.469 & 110.502 & 108.323 & & 74.062 \\
\hline 10/5/07 8:30 & 126.525 & 122.639 & 119.079 & 100.986 & 104.732 & 96.537 & 110.556 & 108.389 & & 74.132 \\
\hline 10/5/07 9:00 & 126.57 & 122.449 & 118.85 & 100.65 & 104.784 & 96.587 & 110.592 & 108.443 & & 74.184 \\
\hline 10/5/07 9:30 & 124.92 & 122.351 & 118.737 & 100.24 & 104.226 & 95.894 & 109.905 & 106.931 & & 74.087 \\
\hline $10 / 5 / 0710: 00$ & 124.766 & 122.29 & 118.681 & 100.09 & 104.045 & 95.707 & 109.732 & 106.718 & & 73.954 \\
\hline 10/5/07 10:30 & 124.696 & 122.261 & 118.649 & 100.007 & 103.955 & 95.618 & 109.642 & 106.618 & & 73.859 \\
\hline 10/5/07 11:00 & 124.653 & 122.238 & 118.618 & 99.959 & 103.893 & 95.556 & 109.591 & 106.552 & & 73.793 \\
\hline 10/5/07 11:30 & 124.625 & 122.211 & 118.596 & 99.93 & 103.87 & 95.531 & 109.557 & 106.517 & & 73.758 \\
\hline 10/5/07 12:00 & 124.603 & 122.195 & 118.577 & 99.909 & 103.841 & 95.507 & 109.537 & 106.49 & & 73.733 \\
\hline 10/5/07 12:30 & 124.58 & 122.172 & 118.558 & 99.89 & 103.813 & 95.481 & 109.525 & 106.466 & & 73.704 \\
\hline 10/5/07 13:00 & 124.563 & 122.154 & 118.54 & 99.871 & 103.799 & 95.465 & 109.512 & 106.446 & & 73.69 \\
\hline 10/5/07 13:30 & 124.537 & 122.14 & 118.528 & 99.856 & 103.782 & 95.45 & 109.503 & 106.428 & & 73.669 \\
\hline $10 / 5 / 0714: 00$ & 124.523 & 122.134 & 118.514 & 99.84 & 103.761 & 95.429 & 109.497 & 106.408 & & 73.648 \\
\hline $10 / 5 / 0714: 30$ & 124.504 & 122.129 & 118.508 & 99.825 & 103.752 & 95.417 & 109.493 & 106.395 & & 73.636 \\
\hline 10/5/07 15:00 & 124.492 & 122.115 & 118.505 & 99.821 & 103.744 & 95.41 & 109.488 & 106.386 & & 73.628 \\
\hline 10/5/07 15:30 & 124.49 & 122.115 & 118.499 & 99.814 & 103.738 & 95.406 & 109.484 & 106.379 & & 73.622 \\
\hline 10/5/07 16:00 & 124.481 & 122.113 & 118.497 & 99.806 & 103.733 & 95.394 & 109.48 & 106.37 & & 73.611 \\
\hline 10/5/07 16:30 & 124.473 & 122.115 & 118.495 & 99.802 & 103.723 & 95.391 & 109.478 & 106.364 & & 73.607 \\
\hline 10/5/07 17:00 & 124.471 & 122.118 & 118.5 & 99.801 & 103.725 & 95.391 & 109.475 & 106.364 & & 73.607 \\
\hline 10/5/07 17:30 & 124.471 & 122.118 & 118.502 & 99.806 & 103.725 & 95.391 & 109.473 & 106.364 & & 73.605 \\
\hline 10/5/07 18:00 & 124.473 & 122.124 & 118.507 & 99.807 & 103.731 & 95.396 & 109.473 & 106.368 & & 73.611 \\
\hline 10/5/07 18:30 & 124.478 & 122.131 & 118.514 & 99.813 & 103.733 & 95.399 & 109.469 & 106.37 & & 73.613 \\
\hline 10/5/07 19:00 & 124.483 & 122.143 & 118.528 & 99.814 & 103.736 & 95.401 & 109.471 & 106.375 & & 73.615 \\
\hline 10/5/07 19:30 & 124.485 & 122.152 & 118.532 & 99.828 & 103.742 & 95.41 & 109.471 & 106.384 & & 73.62 \\
\hline 10/5/07 20:00 & 124.497 & 122.156 & 118.538 & 99.84 & 103.757 & 95.424 & 109.465 & 106.397 & & 73.636 \\
\hline 10/5/07 20:30 & 124.504 & 122.168 & 118.55 & 99.842 & 103.763 & 95.427 & 109.465 & 106.399 & & 73.636 \\
\hline 10/5/07 21:00 & 124.511 & 122.174 & 118.56 & 99.854 & 103.771 & 95.436 & 109.465 & 106.408 & & 73.646 \\
\hline 10/5/07 21:30 & 124.523 & 122.19 & 118.57 & 99.864 & 103.782 & 95.448 & 109.461 & 106.419 & & 73.657 \\
\hline 10/5/07 22:00 & 124.533 & 122.197 & 118.58 & 99.873 & 103.792 & 95.457 & 109.46 & 106.428 & & 73.663 \\
\hline 10/5/07 22:30 & 124.54 & 122.206 & 118.585 & 99.887 & 103.805 & 95.469 & 109.458 & 106.441 & & 73.675 \\
\hline 10/5/07 23:00 & 124.554 & 122.204 & 118.587 & 99.894 & 103.813 & 95.479 & 109.458 & 106.45 & & 73.686 \\
\hline 10/5/07 23:30 & 124.561 & 122.199 & 118.582 & 99.897 & 103.82 & 95.486 & 109.458 & 106.455 & & 73.688 \\
\hline 10/6/07 0:00 & 124.566 & 122.199 & 118.583 & 99.901 & 103.818 & 95.488 & 109.458 & 106.457 & & 73.69 \\
\hline 10/6/07 0:30 & 124.563 & 122.199 & 118.583 & 99.894 & 103.816 & 95.483 & 109.458 & 106.455 & & 73.688 \\
\hline 10/6/07 1:00 & 124.559 & 122.202 & 118.585 & 99.893 & 103.813 & 95.481 & 109.456 & 106.452 & & 73.684 \\
\hline 10/6/07 1:30 & 124.556 & 122.206 & 118.591 & 99.895 & 103.816 & 95.483 & 109.456 & 106.455 & & 73.686 \\
\hline 10/6/07 2:00 & 124.556 & 122.204 & 118.587 & 99.897 & 103.816 & 95.483 & 109.454 & 106.455 & & 73.686 \\
\hline 10/6/07 2:30 & 124.563 & 122.202 & 118.586 & 99.9 & 103.82 & 95.49 & 109.454 & 106.459 & & 73.692 \\
\hline 10/6/07 3:00 & 124.563 & 122.197 & 118.582 & 99.899 & 103.816 & 95.486 & 109.454 & 106.457 & & 73.688 \\
\hline 10/6/07 3:30 & 124.559 & 122.193 & 118.577 & 99.893 & 103.814 & 95.483 & 109.452 & 106.452 & & 73.686 \\
\hline 10/6/07 4:00 & 124.556 & 122.195 & 118.58 & 99.889 & 103.809 & 95.479 & 109.452 & 106.45 & & 73.684 \\
\hline 10/6/07 4:30 & 124.551 & 122.193 & 118.575 & 99.889 & 103.807 & 95.474 & 109.45 & 106.446 & & 73.677 \\
\hline 10/6/07 5:00 & 124.554 & 122.195 & 118.573 & 99.89 & 103.809 & 95.474 & 109.45 & 106.448 & & 73.679 \\
\hline 10/6/07 5:30 & 124.549 & 122.202 & 118.586 & 99.885 & 103.809 & 95.472 & 109.452 & 106.443 & & 73.675 \\
\hline 10/6/07 6:00 & 124.551 & 122.202 & 118.587 & 99.89 & 103.809 & 95.474 & 109.45 & 106.446 & & 73.679 \\
\hline 10/6/07 6:30 & 124.556 & 124.013 & 119.813 & 99.897 & 103.814 & 95.481 & 109.45 & 106.455 & & 73.684 \\
\hline 10/6/07 7:00 & 124.561 & 124.356 & 120.248 & 99.895 & 103.818 & 95.486 & 109.452 & 106.457 & & 73.686 \\
\hline 10/6/07 7:30 & 126.253 & 124.512 & 120.428 & 100.617 & 104.241 & 96.051 & 110.021 & 107.743 & & 73.717 \\
\hline 10/6/07 8:00 & 126.75 & 124.605 & 120.526 & 100.879 & 104.57 & 96.389 & 110.333 & 108.144 & & 73.925 \\
\hline 10/6/07 8:30 & 126.943 & 124.669 & 120.581 & 101.01 & 104.725 & 96.544 & 110.479 & 108.314 & & 74.072 \\
\hline 10/6/07 9:00 & 127.043 & 124.712 & 120.641 & 101.093 & 104.816 & 96.636 & 110.562 & 108.412 & & 74.169 \\
\hline 10/6/07 9:30 & 127.109 & 124.743 & 120.675 & 101.149 & 104.881 & 96.7 & 110.618 & 108.48 & & 74.236 \\
\hline 10/6/07 10:00 & 127.161 & 124.768 & 120.697 & 101.191 & 104.924 & 96.742 & 110.656 & 108.525 & & 74.285 \\
\hline $10 / 6 / 07$ 10:30 & 127.196 & 124.78 & 120.717 & 101.217 & 104.955 & 96.773 & 110.686 & 108.56 & & 74.316 \\
\hline 10/6/07 11:00 & 127.222 & 124.791 & 120.73 & 101.236 & 104.978 & 96.796 & 110.708 & 108.586 & & 74.341 \\
\hline
\end{tabular}


TABLE S1.1 (Cont.)

\begin{tabular}{|c|c|c|c|c|c|c|c|c|c|c|}
\hline \multirow[b]{2}{*}{ Date and Time } & \multicolumn{10}{|c|}{ Depth from Top of Casing (ft) } \\
\hline & MW1D & MW2D & MW3D & MW4D & MW7 & MW9 & Oentrich & MW14D & MW15D & MW16D \\
\hline 10/6/07 11:30 & 127.234 & 124.793 & 120.735 & 101.249 & 104.991 & 96.813 & 110.725 & 108.598 & & 74.355 \\
\hline 10/6/07 12:00 & 127.248 & 124.8 & 120.738 & 101.262 & 105.002 & 96.822 & 110.74 & 108.609 & & 74.37 \\
\hline 10/6/07 12:30 & 127.251 & 124.793 & 120.735 & 101.263 & 105.006 & 96.825 & 110.753 & 108.611 & & 74.376 \\
\hline 10/6/07 13:00 & 127.255 & 124.793 & 120.734 & 101.267 & 105.01 & 96.832 & 110.766 & 108.62 & & 74.382 \\
\hline 10/6/07 13:30 & 127.251 & 123.111 & 119.691 & 101.263 & 105.006 & 96.829 & 110.774 & 108.613 & & 74.38 \\
\hline 10/6/07 14:00 & 127.248 & 122.725 & 119.15 & 101.263 & 105.006 & 96.827 & 110.781 & 108.613 & & 74.382 \\
\hline $10 / 6 / 0714: 30$ & 125.712 & 122.558 & 118.96 & 100.598 & 104.652 & 96.337 & 110.295 & 107.48 & & 74.362 \\
\hline 10/6/07 15:00 & 125.145 & 122.456 & 118.853 & 100.322 & 104.296 & 95.967 & 109.953 & 107.004 & & 74.186 \\
\hline 10/6/07 15:30 & 124.941 & 122.392 & 118.782 & 100.188 & 104.14 & 95.811 & 109.811 & 106.824 & & 74.047 \\
\hline 10/6/07 16:00 & 124.833 & 122.344 & 118.734 & 100.102 & 104.043 & 95.714 & 109.728 & 106.718 & & 73.946 \\
\hline 10/6/07 16:30 & 124.762 & 122.317 & 118.704 & 100.048 & 103.98 & 95.648 & 109.675 & 106.647 & & 73.878 \\
\hline 10/6/07 17:00 & 124.712 & 122.297 & 118.681 & 100.009 & 103.938 & 95.606 & 109.642 & 106.603 & & 73.832 \\
\hline 10/6/07 17:30 & 124.681 & 122.283 & 118.663 & 99.987 & 103.913 & 95.58 & 109.617 & 106.572 & & 73.804 \\
\hline 10/6/07 18:00 & 124.658 & 122.27 & 118.653 & 99.97 & 103.894 & 95.561 & 109.597 & 106.55 & & 73.783 \\
\hline 10/6/07 18:30 & 124.644 & 122.256 & 118.643 & 99.955 & 103.877 & 95.547 & 109.583 & 106.534 & & 73.766 \\
\hline 10/6/07 19:00 & 124.629 & 122.251 & 118.635 & 99.945 & 103.868 & 95.535 & 109.576 & 106.521 & & 73.753 \\
\hline 10/6/07 19:30 & 124.62 & 122.247 & 118.633 & 99.937 & 103.858 & 95.526 & 109.567 & 106.51 & & 73.744 \\
\hline 10/6/07 20:00 & 124.613 & 122.247 & 118.631 & 99.935 & 103.854 & 95.521 & 109.559 & 106.503 & & 73.735 \\
\hline 10/6/07 20:30 & 124.608 & 122.247 & 118.63 & 99.933 & 103.853 & 95.519 & 109.552 & 106.501 & & 73.733 \\
\hline 10/6/07 21:00 & 124.608 & 122.249 & 118.633 & 99.93 & 103.853 & 95.519 & 109.546 & 106.499 & & 73.733 \\
\hline 10/6/07 21:30 & 124.608 & 122.247 & 118.626 & 99.935 & 103.851 & 95.519 & 109.542 & 106.499 & & 73.731 \\
\hline 10/6/07 22:00 & 124.608 & 122.238 & 118.626 & 99.933 & 103.854 & 95.523 & 109.538 & 106.499 & & 73.735 \\
\hline 10/6/07 22:30 & 124.608 & 122.233 & 118.62 & 99.932 & 103.851 & 95.521 & 109.535 & 106.499 & & 73.731 \\
\hline 10/6/07 23:00 & 124.601 & 122.227 & 118.61 & 99.926 & 103.845 & 95.516 & 109.531 & 106.492 & & 73.727 \\
\hline 10/6/07 23:30 & 124.596 & 122.217 & 118.603 & 99.92 & 103.843 & 95.509 & 109.529 & 106.488 & & 73.721 \\
\hline 10/7/07 0:00 & 124.589 & 122.215 & 118.598 & 99.906 & 103.83 & 95.498 & 109.527 & 106.477 & & 73.708 \\
\hline 10/7/07 0:30 & 124.58 & 122.22 & 118.603 & 99.902 & 103.826 & 95.49 & 109.523 & 106.47 & & 73.702 \\
\hline 10/7/07 1:00 & 124.573 & 122.222 & 118.603 & 99.904 & 103.82 & 95.488 & 109.52 & 106.466 & & 73.702 \\
\hline 10/7/07 1:30 & 124.577 & 122.224 & 118.605 & 99.909 & 103.826 & 95.493 & 109.518 & 106.47 & & 73.706 \\
\hline 10/7/07 2:00 & 124.58 & 122.229 & 118.608 & 99.912 & 103.83 & 95.495 & 109.516 & 106.472 & & 73.708 \\
\hline 10/7/07 2:30 & 124.582 & 122.222 & 118.605 & 99.913 & 103.832 & 95.5 & 109.514 & 106.477 & & 73.71 \\
\hline 10/7/07 3:00 & 124.587 & 122.227 & 118.6 & 99.916 & 103.837 & 95.502 & 109.512 & 106.479 & & 73.713 \\
\hline 10/7/07 3:30 & 124.585 & 122.224 & 118.608 & 99.911 & 103.83 & 95.495 & 109.51 & 106.472 & & 73.704 \\
\hline 10/7/07 4:00 & 124.582 & 122.222 & 118.606 & 99.916 & 103.832 & 95.498 & 109.508 & 106.472 & & 73.706 \\
\hline 10/7/07 4:30 & 124.585 & 122.224 & 118.61 & 99.912 & 103.834 & 95.5 & 109.506 & 106.474 & & 73.708 \\
\hline 10/7/07 5:00 & 124.582 & 122.227 & 118.611 & 99.914 & 103.834 & 95.5 & 109.506 & 106.472 & & 73.706 \\
\hline 10/7/07 5:30 & 124.585 & 122.24 & 118.626 & 99.914 & 103.835 & 95.502 & 109.506 & 106.474 & & 73.708 \\
\hline 10/7/07 6:00 & 124.585 & 122.245 & 118.63 & 99.924 & 103.837 & 95.505 & 109.503 & 106.477 & & 73.708 \\
\hline 10/7/07 6:30 & 124.599 & 122.249 & 118.633 & 99.935 & 103.854 & 95.519 & 109.501 & 106.492 & & 73.725 \\
\hline 10/7/07 7:00 & 124.606 & 124.127 & 119.792 & 99.938 & 103.854 & 95.523 & 109.501 & 106.497 & & 73.727 \\
\hline 10/7/07 7:30 & 124.611 & 124.44 & 120.186 & 99.94 & 103.862 & 95.528 & 109.501 & 106.501 & & 73.729 \\
\hline 10/7/07 8:00 & 126.36 & 124.592 & 120.354 & 100.641 & 104.289 & 96.089 & 110.06 & 107.865 & & 73.77 \\
\hline 10/7/07 8:30 & 126.816 & 124.682 & 120.46 & 100.881 & 104.593 & 96.4 & 110.349 & 108.23 & & 73.971 \\
\hline 10/7/07 9:00 & 126.995 & 124.748 & 120.52 & 101.012 & 104.742 & 96.549 & 110.487 & 108.396 & & 74.114 \\
\hline 10/7/07 9:30 & 127.097 & 124.791 & 120.573 & 101.093 & 104.835 & 96.643 & 110.565 & 108.491 & & 74.214 \\
\hline 10/7/07 10:00 & 127.161 & 124.821 & 120.604 & 101.149 & 104.894 & 96.7 & 110.622 & 108.558 & & 74.275 \\
\hline 10/7/07 10:30 & 127.211 & 124.841 & 120.626 & 101.191 & 104.942 & 96.749 & 110.659 & 108.606 & & 74.327 \\
\hline 10/7/07 11:00 & 127.242 & 124.859 & 120.646 & 101.217 & 104.97 & 96.78 & 110.687 & 108.637 & & 74.361 \\
\hline 10/7/07 11:30 & 127.263 & 124.868 & 120.665 & 101.238 & 104.993 & 96.801 & 110.706 & 108.66 & & 74.386 \\
\hline 10/7/07 12:00 & 127.279 & 124.877 & 120.671 & 101.255 & 105.012 & 96.818 & 110.721 & 108.679 & & 74.407 \\
\hline 10/7/07 12:30 & 127.298 & 123.127 & 119.679 & 101.268 & 105.027 & 96.829 & 110.736 & 108.693 & & 74.422 \\
\hline 10/7/07 13:00 & 127.305 & 122.759 & 119.182 & 101.274 & 105.033 & 96.841 & 110.749 & 108.699 & & 74.428 \\
\hline 10/7/07 13:30 & 125.712 & 122.596 & 118.996 & 100.631 & 104.677 & 96.356 & 110.271 & 107.482 & & 74.405 \\
\hline 10/7/07 14:00 & 125.178 & 122.505 & 118.898 & 100.36 & 104.334 & 96.002 & 109.957 & 107.039 & & 74.223 \\
\hline 10/7/07 14:30 & 124.977 & 122.44 & 118.833 & 100.228 & 104.18 & 95.849 & 109.818 & 106.862 & & 74.085 \\
\hline 10/7/07 15:00 & 124.878 & 122.401 & 118.787 & 100.154 & 104.092 & 95.762 & 109.741 & 106.762 & & 73.993 \\
\hline 10/7/07 15:30 & 124.814 & 122.378 & 118.767 & 100.1 & 104.03 & 95.7 & 109.692 & 106.696 & & 73.929 \\
\hline 10/7/07 16:00 & 124.769 & 122.363 & 118.751 & 100.069 & 103.996 & 95.665 & 109.655 & 106.654 & & 73.886 \\
\hline 10/7/07 16:30 & 124.745 & 122.356 & 118.744 & 100.047 & 103.976 & 95.644 & 109.632 & 106.632 & & 73.866 \\
\hline 10/7/07 17:00 & 124.726 & 122.344 & 118.732 & 100.043 & 103.963 & 95.632 & 109.615 & 106.614 & & 73.847 \\
\hline 10/7/07 17:30 & 124.719 & 122.351 & 118.729 & 100.035 & 103.956 & 95.625 & 109.602 & 106.607 & & 73.839 \\
\hline 10/7/07 18:00 & 124.705 & 122.344 & 118.731 & 100.024 & 103.946 & 95.615 & 109.591 & 106.596 & & 73.828 \\
\hline 10/7/07 18:30 & 124.7 & 122.34 & 118.731 & 100.035 & 103.955 & 95.625 & 109.582 & 106.601 & & 73.835 \\
\hline 10/7/07 19:00 & 124.707 & 122.347 & 118.724 & 100.03 & 103.95 & 95.615 & 109.578 & 106.594 & & 73.826 \\
\hline 10/7/07 19:30 & 124.707 & 122.351 & 118.737 & 100.021 & 103.946 & 95.618 & 109.572 & 106.594 & & 73.826 \\
\hline 10/7/07 20:00 & 124.7 & 122.356 & 118.731 & 100.042 & 103.957 & 95.625 & 109.563 & 106.601 & & 73.832 \\
\hline
\end{tabular}


TABLE S1.1 (Cont.)

Depth from Top of Casing (ft)

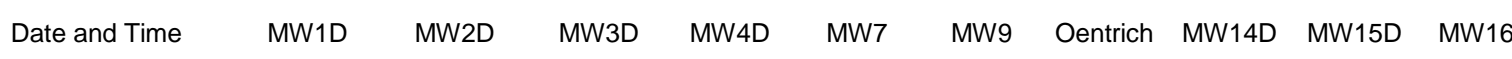

\begin{tabular}{|c|c|c|c|c|c|c|c|c|c|}
\hline $10 / 7 / 0720: 30$ & 124.71 & 122.358 & 118.749 & 100.035 & 103.957 & 95.627 & 109.559 & 106.605 & 73.835 \\
\hline 10/7/07 21:00 & 124.707 & 122.369 & 118.756 & 100.035 & 103.965 & 95.634 & 109.555 & 106.607 & 73.839 \\
\hline 10/7/07 21:30 & 124.707 & 122.353 & 118.741 & 100.048 & 103.969 & 95.637 & 109.553 & 106.616 & 73.847 \\
\hline 10/7/07 22:00 & 124.698 & 122.36 & 118.744 & 100.048 & 103.976 & 95.644 & 109.55 & 106.621 & 73.851 \\
\hline 10/7/07 22:30 & 124.696 & 122.356 & 118.741 & 100.042 & 103.959 & 95.63 & 109.552 & 106.605 & 73.835 \\
\hline 10/7/07 23:00 & 124.684 & 122.365 & 118.751 & 100.045 & 103.974 & 95.644 & 109.544 & 106.616 & 73.841 \\
\hline 10/7/07 23:30 & 124.681 & 122.385 & 118.761 & 100.04 & 103.961 & 95.63 & 109.544 & 106.607 & 73.834 \\
\hline 10/8/07 0:00 & 124.672 & 122.385 & 118.771 & 100.048 & 103.976 & 95.644 & 109.54 & 106.618 & 73.849 \\
\hline 10/8/07 0:30 & 124.66 & 122.388 & 118.764 & 100.081 & 103.995 & 95.665 & 109.535 & 106.634 & 73.861 \\
\hline 10/8/07 1:00 & 124.658 & 122.385 & 118.779 & 100.078 & 103.995 & 95.667 & 109.533 & 106.638 & 73.868 \\
\hline 10/8/07 1:30 & 124.655 & 122.353 & 118.744 & 100.083 & 103.992 & 95.66 & 109.531 & 106.632 & 73.859 \\
\hline $10 / 8 / 072: 00$ & 124.653 & 122.367 & 118.759 & 100.071 & 103.992 & 95.663 & 109.531 & 106.64 & 73.871 \\
\hline $10 / 8 / 072: 30$ & 124.655 & 122.372 & 118.754 & 100.043 & 103.959 & 95.632 & 109.533 & 106.607 & 73.835 \\
\hline 10/8/07 3:00 & 124.644 & 122.372 & 118.756 & 100.061 & 103.978 & 95.646 & 109.527 & 106.623 & 73.853 \\
\hline 10/8/07 3:30 & 124.636 & 122.401 & 118.787 & 100.064 & 103.982 & 95.651 & 109.523 & 106.623 & 73.851 \\
\hline $10 / 8 / 074: 00$ & 124.634 & 122.39 & 118.787 & 100.064 & 103.98 & 95.651 & 109.521 & 106.625 & 73.853 \\
\hline $10 / 8 / 074: 30$ & 124.62 & 122.394 & 118.78 & 100.1 & 104.014 & 95.684 & 109.514 & 106.654 & 73.884 \\
\hline $10 / 8 / 075: 00$ & 124.627 & 122.415 & 118.789 & 100.078 & 103.997 & 95.667 & 109.516 & 106.645 & 73.87 \\
\hline 10/8/07 5:30 & 124.625 & 122.44 & 118.827 & 100.097 & 104.003 & 95.67 & 109.512 & 106.647 & 73.87 \\
\hline 10/8/07 6:00 & 124.613 & 124.335 & 120.168 & 100.114 & 104.026 & 95.696 & 109.508 & 106.665 & 73.892 \\
\hline 10/8/07 6:30 & 124.611 & 124.623 & 120.525 & 100.133 & 104.051 & 95.719 & 109.506 & 106.694 & 73.919 \\
\hline 10/8/07 7:00 & 126.187 & 124.771 & 120.689 & 100.924 & 104.561 & 96.374 & 110.147 & 108.091 & 73.99 \\
\hline 10/8/07 7:30 & 126.442 & 124.861 & 120.787 & 101.146 & 104.839 & 96.655 & 110.415 & 108.414 & 74.186 \\
\hline 10/8/07 8:00 & 126.563 & 124.929 & 120.858 & 101.268 & 104.983 & 96.806 & 110.547 & 108.58 & 74.327 \\
\hline $10 / 8 / 078: 30$ & 126.634 & 123.816 & 120.922 & 101.354 & 105.077 & 96.895 & 110.625 & 108.675 & 74.424 \\
\hline 10/8/07 9:00 & 126.676 & 123.022 & 119.457 & 101.418 & 105.143 & 96.964 & 110.674 & 108.744 & 74.498 \\
\hline 10/8/07 9:30 & 126.173 & 122.834 & 119.238 & 100.998 & 105.189 & 97.018 & 110.674 & 108.803 & 74.558 \\
\hline $10 / 8 / 07 \quad 10: 00$ & 125.06 & 122.739 & 119.135 & 100.615 & 104.597 & 96.268 & 109.965 & 107.307 & 74.44 \\
\hline $10 / 8 / 07 \quad 10: 30$ & 124.909 & 122.682 & 119.071 & 100.467 & 104.42 & 96.089 & 109.797 & 107.103 & 74.306 \\
\hline $10 / 8 / 0711: 00$ & 124.84 & 122.641 & 119.032 & 100.391 & 104.329 & 96 & 109.707 & 107.001 & 74.213 \\
\hline $10 / 8 / 0711: 30$ & 124.802 & 122.612 & 119.001 & 100.348 & 104.275 & 95.945 & 109.659 & 106.939 & 74.155 \\
\hline $10 / 8 / 07 \quad 12: 00$ & 124.774 & 122.585 & 118.975 & 100.312 & 104.237 & 95.908 & 109.621 & 106.897 & 74.118 \\
\hline $10 / 8 / 07 \quad 12: 30$ & 124.757 & 122.569 & 118.955 & 100.286 & 104.209 & 95.879 & 109.597 & 106.869 & 74.085 \\
\hline $10 / 8 / 07$ 13:00 & 124.752 & 122.548 & 118.935 & 100.262 & 104.184 & 95.856 & 109.58 & 106.84 & 74.06 \\
\hline $10 / 8 / 07$ 13:30 & 124.745 & 122.53 & 118.916 & 100.245 & 104.167 & 95.837 & 109.567 & 106.822 & 74.041 \\
\hline $10 / 8 / 07 \quad 14: 00$ & 124.738 & 122.519 & 118.902 & 100.226 & 104.152 & 95.82 & 109.555 & 106.802 & 74.023 \\
\hline $10 / 8 / 07 \quad 14: 30$ & 124.736 & 122.505 & 118.895 & 100.212 & 104.131 & 95.799 & 109.552 & 106.783 & 74.004 \\
\hline $10 / 8 / 07$ 15:00 & 124.738 & 122.499 & 118.888 & 100.202 & 104.121 & 95.792 & 109.544 & 106.773 & 73.996 \\
\hline 10/8/07 15:30 & 124.736 & 122.49 & 118.875 & 100.186 & 104.108 & 95.778 & 109.537 & 106.758 & 73.983 \\
\hline $10 / 8 / 07 \quad 16: 00$ & 124.733 & 122.483 & 118.872 & 100.181 & 104.102 & 95.773 & 109.531 & 106.753 & 73.977 \\
\hline $10 / 8 / 07 \quad 16: 30$ & 124.733 & 122.485 & 118.865 & 100.178 & 104.092 & 95.764 & 109.527 & 106.742 & 73.967 \\
\hline $10 / 8 / 07$ 17:00 & 124.731 & 122.49 & 118.875 & 100.169 & 104.091 & 95.762 & 109.523 & 106.738 & 73.963 \\
\hline $10 / 8 / 07 \quad 17: 30$ & 124.733 & 122.492 & 118.877 & 100.173 & 104.087 & 95.759 & 109.52 & 106.736 & 73.961 \\
\hline $10 / 8 / 07$ 18:00 & 124.731 & 122.494 & 118.877 & 100.178 & 104.095 & 95.764 & 109.516 & 106.74 & 73.967 \\
\hline 10/8/07 18:30 & 124.729 & 122.499 & 118.88 & 100.179 & 104.095 & 95.764 & 109.512 & 106.742 & 73.969 \\
\hline 10/8/07 19:00 & 124.729 & 122.505 & 118.89 & 100.183 & 104.098 & 95.769 & 109.51 & 106.745 & 73.971 \\
\hline $10 / 8 / 07$ 19:30 & 124.726 & 122.512 & 118.896 & 100.188 & 104.102 & 95.773 & 109.508 & 106.749 & 73.975 \\
\hline $10 / 8 / 0720: 00$ & 124.726 & 122.517 & 118.897 & 100.198 & 104.111 & 95.783 & 109.505 & 106.758 & 73.983 \\
\hline $10 / 8 / 0720: 30$ & 124.729 & 122.521 & 118.902 & 100.202 & 104.115 & 95.785 & 109.503 & 106.762 & 73.987 \\
\hline $10 / 8 / 0721: 00$ & 124.729 & 122.533 & 118.912 & 100.204 & 104.119 & 95.787 & 109.501 & 106.767 & 73.992 \\
\hline 10/8/07 21:30 & 124.729 & 122.537 & 118.923 & 100.212 & 104.127 & 95.795 & 109.497 & 106.773 & 73.998 \\
\hline $10 / 8 / 0722: 00$ & 124.731 & 122.539 & 118.928 & 100.223 & 104.138 & 95.806 & 109.497 & 106.784 & 74.006 \\
\hline $10 / 8 / 07$ 22:30 & 124.733 & 122.548 & 118.933 & 100.226 & 104.142 & 95.813 & 109.493 & 106.789 & 74.012 \\
\hline $10 / 8 / 07$ 23:00 & 124.733 & 122.546 & 118.93 & 100.231 & 104.146 & 95.816 & 109.491 & 106.795 & 74.016 \\
\hline $10 / 8 / 07$ 23:30 & 124.738 & 122.546 & 118.93 & 100.236 & 104.151 & 95.823 & 109.49 & 106.8 & 74.023 \\
\hline $10 / 9 / 070: 00$ & 124.738 & 122.548 & 118.928 & 100.235 & 104.151 & 95.823 & 109.488 & 106.798 & 74.021 \\
\hline 10/9/07 0:30 & 124.74 & 122.551 & 118.935 & 100.236 & 104.15 & 95.823 & 109.486 & 106.798 & 74.021 \\
\hline 10/9/07 1:00 & 124.743 & 122.553 & 118.938 & 100.236 & 104.151 & 95.823 & 109.484 & 106.8 & 74.023 \\
\hline 10/9/07 1:30 & 124.745 & 122.551 & 118.935 & 100.241 & 104.159 & 95.825 & 109.48 & 106.802 & 74.027 \\
\hline $10 / 9 / 072: 00$ & 124.745 & 122.553 & 118.933 & 100.243 & 104.159 & 95.83 & 109.478 & 106.807 & 74.029 \\
\hline 10/9/07 2:30 & 124.748 & 122.546 & 118.93 & 100.241 & 104.155 & 95.828 & 109.476 & 106.802 & 74.025 \\
\hline 10/9/07 3:00 & 124.75 & 122.544 & 118.928 & 100.241 & 104.157 & 95.828 & 109.473 & 106.804 & 74.027 \\
\hline 10/9/07 3:30 & 124.755 & 122.542 & 118.928 & 100.233 & 104.15 & 95.82 & 109.475 & 106.795 & 74.018 \\
\hline $10 / 9 / 074: 00$ & 124.755 & 122.542 & 118.923 & 100.231 & 104.148 & 95.818 & 109.473 & 106.796 & 74.018 \\
\hline 10/9/07 4:30 & 124.757 & 122.546 & 118.928 & 100.231 & 104.146 & 95.816 & 109.471 & 106.794 & 74.018 \\
\hline $10 / 9 / 075: 00$ & 124.757 & 122.546 & 118.928 & 100.231 & 104.15 & 95.82 & 109.469 & 106.793 & 74.018 \\
\hline
\end{tabular}


TABLE S1.1 (Cont.)

Depth from Top of Casing (ft)

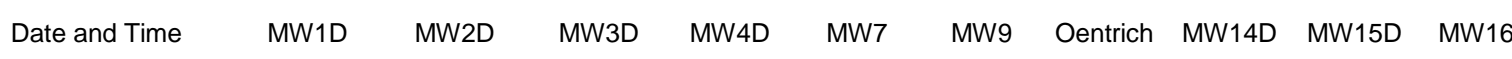

\begin{tabular}{|c|c|c|c|c|c|c|c|c|c|}
\hline 10/9/07 5:30 & 124.762 & 122.548 & 118.925 & 100.236 & 104.15 & 95.823 & 109.465 & 106.795 & 74.021 \\
\hline 10/9/07 6:00 & 124.764 & 124.442 & 120.094 & 100.235 & 104.15 & 95.82 & 109.465 & 106.798 & 74.021 \\
\hline 10/9/07 6:30 & 124.764 & 124.775 & 120.515 & 100.24 & 104.151 & 95.823 & 109.463 & 106.8 & 74.023 \\
\hline 10/9/07 7:00 & 126.322 & 124.92 & 120.685 & 100.951 & 104.587 & 96.391 & 110.019 & 108.172 & 74.072 \\
\hline 10/9/07 7:30 & 126.624 & 125.013 & 120.782 & 101.201 & 104.905 & 96.716 & 110.316 & 108.56 & 74.281 \\
\hline $10 / 9 / 078: 00$ & 126.766 & 125.077 & 120.835 & 101.33 & 105.054 & 96.865 & 110.457 & 108.722 & 74.424 \\
\hline 10/9/07 8:30 & 126.861 & 125.12 & 120.881 & 101.413 & 105.147 & 96.959 & 110.539 & 108.819 & 74.521 \\
\hline 10/9/07 9:00 & 126.929 & 125.149 & 120.916 & 101.47 & 105.21 & 97.02 & 110.592 & 108.885 & 74.587 \\
\hline 10/9/07 9:30 & 126.984 & 125.172 & 120.939 & 101.51 & 105.256 & 97.065 & 110.631 & 108.932 & 74.637 \\
\hline 10/9/07 10:00 & 127.033 & 125.186 & 120.959 & 101.537 & 105.284 & 97.098 & 110.667 & 108.965 & 74.668 \\
\hline 10/9/07 10:30 & 127.078 & 125.195 & 120.971 & 101.558 & 105.307 & 97.119 & 110.689 & 108.987 & 74.692 \\
\hline 10/9/07 11:00 & 127.118 & 125.204 & 120.976 & 101.572 & 105.322 & 97.138 & 110.706 & 109.005 & 74.709 \\
\hline 10/9/07 11:30 & 127.154 & 125.206 & 120.984 & 101.584 & 105.338 & 97.15 & 110.717 & 109.016 & 74.728 \\
\hline 10/9/07 12:00 & 127.189 & 123.381 & 119.88 & 101.592 & 105.345 & 97.157 & 110.727 & 109.023 & 74.736 \\
\hline $10 / 9 / 07 \quad 12: 30$ & 127.248 & 123.065 & 119.482 & 101.597 & 105.351 & 97.162 & 110.736 & 109.027 & 74.746 \\
\hline 10/9/07 13:00 & 125.811 & 122.909 & 119.311 & 100.901 & 104.926 & 96.605 & 110.192 & 107.705 & 74.695 \\
\hline 10/9/07 13:30 & 125.506 & 122.812 & 119.205 & 100.664 & 104.629 & 96.304 & 109.918 & 107.338 & 74.519 \\
\hline $10 / 9 / 07 \quad 14: 00$ & 125.35 & 122.743 & 119.137 & 100.534 & 104.479 & 96.153 & 109.788 & 107.172 & 74.384 \\
\hline $10 / 9 / 07 \quad 14: 30$ & 125.244 & 122.696 & 119.084 & 100.455 & 104.389 & 96.063 & 109.715 & 107.07 & 74.291 \\
\hline 10/9/07 15:00 & 125.171 & 122.66 & 119.049 & 100.395 & 104.325 & 95.997 & 109.668 & 106.999 & 74.223 \\
\hline 10/9/07 15:30 & 125.107 & 122.635 & 119.018 & 100.353 & 104.281 & 95.955 & 109.634 & 106.95 & 74.176 \\
\hline 10/9/07 16:00 & 125.057 & 122.612 & 118.993 & 100.322 & 104.247 & 95.919 & 109.61 & 106.913 & 74.138 \\
\hline 10/9/07 16:30 & 125.017 & 122.601 & 118.968 & 100.3 & 104.22 & 95.894 & 109.591 & 106.884 & 74.112 \\
\hline 10/9/07 17:00 & 124.989 & 122.592 & 118.97 & 100.283 & 104.203 & 95.875 & 109.578 & 106.864 & 74.093 \\
\hline $10 / 9 / 07 \quad 17: 30$ & 124.967 & 122.587 & 118.963 & 100.272 & 104.192 & 95.865 & 109.567 & 106.851 & 74.081 \\
\hline 10/9/07 18:00 & 124.956 & 122.58 & 118.963 & 100.264 & 104.182 & 95.853 & 109.557 & 106.84 & 74.07 \\
\hline 10/9/07 18:30 & 124.946 & 122.58 & 118.963 & 100.262 & 104.176 & 95.849 & 109.55 & 106.835 & 74.066 \\
\hline 10/9/07 19:00 & 124.941 & 122.585 & 118.965 & 100.259 & 104.174 & 95.846 & 109.542 & 106.831 & 74.06 \\
\hline 10/9/07 19:30 & 124.937 & 122.587 & 118.963 & 100.26 & 104.174 & 95.849 & 109.537 & 106.829 & 74.06 \\
\hline 10/9/07 20:00 & 124.934 & 122.587 & 118.97 & 100.264 & 104.178 & 95.851 & 109.531 & 106.833 & 74.064 \\
\hline 10/9/07 20:30 & 124.937 & 122.592 & 118.97 & 100.266 & 104.18 & 95.851 & 109.529 & 106.835 & 74.064 \\
\hline 10/9/07 21:00 & 124.939 & 122.592 & 118.958 & 100.269 & 104.182 & 95.856 & 109.525 & 106.838 & 74.066 \\
\hline 10/9/07 21:30 & 124.944 & 122.594 & 118.96 & 100.272 & 104.186 & 95.858 & 109.521 & 106.84 & 74.068 \\
\hline 10/9/07 22:00 & 124.944 & 122.592 & 118.96 & 100.271 & 104.186 & 95.861 & 109.52 & 106.84 & 74.068 \\
\hline 10/9/07 22:30 & 124.944 & 122.589 & 118.955 & 100.274 & 104.191 & 95.863 & 109.516 & 106.844 & 74.07 \\
\hline 10/9/07 23:00 & 124.939 & 122.587 & 118.953 & 100.271 & 104.188 & 95.858 & 109.512 & 106.842 & 74.07 \\
\hline 10/9/07 23:30 & 124.937 & 122.582 & 118.96 & 100.269 & 104.188 & 95.858 & 109.51 & 106.84 & 74.066 \\
\hline 10/10/07 0:00 & 124.932 & 122.58 & 118.963 & 100.267 & 104.182 & 95.856 & 109.51 & 106.835 & 74.064 \\
\hline $10 / 10 / 070: 30$ & 124.93 & 122.58 & 118.963 & 100.264 & 104.178 & 95.849 & 109.506 & 106.829 & 74.058 \\
\hline 10/10/07 1:00 & 124.922 & 122.582 & 118.963 & 100.26 & 104.176 & 95.851 & 109.505 & 106.829 & 74.058 \\
\hline 10/10/07 1:30 & 124.918 & 122.58 & 118.965 & 100.264 & 104.182 & 95.849 & 109.501 & 106.829 & 74.06 \\
\hline $10 / 10 / 072: 00$ & 124.915 & 122.576 & 118.955 & 100.264 & 104.178 & 95.849 & 109.499 & 106.829 & 74.06 \\
\hline 10/10/07 2:30 & 124.911 & 122.571 & 118.958 & 100.264 & 104.182 & 95.853 & 109.497 & 106.831 & 74.062 \\
\hline 10/10/07 3:00 & 124.908 & 122.569 & 118.95 & 100.26 & 104.178 & 95.846 & 109.495 & 106.826 & 74.058 \\
\hline $10 / 10 / 073: 30$ & 124.906 & 122.569 & 118.953 & 100.254 & 104.172 & 95.844 & 109.493 & 106.822 & 74.052 \\
\hline $10 / 10 / 074: 00$ & 124.901 & 122.569 & 118.953 & 100.252 & 104.169 & 95.844 & 109.491 & 106.82 & 74.052 \\
\hline $10 / 10 / 074: 30$ & 124.899 & 122.573 & 118.955 & 100.254 & 104.169 & 95.844 & 109.491 & 106.818 & 74.05 \\
\hline $10 / 10 / 07$ 5:00 & 124.894 & 122.578 & 118.96 & 100.255 & 104.17 & 95.842 & 109.488 & 106.818 & 74.05 \\
\hline 10/10/07 5:30 & 124.892 & 124.313 & 120.024 & 100.259 & 104.174 & 95.846 & 109.484 & 106.822 & 74.054 \\
\hline 10/10/07 6:00 & 124.887 & 124.712 & 120.598 & 100.267 & 104.178 & 95.851 & 109.484 & 106.826 & 74.059 \\
\hline 10/10/07 6:30 & 126.319 & 124.877 & 120.787 & 100.938 & 104.527 & 96.337 & 109.968 & 107.989 & 74.076 \\
\hline 10/10/07 7:00 & 126.691 & 124.977 & 120.898 & 101.229 & 104.911 & 96.735 & 110.338 & 108.491 & 74.276 \\
\hline $10 / 10 / 077: 30$ & 126.849 & 125.045 & 120.971 & 101.37 & 105.075 & 96.9 & 110.496 & 108.675 & 74.428 \\
\hline $10 / 10 / 07$ 8:00 & 126.946 & 125.093 & 121.024 & 101.458 & 105.178 & 96.997 & 110.588 & 108.781 & 74.533 \\
\hline 10/10/07 8:30 & 127.014 & 125.122 & 121.059 & 101.523 & 105.244 & 97.067 & 110.648 & 108.854 & 74.606 \\
\hline 10/10/07 9:00 & 127.069 & 125.152 & 121.082 & 101.563 & 105.292 & 97.115 & 110.691 & 108.905 & 74.655 \\
\hline 10/10/07 9:30 & 127.111 & 125.177 & 121.11 & 101.594 & 105.326 & 97.15 & 110.725 & 108.939 & 74.69 \\
\hline 10/10/07 10:00 & 127.156 & 123.569 & 120.271 & 101.62 & 105.349 & 97.176 & 110.749 & 108.967 & 74.717 \\
\hline $10 / 10 / 07$ 10:30 & 127.194 & 123.129 & 119.558 & 101.639 & 105.372 & 97.197 & 110.768 & 108.989 & 74.74 \\
\hline $10 / 10 / 0711: 00$ & 125.908 & 122.957 & 119.361 & 101.022 & 105.094 & 96.789 & 110.353 & 108.009 & 74.742 \\
\hline 10/10/07 11:30 & 125.513 & 122.85 & 119.248 & 100.719 & 104.692 & 96.37 & 109.966 & 107.409 & 74.57 \\
\hline $10 / 10 / 07$ 12:00 & 125.362 & 122.775 & 119.17 & 100.581 & 104.527 & 96.202 & 109.816 & 107.221 & 74.43 \\
\hline $10 / 10 / 07$ 12:30 & 125.256 & 122.723 & 119.119 & 100.491 & 104.428 & 96.101 & 109.732 & 107.11 & 74.331 \\
\hline $10 / 10 / 07$ 13:00 & 125.175 & 122.68 & 119.069 & 100.426 & 104.357 & 96.03 & 109.685 & 107.035 & 74.256 \\
\hline $10 / 10 / 07$ 13:30 & 125.109 & 122.641 & 119.033 & 100.379 & 104.309 & 95.983 & 109.649 & 106.982 & 74.207 \\
\hline $10 / 10 / 07$ 14:00 & 125.064 & 122.612 & 119.001 & 100.34 & 104.268 & 95.941 & 109.625 & 106.935 & 74.163 \\
\hline
\end{tabular}


TABLE S1.1 (Cont.)

Depth from Top of Casing (ft)

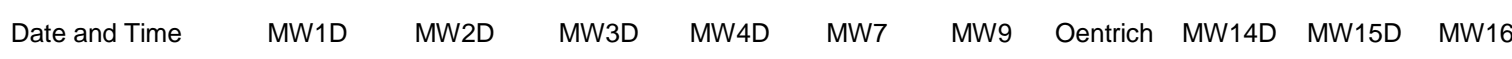

\begin{tabular}{|c|c|c|c|c|c|c|c|c|c|}
\hline 10/10/07 14:30 & 125.024 & 122.589 & 118.978 & 100.302 & 104.23 & 95.901 & 109.602 & 106.893 & 74.122 \\
\hline 10/10/07 15:00 & 124.991 & 122.564 & 118.955 & 100.278 & 104.199 & 95.872 & 109.591 & 106.864 & 74.091 \\
\hline 10/10/07 15:30 & 124.965 & 122.546 & 118.93 & 100.257 & 104.178 & 95.851 & 109.58 & 106.84 & 74.072 \\
\hline 10/10/07 16:00 & 124.944 & 122.537 & 118.918 & 100.235 & 104.159 & 95.83 & 109.572 & 106.818 & 74.052 \\
\hline $10 / 10 / 07$ 16:30 & 124.92 & 122.528 & 118.915 & 100.219 & 104.138 & 95.811 & 109.565 & 106.798 & 74.033 \\
\hline $10 / 10 / 07$ 17:00 & 124.908 & 122.521 & 118.907 & 100.21 & 104.127 & 95.799 & 109.557 & 106.784 & 74.021 \\
\hline $10 / 10 / 07$ 17:30 & 124.894 & 122.517 & 118.9 & 100.205 & 104.123 & 95.795 & 109.552 & 106.778 & 74.014 \\
\hline $10 / 10 / 07$ 18:00 & 124.887 & 122.512 & 118.897 & 100.197 & 104.117 & 95.787 & 109.548 & 106.771 & 74.008 \\
\hline 10/10/07 18:30 & 124.88 & 122.512 & 118.895 & 100.195 & 104.111 & 95.783 & 109.542 & 106.764 & 74.002 \\
\hline $10 / 10 / 07$ 19:00 & 124.875 & 122.512 & 118.895 & 100.19 & 104.108 & 95.78 & 109.54 & 106.76 & 73.996 \\
\hline 10/10/07 19:30 & 124.87 & 122.51 & 118.89 & 100.193 & 104.11 & 95.778 & 109.537 & 106.76 & 73.998 \\
\hline $10 / 10 / 0720: 00$ & 124.87 & 122.514 & 118.897 & 100.191 & 104.108 & 95.78 & 109.535 & 106.76 & 73.996 \\
\hline 10/10/07 20:30 & 124.866 & 122.517 & 118.9 & 100.191 & 104.108 & 95.778 & 109.533 & 106.758 & 73.994 \\
\hline $10 / 10 / 0721: 00$ & 124.863 & 122.519 & 118.902 & 100.195 & 104.108 & 95.78 & 109.531 & 106.76 & 73.996 \\
\hline 10/10/07 21:30 & 124.859 & 122.524 & 118.907 & 100.197 & 104.115 & 95.785 & 109.529 & 106.764 & 74 \\
\hline $10 / 10 / 0722: 00$ & 124.854 & 122.526 & 118.907 & 100.204 & 104.115 & 95.79 & 109.525 & 106.767 & 74.002 \\
\hline 10/10/07 22:30 & 124.849 & 122.53 & 118.912 & 100.204 & 104.121 & 95.795 & 109.523 & 106.773 & 74.006 \\
\hline 10/10/07 23:00 & 124.852 & 122.533 & 118.918 & 100.205 & 104.119 & 95.792 & 109.523 & 106.773 & 74.004 \\
\hline 10/10/07 23:30 & 124.844 & 122.526 & 118.91 & 100.212 & 104.127 & 95.799 & 109.52 & 106.778 & 74.01 \\
\hline $10 / 11 / 070: 00$ & 124.842 & 122.524 & 118.91 & 100.216 & 104.131 & 95.804 & 109.52 & 106.782 & 74.014 \\
\hline 10/11/07 0:30 & 124.844 & 122.521 & 118.907 & 100.21 & 104.125 & 95.799 & 109.52 & 106.776 & 74.008 \\
\hline 10/11/07 1:00 & 124.84 & 122.517 & 118.902 & 100.205 & 104.121 & 95.795 & 109.518 & 106.773 & 74.004 \\
\hline $10 / 11 / 071: 30$ & 124.84 & 122.521 & 118.902 & 100.204 & 104.119 & 95.792 & 109.516 & 106.771 & 74.004 \\
\hline $10 / 11 / 072: 00$ & 124.84 & 122.512 & 118.897 & 100.2 & 104.117 & 95.787 & 109.516 & 106.767 & 74 \\
\hline 10/11/07 2:30 & 124.833 & 122.51 & 118.887 & 100.205 & 104.119 & 95.79 & 109.512 & 106.769 & 74 \\
\hline 10/11/07 3:00 & 124.835 & 122.51 & 118.895 & 100.193 & 104.113 & 95.785 & 109.514 & 106.762 & 73.994 \\
\hline 10/11/07 3:30 & 124.828 & 122.505 & 118.89 & 100.195 & 104.108 & 95.78 & 109.51 & 106.758 & 73.992 \\
\hline $10 / 11 / 074: 00$ & 124.83 & 122.51 & 118.892 & 100.195 & 104.111 & 95.783 & 109.508 & 106.758 & 73.992 \\
\hline $10 / 11 / 074: 30$ & 124.828 & 122.503 & 118.887 & 100.19 & 104.106 & 95.78 & 109.508 & 106.756 & 73.99 \\
\hline $10 / 11 / 075: 00$ & 124.823 & 122.505 & 118.885 & 100.197 & 104.11 & 95.78 & 109.505 & 106.758 & 73.992 \\
\hline 10/11/07 5:30 & 124.826 & 122.51 & 118.89 & 100.19 & 104.102 & 95.773 & 109.506 & 106.753 & 73.983 \\
\hline $10 / 11 / 076: 00$ & 124.823 & 124.288 & 119.88 & 100.192 & 104.106 & 95.776 & 109.505 & 106.751 & 73.985 \\
\hline 10/11/07 6:30 & 124.821 & 124.682 & 120.422 & 100.2 & 104.112 & 95.783 & 109.503 & 106.76 & 73.992 \\
\hline 10/11/07 7:00 & 126.265 & 124.85 & 120.613 & 100.836 & 104.45 & 96.245 & 109.966 & 107.975 & 74.01 \\
\hline 10/11/07 7:30 & 126.631 & 124.948 & 120.727 & 101.125 & 104.82 & 96.629 & 110.319 & 108.463 & 74.211 \\
\hline 10/11/07 8:00 & 126.783 & 125.018 & 120.8 & 101.27 & 104.993 & 96.801 & 110.468 & 108.648 & 74.372 \\
\hline 10/11/07 8:30 & 126.88 & 125.05 & 120.84 & 101.365 & 105.09 & 96.902 & 110.558 & 108.755 & 74.475 \\
\hline 10/11/07 9:00 & 126.951 & 125.068 & 120.858 & 101.418 & 105.161 & 96.973 & 110.618 & 108.83 & 74.546 \\
\hline 10/11/07 9:30 & 127.005 & 123.106 & 119.532 & 101.448 & 105.197 & 97.008 & 110.661 & 108.868 & 74.587 \\
\hline $10 / 11 / 07$ 10:00 & 127.047 & 122.898 & 119.308 & 101.137 & 105.214 & 97.03 & 110.687 & 108.888 & 74.612 \\
\hline $10 / 11 / 07 \quad 10: 30$ & 125.428 & 122.782 & 119.18 & 100.684 & 104.667 & 96.341 & 110.01 & 107.393 & 74.515 \\
\hline 10/11/07 11:00 & 125.265 & 122.7 & 119.091 & 100.519 & 104.471 & 96.146 & 109.826 & 107.163 & 74.368 \\
\hline $10 / 11 / 0711: 30$ & 125.185 & 122.653 & 119.041 & 100.421 & 104.357 & 96.033 & 109.732 & 107.037 & 74.256 \\
\hline $10 / 11 / 0712: 00$ & 125.121 & 122.612 & 119.001 & 100.35 & 104.279 & 95.952 & 109.675 & 106.955 & 74.176 \\
\hline 10/11/07 12:30 & 125.064 & 122.571 & 118.96 & 100.309 & 104.235 & 95.91 & 109.638 & 106.904 & 74.128 \\
\hline $10 / 11 / 0713: 00$ & 125.015 & 122.535 & 118.925 & 100.271 & 104.195 & 95.87 & 109.615 & 106.862 & 74.085 \\
\hline $10 / 11 / 0713: 30$ & 124.963 & 122.514 & 118.902 & 100.233 & 104.157 & 95.83 & 109.6 & 106.822 & 74.047 \\
\hline 10/11/07 14:00 & 124.922 & 122.49 & 118.88 & 100.205 & 104.121 & 95.797 & 109.587 & 106.784 & 74.01 \\
\hline 10/11/07 14:30 & 124.892 & 122.46 & 118.85 & 100.181 & 104.102 & 95.778 & 109.578 & 106.762 & 73.99 \\
\hline $10 / 11 / 07$ 15:00 & 124.873 & 122.444 & 118.827 & 100.159 & 104.079 & 95.757 & 109.57 & 106.738 & 73.966 \\
\hline 10/11/07 15:30 & 124.847 & 122.426 & 118.814 & 100.131 & 104.051 & 95.726 & 109.567 & 106.707 & 73.94 \\
\hline $10 / 11 / 07$ 16:00 & 124.826 & 122.408 & 118.794 & 100.112 & 104.032 & 95.71 & 109.563 & 106.694 & 73.921 \\
\hline $10 / 11 / 0716: 30$ & 124.804 & 122.403 & 118.787 & 100.098 & 104.018 & 95.691 & 109.561 & 106.674 & 73.907 \\
\hline $10 / 11 / 07$ 17:00 & 124.785 & 122.403 & 118.787 & 100.083 & 103.999 & 95.672 & 109.555 & 106.654 & 73.888 \\
\hline $10 / 11 / 07$ 17:30 & 124.769 & 122.399 & 118.781 & 100.083 & 103.997 & 95.67 & 109.55 & 106.649 & 73.886 \\
\hline $10 / 11 / 0718: 00$ & 124.764 & 122.394 & 118.776 & 100.081 & 103.999 & 95.67 & 109.544 & 106.647 & 73.884 \\
\hline 10/11/07 18:30 & 124.762 & 122.397 & 118.784 & 100.079 & 103.993 & 95.665 & 109.542 & 106.645 & 73.882 \\
\hline $10 / 11 / 07$ 19:00 & 124.759 & 122.401 & 118.787 & 100.074 & 103.988 & 95.66 & 109.54 & 106.64 & 73.873 \\
\hline 10/11/07 19:30 & 124.757 & 122.397 & 118.781 & 100.079 & 103.992 & 95.663 & 109.538 & 106.643 & 73.878 \\
\hline 10/11/07 20:00 & 124.757 & 122.394 & 118.781 & 100.076 & 103.995 & 95.667 & 109.537 & 106.647 & 73.882 \\
\hline $10 / 11 / 0720: 30$ & 124.759 & 122.397 & 118.779 & 100.073 & 103.99 & 95.663 & 109.538 & 106.64 & 73.876 \\
\hline 10/11/07 21:00 & 124.757 & 122.399 & 118.784 & 100.074 & 103.992 & 95.663 & 109.537 & 106.64 & 73.874 \\
\hline 10/11/07 21:30 & 124.755 & 122.401 & 118.787 & 100.078 & 103.99 & 95.663 & 109.537 & 106.64 & 73.874 \\
\hline 10/11/07 22:00 & 124.752 & 122.403 & 118.789 & 100.081 & 103.993 & 95.665 & 109.535 & 106.643 & 73.874 \\
\hline $10 / 11 / 0722: 30$ & 124.752 & 122.397 & 118.784 & 100.085 & 103.993 & 95.667 & 109.535 & 106.645 & 73.876 \\
\hline $10 / 11 / 07$ 23:00 & 124.752 & 122.399 & 118.784 & 100.081 & 103.999 & 95.672 & 109.535 & 106.647 & 73.88 \\
\hline
\end{tabular}


TABLE S1.1 (Cont.)

Depth from Top of Casing (ft)

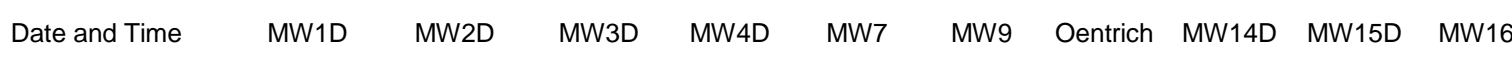

\begin{tabular}{|c|c|c|c|c|c|c|c|c|c|}
\hline $10 / 11 / 0723: 30$ & 124.755 & 122.401 & 118.784 & 100.074 & 103.992 & 95.665 & 109.535 & 106.643 & 73.872 \\
\hline $10 / 12 / 070: 00$ & 124.75 & 122.399 & 118.781 & 100.079 & 103.993 & 95.667 & 109.533 & 106.645 & 73.874 \\
\hline $10 / 12 / 070: 30$ & 124.748 & 122.39 & 118.774 & 100.081 & 103.999 & 95.67 & 109.535 & 106.647 & 73.876 \\
\hline 10/12/07 1:00 & 124.748 & 122.394 & 118.779 & 100.074 & 103.993 & 95.667 & 109.535 & 106.643 & 73.872 \\
\hline 10/12/07 1:30 & 124.75 & 122.394 & 118.781 & 100.071 & 103.982 & 95.658 & 109.535 & 106.632 & 73.861 \\
\hline $10 / 12 / 072: 00$ & 124.748 & 122.392 & 118.779 & 100.076 & 103.986 & 95.663 & 109.533 & 106.639 & 73.867 \\
\hline $10 / 12 / 072: 30$ & 124.745 & 122.381 & 118.771 & 100.076 & 103.99 & 95.667 & 109.531 & 106.64 & 73.87 \\
\hline 10/12/07 3:00 & 124.745 & 122.378 & 118.766 & 100.073 & 103.988 & 95.66 & 109.529 & 106.636 & 73.863 \\
\hline 10/12/07 3:30 & 124.745 & 122.376 & 118.761 & 100.062 & 103.978 & 95.651 & 109.531 & 106.629 & 73.855 \\
\hline 10/12/07 4:00 & 124.745 & 122.372 & 118.761 & 100.061 & 103.978 & 95.651 & 109.529 & 106.625 & 73.853 \\
\hline $10 / 12 / 074: 30$ & 124.745 & 122.367 & 118.754 & 100.057 & 103.974 & 95.646 & 109.531 & 106.621 & 73.849 \\
\hline $10 / 12 / 075: 00$ & 124.745 & 122.36 & 118.751 & 100.054 & 103.969 & 95.641 & 109.529 & 106.618 & 73.845 \\
\hline 10/12/07 5:30 & 124.743 & 122.36 & 118.741 & 100.047 & 103.963 & 95.634 & 109.529 & 106.609 & 73.839 \\
\hline 10/12/07 6:00 & 124.74 & 124.19 & 119.986 & 100.045 & 103.959 & 95.632 & 109.529 & 106.609 & 73.837 \\
\hline $10 / 12 / 076: 30$ & 124.743 & 124.528 & 120.422 & 100.042 & 103.957 & 95.632 & 109.527 & 106.605 & 73.832 \\
\hline $10 / 12 / 077: 00$ & 126.267 & 124.687 & 120.598 & 100.779 & 104.395 & 96.209 & 110.104 & 107.909 & 73.872 \\
\hline $10 / 12 / 07 \quad 7: 30$ & 126.579 & 124.789 & 120.709 & 101.036 & 104.721 & 96.546 & 110.415 & 108.308 & 74.076 \\
\hline $10 / 12 / 078: 00$ & 126.726 & 124.85 & 120.785 & 101.177 & 104.879 & 96.704 & 110.56 & 108.48 & 74.229 \\
\hline 10/12/07 8:30 & 126.818 & 124.895 & 120.823 & 101.261 & 104.98 & 96.801 & 110.644 & 108.586 & 74.331 \\
\hline 10/12/07 9:00 & 126.887 & 123.317 & 120.062 & 101.322 & 105.044 & 96.872 & 110.702 & 108.66 & 74.401 \\
\hline 10/12/07 9:30 & 126.936 & 122.88 & 119.311 & 101.365 & 105.09 & 96.914 & 110.742 & 108.704 & 74.446 \\
\hline $10 / 12 / 0710: 00$ & 125.655 & 122.714 & 119.122 & 100.764 & 104.841 & 96.535 & 110.351 & 107.765 & 74.467 \\
\hline $10 / 12 / 07 \quad 10: 30$ & 125.249 & 122.601 & 119.006 & 100.474 & 104.443 & 96.115 & 109.965 & 107.152 & 74.31 \\
\hline $10 / 12 / 0711: 00$ & 125.112 & 122.539 & 118.928 & 100.347 & 104.289 & 95.96 & 109.818 & 106.973 & 74.18 \\
\hline $10 / 12 / 0711: 30$ & 125.041 & 122.494 & 118.887 & 100.24 & 104.178 & 95.853 & 109.745 & 106.857 & 74.07 \\
\hline $10 / 12 / 0712: 00$ & 124.993 & 122.467 & 118.844 & 100.205 & 104.117 & 95.79 & 109.694 & 106.784 & 74.002 \\
\hline $10 / 12 / 0712: 30$ & 124.967 & 122.433 & 118.827 & 100.16 & 104.077 & 95.752 & 109.664 & 106.745 & 73.963 \\
\hline $10 / 12 / 0713: 00$ & 124.944 & 122.394 & 118.781 & 100.126 & 104.051 & 95.721 & 109.642 & 106.711 & 73.928 \\
\hline 10/12/07 13:30 & 124.932 & 122.376 & 118.764 & 100.095 & 104.018 & 95.696 & 109.629 & 106.683 & 73.899 \\
\hline $10 / 12 / 0714: 00$ & 124.925 & 122.367 & 118.756 & 100.064 & 103.98 & 95.653 & 109.619 & 106.64 & 73.859 \\
\hline $10 / 12 / 0714: 30$ & 124.901 & 122.338 & 118.731 & 100.048 & 103.965 & 95.637 & 109.61 & 106.623 & 73.844 \\
\hline $10 / 12 / 0715: 00$ & 124.87 & 122.324 & 118.713 & 100.042 & 103.953 & 95.627 & 109.604 & 106.612 & 73.832 \\
\hline $10 / 12 / 0715: 30$ & 124.842 & 122.317 & 118.703 & 100.011 & 103.925 & 95.599 & 109.602 & 106.585 & 73.804 \\
\hline $10 / 12 / 0716: 00$ & 124.802 & 122.315 & 118.706 & 100 & 103.915 & 95.592 & 109.597 & 106.572 & 73.795 \\
\hline $10 / 12 / 0716: 30$ & 124.769 & 122.317 & 118.696 & 99.997 & 103.908 & 95.582 & 109.593 & 106.563 & 73.787 \\
\hline $10 / 12 / 07$ 17:00 & 124.74 & 122.304 & 118.693 & 99.992 & 103.91 & 95.582 & 109.589 & 106.561 & 73.789 \\
\hline $10 / 12 / 0717: 30$ & 124.719 & 122.299 & 118.691 & 99.988 & 103.915 & 95.582 & 109.583 & 106.561 & 73.787 \\
\hline $10 / 12 / 0718: 00$ & 124.719 & 122.301 & 118.688 & 99.988 & 103.898 & 95.575 & 109.585 & 106.55 & 73.775 \\
\hline $10 / 12 / 07$ 18:30 & 124.717 & 122.301 & 118.691 & 99.973 & 103.893 & 95.568 & 109.583 & 106.545 & 73.768 \\
\hline $10 / 12 / 0719: 00$ & 124.712 & 122.297 & 118.686 & 99.988 & 103.896 & 95.571 & 109.582 & 106.548 & 73.772 \\
\hline $10 / 12 / 0719: 30$ & 124.71 & 122.295 & 118.676 & 99.981 & 103.893 & 95.564 & 109.58 & 106.543 & 73.766 \\
\hline $10 / 12 / 0720: 00$ & 124.705 & 122.301 & 118.688 & 99.976 & 103.891 & 95.564 & 109.58 & 106.541 & 73.764 \\
\hline $10 / 12 / 0720: 30$ & 124.705 & 122.297 & 118.683 & 99.98 & 103.889 & 95.559 & 109.582 & 106.539 & 73.76 \\
\hline $10 / 12 / 0721: 00$ & 124.703 & 122.285 & 118.681 & 99.976 & 103.896 & 95.571 & 109.578 & 106.548 & 73.77 \\
\hline 10/12/07 21:30 & 124.703 & 122.285 & 118.678 & 99.974 & 103.893 & 95.564 & 109.58 & 106.541 & 73.762 \\
\hline $10 / 12 / 0722: 00$ & 124.707 & 122.283 & 118.671 & 99.966 & 103.881 & 95.552 & 109.582 & 106.532 & 73.75 \\
\hline $10 / 12 / 0722: 30$ & 124.703 & 122.283 & 118.668 & 99.966 & 103.881 & 95.554 & 109.582 & 106.532 & 73.752 \\
\hline $10 / 12 / 0723: 00$ & 124.705 & 122.283 & 118.661 & 99.964 & 103.872 & 95.547 & 109.583 & 106.525 & 73.743 \\
\hline $10 / 12 / 0723: 30$ & 124.7 & 122.281 & 118.683 & 99.962 & 103.875 & 95.547 & 109.583 & 106.525 & 73.746 \\
\hline 10/13/07 0:00 & 124.698 & 122.276 & 118.673 & 99.976 & 103.873 & 95.545 & 109.58 & 106.519 & 73.739 \\
\hline $10 / 13 / 070: 30$ & 124.703 & 122.267 & 118.671 & 99.959 & 103.877 & 95.552 & 109.583 & 106.53 & 73.747 \\
\hline 10/13/07 1:00 & 124.703 & 122.263 & 118.761 & 99.962 & 103.872 & 95.547 & 109.583 & 106.525 & 73.744 \\
\hline 10/13/07 1:30 & 124.703 & 122.267 & 118.645 & 99.967 & 103.864 & 95.54 & 109.585 & 106.519 & 73.737 \\
\hline $10 / 13 / 072: 00$ & 124.688 & 122.24 & 118.62 & 99.942 & 103.864 & 95.535 & 109.58 & 106.51 & 74.513 \\
\hline $10 / 13 / 072: 30$ & 124.688 & 122.236 & 118.605 & 99.959 & 103.862 & 95.535 & 109.576 & 106.506 & 75.043 \\
\hline 10/13/07 3:00 & 124.698 & 122.204 & 118.58 & 99.923 & 103.839 & 95.516 & 109.582 & 106.49 & 74.831 \\
\hline $10 / 13 / 07$ 3:30 & 124.698 & 122.195 & 118.577 & 99.911 & 103.822 & 95.495 & 109.578 & 106.472 & 74.624 \\
\hline $10 / 13 / 074: 00$ & 124.7 & 122.227 & 118.61 & 99.897 & 103.799 & 95.472 & 109.58 & 106.448 & 74.479 \\
\hline $10 / 13 / 074: 30$ & 124.696 & 122.24 & 118.625 & 99.887 & 103.79 & 95.457 & 109.574 & 106.435 & 74.357 \\
\hline 10/13/07 5:00 & 124.686 & 122.236 & 118.625 & 99.912 & 103.832 & 95.502 & 109.567 & 106.474 & 74.312 \\
\hline $10 / 13 / 075: 30$ & 124.688 & 122.236 & 118.625 & 99.924 & 103.839 & 95.514 & 109.565 & 106.483 & 74.246 \\
\hline $10 / 13 / 076: 00$ & 124.691 & 122.238 & 118.625 & 99.918 & 103.835 & 95.507 & 109.568 & 106.481 & 74.13 \\
\hline 10/13/07 6:30 & 124.691 & 124.086 & 119.731 & 99.921 & 103.835 & 95.507 & 109.568 & 106.481 & 74.114 \\
\hline $10 / 13 / 07$ 7:00 & 124.693 & 124.426 & 120.177 & 99.923 & 103.835 & 95.507 & 109.567 & 106.481 & 74.101 \\
\hline $10 / 13 / 07$ 7:30 & 126.208 & 124.585 & 120.349 & 100.608 & 104.237 & 96.04 & 110.09 & 107.807 & 74.114 \\
\hline $10 / 13 / 078: 00$ & 126.513 & 124.678 & 120.452 & 100.86 & 104.561 & 96.374 & 110.408 & 108.21 & 74.25 \\
\hline
\end{tabular}


TABLE S1.1 (Cont.)

\begin{tabular}{|c|c|c|c|c|c|c|c|c|c|c|}
\hline \multirow[b]{2}{*}{ Date and Time } & \multicolumn{10}{|c|}{ Depth from Top of Casing (ft) } \\
\hline & MW1D & MW2D & MW3D & MW4D & MW7 & MW9 & Oentrich & MW14D & MW15D & MW16D \\
\hline 10/13/07 8:30 & 126.643 & 124.737 & 120.52 & 100.998 & 104.719 & 96.53 & 110.55 & 108.381 & & 74.302 \\
\hline $10 / 13 / 07$ 9:00 & 126.724 & 124.782 & 120.566 & 101.075 & 104.812 & 96.622 & 110.637 & 108.478 & & 74.353 \\
\hline 10/13/07 9:30 & 126.773 & 124.812 & 120.596 & 101.132 & 104.873 & 96.681 & 110.695 & 108.544 & & 74.386 \\
\hline $10 / 13 / 0710: 00$ & 126.813 & 124.832 & 120.618 & 101.174 & 104.917 & 96.728 & 110.736 & 108.593 & & 74.403 \\
\hline $10 / 13 / 07 \quad 10: 30$ & 126.842 & 124.843 & 120.636 & 101.203 & 104.949 & 96.766 & 110.768 & 108.624 & & 74.422 \\
\hline $10 / 13 / 0711: 00$ & 126.865 & 124.846 & 120.646 & 101.22 & 104.966 & 96.777 & 110.793 & 108.644 & & 74.453 \\
\hline 10/13/07 11:30 & 126.884 & 124.85 & 120.649 & 101.229 & 104.98 & 96.794 & 110.809 & 108.658 & & 74.469 \\
\hline $10 / 13 / 0712: 00$ & 126.901 & 123.143 & 119.726 & 101.236 & 104.989 & 96.799 & 110.823 & 108.664 & & 74.494 \\
\hline $10 / 13 / 0712: 30$ & 126.917 & 122.759 & 119.18 & 101.239 & 104.991 & 96.806 & 110.836 & 108.671 & & 74.506 \\
\hline $10 / 13 / 0713: 00$ & 125.528 & 122.589 & 118.993 & 100.622 & 104.671 & 96.36 & 110.389 & 107.524 & & 74.186 \\
\hline $10 / 13 / 0713: 30$ & 125.19 & 122.494 & 118.89 & 100.347 & 104.311 & 95.985 & 110.051 & 107.028 & & 73.963 \\
\hline $10 / 13 / 0714: 00$ & 125.055 & 122.426 & 118.814 & 100.212 & 104.157 & 95.83 & 109.91 & 106.847 & & 73.849 \\
\hline $10 / 13 / 0714: 30$ & 124.979 & 122.385 & 118.779 & 100.133 & 104.066 & 95.738 & 109.835 & 106.745 & & 73.775 \\
\hline $10 / 13 / 07$ 15:00 & 124.93 & 122.36 & 118.751 & 100.078 & 104.001 & 95.674 & 109.784 & 106.676 & & 73.733 \\
\hline 10/13/07 15:30 & 124.894 & 122.338 & 118.726 & 100.045 & 103.963 & 95.637 & 109.749 & 106.632 & & 73.712 \\
\hline $10 / 13 / 0716: 00$ & 124.87 & 122.317 & 118.706 & 100.023 & 103.94 & 95.613 & 109.728 & 106.605 & & 73.708 \\
\hline $10 / 13 / 0716: 30$ & 124.856 & 122.313 & 118.696 & 99.999 & 103.916 & 95.592 & 109.711 & 106.581 & & 73.696 \\
\hline $10 / 13 / 0717: 00$ & 124.837 & 122.301 & 118.686 & 99.985 & 103.9 & 95.573 & 109.698 & 106.561 & & 73.696 \\
\hline $10 / 13 / 0717: 30$ & 124.823 & 122.288 & 118.676 & 99.976 & 103.893 & 95.566 & 109.689 & 106.552 & & 73.698 \\
\hline $10 / 13 / 0718: 00$ & 124.811 & 122.283 & 118.676 & 99.966 & 103.887 & 95.561 & 109.681 & 106.543 & & 73.7 \\
\hline $10 / 13 / 0718: 30$ & 124.802 & 122.281 & 118.668 & 99.964 & 103.877 & 95.552 & 109.674 & 106.534 & & 73.694 \\
\hline $10 / 13 / 0719: 00$ & 124.792 & 122.285 & 118.676 & 99.955 & 103.873 & 95.547 & 109.67 & 106.53 & & 73.692 \\
\hline 10/13/07 19:30 & 124.783 & 122.283 & 118.668 & 99.954 & 103.87 & 95.545 & 109.666 & 106.523 & & 73.69 \\
\hline $10 / 13 / 0720: 00$ & 124.776 & 122.29 & 118.661 & 99.964 & 103.876 & 95.549 & 109.66 & 106.53 & & 73.694 \\
\hline $10 / 13 / 0720: 30$ & 124.766 & 122.29 & 118.661 & 99.962 & 103.872 & 95.549 & 109.66 & 106.528 & & 73.692 \\
\hline $10 / 13 / 0721: 00$ & 124.759 & 122.295 & 118.676 & 99.966 & 103.879 & 95.556 & 109.657 & 106.532 & & 73.698 \\
\hline $10 / 13 / 0721: 30$ & 124.755 & 122.295 & 118.676 & 99.967 & 103.881 & 95.554 & 109.655 & 106.532 & & 73.698 \\
\hline $10 / 13 / 0722: 00$ & 124.745 & 122.276 & 118.666 & 99.978 & 103.887 & 95.559 & 109.653 & 106.537 & & 73.7 \\
\hline $10 / 13 / 0722: 30$ & 124.745 & 122.281 & 118.668 & 99.969 & 103.885 & 95.561 & 109.653 & 106.536 & & 73.7 \\
\hline $10 / 13 / 0723: 00$ & 124.743 & 122.285 & 118.673 & 99.955 & 103.864 & 95.54 & 109.653 & 106.519 & & 73.684 \\
\hline $10 / 13 / 0723: 30$ & 124.738 & 122.288 & 118.678 & 99.957 & 103.875 & 95.545 & 109.651 & 106.525 & & 73.689 \\
\hline $10 / 14 / 070: 00$ & 124.733 & 122.283 & 118.673 & 99.966 & 103.875 & 95.552 & 109.651 & 106.528 & & 73.694 \\
\hline $10 / 14 / 070: 30$ & 124.733 & 122.27 & 118.666 & 99.961 & 103.881 & 95.556 & 109.651 & 106.536 & & 73.696 \\
\hline $10 / 14 / 071: 00$ & 124.729 & 122.272 & 118.666 & 99.964 & 103.872 & 95.549 & 109.649 & 106.525 & & 73.69 \\
\hline 10/14/07 1:30 & 124.731 & 122.276 & 118.666 & 99.95 & 103.862 & 95.54 & 109.649 & 106.517 & & 73.684 \\
\hline $10 / 14 / 072: 00$ & 124.726 & 122.263 & 118.661 & 99.954 & 103.864 & 95.54 & 109.649 & 106.519 & & 73.683 \\
\hline $10 / 14 / 072: 30$ & 124.722 & 122.256 & 118.653 & 99.954 & 103.864 & 95.542 & 109.649 & 106.517 & & 73.684 \\
\hline 10/14/07 3:00 & 124.719 & 122.251 & 118.64 & 99.935 & 103.857 & 95.535 & 109.647 & 106.512 & & 73.679 \\
\hline $10 / 14 / 073: 30$ & 124.717 & 122.263 & 118.643 & 99.931 & 103.853 & 95.526 & 109.647 & 106.503 & & 73.671 \\
\hline $10 / 14 / 074: 00$ & 124.71 & 122.283 & 118.673 & 99.926 & 103.849 & 95.521 & 109.645 & 106.497 & & 73.646 \\
\hline $10 / 14 / 074: 30$ & 124.705 & 122.27 & 118.839 & 99.952 & 103.854 & 95.523 & 109.642 & 106.497 & & 73.636 \\
\hline $10 / 14 / 075: 00$ & 124.703 & 122.281 & 118.666 & 99.978 & 103.877 & 95.554 & 109.638 & 106.528 & & 73.684 \\
\hline $10 / 14 / 075: 30$ & 124.703 & 122.281 & 118.676 & 99.95 & 103.862 & 95.535 & 109.638 & 106.508 & & 74.312 \\
\hline $10 / 14 / 076: 00$ & 124.698 & 122.297 & 118.683 & 99.964 & 103.879 & 95.549 & 109.634 & 106.521 & & 74.258 \\
\hline $10 / 14 / 076: 30$ & 124.7 & 122.304 & 118.686 & 99.974 & 103.875 & 95.552 & 109.632 & 106.525 & & 74.178 \\
\hline $10 / 14 / 07$ 7:00 & 124.696 & 122.258 & 118.661 & 99.971 & 103.894 & 95.566 & 109.63 & 106.536 & & 74.155 \\
\hline 10/14/07 7:30 & 124.686 & 124.129 & 119.774 & 99.978 & 103.91 & 95.578 & 109.623 & 106.548 & & 74.149 \\
\hline $10 / 14 / 078: 00$ & 124.707 & 124.469 & 120.218 & 99.954 & 103.843 & 95.521 & 109.64 & 106.499 & & 74.132 \\
\hline $10 / 14 / 078: 30$ & 126.187 & 124.628 & 120.397 & 100.67 & 104.287 & 96.089 & 110.137 & 107.845 & & 74.149 \\
\hline 10/14/07 9:00 & 126.499 & 124.753 & 120.492 & 100.927 & 104.603 & 96.414 & 110.458 & 108.243 & & 74.285 \\
\hline $10 / 14 / 079: 30$ & 126.624 & 124.766 & 120.555 & 101.043 & 104.778 & 96.586 & 110.599 & 108.427 & & 74.37 \\
\hline $10 / 14 / 0710: 00$ & 126.693 & 124.839 & 120.613 & 101.139 & 104.887 & 96.692 & 110.68 & 108.536 & & 74.423 \\
\hline $10 / 14 / 0710: 30$ & 126.754 & 124.868 & 120.671 & 101.168 & 104.915 & 96.728 & 110.746 & 108.584 & & 74.461 \\
\hline $10 / 14 / 0711: 00$ & 126.78 & 123.435 & 120.707 & 101.218 & 104.976 & 96.782 & 110.774 & 108.633 & & 74.482 \\
\hline $10 / 14 / 0711: 30$ & 126.804 & 122.827 & 119.268 & 101.267 & 105.029 & 96.839 & 110.804 & 108.691 & & 74.49 \\
\hline $10 / 14 / 0712: 00$ & 125.702 & 122.673 & 119.026 & 100.791 & 104.934 & 96.655 & 110.571 & 108.095 & & 74.492 \\
\hline $10 / 14 / 0712: 30$ & 125.138 & 122.553 & 118.97 & 100.424 & 104.384 & 96.061 & 110.094 & 107.101 & & 74.396 \\
\hline 10/14/07 13:00 & 124.972 & 122.499 & 118.895 & 100.312 & 104.247 & 95.915 & 109.925 & 106.917 & & 74.283 \\
\hline $10 / 14 / 0713: 30$ & 124.911 & 122.462 & 118.847 & 100.19 & 104.133 & 95.806 & 109.848 & 106.813 & & 74.219 \\
\hline $10 / 14 / 0714: 00$ & 124.859 & 122.431 & 118.822 & 100.148 & 104.079 & 95.752 & 109.796 & 106.749 & & 74.171 \\
\hline $10 / 14 / 0714: 30$ & 124.828 & 122.401 & 118.794 & 100.123 & 104.045 & 95.714 & 109.762 & 106.707 & & 74.136 \\
\hline $10 / 14 / 0715: 00$ & 124.807 & 122.388 & 118.776 & 100.095 & 104.015 & 95.688 & 109.739 & 106.678 & & 74.112 \\
\hline $10 / 14 / 0715: 30$ & 124.795 & 122.385 & 118.781 & 100.069 & 103.986 & 95.658 & 109.726 & 106.647 & & 74.093 \\
\hline $10 / 14 / 0716: 00$ & 124.781 & 122.358 & 118.754 & 100.064 & 103.972 & 95.648 & 109.709 & 106.632 & & 74.074 \\
\hline $10 / 14 / 0716: 30$ & 124.769 & 122.319 & 118.726 & 100.05 & 103.974 & 95.648 & 109.7 & 106.634 & & 74.056 \\
\hline $10 / 14 / 0717: 00$ & 124.764 & 122.306 & 118.691 & 100.503 & 103.944 & 95.615 & 109.692 & 106.599 & & 74.043 \\
\hline
\end{tabular}


TABLE S1.1 (Cont.)

Depth from Top of Casing (ft)

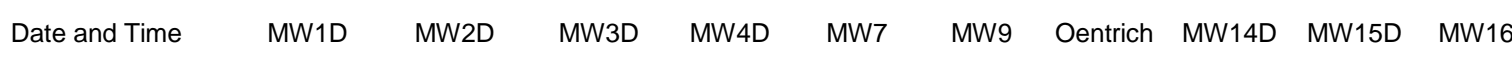

\begin{tabular}{|c|c|c|c|c|c|c|c|c|c|}
\hline 10/14/07 17:30 & 124.759 & 122.313 & 118.708 & 100.512 & 103.925 & 95.599 & 109.689 & 106.581 & 74.029 \\
\hline $10 / 14 / 0718: 00$ & 124.752 & 122.267 & 118.65 & 100.515 & 103.9 & 95.573 & 109.681 & 106.554 & 74.023 \\
\hline $10 / 14 / 0718: 30$ & 124.748 & 122.29 & 118.648 & 100.514 & 103.902 & 95.575 & 109.674 & 106.554 & 74.01 \\
\hline 10/14/07 19:00 & 124.755 & 122.308 & 118.686 & 100.507 & 103.851 & 95.526 & 109.672 & 106.51 & 74.008 \\
\hline 10/14/07 19:30 & 124.736 & 122.299 & 118.691 & 100.472 & 103.881 & 95.552 & 109.655 & 106.523 & 73.992 \\
\hline $10 / 14 / 0720: 00$ & 124.736 & 122.279 & 118.668 & 100.409 & 103.9 & 95.573 & 109.653 & 106.55 & 73.979 \\
\hline $10 / 14 / 0720: 30$ & 124.738 & 122.308 & 118.698 & 100.329 & 103.893 & 95.568 & 109.653 & 106.545 & 73.971 \\
\hline $10 / 14 / 0721: 00$ & 124.74 & 122.315 & 118.706 & 100.183 & 103.87 & 95.542 & 109.655 & 106.523 & 73.965 \\
\hline $10 / 14 / 0721: 30$ & 124.731 & 122.297 & 118.691 & 100.138 & 103.906 & 95.58 & 109.649 & 106.554 & 73.948 \\
\hline $10 / 14 / 0722: 00$ & 124.731 & 122.31 & 118.696 & 99.993 & 103.91 & 95.585 & 109.649 & 106.559 & 73.934 \\
\hline $10 / 14 / 0722: 30$ & 124.733 & 122.297 & 118.683 & 99.974 & 103.891 & 95.566 & 109.649 & 106.543 & 73.93 \\
\hline 10/14/07 23:00 & 124.729 & 122.288 & 118.681 & 99.99 & 103.902 & 95.578 & 109.645 & 106.552 & 73.915 \\
\hline $10 / 14 / 0723: 30$ & 124.729 & 122.197 & 118.615 & 100.009 & 103.889 & 95.566 & 109.647 & 106.541 & 73.909 \\
\hline 10/15/07 0:00 & 124.731 & 122.199 & 118.587 & 100.059 & 103.877 & 95.554 & 109.645 & 106.532 & 73.903 \\
\hline $10 / 15 / 070: 30$ & 124.75 & 122.242 & 118.62 & 100.064 & 103.79 & 95.472 & 109.651 & 106.457 & 73.913 \\
\hline $10 / 15 / 071: 00$ & 124.733 & 122.249 & 118.63 & 100.026 & 103.792 & 95.465 & 109.612 & 106.443 & 73.894 \\
\hline $10 / 15 / 071: 30$ & 124.719 & 122.247 & 118.63 & 99.988 & 103.837 & 95.509 & 109.598 & 106.481 & 73.886 \\
\hline $10 / 15 / 072: 00$ & 124.719 & 122.245 & 118.633 & 99.94 & 103.841 & 95.514 & 109.598 & 106.486 & 73.878 \\
\hline $10 / 15 / 072: 30$ & 124.719 & 122.231 & 118.623 & 99.924 & 103.841 & 95.512 & 109.6 & 106.488 & 73.866 \\
\hline $10 / 15 / 073: 00$ & 124.717 & 122.231 & 118.623 & 99.918 & 103.839 & 95.514 & 109.597 & 106.488 & 73.863 \\
\hline $10 / 15 / 073: 30$ & 124.717 & 122.233 & 118.62 & 99.907 & 103.824 & 95.498 & 109.597 & 106.475 & 73.855 \\
\hline $10 / 15 / 074: 00$ & 124.714 & 122.233 & 118.63 & 99.911 & 103.824 & 95.498 & 109.597 & 106.477 & 73.849 \\
\hline $10 / 15 / 074: 30$ & 124.712 & 122.222 & 118.613 & 99.918 & 103.83 & 95.502 & 109.597 & 106.474 & 73.841 \\
\hline $10 / 15 / 075: 00$ & 124.712 & 122.227 & 118.613 & 99.911 & 103.832 & 95.507 & 109.595 & 106.479 & 73.828 \\
\hline $10 / 15 / 075: 30$ & 124.71 & 122.224 & 118.61 & 99.904 & 103.815 & 95.49 & 109.593 & 106.463 & 73.822 \\
\hline $10 / 15 / 076: 00$ & 124.705 & 124.099 & 119.918 & 99.904 & 103.826 & 95.498 & 109.593 & 106.47 & 73.81 \\
\hline $10 / 15 / 076: 30$ & 124.71 & 124.41 & 120.309 & 99.905 & 103.816 & 95.493 & 109.591 & 106.463 & 73.804 \\
\hline $10 / 15 / 077: 00$ & 126.27 & 124.558 & 120.477 & 100.681 & 104.306 & 96.125 & 110.209 & 107.832 & 73.832 \\
\hline $10 / 15 / 077: 30$ & 126.53 & 124.655 & 120.581 & 100.919 & 104.609 & 96.433 & 110.485 & 108.188 & 73.954 \\
\hline $10 / 15 / 078: 00$ & 126.655 & 124.73 & 120.661 & 101.048 & 104.755 & 96.577 & 110.616 & 108.35 & 74.035 \\
\hline $10 / 15 / 078: 30$ & 126.724 & 124.778 & 120.714 & 101.137 & 104.852 & 96.676 & 110.695 & 108.456 & 74.087 \\
\hline $10 / 15 / 079: 00$ & 126.769 & 124.789 & 120.732 & 101.201 & 104.926 & 96.749 & 110.749 & 108.533 & 74.114 \\
\hline 10/15/07 9:30 & 126.804 & 124.825 & 120.755 & 101.241 & 104.974 & 96.799 & 110.785 & 108.584 & 74.13 \\
\hline $10 / 15 / 07 \quad 10: 00$ & 126.835 & 124.843 & 120.782 & 101.255 & 104.982 & 96.808 & 110.817 & 108.598 & 74.145 \\
\hline $10 / 15 / 07$ 10:30 & 126.849 & 124.868 & 120.805 & 101.286 & 105.02 & 96.843 & 110.836 & 108.631 & 74.153 \\
\hline 10/15/07 $11: 00$ & 126.865 & 123.356 & 120.324 & 101.31 & 105.037 & 96.862 & 110.851 & 108.651 & 74.159 \\
\hline 10/15/07 11:30 & 126.88 & 122.873 & 119.301 & 101.334 & 105.064 & 96.891 & 110.864 & 108.679 & 74.161 \\
\hline $10 / 15 / 07$ 12:00 & 125.662 & 122.687 & 119.096 & 100.76 & 104.86 & 96.568 & 110.515 & 107.874 & 74.163 \\
\hline $10 / 15 / 07$ 12:30 & 125.18 & 122.582 & 118.986 & 100.453 & 104.429 & 96.103 & 110.073 & 107.145 & 74.056 \\
\hline $10 / 15 / 0713: 00$ & 125.034 & 122.517 & 118.91 & 100.305 & 104.254 & 95.929 & 109.918 & 106.948 & 73.961 \\
\hline $10 / 15 / 07$ 13:30 & 124.953 & 122.471 & 118.862 & 100.217 & 104.155 & 95.828 & 109.833 & 106.835 & 73.897 \\
\hline $10 / 15 / 0714: 00$ & 124.901 & 122.435 & 118.829 & 100.164 & 104.091 & 95.764 & 109.777 & 106.764 & 73.845 \\
\hline $10 / 15 / 07 \quad 14: 30$ & 124.866 & 122.415 & 118.804 & 100.126 & 104.049 & 95.724 & 109.741 & 106.718 & 73.806 \\
\hline $10 / 15 / 07$ 15:00 & 124.84 & 122.408 & 118.797 & 100.093 & 104.014 & 95.688 & 109.715 & 106.681 & 73.777 \\
\hline 10/15/07 15:30 & 124.821 & 122.397 & 118.787 & 100.083 & 103.997 & 95.672 & 109.694 & 106.658 & 73.75 \\
\hline 10/15/07 16:00 & 124.804 & 122.383 & 118.771 & 100.078 & 103.992 & 95.663 & 109.675 & 106.649 & 73.727 \\
\hline $10 / 15 / 0716: 30$ & 124.795 & 122.376 & 118.761 & 100.064 & 103.98 & 95.655 & 109.668 & 106.64 & 73.71 \\
\hline 10/15/07 17:00 & 124.783 & 122.385 & 118.769 & 100.054 & 103.969 & 95.644 & 109.66 & 106.627 & 73.696 \\
\hline $10 / 15 / 07$ 17:30 & 124.774 & 122.378 & 118.769 & 100.05 & 103.963 & 95.637 & 109.651 & 106.621 & 73.682 \\
\hline $10 / 15 / 07$ 18:00 & 124.764 & 122.376 & 118.766 & 100.061 & 103.971 & 95.646 & 109.644 & 106.625 & 73.669 \\
\hline $10 / 15 / 07$ 18:30 & 124.759 & 122.374 & 118.764 & 100.054 & 103.969 & 95.644 & 109.64 & 106.621 & 73.653 \\
\hline $10 / 15 / 07$ 19:00 & 124.755 & 122.376 & 118.764 & 100.052 & 103.963 & 95.641 & 109.634 & 106.618 & 73.64 \\
\hline $10 / 15 / 07$ 19:30 & 124.75 & 122.376 & 118.764 & 100.05 & 103.961 & 95.639 & 109.629 & 106.616 & 73.625 \\
\hline $10 / 15 / 0720: 00$ & 124.745 & 122.378 & 118.764 & 100.054 & 103.965 & 95.644 & 109.627 & 106.618 & 73.617 \\
\hline $10 / 15 / 0720: 30$ & 124.74 & 122.378 & 118.764 & 100.054 & 103.963 & 95.644 & 109.623 & 106.616 & 73.607 \\
\hline $10 / 15 / 0721: 00$ & 124.736 & 122.376 & 118.764 & 100.059 & 103.967 & 95.646 & 109.617 & 106.621 & 73.596 \\
\hline 10/15/07 21:30 & 124.733 & 122.381 & 118.766 & 100.054 & 103.971 & 95.646 & 109.615 & 106.621 & 73.586 \\
\hline $10 / 15 / 0722: 00$ & 124.731 & 122.378 & 118.766 & 100.057 & 103.969 & 95.644 & 109.612 & 106.618 & 73.576 \\
\hline $10 / 15 / 0722: 30$ & 124.729 & 122.378 & 118.766 & 100.057 & 103.971 & 95.646 & 109.612 & 106.621 & 73.567 \\
\hline 10/15/07 23:00 & 124.726 & 122.374 & 118.761 & 100.055 & 103.967 & 95.644 & 109.608 & 106.618 & 73.555 \\
\hline $10 / 15 / 0723: 30$ & 124.726 & 122.369 & 118.759 & 100.054 & 103.967 & 95.641 & 109.606 & 106.616 & 73.545 \\
\hline $10 / 16 / 070: 00$ & 124.724 & 122.363 & 118.749 & 100.05 & 103.963 & 95.639 & 109.604 & 106.614 & 73.535 \\
\hline $10 / 16 / 070: 30$ & 124.724 & 122.36 & 118.749 & 100.043 & 103.959 & 95.634 & 109.602 & 106.609 & 73.526 \\
\hline $10 / 16 / 071: 00$ & 124.719 & 122.36 & 118.746 & 100.042 & 103.955 & 95.63 & 109.598 & 106.603 & 73.516 \\
\hline $10 / 16 / 071: 30$ & 124.717 & 122.363 & 118.749 & 100.038 & 103.953 & 95.63 & 109.597 & 106.605 & 73.508 \\
\hline $10 / 16 / 072: 00$ & 124.714 & 122.358 & 118.746 & 100.038 & 103.952 & 95.627 & 109.595 & 106.603 & 73.502 \\
\hline
\end{tabular}


TABLE S1.1 (Cont.)

\begin{tabular}{|c|c|c|c|c|c|c|c|c|c|c|}
\hline \multirow[b]{2}{*}{ Date and Time } & \multicolumn{10}{|c|}{ Depth from Top of Casing (ft) } \\
\hline & MW1D & MW2D & MW3D & MW4D & MW7 & MW9 & Oentrich & MW14D & MW15D & MW16D \\
\hline 10/16/07 2:30 & 124.712 & 122.353 & 118.741 & 100.04 & 103.952 & 95.627 & 109.595 & 106.603 & & 73.495 \\
\hline 10/16/07 3:00 & 124.712 & 122.353 & 118.736 & 100.033 & 103.948 & 95.625 & 109.593 & 106.598 & & 73.491 \\
\hline 10/16/07 3:30 & 124.707 & 122.349 & 118.736 & 100.031 & 103.946 & 95.62 & 109.589 & 106.594 & & 73.483 \\
\hline $10 / 16 / 074: 00$ & 124.705 & 122.349 & 118.739 & 100.031 & 103.948 & 95.622 & 109.589 & 106.596 & & 73.479 \\
\hline $10 / 16 / 074: 30$ & 124.7 & 122.353 & 118.744 & 100.028 & 103.94 & 95.618 & 109.585 & 106.592 & & 73.477 \\
\hline 10/16/07 5:00 & 124.7 & 122.353 & 118.741 & 100.03 & 103.944 & 95.618 & 109.583 & 106.592 & & 73.477 \\
\hline $10 / 16 / 075: 30$ & 124.698 & 123.383 & 118.748 & 100.035 & 103.946 & 95.622 & 109.58 & 106.594 & & 73.477 \\
\hline 10/16/07 6:00 & 124.696 & 124.408 & 120.14 & 100.033 & 103.948 & 95.622 & 109.576 & 106.596 & & 73.477 \\
\hline 10/16/07 6:30 & 124.691 & 124.626 & 120.387 & 100.465 & 103.957 & 95.632 & 109.572 & 106.603 & & 73.477 \\
\hline 10/16/07 7:00 & 126.4 & 124.734 & 120.515 & 100.877 & 104.557 & 96.367 & 110.295 & 108.177 & & 73.586 \\
\hline 10/16/07 7:30 & 126.584 & 124.814 & 120.601 & 101.056 & 104.768 & 96.579 & 110.483 & 108.416 & & 73.733 \\
\hline 10/16/07 8:00 & 126.691 & 123.265 & 120.12 & 101.155 & 104.883 & 96.692 & 110.586 & 108.54 & & 73.832 \\
\hline 10/16/07 8:30 & 126.759 & 122.839 & 119.26 & 101.224 & 104.964 & 96.775 & 110.648 & 108.624 & & 73.901 \\
\hline 10/16/07 9:00 & 125.535 & 122.687 & 119.094 & 100.71 & 104.789 & 96.487 & 110.336 & 107.754 & & 73.946 \\
\hline 10/16/07 9:30 & 125.114 & 122.603 & 118.998 & 100.441 & 104.407 & 96.082 & 109.946 & 107.11 & & 73.855 \\
\hline $10 / 16 / 07$ 10:00 & 124.996 & 122.544 & 118.94 & 100.322 & 104.268 & 95.938 & 109.809 & 106.944 & & 73.762 \\
\hline $10 / 16 / 07 \quad 10: 30$ & 124.937 & 122.505 & 118.895 & 100.252 & 104.182 & 95.856 & 109.734 & 106.851 & & 73.692 \\
\hline 10/16/07 11:00 & 124.908 & 122.476 & 118.867 & 100.198 & 104.125 & 95.799 & 109.69 & 106.793 & & 73.644 \\
\hline $10 / 16 / 0711: 30$ & 124.889 & 122.449 & 118.842 & 100.166 & 104.089 & 95.764 & 109.66 & 106.751 & & 73.605 \\
\hline $10 / 16 / 07 \quad 12: 00$ & 124.875 & 122.428 & 118.819 & 100.14 & 104.062 & 95.736 & 109.647 & 106.72 & & 73.578 \\
\hline $10 / 16 / 07$ 12:30 & 124.863 & 122.408 & 118.794 & 100.116 & 104.035 & 95.714 & 109.629 & 106.694 & & 73.553 \\
\hline 10/16/07 13:00 & 124.849 & 122.383 & 118.764 & 100.093 & 104.014 & 95.691 & 109.621 & 106.671 & & 73.533 \\
\hline $10 / 16 / 07$ 13:30 & 124.837 & 122.369 & 118.744 & 100.076 & 103.993 & 95.67 & 109.613 & 106.652 & & 73.512 \\
\hline $10 / 16 / 07$ 14:00 & 124.828 & 122.353 & 118.729 & 100.054 & 103.972 & 95.646 & 109.608 & 106.629 & & 73.493 \\
\hline $10 / 16 / 07$ 14:30 & 124.821 & 122.344 & 118.716 & 100.04 & 103.955 & 95.632 & 109.606 & 106.612 & & 73.472 \\
\hline 10/16/07 15:00 & 124.814 & 122.329 & 118.701 & 100.026 & 103.944 & 95.618 & 109.598 & 106.601 & & 73.462 \\
\hline $10 / 16 / 07$ 15:30 & 124.807 & 122.315 & 118.686 & 100.016 & 103.931 & 95.606 & 109.597 & 106.585 & & 73.454 \\
\hline $10 / 16 / 0716: 00$ & 124.8 & 122.308 & 118.683 & 100 & 103.915 & 95.592 & 109.591 & 106.57 & & 73.444 \\
\hline 10/16/07 16:30 & 124.788 & 122.299 & 118.688 & 99.99 & 103.904 & 95.578 & 109.587 & 106.556 & & 73.429 \\
\hline $10 / 16 / 07$ 17:00 & 124.776 & 122.288 & 118.683 & 99.983 & 103.9 & 95.575 & 109.583 & 106.552 & & 73.425 \\
\hline $10 / 16 / 07$ 17:30 & 124.762 & 122.276 & 118.668 & 99.976 & 103.891 & 95.568 & 109.583 & 106.543 & & 73.417 \\
\hline $10 / 16 / 0718: 00$ & 124.75 & 122.265 & 118.653 & 99.964 & 103.879 & 95.556 & 109.58 & 106.532 & & 73.407 \\
\hline $10 / 16 / 0718: 30$ & 124.736 & 122.261 & 118.65 & 99.95 & 103.866 & 95.542 & 109.58 & 106.519 & & 73.394 \\
\hline 10/16/07 19:00 & 124.722 & 122.256 & 118.645 & 99.943 & 103.854 & 95.531 & 109.576 & 106.508 & & 73.383 \\
\hline 10/16/07 19:30 & 124.707 & 122.258 & 118.65 & 99.938 & 103.853 & 95.526 & 109.574 & 106.503 & & 73.382 \\
\hline $10 / 16 / 0720: 00$ & 124.696 & 122.258 & 118.648 & 99.936 & 103.849 & 95.521 & 109.572 & 106.499 & & 73.378 \\
\hline 10/16/07 20:30 & 124.684 & 122.263 & 118.65 & 99.938 & 103.851 & 95.526 & 109.57 & 106.501 & & 73.376 \\
\hline 10/16/07 21:00 & 124.679 & 122.258 & 118.65 & 99.936 & 103.853 & 95.526 & 109.567 & 106.501 & & 73.375 \\
\hline $10 / 16 / 0721: 30$ & 124.679 & 122.251 & 118.643 & 99.94 & 103.854 & 95.531 & 109.568 & 106.505 & & 73.374 \\
\hline 10/16/07 22:00 & 124.677 & 122.245 & 118.632 & 99.938 & 103.853 & 95.526 & 109.567 & 106.503 & & 73.371 \\
\hline 10/16/07 22:30 & 124.672 & 122.247 & 118.635 & 99.93 & 103.845 & 95.519 & 109.565 & 106.497 & & 73.365 \\
\hline 10/16/07 23:00 & 124.67 & 122.245 & 118.633 & 99.926 & 103.837 & 95.514 & 109.565 & 106.488 & & 73.353 \\
\hline $10 / 16 / 0723: 30$ & 124.67 & 122.231 & 118.623 & 99.924 & 103.835 & 95.514 & 109.565 & 106.488 & & 73.349 \\
\hline 10/17/07 0:00 & 124.667 & 122.224 & 118.608 & 99.919 & 103.835 & 95.509 & 109.567 & 106.486 & & 73.345 \\
\hline $10 / 17 / 070: 30$ & 124.67 & 122.215 & 118.603 & 99.905 & 103.822 & 95.498 & 109.567 & 106.474 & & 73.336 \\
\hline 10/17/07 1:00 & 124.667 & 122.208 & 118.592 & 99.897 & 103.813 & 95.486 & 109.567 & 106.466 & & 73.328 \\
\hline 10/17/07 1:30 & 124.667 & 122.195 & 118.58 & 99.892 & 103.805 & 95.481 & 109.567 & 106.459 & & 73.32 \\
\hline 10/17/07 2:00 & 124.662 & 122.186 & 118.574 & 99.88 & 103.795 & 95.469 & 109.565 & 106.446 & & 73.314 \\
\hline 10/17/07 2:30 & 124.66 & 122.172 & 118.565 & 99.866 & 103.778 & 95.455 & 109.565 & 106.435 & & 73.303 \\
\hline 10/17/07 3:00 & 124.658 & 122.161 & 118.544 & 99.859 & 103.774 & 95.453 & 109.565 & 106.43 & & 73.299 \\
\hline 10/17/07 3:30 & 124.658 & 122.14 & 118.521 & 99.845 & 103.763 & 95.436 & 109.565 & 106.419 & & 73.293 \\
\hline $10 / 17 / 074: 00$ & 124.66 & 122.134 & 118.514 & 99.831 & 103.752 & 95.42 & 109.565 & 106.401 & & 73.282 \\
\hline 10/17/07 4:30 & 124.66 & 122.129 & 118.529 & 99.802 & 103.729 & 95.399 & 109.567 & 106.381 & & 73.27 \\
\hline $10 / 17 / 075: 00$ & 124.655 & 122.127 & 118.527 & 99.812 & 103.721 & 95.394 & 109.563 & 106.373 & & 73.254 \\
\hline $10 / 17 / 075: 30$ & 124.653 & 122.113 & 118.524 & 99.802 & 103.715 & 95.387 & 109.561 & 106.37 & & 73.249 \\
\hline 10/17/07 6:00 & 124.648 & 123.884 & 119.699 & 99.802 & 103.715 & 95.387 & 109.557 & 106.364 & & 73.239 \\
\hline $10 / 17 / 076: 30$ & 124.646 & 124.208 & 120.117 & 99.788 & 103.708 & 95.382 & 109.557 & 106.359 & & 73.237 \\
\hline $10 / 17 / 077: 00$ & 126.152 & 124.365 & 120.298 & 100.474 & 104.091 & 95.905 & 110.107 & 107.601 & & 73.245 \\
\hline 10/17/07 7:30 & 126.454 & 124.437 & 120.384 & 100.719 & 104.405 & 96.226 & 110.408 & 107.984 & & 73.378 \\
\hline 10/17/07 8:00 & 126.584 & 124.503 & 120.462 & 100.838 & 104.557 & 96.379 & 110.547 & 108.155 & & 73.489 \\
\hline $10 / 17 / 078: 30$ & 126.665 & 124.524 & 120.47 & 100.946 & 104.631 & 96.455 & 110.612 & 108.241 & & 73.57 \\
\hline 10/17/07 9:00 & 126.714 & 124.553 & 120.5 & 100.96 & 104.705 & 96.528 & 110.665 & 108.314 & & 73.63 \\
\hline 10/17/07 9:30 & 126.752 & 124.562 & 120.518 & 100.989 & 104.715 & 96.537 & 110.701 & 108.33 & & 73.671 \\
\hline $10 / 17 / 0710: 00$ & 126.778 & 124.551 & 120.51 & 101.008 & 104.744 & 96.568 & 110.719 & 108.356 & & 73.702 \\
\hline $10 / 17 / 07$ 10:30 & 126.813 & 124.569 & 120.528 & 101.012 & 104.753 & 96.579 & 110.74 & 108.37 & & 73.725 \\
\hline 10/17/07 11:00 & 126.835 & 124.567 & 120.52 & 101.01 & 104.736 & 96.563 & 110.755 & 108.356 & & 73.735 \\
\hline
\end{tabular}


TABLE S1.1 (Cont.)

\begin{tabular}{|c|c|c|c|c|c|c|c|c|c|c|}
\hline \multirow[b]{2}{*}{ Date and Time } & \multicolumn{10}{|c|}{ Depth from Top of Casing (ft) } \\
\hline & MW1D & MW2D & MW3D & MW4D & MW7 & MW9 & Oentrich & MW14D & MW15D & MW16D \\
\hline 10/17/07 11:30 & 126.844 & 122.639 & 119.099 & 101.019 & 104.763 & 96.584 & 110.764 & 108.378 & & 73.739 \\
\hline $10 / 17 / 07$ 12:00 & 126.854 & 122.41 & 118.832 & 100.629 & 104.757 & 96.577 & 110.768 & 108.374 & & 73.737 \\
\hline 10/17/07 12:30 & 125.201 & 122.261 & 118.698 & 100.197 & 104.188 & 95.863 & 110.03 & 106.919 & & 73.663 \\
\hline $10 / 17 / 07$ 13:00 & 125.017 & 122.095 & 118.55 & 100.007 & 103.971 & 95.641 & 109.837 & 106.671 & & 73.992 \\
\hline $10 / 17 / 07$ 13:30 & 124.927 & 122.07 & 118.472 & 99.843 & 103.845 & 95.516 & 109.737 & 106.53 & & 73.95 \\
\hline $10 / 17 / 0714: 00$ & 124.896 & 122.022 & 118.441 & 99.742 & 103.672 & 95.351 & 109.416 & 106.368 & & 73.797 \\
\hline $10 / 17 / 0714: 30$ & 124.842 & 121.977 & 118.383 & 99.716 & 103.647 & 95.318 & 109.369 & 106.317 & & 73.752 \\
\hline $10 / 17 / 07$ 15:00 & 124.821 & 121.952 & 118.351 & 99.678 & 103.596 & 95.271 & 109.345 & 106.271 & & 73.682 \\
\hline 10/17/07 15:30 & 124.804 & 121.925 & 118.328 & 99.619 & 103.556 & 95.229 & 109.324 & 106.222 & & 73.636 \\
\hline $10 / 17 / 07$ 16:00 & 124.79 & 121.905 & 118.31 & 99.601 & 103.527 & 95.201 & 109.311 & 106.191 & & 73.609 \\
\hline $10 / 17 / 07$ 16:30 & 124.781 & 121.871 & 118.278 & 99.587 & 103.508 & 95.179 & 109.3 & 106.169 & & 73.591 \\
\hline 10/17/07 17:00 & 124.771 & 121.857 & 118.24 & 99.563 & 103.487 & 95.158 & 109.292 & 106.147 & & 73.551 \\
\hline $10 / 17 / 07$ 17:30 & 124.762 & 121.85 & 118.235 & 99.532 & 103.458 & 95.125 & 109.279 & 106.114 & & 73.518 \\
\hline $10 / 17 / 07$ 18:00 & 124.755 & 121.855 & 118.242 & 99.521 & 103.439 & 95.109 & 109.264 & 106.094 & & 73.491 \\
\hline 10/17/07 18:30 & 124.748 & 121.837 & 118.245 & 99.528 & 103.438 & 95.109 & 109.255 & 106.089 & & 73.483 \\
\hline $10 / 17 / 07$ 19:00 & 124.74 & 121.812 & 118.222 & 99.52 & 103.441 & 95.113 & 109.251 & 106.096 & & 73.475 \\
\hline $10 / 17 / 07$ 19:30 & 124.736 & 121.791 & 118.202 & 99.499 & 103.424 & 95.097 & 109.247 & 106.08 & & 73.46 \\
\hline $10 / 17 / 0720: 00$ & 124.733 & 121.764 & 118.172 & 99.475 & 103.398 & 95.071 & 109.242 & 106.054 & & 73.446 \\
\hline $10 / 17 / 0720: 30$ & 124.731 & 121.755 & 118.162 & 99.451 & 103.377 & 95.047 & 109.23 & 106.029 & & 73.425 \\
\hline $10 / 17 / 0721: 00$ & 124.726 & 121.762 & 118.159 & 99.433 & 103.354 & 95.024 & 109.217 & 106.007 & & 73.398 \\
\hline $10 / 17 / 0721: 30$ & 124.719 & 121.807 & 118.212 & 99.426 & 103.346 & 95.014 & 109.204 & 105.998 & & 73.367 \\
\hline $10 / 17 / 0722: 00$ & 124.71 & 121.8 & 118.204 & 99.432 & 103.352 & 95.021 & 109.199 & 106.001 & & 73.357 \\
\hline 10/17/07 22:30 & 124.698 & 121.789 & 118.194 & 99.48 & 103.401 & 95.069 & 109.189 & 106.043 & & 73.353 \\
\hline 10/17/07 23:00 & 124.7 & 121.764 & 118.164 & 99.476 & 103.394 & 95.066 & 109.193 & 106.041 & & 73.336 \\
\hline $10 / 17 / 0723: 30$ & 124.698 & 121.759 & 118.159 & 99.456 & 103.382 & 95.052 & 109.193 & 106.029 & & 73.326 \\
\hline 10/18/07 0:00 & 124.698 & 121.75 & 118.154 & 99.432 & 103.354 & 95.024 & 109.197 & 106.001 & & 73.221 \\
\hline 10/18/07 0:30 & 124.696 & 121.746 & 118.152 & 99.428 & 103.35 & 95.021 & 109.195 & 105.996 & & 73.212 \\
\hline 10/18/07 1:00 & 124.693 & 121.737 & 118.144 & 99.421 & 103.342 & 95.012 & 109.197 & 105.99 & & 73.202 \\
\hline 10/18/07 1:30 & 124.691 & 121.728 & 118.134 & 99.418 & 103.342 & 95.01 & 109.197 & 105.987 & & 73.192 \\
\hline $10 / 18 / 072: 00$ & 124.688 & 121.721 & 118.119 & 99.409 & 103.335 & 95.003 & 109.195 & 105.979 & & 73.183 \\
\hline 10/18/07 2:30 & 124.688 & 121.712 & 118.114 & 99.397 & 103.323 & 94.991 & 109.195 & 105.967 & & 73.173 \\
\hline 10/18/07 3:00 & 124.686 & 121.716 & 118.116 & 99.392 & 103.31 & 94.979 & 109.191 & 105.956 & & 73.163 \\
\hline 10/18/07 3:30 & 124.684 & 121.721 & 118.126 & 99.382 & 103.304 & 94.972 & 109.189 & 105.95 & & 73.154 \\
\hline $10 / 18 / 074: 00$ & 124.679 & 121.73 & 118.126 & 99.395 & 103.31 & 94.977 & 109.187 & 105.954 & & 73.148 \\
\hline 10/18/07 4:30 & 124.674 & 121.739 & 118.139 & 99.397 & 103.318 & 94.986 & 109.184 & 105.961 & & 73.142 \\
\hline 10/18/07 5:00 & 124.674 & 121.746 & 118.149 & 99.409 & 103.325 & 94.991 & 109.182 & 105.966 & & 73.136 \\
\hline 10/18/07 5:30 & 124.672 & 121.755 & 118.154 & 99.414 & 103.333 & 95.003 & 109.18 & 105.974 & & 73.133 \\
\hline 10/18/07 6:00 & 124.67 & 123.542 & 119.323 & 99.425 & 103.342 & 95.01 & 109.18 & 105.983 & & 73.13 \\
\hline 10/18/07 6:30 & 124.667 & 123.914 & 119.817 & 99.435 & 103.348 & 95.017 & 109.182 & 105.992 & & 73.121 \\
\hline 10/18/07 7:00 & 126.147 & 124.084 & 120.011 & 100.154 & 103.759 & 95.568 & 109.711 & 107.249 & & 73.138 \\
\hline 10/18/07 7:30 & 126.466 & 124.192 & 120.109 & 100.431 & 104.111 & 95.931 & 110.04 & 107.685 & & 73.28 \\
\hline 10/18/07 8:00 & 126.601 & 124.24 & 120.208 & 100.572 & 104.283 & 96.101 & 110.19 & 107.871 & & 73.386 \\
\hline 10/18/07 8:30 & 126.679 & 124.319 & 120.263 & 100.669 & 104.389 & 96.207 & 110.274 & 107.987 & & 73.45 \\
\hline 10/18/07 9:00 & 126.681 & 124.367 & 120.316 & 100.729 & 104.443 & 96.252 & 110.301 & 107.962 & & 73.491 \\
\hline 10/18/07 9:30 & 126.764 & 122.467 & 118.94 & 100.789 & 104.519 & 96.337 & 110.365 & 108.119 & & 73.516 \\
\hline 10/18/07 10:00 & 126.795 & 122.258 & 118.686 & 100.577 & 104.561 & 96.377 & 110.395 & 108.168 & & 73.535 \\
\hline 10/18/07 10:30 & 125.185 & 122.154 & 118.572 & 100.031 & 104.028 & 95.696 & 109.711 & 106.747 & & 73.475 \\
\hline 10/18/07 11:00 & 124.989 & 122.104 & 118.514 & 99.88 & 103.835 & 95.5 & 109.506 & 106.514 & & 73.374 \\
\hline $10 / 18 / 0711: 30$ & 124.896 & 122.077 & 118.489 & 99.795 & 103.738 & 95.403 & 109.409 & 106.401 & & 73.301 \\
\hline 10/18/07 12:00 & 124.837 & 122.061 & 118.466 & 99.759 & 103.687 & 95.354 & 109.351 & 106.346 & & 73.158 \\
\hline $10 / 18 / 0712: 30$ & 124.792 & 122.05 & 118.454 & 99.738 & 103.662 & 95.333 & 109.309 & 106.317 & & 73.134 \\
\hline 10/18/07 13:00 & 124.762 & 122.047 & 118.451 & 99.725 & 103.649 & 95.318 & 109.287 & 106.302 & & 73.117 \\
\hline 10/18/07 13:30 & 124.74 & 122.045 & 118.456 & 99.718 & 103.636 & 95.304 & 109.272 & 106.286 & & 73.101 \\
\hline $10 / 18 / 07$ 14:00 & 124.714 & 122.05 & 118.449 & 99.725 & 103.64 & 95.309 & 109.259 & 106.286 & & 73.099 \\
\hline $10 / 18 / 07$ 14:30 & 124.7 & 122.056 & 118.459 & 99.726 & 103.637 & 95.309 & 109.251 & 106.286 & & 73.103 \\
\hline 10/18/07 15:00 & 124.686 & 122.075 & 118.479 & 99.733 & 103.643 & 95.314 & 109.242 & 106.288 & & 73.105 \\
\hline $10 / 18 / 07$ 15:30 & 124.672 & 122.086 & 118.497 & 99.735 & 103.651 & 95.321 & 109.24 & 106.295 & & 73.113 \\
\hline 10/18/07 16:00 & 124.655 & 122.1 & 118.502 & 99.756 & 103.672 & 95.34 & 109.232 & 106.315 & & 73.131 \\
\hline $10 / 18 / 07$ 16:30 & 124.644 & 122.109 & 118.517 & 99.769 & 103.685 & 95.354 & 109.229 & 106.326 & & 73.144 \\
\hline $10 / 18 / 07$ 17:00 & 124.639 & 122.122 & 118.529 & 99.783 & 103.696 & 95.366 & 109.225 & 106.335 & & 73.154 \\
\hline $10 / 18 / 07$ 17:30 & 124.632 & 122.131 & 118.535 & 99.795 & 103.706 & 95.38 & 109.225 & 106.35 & & 73.148 \\
\hline $10 / 18 / 07$ 18:00 & 124.625 & 122.14 & 118.54 & 99.807 & 103.719 & 95.394 & 109.225 & 106.364 & & 73.162 \\
\hline $10 / 18 / 07$ 18:30 & 124.622 & 122.149 & 118.555 & 99.816 & 103.733 & 95.403 & 109.223 & 106.373 & & 73.154 \\
\hline 10/18/07 19:00 & 124.618 & 122.161 & 118.567 & 99.824 & 103.736 & 95.408 & 109.225 & 106.379 & & 73.142 \\
\hline 10/18/07 19:30 & 124.613 & 122.172 & 118.57 & 99.837 & 103.75 & 95.42 & 109.227 & 106.39 & & 73.128 \\
\hline $10 / 18 / 0720: 00$ & 124.611 & 122.183 & 118.582 & 99.847 & 103.759 & 95.429 & 109.232 & 106.399 & & 73.119 \\
\hline
\end{tabular}


TABLE S1.1 (Cont.)

\begin{tabular}{|c|c|c|c|c|c|c|c|c|c|c|}
\hline \multirow[b]{2}{*}{ Date and Time } & \multicolumn{10}{|c|}{ Depth from Top of Casing (ft) } \\
\hline & MW1D & MW2D & MW3D & MW4D & MW7 & MW9 & Oentrich & MW14D & MW15D & MW16D \\
\hline 10/18/07 20:30 & 124.608 & 122.19 & 118.592 & 99.856 & 103.767 & 95.441 & 109.242 & 106.41 & & 73.101 \\
\hline $10 / 18 / 0721: 00$ & 124.603 & 122.208 & 118.61 & 99.869 & 103.782 & 95.455 & 109.249 & 106.424 & & 73.103 \\
\hline 10/18/07 21:30 & 124.599 & 122.211 & 118.618 & 99.88 & 103.79 & 95.462 & 109.251 & 106.43 & & 73.082 \\
\hline $10 / 18 / 0722: 00$ & 124.594 & 122.211 & 118.623 & 99.892 & 103.805 & 95.476 & 109.261 & 106.446 & & 73.077 \\
\hline $10 / 18 / 0722: 30$ & 124.594 & 122.213 & 118.623 & 99.897 & 103.809 & 95.481 & 109.272 & 106.452 & & 73.068 \\
\hline 10/18/07 23:00 & 124.589 & 122.213 & 118.618 & 99.897 & 103.814 & 95.483 & 109.272 & 106.455 & & 73.057 \\
\hline $10 / 18 / 0723: 30$ & 124.587 & 122.206 & 118.61 & 99.9 & 103.813 & 95.488 & 109.276 & 106.455 & & 73.041 \\
\hline $10 / 19 / 07$ 0:00 & 124.585 & 122.206 & 118.613 & 99.893 & 103.811 & 95.483 & 109.277 & 106.455 & & 73.03 \\
\hline 10/19/07 0:30 & 124.585 & 122.213 & 118.623 & 99.888 & 103.803 & 95.476 & 109.277 & 106.448 & & 73.02 \\
\hline 10/19/07 1:00 & 124.58 & 122.211 & 118.618 & 99.892 & 103.803 & 95.474 & 109.276 & 106.448 & & 73.012 \\
\hline 10/19/07 1:30 & 124.575 & 122.224 & 118.63 & 99.899 & 103.811 & 95.483 & 109.276 & 106.455 & & 72.997 \\
\hline 10/19/07 2:00 & 124.573 & 122.236 & 118.643 & 99.9 & 103.813 & 95.483 & 109.274 & 106.457 & & 72.989 \\
\hline 10/19/07 2:30 & 124.568 & 122.245 & 118.648 & 99.912 & 103.826 & 95.498 & 109.276 & 106.47 & & 72.974 \\
\hline 10/19/07 3:00 & 124.563 & 122.254 & 118.661 & 99.921 & 103.834 & 95.507 & 109.283 & 106.479 & & 72.954 \\
\hline 10/19/07 3:30 & 124.561 & 122.258 & 118.665 & 99.933 & 103.843 & 95.514 & 109.292 & 106.486 & & 72.944 \\
\hline $10 / 19 / 074: 00$ & 124.559 & 122.258 & 118.668 & 99.94 & 103.853 & 95.526 & 109.302 & 106.497 & & 72.925 \\
\hline $10 / 19 / 074: 30$ & 124.556 & 122.265 & 118.673 & 99.945 & 103.858 & 95.531 & 109.309 & 106.501 & & 72.904 \\
\hline 10/19/07 5:00 & 124.556 & 122.281 & 118.686 & 99.945 & 103.858 & 95.533 & 109.315 & 106.503 & & 72.89 \\
\hline 10/19/07 5:30 & 124.551 & 122.295 & 118.698 & 99.954 & 103.864 & 95.538 & 109.313 & 106.508 & & 72.875 \\
\hline 10/19/07 6:00 & 124.551 & 124.063 & 119.675 & 99.971 & 103.883 & 95.552 & 109.332 & 106.523 & & 72.859 \\
\hline 10/19/07 6:30 & 124.544 & 124.449 & 120.21 & 99.985 & 103.893 & 95.566 & 109.332 & 106.534 & & 72.84 \\
\hline 10/19/07 7:00 & 125.988 & 124.623 & 120.404 & 100.62 & 104.224 & 96.021 & 109.796 & 107.75 & & 72.836 \\
\hline 10/19/07 7:30 & 126.341 & 124.725 & 120.513 & 100.898 & 104.589 & 96.4 & 110.147 & 108.228 & & 72.954 \\
\hline 10/19/07 8:00 & 126.492 & 124.8 & 120.598 & 101.041 & 104.761 & 96.57 & 110.303 & 108.42 & & 73.039 \\
\hline $10 / 19 / 078: 30$ & 126.575 & 124.85 & 120.651 & 101.134 & 104.864 & 96.674 & 110.387 & 108.525 & & 73.097 \\
\hline 10/19/07 9:00 & 126.629 & 124.886 & 120.689 & 101.205 & 104.947 & 96.752 & 110.447 & 108.609 & & 73.13 \\
\hline 10/19/07 9:30 & 126.679 & 123.099 & 119.625 & 101.248 & 104.999 & 96.806 & 110.494 & 108.662 & & 73.152 \\
\hline 10/19/07 10:00 & 126.726 & 122.796 & 119.227 & 101.286 & 105.029 & 96.839 & 110.524 & 108.697 & & 73.175 \\
\hline 10/19/07 10:30 & 125.26 & 122.664 & 119.081 & 100.622 & 104.656 & 96.332 & 110.012 & 107.422 & & 73.169 \\
\hline 10/19/07 11:00 & 124.998 & 122.582 & 118.991 & 100.403 & 104.367 & 96.04 & 109.737 & 107.064 & & 73.099 \\
\hline $10 / 19 / 0711: 30$ & 124.896 & 122.53 & 118.943 & 100.303 & 104.247 & 95.919 & 109.615 & 106.922 & & 73.061 \\
\hline 10/19/07 12:00 & 124.84 & 122.48 & 118.895 & 100.231 & 104.165 & 95.839 & 109.546 & 106.831 & & 73.049 \\
\hline $10 / 19 / 07$ 12:30 & 124.804 & 122.442 & 118.85 & 100.186 & 104.113 & 95.787 & 109.505 & 106.778 & & 73.077 \\
\hline 10/19/07 13:00 & 124.781 & 122.417 & 118.827 & 100.142 & 104.068 & 95.745 & 109.476 & 106.731 & & 73.084 \\
\hline 10/19/07 13:30 & 124.762 & 122.392 & 118.802 & 100.104 & 104.026 & 95.698 & 109.458 & 106.685 & & 73.105 \\
\hline $10 / 19 / 07$ 14:00 & 124.748 & 122.374 & 118.784 & 100.085 & 104.009 & 95.679 & 109.439 & 106.663 & & 73.134 \\
\hline 10/19/07 14:30 & 124.738 & 122.363 & 118.771 & 100.062 & 103.98 & 95.655 & 109.433 & 106.636 & & 73.167 \\
\hline 10/19/07 15:00 & 124.736 & 122.356 & 118.761 & 100.047 & 103.967 & 95.639 & 109.42 & 106.618 & & 73.179 \\
\hline $10 / 19 / 07$ 15:30 & 124.724 & 122.347 & 118.756 & 100.035 & 103.952 & 95.627 & 109.411 & 106.605 & & 73.185 \\
\hline $10 / 19 / 0716: 00$ & 124.719 & 122.34 & 118.749 & 100.028 & 103.944 & 95.62 & 109.403 & 106.601 & & 73.19 \\
\hline 10/19/07 16:30 & 124.717 & 122.329 & 118.736 & 100.023 & 103.936 & 95.608 & 109.401 & 106.592 & & 73.187 \\
\hline $10 / 19 / 07$ 17:00 & 124.707 & 122.324 & 118.728 & 100.016 & 103.934 & 95.606 & 109.394 & 106.585 & & 73.187 \\
\hline 10/19/07 17:30 & 124.705 & 122.319 & 118.724 & 100.007 & 103.923 & 95.594 & 109.39 & 106.572 & & 73.185 \\
\hline 10/19/07 18:00 & 124.7 & 122.317 & 118.726 & 100 & 103.913 & 95.587 & 109.386 & 106.565 & & 73.187 \\
\hline $10 / 19 / 07$ 18:30 & 124.693 & 122.315 & 118.718 & 99.995 & 103.91 & 95.585 & 109.381 & 106.561 & & 73.181 \\
\hline 10/19/07 19:00 & 124.688 & 122.315 & 118.718 & 99.995 & 103.914 & 95.587 & 109.379 & 106.563 & & 73.177 \\
\hline 10/19/07 19:30 & 124.684 & 122.315 & 118.716 & 99.993 & 103.906 & 95.582 & 109.377 & 106.556 & & 73.175 \\
\hline $10 / 19 / 0720: 00$ & 124.679 & 122.315 & 118.718 & 99.992 & 103.906 & 95.582 & 109.373 & 106.556 & & 73.175 \\
\hline 10/19/07 20:30 & 124.674 & 122.304 & 118.708 & 99.992 & 103.908 & 95.585 & 109.369 & 106.556 & & 73.175 \\
\hline 10/19/07 21:00 & 124.672 & 122.299 & 118.688 & 99.992 & 103.908 & 95.582 & 109.366 & 106.557 & & 73.177 \\
\hline $10 / 19 / 0721: 30$ & 124.67 & 122.29 & 118.678 & 99.98 & 103.896 & 95.571 & 109.364 & 106.545 & & 73.175 \\
\hline 10/19/07 22:00 & 124.665 & 122.276 & 118.671 & 99.976 & 103.893 & 95.568 & 109.36 & 106.541 & & 73.171 \\
\hline 10/19/07 22:30 & 124.665 & 122.272 & 118.678 & 99.964 & 103.881 & 95.559 & 109.36 & 106.532 & & 73.167 \\
\hline 10/19/07 23:00 & 124.662 & 122.258 & 118.662 & 99.954 & 103.868 & 95.545 & 109.36 & 106.521 & & 73.159 \\
\hline 10/19/07 23:30 & 124.658 & 122.242 & 118.65 & 99.947 & 103.866 & 95.54 & 109.358 & 106.514 & & 73.154 \\
\hline 10/20/07 0:00 & 124.655 & 122.236 & 118.638 & 99.936 & 103.851 & 95.523 & 109.354 & 106.501 & & 73.146 \\
\hline $10 / 20 / 070: 30$ & 124.655 & 122.229 & 118.632 & 99.918 & 103.835 & 95.509 & 109.354 & 106.488 & & 73.138 \\
\hline 10/20/07 1:00 & 124.651 & 122.224 & 118.63 & 99.914 & 103.83 & 95.5 & 109.351 & 106.479 & & 73.128 \\
\hline 10/20/07 1:30 & 124.644 & 122.224 & 118.628 & 99.907 & 103.822 & 95.495 & 109.347 & 106.472 & & 73.121 \\
\hline 10/20/07 2:00 & 124.641 & 122.222 & 118.623 & 99.902 & 103.817 & 95.49 & 109.345 & 106.468 & & 73.119 \\
\hline 10/20/07 2:30 & 124.636 & 122.215 & 118.62 & 99.904 & 103.82 & 95.49 & 109.343 & 106.468 & & 73.113 \\
\hline 10/20/07 3:00 & 124.632 & 122.213 & 118.614 & 99.902 & 103.818 & 95.49 & 109.339 & 106.466 & & 73.129 \\
\hline 10/20/07 3:30 & 124.629 & 122.213 & 118.612 & 99.897 & 103.813 & 95.483 & 109.339 & 106.461 & & 73.127 \\
\hline $10 / 20 / 074: 00$ & 124.625 & 122.213 & 118.599 & 99.89 & 103.807 & 95.479 & 109.338 & 106.457 & & 73.121 \\
\hline $10 / 20 / 074: 30$ & 124.622 & 122.213 & 118.605 & 99.893 & 103.807 & 95.483 & 109.334 & 106.457 & & 73.123 \\
\hline 10/20/07 5:00 & 124.618 & 122.206 & 118.608 & 99.897 & 103.811 & 95.483 & 109.334 & 106.457 & & 73.125 \\
\hline
\end{tabular}


TABLE S1.1 (Cont.)

\begin{tabular}{|c|c|c|c|c|c|c|c|c|c|c|}
\hline \multirow[b]{2}{*}{ Date and Time } & \multicolumn{10}{|c|}{ Depth from Top of Casing (ft) } \\
\hline & MW1D & MW2D & MW3D & MW4D & MW7 & MW9 & Oentrich & MW14D & MW15D & MW16D \\
\hline 10/20/07 5:30 & 124.615 & 122.208 & 118.607 & 99.893 & 103.809 & 95.483 & 109.33 & 106.457 & & 73.125 \\
\hline 10/20/07 6:00 & 124.613 & 122.208 & 118.61 & 99.883 & 103.801 & 95.476 & 109.33 & 106.45 & & 73.119 \\
\hline 10/20/07 6:30 & 124.611 & 122.204 & 118.607 & 99.887 & 103.801 & 95.474 & 109.326 & 106.45 & & 73.119 \\
\hline 10/20/07 7:00 & 124.606 & 122.199 & 118.607 & 99.887 & 103.801 & 95.474 & 109.324 & 106.45 & & 73.119 \\
\hline $10 / 20 / 07$ 7:30 & 124.603 & 122.197 & 118.598 & 99.885 & 103.797 & 95.472 & 109.322 & 106.446 & & 73.117 \\
\hline 10/20/07 8:00 & 124.601 & 124.133 & 119.994 & 99.88 & 103.795 & 95.472 & 109.322 & 106.443 & & 73.113 \\
\hline 10/20/07 8:30 & 124.601 & 124.39 & 120.306 & 99.874 & 103.792 & 95.462 & 109.322 & 106.442 & & 73.109 \\
\hline 10/20/07 9:00 & 126.239 & 124.517 & 120.447 & 100.684 & 104.327 & 96.148 & 110 & 107.878 & & 73.171 \\
\hline 10/20/07 9:30 & 126.485 & 124.587 & 120.527 & 100.882 & 104.578 & 96.4 & 110.246 & 108.172 & & 73.328 \\
\hline $10 / 20 / 07$ 10:00 & 126.62 & 124.623 & 120.568 & 100.986 & 104.7 & 96.523 & 110.37 & 108.308 & & 73.436 \\
\hline $10 / 20 / 07$ 10:30 & 126.712 & 124.639 & 120.58 & 101.046 & 104.772 & 96.594 & 110.443 & 108.387 & & 73.5 \\
\hline 10/20/07 11:00 & 126.78 & 124.639 & 120.593 & 101.07 & 104.805 & 96.629 & 110.496 & 108.427 & & 73.536 \\
\hline $10 / 20 / 0711: 30$ & 126.889 & 124.646 & 120.596 & 101.084 & 104.82 & 96.643 & 110.533 & 108.443 & & 73.553 \\
\hline $10 / 20 / 07$ 12:00 & 127.073 & 124.646 & 120.593 & 101.082 & 104.825 & 96.65 & 110.562 & 108.451 & & 73.562 \\
\hline 10/20/07 12:30 & 127.112 & 124.632 & 120.581 & 101.087 & 104.829 & 96.648 & 110.58 & 108.454 & & 73.561 \\
\hline $10 / 20 / 07$ 13:00 & 127.119 & 124.619 & 120.571 & 101.081 & 104.829 & 96.65 & 110.599 & 108.454 & & 73.566 \\
\hline $10 / 20 / 07$ 13:30 & 127.109 & 122.982 & 119.693 & 101.068 & 104.816 & 96.636 & 110.612 & 108.441 & & 73.558 \\
\hline $10 / 20 / 07$ 14:00 & 127.099 & 122.53 & 118.973 & 101.058 & 104.799 & 96.622 & 110.622 & 108.429 & & 73.547 \\
\hline $10 / 20 / 0714: 30$ & 125.676 & 122.344 & 118.761 & 100.405 & 104.49 & 96.181 & 110.197 & 107.413 & & 73.526 \\
\hline $10 / 20 / 07$ 15:00 & 125.034 & 122.247 & 118.655 & 100.097 & 104.081 & 95.754 & 109.807 & 106.805 & & 73.377 \\
\hline $10 / 20 / 07$ 15:30 & 124.769 & 122.183 & 118.585 & 99.952 & 103.902 & 95.573 & 109.647 & 106.596 & & 73.241 \\
\hline $10 / 20 / 0716: 00$ & 124.636 & 122.145 & 118.545 & 99.876 & 103.814 & 95.488 & 109.557 & 106.497 & & 73.164 \\
\hline 10/20/07 16:30 & 124.566 & 122.113 & 118.519 & 99.826 & 103.755 & 95.422 & 109.501 & 106.426 & & 73.106 \\
\hline $10 / 20 / 07$ 17:00 & 124.521 & 122.086 & 118.491 & 99.795 & 103.718 & 95.389 & 109.467 & 106.39 & & 73.069 \\
\hline $10 / 20 / 07$ 17:30 & 124.49 & 122.07 & 118.471 & 99.764 & 103.691 & 95.363 & 109.439 & 106.357 & & 73.044 \\
\hline 10/20/07 18:00 & 124.462 & 122.056 & 118.459 & 99.745 & 103.668 & 95.34 & 109.422 & 106.331 & & 73.018 \\
\hline $10 / 20 / 07$ 18:30 & 124.443 & 122.05 & 118.453 & 99.73 & 103.649 & 95.323 & 109.409 & 106.311 & & 73.001 \\
\hline 10/20/07 19:00 & 124.429 & 122.041 & 118.441 & 99.719 & 103.639 & 95.309 & 109.396 & 106.3 & & 72.989 \\
\hline 10/20/07 19:30 & 124.421 & 122.038 & 118.431 & 99.716 & 103.634 & 95.307 & 109.388 & 106.293 & & 72.984 \\
\hline $10 / 20 / 0720: 00$ & 124.414 & 122.022 & 118.426 & 99.709 & 103.626 & 95.297 & 109.381 & 106.286 & & 72.974 \\
\hline $10 / 20 / 0720: 30$ & 124.407 & 122.013 & 118.411 & 99.7 & 103.618 & 95.29 & 109.375 & 106.278 & & 72.968 \\
\hline 10/20/07 21:00 & 124.398 & 121.995 & 118.403 & 99.687 & 103.609 & 95.276 & 109.369 & 106.262 & & 72.958 \\
\hline $10 / 20 / 0721: 30$ & 124.386 & 121.977 & 118.383 & 99.681 & 103.603 & 95.271 & 109.366 & 106.255 & & 72.949 \\
\hline $10 / 20 / 0722: 00$ & 124.377 & 121.95 & 118.36 & 99.661 & 103.582 & 95.252 & 109.36 & 106.24 & & 72.933 \\
\hline 10/20/07 22:30 & 124.355 & 121.941 & 118.34 & 99.64 & 103.563 & 95.236 & 109.343 & 106.218 & & 72.916 \\
\hline $10 / 20 / 0723: 00$ & 124.336 & 121.936 & 118.332 & 99.619 & 103.542 & 95.212 & 109.339 & 106.2 & & 72.898 \\
\hline 10/20/07 23:30 & 124.315 & 121.925 & 118.33 & 99.607 & 103.529 & 95.201 & 109.328 & 106.18 & & 72.879 \\
\hline 10/21/07 0:00 & 124.306 & 121.918 & 118.312 & 99.601 & 103.525 & 95.191 & 109.324 & 106.176 & & 72.877 \\
\hline $10 / 21 / 070: 30$ & 124.299 & 121.918 & 118.312 & 99.588 & 103.508 & 95.182 & 109.319 & 106.162 & & 72.867 \\
\hline $10 / 21 / 071: 00$ & 124.284 & 121.916 & 118.315 & 99.588 & 103.506 & 95.179 & 109.309 & 106.156 & & 72.858 \\
\hline 10/21/07 1:30 & 124.284 & 121.934 & 118.325 & 99.585 & 103.506 & 95.179 & 109.306 & 106.158 & & 72.858 \\
\hline $10 / 21 / 072: 00$ & 124.28 & 121.939 & 118.341 & 99.59 & 103.508 & 95.177 & 109.302 & 106.156 & & 72.856 \\
\hline $10 / 21 / 072: 30$ & 124.287 & 121.943 & 118.337 & 99.604 & 103.521 & 95.191 & 109.298 & 106.169 & & 72.865 \\
\hline 10/21/07 3:00 & 124.301 & 121.943 & 118.342 & 99.611 & 103.529 & 95.201 & 109.296 & 106.176 & & 72.875 \\
\hline $10 / 21 / 073: 30$ & 124.306 & 121.95 & 118.346 & 99.609 & 103.533 & 95.205 & 109.294 & 106.184 & & 72.875 \\
\hline $10 / 21 / 074: 00$ & 124.308 & 121.966 & 118.365 & 99.616 & 103.535 & 95.205 & 109.292 & 106.184 & & 72.877 \\
\hline 10/21/07 4:30 & 124.315 & 121.988 & 118.388 & 99.628 & 103.544 & 95.212 & 109.291 & 106.191 & & 72.884 \\
\hline $10 / 21 / 075: 00$ & 124.327 & 122.02 & 118.415 & 99.649 & 103.559 & 95.229 & 109.289 & 106.207 & & 72.896 \\
\hline 10/21/07 5:30 & 124.343 & 123.855 & 119.502 & 99.669 & 103.582 & 95.252 & 109.285 & 106.226 & & 72.912 \\
\hline 10/21/07 6:00 & 124.369 & 124.217 & 119.983 & 99.706 & 103.616 & 95.288 & 109.281 & 106.257 & & 72.937 \\
\hline $10 / 21 / 076: 30$ & 126.036 & 124.401 & 120.183 & 100.393 & 104.016 & 95.813 & 109.773 & 107.561 & & 72.98 \\
\hline 10/21/07 7:00 & 126.584 & 124.521 & 120.314 & 100.669 & 104.367 & 96.172 & 110.094 & 108.002 & & 73.162 \\
\hline 10/21/07 7:30 & 126.797 & 124.596 & 120.399 & 100.831 & 104.553 & 96.356 & 110.233 & 108.197 & & 73.309 \\
\hline 10/21/07 8:00 & 126.932 & 122.777 & 119.247 & 100.938 & 104.673 & 96.476 & 110.318 & 108.327 & & 73.414 \\
\hline $10 / 21 / 078: 30$ & 127.017 & 122.558 & 118.967 & 101.005 & 104.747 & 96.554 & 110.374 & 108.407 & & 73.487 \\
\hline 10/21/07 9:00 & 125.305 & 122.467 & 118.862 & 100.341 & 104.342 & 96.011 & 109.792 & 107.068 & & 73.495 \\
\hline 10/21/07 9:30 & 125.003 & 122.422 & 118.814 & 100.181 & 104.136 & 95.802 & 109.583 & 106.82 & & 73.396 \\
\hline $10 / 21 / 0710: 00$ & 124.873 & 122.401 & 118.793 & 100.112 & 104.053 & 95.724 & 109.48 & 106.722 & & 73.332 \\
\hline $10 / 21 / 0710: 30$ & 124.807 & 122.394 & 118.778 & 100.088 & 104.011 & 95.677 & 109.428 & 106.669 & & 73.29 \\
\hline $10 / 21 / 0711: 00$ & 124.774 & 122.406 & 118.793 & 100.074 & 103.993 & 95.665 & 109.398 & 106.647 & & 73.272 \\
\hline $10 / 21 / 0711: 30$ & 124.762 & 122.412 & 118.798 & 100.076 & 103.988 & 95.66 & 109.383 & 106.638 & & 73.264 \\
\hline $10 / 21 / 0712: 00$ & 124.766 & 122.417 & 118.808 & 100.09 & 104.001 & 95.672 & 109.377 & 106.652 & & 73.274 \\
\hline $10 / 21 / 0712: 30$ & 124.778 & 122.408 & 118.821 & 100.092 & 104.007 & 95.679 & 109.371 & 106.656 & & 73.276 \\
\hline $10 / 21 / 0713: 00$ & 124.785 & 122.41 & 118.812 & 100.102 & 104.015 & 95.688 & 109.366 & 106.663 & & 73.284 \\
\hline $10 / 21 / 07$ 13:30 & 124.788 & 122.424 & 118.826 & 100.088 & 104.005 & 95.679 & 109.362 & 106.656 & & 73.276 \\
\hline $10 / 21 / 0714: 00$ & 124.783 & 122.431 & 118.831 & 100.095 & 104.001 & 95.674 & 109.36 & 106.649 & & 73.272 \\
\hline
\end{tabular}


TABLE S1.1 (Cont.)

Depth from Top of Casing (ft)

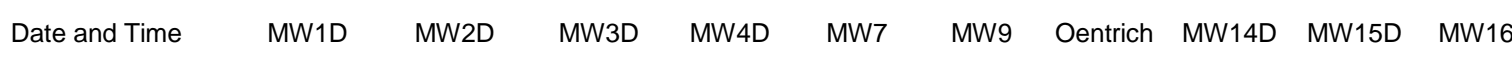

\begin{tabular}{|c|c|c|c|c|c|c|c|c|c|}
\hline $10 / 21 / 0714: 30$ & 124.79 & 122.44 & 118.839 & 100.107 & 104.018 & 95.693 & 109.356 & 106.667 & 73.284 \\
\hline $10 / 21 / 07$ 15:00 & 124.797 & 122.462 & 118.861 & 100.112 & 104.022 & 95.698 & 109.356 & 106.669 & 73.29 \\
\hline $10 / 21 / 07$ 15:30 & 124.807 & 122.49 & 118.887 & 100.126 & 104.037 & 95.712 & 109.358 & 106.683 & 73.301 \\
\hline $10 / 21 / 07 \quad 16: 00$ & 124.823 & 122.505 & 118.904 & 100.15 & 104.058 & 95.733 & 109.354 & 106.702 & 73.32 \\
\hline $10 / 21 / 07 \quad 16: 30$ & 124.849 & 122.521 & 118.919 & 100.178 & 104.085 & 95.759 & 109.381 & 106.732 & 73.344 \\
\hline $10 / 21 / 0717: 00$ & 124.866 & 122.539 & 118.942 & 100.19 & 104.1 & 95.773 & 109.394 & 106.745 & 73.354 \\
\hline $10 / 21 / 07$ 17:30 & 124.882 & 122.564 & 118.972 & 100.217 & 104.115 & 95.792 & 109.461 & 106.763 & 73.369 \\
\hline $10 / 21 / 0718: 00$ & 124.906 & 122.578 & 118.98 & 100.231 & 104.136 & 95.816 & 109.458 & 106.784 & 73.39 \\
\hline $10 / 21 / 07$ 18:30 & 124.93 & 122.605 & 119 & 100.252 & 104.161 & 95.839 & 109.456 & 106.807 & 73.411 \\
\hline $10 / 21 / 0719: 00$ & 124.944 & 122.623 & 119.028 & 100.272 & 104.178 & 95.851 & 109.486 & 106.822 & 73.423 \\
\hline $10 / 21 / 07 \quad 19: 30$ & 124.96 & 122.644 & 119.04 & 100.295 & 104.203 & 95.877 & 109.478 & 106.844 & 73.443 \\
\hline $10 / 21 / 0720: 00$ & 124.989 & 122.66 & 119.068 & 100.309 & 104.224 & 95.901 & 109.476 & 106.869 & 73.469 \\
\hline $10 / 21 / 0720: 30$ & 124.996 & 122.66 & 119.063 & 100.333 & 104.243 & 95.917 & 109.473 & 106.884 & 73.481 \\
\hline $10 / 21 / 0721: 00$ & 125.017 & 122.66 & 119.06 & 100.348 & 104.26 & 95.934 & 109.471 & 106.904 & 73.5 \\
\hline $10 / 21 / 0721: 30$ & 125.029 & 122.648 & 119.04 & 100.352 & 104.253 & 95.929 & 109.469 & 106.902 & 73.498 \\
\hline $10 / 21 / 0722: 00$ & 125.036 & 122.626 & 119.015 & 100.341 & 104.26 & 95.941 & 109.475 & 106.911 & 73.508 \\
\hline $10 / 21 / 0722: 30$ & 125.029 & 122.621 & 119.03 & 100.331 & 104.25 & 95.927 & 109.48 & 106.895 & 73.495 \\
\hline $10 / 21 / 0723: 00$ & 125.024 & 122.648 & 119.035 & 100.317 & 104.224 & 95.894 & 109.488 & 106.869 & 73.472 \\
\hline $10 / 21 / 0723: 30$ & 125.022 & 122.68 & 119.078 & 100.293 & 104.218 & 95.894 & 109.488 & 106.869 & 73.477 \\
\hline $10 / 22 / 070: 00$ & 125.003 & 122.669 & 119.069 & 100.357 & 104.241 & 95.915 & 109.482 & 106.882 & 73.489 \\
\hline 10/22/07 0:30 & 124.998 & 122.68 & 119.081 & 100.374 & 104.275 & 95.955 & 109.484 & 106.922 & 73.526 \\
\hline 10/22/07 1:00 & 125 & 122.691 & 119.101 & 100.353 & 104.266 & 95.941 & 109.491 & 106.913 & 73.516 \\
\hline 10/22/07 1:30 & 124.996 & 122.694 & 119.099 & 100.372 & 104.275 & 95.952 & 109.491 & 106.922 & 73.526 \\
\hline 10/22/07 2:00 & 124.993 & 122.682 & 119.088 & 100.376 & 104.292 & 95.969 & 109.495 & 106.937 & 73.543 \\
\hline $10 / 22 / 072: 30$ & 124.991 & 122.671 & 119.076 & 100.379 & 104.29 & 95.974 & 109.501 & 106.942 & 73.547 \\
\hline 10/22/07 3:00 & 124.991 & 122.678 & 119.078 & 100.372 & 104.277 & 95.957 & 109.505 & 106.928 & 73.534 \\
\hline 10/22/07 3:30 & 124.993 & 122.666 & 119.071 & 100.364 & 104.268 & 95.948 & 109.508 & 106.919 & 73.526 \\
\hline $10 / 22 / 074: 00$ & 124.986 & 122.709 & 119.108 & 100.376 & 104.271 & 95.948 & 109.518 & 106.922 & 73.528 \\
\hline $10 / 22 / 074: 30$ & 124.991 & 122.734 & 119.126 & 100.355 & 104.26 & 95.936 & 109.514 & 106.911 & 73.522 \\
\hline $10 / 22 / 075: 00$ & 124.979 & 122.725 & 119.136 & 100.407 & 104.308 & 95.983 & 109.508 & 106.953 & 73.566 \\
\hline 10/22/07 5:30 & 124.977 & 122.712 & 119.121 & 100.424 & 104.327 & 96.004 & 109.514 & 106.973 & 73.576 \\
\hline 10/22/07 6:00 & 124.982 & 124.578 & 120.232 & 100.388 & 104.329 & 96.007 & 109.521 & 106.977 & 73.584 \\
\hline 10/22/07 6:30 & 124.982 & 124.918 & 120.678 & 100.409 & 104.309 & 95.988 & 109.527 & 106.961 & 73.57 \\
\hline 10/22/07 7:00 & 126.509 & 125.068 & 120.847 & 101.098 & 104.713 & 96.52 & 110.058 & 108.29 & 73.596 \\
\hline $10 / 22 / 077: 30$ & 126.818 & 125.149 & 120.94 & 101.341 & 105.046 & 96.86 & 110.37 & 108.704 & 73.777 \\
\hline 10/22/07 8:00 & 126.965 & 125.233 & 121.026 & 101.477 & 105.193 & 97.006 & 110.517 & 108.865 & 73.897 \\
\hline $10 / 22 / 078: 30$ & 127.057 & 125.261 & 121.051 & 101.558 & 105.262 & 97.074 & 110.609 & 108.945 & 73.964 \\
\hline 10/22/07 9:00 & 127.116 & 125.281 & 121.089 & 101.61 & 105.36 & 97.173 & 110.661 & 109.043 & 74.047 \\
\hline 10/22/07 9:30 & 127.166 & 125.326 & 121.107 & 101.639 & 105.385 & 97.199 & 110.704 & 109.069 & 74.07 \\
\hline $10 / 22 / 07 \quad 10: 00$ & 127.208 & 125.36 & 121.165 & 101.654 & 105.412 & 97.228 & 110.736 & 109.1 & 74.101 \\
\hline $10 / 22 / 07 \quad 10: 30$ & 127.234 & 125.367 & 121.167 & 101.709 & 105.456 & 97.27 & 110.755 & 109.138 & 74.136 \\
\hline $10 / 22 / 0711: 00$ & 127.272 & 125.372 & 121.177 & 101.725 & 105.488 & 97.305 & 110.774 & 109.18 & 74.176 \\
\hline 10/22/07 11:30 & 127.298 & 123.712 & 120.459 & 101.751 & 105.502 & 97.315 & 110.791 & 109.187 & 74.186 \\
\hline 10/22/07 12:00 & 127.324 & 123.263 & 119.706 & 101.744 & 105.507 & 97.322 & 110.997 & 109.195 & 74.198 \\
\hline $10 / 22 / 07 \quad 12: 30$ & 126.04 & 123.068 & 119.491 & 101.136 & 105.224 & 96.924 & 110.625 & 108.172 & 74.171 \\
\hline $10 / 22 / 07$ 13:00 & 125.617 & 122.961 & 119.375 & 100.838 & 104.82 & 96.499 & 110.226 & 107.544 & 74.039 \\
\hline $10 / 22 / 07$ 13:30 & 125.476 & 122.884 & 119.287 & 100.684 & 104.631 & 96.311 & 110.073 & 107.331 & 73.9 \\
\hline $10 / 22 / 0714: 00$ & 125.381 & 122.836 & 119.237 & 100.579 & 104.534 & 96.216 & 109.985 & 107.223 & 73.82 \\
\hline $10 / 22 / 07 \quad 14: 30$ & 125.296 & 122.802 & 119.202 & 100.526 & 104.46 & 96.139 & 109.931 & 107.139 & 73.748 \\
\hline $10 / 22 / 07$ 15:00 & 125.232 & 122.78 & 119.181 & 100.488 & 104.408 & 96.092 & 109.893 & 107.085 & 73.706 \\
\hline $10 / 22 / 07$ 15:30 & 125.182 & 122.755 & 119.161 & 100.455 & 104.378 & 96.059 & 109.867 & 107.048 & 73.673 \\
\hline $10 / 22 / 07$ 16:00 & 125.156 & 122.73 & 119.131 & 100.436 & 104.359 & 96.037 & 109.843 & 107.028 & 73.656 \\
\hline 10/22/07 16:30 & 125.133 & 122.716 & 119.121 & 100.415 & 104.336 & 96.014 & 109.828 & 107.004 & 73.636 \\
\hline $10 / 22 / 07 \quad 17: 00$ & 125.112 & 122.703 & 119.111 & 100.393 & 104.313 & 95.988 & 109.816 & 106.975 & 73.611 \\
\hline $10 / 22 / 07$ 17:30 & 125.093 & 122.696 & 119.093 & 100.383 & 104.3 & 95.976 & 109.805 & 106.964 & 73.601 \\
\hline 10/22/07 18:00 & 125.081 & 122.691 & 119.076 & 100.372 & 104.287 & 95.964 & 109.794 & 106.953 & 73.592 \\
\hline $10 / 22 / 07$ 18:30 & 125.069 & 122.687 & 119.076 & 100.365 & 104.279 & 95.96 & 109.786 & 106.944 & 73.582 \\
\hline $10 / 22 / 07 \quad 19: 00$ & 125.06 & 122.685 & 119.081 & 100.362 & 104.275 & 95.952 & 109.777 & 106.937 & 73.58 \\
\hline 10/22/07 19:30 & 125.055 & 122.675 & 119.06 & 100.359 & 104.273 & 95.945 & 109.771 & 106.933 & 73.578 \\
\hline $10 / 22 / 0720: 00$ & 125.048 & 122.666 & 119.053 & 100.353 & 104.269 & 95.945 & 109.764 & 106.928 & 73.576 \\
\hline 10/22/07 20:30 & 125.041 & 122.66 & 119.058 & 100.345 & 104.26 & 95.936 & 109.76 & 106.922 & 73.565 \\
\hline $10 / 22 / 0721: 00$ & 125.034 & 122.66 & 119.06 & 100.34 & 104.252 & 95.929 & 109.752 & 106.913 & 73.561 \\
\hline $10 / 22 / 0721: 30$ & 125.029 & 122.655 & 119.053 & 100.331 & 104.247 & 95.919 & 109.747 & 106.904 & 73.558 \\
\hline $10 / 22 / 0722: 00$ & 125.019 & 122.653 & 119.053 & 100.334 & 104.245 & 95.924 & 109.743 & 106.904 & 73.555 \\
\hline $10 / 22 / 0722: 30$ & 125.015 & 122.641 & 119.028 & 100.328 & 104.243 & 95.922 & 109.736 & 106.902 & 73.555 \\
\hline $10 / 22 / 0723: 00$ & 125.008 & 122.628 & 119.025 & 100.326 & 104.241 & 95.919 & 109.732 & 106.9 & 73.555 \\
\hline
\end{tabular}


TABLE S1.1 (Cont.)

Depth from Top of Casing (ft)

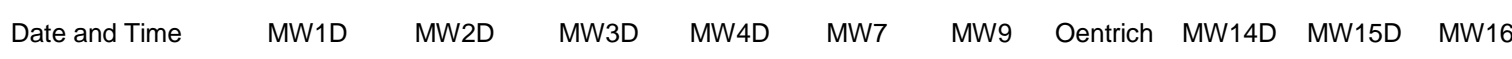

\begin{tabular}{|c|c|c|c|c|c|c|c|c|c|}
\hline 10/22/07 23:30 & 125.003 & 122.617 & 119.02 & 100.312 & 104.228 & 95.905 & 109.726 & 106.886 & 73.541 \\
\hline 10/23/07 0:00 & 124.998 & 122.607 & 119.008 & 100.3 & 104.212 & 95.891 & 109.724 & 106.873 & 73.528 \\
\hline $10 / 23 / 070: 30$ & 124.993 & 122.603 & 118.99 & 100.288 & 104.205 & 95.882 & 109.719 & 106.862 & 73.522 \\
\hline 10/23/07 1:00 & 124.986 & 122.592 & 118.99 & 100.283 & 104.195 & 95.872 & 109.717 & 106.853 & 73.514 \\
\hline 10/23/07 1:30 & 124.982 & 122.585 & 118.99 & 100.276 & 104.191 & 95.87 & 109.711 & 106.849 & 73.512 \\
\hline 10/23/07 2:00 & 124.974 & 122.576 & 118.982 & 100.267 & 104.182 & 95.861 & 109.705 & 106.84 & 73.505 \\
\hline $10 / 23 / 072: 30$ & 124.972 & 122.569 & 118.972 & 100.259 & 104.176 & 95.851 & 109.704 & 106.831 & 73.498 \\
\hline 10/23/07 3:00 & 124.963 & 122.56 & 118.967 & 100.247 & 104.167 & 95.846 & 109.7 & 106.824 & 73.491 \\
\hline $10 / 23 / 073: 30$ & 124.958 & 122.553 & 118.955 & 100.25 & 104.159 & 95.837 & 109.694 & 106.815 & 73.483 \\
\hline $10 / 23 / 074: 00$ & 124.956 & 122.539 & 118.945 & 100.231 & 104.15 & 95.828 & 109.69 & 106.807 & 73.477 \\
\hline $10 / 23 / 074: 30$ & 124.946 & 122.539 & 118.942 & 100.224 & 104.142 & 95.816 & 109.687 & 106.795 & 73.466 \\
\hline 10/23/07 5:00 & 124.944 & 122.526 & 118.924 & 100.216 & 104.132 & 95.806 & 109.687 & 106.789 & 73.458 \\
\hline $10 / 23 / 075: 30$ & 124.937 & 122.528 & 118.929 & 100.212 & 104.131 & 95.809 & 109.681 & 106.784 & 73.456 \\
\hline 10/23/07 6:00 & 124.934 & 124.501 & 120.368 & 100.202 & 104.115 & 95.792 & 109.675 & 106.771 & 73.444 \\
\hline $10 / 23 / 076: 30$ & 124.927 & 124.753 & 120.661 & 100.386 & 104.117 & 95.795 & 109.674 & 106.771 & 73.446 \\
\hline $10 / 23 / 077: 00$ & 126.594 & 124.889 & 120.819 & 101.041 & 104.686 & 96.513 & 110.383 & 108.252 & 73.526 \\
\hline $10 / 23 / 07$ 7:30 & 126.813 & 124.963 & 120.898 & 101.243 & 104.93 & 96.758 & 110.607 & 108.536 & 73.689 \\
\hline $10 / 23 / 07$ 8:00 & 126.934 & 125.022 & 120.958 & 101.351 & 105.069 & 96.895 & 110.723 & 108.686 & 73.809 \\
\hline $10 / 23 / 078: 30$ & 127.012 & 125.05 & 120.996 & 101.425 & 105.144 & 96.966 & 110.796 & 108.762 & 73.876 \\
\hline 10/23/07 9:00 & 127.069 & 125.072 & 121.018 & 101.475 & 105.201 & 97.025 & 110.841 & 108.826 & 73.931 \\
\hline 10/23/07 9:30 & 127.116 & 123.197 & 119.688 & 101.494 & 105.229 & 97.056 & 110.877 & 108.857 & 73.96 \\
\hline $10 / 23 / 07 \quad 10: 00$ & 127.161 & 122.934 & 119.368 & 101.516 & 105.252 & 97.077 & 110.901 & 108.881 & 73.983 \\
\hline $10 / 23 / 07 \quad 10: 30$ & 125.603 & 122.814 & 119.224 & 100.726 & 104.744 & 96.419 & 110.254 & 107.495 & 73.934 \\
\hline $10 / 23 / 0711: 00$ & 125.383 & 122.737 & 119.141 & 100.534 & 104.496 & 96.169 & 110.017 & 107.198 & 73.799 \\
\hline $10 / 23 / 07 \quad 11: 30$ & 125.282 & 122.682 & 119.086 & 100.438 & 104.378 & 96.051 & 109.905 & 107.066 & 73.704 \\
\hline $10 / 23 / 07$ 12:00 & 125.223 & 122.637 & 119.045 & 100.374 & 104.306 & 95.983 & 109.843 & 106.986 & 73.638 \\
\hline $10 / 23 / 07$ 12:30 & 125.166 & 122.598 & 118.995 & 100.329 & 104.26 & 95.934 & 109.797 & 106.933 & 73.594 \\
\hline $10 / 23 / 07$ 13:00 & 125.09 & 122.558 & 118.967 & 100.286 & 104.214 & 95.891 & 109.766 & 106.886 & 73.553 \\
\hline $10 / 23 / 07$ 13:30 & 125.015 & 122.535 & 118.942 & 100.247 & 104.172 & 95.851 & 109.747 & 106.842 & 73.516 \\
\hline $10 / 23 / 07 \quad 14: 00$ & 124.96 & 122.519 & 118.922 & 100.214 & 104.138 & 95.813 & 109.728 & 106.804 & 73.485 \\
\hline $10 / 23 / 07 \quad 14: 30$ & 124.927 & 122.494 & 118.899 & 100.193 & 104.115 & 95.792 & 109.717 & 106.782 & 73.46 \\
\hline $10 / 23 / 07$ 15:00 & 124.904 & 122.483 & 118.884 & 100.179 & 104.1 & 95.778 & 109.704 & 106.764 & 73.445 \\
\hline $10 / 23 / 07$ 15:30 & 124.88 & 122.478 & 118.877 & 100.15 & 104.075 & 95.752 & 109.689 & 106.738 & 73.425 \\
\hline $10 / 23 / 07$ 16:00 & 124.859 & 122.467 & 118.864 & 100.147 & 104.066 & 95.745 & 109.675 & 106.727 & 73.417 \\
\hline $10 / 23 / 07 \quad 16: 30$ & 124.852 & 122.458 & 118.859 & 100.143 & 104.064 & 95.74 & 109.666 & 106.722 & 73.412 \\
\hline $10 / 23 / 07$ 17:00 & 124.842 & 122.456 & 118.856 & 100.128 & 104.049 & 95.726 & 109.664 & 106.709 & 73.398 \\
\hline $10 / 23 / 07 \quad 17: 30$ & 124.83 & 122.458 & 118.861 & 100.124 & 104.043 & 95.719 & 109.659 & 106.702 & 73.392 \\
\hline $10 / 23 / 07$ 18:00 & 124.828 & 122.456 & 118.856 & 100.124 & 104.041 & 95.719 & 109.655 & 106.702 & 73.388 \\
\hline $10 / 23 / 07$ 18:30 & 124.828 & 122.451 & 118.856 & 100.128 & 104.043 & 95.719 & 109.649 & 106.7 & 73.388 \\
\hline $10 / 23 / 07$ 19:00 & 124.828 & 122.458 & 118.861 & 100.124 & 104.045 & 95.719 & 109.649 & 106.701 & 73.386 \\
\hline 10/23/07 19:30 & 124.826 & 122.469 & 118.869 & 100.126 & 104.041 & 95.714 & 109.644 & 106.696 & 73.383 \\
\hline $10 / 23 / 0720: 00$ & 124.828 & 122.476 & 118.877 & 100.138 & 104.045 & 95.721 & 109.64 & 106.703 & 73.388 \\
\hline $10 / 23 / 0720: 30$ & 124.835 & 122.49 & 118.889 & 100.143 & 104.054 & 95.733 & 109.634 & 106.712 & 73.396 \\
\hline 10/23/07 21:00 & 124.842 & 122.499 & 118.899 & 100.152 & 104.064 & 95.743 & 109.632 & 106.72 & 73.404 \\
\hline $10 / 23 / 0721: 30$ & 124.849 & 122.503 & 118.902 & 100.164 & 104.077 & 95.754 & 109.627 & 106.732 & 73.414 \\
\hline $10 / 23 / 0722: 00$ & 124.861 & 122.514 & 118.909 & 100.176 & 104.089 & 95.766 & 109.623 & 106.742 & 73.425 \\
\hline $10 / 23 / 0722: 30$ & 124.863 & 122.521 & 118.919 & 100.181 & 104.095 & 95.771 & 109.621 & 106.747 & 73.429 \\
\hline $10 / 23 / 0723: 00$ & 124.868 & 122.528 & 118.929 & 100.191 & 104.104 & 95.783 & 109.619 & 106.76 & 73.438 \\
\hline $10 / 23 / 0723: 30$ & 124.868 & 122.533 & 118.934 & 100.198 & 104.111 & 95.787 & 109.615 & 106.764 & 73.442 \\
\hline $10 / 24 / 070: 00$ & 124.866 & 122.544 & 118.945 & 100.2 & 104.117 & 95.795 & 109.612 & 106.769 & 73.448 \\
\hline $10 / 24 / 070: 30$ & 124.861 & 122.555 & 118.955 & 100.21 & 104.127 & 95.802 & 109.608 & 106.778 & 73.452 \\
\hline 10/24/07 1:00 & 124.859 & 122.562 & 118.962 & 100.224 & 104.134 & 95.811 & 109.604 & 106.787 & 73.46 \\
\hline 10/24/07 1:30 & 124.854 & 122.573 & 118.975 & 100.236 & 104.148 & 95.823 & 109.6 & 106.8 & 73.47 \\
\hline $10 / 24 / 072: 00$ & 124.854 & 122.587 & 118.987 & 100.243 & 104.155 & 95.832 & 109.598 & 106.807 & 73.479 \\
\hline $10 / 24 / 072: 30$ & 124.852 & 122.601 & 119 & 100.257 & 104.169 & 95.844 & 109.597 & 106.818 & 73.489 \\
\hline 10/24/07 3:00 & 124.849 & 122.605 & 119.008 & 100.271 & 104.18 & 95.861 & 109.593 & 106.831 & 73.5 \\
\hline $10 / 24 / 073: 30$ & 124.849 & 122.619 & 119.018 & 100.281 & 104.194 & 95.872 & 109.593 & 106.845 & 73.51 \\
\hline $10 / 24 / 074: 00$ & 124.847 & 122.628 & 119.03 & 100.288 & 104.203 & 95.879 & 109.591 & 106.851 & 73.512 \\
\hline $10 / 24 / 074: 30$ & 124.849 & 122.63 & 119.033 & 100.302 & 104.214 & 95.891 & 109.591 & 106.862 & 73.522 \\
\hline $10 / 24 / 075: 00$ & 124.852 & 122.641 & 119.038 & 100.307 & 104.224 & 95.898 & 109.591 & 106.873 & 73.53 \\
\hline 10/24/07 5:30 & 124.852 & 122.648 & 119.05 & 100.316 & 104.226 & 95.901 & 109.589 & 106.877 & 73.534 \\
\hline $10 / 24 / 076: 00$ & 124.852 & 124.598 & 120.288 & 100.324 & 104.233 & 95.912 & 109.585 & 106.886 & 73.543 \\
\hline $10 / 24 / 076: 30$ & 124.854 & 124.886 & 120.648 & 100.333 & 104.243 & 95.919 & 109.585 & 106.893 & 73.549 \\
\hline $10 / 24 / 07$ 7:00 & 126.457 & 125.038 & 120.814 & 101.087 & 104.732 & 96.542 & 110.192 & 108.341 & 73.611 \\
\hline $10 / 24 / 07 \quad 7: 30$ & 126.719 & 125.131 & 120.915 & 101.317 & 105.014 & 96.829 & 110.457 & 108.675 & 73.785 \\
\hline $10 / 24 / 07$ 8:00 & 126.854 & 125.195 & 120.988 & 101.439 & 105.163 & 96.978 & 110.586 & 108.834 & 73.907 \\
\hline
\end{tabular}


TABLE S1.1 (Cont.)

Depth from Top of Casing (ft)

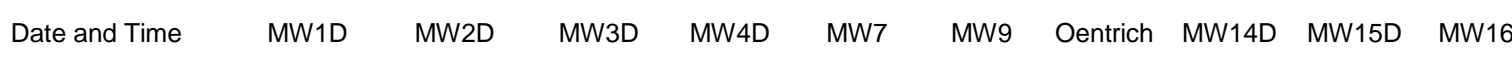

\begin{tabular}{|c|c|c|c|c|c|c|c|c|c|}
\hline 10/24/07 8:30 & 126.941 & 125.242 & 121.039 & 101.527 & 105.258 & 97.074 & 110.667 & 108.936 & 73.993 \\
\hline $10 / 24 / 079: 00$ & 127.005 & 125.283 & 121.074 & 101.585 & 105.324 & 97.14 & 110.721 & 109.007 & 74.055 \\
\hline 10/24/07 9:30 & 127.057 & 125.306 & 121.107 & 101.628 & 105.372 & 97.188 & 110.8 & 109.056 & 74.101 \\
\hline 10/24/07 10:00 & 127.104 & 125.317 & 121.124 & 101.668 & 105.412 & 97.23 & 110.823 & 109.093 & 74.138 \\
\hline $10 / 24 / 07$ 10:30 & 127.145 & 125.324 & 121.129 & 101.689 & 105.44 & 97.256 & 110.841 & 109.122 & 74.165 \\
\hline 10/24/07 11:00 & 127.187 & 125.331 & 121.139 & 101.701 & 105.454 & 97.272 & 110.858 & 109.14 & 74.181 \\
\hline 10/24/07 11:30 & 127.22 & 123.728 & 120.504 & 101.711 & 105.461 & 97.28 & 110.868 & 109.144 & 74.192 \\
\hline $10 / 24 / 0712: 00$ & 127.317 & 123.249 & 119.698 & 101.715 & 105.467 & 97.284 & 110.879 & 109.153 & 74.202 \\
\hline 10/24/07 12:30 & 126.263 & 123.065 & 119.486 & 101.136 & 105.229 & 96.935 & 110.518 & 108.195 & 74.204 \\
\hline 10/24/07 13:00 & 125.721 & 122.952 & 119.368 & 100.824 & 104.804 & 96.485 & 110.113 & 107.53 & 74.059 \\
\hline $10 / 24 / 07$ 13:30 & 125.494 & 122.877 & 119.287 & 100.674 & 104.624 & 96.304 & 109.963 & 107.327 & 73.927 \\
\hline $10 / 24 / 07$ 14:00 & 125.362 & 122.823 & 119.222 & 100.577 & 104.517 & 96.197 & 109.878 & 107.207 & 73.834 \\
\hline $10 / 24 / 0714: 30$ & 125.275 & 122.78 & 119.184 & 100.515 & 104.445 & 96.127 & 109.824 & 107.13 & 73.77 \\
\hline $10 / 24 / 0715: 00$ & 125.213 & 122.746 & 119.149 & 100.465 & 104.391 & 96.073 & 109.786 & 107.07 & 73.72 \\
\hline 10/24/07 15:30 & 125.168 & 122.716 & 119.121 & 100.428 & 104.348 & 96.03 & 109.762 & 107.023 & 73.682 \\
\hline 10/24/07 16:00 & 125.13 & 122.694 & 119.098 & 100.4 & 104.325 & 96.004 & 109.74 & 106.995 & 73.656 \\
\hline $10 / 24 / 0716: 30$ & 125.102 & 122.678 & 119.081 & 100.372 & 104.294 & 95.976 & 109.724 & 106.964 & 73.632 \\
\hline $10 / 24 / 0717: 00$ & 125.078 & 122.673 & 119.076 & 100.353 & 104.275 & 95.952 & 109.713 & 106.942 & 73.611 \\
\hline 10/24/07 17:30 & 125.057 & 122.671 & 119.063 & 100.343 & 104.258 & 95.938 & 109.702 & 106.924 & 73.598 \\
\hline $10 / 24 / 0718: 00$ & 125.045 & 122.664 & 119.06 & 100.34 & 104.252 & 95.934 & 109.692 & 106.919 & 73.592 \\
\hline $10 / 24 / 0718: 30$ & 125.041 & 122.66 & 119.058 & 100.334 & 104.249 & 95.929 & 109.685 & 106.915 & 73.588 \\
\hline 10/24/07 19:00 & 125.034 & 122.657 & 119.058 & 100.328 & 104.241 & 95.922 & 109.677 & 106.906 & 73.58 \\
\hline $10 / 24 / 07$ 19:30 & 125.029 & 122.655 & 119.053 & 100.331 & 104.243 & 95.922 & 109.672 & 106.906 & 73.582 \\
\hline $10 / 24 / 0720: 00$ & 125.026 & 122.648 & 119.048 & 100.324 & 104.239 & 95.919 & 109.666 & 106.904 & 73.578 \\
\hline 10/24/07 20:30 & 125.019 & 122.644 & 119.045 & 100.321 & 104.235 & 95.912 & 109.66 & 106.898 & 73.576 \\
\hline 10/24/07 21:00 & 125.012 & 122.646 & 119.048 & 100.316 & 104.232 & 95.907 & 109.657 & 106.893 & 73.572 \\
\hline $10 / 24 / 0721: 30$ & 125.008 & 122.637 & 119.043 & 100.314 & 104.228 & 95.905 & 109.651 & 106.888 & 73.567 \\
\hline $10 / 24 / 0722: 00$ & 125.003 & 122.63 & 119.03 & 100.312 & 104.231 & 95.905 & 109.649 & 106.891 & 73.569 \\
\hline $10 / 24 / 0722: 30$ & 125 & 122.623 & 119.023 & 100.305 & 104.222 & 95.901 & 109.644 & 106.884 & 73.564 \\
\hline $10 / 24 / 0723: 00$ & 124.996 & 122.621 & 119.023 & 100.297 & 104.213 & 95.891 & 109.642 & 106.875 & 73.555 \\
\hline 10/24/07 23:30 & 124.991 & 122.612 & 119.013 & 100.293 & 104.205 & 95.884 & 109.636 & 106.867 & 73.549 \\
\hline 10/25/07 0:00 & 124.984 & 122.601 & 119.008 & 100.291 & 104.207 & 95.884 & 109.634 & 106.867 & 73.549 \\
\hline $10 / 25 / 070: 30$ & 124.982 & 122.596 & 118.995 & 100.281 & 104.197 & 95.875 & 109.63 & 106.857 & 73.541 \\
\hline 10/25/07 1:00 & 124.974 & 122.587 & 118.987 & 100.271 & 104.188 & 95.865 & 109.627 & 106.849 & 73.536 \\
\hline $10 / 25 / 071: 30$ & 124.967 & 122.578 & 118.982 & 100.266 & 104.182 & 95.858 & 109.621 & 106.84 & 73.528 \\
\hline $10 / 25 / 072: 00$ & 124.965 & 122.567 & 118.967 & 100.255 & 104.174 & 95.849 & 109.619 & 106.831 & 73.522 \\
\hline $10 / 25 / 072: 30$ & 124.963 & 122.555 & 118.957 & 100.25 & 104.165 & 95.842 & 109.615 & 106.825 & 73.516 \\
\hline $10 / 25 / 073: 00$ & 124.956 & 122.548 & 118.947 & 100.236 & 104.157 & 95.832 & 109.612 & 106.813 & 73.505 \\
\hline 10/25/07 3:30 & 124.951 & 122.542 & 118.945 & 100.226 & 104.142 & 95.818 & 109.608 & 106.8 & 73.493 \\
\hline $10 / 25 / 074: 00$ & 124.944 & 122.537 & 118.937 & 100.219 & 104.136 & 95.811 & 109.604 & 106.791 & 73.489 \\
\hline $10 / 25 / 074: 30$ & 124.939 & 122.535 & 118.934 & 100.214 & 104.129 & 95.806 & 109.602 & 106.787 & 73.483 \\
\hline 10/25/07 5:00 & 124.937 & 122.53 & 118.927 & 100.209 & 104.123 & 95.801 & 109.598 & 106.78 & 73.476 \\
\hline $10 / 25 / 075: 30$ & 124.927 & 122.528 & 118.927 & 100.207 & 104.123 & 95.799 & 109.595 & 106.78 & 73.476 \\
\hline $10 / 25 / 076: 00$ & 124.922 & 124.29 & 120.061 & 100.204 & 104.119 & 95.797 & 109.593 & 106.776 & 73.472 \\
\hline 10/25/07 6:30 & 124.92 & 124.657 & 120.56 & 100.2 & 104.117 & 95.792 & 109.591 & 106.771 & 73.466 \\
\hline $10 / 25 / 077: 00$ & 126.386 & 124.816 & 120.741 & 100.898 & 104.49 & 96.308 & 110.113 & 107.987 & 73.479 \\
\hline 10/25/07 7:30 & 126.731 & 124.918 & 120.852 & 101.16 & 104.843 & 96.669 & 110.453 & 108.436 & 73.646 \\
\hline 10/25/07 8:00 & 126.877 & 123.292 & 119.917 & 101.298 & 105.003 & 96.829 & 110.603 & 108.611 & 73.777 \\
\hline 10/25/07 8:30 & 126.972 & 122.927 & 119.363 & 101.386 & 105.102 & 96.928 & 110.693 & 108.719 & 73.863 \\
\hline 10/25/07 9:00 & 125.634 & 122.784 & 119.197 & 100.765 & 104.825 & 96.513 & 110.261 & 107.67 & 73.909 \\
\hline 10/25/07 9:30 & 125.301 & 122.703 & 119.113 & 100.522 & 104.49 & 96.165 & 109.931 & 107.196 & 73.795 \\
\hline $10 / 25 / 0710: 00$ & 125.19 & 122.655 & 119.058 & 100.41 & 104.355 & 96.028 & 109.805 & 107.041 & 73.696 \\
\hline 10/25/07 10:30 & 125.133 & 122.617 & 119.018 & 100.345 & 104.279 & 95.955 & 109.737 & 106.96 & 73.629 \\
\hline 10/25/07 11:00 & 125.102 & 122.58 & 118.982 & 100.302 & 104.228 & 95.903 & 109.698 & 106.9 & 73.582 \\
\hline $10 / 25 / 0711: 30$ & 125.086 & 122.548 & 118.955 & 100.266 & 104.19 & 95.863 & 109.672 & 106.86 & 73.545 \\
\hline 10/25/07 12:00 & 125.067 & 122.517 & 118.922 & 100.238 & 104.159 & 95.835 & 109.655 & 106.827 & 73.516 \\
\hline 10/25/07 12:30 & 125.05 & 122.48 & 118.887 & 100.205 & 104.129 & 95.806 & 109.642 & 106.794 & 73.489 \\
\hline $10 / 25 / 07$ 13:00 & 125.024 & 122.453 & 118.859 & 100.174 & 104.096 & 95.773 & 109.632 & 106.76 & 73.458 \\
\hline 10/25/07 13:30 & 124.97 & 122.426 & 118.831 & 100.136 & 104.06 & 95.738 & 109.627 & 106.727 & 73.427 \\
\hline 10/25/07 14:00 & 124.92 & 122.403 & 118.808 & 100.112 & 104.035 & 95.712 & 109.615 & 106.698 & 73.404 \\
\hline $10 / 25 / 07$ 14:30 & 124.882 & 122.381 & 118.783 & 100.088 & 104.011 & 95.686 & 109.604 & 106.672 & 73.382 \\
\hline 10/25/07 15:00 & 124.847 & 122.372 & 118.776 & 100.062 & 103.988 & 95.667 & 109.595 & 106.649 & 73.363 \\
\hline $10 / 25 / 07$ 15:30 & 124.814 & 122.363 & 118.763 & 100.045 & 103.963 & 95.641 & 109.58 & 106.625 & 73.342 \\
\hline $10 / 25 / 07$ 16:00 & 124.788 & 122.356 & 118.758 & 100.036 & 103.957 & 95.637 & 109.572 & 106.618 & 73.334 \\
\hline $10 / 25 / 0716: 30$ & 124.762 & 122.349 & 118.746 & 100.031 & 103.95 & 95.627 & 109.563 & 106.605 & 73.323 \\
\hline 10/25/07 17:00 & 124.745 & 122.338 & 118.738 & 100.023 & 103.942 & 95.618 & 109.557 & 106.598 & 73.317 \\
\hline
\end{tabular}


TABLE S1.1 (Cont.)

Depth from Top of Casing (ft)

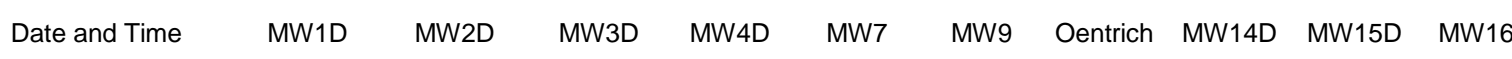

\begin{tabular}{|c|c|c|c|c|c|c|c|c|c|}
\hline $10 / 25 / 0717: 30$ & 124.733 & 122.333 & 118.733 & 100.016 & 103.933 & 95.611 & 109.552 & 106.59 & 73.307 \\
\hline $10 / 25 / 0718: 00$ & 124.722 & 122.331 & 118.73 & 100.004 & 103.923 & 95.599 & 109.548 & 106.579 & 73.297 \\
\hline $10 / 25 / 0718: 30$ & 124.71 & 122.333 & 118.725 & 100.002 & 103.917 & 95.592 & 109.542 & 106.572 & 73.29 \\
\hline $10 / 25 / 07$ 19:00 & 124.7 & 122.338 & 118.733 & 99.999 & 103.919 & 95.589 & 109.54 & 106.572 & 73.29 \\
\hline $10 / 25 / 07$ 19:30 & 124.698 & 122.338 & 118.735 & 100.004 & 103.919 & 95.592 & 109.537 & 106.574 & 73.29 \\
\hline $10 / 25 / 0720: 00$ & 124.693 & 122.338 & 118.735 & 100.009 & 103.925 & 95.601 & 109.533 & 106.579 & 73.297 \\
\hline $10 / 25 / 0720: 30$ & 124.691 & 122.338 & 118.738 & 100.011 & 103.927 & 95.599 & 109.533 & 106.579 & 73.297 \\
\hline $10 / 25 / 0721: 00$ & 124.691 & 122.342 & 118.74 & 100.007 & 103.923 & 95.599 & 109.531 & 106.579 & 73.292 \\
\hline $10 / 25 / 0721: 30$ & 124.686 & 122.349 & 118.746 & 100.009 & 103.927 & 95.599 & 109.531 & 106.579 & 73.295 \\
\hline $10 / 25 / 0722: 00$ & 124.686 & 122.344 & 118.746 & 100.014 & 103.931 & 95.604 & 109.529 & 106.587 & 73.297 \\
\hline $10 / 25 / 0722: 30$ & 124.686 & 122.34 & 118.74 & 100.017 & 103.931 & 95.604 & 109.529 & 106.587 & 73.299 \\
\hline $10 / 25 / 0723: 00$ & 124.686 & 122.338 & 118.74 & 100.016 & 103.934 & 95.604 & 109.531 & 106.585 & 73.299 \\
\hline $10 / 25 / 0723: 30$ & 124.681 & 122.333 & 118.733 & 100.011 & 103.929 & 95.601 & 109.527 & 106.583 & 73.295 \\
\hline $10 / 26 / 070: 00$ & 124.681 & 122.326 & 118.725 & 100.005 & 103.925 & 95.599 & 109.527 & 106.581 & 73.29 \\
\hline 10/26/07 0:30 & 124.677 & 122.324 & 118.725 & 100.004 & 103.921 & 95.592 & 109.527 & 106.576 & 73.288 \\
\hline 10/26/07 1:00 & 124.677 & 122.322 & 118.725 & 99.999 & 103.914 & 95.587 & 109.525 & 106.57 & 73.28 \\
\hline $10 / 26 / 071: 30$ & 124.674 & 122.324 & 118.725 & 99.997 & 103.913 & 95.587 & 109.521 & 106.567 & 73.282 \\
\hline 10/26/07 2:00 & 124.672 & 122.322 & 118.718 & 99.995 & 103.908 & 95.582 & 109.521 & 106.563 & 73.276 \\
\hline 10/26/07 2:30 & 124.667 & 122.317 & 118.72 & 99.999 & 103.914 & 95.587 & 109.52 & 106.565 & 73.282 \\
\hline 10/26/07 3:00 & 124.667 & 122.313 & 118.718 & 99.993 & 103.912 & 95.585 & 109.518 & 106.565 & 73.278 \\
\hline 10/26/07 3:30 & 124.665 & 122.308 & 118.71 & 99.992 & 103.908 & 95.582 & 109.516 & 106.559 & 73.272 \\
\hline $10 / 26 / 074: 00$ & 124.665 & 122.306 & 118.71 & 99.986 & 103.906 & 95.58 & 109.514 & 106.557 & 73.268 \\
\hline $10 / 26 / 074: 30$ & 124.662 & 122.306 & 118.708 & 99.985 & 103.898 & 95.573 & 109.512 & 106.55 & 73.261 \\
\hline $10 / 26 / 075: 00$ & 124.662 & 122.306 & 118.705 & 99.981 & 103.898 & 95.573 & 109.512 & 106.55 & 73.259 \\
\hline $10 / 26 / 075: 30$ & 124.655 & 122.31 & 118.708 & 99.985 & 103.902 & 95.573 & 109.51 & 106.55 & 73.261 \\
\hline $10 / 26 / 076: 00$ & 124.66 & 123.997 & 119.431 & 99.981 & 103.898 & 95.575 & 109.508 & 106.55 & 73.261 \\
\hline $10 / 26 / 076: 30$ & 124.658 & 124.462 & 120.202 & 99.985 & 103.9 & 95.575 & 109.505 & 106.55 & 73.259 \\
\hline 10/26/07 7:00 & 126.019 & 124.644 & 120.416 & 100.586 & 104.154 & 95.945 & 109.884 & 107.601 & 73.264 \\
\hline $10 / 26 / 077: 30$ & 126.457 & 124.75 & 120.535 & 100.894 & 104.584 & 96.396 & 110.301 & 108.232 & 73.425 \\
\hline 10/26/07 8:00 & 126.617 & 124.825 & 120.618 & 101.043 & 104.765 & 96.577 & 110.462 & 108.434 & 73.566 \\
\hline 10/26/07 8:30 & 126.712 & 124.884 & 120.673 & 101.143 & 104.873 & 96.688 & 110.554 & 108.551 & 73.663 \\
\hline 10/26/07 9:00 & 126.783 & 124.914 & 120.711 & 101.211 & 104.949 & 96.763 & 110.614 & 108.631 & 73.733 \\
\hline 10/26/07 9:30 & 126.839 & 124.941 & 120.739 & 101.263 & 105.01 & 96.82 & 110.656 & 108.693 & 73.784 \\
\hline $10 / 26 / 0710: 00$ & 126.88 & 124.957 & 120.761 & 101.292 & 105.041 & 96.851 & 110.684 & 108.724 & 73.815 \\
\hline $10 / 26 / 07 \quad 10: 30$ & 126.92 & 124.97 & 120.777 & 101.318 & 105.069 & 96.881 & 110.706 & 108.755 & 73.844 \\
\hline $10 / 26 / 0711: 00$ & 126.951 & 124.984 & 120.782 & 101.337 & 105.088 & 96.902 & 110.723 & 108.772 & 73.863 \\
\hline 10/26/07 11:30 & 126.981 & 124.991 & 120.794 & 101.351 & 105.105 & 96.919 & 110.736 & 108.788 & 73.882 \\
\hline $10 / 26 / 0712: 00$ & 127.007 & 123.213 & 119.761 & 101.361 & 105.113 & 96.928 & 110.748 & 108.797 & 73.892 \\
\hline $10 / 26 / 0712: 30$ & 127.031 & 122.87 & 119.307 & 101.37 & 105.126 & 96.94 & 110.757 & 108.806 & 73.906 \\
\hline $10 / 26 / 07$ 13:00 & 125.591 & 122.721 & 119.136 & 100.7 & 104.745 & 96.433 & 110.254 & 107.551 & 73.884 \\
\hline $10 / 26 / 0713: 30$ & 125.296 & 122.628 & 119.03 & 100.455 & 104.426 & 96.103 & 109.953 & 107.139 & 73.737 \\
\hline $10 / 26 / 0714: 00$ & 125.171 & 122.569 & 118.972 & 100.331 & 104.279 & 95.955 & 109.824 & 106.973 & 73.621 \\
\hline $10 / 26 / 0714: 30$ & 125.078 & 122.539 & 118.94 & 100.257 & 104.192 & 95.87 & 109.749 & 106.875 & 73.543 \\
\hline $10 / 26 / 0715: 00$ & 125.005 & 122.512 & 118.919 & 100.212 & 104.138 & 95.816 & 109.7 & 106.813 & 73.491 \\
\hline $10 / 26 / 0715: 30$ & 124.944 & 122.499 & 118.899 & 100.19 & 104.112 & 95.787 & 109.664 & 106.782 & 73.464 \\
\hline $10 / 26 / 0716: 00$ & 124.904 & 122.492 & 118.892 & 100.169 & 104.089 & 95.766 & 109.642 & 106.758 & 73.443 \\
\hline $10 / 26 / 0716: 30$ & 124.875 & 122.48 & 118.884 & 100.157 & 104.077 & 95.754 & 109.623 & 106.743 & 73.427 \\
\hline $10 / 26 / 0717: 00$ & 124.859 & 122.483 & 118.887 & 100.152 & 104.066 & 95.747 & 109.61 & 106.731 & 73.419 \\
\hline $10 / 26 / 07$ 17:30 & 124.849 & 122.48 & 118.884 & 100.176 & 104.062 & 95.74 & 109.6 & 106.723 & 73.41 \\
\hline $10 / 26 / 0718: 00$ & 124.847 & 122.49 & 118.884 & 100.15 & 104.064 & 95.743 & 109.602 & 106.727 & 73.408 \\
\hline $10 / 26 / 07$ 18:30 & 124.844 & 122.494 & 118.897 & 100.15 & 104.064 & 95.743 & 109.584 & 106.723 & 73.41 \\
\hline $10 / 26 / 07$ 19:00 & 124.844 & 122.499 & 118.897 & 100.16 & 104.072 & 95.752 & 109.58 & 106.731 & 73.417 \\
\hline $10 / 26 / 0719: 30$ & 124.847 & 122.503 & 118.892 & 100.166 & 104.079 & 95.759 & 109.569 & 106.738 & 73.421 \\
\hline $10 / 26 / 0720: 00$ & 124.847 & 122.508 & 118.892 & 100.171 & 104.081 & 95.762 & 109.561 & 106.74 & 73.423 \\
\hline $10 / 26 / 0720: 30$ & 124.844 & 122.517 & 118.902 & 100.173 & 104.087 & 95.766 & 109.557 & 106.747 & 73.429 \\
\hline $10 / 26 / 0721: 00$ & 124.84 & 122.521 & 118.922 & 100.181 & 104.093 & 95.773 & 109.552 & 106.749 & 73.433 \\
\hline $10 / 26 / 0721: 30$ & 124.835 & 122.528 & 118.929 & 100.19 & 104.1 & 95.778 & 109.548 & 106.758 & 73.439 \\
\hline $10 / 26 / 0722: 00$ & 124.835 & 122.539 & 118.942 & 100.195 & 104.11 & 95.785 & 109.544 & 106.764 & 73.445 \\
\hline $10 / 26 / 0722: 30$ & 124.83 & 122.553 & 118.952 & 100.204 & 104.117 & 95.792 & 109.546 & 106.771 & 73.45 \\
\hline $10 / 26 / 0723: 00$ & 124.83 & 122.562 & 118.965 & 100.216 & 104.125 & 95.802 & 109.544 & 106.78 & 73.458 \\
\hline $10 / 26 / 0723: 30$ & 124.83 & 122.569 & 118.965 & 100.229 & 104.138 & 95.816 & 109.544 & 106.793 & 73.472 \\
\hline $10 / 27 / 070: 00$ & 124.826 & 122.578 & 118.98 & 100.238 & 104.151 & 95.828 & 109.548 & 106.807 & 73.483 \\
\hline $10 / 27 / 070: 30$ & 124.823 & 122.582 & 118.985 & 100.245 & 104.155 & 95.835 & 109.55 & 106.811 & 73.489 \\
\hline 10/27/07 1:00 & 124.823 & 122.58 & 118.98 & 100.252 & 104.165 & 95.844 & 109.552 & 106.82 & 73.497 \\
\hline $10 / 27 / 071: 30$ & 124.823 & 122.587 & 118.97 & 100.255 & 104.169 & 95.846 & 109.552 & 106.825 & 73.499 \\
\hline $10 / 27 / 072: 00$ & 124.823 & 122.594 & 118.992 & 100.255 & 104.169 & 95.844 & 109.548 & 106.822 & 73.497 \\
\hline
\end{tabular}


TABLE S1.1 (Cont.)

Depth from Top of Casing (ft)

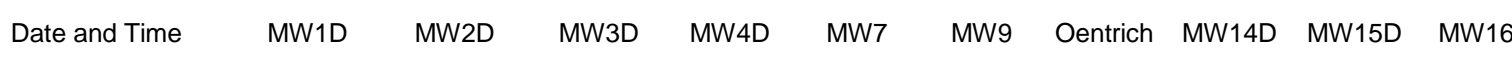

\begin{tabular}{|c|c|c|c|c|c|c|c|c|c|}
\hline 10/27/07 2:30 & 124.823 & 122.596 & 118.985 & 100.264 & 104.174 & 95.853 & 109.548 & 106.829 & 73.505 \\
\hline 10/27/07 3:00 & 124.821 & 122.598 & 119 & 100.271 & 104.184 & 95.863 & 109.544 & 106.833 & 73.512 \\
\hline $10 / 27 / 073: 30$ & 124.821 & 122.603 & 118.987 & 100.272 & 104.188 & 95.865 & 109.54 & 106.84 & 73.516 \\
\hline $10 / 27 / 074: 00$ & 124.823 & 122.612 & 119.013 & 100.272 & 104.188 & 95.868 & 109.538 & 106.842 & 73.52 \\
\hline $10 / 27 / 074: 30$ & 124.823 & 122.623 & 119.02 & 100.285 & 104.19 & 95.868 & 109.535 & 106.844 & 73.52 \\
\hline $10 / 27 / 075: 00$ & 124.826 & 122.637 & 119.035 & 100.29 & 104.203 & 95.879 & 109.531 & 106.851 & 73.526 \\
\hline $10 / 27 / 075: 30$ & 124.826 & 122.646 & 119.048 & 100.303 & 104.212 & 95.891 & 109.531 & 106.867 & 73.536 \\
\hline $10 / 27 / 076: 00$ & 124.823 & 122.664 & 119.066 & 100.317 & 104.23 & 95.905 & 109.58 & 106.877 & 73.551 \\
\hline $10 / 27 / 076: 30$ & 124.823 & 122.671 & 119.071 & 100.331 & 104.237 & 95.917 & 109.582 & 106.888 & 73.559 \\
\hline 10/27/07 7:00 & 124.826 & 124.703 & 120.583 & 100.343 & 104.256 & 95.934 & 109.576 & 106.908 & 73.576 \\
\hline $10 / 27 / 07 \quad 7: 30$ & 124.828 & 124.943 & 120.847 & 100.764 & 104.264 & 95.941 & 109.576 & 106.913 & 73.582 \\
\hline $10 / 27 / 078: 00$ & 126.539 & 125.081 & 121.006 & 101.244 & 104.894 & 96.721 & 110.378 & 108.463 & 73.702 \\
\hline $10 / 27 / 078: 30$ & 126.747 & 125.168 & 121.104 & 101.439 & 105.13 & 96.956 & 110.58 & 108.728 & 73.871 \\
\hline $10 / 27 / 079: 00$ & 126.87 & 125.238 & 121.167 & 101.56 & 105.263 & 97.093 & 110.691 & 108.877 & 73.987 \\
\hline 10/27/07 9:30 & 126.955 & 125.272 & 121.213 & 101.637 & 105.355 & 97.181 & 110.811 & 108.97 & 74.07 \\
\hline $10 / 27 / 0710: 00$ & 127.026 & 125.308 & 121.245 & 101.69 & 105.414 & 97.244 & 110.856 & 109.038 & 74.124 \\
\hline $10 / 27 / 07 \quad 10: 30$ & 127.083 & 125.329 & 121.273 & 101.728 & 105.456 & 97.284 & 110.888 & 109.08 & 74.165 \\
\hline $10 / 27 / 0711: 00$ & 127.133 & 125.344 & 121.296 & 101.761 & 105.488 & 97.317 & 110.911 & 109.114 & 74.194 \\
\hline $10 / 27 / 0711: 30$ & 127.175 & 123.87 & 121.071 & 101.78 & 105.511 & 97.341 & 110.932 & 109.14 & 74.219 \\
\hline $10 / 27 / 07 \quad 12: 00$ & 127.213 & 123.328 & 119.779 & 101.796 & 105.526 & 97.36 & 110.948 & 109.158 & 74.239 \\
\hline $10 / 27 / 07 \quad 12: 30$ & 126.074 & 123.143 & 119.562 & 101.222 & 105.345 & 97.07 & 110.676 & 108.427 & 74.25 \\
\hline 10/27/07 13:00 & 125.606 & 123.031 & 119.441 & 100.898 & 104.879 & 96.561 & 110.205 & 107.611 & 74.124 \\
\hline 10/27/07 13:30 & 125.494 & 122.963 & 119.365 & 100.746 & 104.7 & 96.379 & 110.043 & 107.402 & 73.997 \\
\hline $10 / 27 / 0714: 00$ & 125.414 & 122.916 & 119.323 & 100.655 & 104.593 & 96.273 & 109.951 & 107.283 & 73.909 \\
\hline $10 / 27 / 0714: 30$ & 125.355 & 122.886 & 119.292 & 100.598 & 104.527 & 96.209 & 109.899 & 107.216 & 73.847 \\
\hline $10 / 27 / 07$ 15:00 & 125.303 & 122.866 & 119.267 & 100.562 & 104.485 & 96.165 & 109.863 & 107.168 & 73.807 \\
\hline 10/27/07 15:30 & 125.268 & 122.848 & 119.249 & 100.536 & 104.456 & 96.141 & 109.833 & 107.134 & 73.78 \\
\hline $10 / 27 / 0716: 00$ & 125.244 & 122.832 & 119.234 & 100.517 & 104.437 & 96.12 & 109.814 & 107.11 & 73.764 \\
\hline 10/27/07 16:30 & 125.22 & 122.821 & 119.222 & 100.507 & 104.422 & 96.106 & 109.794 & 107.094 & 73.749 \\
\hline $10 / 27 / 07 \quad 17: 00$ & 125.206 & 122.814 & 119.217 & 100.491 & 104.411 & 96.092 & 109.782 & 107.077 & 73.737 \\
\hline 10/27/07 17:30 & 125.194 & 122.809 & 119.209 & 100.483 & 104.399 & 96.082 & 109.769 & 107.066 & 73.731 \\
\hline $10 / 27 / 07$ 18:00 & 125.182 & 122.809 & 119.212 & 100.479 & 104.393 & 96.075 & 109.762 & 107.057 & 73.725 \\
\hline $10 / 27 / 07$ 18:30 & 125.18 & 122.812 & 119.212 & 100.476 & 104.39 & 96.07 & 109.749 & 107.053 & 73.725 \\
\hline 10/27/07 19:00 & 125.175 & 122.812 & 119.212 & 100.477 & 104.388 & 96.068 & 109.743 & 107.05 & 73.72 \\
\hline 10/27/07 19:30 & 125.173 & 122.807 & 119.212 & 100.479 & 104.392 & 96.07 & 109.731 & 107.052 & 73.722 \\
\hline $10 / 27 / 0720: 00$ & 125.168 & 122.805 & 119.209 & 100.479 & 104.392 & 96.07 & 109.723 & 107.053 & 73.725 \\
\hline $10 / 27 / 0720: 30$ & 125.164 & 122.812 & 119.212 & 100.479 & 104.389 & 96.068 & 109.719 & 107.053 & 73.722 \\
\hline 10/27/07 21:00 & 125.156 & 122.818 & 119.217 & 100.476 & 104.388 & 96.068 & 109.713 & 107.05 & 73.722 \\
\hline $10 / 27 / 0721: 30$ & 125.152 & 122.818 & 119.222 & 100.483 & 104.391 & 96.075 & 109.707 & 107.054 & 73.725 \\
\hline $10 / 27 / 0722: 00$ & 125.147 & 122.818 & 119.219 & 100.49 & 104.397 & 96.077 & 109.706 & 107.061 & 73.733 \\
\hline 10/27/07 22:30 & 125.142 & 122.818 & 119.222 & 100.488 & 104.399 & 96.08 & 109.751 & 107.061 & 73.733 \\
\hline $10 / 27 / 0723: 00$ & 125.14 & 122.814 & 119.219 & 100.49 & 104.399 & 96.077 & 109.747 & 107.061 & 73.735 \\
\hline 10/27/07 23:30 & 125.138 & 122.816 & 119.217 & 100.486 & 104.401 & 96.082 & 109.741 & 107.061 & 73.735 \\
\hline 10/28/07 0:00 & 125.135 & 122.816 & 119.214 & 100.483 & 104.395 & 96.077 & 109.737 & 107.059 & 73.733 \\
\hline $10 / 28 / 070: 30$ & 125.133 & 122.816 & 119.214 & 100.484 & 104.397 & 96.077 & 109.734 & 107.059 & 73.735 \\
\hline $10 / 28 / 071: 00$ & 125.128 & 122.809 & 119.212 & 100.486 & 104.397 & 96.077 & 109.732 & 107.059 & 73.733 \\
\hline $10 / 28 / 071: 30$ & 125.123 & 122.812 & 119.209 & 100.484 & 104.397 & 96.077 & 109.726 & 107.059 & 73.739 \\
\hline $10 / 28 / 072: 00$ & 125.121 & 122.807 & 119.212 & 100.479 & 104.393 & 96.073 & 109.722 & 107.054 & 73.735 \\
\hline $10 / 28 / 072: 30$ & 125.119 & 122.798 & 119.202 & 100.479 & 104.391 & 96.07 & 109.719 & 107.05 & 73.731 \\
\hline $10 / 28 / 073: 00$ & 125.116 & 122.791 & 119.186 & 100.476 & 104.389 & 96.07 & 109.713 & 107.05 & 73.733 \\
\hline $10 / 28 / 073: 30$ & 125.112 & 122.787 & 119.189 & 100.462 & 104.38 & 96.059 & 109.711 & 107.042 & 73.727 \\
\hline $10 / 28 / 074: 00$ & 125.109 & 122.784 & 119.179 & 100.46 & 104.371 & 96.051 & 109.705 & 107.03 & 73.716 \\
\hline $10 / 28 / 074: 30$ & 125.104 & 122.78 & 119.176 & 100.457 & 104.371 & 96.051 & 109.702 & 107.03 & 73.718 \\
\hline $10 / 28 / 075: 00$ & 125.102 & 122.782 & 119.181 & 100.455 & 104.367 & 96.047 & 109.698 & 107.026 & 73.716 \\
\hline $10 / 28 / 075: 30$ & 125.097 & 122.78 & 119.181 & 100.455 & 104.367 & 96.047 & 109.694 & 107.023 & 73.714 \\
\hline $10 / 28 / 076: 00$ & 125.093 & 122.773 & 119.174 & 100.457 & 104.367 & 96.049 & 109.689 & 107.026 & 73.718 \\
\hline $10 / 28 / 076: 30$ & 125.093 & 122.78 & 119.179 & 100.452 & 104.365 & 96.044 & 109.687 & 107.024 & 73.715 \\
\hline $10 / 28 / 077: 00$ & 125.09 & 122.775 & 119.176 & 100.448 & 104.359 & 96.04 & 109.685 & 107.017 & 73.708 \\
\hline $10 / 28 / 077: 30$ & 125.088 & 124.696 & 120.535 & 100.448 & 104.365 & 96.042 & 109.679 & 107.022 & 73.714 \\
\hline $10 / 28 / 078: 00$ & 125.09 & 124.979 & 120.867 & 100.446 & 104.359 & 96.037 & 109.679 & 107.017 & 73.708 \\
\hline $10 / 28 / 078: 30$ & 126.7 & 125.127 & 121.049 & 101.255 & 104.879 & 96.704 & 110.338 & 108.436 & 73.768 \\
\hline $10 / 28 / 07$ 9:00 & 126.955 & 125.215 & 121.142 & 101.475 & 105.161 & 96.989 & 110.635 & 108.762 & 73.944 \\
\hline 10/28/07 9:30 & 127.095 & 125.265 & 121.202 & 101.597 & 105.305 & 97.133 & 110.766 & 108.921 & 74.066 \\
\hline $10 / 28 / 0710: 00$ & 127.189 & 123.417 & 119.912 & 101.672 & 105.387 & 97.221 & 110.845 & 109.014 & 74.143 \\
\hline $10 / 28 / 07 \quad 10: 30$ & 127.258 & 123.154 & 119.585 & 101.718 & 105.44 & 97.275 & 110.894 & 109.067 & 74.192 \\
\hline 10/28/07 11:00 & 125.726 & 123.029 & 119.446 & 100.948 & 104.961 & 96.645 & 110.271 & 107.717 & 74.165 \\
\hline
\end{tabular}


TABLE S1.1 (Cont.)

Depth from Top of Casing (ft)

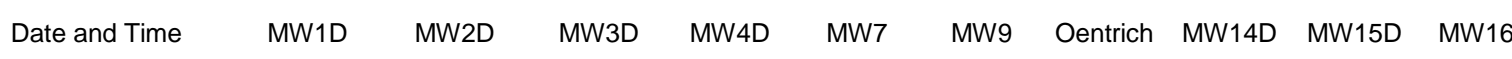

\begin{tabular}{|c|c|c|c|c|c|c|c|c|c|}
\hline 10/28/07 11:30 & 125.511 & 122.943 & 119.353 & 100.753 & 104.711 & 96.393 & 110.038 & 107.416 & 74.035 \\
\hline 10/28/07 12:00 & 125.416 & 122.877 & 119.285 & 100.645 & 104.591 & 96.271 & 109.935 & 107.283 & 73.937 \\
\hline $10 / 28 / 07$ 12:30 & 125.364 & 122.814 & 119.224 & 100.577 & 104.513 & 96.191 & 109.873 & 107.192 & 73.865 \\
\hline 10/28/07 13:00 & 125.305 & 122.782 & 119.184 & 100.517 & 104.443 & 96.125 & 109.833 & 107.123 & 73.807 \\
\hline $10 / 28 / 07$ 13:30 & 125.232 & 122.755 & 119.159 & 100.462 & 104.386 & 96.066 & 109.811 & 107.061 & 73.751 \\
\hline $10 / 28 / 07$ 14:00 & 125.175 & 122.721 & 119.126 & 100.431 & 104.353 & 96.035 & 109.788 & 107.023 & 73.722 \\
\hline $10 / 28 / 07$ 14:30 & 125.145 & 122.694 & 119.098 & 100.403 & 104.329 & 96.011 & 109.775 & 106.999 & 73.7 \\
\hline $10 / 28 / 07$ 15:00 & 125.112 & 122.678 & 119.081 & 100.369 & 104.292 & 95.974 & 109.767 & 106.962 & 73.669 \\
\hline $10 / 28 / 07$ 15:30 & 125.081 & 122.669 & 119.068 & 100.353 & 104.269 & 95.95 & 109.756 & 106.937 & 73.646 \\
\hline $10 / 28 / 07 \quad 16: 00$ & 125.06 & 122.653 & 119.055 & 100.338 & 104.254 & 95.933 & 109.747 & 106.92 & 73.634 \\
\hline $10 / 28 / 07$ 16:30 & 125.043 & 122.637 & 119.035 & 100.329 & 104.245 & 95.924 & 109.741 & 106.909 & 73.623 \\
\hline $10 / 28 / 07$ 17:00 & 125.031 & 122.628 & 119.015 & 100.314 & 104.232 & 95.912 & 109.736 & 106.895 & 73.615 \\
\hline $10 / 28 / 07$ 17:30 & 125.017 & 122.621 & 119.008 & 100.302 & 104.216 & 95.894 & 109.73 & 106.878 & 73.598 \\
\hline $10 / 28 / 07$ 18:00 & 125.008 & 122.614 & 119 & 100.291 & 104.207 & 95.889 & 109.726 & 106.871 & 73.592 \\
\hline $10 / 28 / 0718: 30$ & 124.996 & 122.614 & 118.997 & 100.29 & 104.203 & 95.884 & 109.722 & 106.864 & 73.586 \\
\hline 10/28/07 19:00 & 124.991 & 122.617 & 119.013 & 100.283 & 104.195 & 95.875 & 109.719 & 106.858 & 73.58 \\
\hline $10 / 28 / 07$ 19:30 & 124.986 & 122.614 & 119.018 & 100.286 & 104.195 & 95.877 & 109.715 & 106.855 & 73.578 \\
\hline $10 / 28 / 0720: 00$ & 124.984 & 122.605 & 119.01 & 100.286 & 104.199 & 95.879 & 109.709 & 106.86 & 73.582 \\
\hline $10 / 28 / 0720: 30$ & 124.986 & 122.598 & 118.997 & 100.281 & 104.195 & 95.875 & 109.707 & 106.856 & 73.58 \\
\hline $10 / 28 / 0721: 00$ & 124.984 & 122.598 & 118.997 & 100.267 & 104.188 & 95.865 & 109.705 & 106.849 & 73.572 \\
\hline $10 / 28 / 0721: 30$ & 124.979 & 122.587 & 118.99 & 100.266 & 104.178 & 95.856 & 109.702 & 106.84 & 73.563 \\
\hline $10 / 28 / 0722: 00$ & 124.977 & 122.578 & 118.977 & 100.26 & 104.18 & 95.858 & 109.7 & 106.842 & 73.563 \\
\hline 10/28/07 22:30 & 124.974 & 122.576 & 118.977 & 100.252 & 104.167 & 95.846 & 109.7 & 106.829 & 73.553 \\
\hline 10/28/07 23:00 & 124.972 & 122.571 & 118.972 & 100.241 & 104.159 & 95.837 & 109.698 & 106.818 & 73.543 \\
\hline $10 / 28 / 0723: 30$ & 124.97 & 122.571 & 118.972 & 100.241 & 104.155 & 95.835 & 109.696 & 106.818 & 73.543 \\
\hline 10/29/07 0:00 & 124.965 & 122.56 & 118.96 & 100.236 & 104.152 & 95.83 & 109.692 & 106.811 & 73.541 \\
\hline 10/29/07 0:30 & 124.963 & 122.56 & 118.962 & 100.236 & 104.154 & 95.83 & 109.694 & 106.814 & 73.538 \\
\hline $10 / 29 / 071: 00$ & 124.96 & 122.553 & 118.955 & 100.228 & 104.138 & 95.816 & 109.69 & 106.8 & 73.526 \\
\hline 10/29/07 1:30 & 124.953 & 122.558 & 118.957 & 100.224 & 104.142 & 95.82 & 109.687 & 106.798 & 73.53 \\
\hline $10 / 29 / 072: 00$ & 124.951 & 122.555 & 118.957 & 100.224 & 104.136 & 95.816 & 109.683 & 106.796 & 73.526 \\
\hline $10 / 29 / 072: 30$ & 124.946 & 122.546 & 118.947 & 100.228 & 104.14 & 95.82 & 109.679 & 106.798 & 73.53 \\
\hline 10/29/07 3:00 & 124.944 & 122.537 & 118.929 & 100.222 & 104.138 & 95.818 & 109.679 & 106.798 & 73.526 \\
\hline 10/29/07 3:30 & 124.941 & 122.528 & 118.922 & 100.21 & 104.129 & 95.806 & 109.678 & 106.787 & 73.518 \\
\hline $10 / 29 / 074: 00$ & 124.939 & 122.521 & 118.919 & 100.2 & 104.119 & 95.797 & 109.675 & 106.776 & 73.507 \\
\hline 10/29/07 4:30 & 124.934 & 122.517 & 118.917 & 100.195 & 104.11 & 95.787 & 109.672 & 106.767 & 73.501 \\
\hline 10/29/07 5:00 & 124.93 & 122.503 & 118.904 & 100.188 & 104.108 & 95.783 & 109.67 & 106.763 & 73.497 \\
\hline $10 / 29 / 075: 30$ & 124.927 & 122.499 & 118.902 & 100.181 & 104.098 & 95.778 & 109.668 & 106.756 & 73.489 \\
\hline 10/29/07 6:00 & 124.925 & 124.29 & 119.912 & 100.174 & 104.089 & 95.764 & 109.662 & 106.745 & 73.481 \\
\hline 10/29/07 6:30 & 124.918 & 124.669 & 120.421 & 100.169 & 104.085 & 95.762 & 109.66 & 106.74 & 73.476 \\
\hline $10 / 29 / 07$ 7:00 & 126.383 & 124.83 & 120.603 & 100.829 & 104.432 & 96.233 & 110.135 & 107.982 & 73.485 \\
\hline $10 / 29 / 077: 30$ & 126.733 & 124.927 & 120.711 & 101.094 & 104.789 & 96.603 & 110.479 & 108.445 & 73.656 \\
\hline 10/29/07 8:00 & 126.88 & 124.984 & 120.774 & 101.229 & 104.947 & 96.763 & 110.629 & 108.622 & 73.787 \\
\hline $10 / 29 / 078: 30$ & 126.969 & 125.025 & 120.817 & 101.313 & 105.048 & 96.862 & 110.714 & 108.726 & 73.875 \\
\hline 10/29/07 9:00 & 127.031 & 125.052 & 120.85 & 101.361 & 105.103 & 96.919 & 110.77 & 108.791 & 73.929 \\
\hline 10/29/07 9:30 & 127.083 & 125.081 & 120.872 & 101.399 & 105.145 & 96.956 & 110.808 & 108.833 & 73.964 \\
\hline 10/29/07 10:00 & 127.13 & 125.084 & 120.882 & 101.425 & 105.174 & 96.987 & 110.836 & 108.863 & 73.993 \\
\hline 10/29/07 10:30 & 127.166 & 125.081 & 120.89 & 101.444 & 105.193 & 97.008 & 110.858 & 108.885 & 74.014 \\
\hline 10/29/07 11:00 & 127.199 & 123.512 & 120.479 & 101.451 & 105.205 & 97.018 & 110.875 & 108.895 & 74.026 \\
\hline $10 / 29 / 0711: 30$ & 127.227 & 123.004 & 119.449 & 101.453 & 105.21 & 97.023 & 110.886 & 108.896 & 74.035 \\
\hline $10 / 29 / 07$ 12:00 & 126.017 & 122.823 & 119.239 & 100.881 & 105.003 & 96.711 & 110.562 & 108.035 & 74.033 \\
\hline $10 / 29 / 07$ 12:30 & 125.516 & 122.707 & 119.118 & 100.565 & 104.553 & 96.226 & 110.119 & 107.276 & 73.888 \\
\hline $10 / 29 / 07$ 13:00 & 125.277 & 122.626 & 119.038 & 100.419 & 104.372 & 96.049 & 109.959 & 107.075 & 73.756 \\
\hline $10 / 29 / 07$ 13:30 & 125.123 & 122.573 & 118.98 & 100.322 & 104.266 & 95.943 & 109.869 & 106.953 & 73.66 \\
\hline $10 / 29 / 07 \quad 14: 00$ & 125.029 & 122.53 & 118.934 & 100.257 & 104.188 & 95.865 & 109.813 & 106.871 & 73.59 \\
\hline $10 / 29 / 07$ 14:30 & 124.967 & 122.499 & 118.902 & 100.209 & 104.138 & 95.816 & 109.773 & 106.816 & 73.541 \\
\hline $10 / 29 / 07$ 15:00 & 124.922 & 122.476 & 118.877 & 100.174 & 104.096 & 95.773 & 109.743 & 106.774 & 73.501 \\
\hline $10 / 29 / 07$ 15:30 & 124.885 & 122.458 & 118.859 & 100.147 & 104.068 & 95.745 & 109.719 & 106.738 & 73.474 \\
\hline $10 / 29 / 07 \quad 16: 00$ & 124.859 & 122.437 & 118.841 & 100.126 & 104.047 & 95.724 & 109.702 & 106.718 & 73.454 \\
\hline $10 / 29 / 07 \quad 16: 30$ & 124.837 & 122.422 & 118.826 & 100.109 & 104.028 & 95.707 & 109.687 & 106.694 & 73.437 \\
\hline $10 / 29 / 07$ 17:00 & 124.818 & 122.406 & 118.808 & 100.093 & 104.011 & 95.686 & 109.647 & 106.676 & 73.419 \\
\hline $10 / 29 / 07$ 17:30 & 124.8 & 122.397 & 118.796 & 100.078 & 103.995 & 95.674 & 109.636 & 106.661 & 73.404 \\
\hline $10 / 29 / 07$ 18:00 & 124.785 & 122.392 & 118.796 & 100.064 & 103.98 & 95.66 & 109.629 & 106.645 & 73.39 \\
\hline $10 / 29 / 07$ 18:30 & 124.771 & 122.381 & 118.783 & 100.057 & 103.971 & 95.648 & 109.619 & 106.634 & 73.381 \\
\hline 10/29/07 19:00 & 124.764 & 122.383 & 118.783 & 100.054 & 103.969 & 95.651 & 109.612 & 106.632 & 73.377 \\
\hline $10 / 29 / 07$ 19:30 & 124.757 & 122.378 & 118.778 & 100.047 & 103.961 & 95.637 & 109.608 & 106.621 & 73.367 \\
\hline $10 / 29 / 0720: 00$ & 124.755 & 122.374 & 118.776 & 100.045 & 103.961 & 95.637 & 109.602 & 106.623 & 73.367 \\
\hline
\end{tabular}


TABLE S1.1 (Cont.)

Depth from Top of Casing (ft)

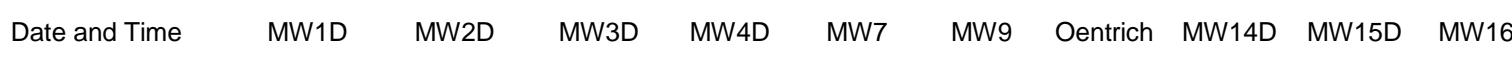

\begin{tabular}{|c|c|c|c|c|c|c|c|c|c|}
\hline $10 / 29 / 0720: 30$ & 124.75 & 122.372 & 118.771 & 100.042 & 103.957 & 95.63 & 109.6 & 106.616 & 73.363 \\
\hline $10 / 29 / 0721: 00$ & 124.745 & 122.372 & 118.773 & 100.035 & 103.952 & 95.627 & 109.597 & 106.61 & 73.359 \\
\hline $10 / 29 / 0721: 30$ & 124.743 & 122.369 & 118.771 & 100.035 & 103.948 & 95.627 & 109.597 & 106.608 & 73.352 \\
\hline $10 / 29 / 0722: 00$ & 124.74 & 122.363 & 118.763 & 100.038 & 103.95 & 95.63 & 109.593 & 106.61 & 73.354 \\
\hline 10/29/07 22:30 & 124.74 & 122.358 & 118.758 & 100.035 & 103.95 & 95.627 & 109.591 & 106.608 & 73.352 \\
\hline $10 / 29 / 0723: 00$ & 124.738 & 122.349 & 118.753 & 100.026 & 103.94 & 95.62 & 109.591 & 106.601 & 73.344 \\
\hline $10 / 29 / 0723: 30$ & 124.733 & 122.344 & 118.743 & 100.023 & 103.938 & 95.615 & 109.589 & 106.597 & 73.34 \\
\hline $10 / 30 / 070: 00$ & 124.729 & 122.335 & 118.735 & 100.011 & 103.931 & 95.608 & 109.585 & 106.588 & 73.334 \\
\hline $10 / 30 / 070: 30$ & 124.722 & 122.331 & 118.73 & 100.005 & 103.921 & 95.599 & 109.582 & 106.579 & 73.323 \\
\hline $10 / 30 / 071: 00$ & 124.719 & 122.324 & 118.725 & 99.999 & 103.914 & 95.592 & 109.58 & 106.574 & 73.317 \\
\hline 10/30/07 1:30 & 124.712 & 122.313 & 118.715 & 99.992 & 103.908 & 95.587 & 109.576 & 106.566 & 73.311 \\
\hline $10 / 30 / 072: 00$ & 124.71 & 122.304 & 118.705 & 99.988 & 103.898 & 95.578 & 109.57 & 106.557 & 73.305 \\
\hline $10 / 30 / 072: 30$ & 124.71 & 122.292 & 118.698 & 99.973 & 103.891 & 95.568 & 109.567 & 106.55 & 73.297 \\
\hline 10/30/07 3:00 & 124.705 & 122.288 & 118.69 & 99.969 & 103.883 & 95.561 & 109.561 & 106.539 & 73.288 \\
\hline $10 / 30 / 073: 30$ & 124.705 & 122.276 & 118.68 & 99.957 & 103.87 & 95.549 & 109.557 & 106.53 & 73.278 \\
\hline $10 / 30 / 074: 00$ & 124.7 & 122.27 & 118.667 & 99.952 & 103.868 & 95.545 & 109.55 & 106.526 & 73.274 \\
\hline $10 / 30 / 074: 30$ & 124.698 & 122.261 & 118.662 & 99.943 & 103.857 & 95.535 & 109.548 & 106.515 & 73.266 \\
\hline $10 / 30 / 075: 00$ & 124.696 & 122.254 & 118.657 & 99.933 & 103.849 & 95.526 & 109.54 & 106.506 & 73.257 \\
\hline 10/30/07 5:30 & 124.691 & 122.249 & 118.65 & 99.923 & 103.841 & 95.519 & 109.535 & 106.499 & 73.249 \\
\hline $10 / 30 / 076: 00$ & 124.686 & 123.582 & 118.64 & 99.921 & 103.838 & 95.514 & 109.529 & 106.49 & 73.245 \\
\hline $10 / 30 / 076: 30$ & 124.681 & 124.292 & 120.167 & 99.916 & 103.832 & 95.509 & 109.527 & 106.488 & 73.239 \\
\hline $10 / 30 / 07$ 7:00 & 125.516 & 124.494 & 120.399 & 100.44 & 103.864 & 95.556 & 109.612 & 106.506 & 73.228 \\
\hline $10 / 30 / 07$ 7:30 & 126.423 & 124.603 & 120.53 & 100.807 & 104.471 & 96.299 & 110.308 & 108.051 & 73.357 \\
\hline $10 / 30 / 07$ 8:00 & 126.603 & 124.671 & 120.61 & 100.97 & 104.673 & 96.497 & 110.488 & 108.277 & 73.507 \\
\hline $10 / 30 / 078: 30$ & 126.709 & 124.714 & 120.648 & 101.062 & 104.78 & 96.608 & 110.59 & 108.394 & 73.605 \\
\hline $10 / 30 / 07$ 9:00 & 126.778 & 124.741 & 120.681 & 101.12 & 104.847 & 96.676 & 110.652 & 108.469 & 73.667 \\
\hline 10/30/07 9:30 & 126.832 & 124.755 & 120.688 & 101.155 & 104.883 & 96.711 & 110.695 & 108.507 & 73.704 \\
\hline $10 / 30 / 0710: 00$ & 126.88 & 124.773 & 120.706 & 101.182 & 104.913 & 96.74 & 110.725 & 108.54 & 73.729 \\
\hline $10 / 30 / 0710: 30$ & 126.915 & 124.778 & 120.714 & 101.198 & 104.934 & 96.759 & 110.744 & 108.56 & 73.749 \\
\hline $10 / 30 / 0711: 00$ & 126.953 & 124.791 & 120.734 & 101.21 & 104.949 & 96.777 & 110.764 & 108.576 & 73.766 \\
\hline $10 / 30 / 0711: 30$ & 127.038 & 122.823 & 119.29 & 101.218 & 104.953 & 96.785 & 110.772 & 108.582 & 73.772 \\
\hline $10 / 30 / 0712: 00$ & 127.199 & 122.58 & 119.003 & 100.826 & 104.968 & 96.796 & 110.783 & 108.596 & 73.784 \\
\hline $10 / 30 / 0712: 30$ & 125.357 & 122.46 & 118.869 & 100.353 & 104.359 & 96.04 & 110.051 & 107.099 & 73.679 \\
\hline $10 / 30 / 0713: 00$ & 125.029 & 122.367 & 118.781 & 100.166 & 104.131 & 95.806 & 109.846 & 106.836 & 73.524 \\
\hline $10 / 30 / 07$ 13:30 & 124.861 & 122.301 & 118.71 & 100.071 & 104.011 & 95.688 & 109.737 & 106.701 & 73.421 \\
\hline $10 / 30 / 0714: 00$ & 124.769 & 122.258 & 118.665 & 99.992 & 103.925 & 95.606 & 109.659 & 106.61 & 73.342 \\
\hline $10 / 30 / 0714: 30$ & 124.696 & 122.22 & 118.627 & 99.93 & 103.86 & 95.542 & 109.602 & 106.541 & 73.282 \\
\hline $10 / 30 / 0715: 00$ & 124.644 & 122.193 & 118.599 & 99.895 & 103.824 & 95.505 & 109.565 & 106.497 & 73.243 \\
\hline $10 / 30 / 07$ 15:30 & 124.606 & 122.174 & 118.579 & 99.862 & 103.786 & 95.465 & 109.535 & 106.457 & 73.208 \\
\hline $10 / 30 / 0716: 00$ & 124.575 & 122.149 & 118.557 & 99.845 & 103.763 & 95.439 & 109.512 & 106.431 & 73.183 \\
\hline $10 / 30 / 0716: 30$ & 124.551 & 122.131 & 118.534 & 99.824 & 103.74 & 95.422 & 109.497 & 106.411 & 73.165 \\
\hline $10 / 30 / 07$ 17:00 & 124.53 & 122.12 & 118.521 & 99.802 & 103.719 & 95.401 & 109.478 & 106.386 & 73.142 \\
\hline $10 / 30 / 0717: 30$ & 124.509 & 122.113 & 118.511 & 99.783 & 103.7 & 95.377 & 109.463 & 106.366 & 73.127 \\
\hline $10 / 30 / 0718: 00$ & 124.492 & 122.111 & 118.514 & 99.775 & 103.693 & 95.373 & 109.452 & 106.355 & 73.115 \\
\hline $10 / 30 / 07$ 18:30 & 124.481 & 122.118 & 118.516 & 99.775 & 103.687 & 95.363 & 109.441 & 106.344 & 73.106 \\
\hline $10 / 30 / 07$ 19:00 & 124.481 & 122.115 & 118.521 & 99.773 & 103.689 & 95.368 & 109.435 & 106.346 & 73.109 \\
\hline $10 / 30 / 07$ 19:30 & 124.483 & 122.109 & 118.511 & 99.781 & 103.693 & 95.373 & 109.431 & 106.351 & 73.111 \\
\hline $10 / 30 / 0720: 00$ & 124.488 & 122.111 & 118.506 & 99.778 & 103.7 & 95.377 & 109.428 & 106.355 & 73.113 \\
\hline $10 / 30 / 0720: 30$ & 124.483 & 122.113 & 118.509 & 99.769 & 103.685 & 95.363 & 109.43 & 106.342 & 73.1 \\
\hline $10 / 30 / 0721: 00$ & 124.476 & 122.111 & 118.516 & 99.773 & 103.687 & 95.363 & 109.426 & 106.342 & 73.098 \\
\hline $10 / 30 / 0721: 30$ & 124.478 & 122.109 & 118.511 & 99.776 & 103.691 & 95.373 & 109.422 & 106.344 & 73.102 \\
\hline $10 / 30 / 0722: 00$ & 124.483 & 122.111 & 118.511 & 99.778 & 103.689 & 95.37 & 109.424 & 106.344 & 73.098 \\
\hline $10 / 30 / 0722: 30$ & 124.483 & 122.104 & 118.506 & 99.775 & 103.689 & 95.37 & 109.422 & 106.346 & 73.098 \\
\hline $10 / 30 / 0723: 00$ & 124.481 & 122.095 & 118.499 & 99.775 & 103.689 & 95.368 & 109.422 & 106.342 & 73.094 \\
\hline $10 / 30 / 0723: 30$ & 124.476 & 122.093 & 118.496 & 99.764 & 103.681 & 95.361 & 109.426 & 106.338 & 73.086 \\
\hline $10 / 31 / 070: 00$ & 124.466 & 122.09 & 118.491 & 99.759 & 103.672 & 95.354 & 109.422 & 106.329 & 73.08 \\
\hline $10 / 31 / 070: 30$ & 124.462 & 122.102 & 118.501 & 99.761 & 103.674 & 95.354 & 109.418 & 106.329 & 73.08 \\
\hline $10 / 31 / 071: 00$ & 124.462 & 122.152 & 118.549 & 99.756 & 103.67 & 95.351 & 109.416 & 106.326 & 73.075 \\
\hline $10 / 31 / 071: 30$ & 124.459 & 122.168 & 118.569 & 99.771 & 103.683 & 95.358 & 109.414 & 106.333 & 73.086 \\
\hline $10 / 31 / 072: 00$ & 124.483 & 122.179 & 118.579 & 99.824 & 103.738 & 95.415 & 109.405 & 106.382 & 73.127 \\
\hline $10 / 31 / 072: 30$ & 124.514 & 122.168 & 118.574 & 99.843 & 103.752 & 95.434 & 109.407 & 106.399 & 73.142 \\
\hline 10/31/07 3:00 & 124.533 & 122.197 & 118.599 & 99.845 & 103.763 & 95.443 & 109.407 & 106.411 & 73.148 \\
\hline $10 / 31 / 073: 30$ & 124.542 & 122.215 & 118.617 & 99.838 & 103.75 & 95.432 & 109.411 & 106.404 & 73.14 \\
\hline $10 / 31 / 074: 00$ & 124.533 & 122.229 & 118.63 & 99.874 & 103.778 & 95.457 & 109.405 & 106.426 & 73.162 \\
\hline $10 / 31 / 074: 30$ & 124.53 & 122.249 & 118.647 & 99.895 & 103.798 & 95.481 & 109.403 & 106.446 & 73.183 \\
\hline $10 / 31 / 075: 00$ & 124.533 & 122.272 & 118.672 & 99.909 & 103.818 & 95.5 & 109.403 & 106.466 & 73.195 \\
\hline
\end{tabular}


TABLE S1.1 (Cont.)

Depth from Top of Casing (ft)

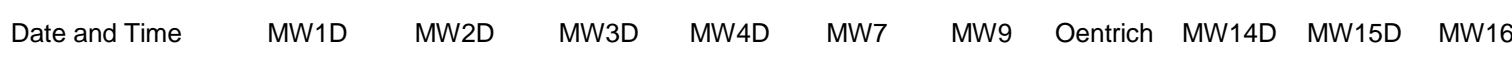

\begin{tabular}{|c|c|c|c|c|c|c|c|c|c|}
\hline 10/31/07 5:30 & 124.535 & 122.299 & 118.7 & 99.93 & 103.832 & 95.512 & 109.401 & 106.481 & 73.208 \\
\hline 10/31/07 6:00 & 124.535 & 123.991 & 119.474 & 99.954 & 103.86 & 95.538 & 109.398 & 106.506 & 73.23 \\
\hline 10/31/07 6:30 & 124.535 & 124.455 & 120.21 & 99.983 & 103.889 & 95.568 & 109.401 & 106.535 & 73.253 \\
\hline 10/31/07 7:00 & 125.892 & 124.651 & 120.429 & 100.595 & 104.165 & 95.96 & 109.796 & 107.611 & 73.27 \\
\hline 10/31/07 7:30 & 126.319 & 124.768 & 120.56 & 100.91 & 104.595 & 96.41 & 110.205 & 108.228 & 73.448 \\
\hline 10/31/07 8:00 & 126.485 & 124.855 & 120.651 & 101.074 & 104.789 & 96.605 & 110.374 & 108.443 & 73.601 \\
\hline 10/31/07 8:30 & 126.589 & 124.92 & 120.729 & 101.187 & 104.911 & 96.728 & 110.477 & 108.571 & 73.71 \\
\hline 10/31/07 9:00 & 126.657 & 124.977 & 120.784 & 101.265 & 105.001 & 96.813 & 110.537 & 108.662 & 73.793 \\
\hline 10/31/07 9:30 & 126.717 & 123.249 & 119.804 & 101.322 & 105.069 & 96.886 & 110.577 & 108.735 & 73.857 \\
\hline $10 / 31 / 07 \quad 10: 00$ & 126.769 & 122.92 & 119.358 & 101.368 & 105.126 & 96.942 & 110.609 & 108.793 & 73.906 \\
\hline $10 / 31 / 07 \quad 10: 30$ & 125.362 & 122.793 & 119.207 & 100.746 & 104.797 & 96.485 & 110.134 & 107.593 & 73.911 \\
\hline $10 / 31 / 07 \quad 11: 00$ & 125.071 & 122.723 & 119.131 & 100.522 & 104.487 & 96.167 & 109.858 & 107.188 & 73.782 \\
\hline $10 / 31 / 0711: 30$ & 124.963 & 122.68 & 119.088 & 100.419 & 104.361 & 96.044 & 109.739 & 107.046 & 73.687 \\
\hline $10 / 31 / 07$ 12:00 & 124.901 & 122.646 & 119.055 & 100.367 & 104.296 & 95.976 & 109.674 & 106.968 & 73.625 \\
\hline $10 / 31 / 07 \quad 12: 30$ & 124.873 & 122.621 & 119.028 & 100.333 & 104.254 & 95.938 & 109.642 & 106.922 & 73.588 \\
\hline $10 / 31 / 07$ 13:00 & 124.863 & 122.601 & 119.005 & 100.303 & 104.224 & 95.905 & 109.617 & 106.888 & 73.559 \\
\hline $10 / 31 / 07$ 13:30 & 124.93 & 122.589 & 119 & 100.281 & 104.201 & 95.884 & 109.602 & 106.864 & 73.536 \\
\hline $10 / 31 / 0714: 00$ & 124.97 & 122.576 & 118.985 & 100.266 & 104.18 & 95.865 & 109.587 & 106.842 & 73.518 \\
\hline $10 / 31 / 07$ 14:30 & 124.965 & 122.571 & 118.975 & 100.252 & 104.167 & 95.853 & 109.578 & 106.829 & 73.507 \\
\hline $10 / 31 / 07$ 15:00 & 124.958 & 122.569 & 118.975 & 100.241 & 104.155 & 95.844 & 109.568 & 106.813 & 73.495 \\
\hline $10 / 31 / 07$ 15:30 & 124.946 & 122.567 & 118.97 & 100.24 & 104.148 & 95.832 & 109.563 & 106.809 & 73.491 \\
\hline $10 / 31 / 07 \quad 16: 00$ & 124.944 & 122.567 & 118.967 & 100.236 & 104.15 & 95.832 & 109.557 & 106.807 & 73.491 \\
\hline $10 / 31 / 07 \quad 16: 30$ & 124.939 & 122.573 & 118.975 & 100.238 & 104.148 & 95.83 & 109.55 & 106.805 & 73.489 \\
\hline $10 / 31 / 07$ 17:00 & 124.939 & 122.576 & 118.979 & 100.24 & 104.152 & 95.832 & 109.548 & 106.807 & 73.491 \\
\hline $10 / 31 / 07$ 17:30 & 124.946 & 122.582 & 118.987 & 100.248 & 104.157 & 95.837 & 109.55 & 106.811 & 73.497 \\
\hline $10 / 31 / 07$ 18:00 & 124.946 & 122.585 & 118.99 & 100.252 & 104.159 & 95.842 & 109.552 & 106.814 & 73.5 \\
\hline $10 / 31 / 07$ 18:30 & 124.951 & 122.585 & 118.992 & 100.255 & 104.165 & 95.849 & 109.561 & 106.82 & 73.505 \\
\hline $10 / 31 / 07$ 19:00 & 124.951 & 122.589 & 118.997 & 100.26 & 104.167 & 95.849 & 109.563 & 106.822 & 73.51 \\
\hline $10 / 31 / 07$ 19:30 & 124.946 & 122.592 & 118.995 & 100.262 & 104.169 & 95.849 & 109.567 & 106.822 & 73.512 \\
\hline $10 / 31 / 0720: 00$ & 124.941 & 122.596 & 118.994 & 100.267 & 104.173 & 95.853 & 109.57 & 106.827 & 73.516 \\
\hline $10 / 31 / 0720: 30$ & 124.941 & 122.594 & 119 & 100.269 & 104.176 & 95.861 & 109.572 & 106.833 & 73.518 \\
\hline $10 / 31 / 0721: 00$ & 124.932 & 122.598 & 119.003 & 100.269 & 104.178 & 95.863 & 109.578 & 106.833 & 73.522 \\
\hline $10 / 31 / 0721: 30$ & 124.93 & 122.598 & 119.002 & 100.269 & 104.174 & 95.861 & 109.578 & 106.831 & 73.52 \\
\hline $10 / 31 / 0722: 00$ & 124.927 & 122.596 & 119 & 100.272 & 104.178 & 95.863 & 109.58 & 106.833 & 73.524 \\
\hline $10 / 31 / 0722: 30$ & 124.925 & 122.601 & 119.002 & 100.271 & 104.178 & 95.865 & 109.582 & 106.836 & 73.524 \\
\hline $10 / 31 / 0723: 00$ & 124.925 & 122.596 & 119.003 & 100.271 & 104.18 & 95.863 & 109.58 & 106.833 & 73.524 \\
\hline $10 / 31 / 0723: 30$ & 124.922 & 122.596 & 119 & 100.272 & 104.178 & 95.863 & 109.58 & 106.833 & 73.522 \\
\hline $11 / 1 / 07$ 0:00 & 124.918 & 122.592 & 118.992 & 100.271 & 104.176 & 95.863 & 109.58 & 106.833 & 73.526 \\
\hline 11/1/07 0:30 & 124.918 & 122.585 & 118.989 & 100.267 & 104.176 & 95.861 & 109.58 & 106.833 & 73.526 \\
\hline 11/1/07 1:00 & 124.918 & 122.58 & 118.982 & 100.264 & 104.173 & 95.858 & 109.58 & 106.829 & 73.524 \\
\hline 11/1/07 1:30 & 124.915 & 122.576 & 118.982 & 100.259 & 104.167 & 95.851 & 109.58 & 106.822 & 73.52 \\
\hline $11 / 1 / 072: 00$ & 124.913 & 122.573 & 118.98 & 100.255 & 104.163 & 95.844 & 109.576 & 106.82 & 73.518 \\
\hline $11 / 1 / 072: 30$ & 124.913 & 122.571 & 118.975 & 100.25 & 104.157 & 95.839 & 109.576 & 106.816 & 73.516 \\
\hline 11/1/07 3:00 & 124.908 & 122.56 & 118.967 & 100.245 & 104.157 & 95.837 & 109.572 & 106.811 & 73.514 \\
\hline 11/1/07 3:30 & 124.908 & 122.555 & 118.962 & 100.243 & 104.151 & 95.837 & 109.57 & 106.807 & 73.512 \\
\hline 11/1/07 4:00 & 124.911 & 122.546 & 118.952 & 100.233 & 104.144 & 95.825 & 109.57 & 106.798 & 73.503 \\
\hline 11/1/07 4:30 & 124.906 & 122.546 & 118.955 & 100.228 & 104.136 & 95.818 & 109.567 & 106.794 & 73.499 \\
\hline 11/1/07 5:00 & 124.901 & 122.539 & 118.95 & 100.221 & 104.131 & 95.813 & 109.565 & 106.785 & 73.493 \\
\hline 11/1/07 5:30 & 124.896 & 122.535 & 118.944 & 100.221 & 104.129 & 95.811 & 109.563 & 106.783 & 73.495 \\
\hline 11/1/07 6:00 & 124.894 & 124.24 & 119.953 & 100.214 & 104.125 & 95.806 & 109.559 & 106.78 & 73.493 \\
\hline 11/1/07 6:30 & 124.894 & 124.648 & 120.557 & 100.209 & 104.121 & 95.799 & 109.559 & 106.776 & 73.485 \\
\hline 11/1/07 7:00 & 126.3 & 124.818 & 120.746 & 100.882 & 104.443 & 96.261 & 110.023 & 107.894 & 73.495 \\
\hline 11/1/07 7:30 & 126.688 & 124.916 & 120.852 & 101.163 & 104.831 & 96.664 & 110.406 & 108.423 & 73.66 \\
\hline $11 / 1 / 078: 00$ & 126.844 & 124.988 & 120.933 & 101.299 & 104.997 & 96.832 & 110.56 & 108.607 & 73.799 \\
\hline 11/1/07 8:30 & 126.939 & 125.025 & 120.976 & 101.389 & 105.098 & 96.931 & 110.648 & 108.713 & 73.888 \\
\hline 11/1/07 9:00 & 127.007 & 125.056 & 121.003 & 101.448 & 105.166 & 97.001 & 110.704 & 108.788 & 73.952 \\
\hline 11/1/07 9:30 & 127.064 & 125.07 & 121.025 & 101.48 & 105.205 & 97.039 & 110.744 & 108.828 & 73.992 \\
\hline $11 / 1 / 07$ 10:00 & 127.114 & 125.09 & 121.039 & 101.506 & 105.233 & 97.065 & 110.774 & 108.861 & 74.016 \\
\hline 11/1/07 10:30 & 127.154 & 125.095 & 121.046 & 101.52 & 105.248 & 97.082 & 110.794 & 108.877 & 74.035 \\
\hline 11/1/07 11:00 & 127.189 & 125.097 & 121.051 & 101.535 & 105.264 & 97.098 & 110.815 & 108.89 & 74.049 \\
\hline 11/1/07 11:30 & 127.225 & 125.093 & 121.049 & 101.539 & 105.269 & 97.103 & 110.828 & 108.899 & 74.057 \\
\hline $11 / 1 / 07$ 12:00 & 127.255 & 125.086 & 121.039 & 101.541 & 105.273 & 97.107 & 110.84 & 108.903 & 74.066 \\
\hline $11 / 1 / 0712: 30$ & 127.336 & 125.07 & 121.026 & 101.535 & 105.271 & 97.105 & 110.849 & 108.901 & 74.068 \\
\hline 11/1/07 13:00 & 127.492 & 125.059 & 121.01 & 101.52 & 105.26 & 97.096 & 110.86 & 108.89 & 74.059 \\
\hline 11/1/07 13:30 & 127.532 & 125.045 & 120.995 & 101.506 & 105.243 & 97.077 & 110.87 & 108.875 & 74.051 \\
\hline $11 / 1 / 0714: 00$ & 127.532 & 125.034 & 120.986 & 101.492 & 105.233 & 97.065 & 110.881 & 108.864 & 74.041 \\
\hline
\end{tabular}


TABLE S1.1 (Cont.)

\begin{tabular}{|c|c|c|c|c|c|c|c|c|c|c|}
\hline \multirow[b]{2}{*}{ Date and Time } & \multicolumn{10}{|c|}{ Depth from Top of Casing (ft) } \\
\hline & MW1D & MW2D & MW3D & MW4D & MW7 & MW9 & Oentrich & MW14D & MW15D & MW16D \\
\hline 11/1/07 14:30 & 127.523 & 125.027 & 120.983 & 101.48 & 105.218 & 97.053 & 110.886 & 108.853 & & 74.035 \\
\hline $11 / 1 / 07$ 15:00 & 127.511 & 125.027 & 120.975 & 101.466 & 105.205 & 97.037 & 110.894 & 108.839 & & 74.026 \\
\hline 11/1/07 15:30 & 127.501 & 125.022 & 120.973 & 101.461 & 105.201 & 97.032 & 110.903 & 108.835 & & 74.022 \\
\hline 11/1/07 16:00 & 127.494 & 125.018 & 120.975 & 101.46 & 105.195 & 97.027 & 110.909 & 108.83 & & 74.022 \\
\hline $11 / 1 / 0716: 30$ & 127.49 & 124.02 & 120.971 & 101.458 & 105.191 & 97.023 & 110.917 & 108.826 & & 74.02 \\
\hline 11/1/07 17:00 & 127.487 & 123.031 & 119.486 & 101.454 & 105.191 & 97.025 & 110.922 & 108.826 & & 74.024 \\
\hline $11 / 1 / 0717: 30$ & 127.485 & 122.816 & 119.241 & 100.977 & 105.191 & 97.023 & 110.922 & 108.824 & & 74.024 \\
\hline 11/1/07 18:00 & 125.561 & 122.696 & 119.11 & 100.569 & 104.563 & 96.245 & 110.164 & 107.305 & & 73.906 \\
\hline 11/1/07 18:30 & 125.256 & 122.626 & 119.035 & 100.403 & 104.359 & 96.04 & 109.976 & 107.07 & & 73.766 \\
\hline 11/1/07 19:00 & 125.104 & 122.578 & 118.984 & 100.31 & 104.245 & 95.927 & 109.871 & 106.946 & & 73.671 \\
\hline 11/1/07 19:30 & 125.017 & 122.542 & 118.944 & 100.252 & 104.18 & 95.861 & 109.807 & 106.869 & & 73.607 \\
\hline 11/1/07 20:00 & 124.963 & 122.514 & 118.917 & 100.21 & 104.134 & 95.818 & 109.766 & 106.818 & & 73.563 \\
\hline $11 / 1 / 0720: 30$ & 124.93 & 122.49 & 118.892 & 100.181 & 104.1 & 95.783 & 109.736 & 106.78 & & 73.53 \\
\hline $11 / 1 / 0721: 00$ & 124.908 & 122.476 & 118.879 & 100.155 & 104.075 & 95.757 & 109.713 & 106.754 & & 73.505 \\
\hline 11/1/07 21:30 & 124.889 & 122.465 & 118.863 & 100.138 & 104.051 & 95.736 & 109.696 & 106.725 & & 73.483 \\
\hline $11 / 1 / 0722: 00$ & 124.873 & 122.462 & 118.848 & 100.126 & 104.041 & 95.724 & 109.681 & 106.712 & & 73.47 \\
\hline $11 / 1 / 0722: 30$ & 124.861 & 122.46 & 118.843 & 100.119 & 104.034 & 95.714 & 109.67 & 106.703 & & 73.462 \\
\hline 11/1/07 23:00 & 124.847 & 122.46 & 118.844 & 100.123 & 104.032 & 95.714 & 109.66 & 106.701 & & 73.46 \\
\hline 11/1/07 23:30 & 124.837 & 122.453 & 118.854 & 100.119 & 104.032 & 95.71 & 109.653 & 106.696 & & 73.456 \\
\hline 11/2/07 0:00 & 124.828 & 122.44 & 118.844 & 100.121 & 104.032 & 95.712 & 109.645 & 106.696 & & 73.456 \\
\hline 11/2/07 0:30 & 124.823 & 122.428 & 118.831 & 100.109 & 104.024 & 95.707 & 109.642 & 106.692 & & 73.452 \\
\hline 11/2/07 1:00 & 124.818 & 122.412 & 118.811 & 100.102 & 104.012 & 95.696 & 109.638 & 106.676 & & 73.439 \\
\hline 11/2/07 1:30 & 124.814 & 122.406 & 118.8 & 100.086 & 104.003 & 95.684 & 109.634 & 106.665 & & 73.427 \\
\hline 11/2/07 2:00 & 124.809 & 122.401 & 118.79 & 100.071 & 103.982 & 95.66 & 109.632 & 106.647 & & 73.408 \\
\hline 11/2/07 2:30 & 124.802 & 122.385 & 118.771 & 100.071 & 103.978 & 95.66 & 109.627 & 106.641 & & 73.406 \\
\hline 11/2/07 3:00 & 124.8 & 122.383 & 118.786 & 100.057 & 103.976 & 95.655 & 109.623 & 106.639 & & 73.402 \\
\hline 11/2/07 3:30 & 124.797 & 122.376 & 118.778 & 100.047 & 103.959 & 95.639 & 109.619 & 106.621 & & 73.388 \\
\hline 11/2/07 4:00 & 124.792 & 122.372 & 118.775 & 100.04 & 103.956 & 95.634 & 109.617 & 106.619 & & 73.383 \\
\hline 11/2/07 4:30 & 124.788 & 122.381 & 118.778 & 100.036 & 103.95 & 95.63 & 109.61 & 106.61 & & 73.378 \\
\hline $11 / 2 / 075: 00$ & 124.783 & 122.378 & 118.78 & 100.033 & 103.948 & 95.625 & 109.608 & 106.608 & & 73.375 \\
\hline 11/2/07 5:30 & 124.776 & 122.381 & 118.78 & 100.045 & 103.957 & 95.639 & 109.604 & 106.614 & & 73.379 \\
\hline 11/2/07 6:00 & 124.771 & 122.378 & 118.778 & 100.043 & 103.954 & 95.637 & 109.602 & 106.614 & & 73.379 \\
\hline 11/2/07 6:30 & 124.769 & 122.381 & 118.783 & 100.043 & 103.955 & 95.637 & 109.602 & 106.614 & & 73.375 \\
\hline 11/2/07 7:00 & 124.764 & 122.388 & 118.788 & 100.042 & 103.954 & 95.637 & 109.597 & 106.612 & & 73.376 \\
\hline 11/2/07 7:30 & 124.759 & 122.406 & 118.795 & 100.04 & 103.957 & 95.639 & 109.595 & 106.614 & & 73.377 \\
\hline 11/2/07 8:00 & 124.755 & 122.424 & 118.824 & 100.057 & 103.967 & 95.646 & 109.593 & 106.623 & & 73.383 \\
\hline 11/2/07 8:30 & 124.752 & 122.433 & 118.833 & 100.086 & 103.984 & 95.66 & 109.589 & 106.634 & & 73.394 \\
\hline 11/2/07 9:00 & 124.75 & 122.446 & 118.846 & 100.097 & 104.005 & 95.688 & 109.585 & 106.659 & & 73.414 \\
\hline 11/2/07 9:30 & 124.755 & 122.451 & 118.854 & 100.104 & 104.013 & 95.693 & 109.583 & 106.667 & & 73.421 \\
\hline 11/2/07 10:00 & 124.762 & 122.46 & 118.861 & 100.117 & 104.024 & 95.705 & 109.582 & 106.678 & & 73.431 \\
\hline 11/2/07 10:30 & 124.769 & 122.465 & 118.863 & 100.126 & 104.032 & 95.717 & 109.58 & 106.687 & & 73.437 \\
\hline 11/2/07 11:00 & 124.776 & 122.469 & 118.874 & 100.133 & 104.043 & 95.724 & 109.58 & 106.694 & & 73.443 \\
\hline 11/2/07 11:30 & 124.785 & 122.474 & 118.877 & 100.136 & 104.045 & 95.731 & 109.576 & 106.698 & & 73.445 \\
\hline 11/2/07 12:00 & 124.792 & 124.399 & 120.257 & 100.142 & 104.051 & 95.738 & 109.576 & 106.707 & & 73.452 \\
\hline $11 / 2 / 0712: 30$ & 124.809 & 124.664 & 120.58 & 100.145 & 104.058 & 95.74 & 109.576 & 106.709 & & 73.456 \\
\hline 11/2/07 13:00 & 126.672 & 124.798 & 120.729 & 100.958 & 104.595 & 96.419 & 110.248 & 108.142 & & 73.524 \\
\hline 11/2/07 13:30 & 127.083 & 124.882 & 120.824 & 101.162 & 104.854 & 96.685 & 110.496 & 108.445 & & 73.691 \\
\hline $11 / 2 / 0714: 00$ & 127.246 & 123.229 & 119.832 & 101.275 & 104.982 & 96.815 & 110.618 & 108.586 & & 73.803 \\
\hline 11/2/07 14:30 & 127.341 & 122.877 & 119.312 & 101.346 & 105.063 & 96.9 & 110.695 & 108.675 & & 73.88 \\
\hline 11/2/07 15:00 & 125.863 & 122.743 & 119.158 & 100.705 & 104.759 & 96.457 & 110.242 & 107.593 & & 73.913 \\
\hline $11 / 2 / 0715: 30$ & 125.336 & 122.678 & 119.09 & 100.474 & 104.439 & 96.122 & 109.923 & 107.141 & & 73.793 \\
\hline $11 / 2 / 0716: 00$ & 125.147 & 122.632 & 119.04 & 100.372 & 104.312 & 95.995 & 109.797 & 106.993 & & 73.698 \\
\hline 11/2/07 16:30 & 125.064 & 122.61 & 119.01 & 100.317 & 104.247 & 95.931 & 109.732 & 106.92 & & 73.64 \\
\hline 11/2/07 17:00 & 125.015 & 122.596 & 119.002 & 100.285 & 104.203 & 95.886 & 109.69 & 106.873 & & 73.598 \\
\hline 11/2/07 17:30 & 124.984 & 122.589 & 118.994 & 100.267 & 104.182 & 95.868 & 109.662 & 106.849 & & 73.578 \\
\hline 11/2/07 18:00 & 124.972 & 122.592 & 118.997 & 100.259 & 104.171 & 95.856 & 109.645 & 106.836 & & 73.563 \\
\hline $11 / 2 / 0718: 30$ & 124.96 & 122.598 & 118.999 & 100.254 & 104.163 & 95.849 & 109.632 & 106.827 & & 73.557 \\
\hline 11/2/07 19:00 & 124.958 & 122.601 & 119.004 & 100.259 & 104.167 & 95.853 & 109.623 & 106.829 & & 73.559 \\
\hline 11/2/07 19:30 & 124.965 & 122.603 & 119.007 & 100.266 & 104.17 & 95.856 & 109.615 & 106.833 & & 73.561 \\
\hline 11/2/07 20:00 & 124.97 & 122.603 & 119.007 & 100.271 & 104.18 & 95.875 & 109.602 & 106.836 & & 73.568 \\
\hline 11/2/07 20:30 & 124.967 & 122.605 & 119.01 & 100.272 & 104.18 & 95.865 & 109.603 & 106.84 & & 73.569 \\
\hline $11 / 2 / 0721: 00$ & 124.963 & 122.605 & 119.012 & 100.274 & 104.178 & 95.867 & 109.595 & 106.838 & & 73.567 \\
\hline 11/2/07 21:30 & 124.956 & 122.614 & 119.018 & 100.276 & 104.182 & 95.868 & 109.591 & 106.84 & & 73.572 \\
\hline 11/2/07 22:00 & 124.951 & 122.619 & 119.025 & 100.276 & 104.182 & 95.87 & 109.587 & 106.842 & & 73.572 \\
\hline 11/2/07 22:30 & 124.946 & 122.623 & 119.027 & 100.286 & 104.19 & 95.875 & 109.584 & 106.849 & & 73.58 \\
\hline 11/2/07 23:00 & 124.941 & 122.623 & 119.032 & 100.288 & 104.195 & 95.879 & 109.589 & 106.856 & & 73.584 \\
\hline
\end{tabular}


TABLE S1.1 (Cont.)

Depth from Top of Casing (ft)

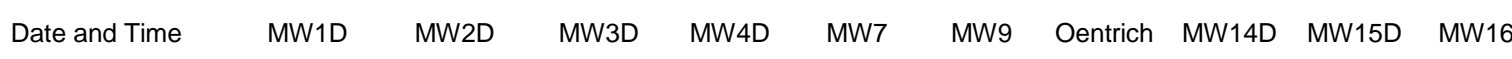

\begin{tabular}{|c|c|c|c|c|c|c|c|c|c|}
\hline 11/2/07 23:30 & 124.937 & 122.626 & 119.035 & 100.295 & 104.203 & 95.886 & 109.587 & 106.86 & 73.588 \\
\hline $11 / 3 / 070: 00$ & 124.937 & 122.626 & 119.032 & 100.297 & 104.205 & 95.886 & 109.585 & 106.86 & 73.59 \\
\hline $11 / 3 / 070: 30$ & 124.932 & 122.626 & 119.03 & 100.295 & 104.205 & 95.889 & 109.585 & 106.862 & 73.59 \\
\hline 11/3/07 1:00 & 124.932 & 122.623 & 119.033 & 100.297 & 104.205 & 95.889 & 109.585 & 106.862 & 73.59 \\
\hline 11/3/07 1:30 & 124.932 & 122.621 & 119.027 & 100.297 & 104.203 & 95.889 & 109.585 & 106.862 & 73.59 \\
\hline $11 / 3 / 07$ 2:00 & 124.93 & 122.614 & 119.014 & 100.298 & 104.203 & 95.886 & 109.585 & 106.86 & 73.588 \\
\hline 11/3/07 2:30 & 124.93 & 122.603 & 119.009 & 100.291 & 104.199 & 95.884 & 109.585 & 106.858 & 73.59 \\
\hline 11/3/07 3:00 & 124.93 & 122.598 & 119.004 & 100.285 & 104.192 & 95.877 & 109.585 & 106.851 & 73.582 \\
\hline 11/3/07 3:30 & 124.927 & 122.594 & 118.999 & 100.279 & 104.182 & 95.865 & 109.583 & 106.84 & 73.574 \\
\hline $11 / 3 / 074: 00$ & 124.93 & 122.594 & 118.997 & 100.267 & 104.176 & 95.861 & 109.583 & 106.833 & 73.567 \\
\hline $11 / 3 / 074: 30$ & 124.925 & 122.598 & 119.004 & 100.266 & 104.173 & 95.858 & 109.58 & 106.833 & 73.567 \\
\hline $11 / 3 / 07$ 5:00 & 124.922 & 122.594 & 119.002 & 100.269 & 104.174 & 95.856 & 109.578 & 106.829 & 73.565 \\
\hline $11 / 3 / 075: 30$ & 124.92 & 122.589 & 118.997 & 100.266 & 104.176 & 95.861 & 109.576 & 106.833 & 73.572 \\
\hline 11/3/07 6:00 & 124.918 & 122.582 & 118.987 & 100.266 & 104.174 & 95.861 & 109.572 & 106.833 & 73.572 \\
\hline $11 / 3 / 076: 30$ & 124.918 & 122.58 & 118.987 & 100.257 & 104.169 & 95.853 & 109.574 & 106.827 & 73.563 \\
\hline $11 / 3 / 077: 00$ & 124.918 & 124.621 & 120.358 & 100.254 & 104.161 & 95.846 & 109.57 & 106.82 & 73.559 \\
\hline $11 / 3 / 07$ 7:30 & 124.915 & 124.823 & 120.6 & 100.643 & 104.159 & 95.844 & 109.57 & 106.818 & 73.559 \\
\hline 11/3/07 8:00 & 126.615 & 124.939 & 120.731 & 101.081 & 104.748 & 96.565 & 110.28 & 108.379 & 73.669 \\
\hline 11/3/07 8:30 & 126.816 & 125.004 & 120.804 & 101.244 & 104.949 & 96.768 & 110.481 & 108.609 & 73.815 \\
\hline 11/3/07 9:00 & 126.925 & 125.047 & 120.851 & 101.344 & 105.069 & 96.891 & 110.584 & 108.74 & 73.925 \\
\hline 11/3/07 9:30 & 127.005 & 125.068 & 120.872 & 101.396 & 105.132 & 96.949 & 110.65 & 108.806 & 73.985 \\
\hline $11 / 3 / 07$ 10:00 & 127.066 & 123.229 & 119.743 & 101.435 & 105.174 & 96.994 & 110.693 & 108.855 & 74.022 \\
\hline $11 / 3 / 07$ 10:30 & 127.114 & 122.941 & 119.372 & 101.454 & 105.201 & 97.018 & 110.725 & 108.879 & 74.049 \\
\hline $11 / 3 / 0711: 00$ & 125.62 & 122.807 & 119.221 & 100.739 & 104.77 & 96.457 & 110.171 & 107.537 & 74.018 \\
\hline 11/3/07 11:30 & 125.379 & 122.709 & 119.115 & 100.538 & 104.491 & 96.174 & 109.918 & 107.199 & 73.875 \\
\hline $11 / 3 / 0712: 00$ & 125.275 & 122.644 & 119.057 & 100.417 & 104.363 & 96.044 & 109.801 & 107.055 & 73.77 \\
\hline $11 / 3 / 0712: 30$ & 125.216 & 122.585 & 118.994 & 100.341 & 104.27 & 95.952 & 109.736 & 106.953 & 73.685 \\
\hline $11 / 3 / 07$ 13:00 & 125.168 & 122.539 & 118.944 & 100.279 & 104.209 & 95.891 & 109.698 & 106.887 & 73.629 \\
\hline $11 / 3 / 0713: 30$ & 125.081 & 122.496 & 118.906 & 100.224 & 104.15 & 95.832 & 109.672 & 106.827 & 73.576 \\
\hline $11 / 3 / 0714: 00$ & 125.003 & 122.469 & 118.876 & 100.181 & 104.102 & 95.785 & 109.657 & 106.776 & 73.532 \\
\hline $11 / 3 / 07 \quad 14: 30$ & 124.932 & 122.442 & 118.848 & 100.147 & 104.062 & 95.747 & 109.644 & 106.738 & 73.495 \\
\hline $11 / 3 / 07$ 15:00 & 124.875 & 122.422 & 118.828 & 100.119 & 104.036 & 95.717 & 109.634 & 106.705 & 73.47 \\
\hline $11 / 3 / 07$ 15:30 & 124.84 & 122.406 & 118.808 & 100.097 & 104.016 & 95.698 & 109.627 & 106.683 & 73.45 \\
\hline $11 / 3 / 07$ 16:00 & 124.809 & 122.394 & 118.8 & 100.079 & 103.99 & 95.672 & 109.621 & 106.659 & 73.429 \\
\hline $11 / 3 / 07$ 16:30 & 124.788 & 122.385 & 118.785 & 100.061 & 103.976 & 95.655 & 109.613 & 106.639 & 73.412 \\
\hline $11 / 3 / 07$ 17:00 & 124.771 & 122.381 & 118.785 & 100.055 & 103.967 & 95.651 & 109.604 & 106.63 & 73.406 \\
\hline $11 / 3 / 07 \quad 17: 30$ & 124.764 & 122.369 & 118.773 & 100.045 & 103.957 & 95.639 & 109.6 & 106.621 & 73.396 \\
\hline $11 / 3 / 07$ 18:00 & 124.755 & 122.356 & 118.757 & 100.04 & 103.957 & 95.637 & 109.598 & 106.616 & 73.394 \\
\hline $11 / 3 / 07$ 18:30 & 124.745 & 122.347 & 118.737 & 100.03 & 103.948 & 95.625 & 109.595 & 106.603 & 73.381 \\
\hline $11 / 3 / 07$ 19:00 & 124.736 & 122.34 & 118.74 & 100.016 & 103.929 & 95.611 & 109.593 & 106.59 & 73.369 \\
\hline 11/3/07 19:30 & 124.724 & 122.34 & 118.747 & 100.007 & 103.919 & 95.601 & 109.587 & 106.579 & 73.359 \\
\hline $11 / 3 / 0720: 00$ & 124.717 & 122.335 & 118.74 & 100.007 & 103.917 & 95.599 & 109.583 & 106.577 & 73.361 \\
\hline $11 / 3 / 0720: 30$ & 124.712 & 122.335 & 118.735 & 100.002 & 103.917 & 95.599 & 109.58 & 106.577 & 73.359 \\
\hline $11 / 3 / 0721: 00$ & 124.71 & 122.344 & 118.747 & 99.999 & 103.913 & 95.592 & 109.576 & 106.57 & 73.352 \\
\hline $11 / 3 / 0721: 30$ & 124.707 & 122.36 & 118.767 & 100.005 & 103.916 & 95.592 & 109.572 & 106.57 & 73.352 \\
\hline 11/3/07 22:00 & 124.7 & 122.369 & 118.778 & 100.017 & 103.925 & 95.604 & 109.57 & 106.579 & 73.361 \\
\hline $11 / 3 / 0722: 30$ & 124.696 & 122.369 & 118.778 & 100.042 & 103.94 & 95.622 & 109.567 & 106.592 & 73.375 \\
\hline $11 / 3 / 0723: 00$ & 124.696 & 122.367 & 118.775 & 100.042 & 103.952 & 95.634 & 109.563 & 106.605 & 73.383 \\
\hline $11 / 3 / 0723: 30$ & 124.696 & 122.365 & 118.775 & 100.043 & 103.948 & 95.63 & 109.565 & 106.605 & 73.381 \\
\hline $11 / 4 / 070: 00$ & 124.693 & 122.365 & 118.77 & 100.036 & 103.946 & 95.63 & 109.563 & 106.603 & 73.377 \\
\hline 11/4/07 0:30 & 124.696 & 122.369 & 118.775 & 100.031 & 103.94 & 95.622 & 109.563 & 106.599 & 73.373 \\
\hline 11/4/07 1:00 & 124.696 & 122.363 & 118.77 & 100.036 & 103.944 & 95.625 & 109.563 & 106.599 & 73.373 \\
\hline 11/4/07 1:30 & 124.693 & 122.36 & 118.767 & 100.042 & 103.95 & 95.632 & 109.559 & 106.605 & 73.377 \\
\hline $11 / 4 / 072: 00$ & 124.693 & 122.358 & 118.76 & 100.036 & 103.942 & 95.622 & 109.559 & 106.599 & 73.373 \\
\hline $11 / 4 / 072: 30$ & 124.691 & 122.353 & 118.747 & 100.031 & 103.944 & 95.622 & 109.559 & 106.597 & 73.371 \\
\hline 11/4/07 3:00 & 124.693 & 122.353 & 118.746 & 100.03 & 103.938 & 95.62 & 109.557 & 106.592 & 73.367 \\
\hline 11/4/07 3:30 & 124.693 & 122.344 & 118.738 & 100.026 & 103.937 & 95.618 & 109.557 & 106.59 & 73.363 \\
\hline 11/4/07 4:00 & 124.693 & 122.349 & 118.738 & 100.023 & 103.933 & 95.613 & 109.557 & 106.588 & 73.363 \\
\hline 11/4/07 4:30 & 124.693 & 122.351 & 118.742 & 100.016 & 103.925 & 95.606 & 109.559 & 106.581 & 73.354 \\
\hline $11 / 4 / 075: 00$ & 124.693 & 122.356 & 118.742 & 100.023 & 103.929 & 95.608 & 109.557 & 106.583 & 73.357 \\
\hline 11/4/07 5:30 & 124.691 & 122.356 & 118.758 & 100.023 & 103.933 & 95.611 & 109.557 & 106.585 & 73.359 \\
\hline $11 / 4 / 076: 00$ & 124.688 & 122.351 & 118.742 & 100.03 & 103.94 & 95.618 & 109.556 & 106.592 & 73.365 \\
\hline 11/4/07 6:30 & 124.691 & 122.344 & 118.737 & 100.026 & 103.937 & 95.618 & 109.553 & 106.592 & 73.363 \\
\hline $11 / 4 / 077: 00$ & 124.691 & 122.338 & 118.743 & 100.024 & 103.937 & 95.615 & 109.555 & 106.588 & 73.361 \\
\hline $11 / 4 / 07$ 7:30 & 124.693 & 123.911 & 119.163 & 100.012 & 103.923 & 95.604 & 109.555 & 106.579 & 73.35 \\
\hline $11 / 4 / 078: 00$ & 124.693 & 124.426 & 120.325 & 100.009 & 103.919 & 95.599 & 109.557 & 106.574 & 73.346 \\
\hline
\end{tabular}


TABLE S1.1 (Cont.)

\begin{tabular}{|c|c|c|c|c|c|c|c|c|c|c|}
\hline \multirow[b]{2}{*}{ Date and Time } & \multicolumn{10}{|c|}{ Depth from Top of Casing (ft) } \\
\hline & MW1D & MW2D & MW3D & MW4D & MW7 & MW9 & Oentrich & MW14D & MW15D & MW16D \\
\hline 11/4/07 8:30 & 125.955 & 124.605 & 120.534 & 100.62 & 104.133 & 95.931 & 109.91 & 107.425 & & 73.344 \\
\hline 11/4/07 9:00 & 126.468 & 124.709 & 120.647 & 100.939 & 104.607 & 96.436 & 110.377 & 108.19 & & 73.501 \\
\hline 11/4/07 9:30 & 126.646 & 124.764 & 120.711 & 101.089 & 104.786 & 96.617 & 110.545 & 108.389 & & 73.644 \\
\hline $11 / 4 / 0710: 00$ & 126.754 & 124.798 & 120.746 & 101.172 & 104.888 & 96.718 & 110.643 & 108.5 & & 73.735 \\
\hline $11 / 4 / 07 \quad 10: 30$ & 126.832 & 124.818 & 120.769 & 101.217 & 104.942 & 96.773 & 110.706 & 108.562 & & 73.791 \\
\hline 11/4/07 11:00 & 126.894 & 124.832 & 120.786 & 101.244 & 104.974 & 96.808 & 110.748 & 108.598 & & 73.822 \\
\hline $11 / 4 / 0711: 30$ & 126.943 & 124.827 & 120.782 & 101.261 & 104.993 & 96.827 & 110.776 & 108.618 & & 73.842 \\
\hline $11 / 4 / 0712: 00$ & 126.988 & 124.818 & 120.777 & 101.274 & 105.005 & 96.836 & 110.796 & 108.633 & & 73.855 \\
\hline 11/4/07 12:30 & 127.142 & 124.809 & 120.769 & 101.267 & 105.003 & 96.834 & 110.811 & 108.631 & & 73.855 \\
\hline 11/4/07 13:00 & 127.27 & 124.802 & 120.761 & 101.256 & 104.995 & 96.827 & 110.817 & 108.624 & & 73.853 \\
\hline 11/4/07 13:30 & 127.284 & 124.793 & 120.749 & 101.243 & 104.984 & 96.813 & 110.821 & 108.613 & & 73.844 \\
\hline $11 / 4 / 0714: 00$ & 127.279 & 124.782 & 120.738 & 101.243 & 104.982 & 96.81 & 110.819 & 108.609 & & 73.842 \\
\hline $11 / 4 / 0714: 30$ & 127.272 & 123.102 & 119.718 & 101.229 & 104.97 & 96.801 & 110.808 & 108.598 & & 73.836 \\
\hline $11 / 4 / 07$ 15:00 & 127.26 & 122.703 & 119.145 & 101.222 & 104.959 & 96.789 & 110.8 & 108.589 & & 73.83 \\
\hline 11/4/07 15:30 & 125.764 & 122.533 & 118.955 & 100.54 & 104.61 & 96.306 & 110.308 & 107.475 & & 73.813 \\
\hline $11 / 4 / 0716: 00$ & 125.19 & 122.433 & 118.849 & 100.264 & 104.239 & 95.919 & 109.95 & 106.962 & & 73.654 \\
\hline $11 / 4 / 0716: 30$ & 124.956 & 122.36 & 118.773 & 100.133 & 104.079 & 95.764 & 109.798 & 106.78 & & 73.53 \\
\hline 11/4/07 17:00 & 124.837 & 122.31 & 118.722 & 100.048 & 103.984 & 95.665 & 109.721 & 106.676 & & 73.441 \\
\hline $11 / 4 / 0717: 30$ & 124.755 & 122.267 & 118.677 & 99.986 & 103.916 & 95.597 & 109.644 & 106.599 & & 73.375 \\
\hline 11/4/07 18:00 & 124.703 & 122.242 & 118.652 & 99.943 & 103.868 & 95.549 & 109.59 & 106.548 & & 73.332 \\
\hline 11/4/07 18:30 & 124.655 & 122.231 & 118.632 & 99.909 & 103.828 & 95.509 & 109.546 & 106.504 & & 73.292 \\
\hline 11/4/07 19:00 & 124.627 & 122.22 & 118.626 & 99.893 & 103.807 & 95.488 & 109.522 & 106.477 & & 73.27 \\
\hline 11/4/07 19:30 & 124.606 & 122.206 & 118.614 & 99.88 & 103.796 & 95.476 & 109.509 & 106.464 & & 73.259 \\
\hline $11 / 4 / 0720: 00$ & 124.596 & 122.195 & 118.601 & 99.873 & 103.786 & 95.465 & 109.495 & 106.453 & & 73.249 \\
\hline 11/4/07 20:30 & 124.587 & 122.174 & 118.579 & 99.859 & 103.773 & 95.455 & 109.49 & 106.439 & & 73.235 \\
\hline $11 / 4 / 0721: 00$ & 124.573 & 122.161 & 118.568 & 99.845 & 103.763 & 95.443 & 109.48 & 106.428 & & 73.226 \\
\hline $11 / 4 / 0721: 30$ & 124.559 & 122.145 & 118.551 & 99.828 & 103.74 & 95.42 & 109.473 & 106.406 & & 73.204 \\
\hline 11/4/07 22:00 & 124.544 & 122.136 & 118.541 & 99.812 & 103.727 & 95.408 & 109.467 & 106.393 & & 73.193 \\
\hline 11/4/07 22:30 & 124.528 & 122.127 & 118.531 & 99.8 & 103.714 & 95.394 & 109.456 & 106.377 & & 73.179 \\
\hline 11/4/07 23:00 & 124.516 & 122.124 & 118.533 & 99.79 & 103.704 & 95.382 & 109.449 & 106.366 & & 73.168 \\
\hline 11/4/07 23:30 & 124.502 & 122.115 & 118.526 & 99.785 & 103.699 & 95.375 & 109.439 & 106.355 & & 73.16 \\
\hline 11/5/07 0:00 & 124.497 & 122.113 & 118.521 & 99.781 & 103.699 & 95.375 & 109.434 & 106.357 & & 73.16 \\
\hline 11/5/07 0:30 & 124.495 & 122.102 & 118.51 & 99.776 & 103.689 & 95.368 & 109.434 & 106.349 & & 73.152 \\
\hline 11/5/07 1:00 & 124.488 & 122.097 & 118.503 & 99.771 & 103.685 & 95.366 & 109.43 & 106.344 & & 73.148 \\
\hline 11/5/07 1:30 & 124.483 & 122.109 & 118.516 & 99.764 & 103.678 & 95.356 & 109.418 & 106.333 & & 73.137 \\
\hline $11 / 5 / 072: 00$ & 124.476 & 122.111 & 118.519 & 99.759 & 103.67 & 95.349 & 109.405 & 106.326 & & 73.131 \\
\hline 11/5/07 2:30 & 124.471 & 122.111 & 118.518 & 99.776 & 103.683 & 95.366 & 109.401 & 106.342 & & 73.142 \\
\hline 11/5/07 3:00 & 124.469 & 122.113 & 118.518 & 99.781 & 103.687 & 95.366 & 109.399 & 106.34 & & 73.14 \\
\hline $11 / 5 / 073: 30$ & 124.469 & 122.124 & 118.521 & 99.776 & 103.685 & 95.365 & 109.398 & 106.342 & & 73.137 \\
\hline $11 / 5 / 074: 00$ & 124.464 & 122.143 & 118.549 & 99.781 & 103.691 & 95.375 & 109.396 & 106.344 & & 73.144 \\
\hline 11/5/07 4:30 & 124.459 & 122.17 & 118.571 & 99.795 & 103.7 & 95.377 & 109.396 & 106.351 & & 73.146 \\
\hline $11 / 5 / 075: 00$ & 124.457 & 122.199 & 118.599 & 99.816 & 103.725 & 95.401 & 109.394 & 106.373 & & 73.166 \\
\hline $11 / 5 / 075: 30$ & 124.452 & 122.217 & 118.619 & 99.849 & 103.75 & 95.427 & 109.392 & 106.397 & & 73.187 \\
\hline 11/5/07 6:00 & 124.452 & 122.245 & 118.649 & 99.874 & 103.778 & 95.46 & 109.386 & 106.426 & & 73.212 \\
\hline $11 / 5 / 076: 30$ & 124.457 & 124.208 & 119.929 & 99.9 & 103.799 & 95.481 & 109.39 & 106.448 & & 73.228 \\
\hline 11/5/07 7:00 & 124.457 & 124.51 & 120.284 & 99.924 & 103.828 & 95.507 & 109.387 & 106.475 & & 73.251 \\
\hline 11/5/07 7:30 & 126.069 & 124.685 & 120.468 & 100.724 & 104.361 & 96.172 & 110.01 & 107.956 & & 73.346 \\
\hline $11 / 5 / 078: 00$ & 126.317 & 124.798 & 120.592 & 100.96 & 104.654 & 96.471 & 110.256 & 108.292 & & 73.545 \\
\hline $11 / 5 / 078: 30$ & 126.445 & 123.8 & 120.685 & 101.113 & 104.829 & 96.645 & 110.393 & 108.476 & & 73.691 \\
\hline 11/5/07 9:00 & 126.532 & 122.923 & 119.38 & 101.215 & 104.942 & 96.758 & 110.477 & 108.598 & & 73.797 \\
\hline 11/5/07 9:30 & 126.603 & 122.766 & 119.193 & 100.831 & 105.026 & 96.839 & 110.53 & 108.684 & & 73.871 \\
\hline 11/5/07 10:00 & 124.974 & 122.696 & 119.11 & 100.503 & 104.483 & 96.165 & 109.844 & 107.192 & & 73.809 \\
\hline 11/5/07 10:30 & 124.837 & 122.655 & 119.065 & 100.395 & 104.338 & 96.016 & 109.69 & 107.019 & & 73.716 \\
\hline 11/5/07 11:00 & 124.774 & 122.635 & 119.042 & 100.34 & 104.269 & 95.955 & 109.625 & 106.942 & & 73.658 \\
\hline 11/5/07 11:30 & 124.748 & 122.626 & 119.035 & 100.309 & 104.23 & 95.915 & 109.593 & 106.898 & & 73.619 \\
\hline 11/5/07 12:00 & 124.783 & 122.614 & 119.022 & 100.3 & 104.211 & 95.894 & 109.58 & 106.871 & & 73.594 \\
\hline $11 / 5 / 0712: 30$ & 124.953 & 122.607 & 119.012 & 100.29 & 104.203 & 95.889 & 109.578 & 106.864 & & 73.59 \\
\hline 11/5/07 13:00 & 124.989 & 122.596 & 119.002 & 100.281 & 104.192 & 95.877 & 109.569 & 106.851 & & 73.576 \\
\hline 11/5/07 13:30 & 124.984 & 122.592 & 118.997 & 100.267 & 104.184 & 95.868 & 109.563 & 106.84 & & 73.569 \\
\hline $11 / 5 / 0714: 00$ & 124.974 & 122.587 & 118.997 & 100.264 & 104.173 & 95.861 & 109.557 & 106.831 & & 73.561 \\
\hline $11 / 5 / 0714: 30$ & 124.967 & 122.587 & 118.992 & 100.26 & 104.171 & 95.856 & 109.552 & 106.825 & & 73.555 \\
\hline 11/5/07 15:00 & 124.967 & 122.587 & 118.987 & 100.26 & 104.171 & 95.856 & 109.55 & 106.825 & & 73.555 \\
\hline $11 / 5 / 07$ 15:30 & 124.963 & 122.592 & 118.984 & 100.259 & 104.163 & 95.849 & 109.546 & 106.82 & & 73.551 \\
\hline 11/5/07 16:00 & 124.96 & 122.596 & 119.002 & 100.262 & 104.169 & 95.851 & 109.544 & 106.822 & & 73.555 \\
\hline $11 / 5 / 0716: 30$ & 124.967 & 122.603 & 119.007 & 100.267 & 104.172 & 95.858 & 109.548 & 106.827 & & 73.559 \\
\hline 11/5/07 17:00 & 124.972 & 122.612 & 119.019 & 100.269 & 104.174 & 95.861 & 109.552 & 106.831 & & 73.563 \\
\hline
\end{tabular}


TABLE S1.1 (Cont.)

Depth from Top of Casing (ft)

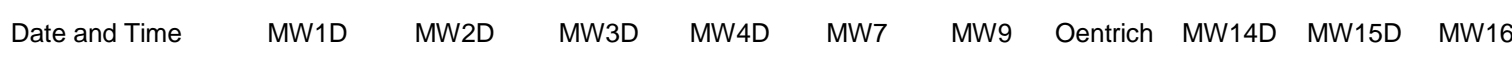

\begin{tabular}{|c|c|c|c|c|c|c|c|c|c|}
\hline 11/5/07 17:30 & 124.972 & 122.623 & 119.03 & 100.283 & 104.178 & 95.865 & 109.555 & 106.833 & 73.567 \\
\hline $11 / 5 / 07$ 18:00 & 124.984 & 122.637 & 119.037 & 100.286 & 104.195 & 95.877 & 109.567 & 106.849 & 73.58 \\
\hline 11/5/07 18:30 & 124.991 & 122.646 & 119.047 & 100.302 & 104.203 & 95.889 & 109.576 & 106.858 & 73.588 \\
\hline 11/5/07 19:00 & 124.998 & 122.655 & 119.062 & 100.314 & 104.214 & 95.901 & 109.583 & 106.869 & 73.598 \\
\hline 11/5/07 19:30 & 124.996 & 122.657 & 119.065 & 100.322 & 104.224 & 95.91 & 109.598 & 106.88 & 73.609 \\
\hline $11 / 5 / 0720: 00$ & 124.993 & 122.664 & 119.072 & 100.331 & 104.235 & 95.919 & 109.608 & 106.889 & 73.617 \\
\hline $11 / 5 / 0720: 30$ & 124.989 & 122.671 & 119.08 & 100.334 & 104.237 & 95.922 & 109.613 & 106.893 & 73.623 \\
\hline $11 / 5 / 0721: 00$ & 124.986 & 122.678 & 119.082 & 100.341 & 104.243 & 95.929 & 109.619 & 106.9 & 73.629 \\
\hline 11/5/07 21:30 & 124.982 & 122.682 & 119.09 & 100.347 & 104.249 & 95.938 & 109.623 & 106.906 & 73.636 \\
\hline $11 / 5 / 0722: 00$ & 124.979 & 122.689 & 119.093 & 100.353 & 104.256 & 95.943 & 109.629 & 106.911 & 73.64 \\
\hline 11/5/07 22:30 & 124.979 & 122.696 & 119.098 & 100.36 & 104.264 & 95.948 & 109.63 & 106.92 & 73.65 \\
\hline $11 / 5 / 0723: 00$ & 124.974 & 122.703 & 119.108 & 100.364 & 104.268 & 95.955 & 109.634 & 106.924 & 73.654 \\
\hline 11/5/07 23:30 & 124.974 & 122.707 & 119.113 & 100.371 & 104.275 & 95.962 & 109.642 & 106.931 & 73.66 \\
\hline $11 / 6 / 07$ 0:00 & 124.974 & 122.714 & 119.12 & 100.381 & 104.283 & 95.971 & 109.647 & 106.94 & 73.671 \\
\hline $11 / 6 / 07$ 0:30 & 124.972 & 122.716 & 119.12 & 100.384 & 104.289 & 95.976 & 109.657 & 106.944 & 73.679 \\
\hline 11/6/07 1:00 & 124.972 & 122.721 & 119.128 & 100.388 & 104.294 & 95.978 & 109.66 & 106.949 & 73.681 \\
\hline $11 / 6 / 07 \quad 1: 30$ & 124.972 & 122.723 & 119.128 & 100.393 & 104.294 & 95.983 & 109.662 & 106.951 & 73.683 \\
\hline $11 / 6 / 072: 00$ & 124.972 & 122.723 & 119.13 & 100.398 & 104.302 & 95.988 & 109.668 & 106.955 & 73.689 \\
\hline 11/6/07 2:30 & 124.972 & 122.725 & 119.13 & 100.395 & 104.302 & 95.99 & 109.674 & 106.96 & 73.694 \\
\hline 11/6/07 3:00 & 124.972 & 122.723 & 119.128 & 100.4 & 104.304 & 95.99 & 109.674 & 106.962 & 73.694 \\
\hline 11/6/07 3:30 & 124.972 & 122.716 & 119.125 & 100.4 & 104.306 & 95.993 & 109.678 & 106.962 & 73.698 \\
\hline $11 / 6 / 074: 00$ & 124.972 & 122.723 & 119.13 & 100.398 & 104.304 & 95.988 & 109.678 & 106.96 & 73.698 \\
\hline $11 / 6 / 074: 30$ & 124.97 & 122.73 & 119.135 & 100.397 & 104.296 & 95.978 & 109.675 & 106.953 & 73.691 \\
\hline $11 / 6 / 07$ 5:00 & 124.972 & 122.732 & 119.138 & 100.402 & 104.304 & 95.988 & 109.676 & 106.96 & 73.698 \\
\hline $11 / 6 / 075: 30$ & 124.97 & 122.737 & 119.145 & 100.407 & 104.31 & 95.995 & 109.679 & 106.964 & 73.708 \\
\hline $11 / 6 / 076: 00$ & 124.97 & 122.743 & 119.151 & 100.412 & 104.312 & 96 & 109.685 & 106.968 & 73.708 \\
\hline 11/6/07 6:30 & 124.972 & 124.061 & 119.153 & 100.415 & 104.317 & 96.002 & 109.689 & 106.975 & 73.714 \\
\hline $11 / 6 / 07$ 7:00 & 124.97 & 124.798 & 120.685 & 100.424 & 104.325 & 96.009 & 109.69 & 106.98 & 73.722 \\
\hline $11 / 6 / 077: 30$ & 125.752 & 124.997 & 120.925 & 100.956 & 104.367 & 96.054 & 109.788 & 106.995 & 73.722 \\
\hline $11 / 6 / 078: 00$ & 126.719 & 125.12 & 121.056 & 101.325 & 104.982 & 96.81 & 110.487 & 108.558 & 73.857 \\
\hline $11 / 6 / 078: 30$ & 126.906 & 125.204 & 121.141 & 101.494 & 105.178 & 97.013 & 110.672 & 108.784 & 74.012 \\
\hline 11/6/07 9:00 & 127.017 & 125.261 & 121.207 & 101.597 & 105.298 & 97.131 & 110.779 & 108.91 & 74.117 \\
\hline 11/6/07 9:30 & 127.099 & 125.299 & 121.25 & 101.67 & 105.382 & 97.216 & 110.857 & 109.001 & 74.194 \\
\hline 11/6/07 10:00 & 127.166 & 125.322 & 121.27 & 101.718 & 105.435 & 97.27 & 110.911 & 109.061 & 74.248 \\
\hline $11 / 6 / 07 \quad 10: 30$ & 127.22 & 125.34 & 121.292 & 101.753 & 105.475 & 97.31 & 110.945 & 109.103 & 74.283 \\
\hline 11/6/07 11:00 & 127.265 & 125.347 & 121.305 & 101.775 & 105.5 & 97.336 & 110.967 & 109.129 & 74.31 \\
\hline 11/6/07 11:30 & 127.303 & 125.351 & 121.308 & 101.785 & 105.515 & 97.353 & 110.988 & 109.145 & 74.326 \\
\hline $11 / 6 / 0712: 00$ & 127.341 & 125.354 & 121.305 & 101.796 & 105.524 & 97.362 & 111.001 & 109.156 & 74.339 \\
\hline $11 / 6 / 07$ 12:30 & 127.371 & 123.825 & 120.801 & 101.794 & 105.524 & 97.364 & 111.014 & 109.158 & 74.347 \\
\hline $11 / 6 / 07$ 13:00 & 127.407 & 123.299 & 119.753 & 101.794 & 105.522 & 97.36 & 111.024 & 109.156 & 74.345 \\
\hline 11/6/07 13:30 & 126.246 & 123.104 & 119.531 & 101.177 & 105.296 & 97.018 & 110.678 & 108.332 & 74.343 \\
\hline $11 / 6 / 07 \quad 14: 00$ & 125.74 & 122.995 & 119.41 & 100.858 & 104.839 & 96.525 & 110.227 & 107.568 & 74.198 \\
\hline 11/6/07 14:30 & 125.565 & 122.923 & 119.334 & 100.705 & 104.652 & 96.337 & 110.066 & 107.358 & 74.062 \\
\hline 11/6/07 15:00 & 125.454 & 122.868 & 119.279 & 100.615 & 104.548 & 96.235 & 109.976 & 107.243 & 73.971 \\
\hline $11 / 6 / 07 \quad 15: 30$ & 125.381 & 122.83 & 119.234 & 100.552 & 104.477 & 96.165 & 109.92 & 107.166 & 73.906 \\
\hline 11/6/07 16:00 & 125.327 & 122.809 & 119.214 & 100.509 & 104.428 & 96.11 & 109.882 & 107.108 & 73.859 \\
\hline $11 / 6 / 07$ 16:30 & 125.291 & 122.784 & 119.193 & 100.477 & 104.393 & 96.08 & 109.854 & 107.07 & 73.826 \\
\hline $11 / 6 / 07$ 17:00 & 125.268 & 122.766 & 119.171 & 100.457 & 104.369 & 96.054 & 109.835 & 107.046 & 73.805 \\
\hline $11 / 6 / 07 \quad 17: 30$ & 125.246 & 122.746 & 119.153 & 100.436 & 104.35 & 96.035 & 109.818 & 107.024 & 73.787 \\
\hline $11 / 6 / 07$ 18:00 & 125.23 & 122.739 & 119.143 & 100.417 & 104.329 & 96.014 & 109.807 & 107.002 & 73.768 \\
\hline $11 / 6 / 07$ 18:30 & 125.216 & 122.732 & 119.13 & 100.403 & 104.312 & 95.997 & 109.797 & 106.984 & 73.753 \\
\hline $11 / 6 / 07$ 19:00 & 125.199 & 122.725 & 119.113 & 100.397 & 104.308 & 95.992 & 109.788 & 106.975 & 73.745 \\
\hline 11/6/07 19:30 & 125.187 & 122.716 & 119.103 & 100.393 & 104.3 & 95.983 & 109.781 & 106.966 & 73.741 \\
\hline $11 / 6 / 0720: 00$ & 125.175 & 122.709 & 119.108 & 100.383 & 104.294 & 95.976 & 109.773 & 106.96 & 73.735 \\
\hline $11 / 6 / 0720: 30$ & 125.168 & 122.709 & 119.113 & 100.376 & 104.285 & 95.969 & 109.767 & 106.951 & 73.727 \\
\hline 11/6/07 21:00 & 125.159 & 122.707 & 119.108 & 100.372 & 104.277 & 95.964 & 109.764 & 106.946 & 73.725 \\
\hline $11 / 6 / 0721: 30$ & 125.149 & 122.707 & 119.108 & 100.371 & 104.275 & 95.962 & 109.758 & 106.942 & 73.72 \\
\hline 11/6/07 22:00 & 125.142 & 122.7 & 119.103 & 100.369 & 104.273 & 95.96 & 109.754 & 106.94 & 73.72 \\
\hline 11/6/07 22:30 & 125.135 & 122.689 & 119.095 & 100.371 & 104.275 & 95.96 & 109.751 & 106.94 & 73.72 \\
\hline 11/6/07 23:00 & 125.126 & 122.682 & 119.09 & 100.362 & 104.272 & 95.955 & 109.745 & 106.935 & 73.716 \\
\hline 11/6/07 23:30 & 125.121 & 122.671 & 119.077 & 100.353 & 104.26 & 95.945 & 109.743 & 106.929 & 73.71 \\
\hline $11 / 7 / 070: 00$ & 125.116 & 122.662 & 119.067 & 100.345 & 104.256 & 95.936 & 109.739 & 106.918 & 73.704 \\
\hline 11/7/07 0:30 & 125.109 & 122.646 & 119.055 & 100.333 & 104.245 & 95.927 & 109.737 & 106.906 & 73.696 \\
\hline 11/7/07 1:00 & 125.102 & 122.632 & 119.035 & 100.324 & 104.232 & 95.917 & 109.734 & 106.898 & 73.683 \\
\hline 11/7/07 1:30 & 125.097 & 122.621 & 119.027 & 100.307 & 104.224 & 95.903 & 109.732 & 106.884 & 73.671 \\
\hline 11/7/07 2:00 & 125.09 & 122.614 & 119.019 & 100.295 & 104.222 & 95.891 & 109.728 & 106.871 & 73.66 \\
\hline
\end{tabular}


TABLE S1.1 (Cont.)

\begin{tabular}{|c|c|c|c|c|c|c|c|c|c|c|}
\hline \multirow[b]{2}{*}{ Date and Time } & \multicolumn{10}{|c|}{ Depth from Top of Casing (ft) } \\
\hline & MW1D & MW2D & MW3D & MW4D & MW7 & MW9 & Oentrich & MW14D & MW15D & MW16D \\
\hline 11/7/07 2:30 & 125.086 & 122.61 & 119.014 & 100.285 & 104.226 & 95.877 & 109.726 & 106.858 & & 73.648 \\
\hline 11/7/07 3:00 & 125.078 & 122.596 & 119.002 & 100.279 & 104.218 & 95.872 & 109.721 & 106.853 & & 73.648 \\
\hline 11/7/07 3:30 & 125.071 & 122.585 & 118.989 & 100.272 & 104.216 & 95.868 & 109.721 & 106.845 & & 73.64 \\
\hline 11/7/07 4:00 & 125.069 & 122.576 & 118.982 & 100.257 & 104.214 & 95.856 & 109.719 & 106.833 & & 73.627 \\
\hline 11/7/07 4:30 & 125.06 & 122.567 & 118.969 & 100.25 & 104.213 & 95.846 & 109.717 & 106.822 & & 73.619 \\
\hline 11/7/07 5:00 & 125.052 & 122.56 & 118.944 & 100.238 & 104.211 & 95.835 & 109.717 & 106.814 & & 73.609 \\
\hline 11/7/07 5:30 & 125.045 & 122.551 & 118.939 & 100.231 & 104.207 & 95.823 & 109.711 & 106.802 & & 73.598 \\
\hline 11/7/07 6:00 & 125.038 & 122.537 & 118.921 & 100.224 & 104.205 & 95.816 & 109.711 & 106.794 & & 73.596 \\
\hline 11/7/07 6:30 & 125.036 & 122.526 & 118.929 & 100.209 & 104.203 & 95.809 & 109.709 & 106.787 & & 73.59 \\
\hline 11/7/07 7:00 & 125.029 & 124.503 & 120.232 & 100.2 & 104.203 & 95.79 & 109.709 & 106.769 & & 73.572 \\
\hline 11/7/07 7:30 & 125.022 & 124.737 & 120.514 & 100.393 & 104.201 & 95.78 & 109.707 & 106.76 & & 73.565 \\
\hline 11/7/07 8:00 & 126.669 & 124.857 & 120.647 & 100.981 & 104.744 & 96.447 & 110.374 & 108.255 & & 73.642 \\
\hline 11/7/07 8:30 & 126.884 & 124.92 & 120.723 & 101.163 & 104.978 & 96.685 & 110.595 & 108.525 & & 73.801 \\
\hline 11/7/07 9:00 & 126.998 & 124.97 & 120.773 & 101.255 & 105.1 & 96.806 & 110.71 & 108.653 & & 73.911 \\
\hline 11/7/07 9:30 & 127.078 & 123.503 & 120.781 & 101.318 & 105.176 & 96.867 & 110.776 & 108.724 & & 73.971 \\
\hline 11/7/07 10:00 & 127.133 & 122.929 & 119.38 & 101.358 & 105.224 & 96.919 & 110.823 & 108.775 & & 74.02 \\
\hline 11/7/07 10:30 & 126.074 & 122.739 & 119.156 & 100.813 & 105.121 & 96.702 & 110.609 & 108.153 & & 74.03 \\
\hline 11/7/07 11:00 & 125.504 & 122.63 & 119.04 & 100.484 & 104.627 & 96.153 & 110.119 & 107.199 & & 73.894 \\
\hline 11/7/07 11:30 & 125.362 & 122.551 & 118.959 & 100.338 & 104.441 & 95.967 & 109.961 & 106.986 & & 73.758 \\
\hline 11/7/07 12:00 & 125.286 & 122.49 & 118.901 & 100.247 & 104.352 & 95.863 & 109.874 & 106.869 & & 73.665 \\
\hline 11/7/07 12:30 & 125.242 & 122.44 & 118.848 & 100.178 & 104.277 & 95.787 & 109.807 & 106.789 & & 73.594 \\
\hline 11/7/07 13:00 & 125.208 & 122.399 & 118.805 & 100.121 & 104.222 & 95.731 & 109.762 & 106.727 & & 73.541 \\
\hline 11/7/07 13:30 & 125.173 & 122.363 & 118.767 & 100.074 & 104.167 & 95.684 & 109.711 & 106.676 & & 73.493 \\
\hline 11/7/07 14:00 & 125.147 & 122.338 & 118.745 & 100.038 & 104.135 & 95.641 & 109.675 & 106.634 & & 73.458 \\
\hline 11/7/07 14:30 & 125.121 & 122.308 & 118.717 & 100.005 & 104.102 & 95.608 & 109.644 & 106.597 & & 73.425 \\
\hline 11/7/07 15:00 & 125.1 & 122.29 & 118.697 & 99.98 & 104.081 & 95.585 & 109.623 & 106.572 & & 73.404 \\
\hline 11/7/07 15:30 & 125.081 & 122.279 & 118.672 & 99.955 & 104.045 & 95.559 & 109.597 & 106.543 & & 73.377 \\
\hline 11/7/07 16:00 & 125.05 & 122.265 & 118.654 & 99.938 & 104.03 & 95.535 & 109.578 & 106.526 & & 73.359 \\
\hline 11/7/07 16:30 & 125 & 122.256 & 118.642 & 99.931 & 104.018 & 95.528 & 109.557 & 106.508 & & 73.344 \\
\hline 11/7/07 17:00 & 124.944 & 122.247 & 118.647 & 99.921 & 104.011 & 95.514 & 109.552 & 106.497 & & 73.334 \\
\hline 11/7/07 17:30 & 124.885 & 122.242 & 118.647 & 99.912 & 103.992 & 95.507 & 109.54 & 106.486 & & 73.326 \\
\hline 11/7/07 18:00 & 124.835 & 122.245 & 118.644 & 99.9 & 103.984 & 95.505 & 109.531 & 106.481 & & 73.317 \\
\hline 11/7/07 18:30 & 124.795 & 122.242 & 118.644 & 99.905 & 103.972 & 95.493 & 109.529 & 106.473 & & 73.309 \\
\hline 11/7/07 19:00 & 124.762 & 122.251 & 118.652 & 99.905 & 103.976 & 95.498 & 109.527 & 106.475 & & 73.309 \\
\hline 11/7/07 19:30 & 124.736 & 122.245 & 118.647 & 99.909 & 103.976 & 95.493 & 109.523 & 106.473 & & 73.305 \\
\hline 11/7/07 20:00 & 124.722 & 122.238 & 118.642 & 99.911 & 103.975 & 95.507 & 109.521 & 106.484 & & 73.317 \\
\hline 11/7/07 20:30 & 124.717 & 122.233 & 118.634 & 99.904 & 103.976 & 95.502 & 109.52 & 106.479 & & 73.311 \\
\hline 11/7/07 21:00 & 124.714 & 122.231 & 118.634 & 99.897 & 103.976 & 95.493 & 109.521 & 106.47 & & 73.303 \\
\hline 11/7/07 21:30 & 124.71 & 122.236 & 118.636 & 99.892 & 103.971 & 95.483 & 109.522 & 106.462 & & 73.295 \\
\hline 11/7/07 22:00 & 124.705 & 122.233 & 118.639 & 99.89 & 103.963 & 95.483 & 109.518 & 106.462 & & 73.292 \\
\hline 11/7/07 22:30 & 124.7 & 122.233 & 118.634 & 99.897 & 103.963 & 95.488 & 109.512 & 106.464 & & 73.295 \\
\hline 11/7/07 23:00 & 124.696 & 122.236 & 118.636 & 99.899 & 103.967 & 95.49 & 109.51 & 106.466 & & 73.295 \\
\hline 11/7/07 23:30 & 124.693 & 122.245 & 118.644 & 99.899 & 103.965 & 95.486 & 109.51 & 106.464 & & 73.292 \\
\hline 11/8/07 0:00 & 124.688 & 122.24 & 118.639 & 99.902 & 103.963 & 95.49 & 109.509 & 106.466 & & 73.292 \\
\hline 11/8/07 0:30 & 124.686 & 122.242 & 118.642 & 99.911 & 103.961 & 95.5 & 109.506 & 106.475 & & 73.303 \\
\hline 11/8/07 1:00 & 124.681 & 122.236 & 118.642 & 99.911 & 103.963 & 95.495 & 109.51 & 106.47 & & 73.295 \\
\hline 11/8/07 1:30 & 124.679 & 122.236 & 118.639 & 99.905 & 103.961 & 95.5 & 109.506 & 106.473 & & 73.299 \\
\hline 11/8/07 2:00 & 124.677 & 122.229 & 118.626 & 99.904 & 103.963 & 95.493 & 109.508 & 106.466 & & 73.29 \\
\hline 11/8/07 2:30 & 124.674 & 122.233 & 118.634 & 99.9 & 103.961 & 95.49 & 109.508 & 106.466 & & 73.288 \\
\hline 11/8/07 3:00 & 124.672 & 122.238 & 118.642 & 99.899 & 103.965 & 95.486 & 109.506 & 106.457 & & 73.28 \\
\hline 11/8/07 3:30 & 124.667 & 122.231 & 118.634 & 99.907 & 103.961 & 95.493 & 109.508 & 106.466 & & 73.286 \\
\hline 11/8/07 4:00 & 124.665 & 122.231 & 118.634 & 99.902 & 103.961 & 95.495 & 109.507 & 106.468 & & 73.288 \\
\hline 11/8/07 4:30 & 124.665 & 122.231 & 118.634 & 99.897 & 103.965 & 95.493 & 109.508 & 106.464 & & 73.282 \\
\hline 11/8/07 5:00 & 124.662 & 122.238 & 118.639 & 99.897 & 103.961 & 95.488 & 109.506 & 106.462 & & 73.28 \\
\hline 11/8/07 5:30 & 124.66 & 122.245 & 118.647 & 99.902 & 103.961 & 95.49 & 109.506 & 106.462 & & 73.278 \\
\hline 11/8/07 6:00 & 124.655 & 122.238 & 118.647 & 99.907 & 103.959 & 95.498 & 109.506 & 106.468 & & 73.282 \\
\hline 11/8/07 6:30 & 124.655 & 122.24 & 118.647 & 99.914 & 103.961 & 95.505 & 109.508 & 106.474 & & 73.29 \\
\hline 11/8/07 7:00 & 124.658 & 124.036 & 119.821 & 99.912 & 103.963 & 95.5 & 109.508 & 106.473 & & 73.282 \\
\hline 11/8/07 7:30 & 124.655 & 124.399 & 120.305 & 99.911 & 103.963 & 95.5 & 109.51 & 106.473 & & 73.282 \\
\hline 11/8/07 8:00 & 126.152 & 124.571 & 120.491 & 100.646 & 104.371 & 96.044 & 110.057 & 107.732 & & 73.307 \\
\hline 11/8/07 8:30 & 126.483 & 124.671 & 120.612 & 100.908 & 104.713 & 96.405 & 110.387 & 108.166 & & 73.491 \\
\hline 11/8/07 9:00 & 126.629 & 124.732 & 120.678 & 101.055 & 104.869 & 96.575 & 110.533 & 108.352 & & 73.634 \\
\hline 11/8/07 9:30 & 126.721 & 124.771 & 120.715 & 101.137 & 104.957 & 96.683 & 110.618 & 108.465 & & 73.733 \\
\hline $11 / 8 / 0710: 00$ & 126.795 & 124.798 & 120.746 & 101.186 & 105.02 & 96.74 & 110.678 & 108.527 & & 73.784 \\
\hline $11 / 8 / 0710: 30$ & 126.851 & 124.827 & 120.773 & 101.22 & 105.064 & 96.78 & 110.719 & 108.569 & & 73.826 \\
\hline 11/8/07 11:00 & 126.896 & 124.852 & 120.799 & 101.246 & 105.094 & 96.803 & 110.749 & 108.596 & & 73.849 \\
\hline
\end{tabular}


TABLE S1.1 (Cont.)

Depth from Top of Casing (ft)

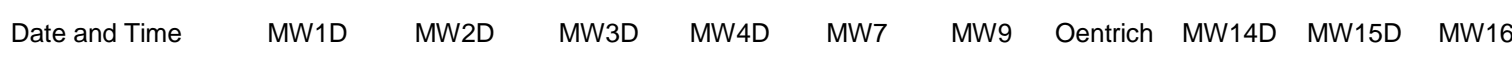

\begin{tabular}{|c|c|c|c|c|c|c|c|c|c|}
\hline 11/8/07 11:30 & 126.939 & 124.864 & 120.819 & 101.279 & 105.119 & 96.834 & 110.772 & 108.624 & 73.875 \\
\hline $11 / 8 / 07$ 12:00 & 126.969 & 124.87 & 120.824 & 101.298 & 105.138 & 96.86 & 110.789 & 108.651 & 73.898 \\
\hline 11/8/07 12:30 & 127.021 & 124.875 & 120.829 & 101.305 & 105.155 & 96.872 & 110.806 & 108.667 & 73.913 \\
\hline $11 / 8 / 0713: 00$ & 127.17 & 124.866 & 120.829 & 101.313 & 105.168 & 96.879 & 110.821 & 108.673 & 73.923 \\
\hline 11/8/07 13:30 & 127.296 & 124.87 & 120.824 & 101.315 & 105.18 & 96.884 & 110.832 & 108.678 & 73.929 \\
\hline $11 / 8 / 0714: 00$ & 127.334 & 123.601 & 120.834 & 101.31 & 105.191 & 96.876 & 110.843 & 108.673 & 73.927 \\
\hline $11 / 8 / 0714: 30$ & 127.343 & 122.868 & 119.324 & 101.315 & 105.201 & 96.874 & 110.851 & 108.669 & 73.927 \\
\hline $11 / 8 / 07$ 15:00 & 126.504 & 122.664 & 119.09 & 100.779 & 105.143 & 96.789 & 110.719 & 108.529 & 73.944 \\
\hline $11 / 8 / 0715: 30$ & 125.44 & 122.56 & 118.977 & 100.412 & 104.559 & 96.084 & 110.087 & 107.135 & 73.801 \\
\hline $11 / 8 / 07 \quad 16: 00$ & 125.14 & 122.487 & 118.901 & 100.26 & 104.369 & 95.891 & 109.905 & 106.911 & 73.665 \\
\hline 11/8/07 16:30 & 124.986 & 122.444 & 118.846 & 100.173 & 104.271 & 95.795 & 109.805 & 106.802 & 73.58 \\
\hline $11 / 8 / 07 \quad 17: 00$ & 124.896 & 122.415 & 118.825 & 100.112 & 104.205 & 95.726 & 109.747 & 106.725 & 73.514 \\
\hline $11 / 8 / 07 \quad 17: 30$ & 124.835 & 122.406 & 118.813 & 100.079 & 104.159 & 95.686 & 109.702 & 106.678 & 73.474 \\
\hline 11/8/07 18:00 & 124.797 & 122.392 & 118.798 & 100.064 & 104.131 & 95.66 & 109.675 & 106.65 & 73.448 \\
\hline 11/8/07 18:30 & 124.778 & 122.392 & 118.8 & 100.059 & 104.11 & 95.655 & 109.653 & 106.639 & 73.439 \\
\hline $11 / 8 / 07 \quad 19: 00$ & 124.769 & 122.388 & 118.79 & 100.052 & 104.096 & 95.637 & 109.64 & 106.623 & 73.423 \\
\hline 11/8/07 19:30 & 124.764 & 122.388 & 118.793 & 100.043 & 104.085 & 95.644 & 109.627 & 106.628 & 73.425 \\
\hline $11 / 8 / 0720: 00$ & 124.757 & 122.378 & 118.783 & 100.043 & 104.075 & 95.637 & 109.619 & 106.616 & 73.419 \\
\hline $11 / 8 / 0720: 30$ & 124.752 & 122.388 & 118.79 & 100.045 & 104.066 & 95.641 & 109.613 & 106.619 & 73.421 \\
\hline 11/8/07 21:00 & 124.75 & 122.383 & 118.785 & 100.038 & 104.064 & 95.63 & 109.61 & 106.612 & 73.41 \\
\hline 11/8/07 21:30 & 124.74 & 122.378 & 118.785 & 100.043 & 104.058 & 95.641 & 109.602 & 106.619 & 73.419 \\
\hline 11/8/07 22:00 & 124.738 & 122.392 & 118.793 & 100.042 & 104.053 & 95.634 & 109.598 & 106.612 & 73.412 \\
\hline 11/8/07 22:30 & 124.736 & 122.385 & 118.793 & 100.045 & 104.049 & 95.632 & 109.597 & 106.61 & 73.41 \\
\hline $11 / 8 / 0723: 00$ & 124.729 & 122.374 & 118.783 & 100.055 & 104.043 & 95.644 & 109.591 & 106.621 & 73.421 \\
\hline $11 / 8 / 0723: 30$ & 124.729 & 122.367 & 118.773 & 100.045 & 104.043 & 95.641 & 109.591 & 106.619 & 73.419 \\
\hline $11 / 9 / 070: 00$ & 124.729 & 122.367 & 118.773 & 100.033 & 104.043 & 95.63 & 109.591 & 106.608 & 73.406 \\
\hline $11 / 9 / 070: 30$ & 124.726 & 122.374 & 118.778 & 100.03 & 104.039 & 95.62 & 109.587 & 106.597 & 73.398 \\
\hline 11/9/07 1:00 & 124.719 & 122.376 & 118.778 & 100.036 & 104.037 & 95.625 & 109.583 & 106.599 & 73.402 \\
\hline 11/9/07 1:30 & 124.717 & 122.374 & 118.78 & 100.042 & 104.033 & 95.632 & 109.58 & 106.605 & 73.41 \\
\hline $11 / 9 / 072: 00$ & 124.714 & 122.376 & 118.778 & 100.043 & 104.032 & 95.632 & 109.58 & 106.605 & 73.406 \\
\hline $11 / 9 / 072: 30$ & 124.712 & 122.385 & 118.785 & 100.042 & 104.028 & 95.634 & 109.578 & 106.608 & 73.408 \\
\hline $11 / 9 / 07$ 3:00 & 124.71 & 122.39 & 118.788 & 100.045 & 104.026 & 95.634 & 109.574 & 106.605 & 73.406 \\
\hline 11/9/07 3:30 & 124.707 & 122.39 & 118.78 & 100.054 & 104.024 & 95.644 & 109.572 & 106.612 & 73.414 \\
\hline 11/9/07 4:00 & 124.705 & 122.394 & 118.785 & 100.059 & 104.02 & 95.646 & 109.571 & 106.619 & 73.419 \\
\hline $11 / 9 / 074: 30$ & 124.705 & 122.399 & 118.803 & 100.055 & 104.02 & 95.648 & 109.567 & 106.619 & 73.421 \\
\hline 11/9/07 5:00 & 124.703 & 122.412 & 118.81 & 100.062 & 104.018 & 95.651 & 109.565 & 106.621 & 73.417 \\
\hline 11/9/07 5:30 & 124.705 & 122.419 & 118.825 & 100.067 & 104.016 & 95.66 & 109.565 & 106.63 & 73.427 \\
\hline 11/9/07 6:00 & 124.705 & 122.428 & 118.83 & 100.085 & 104.014 & 95.672 & 109.561 & 106.641 & 73.437 \\
\hline 11/9/07 6:30 & 124.703 & 122.428 & 118.83 & 100.09 & 104.012 & 95.684 & 109.559 & 106.652 & 73.445 \\
\hline $11 / 9 / 07$ 7:00 & 124.705 & 124.274 & 119.939 & 100.097 & 104.011 & 95.686 & 109.559 & 106.654 & 73.448 \\
\hline 11/9/07 7:30 & 124.705 & 124.619 & 120.383 & 100.098 & 104.015 & 95.688 & 109.559 & 106.659 & 73.45 \\
\hline $11 / 9 / 078: 00$ & 126.222 & 124.771 & 120.544 & 100.819 & 104.424 & 96.235 & 110.094 & 107.993 & 73.487 \\
\hline $11 / 9 / 078: 30$ & 126.52 & 124.861 & 120.642 & 101.065 & 104.726 & 96.575 & 110.385 & 108.396 & 73.685 \\
\hline 11/9/07 9:00 & 126.66 & 123.431 & 120.718 & 101.196 & 104.871 & 96.73 & 110.524 & 108.562 & 73.815 \\
\hline 11/9/07 9:30 & 126.757 & 122.923 & 119.352 & 101.277 & 104.959 & 96.817 & 110.607 & 108.66 & 73.898 \\
\hline 11/9/07 10:00 & 125.686 & 122.773 & 119.176 & 100.784 & 104.868 & 96.645 & 110.398 & 108.047 & 73.962 \\
\hline $11 / 9 / 07 \quad 10: 30$ & 125.164 & 122.694 & 119.09 & 100.507 & 104.424 & 96.16 & 109.946 & 107.185 & 73.861 \\
\hline $11 / 9 / 0711: 00$ & 125.041 & 122.651 & 119.042 & 100.391 & 104.275 & 96.016 & 109.809 & 107.017 & 73.76 \\
\hline 11/9/07 11:30 & 124.986 & 122.617 & 119.009 & 100.334 & 104.199 & 95.943 & 109.738 & 106.933 & 73.696 \\
\hline 11/9/07 12:00 & 124.953 & 122.587 & 118.979 & 100.297 & 104.15 & 95.901 & 109.698 & 106.884 & 73.654 \\
\hline $11 / 9 / 0712: 30$ & 124.939 & 122.567 & 118.969 & 100.267 & 104.121 & 95.868 & 109.675 & 106.853 & 73.621 \\
\hline $11 / 9 / 07$ 13:00 & 124.932 & 122.548 & 118.954 & 100.245 & 104.102 & 95.842 & 109.657 & 106.822 & 73.598 \\
\hline 11/9/07 13:30 & 124.927 & 122.533 & 118.939 & 100.224 & 104.087 & 95.82 & 109.642 & 106.798 & 73.576 \\
\hline $11 / 9 / 07 \quad 14: 00$ & 124.922 & 122.508 & 118.921 & 100.207 & 104.079 & 95.802 & 109.636 & 106.78 & 73.557 \\
\hline 11/9/07 14:30 & 124.913 & 122.499 & 118.904 & 100.191 & 104.07 & 95.787 & 109.627 & 106.765 & 73.547 \\
\hline 11/9/07 15:00 & 124.904 & 122.496 & 118.901 & 100.169 & 104.062 & 95.766 & 109.621 & 106.743 & 73.53 \\
\hline $11 / 9 / 07 \quad 15: 30$ & 124.889 & 122.492 & 118.896 & 100.164 & 104.056 & 95.754 & 109.615 & 106.729 & 73.518 \\
\hline 11/9/07 16:00 & 124.882 & 122.487 & 118.893 & 100.157 & 104.053 & 95.752 & 109.61 & 106.727 & 73.516 \\
\hline 11/9/07 16:30 & 124.873 & 122.483 & 118.891 & 100.157 & 104.047 & 95.75 & 109.604 & 106.723 & 73.514 \\
\hline $11 / 9 / 07 \quad 17: 00$ & 124.868 & 122.474 & 118.878 & 100.152 & 104.043 & 95.747 & 109.6 & 106.718 & 73.512 \\
\hline 11/9/07 17:30 & 124.863 & 122.471 & 118.876 & 100.147 & 104.041 & 95.743 & 109.601 & 106.714 & 73.507 \\
\hline $11 / 9 / 07$ 18:00 & 124.856 & 122.469 & 118.868 & 100.138 & 104.039 & 95.733 & 109.599 & 106.705 & 73.499 \\
\hline 11/9/07 18:30 & 124.849 & 122.469 & 118.871 & 100.136 & 104.037 & 95.731 & 109.595 & 106.703 & 73.499 \\
\hline 11/9/07 19:00 & 124.847 & 122.471 & 118.876 & 100.138 & 104.032 & 95.729 & 109.593 & 106.698 & 73.495 \\
\hline 11/9/07 19:30 & 124.844 & 122.469 & 118.873 & 100.136 & 104.032 & 95.731 & 109.591 & 106.701 & 73.497 \\
\hline $11 / 9 / 0720: 00$ & 124.844 & 122.469 & 118.876 & 100.136 & 104.03 & 95.729 & 109.587 & 106.701 & 73.495 \\
\hline
\end{tabular}


TABLE S1.1 (Cont.)

Depth from Top of Casing (ft)

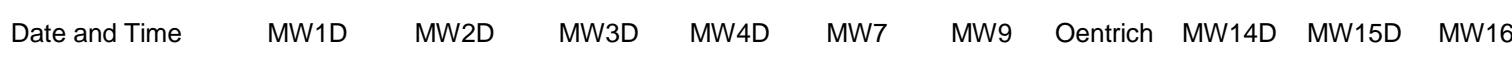

\begin{tabular}{|c|c|c|c|c|c|c|c|c|c|}
\hline 11/9/07 20:30 & 124.84 & 122.467 & 118.873 & 100.14 & 104.03 & 95.731 & 109.585 & 106.701 & 73.497 \\
\hline $11 / 9 / 0721: 00$ & 124.837 & 122.469 & 118.878 & 100.138 & 104.028 & 95.729 & 109.585 & 106.703 & 73.497 \\
\hline 11/9/07 21:30 & 124.837 & 122.467 & 118.875 & 100.136 & 104.026 & 95.726 & 109.585 & 106.698 & 73.497 \\
\hline 11/9/07 22:00 & 124.837 & 122.462 & 118.866 & 100.138 & 104.03 & 95.733 & 109.586 & 106.707 & 73.499 \\
\hline 11/9/07 22:30 & 124.835 & 122.46 & 118.866 & 100.133 & 104.028 & 95.726 & 109.583 & 106.698 & 73.497 \\
\hline $11 / 9 / 0723: 00$ & 124.83 & 122.458 & 118.863 & 100.129 & 104.024 & 95.719 & 109.584 & 106.692 & 73.491 \\
\hline 11/9/07 23:30 & 124.828 & 122.458 & 118.858 & 100.128 & 104.022 & 95.721 & 109.58 & 106.692 & 73.493 \\
\hline 11/10/07 0:00 & 124.826 & 122.449 & 118.856 & 100.126 & 104.02 & 95.717 & 109.58 & 106.69 & 73.491 \\
\hline $11 / 10 / 070: 30$ & 124.823 & 122.446 & 118.853 & 100.126 & 104.022 & 95.714 & 109.578 & 106.687 & 73.491 \\
\hline $11 / 10 / 071: 00$ & 124.821 & 122.446 & 118.846 & 100.117 & 104.018 & 95.707 & 109.578 & 106.683 & 73.487 \\
\hline 11/10/07 1:30 & 124.818 & 122.442 & 118.848 & 100.114 & 104.016 & 95.705 & 109.574 & 106.678 & 73.483 \\
\hline $11 / 10 / 072: 00$ & 124.816 & 122.433 & 118.838 & 100.116 & 104.016 & 95.705 & 109.574 & 106.676 & 73.481 \\
\hline $11 / 10 / 072: 30$ & 124.814 & 122.424 & 118.83 & 100.109 & 104.015 & 95.703 & 109.572 & 106.674 & 73.479 \\
\hline 11/10/07 3:00 & 124.814 & 122.417 & 118.825 & 100.105 & 104.012 & 95.693 & 109.572 & 106.663 & 73.47 \\
\hline 11/10/07 3:30 & 124.811 & 122.401 & 118.808 & 100.088 & 104.012 & 95.684 & 109.572 & 106.654 & 73.462 \\
\hline $11 / 10 / 074: 00$ & 124.809 & 122.394 & 118.803 & 100.081 & 104.013 & 95.677 & 109.57 & 106.647 & 73.456 \\
\hline $11 / 10 / 074: 30$ & 124.804 & 122.392 & 118.795 & 100.066 & 104.009 & 95.665 & 109.57 & 106.634 & 73.443 \\
\hline 11/10/07 5:00 & 124.802 & 122.394 & 118.795 & 100.064 & 104.009 & 95.655 & 109.568 & 106.628 & 73.435 \\
\hline 11/10/07 5:30 & 124.8 & 122.397 & 118.795 & 100.062 & 104.005 & 95.653 & 109.567 & 106.623 & 73.435 \\
\hline $11 / 10 / 076: 00$ & 124.797 & 122.385 & 118.793 & 100.066 & 104.003 & 95.653 & 109.567 & 106.625 & 73.433 \\
\hline 11/10/07 6:30 & 124.792 & 122.374 & 118.783 & 100.064 & 104.003 & 95.658 & 109.563 & 106.625 & 73.437 \\
\hline $11 / 10 / 07$ 7:00 & 124.795 & 122.367 & 118.775 & 100.05 & 104.001 & 95.646 & 109.565 & 106.616 & 73.427 \\
\hline 11/10/07 7:30 & 124.79 & 122.365 & 118.762 & 100.043 & 104.003 & 95.634 & 109.565 & 106.605 & 73.417 \\
\hline 11/10/07 8:00 & 124.788 & 122.363 & 118.769 & 100.036 & 104.001 & 95.629 & 109.565 & 106.601 & 73.41 \\
\hline $11 / 10 / 078: 30$ & 124.785 & 124.329 & 120.048 & 100.035 & 103.999 & 95.627 & 109.563 & 106.594 & 73.406 \\
\hline 11/10/07 9:00 & 124.785 & 124.582 & 120.355 & 100.045 & 104.001 & 95.622 & 109.565 & 106.592 & 73.404 \\
\hline 11/10/07 9:30 & 126.426 & 124.703 & 120.494 & 100.813 & 104.519 & 96.275 & 110.205 & 108.073 & 73.479 \\
\hline $11 / 10 / 07$ 10:00 & 126.667 & 124.771 & 120.569 & 101.003 & 104.772 & 96.523 & 110.447 & 108.361 & 73.644 \\
\hline $11 / 10 / 07$ 10:30 & 126.797 & 124.814 & 120.617 & 101.098 & 104.902 & 96.643 & 110.569 & 108.494 & 73.749 \\
\hline $11 / 10 / 07$ 11:00 & 126.882 & 124.841 & 120.652 & 101.162 & 104.98 & 96.711 & 110.642 & 108.567 & 73.813 \\
\hline 11/10/07 11:30 & 126.946 & 124.857 & 120.665 & 101.194 & 105.033 & 96.754 & 110.687 & 108.611 & 73.857 \\
\hline $11 / 10 / 07$ 12:00 & 126.988 & 124.859 & 120.67 & 101.222 & 105.071 & 96.789 & 110.725 & 108.647 & 73.89 \\
\hline $11 / 10 / 07$ 12:30 & 127.026 & 124.846 & 120.665 & 101.237 & 105.098 & 96.801 & 110.748 & 108.66 & 73.902 \\
\hline $11 / 10 / 07$ 13:00 & 127.062 & 124.821 & 120.637 & 101.23 & 105.119 & 96.803 & 110.764 & 108.664 & 73.911 \\
\hline $11 / 10 / 07$ 13:30 & 127.116 & 124.805 & 120.617 & 101.213 & 105.124 & 96.794 & 110.776 & 108.653 & 73.904 \\
\hline $11 / 10 / 07$ 14:00 & 127.185 & 124.8 & 120.615 & 101.187 & 105.115 & 96.768 & 110.783 & 108.629 & 73.884 \\
\hline $11 / 10 / 07$ 14:30 & 127.222 & 124.78 & 120.602 & 101.182 & 105.094 & 96.752 & 110.755 & 108.613 & 73.878 \\
\hline $11 / 10 / 07$ 15:00 & 127.241 & 122.984 & 119.516 & 101.168 & 105.092 & 96.744 & 110.746 & 108.605 & 73.873 \\
\hline 11/10/07 15:30 & 127.237 & 122.653 & 119.093 & 101.151 & 105.083 & 96.73 & 110.733 & 108.589 & 73.861 \\
\hline $11 / 10 / 07$ 16:00 & 125.653 & 122.494 & 118.914 & 100.455 & 104.666 & 96.188 & 110.197 & 107.301 & 73.818 \\
\hline 11/10/07 16:30 & 125.175 & 122.419 & 118.82 & 100.219 & 104.355 & 95.872 & 109.893 & 106.909 & 73.663 \\
\hline $11 / 10 / 07$ 17:00 & 124.948 & 122.36 & 118.77 & 100.093 & 104.205 & 95.719 & 109.758 & 106.736 & 73.536 \\
\hline $11 / 10 / 07$ 17:30 & 124.818 & 122.315 & 118.72 & 100.04 & 104.123 & 95.646 & 109.675 & 106.65 & 73.466 \\
\hline $11 / 10 / 07$ 18:00 & 124.755 & 122.295 & 118.677 & 99.988 & 104.077 & 95.599 & 109.629 & 106.592 & 73.419 \\
\hline $11 / 10 / 07$ 18:30 & 124.71 & 122.29 & 118.674 & 99.947 & 104.036 & 95.549 & 109.591 & 106.546 & 73.373 \\
\hline $11 / 10 / 07$ 19:00 & 124.67 & 122.279 & 118.664 & 99.94 & 104.007 & 95.535 & 109.565 & 106.521 & 73.352 \\
\hline $11 / 10 / 07$ 19:30 & 124.655 & 122.281 & 118.672 & 99.938 & 103.988 & 95.535 & 109.548 & 106.519 & 73.352 \\
\hline $11 / 10 / 0720: 00$ & 124.653 & 122.267 & 118.664 & 99.931 & 103.975 & 95.521 & 109.535 & 106.504 & 73.338 \\
\hline $11 / 10 / 0720: 30$ & 124.646 & 122.261 & 118.664 & 99.928 & 103.965 & 95.528 & 109.525 & 106.512 & 73.342 \\
\hline $11 / 10 / 0721: 00$ & 124.644 & 122.261 & 118.662 & 99.918 & 103.961 & 95.509 & 109.52 & 106.492 & 73.323 \\
\hline $11 / 10 / 0721: 30$ & 124.634 & 122.258 & 118.649 & 99.918 & 103.956 & 95.507 & 109.516 & 106.488 & 73.319 \\
\hline $11 / 10 / 0722: 00$ & 124.632 & 122.254 & 118.649 & 99.916 & 103.95 & 95.509 & 109.512 & 106.49 & 73.319 \\
\hline 11/10/07 22:30 & 124.629 & 122.247 & 118.649 & 99.911 & 103.946 & 95.505 & 109.508 & 106.484 & 73.313 \\
\hline $11 / 10 / 0723: 00$ & 124.627 & 122.238 & 118.642 & 99.907 & 103.942 & 95.5 & 109.505 & 106.477 & 73.309 \\
\hline $11 / 10 / 07$ 23:30 & 124.622 & 122.233 & 118.636 & 99.902 & 103.942 & 95.497 & 109.505 & 106.473 & 73.305 \\
\hline 11/11/07 0:00 & 124.622 & 122.224 & 118.626 & 99.892 & 103.942 & 95.488 & 109.503 & 106.466 & 73.295 \\
\hline $11 / 11 / 070: 30$ & 124.615 & 122.217 & 118.621 & 99.888 & 103.938 & 95.483 & 109.501 & 106.459 & 73.29 \\
\hline 11/11/07 1:00 & 124.613 & 122.213 & 118.609 & 99.881 & 103.938 & 95.476 & 109.501 & 106.45 & 73.282 \\
\hline 11/11/07 1:30 & 124.611 & 122.202 & 118.606 & 99.873 & 103.935 & 95.469 & 109.497 & 106.444 & 73.276 \\
\hline $11 / 11 / 072: 00$ & 124.606 & 122.197 & 118.601 & 99.871 & 103.935 & 95.462 & 109.495 & 106.437 & 73.27 \\
\hline 11/11/07 2:30 & 124.606 & 122.199 & 118.606 & 99.859 & 103.923 & 95.455 & 109.495 & 106.431 & 73.261 \\
\hline $11 / 11 / 073: 00$ & 124.603 & 122.199 & 118.606 & 99.862 & 103.925 & 95.45 & 109.491 & 106.424 & 73.257 \\
\hline 11/11/07 3:30 & 124.599 & 122.193 & 118.599 & 99.864 & 103.927 & 95.453 & 109.491 & 106.426 & 73.259 \\
\hline $11 / 11 / 074: 00$ & 124.599 & 122.197 & 118.601 & 99.856 & 103.923 & 95.455 & 109.49 & 106.426 & 73.259 \\
\hline 11/11/07 4:30 & 124.599 & 122.188 & 118.594 & 99.859 & 103.921 & 95.446 & 109.488 & 106.419 & 73.249 \\
\hline 11/11/07 5:00 & 124.596 & 122.186 & 118.591 & 99.857 & 103.921 & 95.453 & 109.486 & 106.424 & 73.253 \\
\hline
\end{tabular}


TABLE S1.1 (Cont.)

\begin{tabular}{|c|c|c|c|c|c|c|c|c|c|c|}
\hline \multirow[b]{2}{*}{ Date and Time } & \multicolumn{10}{|c|}{ Depth from Top of Casing (ft) } \\
\hline & MW1D & MW2D & MW3D & MW4D & MW7 & MW9 & Oentrich & MW14D & MW15D & MW16D \\
\hline 11/11/07 5:30 & 124.599 & 122.183 & 118.589 & 99.852 & 103.919 & 95.443 & 109.486 & 106.417 & & 73.247 \\
\hline $11 / 11 / 076: 00$ & 124.601 & 122.181 & 118.584 & 99.849 & 103.912 & 95.443 & 109.486 & 106.413 & & 73.243 \\
\hline 11/11/07 6:30 & 124.603 & 122.186 & 118.586 & 99.847 & 103.915 & 95.439 & 109.48 & 106.408 & & 73.239 \\
\hline 11/11/07 7:00 & 124.606 & 122.186 & 118.591 & 99.84 & 103.906 & 95.434 & 109.48 & 106.406 & & 73.235 \\
\hline $11 / 11 / 07$ 7:30 & 124.606 & 122.19 & 118.59 & 99.849 & 103.906 & 95.441 & 109.475 & 106.408 & & 73.237 \\
\hline 11/11/07 8:00 & 124.603 & 122.188 & 118.596 & 99.852 & 103.906 & 95.443 & 109.473 & 106.413 & & 73.241 \\
\hline $11 / 11 / 078: 30$ & 124.606 & 122.202 & 118.601 & 99.854 & 103.908 & 95.446 & 109.473 & 106.413 & & 73.241 \\
\hline 11/11/07 9:00 & 124.611 & 124.086 & 119.929 & 99.854 & 103.906 & 95.446 & 109.473 & 106.415 & & 73.239 \\
\hline 11/11/07 9:30 & 124.613 & 124.383 & 120.297 & 99.866 & 103.908 & 95.455 & 109.473 & 106.424 & & 73.247 \\
\hline $11 / 11 / 0710: 00$ & 126.189 & 124.521 & 120.458 & 100.66 & 104.399 & 96.098 & 110.102 & 107.805 & & 73.309 \\
\hline $11 / 11 / 07$ 10:30 & 126.449 & 124.612 & 120.552 & 100.888 & 104.681 & 96.395 & 110.374 & 108.148 & & 73.487 \\
\hline $11 / 11 / 0711: 00$ & 126.575 & 124.662 & 120.612 & 101.005 & 104.824 & 96.537 & 110.507 & 108.306 & & 73.611 \\
\hline $11 / 11 / 0711: 30$ & 126.65 & 124.696 & 120.65 & 101.079 & 104.905 & 96.626 & 110.584 & 108.401 & & 73.694 \\
\hline $11 / 11 / 07$ 12:00 & 126.705 & 124.716 & 120.67 & 101.12 & 104.963 & 96.678 & 110.641 & 108.456 & & 73.741 \\
\hline 11/11/07 12:30 & 126.745 & 123.811 & 120.685 & 101.148 & 105.005 & 96.707 & 110.68 & 108.492 & & 73.77 \\
\hline $11 / 11 / 07$ 13:00 & 126.776 & 122.759 & 119.224 & 101.163 & 105.035 & 96.725 & 110.712 & 108.514 & & 73.791 \\
\hline 11/11/07 13:30 & 126.806 & 122.558 & 118.984 & 100.691 & 105.058 & 96.74 & 110.733 & 108.529 & & 73.805 \\
\hline $11 / 11 / 0714: 00$ & 125.154 & 122.453 & 118.863 & 100.305 & 104.452 & 95.983 & 109.991 & 107.026 & & 73.698 \\
\hline $11 / 11 / 0714: 30$ & 124.986 & 122.392 & 118.8 & 100.152 & 104.253 & 95.785 & 109.813 & 106.8 & & 73.563 \\
\hline $11 / 11 / 0715: 00$ & 124.896 & 122.351 & 118.757 & 100.073 & 104.155 & 95.686 & 109.713 & 106.687 & & 73.476 \\
\hline $11 / 11 / 0715: 30$ & 124.842 & 122.322 & 118.73 & 100.024 & 104.095 & 95.636 & 109.657 & 106.625 & & 73.425 \\
\hline $11 / 11 / 0716: 00$ & 124.807 & 122.308 & 118.712 & 99.99 & 104.056 & 95.594 & 109.621 & 106.579 & & 73.383 \\
\hline 11/11/07 16:30 & 124.781 & 122.299 & 118.707 & 99.969 & 104.033 & 95.568 & 109.593 & 106.55 & & 73.359 \\
\hline $11 / 11 / 07$ 17:00 & 124.759 & 122.301 & 118.705 & 99.961 & 104.013 & 95.556 & 109.574 & 106.535 & & 73.344 \\
\hline $11 / 11 / 07$ 17:30 & 124.743 & 122.304 & 118.705 & 99.957 & 103.996 & 95.552 & 109.563 & 106.528 & & 73.338 \\
\hline $11 / 11 / 0718: 00$ & 124.726 & 122.308 & 118.707 & 99.961 & 103.986 & 95.556 & 109.55 & 106.528 & & 73.34 \\
\hline 11/11/07 18:30 & 124.717 & 122.319 & 118.724 & 99.966 & 103.978 & 95.559 & 109.542 & 106.53 & & 73.34 \\
\hline 11/11/07 19:00 & 124.71 & 122.326 & 118.73 & 99.973 & 103.973 & 95.561 & 109.535 & 106.535 & & 73.342 \\
\hline 11/11/07 19:30 & 124.7 & 122.34 & 118.742 & 99.983 & 103.967 & 95.573 & 109.531 & 106.546 & & 73.352 \\
\hline $11 / 11 / 0720: 00$ & 124.696 & 122.349 & 118.752 & 99.993 & 103.963 & 95.58 & 109.525 & 106.552 & & 73.359 \\
\hline $11 / 11 / 0720: 30$ & 124.688 & 122.36 & 118.762 & 100.005 & 103.959 & 95.592 & 109.521 & 106.563 & & 73.369 \\
\hline 11/11/07 21:00 & 124.684 & 122.367 & 118.77 & 100.016 & 103.957 & 95.606 & 109.518 & 106.574 & & 73.377 \\
\hline $11 / 11 / 0721: 30$ & 124.684 & 122.383 & 118.783 & 100.03 & 103.956 & 95.618 & 109.518 & 106.585 & & 73.388 \\
\hline $11 / 11 / 0722: 00$ & 124.681 & 122.392 & 118.798 & 100.036 & 103.954 & 95.622 & 109.516 & 106.592 & & 73.392 \\
\hline 11/11/07 22:30 & 124.679 & 122.41 & 118.815 & 100.054 & 103.952 & 95.639 & 109.514 & 106.608 & & 73.406 \\
\hline 11/11/07 23:00 & 124.679 & 122.422 & 118.825 & 100.066 & 103.952 & 95.648 & 109.51 & 106.619 & & 73.414 \\
\hline 11/11/07 23:30 & 124.677 & 122.433 & 118.838 & 100.083 & 103.959 & 95.672 & 109.508 & 106.639 & & 73.435 \\
\hline 11/12/07 0:00 & 124.679 & 122.437 & 118.843 & 100.093 & 103.955 & 95.681 & 109.508 & 106.65 & & 73.443 \\
\hline 11/12/07 0:30 & 124.681 & 122.437 & 118.851 & 100.1 & 103.956 & 95.691 & 109.514 & 106.659 & & 73.452 \\
\hline 11/12/07 1:00 & 124.686 & 122.44 & 118.848 & 100.105 & 103.954 & 95.695 & 109.514 & 106.665 & & 73.456 \\
\hline 11/12/07 1:30 & 124.688 & 122.444 & 118.853 & 100.109 & 103.954 & 95.7 & 109.516 & 106.667 & & 73.458 \\
\hline $11 / 12 / 072: 00$ & 124.688 & 122.451 & 118.858 & 100.11 & 103.952 & 95.698 & 109.518 & 106.667 & & 73.46 \\
\hline 11/12/07 2:30 & 124.691 & 122.453 & 118.858 & 100.116 & 103.952 & 95.707 & 109.518 & 106.674 & & 73.466 \\
\hline 11/12/07 3:00 & 124.693 & 122.458 & 118.865 & 100.121 & 103.95 & 95.712 & 109.518 & 106.681 & & 73.472 \\
\hline $11 / 12 / 073: 30$ & 124.698 & 122.467 & 118.871 & 100.123 & 103.948 & 95.714 & 109.516 & 106.683 & & 73.474 \\
\hline $11 / 12 / 074: 00$ & 124.698 & 122.469 & 118.878 & 100.133 & 103.946 & 95.719 & 109.518 & 106.687 & & 73.481 \\
\hline 11/12/07 4:30 & 124.698 & 122.48 & 118.883 & 100.142 & 103.944 & 95.728 & 109.514 & 106.694 & & 73.487 \\
\hline $11 / 12 / 075: 00$ & 124.703 & 122.499 & 118.901 & 100.142 & 103.944 & 95.733 & 109.516 & 106.701 & & 73.491 \\
\hline 11/12/07 5:30 & 124.705 & 122.51 & 118.911 & 100.159 & 103.957 & 95.743 & 109.512 & 106.705 & & 73.497 \\
\hline 11/12/07 6:00 & 124.705 & 122.524 & 118.931 & 100.171 & 103.959 & 95.761 & 109.516 & 106.725 & & 73.516 \\
\hline 11/12/07 6:30 & 124.707 & 122.528 & 118.931 & 100.186 & 103.974 & 95.776 & 109.512 & 106.738 & & 73.526 \\
\hline 11/12/07 7:00 & 124.712 & 124.551 & 120.277 & 100.198 & 103.99 & 95.79 & 109.512 & 106.754 & & 73.541 \\
\hline 11/12/07 7:30 & 124.712 & 124.8 & 120.582 & 100.498 & 103.99 & 95.792 & 109.51 & 106.756 & & 73.541 \\
\hline 11/12/07 8:00 & 126.374 & 124.932 & 120.728 & 101.039 & 104.566 & 96.504 & 110.184 & 108.299 & & 73.663 \\
\hline $11 / 12 / 078: 30$ & 126.594 & 125.018 & 120.821 & 101.237 & 104.818 & 96.758 & 110.413 & 108.582 & & 73.84 \\
\hline 11/12/07 9:00 & 126.712 & 125.088 & 120.884 & 101.351 & 104.951 & 96.89 & 110.535 & 108.726 & & 73.958 \\
\hline 11/12/07 9:30 & 126.795 & 125.14 & 120.947 & 101.422 & 105.035 & 96.98 & 110.616 & 108.822 & & 74.041 \\
\hline 11/12/07 10:00 & 126.858 & 125.174 & 120.985 & 101.496 & 105.1 & 97.044 & 110.674 & 108.885 & & 74.101 \\
\hline 11/12/07 10:30 & 126.906 & 125.202 & 121.018 & 101.541 & 105.159 & 97.103 & 110.734 & 108.948 & & 74.152 \\
\hline $11 / 12 / 0711: 00$ & 126.951 & 123.431 & 119.961 & 101.57 & 105.195 & 97.138 & 110.77 & 108.983 & & 74.186 \\
\hline 11/12/07 11:30 & 126.988 & 123.122 & 119.559 & 101.596 & 105.222 & 97.166 & 110.796 & 109.012 & & 74.214 \\
\hline 11/12/07 12:00 & 125.546 & 122.984 & 119.39 & 100.925 & 104.833 & 96.653 & 110.284 & 107.746 & & 74.194 \\
\hline $11 / 12 / 0712: 30$ & 125.277 & 122.893 & 119.292 & 100.712 & 104.55 & 96.36 & 110.006 & 107.378 & & 74.062 \\
\hline 11/12/07 13:00 & 125.173 & 122.843 & 119.239 & 100.602 & 104.418 & 96.226 & 109.888 & 107.23 & & 73.956 \\
\hline 11/12/07 13:30 & 125.119 & 122.816 & 119.208 & 100.526 & 104.344 & 96.139 & 109.82 & 107.135 & & 73.875 \\
\hline $11 / 12 / 07$ 14:00 & 125.088 & 122.782 & 119.178 & 100.488 & 104.294 & 96.094 & 109.775 & 107.079 & & 73.832 \\
\hline
\end{tabular}


TABLE S1.1 (Cont.)

\begin{tabular}{|c|c|c|c|c|c|c|c|c|c|c|}
\hline \multirow[b]{2}{*}{ Date and Time } & \multicolumn{10}{|c|}{ Depth from Top of Casing (ft) } \\
\hline & MW1D & MW2D & MW3D & MW4D & MW7 & MW9 & Oentrich & MW14D & MW15D & MW16D \\
\hline $11 / 12 / 07$ 14:30 & 125.074 & 122.764 & 119.173 & 100.46 & 104.262 & 96.065 & 109.745 & 107.05 & & 73.807 \\
\hline $11 / 12 / 07$ 15:00 & 125.116 & 122.757 & 119.166 & 100.438 & 104.243 & 96.035 & 109.726 & 107.017 & & 73.778 \\
\hline 11/12/07 15:30 & 125.138 & 122.755 & 119.161 & 100.422 & 104.222 & 96.016 & 109.709 & 106.995 & & 73.76 \\
\hline 11/12/07 16:00 & 125.133 & 122.746 & 119.151 & 100.414 & 104.209 & 96.014 & 109.696 & 106.988 & & 73.758 \\
\hline $11 / 12 / 07$ 16:30 & 125.128 & 122.739 & 119.148 & 100.414 & 104.209 & 96.011 & 109.685 & 106.984 & & 73.753 \\
\hline 11/12/07 17:00 & 125.123 & 122.739 & 119.14 & 100.409 & 104.195 & 96.004 & 109.679 & 106.975 & & 73.747 \\
\hline $11 / 12 / 07$ 17:30 & 125.119 & 122.739 & 119.145 & 100.398 & 104.195 & 95.997 & 109.677 & 106.968 & & 73.741 \\
\hline $11 / 12 / 07$ 18:00 & 125.114 & 122.743 & 119.151 & 100.398 & 104.191 & 95.995 & 109.67 & 106.968 & & 73.743 \\
\hline 11/12/07 18:30 & 125.112 & 122.728 & 119.138 & 100.407 & 104.192 & 95.992 & 109.67 & 106.966 & & 73.741 \\
\hline 11/12/07 19:00 & 125.119 & 122.725 & 119.138 & 100.403 & 104.195 & 96 & 109.674 & 106.975 & & 73.749 \\
\hline $11 / 12 / 07$ 19:30 & 125.114 & 122.723 & 119.125 & 100.395 & 104.188 & 95.985 & 109.674 & 106.96 & & 73.737 \\
\hline 11/12/07 20:00 & 125.109 & 122.716 & 119.122 & 100.393 & 104.184 & 95.981 & 109.668 & 106.955 & & 73.735 \\
\hline $11 / 12 / 0720: 30$ & 125.102 & 122.725 & 119.125 & 100.388 & 104.178 & 95.981 & 109.664 & 106.953 & & 73.735 \\
\hline $11 / 12 / 0721: 00$ & 125.1 & 122.734 & 119.138 & 100.374 & 104.176 & 95.978 & 109.664 & 106.951 & & 73.733 \\
\hline 11/12/07 21:30 & 125.093 & 122.723 & 119.128 & 100.398 & 104.172 & 95.976 & 109.659 & 106.949 & & 73.733 \\
\hline $11 / 12 / 0722: 00$ & 125.086 & 122.721 & 119.128 & 100.398 & 104.182 & 95.99 & 109.662 & 106.962 & & 73.745 \\
\hline $11 / 12 / 0722: 30$ & 125.088 & 122.698 & 119.113 & 100.388 & 104.176 & 95.978 & 109.666 & 106.953 & & 73.737 \\
\hline 11/12/07 23:00 & 125.083 & 122.678 & 119.082 & 100.376 & 104.174 & 95.978 & 109.664 & 106.953 & & 73.737 \\
\hline $11 / 12 / 0723: 30$ & 125.083 & 122.673 & 119.082 & 100.357 & 104.173 & 95.957 & 109.664 & 106.933 & & 73.72 \\
\hline 11/13/07 0:00 & 125.081 & 122.657 & 119.065 & 100.331 & 104.174 & 95.931 & 109.664 & 106.906 & & 73.696 \\
\hline 11/13/07 0:30 & 125.074 & 122.637 & 119.045 & 100.328 & 104.171 & 95.931 & 109.66 & 106.902 & & 73.696 \\
\hline $11 / 13 / 071: 00$ & 125.067 & 122.619 & 119.025 & 100.314 & 104.167 & 95.912 & 109.659 & 106.887 & & 73.683 \\
\hline 11/13/07 1:30 & 125.064 & 122.605 & 119.012 & 100.291 & 104.167 & 95.896 & 109.659 & 106.867 & & 73.667 \\
\hline $11 / 13 / 072: 00$ & 125.057 & 122.587 & 118.994 & 100.276 & 104.165 & 95.872 & 109.657 & 106.845 & & 73.646 \\
\hline 11/13/07 2:30 & 125.05 & 122.578 & 118.982 & 100.264 & 104.161 & 95.858 & 109.653 & 106.831 & & 73.64 \\
\hline 11/13/07 3:00 & 125.045 & 122.567 & 118.973 & 100.245 & 104.159 & 95.839 & 109.653 & 106.816 & & 73.623 \\
\hline 11/13/07 3:30 & 125.038 & 122.553 & 118.961 & 100.24 & 104.157 & 95.832 & 109.649 & 106.805 & & 73.617 \\
\hline $11 / 13 / 074: 00$ & 125.031 & 122.546 & 118.951 & 100.226 & 104.155 & 95.818 & 109.649 & 106.796 & & 73.609 \\
\hline 11/13/07 4:30 & 125.024 & 122.533 & 118.946 & 100.214 & 104.152 & 95.809 & 109.645 & 106.783 & & 73.598 \\
\hline $11 / 13 / 075: 00$ & 125.017 & 122.524 & 118.931 & 100.207 & 104.148 & 95.799 & 109.642 & 106.774 & & 73.592 \\
\hline $11 / 13 / 075: 30$ & 125.01 & 122.517 & 118.926 & 100.195 & 104.146 & 95.792 & 109.642 & 106.765 & & 73.584 \\
\hline 11/13/07 6:00 & 125.003 & 122.503 & 118.911 & 100.188 & 104.146 & 95.78 & 109.638 & 106.754 & & 73.574 \\
\hline 11/13/07 6:30 & 124.998 & 122.492 & 118.893 & 100.174 & 104.142 & 95.773 & 109.638 & 106.747 & & 73.569 \\
\hline $11 / 13 / 077: 00$ & 124.991 & 124.385 & 120.211 & 100.164 & 104.142 & 95.759 & 109.636 & 106.732 & & 73.555 \\
\hline 11/13/07 7:30 & 124.984 & 124.66 & 120.559 & 100.154 & 104.14 & 95.745 & 109.634 & 106.721 & & 73.545 \\
\hline 11/13/07 8:00 & 126.577 & 124.78 & 120.7 & 100.944 & 104.643 & 96.384 & 110.278 & 108.106 & & 73.592 \\
\hline 11/13/07 8:30 & 126.835 & 124.855 & 120.783 & 101.146 & 104.923 & 96.664 & 110.543 & 108.43 & & 73.762 \\
\hline 11/13/07 9:00 & 126.962 & 124.904 & 120.851 & 101.251 & 105.062 & 96.787 & 110.674 & 108.565 & & 73.869 \\
\hline 11/13/07 9:30 & 127.043 & 124.929 & 120.879 & 101.31 & 105.144 & 96.865 & 110.753 & 108.646 & & 73.942 \\
\hline 11/13/07 10:00 & 127.102 & 124.934 & 120.894 & 101.354 & 105.197 & 96.914 & 110.802 & 108.698 & & 73.989 \\
\hline 11/13/07 10:30 & 127.149 & 124.945 & 120.894 & 101.373 & 105.227 & 96.933 & 110.84 & 108.724 & & 74.014 \\
\hline 11/13/07 11:00 & 127.192 & 124.941 & 120.899 & 101.373 & 105.237 & 96.945 & 110.866 & 108.737 & & 74.024 \\
\hline $11 / 13 / 0711: 30$ & 127.225 & 124.923 & 120.884 & 101.38 & 105.243 & 96.947 & 110.881 & 108.742 & & 74.028 \\
\hline 11/13/07 12:00 & 127.255 & 124.911 & 120.869 & 101.37 & 105.252 & 96.947 & 110.896 & 108.74 & & 74.033 \\
\hline $11 / 13 / 07$ 12:30 & 127.286 & 124.893 & 120.854 & 101.353 & 105.235 & 96.931 & 110.903 & 108.726 & & 74.02 \\
\hline 11/13/07 13:00 & 127.305 & 124.868 & 120.829 & 101.341 & 105.221 & 96.912 & 110.892 & 108.711 & & 74.012 \\
\hline 11/13/07 13:30 & 127.317 & 122.925 & 119.397 & 101.325 & 105.21 & 96.9 & 110.883 & 108.698 & & 74.002 \\
\hline $11 / 13 / 07$ 14:00 & 127.329 & 122.673 & 119.105 & 100.941 & 105.189 & 96.874 & 110.862 & 108.671 & & 73.983 \\
\hline $11 / 13 / 07$ 14:30 & 125.686 & 122.544 & 118.962 & 100.434 & 104.595 & 96.131 & 110.122 & 107.194 & & 73.871 \\
\hline 11/13/07 15:00 & 125.447 & 122.456 & 118.863 & 100.245 & 104.35 & 95.891 & 109.903 & 106.92 & & 73.714 \\
\hline $11 / 13 / 07$ 15:30 & 125.296 & 122.403 & 118.808 & 100.142 & 104.232 & 95.764 & 109.783 & 106.78 & & 73.607 \\
\hline $11 / 13 / 07$ 16:00 & 125.171 & 122.363 & 118.77 & 100.069 & 104.144 & 95.681 & 109.698 & 106.687 & & 73.526 \\
\hline 11/13/07 16:30 & 125.052 & 122.326 & 118.732 & 100.03 & 104.095 & 95.634 & 109.657 & 106.632 & & 73.479 \\
\hline $11 / 13 / 07$ 17:00 & 124.953 & 122.308 & 118.711 & 99.99 & 104.058 & 95.594 & 109.623 & 106.592 & & 73.443 \\
\hline 11/13/07 17:30 & 124.868 & 122.292 & 118.697 & 99.964 & 104.024 & 95.566 & 109.598 & 106.559 & & 73.41 \\
\hline 11/13/07 18:00 & 124.8 & 122.288 & 118.689 & 99.947 & 104.015 & 95.549 & 109.58 & 106.537 & & 73.394 \\
\hline $11 / 13 / 07$ 18:30 & 124.75 & 122.288 & 118.692 & 99.938 & 103.996 & 95.533 & 109.569 & 106.519 & & 73.377 \\
\hline 11/13/07 19:00 & 124.714 & 122.281 & 118.684 & 99.936 & 103.988 & 95.53 & 109.559 & 106.512 & & 73.371 \\
\hline 11/13/07 19:30 & 124.696 & 122.267 & 118.672 & 99.936 & 103.978 & 95.53 & 109.552 & 106.512 & & 73.371 \\
\hline $11 / 13 / 0720: 00$ & 124.684 & 122.27 & 118.672 & 99.924 & 103.976 & 95.526 & 109.548 & 106.508 & & 73.365 \\
\hline 11/13/07 20:30 & 124.677 & 122.272 & 118.674 & 99.921 & 103.971 & 95.512 & 109.542 & 106.492 & & 73.35 \\
\hline $11 / 13 / 0721: 00$ & 124.67 & 122.279 & 118.674 & 99.924 & 103.965 & 95.514 & 109.539 & 106.497 & & 73.352 \\
\hline $11 / 13 / 0721: 30$ & 124.665 & 122.324 & 118.735 & 99.931 & 103.961 & 95.519 & 109.535 & 106.499 & & 73.352 \\
\hline $11 / 13 / 0722: 00$ & 124.66 & 122.342 & 118.742 & 99.938 & 103.959 & 95.523 & 109.531 & 106.501 & & 73.357 \\
\hline 11/13/07 22:30 & 124.651 & 122.356 & 118.759 & 99.985 & 103.956 & 95.58 & 109.524 & 106.554 & & 73.406 \\
\hline 11/13/07 23:00 & 124.651 & 122.374 & 118.778 & 100.002 & 103.95 & 95.592 & 109.52 & 106.566 & & 73.41 \\
\hline
\end{tabular}


TABLE S1.1 (Cont.)

\begin{tabular}{|c|c|c|c|c|c|c|c|c|c|c|}
\hline \multirow[b]{2}{*}{ Date and Time } & \multicolumn{10}{|c|}{ Depth from Top of Casing (ft) } \\
\hline & MW1D & MW2D & MW3D & MW4D & MW7 & MW9 & Oentrich & MW14D & MW15D & MW16D \\
\hline 11/13/07 23:30 & 124.651 & 122.385 & 118.785 & 100.023 & 103.95 & 95.603 & 109.518 & 106.583 & & 73.423 \\
\hline $11 / 14 / 07$ 0:00 & 124.651 & 122.403 & 118.808 & 100.04 & 103.948 & 95.622 & 109.516 & 106.603 & & 73.437 \\
\hline 11/14/07 0:30 & 124.653 & 122.417 & 118.825 & 100.052 & 103.95 & 95.629 & 109.518 & 106.61 & & 73.443 \\
\hline $11 / 14 / 071: 00$ & 124.651 & 122.419 & 118.825 & 100.074 & 103.948 & 95.653 & 109.514 & 106.632 & & 73.462 \\
\hline $11 / 14 / 071: 30$ & 124.653 & 122.433 & 118.836 & 100.083 & 103.944 & 95.669 & 109.512 & 106.647 & & 73.474 \\
\hline $11 / 14 / 072: 00$ & 124.658 & 122.437 & 118.845 & 100.086 & 103.946 & 95.667 & 109.514 & 106.647 & & 73.472 \\
\hline $11 / 14 / 072: 30$ & 124.658 & 122.456 & 118.858 & 100.1 & 103.946 & 95.684 & 109.512 & 106.661 & & 73.485 \\
\hline 11/14/07 3:00 & 124.662 & 122.469 & 118.876 & 100.114 & 103.944 & 95.688 & 109.518 & 106.667 & & 73.489 \\
\hline 11/14/07 3:30 & 124.66 & 122.49 & 118.893 & 100.128 & 103.942 & 95.705 & 109.518 & 106.683 & & 73.505 \\
\hline $11 / 14 / 074: 00$ & 124.665 & 122.496 & 118.899 & 100.147 & 103.94 & 95.724 & 109.523 & 106.701 & & 73.518 \\
\hline $11 / 14 / 074: 30$ & 124.667 & 122.501 & 118.908 & 100.157 & 103.959 & 95.738 & 109.527 & 106.716 & & 73.53 \\
\hline $11 / 14 / 075: 00$ & 124.67 & 122.514 & 118.921 & 100.16 & 103.956 & 95.745 & 109.527 & 106.725 & & 73.538 \\
\hline $11 / 14 / 075: 30$ & 124.677 & 122.528 & 118.924 & 100.173 & 103.957 & 95.752 & 109.527 & 106.729 & & 73.541 \\
\hline $11 / 14 / 076: 00$ & 124.681 & 122.553 & 118.956 & 100.185 & 103.978 & 95.766 & 109.529 & 106.743 & & 73.553 \\
\hline 11/14/07 6:30 & 124.688 & 122.571 & 118.974 & 100.207 & 103.978 & 95.78 & 109.527 & 106.756 & & 73.565 \\
\hline $11 / 14 / 077: 00$ & 124.696 & 124.351 & 119.98 & 100.226 & 104.001 & 95.804 & 109.527 & 106.78 & & 73.588 \\
\hline $11 / 14 / 07$ 7:30 & 124.703 & 124.743 & 120.511 & 100.24 & 104.018 & 95.823 & 109.527 & 106.796 & & 73.601 \\
\hline 11/14/07 8:00 & 126.147 & 124.92 & 120.705 & 100.924 & 104.376 & 96.308 & 110.006 & 108.033 & & 73.64 \\
\hline $11 / 14 / 078: 30$ & 126.511 & 125.022 & 120.823 & 101.198 & 104.749 & 96.69 & 110.353 & 108.514 & & 73.822 \\
\hline 11/14/07 9:00 & 126.665 & 125.1 & 120.906 & 101.346 & 104.923 & 96.869 & 110.517 & 108.706 & & 73.971 \\
\hline 11/14/07 9:30 & 126.764 & 125.152 & 120.962 & 101.442 & 105.027 & 96.978 & 110.618 & 108.819 & & 74.07 \\
\hline $11 / 14 / 07 \quad 10: 00$ & 126.837 & 125.181 & 120.995 & 101.506 & 105.106 & 97.053 & 110.697 & 108.901 & & 74.14 \\
\hline 11/14/07 10:30 & 126.894 & 123.285 & 119.763 & 101.551 & 105.165 & 97.11 & 110.746 & 108.957 & & 74.192 \\
\hline 11/14/07 11:00 & 126.941 & 123.07 & 119.495 & 101.341 & 105.193 & 97.138 & 110.781 & 108.987 & & 74.219 \\
\hline 11/14/07 11:30 & 125.376 & 122.95 & 119.372 & 100.824 & 104.69 & 96.509 & 110.152 & 107.559 & & 74.167 \\
\hline $11 / 14 / 07$ 12:00 & 125.197 & 122.877 & 119.293 & 100.664 & 104.485 & 96.301 & 109.955 & 107.314 & & 74.043 \\
\hline $11 / 14 / 07$ 12:30 & 125.116 & 122.83 & 119.244 & 100.574 & 104.376 & 96.183 & 109.859 & 107.19 & & 73.944 \\
\hline $11 / 14 / 07$ 13:00 & 125.074 & 122.796 & 119.208 & 100.51 & 104.313 & 96.115 & 109.805 & 107.115 & & 73.882 \\
\hline 11/14/07 13:30 & 125.05 & 122.768 & 119.183 & 100.471 & 104.275 & 96.068 & 109.768 & 107.064 & & 73.838 \\
\hline $11 / 14 / 0714: 00$ & 125.029 & 122.75 & 119.158 & 100.445 & 104.247 & 96.035 & 109.737 & 107.026 & & 73.807 \\
\hline $11 / 14 / 07 \quad 14: 30$ & 125.015 & 122.741 & 119.148 & 100.419 & 104.226 & 96.011 & 109.721 & 106.999 & & 73.784 \\
\hline 11/14/07 15:00 & 125.008 & 122.728 & 119.14 & 100.403 & 104.213 & 95.992 & 109.708 & 106.98 & & 73.77 \\
\hline $11 / 14 / 07$ 15:30 & 125.029 & 122.73 & 119.14 & 100.395 & 104.201 & 95.985 & 109.696 & 106.966 & & 73.76 \\
\hline $11 / 14 / 0716: 00$ & 125.088 & 122.732 & 119.143 & 100.384 & 104.192 & 95.973 & 109.685 & 106.957 & & 73.753 \\
\hline 11/14/07 16:30 & 125.102 & 122.739 & 119.143 & 100.393 & 104.182 & 95.978 & 109.679 & 106.957 & & 73.756 \\
\hline $11 / 14 / 07$ 17:00 & 125.112 & 122.748 & 119.156 & 100.395 & 104.174 & 95.978 & 109.672 & 106.96 & & 73.758 \\
\hline 11/14/07 17:30 & 125.112 & 122.759 & 119.165 & 100.4 & 104.171 & 95.983 & 109.664 & 106.964 & & 73.762 \\
\hline $11 / 14 / 07$ 18:00 & 125.121 & 122.773 & 119.178 & 100.412 & 104.18 & 95.992 & 109.663 & 106.973 & & 73.772 \\
\hline $11 / 14 / 0718: 30$ & 125.128 & 122.787 & 119.188 & 100.426 & 104.19 & 96.007 & 109.678 & 106.984 & & 73.782 \\
\hline 11/14/07 19:00 & 125.13 & 122.802 & 119.205 & 100.441 & 104.205 & 96.021 & 109.693 & 106.997 & & 73.793 \\
\hline 11/14/07 19:30 & 125.126 & 122.809 & 119.215 & 100.459 & 104.216 & 96.037 & 109.702 & 107.015 & & 73.807 \\
\hline $11 / 14 / 0720: 00$ & 125.119 & 122.818 & 119.22 & 100.469 & 104.234 & 96.051 & 109.722 & 107.028 & & 73.822 \\
\hline $11 / 14 / 0720: 30$ & 125.114 & 122.83 & 119.233 & 100.476 & 104.243 & 96.058 & 109.732 & 107.037 & & 73.828 \\
\hline 11/14/07 21:00 & 125.114 & 122.839 & 119.241 & 100.486 & 104.247 & 96.065 & 109.734 & 107.046 & & 73.834 \\
\hline $11 / 14 / 0721: 30$ & 125.109 & 122.85 & 119.253 & 100.496 & 104.26 & 96.077 & 109.741 & 107.057 & & 73.844 \\
\hline $11 / 14 / 0722: 00$ & 125.109 & 122.859 & 119.263 & 100.503 & 104.266 & 96.087 & 109.749 & 107.064 & & 73.851 \\
\hline 11/14/07 22:30 & 125.112 & 122.866 & 119.271 & 100.517 & 104.279 & 96.096 & 109.764 & 107.077 & & 73.863 \\
\hline 11/14/07 23:00 & 125.109 & 122.873 & 119.273 & 100.531 & 104.283 & 96.106 & 109.775 & 107.084 & & 73.871 \\
\hline $11 / 14 / 0723: 30$ & 125.109 & 122.882 & 119.284 & 100.533 & 104.291 & 96.113 & 109.782 & 107.092 & & 73.878 \\
\hline 11/15/07 0:00 & 125.112 & 122.882 & 119.284 & 100.543 & 104.3 & 96.122 & 109.788 & 107.099 & & 73.886 \\
\hline 11/15/07 0:30 & 125.112 & 122.884 & 119.288 & 100.545 & 104.308 & 96.129 & 109.798 & 107.11 & & 73.894 \\
\hline 11/15/07 1:00 & 125.114 & 122.88 & 119.286 & 100.548 & 104.306 & 96.127 & 109.799 & 107.108 & & 73.894 \\
\hline 11/15/07 1:30 & 125.116 & 122.875 & 119.284 & 100.55 & 104.308 & 96.131 & 109.801 & 107.112 & & 73.9 \\
\hline $11 / 15 / 072: 00$ & 125.119 & 122.875 & 119.279 & 100.541 & 104.31 & 96.127 & 109.803 & 107.108 & & 73.896 \\
\hline 11/15/07 2:30 & 125.121 & 122.875 & 119.279 & 100.54 & 104.31 & 96.122 & 109.803 & 107.104 & & 73.894 \\
\hline 11/15/07 3:00 & 125.121 & 122.875 & 119.273 & 100.54 & 104.306 & 96.124 & 109.799 & 107.101 & & 73.894 \\
\hline 11/15/07 3:30 & 125.121 & 122.877 & 119.279 & 100.54 & 104.302 & 96.122 & 109.797 & 107.104 & & 73.896 \\
\hline 11/15/07 4:00 & 125.121 & 122.877 & 119.276 & 100.536 & 104.302 & 96.122 & 109.797 & 107.101 & & 73.894 \\
\hline $11 / 15 / 074: 30$ & 125.121 & 122.877 & 119.278 & 100.541 & 104.304 & 96.124 & 109.797 & 107.104 & & 73.898 \\
\hline $11 / 15 / 07$ 5:00 & 125.121 & 122.868 & 119.276 & 100.541 & 104.302 & 96.124 & 109.797 & 107.101 & & 73.898 \\
\hline 11/15/07 5:30 & 125.123 & 122.864 & 119.268 & 100.538 & 104.302 & 96.124 & 109.796 & 107.101 & & 73.9 \\
\hline 11/15/07 6:00 & 125.123 & 122.864 & 119.269 & 100.529 & 104.304 & 96.12 & 109.796 & 107.099 & & 73.898 \\
\hline $11 / 15 / 076: 30$ & 125.123 & 122.864 & 119.268 & 100.533 & 104.3 & 96.11 & 109.796 & 107.09 & & 73.89 \\
\hline 11/15/07 7:00 & 125.123 & 124.483 & 119.937 & 100.531 & 104.3 & 96.113 & 109.794 & 107.092 & & 73.894 \\
\hline 11/15/07 7:30 & 125.123 & 124.97 & 120.867 & 100.533 & 104.296 & 96.113 & 109.79 & 107.092 & & 73.896 \\
\hline 11/15/07 8:00 & 126.433 & 125.149 & 121.075 & 101.174 & 104.544 & 96.48 & 110.175 & 108.025 & & 73.9 \\
\hline
\end{tabular}


TABLE S1.1 (Cont.)

\begin{tabular}{|c|c|c|c|c|c|c|c|c|c|c|}
\hline \multirow[b]{2}{*}{ Date and Time } & \multicolumn{10}{|c|}{ Depth from Top of Casing (ft) } \\
\hline & MW1D & MW2D & MW3D & MW4D & MW7 & MW9 & Oentrich & MW14D & MW15D & MW16D \\
\hline 11/15/07 8:30 & 126.903 & 125.261 & 121.202 & 101.482 & 105.003 & 96.966 & 110.62 & 108.728 & & 74.07 \\
\hline 11/15/07 9:00 & 127.078 & 125.322 & 121.262 & 101.634 & 105.187 & 97.147 & 110.793 & 108.93 & & 74.217 \\
\hline 11/15/07 9:30 & 127.189 & 125.363 & 121.309 & 101.72 & 105.292 & 97.261 & 110.892 & 109.05 & & 74.316 \\
\hline 11/15/07 10:00 & 127.27 & 125.385 & 121.338 & 101.777 & 105.355 & 97.317 & 110.954 & 109.112 & & 74.372 \\
\hline $11 / 15 / 07$ 10:30 & 127.331 & 125.415 & 121.363 & 101.813 & 105.397 & 97.36 & 110.997 & 109.158 & & 74.413 \\
\hline $11 / 15 / 0711: 00$ & 127.381 & 125.419 & 121.372 & 101.834 & 105.429 & 97.385 & 111.027 & 109.187 & & 74.44 \\
\hline $11 / 15 / 0711: 30$ & 127.423 & 125.419 & 121.375 & 101.856 & 105.45 & 97.411 & 111.047 & 109.209 & & 74.463 \\
\hline $11 / 15 / 07$ 12:00 & 127.461 & 125.408 & 121.371 & 101.856 & 105.469 & 97.418 & 111.062 & 109.216 & & 74.471 \\
\hline 11/15/07 12:30 & 127.497 & 125.394 & 121.358 & 101.858 & 105.484 & 97.421 & 111.077 & 109.22 & & 74.475 \\
\hline 11/15/07 13:00 & 127.525 & 125.385 & 121.338 & 101.847 & 105.498 & 97.414 & 111.091 & 109.216 & & 74.473 \\
\hline $11 / 15 / 07$ 13:30 & 127.549 & 123.655 & 120.218 & 101.828 & 105.511 & 97.397 & 111.103 & 109.198 & & 74.463 \\
\hline $11 / 15 / 0714: 00$ & 127.565 & 123.279 & 119.72 & 101.821 & 105.519 & 97.388 & 111.112 & 109.187 & & 74.456 \\
\hline $11 / 15 / 0714: 30$ & 126.144 & 123.109 & 119.528 & 101.093 & 105.132 & 96.85 & 110.584 & 107.994 & & 74.427 \\
\hline $11 / 15 / 07$ 15:00 & 125.821 & 123.004 & 119.414 & 100.832 & 104.805 & 96.488 & 110.265 & 107.531 & & 74.26 \\
\hline 11/15/07 15:30 & 125.679 & 122.932 & 119.342 & 100.695 & 104.656 & 96.325 & 110.126 & 107.347 & & 74.132 \\
\hline $11 / 15 / 07$ 16:00 & 125.594 & 122.877 & 119.283 & 100.612 & 104.572 & 96.221 & 110.047 & 107.239 & & 74.043 \\
\hline $11 / 15 / 07$ 16:30 & 125.532 & 122.841 & 119.248 & 100.548 & 104.515 & 96.155 & 109.995 & 107.166 & & 73.981 \\
\hline $11 / 15 / 07$ 17:00 & 125.483 & 122.802 & 119.208 & 100.505 & 104.479 & 96.103 & 109.963 & 107.108 & & 73.929 \\
\hline $11 / 15 / 07$ 17:30 & 125.447 & 122.775 & 119.178 & 100.471 & 104.452 & 96.072 & 109.936 & 107.073 & & 73.9 \\
\hline $11 / 15 / 0718: 00$ & 125.419 & 122.753 & 119.153 & 100.436 & 104.433 & 96.035 & 109.92 & 107.033 & & 73.865 \\
\hline $11 / 15 / 0718: 30$ & 125.393 & 122.737 & 119.138 & 100.409 & 104.416 & 96.009 & 109.905 & 107.002 & & 73.84 \\
\hline 11/15/07 19:00 & 125.369 & 122.719 & 119.122 & 100.393 & 104.403 & 95.99 & 109.893 & 106.982 & & 73.824 \\
\hline 11/15/07 19:30 & 125.35 & 122.698 & 119.102 & 100.383 & 104.393 & 95.973 & 109.884 & 106.966 & & 73.807 \\
\hline $11 / 15 / 0720: 00$ & 125.334 & 122.682 & 119.085 & 100.362 & 104.386 & 95.957 & 109.876 & 106.949 & & 73.795 \\
\hline $11 / 15 / 0720: 30$ & 125.315 & 122.662 & 119.064 & 100.345 & 104.251 & 95.938 & 109.869 & 106.929 & & 73.774 \\
\hline 11/15/07 21:00 & 125.301 & 122.644 & 119.045 & 100.328 & 104.294 & 95.917 & 109.86 & 106.911 & & 73.76 \\
\hline 11/15/07 21:30 & 125.286 & 122.626 & 119.03 & 100.305 & 104.296 & 95.898 & 109.854 & 106.891 & & 73.741 \\
\hline 11/15/07 22:00 & 125.275 & 122.612 & 119.014 & 100.286 & 104.296 & 95.879 & 109.848 & 106.871 & & 73.725 \\
\hline 11/15/07 22:30 & 125.26 & 122.598 & 118.999 & 100.269 & 104.293 & 95.863 & 109.841 & 106.851 & & 73.708 \\
\hline 11/15/07 23:00 & 125.246 & 122.576 & 118.977 & 100.255 & 104.291 & 95.849 & 109.835 & 106.84 & & 73.698 \\
\hline $11 / 15 / 0723: 30$ & 125.232 & 122.548 & 118.951 & 100.241 & 104.283 & 95.839 & 109.828 & 106.827 & & 73.683 \\
\hline 11/16/07 0:00 & 125.22 & 122.535 & 118.933 & 100.217 & 104.26 & 95.818 & 109.824 & 106.802 & & 73.663 \\
\hline 11/16/07 0:30 & 125.211 & 122.51 & 118.916 & 100.195 & 104.243 & 95.787 & 109.797 & 106.776 & & 73.638 \\
\hline 11/16/07 1:00 & 125.197 & 122.485 & 118.888 & 100.176 & 104.232 & 95.776 & 109.786 & 106.76 & & 73.625 \\
\hline 11/16/07 1:30 & 125.185 & 122.469 & 118.87 & 100.154 & 104.211 & 95.752 & 109.767 & 106.738 & & 73.607 \\
\hline 11/16/07 2:00 & 125.175 & 122.462 & 118.861 & 100.129 & 104.102 & 95.724 & 109.743 & 106.712 & & 73.582 \\
\hline 11/16/07 2:30 & 125.161 & 122.446 & 118.845 & 100.114 & 104.125 & 95.705 & 109.728 & 106.694 & & 73.563 \\
\hline 11/16/07 3:00 & 125.147 & 122.419 & 118.823 & 100.11 & 104.129 & 95.698 & 109.719 & 106.685 & & 73.559 \\
\hline 11/16/07 3:30 & 125.135 & 122.412 & 118.817 & 100.09 & 104.129 & 95.686 & 109.705 & 106.672 & & 73.543 \\
\hline $11 / 16 / 074: 00$ & 125.126 & 122.399 & 118.807 & 100.066 & 104.045 & 95.655 & 109.687 & 106.645 & & 73.52 \\
\hline 11/16/07 4:30 & 125.109 & 122.388 & 118.789 & 100.064 & 104.085 & 95.651 & 109.672 & 106.639 & & 73.514 \\
\hline $11 / 16 / 07$ 5:00 & 125.097 & 122.372 & 118.774 & 100.04 & 104.091 & 95.636 & 109.664 & 106.623 & & 73.499 \\
\hline 11/16/07 5:30 & 125.086 & 122.365 & 118.767 & 100.028 & 104.087 & 95.63 & 109.649 & 106.614 & & 73.493 \\
\hline 11/16/07 6:00 & 125.071 & 122.349 & 118.754 & 100.026 & 104.068 & 95.611 & 109.638 & 106.599 & & 73.476 \\
\hline $11 / 16 / 076: 30$ & 125.055 & 122.331 & 118.739 & 100.014 & 104.074 & 95.606 & 109.629 & 106.59 & & 73.47 \\
\hline 11/16/07 7:00 & 125.045 & 123.746 & 118.732 & 99.999 & 104.06 & 95.592 & 109.615 & 106.577 & & 73.454 \\
\hline 11/16/07 7:30 & 125.036 & 124.378 & 120.118 & 99.986 & 104.045 & 95.573 & 109.604 & 106.559 & & 73.439 \\
\hline 11/16/07 8:00 & 125.981 & 124.555 & 120.327 & 100.5 & 104.056 & 95.691 & 109.739 & 106.849 & & 73.439 \\
\hline 11/16/07 8:30 & 126.724 & 124.66 & 120.432 & 100.843 & 104.616 & 96.329 & 110.336 & 108.144 & & 73.574 \\
\hline 11/16/07 9:00 & 126.896 & 124.709 & 120.498 & 100.981 & 104.808 & 96.504 & 110.509 & 108.341 & & 73.71 \\
\hline 11/16/07 9:30 & 126.988 & 123.104 & 119.742 & 101.063 & 104.911 & 96.608 & 110.594 & 108.452 & & 73.803 \\
\hline 11/16/07 10:00 & 127.055 & 122.719 & 119.138 & 101.112 & 104.98 & 96.657 & 110.652 & 108.507 & & 73.853 \\
\hline 11/16/07 10:30 & 125.747 & 122.567 & 118.961 & 100.534 & 104.732 & 96.313 & 110.269 & 107.504 & & 73.898 \\
\hline 11/16/07 11:00 & 125.398 & 122.485 & 118.86 & 100.29 & 104.388 & 95.941 & 109.931 & 106.971 & & 73.76 \\
\hline 11/16/07 11:30 & 125.275 & 122.428 & 118.812 & 100.174 & 104.253 & 95.792 & 109.805 & 106.802 & & 73.646 \\
\hline 11/16/07 12:00 & 125.208 & 122.381 & 118.769 & 100.102 & 104.18 & 95.712 & 109.732 & 106.714 & & 73.572 \\
\hline 11/16/07 12:30 & 125.166 & 122.349 & 118.732 & 100.055 & 104.129 & 95.66 & 109.685 & 106.656 & & 73.52 \\
\hline 11/16/07 13:00 & 125.13 & 122.335 & 118.722 & 100.011 & 104.03 & 95.615 & 109.649 & 106.61 & & 73.474 \\
\hline 11/16/07 13:30 & 125.06 & 122.319 & 118.709 & 99.993 & 104.028 & 95.582 & 109.61 & 106.572 & & 73.441 \\
\hline $11 / 16 / 07$ 14:00 & 124.951 & 122.313 & 118.697 & 99.98 & 104.015 & 95.573 & 109.597 & 106.559 & & 73.431 \\
\hline 11/16/07 14:30 & 124.868 & 122.299 & 118.684 & 99.967 & 104.007 & 95.561 & 109.586 & 106.546 & & 73.417 \\
\hline $11 / 16 / 07$ 15:00 & 124.802 & 122.292 & 118.679 & 99.961 & 104.001 & 95.552 & 109.58 & 106.537 & & 73.406 \\
\hline $11 / 16 / 07$ 15:30 & 124.752 & 122.292 & 118.676 & 99.947 & 103.997 & 95.54 & 109.568 & 106.526 & & 73.394 \\
\hline $11 / 16 / 07$ 16:00 & 124.71 & 122.295 & 118.681 & 99.945 & 103.996 & 95.535 & 109.563 & 106.515 & & 73.388 \\
\hline 11/16/07 16:30 & 124.686 & 122.292 & 118.674 & 99.947 & 103.99 & 95.533 & 109.557 & 106.515 & & 73.386 \\
\hline $11 / 16 / 07$ 17:00 & 124.677 & 122.295 & 118.677 & 99.95 & 103.986 & 95.54 & 109.552 & 106.519 & & 73.388 \\
\hline
\end{tabular}


TABLE S1.1 (Cont.)

\begin{tabular}{|c|c|c|c|c|c|c|c|c|c|c|}
\hline \multirow[b]{2}{*}{ Date and Time } & \multicolumn{10}{|c|}{ Depth from Top of Casing (ft) } \\
\hline & MW1D & MW2D & MW3D & MW4D & MW7 & MW9 & Oentrich & MW14D & MW15D & MW16D \\
\hline 11/16/07 17:30 & 124.674 & 122.306 & 118.689 & 99.947 & 103.986 & 95.533 & 109.552 & 106.512 & & 73.381 \\
\hline $11 / 16 / 07$ 18:00 & 124.667 & 122.308 & 118.694 & 99.954 & 103.984 & 95.537 & 109.548 & 106.517 & & 73.383 \\
\hline 11/16/07 18:30 & 124.667 & 122.315 & 118.699 & 99.961 & 103.982 & 95.552 & 109.548 & 106.528 & & 73.394 \\
\hline 11/16/07 19:00 & 124.674 & 122.324 & 118.721 & 99.967 & 103.982 & 95.554 & 109.546 & 106.532 & & 73.396 \\
\hline 11/16/07 19:30 & 124.674 & 122.326 & 118.726 & 99.974 & 103.98 & 95.556 & 109.548 & 106.537 & & 73.398 \\
\hline 11/16/07 20:00 & 124.672 & 122.331 & 118.734 & 99.985 & 103.978 & 95.568 & 109.544 & 106.543 & & 73.406 \\
\hline $11 / 16 / 0720: 30$ & 124.674 & 122.329 & 118.734 & 99.986 & 103.98 & 95.57 & 109.544 & 106.548 & & 73.408 \\
\hline 11/16/07 21:00 & 124.672 & 122.331 & 118.732 & 99.992 & 103.978 & 95.578 & 109.546 & 106.554 & & 73.412 \\
\hline 11/16/07 21:30 & 124.672 & 122.335 & 118.737 & 99.988 & 103.98 & 95.575 & 109.546 & 106.554 & & 73.408 \\
\hline $11 / 16 / 0722: 00$ & 124.674 & 122.333 & 118.739 & 99.99 & 103.98 & 95.575 & 109.544 & 106.554 & & 73.408 \\
\hline $11 / 16 / 0722: 30$ & 124.672 & 122.313 & 118.714 & 99.995 & 103.98 & 95.58 & 109.546 & 106.559 & & 73.412 \\
\hline 11/16/07 23:00 & 124.677 & 122.329 & 118.729 & 99.993 & 103.982 & 95.58 & 109.543 & 106.559 & & 73.41 \\
\hline 11/16/07 23:30 & 124.677 & 122.344 & 118.742 & 99.974 & 103.986 & 95.556 & 109.546 & 106.535 & & 73.39 \\
\hline $11 / 17 / 07$ 0:00 & 124.672 & 122.347 & 118.752 & 99.986 & 103.982 & 95.575 & 109.544 & 106.552 & & 73.406 \\
\hline 11/17/07 0:30 & 124.672 & 122.338 & 118.744 & 100.012 & 103.982 & 95.592 & 109.542 & 106.568 & & 73.419 \\
\hline $11 / 17 / 071: 00$ & 124.674 & 122.342 & 118.747 & 100.004 & 103.984 & 95.596 & 109.544 & 106.572 & & 73.421 \\
\hline 11/17/07 1:30 & 124.677 & 122.338 & 118.744 & 99.999 & 103.984 & 95.585 & 109.546 & 106.563 & & 73.41 \\
\hline $11 / 17 / 072: 00$ & 124.679 & 122.347 & 118.747 & 100 & 103.986 & 95.589 & 109.546 & 106.566 & & 73.41 \\
\hline $11 / 17 / 072: 30$ & 124.681 & 122.363 & 118.767 & 99.997 & 103.986 & 95.587 & 109.548 & 106.561 & & 73.41 \\
\hline 11/17/07 3:00 & 124.679 & 122.369 & 118.772 & 100.017 & 103.988 & 95.597 & 109.544 & 106.57 & & 73.419 \\
\hline 11/17/07 3:30 & 124.677 & 122.369 & 118.777 & 100.033 & 103.984 & 95.613 & 109.544 & 106.585 & & 73.431 \\
\hline $11 / 17 / 074: 00$ & 124.679 & 122.369 & 118.772 & 100.036 & 103.988 & 95.618 & 109.546 & 106.594 & & 73.437 \\
\hline 11/17/07 4:30 & 124.681 & 122.374 & 118.778 & 100.028 & 103.988 & 95.615 & 109.546 & 106.594 & & 73.433 \\
\hline $11 / 17 / 075: 00$ & 124.686 & 122.378 & 118.777 & 100.03 & 103.986 & 95.615 & 109.548 & 106.592 & & 73.433 \\
\hline 11/17/07 5:30 & 124.686 & 122.388 & 118.785 & 100.038 & 103.986 & 95.622 & 109.546 & 106.597 & & 73.439 \\
\hline 11/17/07 6:00 & 124.686 & 122.392 & 118.797 & 100.043 & 103.986 & 95.627 & 109.546 & 106.603 & & 73.443 \\
\hline 11/17/07 6:30 & 124.686 & 122.399 & 118.802 & 100.057 & 103.986 & 95.636 & 109.546 & 106.61 & & 73.45 \\
\hline 11/17/07 7:00 & 124.691 & 122.399 & 118.802 & 100.059 & 103.99 & 95.641 & 109.548 & 106.616 & & 73.454 \\
\hline 11/17/07 7:30 & 124.691 & 122.412 & 118.81 & 100.066 & 103.988 & 95.646 & 109.546 & 106.623 & & 73.46 \\
\hline 11/17/07 8:00 & 124.693 & 124.215 & 120.002 & 100.066 & 103.988 & 95.648 & 109.546 & 106.623 & & 73.462 \\
\hline 11/17/07 8:30 & 124.693 & 124.569 & 120.475 & 100.083 & 103.986 & 95.662 & 109.544 & 106.634 & & 73.472 \\
\hline 11/17/07 9:00 & 126.196 & 124.737 & 120.664 & 100.824 & 104.393 & 96.219 & 110.093 & 107.905 & & 73.507 \\
\hline 11/17/07 9:30 & 126.53 & 124.836 & 120.775 & 101.079 & 104.734 & 96.575 & 110.417 & 108.332 & & 73.694 \\
\hline $11 / 17 / 07$ 10:00 & 126.683 & 124.907 & 120.853 & 101.222 & 104.894 & 96.737 & 110.562 & 108.512 & & 73.83 \\
\hline 11/17/07 10:30 & 126.785 & 124.948 & 120.896 & 101.303 & 104.984 & 96.841 & 110.648 & 108.622 & & 73.923 \\
\hline 11/17/07 11:00 & 126.858 & 124.982 & 120.929 & 101.365 & 105.045 & 96.912 & 110.702 & 108.698 & & 73.987 \\
\hline 11/17/07 11:30 & 126.92 & 124.997 & 120.947 & 101.401 & 105.086 & 96.949 & 110.744 & 108.74 & & 74.024 \\
\hline 11/17/07 12:00 & 126.974 & 125.011 & 120.944 & 101.429 & 105.121 & 96.982 & 110.776 & 108.773 & & 74.055 \\
\hline $11 / 17 / 0712: 30$ & 127.024 & 125.027 & 120.979 & 101.442 & 105.144 & 97.001 & 110.802 & 108.793 & & 74.072 \\
\hline $11 / 17 / 07$ 13:00 & 127.187 & 125.031 & 120.987 & 101.456 & 105.166 & 97.013 & 110.821 & 108.804 & & 74.086 \\
\hline 11/17/07 13:30 & 127.409 & 125.041 & 120.994 & 101.47 & 105.182 & 97.03 & 110.836 & 108.822 & & 74.101 \\
\hline $11 / 17 / 0714: 00$ & 127.487 & 125.054 & 121.012 & 101.473 & 105.197 & 97.037 & 110.849 & 108.83 & & 74.109 \\
\hline $11 / 17 / 0714: 30$ & 127.508 & 124.911 & 121.015 & 101.485 & 105.208 & 97.046 & 110.86 & 108.839 & & 74.119 \\
\hline 11/17/07 15:00 & 127.525 & 123.113 & 119.573 & 101.498 & 105.218 & 97.06 & 110.87 & 108.853 & & 74.136 \\
\hline 11/17/07 15:30 & 127.534 & 122.9 & 119.324 & 101.051 & 105.229 & 97.065 & 110.881 & 108.857 & & 74.142 \\
\hline 11/17/07 16:00 & 125.674 & 122.791 & 119.205 & 100.639 & 104.635 & 96.32 & 110.15 & 107.378 & & 74.039 \\
\hline 11/17/07 16:30 & 125.357 & 122.73 & 119.135 & 100.488 & 104.43 & 96.117 & 109.955 & 107.141 & & 73.906 \\
\hline 11/17/07 17:00 & 125.206 & 122.687 & 119.089 & 100.4 & 104.323 & 96.014 & 109.854 & 107.024 & & 73.818 \\
\hline $11 / 17 / 07$ 17:30 & 125.123 & 122.662 & 119.064 & 100.352 & 104.258 & 95.957 & 109.792 & 106.957 & & 73.762 \\
\hline 11/17/07 18:00 & 125.074 & 122.648 & 119.049 & 100.319 & 104.214 & 95.917 & 109.756 & 106.913 & & 73.722 \\
\hline $11 / 17 / 0718: 30$ & 125.043 & 122.641 & 119.044 & 100.3 & 104.188 & 95.896 & 109.728 & 106.887 & & 73.7 \\
\hline 11/17/07 19:00 & 125.022 & 122.635 & 119.036 & 100.293 & 104.165 & 95.884 & 109.705 & 106.871 & & 73.687 \\
\hline 11/17/07 19:30 & 125.012 & 122.635 & 119.034 & 100.286 & 104.15 & 95.882 & 109.69 & 106.864 & & 73.681 \\
\hline $11 / 17 / 0720: 00$ & 125.01 & 122.63 & 119.031 & 100.29 & 104.14 & 95.875 & 109.679 & 106.86 & & 73.675 \\
\hline $11 / 17 / 0720: 30$ & 125.005 & 122.621 & 119.024 & 100.286 & 104.127 & 95.875 & 109.674 & 106.858 & & 73.677 \\
\hline 11/17/07 21:00 & 125.003 & 122.617 & 119.021 & 100.279 & 104.119 & 95.87 & 109.666 & 106.853 & & 73.671 \\
\hline $11 / 17 / 0721: 30$ & 124.998 & 122.614 & 119.014 & 100.272 & 104.114 & 95.86 & 109.662 & 106.842 & & 73.663 \\
\hline 11/17/07 22:00 & 124.991 & 122.617 & 119.019 & 100.272 & 104.11 & 95.863 & 109.657 & 106.842 & & 73.663 \\
\hline $11 / 17 / 0722: 30$ & 124.984 & 122.619 & 119.019 & 100.272 & 104.102 & 95.858 & 109.653 & 106.838 & & 73.66 \\
\hline 11/17/07 23:00 & 124.979 & 122.621 & 119.021 & 100.272 & 104.096 & 95.858 & 109.649 & 106.842 & & 73.663 \\
\hline 11/17/07 23:30 & 124.972 & 122.619 & 119.021 & 100.279 & 104.096 & 95.86 & 109.645 & 106.842 & & 73.667 \\
\hline 11/18/07 0:00 & 124.97 & 122.614 & 119.019 & 100.279 & 104.091 & 95.865 & 109.644 & 106.847 & & 73.669 \\
\hline $11 / 18 / 070: 30$ & 124.967 & 122.617 & 119.019 & 100.278 & 104.087 & 95.863 & 109.642 & 106.845 & & 73.667 \\
\hline 11/18/07 1:00 & 124.965 & 122.617 & 119.019 & 100.274 & 104.085 & 95.86 & 109.64 & 106.84 & & 73.663 \\
\hline 11/18/07 1:30 & 124.963 & 122.607 & 119.016 & 100.278 & 104.083 & 95.863 & 109.638 & 106.842 & & 73.665 \\
\hline $11 / 18 / 072: 00$ & 124.96 & 122.603 & 119.006 & 100.276 & 104.081 & 95.863 & 109.636 & 106.84 & & 73.665 \\
\hline
\end{tabular}


TABLE S1.1 (Cont.)

Depth from Top of Casing (ft)

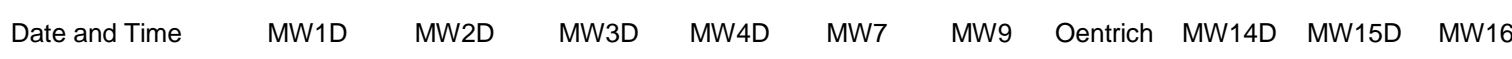

\begin{tabular}{|c|c|c|c|c|c|c|c|c|c|}
\hline $11 / 18 / 072: 30$ & 124.96 & 122.619 & 119.016 & 100.266 & 104.079 & 95.853 & 109.636 & 106.836 & 73.66 \\
\hline 11/18/07 3:00 & 124.958 & 122.612 & 119.019 & 100.266 & 104.079 & 95.846 & 109.634 & 106.827 & 73.652 \\
\hline 11/18/07 3:30 & 124.953 & 122.61 & 119.014 & 100.276 & 104.074 & 95.865 & 109.631 & 106.845 & 73.669 \\
\hline $11 / 18 / 074: 00$ & 124.956 & 122.605 & 119.009 & 100.274 & 104.074 & 95.86 & 109.63 & 106.84 & 73.665 \\
\hline $11 / 18 / 074: 30$ & 124.958 & 122.619 & 119.021 & 100.269 & 104.072 & 95.858 & 109.629 & 106.838 & 73.663 \\
\hline $11 / 18 / 07$ 5:00 & 124.956 & 122.61 & 119.014 & 100.272 & 104.071 & 95.849 & 109.63 & 106.827 & 73.654 \\
\hline $11 / 18 / 075: 30$ & 124.953 & 122.598 & 119.004 & 100.279 & 104.068 & 95.867 & 109.627 & 106.845 & 73.671 \\
\hline $11 / 18 / 076: 00$ & 124.956 & 122.605 & 119.006 & 100.266 & 104.07 & 95.858 & 109.627 & 106.836 & 73.66 \\
\hline $11 / 18 / 076: 30$ & 124.956 & 122.617 & 119.016 & 100.262 & 104.068 & 95.846 & 109.627 & 106.827 & 73.654 \\
\hline 11/18/07 7:00 & 124.953 & 122.603 & 119.006 & 100.271 & 104.066 & 95.856 & 109.623 & 106.831 & 73.66 \\
\hline 11/18/07 7:30 & 124.951 & 122.605 & 119.006 & 100.279 & 104.062 & 95.863 & 109.619 & 106.84 & 73.671 \\
\hline $11 / 18 / 078: 00$ & 124.958 & 122.612 & 119.011 & 100.266 & 104.062 & 95.849 & 109.623 & 106.829 & 73.658 \\
\hline $11 / 18 / 078: 30$ & 124.953 & 124.621 & 120.367 & 100.267 & 104.06 & 95.856 & 109.619 & 106.833 & 73.663 \\
\hline 11/18/07 9:00 & 124.953 & 124.83 & 120.609 & 100.683 & 104.058 & 95.86 & 109.617 & 106.836 & 73.665 \\
\hline 11/18/07 9:30 & 126.631 & 124.943 & 120.732 & 101.099 & 104.645 & 96.572 & 110.327 & 108.374 & 73.778 \\
\hline $11 / 18 / 07$ 10:00 & 126.83 & 125.013 & 120.816 & 101.267 & 104.852 & 96.78 & 110.515 & 108.611 & 73.929 \\
\hline $11 / 18 / 07$ 10:30 & 126.943 & 125.056 & 120.863 & 101.356 & 104.968 & 96.895 & 110.618 & 108.735 & 74.035 \\
\hline 11/18/07 11:00 & 127.017 & 123.161 & 119.631 & 101.418 & 105.035 & 96.966 & 110.684 & 108.813 & 74.105 \\
\hline 11/18/07 11:30 & 127.078 & 122.938 & 119.364 & 101.206 & 105.083 & 97.013 & 110.727 & 108.859 & 74.15 \\
\hline $11 / 18 / 07$ 12:00 & 125.518 & 122.812 & 119.233 & 100.693 & 104.584 & 96.379 & 110.107 & 107.431 & 74.09 \\
\hline $11 / 18 / 07$ 12:30 & 125.341 & 122.73 & 119.14 & 100.531 & 104.38 & 96.162 & 109.912 & 107.181 & 73.96 \\
\hline 11/18/07 13:00 & 125.263 & 122.68 & 119.087 & 100.422 & 104.279 & 96.044 & 109.82 & 107.05 & 73.859 \\
\hline $11 / 18 / 07$ 13:30 & 125.204 & 122.639 & 119.036 & 100.359 & 104.222 & 95.964 & 109.766 & 106.964 & 73.782 \\
\hline $11 / 18 / 07$ 14:00 & 125.14 & 122.614 & 119.014 & 100.307 & 104.186 & 95.915 & 109.73 & 106.909 & 73.733 \\
\hline $11 / 18 / 07$ 14:30 & 125.078 & 122.603 & 118.999 & 100.271 & 104.171 & 95.875 & 109.715 & 106.862 & 73.691 \\
\hline $11 / 18 / 07$ 15:00 & 125.026 & 122.589 & 118.994 & 100.257 & 104.142 & 95.851 & 109.692 & 106.838 & 73.675 \\
\hline 11/18/07 15:30 & 124.998 & 122.585 & 118.989 & 100.243 & 104.133 & 95.837 & 109.681 & 107.026 & \\
\hline $11 / 18 / 07$ 16:00 & 124.977 & 122.58 & 118.984 & 100.24 & 104.123 & 95.825 & 109.672 & & \\
\hline $11 / 18 / 07$ 16:30 & 124.965 & & 118.978 & 100.233 & 104.114 & 95.825 & 109.663 & & 73.698 \\
\hline $11 / 18 / 07$ 17:00 & 124.963 & & 118.996 & 100.235 & 104.11 & 95.82 & 109.659 & 106.864 & 73.723 \\
\hline $11 / 18 / 07$ 17:30 & & 122.509 & 118.989 & 100.229 & 104.104 & 95.818 & 109.65 & 106.854 & 73.725 \\
\hline $11 / 18 / 07$ 18:00 & 125.006 & 122.519 & 118.986 & 100.247 & 104.1 & 95.832 & 109.646 & 106.875 & 73.741 \\
\hline 11/18/07 18:30 & 125.015 & 122.519 & 118.989 & 100.245 & 104.095 & 95.828 & 109.64 & 106.863 & 73.749 \\
\hline 11/18/07 19:00 & 125.013 & 122.523 & 118.984 & 100.246 & 104.091 & 95.832 & 109.636 & 106.866 & 73.76 \\
\hline $11 / 18 / 07$ 19:30 & 125.007 & 122.518 & 118.981 & 100.241 & 104.091 & 95.83 & 109.634 & 106.864 & 73.766 \\
\hline 11/18/07 20:00 & 125.001 & 122.522 & 118.978 & 100.243 & 104.085 & 95.832 & 109.631 & 106.866 & 73.77 \\
\hline $11 / 18 / 0720: 30$ & 124.996 & 122.522 & 118.976 & 100.236 & 104.085 & 95.823 & 109.631 & 106.859 & 73.766 \\
\hline $11 / 18 / 0721: 00$ & 124.993 & 122.524 & 118.973 & 100.236 & 104.083 & 95.825 & 109.629 & 106.858 & 73.772 \\
\hline 11/18/07 21:30 & 124.99 & 122.527 & 118.971 & 100.233 & 104.083 & 95.818 & 109.627 & 106.852 & 73.77 \\
\hline $11 / 18 / 0722: 00$ & 124.988 & 122.533 & 118.981 & 100.233 & 104.081 & 95.816 & 109.627 & 106.851 & 73.772 \\
\hline $11 / 18 / 0722: 30$ & 124.985 & 122.533 & 118.981 & 100.238 & 104.079 & 95.82 & 109.623 & 106.853 & 73.78 \\
\hline 11/18/07 23:00 & 124.983 & 122.529 & 118.978 & 100.238 & 104.077 & 95.823 & 109.621 & 106.862 & 73.789 \\
\hline $11 / 18 / 0723: 30$ & 124.985 & 122.529 & 118.978 & 100.238 & 104.075 & 95.823 & 109.621 & 106.862 & 73.789 \\
\hline 11/19/07 0:00 & 124.982 & 122.513 & 118.963 & 100.238 & 104.074 & 95.82 & 109.621 & 106.857 & 73.791 \\
\hline $11 / 19 / 070: 30$ & 124.98 & 122.51 & 118.963 & 100.231 & 104.074 & 95.823 & 109.619 & 106.855 & 73.791 \\
\hline 11/19/07 1:00 & 124.98 & 122.504 & 118.953 & 100.219 & 104.074 & 95.804 & 109.621 & 106.839 & 73.776 \\
\hline 11/19/07 1:30 & 124.978 & 122.506 & 118.951 & 100.216 & 104.072 & 95.801 & 109.619 & 106.839 & 73.78 \\
\hline $11 / 19 / 072: 00$ & 124.975 & 122.499 & 118.946 & 100.209 & 104.072 & 95.794 & 109.617 & 106.83 & 73.775 \\
\hline $11 / 19 / 072: 30$ & 124.973 & 122.497 & 118.931 & 100.212 & 104.07 & 95.797 & 109.617 & 106.83 & 73.779 \\
\hline 11/19/07 3:00 & 124.973 & 122.49 & 118.928 & 100.205 & 104.072 & 95.792 & 109.617 & 106.828 & 73.776 \\
\hline 11/19/07 3:30 & 124.97 & 122.485 & 118.921 & 100.198 & 104.07 & 95.787 & 109.617 & 106.826 & 73.777 \\
\hline $11 / 19 / 074: 00$ & 124.97 & 122.469 & 118.921 & 100.197 & 104.07 & 95.783 & 109.617 & 106.817 & 73.77 \\
\hline 11/19/07 4:30 & 124.968 & 122.46 & 118.913 & 100.19 & 104.072 & 95.776 & 109.615 & 106.812 & 73.768 \\
\hline $11 / 19 / 075: 00$ & 124.968 & 122.463 & 118.913 & 100.174 & 104.072 & 95.764 & 109.617 & 106.797 & 73.756 \\
\hline 11/19/07 5:30 & 124.965 & 122.465 & 118.908 & 100.169 & 104.068 & 95.754 & 109.619 & 106.788 & 73.747 \\
\hline $11 / 19 / 076: 00$ & 124.963 & 122.465 & 118.898 & 100.167 & 104.068 & 95.757 & 109.617 & 106.79 & 73.752 \\
\hline $11 / 19 / 076: 30$ & 124.958 & 124.023 & 119.243 & 100.171 & 104.068 & 95.757 & 109.615 & 106.79 & 73.754 \\
\hline 11/19/07 7:00 & 124.961 & 124.54 & 120.485 & 100.169 & 104.066 & 95.759 & 109.617 & 106.79 & 73.756 \\
\hline 11/19/07 7:30 & 126.478 & 124.732 & 120.702 & 100.787 & 104.275 & 96.075 & 109.959 & 107.609 & 73.756 \\
\hline $11 / 19 / 078: 00$ & 127.095 & 124.839 & 120.823 & 101.108 & 104.751 & 96.589 & 110.432 & 108.386 & 73.923 \\
\hline 11/19/07 8:30 & 127.287 & 124.916 & & & 104.936 & 96.78 & 110.603 & 108.607 & 74.081 \\
\hline 11/19/07 9:00 & 127.393 & 124.948 & & & 105.035 & 96.886 & 110.701 & 108.722 & 74.182 \\
\hline 11/19/07 9:30 & 127.471 & 124.986 & 121.403 & & 105.109 & 96.964 & 110.772 & 108.796 & 74.252 \\
\hline 11/19/07 10:00 & 127.514 & 125.009 & 121.444 & 101.567 & 105.153 & 96.992 & 110.81 & 108.84 & 74.296 \\
\hline $11 / 19 / 07$ 10:30 & 127.544 & 125.034 & 121.466 & 101.611 & 105.191 & 97.032 & 110.843 & 108.88 & 74.335 \\
\hline 11/19/07 11:00 & 127.568 & 125.05 & 121.489 & 101.625 & 105.21 & 97.06 & 110.864 & 108.909 & 74.378 \\
\hline
\end{tabular}


TABLE S1.1 (Cont.)

Depth from Top of Casing (ft)

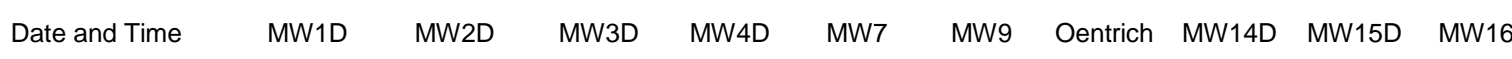

\begin{tabular}{|c|c|c|c|c|c|c|c|c|c|}
\hline 11/19/07 11:30 & 127.585 & 125.048 & 121.504 & 101.623 & 105.225 & 97.081 & 110.881 & 108.931 & 74.407 \\
\hline $11 / 19 / 07$ 12:00 & 127.596 & 125.041 & 121.484 & 101.632 & 105.233 & 97.096 & 110.879 & 108.951 & 74.432 \\
\hline $11 / 19 / 07$ 12:30 & 127.613 & 125.048 & 121.493 & 101.642 & 105.25 & 97.093 & 110.9 & 108.955 & 74.434 \\
\hline 11/19/07 13:00 & 127.617 & 123.769 & 121.502 & 101.652 & 105.26 & 97.081 & 110.907 & 108.942 & 74.429 \\
\hline 11/19/07 13:30 & 127.636 & 123.054 & 120.004 & 101.609 & & 97.086 & 110.911 & 108.948 & 74.44 \\
\hline $11 / 19 / 0714: 00$ & 126.575 & 122.843 & 119.767 & 101.079 & & & 110.775 & 108.762 & 74.453 \\
\hline $11 / 19 / 07$ 14:30 & 125.585 & 122.716 & 119.628 & 100.733 & 104.729 & 96.245 & 110.157 & 107.416 & 74.316 \\
\hline $11 / 19 / 07$ 15:00 & 125.379 & 122.648 & 119.552 & 100.55 & 104.551 & 96.038 & 109.982 & 107.186 & 74.165 \\
\hline $11 / 19 / 07$ 15:30 & 125.268 & 122.61 & 119.507 & 100.473 & 104.442 & 95.919 & 109.888 & 107.056 & 74.057 \\
\hline $11 / 19 / 07$ 16:00 & 125.199 & 122.574 & 119.473 & 100.414 & 104.369 & 95.856 & 109.826 & 106.982 & 73.992 \\
\hline 11/19/07 16:30 & 125.152 & 122.553 & 119.453 & 100.378 & 104.326 & 95.818 & 109.786 & 106.94 & 73.954 \\
\hline $11 / 19 / 07$ 17:00 & 125.121 & 122.535 & 119.43 & 100.353 & 104.29 & 95.789 & 109.76 & 106.905 & 73.921 \\
\hline $11 / 19 / 07$ 17:30 & 125.1 & 122.519 & 119.418 & 100.334 & 104.269 & 95.767 & 109.743 & 106.88 & 73.898 \\
\hline 11/19/07 18:00 & 125.086 & 122.51 & 119.402 & 100.321 & 104.251 & 95.753 & 109.732 & 106.863 & 73.878 \\
\hline $11 / 19 / 07$ 18:30 & 125.072 & 122.499 & 119.392 & 100.309 & 104.239 & 95.741 & 109.719 & 106.849 & 73.866 \\
\hline $11 / 19 / 07$ 19:00 & 125.057 & 122.492 & 119.384 & 100.3 & 104.231 & 95.731 & 109.711 & 106.836 & 73.857 \\
\hline 11/19/07 19:30 & 125.052 & 122.483 & 119.377 & 100.293 & 104.221 & 95.721 & 109.706 & 106.827 & 73.844 \\
\hline $11 / 19 / 0720: 00$ & 125.043 & 122.474 & 119.367 & 100.253 & 104.212 & 95.712 & 109.698 & 106.816 & 73.839 \\
\hline $11 / 19 / 0720: 30$ & 125.039 & 122.471 & 119.358 & 100.251 & 104.206 & 95.705 & 109.694 & 106.809 & 73.828 \\
\hline $11 / 19 / 0721: 00$ & 125.031 & 122.462 & 119.353 & 100.246 & 104.196 & 95.695 & 109.691 & 106.801 & 73.822 \\
\hline $11 / 19 / 0721: 30$ & 125.026 & 122.462 & 119.353 & 100.242 & 104.196 & 95.693 & 109.683 & 106.801 & 73.822 \\
\hline $11 / 19 / 0722: 00$ & 125.022 & 122.456 & 119.346 & 100.237 & 104.187 & 95.683 & 109.679 & 106.79 & 73.811 \\
\hline $11 / 19 / 07$ 22:30 & 125.017 & 122.444 & 119.336 & 100.234 & 104.187 & 95.683 & 109.677 & 106.787 & 73.811 \\
\hline $11 / 19 / 0723: 00$ & 125.012 & 122.437 & 119.331 & 100.23 & 104.181 & 95.678 & 109.675 & 106.783 & 73.803 \\
\hline $11 / 19 / 0723: 30$ & 125.013 & 122.433 & 119.323 & 100.228 & 104.175 & 95.671 & 109.674 & 106.774 & 73.797 \\
\hline 11/20/07 0:00 & 125.008 & 122.415 & 119.307 & 100.223 & 104.164 & 95.661 & 109.67 & 106.765 & 73.789 \\
\hline $11 / 20 / 070: 30$ & 125.008 & 122.401 & 119.295 & 100.221 & 104.154 & 95.654 & 109.668 & 106.759 & 73.783 \\
\hline $11 / 20 / 071: 00$ & 125.003 & 122.385 & 119.275 & 100.171 & 104.147 & 95.64 & 109.666 & 106.743 & 73.768 \\
\hline 11/20/07 1:30 & 125 & 122.374 & 119.267 & 100.183 & 104.131 & 95.626 & 109.662 & 106.732 & 73.756 \\
\hline $11 / 20 / 072: 00$ & 125 & 122.365 & 119.26 & 100.181 & 104.114 & 95.612 & 109.659 & 106.712 & 73.741 \\
\hline $11 / 20 / 072: 30$ & 124.998 & 122.358 & 119.249 & 100.142 & 104.103 & 95.6 & 109.651 & 106.703 & 73.731 \\
\hline $11 / 20 / 073: 00$ & 124.994 & 122.349 & 119.239 & 100.145 & 104.099 & 95.595 & 109.649 & 106.694 & 73.727 \\
\hline $11 / 20 / 073: 30$ & 124.991 & 122.349 & 119.234 & 100.145 & 104.088 & 95.583 & 109.649 & 106.688 & 73.717 \\
\hline $11 / 20 / 074: 00$ & 124.989 & 122.335 & 119.232 & 100.144 & 104.08 & 95.576 & 109.645 & 106.677 & 73.708 \\
\hline $11 / 20 / 074: 30$ & 124.986 & 122.333 & 119.219 & 100.142 & 104.074 & 95.571 & 109.644 & 106.672 & 73.704 \\
\hline $11 / 20 / 075: 00$ & 124.986 & 122.333 & 119.222 & 100.095 & 104.068 & 95.564 & 109.644 & 106.663 & 73.696 \\
\hline $11 / 20 / 075: 30$ & 124.986 & 122.335 & 119.224 & 100.107 & 104.063 & 95.56 & 109.64 & 106.661 & 73.692 \\
\hline $11 / 20 / 076: 00$ & 124.984 & 122.351 & 119.239 & 100.107 & 104.067 & 95.557 & 109.636 & 106.659 & 73.691 \\
\hline $11 / 20 / 076: 30$ & 124.982 & 123.753 & 119.242 & 100.107 & 104.07 & 95.564 & 109.634 & 106.663 & 73.696 \\
\hline $11 / 20 / 077: 00$ & 124.977 & 124.406 & 120.782 & 100.109 & 104.088 & 95.585 & 109.634 & 106.681 & 73.713 \\
\hline $11 / 20 / 077: 30$ & 126.208 & 124.601 & 121.011 & 100.679 & 104.181 & 95.739 & 109.829 & 107.004 & 73.706 \\
\hline $11 / 20 / 07$ 8:00 & 127.064 & 124.712 & 121.117 & 101.022 & 104.759 & 96.399 & 110.419 & 108.25 & 73.87 \\
\hline 11/20/07 8:30 & 127.268 & 124.782 & 121.217 & 101.175 & 104.948 & 96.594 & 110.603 & 108.479 & 74.023 \\
\hline $11 / 20 / 079: 00$ & 127.376 & 122.934 & 119.905 & 101.265 & 105.06 & 96.705 & 110.704 & 108.6 & 74.128 \\
\hline 11/20/07 9:30 & 127.447 & 122.705 & 119.62 & 101.222 & 105.127 & 96.773 & 110.766 & 108.674 & 74.2 \\
\hline 11/20/07 10:00 & 125.568 & 122.601 & 119.501 & 100.546 & 104.638 & 96.134 & 110.134 & 107.312 & 74.161 \\
\hline $11 / 20 / 07$ 10:30 & 125.317 & 122.546 & 119.446 & 100.384 & 104.419 & 95.918 & 109.929 & 107.051 & 74.039 \\
\hline $11 / 20 / 0711: 00$ & 125.208 & 122.512 & 119.406 & 100.303 & 104.323 & 95.816 & 109.831 & 106.94 & 73.956 \\
\hline 11/20/07 11:30 & 125.142 & 122.499 & 119.392 & 100.254 & 104.272 & 95.767 & 109.775 & 106.882 & 73.907 \\
\hline $11 / 20 / 07$ 12:00 & 125.104 & 122.496 & 119.387 & 100.219 & 104.236 & 95.734 & 109.739 & 106.843 & 73.87 \\
\hline $11 / 20 / 07$ 12:30 & 125.071 & 122.494 & 119.387 & 100.2 & 104.224 & 95.722 & 109.717 & 106.827 & 73.855 \\
\hline $11 / 20 / 07$ 13:00 & 125.043 & 122.496 & 119.385 & 100.185 & 104.224 & 95.724 & 109.698 & 106.825 & 73.855 \\
\hline $11 / 20 / 07$ 13:30 & 125.024 & 122.501 & 119.392 & 100.174 & 104.226 & 95.727 & 109.689 & 106.827 & 73.853 \\
\hline $11 / 20 / 07 \quad 14: 00$ & 125.017 & 122.506 & 119.397 & 100.167 & 104.232 & 95.727 & 109.677 & 106.825 & 73.853 \\
\hline 11/20/07 14:30 & 125.01 & 122.508 & 119.395 & 100.164 & 104.234 & 95.734 & 109.67 & 106.829 & 73.853 \\
\hline $11 / 20 / 07$ 15:00 & 125.003 & 122.517 & 119.407 & 100.159 & 104.24 & 95.738 & 109.668 & 106.836 & 73.857 \\
\hline $11 / 20 / 07$ 15:30 & 125 & 122.533 & 119.41 & 100.155 & 104.239 & 95.738 & 109.662 & 106.836 & 73.857 \\
\hline 11/20/07 16:00 & 124.996 & 122.555 & 119.438 & 100.241 & 104.253 & 95.75 & 109.659 & 106.849 & 73.868 \\
\hline $11 / 20 / 07$ 16:30 & 124.996 & 122.569 & 119.453 & 100.267 & 104.266 & 95.764 & 109.653 & 106.858 & 73.88 \\
\hline $11 / 20 / 07$ 17:00 & 124.993 & 122.58 & 119.468 & 100.288 & 104.293 & 95.79 & 109.653 & 106.885 & 73.903 \\
\hline $11 / 20 / 07$ 17:30 & 124.993 & 122.598 & 119.486 & 100.302 & 104.306 & 95.807 & 109.649 & 106.9 & 73.913 \\
\hline $11 / 20 / 07$ 18:00 & 124.991 & 122.619 & 119.508 & 100.316 & 104.316 & 95.816 & 109.65 & 106.909 & 73.923 \\
\hline $11 / 20 / 07$ 18:30 & 124.991 & 122.63 & 119.516 & 100.333 & 104.337 & 95.835 & 109.652 & 106.929 & 73.94 \\
\hline $11 / 20 / 07$ 19:00 & 124.991 & 122.639 & 119.523 & 100.352 & 104.356 & 95.856 & 109.655 & 106.951 & 73.96 \\
\hline $11 / 20 / 07$ 19:30 & 124.989 & 122.646 & 119.531 & 100.362 & 104.367 & 95.87 & 109.657 & 106.962 & 73.969 \\
\hline $11 / 20 / 0720: 00$ & 124.989 & 122.646 & 119.536 & 100.369 & 104.375 & 95.875 & 109.657 & 106.969 & 73.975 \\
\hline
\end{tabular}


TABLE S1.1 (Cont.)

\begin{tabular}{|c|c|c|c|c|c|c|c|c|c|c|}
\hline \multirow[b]{2}{*}{ Date and Time } & \multicolumn{10}{|c|}{ Depth from Top of Casing (ft) } \\
\hline & MW1D & MW2D & MW3D & MW4D & MW7 & MW9 & Oentrich & MW14D & MW15D & MW16D \\
\hline $11 / 20 / 0720: 30$ & 124.986 & 122.635 & 119.526 & 100.378 & 104.386 & 95.882 & 109.655 & 106.978 & & 73.985 \\
\hline $11 / 20 / 0721: 00$ & 124.989 & 122.635 & 119.523 & 100.371 & 104.384 & 95.884 & 109.657 & 106.978 & & 73.985 \\
\hline $11 / 20 / 0721: 30$ & 124.991 & 122.648 & 119.531 & 100.36 & 104.371 & 95.873 & 109.659 & 106.969 & & 73.977 \\
\hline $11 / 20 / 0722: 00$ & 124.986 & 122.653 & 119.539 & 100.365 & 104.371 & 95.877 & 109.659 & 106.969 & & 73.977 \\
\hline $11 / 20 / 0722: 30$ & 124.979 & 122.657 & 119.544 & 100.376 & 104.384 & 95.884 & 109.653 & 106.978 & & 73.99 \\
\hline $11 / 20 / 0723: 00$ & 124.979 & 122.669 & 119.564 & 100.381 & 104.388 & 95.889 & 109.649 & 106.984 & & 73.994 \\
\hline $11 / 20 / 0723: 30$ & 124.974 & 122.673 & 119.561 & 100.393 & 104.397 & 95.894 & 109.649 & 106.989 & & 74 \\
\hline $11 / 21 / 070: 00$ & 124.972 & 122.675 & 119.566 & 100.398 & 104.411 & 95.908 & 109.647 & 107.004 & & 74.014 \\
\hline 11/21/07 0:30 & 124.97 & 122.682 & 119.569 & 100.403 & 104.413 & 95.915 & 109.645 & 107.006 & & 74.018 \\
\hline $11 / 21 / 071: 00$ & 124.97 & 122.696 & 119.584 & 100.409 & 104.415 & 95.917 & 109.649 & 107.009 & & 74.021 \\
\hline 11/21/07 1:30 & 124.965 & 122.687 & 119.589 & 100.417 & 104.42 & 95.922 & 109.645 & 107.015 & & 74.027 \\
\hline $11 / 21 / 072: 00$ & 124.961 & 122.691 & 119.586 & 100.426 & 104.436 & 95.936 & 109.649 & 107.029 & & 74.039 \\
\hline $11 / 21 / 072: 30$ & 124.965 & 122.689 & 119.584 & 100.417 & 104.426 & 95.929 & 109.655 & 107.022 & & 74.033 \\
\hline 11/21/07 3:00 & 124.96 & 122.691 & 119.586 & 100.419 & 104.428 & 95.927 & 109.651 & 107.022 & & 74.033 \\
\hline 11/21/07 3:30 & 124.958 & 122.698 & 119.581 & 100.417 & 104.43 & 95.929 & 109.651 & 107.024 & & 74.035 \\
\hline $11 / 21 / 074: 00$ & 124.956 & 122.7 & 119.594 & 100.424 & 104.432 & 95.931 & 109.649 & 107.022 & & 74.037 \\
\hline $11 / 21 / 074: 30$ & 124.956 & 122.721 & 119.599 & 100.433 & 104.437 & 95.938 & 109.651 & 107.033 & & 74.043 \\
\hline $11 / 21 / 075: 00$ & 124.953 & 122.716 & 119.599 & 100.432 & 104.445 & 95.943 & 109.653 & 107.04 & & 74.05 \\
\hline $11 / 21 / 075: 30$ & 124.946 & 122.723 & 119.604 & 100.443 & 104.455 & 95.955 & 109.657 & 107.046 & & 74.058 \\
\hline $11 / 21 / 076: 00$ & 124.948 & 122.73 & 119.609 & 100.445 & 104.451 & 95.953 & 109.674 & 107.044 & & 74.058 \\
\hline 11/21/07 6:30 & 124.946 & 122.741 & 119.627 & 100.457 & 104.464 & 95.962 & 109.677 & 107.051 & & 74.068 \\
\hline $11 / 21 / 07$ 7:00 & 124.944 & 124.687 & 120.882 & 100.457 & 104.464 & 95.964 & 109.681 & 107.057 & & 74.072 \\
\hline 11/21/07 7:30 & 124.941 & 124.975 & 121.227 & 100.481 & 104.481 & 95.983 & 109.689 & 107.075 & & 74.089 \\
\hline $11 / 21 / 078: 00$ & 126.884 & 125.109 & 121.365 & 101.254 & 104.99 & 96.62 & 110.318 & 108.496 & & 74.169 \\
\hline 11/21/07 8:30 & 127.175 & 125.195 & 121.476 & 101.466 & 105.266 & 96.898 & 110.586 & 108.846 & & 74.355 \\
\hline 11/21/07 9:00 & 127.312 & 125.258 & 121.544 & 101.58 & 105.405 & 97.039 & 110.721 & 109.003 & & 74.482 \\
\hline 11/21/07 9:30 & 127.393 & 125.295 & 121.577 & 101.659 & 105.488 & 97.126 & 110.804 & 109.094 & & 74.568 \\
\hline $11 / 21 / 0710: 00$ & 127.447 & 125.338 & 121.625 & 101.711 & 105.555 & 97.19 & 110.86 & 109.16 & & 74.635 \\
\hline $11 / 21 / 0710: 30$ & 127.478 & 125.381 & 121.668 & 101.751 & 105.589 & 97.228 & 110.898 & 109.198 & & 74.676 \\
\hline $11 / 21 / 0711: 00$ & 127.502 & 125.419 & 121.711 & 101.796 & 105.635 & 97.273 & 110.936 & 109.243 & & 74.723 \\
\hline $11 / 21 / 0711: 30$ & 127.516 & 125.428 & 121.728 & 101.842 & 105.679 & 97.315 & 110.975 & 109.285 & & 74.769 \\
\hline $11 / 21 / 0712: 00$ & 127.532 & 125.433 & 121.728 & 101.873 & 105.715 & 97.355 & 111.013 & 109.327 & & 74.808 \\
\hline $11 / 21 / 0712: 30$ & 127.546 & 125.44 & 121.741 & 101.883 & 105.73 & 97.367 & 111.033 & 109.342 & & 74.823 \\
\hline $11 / 21 / 07$ 13:00 & 127.551 & 125.446 & 121.751 & 101.89 & 105.736 & 97.374 & 111.048 & 109.346 & & 74.835 \\
\hline 11/21/07 13:30 & 127.558 & 125.458 & 121.761 & 101.896 & 105.745 & 97.383 & 111.055 & 109.355 & & 74.85 \\
\hline $11 / 21 / 0714: 00$ & 127.563 & 125.469 & 121.776 & 101.907 & 105.753 & 97.39 & 111.061 & 109.36 & & 74.86 \\
\hline $11 / 21 / 0714: 30$ & 127.563 & 125.478 & 121.781 & 101.921 & 105.77 & 97.409 & 111.069 & 109.378 & & 74.883 \\
\hline $11 / 21 / 0715: 00$ & 127.57 & 123.868 & 121.013 & 101.933 & 105.78 & 97.419 & 111.082 & 109.388 & & 74.897 \\
\hline $11 / 21 / 0715: 30$ & 127.573 & 123.458 & 120.38 & 101.943 & 105.787 & 97.426 & 111.093 & 109.395 & & 74.905 \\
\hline $11 / 21 / 0716: 00$ & 126.357 & 123.299 & 120.201 & 101.335 & 105.519 & 97.046 & 110.701 & 108.41 & & 74.905 \\
\hline $11 / 21 / 0716: 30$ & 125.856 & 123.213 & 120.105 & 101.081 & 105.157 & 96.657 & 110.333 & 107.826 & & 74.767 \\
\hline $11 / 21 / 0717: 00$ & 125.684 & 123.174 & 120.058 & 100.961 & 105.003 & 96.502 & 110.192 & 107.644 & & 74.651 \\
\hline $11 / 21 / 0717: 30$ & 125.587 & 123.145 & 120.032 & 100.898 & 104.919 & 96.422 & 110.113 & 107.553 & & 74.573 \\
\hline $11 / 21 / 0718: 00$ & 125.523 & 123.131 & 120.015 & 100.87 & 104.881 & 96.384 & 110.076 & 107.506 & & 74.533 \\
\hline $11 / 21 / 0718: 30$ & 125.556 & 123.113 & 120 & 100.848 & 104.858 & 96.363 & 110.058 & 107.48 & & 74.506 \\
\hline $11 / 21 / 0719: 00$ & 125.525 & 123.104 & 119.992 & 100.836 & 104.845 & 96.346 & 110.045 & 107.463 & & 74.49 \\
\hline 11/21/07 19:30 & 125.502 & 123.095 & 119.977 & 100.824 & 104.83 & 96.334 & 110.034 & 107.447 & & 74.471 \\
\hline $11 / 21 / 0720: 00$ & 125.483 & 123.093 & 119.974 & 100.812 & 104.82 & 96.327 & 110.032 & 107.44 & & 74.465 \\
\hline $11 / 21 / 0720: 30$ & 125.466 & 123.084 & 119.972 & 100.812 & 104.814 & 96.316 & 110.017 & 107.427 & & 74.455 \\
\hline $11 / 21 / 0721: 00$ & 125.454 & 123.088 & 119.972 & 100.803 & 104.811 & 96.313 & 110.015 & 107.425 & & 74.453 \\
\hline $11 / 21 / 0721: 30$ & 125.445 & 123.095 & 119.976 & 100.8 & 104.805 & 96.306 & 110.006 & 107.418 & & 74.446 \\
\hline $11 / 21 / 0722: 00$ & 125.433 & 123.088 & 119.976 & 100.807 & 104.807 & 96.309 & 110.004 & 107.42 & & 74.449 \\
\hline $11 / 21 / 0722: 30$ & 125.421 & 123.086 & 119.974 & 100.811 & 104.816 & 96.318 & 110.017 & 107.427 & & 74.457 \\
\hline $11 / 21 / 0723: 00$ & 125.416 & 123.079 & 119.964 & 100.807 & 104.811 & 96.313 & 110.017 & 107.422 & & 74.451 \\
\hline $11 / 21 / 0723: 30$ & 125.409 & 123.072 & 119.959 & 100.803 & 104.807 & 96.311 & 110.013 & 107.418 & & 74.451 \\
\hline 11/22/07 0:00 & 125.4 & 123.07 & 119.954 & 100.796 & 104.803 & 96.304 & 110.008 & 107.411 & & 74.442 \\
\hline $11 / 22 / 070: 30$ & 125.393 & 123.065 & 119.952 & 100.793 & 104.799 & 96.299 & 110 & 107.407 & & 74.44 \\
\hline 11/22/07 1:00 & 125.383 & 123.061 & 119.944 & 100.791 & 104.793 & 96.297 & 109.995 & 107.401 & & 74.438 \\
\hline 11/22/07 1:30 & 125.376 & 123.054 & 119.941 & 100.782 & 104.791 & 96.292 & 109.993 & 107.398 & & 74.438 \\
\hline $11 / 22 / 072: 00$ & 125.372 & 123.052 & 119.936 & 100.78 & 104.788 & 96.287 & 109.987 & 107.391 & & 74.43 \\
\hline $11 / 22 / 072: 30$ & 125.364 & 123.059 & 119.944 & 100.777 & 104.782 & 96.282 & 109.983 & 107.387 & & 74.426 \\
\hline $11 / 22 / 07$ 3:00 & 125.36 & 123.056 & 119.944 & 100.774 & 104.78 & 96.28 & 109.976 & 107.385 & & 74.425 \\
\hline 11/22/07 3:30 & 125.35 & 123.059 & 119.941 & 100.779 & 104.786 & 96.285 & 109.974 & 107.389 & & 74.432 \\
\hline $11 / 22 / 074: 00$ & 125.346 & 123.059 & 119.941 & 100.781 & 104.784 & 96.287 & 109.974 & 107.389 & & 74.432 \\
\hline $11 / 22 / 074: 30$ & 125.341 & 123.054 & 119.938 & 100.779 & 104.786 & 96.287 & 109.974 & 107.391 & & 74.432 \\
\hline $11 / 22 / 07$ 5:00 & 125.334 & 123.056 & 119.938 & 100.78 & 104.784 & 96.285 & 109.972 & 107.389 & & 74.432 \\
\hline
\end{tabular}


TABLE S1.1 (Cont.)

\begin{tabular}{|c|c|c|c|c|c|c|c|c|c|c|}
\hline \multirow[b]{2}{*}{ Date and Time } & \multicolumn{10}{|c|}{ Depth from Top of Casing (ft) } \\
\hline & MW1D & MW2D & MW3D & MW4D & MW7 & MW9 & Oentrich & MW14D & MW15D & MW16D \\
\hline 11/22/07 5:30 & 125.331 & 123.056 & 119.941 & 100.776 & 104.784 & 96.285 & 109.97 & 107.387 & & 74.43 \\
\hline $11 / 22 / 076: 00$ & 125.327 & 123.056 & 119.944 & 100.779 & 104.78 & 96.282 & 109.97 & 107.387 & & 74.43 \\
\hline 11/22/07 6:30 & 125.322 & 123.056 & 119.941 & 100.779 & 104.784 & 96.285 & 109.974 & 107.389 & & 74.43 \\
\hline 11/22/07 7:00 & 125.317 & 123.065 & 119.944 & 100.777 & 104.786 & 96.285 & 109.978 & 107.391 & & 74.432 \\
\hline $11 / 22 / 07$ 7:30 & 125.312 & 123.068 & 119.951 & 100.782 & 104.786 & 96.282 & 109.978 & 107.389 & & 74.434 \\
\hline 11/22/07 8:00 & 125.312 & 123.075 & 119.959 & 100.789 & 104.789 & 96.287 & 109.983 & 107.394 & & 74.434 \\
\hline 11/22/07 8:30 & 125.305 & 124.807 & 121.035 & 100.793 & 104.795 & 96.294 & 109.98 & 107.4 & & 74.442 \\
\hline 11/22/07 9:00 & 125.298 & 125.199 & 121.587 & 100.798 & 104.801 & 96.304 & 109.983 & 107.407 & & 74.451 \\
\hline 11/22/07 9:30 & 127.012 & 125.374 & 121.773 & 101.508 & 105.163 & 96.796 & 110.481 & 108.498 & & 74.467 \\
\hline 11/22/07 10:00 & 127.433 & 125.474 & 121.886 & 101.78 & 105.542 & 97.188 & 110.839 & 109.067 & & 74.657 \\
\hline $11 / 22 / 07$ 10:30 & 127.596 & 125.539 & 121.965 & 101.921 & 105.711 & 97.36 & 111.003 & 109.258 & & 74.804 \\
\hline 11/22/07 11:00 & 127.693 & 125.587 & 122.015 & 102.006 & 105.81 & 97.461 & 111.108 & 109.368 & & 74.903 \\
\hline $11 / 22 / 0711: 30$ & 127.773 & 125.612 & 122.043 & 102.066 & 105.88 & 97.529 & 111.172 & 109.441 & & 74.969 \\
\hline $11 / 22 / 07$ 12:00 & 127.823 & 123.682 & 120.634 & 102.102 & 105.924 & 97.577 & 111.219 & 109.49 & & 75.019 \\
\hline 11/22/07 12:30 & 127.856 & 123.446 & 120.357 & 101.751 & 105.947 & 97.598 & 111.251 & 109.514 & & 75.046 \\
\hline 11/22/07 13:00 & 126.036 & 123.319 & 120.218 & 101.27 & 105.372 & 96.876 & 110.552 & 108.075 & & 74.953 \\
\hline 11/22/07 13:30 & 125.792 & 123.236 & 120.13 & 101.092 & 105.147 & 96.65 & 110.349 & 107.81 & & 74.808 \\
\hline 11/22/07 14:00 & 125.679 & 123.188 & 120.075 & 100.989 & 105.027 & 96.525 & 110.25 & 107.664 & & 74.702 \\
\hline $11 / 22 / 07$ 14:30 & 125.603 & 123.149 & 120.037 & 100.923 & 104.946 & 96.447 & 110.188 & 107.575 & & 74.626 \\
\hline 11/22/07 15:00 & 125.554 & 123.124 & 120.004 & 100.88 & 104.902 & 96.398 & 110.149 & 107.522 & & 74.581 \\
\hline 11/22/07 15:30 & 125.52 & 123.109 & 119.991 & 100.846 & 104.864 & 96.365 & 110.12 & 107.484 & & 74.544 \\
\hline $11 / 22 / 07$ 16:00 & 125.494 & 123.099 & 119.979 & 100.827 & 104.841 & 96.341 & 110.098 & 107.456 & & 74.519 \\
\hline 11/22/07 16:30 & 125.478 & 123.095 & 119.974 & 100.815 & 104.826 & 96.327 & 110.081 & 107.44 & & 74.502 \\
\hline 11/22/07 17:00 & 125.459 & 123.088 & 119.971 & 100.811 & 104.816 & 96.318 & 110.068 & 107.429 & & 74.494 \\
\hline 11/22/07 17:30 & 125.447 & 123.088 & 119.969 & 100.805 & 104.814 & 96.313 & 110.057 & 107.427 & & 74.49 \\
\hline 11/22/07 18:00 & 125.435 & 123.093 & 119.974 & 100.801 & 104.807 & 96.311 & 110.049 & 107.42 & & 74.484 \\
\hline 11/22/07 18:30 & 125.426 & 123.09 & 119.974 & 100.805 & 104.808 & 96.306 & 110.042 & 107.418 & & 74.482 \\
\hline 11/22/07 19:00 & 125.419 & 123.09 & 119.966 & 100.81 & 104.814 & 96.315 & 110.034 & 107.427 & & 74.488 \\
\hline 11/22/07 19:30 & 125.414 & 123.086 & 119.966 & 100.806 & 104.81 & 96.313 & 110.028 & 107.425 & & 74.484 \\
\hline $11 / 22 / 0720: 00$ & 125.407 & 123.086 & 119.969 & 100.807 & 104.812 & 96.313 & 110.023 & 107.422 & & 74.484 \\
\hline $11 / 22 / 0720: 30$ & 125.402 & 123.086 & 119.969 & 100.801 & 104.808 & 96.308 & 110.019 & 107.42 & & 74.482 \\
\hline $11 / 22 / 0721: 00$ & 125.398 & 123.086 & 119.969 & 100.801 & 104.808 & 96.311 & 110.013 & 107.42 & & 74.48 \\
\hline 11/22/07 21:30 & 125.39 & 123.086 & 119.969 & 100.805 & 104.808 & 96.306 & 110.01 & 107.418 & & 74.479 \\
\hline $11 / 22 / 0722: 00$ & 125.386 & 123.084 & 119.964 & 100.805 & 104.808 & 96.311 & 110.006 & 107.42 & & 74.484 \\
\hline 11/22/07 22:30 & 125.383 & 123.079 & 119.964 & 100.801 & 104.807 & 96.308 & 110.004 & 107.418 & & 74.482 \\
\hline 11/22/07 23:00 & 125.379 & 123.075 & 119.954 & 100.801 & 104.807 & 96.306 & 109.998 & 107.416 & & 74.48 \\
\hline 11/22/07 23:30 & 125.376 & 123.068 & 119.946 & 100.795 & 104.801 & 96.301 & 109.995 & 107.411 & & 74.475 \\
\hline 11/23/07 0:00 & 125.369 & 123.063 & 119.944 & 100.791 & 104.797 & 96.299 & 109.991 & 107.407 & & 74.471 \\
\hline $11 / 23 / 070: 30$ & 125.364 & 123.052 & 119.938 & 100.781 & 104.791 & 96.292 & 109.989 & 107.4 & & 74.465 \\
\hline $11 / 23 / 071: 00$ & 125.36 & 123.043 & 119.921 & 100.779 & 104.786 & 96.287 & 109.985 & 107.396 & & 74.463 \\
\hline 11/23/07 1:30 & 125.357 & 123.027 & 119.913 & 100.767 & 104.776 & 96.278 & 109.981 & 107.387 & & 74.455 \\
\hline $11 / 23 / 072: 00$ & 125.355 & 123.02 & 119.903 & 100.758 & 104.767 & 96.264 & 109.976 & 107.371 & & 74.442 \\
\hline 11/23/07 2:30 & 125.348 & 123.013 & 119.896 & 100.741 & 104.753 & 96.252 & 109.976 & 107.363 & & 74.434 \\
\hline 11/23/07 3:00 & 125.343 & 123.013 & 119.896 & 100.739 & 104.744 & 96.245 & 109.972 & 107.354 & & 74.423 \\
\hline $11 / 23 / 073: 30$ & 125.338 & 122.991 & 119.875 & 100.729 & 104.738 & 96.238 & 109.968 & 107.345 & & 74.419 \\
\hline $11 / 23 / 074: 00$ & 125.331 & 122.977 & 119.863 & 100.727 & 104.736 & 96.235 & 109.963 & 107.345 & & 74.417 \\
\hline 11/23/07 4:30 & 125.338 & 122.972 & 119.853 & 100.705 & 104.713 & 96.212 & 109.965 & 107.323 & & 74.393 \\
\hline $11 / 23 / 075: 00$ & 125.331 & 122.966 & 119.85 & 100.693 & 104.704 & 96.202 & 109.959 & 107.309 & & 74.384 \\
\hline $11 / 23 / 075: 30$ & 125.327 & 122.954 & 119.838 & 100.689 & 104.694 & 96.195 & 109.957 & 107.303 & & 74.376 \\
\hline 11/23/07 6:00 & 125.322 & 122.948 & 119.835 & 100.684 & 104.692 & 96.193 & 109.955 & 107.298 & & 74.376 \\
\hline $11 / 23 / 076: 30$ & 125.322 & 122.941 & 119.823 & 100.672 & 104.679 & 96.179 & 109.951 & 107.287 & & 74.361 \\
\hline 11/23/07 7:00 & 125.32 & 122.943 & 119.825 & 100.665 & 104.674 & 96.174 & 109.951 & 107.281 & & 74.357 \\
\hline 11/23/07 7:30 & 125.315 & 124.394 & 119.823 & 100.658 & 104.666 & 96.162 & 109.948 & 107.272 & & 74.347 \\
\hline 11/23/07 8:00 & 125.31 & 125.034 & 121.256 & 100.662 & 104.668 & 96.167 & 109.946 & 107.274 & & 74.351 \\
\hline $11 / 23 / 078: 30$ & 126.513 & 125.224 & 121.483 & 101.206 & 104.752 & 96.297 & 110.115 & 107.602 & & 74.353 \\
\hline 11/23/07 9:00 & 127.4 & 125.324 & 121.597 & 101.547 & 105.309 & 96.942 & 110.701 & 108.886 & & 74.502 \\
\hline 11/23/07 9:30 & 127.603 & 125.383 & 121.662 & 101.697 & 105.5 & 97.138 & 110.879 & 109.112 & & 74.655 \\
\hline 11/23/07 10:00 & 127.724 & 125.422 & 121.7 & 101.776 & 105.604 & 97.239 & 110.978 & 109.22 & & 74.759 \\
\hline $11 / 23 / 07$ 10:30 & 127.799 & 125.444 & 121.723 & 101.826 & 105.663 & 97.298 & 111.042 & 109.284 & & 74.816 \\
\hline $11 / 23 / 0711: 00$ & 127.844 & 125.465 & 121.753 & 101.858 & 105.698 & 97.334 & 111.085 & 109.322 & & 74.855 \\
\hline 11/23/07 11:30 & 127.875 & 125.481 & 121.763 & 101.885 & 105.72 & 97.357 & 111.114 & 109.346 & & 74.878 \\
\hline $11 / 23 / 07$ 12:00 & 127.896 & 125.46 & 121.745 & 101.904 & 105.747 & 97.388 & 111.136 & 109.373 & & 74.911 \\
\hline $11 / 23 / 0712: 30$ & 127.915 & 125.456 & 121.748 & 101.897 & 105.751 & 97.39 & 111.155 & 109.382 & & 74.919 \\
\hline $11 / 23 / 07$ 13:00 & 127.932 & 125.446 & 121.738 & 101.89 & 105.741 & 97.379 & 111.17 & 109.368 & & 74.913 \\
\hline 11/23/07 13:30 & 127.943 & 125.437 & 121.725 & 101.887 & 105.74 & 97.376 & 111.181 & 109.366 & & 74.918 \\
\hline $11 / 23 / 07$ 14:00 & 127.951 & 125.424 & 121.718 & 101.881 & 105.73 & 97.367 & 111.193 & 109.357 & & 74.911 \\
\hline
\end{tabular}


TABLE S1.1 (Cont.)

Depth from Top of Casing (ft)

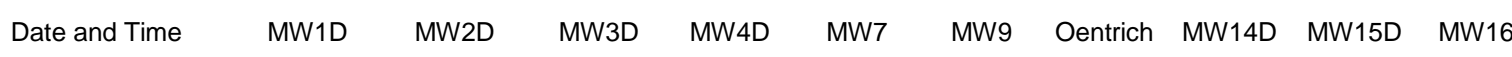

\begin{tabular}{|c|c|c|c|c|c|c|c|c|c|}
\hline $11 / 23 / 07$ 14:30 & 127.956 & 125.417 & 121.713 & 101.868 & 105.724 & 97.36 & 111.2 & 109.348 & 74.909 \\
\hline $11 / 23 / 07$ 15:00 & 127.96 & 125.419 & 121.713 & 101.864 & 105.711 & 97.348 & 111.208 & 109.335 & 74.903 \\
\hline 11/23/07 15:30 & 127.96 & 123.968 & 121.71 & 101.863 & 105.709 & 97.346 & 111.213 & 109.333 & 74.905 \\
\hline $11 / 23 / 07$ 16:00 & 127.96 & 123.397 & 120.319 & 101.857 & 105.715 & 97.348 & 111.217 & 109.335 & 74.912 \\
\hline 11/23/07 16:30 & 126.62 & 123.211 & 120.112 & 101.309 & 105.566 & 97.119 & 110.975 & 108.713 & 74.91 \\
\hline $11 / 23 / 07$ 17:00 & 125.91 & 123.102 & 119.994 & 100.996 & 105.083 & 96.582 & 110.481 & 107.784 & 74.765 \\
\hline $11 / 23 / 07$ 17:30 & 125.712 & 123.031 & 119.921 & 100.853 & 104.906 & 96.405 & 110.316 & 107.56 & 74.632 \\
\hline $11 / 23 / 07$ 18:00 & 125.608 & 122.991 & 119.868 & 100.765 & 104.801 & 96.299 & 110.226 & 107.442 & 74.533 \\
\hline $11 / 23 / 07$ 18:30 & 125.542 & 122.959 & 119.84 & 100.705 & 104.736 & 96.235 & 110.169 & 107.371 & 74.467 \\
\hline $11 / 23 / 07$ 19:00 & 125.494 & 122.932 & 119.812 & 100.674 & 104.694 & 96.195 & 110.132 & 107.325 & 74.423 \\
\hline 11/23/07 19:30 & 125.464 & 122.909 & 119.79 & 100.65 & 104.664 & 96.167 & 110.105 & 107.292 & 74.39 \\
\hline $11 / 23 / 0720: 00$ & 125.442 & 122.9 & 119.777 & 100.624 & 104.639 & 96.139 & 110.087 & 107.263 & 74.361 \\
\hline $11 / 23 / 0720: 30$ & 125.424 & 122.891 & 119.765 & 100.605 & 104.618 & 96.122 & 110.072 & 107.241 & 74.341 \\
\hline $11 / 23 / 0721: 00$ & 125.409 & 122.889 & 119.765 & 100.598 & 104.607 & 96.108 & 110.06 & 107.228 & 74.326 \\
\hline $11 / 23 / 0721: 30$ & 125.4 & 122.889 & 119.75 & 100.594 & 104.599 & 96.101 & 110.049 & 107.219 & 74.316 \\
\hline $11 / 23 / 0722: 00$ & 125.388 & 122.886 & 119.75 & 100.593 & 104.603 & 96.101 & 110.042 & 107.216 & 74.314 \\
\hline 11/23/07 22:30 & 125.381 & 122.877 & 119.739 & 100.593 & 104.599 & 96.101 & 110.036 & 107.216 & 74.312 \\
\hline $11 / 23 / 0723: 00$ & 125.374 & 122.873 & 119.739 & 100.589 & 104.597 & 96.096 & 110.032 & 107.214 & 74.308 \\
\hline $11 / 23 / 0723: 30$ & 125.367 & 122.868 & 119.734 & 100.586 & 104.593 & 96.094 & 110.028 & 107.208 & 74.303 \\
\hline $11 / 24 / 070: 00$ & 125.36 & 122.866 & 119.744 & 100.581 & 104.588 & 96.092 & 110.023 & 107.203 & 74.298 \\
\hline $11 / 24 / 070: 30$ & 125.355 & 122.859 & 119.739 & 100.577 & 104.586 & 96.087 & 110.019 & 107.199 & 74.293 \\
\hline $11 / 24 / 071: 00$ & 125.35 & 122.848 & 119.727 & 100.574 & 104.582 & 96.082 & 110.015 & 107.194 & 74.287 \\
\hline $11 / 24 / 071: 30$ & 125.346 & 122.839 & 119.719 & 100.565 & 104.576 & 96.075 & 110.012 & 107.188 & 74.283 \\
\hline $11 / 24 / 072: 00$ & 125.341 & 122.832 & 119.712 & 100.558 & 104.569 & 96.068 & 110.008 & 107.179 & 74.27 \\
\hline $11 / 24 / 072: 30$ & 125.336 & 122.825 & 119.707 & 100.55 & 104.559 & 96.059 & 110.006 & 107.168 & 74.262 \\
\hline $11 / 24 / 073: 00$ & 125.331 & 122.821 & 119.699 & 100.541 & 104.551 & 96.054 & 110.002 & 107.161 & 74.254 \\
\hline $11 / 24 / 073: 30$ & 125.327 & 122.814 & 119.697 & 100.536 & 104.544 & 96.044 & 110 & 107.154 & 74.248 \\
\hline $11 / 24 / 074: 00$ & 125.324 & 122.807 & 119.689 & 100.533 & 104.544 & 96.042 & 109.997 & 107.15 & 74.242 \\
\hline $11 / 24 / 074: 30$ & 125.32 & 122.802 & 119.684 & 100.524 & 104.536 & 96.037 & 109.995 & 107.146 & 74.238 \\
\hline 11/24/07 5:00 & 125.317 & 122.8 & 119.679 & 100.521 & 104.532 & 96.03 & 109.991 & 107.137 & 74.229 \\
\hline $11 / 24 / 075: 30$ & 125.312 & 122.796 & 119.671 & 100.515 & 104.525 & 96.023 & 109.987 & 107.13 & 74.223 \\
\hline $11 / 24 / 076: 00$ & 125.31 & 122.798 & 119.671 & 100.515 & 104.523 & 96.021 & 109.985 & 107.128 & 74.221 \\
\hline $11 / 24 / 076: 30$ & 125.308 & 122.796 & 119.671 & 100.509 & 104.517 & 96.016 & 109.981 & 107.123 & 74.213 \\
\hline $11 / 24 / 077: 00$ & 125.303 & 122.8 & 119.676 & 100.512 & 104.521 & 96.018 & 109.981 & 107.128 & 74.217 \\
\hline $11 / 24 / 077: 30$ & 125.303 & 124.465 & 120.576 & 100.517 & 104.519 & 96.014 & 109.98 & 107.123 & 74.212 \\
\hline $11 / 24 / 078: 00$ & 125.301 & 124.895 & 121.277 & 100.519 & 104.523 & 96.021 & 109.98 & 107.128 & 74.214 \\
\hline $11 / 24 / 078: 30$ & 126.849 & 125.066 & 121.476 & 101.189 & 104.822 & 96.445 & 110.408 & 108.157 & 74.227 \\
\hline 11/24/07 9:00 & 126.979 & 125.154 & 121.576 & 101.475 & 105.233 & 96.879 & 110.815 & 108.751 & 74.401 \\
\hline 11/24/07 9:30 & 127.341 & 125.213 & 121.637 & 101.61 & 105.404 & 97.048 & 110.968 & 108.952 & 74.546 \\
\hline $11 / 24 / 07 \quad 10: 00$ & 127.445 & 125.261 & 121.687 & 101.684 & 105.494 & 97.14 & 111.063 & 109.05 & 74.632 \\
\hline $11 / 24 / 07 \quad 10: 30$ & 127.508 & 125.295 & 121.723 & 101.734 & 105.553 & 97.197 & 111.123 & 109.112 & 74.692 \\
\hline $11 / 24 / 07 \quad 11: 00$ & 127.551 & 125.31 & 121.74 & 101.775 & 105.599 & 97.244 & 111.165 & 109.162 & 74.74 \\
\hline $11 / 24 / 07$ 11:30 & 127.582 & 125.324 & 121.76 & 101.804 & 105.633 & 97.282 & 111.193 & 109.198 & 74.779 \\
\hline $11 / 24 / 07 \quad 12: 00$ & 127.605 & 123.505 & 120.488 & 101.821 & 105.648 & 97.296 & 111.217 & 109.213 & 74.796 \\
\hline $11 / 24 / 07$ 12:30 & 127.624 & 123.224 & 120.135 & 101.835 & 105.663 & 97.313 & 111.236 & 109.229 & 74.814 \\
\hline $11 / 24 / 07$ 13:00 & 126.038 & 123.086 & 119.981 & 101.058 & 105.182 & 96.69 & 110.622 & 107.929 & 74.761 \\
\hline $11 / 24 / 07$ 13:30 & 125.733 & 123.009 & 119.898 & 100.863 & 104.919 & 96.417 & 110.367 & 107.575 & 74.613 \\
\hline $11 / 24 / 0714: 00$ & 125.598 & 122.943 & 119.833 & 100.764 & 104.788 & 96.285 & 110.252 & 107.427 & 74.5 \\
\hline $11 / 24 / 07$ 14:30 & 125.518 & 122.904 & 119.79 & 100.701 & 104.713 & 96.214 & 110.182 & 107.345 & 74.428 \\
\hline $11 / 24 / 07$ 15:00 & 125.459 & 122.877 & 119.755 & 100.66 & 104.65 & 96.15 & 110.137 & 107.278 & 74.364 \\
\hline $11 / 24 / 07$ 15:30 & 125.416 & 122.868 & 119.744 & 100.631 & 104.614 & 96.115 & 110.105 & 107.239 & 74.326 \\
\hline $11 / 24 / 07$ 16:00 & 125.39 & 122.855 & 119.737 & 100.61 & 104.588 & 96.087 & 110.085 & 107.208 & 74.298 \\
\hline $11 / 24 / 07 \quad 16: 30$ & 125.367 & 122.836 & 119.719 & 100.596 & 104.58 & 96.08 & 110.066 & 107.197 & 74.287 \\
\hline $11 / 24 / 07 \quad 17: 00$ & 125.355 & 122.825 & 119.704 & 100.584 & 104.569 & 96.068 & 110.055 & 107.185 & 74.273 \\
\hline $11 / 24 / 07$ 17:30 & 125.346 & 122.818 & 119.697 & 100.574 & 104.55 & 96.051 & 110.045 & 107.166 & 74.254 \\
\hline $11 / 24 / 0718: 00$ & 125.336 & 122.809 & 119.692 & 100.569 & 104.542 & 96.04 & 110.038 & 107.154 & 74.241 \\
\hline $11 / 24 / 07$ 18:30 & 125.327 & 122.805 & 119.687 & 100.562 & 104.538 & 96.037 & 110.032 & 107.148 & 74.238 \\
\hline $11 / 24 / 07$ 19:00 & 125.322 & 122.798 & 119.681 & 100.558 & 104.527 & 96.03 & 110.027 & 107.139 & 74.225 \\
\hline $11 / 24 / 07$ 19:30 & 125.317 & 122.791 & 119.679 & 100.555 & 104.521 & 96.023 & 110.025 & 107.132 & 74.219 \\
\hline $11 / 24 / 0720: 00$ & 125.312 & 122.784 & 119.676 & 100.55 & 104.519 & 96.021 & 110.021 & 107.128 & 74.215 \\
\hline $11 / 24 / 0720: 30$ & 125.31 & 122.775 & 119.674 & 100.548 & 104.51 & 96.011 & 110.019 & 107.121 & 74.204 \\
\hline $11 / 24 / 0721: 00$ & 125.305 & 122.768 & 119.669 & 100.545 & 104.504 & 96.004 & 110.017 & 107.115 & 74.198 \\
\hline $11 / 24 / 0721: 30$ & 125.303 & 122.771 & 119.664 & 100.541 & 104.492 & 95.995 & 110.013 & 107.104 & 74.188 \\
\hline $11 / 24 / 0722: 00$ & 125.301 & 122.762 & 119.664 & 100.538 & 104.487 & 95.99 & 110.008 & 107.097 & 74.184 \\
\hline $11 / 24 / 0722: 30$ & 125.296 & 122.762 & 119.659 & 100.538 & 104.489 & 95.99 & 110.004 & 107.095 & 74.184 \\
\hline $11 / 24 / 0723: 00$ & 125.296 & 122.757 & 119.656 & 100.534 & 104.483 & 95.981 & 110.004 & 107.09 & 74.173 \\
\hline
\end{tabular}


TABLE S1.1 (Cont.)

Depth from Top of Casing (ft)

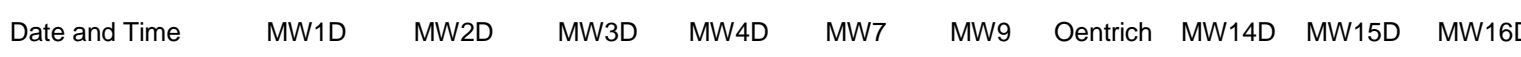

\begin{tabular}{|c|c|c|c|c|c|c|c|c|c|}
\hline 11/24/07 23:30 & 125.291 & 122.748 & 119.654 & 100.534 & 104.479 & 95.981 & 109.998 & 107.088 & 74.174 \\
\hline 11/25/07 0:00 & 125.289 & 122.743 & 119.651 & 100.531 & 104.475 & 95.978 & 109.998 & 107.086 & 74.171 \\
\hline 11/25/07 0:30 & 125.286 & 122.73 & 119.646 & 100.531 & 104.468 & 95.971 & 109.997 & 107.077 & 74.163 \\
\hline $11 / 25 / 071: 00$ & 125.284 & 122.709 & 119.641 & 100.527 & 104.464 & 95.967 & 109.995 & 107.073 & 74.159 \\
\hline $11 / 25 / 071: 30$ & 125.284 & 122.696 & 119.631 & 100.453 & 104.452 & 95.955 & 109.991 & 107.061 & 74.149 \\
\hline $11 / 25 / 072: 00$ & 125.284 & 122.696 & 119.616 & 100.479 & 104.432 & 95.934 & 109.989 & 107.042 & 74.128 \\
\hline 11/25/07 2:30 & 125.279 & 122.691 & 119.616 & 100.479 & 104.418 & 95.919 & 109.98 & 107.026 & 74.115 \\
\hline 11/25/07 3:00 & 125.275 & 122.687 & 119.608 & 100.479 & 104.418 & 95.922 & 109.961 & 107.026 & 74.115 \\
\hline 11/25/07 3:30 & 125.272 & 122.685 & 119.603 & 100.477 & 104.412 & 95.915 & 109.948 & 107.017 & 74.107 \\
\hline $11 / 25 / 074: 00$ & 125.27 & 122.678 & 119.596 & 100.474 & 104.411 & 95.915 & 109.946 & 107.017 & 74.105 \\
\hline $11 / 25 / 074: 30$ & 125.265 & 122.66 & 119.596 & 100.474 & 104.409 & 95.912 & 109.944 & 107.013 & 74.103 \\
\hline $11 / 25 / 075: 00$ & 125.263 & 122.657 & 119.583 & 100.471 & 104.405 & 95.905 & 109.942 & 107.008 & 74.099 \\
\hline $11 / 25 / 075: 30$ & 125.268 & 122.646 & 119.581 & 100.374 & 104.384 & 95.884 & 109.929 & 106.991 & 74.078 \\
\hline $11 / 25 / 076: 00$ & 125.26 & 122.641 & 119.568 & 100.369 & 104.38 & 95.882 & 109.918 & 106.984 & 74.074 \\
\hline 11/25/07 6:30 & 125.26 & 122.637 & 119.566 & 100.362 & 104.372 & 95.87 & 109.912 & 106.975 & 74.064 \\
\hline $11 / 25 / 077: 00$ & 125.258 & 122.635 & 119.563 & 100.359 & 104.367 & 95.868 & 109.91 & 106.968 & 74.058 \\
\hline $11 / 25 / 077: 30$ & 125.256 & 122.635 & 119.561 & 100.358 & 104.363 & 95.865 & 109.901 & 106.966 & 74.053 \\
\hline $11 / 25 / 078: 00$ & 125.256 & 124.004 & 119.558 & 100.352 & 104.361 & 95.863 & 109.899 & 106.964 & 74.053 \\
\hline 11/25/07 8:30 & 125.253 & 124.691 & 120.957 & 100.355 & 104.361 & 95.865 & 109.901 & 106.964 & 74.051 \\
\hline $11 / 25 / 079: 00$ & 126.286 & 124.886 & 121.173 & 100.879 & 104.411 & 95.927 & 110 & 107.059 & 74.051 \\
\hline 11/25/07 9:30 & 127.057 & 124.993 & 121.277 & 101.22 & 104.989 & 96.62 & 110.641 & 108.551 & 74.196 \\
\hline 11/25/07 10:00 & 127.255 & 125.045 & 121.35 & 101.373 & 105.184 & 96.82 & 110.819 & 108.773 & 74.355 \\
\hline 11/25/07 10:30 & 127.369 & 125.088 & 121.408 & 101.46 & 105.292 & 96.926 & 110.918 & 108.888 & 74.457 \\
\hline $11 / 25 / 0711: 00$ & 127.442 & 125.113 & 121.445 & 101.513 & 105.347 & 96.985 & 110.982 & 108.952 & 74.515 \\
\hline 11/25/07 11:30 & 127.49 & 125.129 & 121.471 & 101.551 & 105.387 & 97.023 & 111.024 & 108.994 & 74.558 \\
\hline $11 / 25 / 0712: 00$ & 127.53 & 125.124 & 121.481 & 101.568 & 105.412 & 97.051 & 111.05 & 109.023 & 74.591 \\
\hline $11 / 25 / 0712: 30$ & 127.556 & 125.134 & 121.486 & 101.58 & 105.427 & 97.065 & 111.067 & 109.038 & 74.608 \\
\hline $11 / 25 / 07$ 13:00 & 127.579 & 125.134 & 121.491 & 101.582 & 105.433 & 97.067 & 111.072 & 109.043 & 74.616 \\
\hline 11/25/07 13:30 & 127.594 & 125.129 & 121.488 & 101.585 & 105.437 & 97.07 & 111.074 & 109.045 & 74.622 \\
\hline 11/25/07 14:00 & 127.605 & 123.34 & 120.39 & 101.583 & 105.439 & 97.074 & 111.076 & 109.047 & 74.628 \\
\hline $11 / 25 / 0714: 30$ & 127.61 & 123.022 & 119.994 & 101.582 & 105.439 & 97.07 & 111.072 & 109.045 & 74.63 \\
\hline $11 / 25 / 07$ 15:00 & 125.814 & 122.88 & 119.823 & 100.867 & 105.016 & 96.523 & 110.528 & 107.781 & 74.585 \\
\hline 11/25/07 15:30 & 125.494 & 122.789 & 119.734 & 100.645 & 104.715 & 96.216 & 110.241 & 107.374 & 74.415 \\
\hline $11 / 25 / 0716: 00$ & 125.353 & 122.743 & 119.671 & 100.531 & 104.58 & 96.082 & 110.109 & 107.221 & 74.301 \\
\hline 11/25/07 16:30 & 125.27 & 122.712 & 119.629 & 100.471 & 104.491 & 95.993 & 110.032 & 107.123 & 74.212 \\
\hline 11/25/07 17:00 & 125.216 & 122.68 & 119.606 & 100.428 & 104.451 & 95.95 & 109.981 & 107.075 & 74.169 \\
\hline 11/25/07 17:30 & 125.178 & 122.653 & 119.583 & 100.407 & 104.422 & 95.922 & 109.95 & 107.042 & 74.136 \\
\hline 11/25/07 18:00 & 125.156 & 122.657 & 119.561 & 100.376 & 104.392 & 95.891 & 109.931 & 107.008 & 74.103 \\
\hline 11/25/07 18:30 & 125.138 & 122.655 & 119.545 & 100.359 & 104.365 & 95.868 & 109.914 & 106.984 & 74.078 \\
\hline 11/25/07 19:00 & 125.119 & 122.655 & 119.543 & 100.371 & 104.372 & 95.872 & 109.895 & 106.986 & 74.081 \\
\hline 11/25/07 19:30 & 125.112 & 122.657 & 119.543 & 100.365 & 104.371 & 95.872 & 109.889 & 106.984 & 74.076 \\
\hline $11 / 25 / 0720: 00$ & 125.107 & 122.655 & 119.545 & 100.365 & 104.369 & 95.87 & 109.884 & 106.982 & 74.07 \\
\hline 11/25/07 20:30 & 125.095 & 122.648 & 119.54 & 100.372 & 104.378 & 95.879 & 109.878 & 106.984 & 74.074 \\
\hline $11 / 25 / 0721: 00$ & 125.093 & 122.651 & 119.54 & 100.365 & 104.372 & 95.872 & 109.873 & 106.982 & 74.068 \\
\hline $11 / 25 / 0721: 30$ & 125.088 & 122.648 & 119.535 & 100.36 & 104.369 & 95.87 & 109.871 & 106.98 & 74.064 \\
\hline $11 / 25 / 0722: 00$ & 125.083 & 122.639 & 119.533 & 100.36 & 104.372 & 95.872 & 109.869 & 106.98 & 74.064 \\
\hline 11/25/07 22:30 & 125.081 & 122.637 & 119.525 & 100.362 & 104.369 & 95.868 & 109.869 & 106.975 & 74.057 \\
\hline 11/25/07 23:00 & 125.081 & 122.637 & 119.525 & 100.35 & 104.357 & 95.858 & 109.867 & 106.968 & 74.05 \\
\hline 11/25/07 23:30 & 125.078 & 122.635 & 119.523 & 100.353 & 104.357 & 95.858 & 109.863 & 106.964 & 74.047 \\
\hline 11/26/07 0:00 & 125.076 & 122.644 & 119.523 & 100.35 & 104.359 & 95.861 & 109.865 & 106.966 & 74.047 \\
\hline 11/26/07 0:30 & 125.071 & 122.635 & 119.528 & 100.357 & 104.353 & 95.856 & 109.861 & 106.96 & 74.043 \\
\hline $11 / 26 / 071: 00$ & 125.069 & 122.639 & 119.522 & 100.357 & 104.365 & 95.865 & 109.859 & 106.968 & 74.054 \\
\hline 11/26/07 1:30 & 125.069 & 122.644 & 119.528 & 100.348 & 104.359 & 95.861 & 109.861 & 106.964 & 74.045 \\
\hline 11/26/07 2:00 & 125.067 & 122.651 & 119.532 & 100.357 & 104.361 & 95.863 & 109.858 & 106.964 & 74.047 \\
\hline 11/26/07 2:30 & 125.064 & 122.653 & 119.54 & 100.36 & 104.363 & 95.868 & 109.858 & 106.968 & 74.05 \\
\hline 11/26/07 3:00 & 125.06 & 122.664 & 119.548 & 100.369 & 104.372 & 95.875 & 109.854 & 106.975 & 74.058 \\
\hline $11 / 26 / 073: 30$ & 125.06 & 122.673 & 119.553 & 100.374 & 104.376 & 95.879 & 109.852 & 106.982 & 74.059 \\
\hline $11 / 26 / 074: 00$ & 125.057 & 122.68 & 119.543 & 100.388 & 104.388 & 95.886 & 109.852 & 106.986 & 74.066 \\
\hline 11/26/07 4:30 & 125.052 & 122.685 & 119.561 & 100.39 & 104.395 & 95.894 & 109.85 & 106.995 & 74.072 \\
\hline $11 / 26 / 075: 00$ & 125.05 & 122.703 & 119.553 & 100.403 & 104.407 & 95.905 & 109.848 & 107.006 & 74.082 \\
\hline 11/26/07 5:30 & 125.052 & 122.705 & 119.561 & 100.415 & 104.412 & 95.912 & 109.846 & 107.013 & 74.086 \\
\hline $11 / 26 / 076: 00$ & 125.045 & 122.705 & 119.561 & 100.424 & 104.43 & 95.931 & 109.843 & 107.028 & 74.103 \\
\hline 11/26/07 6:30 & 125.045 & 122.716 & 119.56 & 100.424 & 104.432 & 95.934 & 109.843 & 107.03 & 74.103 \\
\hline 11/26/07 7:00 & 125.048 & 124.723 & 121.05 & 100.428 & 104.43 & 95.934 & 109.844 & 107.033 & 74.103 \\
\hline 11/26/07 7:30 & 125.043 & 124.961 & 121.307 & 100.901 & 104.443 & 95.948 & 109.841 & 107.044 & 74.113 \\
\hline 11/26/07 8:00 & 127.066 & 125.095 & 121.468 & 101.336 & 105.071 & 96.719 & 110.582 & 108.565 & 74.25 \\
\hline
\end{tabular}


TABLE S1.1 (Cont.)

Depth from Top of Casing (ft)

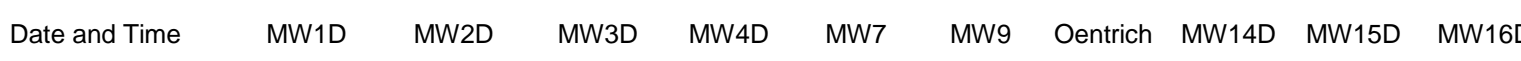

\begin{tabular}{|c|c|c|c|c|c|c|c|c|c|}
\hline 11/26/07 8:30 & 127.303 & 125.193 & 121.554 & 101.53 & 105.305 & 96.954 & 110.785 & 108.83 & 74.434 \\
\hline 11/26/07 9:00 & 127.428 & 125.258 & 121.639 & 101.644 & 105.443 & 97.096 & 110.9 & 108.988 & 74.564 \\
\hline $11 / 26 / 079: 30$ & 127.501 & 125.299 & 121.684 & 101.733 & 105.54 & 97.19 & 110.973 & 109.085 & 74.655 \\
\hline 11/26/07 10:00 & 127.551 & 123.623 & 120.601 & 101.792 & 105.606 & 97.258 & 111.029 & 109.156 & 74.726 \\
\hline 11/26/07 10:30 & 127.591 & 123.297 & 120.163 & 101.828 & 105.646 & 97.303 & 111.072 & 109.2 & 74.767 \\
\hline $11 / 26 / 0711: 00$ & 126.215 & 123.156 & 120.004 & 101.146 & 105.294 & 96.815 & 110.573 & 108.091 & 74.775 \\
\hline $11 / 26 / 0711: 30$ & 125.816 & 123.072 & 119.92 & 100.931 & 104.995 & 96.504 & 110.278 & 107.65 & 74.643 \\
\hline $11 / 26 / 0712: 00$ & 125.665 & 123.018 & 119.865 & 100.822 & 104.862 & 96.37 & 110.156 & 107.498 & 74.535 \\
\hline $11 / 26 / 07$ 12:30 & 125.58 & 122.986 & 119.835 & 100.755 & 104.778 & 96.29 & 110.087 & 107.405 & 74.457 \\
\hline 11/26/07 13:00 & 125.523 & 122.966 & 119.812 & 100.715 & 104.73 & 96.242 & 110.043 & 107.354 & 74.409 \\
\hline 11/26/07 13:30 & 125.49 & 122.948 & 119.797 & 100.688 & 104.698 & 96.209 & 110.015 & 107.318 & 74.376 \\
\hline $11 / 26 / 0714: 00$ & 125.461 & 122.938 & 119.787 & 100.669 & 104.679 & 96.191 & 109.995 & 107.294 & 74.355 \\
\hline $11 / 26 / 0714: 30$ & 125.442 & 122.929 & 119.777 & 100.657 & 104.662 & 96.172 & 109.98 & 107.276 & 74.336 \\
\hline $11 / 26 / 07$ 15:00 & 125.428 & 122.923 & 119.77 & 100.652 & 104.652 & 96.165 & 109.97 & 107.265 & 74.326 \\
\hline $11 / 26 / 07$ 15:30 & 125.416 & 122.929 & 119.761 & 100.641 & 104.649 & 96.158 & 109.963 & 107.261 & 74.322 \\
\hline $11 / 26 / 0716: 00$ & 125.407 & 122.936 & 119.76 & 100.638 & 104.645 & 96.15 & 109.955 & 107.252 & 74.314 \\
\hline $11 / 26 / 07 \quad 16: 30$ & 125.395 & 122.945 & 119.756 & 100.65 & 104.649 & 96.158 & 109.95 & 107.256 & 74.322 \\
\hline 11/26/07 17:00 & 125.39 & 122.952 & 119.8 & 100.658 & 104.654 & 96.162 & 109.95 & 107.261 & 74.326 \\
\hline $11 / 26 / 07$ 17:30 & 125.383 & 122.957 & 119.797 & 100.674 & 104.67 & 96.176 & 109.957 & 107.272 & 74.337 \\
\hline $11 / 26 / 07$ 18:00 & 125.383 & 122.968 & 119.795 & 100.672 & 104.67 & 96.183 & 109.963 & 107.276 & 74.339 \\
\hline $11 / 26 / 07$ 18:30 & 125.381 & 122.97 & 119.797 & 100.677 & 104.675 & 96.186 & 109.965 & 107.283 & 74.341 \\
\hline 11/26/07 19:00 & 125.374 & 122.972 & 119.8 & 100.688 & 104.689 & 96.2 & 109.962 & 107.294 & 74.355 \\
\hline 11/26/07 19:30 & 125.372 & 122.977 & 119.8 & 100.693 & 104.69 & 96.2 & 109.963 & 107.296 & 74.353 \\
\hline $11 / 26 / 0720: 00$ & 125.367 & 122.975 & 119.802 & 100.693 & 104.694 & 96.205 & 109.967 & 107.301 & 74.36 \\
\hline $11 / 26 / 0720: 30$ & 125.364 & 122.977 & 119.805 & 100.698 & 104.696 & 96.209 & 109.967 & 107.303 & 74.363 \\
\hline $11 / 26 / 0721: 00$ & 125.362 & 122.984 & 119.804 & 100.695 & 104.698 & 96.209 & 109.966 & 107.303 & 74.364 \\
\hline $11 / 26 / 0721: 30$ & 125.357 & 122.979 & 119.81 & 100.698 & 104.7 & 96.209 & 109.966 & 107.305 & 74.366 \\
\hline $11 / 26 / 0722: 00$ & 125.355 & 122.977 & 119.812 & 100.701 & 104.702 & 96.209 & 109.966 & 107.307 & 74.368 \\
\hline 11/26/07 22:30 & 125.355 & 122.982 & 119.812 & 100.699 & 104.696 & 96.209 & 109.968 & 107.305 & 74.364 \\
\hline $11 / 26 / 0723: 00$ & 125.353 & 122.979 & 119.814 & 100.696 & 104.698 & 96.209 & 109.968 & 107.305 & 74.364 \\
\hline $11 / 26 / 0723: 30$ & 125.348 & 122.979 & 119.814 & 100.7 & 104.702 & 96.212 & 109.968 & 107.307 & 74.37 \\
\hline $11 / 27 / 070: 00$ & 125.348 & 122.982 & 119.814 & 100.698 & 104.698 & 96.207 & 109.968 & 107.307 & 74.368 \\
\hline $11 / 27 / 070: 30$ & 125.346 & 122.975 & 119.82 & 100.7 & 104.7 & 96.209 & 109.966 & 107.309 & 74.372 \\
\hline $11 / 27 / 071: 00$ & 125.343 & 122.966 & 119.82 & 100.693 & 104.704 & 96.214 & 109.965 & 107.314 & 74.376 \\
\hline $11 / 27 / 071: 30$ & 125.343 & 122.957 & 119.82 & 100.693 & 104.694 & 96.205 & 109.965 & 107.303 & 74.366 \\
\hline $11 / 27 / 072: 00$ & 125.341 & 122.959 & 119.817 & 100.681 & 104.689 & 96.198 & 109.965 & 107.298 & 74.359 \\
\hline $11 / 27 / 072: 30$ & 125.338 & 122.963 & 119.809 & 100.674 & 104.677 & 96.183 & 109.963 & 107.283 & 74.349 \\
\hline $11 / 27 / 073: 00$ & 125.331 & 122.963 & 119.81 & 100.679 & 104.679 & 96.191 & 109.961 & 107.285 & 74.353 \\
\hline 11/27/07 3:30 & 125.329 & 122.957 & 119.815 & 100.684 & 104.685 & 96.195 & 109.955 & 107.292 & 74.362 \\
\hline $11 / 27 / 074: 00$ & 125.324 & 122.948 & 119.815 & 100.683 & 104.689 & 96.198 & 109.953 & 107.292 & 74.359 \\
\hline $11 / 27 / 074: 30$ & 125.327 & 122.938 & 119.812 & 100.679 & 104.679 & 96.191 & 109.953 & 107.285 & 74.355 \\
\hline $11 / 27 / 07$ 5:00 & 125.322 & 122.934 & 119.812 & 100.66 & 104.671 & 96.183 & 109.951 & 107.276 & 74.349 \\
\hline $11 / 27 / 075: 30$ & 125.322 & 122.934 & 119.807 & 100.655 & 104.66 & 96.169 & 109.951 & 107.267 & 74.339 \\
\hline $11 / 27 / 076: 00$ & 125.317 & 122.929 & 119.8 & 100.652 & 104.656 & 96.165 & 109.948 & 107.263 & 74.335 \\
\hline $11 / 27 / 076: 30$ & 125.312 & 122.932 & 119.802 & 100.645 & 104.656 & 96.165 & 109.946 & 107.261 & 74.335 \\
\hline $11 / 27 / 07$ 7:00 & 125.308 & 124.891 & 121.095 & 100.652 & 104.654 & 96.16 & 109.942 & 107.256 & 74.333 \\
\hline $11 / 27 / 07$ 7:30 & 125.305 & 125.124 & 121.372 & 100.925 & 104.654 & 96.162 & 109.94 & 107.261 & 74.335 \\
\hline $11 / 27 / 078: 00$ & 127.274 & 125.263 & 121.5 & 101.447 & 105.193 & 96.829 & 110.597 & 108.729 & 74.428 \\
\hline $11 / 27 / 078: 30$ & 127.525 & 125.344 & 121.583 & 101.625 & 105.423 & 97.067 & 110.819 & 109.01 & 74.595 \\
\hline 11/27/07 9:00 & 127.646 & 125.385 & 121.645 & 101.753 & 105.568 & 97.211 & 110.928 & 109.158 & 74.732 \\
\hline 11/27/07 9:30 & 127.728 & 125.419 & 121.682 & 101.818 & 105.654 & 97.291 & 110.999 & 109.249 & 74.814 \\
\hline $11 / 27 / 07 \quad 10: 00$ & 127.787 & 123.857 & 121.098 & 101.854 & 105.684 & 97.327 & 111.052 & 109.293 & 74.853 \\
\hline $11 / 27 / 07$ 10:30 & 127.816 & 123.399 & 120.318 & 101.878 & 105.724 & 97.369 & 111.082 & 109.333 & 74.897 \\
\hline 11/27/07 11:00 & 126.52 & 123.226 & 120.139 & 101.29 & 105.498 & 97.037 & 110.749 & 108.439 & 74.903 \\
\hline $11 / 27 / 0711: 30$ & 125.934 & 123.122 & 120.044 & 101.02 & 105.092 & 96.601 & 110.361 & 107.783 & 74.759 \\
\hline $11 / 27 / 07$ 12:00 & 125.766 & 123.043 & 119.986 & 100.884 & 104.927 & 96.433 & 110.218 & 107.571 & 74.628 \\
\hline $11 / 27 / 07$ 12:30 & 125.674 & 122.982 & 119.94 & 100.793 & 104.828 & 96.337 & 110.141 & 107.462 & 74.533 \\
\hline 11/27/07 13:00 & 125.62 & 122.936 & 119.905 & 100.722 & 104.746 & 96.257 & 110.096 & 107.376 & 74.455 \\
\hline $11 / 27 / 07$ 13:30 & 125.577 & 122.886 & 119.87 & 100.667 & 104.69 & 96.195 & 110.066 & 107.314 & 74.396 \\
\hline $11 / 27 / 07$ 14:00 & 125.549 & 122.855 & 119.832 & 100.62 & 104.639 & 96.15 & 110.045 & 107.263 & 74.351 \\
\hline $11 / 27 / 0714: 30$ & 125.53 & 122.823 & 119.79 & 100.583 & 104.597 & 96.108 & 110.032 & 107.223 & 74.31 \\
\hline $11 / 27 / 07$ 15:00 & 125.516 & 122.784 & 119.751 & 100.55 & 104.563 & 96.075 & 110.015 & 107.185 & 74.276 \\
\hline $11 / 27 / 07$ 15:30 & 125.506 & 122.759 & 119.736 & 100.517 & 104.532 & 96.037 & 110 & 107.148 & 74.242 \\
\hline $11 / 27 / 0716: 00$ & 125.494 & 122.734 & 119.714 & 100.484 & 104.498 & 96.007 & 109.981 & 107.115 & 74.211 \\
\hline $11 / 27 / 07 \quad 16: 30$ & 125.487 & 122.705 & 119.678 & 100.462 & 104.473 & 95.983 & 109.965 & 107.092 & 74.192 \\
\hline $11 / 27 / 07$ 17:00 & 125.461 & 122.68 & 119.659 & 100.434 & 104.447 & 95.957 & 109.953 & 107.061 & 74.165 \\
\hline
\end{tabular}


TABLE S1.1 (Cont.)

Depth from Top of Casing (ft)

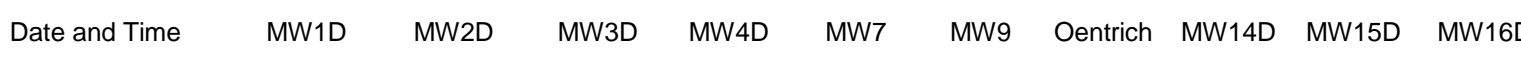

\begin{tabular}{|c|c|c|c|c|c|c|c|c|c|}
\hline 11/27/07 17:30 & 125.13 & 122.655 & 119.631 & 100.407 & 104.416 & 95.929 & 109.946 & 107.03 & 74.136 \\
\hline $11 / 27 / 0718: 00$ & 125.104 & 122.641 & 119.535 & 100.384 & 104.395 & 95.905 & 109.933 & 107.008 & 74.113 \\
\hline $11 / 27 / 0718: 30$ & 125.083 & 122.63 & 119.53 & 100.359 & 104.371 & 95.882 & 109.921 & 106.984 & 74.091 \\
\hline 11/27/07 19:00 & 125.067 & 122.623 & 119.525 & 100.347 & 104.359 & 95.868 & 109.908 & 106.968 & 74.078 \\
\hline $11 / 27 / 07$ 19:30 & 125.052 & 122.626 & 119.52 & 100.339 & 104.346 & 95.856 & 109.899 & 106.955 & 74.064 \\
\hline $11 / 27 / 0720: 00$ & 125.045 & 122.632 & 119.525 & 100.339 & 104.342 & 95.851 & 109.889 & 106.949 & 74.059 \\
\hline $11 / 27 / 0720: 30$ & 125.043 & 122.641 & 119.525 & 100.348 & 104.342 & 95.851 & 109.882 & 106.949 & 74.06 \\
\hline $11 / 27 / 0721: 00$ & 125.043 & 122.637 & 119.53 & 100.353 & 104.35 & 95.858 & 109.878 & 106.957 & 74.064 \\
\hline 11/27/07 21:30 & 125.038 & 122.626 & 119.518 & 100.355 & 104.361 & 95.87 & 109.874 & 106.968 & 74.076 \\
\hline 11/27/07 22:00 & 125.038 & 122.614 & 119.512 & 100.35 & 104.357 & 95.865 & 109.874 & 106.966 & 74.068 \\
\hline $11 / 27 / 0722: 30$ & 125.041 & 122.596 & 119.502 & 100.338 & 104.344 & 95.853 & 109.874 & 106.953 & 74.054 \\
\hline $11 / 27 / 0723: 00$ & 125.043 & 122.571 & 119.474 & 100.324 & 104.334 & 95.844 & 109.874 & 106.944 & 74.045 \\
\hline 11/27/07 23:30 & 125.045 & 122.555 & 119.467 & 100.303 & 104.313 & 95.823 & 109.871 & 106.922 & 74.024 \\
\hline $11 / 28 / 070: 00$ & 125.043 & 122.558 & 119.467 & 100.278 & 104.289 & 95.797 & 109.869 & 106.9 & 73.999 \\
\hline 11/28/07 0:30 & 125.043 & 122.542 & 119.464 & 100.267 & 104.27 & 95.778 & 109.839 & 106.878 & 73.985 \\
\hline $11 / 28 / 071: 00$ & 125.038 & 122.528 & 119.464 & 100.264 & 104.273 & 95.78 & 109.835 & 106.88 & 73.985 \\
\hline $11 / 28 / 071: 30$ & 125.043 & 122.51 & 119.451 & 100.248 & 104.262 & 95.769 & 109.828 & 106.873 & 73.973 \\
\hline $11 / 28 / 072: 00$ & 125.041 & 122.51 & 119.441 & 100.233 & 104.245 & 95.752 & 109.811 & 106.856 & 73.956 \\
\hline $11 / 28 / 072: 30$ & 125.041 & 122.499 & 119.439 & 100.217 & 104.226 & 95.733 & 109.788 & 106.836 & 73.94 \\
\hline $11 / 28 / 073: 00$ & 125.036 & 122.496 & 119.431 & 100.219 & 104.226 & 95.733 & 109.788 & 106.833 & 73.937 \\
\hline 11/28/07 3:30 & 125.036 & 122.496 & 119.426 & 100.214 & 104.216 & 95.724 & 109.777 & 106.82 & 73.929 \\
\hline $11 / 28 / 074: 00$ & 125.034 & 122.483 & 119.424 & 100.21 & 104.214 & 95.721 & 109.771 & 106.822 & 73.927 \\
\hline $11 / 28 / 074: 30$ & 125.034 & 122.487 & 119.414 & 100.21 & 104.216 & 95.721 & 109.773 & 106.82 & 73.925 \\
\hline $11 / 28 / 075: 00$ & 125.034 & 122.499 & 119.409 & 100.196 & 104.201 & 95.707 & 109.766 & 106.807 & 73.911 \\
\hline 11/28/07 5:30 & 125.031 & 122.512 & 119.394 & 100.212 & 104.207 & 95.717 & 109.764 & 106.811 & 73.919 \\
\hline $11 / 28 / 076: 00$ & 125.026 & 123.923 & 119.409 & 100.222 & 104.22 & 95.724 & 109.762 & 106.82 & 73.925 \\
\hline $11 / 28 / 076: 30$ & 125.026 & 124.578 & 120.951 & 100.234 & 104.235 & 95.743 & 109.762 & 106.836 & 73.94 \\
\hline $11 / 28 / 077: 00$ & 126.272 & 124.809 & 121.21 & 100.822 & 104.342 & 95.908 & 109.965 & 107.179 & 73.942 \\
\hline 11/28/07 7:30 & 127.125 & 124.929 & 121.341 & 101.178 & 104.919 & 96.572 & 110.55 & 108.425 & 74.113 \\
\hline $11 / 28 / 078: 00$ & 127.326 & 125.038 & 121.44 & 101.368 & 105.151 & 96.806 & 110.727 & 108.68 & 74.305 \\
\hline 11/28/07 8:30 & 127.442 & 125.113 & 121.488 & 101.477 & 105.271 & 96.926 & 110.83 & 108.808 & 74.417 \\
\hline $11 / 28 / 079: 00$ & 127.511 & 125.168 & 121.548 & 101.572 & 105.382 & 97.037 & 110.89 & 108.928 & 74.525 \\
\hline 11/28/07 9:30 & 127.558 & 123.36 & 120.24 & 101.647 & 105.458 & 97.115 & 110.933 & 109.007 & 74.603 \\
\hline $11 / 28 / 0710: 00$ & 127.594 & 123.136 & 119.966 & 101.545 & 105.511 & 97.169 & 110.969 & 109.067 & 74.655 \\
\hline $11 / 28 / 0710: 30$ & 125.688 & 123.043 & 119.842 & 100.946 & 105.048 & 96.561 & 110.342 & 107.765 & 74.637 \\
\hline $11 / 28 / 0711: 00$ & 125.428 & 122.993 & 119.787 & 100.787 & 104.839 & 96.351 & 110.139 & 107.484 & 74.518 \\
\hline 11/28/07 11:30 & 125.31 & 122.957 & 119.749 & 100.724 & 104.748 & 96.259 & 110.042 & 107.376 & 74.438 \\
\hline $11 / 28 / 0712: 00$ & 125.237 & 122.927 & 119.726 & 100.684 & 104.704 & 96.214 & 109.987 & 107.325 & 74.392 \\
\hline $11 / 28 / 0712: 30$ & 125.197 & 122.911 & 119.714 & 100.655 & 104.67 & 96.183 & 109.953 & 107.289 & 74.357 \\
\hline $11 / 28 / 0713: 00$ & 125.171 & 122.898 & 119.706 & 100.632 & 104.643 & 96.153 & 109.931 & 107.256 & 74.324 \\
\hline 11/28/07 13:30 & 125.149 & 122.904 & 119.699 & 100.622 & 104.626 & 96.136 & 109.916 & 107.241 & 74.308 \\
\hline $11 / 28 / 0714: 00$ & 125.138 & 122.904 & 119.693 & 100.612 & 104.612 & 96.127 & 109.905 & 107.225 & 74.293 \\
\hline $11 / 28 / 0714: 30$ & 125.121 & 122.909 & 119.693 & 100.613 & 104.622 & 96.136 & 109.893 & 107.232 & 74.302 \\
\hline $11 / 28 / 0715: 00$ & 125.119 & 122.911 & 119.696 & 100.624 & 104.62 & 96.132 & 109.886 & 107.228 & 74.295 \\
\hline $11 / 28 / 0715: 30$ & 125.114 & 122.927 & 119.696 & 100.622 & 104.626 & 96.139 & 109.88 & 107.234 & 74.302 \\
\hline $11 / 28 / 0716: 00$ & 125.102 & 122.927 & 119.741 & 100.632 & 104.628 & 96.139 & 109.876 & 107.234 & 74.302 \\
\hline 11/28/07 16:30 & 125.097 & 122.934 & 119.739 & 100.648 & 104.645 & 96.158 & 109.871 & 107.252 & 74.32 \\
\hline $11 / 28 / 0717: 00$ & 125.095 & 122.938 & 119.741 & 100.65 & 104.647 & 96.16 & 109.886 & 107.254 & 74.32 \\
\hline $11 / 28 / 0717: 30$ & 125.09 & 122.954 & 119.741 & 100.653 & 104.654 & 96.165 & 109.893 & 107.263 & 74.329 \\
\hline $11 / 28 / 0718: 00$ & 125.088 & 122.963 & 119.746 & 100.663 & 104.656 & 96.167 & 109.895 & 107.263 & 74.331 \\
\hline $11 / 28 / 0718: 30$ & 125.119 & 122.972 & 119.749 & 100.676 & 104.675 & 96.188 & 109.91 & 107.281 & 74.345 \\
\hline $11 / 28 / 07$ 19:00 & 125.116 & 122.984 & 119.736 & 100.687 & 104.685 & 96.195 & 109.923 & 107.289 & 74.353 \\
\hline 11/28/07 19:30 & 125.14 & 123.004 & 119.754 & 100.693 & 104.69 & 96.205 & 109.935 & 107.298 & 74.361 \\
\hline $11 / 28 / 0720: 00$ & 125.138 & 123.018 & 119.756 & 100.712 & 104.702 & 96.216 & 109.944 & 107.309 & 74.372 \\
\hline $11 / 28 / 0720: 30$ & 125.164 & 123.029 & 119.84 & 100.732 & 104.725 & 96.233 & 109.959 & 107.327 & 74.391 \\
\hline $11 / 28 / 0721: 00$ & 125.275 & 123.027 & 119.838 & 100.744 & 104.738 & 96.252 & 109.983 & 107.343 & 74.405 \\
\hline $11 / 28 / 0721: 30$ & 125.27 & 123.031 & 119.842 & 100.755 & 104.749 & 96.261 & 109.991 & 107.354 & 74.415 \\
\hline $11 / 28 / 07$ 22:00 & 125.27 & 123.031 & 119.847 & 100.753 & 104.749 & 96.261 & 109.997 & 107.356 & 74.415 \\
\hline 11/28/07 22:30 & 125.268 & 123.043 & 119.847 & 100.751 & 104.755 & 96.266 & 109.995 & 107.36 & 74.42 \\
\hline $11 / 28 / 07$ 23:00 & 125.265 & 123.041 & 119.845 & 100.76 & 104.751 & 96.261 & 109.993 & 107.358 & 74.417 \\
\hline 11/28/07 23:30 & 125.26 & 123.05 & 119.855 & 100.767 & 104.765 & 96.278 & 110.002 & 107.371 & 74.432 \\
\hline 11/29/07 0:00 & 125.263 & 123.05 & 119.857 & 100.765 & 104.761 & 96.275 & 110.006 & 107.369 & 74.432 \\
\hline 11/29/07 0:30 & 125.258 & 123.047 & 119.86 & 100.777 & 104.77 & 96.282 & 110.01 & 107.378 & 74.438 \\
\hline 11/29/07 1:00 & 125.258 & 123.038 & 119.865 & 100.774 & 104.77 & 96.285 & 110.021 & 107.38 & 74.44 \\
\hline 11/29/07 1:30 & 125.258 & 123.036 & 119.867 & 100.768 & 104.765 & 96.278 & 110.019 & 107.376 & 74.436 \\
\hline $11 / 29 / 072: 00$ & 125.258 & 123.027 & 119.867 & 100.753 & 104.759 & 96.271 & 110.019 & 107.369 & 74.432 \\
\hline
\end{tabular}


TABLE S1.1 (Cont.)

Depth from Top of Casing (ft)

$\begin{array}{lllllllllll}\text { Date and Time } & \text { MW1D } & \text { MW2D } & \text { MW3D } & \text { MW4D } & \text { MW7 } & \text { MW9 } & \text { Oentrich } & \text { MW14D } & \text { MW15D } & \text { MW16 }\end{array}$

\begin{tabular}{|c|c|c|c|c|c|c|c|c|c|}
\hline 11/29/07 2:30 & 125.253 & 123.02 & 119.872 & 100.751 & 104.755 & 96.266 & 110.017 & 107.363 & 74.428 \\
\hline $11 / 29 / 073: 00$ & 125.256 & 123.009 & 119.872 & 100.744 & 104.748 & 96.261 & 110.015 & 107.358 & 74.425 \\
\hline 11/29/07 3:30 & 125.251 & 122.986 & 119.875 & 100.741 & 104.74 & 96.252 & 110.013 & 107.347 & 74.417 \\
\hline $11 / 29 / 074: 00$ & 125.251 & 122.988 & 119.867 & 100.725 & 104.73 & 96.24 & 110.013 & 107.338 & 74.407 \\
\hline $11 / 29 / 074: 30$ & 125.251 & 123.002 & 119.865 & 100.706 & 104.706 & 96.214 & 110.013 & 107.316 & 74.386 \\
\hline $11 / 29 / 07$ 5:00 & 125.246 & 123 & 119.86 & 100.72 & 104.706 & 96.216 & 110.01 & 107.316 & 74.391 \\
\hline 11/29/07 5:30 & 125.239 & 123 & 119.857 & 100.72 & 104.719 & 96.228 & 110.004 & 107.327 & 74.401 \\
\hline $11 / 29 / 076: 00$ & 125.237 & 122.991 & 119.857 & 100.72 & 104.717 & 96.231 & 110.002 & 107.325 & 74.401 \\
\hline 11/29/07 6:30 & 125.234 & 122.995 & 119.852 & 100.72 & 104.719 & 96.231 & 110 & 107.325 & 74.403 \\
\hline 11/29/07 7:00 & 125.234 & 125.007 & 121.198 & 100.715 & 104.709 & 96.221 & 109.998 & 107.318 & 74.395 \\
\hline $11 / 29 / 07$ 7:30 & 125.227 & 125.213 & 121.45 & 101.132 & 104.717 & 96.226 & 109.995 & 107.323 & 74.401 \\
\hline $11 / 29 / 078: 00$ & 127.265 & 125.315 & 121.578 & 101.549 & 105.296 & 96.938 & 110.693 & 108.846 & 74.519 \\
\hline $11 / 29 / 078: 30$ & 127.49 & 125.376 & 121.654 & 101.706 & 105.505 & 97.147 & 110.892 & 109.098 & 74.682 \\
\hline 11/29/07 9:00 & 127.605 & 125.415 & 121.702 & 101.787 & 105.612 & 97.253 & 110.999 & 109.211 & 74.783 \\
\hline 11/29/07 9:30 & 127.674 & 125.437 & 121.732 & 101.846 & 105.677 & 97.317 & 111.063 & 109.278 & 74.85 \\
\hline $11 / 29 / 07$ 10:00 & 127.719 & 125.462 & 121.752 & 101.876 & 105.713 & 97.36 & 111.108 & 109.324 & 74.895 \\
\hline $11 / 29 / 0710: 30$ & 127.757 & 125.478 & 121.772 & 101.9 & 105.736 & 97.381 & 111.14 & 109.344 & 74.92 \\
\hline $11 / 29 / 0711: 00$ & 127.776 & 125.478 & 121.787 & 101.928 & 105.766 & 97.409 & 111.162 & 109.373 & 74.953 \\
\hline 11/29/07 11:30 & 127.794 & 125.469 & 121.795 & 101.937 & 105.778 & 97.423 & 111.181 & 109.388 & 74.969 \\
\hline $11 / 29 / 0712: 00$ & 127.811 & 125.458 & 121.81 & 101.935 & 105.781 & 97.428 & 111.196 & 109.393 & 74.977 \\
\hline $11 / 29 / 0712: 30$ & 127.82 & 125.193 & 121.813 & 101.919 & 105.772 & 97.414 & 111.209 & 109.379 & 74.972 \\
\hline $11 / 29 / 0713: 00$ & 127.828 & 123.508 & 120.51 & 101.914 & 105.759 & 97.404 & 111.219 & 109.368 & 74.967 \\
\hline $11 / 29 / 0713: 30$ & 127.835 & 123.285 & 120.258 & 101.485 & 105.76 & 97.407 & 111.229 & 109.371 & 74.973 \\
\hline $11 / 29 / 0714: 00$ & 125.868 & 123.152 & 120.132 & 101.087 & 105.184 & 96.697 & 110.541 & 107.889 & 74.852 \\
\hline $11 / 29 / 0714: 30$ & 125.65 & 123.072 & 120.054 & 100.917 & 104.97 & 96.483 & 110.359 & 107.628 & 74.698 \\
\hline $11 / 29 / 0715: 00$ & 125.542 & 123.025 & 119.993 & 100.808 & 104.843 & 96.358 & 110.261 & 107.491 & 74.582 \\
\hline $11 / 29 / 0715: 30$ & 125.473 & 122.963 & 119.958 & 100.746 & 104.761 & 96.275 & 110.201 & 107.4 & 74.502 \\
\hline $11 / 29 / 0716: 00$ & 125.428 & 122.936 & 119.92 & 100.699 & 104.719 & 96.231 & 110.16 & 107.356 & 74.457 \\
\hline $11 / 29 / 0716: 30$ & 125.398 & 122.918 & 119.882 & 100.641 & 104.66 & 96.172 & 110.132 & 107.292 & 74.396 \\
\hline $11 / 29 / 07$ 17:00 & 125.369 & 122.902 & 119.865 & 100.624 & 104.635 & 96.146 & 110.105 & 107.261 & 74.372 \\
\hline $11 / 29 / 0717: 30$ & 125.35 & 122.889 & 119.842 & 100.615 & 104.624 & 96.132 & 110.089 & 107.247 & 74.358 \\
\hline $11 / 29 / 0718: 00$ & 125.336 & 122.898 & 119.822 & 100.598 & 104.607 & 96.12 & 110.077 & 107.228 & 74.339 \\
\hline $11 / 29 / 0718: 30$ & 125.327 & 122.891 & 119.804 & 100.594 & 104.593 & 96.103 & 110.064 & 107.212 & 74.324 \\
\hline $11 / 29 / 07$ 19:00 & 125.312 & 122.886 & 119.792 & 100.6 & 104.605 & 96.117 & 110.055 & 107.221 & 74.332 \\
\hline $11 / 29 / 07$ 19:30 & 125.305 & 122.884 & 119.782 & 100.598 & 104.603 & 96.11 & 110.049 & 107.219 & 74.328 \\
\hline $11 / 29 / 0720: 00$ & 125.301 & 122.884 & 119.774 & 100.586 & 104.595 & 96.103 & 110.043 & 107.21 & 74.32 \\
\hline $11 / 29 / 0720: 30$ & 125.294 & 122.877 & 119.769 & 100.589 & 104.59 & 96.101 & 110.04 & 107.205 & 74.316 \\
\hline $11 / 29 / 0721: 00$ & 125.289 & 122.877 & 119.764 & 100.591 & 104.595 & 96.103 & 110.036 & 107.208 & 74.316 \\
\hline 11/29/07 21:30 & 125.286 & 122.882 & 119.756 & 100.584 & 104.588 & 96.099 & 110.032 & 107.205 & 74.312 \\
\hline $11 / 29 / 0722: 00$ & 125.279 & 122.884 & 119.749 & 100.586 & 104.59 & 96.099 & 110.028 & 107.201 & 74.31 \\
\hline $11 / 29 / 0722: 30$ & 125.277 & 122.886 & 119.751 & 100.589 & 104.593 & 96.103 & 110.027 & 107.205 & 74.314 \\
\hline 11/29/07 23:00 & 125.275 & 122.889 & 119.744 & 100.596 & 104.595 & 96.106 & 110.025 & 107.208 & 74.314 \\
\hline $11 / 29 / 0723: 30$ & 125.27 & 122.9 & 119.739 & 100.6 & 104.597 & 96.108 & 110.025 & 107.21 & 74.314 \\
\hline 11/30/07 0:00 & 125.27 & 122.909 & 119.739 & 100.603 & 104.603 & 96.11 & 110.021 & 107.212 & 74.316 \\
\hline 11/30/07 0:30 & 125.265 & 122.925 & 119.739 & 100.617 & 104.614 & 96.125 & 110.017 & 107.223 & 74.328 \\
\hline $11 / 30 / 071: 00$ & 125.263 & 122.968 & 119.729 & 100.629 & 104.624 & 96.134 & 110.017 & 107.234 & 74.337 \\
\hline $11 / 30 / 071: 30$ & 125.26 & 122.975 & 119.744 & 100.646 & 104.639 & 96.148 & 110.013 & 107.247 & 74.349 \\
\hline 11/30/07 2:00 & 125.256 & 122.995 & 119.749 & 100.681 & 104.679 & 96.19 & 110.01 & 107.289 & 74.386 \\
\hline 11/30/07 2:30 & 125.256 & 123.013 & 119.756 & 100.696 & 104.687 & 96.198 & 110.01 & 107.296 & 74.39 \\
\hline $11 / 30 / 073: 00$ & 125.251 & 123.029 & 119.766 & 100.72 & 104.713 & 96.224 & 110.012 & 107.318 & 74.415 \\
\hline 11/30/07 3:30 & 125.249 & 123.045 & 119.774 & 100.736 & 104.73 & 96.245 & 110.013 & 107.336 & 74.429 \\
\hline $11 / 30 / 074: 00$ & 125.249 & 123.061 & 119.789 & 100.756 & 104.746 & 96.259 & 110.013 & 107.356 & 74.444 \\
\hline 11/30/07 4:30 & 125.249 & 123.077 & 119.789 & 100.765 & 104.761 & 96.275 & 110.012 & 107.371 & 74.458 \\
\hline 11/30/07 5:00 & 125.244 & 123.086 & 119.797 & 100.784 & 104.776 & 96.29 & 110.008 & 107.385 & 74.473 \\
\hline $11 / 30 / 075: 30$ & 125.242 & 123.099 & 119.804 & 100.796 & 104.795 & 96.306 & 110.025 & 107.402 & 74.49 \\
\hline 11/30/07 6:00 & 125.239 & 123.106 & 119.817 & 100.811 & 104.805 & 96.318 & 110.036 & 107.413 & 74.498 \\
\hline 11/30/07 6:30 & 125.239 & 124.462 & 119.895 & 100.817 & 104.816 & 96.327 & 110.045 & 107.425 & 74.508 \\
\hline $11 / 30 / 07$ 7:00 & 125.336 & 125.163 & 121.412 & 100.827 & 104.826 & 96.339 & 110.049 & 107.433 & 74.519 \\
\hline 11/30/07 7:30 & 126.369 & 125.374 & 121.677 & 101.404 & 104.898 & 96.433 & 110.209 & 107.54 & 74.529 \\
\hline 11/30/07 8:00 & 127.407 & 125.483 & 121.823 & 101.768 & 105.507 & 97.164 & 110.864 & 109.016 & 74.699 \\
\hline $11 / 30 / 078: 30$ & 127.615 & 124.29 & 121.898 & 101.94 & 105.717 & 97.374 & 111.065 & 109.255 & 74.87 \\
\hline 11/30/07 9:00 & 127.726 & 123.628 & 120.464 & 102.033 & 105.831 & 97.496 & 111.185 & 109.384 & 74.979 \\
\hline 11/30/07 9:30 & 127.057 & 123.467 & 120.27 & 101.556 & 105.85 & 97.482 & 111.174 & 109.304 & 75.068 \\
\hline $11 / 30 / 07$ 10:00 & 126.071 & 123.385 & 120.172 & 101.249 & 105.321 & 96.836 & 110.522 & 107.998 & 74.969 \\
\hline $11 / 30 / 07$ 10:30 & 125.884 & 123.335 & 120.119 & 101.134 & 105.172 & 96.686 & 110.366 & 107.821 & 74.87 \\
\hline $11 / 30 / 0711: 00$ & 125.79 & 123.299 & 120.094 & 101.072 & 105.09 & 96.605 & 110.303 & 107.721 & 74.797 \\
\hline
\end{tabular}


TABLE S1.1 (Cont.)

\begin{tabular}{|c|c|c|c|c|c|c|c|c|c|c|}
\hline \multirow[b]{2}{*}{ Date and Time } & \multicolumn{10}{|c|}{ Depth from Top of Casing (ft) } \\
\hline & MW1D & MW2D & MW3D & MW4D & MW7 & MW9 & Oentrich & MW14D & MW15D & MW16D \\
\hline 11/30/07 11:30 & 125.733 & 123.267 & 120.079 & 101.029 & 105.045 & 96.556 & 110.261 & 107.668 & & 74.75 \\
\hline $11 / 30 / 07$ 12:00 & 125.698 & 123.236 & 120.064 & 101.004 & 105.01 & 96.523 & 110.235 & 107.635 & & 74.717 \\
\hline 11/30/07 12:30 & 125.679 & 123.217 & 120.049 & 100.97 & 104.974 & 96.49 & 110.218 & 107.597 & & 74.68 \\
\hline $11 / 30 / 07$ 13:00 & 125.662 & 123.199 & 120.046 & 100.936 & 104.946 & 96.462 & 110.205 & 107.568 & & 74.651 \\
\hline $11 / 30 / 07$ 13:30 & 125.648 & 123.172 & 120.039 & 100.931 & 104.927 & 96.443 & 110.194 & 107.546 & & 74.635 \\
\hline $11 / 30 / 07$ 14:00 & 125.636 & 123.17 & 120.023 & 100.901 & 104.915 & 96.431 & 110.184 & 107.533 & & 74.62 \\
\hline $11 / 30 / 07$ 14:30 & 125.632 & 123.152 & 120.023 & 100.887 & 104.885 & 96.4 & 110.179 & 107.504 & & 74.595 \\
\hline $11 / 30 / 07$ 15:00 & 125.62 & 123.131 & 120.018 & 100.877 & 104.881 & 96.395 & 110.171 & 107.5 & & 74.593 \\
\hline 11/30/07 15:30 & 125.615 & 123.111 & 120.013 & 100.855 & 104.862 & 96.377 & 110.167 & 107.48 & & 74.573 \\
\hline $11 / 30 / 07$ 16:00 & 125.613 & 123.102 & 120.003 & 100.825 & 104.845 & 96.358 & 110.164 & 107.46 & & 74.556 \\
\hline $11 / 30 / 07$ 16:30 & 125.606 & 123.086 & 119.996 & 100.825 & 104.822 & 96.337 & 110.162 & 107.438 & & 74.539 \\
\hline 11/30/07 17:00 & 125.598 & 123.075 & 119.988 & 100.805 & 104.816 & 96.33 & 110.156 & 107.431 & & 74.533 \\
\hline $11 / 30 / 07$ 17:30 & 125.594 & 123.061 & 119.978 & 100.798 & 104.799 & 96.313 & 110.152 & 107.416 & & 74.518 \\
\hline $11 / 30 / 07$ 18:00 & 125.589 & 123.05 & 119.966 & 100.789 & 104.788 & 96.301 & 110.152 & 107.405 & & 74.508 \\
\hline 11/30/07 18:30 & 125.584 & 123.059 & 119.958 & 100.77 & 104.776 & 96.292 & 110.147 & 107.391 & & 74.498 \\
\hline 11/30/07 19:00 & 125.577 & 123.045 & 119.95 & 100.767 & 104.761 & 96.275 & 110.147 & 107.378 & & 74.484 \\
\hline $11 / 30 / 07$ 19:30 & 125.57 & 123.025 & 119.948 & 100.77 & 104.77 & 96.282 & 110.141 & 107.382 & & 74.492 \\
\hline $11 / 30 / 0720: 00$ & 125.57 & 123.007 & 119.938 & 100.758 & 104.763 & 96.275 & 110.139 & 107.376 & & 74.483 \\
\hline $11 / 30 / 0720: 30$ & 125.568 & 122.991 & 119.933 & 100.736 & 104.74 & 96.254 & 110.139 & 107.356 & & 74.463 \\
\hline $11 / 30 / 0721: 00$ & 125.565 & 122.984 & 119.918 & 100.717 & 104.725 & 96.235 & 110.137 & 107.338 & & 74.449 \\
\hline $11 / 30 / 0721: 30$ & 125.561 & 122.977 & 119.908 & 100.696 & 104.702 & 96.214 & 110.137 & 107.318 & & 74.429 \\
\hline 11/30/07 22:00 & 125.556 & 122.963 & 119.9 & 100.696 & 104.698 & 96.209 & 110.134 & 107.312 & & 74.426 \\
\hline 11/30/07 22:30 & 125.551 & 122.938 & 119.897 & 100.684 & 104.692 & 96.204 & 110.13 & 107.305 & & 74.422 \\
\hline 11/30/07 23:00 & 125.551 & 122.92 & 119.885 & 100.67 & 104.681 & 96.191 & 110.124 & 107.294 & & 74.409 \\
\hline 11/30/07 23:30 & 125.551 & 122.893 & 119.877 & 100.648 & 104.652 & 96.165 & 110.122 & 107.267 & & 74.384 \\
\hline 12/1/07 0:00 & 125.551 & 122.88 & 119.86 & 100.626 & 104.63 & 96.143 & 110.119 & 107.247 & & 74.363 \\
\hline 12/1/07 0:30 & 125.551 & 122.857 & 119.852 & 100.596 & 104.601 & 96.113 & 110.111 & 107.216 & & 74.335 \\
\hline 12/1/07 1:00 & 125.549 & 122.839 & 119.837 & 100.586 & 104.597 & 96.108 & 110.102 & 107.214 & & 74.332 \\
\hline 12/1/07 1:30 & 125.546 & 122.823 & 119.824 & 100.562 & 104.571 & 96.082 & 110.092 & 107.185 & & 74.306 \\
\hline $12 / 1 / 072: 00$ & 125.542 & 122.8 & 119.812 & 100.543 & 104.55 & 96.061 & 110.085 & 107.163 & & 74.287 \\
\hline $12 / 1 / 072: 30$ & 125.539 & 122.784 & 119.797 & 100.524 & 104.534 & 96.044 & 110.057 & 107.146 & & 74.272 \\
\hline 12/1/07 3:00 & 125.539 & 122.766 & 119.782 & 100.505 & 104.513 & 96.026 & 110.036 & 107.128 & & 74.252 \\
\hline 12/1/07 3:30 & 125.513 & 122.73 & 119.769 & 100.491 & 104.498 & 96.009 & 110.023 & 107.11 & & 74.238 \\
\hline $12 / 1 / 074: 00$ & 125.511 & 122.714 & 119.746 & 100.469 & 104.479 & 95.99 & 110.006 & 107.092 & & 74.221 \\
\hline 12/1/07 4:30 & 125.518 & 122.68 & 119.739 & 100.429 & 104.445 & 95.957 & 109.976 & 107.061 & & 74.186 \\
\hline 12/1/07 5:00 & 125.513 & 122.662 & 119.719 & 100.412 & 104.428 & 95.936 & 109.94 & 107.039 & & 74.169 \\
\hline 12/1/07 5:30 & 125.516 & 122.623 & 119.698 & 100.384 & 104.393 & 95.905 & 109.923 & 107.008 & & 74.136 \\
\hline 12/1/07 6:00 & 125.516 & 122.589 & 119.671 & 100.358 & 104.374 & 95.886 & 109.908 & 106.986 & & 74.122 \\
\hline $12 / 1 / 076: 30$ & 125.52 & 122.562 & 119.643 & 100.32 & 104.334 & 95.844 & 109.863 & 106.949 & & 74.082 \\
\hline 12/1/07 7:00 & 125.52 & 122.542 & 119.618 & 100.289 & 104.304 & 95.813 & 109.839 & 106.92 & & 74.056 \\
\hline 12/1/07 7:30 & 125.52 & 122.51 & 119.467 & 100.262 & 104.275 & 95.785 & 109.801 & 106.887 & & 74.025 \\
\hline $12 / 1 / 078: 00$ & 125.518 & 122.476 & 119.459 & 100.241 & 104.253 & 95.762 & 109.779 & 106.864 & & 74.004 \\
\hline $12 / 1 / 078: 30$ & 125.518 & 124.401 & 120.719 & 100.207 & 104.226 & 95.738 & 109.751 & 106.836 & & 73.977 \\
\hline 12/1/07 9:00 & 125.523 & 124.616 & 120.996 & 100.422 & 104.19 & 95.7 & 109.732 & 106.802 & & 73.944 \\
\hline 12/1/07 9:30 & 127.156 & 124.723 & 121.12 & 100.948 & 104.694 & 96.332 & 110.342 & 108.237 & & 74.006 \\
\hline $12 / 1 / 07$ 10:00 & 127.308 & 124.773 & 121.183 & 101.106 & 104.911 & 96.551 & 110.543 & 108.498 & & 74.161 \\
\hline 12/1/07 10:30 & 127.473 & 124.807 & 121.213 & 101.191 & 105.02 & 96.662 & 110.652 & 108.618 & & 74.266 \\
\hline 12/1/07 11:00 & 127.551 & 124.821 & 121.241 & 101.234 & 105.069 & 96.711 & 110.695 & 108.671 & & 74.318 \\
\hline $12 / 1 / 0711: 30$ & 127.601 & 124.818 & 121.238 & 101.267 & 105.104 & 96.747 & 110.719 & 108.706 & & 74.355 \\
\hline 12/1/07 12:00 & 127.634 & 124.818 & 121.236 & 101.272 & 105.123 & 96.766 & 110.746 & 108.731 & & 74.38 \\
\hline $12 / 1 / 0712: 30$ & 127.664 & 123.378 & 121.225 & 101.266 & 105.119 & 96.756 & 110.733 & 108.726 & & 74.38 \\
\hline $12 / 1 / 07$ 13:00 & 127.688 & 122.78 & 119.824 & 101.263 & 105.119 & 96.761 & 110.751 & 108.726 & & 74.38 \\
\hline 12/1/07 13:30 & 126.428 & 122.578 & 119.593 & 100.694 & 104.972 & 96.542 & 110.517 & 108.193 & & 74.362 \\
\hline $12 / 1 / 0714: 00$ & 125.695 & 122.451 & 119.467 & 100.367 & 104.462 & 95.971 & 109.985 & 107.137 & & 74.202 \\
\hline $12 / 1 / 0714: 30$ & 125.513 & 122.365 & 119.346 & 100.212 & 104.268 & 95.769 & 109.788 & 106.915 & & 74.045 \\
\hline 12/1/07 15:00 & 125.421 & 122.31 & 119.295 & 100.098 & 104.142 & 95.653 & 109.677 & 106.783 & & 73.927 \\
\hline $12 / 1 / 0715: 30$ & 125.367 & 122.267 & 119.252 & 100.04 & 104.051 & 95.556 & 109.578 & 106.683 & & 73.832 \\
\hline 12/1/07 16:00 & 125.32 & 122.256 & 119.215 & 99.979 & 103.999 & 95.507 & 109.527 & 106.628 & & 73.779 \\
\hline $12 / 1 / 0716: 30$ & 125.251 & 122.267 & 119.172 & 99.936 & 103.963 & 95.467 & 109.484 & 106.588 & & 73.741 \\
\hline $12 / 1 / 07$ 17:00 & 125.223 & 122.265 & 119.164 & 99.971 & 103.965 & 95.465 & 109.443 & 106.57 & & 73.729 \\
\hline 12/1/07 17:30 & 125.211 & 122.267 & 119.149 & 99.96 & 103.973 & 95.476 & 109.431 & 106.588 & & 73.737 \\
\hline $12 / 1 / 07$ 18:00 & 125.204 & 122.276 & 119.154 & 99.957 & 103.969 & 95.469 & 109.428 & 106.581 & & 73.729 \\
\hline $12 / 1 / 0718: 30$ & 125.197 & 122.281 & 119.157 & 99.972 & 103.973 & 95.481 & 109.422 & 106.585 & & 73.729 \\
\hline $12 / 1 / 07$ 19:00 & 125.189 & 122.295 & 119.157 & 99.978 & 103.98 & 95.486 & 109.418 & 106.588 & & 73.733 \\
\hline 12/1/07 19:30 & 125.182 & 122.308 & 119.162 & 99.986 & 103.99 & 95.495 & 109.414 & 106.599 & & 73.741 \\
\hline $12 / 1 / 0720: 00$ & 125.178 & 122.342 & 119.172 & 100 & 104.005 & 95.509 & 109.411 & 106.612 & & 73.747 \\
\hline
\end{tabular}


TABLE S1.1 (Cont.)

\begin{tabular}{|c|c|c|c|c|c|c|c|c|c|c|}
\hline \multirow[b]{2}{*}{ Date and Time } & \multicolumn{10}{|c|}{ Depth from Top of Casing (ft) } \\
\hline & MW1D & MW2D & MW3D & MW4D & MW7 & MW9 & Oentrich & MW14D & MW15D & MW16D \\
\hline 12/1/07 20:30 & 125.175 & 122.376 & 119.23 & 100.026 & 104.016 & 95.521 & 109.411 & 106.621 & & 73.756 \\
\hline 12/1/07 21:00 & 125.171 & 122.408 & 119.257 & 100.071 & 104.049 & 95.554 & 109.414 & 106.652 & & 73.782 \\
\hline 12/1/07 21:30 & 125.161 & 122.435 & 119.278 & 100.098 & 104.095 & 95.601 & 109.42 & 106.692 & & 73.82 \\
\hline $12 / 1 / 0722: 00$ & 125.159 & 122.462 & 119.31 & 100.124 & 104.123 & 95.632 & 109.439 & 106.725 & & 73.849 \\
\hline $12 / 1 / 0722: 30$ & 125.156 & 122.476 & 119.328 & 100.158 & 104.152 & 95.66 & 109.452 & 106.749 & & 73.869 \\
\hline $12 / 1 / 0723: 00$ & 125.156 & 122.485 & 119.343 & 100.186 & 104.184 & 95.693 & 109.482 & 106.783 & & 73.896 \\
\hline 12/1/07 23:30 & 125.159 & 122.51 & 119.351 & 100.198 & 104.197 & 95.707 & 109.499 & 106.796 & & 73.909 \\
\hline $12 / 2 / 07$ 0:00 & 125.159 & 122.519 & 119.373 & 100.21 & 104.201 & 95.712 & 109.512 & 106.802 & & 73.911 \\
\hline 12/2/07 0:30 & 125.156 & 122.533 & 119.394 & 100.231 & 104.228 & 95.738 & 109.518 & 106.825 & & 73.931 \\
\hline 12/2/07 1:00 & 125.159 & 122.553 & 119.404 & 100.246 & 104.237 & 95.747 & 109.533 & 106.838 & & 73.942 \\
\hline 12/2/07 1:30 & 125.161 & 122.555 & 119.414 & 100.253 & 104.253 & 95.769 & 109.548 & 106.858 & & 73.954 \\
\hline 12/2/07 2:00 & 125.159 & 122.564 & 119.416 & 100.27 & 104.273 & 95.787 & 109.565 & 106.873 & & 73.971 \\
\hline $12 / 2 / 072: 30$ & 125.161 & 122.578 & 119.431 & 100.277 & 104.277 & 95.79 & 109.571 & 106.875 & & 73.971 \\
\hline 12/2/07 3:00 & 125.161 & 122.598 & 119.451 & 100.286 & 104.285 & 95.797 & 109.574 & 106.887 & & 73.981 \\
\hline 12/2/07 3:30 & 125.159 & 122.614 & 119.462 & 100.301 & 104.3 & 95.811 & 109.586 & 106.9 & & 73.994 \\
\hline $12 / 2 / 074: 00$ & 125.159 & 122.639 & 119.482 & 100.319 & 104.323 & 95.832 & 109.595 & 106.92 & & 74.014 \\
\hline $12 / 2 / 074: 30$ & 125.161 & 122.664 & 119.489 & 100.339 & 104.334 & 95.849 & 109.597 & 106.933 & & 74.026 \\
\hline 12/2/07 5:00 & 125.159 & 122.696 & 119.509 & 100.369 & 104.357 & 95.872 & 109.6 & 106.956 & & 74.045 \\
\hline $12 / 2 / 075: 30$ & 125.159 & 122.714 & 119.514 & 100.398 & 104.384 & 95.898 & 109.606 & 106.982 & & 74.07 \\
\hline $12 / 2 / 076: 00$ & 125.156 & 122.73 & 119.535 & 100.42 & 104.414 & 95.931 & 109.659 & 107.013 & & 74.101 \\
\hline 12/2/07 6:30 & 125.159 & 122.748 & 119.55 & 100.439 & 104.432 & 95.945 & 109.685 & 107.028 & & 74.112 \\
\hline $12 / 2 / 07$ 7:00 & 125.161 & 122.777 & 119.555 & 100.453 & 104.452 & 95.964 & 109.698 & 107.05 & & 74.134 \\
\hline 12/2/07 7:30 & 125.159 & 122.796 & 119.598 & 100.479 & 104.471 & 95.985 & 109.713 & 107.07 & & 74.151 \\
\hline 12/2/07 8:00 & 125.164 & 124.483 & 120.381 & 100.51 & 104.504 & 96.014 & 109.721 & 107.097 & & 74.18 \\
\hline 12/2/07 8:30 & 125.164 & 124.948 & 121.067 & 100.538 & 104.515 & 96.03 & 109.73 & 107.115 & & 74.192 \\
\hline 12/2/07 9:00 & 126.79 & 125.118 & 121.253 & 101.189 & 104.81 & 96.433 & 110.135 & 108.175 & & 74.227 \\
\hline 12/2/07 9:30 & 127.26 & 125.227 & 121.359 & 101.485 & 105.258 & 96.9 & 110.537 & 108.804 & & 74.444 \\
\hline $12 / 2 / 07$ 10:00 & 127.419 & 125.313 & 121.442 & 101.642 & 105.441 & 97.081 & 110.69 & 109.005 & & 74.606 \\
\hline 12/2/07 10:30 & 127.516 & 125.39 & 121.596 & 101.742 & 105.555 & 97.197 & 110.783 & 109.127 & & 74.723 \\
\hline 12/2/07 11:00 & 127.575 & 125.424 & 121.634 & 101.819 & 105.637 & 97.282 & 110.866 & 109.213 & & 74.808 \\
\hline 12/2/07 11:30 & 127.615 & 125.456 & 121.659 & 101.89 & 105.719 & 97.364 & 110.977 & 109.3 & & 74.891 \\
\hline $12 / 2 / 07$ 12:00 & 127.653 & 125.487 & 121.682 & 101.911 & 105.753 & 97.4 & 111.014 & 109.337 & & 74.926 \\
\hline $12 / 2 / 07$ 12:30 & 127.672 & 125.51 & 121.704 & 101.952 & 105.783 & 97.43 & 111.031 & 109.366 & & 74.961 \\
\hline 12/2/07 13:00 & 127.688 & 123.719 & 120.55 & 101.983 & 105.819 & 97.466 & 111.05 & 109.404 & & 75 \\
\hline 12/2/07 13:30 & 127.785 & 123.48 & 120.248 & 101.935 & 105.844 & 97.489 & 111.063 & 109.426 & & 75.029 \\
\hline $12 / 2 / 0714: 00$ & 126.012 & 123.369 & 120.127 & 101.296 & 105.395 & 96.914 & 110.477 & 108.113 & & 74.984 \\
\hline 12/2/07 14:30 & 125.75 & 123.319 & 120.059 & 101.122 & 105.17 & 96.688 & 110.259 & 107.834 & & 74.852 \\
\hline 12/2/07 15:00 & 125.622 & 123.279 & 120.016 & 101.054 & 105.064 & 96.579 & 110.16 & 107.699 & & 74.757 \\
\hline $12 / 2 / 0715: 30$ & 125.546 & 123.276 & 120.011 & 101.01 & 105.022 & 96.537 & 110.181 & 107.648 & & 74.715 \\
\hline $12 / 2 / 0716: 00$ & 125.504 & 123.27 & 120.001 & 100.975 & 104.982 & 96.497 & 110.143 & 107.608 & & 74.674 \\
\hline 12/2/07 16:30 & 125.466 & 123.279 & 119.993 & 100.987 & 104.982 & 96.495 & 110.113 & 107.597 & & 74.668 \\
\hline 12/2/07 17:00 & 125.445 & 123.288 & 120.036 & 100.98 & 104.978 & 96.495 & 110.094 & 107.593 & & 74.661 \\
\hline $12 / 2 / 07$ 17:30 & 125.424 & 123.292 & 120.036 & 100.999 & 104.987 & 96.504 & 110.078 & 107.604 & & 74.672 \\
\hline 12/2/07 18:00 & 125.499 & 123.308 & 120.029 & 100.998 & 104.997 & 96.516 & 110.139 & 107.613 & & 74.68 \\
\hline $12 / 2 / 0718: 30$ & 125.487 & 123.326 & 120.061 & 101.011 & 105.001 & 96.516 & 110.145 & 107.615 & & 74.682 \\
\hline 12/2/07 19:00 & 125.476 & 123.324 & 120.064 & 101.027 & 105.016 & 96.535 & 110.136 & 107.63 & & 74.696 \\
\hline 12/2/07 19:30 & 125.466 & 123.328 & 120.066 & 101.039 & 105.035 & 96.551 & 110.128 & 107.648 & & 74.715 \\
\hline 12/2/07 20:00 & 125.461 & 123.344 & 120.064 & 101.041 & 105.035 & 96.551 & 110.123 & 107.648 & & 74.713 \\
\hline $12 / 2 / 0720: 30$ & 125.558 & 123.344 & 120.064 & 101.054 & 105.039 & 96.556 & 110.117 & 107.653 & & 74.717 \\
\hline 12/2/07 21:00 & 125.551 & 123.351 & 120.097 & 101.065 & 105.056 & 96.572 & 110.109 & 107.67 & & 74.734 \\
\hline $12 / 2 / 0721: 30$ & 125.544 & 123.342 & 120.099 & 101.066 & 105.054 & 96.572 & 110.106 & 107.668 & & 74.733 \\
\hline 12/2/07 22:00 & 125.537 & 123.333 & 120.102 & 101.07 & 105.064 & 96.579 & 110.098 & 107.677 & & 74.746 \\
\hline 12/2/07 22:30 & 125.535 & 123.335 & 120.102 & 101.058 & 105.056 & 96.575 & 110.094 & 107.67 & & 74.74 \\
\hline 12/2/07 23:00 & 125.528 & 123.349 & 120.099 & 101.054 & 105.046 & 96.563 & 110.09 & 107.661 & & 74.731 \\
\hline 12/2/07 23:30 & 125.523 & 123.351 & 120.102 & 101.058 & 105.048 & 96.565 & 110.085 & 107.661 & & 74.738 \\
\hline 12/3/07 0:00 & 125.516 & 123.349 & 120.104 & 101.07 & 105.062 & 96.579 & 110.079 & 107.677 & & 74.752 \\
\hline $12 / 3 / 07$ 0:30 & 125.513 & 123.331 & 120.112 & 101.073 & 105.066 & 96.584 & 110.075 & 107.679 & & 74.757 \\
\hline 12/3/07 1:00 & 125.509 & 123.31 & 120.109 & 101.066 & 105.064 & 96.582 & 110.07 & 107.679 & & 74.756 \\
\hline 12/3/07 1:30 & 125.506 & 123.29 & 120.114 & 101.042 & 105.048 & 96.563 & 110.068 & 107.661 & & 74.74 \\
\hline $12 / 3 / 072: 00$ & 125.504 & 123.274 & 120.112 & 101.023 & 105.024 & 96.539 & 110.066 & 107.639 & & 74.719 \\
\hline $12 / 3 / 072: 30$ & 125.504 & 123.27 & 120.102 & 100.998 & 105.001 & 96.518 & 110.062 & 107.617 & & 74.702 \\
\hline 12/3/07 3:00 & 125.494 & 123.272 & 120.094 & 100.991 & 104.987 & 96.502 & 110.059 & 107.599 & & 74.688 \\
\hline $12 / 3 / 073: 30$ & 125.49 & 123.279 & 120.092 & 100.989 & 104.986 & 96.499 & 110.055 & 107.595 & & 74.688 \\
\hline $12 / 3 / 074: 00$ & 125.485 & 123.283 & 120.089 & 100.987 & 104.986 & 96.502 & 110.049 & 107.599 & & 74.694 \\
\hline $12 / 3 / 074: 30$ & 125.478 & 123.276 & 120.086 & 100.994 & 104.991 & 96.506 & 110.043 & 107.604 & & 74.699 \\
\hline $12 / 3 / 075: 00$ & 125.473 & 123.276 & 120.084 & 101.001 & 104.993 & 96.509 & 110.04 & 107.606 & & 74.703 \\
\hline
\end{tabular}


TABLE S1.1 (Cont.)

Depth from Top of Casing (ft)

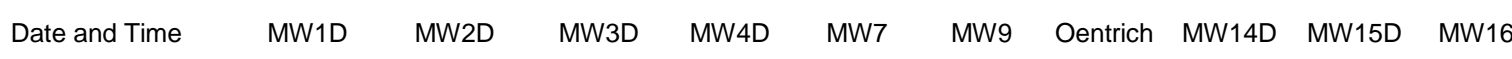

\begin{tabular}{|c|c|c|c|c|c|c|c|c|c|}
\hline 12/3/07 5:30 & 125.471 & 123.265 & 120.084 & 100.991 & 104.989 & 96.504 & 110.038 & 107.604 & 74.699 \\
\hline 12/3/07 6:00 & 125.466 & 123.27 & 120.081 & 100.992 & 104.991 & 96.504 & 110.036 & 107.604 & 74.701 \\
\hline 12/3/07 6:30 & 125.466 & 123.26 & 120.079 & 100.979 & 104.978 & 96.492 & 110.034 & 107.591 & 74.69 \\
\hline $12 / 3 / 077: 00$ & 125.461 & 125.179 & 121.364 & 100.984 & 104.982 & 96.497 & 110.027 & 107.595 & 74.697 \\
\hline 12/3/07 7:30 & 125.461 & 125.406 & 121.634 & 101.275 & 104.974 & 96.49 & 110.025 & 107.591 & 74.692 \\
\hline $12 / 3 / 078: 00$ & 127.43 & 125.515 & 121.767 & 101.745 & 105.481 & 97.124 & 110.684 & 109.023 & 74.756 \\
\hline $12 / 3 / 078: 30$ & 127.664 & 125.596 & 121.84 & 101.909 & 105.711 & 97.357 & 110.89 & 109.293 & 74.93 \\
\hline $12 / 3 / 079: 00$ & 127.785 & 124.186 & 121.888 & 102 & 105.819 & 97.463 & 111.001 & 109.41 & 75.034 \\
\hline $12 / 3 / 079: 30$ & 127.858 & 123.655 & 120.543 & 102.071 & 105.899 & 97.546 & 111.067 & 109.499 & 75.12 \\
\hline $12 / 3 / 07$ 10:00 & 126.787 & 123.483 & 120.359 & 101.559 & 105.797 & 97.364 & 110.924 & 108.892 & 75.168 \\
\hline 12/3/07 10:30 & 126.081 & 123.392 & 120.255 & 101.263 & 105.338 & 96.855 & 110.406 & 108.025 & 75.04 \\
\hline $12 / 3 / 0711: 00$ & 125.908 & 123.317 & 120.205 & 101.139 & 105.172 & 96.683 & 110.261 & 107.827 & 74.912 \\
\hline $12 / 3 / 0711: 30$ & 125.823 & 123.251 & 120.165 & 101.048 & 105.086 & 96.601 & 110.184 & 107.726 & 74.833 \\
\hline $12 / 3 / 07$ 12:00 & 125.769 & 123.204 & 120.137 & 100.998 & 105.014 & 96.528 & 110.139 & 107.646 & 74.758 \\
\hline $12 / 3 / 0712: 30$ & 125.738 & 123.149 & 120.109 & 100.93 & 104.953 & 96.466 & 110.111 & 107.586 & 74.702 \\
\hline $12 / 3 / 07$ 13:00 & 125.71 & 123.102 & 120.084 & 100.887 & 104.902 & 96.417 & 110.092 & 107.529 & 74.651 \\
\hline 12/3/07 13:30 & 125.693 & 123.077 & 120.041 & 100.832 & 104.847 & 96.363 & 110.075 & 107.475 & 74.599 \\
\hline $12 / 3 / 0714: 00$ & 125.674 & 123.054 & 120.016 & 100.789 & 104.799 & 96.315 & 110.066 & 107.429 & 74.554 \\
\hline $12 / 3 / 07 \quad 14: 30$ & 125.655 & 123.034 & 120.011 & 100.77 & 104.778 & 96.29 & 110.06 & 107.4 & 74.533 \\
\hline $12 / 3 / 07$ 15:00 & 125.646 & 123 & 119.998 & 100.755 & 104.757 & 96.266 & 110.049 & 107.378 & 74.512 \\
\hline $12 / 3 / 07$ 15:30 & 125.636 & 122.979 & 119.978 & 100.729 & 104.736 & 96.249 & 110.043 & 107.358 & 74.494 \\
\hline $12 / 3 / 07$ 16:00 & 125.634 & 122.961 & 119.96 & 100.684 & 104.7 & 96.212 & 110.043 & 107.323 & 74.457 \\
\hline $12 / 3 / 07$ 16:30 & 125.624 & 122.948 & 119.94 & 100.674 & 104.685 & 96.195 & 110.028 & 107.305 & 74.444 \\
\hline $12 / 3 / 07$ 17:00 & 125.622 & 122.932 & 119.925 & 100.656 & 104.666 & 96.176 & 110.025 & 107.283 & 74.423 \\
\hline $12 / 3 / 07 \quad 17: 30$ & 125.617 & 122.909 & 119.903 & 100.655 & 104.652 & 96.165 & 110.017 & 107.272 & 74.411 \\
\hline $12 / 3 / 07$ 18:00 & 125.615 & 122.909 & 119.887 & 100.624 & 104.635 & 96.148 & 110.012 & 107.259 & 74.395 \\
\hline $12 / 3 / 07$ 18:30 & 125.61 & 122.907 & 119.872 & 100.61 & 104.614 & 96.125 & 110 & 107.234 & 74.374 \\
\hline $12 / 3 / 07$ 19:00 & 125.606 & 122.909 & 119.855 & 100.613 & 104.612 & 96.122 & 109.985 & 107.232 & 74.374 \\
\hline $12 / 3 / 07 \quad 19: 30$ & 125.601 & 122.907 & 119.842 & 100.61 & 104.61 & 96.125 & 109.98 & 107.228 & 74.37 \\
\hline $12 / 3 / 07$ 20:00 & 125.596 & 122.902 & 119.827 & 100.615 & 104.616 & 96.125 & 109.976 & 107.228 & 74.372 \\
\hline $12 / 3 / 0720: 30$ & 125.594 & 122.9 & 119.822 & 100.612 & 104.614 & 96.125 & 109.972 & 107.228 & 74.37 \\
\hline $12 / 3 / 0721: 00$ & 125.594 & 122.886 & 119.814 & 100.61 & 104.609 & 96.122 & 109.972 & 107.225 & 74.365 \\
\hline $12 / 3 / 0721: 30$ & 125.591 & 122.88 & 119.804 & 100.601 & 104.607 & 96.117 & 109.97 & 107.223 & 74.361 \\
\hline $12 / 3 / 0722: 00$ & 125.591 & 122.88 & 119.794 & 100.584 & 104.593 & 96.106 & 109.97 & 107.212 & 74.349 \\
\hline $12 / 3 / 0722: 30$ & 125.587 & 122.875 & 119.784 & 100.586 & 104.588 & 96.099 & 109.968 & 107.201 & 74.345 \\
\hline $12 / 3 / 07$ 23:00 & 125.584 & 122.859 & 119.779 & 100.581 & 104.586 & 96.099 & 109.966 & 107.203 & 74.343 \\
\hline $12 / 3 / 0723: 30$ & 125.582 & 122.846 & 119.771 & 100.577 & 104.582 & 96.094 & 109.965 & 107.197 & 74.334 \\
\hline $12 / 4 / 070: 00$ & 125.582 & 122.836 & 119.761 & 100.563 & 104.567 & 96.077 & 109.959 & 107.183 & 74.32 \\
\hline $12 / 4 / 070: 30$ & 125.568 & 122.827 & 119.751 & 100.55 & 104.553 & 96.066 & 109.95 & 107.168 & 74.306 \\
\hline 12/4/07 1:00 & 125.561 & 122.809 & 119.749 & 100.538 & 104.546 & 96.059 & 109.944 & 107.161 & 74.299 \\
\hline $12 / 4 / 071: 30$ & 125.256 & 122.802 & 119.739 & 100.534 & 104.536 & 96.049 & 109.935 & 107.152 & 74.291 \\
\hline $12 / 4 / 072: 00$ & 125.263 & 122.809 & 119.724 & 100.517 & 104.519 & 96.03 & 109.933 & 107.135 & 74.272 \\
\hline 12/4/07 2:30 & 125.263 & 122.809 & 119.716 & 100.519 & 104.511 & 96.021 & 109.921 & 107.123 & 74.264 \\
\hline $12 / 4 / 073: 00$ & 125.258 & 122.8 & 119.709 & 100.531 & 104.521 & 96.033 & 109.918 & 107.13 & 74.27 \\
\hline 12/4/07 3:30 & 125.26 & 122.798 & 119.698 & 100.517 & 104.519 & 96.033 & 109.92 & 107.132 & 74.27 \\
\hline $12 / 4 / 074: 00$ & 125.263 & 122.775 & 119.698 & 100.51 & 104.511 & 96.026 & 109.921 & 107.126 & 74.264 \\
\hline $12 / 4 / 074: 30$ & 125.26 & 122.764 & 119.693 & 100.5 & 104.511 & 96.023 & 109.918 & 107.119 & 74.258 \\
\hline $12 / 4 / 075: 00$ & 125.268 & 122.757 & 119.683 & 100.472 & 104.481 & 95.995 & 109.912 & 107.097 & 74.231 \\
\hline $12 / 4 / 075: 30$ & 125.263 & 122.757 & 119.676 & 100.467 & 104.473 & 95.983 & 109.899 & 107.084 & 74.221 \\
\hline $12 / 4 / 076: 00$ & 125.26 & 122.764 & 119.668 & 100.472 & 104.468 & 95.981 & 109.893 & 107.079 & 74.219 \\
\hline $12 / 4 / 076: 30$ & 125.26 & 122.764 & 119.658 & 100.472 & 104.47 & 95.981 & 109.895 & 107.079 & 74.217 \\
\hline $12 / 4 / 077: 00$ & 125.258 & 124.496 & 120.734 & 100.472 & 104.475 & 95.983 & 109.891 & 107.084 & 74.223 \\
\hline $12 / 4 / 077: 30$ & 125.258 & 124.886 & 121.256 & 100.474 & 104.477 & 95.988 & 109.891 & 107.086 & 74.223 \\
\hline $12 / 4 / 078: 00$ & 127.003 & 125.045 & 121.432 & 101.199 & 104.841 & 96.483 & 110.393 & 108.21 & 74.243 \\
\hline $12 / 4 / 078: 30$ & 127.416 & 125.129 & 121.543 & 101.456 & 105.218 & 96.874 & 110.744 & 108.74 & 74.444 \\
\hline $12 / 4 / 079: 00$ & 127.584 & 125.193 & 121.609 & 101.587 & 105.378 & 97.037 & 110.898 & 108.923 & 74.585 \\
\hline $12 / 4 / 079: 30$ & 127.688 & 125.236 & 121.649 & 101.661 & 105.462 & 97.122 & 110.99 & 109.021 & 74.672 \\
\hline $12 / 4 / 07$ 10:00 & 127.747 & 125.245 & 121.682 & 101.713 & 105.526 & 97.183 & 111.048 & 109.083 & 74.733 \\
\hline $12 / 4 / 07 \quad 10: 30$ & 127.794 & 125.249 & 121.707 & 101.749 & 105.568 & 97.228 & 111.089 & 109.131 & 74.783 \\
\hline $12 / 4 / 0711: 00$ & 127.83 & 125.233 & 121.724 & 101.754 & 105.576 & 97.237 & 111.121 & 109.145 & 74.795 \\
\hline $12 / 4 / 0711: 30$ & 127.858 & 125.222 & 121.739 & 101.751 & 105.58 & 97.239 & 111.131 & 109.147 & 74.802 \\
\hline $12 / 4 / 07 \quad 12: 00$ & 127.882 & 125.267 & 121.73 & 101.73 & 105.564 & 97.225 & 111.144 & 109.136 & 74.791 \\
\hline $12 / 4 / 07 \quad 12: 30$ & 127.896 & 125.256 & 121.737 & 101.738 & 105.551 & 97.209 & 111.161 & 109.118 & 74.781 \\
\hline $12 / 4 / 07$ 13:00 & 127.896 & 123.403 & 120.429 & 101.778 & 105.602 & 97.261 & 111.166 & 109.162 & 74.835 \\
\hline $12 / 4 / 07$ 13:30 & 127.92 & 123.111 & 120.103 & 101.647 & 105.589 & 97.249 & 111.189 & 109.16 & 74.822 \\
\hline $12 / 4 / 07 \quad 14: 00$ & 126.038 & 122.957 & 119.953 & 100.937 & 105.064 & 96.584 & 110.547 & 107.808 & 74.736 \\
\hline
\end{tabular}


TABLE S1.1 (Cont.)

\begin{tabular}{|c|c|c|c|c|c|c|c|c|c|c|}
\hline \multirow[b]{2}{*}{ Date and Time } & \multicolumn{10}{|c|}{ Depth from Top of Casing (ft) } \\
\hline & MW1D & MW2D & MW3D & MW4D & MW7 & MW9 & Oentrich & MW14D & MW15D & MW16D \\
\hline $12 / 4 / 07$ 14:30 & 125.759 & 122.855 & 119.857 & 100.72 & 104.786 & 96.299 & 110.312 & 107.453 & & 74.554 \\
\hline $12 / 4 / 07$ 15:00 & 125.627 & 122.793 & 119.792 & 100.591 & 104.639 & 96.148 & 110.196 & 107.287 & & 74.419 \\
\hline 12/4/07 15:30 & 125.549 & 122.755 & 119.739 & 100.512 & 104.54 & 96.054 & 109.966 & 107.183 & & 74.324 \\
\hline $12 / 4 / 0716: 00$ & 125.499 & 122.737 & 119.696 & 100.463 & 104.481 & 95.993 & 109.92 & 107.115 & & 74.262 \\
\hline $12 / 4 / 07 \quad 16: 30$ & 125.461 & 122.703 & 119.673 & 100.441 & 104.449 & 95.957 & 109.886 & 107.077 & & 74.225 \\
\hline $12 / 4 / 07$ 17:00 & 125.435 & 122.696 & 119.646 & 100.42 & 104.43 & 95.941 & 109.863 & 107.055 & & 74.204 \\
\hline $12 / 4 / 0717: 30$ & 125.421 & 122.675 & 119.628 & 100.398 & 104.395 & 95.908 & 109.848 & 107.022 & & 74.167 \\
\hline $12 / 4 / 07$ 18:00 & 125.405 & 122.671 & 119.608 & 100.388 & 104.393 & 95.905 & 109.835 & 107.015 & & 74.163 \\
\hline 12/4/07 18:30 & 125.398 & 122.662 & 119.59 & 100.37 & 104.376 & 95.889 & 109.828 & 106.997 & & 74.144 \\
\hline $12 / 4 / 07$ 19:00 & 125.386 & 122.657 & 119.577 & 100.369 & 104.369 & 95.879 & 109.816 & 106.988 & & 74.134 \\
\hline 12/4/07 19:30 & 125.381 & 122.651 & 119.567 & 100.365 & 104.361 & 95.87 & 109.811 & 106.977 & & 74.124 \\
\hline 12/4/07 20:00 & 125.376 & 122.637 & 119.555 & 100.353 & 104.359 & 95.87 & 109.805 & 106.977 & & 74.121 \\
\hline $12 / 4 / 0720: 30$ & 125.367 & 122.623 & 119.545 & 100.348 & 104.352 & 95.863 & 109.801 & 106.966 & & 74.111 \\
\hline 12/4/07 21:00 & 125.362 & 122.619 & 119.535 & 100.336 & 104.346 & 95.856 & 109.797 & 106.96 & & 74.103 \\
\hline 12/4/07 21:30 & 125.052 & 122.63 & 119.522 & 100.32 & 104.327 & 95.839 & 109.796 & 106.944 & & 74.086 \\
\hline $12 / 4 / 0722: 00$ & 125.045 & 122.626 & 119.512 & 100.326 & 104.323 & 95.837 & 109.788 & 106.94 & & 74.084 \\
\hline $12 / 4 / 0722: 30$ & 125.043 & 122.623 & 119.504 & 100.334 & 104.336 & 95.849 & 109.784 & 106.949 & & 74.093 \\
\hline $12 / 4 / 0723: 00$ & 125.045 & 122.623 & 119.499 & 100.334 & 104.329 & 95.842 & 109.784 & 106.942 & & 74.084 \\
\hline 12/4/07 23:30 & 125.045 & 122.621 & 119.497 & 100.332 & 104.329 & 95.839 & 109.782 & 106.942 & & 74.084 \\
\hline $12 / 5 / 070: 00$ & 125.041 & 122.63 & 119.489 & 100.334 & 104.331 & 95.844 & 109.781 & 106.944 & & 74.084 \\
\hline 12/5/07 0:30 & 125.041 & 122.639 & 119.482 & 100.329 & 104.331 & 95.842 & 109.781 & 106.942 & & 74.082 \\
\hline 12/5/07 1:00 & 125.038 & 122.653 & 119.479 & 100.345 & 104.338 & 95.849 & 109.779 & 106.946 & & 74.086 \\
\hline 12/5/07 1:30 & 125.041 & 122.664 & 119.477 & 100.351 & 104.352 & 95.863 & 109.777 & 106.96 & & 74.099 \\
\hline 12/5/07 2:00 & 125.036 & 122.685 & 119.476 & 100.369 & 104.365 & 95.877 & 109.775 & 106.971 & & 74.109 \\
\hline $12 / 5 / 072: 30$ & 125.038 & 122.721 & 119.476 & 100.384 & 104.376 & 95.889 & 109.775 & 106.984 & & 74.119 \\
\hline 12/5/07 3:00 & 125.036 & 122.741 & 119.479 & 100.405 & 104.395 & 95.91 & 109.773 & 107.004 & & 74.134 \\
\hline 12/5/07 3:30 & 125.034 & 122.766 & 119.479 & 100.446 & 104.432 & 95.945 & 109.773 & 107.035 & & 74.167 \\
\hline 12/5/07 4:00 & 125.034 & 122.802 & 119.492 & 100.462 & 104.454 & 95.974 & 109.771 & 107.059 & & 74.19 \\
\hline 12/5/07 4:30 & 125.034 & 122.825 & 119.507 & 100.488 & 104.481 & 95.995 & 109.773 & 107.084 & & 74.21 \\
\hline $12 / 5 / 075: 00$ & 125.031 & 122.852 & 119.52 & 100.517 & 104.511 & 96.026 & 109.79 & 107.112 & & 74.237 \\
\hline 12/5/07 5:30 & 125.031 & 122.884 & 119.535 & 100.555 & 104.538 & 96.054 & 109.797 & 107.141 & & 74.262 \\
\hline $12 / 5 / 076: 00$ & 125.034 & 122.918 & 119.55 & 100.579 & 104.571 & 96.082 & 109.809 & 107.17 & & 74.289 \\
\hline 12/5/07 6:30 & 125.031 & 122.941 & 119.58 & 100.608 & 104.603 & 96.12 & 109.815 & 107.203 & & 74.32 \\
\hline $12 / 5 / 07$ 7:00 & 125.187 & 124.725 & 120.767 & 100.643 & 104.637 & 96.15 & 109.813 & 107.234 & & 74.349 \\
\hline 12/5/07 7:30 & 125.145 & 125.088 & 121.349 & 100.667 & 104.658 & 96.176 & 109.867 & 107.263 & & 74.372 \\
\hline 12/5/07 8:00 & 126.948 & 125.274 & 121.528 & 101.418 & 105.077 & 96.728 & 110.408 & 108.465 & & 74.413 \\
\hline 12/5/07 8:30 & 127.319 & 125.394 & 121.636 & 101.685 & 105.429 & 97.096 & 110.759 & 108.95 & & 74.622 \\
\hline 12/5/07 9:00 & 127.579 & 125.478 & 121.742 & 101.837 & 105.616 & 97.277 & 110.941 & 109.156 & & 74.789 \\
\hline 12/5/07 9:30 & 127.664 & 123.766 & 120.588 & 101.938 & 105.736 & 97.4 & 111.039 & 109.28 & & 74.909 \\
\hline $12 / 5 / 07$ 10:00 & 127.724 & 123.494 & 120.225 & 102.018 & 105.818 & 97.482 & 111.118 & 109.368 & & 74.994 \\
\hline 12/5/07 10:30 & 126.092 & 123.385 & 120.092 & 101.313 & 105.435 & 96.964 & 110.614 & 108.193 & & 75.007 \\
\hline 12/5/07 11:00 & 125.771 & 123.319 & 120.023 & 101.142 & 105.184 & 96.702 & 110.363 & 107.834 & & 74.884 \\
\hline 12/5/07 11:30 & 125.641 & 123.27 & 119.991 & 101.058 & 105.081 & 96.598 & 110.267 & 107.717 & & 74.802 \\
\hline 12/5/07 12:00 & 125.57 & 123.224 & 119.971 & 101.004 & 105.022 & 96.539 & 110.205 & 107.648 & & 74.742 \\
\hline $12 / 5 / 0712: 30$ & 125.525 & 123.213 & 119.95 & 100.956 & 104.97 & 96.49 & 110.165 & 107.597 & & 74.69 \\
\hline 12/5/07 13:00 & 125.502 & 123.192 & 119.938 & 100.929 & 104.928 & 96.447 & 110.141 & 107.555 & & 74.649 \\
\hline 12/5/07 13:30 & 125.473 & 123.177 & 119.938 & 100.917 & 104.915 & 96.436 & 110.122 & 107.537 & & 74.635 \\
\hline $12 / 5 / 0714: 00$ & 125.461 & 123.161 & 119.932 & 100.901 & 104.9 & 96.414 & 110.109 & 107.518 & & 74.618 \\
\hline $12 / 5 / 0714: 30$ & 125.447 & 123.149 & 119.93 & 100.882 & 104.885 & 96.403 & 110.098 & 107.504 & & 74.603 \\
\hline 12/5/07 15:00 & 125.44 & 123.147 & 119.925 & 100.87 & 104.873 & 96.391 & 110.09 & 107.489 & & 74.591 \\
\hline $12 / 5 / 0715: 30$ & 125.433 & 123.127 & 119.925 & 100.861 & 104.856 & 96.372 & 110.081 & 107.473 & & 74.576 \\
\hline $12 / 5 / 07$ 16:00 & 125.424 & 123.138 & 119.915 & 100.853 & 104.856 & 96.372 & 110.075 & 107.471 & & 74.576 \\
\hline 12/5/07 16:30 & 125.421 & 123.122 & 119.923 & 100.841 & 104.835 & 96.353 & 110.073 & 107.456 & & 74.56 \\
\hline $12 / 5 / 07$ 17:00 & 125.414 & 123.113 & 119.92 & 100.853 & 104.848 & 96.367 & 110.064 & 107.464 & & 74.572 \\
\hline $12 / 5 / 07$ 17:30 & 125.414 & 123.129 & 119.91 & 100.836 & 104.828 & 96.346 & 110.064 & 107.447 & & 74.551 \\
\hline 12/5/07 18:00 & 125.412 & 123.133 & 119.91 & 100.829 & 104.82 & 96.334 & 110.062 & 107.436 & & 74.543 \\
\hline $12 / 5 / 0718: 30$ & 125.405 & 123.131 & 119.908 & 100.846 & 104.835 & 96.353 & 110.055 & 107.451 & & 74.562 \\
\hline 12/5/07 19:00 & 125.402 & 123.113 & 119.91 & 100.853 & 104.839 & 96.358 & 110.053 & 107.456 & & 74.566 \\
\hline 12/5/07 19:30 & 125.4 & 123.086 & 119.915 & 100.842 & 104.839 & 96.356 & 110.047 & 107.458 & & 74.566 \\
\hline 12/5/07 20:00 & 125.4 & 123.097 & 119.908 & 100.824 & 104.822 & 96.339 & 110.051 & 107.44 & & 74.55 \\
\hline $12 / 5 / 0720: 30$ & 125.402 & 123.124 & 119.9 & 100.808 & 104.788 & 96.304 & 110.051 & 107.409 & & 74.518 \\
\hline $12 / 5 / 0721: 00$ & 125.393 & 123.102 & 119.903 & 100.815 & 104.805 & 96.32 & 110.043 & 107.42 & & 74.537 \\
\hline 12/5/07 21:30 & 125.383 & 123.102 & 119.903 & 100.841 & 104.833 & 96.348 & 110.036 & 107.444 & & 74.564 \\
\hline 12/5/07 22:00 & 125.39 & 123.095 & 119.903 & 100.81 & 104.807 & 96.325 & 110.04 & 107.427 & & 74.542 \\
\hline 12/5/07 22:30 & 125.383 & 123.081 & 119.9 & 100.813 & 104.81 & 96.327 & 110.034 & 107.427 & & 74.543 \\
\hline 12/5/07 23:00 & 125.383 & 123.072 & 119.897 & 100.801 & 104.803 & 96.318 & 110.034 & 107.418 & & 74.537 \\
\hline
\end{tabular}


TABLE S1.1 (Cont.)

Depth from Top of Casing (ft)

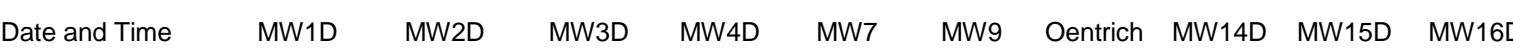

\begin{tabular}{|c|c|c|c|c|c|c|c|c|c|}
\hline 12/5/07 23:30 & 125.381 & 123.07 & 119.887 & 100.791 & 104.791 & 96.308 & 110.032 & 107.409 & 74.529 \\
\hline 12/6/07 0:00 & 125.379 & 123.061 & 119.89 & 100.782 & 104.782 & 96.297 & 110.03 & 107.4 & 74.52 \\
\hline 12/6/07 0:30 & 125.376 & 123.034 & 119.885 & 100.782 & 104.778 & 96.292 & 110.028 & 107.394 & 74.516 \\
\hline 12/6/07 1:00 & 125.374 & 123.013 & 119.885 & 100.767 & 104.772 & 96.285 & 110.027 & 107.385 & 74.51 \\
\hline 12/6/07 1:30 & 125.379 & 122.993 & 119.875 & 100.739 & 104.746 & 96.264 & 110.028 & 107.365 & 74.489 \\
\hline 12/6/07 2:00 & 125.374 & 122.961 & 119.867 & 100.718 & 104.723 & 96.238 & 110.025 & 107.338 & 74.467 \\
\hline 12/6/07 2:30 & 125.374 & 122.945 & 119.857 & 100.686 & 104.702 & 96.216 & 110.025 & 107.32 & 74.448 \\
\hline 12/6/07 3:00 & 125.374 & 122.954 & 119.842 & 100.656 & 104.671 & 96.188 & 110.025 & 107.294 & 74.424 \\
\hline 12/6/07 3:30 & 125.367 & 122.943 & 119.834 & 100.66 & 104.654 & 96.167 & 110.021 & 107.267 & 74.405 \\
\hline 12/6/07 4:00 & 125.36 & 122.941 & 119.824 & 100.67 & 104.664 & 96.176 & 110.017 & 107.276 & 74.413 \\
\hline 12/6/07 4:30 & 125.362 & 122.927 & 119.819 & 100.658 & 104.65 & 96.165 & 110.015 & 107.265 & 74.403 \\
\hline 12/6/07 5:00 & 125.36 & 122.929 & 119.804 & 100.65 & 104.652 & 96.167 & 110.015 & 107.267 & 74.405 \\
\hline 12/6/07 5:30 & 125.357 & 122.938 & 119.797 & 100.632 & 104.635 & 96.148 & 110.012 & 107.247 & 74.386 \\
\hline 12/6/07 6:00 & 125.353 & 122.932 & 119.792 & 100.651 & 104.635 & 96.148 & 110.008 & 107.247 & 74.386 \\
\hline 12/6/07 6:30 & 125.35 & 122.914 & 119.789 & 100.648 & 104.649 & 96.16 & 110.008 & 107.259 & 74.398 \\
\hline 12/6/07 7:00 & 125.355 & 124.859 & 121.039 & 100.636 & 104.643 & 96.155 & 110.006 & 107.256 & 74.393 \\
\hline 12/6/07 7:30 & 125.357 & 125.084 & 121.336 & 100.834 & 104.62 & 96.134 & 110.01 & 107.236 & 74.372 \\
\hline 12/6/07 8:00 & 127.296 & 125.195 & 121.475 & 101.421 & 105.155 & 96.794 & 110.646 & 108.689 & 74.471 \\
\hline 12/6/07 8:30 & 127.558 & 125.263 & 121.548 & 101.58 & 105.372 & 97.022 & 110.879 & 108.965 & 74.628 \\
\hline 12/6/07 9:00 & 127.693 & 125.304 & 121.596 & 101.675 & 105.486 & 97.131 & 110.99 & 109.089 & 74.735 \\
\hline 12/6/07 9:30 & 127.771 & 125.333 & 121.621 & 101.733 & 105.557 & 97.206 & 111.059 & 109.165 & 74.81 \\
\hline 12/6/07 10:00 & 127.818 & 125.335 & 121.649 & 101.766 & 105.599 & 97.246 & 111.104 & 109.207 & 74.855 \\
\hline $12 / 6 / 07 \quad 10: 30$ & 127.849 & 125.329 & 121.661 & 101.79 & 105.623 & 97.268 & 111.131 & 109.229 & 74.884 \\
\hline 12/6/07 11:00 & 127.88 & 125.342 & 121.661 & 101.783 & 105.639 & 97.284 & 111.155 & 109.249 & 74.901 \\
\hline 12/6/07 11:30 & 127.899 & 125.358 & 121.669 & 101.79 & 105.629 & 97.277 & 111.17 & 109.24 & 74.901 \\
\hline $12 / 6 / 07$ 12:00 & 127.908 & 123.839 & 121.215 & 101.804 & 105.646 & 97.291 & 111.179 & 109.255 & 74.922 \\
\hline 12/6/07 12:30 & 127.913 & 123.349 & 120.291 & 101.816 & 105.663 & 97.305 & 111.183 & 109.264 & 74.936 \\
\hline 12/6/07 13:00 & 126.471 & 123.163 & 120.092 & 101.225 & 105.448 & 96.997 & 110.877 & 108.445 & 74.934 \\
\hline 12/6/07 13:30 & 125.908 & 123.056 & 119.966 & 100.941 & 105.024 & 96.539 & 110.458 & 107.723 & 74.775 \\
\hline $12 / 6 / 07$ 14:00 & 125.731 & 122.986 & 119.91 & 100.798 & 104.843 & 96.356 & 110.306 & 107.5 & 74.63 \\
\hline 12/6/07 14:30 & 125.632 & 122.938 & 119.857 & 100.715 & 104.742 & 96.254 & 110.224 & 107.387 & 74.531 \\
\hline 12/6/07 15:00 & 125.568 & 122.909 & 119.822 & 100.66 & 104.675 & 96.188 & 110.173 & 107.314 & 74.463 \\
\hline 12/6/07 15:30 & 125.528 & 122.891 & 119.789 & 100.617 & 104.633 & 96.148 & 110.139 & 107.267 & 74.417 \\
\hline 12/6/07 16:00 & 125.502 & 122.886 & 119.764 & 100.6 & 104.601 & 96.115 & 110.117 & 107.232 & 74.378 \\
\hline $12 / 6 / 07 \quad 16: 30$ & 125.48 & 122.889 & 119.746 & 100.587 & 104.588 & 96.101 & 110.1 & 107.214 & 74.363 \\
\hline 12/6/07 17:00 & 125.459 & 122.879 & 119.731 & 100.586 & 104.584 & 96.099 & 110.085 & 107.21 & 74.359 \\
\hline 12/6/07 17:30 & 125.445 & 122.877 & 119.718 & 100.584 & 104.586 & 96.099 & 110.073 & 107.208 & 74.357 \\
\hline $12 / 6 / 07$ 18:00 & 125.44 & 122.884 & 119.703 & 100.574 & 104.576 & 96.092 & 110.068 & 107.199 & 74.347 \\
\hline 12/6/07 18:30 & 125.431 & 122.891 & 119.693 & 100.586 & 104.578 & 96.089 & 110.062 & 107.197 & 74.345 \\
\hline 12/6/07 19:00 & 125.424 & 122.9 & 119.696 & 100.591 & 104.588 & 96.098 & 110.057 & 107.205 & 74.353 \\
\hline 12/6/07 19:30 & 125.419 & 122.911 & 119.69 & 100.598 & 104.591 & 96.106 & 110.051 & 107.212 & 74.357 \\
\hline 12/6/07 20:00 & 125.412 & 122.927 & 119.688 & 100.61 & 104.599 & 96.113 & 110.049 & 107.219 & 74.361 \\
\hline 12/6/07 20:30 & 125.409 & 122.943 & 119.69 & 100.625 & 104.614 & 96.129 & 110.045 & 107.232 & 74.376 \\
\hline $12 / 6 / 0721: 00$ & 125.405 & 122.938 & 119.696 & 100.644 & 104.63 & 96.143 & 110.042 & 107.247 & 74.386 \\
\hline 12/6/07 21:30 & 125.4 & 122.941 & 119.695 & 100.648 & 104.645 & 96.16 & 110.04 & 107.259 & 74.401 \\
\hline 12/6/07 22:00 & 125.402 & 122.941 & 119.698 & 100.648 & 104.643 & 96.155 & 110.04 & 107.259 & 74.396 \\
\hline 12/6/07 22:30 & 125.4 & 122.936 & 119.7 & 100.656 & 104.643 & 96.158 & 110.038 & 107.261 & 74.396 \\
\hline 12/6/07 23:00 & 125.395 & 122.932 & 119.703 & 100.65 & 104.649 & 96.164 & 110.038 & 107.265 & 74.401 \\
\hline 12/6/07 23:30 & 125.398 & 122.952 & 119.69 & 100.639 & 104.639 & 96.155 & 110.038 & 107.256 & 74.392 \\
\hline 12/7/07 0:00 & 125.395 & 122.977 & 119.698 & 100.648 & 104.635 & 96.153 & 110.036 & 107.252 & 74.388 \\
\hline 12/7/07 0:30 & 125.386 & 122.97 & 119.71 & 100.67 & 104.66 & 96.172 & 110.03 & 107.27 & 74.409 \\
\hline 12/7/07 1:00 & 125.381 & 122.963 & 119.713 & 100.693 & 104.683 & 96.197 & 110.027 & 107.296 & 74.434 \\
\hline 12/7/07 1:30 & 125.383 & 122.966 & 119.713 & 100.679 & 104.677 & 96.193 & 110.028 & 107.289 & 74.425 \\
\hline $12 / 7 / 072: 00$ & 125.383 & 122.963 & 119.718 & 100.674 & 104.671 & 96.188 & 110.028 & 107.287 & 74.419 \\
\hline 12/7/07 2:30 & 125.381 & 122.957 & 119.723 & 100.672 & 104.673 & 96.188 & 110.028 & 107.287 & 74.421 \\
\hline 12/7/07 3:00 & 125.381 & 122.984 & 119.718 & 100.668 & 104.671 & 96.186 & 110.027 & 107.285 & 74.419 \\
\hline 12/7/07 3:30 & 125.379 & 122.97 & 119.729 & 100.682 & 104.664 & 96.179 & 110.027 & 107.278 & 74.411 \\
\hline 12/7/07 4:00 & 125.372 & 122.966 & 119.726 & 100.699 & 104.694 & 96.207 & 110.021 & 107.305 & 74.438 \\
\hline $12 / 7 / 074: 30$ & 125.376 & 122.968 & 119.724 & 100.682 & 104.679 & 96.197 & 110.023 & 107.296 & 74.425 \\
\hline $12 / 7 / 075: 00$ & 125.374 & 122.975 & 119.729 & 100.677 & 104.675 & 96.191 & 110.023 & 107.289 & 74.423 \\
\hline 12/7/07 5:30 & 125.372 & 122.979 & 119.73 & 100.679 & 104.675 & 96.191 & 110.021 & 107.289 & 74.423 \\
\hline 12/7/07 6:00 & 125.369 & 122.975 & 119.733 & 100.691 & 104.683 & 96.198 & 110.021 & 107.294 & 74.429 \\
\hline 12/7/07 6:30 & 125.367 & 122.982 & 119.738 & 100.691 & 104.69 & 96.204 & 110.021 & 107.305 & 74.436 \\
\hline $12 / 7 / 07$ 7:00 & 125.369 & 124.723 & 120.718 & 100.691 & 104.681 & 96.198 & 110.021 & 107.296 & 74.429 \\
\hline $12 / 7 / 077: 30$ & 125.367 & 125.081 & 121.207 & 100.696 & 104.692 & 96.207 & 110.017 & 107.305 & 74.436 \\
\hline & & & & & & & & & \\
\hline
\end{tabular}


TABLE S1.1 (Cont.)

Depth from Top of Casing (ft)

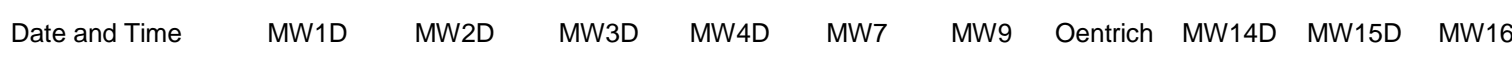

\begin{tabular}{|c|c|c|c|c|c|c|c|c|c|}
\hline 12/7/07 8:30 & 127.482 & 123.446 & 120.27 & 101.601 & 105.385 & 97.027 & 110.811 & 108.95 & 74.644 \\
\hline 12/7/07 9:00 & 127.641 & 123.26 & 120.025 & 101.561 & 105.524 & 97.171 & 110.96 & 109.112 & 74.773 \\
\hline 12/7/07 9:30 & 125.849 & 123.195 & 119.928 & 101.053 & 105.13 & 96.653 & 110.423 & 107.839 & 74.795 \\
\hline $12 / 7 / 07$ 10:00 & 125.639 & 123.149 & 119.882 & 100.918 & 104.957 & 96.469 & 110.242 & 107.597 & 74.7 \\
\hline $12 / 7 / 07 \quad 10: 30$ & 125.544 & 123.131 & 119.851 & 100.872 & 104.887 & 96.403 & 110.164 & 107.515 & 74.644 \\
\hline 12/7/07 11:00 & 125.497 & 123.131 & 119.834 & 100.846 & 104.848 & 96.367 & 110.124 & 107.475 & 74.607 \\
\hline 12/7/07 11:30 & 125.466 & 123.09 & 119.836 & 100.839 & 104.829 & 96.348 & 110.1 & 107.453 & 74.582 \\
\hline $12 / 7 / 07 \quad 12: 00$ & 125.442 & 123.061 & 119.836 & 100.825 & 104.835 & 96.355 & 110.094 & 107.456 & 74.585 \\
\hline $12 / 7 / 07$ 12:30 & 125.438 & 123.041 & 119.826 & 100.791 & 104.789 & 96.311 & 110.089 & 107.416 & 74.537 \\
\hline $12 / 7 / 07$ 13:00 & 125.431 & 123.031 & 119.822 & 100.765 & 104.763 & 96.282 & 110.08 & 107.385 & 74.512 \\
\hline $12 / 7 / 07$ 13:30 & 125.424 & 123.025 & 119.809 & 100.744 & 104.746 & 96.264 & 110.07 & 107.367 & 74.498 \\
\hline $12 / 7 / 07 \quad 14: 00$ & 125.414 & 123.016 & 119.806 & 100.737 & 104.736 & 96.257 & 110.065 & 107.356 & 74.489 \\
\hline $12 / 7 / 07$ 14:30 & 125.409 & 123.007 & 119.807 & 100.732 & 104.73 & 96.245 & 110.062 & 107.347 & 74.481 \\
\hline $12 / 7 / 07$ 15:00 & 125.405 & 123.011 & 119.801 & 100.722 & 104.719 & 96.235 & 110.057 & 107.336 & 74.471 \\
\hline $12 / 7 / 07 \quad 15: 30$ & 125.405 & 123.027 & 119.787 & 100.713 & 104.709 & 96.226 & 110.057 & 107.329 & 74.465 \\
\hline 12/7/07 16:00 & 125.395 & 123.029 & 119.792 & 100.72 & 104.717 & 96.23 & 110.051 & 107.329 & 74.467 \\
\hline 12/7/07 16:30 & 125.39 & 123.036 & 119.787 & 100.736 & 104.734 & 96.252 & 110.047 & 107.347 & 74.487 \\
\hline $12 / 7 / 07$ 17:00 & 125.388 & 123.036 & 119.793 & 100.739 & 104.734 & 96.252 & 110.043 & 107.349 & 74.487 \\
\hline $12 / 7 / 07$ 17:30 & 125.388 & 123.043 & 119.794 & 100.748 & 104.742 & 96.261 & 110.042 & 107.358 & 74.494 \\
\hline 12/7/07 18:00 & 125.386 & 123.061 & 119.792 & 100.76 & 104.744 & 96.259 & 110.04 & 107.358 & 74.494 \\
\hline 12/7/07 18:30 & 125.383 & 123.056 & 119.802 & 100.756 & 104.748 & 96.266 & 110.042 & 107.363 & 74.498 \\
\hline $12 / 7 / 07 \quad 19: 00$ & 125.381 & 123.075 & 119.799 & 100.782 & 104.769 & 96.285 & 110.036 & 107.382 & 74.514 \\
\hline 12/7/07 19:30 & 125.383 & 123.077 & 119.804 & 100.779 & 104.761 & 96.278 & 110.04 & 107.38 & 74.508 \\
\hline $12 / 7 / 0720: 00$ & 125.379 & 123.084 & 119.807 & 100.791 & 104.778 & 96.297 & 110.036 & 107.394 & 74.527 \\
\hline $12 / 7 / 0720: 30$ & 125.376 & 123.09 & 119.812 & 100.789 & 104.784 & 96.304 & 110.038 & 107.4 & 74.533 \\
\hline 12/7/07 21:00 & 125.374 & 123.09 & 119.819 & 100.798 & 104.791 & 96.308 & 110.042 & 107.405 & 74.537 \\
\hline $12 / 7 / 0721: 30$ & 125.374 & 123.109 & 119.821 & 100.806 & 104.793 & 96.311 & 110.045 & 107.409 & 74.541 \\
\hline $12 / 7 / 0722: 00$ & 125.376 & 123.102 & 119.827 & 100.81 & 104.793 & 96.313 & 110.045 & 107.413 & 74.543 \\
\hline 12/7/07 22:30 & 125.369 & 123.113 & 119.824 & 100.806 & 104.812 & 96.33 & 110.042 & 107.427 & 74.56 \\
\hline 12/7/07 23:00 & 125.369 & 123.122 & 119.832 & 100.827 & 104.807 & 96.323 & 110.043 & 107.422 & 74.554 \\
\hline $12 / 7 / 0723: 30$ & 125.367 & 123.127 & 119.839 & 100.824 & 104.82 & 96.337 & 110.042 & 107.434 & 74.566 \\
\hline $12 / 8 / 070: 00$ & 125.364 & 123.131 & 119.842 & 100.842 & 104.833 & 96.351 & 110.038 & 107.444 & 74.578 \\
\hline $12 / 8 / 070: 30$ & 125.362 & 123.133 & 119.844 & 100.844 & 104.835 & 96.353 & 110.034 & 107.444 & 74.58 \\
\hline $12 / 8 / 07$ 1:00 & 125.36 & 123.127 & 119.849 & 100.861 & 104.839 & 96.358 & 110.034 & 107.451 & 74.585 \\
\hline $12 / 8 / 07 \quad 1: 30$ & 125.362 & 123.14 & 119.852 & 100.844 & 104.847 & 96.367 & 110.034 & 107.464 & 74.593 \\
\hline $12 / 8 / 072: 00$ & 125.36 & 123.133 & 119.857 & 100.848 & 104.833 & 96.353 & 110.034 & 107.451 & 74.58 \\
\hline $12 / 8 / 072: 30$ & 125.353 & 123.129 & 119.862 & 100.849 & 104.852 & 96.37 & 110.028 & 107.462 & 74.597 \\
\hline $12 / 8 / 07$ 3:00 & 125.355 & 123.131 & 119.851 & 100.842 & 104.845 & 96.363 & 110.028 & 107.46 & 74.593 \\
\hline 12/8/07 3:30 & 125.35 & 123.138 & 119.864 & 100.842 & 104.839 & 96.358 & 110.027 & 107.453 & 74.589 \\
\hline $12 / 8 / 074: 00$ & 125.35 & 123.136 & 119.869 & 100.848 & 104.843 & 96.363 & 110.027 & 107.46 & 74.595 \\
\hline $12 / 8 / 074: 30$ & 125.346 & 123.145 & 119.869 & 100.849 & 104.85 & 96.367 & 110.021 & 107.464 & 74.599 \\
\hline $12 / 8 / 07$ 5:00 & 125.346 & 123.152 & 119.872 & 100.849 & 104.843 & 96.363 & 110.021 & 107.458 & 74.593 \\
\hline $12 / 8 / 075: 30$ & 125.341 & 123.152 & 119.875 & 100.858 & 104.856 & 96.374 & 110.017 & 107.469 & 74.603 \\
\hline $12 / 8 / 076: 00$ & 125.341 & 123.156 & 119.879 & 100.863 & 104.864 & 96.381 & 110.015 & 107.478 & 74.611 \\
\hline $12 / 8 / 076: 30$ & 125.338 & 123.168 & 119.882 & 100.867 & 104.862 & 96.381 & 110.045 & 107.475 & 74.609 \\
\hline $12 / 8 / 07$ 7:00 & 125.338 & 123.179 & 119.885 & 100.872 & 104.866 & 96.386 & 110.046 & 107.48 & 74.616 \\
\hline $12 / 8 / 07$ 7:30 & 125.331 & 123.19 & 119.885 & 100.884 & 104.877 & 96.396 & 110.04 & 107.491 & 74.626 \\
\hline $12 / 8 / 078: 00$ & 125.329 & 125.172 & 121.344 & 100.896 & 104.888 & 96.412 & 110.073 & 107.506 & 74.639 \\
\hline $12 / 8 / 078: 30$ & 125.329 & 125.408 & 121.623 & 101.325 & 104.898 & 96.417 & 110.104 & 107.511 & 74.644 \\
\hline $12 / 8 / 07$ 9:00 & 127.355 & 125.546 & 121.762 & 101.782 & 105.498 & 97.159 & 110.828 & 109.005 & 74.764 \\
\hline $12 / 8 / 079: 30$ & 127.591 & 125.614 & 121.852 & 101.966 & 105.736 & 97.4 & 111.039 & 109.284 & 74.953 \\
\hline $12 / 8 / 07 \quad 10: 00$ & 127.714 & 125.669 & 121.918 & 102.087 & 105.875 & 97.539 & 111.151 & 109.433 & 75.089 \\
\hline $12 / 8 / 07 \quad 10: 30$ & 127.787 & 125.707 & 121.958 & 102.138 & 105.941 & 97.607 & 111.223 & 109.508 & 75.159 \\
\hline 12/8/07 11:00 & 127.837 & 125.728 & 121.993 & 102.188 & 105.997 & 97.661 & 111.27 & 109.565 & 75.22 \\
\hline $12 / 8 / 07 \quad 11: 30$ & 127.873 & 125.746 & 122.021 & 102.221 & 106.033 & 97.699 & 111.304 & 109.605 & 75.261 \\
\hline $12 / 8 / 07 \quad 12: 00$ & 127.899 & 125.757 & 122.045 & 102.242 & 106.056 & 97.725 & 111.328 & 109.63 & 75.287 \\
\hline $12 / 8 / 07 \quad 12: 30$ & 127.917 & 125.759 & 122.067 & 102.254 & 106.075 & 97.746 & 111.348 & 109.65 & 75.308 \\
\hline $12 / 8 / 07$ 13:00 & 127.936 & 125.769 & 122.075 & 102.266 & 106.084 & 97.756 & 111.365 & 109.661 & 75.321 \\
\hline $12 / 8 / 07$ 13:30 & 127.953 & 125.782 & 122.087 & 102.269 & 106.088 & 97.753 & 111.379 & 109.663 & 75.325 \\
\hline $12 / 8 / 0714: 00$ & 127.962 & 125.8 & 122.102 & 102.278 & 106.096 & 97.763 & 111.39 & 109.672 & 75.337 \\
\hline $12 / 8 / 07 \quad 14: 30$ & 127.972 & 125.798 & 122.118 & 102.297 & 106.109 & 97.775 & 111.395 & 109.683 & 75.354 \\
\hline $12 / 8 / 07$ 15:00 & 127.984 & 124.349 & 121.898 & 102.302 & 106.128 & 97.798 & 111.408 & 109.709 & 75.374 \\
\hline $12 / 8 / 07 \quad 15: 30$ & 127.993 & 123.827 & 120.623 & 102.297 & 106.122 & 97.791 & 111.416 & 109.703 & 75.373 \\
\hline $12 / 8 / 07$ 16:00 & 126.594 & 123.639 & 120.411 & 101.704 & 105.943 & 97.513 & 111.185 & 109.003 & 75.381 \\
\hline $12 / 8 / 07 \quad 16: 30$ & 126.048 & 123.548 & 120.297 & 101.409 & 105.488 & 97.006 & 110.635 & 108.188 & 75.242 \\
\hline $12 / 8 / 07 \quad 17: 00$ & 125.936 & 123.478 & 120.235 & 101.277 & 105.311 & 96.832 & 110.466 & 107.98 & 75.104 \\
\hline
\end{tabular}


TABLE S1.1 (Cont.)

Depth from Top of Casing (ft)

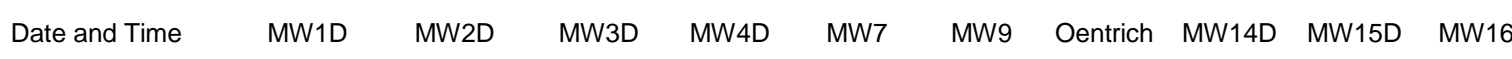

\begin{tabular}{|c|c|c|c|c|c|c|c|c|c|}
\hline 12/8/07 17:30 & 125.814 & 123.449 & 120.187 & 101.201 & 105.224 & 96.742 & 110.374 & 107.885 & 75.019 \\
\hline 12/8/07 18:00 & 125.901 & 123.421 & 120.151 & 101.149 & 105.157 & 96.676 & 110.318 & 107.814 & 74.953 \\
\hline 12/8/07 18:30 & 125.797 & 123.412 & 120.116 & 101.127 & 105.134 & 96.65 & 110.31 & 107.777 & 74.924 \\
\hline 12/8/07 19:00 & 125.766 & 123.392 & 120.116 & 101.101 & 105.1 & 96.619 & 110.282 & 107.743 & 74.888 \\
\hline 12/8/07 19:30 & 125.738 & 123.381 & 120.108 & 101.101 & 105.094 & 96.612 & 110.257 & 107.73 & 74.879 \\
\hline $12 / 8 / 0720: 00$ & 125.726 & 123.372 & 120.101 & 101.08 & 105.077 & 96.596 & 110.269 & 107.717 & 74.859 \\
\hline $12 / 8 / 0720: 30$ & 125.707 & 123.363 & 120.093 & 101.075 & 105.067 & 96.584 & 110.256 & 107.701 & 74.847 \\
\hline $12 / 8 / 0721: 00$ & 125.698 & 123.344 & 120.091 & 101.056 & 105.062 & 96.582 & 110.246 & 107.697 & 74.845 \\
\hline $12 / 8 / 0721: 30$ & 125.688 & 123.36 & 120.083 & 101.056 & 105.054 & 96.572 & 110.237 & 107.688 & 74.837 \\
\hline 12/8/07 22:00 & 125.681 & 123.36 & 120.076 & 101.039 & 105.039 & 96.558 & 110.229 & 107.672 & 74.82 \\
\hline 12/8/07 22:30 & 125.662 & 123.353 & 120.073 & 101.051 & 105.056 & 96.572 & 110.227 & 107.679 & 74.835 \\
\hline $12 / 8 / 0723: 00$ & 125.658 & 123.342 & 120.076 & 101.06 & 105.056 & 96.575 & 110.231 & 107.684 & 74.837 \\
\hline 12/8/07 23:30 & 125.655 & 123.328 & 120.076 & 101.053 & 105.046 & 96.565 & 110.237 & 107.679 & 74.828 \\
\hline 12/9/07 0:00 & 125.648 & 123.326 & 120.071 & 101.032 & 105.037 & 96.556 & 110.231 & 107.668 & 74.821 \\
\hline 12/9/07 0:30 & 125.646 & 123.331 & 120.066 & 101.027 & 105.024 & 96.542 & 110.229 & 107.655 & 74.808 \\
\hline 12/9/07 1:00 & 125.639 & 123.313 & 120.061 & 101.03 & 105.024 & 96.539 & 110.222 & 107.65 & 74.806 \\
\hline 12/9/07 1:30 & 125.629 & 123.31 & 120.063 & 101.032 & 105.026 & 96.546 & 110.216 & 107.655 & 74.812 \\
\hline $12 / 9 / 072: 00$ & 125.632 & 123.281 & 120.063 & 101.001 & 105.01 & 96.532 & 110.214 & 107.642 & 74.795 \\
\hline $12 / 9 / 072: 30$ & 125.622 & 123.283 & 120.051 & 101.001 & 105.01 & 96.528 & 110.209 & 107.635 & 74.793 \\
\hline 12/9/07 3:00 & 125.627 & 123.317 & 120.035 & 100.975 & 104.984 & 96.502 & 110.207 & 107.615 & 74.771 \\
\hline 12/9/07 3:30 & 125.62 & 123.279 & 120.048 & 100.992 & 104.972 & 96.485 & 110.205 & 107.597 & 74.758 \\
\hline $12 / 9 / 074: 00$ & 125.603 & 123.283 & 120.04 & 100.941 & 105.022 & 96.539 & 110.218 & 107.646 & 74.81 \\
\hline $12 / 9 / 074: 30$ & 125.603 & 123.29 & 120.033 & 100.992 & 104.98 & 96.497 & 110.218 & 107.599 & 74.766 \\
\hline $12 / 9 / 075: 00$ & 125.603 & 123.279 & 120.033 & 100.979 & 104.98 & 96.497 & 110.216 & 107.606 & 74.769 \\
\hline $12 / 9 / 075: 30$ & 125.596 & 123.267 & 120.035 & 100.992 & 104.991 & 96.504 & 110.212 & 107.615 & 74.777 \\
\hline $12 / 9 / 076: 00$ & 125.596 & 123.279 & 120.03 & 100.991 & 104.982 & 96.495 & 110.212 & 107.606 & 74.766 \\
\hline $12 / 9 / 076: 30$ & 125.596 & 123.31 & 120.018 & 100.963 & 104.97 & 96.485 & 110.212 & 107.597 & 74.758 \\
\hline $12 / 9 / 07$ 7:00 & 125.589 & 123.304 & 120.028 & 100.996 & 104.976 & 96.49 & 110.207 & 107.599 & 74.762 \\
\hline $12 / 9 / 077: 30$ & 125.58 & 123.308 & 120.033 & 101.01 & 105.01 & 96.528 & 110.201 & 107.63 & 74.795 \\
\hline $12 / 9 / 078: 00$ & 125.589 & 123.294 & 120.043 & 101.02 & 104.986 & 96.506 & 110.205 & 107.622 & 74.773 \\
\hline $12 / 9 / 078: 30$ & 125.58 & 123.294 & 120.04 & 101.008 & 105.006 & 96.52 & 110.199 & 107.628 & 74.787 \\
\hline 12/9/07 9:00 & 125.58 & 125.084 & 121.076 & 100.984 & 104.995 & 96.511 & 110.199 & 107.619 & 74.779 \\
\hline 12/9/07 9:30 & 125.575 & 125.412 & 121.512 & 101.004 & 104.993 & 96.506 & 110.196 & 107.613 & 74.775 \\
\hline $12 / 9 / 07 \quad 10: 00$ & 127.336 & 125.551 & 121.691 & 101.695 & 105.385 & 97.025 & 110.706 & 108.837 & 74.82 \\
\hline $12 / 9 / 07 \quad 10: 30$ & 127.681 & 125.655 & 121.782 & 101.928 & 105.713 & 97.36 & 111.01 & 109.291 & 75.012 \\
\hline $12 / 9 / 07 \quad 11: 00$ & 127.832 & 125.689 & 121.842 & 102.057 & 105.846 & 97.494 & 111.153 & 109.441 & 75.132 \\
\hline $12 / 9 / 0711: 30$ & 127.915 & 123.845 & 120.674 & 102.135 & 105.957 & 97.603 & 111.232 & 109.554 & 75.244 \\
\hline $12 / 9 / 0712: 00$ & 127.977 & 123.621 & 120.406 & 101.925 & 105.989 & 97.642 & 111.294 & 109.603 & 75.287 \\
\hline $12 / 9 / 07 \quad 12: 30$ & 126.133 & 123.496 & 120.285 & 101.409 & 105.513 & 97.032 & 110.697 & 108.237 & 75.227 \\
\hline $12 / 9 / 07$ 13:00 & 125.899 & 123.428 & 120.202 & 101.246 & 105.298 & 96.818 & 110.498 & 107.967 & 75.095 \\
\hline $12 / 9 / 07$ 13:30 & 125.792 & 123.39 & 120.156 & 101.151 & 105.176 & 96.695 & 110.404 & 107.83 & 74.986 \\
\hline $12 / 9 / 07 \quad 14: 00$ & 125.728 & 123.358 & 120.126 & 101.097 & 105.113 & 96.629 & 110.346 & 107.757 & 74.919 \\
\hline 12/9/07 14:30 & 125.686 & 123.331 & 120.106 & 101.072 & 105.075 & 96.589 & 110.31 & 107.712 & 74.88 \\
\hline $12 / 9 / 07 \quad 15: 00$ & 125.66 & 123.31 & 120.088 & 101.041 & 105.046 & 96.561 & 110.286 & 107.684 & 74.851 \\
\hline $12 / 9 / 07 \quad 15: 30$ & 125.641 & 123.306 & 120.078 & 101.018 & 105.02 & 96.535 & 110.269 & 107.655 & 74.822 \\
\hline 12/9/07 16:00 & 125.624 & 123.294 & 120.076 & 101.004 & 105.005 & 96.518 & 110.256 & 107.637 & 74.804 \\
\hline 12/9/07 16:30 & 125.608 & 123.292 & 120.068 & 100.998 & 104.995 & 96.511 & 110.246 & 107.626 & 74.795 \\
\hline $12 / 9 / 07 \quad 17: 00$ & 125.601 & 123.283 & 120.061 & 100.992 & 104.989 & 96.504 & 110.237 & 107.619 & 74.787 \\
\hline $12 / 9 / 07 \quad 17: 30$ & 125.594 & 123.281 & 120.051 & 100.985 & 104.984 & 96.499 & 110.229 & 107.613 & 74.785 \\
\hline $12 / 9 / 07$ 18:00 & 125.587 & 123.276 & 120.056 & 100.98 & 104.976 & 96.49 & 110.224 & 107.606 & 74.773 \\
\hline $12 / 9 / 07$ 18:30 & 125.582 & 123.272 & 120.048 & 100.972 & 104.974 & 96.49 & 110.22 & 107.602 & 74.773 \\
\hline 12/9/07 19:00 & 125.584 & 123.27 & 120.04 & 100.967 & 104.97 & 96.485 & 110.22 & 107.602 & 74.764 \\
\hline $12 / 9 / 07 \quad 19: 30$ & 125.58 & 123.274 & 120.038 & 100.965 & 104.967 & 96.48 & 110.218 & 107.595 & 74.762 \\
\hline 12/9/07 20:00 & 125.572 & 123.267 & 120.035 & 100.972 & 104.963 & 96.478 & 110.212 & 107.591 & 74.76 \\
\hline 12/9/07 20:30 & 125.57 & 123.256 & 120.035 & 100.97 & 104.968 & 96.485 & 110.209 & 107.597 & 74.764 \\
\hline $12 / 9 / 0721: 00$ & 125.565 & 123.26 & 120.03 & 100.965 & 104.965 & 96.48 & 110.205 & 107.591 & 74.76 \\
\hline 12/9/07 21:30 & 125.565 & 123.258 & 120.028 & 100.956 & 104.947 & 96.464 & 110.205 & 107.577 & 74.744 \\
\hline $12 / 9 / 0722: 00$ & 125.561 & 123.258 & 120.025 & 100.954 & 104.955 & 96.471 & 110.203 & 107.582 & 74.752 \\
\hline $12 / 9 / 0722: 30$ & 125.561 & 123.265 & 120.023 & 100.958 & 104.951 & 96.466 & 110.199 & 107.58 & 74.748 \\
\hline 12/9/07 23:00 & 125.556 & 123.256 & 120.015 & 100.961 & 104.951 & 96.466 & 110.197 & 107.58 & 74.748 \\
\hline 12/9/07 23:30 & 125.554 & 123.242 & 120.02 & 100.963 & 104.959 & 96.473 & 110.196 & 107.586 & 74.754 \\
\hline $12 / 10 / 070: 00$ & 125.554 & 123.24 & 120.015 & 100.954 & 104.951 & 96.466 & 110.196 & 107.58 & 74.748 \\
\hline $12 / 10 / 070: 30$ & 125.556 & 123.226 & 120.015 & 100.937 & 104.934 & 96.455 & 110.194 & 107.566 & 74.733 \\
\hline $12 / 10 / 071: 00$ & 125.551 & 123.215 & 120.013 & 100.937 & 104.934 & 96.45 & 110.192 & 107.562 & 74.731 \\
\hline $12 / 10 / 071: 30$ & 125.551 & 123.206 & 120.008 & 100.923 & 104.921 & 96.438 & 110.19 & 107.551 & 74.719 \\
\hline $12 / 10 / 072: 00$ & 125.549 & 123.206 & 120.008 & 100.91 & 104.907 & 96.422 & 110.19 & 107.535 & 74.707 \\
\hline
\end{tabular}


TABLE S1.1 (Cont.)

Depth from Top of Casing (ft)

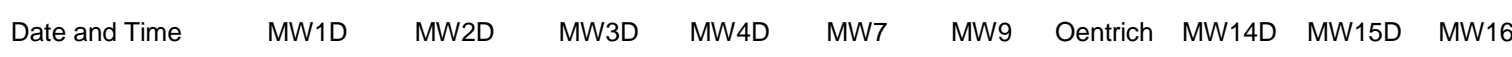

\begin{tabular}{|c|c|c|c|c|c|c|c|c|c|}
\hline $12 / 10 / 072: 30$ & 125.544 & 123.206 & 119.998 & 100.908 & 104.904 & 96.419 & 110.186 & 107.529 & 74.702 \\
\hline 12/10/07 3:00 & 125.542 & 123.202 & 119.98 & 100.908 & 104.902 & 96.417 & 110.184 & 107.526 & 74.698 \\
\hline 12/10/07 3:30 & 125.539 & 123.181 & 119.98 & 100.908 & 104.9 & 96.414 & 110.182 & 107.526 & 74.698 \\
\hline $12 / 10 / 074: 00$ & 125.539 & 123.174 & 119.985 & 100.896 & 104.896 & 96.412 & 110.182 & 107.524 & 74.696 \\
\hline 12/10/07 4:30 & 125.539 & 123.172 & 119.985 & 100.886 & 104.881 & 96.396 & 110.181 & 107.506 & 74.678 \\
\hline $12 / 10 / 075: 00$ & 125.537 & 123.154 & 119.98 & 100.88 & 104.871 & 96.389 & 110.179 & 107.498 & 74.671 \\
\hline $12 / 10 / 075: 30$ & 125.532 & 123.152 & 119.972 & 100.87 & 104.869 & 96.384 & 110.179 & 107.493 & 74.668 \\
\hline $12 / 10 / 076: 00$ & 125.535 & 123.145 & 119.965 & 100.853 & 104.85 & 96.365 & 110.179 & 107.475 & 74.651 \\
\hline $12 / 10 / 076: 30$ & 125.53 & 123.143 & 119.96 & 100.856 & 104.848 & 96.363 & 110.175 & 107.471 & 74.649 \\
\hline 12/10/07 7:00 & 125.53 & 124.927 & 121.109 & 100.841 & 104.841 & 96.358 & 110.177 & 107.469 & 74.642 \\
\hline 12/10/07 7:30 & 125.528 & 125.276 & 121.565 & 100.851 & 104.837 & 96.353 & 110.171 & 107.462 & 74.639 \\
\hline $12 / 10 / 078: 00$ & 127.3 & 125.44 & 121.744 & 101.604 & 105.26 & 96.912 & 110.712 & 108.671 & 74.684 \\
\hline $12 / 10 / 078: 30$ & 127.674 & 125.528 & 121.84 & 101.856 & 105.597 & 97.258 & 111.031 & 109.138 & 74.878 \\
\hline 12/10/07 9:00 & 127.835 & 125.587 & 121.905 & 101.973 & 105.76 & 97.423 & 111.176 & 109.32 & 75.029 \\
\hline 12/10/07 9:30 & 127.927 & 125.63 & 121.946 & 102.059 & 105.852 & 97.517 & 111.262 & 109.419 & 75.124 \\
\hline $12 / 10 / 07$ 10:00 & 127.988 & 125.66 & 121.978 & 102.102 & 105.911 & 97.574 & 111.318 & 109.481 & 75.184 \\
\hline 12/10/07 10:30 & 128.036 & 123.78 & 120.635 & 102.142 & 105.949 & 97.607 & 111.356 & 109.517 & 75.221 \\
\hline $12 / 10 / 0711: 00$ & 128.071 & 123.546 & 120.355 & 101.823 & 105.981 & 97.645 & 111.386 & 109.559 & 75.263 \\
\hline $12 / 10 / 07$ 11:30 & 126.166 & 123.406 & 120.229 & 101.339 & 105.439 & 96.957 & 110.717 & 108.162 & 75.174 \\
\hline $12 / 10 / 07$ 12:00 & 125.92 & 123.324 & 120.151 & 101.166 & 105.214 & 96.728 & 110.509 & 107.889 & 75.027 \\
\hline $12 / 10 / 07$ 12:30 & 125.809 & 123.26 & 120.106 & 101.054 & 105.079 & 96.598 & 110.408 & 107.739 & 74.903 \\
\hline $12 / 10 / 07$ 13:00 & 125.738 & 123.222 & 120.063 & 100.987 & 105.001 & 96.518 & 110.34 & 107.651 & 74.824 \\
\hline $12 / 10 / 07$ 13:30 & 125.695 & 123.195 & 120.033 & 100.929 & 104.942 & 96.457 & 110.301 & 107.586 & 74.76 \\
\hline $12 / 10 / 07$ 14:00 & 125.662 & 123.168 & 120.01 & 100.898 & 104.906 & 96.419 & 110.271 & 107.546 & 74.723 \\
\hline $12 / 10 / 0714: 30$ & 125.639 & 123.143 & 119.995 & 100.875 & 104.877 & 96.393 & 110.25 & 107.518 & 74.694 \\
\hline $12 / 10 / 07$ 15:00 & 125.62 & 123.124 & 119.98 & 100.844 & 104.854 & 96.372 & 110.233 & 107.491 & 74.669 \\
\hline $12 / 10 / 07$ 15:30 & 125.613 & 123.131 & 119.962 & 100.829 & 104.828 & 96.344 & 110.204 & 107.464 & 74.642 \\
\hline $12 / 10 / 07$ 16:00 & 125.601 & 123.111 & 119.945 & 100.82 & 104.81 & 96.325 & 110.195 & 107.442 & 74.624 \\
\hline $12 / 10 / 07$ 16:30 & 125.587 & 123.09 & 119.94 & 100.82 & 104.826 & 96.341 & 110.188 & 107.453 & 74.634 \\
\hline $12 / 10 / 07$ 17:00 & 125.584 & 123.09 & 119.927 & 100.801 & 104.803 & 96.318 & 110.188 & 107.433 & 74.611 \\
\hline $12 / 10 / 07$ 17:30 & 125.582 & 123.095 & 119.914 & 100.786 & 104.782 & 96.299 & 110.184 & 107.416 & 74.595 \\
\hline $12 / 10 / 07$ 18:00 & 125.572 & 123.07 & 119.909 & 100.786 & 104.782 & 96.296 & 110.179 & 107.411 & 74.593 \\
\hline $12 / 10 / 07$ 18:30 & 125.565 & 123.075 & 119.899 & 100.787 & 104.788 & 96.304 & 110.175 & 107.413 & 74.597 \\
\hline $12 / 10 / 07$ 19:00 & 125.568 & 123.09 & 119.879 & 100.765 & 104.763 & 96.278 & 110.175 & 107.391 & 74.57 \\
\hline 12/10/07 19:30 & 125.561 & 123.07 & 119.887 & 100.772 & 104.763 & 96.278 & 110.167 & 107.391 & 74.572 \\
\hline $12 / 10 / 0720: 00$ & 125.558 & 123.063 & 119.879 & 100.77 & 104.784 & 96.299 & 110.164 & 107.413 & 74.591 \\
\hline $12 / 10 / 0720: 30$ & 125.556 & 123.034 & 119.882 & 100.772 & 104.759 & 96.275 & 110.164 & 107.385 & 74.564 \\
\hline $12 / 10 / 0721: 00$ & 125.556 & 123.011 & 119.874 & 100.751 & 104.755 & 96.271 & 110.164 & 107.382 & 74.56 \\
\hline $12 / 10 / 0721: 30$ & 125.558 & 123.041 & 119.851 & 100.729 & 104.723 & 96.24 & 110.162 & 107.351 & 74.527 \\
\hline $12 / 10 / 0722: 00$ & 125.563 & 123.045 & 119.844 & 100.72 & 104.702 & 96.221 & 110.164 & 107.332 & 74.51 \\
\hline $12 / 10 / 0722: 30$ & 125.546 & 123.047 & 119.841 & 100.748 & 104.736 & 96.252 & 110.154 & 107.358 & 74.539 \\
\hline $12 / 10 / 07$ 23:00 & 125.546 & 123.029 & 119.836 & 100.744 & 104.738 & 96.252 & 110.154 & 107.367 & 74.541 \\
\hline 12/10/07 23:30 & 125.544 & 122.995 & 119.836 & 100.737 & 104.742 & 96.254 & 110.152 & 107.365 & 74.543 \\
\hline $12 / 11 / 070: 00$ & 125.549 & 123.029 & 119.821 & 100.717 & 104.721 & 96.237 & 110.156 & 107.349 & 74.653 \\
\hline $12 / 11 / 070: 30$ & 125.554 & 123.016 & 119.824 & 100.698 & 104.69 & 96.207 & 110.158 & 107.323 & 74.874 \\
\hline $12 / 11 / 071: 00$ & 125.537 & 123.007 & 119.819 & 100.736 & 104.727 & 96.24 & 110.149 & 107.345 & 74.702 \\
\hline 12/11/07 1:30 & 125.537 & 123.011 & 119.811 & 100.698 & 104.721 & 96.235 & 110.15 & 107.343 & 74.721 \\
\hline $12 / 11 / 072: 00$ & 125.539 & 122.997 & 119.816 & 100.78 & 104.702 & 96.214 & 110.15 & 107.32 & 74.678 \\
\hline $12 / 11 / 072: 30$ & 125.537 & 122.997 & 119.806 & 100.701 & 104.708 & 96.219 & 110.15 & 107.325 & 74.731 \\
\hline 12/11/07 3:00 & 125.542 & 123 & 119.788 & 100.715 & 104.687 & 96.2 & 110.15 & 107.307 & 74.698 \\
\hline 12/11/07 3:30 & 125.539 & 123 & 119.783 & 100.693 & 104.692 & 96.207 & 110.15 & 107.312 & 74.7 \\
\hline $12 / 11 / 074: 00$ & 125.537 & 122.993 & 119.783 & 100.67 & 104.702 & 96.216 & 110.149 & 107.325 & 74.707 \\
\hline $12 / 11 / 074: 30$ & 125.53 & 122.991 & 119.783 & 100.698 & 104.698 & 96.207 & 110.147 & 107.309 & 74.698 \\
\hline 12/11/07 5:00 & 125.532 & 123.011 & 119.766 & 100.696 & 104.7 & 96.214 & 110.147 & 107.32 & 74.704 \\
\hline $12 / 11 / 075: 30$ & 125.537 & 122.97 & 119.781 & 100.699 & 104.67 & 96.186 & 110.147 & 107.296 & 74.671 \\
\hline $12 / 11 / 076: 00$ & 125.528 & 122.972 & 119.763 & 100.815 & 104.708 & 96.223 & 110.145 & 107.334 & 74.715 \\
\hline $12 / 11 / 076: 30$ & 125.535 & 122.961 & 119.768 & 100.846 & 104.67 & 96.181 & 110.149 & 107.289 & 74.686 \\
\hline $12 / 11 / 07$ 7:00 & 125.53 & 123.025 & 119.743 & 100.891 & 104.675 & 96.193 & 110.145 & 107.298 & 74.699 \\
\hline $12 / 11 / 077: 30$ & 125.532 & 124.682 & 120.59 & 100.83 & 104.666 & 96.176 & 110.147 & 107.283 & 74.688 \\
\hline 12/11/07 8:00 & 125.511 & 125.17 & 121.288 & 100.908 & 104.729 & 96.24 & 110.13 & 107.34 & 74.769 \\
\hline $12 / 11 / 078: 30$ & 127.116 & 123.358 & 120.234 & 101.459 & 104.972 & 96.593 & 110.496 & 108.292 & 74.783 \\
\hline 12/11/07 9:00 & 127.525 & 123.247 & 120.134 & 101.421 & 105.353 & 97.084 & 110.896 & 108.996 & 74.971 \\
\hline $12 / 11 / 079: 30$ & 125.733 & 123.154 & 120.093 & 101.151 & 105.027 & 96.563 & 110.368 & 107.732 & 75.029 \\
\hline $12 / 11 / 07$ 10:00 & 125.601 & 123.115 & 120.056 & 101.061 & 104.927 & 96.457 & 110.254 & 107.577 & 74.965 \\
\hline $12 / 11 / 07 \quad 10: 30$ & 125.556 & 123.081 & 120.028 & 100.991 & 104.875 & 96.362 & 110.212 & 107.484 & 74.874 \\
\hline $12 / 11 / 0711: 00$ & 125.528 & 123.043 & 120.005 & 100.953 & 104.852 & 96.327 & 110.186 & 107.447 & 74.833 \\
\hline
\end{tabular}


TABLE S1.1 (Cont.)

\begin{tabular}{|c|c|c|c|c|c|c|c|c|c|c|}
\hline \multirow[b]{2}{*}{ Date and Time } & \multicolumn{10}{|c|}{ Depth from Top of Casing (ft) } \\
\hline & MW1D & MW2D & MW3D & MW4D & MW7 & MW9 & Oentrich & MW14D & MW15D & MW16D \\
\hline $12 / 11 / 0711: 30$ & 125.513 & 123.113 & 119.972 & 100.913 & 104.782 & 96.299 & 110.173 & 107.413 & & 74.798 \\
\hline $12 / 11 / 07$ 12:00 & 125.504 & 123.099 & 119.952 & 100.891 & 104.746 & 96.261 & 110.164 & 107.376 & & 74.757 \\
\hline 12/11/07 12:30 & 125.478 & 123.081 & 119.95 & 100.923 & 104.789 & 96.301 & 110.147 & 107.409 & & 74.787 \\
\hline $12 / 11 / 07$ 13:00 & 125.473 & 123.054 & 119.94 & 100.898 & 104.793 & 96.301 & 110.143 & 107.409 & & 74.785 \\
\hline $12 / 11 / 07$ 13:30 & 125.483 & 123.052 & 119.922 & 100.891 & 104.778 & 96.292 & 110.145 & 107.407 & & 74.773 \\
\hline $12 / 11 / 0714: 00$ & 125.478 & 123.061 & 119.907 & 100.87 & 104.749 & 96.264 & 110.149 & 107.374 & & 74.744 \\
\hline $12 / 11 / 0714: 30$ & 125.476 & 123.079 & 119.897 & 100.861 & 104.753 & 96.27 & 110.141 & 107.38 & & 74.752 \\
\hline $12 / 11 / 07$ 15:00 & 125.468 & 123.063 & 119.902 & 100.865 & 104.767 & 96.282 & 110.137 & 107.394 & & 74.764 \\
\hline 12/11/07 15:30 & 125.461 & 124.982 & 121.114 & 100.867 & 104.784 & 96.296 & 110.132 & 107.402 & & 74.773 \\
\hline $12 / 11 / 0716: 00$ & 125.466 & 125.256 & 121.419 & 100.877 & 104.753 & 96.261 & 110.134 & 107.371 & & 74.735 \\
\hline $12 / 11 / 07$ 16:30 & 127.378 & 125.419 & 121.55 & 101.477 & 105.26 & 96.898 & 110.746 & 108.795 & & 74.826 \\
\hline $12 / 11 / 0717: 00$ & 127.65 & 125.501 & 121.636 & 101.661 & 105.526 & 97.171 & 110.99 & 109.129 & & 75.017 \\
\hline $12 / 11 / 0717: 30$ & 127.776 & 125.56 & 121.691 & 101.785 & 105.7 & 97.345 & 111.102 & 109.306 & & 75.165 \\
\hline $12 / 11 / 07$ 18:00 & 127.856 & 125.621 & 121.724 & 101.863 & 105.776 & 97.423 & 111.179 & 109.393 & & 75.258 \\
\hline 12/11/07 18:30 & 127.906 & 125.655 & 121.754 & 101.911 & 105.84 & 97.487 & 111.226 & 109.455 & & 75.323 \\
\hline $12 / 11 / 07$ 19:00 & 127.939 & 125.685 & 121.782 & 101.949 & 105.905 & 97.553 & 111.262 & 109.519 & & 75.387 \\
\hline $12 / 11 / 07$ 19:30 & 127.965 & 125.723 & 121.898 & 101.969 & 105.938 & 97.586 & 111.296 & 109.557 & & 75.422 \\
\hline $12 / 11 / 0720: 00$ & 127.986 & 125.735 & 121.91 & 102.038 & 105.964 & 97.614 & 111.316 & 109.585 & & 75.449 \\
\hline $12 / 11 / 0720: 30$ & 127.995 & 125.741 & 121.92 & 102.04 & 106.01 & 97.659 & 111.337 & 109.63 & & 75.492 \\
\hline $12 / 11 / 0721: 00$ & 128.012 & 125.755 & 121.923 & 102.068 & 106.023 & 97.668 & 111.352 & 109.641 & & 75.505 \\
\hline $12 / 11 / 0721: 30$ & 128.019 & 125.764 & 121.943 & 102.099 & 106.031 & 97.678 & 111.362 & 109.647 & & 75.513 \\
\hline $12 / 11 / 0722: 00$ & 128.026 & 125.773 & 121.951 & 102.09 & 106.044 & 97.692 & 111.373 & 109.663 & & 75.529 \\
\hline 12/11/07 22:30 & 128.031 & 125.784 & 121.948 & 102.126 & 106.056 & 97.704 & 111.382 & 109.672 & & 75.542 \\
\hline $12 / 11 / 0723: 00$ & 128.036 & 124.029 & 120.925 & 102.224 & 106.065 & 97.713 & 111.393 & 109.683 & & 75.554 \\
\hline $12 / 11 / 0723: 30$ & 128.038 & 123.739 & 120.549 & 102.24 & 106.08 & 97.73 & 111.408 & 109.696 & & 75.571 \\
\hline 12/12/07 0:00 & 126.279 & 123.603 & 120.391 & 101.545 & 105.671 & 97.197 & 110.875 & 108.452 & & 75.529 \\
\hline $12 / 12 / 070: 30$ & 125.96 & 123.535 & 120.3 & 101.346 & 105.397 & 96.917 & 110.625 & 108.089 & & 75.376 \\
\hline $12 / 12 / 071: 00$ & 125.884 & 123.476 & 120.252 & 101.242 & 105.269 & 96.787 & 110.505 & 107.938 & & 75.261 \\
\hline 12/12/07 1:30 & 125.799 & 123.435 & 120.207 & 101.192 & 105.206 & 96.723 & 110.43 & 107.865 & & 75.194 \\
\hline $12 / 12 / 072: 00$ & 125.75 & 123.412 & 120.179 & 101.137 & 105.149 & 96.669 & 110.389 & 107.808 & & 75.134 \\
\hline $12 / 12 / 072: 30$ & 125.71 & 123.401 & 120.151 & 101.11 & 105.113 & 96.629 & 110.357 & 107.759 & & 75.091 \\
\hline $12 / 12 / 073: 00$ & 125.681 & 123.397 & 120.146 & 101.096 & 105.09 & 96.608 & 110.331 & 107.732 & & 75.041 \\
\hline 12/12/07 3:30 & 125.658 & 123.383 & 120.139 & 101.085 & 105.085 & 96.603 & 110.314 & 107.723 & & 75.025 \\
\hline $12 / 12 / 074: 00$ & 125.641 & 123.367 & 120.144 & 101.082 & 105.079 & 96.593 & 110.301 & 107.715 & & 75.01 \\
\hline 12/12/07 4:30 & 125.629 & 123.347 & 120.134 & 101.068 & 105.067 & 96.579 & 110.291 & 107.702 & & 75 \\
\hline $12 / 12 / 075: 00$ & 125.617 & 123.335 & 120.121 & 101.048 & 105.05 & 96.568 & 110.284 & 107.686 & & 74.99 \\
\hline 12/12/07 5:30 & 125.608 & 123.317 & 120.114 & 101.035 & 105.031 & 96.549 & 110.274 & 107.666 & & 74.984 \\
\hline 12/12/07 6:00 & 125.601 & 123.313 & 120.098 & 101.022 & 105.02 & 96.537 & 110.269 & 107.653 & & 74.973 \\
\hline $12 / 12 / 076: 30$ & 125.596 & 123.294 & 120.091 & 101.004 & 105.005 & 96.523 & 110.263 & 107.637 & & 74.948 \\
\hline 12/12/07 7:00 & 125.589 & 123.274 & 120.083 & 101.003 & 104.997 & 96.511 & 110.256 & 107.628 & & 74.934 \\
\hline 12/12/07 7:30 & 125.584 & 123.265 & 120.073 & 100.982 & 104.984 & 96.499 & 110.254 & 107.615 & & 74.915 \\
\hline $12 / 12 / 078: 00$ & 125.582 & 123.274 & 120.058 & 100.956 & 104.961 & 96.478 & 110.252 & 107.593 & & 74.888 \\
\hline 12/12/07 8:30 & 125.575 & 123.297 & 120.045 & 100.968 & 104.953 & 96.471 & 110.246 & 107.584 & & 74.872 \\
\hline 12/12/07 9:00 & 125.568 & 123.301 & 120.038 & 100.97 & 104.965 & 96.48 & 110.239 & 107.595 & & 74.864 \\
\hline $12 / 12 / 07$ 9:30 & 125.561 & 123.306 & 120.035 & 100.984 & 104.98 & 96.494 & 110.231 & 107.606 & & 74.859 \\
\hline $12 / 12 / 07$ 10:00 & 125.554 & 123.304 & 120.033 & 101.008 & 104.991 & 96.509 & 110.227 & 107.617 & & 74.853 \\
\hline 12/12/07 10:30 & 125.551 & 123.288 & 120.018 & 101.003 & 104.997 & 96.513 & 110.224 & 107.626 & & 74.849 \\
\hline $12 / 12 / 0711: 00$ & 125.549 & 123.27 & 120.025 & 100.985 & 104.993 & 96.506 & 110.22 & 107.619 & & 74.847 \\
\hline 12/12/07 11:30 & 125.546 & 123.258 & 120.023 & 100.97 & 104.974 & 96.49 & 110.218 & 107.604 & & 74.847 \\
\hline 12/12/07 12:00 & 125.546 & 123.236 & 120.028 & 100.953 & 104.963 & 96.476 & 110.218 & 107.588 & & 74.849 \\
\hline $12 / 12 / 07$ 12:30 & 125.544 & 123.208 & 120.028 & 100.932 & 104.942 & 96.459 & 110.218 & 107.573 & & 74.839 \\
\hline 12/12/07 13:00 & 125.546 & 123.183 & 120.018 & 100.91 & 104.925 & 96.438 & 110.216 & 107.555 & & 74.812 \\
\hline 12/12/07 13:30 & 125.544 & 123.158 & 120.008 & 100.891 & 104.9 & 96.414 & 110.214 & 107.529 & & 74.795 \\
\hline $12 / 12 / 07$ 14:00 & 125.544 & 123.145 & 119.99 & 100.872 & 104.873 & 96.388 & 110.214 & 107.502 & & 74.773 \\
\hline $12 / 12 / 07$ 14:30 & 125.542 & 123.136 & 119.97 & 100.863 & 104.848 & 96.362 & 110.205 & 107.478 & & 74.744 \\
\hline 12/12/07 15:00 & 125.542 & 123.124 & 119.957 & 100.858 & 104.835 & 96.351 & 110.201 & 107.464 & & 74.735 \\
\hline $12 / 12 / 07$ 15:30 & 125.537 & 123.118 & 119.947 & 100.853 & 104.828 & 96.341 & 110.196 & 107.453 & & 74.738 \\
\hline $12 / 12 / 07$ 16:00 & 125.535 & 123.109 & 119.935 & 100.848 & 104.816 & 96.329 & 110.192 & 107.442 & & 74.733 \\
\hline $12 / 12 / 0716: 30$ & 125.53 & 123.099 & 119.919 & 100.842 & 104.807 & 96.322 & 110.184 & 107.432 & & 74.731 \\
\hline $12 / 12 / 07$ 17:00 & 125.53 & 123.088 & 119.917 & 100.837 & 104.799 & 96.313 & 110.179 & 107.425 & & 74.717 \\
\hline $12 / 12 / 07$ 17:30 & 125.525 & 123.077 & 119.902 & 100.791 & 104.789 & 96.306 & 110.177 & 107.418 & & 74.711 \\
\hline $12 / 12 / 07$ 18:00 & 125.525 & 123.072 & 119.892 & 100.775 & 104.778 & 96.294 & 110.171 & 107.405 & & 74.702 \\
\hline $12 / 12 / 0718: 30$ & 125.525 & 123.063 & 119.879 & 100.768 & 104.769 & 96.282 & 110.164 & 107.394 & & 74.688 \\
\hline 12/12/07 19:00 & 125.523 & 123.05 & 119.872 & 100.763 & 104.761 & 96.278 & 110.158 & 107.387 & & 74.684 \\
\hline 12/12/07 19:30 & 125.52 & 123.043 & 119.862 & 100.755 & 104.753 & 96.268 & 110.154 & 107.378 & & 74.682 \\
\hline $12 / 12 / 0720: 00$ & 125.52 & 123.038 & 119.851 & 100.744 & 104.74 & 96.256 & 110.128 & 107.367 & & 74.669 \\
\hline
\end{tabular}


TABLE S1.1 (Cont.)

Depth from Top of Casing (ft)

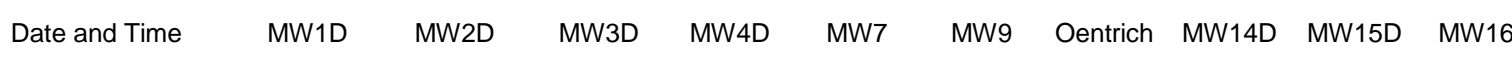

\begin{tabular}{|c|c|c|c|c|c|c|c|c|c|}
\hline $12 / 12 / 0720: 30$ & 125.52 & 123.036 & 119.841 & 100.737 & 104.732 & 96.249 & 110.12 & 107.358 & 74.659 \\
\hline 12/12/07 21:00 & 125.516 & 123.031 & 119.829 & 100.739 & 104.732 & 96.247 & 110.117 & 107.356 & 74.649 \\
\hline $12 / 12 / 0721: 30$ & 125.516 & 123.031 & 119.824 & 100.73 & 104.727 & 96.245 & 110.113 & 107.351 & 74.642 \\
\hline $12 / 12 / 0722: 00$ & 125.516 & 123.027 & 119.821 & 100.727 & 104.727 & 96.235 & 110.109 & 107.347 & 74.63 \\
\hline 12/12/07 22:30 & 125.513 & 123.027 & 119.814 & 100.729 & 104.729 & 96.24 & 110.111 & 107.349 & 74.626 \\
\hline $12 / 12 / 0723: 00$ & 125.513 & 123.027 & 119.849 & 100.718 & 104.723 & 96.235 & 110.111 & 107.345 & 74.616 \\
\hline $12 / 12 / 0723: 30$ & 125.513 & 123.018 & 119.844 & 100.724 & 104.719 & 96.233 & 110.109 & 107.34 & 74.609 \\
\hline 12/13/07 0:00 & 125.513 & 123.009 & 119.841 & 100.722 & 104.721 & 96.233 & 110.109 & 107.343 & 74.601 \\
\hline $12 / 13 / 070: 30$ & 125.513 & 122.993 & 119.841 & 100.713 & 104.711 & 96.228 & 110.109 & 107.336 & 74.595 \\
\hline $12 / 13 / 071: 00$ & 125.513 & 122.988 & 119.831 & 100.701 & 104.704 & 96.219 & 110.107 & 107.327 & 74.576 \\
\hline 12/13/07 1:30 & 125.516 & 122.986 & 119.821 & 100.684 & 104.685 & 96.202 & 110.105 & 107.309 & 74.547 \\
\hline $12 / 13 / 072: 00$ & 125.511 & 122.986 & 119.811 & 100.684 & 104.683 & 96.197 & 110.105 & 107.307 & 74.547 \\
\hline $12 / 13 / 072: 30$ & 125.511 & 122.986 & 119.799 & 100.681 & 104.679 & 96.193 & 110.104 & 107.301 & 74.546 \\
\hline 12/13/07 3:00 & 125.506 & 122.984 & 119.791 & 100.681 & 104.677 & 96.193 & 110.102 & 107.298 & 74.541 \\
\hline 12/13/07 3:30 & 125.502 & 122.97 & 119.778 & 100.682 & 104.681 & 96.195 & 110.1 & 107.303 & 74.542 \\
\hline $12 / 13 / 074: 00$ & 125.499 & 122.975 & 119.809 & 100.679 & 104.675 & 96.19 & 110.1 & 107.296 & 74.537 \\
\hline 12/13/07 4:30 & 125.497 & 122.97 & 119.804 & 100.667 & 104.662 & 96.176 & 110.1 & 107.283 & 74.533 \\
\hline $12 / 13 / 075: 00$ & 125.492 & 122.975 & 119.799 & 100.67 & 104.666 & 96.181 & 110.096 & 107.285 & 74.444 \\
\hline $12 / 13 / 075: 30$ & 125.492 & 122.977 & 119.786 & 100.67 & 104.666 & 96.179 & 110.098 & 107.285 & 74.444 \\
\hline $12 / 13 / 076: 00$ & 125.487 & 122.991 & 119.799 & 100.675 & 104.671 & 96.183 & 110.094 & 107.287 & 74.446 \\
\hline $12 / 13 / 076: 30$ & 125.485 & 123.013 & 119.804 & 100.677 & 104.673 & 96.188 & 110.094 & 107.289 & 74.446 \\
\hline $12 / 13 / 077: 00$ & 125.48 & 123.02 & 119.816 & 100.698 & 104.694 & 96.207 & 110.092 & 107.312 & 74.467 \\
\hline $12 / 13 / 077: 30$ & 125.478 & 124.997 & 121.258 & 100.718 & 104.711 & 96.226 & 110.09 & 107.327 & 74.481 \\
\hline 12/13/07 8:00 & 125.478 & 125.227 & 121.547 & 101.163 & 104.717 & 96.233 & 110.089 & 107.334 & 74.485 \\
\hline $12 / 13 / 078: 30$ & 127.116 & 125.369 & 121.696 & 101.608 & 105.321 & 96.98 & 110.819 & 108.822 & 74.611 \\
\hline 12/13/07 9:00 & 127.374 & 125.451 & 121.774 & 101.787 & 105.547 & 97.211 & 111.031 & 109.098 & 74.789 \\
\hline $12 / 13 / 079: 30$ & 127.501 & 125.521 & 121.82 & 101.897 & 105.688 & 97.348 & 111.138 & 109.244 & 74.924 \\
\hline $12 / 13 / 07 \quad 10: 00$ & 127.579 & 125.571 & 121.872 & 101.98 & 105.77 & 97.433 & 111.208 & 109.335 & 75.008 \\
\hline $12 / 13 / 07 \quad 10: 30$ & 127.627 & 125.612 & 121.893 & 102.04 & 105.842 & 97.506 & 111.253 & 109.41 & 75.081 \\
\hline $12 / 13 / 07 \quad 11: 00$ & 127.662 & 125.644 & 121.915 & 102.087 & 105.896 & 97.56 & 111.287 & 109.466 & 75.139 \\
\hline $12 / 13 / 07$ 11:30 & 127.693 & 125.669 & 121.941 & 102.128 & 105.938 & 97.602 & 111.313 & 109.508 & 75.18 \\
\hline $12 / 13 / 07$ 12:00 & 127.716 & 125.68 & 121.961 & 102.157 & 105.966 & 97.631 & 111.335 & 109.539 & 75.209 \\
\hline $12 / 13 / 07 \quad 12: 30$ & 127.79 & 125.682 & 121.973 & 102.174 & 105.993 & 97.657 & 111.354 & 109.563 & 75.234 \\
\hline $12 / 13 / 07$ 13:00 & 127.896 & 125.691 & 121.983 & 102.181 & 106.004 & 97.668 & 111.371 & 109.579 & 75.248 \\
\hline $12 / 13 / 07$ 13:30 & 127.908 & 125.696 & 121.993 & 102.186 & 106.006 & 97.671 & 111.384 & 109.581 & 75.261 \\
\hline $12 / 13 / 07 \quad 14: 00$ & 127.92 & 125.703 & 121.996 & 102.193 & 106.014 & 97.678 & 111.394 & 109.588 & 75.277 \\
\hline $12 / 13 / 0714: 30$ & 127.929 & 123.823 & 120.625 & 102.202 & 106.018 & 97.683 & 111.405 & 109.594 & 75.287 \\
\hline $12 / 13 / 07$ 15:00 & 127.936 & 123.587 & 120.345 & 101.83 & 106.027 & 97.69 & 111.411 & 109.601 & 75.298 \\
\hline $12 / 13 / 07$ 15:30 & 126.289 & 123.474 & 120.204 & 101.37 & 105.471 & 96.994 & 110.719 & 108.202 & 75.226 \\
\hline $12 / 13 / 07$ 16:00 & 126.076 & 123.403 & 120.126 & 101.203 & 105.246 & 96.766 & 110.505 & 107.927 & 75.099 \\
\hline $12 / 13 / 07 \quad 16: 30$ & 125.946 & 123.358 & 120.071 & 101.116 & 105.14 & 96.659 & 110.396 & 107.808 & 75.017 \\
\hline $12 / 13 / 07$ 17:00 & 125.863 & 123.331 & 120.035 & 101.065 & 105.077 & 96.593 & 110.331 & 107.728 & 74.963 \\
\hline $12 / 13 / 07$ 17:30 & 125.811 & 123.313 & 120.01 & 101.029 & 105.033 & 96.551 & 110.288 & 107.681 & 74.926 \\
\hline $12 / 13 / 07$ 18:00 & 125.769 & 123.294 & 119.972 & 101.01 & 105.008 & 96.525 & 110.257 & 107.65 & 74.893 \\
\hline $12 / 13 / 07$ 18:30 & 125.74 & 123.279 & 119.96 & 100.994 & 104.993 & 96.511 & 110.242 & 107.63 & 74.868 \\
\hline $12 / 13 / 07$ 19:00 & 125.724 & 123.276 & 119.945 & 100.977 & 104.976 & 96.494 & 110.229 & 107.615 & 74.852 \\
\hline $12 / 13 / 07$ 19:30 & 125.707 & 123.272 & 119.935 & 100.968 & 104.963 & 96.483 & 110.216 & 107.599 & 74.812 \\
\hline $12 / 13 / 0720: 00$ & 125.693 & 123.263 & 119.937 & 100.968 & 104.959 & 96.478 & 110.207 & 107.593 & 74.8 \\
\hline $12 / 13 / 0720: 30$ & 125.681 & 123.26 & 119.932 & 100.963 & 104.957 & 96.476 & 110.211 & 107.591 & 74.789 \\
\hline $12 / 13 / 0721: 00$ & 125.674 & 123.251 & 119.922 & 100.958 & 104.949 & 96.466 & 110.211 & 107.582 & 74.779 \\
\hline $12 / 13 / 0721: 30$ & 125.667 & 123.247 & 119.925 & 100.949 & 104.947 & 96.464 & 110.205 & 107.577 & 74.773 \\
\hline $12 / 13 / 0722: 00$ & 125.66 & 123.242 & 119.922 & 100.946 & 104.938 & 96.457 & 110.201 & 107.571 & 74.764 \\
\hline $12 / 13 / 0722: 30$ & 125.655 & 123.247 & 119.912 & 100.941 & 104.932 & 96.452 & 110.196 & 107.564 & 74.759 \\
\hline $12 / 13 / 07$ 23:00 & 125.65 & 123.247 & 119.914 & 100.941 & 104.928 & 96.447 & 110.192 & 107.56 & 74.75 \\
\hline 12/13/07 23:30 & 125.643 & 123.245 & 119.914 & 100.942 & 104.936 & 96.454 & 110.188 & 107.564 & 74.742 \\
\hline $12 / 14 / 070: 00$ & 125.639 & 123.24 & 119.909 & 100.946 & 104.936 & 96.455 & 110.184 & 107.568 & 74.735 \\
\hline $12 / 14 / 070: 30$ & 125.636 & 123.24 & 119.907 & 100.941 & 104.932 & 96.452 & 110.184 & 107.562 & 74.727 \\
\hline $12 / 14 / 071: 00$ & 125.632 & 123.24 & 119.907 & 100.941 & 104.93 & 96.447 & 110.182 & 107.56 & 74.721 \\
\hline 12/14/07 1:30 & 125.629 & 123.24 & 119.909 & 100.939 & 104.928 & 96.445 & 110.181 & 107.557 & 74.715 \\
\hline $12 / 14 / 072: 00$ & 125.624 & 123.24 & 119.907 & 100.932 & 104.93 & 96.447 & 110.177 & 107.557 & 74.709 \\
\hline $12 / 14 / 072: 30$ & 125.62 & 123.249 & 119.899 & 100.937 & 104.93 & 96.45 & 110.175 & 107.555 & 74.702 \\
\hline 12/14/07 3:00 & 125.615 & 123.247 & 119.904 & 100.944 & 104.934 & 96.45 & 110.171 & 107.557 & 74.698 \\
\hline $12 / 14 / 073: 30$ & 125.613 & 123.24 & 119.904 & 100.946 & 104.94 & 96.459 & 110.169 & 107.566 & 74.692 \\
\hline $12 / 14 / 074: 00$ & 125.608 & 123.236 & 119.904 & 100.946 & 104.94 & 96.459 & 110.169 & 107.564 & 74.688 \\
\hline $12 / 14 / 074: 30$ & 125.606 & 123.222 & 119.907 & 100.937 & 104.932 & 96.452 & 110.168 & 107.56 & 74.684 \\
\hline $12 / 14 / 075: 00$ & 125.601 & 123.213 & 119.907 & 100.932 & 104.928 & 96.45 & 110.164 & 107.555 & 74.682 \\
\hline
\end{tabular}


TABLE S1.1 (Cont.)

Depth from Top of Casing (ft)

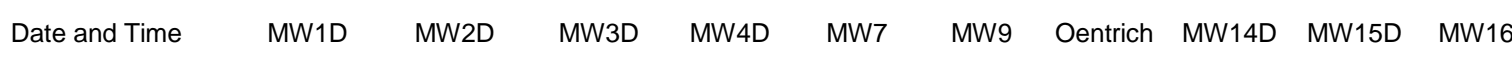

\begin{tabular}{|c|c|c|c|c|c|c|c|c|c|}
\hline $12 / 14 / 075: 30$ & 125.603 & 123.217 & 119.894 & 100.922 & 104.913 & 96.431 & 110.164 & 107.542 & 74.68 \\
\hline $12 / 14 / 076: 00$ & 125.596 & 123.215 & 119.897 & 100.915 & 104.907 & 96.426 & 110.16 & 107.533 & 74.675 \\
\hline $12 / 14 / 076: 30$ & 125.591 & 123.222 & 119.892 & 100.915 & 104.913 & 96.431 & 110.154 & 107.537 & 74.671 \\
\hline $12 / 14 / 077: 00$ & 125.589 & 124.766 & 119.957 & 100.922 & 104.909 & 96.431 & 110.153 & 107.535 & 74.665 \\
\hline $12 / 14 / 077: 30$ & 125.584 & 125.27 & 121.31 & 100.923 & 104.919 & 96.436 & 110.149 & 107.54 & 74.663 \\
\hline $12 / 14 / 078: 00$ & 127.038 & 125.437 & 121.512 & 101.509 & 105.09 & 96.702 & 110.432 & 108.277 & 74.661 \\
\hline $12 / 14 / 078: 30$ & 127.627 & 125.537 & 121.628 & 101.802 & 105.562 & 97.209 & 110.896 & 109.131 & 74.791 \\
\hline 12/14/07 9:00 & 127.809 & 125.598 & 121.696 & 101.938 & 105.736 & 97.388 & 111.057 & 109.331 & 74.917 \\
\hline 12/14/07 9:30 & 127.913 & 125.644 & 121.736 & 102.014 & 105.833 & 97.482 & 111.15 & 109.435 & 75.01 \\
\hline $12 / 14 / 07 \quad 10: 00$ & 127.981 & 123.995 & 120.839 & 102.076 & 105.896 & 97.543 & 111.209 & 109.501 & 75.077 \\
\hline $12 / 14 / 07 \quad 10: 30$ & 128.024 & 123.653 & 120.365 & 102.114 & 105.939 & 97.588 & 111.249 & 109.548 & 75.13 \\
\hline $12 / 14 / 0711: 00$ & 126.383 & 123.508 & 120.207 & 101.48 & 105.635 & 97.171 & 110.821 & 108.468 & 75.155 \\
\hline $12 / 14 / 0711: 30$ & 125.996 & 123.419 & 120.124 & 101.263 & 105.323 & 96.843 & 110.509 & 108.002 & 75.064 \\
\hline $12 / 14 / 07$ 12:00 & 125.849 & 123.358 & 120.076 & 101.154 & 105.184 & 96.702 & 110.385 & 107.847 & 74.984 \\
\hline $12 / 14 / 07$ 12:30 & 125.769 & 123.315 & 120.04 & 101.089 & 105.1 & 96.617 & 110.316 & 107.75 & 74.922 \\
\hline $12 / 14 / 07$ 13:00 & 125.719 & 123.478 & 120.015 & 101.027 & 105.039 & 96.556 & 110.273 & 107.681 & 74.884 \\
\hline $12 / 14 / 07$ 13:30 & 125.686 & 125.211 & 121.323 & 100.989 & 105.001 & 96.52 & 110.243 & 107.642 & 74.864 \\
\hline $12 / 14 / 0714: 00$ & 125.662 & 125.394 & 121.542 & 101.402 & 104.957 & 96.476 & 110.224 & 107.595 & 74.851 \\
\hline $12 / 14 / 0714: 30$ & 127.634 & 125.485 & 121.651 & 101.749 & 105.505 & 97.152 & 110.9 & 109.072 & 74.926 \\
\hline $12 / 14 / 07$ 15:00 & 127.844 & 125.544 & 121.71 & 101.89 & 105.69 & 97.341 & 111.074 & 109.284 & 75.043 \\
\hline $12 / 14 / 0715: 30$ & 127.948 & 123.678 & 120.497 & 101.962 & 105.781 & 97.433 & 111.172 & 109.384 & 75.126 \\
\hline $12 / 14 / 07 \quad 16: 00$ & 128.012 & 123.46 & 120.297 & 101.659 & 105.84 & 97.492 & 111.23 & 109.448 & 75.174 \\
\hline $12 / 14 / 0716: 30$ & 126.121 & 123.356 & 120.182 & 101.253 & 105.34 & 96.862 & 110.616 & 108.058 & 75.12 \\
\hline $12 / 14 / 0717: 00$ & 125.906 & 123.292 & 120.106 & 101.091 & 105.132 & 96.652 & 110.436 & 107.814 & 74.922 \\
\hline $12 / 14 / 07$ 17:30 & 125.799 & 123.265 & 120.056 & 101.011 & 105.033 & 96.551 & 110.346 & 107.686 & 74.826 \\
\hline $12 / 14 / 0718: 00$ & 125.738 & 123.242 & 120.03 & 100.965 & 104.972 & 96.49 & 110.295 & 107.619 & 74.764 \\
\hline $12 / 14 / 07$ 18:30 & 125.693 & 123.24 & 120.003 & 100.939 & 104.947 & 96.469 & 110.257 & 107.588 & 74.735 \\
\hline $12 / 14 / 07$ 19:00 & 125.665 & 123.236 & 119.977 & 100.93 & 104.925 & 96.443 & 110.233 & 107.562 & 74.709 \\
\hline $12 / 14 / 07$ 19:30 & 125.643 & 123.231 & 119.972 & 100.93 & 104.925 & 96.44 & 110.214 & 107.555 & 74.702 \\
\hline $12 / 14 / 0720: 00$ & 125.629 & 123.215 & 119.965 & 100.93 & 104.923 & 96.443 & 110.201 & 107.555 & 74.702 \\
\hline $12 / 14 / 0720: 30$ & 125.617 & 123.183 & 119.96 & 100.922 & 104.917 & 96.436 & 110.19 & 107.549 & 74.692 \\
\hline $12 / 14 / 0721: 00$ & 125.608 & 123.168 & 119.95 & 100.899 & 104.906 & 96.426 & 110.184 & 107.537 & 74.68 \\
\hline $12 / 14 / 0721: 30$ & 125.606 & 123.165 & 119.937 & 100.872 & 104.871 & 96.391 & 110.181 & 107.504 & 74.644 \\
\hline $12 / 14 / 0722: 00$ & 125.601 & 123.143 & 119.935 & 100.861 & 104.856 & 96.379 & 110.179 & 107.491 & 74.632 \\
\hline $12 / 14 / 0722: 30$ & 125.594 & 123.143 & 119.917 & 100.855 & 104.856 & 96.374 & 110.173 & 107.487 & 74.63 \\
\hline $12 / 14 / 0723: 00$ & 125.591 & 123.14 & 119.907 & 100.844 & 104.837 & 96.355 & 110.171 & 107.469 & 74.611 \\
\hline $12 / 14 / 0723: 30$ & 125.584 & 123.12 & 119.911 & 100.841 & 104.837 & 96.358 & 110.165 & 107.469 & 74.616 \\
\hline $12 / 15 / 07$ 0:00 & 125.577 & 123.106 & 119.912 & 100.832 & 104.837 & 96.355 & 110.16 & 107.467 & 74.611 \\
\hline $12 / 15 / 070: 30$ & 125.58 & 123.109 & 119.906 & 100.81 & 104.81 & 96.329 & 110.158 & 107.444 & 74.587 \\
\hline 12/15/07 1:00 & 125.575 & 123.109 & 119.899 & 100.808 & 104.797 & 96.315 & 110.152 & 107.427 & 74.572 \\
\hline 12/15/07 1:30 & 125.565 & 123.104 & 119.907 & 100.805 & 104.801 & 96.318 & 110.147 & 107.425 & 74.568 \\
\hline $12 / 15 / 072: 00$ & 125.563 & 123.104 & 119.894 & 100.803 & 104.803 & 96.32 & 110.145 & 107.429 & 74.572 \\
\hline $12 / 15 / 07$ 2:30 & 125.561 & 123.104 & 119.884 & 100.798 & 104.795 & 96.315 & 110.143 & 107.422 & 74.566 \\
\hline 12/15/07 3:00 & 125.556 & 123.102 & 119.877 & 100.798 & 104.797 & 96.313 & 110.139 & 107.422 & 74.566 \\
\hline $12 / 15 / 073: 30$ & 125.554 & 123.095 & 119.869 & 100.803 & 104.799 & 96.315 & 110.137 & 107.422 & 74.566 \\
\hline $12 / 15 / 074: 00$ & 125.551 & 123.081 & 119.867 & 100.799 & 104.797 & 96.313 & 110.135 & 107.42 & 74.564 \\
\hline $12 / 15 / 074: 30$ & 125.551 & 123.061 & 119.864 & 100.786 & 104.788 & 96.306 & 110.135 & 107.413 & 74.556 \\
\hline $12 / 15 / 07$ 5:00 & 125.549 & 123.047 & 119.876 & 100.774 & 104.776 & 96.294 & 110.132 & 107.402 & 74.541 \\
\hline $12 / 15 / 075: 30$ & 125.549 & 123.031 & 119.869 & 100.753 & 104.755 & 96.273 & 110.128 & 107.38 & 74.523 \\
\hline $12 / 15 / 076: 00$ & 125.544 & 123.027 & 119.864 & 100.743 & 104.742 & 96.259 & 110.12 & 107.363 & 74.508 \\
\hline $12 / 15 / 076: 30$ & 125.542 & 123.027 & 119.849 & 100.729 & 104.727 & 96.245 & 110.119 & 107.351 & 74.496 \\
\hline $12 / 15 / 07$ 7:00 & 125.537 & 123.027 & 119.844 & 100.729 & 104.725 & 96.24 & 110.111 & 107.345 & 74.492 \\
\hline $12 / 15 / 07 \quad 7: 30$ & 125.532 & 124.562 & 119.892 & 100.725 & 104.725 & 96.24 & 110.109 & 107.345 & 74.489 \\
\hline $12 / 15 / 078: 00$ & 125.53 & 125.088 & 121.371 & 100.732 & 104.721 & 96.237 & 110.107 & 107.343 & 74.49 \\
\hline $12 / 15 / 07$ 8:30 & 127.003 & 125.279 & 121.578 & 101.366 & 104.923 & 96.542 & 110.425 & 108.091 & 74.498 \\
\hline 12/15/07 9:00 & 127.629 & 125.381 & 121.686 & 101.673 & 105.41 & 97.07 & 110.907 & 108.937 & 74.675 \\
\hline 12/15/07 9:30 & 127.818 & 125.444 & 121.754 & 101.823 & 105.601 & 97.263 & 111.076 & 109.151 & 74.845 \\
\hline $12 / 15 / 07$ 10:00 & 127.927 & 125.49 & 121.799 & 101.906 & 105.705 & 97.369 & 111.17 & 109.267 & 74.948 \\
\hline $12 / 15 / 07$ 10:30 & 127.988 & 125.519 & 121.835 & 101.964 & 105.77 & 97.433 & 111.234 & 109.335 & 75.015 \\
\hline $12 / 15 / 0711: 00$ & 128.036 & 125.535 & 121.857 & 102 & 105.814 & 97.48 & 111.275 & 109.384 & 75.064 \\
\hline $12 / 15 / 0711: 30$ & 128.069 & 125.546 & 121.875 & 102.023 & 105.842 & 97.508 & 111.309 & 109.415 & 75.095 \\
\hline $12 / 15 / 07$ 12:00 & 128.095 & 124.251 & 121.887 & 102.04 & 105.858 & 97.522 & 111.333 & 109.43 & 75.114 \\
\hline $12 / 15 / 07$ 12:30 & 128.114 & 123.569 & 120.423 & 102.045 & 105.871 & 97.536 & 111.352 & 109.446 & 75.128 \\
\hline $12 / 15 / 07$ 13:00 & 127.029 & 123.367 & 120.198 & 101.471 & 105.785 & 97.409 & 111.27 & 109.196 & 75.124 \\
\hline $12 / 15 / 07$ 13:30 & 126.116 & 123.256 & 120.088 & 101.142 & 105.227 & 96.749 & 110.599 & 107.934 & 74.975 \\
\hline $12 / 15 / 0714: 00$ & 125.913 & 123.181 & 120.02 & 100.991 & 105.035 & 96.554 & 110.411 & 107.702 & 74.826 \\
\hline
\end{tabular}


TABLE S1.1 (Cont.)

\begin{tabular}{|c|c|c|c|c|c|c|c|c|c|c|}
\hline \multirow[b]{2}{*}{ Date and Time } & \multicolumn{10}{|c|}{ Depth from Top of Casing (ft) } \\
\hline & MW1D & MW2D & MW3D & MW4D & MW7 & MW9 & Oentrich & MW14D & MW15D & MW16D \\
\hline $12 / 15 / 07$ 14:30 & 125.804 & 123.133 & 119.975 & 100.901 & 104.928 & 96.45 & 110.316 & 107.582 & & 74.721 \\
\hline $12 / 15 / 07$ 15:00 & 125.738 & 123.111 & 119.94 & 100.844 & 104.86 & 96.379 & 110.259 & 107.506 & & 74.649 \\
\hline 12/15/07 15:30 & 125.693 & 123.088 & 119.919 & 100.808 & 104.816 & 96.334 & 110.218 & 107.456 & & 74.601 \\
\hline $12 / 15 / 07$ 16:00 & 125.66 & 123.079 & 119.896 & 100.789 & 104.789 & 96.308 & 110.192 & 107.425 & & 74.572 \\
\hline $12 / 15 / 07$ 16:30 & 125.639 & 124.823 & 120.89 & 100.774 & 104.772 & 96.292 & 110.173 & 107.407 & & 74.549 \\
\hline $12 / 15 / 07$ 17:00 & 125.62 & 125.154 & 121.34 & 100.765 & 104.767 & 96.287 & 110.156 & 107.398 & & 74.543 \\
\hline $12 / 15 / 07$ 17:30 & 127.362 & 125.295 & 121.5 & 101.44 & 105.117 & 96.756 & 110.627 & 108.565 & & 74.551 \\
\hline $12 / 15 / 07$ 18:00 & 127.731 & 125.381 & 121.583 & 101.67 & 105.443 & 97.093 & 110.941 & 109.027 & & 74.738 \\
\hline 12/15/07 18:30 & 127.877 & 125.431 & 121.637 & 101.78 & 105.583 & 97.235 & 111.078 & 109.182 & & 74.868 \\
\hline $12 / 15 / 07$ 19:00 & 127.955 & 125.469 & 121.676 & 101.854 & 105.673 & 97.324 & 111.151 & 109.278 & & 74.959 \\
\hline $12 / 15 / 07$ 19:30 & 128.01 & 123.777 & 120.7 & 101.9 & 105.726 & 97.378 & 111.202 & 109.335 & & 75.017 \\
\hline 12/15/07 20:00 & 128.043 & 123.458 & 120.272 & 101.935 & 105.766 & 97.418 & 111.236 & 109.375 & & 75.056 \\
\hline $12 / 15 / 0720: 30$ & 126.357 & 123.324 & 120.111 & 101.272 & 105.422 & 96.952 & 110.768 & 108.235 & & 75.05 \\
\hline $12 / 15 / 0721: 00$ & 125.998 & 123.247 & 120.027 & 101.072 & 105.125 & 96.643 & 110.479 & 107.814 & & 74.901 \\
\hline 12/15/07 21:30 & 125.854 & 123.202 & 119.969 & 100.968 & 104.999 & 96.516 & 110.357 & 107.655 & & 74.791 \\
\hline $12 / 15 / 0722: 00$ & 125.771 & 123.168 & 119.934 & 100.91 & 104.925 & 96.445 & 110.288 & 107.573 & & 74.715 \\
\hline $12 / 15 / 0722: 30$ & 125.717 & 123.147 & 119.909 & 100.873 & 104.883 & 96.398 & 110.241 & 107.522 & & 74.663 \\
\hline $12 / 15 / 0723: 00$ & 125.684 & 123.122 & 119.896 & 100.844 & 104.848 & 96.367 & 110.214 & 107.487 & & 74.626 \\
\hline $12 / 15 / 0723: 30$ & 125.658 & 123.111 & 119.876 & 100.827 & 104.833 & 96.348 & 110.194 & 107.465 & & 74.605 \\
\hline $12 / 16 / 07$ 0:00 & 125.643 & 123.104 & 119.866 & 100.81 & 104.807 & 96.327 & 110.181 & 107.44 & & 74.58 \\
\hline 12/16/07 0:30 & 125.629 & 123.093 & 119.858 & 100.798 & 104.797 & 96.315 & 110.167 & 107.429 & & 74.57 \\
\hline $12 / 16 / 071: 00$ & 125.615 & 123.088 & 119.848 & 100.793 & 104.789 & 96.311 & 110.16 & 107.42 & & 74.56 \\
\hline 12/16/07 1:30 & 125.61 & 123.081 & 119.841 & 100.787 & 104.78 & 96.299 & 110.154 & 107.411 & & 74.549 \\
\hline 12/16/07 2:00 & 125.601 & 123.077 & 119.836 & 100.779 & 104.776 & 96.296 & 110.147 & 107.405 & & 74.543 \\
\hline 12/16/07 2:30 & 125.594 & 123.081 & 119.831 & 100.777 & 104.769 & 96.287 & 110.143 & 107.398 & & 74.537 \\
\hline 12/16/07 3:00 & 125.589 & 123.086 & 119.858 & 100.775 & 104.767 & 96.285 & 110.139 & 107.394 & & 74.533 \\
\hline 12/16/07 3:30 & 125.584 & 123.084 & 119.853 & 100.782 & 104.774 & 96.289 & 110.134 & 107.398 & & 74.539 \\
\hline $12 / 16 / 074: 00$ & 125.58 & 123.075 & 119.853 & 100.784 & 104.776 & 96.292 & 110.13 & 107.402 & & 74.541 \\
\hline 12/16/07 4:30 & 125.577 & 123.079 & 119.871 & 100.777 & 104.774 & 96.292 & 110.128 & 107.398 & & 74.537 \\
\hline $12 / 16 / 075: 00$ & 125.575 & 123.077 & 119.864 & 100.775 & 104.767 & 96.285 & 110.126 & 107.392 & & 74.531 \\
\hline $12 / 16 / 075: 30$ & 125.568 & 123.065 & 119.863 & 100.78 & 104.774 & 96.292 & 110.122 & 107.398 & & 74.535 \\
\hline $12 / 16 / 076: 00$ & 125.565 & 123.072 & 119.851 & 100.775 & 104.774 & 96.292 & 110.119 & 107.396 & & 74.535 \\
\hline $12 / 16 / 076: 30$ & 125.563 & 123.075 & 119.856 & 100.768 & 104.757 & 96.275 & 110.119 & 107.383 & & 74.52 \\
\hline $12 / 16 / 07$ 7:00 & 125.556 & 123.072 & 119.851 & 100.774 & 104.769 & 96.289 & 110.113 & 107.387 & & 74.527 \\
\hline 12/16/07 7:30 & 125.554 & 123.077 & 119.849 & 100.774 & 104.77 & 96.29 & 110.111 & 107.391 & & 74.531 \\
\hline 12/16/07 8:00 & 125.551 & 124.855 & 120.876 & 100.774 & 104.772 & 96.287 & 110.109 & 107.389 & & 74.529 \\
\hline 12/16/07 8:30 & 125.549 & 125.197 & 121.332 & 100.787 & 104.77 & 96.29 & 110.107 & 107.391 & & 74.529 \\
\hline 12/16/07 9:00 & 127.319 & 125.344 & 121.5 & 101.475 & 105.145 & 96.787 & 110.599 & 108.596 & & 74.558 \\
\hline 12/16/07 9:30 & 127.69 & 125.433 & 121.588 & 101.709 & 105.482 & 97.131 & 110.911 & 109.067 & & 74.758 \\
\hline $12 / 16 / 07$ 10:00 & 127.846 & 125.485 & 121.653 & 101.83 & 105.627 & 97.279 & 111.048 & 109.229 & & 74.895 \\
\hline 12/16/07 10:30 & 127.936 & 125.517 & 121.694 & 101.907 & 105.722 & 97.371 & 111.132 & 109.329 & & 74.99 \\
\hline $12 / 16 / 0711: 00$ & 127.993 & 125.56 & 121.71 & 101.949 & 105.776 & 97.426 & 111.185 & 109.384 & & 75.046 \\
\hline $12 / 16 / 0711: 30$ & 128.031 & 125.58 & 121.758 & 101.981 & 105.808 & 97.459 & 111.224 & 109.422 & & 75.081 \\
\hline $12 / 16 / 07$ 12:00 & 128.055 & 125.592 & 121.771 & 102.023 & 105.85 & 97.496 & 111.247 & 109.459 & & 75.122 \\
\hline $12 / 16 / 07$ 12:30 & 128.078 & 125.605 & 121.784 & 102.03 & 105.869 & 97.52 & 111.27 & 109.484 & & 75.147 \\
\hline 12/16/07 13:00 & 128.097 & 125.608 & 121.804 & 102.047 & 105.884 & 97.534 & 111.286 & 109.499 & & 75.163 \\
\hline 12/16/07 13:30 & 128.109 & 124.46 & 121.809 & 102.057 & 105.898 & 97.548 & 111.3 & 109.512 & & 75.18 \\
\hline $12 / 16 / 0714: 00$ & 128.123 & 123.666 & 120.489 & 102.059 & 105.896 & 97.546 & 111.311 & 109.512 & & 75.18 \\
\hline $12 / 16 / 07$ 14:30 & 127.558 & 123.478 & 120.254 & 101.571 & 105.901 & 97.555 & 111.317 & 109.519 & & 75.192 \\
\hline 12/16/07 15:00 & 126.208 & 123.378 & 120.146 & 101.244 & 105.324 & 96.846 & 110.62 & 108.034 & & 75.066 \\
\hline $12 / 16 / 07$ 15:30 & 125.996 & 123.31 & 120.073 & 101.106 & 105.145 & 96.664 & 110.445 & 107.823 & & 74.932 \\
\hline $12 / 16 / 0716: 00$ & 125.892 & 123.274 & 120.022 & 101.025 & 105.046 & 96.568 & 110.351 & 107.702 & & 74.835 \\
\hline 12/16/07 16:30 & 125.825 & 123.251 & 119.993 & 100.979 & 104.984 & 96.504 & 110.295 & 107.631 & & 74.764 \\
\hline $12 / 16 / 07$ 17:00 & 125.78 & 125.174 & 121.259 & 100.951 & 104.953 & 96.471 & 110.258 & 107.595 & & 74.731 \\
\hline $12 / 16 / 07$ 17:30 & 125.75 & 125.378 & 121.534 & 101.332 & 104.932 & 96.45 & 110.231 & 107.571 & & 74.707 \\
\hline 12/16/07 18:00 & 127.669 & 125.471 & 121.646 & 101.732 & 105.473 & 97.114 & 110.883 & 109.021 & & 74.797 \\
\hline $12 / 16 / 07$ 18:30 & 127.873 & 125.537 & 121.706 & 101.885 & 105.677 & 97.322 & 111.065 & 109.262 & & 74.959 \\
\hline 12/16/07 19:00 & 127.974 & 124.045 & 121.169 & 101.962 & 105.778 & 97.426 & 111.165 & 109.375 & & 75.06 \\
\hline 12/16/07 19:30 & 128.031 & 123.612 & 120.401 & 102.014 & 105.842 & 97.489 & 111.219 & 109.441 & & 75.126 \\
\hline $12 / 16 / 0720: 00$ & 126.589 & 123.462 & 120.221 & 101.49 & 105.661 & 97.213 & 110.932 & 108.614 & & 75.168 \\
\hline $12 / 16 / 0720: 30$ & 126.092 & 123.367 & 120.129 & 101.213 & 105.275 & 96.799 & 110.524 & 107.965 & & 75.031 \\
\hline $12 / 16 / 0721: 00$ & 125.929 & 123.31 & 120.071 & 101.097 & 105.134 & 96.653 & 110.39 & 107.801 & & 74.917 \\
\hline $12 / 16 / 0721: 30$ & 125.847 & 123.279 & 120.025 & 101.027 & 105.043 & 96.563 & 110.32 & 107.692 & & 74.828 \\
\hline $12 / 16 / 0722: 00$ & 125.795 & 123.242 & 120 & 100.985 & 104.989 & 96.509 & 110.275 & 107.633 & & 74.773 \\
\hline $12 / 16 / 0722: 30$ & 125.754 & 123.229 & 119.977 & 100.953 & 104.961 & 96.48 & 110.243 & 107.602 & & 74.74 \\
\hline 12/16/07 23:00 & 125.733 & 123.222 & 119.962 & 100.925 & 104.925 & 96.443 & 110.226 & 107.562 & & 74.7 \\
\hline
\end{tabular}


TABLE S1.1 (Cont.)

Depth from Top of Casing (ft)

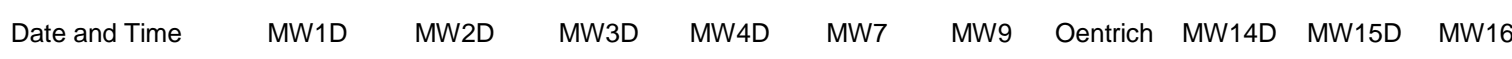

\begin{tabular}{|c|c|c|c|c|c|c|c|c|c|}
\hline 12/16/07 23:30 & 125.714 & 123.211 & 119.957 & 100.918 & 104.913 & 96.433 & 110.209 & 107.549 & 74.684 \\
\hline 12/17/07 0:00 & 125.695 & 123.192 & 119.941 & 100.915 & 104.907 & 96.426 & 110.194 & 107.54 & 74.678 \\
\hline 12/17/07 0:30 & 125.686 & 123.181 & 119.93 & 100.899 & 104.896 & 96.414 & 110.184 & 107.531 & 74.665 \\
\hline 12/17/07 1:00 & 125.676 & 123.163 & 119.926 & 100.882 & 104.881 & 96.4 & 110.179 & 107.513 & 74.649 \\
\hline 12/17/07 1:30 & 125.672 & 123.158 & 119.911 & 100.87 & 104.869 & 96.386 & 110.173 & 107.5 & 74.636 \\
\hline $12 / 17 / 072: 00$ & 125.667 & 123.152 & 119.911 & 100.853 & 104.848 & 96.367 & 110.168 & 107.48 & 74.618 \\
\hline $12 / 17 / 072: 30$ & 125.658 & 123.149 & 119.904 & 100.849 & 104.848 & 96.363 & 110.162 & 107.475 & 74.613 \\
\hline 12/17/07 3:00 & 125.653 & 123.14 & 119.899 & 100.851 & 104.843 & 96.36 & 110.156 & 107.469 & 74.607 \\
\hline 12/17/07 3:30 & 125.648 & 123.136 & 119.892 & 100.842 & 104.841 & 96.36 & 110.152 & 107.469 & 74.607 \\
\hline $12 / 17 / 074: 00$ & 125.646 & 123.131 & 119.886 & 100.837 & 104.831 & 96.351 & 110.149 & 107.458 & 74.597 \\
\hline $12 / 17 / 074: 30$ & 125.641 & 123.12 & 119.884 & 100.832 & 104.828 & 96.346 & 110.145 & 107.453 & 74.593 \\
\hline $12 / 17 / 075: 00$ & 125.636 & 123.115 & 119.876 & 100.827 & 104.82 & 96.339 & 110.143 & 107.447 & 74.587 \\
\hline $12 / 17 / 075: 30$ & 125.636 & 123.111 & 119.872 & 100.813 & 104.808 & 96.327 & 110.141 & 107.438 & 74.574 \\
\hline $12 / 17 / 076: 00$ & 125.632 & 123.106 & 119.869 & 100.811 & 104.803 & 96.323 & 110.135 & 107.429 & 74.568 \\
\hline $12 / 17 / 076: 30$ & 125.627 & 123.104 & 119.859 & 100.808 & 104.803 & 96.32 & 110.132 & 107.427 & 74.568 \\
\hline $12 / 17 / 077: 00$ & 125.622 & 123.102 & 119.856 & 100.808 & 104.797 & 96.315 & 110.13 & 107.422 & 74.56 \\
\hline 12/17/07 7:30 & 125.622 & 123.095 & 119.849 & 100.803 & 104.797 & 96.315 & 110.128 & 107.422 & 74.56 \\
\hline $12 / 17 / 078: 00$ & 125.617 & 123.09 & 119.844 & 100.803 & 104.795 & 96.313 & 110.126 & 107.418 & 74.558 \\
\hline $12 / 17 / 078: 30$ & 125.615 & 123.086 & 119.838 & 100.796 & 104.789 & 96.308 & 110.124 & 107.413 & 74.554 \\
\hline 12/17/07 9:00 & 125.613 & 123.079 & 119.838 & 100.791 & 104.784 & 96.303 & 110.123 & 107.409 & 74.547 \\
\hline 12/17/07 9:30 & 125.608 & 123.077 & 119.833 & 100.784 & 104.784 & 96.299 & 110.119 & 107.405 & 74.543 \\
\hline $12 / 17 / 07 \quad 10: 00$ & 125.608 & 123.079 & 119.828 & 100.78 & 104.786 & 96.294 & 110.119 & 107.398 & 74.537 \\
\hline $12 / 17 / 07$ 10:30 & 125.606 & 123.072 & 119.826 & 100.777 & 104.782 & 96.289 & 110.115 & 107.394 & 74.535 \\
\hline $12 / 17 / 07 \quad 11: 00$ & 125.603 & 124.954 & 121.172 & 100.777 & 104.78 & 96.289 & 110.113 & 107.396 & 74.535 \\
\hline $12 / 17 / 0711: 30$ & 125.601 & 125.208 & 121.494 & 100.917 & 104.78 & 96.287 & 110.111 & 107.389 & 74.529 \\
\hline $12 / 17 / 0712: 00$ & 127.518 & 125.322 & 121.642 & 101.583 & 105.305 & 96.938 & 110.77 & 108.764 & 74.597 \\
\hline $12 / 17 / 07$ 12:30 & 127.797 & 125.39 & 121.731 & 101.763 & 105.562 & 97.195 & 111.018 & 109.076 & 74.775 \\
\hline $12 / 17 / 07$ 13:00 & 127.932 & 125.422 & 121.781 & 101.852 & 105.696 & 97.308 & 111.142 & 109.202 & 74.884 \\
\hline $12 / 17 / 07$ 13:30 & 128.01 & 125.444 & 121.816 & 101.9 & 105.776 & 97.376 & 111.217 & 109.278 & 74.959 \\
\hline $12 / 17 / 07 \quad 14: 00$ & 128.064 & 125.456 & 121.847 & 101.93 & 105.829 & 97.412 & 111.268 & 109.315 & 75 \\
\hline $12 / 17 / 07$ 14:30 & 128.097 & 125.458 & 121.865 & 101.947 & 105.867 & 97.433 & 111.305 & 109.338 & 75.025 \\
\hline $12 / 17 / 07$ 15:00 & 128.128 & 125.456 & 121.874 & 101.957 & 105.894 & 97.44 & 111.332 & 109.348 & 75.037 \\
\hline $12 / 17 / 07 \quad 15: 30$ & 128.147 & 125.456 & 121.882 & 101.956 & 105.913 & 97.445 & 111.352 & 109.353 & 75.046 \\
\hline $12 / 17 / 07 \quad 16: 00$ & 128.161 & 125.46 & 121.889 & 101.956 & 105.918 & 97.447 & 111.369 & 109.355 & 75.05 \\
\hline $12 / 17 / 07$ 16:30 & 128.173 & 125.456 & 121.897 & 101.956 & 105.917 & 97.444 & 111.38 & 109.355 & 75.052 \\
\hline $12 / 17 / 07 \quad 17: 00$ & 128.182 & 125.446 & 121.9 & 101.959 & 105.92 & 97.444 & 111.39 & 109.353 & 75.054 \\
\hline $12 / 17 / 07$ 17:30 & 128.194 & 124.186 & 121.9 & 101.95 & 105.928 & 97.447 & 111.401 & 109.353 & 75.058 \\
\hline $12 / 17 / 07$ 18:00 & 128.203 & 123.489 & 120.435 & 101.947 & 105.92 & 97.44 & 111.403 & 109.346 & 75.052 \\
\hline $12 / 17 / 07$ 18:30 & 127.159 & 123.297 & 120.209 & 101.387 & 105.858 & 97.341 & 111.296 & 109.174 & 75.054 \\
\hline $12 / 17 / 07 \quad 19: 00$ & 126.227 & 123.199 & 120.083 & 101.058 & 105.298 & 96.662 & 110.599 & 107.857 & 74.911 \\
\hline $12 / 17 / 07$ 19:30 & 126.017 & 123.138 & 120.007 & 100.92 & 105.104 & 96.478 & 110.417 & 107.626 & 74.764 \\
\hline $12 / 17 / 0720: 00$ & 125.903 & 123.084 & 119.954 & 100.844 & 105.006 & 96.384 & 110.321 & 107.522 & 74.673 \\
\hline $12 / 17 / 0720: 30$ & 125.837 & 123.059 & 119.911 & 100.794 & 104.944 & 96.323 & 110.267 & 107.456 & 74.607 \\
\hline $12 / 17 / 0721: 00$ & 125.795 & 123.029 & 119.889 & 100.758 & 104.906 & 96.278 & 110.226 & 107.403 & 74.556 \\
\hline $12 / 17 / 0721: 30$ & 125.759 & 123.009 & 119.866 & 100.729 & 104.879 & 96.254 & 110.199 & 107.376 & 74.527 \\
\hline $12 / 17 / 0722: 00$ & 125.736 & 122.991 & 119.846 & 100.708 & 104.852 & 96.228 & 110.181 & 107.347 & 74.498 \\
\hline $12 / 17 / 0722: 30$ & 125.721 & 122.966 & 119.833 & 100.687 & 104.837 & 96.209 & 110.167 & 107.327 & 74.479 \\
\hline $12 / 17 / 0723: 00$ & 125.705 & 122.948 & 119.818 & 100.67 & 104.818 & 96.19 & 110.156 & 107.308 & 74.456 \\
\hline $12 / 17 / 0723: 30$ & 125.698 & 122.934 & 119.808 & 100.651 & 104.803 & 96.165 & 110.149 & 107.281 & 74.432 \\
\hline $12 / 18 / 070: 00$ & 125.686 & 122.929 & 119.795 & 100.632 & 104.786 & 96.15 & 110.143 & 107.265 & 74.415 \\
\hline $12 / 18 / 070: 30$ & 125.679 & 122.92 & 119.783 & 100.622 & 104.772 & 96.136 & 110.137 & 107.25 & 74.401 \\
\hline 12/18/07 1:00 & 125.669 & 122.911 & 119.773 & 100.615 & 104.765 & 96.132 & 110.132 & 107.243 & 74.394 \\
\hline $12 / 18 / 071: 30$ & 125.665 & 122.902 & 119.765 & 100.612 & 104.759 & 96.124 & 110.126 & 107.234 & 74.384 \\
\hline 12/18/07 2:00 & 125.66 & 122.902 & 119.755 & 100.601 & 104.749 & 96.117 & 110.124 & 107.225 & 74.376 \\
\hline 12/18/07 2:30 & 125.655 & 122.891 & 119.747 & 100.593 & 104.738 & 96.108 & 110.12 & 107.217 & 74.365 \\
\hline 12/18/07 3:00 & 125.65 & 122.898 & 119.735 & 100.594 & 104.738 & 96.11 & 110.117 & 107.217 & 74.363 \\
\hline 12/18/07 3:30 & 125.648 & 122.9 & 119.725 & 100.587 & 104.727 & 96.094 & 110.117 & 107.199 & 74.347 \\
\hline 12/18/07 4:00 & 125.643 & 122.895 & 119.715 & 100.598 & 104.727 & 96.101 & 110.113 & 107.208 & 74.353 \\
\hline $12 / 18 / 074: 30$ & 125.641 & 122.895 & 119.717 & 100.593 & 104.727 & 96.108 & 110.113 & 107.215 & 74.361 \\
\hline $12 / 18 / 07$ 5:00 & 125.641 & 122.898 & 119.71 & 100.586 & 104.725 & 96.103 & 110.111 & 107.21 & 74.357 \\
\hline $12 / 18 / 075: 30$ & 125.636 & 122.895 & 119.707 & 100.594 & 104.725 & 96.106 & 110.107 & 107.208 & 74.353 \\
\hline 12/18/07 6:00 & 125.634 & 122.898 & 119.705 & 100.596 & 104.723 & 96.106 & 110.105 & 107.212 & 74.355 \\
\hline $12 / 18 / 076: 30$ & 125.632 & 122.904 & 119.697 & 100.591 & 104.721 & 96.106 & 110.106 & 107.21 & 74.355 \\
\hline $12 / 18 / 077: 00$ & 125.629 & 122.92 & 119.692 & 100.605 & 104.721 & 96.106 & 110.104 & 107.208 & 74.349 \\
\hline $12 / 18 / 077: 30$ & 125.627 & 122.927 & 119.689 & 100.613 & 104.721 & 96.117 & 110.1 & 107.219 & 74.357 \\
\hline $12 / 18 / 078: 00$ & 125.622 & 122.929 & 119.687 & 100.624 & 104.721 & 96.129 & 110.1 & 107.23 & 74.37 \\
\hline
\end{tabular}


TABLE S1.1 (Cont.)

\begin{tabular}{|c|c|c|c|c|c|c|c|c|c|c|}
\hline \multirow[b]{2}{*}{ Date and Time } & \multicolumn{10}{|c|}{ Depth from Top of Casing (ft) } \\
\hline & MW1D & MW2D & MW3D & MW4D & MW7 & MW9 & Oentrich & MW14D & MW15D & MW16D \\
\hline 12/18/07 8:30 & 125.617 & 122.932 & 119.679 & 100.627 & 104.717 & 96.139 & 110.098 & 107.237 & & 74.376 \\
\hline 12/18/07 9:00 & 125.617 & 122.95 & 119.705 & 100.632 & 104.719 & 96.139 & 110.096 & 107.239 & & 74.378 \\
\hline 12/18/07 9:30 & 125.615 & 122.952 & 119.715 & 100.639 & 104.715 & 96.15 & 110.094 & 107.247 & & 74.386 \\
\hline $12 / 18 / 07 \quad 10: 00$ & 125.61 & 122.963 & 119.712 & 100.653 & 104.713 & 96.162 & 110.092 & 107.261 & & 74.396 \\
\hline $12 / 18 / 07$ 10:30 & 125.613 & 122.984 & 119.74 & 100.662 & 104.717 & 96.164 & 110.094 & 107.261 & & 74.396 \\
\hline $12 / 18 / 0711: 00$ & 125.608 & 122.991 & 119.745 & 100.675 & 104.713 & 96.179 & 110.092 & 107.274 & & 74.409 \\
\hline $12 / 18 / 0711: 30$ & 125.608 & 122.993 & 119.742 & 100.689 & 104.715 & 96.2 & 110.091 & 107.296 & & 74.427 \\
\hline $12 / 18 / 07$ 12:00 & 125.606 & 122.982 & 119.74 & 100.698 & 104.713 & 96.209 & 110.091 & 107.305 & & 74.436 \\
\hline 12/18/07 12:30 & 125.606 & 122.991 & 119.74 & 100.694 & 104.713 & 96.207 & 110.092 & 107.305 & & 74.436 \\
\hline $12 / 18 / 07$ 13:00 & 125.608 & 122.997 & 119.742 & 100.687 & 104.715 & 96.2 & 110.093 & 107.299 & & 74.425 \\
\hline $12 / 18 / 07$ 13:30 & 125.603 & 122.991 & 119.732 & 100.699 & 104.715 & 96.207 & 110.093 & 107.303 & & 74.429 \\
\hline 12/18/07 14:00 & 125.603 & 122.984 & 119.727 & 100.694 & 104.715 & 96.214 & 110.09 & 107.31 & & 74.434 \\
\hline $12 / 18 / 0714: 30$ & 125.606 & 122.988 & 119.717 & 100.694 & 104.719 & 96.207 & 110.086 & 107.301 & & 74.425 \\
\hline $12 / 18 / 07$ 15:00 & 125.606 & 122.995 & 119.712 & 100.691 & 104.715 & 96.2 & 110.087 & 107.294 & & 74.421 \\
\hline 12/18/07 15:30 & 125.601 & 123.007 & 119.707 & 100.696 & 104.717 & 96.207 & 110.087 & 107.301 & & 74.425 \\
\hline $12 / 18 / 07$ 16:00 & 125.598 & 123.018 & 119.725 & 100.701 & 104.715 & 96.212 & 110.085 & 107.308 & & 74.432 \\
\hline $12 / 18 / 07$ 16:30 & 125.596 & 123.025 & 119.725 & 100.717 & 104.711 & 96.221 & 110.081 & 107.316 & & 74.425 \\
\hline $12 / 18 / 07$ 17:00 & 125.591 & 123.031 & 119.727 & 100.727 & 104.711 & 96.233 & 110.081 & 107.33 & & 74.419 \\
\hline $12 / 18 / 07$ 17:30 & 125.594 & 123.047 & 119.722 & 100.732 & 104.711 & 96.242 & 110.079 & 107.336 & & 74.417 \\
\hline $12 / 18 / 0718: 00$ & 125.589 & 123.047 & 119.727 & 100.749 & 104.711 & 96.247 & 110.079 & 107.341 & & 74.415 \\
\hline $12 / 18 / 07$ 18:30 & 125.589 & 123.052 & 119.727 & 100.753 & 104.709 & 96.263 & 110.079 & 107.361 & & 74.413 \\
\hline 12/18/07 19:00 & 125.589 & 123.056 & 119.722 & 100.748 & 104.709 & 96.266 & 110.077 & 107.358 & & 74.413 \\
\hline 12/18/07 19:30 & 125.584 & 123.068 & 119.712 & 100.76 & 104.709 & 96.266 & 110.077 & 107.361 & & 74.415 \\
\hline $12 / 18 / 0720: 00$ & 125.582 & 123.068 & 119.74 & 100.765 & 104.708 & 96.275 & 110.076 & 107.37 & & 74.415 \\
\hline 12/18/07 20:30 & 125.58 & 123.07 & 119.742 & 100.774 & 104.708 & 96.285 & 110.074 & 107.378 & & 74.415 \\
\hline 12/18/07 21:00 & 125.58 & 123.075 & 119.737 & 100.772 & 104.704 & 96.287 & 110.074 & 107.382 & & 74.417 \\
\hline $12 / 18 / 0721: 30$ & 125.577 & 123.086 & 119.74 & 100.777 & 104.704 & 96.29 & 110.072 & 107.385 & & 74.417 \\
\hline 12/18/07 22:00 & 125.575 & 123.088 & 119.74 & 100.787 & 104.704 & 96.294 & 110.068 & 107.389 & & 74.417 \\
\hline 12/18/07 22:30 & 125.572 & 123.079 & 119.742 & 100.796 & 104.704 & 96.304 & 110.068 & 107.398 & & 74.417 \\
\hline $12 / 18 / 0723: 00$ & 125.568 & 123.079 & 119.737 & 100.794 & 104.7 & 96.306 & 110.068 & 107.401 & & 74.419 \\
\hline $12 / 18 / 0723: 30$ & 125.57 & 123.081 & 119.742 & 100.789 & 104.704 & 96.299 & 110.066 & 107.396 & & 74.419 \\
\hline 12/19/07 0:00 & 125.565 & 123.077 & 119.745 & 100.791 & 104.7 & 96.299 & 110.866 & 107.394 & & 74.417 \\
\hline $12 / 19 / 070: 30$ & 125.568 & 123.068 & 119.745 & 100.791 & 104.7 & 96.301 & 110.866 & 107.398 & & 74.415 \\
\hline 12/19/07 1:00 & 125.565 & 123.059 & 119.747 & 100.784 & 104.698 & 96.297 & 110.866 & 107.392 & & 74.413 \\
\hline $12 / 19 / 071: 30$ & 125.565 & 123.054 & 119.745 & 100.774 & 104.7 & 96.29 & 110.862 & 107.385 & & 74.411 \\
\hline 12/19/07 2:00 & 125.565 & 123.054 & 119.742 & 100.763 & 104.698 & 96.28 & 110.864 & 107.376 & & 74.409 \\
\hline 12/19/07 2:30 & 125.561 & 123.059 & 119.74 & 100.758 & 104.696 & 96.273 & 110.862 & 107.37 & & 74.405 \\
\hline 12/19/07 3:00 & 125.561 & 123.054 & 119.74 & 100.763 & 104.696 & 96.275 & 110.862 & 107.37 & & 74.398 \\
\hline 12/19/07 3:30 & 125.556 & 123.05 & 119.732 & 100.763 & 104.696 & 96.278 & 110.858 & 107.372 & & 74.394 \\
\hline $12 / 19 / 074: 00$ & 125.554 & 123.045 & 119.737 & 100.762 & 104.692 & 96.27 & 110.859 & 107.367 & & 74.39 \\
\hline $12 / 19 / 074: 30$ & 125.554 & 123.038 & 119.73 & 100.751 & 104.692 & 96.271 & 110.859 & 107.365 & & 74.386 \\
\hline $12 / 19 / 075: 00$ & 125.549 & 123.025 & 119.735 & 100.748 & 104.694 & 96.266 & 110.857 & 107.358 & & 74.382 \\
\hline 12/19/07 5:30 & 125.551 & 123.029 & 119.732 & 100.739 & 104.69 & 96.259 & 110.857 & 107.352 & & 74.376 \\
\hline $12 / 19 / 076: 00$ & 125.551 & 123.027 & 119.725 & 100.73 & 104.692 & 96.245 & 110.857 & 107.341 & & 74.374 \\
\hline 12/19/07 6:30 & 125.549 & 123.022 & 119.725 & 100.736 & 104.692 & 96.247 & 110.855 & 107.341 & & 74.365 \\
\hline 12/19/07 7:00 & 125.546 & 124.952 & 121.138 & 100.73 & 104.69 & 96.247 & 110.853 & 107.343 & & 74.361 \\
\hline $12 / 19 / 077: 30$ & 125.546 & 125.19 & 121.411 & 101.116 & 104.689 & 96.245 & 110.851 & 107.339 & & 74.456 \\
\hline 12/19/07 8:00 & 127.13 & 125.306 & 121.544 & 101.576 & 105.262 & 96.947 & 111.557 & 108.775 & & 74.564 \\
\hline 12/19/07 8:30 & 127.39 & 125.369 & 121.625 & 101.749 & 105.492 & 97.185 & 111.675 & 109.059 & & 74.748 \\
\hline 12/19/07 9:00 & 127.518 & 125.412 & 121.68 & 101.837 & 105.614 & 97.301 & 111.788 & 109.189 & & 74.862 \\
\hline 12/19/07 9:30 & 127.589 & 123.614 & 120.395 & 101.895 & 105.686 & 97.369 & 111.857 & 109.26 & & 74.936 \\
\hline 12/19/07 10:00 & 127.641 & 123.372 & 120.09 & 101.751 & 105.734 & 97.416 & 111.902 & 109.311 & & 74.986 \\
\hline $12 / 19 / 07$ 10:30 & 125.818 & 123.251 & & 101.173 & 105.25 & 96.803 & 111.282 & 108.009 & & 74.948 \\
\hline 12/19/07 11:00 & 125.627 & 123.179 & & 101.001 & 105.022 & 96.57 & 111.061 & 107.71 & & 74.806 \\
\hline 12/19/07 11:30 & 125.511 & 123.122 & & 100.911 & 104.915 & 96.452 & 110.959 & 107.578 & & 74.7 \\
\hline $12 / 19 / 07$ 12:00 & & 123.077 & & 100.853 & 104.852 & 96.381 & 110.903 & & & 74.628 \\
\hline $12 / 19 / 07$ 12:30 & 125.606 & 123.034 & & 100.798 & 104.816 & 96.327 & 110.868 & & & 74.568 \\
\hline 12/19/07 13:00 & 125.589 & 122.995 & & 100.753 & 104.789 & 96.28 & 110.845 & & & 74.518 \\
\hline $12 / 19 / 07$ 13:30 & 125.58 & 122.972 & & 100.708 & 104.772 & 96.24 & 110.83 & & & 74.477 \\
\hline 12/19/07 14:00 & 125.57 & 122.945 & & 100.686 & 104.761 & 96.2 & 110.819 & & & 74.438 \\
\hline $12 / 19 / 0714: 30$ & 125.558 & 122.92 & & 100.651 & 104.751 & 96.186 & 110.811 & & & 74.417 \\
\hline $12 / 19 / 07$ 15:00 & 125.549 & 122.909 & & 100.629 & 104.746 & 96.157 & 110.805 & & & 74.386 \\
\hline $12 / 19 / 07$ 15:30 & 125.542 & 122.891 & & 100.61 & & 96.134 & 110.8 & & 80.853 & 74.382 \\
\hline $12 / 19 / 07$ 16:00 & 125.537 & 122.886 & & 100.598 & 104.475 & 96.124 & 110.794 & & & 74.376 \\
\hline $12 / 19 / 07 \quad 16: 30$ & 125.532 & 122.88 & & 100.581 & 104.456 & 96.103 & 111.138 & & & 74.37 \\
\hline
\end{tabular}


TABLE S1.1 (Cont.)

\begin{tabular}{|c|c|c|c|c|c|c|c|c|c|c|}
\hline \multirow[b]{2}{*}{ Date and Time } & \multicolumn{10}{|c|}{ Depth from Top of Casing (ft) } \\
\hline & MW1D & MW2D & MW3D & MW4D & MW7 & MW9 & Oentrich & MW14D & MW15D & MW16D \\
\hline 12/19/07 17:00 & 125.525 & 122.873 & & 100.577 & 104.452 & 96.098 & 111.183 & & 80.834 & 74.359 \\
\hline $12 / 19 / 07$ 17:30 & 125.523 & 122.868 & & 100.572 & 104.447 & 96.091 & 110.949 & & & 74.355 \\
\hline 12/19/07 18:00 & 125.52 & 122.868 & & 100.563 & 104.441 & 96.087 & 110.962 & & & 74.349 \\
\hline $12 / 19 / 07$ 18:30 & 125.513 & 122.87 & & 100.563 & 104.437 & 96.082 & 110.789 & & 80.79 & 74.336 \\
\hline $12 / 19 / 07$ 19:00 & 125.509 & 122.868 & & 100.562 & 104.437 & 96.082 & 110.789 & & & 74.326 \\
\hline 12/19/07 19:30 & 125.502 & 122.861 & & 100.565 & 104.439 & 96.082 & 110.787 & & & 74.322 \\
\hline $12 / 19 / 0720: 00$ & 125.497 & 122.859 & & 100.562 & 104.439 & 96.084 & 110.787 & & 80.756 & 74.318 \\
\hline $12 / 19 / 0720: 30$ & 125.494 & 122.843 & & 100.553 & 104.433 & 96.08 & 110.785 & & & 74.314 \\
\hline 12/19/07 21:00 & 125.492 & 122.836 & & 100.546 & 104.43 & 96.077 & 110.785 & & & 74.31 \\
\hline $12 / 19 / 0721: 30$ & 125.49 & 122.83 & & 100.531 & 104.412 & 96.056 & 110.776 & & 80.741 & 74.308 \\
\hline $12 / 19 / 0722: 00$ & 125.487 & 122.827 & & 100.529 & 104.405 & 96.051 & 110.772 & & & 74.303 \\
\hline 12/19/07 22:30 & 125.483 & 122.812 & & 100.52 & 104.399 & 96.044 & 110.765 & & & 74.299 \\
\hline 12/19/07 23:00 & 125.478 & 122.823 & & 100.519 & 104.395 & 96.042 & 110.757 & & 80.719 & 74.293 \\
\hline $12 / 19 / 0723: 30$ & 125.476 & 122.821 & & 100.506 & 104.382 & 96.028 & 110.757 & & & 74.287 \\
\hline 12/20/07 0:00 & 125.468 & 122.809 & & 100.524 & 104.399 & 96.042 & 110.752 & & & 74.279 \\
\hline $12 / 20 / 070: 30$ & 125.466 & 122.8 & & 100.519 & 104.388 & 96.035 & 110.752 & & 80.699 & 74.272 \\
\hline $12 / 20 / 071: 00$ & 125.461 & 122.787 & & 100.498 & 104.38 & 96.028 & 110.755 & & & 74.266 \\
\hline 12/20/07 1:30 & 125.454 & 122.787 & & 100.494 & 104.372 & 96.018 & 110.746 & & & 74.26 \\
\hline $12 / 20 / 072: 00$ & 125.452 & 122.782 & & 100.486 & 104.359 & 96.007 & 110.74 & & 80.675 & 74.25 \\
\hline $12 / 20 / 072: 30$ & 125.445 & 122.784 & & 100.484 & 104.361 & 96.009 & 110.737 & & & 74.241 \\
\hline 12/20/07 3:00 & 125.435 & 122.782 & & 100.481 & 104.357 & 96.002 & 110.737 & & & 74.231 \\
\hline 12/20/07 3:30 & 125.428 & 122.78 & & 100.486 & 104.357 & 96.004 & 110.733 & & 80.65 & 74.223 \\
\hline 12/20/07 4:00 & 125.424 & 122.775 & & 100.482 & 104.355 & 96.004 & 110.735 & & & 74.214 \\
\hline $12 / 20 / 074: 30$ & 125.416 & 122.771 & & 100.477 & 104.353 & 95.999 & 110.735 & & & 74.206 \\
\hline $12 / 20 / 075: 00$ & 125.412 & 122.766 & & 100.474 & 104.348 & 95.992 & 110.725 & & 80.636 & 74.2 \\
\hline 12/20/07 5:30 & 125.405 & 122.773 & & 100.474 & 104.348 & 95.995 & 110.727 & & & 74.192 \\
\hline $12 / 20 / 076: 00$ & 125.398 & 122.78 & & 100.47 & 104.346 & 95.988 & 110.727 & & & 74.188 \\
\hline $12 / 20 / 076: 30$ & 125.393 & 122.789 & & 100.474 & 104.346 & 95.992 & 110.723 & & 80.641 & 74.179 \\
\hline 12/20/07 7:00 & 125.383 & 124.728 & & 100.484 & 104.355 & 95.999 & 110.721 & & & 74.173 \\
\hline $12 / 20 / 077: 30$ & 125.376 & 124.968 & & 100.811 & 104.363 & 96.009 & 110.721 & & & 74.165 \\
\hline 12/20/07 8:00 & 126.993 & 125.102 & & 101.294 & 104.896 & 96.669 & 111.373 & & 80.611 & 74.243 \\
\hline $12 / 20 / 078: 30$ & 127.199 & 125.174 & & 101.47 & 105.134 & 96.912 & 111.591 & & & 74.386 \\
\hline 12/20/07 9:00 & 127.303 & 125.236 & & 101.575 & 105.267 & 97.046 & 111.703 & & & 74.487 \\
\hline 12/20/07 9:30 & 127.371 & 125.27 & & 101.647 & 105.345 & 97.121 & 111.773 & & 80.668 & 74.57 \\
\hline 12/20/07 10:00 & 127.419 & 125.308 & & 101.695 & 105.404 & 97.18 & 111.82 & & & 74.634 \\
\hline $12 / 20 / 07$ 10:30 & 127.461 & 125.347 & & 101.74 & 105.441 & 97.22 & 111.852 & & & 74.69 \\
\hline 12/20/07 11:00 & 127.501 & 125.354 & & 101.775 & 105.481 & 97.258 & 111.876 & & 80.719 & 74.74 \\
\hline 12/20/07 11:30 & 127.525 & 125.354 & & 101.802 & 105.522 & 97.296 & 111.893 & & & 74.785 \\
\hline $12 / 20 / 07$ 12:00 & 127.549 & 123.555 & & 101.809 & 105.528 & 97.305 & 111.91 & & & 74.824 \\
\hline $12 / 20 / 07$ 12:30 & 127.572 & 123.279 & & 101.792 & 105.53 & 97.308 & 111.923 & & 80.802 & 74.866 \\
\hline 12/20/07 13:00 & 126.081 & 123.145 & & 101.082 & 105.085 & 96.737 & 111.352 & & & 74.831 \\
\hline $12 / 20 / 07$ 13:30 & 125.858 & 123.059 & & 100.889 & 104.826 & 96.471 & 111.117 & & & 74.721 \\
\hline $12 / 20 / 07$ 14:00 & 125.757 & 123.011 & & 100.784 & 104.692 & 96.341 & 111.009 & & 80.8 & 74.642 \\
\hline $12 / 20 / 07$ 14:30 & 125.691 & 122.97 & & 100.715 & 104.612 & 96.261 & 110.945 & & & 74.582 \\
\hline $12 / 20 / 07$ 15:00 & 125.641 & 122.95 & & 100.677 & 104.567 & 96.212 & 110.903 & & & 74.539 \\
\hline $12 / 20 / 07$ 15:30 & 125.608 & 122.941 & & 100.643 & 104.529 & 96.174 & 110.875 & & 80.763 & 74.502 \\
\hline 12/20/07 16:00 & 125.58 & 122.929 & & 100.631 & 104.508 & 96.155 & 110.856 & & & 74.467 \\
\hline $12 / 20 / 07$ 16:30 & 125.558 & 122.923 & & 100.624 & 104.504 & 96.15 & 110.84 & & & 74.444 \\
\hline $12 / 20 / 07$ 17:00 & 125.539 & 122.918 & & 100.617 & 104.492 & 96.139 & 110.83 & & 80.734 & 74.432 \\
\hline 12/20/07 17:30 & 125.528 & 122.914 & & 100.608 & 104.485 & 96.131 & 110.823 & & & 74.425 \\
\hline $12 / 20 / 07$ 18:00 & 125.513 & 122.911 & & 100.606 & 104.483 & 96.129 & 110.817 & & & 74.421 \\
\hline $12 / 20 / 07$ 18:30 & 125.502 & 122.902 & & 100.606 & 104.481 & 96.129 & 110.813 & & 80.716 & 74.409 \\
\hline 12/20/07 19:00 & 125.494 & 122.898 & & 100.601 & 104.475 & 96.124 & 110.809 & & & 74.365 \\
\hline $12 / 20 / 07$ 19:30 & 125.487 & 122.893 & & 100.587 & 104.47 & 96.115 & 110.806 & & & 74.355 \\
\hline 12/20/07 20:00 & 125.478 & 122.891 & & 100.589 & 104.462 & 96.11 & 110.804 & & 80.707 & 74.345 \\
\hline $12 / 20 / 0720: 30$ & 125.468 & 122.889 & & 100.587 & 104.462 & 96.108 & 110.802 & & & 74.341 \\
\hline $12 / 20 / 0721: 00$ & 125.461 & 122.875 & & 100.584 & 104.46 & 96.103 & 110.8 & & & 74.336 \\
\hline 12/20/07 21:30 & 125.457 & 122.87 & & 100.582 & 104.456 & 96.106 & 110.796 & & 80.702 & 74.332 \\
\hline 12/20/07 22:00 & 125.452 & 122.875 & & 100.57 & 104.443 & 96.091 & 110.796 & & & 74.328 \\
\hline $12 / 20 / 0722: 30$ & 125.447 & 122.873 & & 100.569 & 104.439 & 96.087 & 110.794 & & & 74.32 \\
\hline 12/20/07 23:00 & 125.438 & 122.877 & & 100.567 & 104.443 & 96.089 & 110.791 & & 80.69 & 74.316 \\
\hline $12 / 20 / 0723: 30$ & 125.431 & 122.873 & & 100.572 & 104.441 & 96.089 & 110.791 & & & 74.314 \\
\hline $12 / 21 / 070: 00$ & 125.414 & 122.859 & & 100.574 & 104.451 & 96.096 & 110.787 & & & 74.316 \\
\hline $12 / 21 / 070: 30$ & 125.39 & 122.85 & & 100.567 & 104.443 & 96.091 & 110.787 & & 80.677 & 74.314 \\
\hline $12 / 21 / 071: 00$ & 125.357 & 122.832 & & 100.551 & 104.43 & 96.075 & 110.787 & & & 74.308 \\
\hline $12 / 21 / 071: 30$ & 125.324 & 122.82 & & 100.543 & 104.422 & 96.068 & 110.787 & & & 74.297 \\
\hline
\end{tabular}


TABLE S1.1 (Cont.)

\begin{tabular}{|c|c|c|c|c|c|c|c|c|c|c|}
\hline \multirow[b]{2}{*}{ Date and Time } & \multicolumn{10}{|c|}{ Depth from Top of Casing (ft) } \\
\hline & MW1D & MW2D & MW3D & MW4D & MW7 & MW9 & Oentrich & MW14D & MW15D & MW16D \\
\hline $12 / 21 / 072: 00$ & 125.298 & 122.812 & & 100.524 & 104.405 & 96.049 & 110.787 & & 80.66 & 74.285 \\
\hline $12 / 21 / 072: 30$ & 125.272 & 122.816 & & 100.513 & 104.392 & 96.037 & 110.778 & & & 74.27 \\
\hline $12 / 21 / 07$ 3:00 & 125.256 & 122.823 & & 100.51 & 104.386 & 96.032 & 110.77 & & & 74.258 \\
\hline $12 / 21 / 073: 30$ & 125.249 & 122.807 & & 100.522 & 104.388 & 96.037 & 110.766 & & 80.631 & 74.252 \\
\hline $12 / 21 / 074: 00$ & 125.253 & 122.791 & & 100.517 & 104.395 & 96.042 & 110.767 & & & 74.256 \\
\hline $12 / 21 / 074: 30$ & 125.251 & 122.791 & & 100.501 & 104.382 & 96.03 & 110.768 & & & 74.252 \\
\hline $12 / 21 / 075: 00$ & 125.242 & 122.789 & & 100.491 & 104.365 & 96.011 & 110.755 & & 80.619 & 74.239 \\
\hline $12 / 21 / 075: 30$ & 125.227 & 122.78 & & 100.496 & 104.365 & 96.011 & 110.749 & & & 74.229 \\
\hline $12 / 21 / 076: 00$ & 125.23 & 122.782 & & 100.484 & 104.363 & 96.009 & 110.748 & & & 74.227 \\
\hline $12 / 21 / 076: 30$ & 125.223 & 122.782 & & 100.479 & 104.353 & 95.997 & 110.744 & & 80.599 & 74.219 \\
\hline $12 / 21 / 077: 00$ & 125.218 & 124.372 & & 100.484 & 104.357 & 96.002 & 110.737 & & & 74.212 \\
\hline $12 / 21 / 07 \quad 7: 30$ & 125.218 & 124.812 & & 100.479 & 104.357 & 96.002 & 110.738 & & & 74.212 \\
\hline $12 / 21 / 078: 00$ & 126.563 & 124.979 & & 101.132 & 104.61 & 96.372 & 111.131 & & 80.58 & 74.212 \\
\hline $12 / 21 / 078: 30$ & 127.163 & 125.066 & & 101.397 & 105.026 & 96.813 & 111.54 & & & 74.372 \\
\hline 12/21/07 9:00 & 127.426 & 123.957 & & 101.528 & 105.191 & 96.982 & 111.7 & & & 74.523 \\
\hline $12 / 21 / 07$ 9:30 & 127.565 & 123.215 & & 101.604 & 105.284 & 97.074 & 111.79 & & 80.643 & 74.626 \\
\hline $12 / 21 / 07 \quad 10: 00$ & 126.979 & 123.031 & & 101.104 & 105.317 & 97.124 & 111.786 & & & 74.694 \\
\hline $12 / 21 / 07 \quad 10: 30$ & 125.816 & 122.938 & & 100.801 & 104.751 & 96.4 & 111.123 & & & 74.609 \\
\hline $12 / 21 / 0711: 00$ & 125.648 & 122.875 & & 100.675 & 104.586 & 96.233 & 110.962 & & 80.653 & 74.485 \\
\hline $12 / 21 / 0711: 30$ & 125.577 & 122.83 & & 100.596 & 104.496 & 96.143 & 110.883 & & & 74.392 \\
\hline $12 / 21 / 07 \quad 12: 00$ & 125.535 & 122.791 & & 100.543 & 104.437 & 96.082 & 110.819 & & & 74.314 \\
\hline $12 / 21 / 07 \quad 12: 30$ & 125.506 & 122.762 & & 100.503 & 104.392 & 96.039 & 110.782 & & 80.619 & 74.27 \\
\hline $12 / 21 / 07$ 13:00 & 125.473 & 122.739 & & 100.47 & 104.353 & 96.002 & 110.75 & & & 74.231 \\
\hline $12 / 21 / 07$ 13:30 & 125.438 & 122.709 & & 100.446 & 104.325 & 95.971 & 110.71 & & & 74.196 \\
\hline $12 / 21 / 0714: 00$ & 125.405 & 122.696 & & 100.42 & 104.308 & 95.952 & 110.699 & & 80.602 & 74.179 \\
\hline $12 / 21 / 07 \quad 14: 30$ & 125.383 & 122.685 & & 100.393 & 104.277 & 95.922 & 110.678 & & & 74.159 \\
\hline $12 / 21 / 07$ 15:00 & 125.353 & 122.68 & & 100.384 & 104.264 & 95.912 & 110.658 & & & 74.138 \\
\hline $12 / 21 / 07$ 15:30 & 125.329 & 122.682 & & 100.377 & 104.254 & 95.9 & 110.65 & & 80.719 & 74.128 \\
\hline $12 / 21 / 07$ 16:00 & 125.303 & 122.68 & & 100.379 & 104.251 & 95.896 & 110.642 & & & 74.121 \\
\hline $12 / 21 / 07 \quad 16: 30$ & 125.279 & 122.689 & & 100.376 & 104.253 & 95.898 & 110.639 & & & 74.121 \\
\hline $12 / 21 / 07 \quad 17: 00$ & 125.258 & 122.696 & & 100.384 & 104.251 & 95.898 & 110.639 & & 80.626 & 74.117 \\
\hline $12 / 21 / 07$ 17:30 & 125.152 & 122.709 & & 100.386 & 104.258 & 95.905 & 110.637 & & & 74.128 \\
\hline $12 / 21 / 07 \quad 18: 00$ & 125.13 & 122.723 & & 100.396 & 104.268 & 95.912 & 110.633 & & & 74.134 \\
\hline $12 / 21 / 07$ 18:30 & 125.138 & 122.732 & & 100.415 & 104.283 & 95.926 & 110.631 & & 80.531 & 74.144 \\
\hline $12 / 21 / 07 \quad 19: 00$ & 125.154 & 122.746 & & 100.42 & 104.296 & 95.94 & 110.629 & & & 74.159 \\
\hline $12 / 21 / 07 \quad 19: 30$ & 125.159 & 122.757 & & 100.436 & 104.302 & 95.945 & 110.629 & & & 74.161 \\
\hline $12 / 21 / 0720: 00$ & 125.175 & 122.764 & & 100.446 & 104.321 & 95.966 & 110.628 & & 80.504 & 74.179 \\
\hline $12 / 21 / 0720: 30$ & 125.187 & 122.775 & & 100.46 & 104.331 & 95.973 & 110.629 & & & 74.188 \\
\hline $12 / 21 / 0721: 00$ & 125.201 & 122.782 & & 100.472 & 104.34 & 95.983 & 110.635 & & & 74.202 \\
\hline $12 / 21 / 0721: 30$ & 125.208 & 122.791 & & 100.477 & 104.348 & 95.995 & 110.645 & & 80.509 & 74.208 \\
\hline $12 / 21 / 0722: 00$ & 125.213 & 122.8 & & 100.488 & 104.355 & 96.002 & 110.65 & & & 74.212 \\
\hline $12 / 21 / 0722: 30$ & 125.225 & 122.807 & & 100.494 & 104.365 & 96.011 & 110.656 & & & 74.221 \\
\hline $12 / 21 / 0723: 00$ & 125.234 & 122.814 & & 100.513 & 104.38 & 96.025 & 110.665 & & 80.521 & 74.231 \\
\hline $12 / 21 / 0723: 30$ & 125.249 & 122.855 & & 100.52 & 104.39 & 96.037 & 110.684 & & & 74.274 \\
\hline $12 / 22 / 070: 00$ & 125.253 & 122.875 & & 100.534 & 104.392 & 96.037 & 110.684 & & & 74.341 \\
\hline $12 / 22 / 070: 30$ & 125.277 & 122.911 & & 100.569 & 104.435 & 96.082 & 110.714 & & 80.528 & 74.392 \\
\hline $12 / 22 / 071: 00$ & 125.305 & 122.911 & & 100.596 & 104.454 & 96.101 & 110.735 & & & 74.44 \\
\hline 12/22/07 1:30 & 125.336 & 122.927 & & 100.618 & 104.489 & 96.134 & 110.763 & & & 74.483 \\
\hline $12 / 22 / 07$ 2:00 & 125.36 & 122.932 & & 100.624 & 104.496 & 96.148 & 110.778 & & 80.565 & 74.525 \\
\hline $12 / 22 / 072: 30$ & 125.362 & 122.925 & & 100.636 & 104.51 & 96.157 & 110.783 & & & 74.562 \\
\hline $12 / 22 / 07$ 3:00 & 125.372 & 122.948 & & 100.636 & 104.511 & 96.162 & 110.783 & & & 74.595 \\
\hline $12 / 22 / 073: 30$ & 125.376 & 122.975 & & 100.639 & 104.511 & 96.16 & 110.795 & & 80.572 & 74.624 \\
\hline $12 / 22 / 074: 00$ & 125.39 & 123.004 & & 100.66 & 104.531 & 96.183 & 110.802 & & & 74.647 \\
\hline $12 / 22 / 074: 30$ & 125.409 & 123.034 & & 100.689 & 104.559 & 96.209 & 110.834 & & & 74.669 \\
\hline $12 / 22 / 075: 00$ & 125.431 & 123.029 & & 100.724 & 104.584 & 96.233 & 110.859 & & 80.587 & 74.69 \\
\hline $12 / 22 / 075: 30$ & 125.459 & 123.031 & & 100.743 & 104.614 & 96.263 & 110.885 & & & 74.711 \\
\hline $12 / 22 / 076: 00$ & 125.473 & 123.034 & & 100.734 & 104.61 & 96.263 & 110.892 & & & 74.735 \\
\hline $12 / 22 / 076: 30$ & 125.471 & 123.02 & & 100.746 & 104.612 & 96.263 & 110.892 & & 80.611 & 74.754 \\
\hline $12 / 22 / 07$ 7:00 & 125.478 & 123.018 & & 100.736 & 104.614 & 96.263 & 110.896 & & & 74.773 \\
\hline $12 / 22 / 07 \quad 7: 30$ & 125.468 & 123.056 & & 100.724 & 104.603 & 96.252 & 110.9 & & & 74.787 \\
\hline $12 / 22 / 07$ 8:00 & 125.459 & 123.084 & & 100.729 & 104.595 & 96.245 & 110.898 & & 80.616 & 74.795 \\
\hline $12 / 22 / 07$ 8:30 & 125.478 & 125.072 & & 100.784 & 104.639 & 96.289 & 110.907 & & & 74.804 \\
\hline $12 / 22 / 07$ 9:00 & 125.518 & 125.283 & & 101.228 & 104.664 & 96.313 & 110.939 & & & 74.814 \\
\hline $12 / 22 / 079: 30$ & 127.504 & 125.399 & & 101.628 & 105.246 & 97.022 & 112.432 & & 80.655 & 74.913 \\
\hline $12 / 22 / 07 \quad 10: 00$ & 127.771 & 125.469 & & 101.79 & 105.462 & 97.239 & 112.637 & & & 75.041 \\
\hline $12 / 22 / 07 \quad 10: 30$ & 127.894 & 125.521 & & 101.897 & 105.574 & 97.352 & 112.748 & & & 75.118 \\
\hline
\end{tabular}


TABLE S1.1 (Cont.)

\begin{tabular}{|c|c|c|c|c|c|c|c|c|c|c|}
\hline \multirow[b]{2}{*}{ Date and Time } & \multicolumn{10}{|c|}{ Depth from Top of Casing (ft) } \\
\hline & MW1D & MW2D & MW3D & MW4D & MW7 & MW9 & Oentrich & MW14D & MW15D & MW16D \\
\hline 12/22/07 11:00 & 127.974 & 125.551 & & 101.947 & 105.646 & 97.426 & 112.171 & & 80.758 & 75.168 \\
\hline $12 / 22 / 07$ 11:30 & 128.019 & 123.675 & & 101.983 & 105.698 & 97.475 & 112.212 & & & 75.205 \\
\hline 12/22/07 12:00 & 128.047 & 123.44 & & 101.671 & 105.73 & 97.508 & 112.242 & & & 75.232 \\
\hline $12 / 22 / 07$ 12:30 & 126.189 & 123.326 & & 101.239 & 105.22 & 96.874 & 111.625 & & 80.819 & 75.174 \\
\hline $12 / 22 / 07$ 13:00 & 125.901 & 123.242 & & 101.072 & 104.997 & 96.648 & 111.428 & & & 75.077 \\
\hline 12/22/07 13:30 & 125.773 & 123.19 & & 100.979 & 104.885 & 96.535 & 111.336 & & & 75.002 \\
\hline $12 / 22 / 07$ 14:00 & 125.695 & 123.165 & & 100.903 & 104.808 & 96.457 & 111.281 & & 80.787 & 74.955 \\
\hline $12 / 22 / 07$ 14:30 & 125.634 & 123.143 & & 100.868 & 104.757 & 96.405 & 111.247 & & & 74.922 \\
\hline $12 / 22 / 07$ 15:00 & 125.608 & 123.133 & & 100.848 & 104.73 & 96.384 & 111.219 & & & 74.897 \\
\hline $12 / 22 / 07$ 15:30 & 125.584 & 123.133 & & 100.83 & 104.709 & 96.36 & 111.2 & & 80.763 & 74.878 \\
\hline $12 / 22 / 07$ 16:00 & 125.575 & 123.129 & & 100.825 & 104.704 & 96.355 & 111.184 & & & 74.864 \\
\hline 12/22/07 16:30 & 125.568 & 123.124 & & 100.83 & 104.702 & 96.351 & 111.168 & & & 74.851 \\
\hline 12/22/07 17:00 & 125.568 & 123.124 & & 100.824 & 104.7 & 96.348 & 111.165 & & 80.765 & 74.843 \\
\hline $12 / 22 / 07$ 17:30 & 125.561 & 123.12 & & 100.822 & 104.694 & 96.344 & 111.157 & & & 74.837 \\
\hline 12/22/07 18:00 & 125.558 & 123.12 & & 100.824 & 104.692 & 96.344 & 111.15 & & & 74.831 \\
\hline $12 / 22 / 07$ 18:30 & 125.561 & 123.118 & & 100.82 & 104.692 & 96.339 & 111.144 & & 80.773 & 74.826 \\
\hline $12 / 22 / 07$ 19:00 & 125.558 & 123.113 & & 100.818 & 104.69 & 96.341 & 111.14 & & & 74.822 \\
\hline 12/22/07 19:30 & 125.554 & 123.104 & & 100.815 & 104.689 & 96.339 & 111.138 & & & 74.818 \\
\hline $12 / 22 / 0720: 00$ & 125.551 & 123.095 & & 100.81 & 104.683 & 96.334 & 111.135 & & 80.775 & 74.814 \\
\hline $12 / 22 / 0720: 30$ & 125.542 & 123.095 & & 100.801 & 104.673 & 96.322 & 111.133 & & & 74.812 \\
\hline 12/22/07 21:00 & 125.535 & 123.086 & & 100.793 & 104.664 & 96.315 & 111.131 & & & 74.81 \\
\hline $12 / 22 / 0721: 30$ & 125.528 & 123.081 & & 100.789 & 104.664 & 96.308 & 111.127 & & 80.77 & 74.804 \\
\hline 12/22/07 22:00 & 125.525 & 123.077 & & 100.784 & 104.654 & 96.303 & 111.125 & & & 74.802 \\
\hline 12/22/07 22:30 & 125.518 & 123.072 & & 100.777 & 104.65 & 96.296 & 111.123 & & & 74.797 \\
\hline $12 / 22 / 0723: 00$ & 125.516 & 123.065 & & 100.774 & 104.647 & 96.294 & 111.12 & & 80.775 & 74.791 \\
\hline 12/22/07 23:30 & 125.506 & 123.059 & & 100.767 & 104.641 & 96.289 & 111.118 & & & 74.787 \\
\hline 12/23/07 0:00 & 125.502 & 123.047 & & 100.763 & 104.637 & 96.282 & 111.116 & & & 74.783 \\
\hline $12 / 23 / 070: 30$ & 125.499 & 123.038 & & 100.756 & 104.631 & 96.278 & 111.112 & & 80.768 & 74.777 \\
\hline 12/23/07 1:00 & 125.487 & 123.029 & & 100.746 & 104.62 & 96.268 & 111.11 & & & 74.773 \\
\hline $12 / 23 / 071: 30$ & 125.478 & 123.031 & & 100.734 & 104.61 & 96.254 & 111.108 & & & 74.766 \\
\hline $12 / 23 / 07$ 2:00 & 125.464 & 123.038 & & 100.73 & 104.599 & 96.245 & 111.107 & & 80.758 & 74.764 \\
\hline $12 / 23 / 072: 30$ & 125.464 & 123.047 & & 100.736 & 104.603 & 96.247 & 111.101 & & & 74.756 \\
\hline 12/23/07 3:00 & 125.473 & 123.043 & & 100.741 & 104.61 & 96.256 & 111.099 & & & 74.75 \\
\hline $12 / 23 / 073: 30$ & 125.483 & 123.041 & & 100.744 & 104.622 & 96.266 & 111.093 & & 80.756 & 74.746 \\
\hline 12/23/07 4:00 & 125.48 & 123.036 & & 100.743 & 104.618 & 96.263 & 111.092 & & & 74.742 \\
\hline $12 / 23 / 074: 30$ & 125.476 & 123.038 & & 100.734 & 104.614 & 96.261 & 111.09 & & & 74.738 \\
\hline 12/23/07 5:00 & 125.468 & 123.043 & & 100.741 & 104.614 & 96.256 & 111.086 & & 80.743 & 74.731 \\
\hline 12/23/07 5:30 & 125.473 & 123.041 & & 100.741 & 104.612 & 96.259 & 111.086 & & & 74.723 \\
\hline $12 / 23 / 076: 00$ & 125.476 & 123.041 & & 100.744 & 104.616 & 96.261 & 111.082 & & & 74.719 \\
\hline $12 / 23 / 076: 30$ & 125.478 & 123.045 & & 100.744 & 104.614 & 96.261 & 111.08 & & 80.743 & 74.713 \\
\hline 12/23/07 7:00 & 125.476 & 123.047 & & 100.744 & 104.618 & 96.263 & 111.078 & & & 74.707 \\
\hline $12 / 23 / 077: 30$ & 125.478 & 123.047 & & 100.749 & 104.62 & 96.263 & 111.076 & & & 74.7 \\
\hline 12/23/07 8:00 & 125.48 & 123.05 & & 100.748 & 104.62 & 96.266 & 111.074 & & 80.738 & 74.694 \\
\hline 12/23/07 8:30 & 125.48 & 123.056 & & 100.748 & 104.62 & 96.266 & 111.076 & & & 74.688 \\
\hline $12 / 23 / 079: 00$ & 125.48 & 125.036 & & 100.751 & 104.622 & 96.268 & 111.074 & & & 74.684 \\
\hline 12/23/07 9:30 & 125.487 & 125.274 & & 101.223 & 104.631 & 96.278 & 111.071 & & 80.741 & 74.675 \\
\hline 12/23/07 10:00 & 127.492 & 125.406 & & 101.652 & 105.241 & 97.03 & 111.794 & & & 74.764 \\
\hline $12 / 23 / 07$ 10:30 & 127.778 & 125.487 & & 101.84 & 105.477 & 97.27 & 112.021 & & & 74.899 \\
\hline $12 / 23 / 0711: 00$ & 127.917 & 125.537 & & 101.942 & 105.61 & 97.402 & 112.147 & & 80.826 & 74.996 \\
\hline $12 / 23 / 0711: 30$ & 128.003 & 125.567 & & 102.011 & 105.69 & 97.484 & 112.224 & & & 75.062 \\
\hline $12 / 23 / 07$ 12:00 & 128.059 & 125.592 & & 102.05 & 105.745 & 97.539 & 112.28 & & & 75.108 \\
\hline $12 / 23 / 0712: 30$ & 128.09 & 125.61 & & 102.085 & 105.77 & 97.567 & 112.31 & & 80.897 & 75.147 \\
\hline 12/23/07 13:00 & 128.125 & 125.626 & & 102.1 & 105.797 & 97.591 & 112.34 & & & 75.176 \\
\hline $12 / 23 / 07$ 13:30 & 128.133 & 125.635 & & 102.118 & 105.812 & 97.607 & 112.353 & & & 75.201 \\
\hline $12 / 23 / 07$ 14:00 & 128.151 & 125.648 & & 102.131 & 105.829 & 97.624 & 112.368 & & 80.924 & 75.217 \\
\hline $12 / 23 / 07$ 14:30 & 128.159 & 125.66 & & 102.135 & 105.84 & 97.633 & 112.381 & & & 75.234 \\
\hline $12 / 23 / 07$ 15:00 & 128.168 & 125.673 & & 102.15 & 105.85 & 97.645 & 112.391 & & & 75.246 \\
\hline 12/23/07 15:30 & 128.185 & 125.689 & & 102.169 & 105.865 & 97.661 & 112.4 & & 80.966 & 75.256 \\
\hline $12 / 23 / 07$ 16:00 & 128.199 & 123.904 & & 102.178 & 105.879 & 97.673 & 112.408 & & & 75.269 \\
\hline $12 / 23 / 07 \quad 16: 30$ & 128.206 & 123.628 & & 102.18 & 105.894 & 97.69 & 112.413 & & & 75.277 \\
\hline 12/23/07 17:00 & 126.438 & 123.501 & & 101.415 & 105.422 & 97.079 & 111.809 & & 81.032 & 75.232 \\
\hline $12 / 23 / 07$ 17:30 & 126.078 & 123.417 & & 101.227 & 105.163 & 96.81 & 111.555 & & & 75.106 \\
\hline $12 / 23 / 07$ 18:00 & 125.941 & 123.367 & & 101.137 & 105.041 & 96.688 & 111.437 & & & 75.012 \\
\hline $12 / 23 / 0718: 30$ & 125.858 & 123.335 & & 101.07 & 104.963 & 96.612 & 111.368 & & 81.005 & 74.944 \\
\hline $12 / 23 / 07$ 19:00 & 125.806 & 123.319 & & 101.03 & 104.917 & 96.565 & 111.32 & & & 74.897 \\
\hline $12 / 23 / 07$ 19:30 & 125.771 & 123.313 & & 101.01 & 104.888 & 96.537 & 111.291 & & & 74.859 \\
\hline
\end{tabular}


TABLE S1.1 (Cont.)

\begin{tabular}{|c|c|c|c|c|c|c|c|c|c|c|}
\hline \multirow[b]{2}{*}{ Date and Time } & \multicolumn{10}{|c|}{ Depth from Top of Casing (ft) } \\
\hline & MW1D & MW2D & MW3D & MW4D & MW7 & MW9 & Oentrich & MW14D & MW15D & MW16D \\
\hline $12 / 23 / 0720: 00$ & 125.752 & 123.306 & & 100.996 & 104.869 & 96.518 & 111.277 & & 80.966 & 74.828 \\
\hline $12 / 23 / 0720: 30$ & 125.745 & 123.299 & & 100.994 & 104.866 & 96.513 & 111.264 & & & 74.808 \\
\hline 12/23/07 21:00 & 125.738 & 123.29 & & 100.987 & 104.862 & 96.511 & 111.27 & & & 74.789 \\
\hline $12 / 23 / 0721: 30$ & 125.728 & 123.279 & & 100.979 & 104.854 & 96.502 & 111.262 & & 80.941 & 74.777 \\
\hline $12 / 23 / 0722: 00$ & 125.721 & 123.274 & & 100.972 & 104.845 & 96.494 & 111.257 & & & 74.765 \\
\hline $12 / 23 / 0722: 30$ & 125.714 & 123.272 & & 100.965 & 104.839 & 96.49 & 111.251 & & & 74.752 \\
\hline $12 / 23 / 0723: 00$ & 125.712 & 123.27 & & 100.96 & 104.837 & 96.483 & 111.245 & & 80.932 & 74.744 \\
\hline $12 / 23 / 0723: 30$ & 125.705 & 123.267 & & 100.96 & 104.831 & 96.483 & 111.24 & & & 74.733 \\
\hline 12/24/07 0:00 & 125.702 & 123.26 & & 100.96 & 104.831 & 96.48 & 111.238 & & & 74.723 \\
\hline $12 / 24 / 070: 30$ & 125.7 & 123.251 & & 100.953 & 104.829 & 96.476 & 111.236 & & 80.932 & 74.715 \\
\hline $12 / 24 / 071: 00$ & 125.695 & 123.242 & & 100.946 & 104.822 & 96.469 & 111.232 & & & 74.709 \\
\hline 12/24/07 1:30 & 125.688 & 123.233 & & 100.942 & 104.816 & 96.464 & 111.23 & & & 74.7 \\
\hline $12 / 24 / 072: 00$ & 125.681 & 123.229 & & 100.93 & 104.807 & 96.454 & 111.229 & & 80.919 & 74.694 \\
\hline $12 / 24 / 072: 30$ & 125.669 & 123.231 & & 100.923 & 104.797 & 96.445 & 111.223 & & & 74.686 \\
\hline 12/24/07 3:00 & 125.665 & 123.226 & & 100.92 & 104.795 & 96.443 & 111.217 & & & 74.678 \\
\hline $12 / 24 / 073: 30$ & 125.662 & 123.222 & & 100.923 & 104.799 & 96.447 & 111.212 & & 80.907 & 74.667 \\
\hline $12 / 24 / 074: 00$ & 125.66 & 123.213 & & 100.917 & 104.793 & 96.443 & 111.208 & & & 74.661 \\
\hline $12 / 24 / 074: 30$ & 125.66 & 123.208 & & 100.911 & 104.786 & 96.433 & 111.206 & & & 74.653 \\
\hline $12 / 24 / 075: 00$ & 125.65 & 123.204 & & 100.903 & 104.78 & 96.426 & 111.202 & & 80.9 & 74.647 \\
\hline $12 / 24 / 075: 30$ & 125.641 & 123.202 & & 100.901 & 104.774 & 96.421 & 111.2 & & & 74.639 \\
\hline $12 / 24 / 076: 00$ & 125.641 & 123.199 & & 100.898 & 104.77 & 96.421 & 111.196 & & & 74.632 \\
\hline $12 / 24 / 076: 30$ & 125.636 & 123.197 & & 100.891 & 104.767 & 96.417 & 111.191 & & 80.885 & 74.624 \\
\hline $12 / 24 / 07$ 7:00 & 125.636 & 124.814 & & 100.892 & 104.77 & 96.414 & 111.189 & & & 74.618 \\
\hline $12 / 24 / 077: 30$ & 125.632 & 125.231 & & 100.892 & 104.763 & 96.412 & 111.187 & & & 74.611 \\
\hline $12 / 24 / 078: 00$ & 127.081 & 123.551 & & 101.511 & 105.018 & 96.77 & 111.555 & & 80.883 & 74.607 \\
\hline $12 / 24 / 078: 30$ & 127.702 & 123.381 & & 101.628 & 105.416 & 97.187 & 111.94 & & & 74.742 \\
\hline 12/24/07 9:00 & 126.05 & 123.315 & & 101.139 & 105.1 & 96.751 & 111.495 & & & 74.791 \\
\hline 12/24/07 9:30 & 125.828 & 123.274 & & 101.032 & 104.938 & 96.586 & 111.343 & & 80.924 & 74.733 \\
\hline $12 / 24 / 07$ 10:00 & 125.754 & 123.254 & & 100.984 & 104.875 & 96.523 & 111.279 & & & 74.688 \\
\hline $12 / 24 / 07 \quad 10: 30$ & 125.714 & 123.238 & & 100.956 & 104.837 & 96.483 & 111.245 & & & 74.657 \\
\hline $12 / 24 / 07$ 11:00 & 125.688 & 123.224 & & 100.937 & 104.816 & 96.464 & 111.223 & & 80.914 & 74.632 \\
\hline $12 / 24 / 0711: 30$ & 125.672 & 123.208 & & 100.922 & 104.801 & 96.45 & 111.208 & & & 74.613 \\
\hline $12 / 24 / 07$ 12:00 & 125.66 & 123.188 & & 100.911 & 104.788 & 96.438 & 111.2 & & & 74.599 \\
\hline $12 / 24 / 07$ 12:30 & 125.646 & 123.168 & & 100.892 & 104.769 & 96.419 & 111.195 & & 80.927 & 74.589 \\
\hline $12 / 24 / 07$ 13:00 & 125.624 & 123.149 & & 100.875 & 104.751 & 96.398 & 111.189 & & & 74.578 \\
\hline $12 / 24 / 07$ 13:30 & 125.608 & 123.138 & & 100.849 & 104.732 & 96.379 & 111.185 & & & 74.57 \\
\hline $12 / 24 / 07$ 14:00 & 125.589 & 123.124 & & 100.837 & 104.715 & 96.36 & 111.18 & & 80.927 & 74.558 \\
\hline $12 / 24 / 0714: 30$ & 125.575 & 123.118 & & 100.822 & 104.702 & 96.348 & 111.178 & & & 74.545 \\
\hline $12 / 24 / 07$ 15:00 & 125.561 & 123.104 & & 100.813 & 104.69 & 96.337 & 111.174 & & & 74.535 \\
\hline $12 / 24 / 07$ 15:30 & 125.554 & 123.088 & & 100.803 & 104.683 & 96.329 & 111.17 & & 80.944 & 74.525 \\
\hline $12 / 24 / 0716: 00$ & 125.539 & 123.075 & & 100.789 & 104.668 & 96.313 & 111.17 & & & 74.514 \\
\hline $12 / 24 / 07 \quad 16: 30$ & 125.525 & 123.068 & & 100.772 & 104.652 & 96.299 & 111.166 & & & 74.504 \\
\hline $12 / 24 / 07$ 17:00 & 125.513 & 123.059 & & 100.762 & 104.641 & 96.287 & 111.163 & & 80.895 & 74.494 \\
\hline $12 / 24 / 07$ 17:30 & 125.504 & 123.043 & & 100.751 & 104.633 & 96.28 & 111.148 & & & 74.483 \\
\hline $12 / 24 / 0718: 00$ & 125.494 & 123.029 & & 100.743 & 104.622 & 96.268 & 111.142 & & & 74.473 \\
\hline $12 / 24 / 0718: 30$ & 125.476 & 123.02 & & 100.729 & 104.607 & 96.252 & 111.129 & & 80.863 & 74.465 \\
\hline $12 / 24 / 07$ 19:00 & 125.466 & 123.016 & & 100.717 & 104.597 & 96.242 & 111.122 & & & 74.455 \\
\hline 12/24/07 19:30 & 125.457 & 123.011 & & 100.71 & 104.588 & 96.233 & 111.118 & & & 74.444 \\
\hline $12 / 24 / 0720: 00$ & 125.452 & 123.011 & & 100.703 & 104.584 & 96.228 & 111.112 & & 80.839 & 74.434 \\
\hline $12 / 24 / 0720: 30$ & 125.445 & 123.018 & & 100.705 & 104.578 & 96.226 & 111.103 & & & 74.425 \\
\hline $12 / 24 / 0721: 00$ & 125.447 & 123.011 & & 100.706 & 104.578 & 96.223 & 111.101 & & & 74.415 \\
\hline $12 / 24 / 0721: 30$ & 125.45 & 123.004 & & 100.705 & 104.582 & 96.228 & 111.097 & & 80.819 & 74.407 \\
\hline 12/24/07 22:00 & 125.447 & 123.007 & & 100.703 & 104.58 & 96.226 & 111.099 & & & 74.401 \\
\hline $12 / 24 / 0722: 30$ & 125.44 & 123 & & 100.694 & 104.571 & 96.216 & 111.097 & & & 74.392 \\
\hline $12 / 24 / 0723: 00$ & 125.438 & 122.988 & & 100.694 & 104.571 & 96.216 & 111.093 & & 80.809 & 74.386 \\
\hline 12/24/07 23:30 & 125.433 & 122.979 & & 100.684 & 104.565 & 96.212 & 111.092 & & & 74.38 \\
\hline $12 / 25 / 07$ 0:00 & 125.424 & 122.97 & & 100.675 & 104.553 & 96.2 & 111.088 & & & 74.374 \\
\hline 12/25/07 0:30 & 125.414 & 122.959 & & 100.67 & 104.546 & 96.19 & 111.076 & & 80.797 & 74.367 \\
\hline $12 / 25 / 071: 00$ & 125.405 & 122.948 & & 100.655 & 104.534 & 96.181 & 111.065 & & & 74.359 \\
\hline $12 / 25 / 071: 30$ & 125.393 & 122.945 & & 100.644 & 104.525 & 96.172 & 111.054 & & & 74.353 \\
\hline $12 / 25 / 072: 00$ & 125.383 & 122.938 & & 100.636 & 104.517 & 96.157 & 111.046 & & 80.778 & 74.345 \\
\hline $12 / 25 / 072: 30$ & 125.376 & 122.934 & & 100.629 & 104.51 & 96.153 & 111.037 & & & 74.336 \\
\hline $12 / 25 / 073: 00$ & 125.367 & 122.934 & & 100.627 & 104.502 & 96.146 & 111.031 & & & 74.328 \\
\hline $12 / 25 / 073: 30$ & 125.367 & 122.927 & & 100.629 & 104.504 & 96.143 & 111.03 & & 80.751 & 74.322 \\
\hline $12 / 25 / 074: 00$ & 125.367 & 122.925 & & 100.622 & 104.504 & 96.146 & 111.028 & & & 74.312 \\
\hline $12 / 25 / 074: 30$ & 125.357 & 122.918 & & 100.62 & 104.498 & 96.139 & 111.024 & & & 74.303 \\
\hline
\end{tabular}


TABLE S1.1 (Cont.)

\begin{tabular}{|c|c|c|c|c|c|c|c|c|c|c|}
\hline \multirow[b]{2}{*}{ Date and Time } & \multicolumn{10}{|c|}{ Depth from Top of Casing (ft) } \\
\hline & MW1D & MW2D & MW3D & MW4D & MW7 & MW9 & Oentrich & MW14D & MW15D & MW16D \\
\hline $12 / 25 / 075: 00$ & 125.355 & 122.907 & & 100.613 & 104.491 & 96.134 & 111.022 & & 80.746 & 74.297 \\
\hline $12 / 25 / 075: 30$ & 125.35 & 122.9 & & 100.603 & 104.485 & 96.129 & 111.016 & & & 74.291 \\
\hline $12 / 25 / 076: 00$ & 125.341 & 122.902 & & 100.596 & 104.475 & 96.117 & 111.007 & & & 74.283 \\
\hline $12 / 25 / 076: 30$ & 125.331 & 122.911 & & 100.594 & 104.471 & 96.113 & 110.998 & & 80.712 & 74.274 \\
\hline $12 / 25 / 07$ 7:00 & 125.331 & 122.914 & & 100.598 & 104.47 & 96.113 & 110.996 & & & 74.266 \\
\hline $12 / 25 / 077: 30$ & 125.338 & 122.918 & & 100.61 & 104.481 & 96.122 & 110.994 & & & 74.26 \\
\hline 12/25/07 8:00 & 125.343 & 122.92 & & 100.61 & 104.485 & 96.129 & 110.992 & & 80.69 & 74.254 \\
\hline $12 / 25 / 078: 30$ & 125.348 & 124.746 & & 100.613 & 104.487 & 96.131 & 110.992 & & & 74.25 \\
\hline 12/25/07 9:00 & 125.353 & 125.059 & & 100.62 & 104.494 & 96.136 & 110.992 & & & 74.243 \\
\hline $12 / 25 / 079: 30$ & 127.116 & 125.211 & & 101.347 & 104.909 & 96.676 & 111.521 & & 80.68 & 74.272 \\
\hline $12 / 25 / 07$ 10:00 & 127.537 & 125.295 & & 101.57 & 105.22 & 96.994 & 111.813 & & & 74.421 \\
\hline 12/25/07 10:30 & 127.698 & 125.347 & & 101.692 & 105.372 & 97.145 & 111.946 & & & 74.531 \\
\hline $12 / 25 / 0711: 00$ & 127.785 & 125.378 & & 101.759 & 105.456 & 97.232 & 112.027 & & 80.746 & 74.607 \\
\hline $12 / 25 / 07$ 11:30 & 127.839 & 125.392 & & 101.806 & 105.509 & 97.286 & 112.075 & & & 74.661 \\
\hline $12 / 25 / 0712: 00$ & 127.875 & 125.397 & & 101.826 & 105.545 & 97.322 & 112.113 & & & 74.702 \\
\hline $12 / 25 / 0712: 30$ & 127.894 & 125.401 & & 101.844 & 105.555 & 97.331 & 112.143 & & 80.814 & 74.733 \\
\hline $12 / 25 / 07$ 13:00 & 127.913 & 125.394 & & 101.849 & 105.566 & 97.343 & 112.166 & & & 74.758 \\
\hline $12 / 25 / 07$ 13:30 & 127.927 & 125.39 & & 101.847 & 105.572 & 97.345 & 112.182 & & & 74.779 \\
\hline $12 / 25 / 07$ 14:00 & 127.941 & 123.703 & & 101.844 & 105.566 & 97.341 & 112.19 & & 80.846 & 74.8 \\
\hline $12 / 25 / 07 \quad 14: 30$ & 127.946 & 123.36 & & 101.838 & 105.566 & 97.341 & 112.184 & & & 74.818 \\
\hline $12 / 25 / 07$ 15:00 & 126.563 & 123.226 & & 101.166 & 105.212 & 96.869 & 111.709 & & & 74.814 \\
\hline $12 / 25 / 07$ 15:30 & 126.258 & 123.152 & & 100.958 & 104.896 & 96.542 & 111.401 & & 80.868 & 74.702 \\
\hline $12 / 25 / 0716: 00$ & 126.133 & 123.104 & & 100.86 & 104.77 & 96.412 & 111.268 & & & 74.622 \\
\hline $12 / 25 / 07$ 16:30 & 126.064 & 123.072 & & 100.803 & 104.698 & 96.344 & 111.197 & & & 74.578 \\
\hline $12 / 25 / 07$ 17:00 & 126.019 & 123.056 & & 100.767 & 104.654 & 96.301 & 111.148 & & 80.829 & 74.562 \\
\hline $12 / 25 / 07$ 17:30 & 125.986 & 123.052 & & 100.744 & 104.628 & 96.275 & 111.118 & & & 74.554 \\
\hline $12 / 25 / 07$ 18:00 & 125.962 & 123.045 & & 100.734 & 104.612 & 96.259 & 111.093 & & & 74.556 \\
\hline $12 / 25 / 0718: 30$ & 125.946 & 123.038 & & 100.732 & 104.607 & 96.254 & 111.076 & & 80.792 & 74.564 \\
\hline 12/25/07 19:00 & 125.934 & 123.036 & & 100.729 & 104.605 & 96.249 & 111.063 & & & 74.572 \\
\hline $12 / 25 / 07$ 19:30 & 125.927 & 123.052 & & 100.722 & 104.599 & 96.242 & 111.056 & & & 74.585 \\
\hline $12 / 25 / 0720: 00$ & 125.92 & 123.052 & & 100.73 & 104.599 & 96.242 & 111.048 & & 80.778 & 74.595 \\
\hline $12 / 25 / 0720: 30$ & 125.91 & 123.052 & & 100.739 & 104.616 & 96.263 & 111.05 & & & 74.605 \\
\hline $12 / 25 / 0721: 00$ & 125.906 & 123.054 & & 100.744 & 104.616 & 96.263 & 111.048 & & & 74.618 \\
\hline $12 / 25 / 0721: 30$ & 125.896 & 123.054 & & 100.743 & 104.616 & 96.261 & 111.048 & & 80.78 & 74.628 \\
\hline 12/25/07 22:00 & 125.892 & 123.054 & & 100.746 & 104.618 & 96.263 & 111.048 & & & 74.638 \\
\hline $12 / 25 / 0722: 30$ & 125.889 & 123.059 & & 100.744 & 104.616 & 96.263 & 111.048 & & & 74.649 \\
\hline $12 / 25 / 0723: 00$ & 125.887 & 123.063 & & 100.748 & 104.618 & 96.266 & 111.048 & & 80.775 & 74.657 \\
\hline 12/25/07 23:30 & 125.88 & 123.061 & & 100.751 & 104.624 & 96.27 & 111.048 & & & 74.663 \\
\hline 12/26/07 0:00 & 125.877 & 123.061 & & 100.755 & 104.63 & 96.275 & 111.052 & & & 74.669 \\
\hline 12/26/07 0:30 & 125.873 & 123.061 & & 100.751 & 104.628 & 96.273 & 111.056 & & 80.775 & 74.673 \\
\hline 12/26/07 1:00 & 125.868 & 123.056 & & 100.753 & 104.628 & 96.273 & 111.054 & & & 74.678 \\
\hline $12 / 26 / 071: 30$ & 125.866 & 123.056 & & 100.753 & 104.626 & 96.27 & 111.056 & & & 74.682 \\
\hline $12 / 26 / 072: 00$ & 125.861 & 123.045 & & 100.755 & 104.624 & 96.27 & 111.054 & & 80.77 & 74.684 \\
\hline 12/26/07 2:30 & 125.854 & 123.047 & & 100.748 & 104.624 & 96.27 & 111.052 & & & 74.686 \\
\hline $12 / 26 / 073: 00$ & 125.851 & 123.043 & & 100.739 & 104.61 & 96.259 & 111.054 & & & 74.688 \\
\hline 12/26/07 3:30 & 125.844 & 123.047 & & 100.741 & 104.614 & 96.261 & 111.05 & & 80.77 & 74.686 \\
\hline $12 / 26 / 074: 00$ & 125.84 & 123.029 & & 100.737 & 104.612 & 96.259 & 111.048 & & & 74.684 \\
\hline $12 / 26 / 074: 30$ & 125.832 & 123.009 & & 100.736 & 104.618 & 96.263 & 111.046 & & & 74.682 \\
\hline $12 / 26 / 07$ 5:00 & 125.832 & 123.016 & & 100.712 & 104.601 & 96.245 & 111.046 & & 80.756 & 74.68 \\
\hline 12/26/07 5:30 & 125.832 & 123.036 & & 100.708 & 104.574 & 96.221 & 111.046 & & & 74.675 \\
\hline $12 / 26 / 076: 00$ & 125.825 & 123.041 & & 100.722 & 104.584 & 96.228 & 111.042 & & & 74.667 \\
\hline $12 / 26 / 076: 30$ & 125.818 & 123.031 & & 100.737 & 104.607 & 96.249 & 111.037 & & 80.741 & 74.659 \\
\hline 12/26/07 7:00 & 125.816 & 124.721 & & 100.729 & 104.609 & 96.254 & 111.041 & & & 74.655 \\
\hline $12 / 26 / 077: 30$ & 125.816 & 125.09 & & 100.724 & 104.603 & 96.247 & 111.041 & & & 74.649 \\
\hline $12 / 26 / 078: 00$ & 127.215 & 125.251 & & 101.439 & 104.944 & 96.714 & 111.518 & & 80.721 & 74.655 \\
\hline 12/26/07 8:30 & 127.558 & 125.344 & & 101.673 & 105.305 & 97.091 & 111.873 & & & 74.804 \\
\hline 12/26/07 9:00 & 127.693 & 124.236 & & 101.802 & 105.465 & 97.253 & 112.019 & & & 74.917 \\
\hline 12/26/07 9:30 & 127.771 & 123.492 & & 101.88 & 105.559 & 97.345 & 112.107 & & 80.802 & 74.994 \\
\hline $12 / 26 / 07$ 10:00 & 127.185 & 123.324 & & 101.375 & 105.593 & 97.4 & 112.145 & & & 75.05 \\
\hline $12 / 26 / 07 \quad 10: 30$ & 126.201 & 123.233 & & 101.077 & 105.037 & 96.683 & 111.448 & & & 74.975 \\
\hline $12 / 26 / 0711: 00$ & 126.052 & 123.177 & & 100.96 & 104.877 & 96.52 & 111.291 & & 80.814 & 74.88 \\
\hline $12 / 26 / 0711: 30$ & 125.977 & 123.138 & & 100.886 & 104.789 & 96.433 & 111.212 & & & 74.812 \\
\hline $12 / 26 / 07$ 12:00 & 125.934 & 123.106 & & 100.841 & 104.734 & 96.379 & 111.165 & & & 74.764 \\
\hline $12 / 26 / 0712: 30$ & 125.908 & 123.09 & & 100.811 & 104.694 & 96.344 & 111.137 & & 80.804 & 74.729 \\
\hline $12 / 26 / 07$ 13:00 & 125.887 & 123.07 & & 100.787 & 104.671 & 96.315 & 111.116 & & & 74.704 \\
\hline $12 / 26 / 07$ 13:30 & 125.87 & 123.059 & & 100.772 & 104.652 & 96.299 & 111.101 & & & 74.682 \\
\hline
\end{tabular}


TABLE S1.1 (Cont.)

\begin{tabular}{|c|c|c|c|c|c|c|c|c|c|c|}
\hline \multirow[b]{2}{*}{ Date and Time } & \multicolumn{10}{|c|}{ Depth from Top of Casing (ft) } \\
\hline & MW1D & MW2D & MW3D & MW4D & MW7 & MW9 & Oentrich & MW14D & MW15D & MW16D \\
\hline $12 / 26 / 07$ 14:00 & 125.863 & 123.047 & & 100.749 & 104.631 & 96.28 & 111.092 & & 80.792 & 74.663 \\
\hline $12 / 26 / 07$ 14:30 & 125.854 & 123.041 & & 100.744 & 104.62 & 96.266 & 111.084 & & & 74.649 \\
\hline $12 / 26 / 07$ 15:00 & 125.847 & 123.038 & & 100.736 & 104.612 & 96.256 & 111.076 & & & 74.634 \\
\hline $12 / 26 / 07$ 15:30 & 125.84 & 123.034 & & 100.729 & 104.607 & 96.252 & 111.071 & & 80.758 & 74.622 \\
\hline $12 / 26 / 07$ 16:00 & 125.835 & 123.031 & & 100.727 & 104.601 & 96.247 & 111.067 & & & 74.611 \\
\hline $12 / 26 / 07$ 16:30 & 125.83 & 123.031 & & 100.725 & 104.601 & 96.245 & 111.061 & & & 74.601 \\
\hline $12 / 26 / 07$ 17:00 & 125.825 & 123.034 & & 100.725 & 104.597 & 96.245 & 111.06 & & 80.748 & 74.593 \\
\hline $12 / 26 / 07$ 17:30 & 125.821 & 123.036 & & 100.725 & 104.599 & 96.247 & 111.056 & & & 74.587 \\
\hline 12/26/07 18:00 & 125.816 & 123.036 & & 100.729 & 104.601 & 96.247 & 111.054 & & & 74.58 \\
\hline $12 / 26 / 0718: 30$ & 125.814 & 123.043 & & 100.732 & 104.607 & 96.252 & 111.021 & & 80.741 & 74.574 \\
\hline $12 / 26 / 07$ 19:00 & 125.809 & 123.045 & & 100.734 & 104.605 & 96.249 & 111.016 & & & 74.57 \\
\hline 12/26/07 19:30 & 125.804 & 123.05 & & 100.743 & 104.612 & 96.256 & 111.015 & & & 74.564 \\
\hline $12 / 26 / 0720: 00$ & 125.802 & 123.054 & & 100.739 & 104.614 & 96.259 & 111.017 & & 80.736 & 74.562 \\
\hline $12 / 26 / 0720: 30$ & 125.799 & 123.063 & & 100.751 & 104.618 & 96.263 & 111.017 & & & 74.558 \\
\hline 12/26/07 21:00 & 125.797 & 123.065 & & 100.756 & 104.622 & 96.268 & 111.018 & & & 74.554 \\
\hline $12 / 26 / 0721: 30$ & 125.79 & 123.056 & & 100.76 & 104.631 & 96.275 & 111.028 & & 80.734 & 74.549 \\
\hline $12 / 26 / 0722: 00$ & 125.721 & 123.059 & & 100.763 & 104.637 & 96.28 & 111.031 & & & 74.545 \\
\hline $12 / 26 / 0722: 30$ & 125.643 & 123.059 & & 100.753 & 104.626 & 96.27 & 111.035 & & & 74.541 \\
\hline 12/26/07 23:00 & 125.577 & 123.065 & & 100.755 & 104.626 & 96.27 & 111.033 & & 80.741 & 74.535 \\
\hline $12 / 26 / 0723: 30$ & 125.537 & 123.063 & & 100.756 & 104.626 & 96.27 & 111.035 & & & 74.529 \\
\hline 12/27/07 0:00 & 125.516 & 123.056 & & 100.763 & 104.633 & 96.28 & 111.035 & & & 74.523 \\
\hline $12 / 27 / 070: 30$ & 125.511 & 123.059 & & 100.762 & 104.637 & 96.28 & 111.043 & & 80.736 & 74.516 \\
\hline 12/27/07 1:00 & 125.506 & 123.05 & & 100.755 & 104.626 & 96.27 & 111.043 & & & 74.514 \\
\hline $12 / 27 / 071: 30$ & 125.499 & 123.054 & & 100.743 & 104.631 & 96.273 & 111.041 & & & 74.504 \\
\hline $12 / 27 / 072: 00$ & 125.494 & 123.065 & & 100.748 & 104.618 & 96.263 & 111.043 & & 80.731 & 74.498 \\
\hline 12/27/07 2:30 & 125.49 & 123.072 & & 100.756 & 104.624 & 96.27 & 111.041 & & & 74.492 \\
\hline 12/27/07 3:00 & 125.49 & 123.072 & & 100.763 & 104.635 & 96.28 & 111.037 & & & 74.483 \\
\hline 12/27/07 3:30 & 125.49 & 123.079 & & 100.772 & 104.645 & 96.289 & 111.041 & & 80.729 & 74.477 \\
\hline $12 / 27 / 074: 00$ & 125.497 & 123.077 & & 100.775 & 104.645 & 96.292 & 111.048 & & & 74.471 \\
\hline $12 / 27 / 074: 30$ & 125.497 & 123.07 & & 100.775 & 104.65 & 96.294 & 111.05 & & & 74.465 \\
\hline 12/27/07 5:00 & 125.502 & 123.075 & & 100.77 & 104.647 & 96.292 & 111.052 & & 80.738 & 74.458 \\
\hline $12 / 27 / 075: 30$ & 125.502 & 123.081 & & 100.768 & 104.641 & 96.285 & 111.052 & & & 74.454 \\
\hline $12 / 27 / 076: 00$ & 125.499 & 123.102 & & 100.774 & 104.643 & 96.287 & 111.05 & & & 74.446 \\
\hline $12 / 27 / 076: 30$ & 125.502 & 123.104 & & 100.789 & 104.656 & 96.296 & 111.05 & & 80.734 & 74.44 \\
\hline 12/27/07 7:00 & 125.504 & 124.458 & & 100.801 & 104.673 & 96.318 & 111.071 & & & 74.436 \\
\hline $12 / 27 / 077: 30$ & 125.513 & 125.124 & & 100.803 & 104.679 & 96.322 & 111.078 & & & 74.432 \\
\hline 12/27/07 8:00 & 126.428 & 125.31 & & 101.399 & 104.748 & 96.419 & 111.234 & & 80.729 & 74.427 \\
\hline 12/27/07 8:30 & 127.331 & 125.415 & & 101.723 & 105.334 & 97.119 & 111.856 & & & 74.547 \\
\hline $12 / 27 / 079: 00$ & 127.617 & 125.496 & & 101.859 & 105.519 & 97.308 & 112.04 & & & 74.675 \\
\hline 12/27/07 9:30 & 127.797 & 125.56 & & 101.957 & 105.623 & 97.414 & 112.135 & & 80.807 & 74.76 \\
\hline $12 / 27 / 07$ 10:00 & 127.925 & 125.592 & & 102.026 & 105.703 & 97.494 & 112.201 & & & 74.818 \\
\hline $12 / 27 / 07$ 10:30 & 128.012 & 123.936 & & 102.083 & 105.766 & 97.555 & 112.269 & & & 74.859 \\
\hline $12 / 27 / 0711: 00$ & 128.078 & 123.623 & & 102.1 & 105.799 & 97.588 & 112.299 & & 80.883 & 74.893 \\
\hline 12/27/07 11:30 & 126.662 & 123.426 & & 101.413 & 105.448 & 97.11 & 111.814 & & & 74.895 \\
\hline $12 / 27 / 0712: 00$ & 126.211 & 123.34 & & 101.18 & 105.132 & 96.78 & 111.503 & & & 74.775 \\
\hline $12 / 27 / 07$ 12:30 & 125.981 & 123.283 & & 101.063 & 104.972 & 96.617 & 111.375 & & 80.888 & 74.682 \\
\hline 12/27/07 13:00 & 125.837 & 123.229 & & 100.989 & 104.892 & 96.539 & 111.304 & & & 74.613 \\
\hline 12/27/07 13:30 & 125.752 & 123.192 & & 100.94 & 104.835 & 96.48 & 111.258 & & & 74.564 \\
\hline $12 / 27 / 0714: 00$ & 125.695 & 123.168 & & 100.901 & 104.784 & 96.431 & 111.23 & & 80.856 & 74.527 \\
\hline $12 / 27 / 0714: 30$ & 125.648 & 123.156 & & 100.867 & 104.751 & 96.398 & 111.208 & & & 74.496 \\
\hline $12 / 27 / 07$ 15:00 & 125.615 & 123.138 & & 100.841 & 104.725 & 96.37 & 111.193 & & & 74.469 \\
\hline $12 / 27 / 07$ 15:30 & 125.594 & 123.115 & & 100.832 & 104.715 & 96.362 & 111.18 & & 80.839 & 74.448 \\
\hline $12 / 27 / 0716: 00$ & 125.58 & 123.113 & & 100.815 & 104.702 & 96.346 & 111.17 & & & 74.427 \\
\hline $12 / 27 / 07 \quad 16: 30$ & 125.561 & 123.12 & & 100.801 & 104.681 & 96.325 & 111.165 & & & 74.413 \\
\hline 12/27/07 17:00 & 125.546 & 123.12 & & 100.804 & 104.677 & 96.318 & 111.157 & & 80.822 & 74.396 \\
\hline 12/27/07 17:30 & 125.546 & 123.118 & & 100.813 & 104.687 & 96.329 & 111.148 & & & 74.38 \\
\hline $12 / 27 / 0718: 00$ & 125.549 & 123.109 & & 100.82 & 104.683 & 96.329 & 111.144 & & & 74.37 \\
\hline 12/27/07 18:30 & 125.558 & 123.109 & & 100.803 & 104.683 & 96.329 & 111.138 & & 80.814 & 74.361 \\
\hline 12/27/07 19:00 & 125.549 & 123.093 & & 100.798 & 104.673 & 96.318 & 111.136 & & & 74.353 \\
\hline $12 / 27 / 07$ 19:30 & 125.539 & 123.099 & & 100.803 & 104.675 & 96.322 & 111.131 & & & 74.345 \\
\hline $12 / 27 / 0720: 00$ & 125.539 & 123.118 & & 100.789 & 104.662 & 96.313 & 111.129 & & 80.807 & 74.339 \\
\hline $12 / 27 / 0720: 30$ & 125.532 & 123.102 & & 100.797 & 104.67 & 96.313 & 111.125 & & & 74.33 \\
\hline $12 / 27 / 0721: 00$ & 125.535 & 123.081 & & 100.808 & 104.685 & 96.329 & 111.121 & & & 74.328 \\
\hline $12 / 27 / 0721: 30$ & 125.544 & 123.068 & & 100.796 & 104.668 & 96.313 & 111.121 & & 80.804 & 74.334 \\
\hline $12 / 27 / 0722: 00$ & 125.535 & 123.054 & & 100.765 & 104.649 & 96.292 & 111.123 & & & 74.341 \\
\hline $12 / 27 / 0722: 30$ & 125.516 & 123.072 & & 100.753 & 104.635 & 96.28 & 111.119 & & & 74.345 \\
\hline
\end{tabular}


TABLE S1.1 (Cont.)

\begin{tabular}{|c|c|c|c|c|c|c|c|c|c|c|}
\hline \multirow[b]{2}{*}{ Date and Time } & \multicolumn{10}{|c|}{ Depth from Top of Casing (ft) } \\
\hline & MW1D & MW2D & MW3D & MW4D & MW7 & MW9 & Oentrich & MW14D & MW15D & MW16D \\
\hline $12 / 27 / 0723: 00$ & 125.499 & 123.077 & & 100.763 & 104.626 & 96.268 & 111.108 & & 80.787 & 74.347 \\
\hline $12 / 27 / 0723: 30$ & 125.499 & 123.068 & & 100.761 & 104.641 & 96.287 & 111.103 & & & 74.349 \\
\hline 12/28/07 0:00 & 125.506 & 123.052 & & 100.76 & 104.647 & 96.292 & 111.101 & & & 74.353 \\
\hline $12 / 28 / 070: 30$ & 125.504 & 123.052 & & 100.744 & 104.633 & 96.28 & 111.101 & & 80.78 & 74.359 \\
\hline $12 / 28 / 071: 00$ & 125.494 & 123.045 & & 100.741 & 104.622 & 96.268 & 111.099 & & & 74.363 \\
\hline $12 / 28 / 071: 30$ & 125.487 & 123.025 & & 100.739 & 104.62 & 96.266 & 111.097 & & & 74.365 \\
\hline $12 / 28 / 072: 00$ & 125.485 & 123.02 & & 100.73 & 104.614 & 96.261 & 111.097 & & 80.768 & 74.365 \\
\hline $12 / 28 / 072: 30$ & 125.478 & 123.016 & & 100.713 & 104.593 & 96.242 & 111.089 & & & 74.365 \\
\hline 12/28/07 3:00 & 125.459 & 123.004 & & 100.708 & 104.588 & 96.235 & 111.078 & & & 74.359 \\
\hline $12 / 28 / 073: 30$ & 125.452 & 122.993 & & 100.703 & 104.582 & 96.23 & 111.075 & & 80.751 & 74.355 \\
\hline $12 / 28 / 074: 00$ & 125.445 & 122.991 & & 100.696 & 104.572 & 96.219 & 111.069 & & & 74.353 \\
\hline 12/28/07 4:30 & 125.438 & 122.982 & & 100.684 & 104.559 & 96.204 & 111.061 & & & 74.347 \\
\hline $12 / 28 / 075: 00$ & 125.428 & 122.977 & & 100.679 & 104.555 & 96.204 & 111.052 & & 80.726 & 74.341 \\
\hline $12 / 28 / 075: 30$ & 125.421 & 122.977 & & 100.672 & 104.55 & 96.195 & 111.048 & & & 74.336 \\
\hline $12 / 28 / 076: 00$ & 125.414 & 122.982 & & 100.67 & 104.546 & 96.19 & 111.045 & & & 74.33 \\
\hline $12 / 28 / 076: 30$ & 125.412 & 122.984 & & 100.675 & 104.55 & 96.193 & 111.041 & & 80.714 & 74.326 \\
\hline $12 / 28 / 07$ 7:00 & 125.414 & 124.875 & & 100.677 & 104.551 & 96.195 & 111.039 & & & 74.326 \\
\hline $12 / 28 / 077: 30$ & 125.416 & 125.14 & & 100.887 & 104.553 & 96.2 & 111.037 & & & 74.326 \\
\hline $12 / 28 / 078: 00$ & 127.125 & 125.267 & & 101.514 & 105.088 & 96.869 & 111.69 & & 80.704 & 74.392 \\
\hline $12 / 28 / 078: 30$ & 127.518 & 125.351 & & 101.704 & 105.347 & 97.133 & 111.935 & & & 74.556 \\
\hline 12/28/07 9:00 & 127.728 & 125.412 & & 101.806 & 105.473 & 97.263 & 112.057 & & & 74.68 \\
\hline $12 / 28 / 07$ 9:30 & 127.842 & 125.458 & & 101.88 & 105.561 & 97.35 & 112.128 & & 80.782 & 74.769 \\
\hline 12/28/07 10:00 & 127.915 & 125.496 & & 101.935 & 105.62 & 97.407 & 112.177 & & & 74.837 \\
\hline $12 / 28 / 07$ 10:30 & 127.967 & 125.521 & & 101.976 & 105.667 & 97.456 & 112.211 & & & 74.886 \\
\hline $12 / 28 / 0711: 00$ & 128.007 & 124.886 & & 102.011 & 105.705 & 97.494 & 112.237 & & 80.856 & 74.924 \\
\hline 12/28/07 11:30 & 128.038 & 123.626 & & 102.031 & 105.73 & 97.52 & 112.256 & & & 74.957 \\
\hline $12 / 28 / 07$ 12:00 & 128.062 & 123.41 & & 101.535 & 105.751 & 97.543 & 112.273 & & & 74.996 \\
\hline $12 / 28 / 07$ 12:30 & 126.265 & 123.306 & & 101.184 & 105.155 & 96.806 & 111.555 & & 80.88 & 74.905 \\
\hline 12/28/07 13:00 & 125.948 & 123.231 & & 101.03 & 104.955 & 96.601 & 111.373 & & & 74.777 \\
\hline $12 / 28 / 07$ 13:30 & 125.78 & 123.19 & & 100.946 & 104.854 & 96.499 & 111.277 & & & 74.678 \\
\hline $12 / 28 / 07$ 14:00 & 125.691 & 123.165 & & 100.891 & 104.784 & 96.428 & 111.221 & & 80.836 & 74.655 \\
\hline $12 / 28 / 07$ 14:30 & 125.636 & 123.145 & & 100.86 & 104.742 & 96.386 & 111.183 & & & 74.622 \\
\hline $12 / 28 / 07$ 15:00 & 125.603 & 123.131 & & 100.837 & 104.719 & 96.365 & 111.157 & & & 74.597 \\
\hline $12 / 28 / 07$ 15:30 & 125.58 & 123.131 & & 100.817 & 104.7 & 96.346 & 111.138 & & 80.814 & 74.574 \\
\hline 12/28/07 16:00 & 125.563 & 123.129 & & 100.813 & 104.687 & 96.329 & 111.125 & & & 74.556 \\
\hline $12 / 28 / 07$ 16:30 & 125.558 & 123.12 & & 100.818 & 104.69 & 96.337 & 111.114 & & & 74.56 \\
\hline $12 / 28 / 07$ 17:00 & 125.558 & 123.109 & & 100.813 & 104.687 & 96.334 & 111.104 & & 80.807 & 74.556 \\
\hline 12/28/07 17:30 & 125.554 & 123.104 & & 100.803 & 104.681 & 96.327 & 111.099 & & & 74.547 \\
\hline $12 / 28 / 07$ 18:00 & 125.546 & 123.097 & & 100.796 & 104.67 & 96.315 & 111.095 & & & 74.535 \\
\hline $12 / 28 / 07$ 18:30 & 125.539 & 123.099 & & 100.794 & 104.666 & 96.308 & 111.089 & & 80.797 & 74.531 \\
\hline 12/28/07 19:00 & 125.537 & 123.109 & & 100.787 & 104.662 & 96.306 & 111.086 & & & 74.508 \\
\hline $12 / 28 / 07$ 19:30 & 125.53 & 123.106 & & 100.794 & 104.662 & 96.308 & 111.08 & & & 74.479 \\
\hline $12 / 28 / 0720: 00$ & 125.535 & 123.106 & & 100.803 & 104.671 & 96.318 & 111.082 & & 80.787 & 74.452 \\
\hline $12 / 28 / 0720: 30$ & 125.542 & 123.111 & & 100.798 & 104.673 & 96.318 & 111.084 & & & 74.429 \\
\hline $12 / 28 / 0721: 00$ & 125.539 & 123.095 & & 100.801 & 104.67 & 96.318 & 111.082 & & & 74.411 \\
\hline $12 / 28 / 0721: 30$ & 125.539 & 123.093 & & 100.799 & 104.679 & 96.32 & 111.084 & & 80.785 & 74.39 \\
\hline 12/28/07 22:00 & 125.537 & 123.104 & & 100.786 & 104.66 & 96.303 & 111.086 & & & 74.378 \\
\hline $12 / 28 / 0722: 30$ & 125.525 & 123.099 & & 100.796 & 104.656 & 96.301 & 111.084 & & & 74.361 \\
\hline 12/28/07 23:00 & 125.53 & 123.099 & & 100.794 & 104.67 & 96.315 & 111.08 & & 80.782 & 74.345 \\
\hline 12/28/07 23:30 & 125.535 & 123.099 & & 100.794 & 104.668 & 96.313 & 111.08 & & & 74.332 \\
\hline $12 / 29 / 07$ 0:00 & 125.532 & 123.097 & & 100.793 & 104.668 & 96.311 & 111.078 & & & 74.318 \\
\hline 12/29/07 0:30 & 125.535 & 123.104 & & 100.791 & 104.664 & 96.313 & 111.076 & & 80.778 & 74.308 \\
\hline 12/29/07 1:00 & 125.532 & 123.102 & & 100.793 & 104.666 & 96.311 & 111.074 & & & 74.297 \\
\hline $12 / 29 / 071: 30$ & 125.532 & 123.095 & & 100.798 & 104.67 & 96.315 & 111.074 & & & 74.285 \\
\hline 12/29/07 2:00 & 125.537 & 123.093 & & 100.793 & 104.666 & 96.313 & 111.073 & & 80.778 & 74.386 \\
\hline 12/29/07 2:30 & 125.532 & 123.088 & & 100.787 & 104.66 & 96.308 & 111.073 & & & 74.523 \\
\hline 12/29/07 3:00 & 125.53 & 123.093 & & 100.786 & 104.658 & 96.303 & 111.073 & & & 74.52 \\
\hline 12/29/07 3:30 & 125.525 & 123.09 & & 100.784 & 104.654 & 96.299 & 111.071 & & 80.77 & 74.516 \\
\hline $12 / 29 / 074: 00$ & 125.523 & 123.088 & & 100.787 & 104.658 & 96.303 & 111.071 & & & 74.52 \\
\hline $12 / 29 / 074: 30$ & 125.528 & 123.081 & & 100.784 & 104.66 & 96.306 & 111.069 & & & 74.523 \\
\hline 12/29/07 5:00 & 125.523 & 123.077 & & 100.777 & 104.654 & 96.299 & 111.067 & & 80.768 & 74.516 \\
\hline $12 / 29 / 075: 30$ & 125.518 & 123.07 & & 100.774 & 104.647 & 96.292 & 111.067 & & & 74.51 \\
\hline $12 / 29 / 076: 00$ & 125.513 & 123.063 & & 100.768 & 104.645 & 96.292 & 111.063 & & & 74.508 \\
\hline $12 / 29 / 076: 30$ & 125.509 & 123.063 & & 100.763 & 104.641 & 96.285 & 111.065 & & 80.76 & 74.502 \\
\hline 12/29/07 7:00 & 125.504 & 123.065 & & 100.756 & 104.63 & 96.278 & 111.063 & & & 74.496 \\
\hline $12 / 29 / 077: 30$ & 125.499 & 123.065 & & 100.756 & 104.63 & 96.278 & 111.059 & & & 74.496 \\
\hline
\end{tabular}


TABLE S1.1 (Cont.)

\begin{tabular}{|c|c|c|c|c|c|c|c|c|c|c|}
\hline \multirow[b]{2}{*}{ Date and Time } & \multicolumn{10}{|c|}{ Depth from Top of Casing (ft) } \\
\hline & MW1D & MW2D & MW3D & MW4D & MW7 & MW9 & Oentrich & MW14D & MW15D & MW16D \\
\hline 12/29/07 8:00 & 125.494 & 124.791 & & 100.76 & 104.631 & 96.275 & 111.057 & & 80.748 & 74.494 \\
\hline $12 / 29 / 078: 30$ & 125.497 & 125.14 & & 100.76 & 104.635 & 96.278 & 111.057 & & & 74.496 \\
\hline 12/29/07 9:00 & 126.993 & 125.297 & & 101.433 & 104.972 & 96.733 & 111.512 & & & 74.518 \\
\hline $12 / 29 / 079: 30$ & 127.508 & 125.385 & & 101.661 & 105.315 & 97.084 & 111.846 & & 80.758 & 74.704 \\
\hline $12 / 29 / 07 \quad 10: 00$ & 127.738 & 125.442 & & 101.788 & 105.473 & 97.244 & 111.987 & & & 74.853 \\
\hline $12 / 29 / 07$ 10:30 & 127.858 & 125.487 & & 101.866 & 105.559 & 97.336 & 112.068 & & & 74.948 \\
\hline $12 / 29 / 0711: 00$ & 127.929 & 123.61 & & 101.912 & 105.621 & 97.395 & 112.12 & & 80.824 & 75.01 \\
\hline $12 / 29 / 07$ 11:30 & 127.974 & 123.394 & & 101.563 & 105.66 & 97.435 & 112.16 & & & 75.052 \\
\hline 12/29/07 12:00 & 126.237 & 123.27 & & 101.166 & 105.145 & 96.794 & 111.534 & & & 74.969 \\
\hline $12 / 29 / 07$ 12:30 & 125.906 & 123.192 & & 101.011 & 104.94 & 96.584 & 111.347 & & 80.851 & 74.822 \\
\hline $12 / 29 / 07$ 13:00 & 125.747 & 123.145 & & 100.911 & 104.822 & 96.469 & 111.258 & & & 74.711 \\
\hline 12/29/07 13:30 & 125.653 & 123.115 & & 100.848 & 104.744 & 96.391 & 111.206 & & & 74.63 \\
\hline $12 / 29 / 0714: 00$ & 125.589 & 123.09 & & 100.811 & 104.696 & 96.344 & 111.17 & & 80.809 & 74.576 \\
\hline $12 / 29 / 07$ 14:30 & 125.554 & 123.07 & & 100.782 & 104.67 & 96.318 & 111.146 & & & 74.547 \\
\hline 12/29/07 15:00 & 125.528 & 123.052 & & 100.767 & 104.647 & 96.294 & 111.129 & & & 74.523 \\
\hline $12 / 29 / 07$ 15:30 & 125.511 & 123.05 & & 100.746 & 104.63 & 96.275 & 111.116 & & 80.785 & 74.5 \\
\hline $12 / 29 / 07$ 16:00 & 125.492 & 123.043 & & 100.737 & 104.612 & 96.259 & 111.097 & & & 74.471 \\
\hline $12 / 29 / 07$ 16:30 & 125.483 & 123.031 & & 100.732 & 104.612 & 96.256 & 111.089 & & & 74.444 \\
\hline $12 / 29 / 07$ 17:00 & 125.478 & 123.031 & & 100.725 & 104.599 & 96.245 & 111.084 & & 80.768 & 74.423 \\
\hline $12 / 29 / 07 \quad 17: 30$ & 125.468 & 123.022 & & 100.718 & 104.593 & 96.24 & 111.078 & & & 74.401 \\
\hline $12 / 29 / 07$ 18:00 & 125.464 & 123.029 & & 100.715 & 104.595 & 96.237 & 111.054 & & & 74.38 \\
\hline $12 / 29 / 07$ 18:30 & 125.461 & 123.031 & & 100.717 & 104.586 & 96.23 & 111.045 & & 80.751 & 74.363 \\
\hline 12/29/07 19:00 & 125.461 & 123.034 & & 100.715 & 104.591 & 96.237 & 111.041 & & & 74.347 \\
\hline 12/29/07 19:30 & 125.461 & 123.029 & & 100.727 & 104.599 & 96.245 & 111.036 & & & 74.33 \\
\hline $12 / 29 / 0720: 00$ & 125.468 & 123.031 & & 100.727 & 104.603 & 96.247 & 111.034 & & 80.738 & 74.318 \\
\hline $12 / 29 / 0720: 30$ & 125.468 & 123.025 & & 100.722 & 104.597 & 96.242 & 111.032 & & & 74.303 \\
\hline $12 / 29 / 0721: 00$ & 125.466 & 123.016 & & 100.722 & 104.599 & 96.242 & 111.03 & & & 74.291 \\
\hline $12 / 29 / 0721: 30$ & 125.464 & 123.002 & & 100.712 & 104.591 & 96.235 & 111.031 & & 80.731 & 74.281 \\
\hline 12/29/07 22:00 & 125.454 & 122.993 & & 100.703 & 104.58 & 96.226 & 111.03 & & & 74.27 \\
\hline $12 / 29 / 0722: 30$ & 125.447 & 122.982 & & 100.689 & 104.569 & 96.214 & 111.03 & & & 74.26 \\
\hline 12/29/07 23:00 & 125.433 & 122.977 & & 100.682 & 104.557 & 96.202 & 111.028 & & 80.716 & 74.25 \\
\hline $12 / 29 / 0723: 30$ & 125.424 & 122.972 & & 100.672 & 104.548 & 96.193 & 111.027 & & & 74.239 \\
\hline 12/30/07 0:00 & 125.416 & 122.966 & & 100.672 & 104.544 & 96.188 & 111.027 & & & 74.229 \\
\hline 12/30/07 0:30 & 125.414 & 122.968 & & 100.663 & 104.542 & 96.186 & 111.026 & & 80.702 & 74.219 \\
\hline 12/30/07 1:00 & 125.407 & 122.952 & & 100.662 & 104.534 & 96.176 & 111.024 & & & 74.21 \\
\hline 12/30/07 1:30 & 125.402 & 122.945 & & 100.656 & 104.536 & 96.176 & 111.022 & & & 74.2 \\
\hline 12/30/07 2:00 & 125.395 & 122.936 & & 100.643 & 104.521 & 96.167 & 111.009 & & 80.69 & 74.192 \\
\hline 12/30/07 2:30 & 125.386 & 122.932 & & 100.634 & 104.513 & 96.157 & 110.999 & & & 74.183 \\
\hline 12/30/07 3:00 & 125.376 & 122.927 & & 100.627 & 104.506 & 96.15 & 110.99 & & & 74.173 \\
\hline 12/30/07 3:30 & 125.367 & 122.925 & & 100.624 & 104.498 & 96.146 & 110.982 & & 80.668 & 74.165 \\
\hline $12 / 30 / 074: 00$ & 125.364 & 122.916 & & 100.622 & 104.496 & 96.141 & 110.982 & & & 74.155 \\
\hline $12 / 30 / 074: 30$ & 125.364 & 122.909 & & 100.612 & 104.492 & 96.139 & 110.979 & & & 74.186 \\
\hline $12 / 30 / 075: 00$ & 125.357 & 122.893 & & 100.608 & 104.485 & 96.131 & 110.975 & & 80.653 & 74.227 \\
\hline 12/30/07 5:30 & 125.348 & 122.882 & & 100.598 & 104.475 & 96.122 & 110.964 & & & 74.258 \\
\hline $12 / 30 / 076: 00$ & 125.336 & 122.873 & & 100.584 & 104.462 & 96.108 & 110.95 & & & 74.328 \\
\hline $12 / 30 / 076: 30$ & 125.324 & 122.864 & & 100.574 & 104.452 & 96.098 & 110.941 & & 80.636 & 74.318 \\
\hline 12/30/07 7:00 & 125.312 & 122.852 & & 100.565 & 104.439 & 96.082 & 110.926 & & & 74.305 \\
\hline 12/30/07 7:30 & 125.303 & 122.85 & & 100.555 & 104.433 & 96.075 & 110.922 & & & 74.301 \\
\hline $12 / 30 / 07$ 8:00 & 125.296 & 122.841 & & 100.546 & 104.42 & 96.063 & 110.913 & & 80.616 & 74.289 \\
\hline 12/30/07 8:30 & 125.286 & 124.313 & & 100.539 & 104.416 & 96.058 & 110.909 & & & 74.285 \\
\hline 12/30/07 9:00 & 125.282 & 124.882 & & 100.536 & 104.412 & 96.054 & 110.909 & & & 74.279 \\
\hline 12/30/07 9:30 & 126.404 & 125.066 & & 101.113 & 104.536 & 96.242 & 111.133 & & 80.592 & 74.279 \\
\hline 12/30/07 10:00 & 127.229 & 125.168 & & 101.413 & 105.046 & 96.815 & 111.649 & & & 74.448 \\
\hline $12 / 30 / 07$ 10:30 & 127.508 & 125.227 & & 101.556 & 105.231 & 97.004 & 111.818 & & & 74.613 \\
\hline 12/30/07 11:00 & 127.646 & 125.263 & & 101.637 & 105.336 & 97.11 & 111.914 & & 80.626 & 74.723 \\
\hline 12/30/07 11:30 & 127.714 & 125.288 & & 101.69 & 105.395 & 97.169 & 111.972 & & & 74.783 \\
\hline $12 / 30 / 07$ 12:00 & 127.761 & 125.299 & & 101.716 & 105.431 & 97.206 & 112.015 & & & 74.826 \\
\hline 12/30/07 12:30 & 127.783 & 125.308 & & 101.744 & 105.458 & 97.232 & 112.045 & & 80.675 & 74.855 \\
\hline 12/30/07 13:00 & 127.804 & 125.31 & & 101.754 & 105.473 & 97.249 & 112.068 & & & 74.876 \\
\hline $12 / 30 / 07$ 13:30 & 127.813 & 125.308 & & 101.754 & 105.482 & 97.256 & 112.086 & & & 74.886 \\
\hline $12 / 30 / 07$ 14:00 & 127.816 & 123.787 & & 101.761 & 105.484 & 97.258 & 112.064 & & 80.716 & 74.891 \\
\hline $12 / 30 / 07$ 14:30 & 127.813 & 123.328 & & 101.763 & 105.486 & 97.258 & 112.058 & & & 74.893 \\
\hline $12 / 30 / 07$ 15:00 & 126.539 & 123.165 & & 101.161 & 105.265 & 96.94 & 111.722 & & & 74.895 \\
\hline $12 / 30 / 07$ 15:30 & 125.884 & 123.081 & & 100.911 & 104.866 & 96.509 & 111.32 & & 80.731 & 74.738 \\
\hline $12 / 30 / 07$ 16:00 & 125.641 & 123.036 & & 100.794 & 104.709 & 96.353 & 111.174 & & & 74.605 \\
\hline $12 / 30 / 07 \quad 16: 30$ & 125.53 & 123.009 & & 100.728 & 104.63 & 96.27 & 111.091 & & & 74.52 \\
\hline
\end{tabular}


TABLE S1.1 (Cont.)

\begin{tabular}{|c|c|c|c|c|c|c|c|c|c|c|}
\hline \multirow[b]{2}{*}{ Date and Time } & \multicolumn{10}{|c|}{ Depth from Top of Casing (ft) } \\
\hline & MW1D & MW2D & MW3D & MW4D & MW7 & MW9 & Oentrich & MW14D & MW15D & MW16D \\
\hline 12/30/07 17:00 & 125.468 & 122.984 & & 100.696 & 104.582 & 96.226 & 111.041 & & 80.692 & 74.467 \\
\hline $12 / 30 / 0717: 30$ & 125.44 & 122.961 & & 100.677 & 104.563 & 96.209 & 111.009 & & & 74.442 \\
\hline 12/30/07 18:00 & 125.424 & 122.968 & & 100.66 & 104.54 & 96.186 & 110.988 & & & 74.415 \\
\hline $12 / 30 / 07$ 18:30 & 125.402 & 122.95 & & 100.641 & 104.521 & 96.162 & 110.971 & & 80.668 & 74.392 \\
\hline 12/30/07 19:00 & 125.39 & 122.948 & & 100.656 & 104.527 & 96.169 & 110.956 & & & 74.392 \\
\hline 12/30/07 19:30 & 125.4 & 122.934 & & 100.639 & 104.513 & 96.16 & 110.949 & & & 74.386 \\
\hline 12/30/07 20:00 & 125.381 & 122.936 & & 100.634 & 104.511 & 96.155 & 110.943 & & 80.65 & 74.374 \\
\hline 12/30/07 20:30 & 125.374 & 122.941 & & 100.631 & 104.492 & 96.139 & 110.939 & & & 74.357 \\
\hline 12/30/07 21:00 & 125.374 & 122.938 & & 100.625 & 104.5 & 96.146 & 110.934 & & & 74.367 \\
\hline $12 / 30 / 0721: 30$ & 125.374 & 122.936 & & 100.632 & 104.506 & 96.15 & 110.926 & & 80.638 & 74.37 \\
\hline 12/30/07 22:00 & 125.374 & 122.941 & & 100.632 & 104.5 & 96.146 & 110.924 & & & 74.361 \\
\hline 12/30/07 22:30 & 125.374 & 122.927 & & 100.634 & 104.504 & 96.148 & 110.92 & & & 74.365 \\
\hline 12/30/07 23:00 & 125.379 & 122.952 & & 100.627 & 104.508 & 96.153 & 110.919 & & 80.631 & 74.367 \\
\hline 12/30/07 23:30 & 125.367 & 122.938 & & 100.627 & 104.491 & 96.136 & 110.919 & & & 74.349 \\
\hline 12/31/07 0:00 & 125.372 & 122.929 & & 100.643 & 104.519 & 96.162 & 110.911 & & & 74.374 \\
\hline 12/31/07 0:30 & 125.381 & 122.9 & & 100.631 & 104.513 & 96.153 & 110.913 & & 80.626 & 74.365 \\
\hline 12/31/07 1:00 & 125.376 & 122.861 & & 100.612 & 104.498 & 96.143 & 110.915 & & & 74.359 \\
\hline $12 / 31 / 071: 30$ & 125.353 & 122.836 & & 100.581 & 104.466 & 96.113 & 110.917 & & & 74.326 \\
\hline 12/31/07 2:00 & 125.324 & 122.841 & & 100.522 & 104.428 & 96.077 & 110.922 & & 80.616 & 74.291 \\
\hline 12/31/07 2:30 & 125.279 & 122.848 & & 100.531 & 104.399 & 96.047 & 110.87 & & & 74.268 \\
\hline 12/31/07 3:00 & 125.272 & 122.868 & & 100.543 & 104.407 & 96.054 & 110.868 & & & 74.266 \\
\hline 12/31/07 3:30 & 125.277 & 122.857 & & 100.562 & 104.412 & 96.054 & 110.866 & & 80.58 & 74.266 \\
\hline 12/31/07 4:00 & 125.298 & 122.882 & & 100.551 & 104.441 & 96.087 & 110.86 & & & 74.299 \\
\hline $12 / 31 / 074: 30$ & 125.298 & 122.886 & & 100.562 & 104.424 & 96.068 & 110.864 & & & 74.283 \\
\hline 12/31/07 5:00 & 125.303 & 122.902 & & 100.57 & 104.454 & 96.096 & 110.857 & & 80.575 & 74.308 \\
\hline 12/31/07 5:30 & 125.32 & 122.918 & & 100.581 & 104.452 & 96.101 & 110.86 & & & 74.312 \\
\hline $12 / 31 / 076: 00$ & 125.329 & 122.932 & & 100.61 & 104.473 & 96.12 & 110.866 & & & 74.324 \\
\hline $12 / 31 / 076: 30$ & 125.348 & 122.952 & & 100.624 & 104.487 & 96.136 & 110.876 & & 80.577 & 74.328 \\
\hline 12/31/07 7:00 & 125.36 & 124.898 & & 100.636 & 104.502 & 96.15 & 110.887 & & & 74.312 \\
\hline $12 / 31 / 077: 30$ & 125.376 & 125.136 & & 101.097 & 104.529 & 96.174 & 110.906 & & & 74.297 \\
\hline 12/31/07 8:00 & 127.241 & 125.265 & & 101.532 & 105.117 & 96.902 & 111.627 & & 80.587 & 74.38 \\
\hline 12/31/07 8:30 & 127.596 & 125.354 & & 101.707 & 105.353 & 97.143 & 111.854 & & & 74.51 \\
\hline 12/31/07 9:00 & 127.761 & 123.637 & & 101.814 & 105.481 & 97.27 & 111.97 & & & 74.597 \\
\hline 12/31/07 9:30 & 127.858 & 123.387 & & 101.892 & 105.57 & 97.362 & 112.053 & & 80.704 & 74.651 \\
\hline 12/31/07 10:00 & 126.272 & 123.292 & & 101.168 & 105.166 & 96.822 & 111.512 & & & 74.636 \\
\hline $12 / 31 / 07$ 10:30 & 125.88 & 123.247 & & 101.018 & 104.942 & 96.586 & 111.294 & & & 74.518 \\
\hline 12/31/07 11:00 & 125.738 & 123.217 & & 100.951 & 104.847 & 96.497 & 111.212 & & 80.712 & 74.514 \\
\hline 12/31/07 11:30 & 125.681 & 123.199 & & 100.92 & 104.808 & 96.452 & 111.168 & & & 74.483 \\
\hline $12 / 31 / 07$ 12:00 & 125.658 & 123.188 & & 100.899 & 104.778 & 96.428 & 111.153 & & & 74.438 \\
\hline $12 / 31 / 07$ 12:30 & 125.639 & 123.183 & & 100.889 & 104.761 & 96.41 & 111.134 & & 80.724 & 74.405 \\
\hline 12/31/07 13:00 & 125.627 & 123.179 & & 100.879 & 104.753 & 96.4 & 111.127 & & & 74.378 \\
\hline $12 / 31 / 07$ 13:30 & 125.62 & 123.181 & & 100.875 & 104.749 & 96.395 & 111.119 & & & 74.357 \\
\hline 12/31/07 14:00 & 125.615 & 123.195 & & 100.875 & 104.744 & 96.393 & 111.119 & & 80.734 & 74.339 \\
\hline $12 / 31 / 07$ 14:30 & 125.615 & 123.206 & & 100.879 & 104.749 & 96.395 & 111.123 & & & 74.324 \\
\hline $12 / 31 / 07$ 15:00 & 125.606 & 123.22 & & 100.892 & 104.759 & 96.407 & 111.125 & & & 74.308 \\
\hline $12 / 31 / 07$ 15:30 & 125.601 & 123.245 & & 100.91 & 104.776 & 96.421 & 111.144 & & 80.753 & 74.301 \\
\hline 12/31/07 16:00 & 125.598 & 123.265 & & 100.927 & 104.789 & 96.436 & 111.148 & & & 74.291 \\
\hline $12 / 31 / 07$ 16:30 & 125.594 & 123.288 & & 100.951 & 104.814 & 96.461 & 111.176 & & & 74.281 \\
\hline $12 / 31 / 0717: 00$ & 125.591 & 123.304 & & 100.975 & 104.835 & 96.483 & 111.195 & & 80.782 & 74.272 \\
\hline 12/31/07 17:30 & 125.589 & 123.319 & & 100.996 & 104.856 & 96.506 & 111.219 & & & 74.264 \\
\hline $12 / 31 / 0718: 00$ & 125.589 & 123.333 & & 101.01 & 104.873 & 96.523 & 111.238 & & & 74.256 \\
\hline 12/31/07 18:30 & 125.589 & 123.349 & & 101.025 & 104.887 & 96.537 & 111.251 & & 80.814 & 74.248 \\
\hline 12/31/07 19:00 & 125.589 & 123.369 & & 101.039 & 104.9 & 96.549 & 111.26 & & & 74.241 \\
\hline $12 / 31 / 07$ 19:30 & 125.632 & 123.383 & & 101.06 & 104.921 & 96.568 & 111.281 & & & 74.235 \\
\hline 12/31/07 20:00 & 125.634 & 123.399 & & 101.077 & 104.94 & 96.586 & 111.287 & & 80.848 & 74.227 \\
\hline $12 / 31 / 0720: 30$ & 125.641 & 123.417 & & 101.092 & 104.957 & 96.605 & 111.313 & & & 74.221 \\
\hline $12 / 31 / 0721: 00$ & 125.65 & 123.431 & & 101.108 & 104.97 & 96.617 & 111.328 & & & 74.217 \\
\hline 12/31/07 21:30 & 125.66 & 123.435 & & 101.122 & 104.987 & 96.636 & 111.343 & & 80.885 & 74.208 \\
\hline $12 / 31 / 0722: 00$ & 125.679 & 123.442 & & 101.135 & 105.001 & 96.65 & 111.356 & & & 74.204 \\
\hline $12 / 31 / 0722: 30$ & 125.698 & 123.446 & & 101.137 & 105.005 & 96.655 & 111.365 & & & 74.198 \\
\hline 12/31/07 23:00 & 125.717 & 123.458 & & 101.147 & 105.014 & 96.659 & 111.368 & & 80.91 & 74.192 \\
\hline $12 / 31 / 0723: 30$ & 125.736 & 123.469 & & 101.149 & 105.02 & 96.671 & 111.379 & & & 74.188 \\
\hline $1 / 1 / 080: 00$ & 125.754 & 123.474 & & 101.165 & 105.029 & 96.676 & 111.381 & & & 74.181 \\
\hline 1/1/08 0:30 & 125.771 & 123.476 & & 101.173 & 105.037 & 96.688 & 111.398 & & 80.939 & 74.175 \\
\hline 1/1/08 1:00 & 125.79 & 123.478 & & 101.18 & 105.045 & 96.695 & 111.401 & & & 74.167 \\
\hline 1/1/08 1:30 & 125.809 & 123.483 & & 101.18 & 105.045 & 96.697 & 111.407 & & & 74.165 \\
\hline
\end{tabular}


TABLE S1.1 (Cont.)

\begin{tabular}{|c|c|c|c|c|c|c|c|c|c|c|}
\hline \multirow[b]{2}{*}{ Date and Time } & \multicolumn{10}{|c|}{ Depth from Top of Casing (ft) } \\
\hline & MW1D & MW2D & MW3D & MW4D & MW7 & MW9 & Oentrich & MW14D & MW15D & MW16D \\
\hline 1/1/08 2:00 & 125.823 & 123.485 & & 101.177 & 105.046 & 96.697 & 111.405 & & 80.956 & 74.159 \\
\hline $1 / 1 / 082: 30$ & 125.835 & 123.501 & & 101.184 & 105.052 & 96.702 & 111.403 & & & 74.152 \\
\hline 1/1/08 3:00 & 125.847 & 123.517 & & 101.196 & 105.054 & 96.704 & 111.407 & & & 74.144 \\
\hline 1/1/08 3:30 & 125.854 & 123.528 & & 101.204 & 105.071 & 96.721 & 111.424 & & 80.988 & 74.138 \\
\hline $1 / 1 / 084: 00$ & 125.868 & 123.539 & & 101.223 & 105.086 & 96.737 & 111.439 & & & 74.13 \\
\hline $1 / 1 / 084: 30$ & 125.88 & 123.546 & & 101.232 & 105.096 & 96.747 & 111.452 & & & 74.124 \\
\hline 1/1/08 5:00 & 125.892 & 123.555 & & 101.244 & 105.109 & 96.758 & 111.459 & & 81.017 & 74.115 \\
\hline 1/1/08 5:30 & 125.906 & 123.56 & & 101.251 & 105.119 & 96.766 & 111.469 & & & 74.196 \\
\hline 1/1/08 6:00 & 125.918 & 123.569 & & 101.258 & 105.123 & 96.773 & 111.474 & & & 74.347 \\
\hline 1/1/08 6:30 & 125.929 & 123.585 & & 101.261 & 105.128 & 96.78 & 111.48 & & 81.042 & 74.545 \\
\hline $1 / 1 / 087: 00$ & 125.939 & 123.596 & & 101.277 & 105.138 & 96.787 & 111.488 & & & 74.678 \\
\hline 1/1/08 7:30 & 125.951 & 123.601 & & 101.287 & 105.155 & 96.803 & 111.502 & & & 74.816 \\
\hline 1/1/08 8:00 & 125.96 & 123.607 & & 101.295 & 105.166 & 96.813 & 111.514 & & 81.078 & 74.903 \\
\hline 1/1/08 8:30 & 125.974 & 123.61 & & 101.303 & 105.17 & 96.817 & 111.517 & & & 74.95 \\
\hline 1/1/08 9:00 & 125.984 & 123.621 & & 101.309 & 105.178 & 96.824 & 111.523 & & & 74.967 \\
\hline 1/1/08 9:30 & 125.996 & 125.476 & & 101.313 & 105.18 & 96.824 & 111.527 & & 81.1 & 74.986 \\
\hline $1 / 1 / 08$ 10:00 & 126.003 & 125.773 & & 101.323 & 105.189 & 96.839 & 111.537 & & & 74.986 \\
\hline 1/1/08 10:30 & 127.575 & 125.918 & & 102.074 & 105.641 & 97.409 & 112.088 & & & 75.031 \\
\hline 1/1/08 11:00 & 127.925 & 126.002 & & 102.286 & 105.934 & 97.713 & 112.38 & & 81.174 & 75.209 \\
\hline 1/1/08 11:30 & 128.13 & 126.052 & & 102.402 & 106.08 & 97.857 & 112.519 & & & 75.347 \\
\hline $1 / 1 / 08 \quad 12: 00$ & 128.277 & 126.088 & & 102.471 & 106.166 & 97.947 & 112.588 & & & 75.451 \\
\hline 1/1/08 12:30 & 128.378 & 126.106 & & 102.512 & 106.214 & 97.996 & 112.633 & & 81.259 & 75.523 \\
\hline $1 / 1 / 0813: 00$ & 128.454 & 126.12 & & 102.541 & 106.252 & 98.029 & 112.663 & & & 75.569 \\
\hline 1/1/08 13:30 & 128.513 & 126.134 & & 102.56 & 106.267 & 98.048 & 112.69 & & & 75.604 \\
\hline 1/1/08 14:00 & 128.553 & 126.143 & & 102.567 & 106.284 & 98.067 & 112.707 & & 81.318 & 75.629 \\
\hline 1/1/08 14:30 & 128.582 & 126.154 & & 102.581 & 106.299 & 98.081 & 112.718 & & & 75.643 \\
\hline $1 / 1 / 08$ 15:00 & 128.601 & 126.154 & & 102.593 & 106.311 & 98.09 & 112.727 & & & 75.655 \\
\hline 1/1/08 15:30 & 128.62 & 124.587 & & 102.603 & 106.32 & 98.1 & 112.734 & & 81.384 & 75.67 \\
\hline $1 / 1 / 08$ 16:00 & 128.629 & 124.177 & & 102.605 & 106.322 & 98.102 & 112.738 & & & 75.699 \\
\hline $1 / 1 / 0816: 30$ & 127.341 & 124.016 & & 102 & 106.077 & 97.753 & 112.417 & & & 75.705 \\
\hline $1 / 1 / 08 \quad 17: 00$ & 126.863 & 123.929 & & 101.757 & 105.707 & 97.355 & 112.017 & & 81.403 & 75.573 \\
\hline $1 / 1 / 08$ 17:30 & 126.648 & 123.87 & & 101.637 & 105.553 & 97.202 & 111.869 & & & 75.449 \\
\hline 1/1/08 18:00 & 126.506 & 123.834 & & 101.571 & 105.467 & 97.114 & 111.786 & & & 75.36 \\
\hline 1/1/08 18:30 & 126.412 & 123.807 & & 101.525 & 105.41 & 97.06 & 111.739 & & 81.401 & 75.3 \\
\hline 1/1/08 19:00 & 126.343 & 123.793 & & 101.496 & 105.38 & 97.027 & 111.703 & & & 75.258 \\
\hline 1/1/08 19:30 & 126.293 & 123.784 & & 101.48 & 105.355 & 97.004 & 111.681 & & & 75.23 \\
\hline $1 / 1 / 0820: 00$ & 126.256 & 123.775 & & 101.468 & 105.343 & 96.992 & 111.67 & & 81.406 & 75.215 \\
\hline $1 / 1 / 0820: 30$ & 126.232 & 123.768 & & 101.461 & 105.336 & 96.982 & 111.664 & & & 75.205 \\
\hline 1/1/08 21:00 & 126.215 & 123.764 & & 101.454 & 105.326 & 96.978 & 111.66 & & & 75.199 \\
\hline 1/1/08 21:30 & 126.204 & 123.755 & & 101.449 & 105.324 & 96.973 & 111.655 & & 81.413 & 75.194 \\
\hline $1 / 1 / 0822: 00$ & 126.189 & 123.75 & & 101.442 & 105.319 & 96.968 & 111.645 & & & 75.188 \\
\hline 1/1/08 22:30 & 126.185 & 123.746 & & 101.435 & 105.311 & 96.959 & 111.641 & & & 75.182 \\
\hline 1/1/08 23:00 & 126.178 & 123.743 & & 101.433 & 105.307 & 96.954 & 111.636 & & 81.418 & 75.18 \\
\hline 1/1/08 23:30 & 126.17 & 123.739 & & 101.427 & 105.304 & 96.949 & 111.632 & & & 75.176 \\
\hline $1 / 2 / 08 \quad 0: 00$ & 126.166 & 123.732 & & 101.423 & 105.302 & 96.949 & 111.627 & & & 75.174 \\
\hline 1/2/08 0:30 & 126.163 & 123.725 & & 101.423 & 105.296 & 96.945 & 111.621 & & 81.425 & 75.17 \\
\hline 1/2/08 1:00 & 126.161 & 123.718 & & 101.411 & 105.29 & 96.938 & 111.621 & & & 75.168 \\
\hline 1/2/08 1:30 & 126.154 & 123.716 & & 101.408 & 105.284 & 96.931 & 111.613 & & & 75.161 \\
\hline 1/2/08 2:00 & 126.149 & 123.714 & & 101.404 & 105.281 & 96.926 & 111.608 & & 81.423 & 75.155 \\
\hline $1 / 2 / 082: 30$ & 126.147 & 123.714 & & 101.401 & 105.275 & 96.923 & 111.604 & & & 75.153 \\
\hline 1/2/08 3:00 & 126.142 & 123.725 & & 101.401 & 105.271 & 96.919 & 111.598 & & & 75.149 \\
\hline 1/2/08 3:30 & 126.137 & 123.716 & & 101.402 & 105.269 & 96.914 & 111.598 & & 81.425 & 75.149 \\
\hline $1 / 2 / 084: 00$ & 126.137 & 123.714 & & 101.411 & 105.281 & 96.928 & 111.606 & & & 75.155 \\
\hline 1/2/08 4:30 & 126.14 & 123.709 & & 101.399 & 105.275 & 96.926 & 111.609 & & & 75.157 \\
\hline 1/2/08 5:00 & 126.14 & 123.716 & & 101.397 & 105.273 & 96.921 & 111.604 & & 81.428 & 75.153 \\
\hline 1/2/08 5:30 & 126.135 & 123.721 & & 101.397 & 105.267 & 96.916 & 111.598 & & & 75.153 \\
\hline 1/2/08 6:00 & 126.133 & 123.73 & & 101.404 & 105.275 & 96.923 & 111.6 & & & 75.155 \\
\hline $1 / 2 / 086: 30$ & 126.135 & 123.732 & & 101.411 & 105.283 & 96.928 & 111.604 & & 81.443 & 75.159 \\
\hline 1/2/08 7:00 & 126.14 & 124.993 & & 101.42 & 105.29 & 96.938 & 111.615 & & & 75.17 \\
\hline 1/2/08 7:30 & 126.144 & 125.739 & & 101.42 & 105.294 & 96.94 & 111.621 & & & 75.174 \\
\hline 1/2/08 8:00 & 126.837 & 125.936 & & 101.995 & 105.324 & 96.956 & 111.694 & & 81.452 & 75.178 \\
\hline 1/2/08 8:30 & 127.96 & 124.167 & & 102.323 & 105.932 & 97.723 & 112.381 & & & 75.316 \\
\hline 1/2/08 9:00 & 128.234 & 123.986 & & 102.157 & 106.128 & 97.923 & 112.571 & & & 75.484 \\
\hline 1/2/08 9:30 & 126.745 & 123.904 & & 101.737 & 105.701 & 97.357 & 112.015 & & 81.511 & 75.515 \\
\hline 1/2/08 10:00 & 126.518 & 123.859 & & 101.621 & 105.532 & 97.183 & 111.839 & & & 75.426 \\
\hline $1 / 2 / 08 \quad 10: 30$ & 126.407 & 123.834 & & 101.559 & 105.458 & 97.105 & 111.771 & & & 75.362 \\
\hline
\end{tabular}


TABLE S1.1 (Cont.)

Depth from Top of Casing (ft)

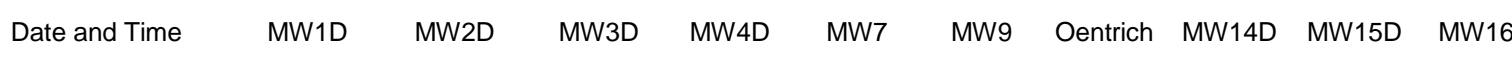

\begin{tabular}{|c|c|c|c|c|c|c|c|c|}
\hline 1/2/08 11:00 & 126.341 & 123.807 & 101.528 & 105.412 & 97.06 & 111.728 & 81.516 & 75.314 \\
\hline 1/2/08 11:30 & 126.298 & 123.777 & 101.501 & 105.385 & 97.032 & 111.709 & & 75.287 \\
\hline $1 / 2 / 0812: 00$ & 126.267 & 123.748 & 101.475 & 105.361 & 97.006 & 111.688 & & 75.263 \\
\hline $1 / 2 / 0812: 30$ & 126.244 & 123.73 & 101.444 & 105.33 & 96.98 & 111.675 & 81.506 & 75.236 \\
\hline 1/2/08 13:00 & 126.22 & 123.712 & 101.423 & 105.304 & 96.952 & 111.664 & & 75.209 \\
\hline $1 / 2 / 0813: 30$ & 126.199 & 123.694 & 101.402 & 105.284 & 96.933 & 111.655 & & 75.188 \\
\hline $1 / 2 / 0814: 00$ & 126.178 & 123.673 & 101.384 & 105.267 & 96.912 & 111.645 & 81.494 & 75.17 \\
\hline $1 / 2 / 08 \quad 14: 30$ & 126.156 & 123.655 & 101.366 & 105.246 & 96.893 & 111.64 & & 75.155 \\
\hline $1 / 2 / 0815: 00$ & 126.137 & 123.63 & 101.346 & 105.227 & 96.874 & 111.632 & & 75.134 \\
\hline $1 / 2 / 0815: 30$ & 126.118 & 123.612 & 101.327 & 105.21 & 96.855 & 111.627 & 81.474 & 75.12 \\
\hline 1/2/08 16:00 & 126.102 & 123.598 & 101.301 & 105.185 & 96.834 & 111.621 & & 75.099 \\
\hline $1 / 2 / 0816: 30$ & 126.081 & 123.58 & 101.284 & 105.166 & 96.813 & 111.593 & & 75.079 \\
\hline 1/2/08 17:00 & 126.059 & 123.569 & 101.272 & 105.151 & 96.796 & 111.58 & 81.472 & 75.064 \\
\hline 1/2/08 17:30 & 126.045 & 123.56 & 101.253 & 105.134 & 96.782 & 111.567 & & 75.052 \\
\hline $1 / 2 / 0818: 00$ & 126.026 & 123.553 & 101.246 & 105.126 & 96.773 & 111.551 & & 75.037 \\
\hline $1 / 2 / 0818: 30$ & 126.01 & 123.539 & 101.239 & 105.117 & 96.763 & 111.542 & 81.443 & 75.027 \\
\hline 1/2/08 19:00 & 126 & 123.528 & 101.227 & 105.107 & 96.756 & 111.534 & & 75.021 \\
\hline 1/2/08 19:30 & 125.991 & 123.517 & 101.215 & 105.096 & 96.742 & 111.523 & & 75.01 \\
\hline $1 / 2 / 0820: 00$ & 125.979 & 123.505 & 101.204 & 105.083 & 96.733 & 111.514 & 81.421 & 75 \\
\hline $1 / 2 / 0820: 30$ & 125.97 & 123.496 & 101.194 & 105.075 & 96.721 & 111.506 & & 74.992 \\
\hline $1 / 2 / 0821: 00$ & 125.958 & 123.483 & 101.178 & 105.062 & 96.709 & 111.495 & & 74.979 \\
\hline $1 / 2 / 0821: 30$ & 125.946 & 123.471 & 101.168 & 105.052 & 96.695 & 111.484 & 81.406 & 74.969 \\
\hline 1/2/08 22:00 & 125.934 & 123.453 & 101.158 & 105.039 & 96.681 & 111.471 & & 74.957 \\
\hline $1 / 2 / 0822: 30$ & 125.922 & 123.433 & 101.146 & 105.029 & 96.674 & 111.461 & & 74.948 \\
\hline $1 / 2 / 0823: 00$ & 125.91 & 123.415 & 101.123 & 105.01 & 96.657 & 111.442 & 81.379 & 74.932 \\
\hline $1 / 2 / 0823: 30$ & 125.896 & 123.399 & 101.11 & 104.989 & 96.636 & 111.422 & & 74.913 \\
\hline $1 / 3 / 080: 00$ & 125.88 & 123.378 & 101.087 & 104.97 & 96.615 & 111.405 & & 74.899 \\
\hline $1 / 3 / 080: 30$ & 125.861 & 123.356 & 101.07 & 104.953 & 96.598 & 111.388 & 81.347 & 74.878 \\
\hline $1 / 3 / 081: 00$ & 125.844 & 123.337 & 101.051 & 104.936 & 96.582 & 111.382 & & 74.864 \\
\hline 1/3/08 1:30 & 125.828 & 123.319 & 101.029 & 104.915 & 96.558 & 111.358 & & 74.843 \\
\hline 1/3/08 2:00 & 125.809 & 123.299 & 101.01 & 104.896 & 96.537 & 111.336 & 81.315 & 74.82 \\
\hline $1 / 3 / 082: 30$ & 125.788 & 123.281 & 100.991 & 104.881 & 96.52 & 111.318 & & 74.804 \\
\hline 1/3/08 3:00 & 125.769 & 123.274 & 100.973 & 104.858 & 96.499 & 111.3 & & 74.785 \\
\hline 1/3/08 3:30 & 125.752 & 123.256 & 100.956 & 104.841 & 96.485 & 111.287 & 81.274 & 74.771 \\
\hline $1 / 3 / 084: 00$ & 125.731 & 123.231 & 100.953 & 104.835 & 96.476 & 111.272 & & 74.758 \\
\hline $1 / 3 / 084: 30$ & 125.721 & 123.217 & 100.923 & 104.814 & 96.457 & 111.258 & & 74.746 \\
\hline $1 / 3 / 085: 00$ & 125.705 & 123.202 & 100.906 & 104.789 & 96.433 & 111.232 & 81.242 & 74.721 \\
\hline 1/3/08 5:30 & 125.686 & 123.186 & 100.89 & 104.776 & 96.417 & 111.219 & & 74.704 \\
\hline $1 / 3 / 086: 00$ & 125.667 & 123.174 & 100.875 & 104.759 & 96.405 & 111.204 & & 74.69 \\
\hline 1/3/08 6:30 & 125.65 & 123.161 & 100.858 & 104.742 & 96.384 & 111.187 & 81.203 & 74.671 \\
\hline $1 / 3 / 087: 00$ & 125.636 & 124.732 & 100.853 & 104.73 & 96.377 & 111.176 & & 74.659 \\
\hline $1 / 3 / 087: 30$ & 125.622 & 125.193 & 100.834 & 104.723 & 96.362 & 111.168 & & 74.649 \\
\hline 1/3/08 8:00 & 126.915 & 125.358 & 101.487 & 104.947 & 96.704 & 111.527 & 81.176 & 74.634 \\
\hline 1/3/08 8:30 & 127.497 & 125.446 & 101.755 & 105.382 & 97.169 & 111.955 & & 74.797 \\
\hline 1/3/08 9:00 & 127.738 & 125.499 & 101.881 & 105.545 & 97.331 & 112.107 & & 74.948 \\
\hline 1/3/08 9:30 & 127.88 & 125.528 & 101.954 & 105.633 & 97.423 & 112.193 & 81.215 & 75.048 \\
\hline 1/3/08 10:00 & 127.969 & 124.53 & 101.997 & 105.688 & 97.477 & 112.242 & & 75.106 \\
\hline $1 / 3 / 08 \quad 10: 30$ & 128.029 & 123.614 & 102.019 & 105.719 & 97.508 & 112.276 & & 75.143 \\
\hline 1/3/08 11:00 & 128.062 & 123.406 & 101.494 & 105.738 & 97.527 & 112.293 & 81.23 & 75.165 \\
\hline $1 / 3 / 0811: 30$ & 126.3 & 123.281 & 101.16 & 105.134 & 96.78 & 111.549 & & 75.048 \\
\hline $1 / 3 / 08$ 12:00 & 126.022 & 123.192 & 101.003 & 104.938 & 96.584 & 111.367 & & 74.897 \\
\hline 1/3/08 12:30 & 125.851 & 123.131 & 100.898 & 104.82 & 96.459 & 111.26 & 81.164 & 74.779 \\
\hline 1/3/08 13:00 & 125.731 & 123.077 & 100.825 & 104.734 & 96.379 & 111.176 & & 74.686 \\
\hline 1/3/08 13:30 & 125.648 & 123.05 & 100.775 & 104.673 & 96.32 & 111.119 & & 74.622 \\
\hline 1/3/08 14:00 & 125.582 & 123.031 & 100.73 & 104.622 & 96.266 & 111.071 & 81.103 & 74.57 \\
\hline $1 / 3 / 08 \quad 14: 30$ & 125.53 & 123.013 & 100.706 & 104.595 & 96.235 & 111.041 & & 74.533 \\
\hline $1 / 3 / 08$ 15:00 & 125.492 & 123.004 & 100.693 & 104.58 & 96.221 & 111.022 & & 74.51 \\
\hline 1/3/08 15:30 & 125.471 & 122.988 & 100.682 & 104.567 & 96.209 & 111.012 & 81.061 & 74.494 \\
\hline $1 / 3 / 0816: 00$ & 125.452 & 122.977 & 100.667 & 104.555 & 96.197 & 111.005 & & 74.483 \\
\hline $1 / 3 / 0816: 30$ & 125.438 & 122.968 & 100.658 & 104.54 & 96.179 & 110.992 & & 74.467 \\
\hline 1/3/08 17:00 & 125.428 & 122.966 & 100.646 & 104.529 & 96.172 & 110.979 & 81.022 & 74.452 \\
\hline $1 / 3 / 08 \quad 17: 30$ & 125.414 & 122.966 & 100.644 & 104.521 & 96.162 & 110.975 & & 74.444 \\
\hline $1 / 3 / 0818: 00$ & 125.407 & 122.966 & 100.644 & 104.525 & 96.164 & 110.971 & & 74.44 \\
\hline $1 / 3 / 0818: 30$ & 125.402 & 122.957 & 100.646 & 104.523 & 96.164 & 110.973 & 81 & 74.44 \\
\hline 1/3/08 19:00 & 125.402 & 122.954 & 100.646 & 104.521 & 96.164 & 110.971 & & 74.438 \\
\hline 1/3/08 19:30 & 125.4 & 122.952 & 100.637 & 104.513 & 96.155 & 110.971 & & 74.429 \\
\hline
\end{tabular}


TABLE S1.1 (Cont.)

\begin{tabular}{|c|c|c|c|c|c|c|c|c|c|c|}
\hline \multirow[b]{2}{*}{ Date and Time } & \multicolumn{10}{|c|}{ Depth from Top of Casing (ft) } \\
\hline & MW1D & MW2D & MW3D & MW4D & MW7 & MW9 & Oentrich & MW14D & MW15D & MW16D \\
\hline 1/3/08 20:00 & 125.395 & 122.947 & & 100.634 & 104.515 & 96.155 & 110.965 & & 80.973 & 74.425 \\
\hline $1 / 3 / 0820: 30$ & 125.39 & 122.941 & & 100.632 & 104.511 & 96.153 & 110.962 & & & 74.421 \\
\hline 1/3/08 21:00 & 125.388 & 122.929 & & 100.624 & 104.504 & 96.146 & 110.96 & & & 74.415 \\
\hline $1 / 3 / 0821: 30$ & 125.381 & 122.918 & & 100.62 & 104.5 & 96.139 & 110.954 & & 80.944 & 74.407 \\
\hline $1 / 3 / 0822: 00$ & 125.379 & 122.916 & & 100.61 & 104.491 & 96.131 & 110.949 & & & 74.398 \\
\hline $1 / 3 / 0822: 30$ & 125.372 & 122.911 & & 100.6 & 104.477 & 96.12 & 110.941 & & & 74.39 \\
\hline 1/3/08 23:00 & 125.362 & 122.909 & & 100.594 & 104.471 & 96.115 & 110.935 & & 80.912 & 74.38 \\
\hline 1/3/08 23:30 & 125.355 & 122.907 & & 100.589 & 104.47 & 96.11 & 110.937 & & & 74.376 \\
\hline 1/4/08 0:00 & 125.348 & 122.898 & & 100.589 & 104.466 & 96.108 & 110.935 & & & 74.372 \\
\hline 1/4/08 0:30 & 125.346 & 122.889 & & 100.586 & 104.47 & 96.11 & 110.937 & & 80.888 & 74.37 \\
\hline 1/4/08 1:00 & 125.341 & 122.882 & & 100.579 & 104.456 & 96.101 & 110.941 & & & 74.361 \\
\hline 1/4/08 1:30 & 125.334 & 122.875 & & 100.567 & 104.449 & 96.091 & 110.943 & & & 74.353 \\
\hline $1 / 4 / 082: 00$ & 125.329 & 122.873 & & 100.569 & 104.449 & 96.087 & 110.943 & & 80.858 & 74.347 \\
\hline $1 / 4 / 082: 30$ & 125.324 & 122.868 & & 100.562 & 104.441 & 96.082 & 110.945 & & & 74.341 \\
\hline 1/4/08 3:00 & 125.32 & 122.857 & & 100.56 & 104.435 & 96.077 & 110.947 & & & 74.332 \\
\hline 1/4/08 3:30 & 125.315 & 122.852 & & 100.551 & 104.432 & 96.075 & 110.947 & & 80.841 & 74.33 \\
\hline $1 / 4 / 084: 00$ & 125.308 & 122.843 & & 100.541 & 104.42 & 96.063 & 110.95 & & & 74.318 \\
\hline 1/4/08 4:30 & 125.301 & 122.841 & & 100.536 & 104.414 & 96.058 & 110.953 & & & 74.31 \\
\hline 1/4/08 5:00 & 125.291 & 122.834 & & 100.531 & 104.407 & 96.051 & 110.954 & & 80.809 & 74.301 \\
\hline 1/4/08 5:30 & 125.289 & 122.825 & & 100.531 & 104.407 & 96.047 & 110.956 & & & 74.299 \\
\hline $1 / 4 / 086: 00$ & 125.284 & 122.823 & & 100.522 & 104.399 & 96.042 & 110.958 & & & 74.293 \\
\hline 1/4/08 6:30 & 125.279 & 122.818 & & 100.513 & 104.39 & 96.03 & 110.962 & & 80.77 & 74.285 \\
\hline 1/4/08 7:00 & 125.27 & 124.746 & & 100.513 & 104.386 & 96.03 & 110.964 & & & 74.276 \\
\hline 1/4/08 7:30 & 125.265 & 124.975 & & 100.82 & 104.384 & 96.028 & 110.966 & & & 74.272 \\
\hline 1/4/08 8:00 & 126.984 & 125.102 & & 101.306 & 104.907 & 96.674 & 111.609 & & 80.773 & 74.363 \\
\hline $1 / 4 / 088: 30$ & 127.319 & 125.165 & & 101.47 & 105.134 & 96.905 & 111.835 & & & 74.543 \\
\hline 1/4/08 9:00 & 127.506 & 125.208 & & 101.568 & 105.262 & 97.037 & 111.951 & & & 74.665 \\
\hline 1/4/08 9:30 & 127.615 & 125.249 & & 101.621 & 105.332 & 97.103 & 112.025 & & 80.819 & 74.742 \\
\hline 1/4/08 10:00 & 127.679 & 125.272 & & 101.67 & 105.374 & 97.147 & 112.072 & & & 74.791 \\
\hline $1 / 4 / 08 \quad 10: 30$ & 127.728 & 125.297 & & 101.706 & 105.414 & 97.19 & 112.105 & & & 74.833 \\
\hline 1/4/08 11:00 & 127.764 & 125.299 & & 101.726 & 105.441 & 97.213 & 112.134 & & 80.851 & 74.862 \\
\hline 1/4/08 11:30 & 127.785 & 125.304 & & 101.744 & 105.465 & 97.237 & 112.152 & & & 74.886 \\
\hline $1 / 4 / 08 \quad 12: 00$ & 127.804 & 125.297 & & 101.744 & 105.473 & 97.249 & 112.169 & & & 74.903 \\
\hline $1 / 4 / 0812: 30$ & 127.811 & 123.476 & & 101.747 & 105.473 & 97.246 & 112.186 & & 80.873 & 74.903 \\
\hline 1/4/08 13:00 & 127.813 & 123.211 & & 101.599 & 105.471 & 97.244 & 112.199 & & & 74.903 \\
\hline $1 / 4 / 0813: 30$ & 126.189 & 123.075 & & 100.983 & 104.993 & 96.645 & 111.623 & & & 74.826 \\
\hline $1 / 4 / 0814: 00$ & 125.802 & 122.991 & & 100.803 & 104.744 & 96.391 & 111.39 & & 80.839 & 74.659 \\
\hline 1/4/08 14:30 & 125.606 & 122.947 & & 100.701 & 104.614 & 96.259 & 111.285 & & & 74.535 \\
\hline $1 / 4 / 08 \quad 15: 00$ & 125.485 & 122.918 & & 100.641 & 104.536 & 96.179 & 111.223 & & & 74.446 \\
\hline $1 / 4 / 08 \quad 15: 30$ & 125.414 & 122.893 & & 100.612 & 104.498 & 96.143 & 111.182 & & 80.797 & 74.398 \\
\hline 1/4/08 16:00 & 125.374 & 122.875 & & 100.586 & 104.47 & 96.115 & 111.155 & & & 74.37 \\
\hline $1 / 4 / 0816: 30$ & 125.346 & 122.85 & & 100.558 & 104.447 & 96.089 & 111.138 & & & 74.341 \\
\hline $1 / 4 / 08 \quad 17: 00$ & 125.324 & 122.846 & & 100.541 & 104.43 & 96.072 & 111.112 & & 80.773 & 74.318 \\
\hline 1/4/08 17:30 & 125.303 & 122.846 & & 100.525 & 104.405 & 96.049 & 111.104 & & & 74.293 \\
\hline $1 / 4 / 08 \quad 18: 00$ & 125.284 & 122.832 & & 100.532 & 104.403 & 96.047 & 111.096 & & & 74.281 \\
\hline $1 / 4 / 08$ 18:30 & 125.282 & 122.823 & & 100.531 & 104.407 & 96.051 & 111.093 & & 80.748 & 74.283 \\
\hline 1/4/08 19:00 & 125.282 & 122.827 & & 100.512 & 104.393 & 96.037 & 111.092 & & & 74.281 \\
\hline 1/4/08 19:30 & 125.27 & 122.825 & & 100.503 & 104.382 & 96.028 & 111.09 & & & 74.264 \\
\hline $1 / 4 / 0820: 00$ & 125.263 & 122.818 & & 100.508 & 104.386 & 96.03 & 111.088 & & 80.729 & 74.26 \\
\hline 1/4/08 20:30 & 125.26 & 122.811 & & 100.512 & 104.384 & 96.03 & 111.088 & & & 74.258 \\
\hline 1/4/08 21:00 & 125.26 & 122.8 & & 100.501 & 104.38 & 96.025 & 111.086 & & & 74.256 \\
\hline $1 / 4 / 0821: 30$ & 125.256 & 122.789 & & 100.491 & 104.372 & 96.018 & 111.088 & & 80.712 & 74.252 \\
\hline 1/4/08 22:00 & 125.246 & 122.782 & & 100.484 & 104.363 & 96.009 & 111.088 & & & 74.239 \\
\hline 1/4/08 22:30 & 125.242 & 122.78 & & 100.47 & 104.35 & 95.995 & 111.09 & & & 74.233 \\
\hline 1/4/08 23:00 & 125.23 & 122.775 & & 100.469 & 104.346 & 95.99 & 111.09 & & 80.687 & 74.223 \\
\hline 1/4/08 23:30 & 125.223 & 122.759 & & 100.474 & 104.34 & 95.985 & 111.09 & & & 74.217 \\
\hline $1 / 5 / 08 \quad 0: 00$ & 125.22 & 122.748 & & 100.463 & 104.34 & 95.985 & 111.08 & & & 74.214 \\
\hline 1/5/08 0:30 & 125.213 & 122.737 & & 100.448 & 104.327 & 95.969 & 111.065 & & 80.66 & 74.206 \\
\hline 1/5/08 1:00 & 125.201 & 122.728 & & 100.431 & 104.313 & 95.959 & 111.058 & & & 74.196 \\
\hline 1/5/08 1:30 & 125.19 & 122.739 & & 100.42 & 104.3 & 95.945 & 111.048 & & & 74.181 \\
\hline 1/5/08 2:00 & 125.178 & 122.741 & & 100.422 & 104.293 & 95.938 & 111.039 & & 80.631 & 74.171 \\
\hline 1/5/08 2:30 & 125.171 & 122.732 & & 100.431 & 104.308 & 95.95 & 111.033 & & & 74.167 \\
\hline 1/5/08 3:00 & 125.173 & 122.73 & & 100.438 & 104.308 & 95.948 & 111.035 & & & 74.169 \\
\hline $1 / 5 / 083: 30$ & 125.178 & 122.723 & & 100.426 & 104.298 & 95.943 & 111.035 & & 80.616 & 74.171 \\
\hline 1/5/08 4:00 & 125.173 & 122.716 & & 100.426 & 104.296 & 95.94 & 111.033 & & & 74.165 \\
\hline 1/5/08 4:30 & 125.168 & 122.707 & & 100.415 & 104.287 & 95.933 & 111.031 & & & 74.161 \\
\hline
\end{tabular}


TABLE S1.1 (Cont.)

\begin{tabular}{|c|c|c|c|c|c|c|c|c|c|c|}
\hline \multirow[b]{2}{*}{ Date and Time } & \multicolumn{10}{|c|}{ Depth from Top of Casing (ft) } \\
\hline & MW1D & MW2D & MW3D & MW4D & MW7 & MW9 & Oentrich & MW14D & MW15D & MW16D \\
\hline 1/5/08 5:00 & 125.164 & 122.696 & & 100.403 & 104.283 & 95.926 & 111.027 & & 80.606 & 74.152 \\
\hline 1/5/08 5:30 & 125.156 & 122.689 & & 100.395 & 104.273 & 95.919 & 111.024 & & & 74.144 \\
\hline 1/5/08 6:00 & 125.147 & 122.698 & & 100.386 & 104.26 & 95.905 & 111.016 & & & 74.132 \\
\hline 1/5/08 6:30 & 125.138 & 122.716 & & 100.382 & 104.254 & 95.898 & 111.009 & & 80.582 & 74.124 \\
\hline $1 / 5 / 087: 00$ & 125.133 & 122.734 & & 100.4 & 104.268 & 95.91 & 111.005 & & & 74.121 \\
\hline 1/5/08 7:30 & 125.138 & 122.748 & & 100.427 & 104.281 & 95.926 & 111.005 & & & 74.134 \\
\hline 1/5/08 8:00 & 125.156 & 122.746 & & 100.434 & 104.3 & 95.945 & 111.005 & & 80.57 & 74.152 \\
\hline 1/5/08 8:30 & 125.171 & 124.621 & & 100.453 & 104.315 & 95.959 & 111.007 & & & 74.169 \\
\hline 1/5/08 9:00 & 125.185 & 124.848 & & 100.658 & 104.313 & 95.962 & 111.009 & & & 74.177 \\
\hline 1/5/08 9:30 & 126.891 & 124.968 & & 101.202 & 104.803 & 96.572 & 111.623 & & 80.582 & 74.243 \\
\hline $1 / 5 / 08$ 10:00 & 127.253 & 125.038 & & 101.363 & 105.027 & 96.801 & 111.861 & & & 74.413 \\
\hline 1/5/08 10:30 & 127.421 & 125.079 & & 101.456 & 105.147 & 96.921 & 111.978 & & & 74.529 \\
\hline $1 / 5 / 0811: 00$ & 127.518 & 123.217 & & 101.52 & 105.22 & 96.994 & 112.049 & & 80.624 & 74.611 \\
\hline 1/5/08 11:30 & 127.577 & 123.004 & & 101.156 & 105.265 & 97.041 & 112.096 & & & 74.663 \\
\hline 1/5/08 12:00 & 125.851 & 122.884 & & 100.779 & 104.761 & 96.41 & 111.472 & & & 74.593 \\
\hline $1 / 5 / 0812: 30$ & 125.544 & 122.825 & & 100.624 & 104.555 & 96.2 & 111.292 & & 80.609 & 74.456 \\
\hline $1 / 5 / 08$ 13:00 & 125.383 & 122.768 & & 100.531 & 104.439 & 96.084 & 111.193 & & & 74.351 \\
\hline 1/5/08 13:30 & 125.291 & 122.732 & & 100.482 & 104.378 & 96.023 & 111.131 & & & 74.276 \\
\hline $1 / 5 / 0814: 00$ & 125.239 & 122.723 & & 100.436 & 104.331 & 95.978 & 111.095 & & 80.57 & 74.223 \\
\hline $1 / 5 / 08$ 14:30 & 125.197 & 122.689 & & 100.412 & 104.293 & 95.94 & 111.063 & & & 74.179 \\
\hline $1 / 5 / 08$ 15:00 & 125.171 & 122.678 & & 100.405 & 104.285 & 95.929 & 111.044 & & & 74.152 \\
\hline $1 / 5 / 08$ 15:30 & 125.152 & 122.669 & & 100.376 & 104.251 & 95.898 & 111.027 & & 80.54 & 74.14 \\
\hline $1 / 5 / 08$ 16:00 & 125.135 & 122.662 & & 100.363 & 104.241 & 95.889 & 111.022 & & & 74.126 \\
\hline $1 / 5 / 0816: 30$ & 125.13 & 122.666 & & 100.355 & 104.232 & 95.882 & 111.014 & & & 74.115 \\
\hline $1 / 5 / 08 \quad 17: 00$ & 125.128 & 122.669 & & 100.357 & 104.23 & 95.875 & 110.999 & & 80.516 & 74.107 \\
\hline 1/5/08 17:30 & 125.123 & 122.662 & & 100.365 & 104.232 & 95.877 & 110.994 & & & 74.103 \\
\hline $1 / 5 / 08$ 18:00 & 125.121 & 122.666 & & 100.358 & 104.234 & 95.884 & 110.994 & & & 74.113 \\
\hline $1 / 5 / 08$ 18:30 & 125.123 & 122.66 & & 100.36 & 104.228 & 95.875 & 110.992 & & 80.504 & 74.146 \\
\hline 1/5/08 19:00 & 125.123 & 122.666 & & 100.355 & 104.232 & 95.877 & 110.99 & & & 74.181 \\
\hline $1 / 5 / 08$ 19:30 & 125.123 & 122.653 & & 100.355 & 104.228 & 95.875 & 110.988 & & & 74.235 \\
\hline $1 / 5 / 0820: 00$ & 125.123 & 122.648 & & 100.357 & 104.235 & 95.879 & 110.986 & & 80.496 & 74.339 \\
\hline $1 / 5 / 0820: 30$ & 125.128 & 122.644 & & 100.346 & 104.22 & 95.87 & 110.984 & & & 74.438 \\
\hline $1 / 5 / 0821: 00$ & 125.13 & 122.646 & & 100.336 & 104.211 & 95.856 & 110.981 & & & 74.512 \\
\hline $1 / 5 / 0821: 30$ & 125.128 & 122.63 & & 100.345 & 104.209 & 95.856 & 110.979 & & 80.482 & 74.568 \\
\hline 1/5/08 22:00 & 125.13 & 122.623 & & 100.339 & 104.216 & 95.86 & 110.977 & & & 74.622 \\
\hline 1/5/08 22:30 & 125.135 & 122.621 & & 100.32 & 104.195 & 95.844 & 110.969 & & & 74.667 \\
\hline 1/5/08 23:00 & 125.133 & 122.621 & & 100.314 & 104.192 & 95.839 & 110.962 & & 80.462 & 74.702 \\
\hline 1/5/08 23:30 & 125.135 & 122.617 & & 100.315 & 104.19 & 95.834 & 110.96 & & & 74.731 \\
\hline $1 / 6 / 080: 00$ & 125.133 & 122.621 & & 100.317 & 104.19 & 95.834 & 110.956 & & & 74.756 \\
\hline 1/6/08 0:30 & 125.138 & 122.632 & & 100.319 & 104.186 & 95.83 & 110.956 & & 80.45 & 74.777 \\
\hline 1/6/08 1:00 & 125.135 & 122.632 & & 100.32 & 104.192 & 95.842 & 110.956 & & & 74.795 \\
\hline 1/6/08 1:30 & 125.133 & 122.632 & & 100.326 & 104.203 & 95.849 & 110.952 & & & 74.81 \\
\hline 1/6/08 2:00 & 125.135 & 122.628 & & 100.334 & 104.201 & 95.849 & 110.954 & & 80.438 & 74.822 \\
\hline 1/6/08 2:30 & 125.135 & 122.639 & & 100.322 & 104.203 & 95.849 & 110.954 & & & 74.835 \\
\hline 1/6/08 3:00 & 125.135 & 122.646 & & 100.329 & 104.203 & 95.849 & 110.954 & & & 74.845 \\
\hline 1/6/08 3:30 & 125.138 & 122.655 & & 100.343 & 104.209 & 95.856 & 110.954 & & 80.428 & 74.859 \\
\hline 1/6/08 4:00 & 125.135 & 122.664 & & 100.348 & 104.218 & 95.867 & 110.954 & & & 74.886 \\
\hline $1 / 6 / 084: 30$ & 125.138 & 122.664 & & 100.368 & 104.224 & 95.872 & 110.956 & & & 74.924 \\
\hline 1/6/08 5:00 & 125.135 & 122.678 & & 100.363 & 104.245 & 95.893 & 110.956 & & 80.426 & 74.961 \\
\hline 1/6/08 5:30 & 125.138 & 122.687 & & 100.375 & 104.235 & 95.884 & 110.958 & & & 75 \\
\hline $1 / 6 / 086: 00$ & 125.142 & 122.712 & & 100.381 & 104.249 & 95.898 & 110.958 & & & 75.031 \\
\hline 1/6/08 6:30 & 125.14 & 122.723 & & 100.396 & 104.26 & 95.907 & 110.958 & & 80.423 & 75.056 \\
\hline 1/6/08 7:00 & 125.135 & 122.743 & & 100.424 & 104.285 & 95.929 & 110.956 & & & 75.077 \\
\hline $1 / 6 / 087: 30$ & 125.138 & 122.762 & & 100.439 & 104.298 & 95.945 & 110.958 & & & 75.097 \\
\hline 1/6/08 8:00 & 125.138 & 122.768 & & 100.456 & 104.317 & 95.962 & 110.958 & & 80.45 & 75.112 \\
\hline 1/6/08 8:30 & 125.138 & 124.317 & & 100.468 & 104.334 & 95.983 & 110.958 & & & 75.128 \\
\hline $1 / 6 / 089: 00$ & 125.142 & 124.809 & & 100.472 & 104.338 & 95.988 & 110.96 & & & 75.145 \\
\hline $1 / 6 / 089: 30$ & 126.407 & 125.007 & & 101.13 & 104.561 & 96.313 & 111.311 & & 80.455 & 75.159 \\
\hline $1 / 6 / 08 \quad 10: 00$ & 126.91 & 125.113 & & 101.421 & 105.024 & 96.81 & 111.764 & & & 75.321 \\
\hline $1 / 6 / 08 \quad 10: 30$ & 127.109 & 125.193 & & 101.573 & 105.22 & 97.011 & 111.927 & & & 75.463 \\
\hline 1/6/08 11:00 & 127.227 & 125.238 & & 101.664 & 105.326 & 97.121 & 112.023 & & 80.543 & 75.567 \\
\hline $1 / 6 / 0811: 30$ & 127.281 & 125.274 & & 101.726 & 105.408 & 97.202 & 112.081 & & & 75.643 \\
\hline $1 / 6 / 08 \quad 12: 00$ & 127.322 & 123.773 & & 101.771 & 105.452 & 97.244 & 112.122 & & & 75.705 \\
\hline 1/6/08 12:30 & 127.352 & 123.315 & & 101.794 & 105.49 & 97.284 & 112.152 & & 80.604 & 75.761 \\
\hline $1 / 6 / 08$ 13:00 & 126.126 & 123.154 & & 101.159 & 105.256 & 96.935 & 111.794 & & & 75.8 \\
\hline $1 / 6 / 08$ 13:30 & 125.702 & 123.07 & & 100.91 & 104.858 & 96.509 & 111.399 & & & 75.701 \\
\hline
\end{tabular}


TABLE S1.1 (Cont.)

\begin{tabular}{|c|c|c|c|c|c|c|c|c|c|c|}
\hline \multirow[b]{2}{*}{ Date and Time } & \multicolumn{10}{|c|}{ Depth from Top of Casing (ft) } \\
\hline & MW1D & MW2D & MW3D & MW4D & MW7 & MW9 & Oentrich & MW14D & MW15D & MW16D \\
\hline 1/6/08 14:00 & 125.57 & 123.013 & & 100.789 & 104.706 & 96.353 & 111.255 & & 80.597 & 75.614 \\
\hline $1 / 6 / 08 \quad 14: 30$ & 125.494 & 122.984 & & 100.765 & 104.63 & 96.273 & 111.176 & & & 75.558 \\
\hline 1/6/08 15:00 & 125.454 & 122.959 & & 100.741 & 104.572 & 96.219 & 111.129 & & & 75.523 \\
\hline $1 / 6 / 08 \quad 15: 30$ & 125.426 & 122.943 & & 100.732 & 104.546 & 96.193 & 111.097 & & 80.587 & 75.5 \\
\hline $1 / 6 / 08$ 16:00 & 125.407 & 122.938 & & 100.703 & 104.523 & 96.172 & 111.075 & & & 75.484 \\
\hline 1/6/08 16:30 & 125.398 & 122.932 & & 100.674 & 104.506 & 96.155 & 111.063 & & & 75.455 \\
\hline $1 / 6 / 08 \quad 17: 00$ & 125.386 & 122.929 & & 100.656 & 104.502 & 96.153 & 111.052 & & 80.589 & 75.445 \\
\hline 1/6/08 17:30 & 125.374 & 122.938 & & 100.643 & 104.498 & 96.146 & 111.043 & & & 75.438 \\
\hline 1/6/08 18:00 & 125.367 & 122.948 & & 100.634 & 104.498 & 96.146 & 111.035 & & & 75.428 \\
\hline $1 / 6 / 0818: 30$ & 125.357 & 122.95 & & 100.641 & 104.506 & 96.153 & 111.027 & & 80.592 & 75.426 \\
\hline 1/6/08 19:00 & 125.353 & 122.952 & & 100.651 & 104.513 & 96.164 & 111.024 & & & 75.428 \\
\hline 1/6/08 19:30 & 125.35 & 122.963 & & 100.649 & 104.523 & 96.169 & 111.02 & & & 75.432 \\
\hline $1 / 6 / 0820: 00$ & 125.348 & 122.95 & & 100.651 & 104.523 & 96.167 & 111.018 & & 80.604 & 75.436 \\
\hline $1 / 6 / 0820: 30$ & 125.343 & 122.932 & & 100.662 & 104.532 & 96.179 & 111.014 & & & 75.438 \\
\hline 1/6/08 21:00 & 125.346 & 122.934 & & 100.636 & 104.521 & 96.167 & 111.014 & & & 75.436 \\
\hline $1 / 6 / 0821: 30$ & 125.348 & 122.907 & & 100.627 & 104.498 & 96.148 & 111.016 & & 80.606 & 75.416 \\
\hline $1 / 6 / 0822: 00$ & 125.343 & 122.898 & & 100.625 & 104.504 & 96.153 & 111.014 & & & 75.416 \\
\hline $1 / 6 / 0822: 30$ & 125.348 & 122.895 & & 100.6 & 104.473 & 96.12 & 111.016 & & & 75.383 \\
\hline $1 / 6 / 0823: 00$ & 125.346 & 122.891 & & 100.591 & 104.466 & 96.113 & 111.015 & & 80.602 & 75.366 \\
\hline 1/6/08 23:30 & 125.341 & 122.891 & & 100.591 & 104.464 & 96.11 & 111.011 & & & 75.358 \\
\hline 1/7/08 0:00 & 125.338 & 122.877 & & 100.584 & 104.46 & 96.108 & 111.009 & & & 75.354 \\
\hline 1/7/08 0:30 & 125.336 & 122.861 & & 100.582 & 104.458 & 96.106 & 111.009 & & 80.599 & 75.345 \\
\hline 1/7/08 1:00 & 125.338 & 122.848 & & 100.57 & 104.449 & 96.096 & 111.009 & & & 75.341 \\
\hline 1/7/08 1:30 & 125.336 & 122.83 & & 100.556 & 104.432 & 96.08 & 111.009 & & & 75.325 \\
\hline 1/7/08 2:00 & 125.334 & 122.891 & & 100.538 & 104.416 & 96.063 & 111.009 & & 80.58 & 75.31 \\
\hline 1/7/08 2:30 & 125.334 & 122.891 & & 100.543 & 104.401 & 96.051 & 111.009 & & & 75.3 \\
\hline 1/7/08 3:00 & 125.322 & 122.855 & & 100.581 & 104.47 & 96.117 & 110.998 & & & 75.296 \\
\hline 1/7/08 3:30 & 125.327 & 122.814 & & 100.56 & 104.471 & 96.12 & 110.998 & & 80.592 & 75.31 \\
\hline 1/7/08 4:00 & 125.331 & 122.8 & & 100.546 & 104.428 & 96.075 & 111.003 & & & 75.306 \\
\hline 1/7/08 4:30 & 125.341 & 122.787 & & 100.515 & 104.386 & 96.037 & 111.009 & & & 75.298 \\
\hline 1/7/08 5:00 & 125.334 & 122.784 & & 100.496 & 104.369 & 96.014 & 111.005 & & 80.55 & 75.256 \\
\hline $1 / 7 / 085: 30$ & 125.334 & 122.809 & & 100.482 & 104.357 & 96.004 & 111.005 & & & 75.254 \\
\hline 1/7/08 6:00 & 125.331 & 122.787 & & 100.479 & 104.352 & 95.995 & 111.003 & & & 75.238 \\
\hline 1/7/08 6:30 & 125.324 & 122.796 & & 100.491 & 104.38 & 96.023 & 110.998 & & 80.545 & 75.24 \\
\hline 1/7/08 7:00 & 125.331 & 124.614 & & 100.475 & 104.355 & 96.002 & 111.003 & & & 75.244 \\
\hline 1/7/08 7:30 & 125.327 & 124.939 & & 100.474 & 104.369 & 96.014 & 111 & & & 75.24 \\
\hline 1/7/08 8:00 & 126.825 & 125.077 & & 101.194 & 104.791 & 96.553 & 111.535 & & 80.55 & 75.281 \\
\hline 1/7/08 8:30 & 127.088 & 125.181 & & 101.442 & 105.111 & 96.886 & 111.813 & & & 75.447 \\
\hline 1/7/08 9:00 & 127.203 & 125.249 & & 101.554 & 105.258 & 97.03 & 111.946 & & & 75.571 \\
\hline 1/7/08 9:30 & 127.27 & 125.27 & & 101.649 & 105.359 & 97.133 & 112.019 & & 80.633 & 75.658 \\
\hline 1/7/08 10:00 & 127.312 & 123.421 & & 101.697 & 105.429 & 97.206 & 112.068 & & & 75.726 \\
\hline $1 / 7 / 08 \quad 10: 30$ & 127.35 & 123.245 & & 101.419 & 105.454 & 97.23 & 112.105 & & & 75.782 \\
\hline 1/7/08 11:00 & 125.773 & 123.14 & & 101.145 & 104.965 & 96.612 & 111.459 & & 80.672 & 75.726 \\
\hline 1/7/08 11:30 & 125.606 & 123.088 & & 101.037 & 104.799 & 96.447 & 111.281 & & & 75.633 \\
\hline $1 / 7 / 08 \quad 12: 00$ & 125.53 & 123.045 & & 100.98 & 104.696 & 96.344 & 111.195 & & & 75.575 \\
\hline 1/7/08 12:30 & 125.487 & 123.022 & & 100.944 & 104.645 & 96.294 & 111.142 & & 80.665 & 75.531 \\
\hline 1/7/08 13:00 & 125.459 & 123 & & 100.789 & 104.609 & 96.254 & 111.11 & & & 75.502 \\
\hline 1/7/08 13:30 & 125.442 & 122.977 & & 100.743 & 104.58 & 96.228 & 111.089 & & & 75.476 \\
\hline 1/7/08 14:00 & 125.433 & 122.979 & & 100.723 & 104.563 & 96.212 & 111.074 & & 80.653 & 75.463 \\
\hline 1/7/08 14:30 & 125.424 & 122.968 & & 100.718 & 104.542 & 96.193 & 111.052 & & & 75.453 \\
\hline $1 / 7 / 08 \quad 15: 00$ & 125.412 & 122.968 & & 100.73 & 104.544 & 96.193 & 111.04 & & & 75.442 \\
\hline 1/7/08 15:30 & 125.405 & 122.968 & & 100.711 & 104.534 & 96.183 & 111.035 & & 80.646 & 75.44 \\
\hline 1/7/08 16:00 & 125.398 & 122.972 & & 100.712 & 104.534 & 96.183 & 111.03 & & & 75.438 \\
\hline 1/7/08 16:30 & 125.39 & 122.984 & & 100.746 & 104.532 & 96.179 & 111.026 & & & 75.438 \\
\hline 1/7/08 17:00 & 125.383 & 122.991 & & 100.782 & 104.542 & 96.188 & 111.022 & & 80.648 & 75.438 \\
\hline 1/7/08 17:30 & 125.376 & 122.991 & & 100.794 & 104.55 & 96.197 & 111.018 & & & 75.438 \\
\hline $1 / 7 / 08$ 18:00 & 125.372 & 122.997 & & 100.773 & 104.559 & 96.209 & 111.016 & & & 75.442 \\
\hline 1/7/08 18:30 & 125.367 & 122.993 & & 100.751 & 104.561 & 96.207 & 111.015 & & 80.658 & 75.445 \\
\hline 1/7/08 19:00 & 125.362 & 122.995 & & 100.765 & 104.567 & 96.212 & 111.011 & & & 75.447 \\
\hline 1/7/08 19:30 & 125.362 & 123.002 & & 100.746 & 104.561 & 96.212 & 111.011 & & & 75.451 \\
\hline 1/7/08 20:00 & 125.36 & 123.009 & & 100.735 & 104.565 & 96.214 & 111.009 & & 80.663 & 75.447 \\
\hline 1/7/08 20:30 & 125.357 & 123.009 & & 100.728 & 104.571 & 96.219 & 111.007 & & & 75.447 \\
\hline 1/7/08 21:00 & 125.36 & 123.011 & & 100.729 & 104.58 & 96.226 & 111.005 & & & 75.449 \\
\hline 1/7/08 21:30 & 125.36 & 122.995 & & 100.725 & 104.578 & 96.226 & 111.003 & & 80.677 & 75.451 \\
\hline 1/7/08 22:00 & 125.36 & 122.997 & & 100.722 & 104.58 & 96.226 & 111.001 & & & 75.451 \\
\hline 1/7/08 22:30 & 125.364 & 122.982 & & 100.717 & 104.563 & 96.212 & 111.003 & & & 75.451 \\
\hline
\end{tabular}


TABLE S1.1 (Cont.)

\begin{tabular}{|c|c|c|c|c|c|c|c|c|c|c|}
\hline \multirow[b]{2}{*}{ Date and Time } & \multicolumn{10}{|c|}{ Depth from Top of Casing (ft) } \\
\hline & MW1D & MW2D & MW3D & MW4D & MW7 & MW9 & Oentrich & MW14D & MW15D & MW16D \\
\hline 1/7/08 23:00 & 125.362 & 122.97 & & 100.712 & 104.565 & 96.209 & 111.001 & & 80.68 & 75.445 \\
\hline 1/7/08 23:30 & 125.364 & 122.936 & & 100.706 & 104.551 & 96.195 & 111.003 & & & 75.44 \\
\hline 1/8/08 0:00 & 125.367 & 122.948 & & 100.697 & 104.54 & 96.188 & 111.003 & & & 75.436 \\
\hline 1/8/08 0:30 & 125.367 & 122.936 & & 100.673 & 104.502 & 96.146 & 111.005 & & 80.66 & 75.401 \\
\hline 1/8/08 1:00 & 125.36 & 122.932 & & 100.68 & 104.513 & 96.157 & 110.997 & & & 75.395 \\
\hline 1/8/08 1:30 & 125.362 & 122.92 & & 100.672 & 104.506 & 96.153 & 110.999 & & & 75.397 \\
\hline 1/8/08 2:00 & 125.364 & 122.904 & & 100.663 & 104.5 & 96.146 & 110.996 & & 80.66 & 75.389 \\
\hline 1/8/08 2:30 & 125.367 & 122.959 & & 100.658 & 104.492 & 96.136 & 110.999 & & & 75.385 \\
\hline 1/8/08 3:00 & 125.367 & 123.009 & & 100.642 & 104.473 & 96.117 & 110.994 & & & 75.374 \\
\hline 1/8/08 3:30 & 125.348 & 122.988 & & 100.682 & 104.531 & 96.172 & 110.983 & & 80.668 & 75.364 \\
\hline $1 / 8 / 084: 00$ & 125.348 & 122.977 & & 100.701 & 104.584 & 96.228 & 110.979 & & & 75.372 \\
\hline 1/8/08 4:30 & 125.355 & 122.995 & & 100.701 & 104.563 & 96.207 & 110.982 & & & 75.387 \\
\hline 1/8/08 5:00 & 125.357 & 122.997 & & 100.697 & 104.548 & 96.193 & 110.984 & & 80.663 & 75.397 \\
\hline 1/8/08 5:30 & 125.353 & 123.013 & & 100.698 & 104.565 & 96.212 & 110.977 & & & 75.401 \\
\hline 1/8/08 6:00 & 125.355 & 123.02 & & 100.693 & 104.572 & 96.216 & 110.977 & & & 75.409 \\
\hline 1/8/08 6:30 & 125.353 & 123.045 & & 100.708 & 104.582 & 96.228 & 110.973 & & 80.677 & 75.414 \\
\hline 1/8/08 7:00 & 125.355 & 124.982 & & 100.715 & 104.593 & 96.24 & 110.971 & & & 75.409 \\
\hline 1/8/08 7:30 & 125.362 & 125.236 & & 101.039 & 104.62 & 96.266 & 110.967 & & & 74.866 \\
\hline 1/8/08 8:00 & 127.066 & 125.365 & & 101.588 & 105.197 & 96.98 & 111.647 & & 80.721 & 74.994 \\
\hline 1/8/08 8:30 & 127.428 & 125.446 & & 101.786 & 105.444 & 97.237 & 111.874 & & & 75.194 \\
\hline 1/8/08 9:00 & 127.603 & 125.508 & & 101.89 & 105.574 & 97.362 & 111.989 & & & 75.389 \\
\hline 1/8/08 9:30 & 127.667 & 125.553 & & 101.941 & 105.658 & 97.449 & 112.06 & & 80.8 & 75.414 \\
\hline 1/8/08 10:00 & 127.712 & 125.589 & & 101.976 & 105.719 & 97.51 & 112.109 & & & 75.49 \\
\hline 1/8/08 10:30 & 127.743 & 125.621 & & 102.007 & 105.764 & 97.555 & 112.141 & & & 75.54 \\
\hline 1/8/08 11:00 & 127.761 & 125.66 & & 102.047 & 105.799 & 97.591 & 112.167 & & 80.88 & 75.591 \\
\hline 1/8/08 11:30 & 127.783 & 124.034 & & 102.074 & 105.835 & 97.624 & 112.184 & & & 75.635 \\
\hline 1/8/08 12:00 & 127.799 & 123.664 & & 102.109 & 105.871 & 97.661 & 112.199 & & & 75.649 \\
\hline $1 / 8 / 0812: 30$ & 126.442 & 123.512 & & 101.566 & 105.54 & 97.206 & 111.726 & & 80.927 & 75.624 \\
\hline 1/8/08 13:00 & 126.116 & 123.424 & & 101.319 & 105.201 & 96.85 & 111.395 & & & 75.5 \\
\hline $1 / 8 / 0813: 30$ & 125.984 & 123.363 & & 101.206 & 105.056 & 96.704 & 111.257 & & & 75.327 \\
\hline 1/8/08 14:00 & 125.896 & 123.331 & & 101.127 & 104.97 & 96.619 & 111.181 & & 80.905 & 75.24 \\
\hline 1/8/08 14:30 & 125.83 & 123.315 & & 101.082 & 104.917 & 96.565 & 111.131 & & & 75.263 \\
\hline $1 / 8 / 08 \quad 15: 00$ & 125.783 & 123.322 & & 101.066 & 104.885 & 96.535 & 111.097 & & & 75.194 \\
\hline 1/8/08 15:30 & 125.747 & 123.324 & & 101.053 & 104.868 & 96.516 & 111.073 & & 80.902 & 75.139 \\
\hline 1/8/08 16:00 & 125.74 & 123.319 & & 101.053 & 104.877 & 96.525 & 111.05 & & & 75.151 \\
\hline 1/8/08 16:30 & 125.74 & 123.328 & & 101.052 & 104.885 & 96.532 & 111.037 & & & 75.172 \\
\hline 1/8/08 17:00 & 125.743 & 123.319 & & 101.097 & 104.881 & 96.527 & 111.024 & & 80.914 & 75.149 \\
\hline 1/8/08 17:30 & 125.747 & 123.317 & & 101.113 & 104.89 & 96.539 & 111.014 & & & 75.188 \\
\hline $1 / 8 / 08 \quad 18: 00$ & 125.754 & 123.313 & & 101.111 & 104.883 & 96.53 & 111.007 & & & 75.18 \\
\hline 1/8/08 18:30 & 125.75 & 123.308 & & 101.099 & 104.881 & 96.527 & 111.001 & & 80.922 & 75.176 \\
\hline 1/8/08 19:00 & 125.747 & 123.31 & & 101.094 & 104.877 & 96.525 & 110.996 & & & 75.157 \\
\hline 1/8/08 19:30 & 125.743 & 123.313 & & 101.087 & 104.869 & 96.518 & 110.99 & & & 75.19 \\
\hline $1 / 8 / 0820: 00$ & 125.74 & 123.313 & & 101.085 & 104.871 & 96.518 & 110.984 & & 80.927 & 75.17 \\
\hline 1/8/08 20:30 & 125.74 & 123.306 & & 101.071 & 104.877 & 96.525 & 110.97 & & & 75.221 \\
\hline $1 / 8 / 0821: 00$ & 125.743 & 123.299 & & 101.063 & 104.877 & 96.525 & 110.957 & & & 75.126 \\
\hline 1/8/08 21:30 & 125.745 & 123.308 & & 101.049 & 104.868 & 96.513 & 110.952 & & 80.932 & 75.118 \\
\hline 1/8/08 22:00 & 125.738 & 123.304 & & 101.039 & 104.862 & 96.509 & 110.95 & & & 75.108 \\
\hline 1/8/08 22:30 & 125.736 & 123.297 & & 101.037 & 104.868 & 96.516 & 110.944 & & & 75.178 \\
\hline 1/8/08 23:00 & 125.738 & 123.29 & & 101.032 & 104.868 & 96.516 & 110.942 & & 80.944 & 75.151 \\
\hline 1/8/08 23:30 & 125.736 & 123.279 & & 101.023 & 104.864 & 96.509 & 110.94 & & & 75.211 \\
\hline 1/9/08 0:00 & 125.733 & 123.267 & & 101.017 & 104.856 & 96.502 & 110.936 & & & 75.246 \\
\hline 1/9/08 0:30 & 125.726 & 123.254 & & 101.01 & 104.841 & 96.49 & 110.936 & & 80.944 & 75.178 \\
\hline 1/9/08 1:00 & 125.717 & 123.242 & & 100.991 & 104.829 & 96.478 & 110.934 & & & 75.108 \\
\hline 1/9/08 1:30 & 125.702 & 123.226 & & 100.98 & 104.82 & 96.466 & 110.93 & & & 75.157 \\
\hline 1/9/08 2:00 & 125.691 & 123.215 & & 100.97 & 104.808 & 96.454 & 110.926 & & 80.929 & 75.184 \\
\hline 1/9/08 2:30 & 125.679 & 123.206 & & 100.953 & 104.791 & 96.436 & 110.923 & & & 75.072 \\
\hline 1/9/08 3:00 & 125.665 & 123.204 & & 100.939 & 104.782 & 96.426 & 110.921 & & & 75.021 \\
\hline 1/9/08 3:30 & 125.653 & 123.195 & & 100.932 & 104.77 & 96.414 & 110.917 & & 80.919 & 75.017 \\
\hline 1/9/08 4:00 & 125.643 & 123.172 & & 100.929 & 104.769 & 96.412 & 110.911 & & & 74.994 \\
\hline $1 / 9 / 084: 30$ & 125.639 & 123.161 & & 100.918 & 104.763 & 96.405 & 110.908 & & & 75.004 \\
\hline $1 / 9 / 085: 00$ & 125.627 & 123.149 & & 100.898 & 104.736 & 96.381 & 110.907 & & 80.902 & 74.919 \\
\hline 1/9/08 5:30 & 125.613 & 123.136 & & 100.886 & 104.729 & 96.372 & 110.904 & & & 74.924 \\
\hline 1/9/08 6:00 & 125.596 & 123.12 & & 100.875 & 104.713 & 96.358 & 110.902 & & & 74.95 \\
\hline 1/9/08 6:30 & 125.584 & 123.115 & & 100.863 & 104.7 & 96.346 & 110.898 & & 80.88 & 74.961 \\
\hline 1/9/08 7:00 & 125.572 & 124.791 & & 100.846 & 104.687 & 96.329 & 110.898 & & & 74.965 \\
\hline 1/9/08 7:30 & 125.558 & 125.168 & & 100.842 & 104.681 & 96.325 & 110.894 & & & 74.961 \\
\hline
\end{tabular}


TABLE S1.1 (Cont.)

\begin{tabular}{|c|c|c|c|c|c|c|c|c|c|c|}
\hline \multirow[b]{2}{*}{ Date and Time } & \multicolumn{10}{|c|}{ Depth from Top of Casing (ft) } \\
\hline & MW1D & MW2D & MW3D & MW4D & MW7 & MW9 & Oentrich & MW14D & MW15D & MW16D \\
\hline 1/9/08 8:00 & 126.986 & 125.326 & & 101.389 & 104.972 & 96.728 & 111.311 & & 80.873 & 74.667 \\
\hline 1/9/08 8:30 & 127.52 & 125.419 & & 101.652 & 105.338 & 97.112 & 111.668 & & & 74.727 \\
\hline 1/9/08 9:00 & 127.757 & 125.483 & & 101.79 & 105.498 & 97.27 & 111.813 & & & 74.872 \\
\hline 1/9/08 9:30 & 127.884 & 125.51 & & 101.875 & 105.593 & 97.369 & 111.897 & & 80.932 & 74.965 \\
\hline 1/9/08 10:00 & 127.962 & 125.53 & & 101.937 & 105.658 & 97.433 & 111.948 & & & 75.031 \\
\hline 1/9/08 10:30 & 128.007 & 123.811 & & 101.954 & 105.688 & 97.461 & 111.987 & & & 75.083 \\
\hline 1/9/08 11:00 & 128.029 & 123.496 & & 101.973 & 105.705 & 97.48 & 112.015 & & 80.968 & 75.11 \\
\hline 1/9/08 11:30 & 126.615 & 123.344 & & 101.356 & 105.328 & 96.982 & 111.519 & & & 75.101 \\
\hline 1/9/08 12:00 & 126.338 & 123.236 & & 101.137 & 105.031 & 96.676 & 111.249 & & & 74.998 \\
\hline $1 / 9 / 0812: 30$ & 126.22 & 123.158 & & 101.018 & 104.888 & 96.53 & 111.133 & & 80.946 & 74.919 \\
\hline 1/9/08 13:00 & 126.152 & 123.09 & & 100.918 & 104.784 & 96.431 & 111.069 & & & 74.872 \\
\hline 1/9/08 13:30 & 126.057 & 123.041 & & 100.851 & 104.709 & 96.351 & 111.027 & & & 74.822 \\
\hline 1/9/08 14:00 & 125.868 & 122.997 & & 100.789 & 104.643 & 96.285 & 111.001 & & 80.888 & 74.793 \\
\hline 1/9/08 14:30 & 125.695 & 122.975 & & 100.747 & 104.593 & 96.237 & 110.982 & & & 74.789 \\
\hline 1/9/08 15:00 & 125.563 & 122.948 & & 100.716 & 104.555 & 96.197 & 110.967 & & & 74.777 \\
\hline 1/9/08 15:30 & 125.478 & 122.932 & & 100.71 & 104.531 & 96.172 & 110.954 & & 80.824 & 74.766 \\
\hline 1/9/08 16:00 & 125.412 & 122.92 & & 100.706 & 104.506 & 96.148 & 110.947 & & & 74.758 \\
\hline 1/9/08 16:30 & 125.376 & 122.916 & & 100.67 & 104.491 & 96.131 & 110.937 & & & 74.75 \\
\hline 1/9/08 17:00 & 125.362 & 122.909 & & 100.665 & 104.479 & 96.122 & 110.93 & & 80.785 & 74.744 \\
\hline 1/9/08 17:30 & 125.355 & 122.907 & & 100.697 & 104.479 & 96.117 & 110.924 & & & 74.738 \\
\hline $1 / 9 / 08 \quad 18: 00$ & 125.35 & 122.9 & & 100.717 & 104.471 & 96.113 & 110.92 & & & 74.729 \\
\hline 1/9/08 18:30 & 125.343 & 122.891 & & 100.713 & 104.468 & 96.108 & 110.917 & & 80.758 & 74.719 \\
\hline $1 / 9 / 08 \quad 19: 00$ & 125.338 & 122.9 & & 100.711 & 104.462 & 96.106 & 110.915 & & & 74.711 \\
\hline 1/9/08 19:30 & 125.334 & 122.902 & & 100.704 & 104.456 & 96.101 & 110.913 & & & 74.707 \\
\hline $1 / 9 / 0820: 00$ & 125.331 & 122.893 & & 100.701 & 104.464 & 96.108 & 110.909 & & 80.738 & 74.702 \\
\hline $1 / 9 / 0820: 30$ & 125.341 & 122.884 & & 100.696 & 104.468 & 96.11 & 110.907 & & & 74.698 \\
\hline 1/9/08 21:00 & 125.334 & 122.886 & & 100.691 & 104.454 & 96.101 & 110.907 & & & 74.694 \\
\hline 1/9/08 21:30 & 125.327 & 122.884 & & 100.689 & 104.447 & 96.091 & 110.905 & & 80.714 & 74.688 \\
\hline 1/9/08 22:00 & 125.322 & 122.882 & & 100.686 & 104.449 & 96.094 & 110.905 & & & 74.686 \\
\hline 1/9/08 22:30 & 125.324 & 122.873 & & 100.682 & 104.447 & 96.091 & 110.905 & & & 74.684 \\
\hline 1/9/08 23:00 & 125.322 & 122.87 & & 100.681 & 104.447 & 96.091 & 110.904 & & 80.702 & 74.682 \\
\hline 1/9/08 23:30 & 125.312 & 122.864 & & 100.672 & 104.441 & 96.082 & 110.904 & & & 74.68 \\
\hline $1 / 10 / 080: 00$ & 125.308 & 122.841 & & 100.663 & 104.433 & 96.077 & 110.904 & & & 74.678 \\
\hline 1/10/08 0:30 & 125.305 & 122.83 & & 100.66 & 104.433 & 96.075 & 110.904 & & 80.68 & 74.675 \\
\hline 1/10/08 1:00 & 125.294 & 122.812 & & 100.644 & 104.409 & 96.054 & 110.905 & & & 74.673 \\
\hline 1/10/08 1:30 & 125.275 & 122.805 & & 100.637 & 104.397 & 96.039 & 110.904 & & & 74.667 \\
\hline 1/10/08 2:00 & 125.26 & 122.787 & & 100.617 & 104.378 & 96.025 & 110.905 & & 80.648 & 74.665 \\
\hline 1/10/08 2:30 & 125.249 & 122.787 & & 100.608 & 104.372 & 96.018 & 110.905 & & & 74.661 \\
\hline 1/10/08 3:00 & 125.237 & 122.784 & & 100.593 & 104.353 & 95.999 & 110.904 & & & 74.657 \\
\hline 1/10/08 3:30 & 125.223 & 122.768 & & 100.596 & 104.355 & 95.997 & 110.902 & & 80.619 & 74.651 \\
\hline 1/10/08 4:00 & 125.213 & 122.766 & & 100.594 & 104.352 & 95.99 & 110.9 & & & 74.647 \\
\hline 1/10/08 4:30 & 125.216 & 122.75 & & 100.594 & 104.336 & 95.981 & 110.902 & & & 74.642 \\
\hline 1/10/08 5:00 & 125.211 & 122.757 & & 100.598 & 104.336 & 95.981 & 110.902 & & 80.587 & 74.64 \\
\hline 1/10/08 5:30 & 125.199 & 122.764 & & 100.592 & 104.319 & 95.959 & 110.9 & & & 74.636 \\
\hline $1 / 10 / 086: 00$ & 125.194 & 122.766 & & 100.593 & 104.327 & 95.966 & 110.898 & & & 74.628 \\
\hline 1/10/08 6:30 & 125.199 & 122.773 & & 100.591 & 104.334 & 95.978 & 110.894 & & 80.567 & 74.626 \\
\hline 1/10/08 7:00 & 125.206 & 124.587 & & 100.591 & 104.338 & 95.981 & 110.896 & & & 74.626 \\
\hline 1/10/08 7:30 & 125.208 & 124.895 & & 100.589 & 104.346 & 95.988 & 110.892 & & & 74.626 \\
\hline 1/10/08 8:00 & 126.91 & 125.038 & & 101.278 & 104.808 & 96.582 & 111.488 & & 80.57 & 74.669 \\
\hline 1/10/08 8:30 & 127.362 & 125.122 & & 101.512 & 105.111 & 96.893 & 111.764 & & & 74.826 \\
\hline 1/10/08 9:00 & 127.549 & 125.188 & & 101.624 & 105.254 & 97.039 & 111.897 & & & 74.942 \\
\hline 1/10/08 9:30 & 127.643 & 123.421 & & 101.697 & 105.34 & 97.124 & 111.978 & & 80.624 & 75.021 \\
\hline 1/10/08 10:00 & 127.705 & 123.186 & & 101.559 & 105.404 & 97.19 & 112.03 & & & 75.068 \\
\hline $1 / 10 / 0810: 30$ & 126.029 & 123.093 & & 100.987 & 104.953 & 96.603 & 111.431 & & & 75.039 \\
\hline 1/10/08 11:00 & 125.672 & 123.038 & & 100.825 & 104.738 & 96.384 & 111.206 & & 80.628 & 74.922 \\
\hline 1/10/08 11:30 & 125.542 & 123.004 & & 100.744 & 104.647 & 96.292 & 111.101 & & & 74.841 \\
\hline $1 / 10 / 08$ 12:00 & 125.483 & 122.97 & & 100.696 & 104.595 & 96.24 & 111.046 & & & 74.785 \\
\hline 1/10/08 12:30 & 125.445 & 122.95 & & 100.668 & 104.565 & 96.209 & 111.011 & & 80.619 & 74.754 \\
\hline 1/10/08 13:00 & 125.426 & 122.932 & & 100.65 & 104.531 & 96.176 & 110.988 & & & 74.738 \\
\hline 1/10/08 13:30 & 125.416 & 122.923 & & 100.636 & 104.511 & 96.157 & 110.973 & & & 74.727 \\
\hline 1/10/08 14:00 & 125.407 & 122.916 & & 100.627 & 104.5 & 96.143 & 110.962 & & 80.606 & 74.727 \\
\hline $1 / 10 / 0814: 30$ & 125.402 & 122.914 & & 100.617 & 104.481 & 96.127 & 110.954 & & & 74.731 \\
\hline $1 / 10 / 0815: 00$ & 125.39 & 122.916 & & 100.611 & 104.481 & 96.127 & 110.945 & & & 74.729 \\
\hline $1 / 10 / 0815: 30$ & 125.383 & 122.923 & & 100.606 & 104.485 & 96.129 & 110.939 & & 80.594 & 74.727 \\
\hline 1/10/08 16:00 & 125.376 & 122.941 & & 100.603 & 104.487 & 96.129 & 110.934 & & & 74.721 \\
\hline $1 / 10 / 0816: 30$ & 125.369 & 122.948 & & 100.598 & 104.492 & 96.139 & 110.928 & & & 74.713 \\
\hline
\end{tabular}


TABLE S1.1 (Cont.)

\begin{tabular}{|c|c|c|c|c|c|c|c|c|c|c|}
\hline \multirow[b]{2}{*}{ Date and Time } & \multicolumn{10}{|c|}{ Depth from Top of Casing (ft) } \\
\hline & MW1D & MW2D & MW3D & MW4D & MW7 & MW9 & Oentrich & MW14D & MW15D & MW16D \\
\hline 1/10/08 17:00 & 125.362 & 122.954 & & 100.592 & 104.51 & 96.157 & 110.924 & & 80.594 & 74.707 \\
\hline $1 / 10 / 0817: 30$ & 125.36 & 122.966 & & 100.591 & 104.517 & 96.164 & 110.921 & & & 74.7 \\
\hline 1/10/08 18:00 & 125.355 & 122.972 & & 100.591 & 104.525 & 96.174 & 110.919 & & & 74.692 \\
\hline $1 / 10 / 0818: 30$ & 125.35 & 122.972 & & 100.591 & 104.538 & 96.183 & 110.915 & & 80.597 & 74.684 \\
\hline 1/10/08 19:00 & 125.35 & 122.982 & & 100.591 & 104.544 & 96.19 & 110.913 & & & 74.675 \\
\hline 1/10/08 19:30 & 125.35 & 122.984 & & 100.589 & 104.542 & 96.188 & 110.913 & & & 74.671 \\
\hline 1/10/08 20:00 & 125.348 & 122.986 & & 100.61 & 104.551 & 96.197 & 110.909 & & 80.594 & 74.667 \\
\hline $1 / 10 / 0820: 30$ & 125.35 & 122.986 & & 100.603 & 104.554 & 96.2 & 110.909 & & & 74.661 \\
\hline 1/10/08 21:00 & 125.348 & 122.991 & & 100.603 & 104.557 & 96.202 & 110.908 & & & 74.655 \\
\hline 1/10/08 21:30 & 125.348 & 122.993 & & 100.601 & 104.555 & 96.197 & 110.908 & & 80.592 & 74.649 \\
\hline 1/10/08 22:00 & 125.348 & 123 & & 100.604 & 104.561 & 96.202 & 110.904 & & & 74.647 \\
\hline 1/10/08 22:30 & 125.348 & 123.004 & & 100.613 & 104.565 & 96.209 & 110.904 & & & 74.642 \\
\hline 1/10/08 23:00 & 125.348 & 123.002 & & 100.616 & 104.571 & 96.214 & 110.902 & & 80.597 & 74.638 \\
\hline 1/10/08 23:30 & 125.348 & 123.004 & & 100.622 & 104.576 & 96.219 & 110.9 & & & 74.636 \\
\hline 1/11/08 0:00 & 125.353 & 123 & & 100.618 & 104.574 & 96.216 & 110.9 & & & 74.63 \\
\hline $1 / 11 / 080: 30$ & 125.353 & 122.995 & & 100.62 & 104.574 & 96.216 & 110.9 & & 80.604 & 74.622 \\
\hline 1/11/08 1:00 & 125.355 & 122.979 & & 100.618 & 104.571 & 96.216 & 110.9 & & & 74.618 \\
\hline 1/11/08 1:30 & 125.357 & 122.963 & & 100.618 & 104.565 & 96.209 & 110.9 & & & 74.609 \\
\hline $1 / 11 / 082: 00$ & 125.362 & 122.954 & & 100.615 & 104.55 & 96.193 & 110.9 & & 80.597 & 74.603 \\
\hline $1 / 11 / 082: 30$ & 125.367 & 122.95 & & 100.613 & 104.532 & 96.179 & 110.9 & & & 74.597 \\
\hline 1/11/08 3:00 & 125.364 & 122.943 & & 100.61 & 104.525 & 96.169 & 110.9 & & & 74.589 \\
\hline 1/11/08 3:30 & 125.362 & 122.943 & & 100.606 & 104.523 & 96.167 & 110.896 & & 80.58 & 74.58 \\
\hline 1/11/08 4:00 & 125.36 & 122.938 & & 100.603 & 104.515 & 96.157 & 110.894 & & & 74.57 \\
\hline 1/11/08 4:30 & 125.357 & 122.941 & & 100.601 & 104.511 & 96.155 & 110.892 & & & 74.562 \\
\hline $1 / 11 / 085: 00$ & 125.36 & 122.936 & & 100.598 & 104.511 & 96.155 & 110.889 & & 80.57 & 74.556 \\
\hline 1/11/08 5:30 & 125.357 & 122.927 & & 100.598 & 104.513 & 96.157 & 110.885 & & & 74.551 \\
\hline 1/11/08 6:00 & 125.357 & 122.923 & & 100.596 & 104.508 & 96.153 & 110.883 & & & 74.545 \\
\hline 1/11/08 6:30 & 125.357 & 122.916 & & 100.592 & 104.498 & 96.143 & 110.883 & & 80.555 & 74.537 \\
\hline 1/11/08 7:00 & 125.355 & 124.762 & & 100.591 & 104.494 & 96.139 & 110.881 & & & 74.527 \\
\hline $1 / 11 / 087: 30$ & 125.357 & 125.031 & & 100.587 & 104.489 & 96.131 & 110.881 & & & 74.518 \\
\hline 1/11/08 8:00 & 126.91 & 125.172 & & 101.307 & 104.936 & 96.702 & 111.45 & & 80.562 & 74.556 \\
\hline 1/11/08 8:30 & 127.232 & 125.247 & & 101.509 & 105.203 & 96.975 & 111.719 & & & 74.7 \\
\hline 1/11/08 9:00 & 127.433 & 125.301 & & 101.623 & 105.347 & 97.121 & 111.833 & & & 74.804 \\
\hline 1/11/08 9:30 & 127.579 & 125.342 & & 101.686 & 105.425 & 97.197 & 111.908 & & 80.616 & 74.878 \\
\hline 1/11/08 10:00 & 127.683 & 125.365 & & 101.738 & 105.481 & 97.251 & 111.953 & & & 74.93 \\
\hline $1 / 11 / 0810: 30$ & 127.761 & 125.381 & & 101.771 & 105.521 & 97.294 & 111.985 & & & 74.969 \\
\hline $1 / 11 / 0811: 00$ & 127.811 & 125.397 & & 101.795 & 105.543 & 97.317 & 112.012 & & 80.663 & 75 \\
\hline 1/11/08 11:30 & 127.832 & 125.401 & & 101.812 & 105.564 & 97.338 & 112.03 & & & 75.029 \\
\hline $1 / 11 / 0812: 00$ & 127.854 & 125.399 & & 101.826 & 105.576 & 97.352 & 112.047 & & & 75.05 \\
\hline 1/11/08 12:30 & 127.868 & 123.623 & & 101.835 & 105.587 & 97.357 & 112.058 & & 80.699 & 75.072 \\
\hline 1/11/08 13:00 & 127.887 & 123.328 & & 101.842 & 105.585 & 97.36 & 112.07 & & & 75.101 \\
\hline 1/11/08 13:30 & 126.407 & 123.19 & & 101.114 & 105.148 & 96.799 & 111.521 & & & 75.056 \\
\hline $1 / 11 / 0814: 00$ & 126.095 & 123.099 & & 100.93 & 104.869 & 96.513 & 111.273 & & 80.68 & 74.957 \\
\hline $1 / 11 / 0814: 30$ & 125.875 & 123.041 & & 100.834 & 104.736 & 96.379 & 111.155 & & & 74.886 \\
\hline $1 / 11 / 0815: 00$ & 125.719 & 123.009 & & 100.773 & 104.649 & 96.294 & 111.092 & & & 74.826 \\
\hline 1/11/08 15:30 & 125.598 & 122.984 & & 100.732 & 104.597 & 96.24 & 111.046 & & 80.643 & 74.781 \\
\hline 1/11/08 16:00 & 125.516 & 122.972 & & 100.704 & 104.563 & 96.207 & 111.014 & & & 74.74 \\
\hline $1 / 11 / 0816: 30$ & 125.438 & 122.963 & & 100.684 & 104.542 & 96.183 & 110.994 & & & 74.711 \\
\hline 1/11/08 17:00 & 125.409 & 122.966 & & 100.672 & 104.532 & 96.176 & 110.975 & & 80.621 & 74.698 \\
\hline 1/11/08 17:30 & 125.405 & 122.97 & & 100.662 & 104.527 & 96.169 & 110.964 & & & 74.704 \\
\hline $1 / 11 / 0818: 00$ & 125.402 & 122.972 & & 100.655 & 104.527 & 96.172 & 110.95 & & & 74.702 \\
\hline 1/11/08 18:30 & 125.402 & 122.961 & & 100.648 & 104.532 & 96.176 & 110.943 & & 80.614 & 74.692 \\
\hline 1/11/08 19:00 & 125.409 & 122.966 & & 100.641 & 104.54 & 96.186 & 110.935 & & & 74.68 \\
\hline 1/11/08 19:30 & 125.405 & 122.966 & & 100.636 & 104.531 & 96.174 & 110.93 & & & 74.669 \\
\hline 1/11/08 20:00 & 125.402 & 122.972 & & 100.632 & 104.532 & 96.179 & 110.926 & & 80.606 & 74.659 \\
\hline 1/11/08 20:30 & 125.4 & 122.977 & & 100.629 & 104.532 & 96.176 & 110.92 & & & 74.651 \\
\hline 1/11/08 21:00 & 125.402 & 122.979 & & 100.625 & 104.542 & 96.183 & 110.915 & & & 74.644 \\
\hline 1/11/08 21:30 & 125.407 & 122.984 & & 100.622 & 104.548 & 96.19 & 110.911 & & 80.602 & 74.64 \\
\hline 1/11/08 22:00 & 125.409 & 122.988 & & 100.636 & 104.546 & 96.19 & 110.909 & & & 74.636 \\
\hline 1/11/08 22:30 & 125.414 & 122.991 & & 100.634 & 104.555 & 96.202 & 110.905 & & & 74.632 \\
\hline 1/11/08 23:00 & 125.419 & 123.002 & & 100.63 & 104.557 & 96.202 & 110.904 & & 80.599 & 74.628 \\
\hline 1/11/08 23:30 & 125.426 & 123.004 & & 100.631 & 104.561 & 96.204 & 110.9 & & & 74.622 \\
\hline $1 / 12 / 080: 00$ & 125.428 & 123.004 & & 100.63 & 104.571 & 96.214 & 110.898 & & & 74.616 \\
\hline $1 / 12 / 08 \quad 0: 30$ & 125.435 & 123.007 & & 100.627 & 104.571 & 96.216 & 110.896 & & 80.606 & 74.611 \\
\hline 1/12/08 1:00 & 125.44 & 123.02 & & 100.627 & 104.572 & 96.219 & 110.894 & & & 74.605 \\
\hline 1/12/08 1:30 & 125.438 & 123.025 & & 100.637 & 104.576 & 96.221 & 110.893 & & & 74.603 \\
\hline
\end{tabular}


TABLE S1.1 (Cont.)

Depth from Top of Casing (ft)

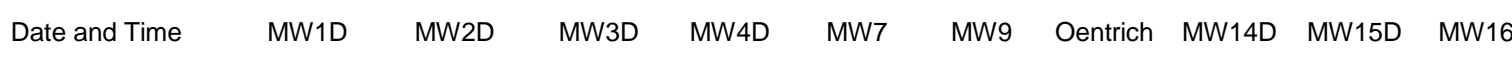

\begin{tabular}{|c|c|c|c|c|c|c|c|c|}
\hline 1/12/08 2:00 & 125.447 & 123.036 & 100.644 & 104.588 & 96.235 & 110.889 & 80.611 & 74.593 \\
\hline 1/12/08 2:30 & 125.452 & 123.045 & 100.651 & 104.593 & 96.237 & 110.887 & & 74.589 \\
\hline 1/12/08 3:00 & 125.459 & 123.056 & 100.665 & 104.607 & 96.252 & 110.883 & & 74.58 \\
\hline 1/12/08 3:30 & 125.471 & 123.056 & 100.672 & 104.618 & 96.261 & 110.879 & 80.616 & 74.574 \\
\hline 1/12/08 4:00 & 125.48 & 123.063 & 100.684 & 104.624 & 96.273 & 110.879 & & 74.566 \\
\hline $1 / 12 / 084: 30$ & 125.487 & 123.072 & 100.684 & 104.626 & 96.273 & 110.877 & & 74.562 \\
\hline $1 / 12 / 085: 00$ & 125.492 & 123.075 & 100.692 & 104.635 & 96.282 & 110.873 & 80.621 & 74.554 \\
\hline 1/12/08 5:30 & 125.499 & 123.077 & 100.703 & 104.645 & 96.289 & 110.872 & & 74.547 \\
\hline $1 / 12 / 086: 00$ & 125.509 & 123.088 & 100.701 & 104.649 & 96.294 & 110.87 & & 74.543 \\
\hline 1/12/08 6:30 & 125.511 & 123.097 & 100.71 & 104.65 & 96.296 & 110.868 & 80.631 & 74.539 \\
\hline 1/12/08 7:00 & 125.516 & 123.106 & 100.72 & 104.66 & 96.308 & 110.864 & & 74.533 \\
\hline $1 / 12 / 087: 30$ & 125.523 & 123.12 & 100.732 & 104.67 & 96.315 & 110.861 & & 74.525 \\
\hline 1/12/08 8:00 & 125.535 & 124.748 & 100.746 & 104.683 & 96.327 & 110.859 & 80.636 & 74.52 \\
\hline 1/12/08 8:30 & 125.546 & 125.179 & 100.747 & 104.694 & 96.339 & 110.855 & & 74.514 \\
\hline 1/12/08 9:00 & 126.948 & 125.356 & 101.445 & 104.989 & 96.754 & 111.281 & & 74.514 \\
\hline 1/12/08 9:30 & 127.553 & 125.465 & 101.709 & 105.399 & 97.183 & 111.668 & 80.682 & 74.663 \\
\hline $1 / 12 / 0810: 00$ & 127.825 & 124 & 101.85 & 105.574 & 97.362 & 111.818 & & 74.785 \\
\hline $1 / 12 / 08 \quad 10: 30$ & 127.96 & 123.587 & 101.943 & 105.684 & 97.475 & 111.888 & & 74.87 \\
\hline 1/12/08 11:00 & 126.754 & 123.449 & 101.34 & 105.502 & 97.176 & 111.555 & 80.756 & 74.919 \\
\hline $1 / 12 / 0811: 30$ & 126.352 & 123.372 & 101.123 & 105.14 & 96.787 & 111.188 & & 74.82 \\
\hline $1 / 12 / 0812: 00$ & 126.215 & 123.317 & 101.021 & 105.005 & 96.652 & 111.054 & & 74.729 \\
\hline $1 / 12 / 08$ 12:30 & 126.14 & 123.283 & 100.966 & 104.932 & 96.582 & 110.984 & 80.753 & 74.667 \\
\hline $1 / 12 / 0813: 00$ & 126.05 & 123.254 & 100.928 & 104.879 & 96.525 & 110.943 & & 74.628 \\
\hline $1 / 12 / 08$ 13:30 & 125.944 & 123.233 & 100.903 & 104.847 & 96.492 & 110.915 & & 74.607 \\
\hline $1 / 12 / 0814: 00$ & 125.837 & 123.231 & 100.884 & 104.818 & 96.464 & 110.896 & 80.741 & 74.595 \\
\hline $1 / 12 / 0814: 30$ & 125.757 & 123.224 & 100.87 & 104.801 & 96.45 & 110.883 & & 74.585 \\
\hline $1 / 12 / 08$ 15:00 & 125.705 & 123.222 & 100.86 & 104.799 & 96.445 & 110.87 & & 74.576 \\
\hline 1/12/08 15:30 & 125.676 & 123.22 & 100.849 & 104.793 & 96.44 & 110.861 & 80.748 & 74.58 \\
\hline $1 / 12 / 08$ 16:00 & 125.662 & 123.222 & 100.848 & 104.791 & 96.438 & 110.853 & & 74.58 \\
\hline 1/12/08 16:30 & 125.658 & 123.224 & 100.842 & 104.789 & 96.436 & 110.846 & & 74.566 \\
\hline $1 / 12 / 08 \quad 17: 00$ & 125.658 & 123.226 & 100.846 & 104.791 & 96.438 & 110.836 & 80.751 & 74.549 \\
\hline 1/12/08 17:30 & 125.66 & 123.231 & 100.853 & 104.795 & 96.44 & 110.832 & & 74.539 \\
\hline $1 / 12 / 08$ 18:00 & 125.665 & 123.236 & 100.858 & 104.797 & 96.443 & 110.827 & & 74.531 \\
\hline $1 / 12 / 08$ 18:30 & 125.665 & 123.247 & 100.863 & 104.801 & 96.447 & 110.819 & 80.758 & 74.52 \\
\hline 1/12/08 19:00 & 125.672 & 123.249 & 100.865 & 104.81 & 96.454 & 110.814 & & 74.514 \\
\hline $1 / 12 / 08 \quad 19: 30$ & 125.679 & 123.247 & 100.872 & 104.814 & 96.461 & 110.808 & & 74.506 \\
\hline $1 / 12 / 0820: 00$ & 125.684 & 123.245 & 100.873 & 104.822 & 96.466 & 110.806 & 80.77 & 74.504 \\
\hline $1 / 12 / 0820: 30$ & 125.686 & 123.24 & 100.872 & 104.82 & 96.466 & 110.813 & & 74.498 \\
\hline 1/12/08 21:00 & 125.684 & 123.24 & 100.868 & 104.816 & 96.464 & 110.821 & & 74.492 \\
\hline $1 / 12 / 0821: 30$ & 125.681 & 123.245 & 100.87 & 104.814 & 96.459 & 110.819 & 80.77 & 74.487 \\
\hline $1 / 12 / 0822: 00$ & 125.679 & 123.247 & 100.867 & 104.812 & 96.459 & 110.819 & & 74.477 \\
\hline 1/12/08 22:30 & 125.681 & 123.24 & 100.872 & 104.816 & 96.464 & 110.817 & & 74.473 \\
\hline 1/12/08 23:00 & 125.684 & 123.24 & 100.87 & 104.818 & 96.464 & 110.823 & 80.775 & 74.467 \\
\hline $1 / 12 / 0823: 30$ & 125.681 & 123.233 & 100.867 & 104.812 & 96.457 & 110.823 & & 74.463 \\
\hline $1 / 13 / 080: 00$ & 125.681 & 123.229 & 100.865 & 104.812 & 96.459 & 110.823 & & 74.458 \\
\hline $1 / 13 / 080: 30$ & 125.674 & 123.222 & 100.861 & 104.805 & 96.452 & 110.821 & 80.78 & 74.45 \\
\hline 1/13/08 1:00 & 125.672 & 123.215 & 100.859 & 104.799 & 96.447 & 110.819 & & 74.446 \\
\hline 1/13/08 1:30 & 125.667 & 123.213 & 100.856 & 104.793 & 96.44 & 110.817 & & 74.446 \\
\hline $1 / 13 / 082: 00$ & 125.658 & 123.215 & 100.853 & 104.788 & 96.433 & 110.816 & 80.78 & 74.442 \\
\hline $1 / 13 / 082: 30$ & 125.655 & 123.217 & 100.849 & 104.784 & 96.428 & 110.812 & & 74.44 \\
\hline 1/13/08 3:00 & 125.655 & 123.215 & 100.846 & 104.788 & 96.431 & 110.808 & & 74.44 \\
\hline $1 / 13 / 083: 30$ & 125.655 & 123.213 & 100.846 & 104.789 & 96.433 & 110.806 & 80.785 & 74.44 \\
\hline 1/13/08 4:00 & 125.66 & 123.208 & 100.844 & 104.788 & 96.433 & 110.806 & & 74.442 \\
\hline $1 / 13 / 084: 30$ & 125.653 & 123.206 & 100.841 & 104.784 & 96.428 & 110.804 & & 74.448 \\
\hline 1/13/08 5:00 & 125.648 & 123.204 & 100.837 & 104.782 & 96.426 & 110.8 & 80.785 & 74.456 \\
\hline $1 / 13 / 085: 30$ & 125.648 & 123.201 & 100.834 & 104.78 & 96.424 & 110.799 & & 74.463 \\
\hline 1/13/08 6:00 & 125.643 & 123.199 & 100.83 & 104.778 & 96.421 & 110.797 & & 74.475 \\
\hline 1/13/08 6:30 & 125.641 & 123.204 & 100.83 & 104.774 & 96.417 & 110.793 & 80.782 & 74.485 \\
\hline $1 / 13 / 087: 00$ & 125.639 & 123.202 & 100.828 & 104.772 & 96.417 & 110.793 & & 74.498 \\
\hline $1 / 13 / 08$ 7:30 & 125.639 & 123.204 & 100.83 & 104.776 & 96.419 & 110.791 & & 74.502 \\
\hline 1/13/08 8:00 & 125.639 & 123.208 & 100.83 & 104.776 & 96.421 & 110.791 & 80.782 & 74.512 \\
\hline 1/13/08 8:30 & 125.641 & 123.21 & 100.832 & 104.776 & 96.421 & 110.789 & & 74.523 \\
\hline $1 / 13 / 089: 00$ & 125.643 & 125.168 & 100.842 & 104.782 & 96.426 & 110.785 & & 74.533 \\
\hline 1/13/08 9:30 & 125.653 & 125.385 & 101.257 & 104.786 & 96.431 & 110.783 & 80.795 & 74.541 \\
\hline $1 / 13 / 08$ 10:00 & 127.511 & 125.501 & 101.661 & 105.338 & 97.107 & 111.465 & & 74.64 \\
\hline $1 / 13 / 08 \quad 10: 30$ & 127.844 & 125.578 & 101.823 & 105.557 & 97.331 & 111.672 & & 74.795 \\
\hline
\end{tabular}


TABLE S1.1 (Cont.)

\begin{tabular}{|c|c|c|c|c|c|c|c|c|c|c|}
\hline \multirow[b]{2}{*}{ Date and Time } & \multicolumn{10}{|c|}{ Depth from Top of Casing (ft) } \\
\hline & MW1D & MW2D & MW3D & MW4D & MW7 & MW9 & Oentrich & MW14D & MW15D & MW16D \\
\hline 1/13/08 11:00 & 127.981 & 125.612 & & 101.919 & 105.673 & 97.449 & 111.782 & & 80.853 & 74.911 \\
\hline $1 / 13 / 0811: 30$ & 128.05 & 125.628 & & 101.979 & 105.751 & 97.525 & 111.858 & & & 75.002 \\
\hline 1/13/08 12:00 & 128.097 & 125.639 & & 102.016 & 105.791 & 97.56 & 111.903 & & & 75.064 \\
\hline $1 / 13 / 0812: 30$ & 128.133 & 125.642 & & 102.041 & 105.804 & 97.579 & 111.935 & & 80.9 & 75.157 \\
\hline $1 / 13 / 0813: 00$ & 128.156 & 125.644 & & 102.059 & 105.812 & 97.586 & 111.957 & & & 75.18 \\
\hline $1 / 13 / 08$ 13:30 & 128.166 & 125.644 & & 102.069 & 105.814 & 97.591 & 111.974 & & & 75.186 \\
\hline $1 / 13 / 0814: 00$ & 128.168 & 125.637 & & 102.078 & 105.821 & 97.595 & 111.987 & & 80.932 & 75.192 \\
\hline $1 / 13 / 0814: 30$ & 128.159 & 125.642 & & 102.083 & 105.819 & 97.595 & 111.998 & & & 75.196 \\
\hline $1 / 13 / 08$ 15:00 & 128.152 & 123.798 & & 102.09 & 105.816 & 97.588 & 112.006 & & & 75.194 \\
\hline $1 / 13 / 0815: 30$ & 128.147 & 123.56 & & 101.778 & 105.823 & 97.595 & 112.013 & & 80.958 & 75.201 \\
\hline $1 / 13 / 0816: 00$ & 126.506 & 123.444 & & 101.321 & 105.321 & 96.971 & 111.394 & & & 75.12 \\
\hline 1/13/08 16:30 & 126.111 & 123.376 & & 101.158 & 105.1 & 96.742 & 111.178 & & & 74.965 \\
\hline $1 / 13 / 08$ 17:00 & 125.92 & 123.328 & & 101.07 & 104.987 & 96.631 & 111.069 & & 80.924 & 74.859 \\
\hline $1 / 13 / 0817: 30$ & 125.823 & 123.299 & & 101.014 & 104.921 & 96.563 & 111.005 & & & 74.785 \\
\hline $1 / 13 / 08$ 18:00 & 125.771 & 123.279 & & 100.975 & 104.877 & 96.52 & 110.947 & & & 74.74 \\
\hline $1 / 13 / 0818: 30$ & 125.738 & 123.258 & & 100.949 & 104.854 & 96.499 & 110.915 & & 80.905 & 74.711 \\
\hline 1/13/08 19:00 & 125.717 & 123.242 & & 100.93 & 104.833 & 96.478 & 110.895 & & & 74.69 \\
\hline 1/13/08 19:30 & 125.698 & 123.229 & & 100.913 & 104.814 & 96.459 & 110.881 & & & 74.671 \\
\hline $1 / 13 / 0820: 00$ & 125.684 & 123.224 & & 100.901 & 104.799 & 96.445 & 110.868 & & 80.888 & 74.653 \\
\hline $1 / 13 / 0820: 30$ & 125.669 & 123.22 & & 100.89 & 104.789 & 96.433 & 110.861 & & & 74.638 \\
\hline $1 / 13 / 0821: 00$ & 125.66 & 123.217 & & 100.884 & 104.786 & 96.426 & 110.849 & & & 74.63 \\
\hline 1/13/08 21:30 & 125.653 & 123.215 & & 100.878 & 104.782 & 96.426 & 110.842 & & 80.875 & 74.626 \\
\hline 1/13/08 22:00 & 125.65 & 123.224 & & 100.871 & 104.782 & 96.421 & 110.834 & & & 74.624 \\
\hline $1 / 13 / 0822: 30$ & 125.648 & 123.231 & & 100.868 & 104.776 & 96.421 & 110.829 & & & 74.618 \\
\hline $1 / 13 / 0823: 00$ & 125.65 & 123.235 & & 100.868 & 104.786 & 96.428 & 110.823 & & 80.866 & 74.62 \\
\hline 1/13/08 23:30 & 125.66 & 123.245 & & 100.863 & 104.795 & 96.44 & 110.817 & & & 74.63 \\
\hline $1 / 14 / 080: 00$ & 125.667 & 123.247 & & 100.861 & 104.803 & 96.445 & 110.812 & & & 74.634 \\
\hline 1/14/08 0:30 & 125.669 & 123.249 & & 100.867 & 104.81 & 96.452 & 110.806 & & 80.87 & 74.636 \\
\hline 1/14/08 1:00 & 125.679 & 123.254 & & 100.872 & 104.816 & 96.457 & 110.808 & & & 74.642 \\
\hline $1 / 14 / 08 \quad 1: 30$ & 125.681 & 123.263 & & 100.871 & 104.816 & 96.461 & 110.813 & & & 74.647 \\
\hline $1 / 14 / 082: 00$ & 125.688 & 123.267 & & 100.873 & 104.82 & 96.464 & 110.819 & & 80.873 & 74.647 \\
\hline $1 / 14 / 082: 30$ & 125.691 & 123.276 & & 100.885 & 104.829 & 96.473 & 110.828 & & & 74.653 \\
\hline 1/14/08 3:00 & 125.7 & 123.276 & & 100.892 & 104.833 & 96.478 & 110.838 & & & 74.659 \\
\hline 1/14/08 3:30 & 125.705 & 123.27 & & 100.899 & 104.841 & 96.487 & 110.843 & & 80.883 & 74.665 \\
\hline 1/14/08 4:00 & 125.71 & 123.265 & & 100.899 & 104.841 & 96.485 & 110.847 & & & 74.667 \\
\hline 1/14/08 4:30 & 125.712 & 123.263 & & 100.894 & 104.835 & 96.48 & 110.843 & & & 74.667 \\
\hline 1/14/08 5:00 & 125.7 & 123.265 & & 100.889 & 104.832 & 96.476 & 110.842 & & 80.878 & 74.657 \\
\hline 1/14/08 5:30 & 125.7 & 123.267 & & 100.885 & 104.829 & 96.476 & 110.838 & & & 74.657 \\
\hline $1 / 14 / 086: 00$ & 125.698 & 123.274 & & 100.89 & 104.833 & 96.478 & 110.834 & & & 74.657 \\
\hline 1/14/08 6:30 & 125.705 & 123.288 & & 100.891 & 104.837 & 96.483 & 110.843 & & 80.878 & 74.665 \\
\hline 1/14/08 7:00 & 125.705 & 125.165 & & 100.896 & 104.841 & 96.487 & 110.845 & & & 74.665 \\
\hline $1 / 14 / 087: 30$ & 125.712 & 125.424 & & 101.184 & 104.858 & 96.504 & 110.858 & & & 74.673 \\
\hline $1 / 14 / 088: 00$ & 127.461 & 125.555 & & 101.742 & 105.382 & 97.164 & 111.521 & & 80.907 & 74.75 \\
\hline 1/14/08 8:30 & 127.868 & 125.639 & & 101.924 & 105.637 & 97.423 & 111.763 & & & 74.938 \\
\hline $1 / 14 / 089: 00$ & 128.052 & 125.703 & & 102.031 & 105.77 & 97.56 & 111.889 & & & 75.072 \\
\hline 1/14/08 9:30 & 128.149 & 123.972 & & 102.109 & 105.856 & 97.647 & 111.97 & & 80.976 & 75.163 \\
\hline 1/14/08 10:00 & 128.215 & 123.705 & & 102.157 & 105.917 & 97.708 & 112.019 & & & 75.234 \\
\hline $1 / 14 / 0810: 30$ & 126.624 & 123.585 & & 101.413 & 105.496 & 97.154 & 111.472 & & & 75.225 \\
\hline $1 / 14 / 0811: 00$ & 126.211 & 123.508 & & 101.246 & 105.25 & 96.898 & 111.232 & & 80.993 & 75.101 \\
\hline 1/14/08 11:30 & 126.048 & 123.453 & & 101.154 & 105.136 & 96.784 & 111.133 & & & 74.998 \\
\hline $1 / 14 / 0812: 00$ & 125.974 & 123.412 & & 101.099 & 105.066 & 96.711 & 111.067 & & & 74.919 \\
\hline 1/14/08 12:30 & 125.927 & 123.381 & & 101.063 & 105.008 & 96.657 & 111.027 & & 80.976 & 74.862 \\
\hline 1/14/08 13:00 & 125.889 & 123.353 & & 101.035 & 104.97 & 96.617 & 111.001 & & & 74.82 \\
\hline 1/14/08 13:30 & 125.858 & 123.322 & & 101.014 & 104.94 & 96.591 & 110.982 & & & 74.791 \\
\hline 1/14/08 14:00 & 125.828 & 123.303 & & 100.996 & 104.909 & 96.56 & 110.967 & & 80.958 & 74.764 \\
\hline $1 / 14 / 0814: 30$ & 125.799 & 123.292 & & 100.978 & 104.885 & 96.532 & 110.958 & & & 74.735 \\
\hline $1 / 14 / 08$ 15:00 & 125.771 & 123.279 & & 100.968 & 104.866 & 96.513 & 110.949 & & & 74.717 \\
\hline $1 / 14 / 0815: 30$ & 125.747 & 123.272 & & 100.961 & 104.854 & 96.499 & 110.939 & & 80.939 & 74.702 \\
\hline $1 / 14 / 0816: 00$ & 125.731 & 123.274 & & 100.952 & 104.843 & 96.49 & 110.932 & & & 74.696 \\
\hline $1 / 14 / 0816: 30$ & 125.717 & 123.274 & & 100.946 & 104.835 & 96.48 & 110.924 & & & 74.688 \\
\hline 1/14/08 17:00 & 125.707 & 123.267 & & 100.94 & 104.841 & 96.487 & 110.919 & & 80.936 & 74.69 \\
\hline $1 / 14 / 08$ 17:30 & 125.707 & 123.258 & & 100.939 & 104.839 & 96.487 & 110.911 & & & 74.69 \\
\hline $1 / 14 / 0818: 00$ & 125.707 & 123.258 & & 100.934 & 104.835 & 96.48 & 110.907 & & & 74.686 \\
\hline 1/14/08 18:30 & 125.698 & 123.247 & & 100.927 & 104.826 & 96.471 & 110.904 & & 80.924 & 74.675 \\
\hline 1/14/08 19:00 & 125.693 & 123.242 & & 100.923 & 104.822 & 96.466 & 110.898 & & & 74.671 \\
\hline 1/14/08 19:30 & 125.686 & 123.231 & & 100.917 & 104.814 & 96.457 & 110.894 & & & 74.665 \\
\hline
\end{tabular}


TABLE S1.1 (Cont.)

\begin{tabular}{|c|c|c|c|c|c|c|c|c|c|c|}
\hline \multirow[b]{2}{*}{ Date and Time } & \multicolumn{10}{|c|}{ Depth from Top of Casing (ft) } \\
\hline & MW1D & MW2D & MW3D & MW4D & MW7 & MW9 & Oentrich & MW14D & MW15D & MW16D \\
\hline $1 / 14 / 0820: 00$ & 125.676 & 123.22 & & 100.913 & 104.808 & 96.454 & 110.89 & & 80.917 & 74.657 \\
\hline $1 / 14 / 0820: 30$ & 125.672 & 123.206 & & 100.906 & 104.799 & 96.443 & 110.889 & & & 74.651 \\
\hline 1/14/08 21:00 & 125.662 & 123.197 & & 100.901 & 104.788 & 96.431 & 110.885 & & & 74.64 \\
\hline $1 / 14 / 0821: 30$ & 125.646 & 123.188 & & 100.898 & 104.774 & 96.421 & 110.881 & & 80.897 & 74.624 \\
\hline 1/14/08 22:00 & 125.639 & 123.177 & & 100.892 & 104.765 & 96.41 & 110.877 & & & 74.62 \\
\hline 1/14/08 22:30 & 125.632 & 123.179 & & 100.889 & 104.759 & 96.405 & 110.875 & & & 74.613 \\
\hline 1/14/08 23:00 & 125.62 & 123.177 & & 100.886 & 104.746 & 96.391 & 110.873 & & 80.888 & 74.599 \\
\hline $1 / 14 / 0823: 30$ & 125.613 & 123.161 & & 100.882 & 104.748 & 96.393 & 110.868 & & & 74.597 \\
\hline 1/15/08 0:00 & 125.615 & 123.143 & & 100.88 & 104.742 & 96.386 & 110.866 & & & 74.595 \\
\hline 1/15/08 0:30 & 125.606 & 123.122 & & 100.875 & 104.729 & 96.374 & 110.866 & & 80.866 & 74.585 \\
\hline 1/15/08 1:00 & 125.591 & 123.115 & & 100.868 & 104.709 & 96.355 & 110.864 & & & 74.568 \\
\hline 1/15/08 1:30 & 125.568 & 123.102 & & 100.861 & 104.689 & 96.334 & 110.862 & & & 74.547 \\
\hline $1 / 15 / 082: 00$ & 125.554 & 123.09 & & 100.858 & 104.685 & 96.327 & 110.86 & & 80.839 & 74.539 \\
\hline 1/15/08 2:30 & 125.544 & 123.079 & & 100.856 & 104.671 & 96.315 & 110.858 & & & 74.529 \\
\hline 1/15/08 3:00 & 125.532 & 123.065 & & 100.846 & 104.656 & 96.303 & 110.857 & & & 74.516 \\
\hline 1/15/08 3:30 & 125.523 & 123.061 & & 100.842 & 104.647 & 96.292 & 110.855 & & 80.814 & 74.506 \\
\hline 1/15/08 4:00 & 125.506 & 123.038 & & 100.817 & 104.634 & 96.275 & 110.853 & & & 74.492 \\
\hline 1/15/08 4:30 & 125.502 & 123.016 & & 100.815 & 104.63 & 96.273 & 110.851 & & & 74.489 \\
\hline $1 / 15 / 085: 00$ & 125.487 & 123 & & 100.778 & 104.607 & 96.249 & 110.851 & & 80.792 & 74.469 \\
\hline 1/15/08 5:30 & 125.461 & 122.975 & & 100.782 & 104.584 & 96.228 & 110.851 & & & 74.446 \\
\hline 1/15/08 6:00 & 125.438 & 122.959 & & 100.753 & 104.569 & 96.212 & 110.849 & & & 74.427 \\
\hline 1/15/08 6:30 & 125.424 & 122.943 & & 100.753 & 104.544 & 96.19 & 110.851 & & 80.753 & 74.409 \\
\hline 1/15/08 7:00 & 125.405 & 124.721 & & 100.725 & 104.527 & 96.169 & 110.849 & & & 74.392 \\
\hline 1/15/08 7:30 & 125.39 & 125.011 & & 100.725 & 104.513 & 96.155 & 110.847 & & & 74.378 \\
\hline 1/15/08 8:00 & 127.012 & 125.129 & & 101.419 & 104.904 & 96.664 & 111.373 & & 80.741 & 74.398 \\
\hline 1/15/08 8:30 & 127.466 & 125.199 & & 101.624 & 105.191 & 96.961 & 111.66 & & & 74.582 \\
\hline 1/15/08 9:00 & 127.624 & 125.242 & & 101.705 & 105.311 & 97.084 & 111.794 & & & 74.7 \\
\hline 1/15/08 9:30 & 127.695 & 125.274 & & 101.773 & 105.382 & 97.154 & 111.87 & & 80.768 & 74.779 \\
\hline 1/15/08 10:00 & 127.74 & 125.279 & & 101.786 & 105.427 & 97.195 & 111.92 & & & 74.826 \\
\hline $1 / 15 / 0810: 30$ & 127.776 & 125.283 & & 101.821 & 105.458 & 97.228 & 111.951 & & & 74.862 \\
\hline 1/15/08 11:00 & 127.792 & 125.285 & & 101.805 & 105.469 & 97.239 & 111.978 & & 80.785 & 74.88 \\
\hline $1 / 15 / 0811: 30$ & 127.797 & 123.378 & & 101.833 & 105.471 & 97.242 & 111.989 & & & 74.884 \\
\hline $1 / 15 / 08$ 12:00 & 127.804 & 123.145 & & 101.363 & 105.479 & 97.246 & 112.004 & & & 74.893 \\
\hline $1 / 15 / 0812: 30$ & 126.104 & 123.013 & & 100.982 & 104.91 & 96.556 & 111.349 & & 80.765 & 74.76 \\
\hline 1/15/08 13:00 & 125.79 & 122.927 & & 100.816 & 104.689 & 96.332 & 111.161 & & & 74.589 \\
\hline 1/15/08 13:30 & 125.594 & 122.861 & & 100.711 & 104.565 & 96.204 & 111.067 & & & 74.463 \\
\hline $1 / 15 / 08$ 14:00 & 125.464 & 122.816 & & 100.636 & 104.477 & 96.122 & 111.011 & & 80.692 & 74.374 \\
\hline 1/15/08 14:30 & 125.367 & 122.787 & & 100.616 & 104.414 & 96.058 & 110.971 & & & 74.308 \\
\hline $1 / 15 / 08$ 15:00 & 125.296 & 122.759 & & 100.569 & 104.372 & 96.011 & 110.947 & & & 74.26 \\
\hline $1 / 15 / 08$ 15:30 & 125.249 & 122.732 & & 100.515 & 104.342 & 95.983 & 110.928 & & 80.638 & 74.225 \\
\hline 1/15/08 16:00 & 125.218 & 122.712 & & 100.515 & 104.313 & 95.957 & 110.915 & & & 74.198 \\
\hline $1 / 15 / 0816: 30$ & 125.19 & 122.698 & & 100.468 & 104.293 & 95.933 & 110.905 & & & 74.173 \\
\hline 1/15/08 17:00 & 125.166 & 122.694 & & 100.472 & 104.27 & 95.91 & 110.898 & & 80.589 & 74.15 \\
\hline 1/15/08 17:30 & 125.138 & 122.691 & & 100.468 & 104.258 & 95.898 & 110.892 & & & 74.138 \\
\hline $1 / 15 / 0818: 00$ & 125.128 & 122.687 & & 100.465 & 104.258 & 95.898 & 110.887 & & & 74.13 \\
\hline $1 / 15 / 0818: 30$ & 125.13 & 122.685 & & 100.461 & 104.256 & 95.896 & 110.883 & & 80.56 & 74.13 \\
\hline 1/15/08 19:00 & 125.123 & 122.669 & & 100.46 & 104.253 & 95.896 & 110.879 & & & 74.124 \\
\hline 1/15/08 19:30 & 125.123 & 122.662 & & 100.458 & 104.253 & 95.891 & 110.875 & & & 74.121 \\
\hline 1/15/08 20:00 & 125.109 & 122.662 & & 100.411 & 104.239 & 95.877 & 110.875 & & 80.526 & 74.107 \\
\hline 1/15/08 20:30 & 125.1 & 122.662 & & 100.426 & 104.232 & 95.872 & 110.873 & & & 74.099 \\
\hline 1/15/08 21:00 & 125.1 & 122.673 & & 100.427 & 104.235 & 95.877 & 110.872 & & & 74.099 \\
\hline 1/15/08 21:30 & 125.097 & 122.671 & & 100.427 & 104.232 & 95.872 & 110.87 & & 80.496 & 74.095 \\
\hline 1/15/08 22:00 & 125.109 & 122.669 & & 100.426 & 104.245 & 95.886 & 110.866 & & & 74.107 \\
\hline 1/15/08 22:30 & 125.112 & 122.666 & & 100.427 & 104.243 & 95.884 & 110.866 & & & 74.105 \\
\hline 1/15/08 23:00 & 125.107 & 122.662 & & 100.427 & 104.239 & 95.882 & 110.866 & & 80.474 & 74.101 \\
\hline 1/15/08 23:30 & 125.104 & 122.655 & & 100.427 & 104.239 & 95.882 & 110.866 & & & 74.099 \\
\hline $1 / 16 / 080: 00$ & 125.1 & 122.655 & & 100.429 & 104.235 & 95.875 & 110.87 & & & 74.09 \\
\hline 1/16/08 0:30 & 125.097 & 122.653 & & 100.429 & 104.23 & 95.87 & 110.868 & & 80.448 & 74.088 \\
\hline 1/16/08 1:00 & 125.093 & 122.655 & & 100.429 & 104.226 & 95.87 & 110.868 & & & 74.084 \\
\hline 1/16/08 1:30 & 125.09 & 122.666 & & 100.429 & 104.226 & 95.867 & 110.868 & & & 74.08 \\
\hline $1 / 16 / 082: 00$ & 125.093 & 122.689 & & 100.429 & 104.228 & 95.867 & 110.868 & & 80.428 & 74.08 \\
\hline 1/16/08 2:30 & 125.102 & 122.719 & & 100.43 & 104.241 & 95.882 & 110.864 & & & 74.09 \\
\hline 1/16/08 3:00 & 125.114 & 122.725 & & 100.434 & 104.26 & 95.9 & 110.864 & & & 74.107 \\
\hline 1/16/08 3:30 & 125.151 & 122.732 & & 100.434 & 104.294 & 95.933 & 110.862 & & 80.428 & 74.138 \\
\hline 1/16/08 4:00 & 125.168 & 122.741 & & 100.436 & 104.302 & 95.948 & 110.864 & & & 74.148 \\
\hline 1/16/08 4:30 & 125.168 & 122.753 & & 100.436 & 104.31 & 95.952 & 110.862 & & & 74.148 \\
\hline
\end{tabular}


TABLE S1.1 (Cont.)

Depth from Top of Casing (ft)

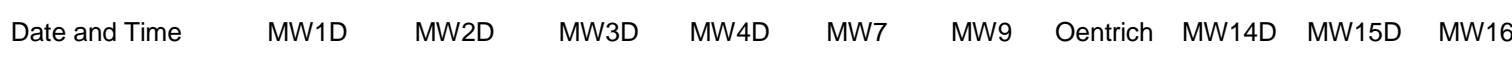

\begin{tabular}{|c|c|c|c|c|c|c|c|c|}
\hline 1/16/08 5:00 & 125.175 & 122.766 & 100.437 & 104.317 & 95.959 & 110.862 & 80.423 & 74.155 \\
\hline 1/16/08 5:30 & 125.192 & 122.78 & 100.437 & 104.329 & 95.971 & 110.862 & & 74.165 \\
\hline $1 / 16 / 086: 00$ & 125.201 & 122.796 & 100.439 & 104.342 & 95.985 & 110.861 & & 74.177 \\
\hline 1/16/08 6:30 & 125.22 & 122.812 & 100.439 & 104.359 & 96.002 & 110.861 & 80.428 & 74.194 \\
\hline 1/16/08 7:00 & 125.23 & 124.585 & 100.439 & 104.374 & 96.016 & 110.858 & & 74.202 \\
\hline $1 / 16 / 087: 30$ & 125.249 & 124.932 & 100.527 & 104.39 & 96.032 & 110.859 & & 74.219 \\
\hline 1/16/08 8:00 & 126.913 & 125.086 & 101.294 & 104.822 & 96.593 & 111.398 & 80.452 & 74.272 \\
\hline 1/16/08 8:30 & 127.421 & 125.181 & 101.525 & 105.153 & 96.937 & 111.709 & & 74.479 \\
\hline 1/16/08 9:00 & 127.608 & 123.392 & 101.655 & 105.311 & 97.1 & 111.85 & & 74.636 \\
\hline 1/16/08 9:30 & 127.705 & 123.192 & 101.349 & 105.406 & 97.192 & 111.935 & 80.531 & 74.727 \\
\hline 1/16/08 10:00 & 125.918 & 123.118 & 100.961 & 104.94 & 96.593 & 111.307 & & 74.711 \\
\hline $1 / 16 / 08 \quad 10: 30$ & 125.653 & 123.084 & 100.846 & 104.755 & 96.4 & 111.12 & & 74.593 \\
\hline $1 / 16 / 0811: 00$ & 125.563 & 123.054 & 100.787 & 104.685 & 96.329 & 111.03 & 80.531 & 74.52 \\
\hline 1/16/08 11:30 & 125.525 & 123.034 & 100.763 & 104.654 & 96.299 & 110.977 & & 74.487 \\
\hline $1 / 16 / 0812: 00$ & 125.499 & 123.018 & 100.739 & 104.624 & 96.27 & 110.949 & & 74.452 \\
\hline $1 / 16 / 0812: 30$ & 125.476 & 122.993 & 100.732 & 104.605 & 96.252 & 110.928 & 80.536 & 74.427 \\
\hline 1/16/08 13:00 & 125.471 & 122.988 & 100.708 & 104.591 & 96.24 & 110.915 & & 74.419 \\
\hline $1 / 16 / 08$ 13:30 & 125.438 & 122.982 & 100.68 & 104.565 & 96.209 & 110.909 & & 74.386 \\
\hline $1 / 16 / 08 \quad 14: 00$ & 125.433 & 122.968 & 100.675 & 104.565 & 96.212 & 110.898 & 80.531 & 74.388 \\
\hline $1 / 16 / 0814: 30$ & 125.424 & 122.975 & 100.677 & 104.553 & 96.197 & 110.896 & & 74.372 \\
\hline $1 / 16 / 08$ 15:00 & 125.416 & 122.977 & 100.679 & 104.546 & 96.193 & 110.893 & & 74.367 \\
\hline $1 / 16 / 08$ 15:30 & 125.412 & 122.979 & 100.682 & 104.55 & 96.193 & 110.887 & 80.531 & 74.365 \\
\hline $1 / 16 / 0816: 00$ & 125.424 & 122.97 & 100.679 & 104.554 & 96.2 & 110.883 & & 74.378 \\
\hline 1/16/08 16:30 & 125.419 & 122.979 & 100.672 & 104.555 & 96.202 & 110.877 & & 74.374 \\
\hline $1 / 16 / 08$ 17:00 & 125.412 & 122.986 & 100.68 & 104.546 & 96.193 & 110.877 & 80.531 & 74.365 \\
\hline $1 / 16 / 08$ 17:30 & 125.424 & 122.986 & 100.685 & 104.557 & 96.204 & 110.874 & & 74.378 \\
\hline $1 / 16 / 08$ 18:00 & 125.426 & 122.991 & 100.691 & 104.561 & 96.207 & 110.872 & & 74.38 \\
\hline 1/16/08 18:30 & 125.433 & 122.993 & 100.692 & 104.567 & 96.214 & 110.868 & 80.536 & 74.386 \\
\hline 1/16/08 19:00 & 125.433 & 123.004 & 100.697 & 104.569 & 96.216 & 110.864 & & 74.388 \\
\hline 1/16/08 19:30 & 125.435 & 122.997 & 100.703 & 104.572 & 96.219 & 110.864 & & 74.39 \\
\hline $1 / 16 / 0820: 00$ & 125.442 & 123.007 & 100.704 & 104.58 & 96.226 & 110.859 & 80.545 & 74.398 \\
\hline $1 / 16 / 0820: 30$ & 125.442 & 123.006 & 100.706 & 104.582 & 96.223 & 110.859 & & 74.398 \\
\hline $1 / 16 / 0821: 00$ & 125.445 & 123.009 & 100.71 & 104.582 & 96.23 & 110.859 & & 74.403 \\
\hline 1/16/08 21:30 & 125.447 & 123.004 & 100.712 & 104.584 & 96.23 & 110.855 & 80.55 & 74.403 \\
\hline 1/16/08 22:00 & 125.447 & 123.004 & 100.715 & 104.586 & 96.233 & 110.853 & & 74.403 \\
\hline 1/16/08 22:30 & 125.447 & 123.002 & 100.711 & 104.582 & 96.23 & 110.853 & & 74.401 \\
\hline 1/16/08 23:00 & 125.447 & 122.995 & 100.708 & 104.586 & 96.228 & 110.849 & 80.548 & 74.403 \\
\hline 1/16/08 23:30 & 125.44 & 122.991 & 100.703 & 104.58 & 96.226 & 110.847 & & 74.398 \\
\hline 1/17/08 0:00 & 125.438 & 122.993 & 100.697 & 104.572 & 96.219 & 110.845 & & 74.392 \\
\hline 1/17/08 0:30 & 125.431 & 122.993 & 100.699 & 104.571 & 96.216 & 110.845 & 80.543 & 74.39 \\
\hline $1 / 17 / 081: 00$ & 125.433 & 122.986 & 100.701 & 104.572 & 96.216 & 110.842 & & 74.394 \\
\hline $1 / 17 / 081: 30$ & 125.435 & 122.984 & 100.696 & 104.571 & 96.216 & 110.842 & & 74.394 \\
\hline 1/17/08 2:00 & 125.428 & 122.975 & 100.689 & 104.565 & 96.212 & 110.84 & 80.538 & 74.386 \\
\hline $1 / 17 / 082: 30$ & 125.424 & 122.966 & 100.685 & 104.563 & 96.207 & 110.838 & & 74.384 \\
\hline $1 / 17 / 083: 00$ & 125.419 & 122.961 & 100.677 & 104.557 & 96.2 & 110.838 & & 74.378 \\
\hline 1/17/08 3:30 & 125.407 & 122.95 & 100.672 & 104.546 & 96.19 & 110.836 & 80.533 & 74.37 \\
\hline 1/17/08 4:00 & 125.402 & 122.941 & 100.66 & 104.538 & 96.183 & 110.834 & & 74.363 \\
\hline 1/17/08 4:30 & 125.39 & 122.932 & 100.651 & 104.527 & 96.172 & 110.834 & & 74.353 \\
\hline 1/17/08 5:00 & 125.383 & 122.923 & 100.642 & 104.521 & 96.162 & 110.834 & 80.521 & 74.347 \\
\hline $1 / 17 / 085: 30$ & 125.376 & 122.918 & 100.632 & 104.51 & 96.153 & 110.832 & & 74.336 \\
\hline $1 / 17 / 086: 00$ & 125.364 & 122.918 & 100.625 & 104.502 & 96.141 & 110.831 & & 74.328 \\
\hline 1/17/08 6:30 & 125.357 & 122.914 & 100.629 & 104.496 & 96.139 & 110.829 & 80.514 & 74.324 \\
\hline $1 / 17 / 087: 00$ & 125.357 & 124.578 & 100.623 & 104.496 & 96.139 & 110.827 & & 74.324 \\
\hline $1 / 17 / 087: 30$ & 125.355 & 124.977 & 100.618 & 104.494 & 96.136 & 110.825 & & 74.322 \\
\hline 1/17/08 8:00 & 126.823 & 125.138 & 101.273 & 104.77 & 96.52 & 111.225 & 80.516 & 74.326 \\
\hline $1 / 17 / 088: 30$ & 127.44 & 125.236 & 101.518 & 105.157 & 96.926 & 111.598 & & 74.516 \\
\hline 1/17/08 9:00 & 127.624 & 125.292 & 101.649 & 105.319 & 97.091 & 111.747 & & 74.671 \\
\hline 1/17/08 9:30 & 127.731 & 125.338 & 101.724 & 105.423 & 97.195 & 111.829 & 80.587 & 74.777 \\
\hline $1 / 17 / 0810: 00$ & 127.79 & 125.367 & 101.778 & 105.481 & 97.251 & 111.884 & & 74.839 \\
\hline $1 / 17 / 08 \quad 10: 30$ & 127.839 & 125.39 & 101.814 & 105.522 & 97.294 & 111.92 & & 74.882 \\
\hline $1 / 17 / 0811: 00$ & 127.868 & 125.399 & 101.845 & 105.557 & 97.329 & 111.946 & 80.638 & 74.917 \\
\hline 1/17/08 11:30 & 127.891 & 125.403 & 101.861 & 105.576 & 97.348 & 111.965 & & 74.94 \\
\hline $1 / 17 / 0812: 00$ & 127.906 & 125.406 & 101.867 & 105.589 & 97.362 & 111.981 & & 74.959 \\
\hline 1/17/08 12:30 & 127.91 & 124.106 & 101.873 & 105.597 & 97.369 & 111.993 & 80.68 & 74.967 \\
\hline 1/17/08 13:00 & 127.91 & 123.446 & 101.869 & 105.595 & 97.364 & 112.006 & & 74.965 \\
\hline $1 / 17 / 0813: 30$ & 126.974 & 123.256 & 101.311 & 105.522 & 97.27 & 111.942 & & 74.965 \\
\hline
\end{tabular}


TABLE S1.1 (Cont.)

\begin{tabular}{|c|c|c|c|c|c|c|c|c|c|c|}
\hline \multirow[b]{2}{*}{ Date and Time } & \multicolumn{10}{|c|}{ Depth from Top of Casing (ft) } \\
\hline & MW1D & MW2D & MW3D & MW4D & MW7 & MW9 & Oentrich & MW14D & MW15D & MW16D \\
\hline 1/17/08 14:00 & 125.944 & 123.147 & & 101.015 & 104.982 & 96.629 & 111.313 & & 80.672 & 74.81 \\
\hline $1 / 17 / 0814: 30$ & 125.71 & 123.074 & & 100.877 & 104.803 & 96.445 & 111.148 & & & 74.663 \\
\hline 1/17/08 15:00 & 125.594 & 123.029 & & 100.793 & 104.7 & 96.341 & 111.063 & & & 74.558 \\
\hline $1 / 17 / 0815: 30$ & 125.523 & 122.997 & & 100.732 & 104.634 & 96.273 & 111.009 & & 80.638 & 74.485 \\
\hline 1/17/08 16:00 & 125.471 & 122.975 & & 100.703 & 104.59 & 96.23 & 110.971 & & & 74.438 \\
\hline $1 / 17 / 0816: 30$ & 125.438 & 122.954 & & 100.673 & 104.559 & 96.202 & 110.947 & & & 74.405 \\
\hline $1 / 17 / 0817: 00$ & 125.414 & 122.938 & & 100.654 & 104.534 & 96.176 & 110.928 & & 80.614 & 74.38 \\
\hline $1 / 17 / 0817: 30$ & 125.393 & 122.932 & & 100.637 & 104.517 & 96.157 & 110.917 & & & 74.361 \\
\hline 1/17/08 18:00 & 125.379 & 122.913 & & 100.625 & 104.502 & 96.143 & 110.904 & & & 74.345 \\
\hline 1/17/08 18:30 & 125.369 & 122.907 & & 100.618 & 104.498 & 96.139 & 110.896 & & 80.599 & 74.339 \\
\hline 1/17/08 19:00 & 125.357 & 122.902 & & 100.603 & 104.481 & 96.122 & 110.889 & & & 74.324 \\
\hline 1/17/08 19:30 & 125.343 & 122.895 & & 100.598 & 104.476 & 96.12 & 110.883 & & & 74.314 \\
\hline $1 / 17 / 0820: 00$ & 125.338 & 122.889 & & 100.594 & 104.47 & 96.113 & 110.877 & & 80.599 & 74.31 \\
\hline $1 / 17 / 0820: 30$ & 125.334 & 122.884 & & 100.589 & 104.466 & 96.106 & 110.874 & & & 74.303 \\
\hline 1/17/08 21:00 & 125.327 & 122.88 & & 100.585 & 104.46 & 96.103 & 110.868 & & & 74.299 \\
\hline $1 / 17 / 0821: 30$ & 125.324 & 122.875 & & 100.579 & 104.456 & 96.096 & 110.866 & & 80.57 & 74.297 \\
\hline 1/17/08 22:00 & 125.32 & 122.868 & & 100.577 & 104.451 & 96.094 & 110.862 & & & 74.291 \\
\hline 1/17/08 22:30 & 125.315 & 122.864 & & 100.57 & 104.447 & 96.089 & 110.859 & & & 74.287 \\
\hline 1/17/08 23:00 & 125.308 & 122.861 & & 100.563 & 104.443 & 96.082 & 110.857 & & 80.555 & 74.281 \\
\hline 1/17/08 23:30 & 125.303 & 122.859 & & 100.563 & 104.436 & 96.077 & 110.851 & & & 74.276 \\
\hline $1 / 18 / 080: 00$ & 125.301 & 122.855 & & 100.561 & 104.436 & 96.075 & 110.849 & & & 74.272 \\
\hline 1/18/08 0:30 & 125.296 & 122.85 & & 100.556 & 104.432 & 96.072 & 110.846 & & 80.545 & 74.27 \\
\hline 1/18/08 1:00 & 125.291 & 122.839 & & 100.555 & 104.43 & 96.072 & 110.844 & & & 74.266 \\
\hline 1/18/08 1:30 & 125.289 & 122.834 & & 100.546 & 104.424 & 96.068 & 110.842 & & & 74.26 \\
\hline $1 / 18 / 082: 00$ & 125.279 & 122.839 & & 100.537 & 104.415 & 96.056 & 110.842 & & 80.526 & 74.252 \\
\hline 1/18/08 2:30 & 125.272 & 122.834 & & 100.539 & 104.413 & 96.054 & 110.838 & & & 74.245 \\
\hline 1/18/08 3:00 & 125.275 & 122.839 & & 100.542 & 104.415 & 96.056 & 110.834 & & & 74.25 \\
\hline 1/18/08 3:30 & 125.277 & 122.832 & & 100.539 & 104.415 & 96.056 & 110.834 & & 80.516 & 74.248 \\
\hline 1/18/08 4:00 & 125.277 & 122.83 & & 100.539 & 104.416 & 96.056 & 110.83 & & & 74.25 \\
\hline 1/18/08 4:30 & 125.272 & 122.823 & & 100.536 & 104.413 & 96.051 & 110.829 & & & 74.243 \\
\hline 1/18/08 5:00 & 125.27 & 122.839 & & 100.532 & 104.407 & 96.049 & 110.827 & & 80.518 & 74.241 \\
\hline 1/18/08 5:30 & 125.263 & 122.843 & & 100.536 & 104.401 & 96.042 & 110.829 & & & 74.235 \\
\hline 1/18/08 6:00 & 125.275 & 122.848 & & 100.546 & 104.415 & 96.056 & 110.825 & & & 74.245 \\
\hline 1/18/08 6:30 & 125.279 & 122.857 & & 100.544 & 104.422 & 96.063 & 110.823 & & 80.514 & 74.252 \\
\hline 1/18/08 7:00 & 125.282 & 124.603 & & 100.558 & 104.426 & 96.065 & 110.821 & & & 74.254 \\
\hline 1/18/08 7:30 & 125.298 & 124.959 & & 100.563 & 104.436 & 96.08 & 110.819 & & & 74.268 \\
\hline 1/18/08 8:00 & 126.934 & 125.127 & & 101.326 & 104.841 & 96.612 & 111.349 & & 80.518 & 74.299 \\
\hline 1/18/08 8:30 & 127.449 & 125.238 & & 101.562 & 105.186 & 96.971 & 111.662 & & & 74.512 \\
\hline 1/18/08 9:00 & 127.636 & 125.324 & & 101.7 & 105.355 & 97.136 & 111.803 & & & 74.671 \\
\hline 1/18/08 9:30 & 127.754 & 123.995 & & 101.798 & 105.467 & 97.251 & 111.886 & & 80.619 & 74.787 \\
\hline 1/18/08 10:00 & 127.837 & 123.478 & & 101.873 & 105.551 & 97.338 & 111.933 & & & 74.874 \\
\hline $1 / 18 / 0810: 30$ & 126.764 & 123.344 & & 101.314 & 105.464 & 97.171 & 111.702 & & & 74.942 \\
\hline 1/18/08 11:00 & 125.953 & 123.285 & & 101.085 & 105.035 & 96.681 & 111.217 & & 80.653 & 74.841 \\
\hline 1/18/08 11:30 & 125.79 & 123.254 & & 101.002 & 104.906 & 96.551 & 111.065 & & & 74.742 \\
\hline $1 / 18 / 0812: 00$ & 125.728 & 123.226 & & 100.963 & 104.852 & 96.497 & 110.984 & & & 74.686 \\
\hline $1 / 18 / 0812: 30$ & 125.695 & 123.204 & & 100.937 & 104.824 & 96.471 & 110.932 & & 80.67 & 74.651 \\
\hline 1/18/08 13:00 & 125.672 & 123.192 & & 100.913 & 104.799 & 96.447 & 110.895 & & & 74.622 \\
\hline 1/18/08 13:30 & 125.646 & 123.181 & & 100.899 & 104.774 & 96.421 & 110.875 & & & 74.597 \\
\hline $1 / 18 / 08$ 14:00 & 125.636 & 123.176 & & 100.885 & 104.765 & 96.412 & 110.862 & & 80.675 & 74.587 \\
\hline 1/18/08 14:30 & 125.627 & 123.168 & & 100.885 & 104.755 & 96.405 & 110.852 & & & 74.576 \\
\hline $1 / 18 / 08$ 15:00 & 125.624 & 123.174 & & 100.87 & 104.753 & 96.4 & 110.844 & & & 74.57 \\
\hline 1/18/08 15:30 & 125.61 & 123.172 & & 100.875 & 104.744 & 96.388 & 110.836 & & 80.692 & 74.56 \\
\hline 1/18/08 16:00 & 125.615 & 123.179 & & 100.875 & 104.749 & 96.393 & 110.829 & & & 74.566 \\
\hline $1 / 18 / 0816: 30$ & 125.613 & 123.186 & & 100.878 & 104.75 & 96.393 & 110.823 & & & 74.566 \\
\hline 1/18/08 17:00 & 125.617 & 123.192 & & 100.885 & 104.757 & 96.403 & 110.819 & & 80.697 & 74.572 \\
\hline 1/18/08 17:30 & 125.624 & 123.19 & & 100.894 & 104.761 & 96.41 & 110.816 & & & 74.574 \\
\hline $1 / 18 / 0818: 00$ & 125.632 & 123.204 & & 100.897 & 104.769 & 96.414 & 110.81 & & & 74.582 \\
\hline 1/18/08 18:30 & 125.634 & 123.238 & & 100.899 & 104.769 & 96.414 & 110.808 & & 80.712 & 74.587 \\
\hline 1/18/08 19:00 & 125.634 & 123.26 & & 100.928 & 104.776 & 96.421 & 110.802 & & & 74.591 \\
\hline 1/18/08 19:30 & 125.662 & 123.272 & & 100.954 & 104.811 & 96.452 & 110.793 & & & 74.62 \\
\hline 1/18/08 20:00 & 125.686 & 123.288 & & 100.977 & 104.837 & 96.48 & 110.789 & & 80.748 & 74.644 \\
\hline $1 / 18 / 0820: 30$ & 125.71 & 123.285 & & 100.998 & 104.847 & 96.494 & 110.81 & & & 74.661 \\
\hline 1/18/08 21:00 & 125.728 & 123.285 & & 100.996 & 104.866 & 96.511 & 110.853 & & & 74.678 \\
\hline 1/18/08 21:30 & 125.724 & 123.304 & & 100.996 & 104.86 & 96.506 & 110.853 & & 80.773 & 74.673 \\
\hline 1/18/08 22:00 & 125.726 & 123.306 & & 101.008 & 104.866 & 96.509 & 110.857 & & & 74.678 \\
\hline 1/18/08 22:30 & 125.738 & 123.315 & & 101.013 & 104.879 & 96.525 & 110.87 & & & 74.692 \\
\hline
\end{tabular}


TABLE S1.1 (Cont.)

\begin{tabular}{|c|c|c|c|c|c|c|c|c|c|c|}
\hline \multirow[b]{2}{*}{ Date and Time } & \multicolumn{10}{|c|}{ Depth from Top of Casing (ft) } \\
\hline & MW1D & MW2D & MW3D & MW4D & MW7 & MW9 & Oentrich & MW14D & MW15D & MW16D \\
\hline 1/18/08 23:00 & 125.745 & 123.319 & & 101.02 & 104.885 & 96.53 & 110.874 & & 80.8 & 74.698 \\
\hline 1/18/08 23:30 & 125.75 & 123.322 & & 101.025 & 104.894 & 96.539 & 110.881 & & & 74.707 \\
\hline 1/19/08 0:00 & 125.757 & 123.324 & & 101.032 & 104.898 & 96.544 & 110.891 & & & 74.713 \\
\hline 1/19/08 0:30 & 125.764 & 123.315 & & 101.035 & 104.904 & 96.546 & 110.896 & & 80.814 & 74.719 \\
\hline 1/19/08 1:00 & 125.766 & 123.292 & & 101.03 & 104.904 & 96.549 & 110.9 & & & 74.721 \\
\hline 1/19/08 1:30 & 125.759 & 123.285 & & 101.018 & 104.892 & 96.542 & 110.898 & & & 74.713 \\
\hline 1/19/08 2:00 & 125.74 & 123.274 & & 100.997 & 104.875 & 96.518 & 110.894 & & 80.819 & 74.696 \\
\hline 1/19/08 2:30 & 125.728 & 123.267 & & 100.985 & 104.864 & 96.509 & 110.89 & & & 74.688 \\
\hline 1/19/08 3:00 & 125.717 & 123.274 & & 100.978 & 104.856 & 96.499 & 110.887 & & & 74.68 \\
\hline 1/19/08 3:30 & 125.705 & 123.279 & & 100.977 & 104.845 & 96.487 & 110.883 & & 80.817 & 74.669 \\
\hline 1/19/08 4:00 & 125.712 & 123.274 & & 100.983 & 104.856 & 96.499 & 110.876 & & & 74.682 \\
\hline 1/19/08 4:30 & 125.714 & 123.267 & & 100.987 & 104.854 & 96.499 & 110.872 & & & 74.682 \\
\hline $1 / 19 / 085: 00$ & 125.714 & 123.265 & & 100.982 & 104.852 & 96.497 & 110.868 & & 80.824 & 74.684 \\
\hline 1/19/08 5:30 & 125.71 & 123.26 & & 100.973 & 104.848 & 96.492 & 110.866 & & & 74.678 \\
\hline 1/19/08 6:00 & 125.705 & 123.258 & & 100.971 & 104.845 & 96.49 & 110.862 & & & 74.675 \\
\hline 1/19/08 6:30 & 125.7 & 123.256 & & 100.961 & 104.839 & 96.483 & 110.858 & & 80.824 & 74.669 \\
\hline $1 / 19 / 08$ 7:00 & 125.693 & 123.256 & & 100.965 & 104.837 & 96.483 & 110.864 & & & 74.667 \\
\hline 1/19/08 7:30 & 125.693 & 123.258 & & 100.961 & 104.831 & 96.476 & 110.864 & & & 74.665 \\
\hline 1/19/08 8:00 & 125.693 & 123.26 & & 100.961 & 104.835 & 96.478 & 110.864 & & 80.831 & 74.667 \\
\hline 1/19/08 8:30 & 125.695 & 123.26 & & 100.965 & 104.835 & 96.48 & 110.862 & & & 74.669 \\
\hline 1/19/08 9:00 & 125.698 & 125.197 & & 100.968 & 104.839 & 96.48 & 110.864 & & & 74.669 \\
\hline 1/19/08 9:30 & 125.7 & 125.424 & & 101.361 & 104.839 & 96.485 & 110.868 & & 80.844 & 74.673 \\
\hline 1/19/08 10:00 & 127.598 & 125.539 & & 101.778 & 105.378 & 97.145 & 111.523 & & & 74.777 \\
\hline $1 / 19 / 0810: 30$ & 127.901 & 125.621 & & 101.943 & 105.606 & 97.378 & 111.734 & & & 74.961 \\
\hline $1 / 19 / 0811: 00$ & 128.031 & 125.673 & & 102.043 & 105.722 & 97.494 & 111.846 & & 80.919 & 75.081 \\
\hline 1/19/08 11:30 & 128.116 & 125.701 & & 102.109 & 105.808 & 97.584 & 111.923 & & & 75.17 \\
\hline 1/19/08 12:00 & 128.166 & 125.714 & & 102.152 & 105.861 & 97.633 & 111.972 & & & 75.223 \\
\hline 1/19/08 12:30 & 128.199 & 125.712 & & 102.169 & 105.888 & 97.661 & 112.006 & & 80.978 & 75.254 \\
\hline 1/19/08 13:00 & 128.213 & 125.705 & & 102.179 & 105.898 & 97.668 & 112.03 & & & 75.269 \\
\hline 1/19/08 13:30 & 128.22 & 123.832 & & 102.178 & 105.901 & 97.675 & 112.047 & & & 75.277 \\
\hline $1 / 19 / 0814: 00$ & 128.213 & 123.582 & & 101.809 & 105.892 & 97.666 & 112.062 & & 81.007 & 75.273 \\
\hline $1 / 19 / 0814: 30$ & 126.371 & 123.453 & & 101.361 & 105.367 & 97.015 & 111.437 & & & 75.17 \\
\hline 1/19/08 15:00 & 126.043 & 123.374 & & 101.19 & 105.132 & 96.775 & 111.226 & & & 75 \\
\hline $1 / 19 / 0815: 30$ & 125.906 & 123.322 & & 101.092 & 105.006 & 96.648 & 111.125 & & 80.963 & 74.88 \\
\hline 1/19/08 16:00 & 125.818 & 123.285 & & 101.032 & 104.932 & 96.572 & 111.061 & & & 74.8 \\
\hline $1 / 19 / 0816: 30$ & 125.766 & 123.263 & & 100.992 & 104.881 & 96.525 & 111.02 & & & 74.748 \\
\hline $1 / 19 / 08$ 17:00 & 125.726 & 123.238 & & 100.959 & 104.847 & 96.492 & 110.992 & & 80.939 & 74.709 \\
\hline 1/19/08 17:30 & 125.7 & 123.226 & & 100.944 & 104.826 & 96.469 & 110.969 & & & 74.686 \\
\hline 1/19/08 18:00 & 125.681 & 123.211 & & 100.923 & 104.803 & 96.447 & 110.954 & & & 74.663 \\
\hline 1/19/08 18:30 & 125.667 & 123.195 & & 100.909 & 104.793 & 96.438 & 110.943 & & 80.929 & 74.653 \\
\hline 1/19/08 19:00 & 125.65 & 123.19 & & 100.897 & 104.78 & 96.421 & 110.934 & & & 74.636 \\
\hline 1/19/08 19:30 & 125.639 & 123.19 & & 100.884 & 104.767 & 96.407 & 110.924 & & & 74.622 \\
\hline 1/19/08 20:00 & 125.629 & 123.181 & & 100.882 & 104.759 & 96.4 & 110.919 & & 80.912 & 74.616 \\
\hline 1/19/08 20:30 & 125.624 & 123.165 & & 100.885 & 104.761 & 96.4 & 110.911 & & & 74.613 \\
\hline 1/19/08 21:00 & 125.622 & 123.172 & & 100.871 & 104.749 & 96.393 & 110.907 & & & 74.609 \\
\hline 1/19/08 21:30 & 125.613 & 123.168 & & 100.865 & 104.738 & 96.381 & 110.904 & & 80.905 & 74.597 \\
\hline 1/19/08 22:00 & 125.603 & 123.161 & & 100.863 & 104.744 & 96.381 & 110.896 & & & 74.595 \\
\hline 1/19/08 22:30 & 125.606 & 123.158 & & 100.858 & 104.738 & 96.379 & 110.892 & & & 74.597 \\
\hline 1/19/08 23:00 & 125.601 & 123.156 & & 100.856 & 104.732 & 96.374 & 110.889 & & 80.902 & 74.591 \\
\hline 1/19/08 23:30 & 125.598 & 123.156 & & 100.856 & 104.734 & 96.377 & 110.883 & & & 74.591 \\
\hline $1 / 20 / 080: 00$ & 125.596 & 123.152 & & 100.856 & 104.73 & 96.372 & 110.879 & & & 74.589 \\
\hline 1/20/08 0:30 & 125.596 & 123.136 & & 100.856 & 104.73 & 96.372 & 110.875 & & 80.897 & 74.589 \\
\hline 1/20/08 1:00 & 125.594 & 123.115 & & 100.846 & 104.729 & 96.367 & 110.872 & & & 74.587 \\
\hline $1 / 20 / 081: 30$ & 125.58 & 123.122 & & 100.828 & 104.711 & 96.351 & 110.87 & & & 74.572 \\
\hline 1/20/08 2:00 & 125.563 & 123.102 & & 100.82 & 104.69 & 96.329 & 110.87 & & 80.875 & 74.551 \\
\hline 1/20/08 2:30 & 125.563 & 123.111 & & 100.815 & 104.696 & 96.337 & 110.862 & & & 74.558 \\
\hline 1/20/08 3:00 & 125.544 & 123.111 & & 100.799 & 104.679 & 96.318 & 110.862 & & & 74.537 \\
\hline 1/20/08 3:30 & 125.539 & 123.104 & & 100.811 & 104.685 & 96.325 & 110.855 & & 80.861 & 74.539 \\
\hline $1 / 20 / 084: 00$ & 125.544 & 123.095 & & 100.811 & 104.685 & 96.325 & 110.853 & & & 74.543 \\
\hline 1/20/08 4:30 & 125.542 & 123.097 & & 100.804 & 104.679 & 96.32 & 110.849 & & & 74.537 \\
\hline 1/20/08 5:00 & 125.537 & 123.093 & & 100.796 & 104.67 & 96.311 & 110.849 & & 80.848 & 74.531 \\
\hline $1 / 20 / 085: 30$ & 125.535 & 123.093 & & 100.797 & 104.675 & 96.318 & 110.843 & & & 74.533 \\
\hline $1 / 20 / 086: 00$ & 125.532 & 123.095 & & 100.791 & 104.668 & 96.311 & 110.843 & & & 74.527 \\
\hline $1 / 20 / 086: 30$ & 125.528 & 123.095 & & 100.796 & 104.67 & 96.311 & 110.84 & & 80.836 & 74.525 \\
\hline $1 / 20 / 087: 00$ & 125.532 & 123.093 & & 100.794 & 104.673 & 96.313 & 110.836 & & & 74.527 \\
\hline $1 / 20 / 087: 30$ & 125.53 & 123.095 & & 100.797 & 104.672 & 96.313 & 110.834 & & & 74.525 \\
\hline
\end{tabular}


TABLE S1.1 (Cont.)

Depth from Top of Casing (ft)

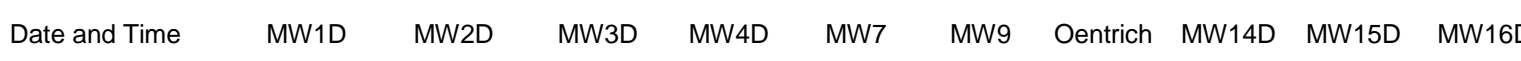

\begin{tabular}{|c|c|c|c|c|c|c|c|c|}
\hline 1/20/08 8:00 & 125.532 & 123.095 & 100.799 & 104.671 & 96.311 & 110.834 & 80.831 & 74.525 \\
\hline $1 / 20 / 088: 30$ & 125.535 & 124.762 & 100.799 & 104.672 & 96.313 & 110.83 & & 74.527 \\
\hline $1 / 20 / 089: 00$ & 125.537 & 125.172 & 100.803 & 104.672 & 96.313 & 110.83 & & 74.529 \\
\hline 1/20/08 9:30 & 127.045 & 125.333 & 101.519 & 104.997 & 96.761 & 111.283 & 80.839 & 74.541 \\
\hline $1 / 20 / 08 \quad 10: 00$ & 127.657 & 125.424 & 101.769 & 105.393 & 97.176 & 111.658 & & 74.744 \\
\hline $1 / 20 / 0810: 30$ & 127.849 & 125.478 & 101.893 & 105.553 & 97.338 & 111.807 & & 74.899 \\
\hline $1 / 20 / 0811: 00$ & 127.946 & 125.512 & 101.964 & 105.642 & 97.428 & 111.895 & 80.907 & 74.996 \\
\hline $1 / 20 / 0811: 30$ & 128 & 123.814 & 102.01 & 105.7 & 97.484 & 111.951 & & 75.052 \\
\hline $1 / 20 / 0812: 00$ & 128.04 & 123.467 & 102.033 & 105.732 & 97.515 & 111.991 & & 75.089 \\
\hline $1 / 20 / 0812: 30$ & 126.423 & 123.308 & 101.282 & 105.334 & 96.992 & 111.469 & 80.946 & 75.068 \\
\hline $1 / 20 / 08$ 13:00 & 125.946 & 123.211 & 101.061 & 105.014 & 96.659 & 111.189 & & 74.891 \\
\hline $1 / 20 / 0813: 30$ & 125.764 & 123.147 & 100.937 & 104.862 & 96.504 & 111.067 & & 74.748 \\
\hline $1 / 20 / 0814: 00$ & 125.66 & 123.106 & 100.865 & 104.771 & 96.414 & 110.997 & 80.878 & 74.653 \\
\hline $1 / 20 / 0814: 30$ & 125.591 & 123.07 & 100.815 & 104.708 & 96.353 & 110.956 & & 74.587 \\
\hline $1 / 20 / 0815: 00$ & 125.551 & 123.045 & 100.778 & 104.668 & 96.311 & 110.926 & & 74.545 \\
\hline $1 / 20 / 08$ 15:30 & 125.513 & 123.027 & 100.747 & 104.634 & 96.275 & 110.905 & 80.844 & 74.512 \\
\hline $1 / 20 / 08$ 16:00 & 125.485 & 123.013 & 100.727 & 104.609 & 96.254 & 110.892 & & 74.483 \\
\hline $1 / 20 / 0816: 30$ & 125.466 & 123 & 100.715 & 104.595 & 96.237 & 110.881 & & 74.467 \\
\hline $1 / 20 / 08$ 17:00 & 125.452 & 122.995 & 100.701 & 104.582 & 96.221 & 110.872 & 80.817 & 74.452 \\
\hline $1 / 20 / 0817: 30$ & 125.44 & 122.991 & 100.691 & 104.569 & 96.212 & 110.864 & & 74.442 \\
\hline $1 / 20 / 08$ 18:00 & 125.431 & 123.002 & 100.687 & 104.571 & 96.209 & 110.86 & & 74.436 \\
\hline $1 / 20 / 0818: 30$ & 125.431 & 123.011 & 100.691 & 104.563 & 96.207 & 110.853 & 80.802 & 74.432 \\
\hline $1 / 20 / 08$ 19:00 & 125.438 & 123.013 & 100.706 & 104.575 & 96.214 & 110.847 & & 74.438 \\
\hline $1 / 20 / 08$ 19:30 & 125.45 & 123.018 & 100.71 & 104.586 & 96.226 & 110.843 & & 74.448 \\
\hline $1 / 20 / 0820: 00$ & 125.454 & 123.013 & 100.715 & 104.588 & 96.228 & 110.842 & 80.802 & 74.45 \\
\hline $1 / 20 / 0820: 30$ & 125.459 & 123.007 & 100.713 & 104.594 & 96.235 & 110.838 & & 74.454 \\
\hline $1 / 20 / 0821: 00$ & 125.454 & 123.002 & 100.711 & 104.588 & 96.23 & 110.836 & & 74.45 \\
\hline $1 / 20 / 0821: 30$ & 125.45 & 122.997 & 100.708 & 104.582 & 96.223 & 110.836 & 80.795 & 74.442 \\
\hline 1/20/08 22:00 & 125.447 & 122.979 & 100.699 & 104.58 & 96.219 & 110.834 & & 74.442 \\
\hline $1 / 20 / 0822: 30$ & 125.438 & 122.979 & 100.691 & 104.573 & 96.214 & 110.832 & & 74.434 \\
\hline $1 / 20 / 0823: 00$ & 125.424 & 122.968 & 100.68 & 104.555 & 96.197 & 110.834 & 80.778 & 74.419 \\
\hline $1 / 20 / 0823: 30$ & 125.419 & 122.954 & 100.675 & 104.553 & 96.195 & 110.83 & & 74.415 \\
\hline $1 / 21 / 080: 00$ & 125.412 & 122.945 & 100.665 & 104.55 & 96.188 & 110.83 & & 74.409 \\
\hline $1 / 21 / 080: 30$ & 125.4 & 122.92 & 100.654 & 104.532 & 96.174 & 110.83 & 80.765 & 74.396 \\
\hline $1 / 21 / 081: 00$ & 125.39 & 122.904 & 100.639 & 104.521 & 96.164 & 110.828 & & 74.386 \\
\hline $1 / 21 / 081: 30$ & 125.372 & 122.893 & 100.615 & 104.498 & 96.141 & 110.828 & & 74.367 \\
\hline $1 / 21 / 082: 00$ & 125.348 & 122.884 & 100.603 & 104.481 & 96.122 & 110.827 & 80.734 & 74.347 \\
\hline $1 / 21 / 082: 30$ & 125.338 & 122.857 & 100.587 & 104.471 & 96.115 & 110.827 & & 74.339 \\
\hline $1 / 21 / 083: 00$ & 125.324 & 122.846 & 100.575 & 104.464 & 96.101 & 110.823 & & 74.326 \\
\hline $1 / 21 / 083: 30$ & 125.301 & 122.836 & 100.551 & 104.433 & 96.075 & 110.819 & 80.699 & 74.301 \\
\hline $1 / 21 / 084: 00$ & 125.289 & 122.834 & 100.544 & 104.426 & 96.065 & 110.817 & & 74.293 \\
\hline $1 / 21 / 084: 30$ & 125.279 & 122.827 & 100.534 & 104.418 & 96.058 & 110.813 & & 74.283 \\
\hline $1 / 21 / 085: 00$ & 125.272 & 122.812 & 100.53 & 104.412 & 96.051 & 110.812 & 80.672 & 74.276 \\
\hline $1 / 21 / 085: 30$ & 125.268 & 122.814 & 100.523 & 104.405 & 96.047 & 110.81 & & 74.27 \\
\hline $1 / 21 / 086: 00$ & 125.258 & 122.809 & 100.515 & 104.392 & 96.032 & 110.808 & & 74.256 \\
\hline $1 / 21 / 086: 30$ & 125.253 & 122.809 & 100.513 & 104.395 & 96.032 & 110.808 & 80.648 & 74.258 \\
\hline $1 / 21 / 087: 00$ & 125.249 & 124.641 & 100.51 & 104.39 & 96.025 & 110.804 & & 74.25 \\
\hline $1 / 21 / 087: 30$ & 125.246 & 124.916 & 100.508 & 104.388 & 96.028 & 110.804 & & 74.25 \\
\hline $1 / 21 / 088: 00$ & 127.005 & 125.043 & 101.256 & 104.831 & 96.589 & 111.371 & 80.646 & 74.297 \\
\hline $1 / 21 / 088: 30$ & 127.397 & 125.115 & 101.444 & 105.104 & 96.869 & 111.634 & & 74.489 \\
\hline $1 / 21 / 089: 00$ & 127.537 & 125.165 & 101.542 & 105.231 & 96.999 & 111.76 & & 74.618 \\
\hline 1/21/08 9:30 & 127.612 & 125.206 & 101.605 & 105.305 & 97.074 & 111.835 & 80.692 & 74.698 \\
\hline $1 / 21 / 0810: 00$ & 127.662 & 124.353 & 101.65 & 105.357 & 97.126 & 111.884 & & 74.752 \\
\hline $1 / 21 / 08 \quad 10: 30$ & 127.707 & 123.322 & 101.685 & 105.399 & 97.169 & 111.917 & & 74.797 \\
\hline $1 / 21 / 0811: 00$ & 127.736 & 123.133 & 101.201 & 105.429 & 97.197 & 111.942 & 80.726 & 74.828 \\
\hline 1/21/08 11:30 & 125.818 & 123.027 & 100.892 & 104.869 & 96.513 & 111.264 & & 74.715 \\
\hline $1 / 21 / 0812: 00$ & 125.582 & 122.948 & 100.759 & 104.689 & 96.329 & 111.097 & & 74.572 \\
\hline $1 / 21 / 08 \quad 12: 30$ & 125.473 & 122.902 & 100.673 & 104.586 & 96.228 & 111.012 & 80.69 & 74.469 \\
\hline $1 / 21 / 08$ 13:00 & 125.398 & 122.873 & 100.61 & 104.51 & 96.15 & 110.964 & & 74.39 \\
\hline $1 / 21 / 0813: 30$ & 125.341 & 122.85 & 100.577 & 104.466 & 96.106 & 110.928 & & 74.341 \\
\hline $1 / 21 / 0814: 00$ & 125.312 & 122.832 & 100.553 & 104.437 & 96.075 & 110.907 & 80.653 & 74.308 \\
\hline $1 / 21 / 0814: 30$ & 125.289 & 122.818 & 100.534 & 104.42 & 96.058 & 110.89 & & 74.287 \\
\hline $1 / 21 / 0815: 00$ & 125.27 & 122.825 & 100.52 & 104.405 & 96.042 & 110.879 & & 74.268 \\
\hline $1 / 21 / 08$ 15:30 & 125.256 & 122.825 & 100.513 & 104.393 & 96.03 & 110.87 & 80.626 & 74.254 \\
\hline $1 / 21 / 08$ 16:00 & 125.256 & 122.832 & 100.523 & 104.395 & 96.032 & 110.86 & & 74.256 \\
\hline 1/21/08 16:30 & 125.263 & 122.836 & 100.527 & 104.403 & 96.039 & 110.853 & & 74.264 \\
\hline
\end{tabular}


TABLE S1.1 (Cont.)

\begin{tabular}{|c|c|c|c|c|c|c|c|c|c|c|}
\hline \multirow[b]{2}{*}{ Date and Time } & \multicolumn{10}{|c|}{ Depth from Top of Casing (ft) } \\
\hline & MW1D & MW2D & MW3D & MW4D & MW7 & MW9 & Oentrich & MW14D & MW15D & MW16D \\
\hline 1/21/08 17:00 & 125.268 & 122.857 & & 100.537 & 104.409 & 96.047 & 110.849 & & 80.619 & 74.266 \\
\hline $1 / 21 / 0817: 30$ & 125.272 & 122.873 & & 100.548 & 104.412 & 96.051 & 110.845 & & & 74.266 \\
\hline $1 / 21 / 0818: 00$ & 125.289 & 122.9 & & 100.56 & 104.435 & 96.077 & 110.84 & & & 74.287 \\
\hline $1 / 21 / 0818: 30$ & 125.301 & 122.92 & & 100.592 & 104.451 & 96.089 & 110.836 & & 80.624 & 74.297 \\
\hline $1 / 21 / 0819: 00$ & 125.334 & 122.925 & & 100.61 & 104.479 & 96.12 & 110.832 & & & 74.326 \\
\hline $1 / 21 / 0819: 30$ & 125.355 & 122.941 & & 100.62 & 104.5 & 96.141 & 110.832 & & & 74.343 \\
\hline $1 / 21 / 0820: 00$ & 125.362 & 122.952 & & 100.634 & 104.506 & 96.148 & 110.832 & & 80.641 & 74.349 \\
\hline $1 / 21 / 0820: 30$ & 125.376 & 122.961 & & 100.649 & 104.521 & 96.164 & 110.83 & & & 74.363 \\
\hline $1 / 21 / 0821: 00$ & 125.386 & 122.979 & & 100.66 & 104.531 & 96.172 & 110.832 & & & 74.372 \\
\hline $1 / 21 / 0821: 30$ & 125.4 & 122.993 & & 100.673 & 104.542 & 96.183 & 110.832 & & 80.65 & 74.38 \\
\hline $1 / 21 / 0822: 00$ & 125.414 & 123.007 & & 100.689 & 104.557 & 96.202 & 110.834 & & & 74.398 \\
\hline $1 / 21 / 0822: 30$ & 125.428 & 123.013 & & 100.706 & 104.571 & 96.212 & 110.836 & & & 74.409 \\
\hline $1 / 21 / 0823: 00$ & 125.447 & 123.027 & & 100.718 & 104.588 & 96.23 & 110.84 & & 80.672 & 74.425 \\
\hline $1 / 21 / 0823: 30$ & 125.452 & 123.043 & & 100.725 & 104.595 & 96.237 & 110.834 & & & 74.429 \\
\hline $1 / 22 / 080: 00$ & 125.464 & 123.056 & & 100.747 & 104.609 & 96.252 & 110.834 & & & 74.442 \\
\hline 1/22/08 0:30 & 125.478 & 123.07 & & 100.753 & 104.622 & 96.268 & 110.832 & & 80.687 & 74.458 \\
\hline $1 / 22 / 08$ 1:00 & 125.487 & 123.068 & & 100.775 & 104.637 & 96.278 & 110.83 & & & 74.467 \\
\hline 1/22/08 1:30 & 125.506 & 123.079 & & 100.775 & 104.651 & 96.294 & 110.827 & & & 74.483 \\
\hline $1 / 22 / 082: 00$ & 125.506 & 123.086 & & 100.778 & 104.649 & 96.292 & 110.827 & & 80.714 & 74.479 \\
\hline $1 / 22 / 082: 30$ & 125.516 & 123.093 & & 100.789 & 104.66 & 96.308 & 110.823 & & & 74.492 \\
\hline 1/22/08 3:00 & 125.523 & 123.099 & & 100.799 & 104.67 & 96.315 & 110.821 & & & 74.5 \\
\hline 1/22/08 3:30 & 125.53 & 123.102 & & 100.804 & 104.674 & 96.32 & 110.819 & & 80.716 & 74.506 \\
\hline 1/22/08 4:00 & 125.537 & 123.099 & & 100.811 & 104.681 & 96.325 & 110.817 & & & 74.514 \\
\hline 1/22/08 4:30 & 125.542 & 123.099 & & 100.809 & 104.685 & 96.327 & 110.815 & & & 74.516 \\
\hline 1/22/08 5:00 & 125.539 & 123.099 & & 100.809 & 104.683 & 96.327 & 110.813 & & 80.734 & 74.514 \\
\hline 1/22/08 5:30 & 125.537 & 123.086 & & 100.808 & 104.683 & 96.325 & 110.814 & & & 74.514 \\
\hline 1/22/08 6:00 & 125.537 & 123.093 & & 100.803 & 104.683 & 96.325 & 110.812 & & & 74.514 \\
\hline 1/22/08 6:30 & 125.53 & 123.097 & & 100.799 & 104.67 & 96.313 & 110.81 & & 80.724 & 74.504 \\
\hline $1 / 22 / 08$ 7:00 & 125.53 & 124.932 & & 100.806 & 104.675 & 96.318 & 110.806 & & & 74.508 \\
\hline $1 / 22 / 087: 30$ & 125.537 & 125.231 & & 100.806 & 104.679 & 96.322 & 110.804 & & & 74.514 \\
\hline 1/22/08 8:00 & 127.319 & 125.367 & & 101.618 & 105.161 & 96.938 & 111.412 & & 80.751 & 74.574 \\
\hline 1/22/08 8:30 & 127.731 & 125.446 & & 101.819 & 105.452 & 97.235 & 111.683 & & & 74.777 \\
\hline 1/22/08 9:00 & 127.884 & 125.501 & & 101.928 & 105.587 & 97.374 & 111.809 & & & 74.913 \\
\hline 1/22/08 9:30 & 127.969 & 125.535 & & 101.991 & 105.669 & 97.456 & 111.887 & & 80.824 & 74.998 \\
\hline $1 / 22 / 0810: 00$ & 128.024 & 125.562 & & 102.035 & 105.724 & 97.51 & 111.938 & & & 75.056 \\
\hline $1 / 22 / 0810: 30$ & 128.062 & 125.583 & & 102.06 & 105.755 & 97.541 & 111.974 & & & 75.091 \\
\hline $1 / 22 / 0811: 00$ & 128.088 & 125.598 & & 102.085 & 105.784 & 97.567 & 111.989 & & 80.883 & 75.118 \\
\hline $1 / 22 / 0811: 30$ & 128.109 & 125.608 & & 102.103 & 105.803 & 97.588 & 112.004 & & & 75.141 \\
\hline $1 / 22 / 0812: 00$ & 128.125 & 123.739 & & 102.114 & 105.819 & 97.605 & 112.021 & & & 75.157 \\
\hline $1 / 22 / 0812: 30$ & 128.135 & 123.478 & & 101.731 & 105.827 & 97.614 & 112.035 & & 80.934 & 75.17 \\
\hline $1 / 22 / 0813: 00$ & 126.279 & 123.335 & & 101.263 & 105.269 & 96.919 & 111.375 & & & 75.064 \\
\hline $1 / 22 / 0813: 30$ & 125.941 & 123.242 & & 101.08 & 105.026 & 96.667 & 111.159 & & & 74.888 \\
\hline $1 / 22 / 0814: 00$ & 125.788 & 123.179 & & 100.968 & 104.885 & 96.53 & 111.054 & & 80.885 & 74.758 \\
\hline $1 / 22 / 0814: 30$ & 125.691 & 123.131 & & 100.892 & 104.799 & 96.44 & 110.99 & & & 74.667 \\
\hline $1 / 22 / 0815: 00$ & 125.622 & 123.095 & & 100.842 & 104.738 & 96.381 & 110.95 & & & 74.601 \\
\hline $1 / 22 / 0815: 30$ & 125.575 & 123.068 & & 100.803 & 104.692 & 96.334 & 110.922 & & 80.851 & 74.554 \\
\hline $1 / 22 / 0816: 00$ & 125.539 & 123.043 & & 100.773 & 104.66 & 96.301 & 110.9 & & & 74.52 \\
\hline $1 / 22 / 0816: 30$ & 125.511 & 123.027 & & 100.751 & 104.634 & 96.275 & 110.885 & & & 74.496 \\
\hline $1 / 22 / 0817: 00$ & 125.487 & 123.016 & & 100.725 & 104.612 & 96.252 & 110.873 & & 80.826 & 74.471 \\
\hline $1 / 22 / 0817: 30$ & 125.464 & 122.997 & & 100.715 & 104.595 & 96.235 & 110.862 & & & 74.452 \\
\hline $1 / 22 / 0818: 00$ & 125.454 & 122.986 & & 100.701 & 104.588 & 96.228 & 110.853 & & & 74.444 \\
\hline $1 / 22 / 0818: 30$ & 125.435 & 122.972 & & 100.684 & 104.567 & 96.209 & 110.847 & & 80.8 & 74.425 \\
\hline $1 / 22 / 08$ 19:00 & 125.426 & 122.954 & & 100.675 & 104.557 & 96.198 & 110.843 & & & 74.415 \\
\hline $1 / 22 / 0819: 30$ & 125.409 & 122.943 & & 100.658 & 104.544 & 96.181 & 110.84 & & & 74.401 \\
\hline $1 / 22 / 0820: 00$ & 125.395 & 122.929 & & 100.647 & 104.527 & 96.167 & 110.836 & & 80.778 & 74.384 \\
\hline $1 / 22 / 0820: 30$ & 125.386 & 122.916 & & 100.635 & 104.519 & 96.157 & 110.832 & & & 74.374 \\
\hline $1 / 22 / 0821: 00$ & 125.372 & 122.904 & & 100.618 & 104.502 & 96.141 & 110.83 & & & 74.361 \\
\hline $1 / 22 / 0821: 30$ & 125.357 & 122.893 & & 100.608 & 104.487 & 96.127 & 110.828 & & 80.76 & 74.347 \\
\hline $1 / 22 / 0822: 00$ & 125.343 & 122.884 & & 100.594 & 104.479 & 96.12 & 110.825 & & & 74.336 \\
\hline 1/22/08 22:30 & 125.334 & 122.882 & & 100.589 & 104.47 & 96.108 & 110.823 & & & 74.326 \\
\hline 1/22/08 23:00 & 125.327 & 122.87 & & 100.58 & 104.458 & 96.099 & 110.821 & & 80.738 & 74.32 \\
\hline 1/22/08 23:30 & 125.32 & 122.861 & & 100.577 & 104.46 & 96.096 & 110.817 & & & 74.316 \\
\hline $1 / 23 / 080: 00$ & 125.315 & 122.857 & & 100.568 & 104.449 & 96.089 & 110.815 & & & 74.31 \\
\hline $1 / 23 / 080: 30$ & 125.303 & 122.843 & & 100.554 & 104.437 & 96.077 & 110.812 & & 80.724 & 74.299 \\
\hline $1 / 23 / 081: 00$ & 125.291 & 122.836 & & 100.549 & 104.432 & 96.07 & 110.808 & & & 74.287 \\
\hline 1/23/08 1:30 & 125.284 & 122.825 & & 100.536 & 104.418 & 96.058 & 110.808 & & & 74.281 \\
\hline
\end{tabular}


TABLE S1.1 (Cont.)

\begin{tabular}{|c|c|c|c|c|c|c|c|c|c|c|}
\hline \multirow[b]{2}{*}{ Date and Time } & \multicolumn{10}{|c|}{ Depth from Top of Casing (ft) } \\
\hline & MW1D & MW2D & MW3D & MW4D & MW7 & MW9 & Oentrich & MW14D & MW15D & MW16D \\
\hline 1/23/08 2:00 & 125.27 & 122.816 & & 100.532 & 104.412 & 96.049 & 110.806 & & 80.704 & 74.268 \\
\hline $1 / 23 / 082: 30$ & 125.265 & 122.805 & & 100.52 & 104.401 & 96.042 & 110.802 & & & 74.264 \\
\hline 1/23/08 3:00 & 125.251 & 122.791 & & 100.51 & 104.393 & 96.03 & 110.8 & & & 74.25 \\
\hline $1 / 23 / 083: 30$ & 125.244 & 122.766 & & 100.503 & 104.382 & 96.021 & 110.8 & & 80.682 & 74.243 \\
\hline $1 / 23 / 08$ 4:00 & 125.232 & 122.746 & & 100.486 & 104.369 & 96.009 & 110.798 & & & 74.229 \\
\hline $1 / 23 / 084: 30$ & 125.213 & 122.741 & & 100.456 & 104.344 & 95.983 & 110.797 & & & 74.206 \\
\hline $1 / 23 / 085: 00$ & 125.187 & 122.737 & & 100.442 & 104.325 & 95.962 & 110.795 & & 80.643 & 74.186 \\
\hline 1/23/08 5:30 & 125.178 & 122.728 & & 100.437 & 104.319 & 95.957 & 110.793 & & & 74.179 \\
\hline 1/23/08 6:00 & 125.173 & 122.732 & & 100.436 & 104.319 & 95.955 & 110.791 & & & 74.177 \\
\hline $1 / 23 / 086: 30$ & 125.168 & 122.748 & & 100.43 & 104.31 & 95.948 & 110.789 & & 80.619 & 74.171 \\
\hline $1 / 23 / 08$ 7:00 & 125.164 & 124.632 & & 100.439 & 104.313 & 95.948 & 110.785 & & & 74.169 \\
\hline 1/23/08 7:30 & 125.178 & 124.879 & & 100.72 & 104.329 & 95.966 & 110.781 & & & 74.186 \\
\hline $1 / 23 / 088: 00$ & 127.026 & 125.002 & & 101.24 & 104.833 & 96.593 & 111.39 & & 80.633 & 74.272 \\
\hline 1/23/08 8:30 & 127.367 & 125.075 & & 101.411 & 105.077 & 96.846 & 111.626 & & & 74.463 \\
\hline 1/23/08 9:00 & 127.494 & 125.143 & & 101.514 & 105.201 & 96.966 & 111.745 & & & 74.58 \\
\hline 1/23/08 9:30 & 127.575 & 123.313 & & 101.574 & 105.277 & 97.046 & 111.814 & & 80.685 & 74.665 \\
\hline $1 / 23 / 0810: 00$ & 127.634 & 123.111 & & 101.242 & 105.346 & 97.114 & 111.857 & & & 74.733 \\
\hline $1 / 23 / 0810: 30$ & 125.823 & 123.027 & & 100.88 & 104.864 & 96.509 & 111.241 & & & 74.68 \\
\hline $1 / 23 / 0811: 00$ & 125.568 & 122.975 & & 100.753 & 104.674 & 96.318 & 111.059 & & 80.685 & 74.543 \\
\hline $1 / 23 / 0811: 30$ & 125.473 & 122.936 & & 100.692 & 104.594 & 96.235 & 110.971 & & & 74.465 \\
\hline $1 / 23 / 08$ 12:00 & 125.416 & 122.918 & & 100.653 & 104.544 & 96.183 & 110.92 & & & 74.405 \\
\hline $1 / 23 / 0812: 30$ & 125.381 & 123.684 & & 100.623 & 104.51 & 96.148 & 110.889 & & 80.675 & 74.365 \\
\hline $1 / 23 / 08$ 13:00 & 125.362 & 123.054 & & 100.608 & 104.491 & 96.134 & 110.866 & & & 74.347 \\
\hline $1 / 23 / 0813: 30$ & 125.35 & 122.959 & & 101.082 & 104.481 & 96.124 & 110.855 & & & 74.336 \\
\hline $1 / 23 / 0814: 00$ & 125.556 & 122.934 & & 100.687 & 104.618 & 96.263 & 110.979 & & 80.682 & 74.398 \\
\hline 1/23/08 14:30 & 125.409 & 122.925 & & 100.644 & 104.533 & 96.176 & 110.89 & & & 74.376 \\
\hline $1 / 23 / 08$ 15:00 & 125.379 & 122.934 & & 100.629 & 104.511 & 96.15 & 110.862 & & & 74.359 \\
\hline $1 / 23 / 0815: 30$ & 125.369 & 122.959 & & 100.627 & 104.502 & 96.146 & 110.843 & & 80.677 & 74.351 \\
\hline 1/23/08 16:00 & 125.372 & 122.986 & & 100.646 & 104.511 & 96.155 & 110.832 & & & 74.357 \\
\hline $1 / 23 / 0816: 30$ & 125.395 & 123.007 & & 100.672 & 104.54 & 96.183 & 110.828 & & & 74.382 \\
\hline $1 / 23 / 08$ 17:00 & 125.419 & 123.025 & & 100.699 & 104.569 & 96.214 & 110.821 & & 80.699 & 74.409 \\
\hline $1 / 23 / 0817: 30$ & 125.442 & 123.041 & & 100.72 & 104.588 & 96.23 & 110.825 & & & 74.423 \\
\hline $1 / 23 / 0818: 00$ & 125.461 & 123.05 & & 100.739 & 104.607 & 96.249 & 110.819 & & & 74.44 \\
\hline $1 / 23 / 0818: 30$ & 125.478 & 123.063 & & 100.754 & 104.622 & 96.268 & 110.813 & & 80.721 & 74.454 \\
\hline 1/23/08 19:00 & 125.49 & 123.07 & & 100.763 & 104.634 & 96.275 & 110.812 & & & 74.465 \\
\hline $1 / 23 / 0819: 30$ & 125.502 & 123.081 & & 100.778 & 104.647 & 96.289 & 110.808 & & & 74.477 \\
\hline 1/23/08 20:00 & 125.511 & 123.084 & & 100.784 & 104.652 & 96.296 & 110.808 & & 80.734 & 74.483 \\
\hline 1/23/08 20:30 & 125.518 & 123.093 & & 100.791 & 104.662 & 96.308 & 110.806 & & & 74.492 \\
\hline $1 / 23 / 0821: 00$ & 125.523 & 123.097 & & 100.801 & 104.666 & 96.308 & 110.804 & & & 74.494 \\
\hline $1 / 23 / 0821: 30$ & 125.532 & 123.104 & & 100.803 & 104.677 & 96.318 & 110.8 & & 80.748 & 74.504 \\
\hline 1/23/08 22:00 & 125.537 & 123.109 & & 100.804 & 104.681 & 96.32 & 110.8 & & & 74.508 \\
\hline $1 / 23 / 0822: 30$ & 125.542 & 123.109 & & 100.813 & 104.685 & 96.327 & 110.798 & & & 74.514 \\
\hline 1/23/08 23:00 & 125.546 & 123.115 & & 100.816 & 104.689 & 96.334 & 110.797 & & 80.765 & 74.516 \\
\hline 1/23/08 23:30 & 125.549 & 123.115 & & 100.816 & 104.689 & 96.329 & 110.795 & & & 74.518 \\
\hline $1 / 24 / 080: 00$ & 125.551 & 123.109 & & 100.823 & 104.694 & 96.337 & 110.795 & & & 74.523 \\
\hline 1/24/08 0:30 & 125.554 & 123.113 & & 100.818 & 104.696 & 96.337 & 110.791 & & 80.78 & 74.525 \\
\hline 1/24/08 1:00 & 125.549 & 123.111 & & 100.818 & 104.687 & 96.329 & 110.791 & & & 74.52 \\
\hline 1/24/08 1:30 & 125.551 & 123.106 & & 100.82 & 104.694 & 96.337 & 110.787 & & & 74.523 \\
\hline $1 / 24 / 082: 00$ & 125.549 & 123.106 & & 100.815 & 104.694 & 96.334 & 110.785 & & 80.804 & 74.523 \\
\hline 1/24/08 2:30 & 125.546 & 123.113 & & 100.815 & 104.689 & 96.329 & 110.784 & & & 74.518 \\
\hline $1 / 24 / 083: 00$ & 125.546 & 123.115 & & 100.816 & 104.689 & 96.332 & 110.78 & & & 74.523 \\
\hline $1 / 24 / 083: 30$ & 125.551 & 123.118 & & 100.825 & 104.694 & 96.339 & 110.776 & & 80.792 & 74.529 \\
\hline $1 / 24 / 084: 00$ & 125.554 & 123.111 & & 100.827 & 104.702 & 96.344 & 110.774 & & & 74.533 \\
\hline $1 / 24 / 084: 30$ & 125.554 & 123.111 & & 100.827 & 104.7 & 96.344 & 110.77 & & & 74.533 \\
\hline 1/24/08 5:00 & 125.554 & 123.109 & & 100.818 & 104.694 & 96.337 & 110.769 & & 80.8 & 74.531 \\
\hline 1/24/08 5:30 & 125.546 & 123.113 & & 100.818 & 104.694 & 96.334 & 110.765 & & & 74.527 \\
\hline $1 / 24 / 086: 00$ & 125.549 & 123.109 & & 100.822 & 104.691 & 96.332 & 110.763 & & & 74.529 \\
\hline 1/24/08 6:30 & 125.551 & 123.109 & & 100.82 & 104.698 & 96.339 & 110.759 & & 80.802 & 74.533 \\
\hline $1 / 24 / 087: 00$ & 125.549 & 124.567 & & 100.822 & 104.694 & 96.334 & 110.757 & & & 74.529 \\
\hline $1 / 24 / 087: 30$ & 125.546 & 125.147 & & 100.827 & 104.696 & 96.337 & 110.754 & & & 74.531 \\
\hline $1 / 24 / 088: 00$ & 126.721 & 125.331 & & 101.468 & 104.847 & 96.56 & 111.012 & & 80.809 & 74.537 \\
\hline $1 / 24 / 088: 30$ & 127.624 & 125.449 & & 101.764 & 105.368 & 97.15 & 111.538 & & & 74.717 \\
\hline $1 / 24 / 089: 00$ & 127.842 & 125.519 & & 101.909 & 105.557 & 97.338 & 111.707 & & & 74.882 \\
\hline 1/24/08 9:30 & 127.96 & 125.571 & & 102 & 105.671 & 97.454 & 111.801 & & 80.895 & 74.998 \\
\hline $1 / 24 / 0810: 00$ & 128.043 & 123.714 & & 102.059 & 105.744 & 97.527 & 111.865 & & & 75.072 \\
\hline $1 / 24 / 0810: 30$ & 128.095 & 123.492 & & 101.664 & 105.797 & 97.581 & 111.905 & & & 75.126 \\
\hline
\end{tabular}


TABLE S1.1 (Cont.)

\begin{tabular}{|c|c|c|c|c|c|c|c|c|c|c|}
\hline \multirow[b]{2}{*}{ Date and Time } & \multicolumn{10}{|c|}{ Depth from Top of Casing (ft) } \\
\hline & MW1D & MW2D & MW3D & MW4D & MW7 & MW9 & Oentrich & MW14D & MW15D & MW16D \\
\hline 1/24/08 11:00 & 126.232 & 123.374 & & 101.27 & 105.26 & 96.907 & 111.236 & & 80.932 & 75.052 \\
\hline $1 / 24 / 0811: 30$ & 125.948 & 123.306 & & 101.114 & 105.05 & 96.692 & 111.039 & & & 74.903 \\
\hline 1/24/08 12:00 & 125.823 & 123.258 & & 101.033 & 104.938 & 96.582 & 110.945 & & & 74.795 \\
\hline $1 / 24 / 0812: 30$ & 125.754 & 123.215 & & 100.975 & 104.873 & 96.516 & 110.889 & & 80.914 & 74.729 \\
\hline $1 / 24 / 0813: 00$ & 125.702 & 123.188 & & 100.932 & 104.828 & 96.471 & 110.851 & & & 74.678 \\
\hline $1 / 24 / 0813: 30$ & 125.662 & 123.163 & & 100.899 & 104.786 & 96.428 & 110.828 & & & 74.636 \\
\hline $1 / 24 / 0814: 00$ & 125.632 & 123.136 & & 100.882 & 104.759 & 96.4 & 110.81 & & 80.902 & 74.607 \\
\hline $1 / 24 / 0814: 30$ & 125.608 & 123.122 & & 100.851 & 104.734 & 96.374 & 110.799 & & & 74.582 \\
\hline $1 / 24 / 0815: 00$ & 125.58 & 123.109 & & 100.83 & 104.71 & 96.351 & 110.789 & & & 74.558 \\
\hline $1 / 24 / 0815: 30$ & 125.563 & 123.09 & & 100.818 & 104.696 & 96.339 & 110.78 & & 80.885 & 74.543 \\
\hline $1 / 24 / 0816: 00$ & 125.551 & 123.077 & & 100.803 & 104.683 & 96.325 & 110.774 & & & 74.533 \\
\hline $1 / 24 / 0816: 30$ & 125.535 & 123.056 & & 100.784 & 104.666 & 96.306 & 110.766 & & & 74.516 \\
\hline $1 / 24 / 0817: 00$ & 125.518 & 123.047 & & 100.775 & 104.656 & 96.296 & 110.761 & & 80.87 & 74.506 \\
\hline $1 / 24 / 0817: 30$ & 125.504 & 123.043 & & 100.754 & 104.635 & 96.278 & 110.759 & & & 74.487 \\
\hline $1 / 24 / 0818: 00$ & 125.483 & 123.05 & & 100.746 & 104.62 & 96.263 & 110.753 & & & 74.471 \\
\hline $1 / 24 / 0818: 30$ & 125.48 & 123.041 & & 100.744 & 104.62 & 96.261 & 110.735 & & 80.853 & 74.471 \\
\hline $1 / 24 / 08$ 19:00 & 125.485 & 123.025 & & 100.751 & 104.63 & 96.268 & 110.73 & & & 74.481 \\
\hline $1 / 24 / 0819: 30$ & 125.485 & 123.022 & & 100.741 & 104.622 & 96.266 & 110.728 & & & 74.473 \\
\hline $1 / 24 / 0820: 00$ & 125.468 & 123.013 & & 100.727 & 104.603 & 96.245 & 110.73 & & 80.858 & 74.458 \\
\hline $1 / 24 / 0820: 30$ & 125.464 & 123.016 & & 100.72 & 104.601 & 96.242 & 110.726 & & & 74.452 \\
\hline $1 / 24 / 0821: 00$ & 125.454 & 123.004 & & 100.72 & 104.594 & 96.235 & 110.728 & & & 74.446 \\
\hline $1 / 24 / 0821: 30$ & 125.461 & 123.013 & & 100.713 & 104.599 & 96.24 & 110.724 & & 80.839 & 74.452 \\
\hline 1/24/08 22:00 & 125.445 & 123.007 & & 100.711 & 104.584 & 96.223 & 110.724 & & & 74.434 \\
\hline $1 / 24 / 0822: 30$ & 125.452 & 122.995 & & 100.72 & 104.597 & 96.233 & 110.72 & & & 74.442 \\
\hline $1 / 24 / 0823: 00$ & 125.447 & 122.982 & & 100.706 & 104.586 & 96.223 & 110.72 & & 80.829 & 74.438 \\
\hline 1/24/08 23:30 & 125.438 & 122.975 & & 100.687 & 104.575 & 96.216 & 110.72 & & & 74.427 \\
\hline $1 / 25 / 080: 00$ & 125.419 & 122.966 & & 100.679 & 104.559 & 96.2 & 110.72 & & & 74.411 \\
\hline 1/25/08 0:30 & 125.412 & 122.954 & & 100.673 & 104.552 & 96.193 & 110.718 & & 80.814 & 74.405 \\
\hline 1/25/08 1:00 & 125.409 & 122.938 & & 100.672 & 104.546 & 96.188 & 110.718 & & & 74.401 \\
\hline $1 / 25 / 081: 30$ & 125.4 & 122.945 & & 100.653 & 104.535 & 96.174 & 110.716 & & & 74.39 \\
\hline $1 / 25 / 082: 00$ & 125.388 & 122.925 & & 100.635 & 104.519 & 96.162 & 110.716 & & 80.802 & 74.378 \\
\hline $1 / 25 / 082: 30$ & 125.376 & 122.914 & & 100.639 & 104.529 & 96.167 & 110.71 & & & 74.378 \\
\hline $1 / 25 / 083: 00$ & 125.374 & 122.904 & & 100.623 & 104.508 & 96.153 & 110.714 & & & 74.365 \\
\hline $1 / 25 / 083: 30$ & 125.353 & 122.866 & & 100.62 & 104.493 & 96.131 & 110.71 & & 80.785 & 74.349 \\
\hline 1/25/08 4:00 & 125.35 & 122.855 & & 100.591 & 104.483 & 96.124 & 110.712 & & & 74.343 \\
\hline 1/25/08 4:30 & 125.31 & 122.832 & & 100.563 & 104.445 & 96.084 & 110.714 & & & 74.301 \\
\hline $1 / 25 / 085: 00$ & 125.298 & 122.805 & & 100.548 & 104.436 & 96.075 & 110.712 & & 80.756 & 74.295 \\
\hline 1/25/08 5:30 & 125.279 & 122.796 & & 100.529 & 104.415 & 96.051 & 110.714 & & & 74.274 \\
\hline $1 / 25 / 086: 00$ & 125.251 & 122.793 & & 100.499 & 104.386 & 96.023 & 110.71 & & & 74.245 \\
\hline $1 / 25 / 086: 30$ & 125.23 & 122.787 & & 100.494 & 104.373 & 96.009 & 110.705 & & 80.721 & 74.231 \\
\hline $1 / 25 / 087: 00$ & 125.23 & 124.605 & & 100.489 & 104.373 & 96.011 & 110.703 & & & 74.235 \\
\hline $1 / 25 / 087: 30$ & 125.225 & 124.914 & & 100.491 & 104.369 & 96.007 & 110.701 & & & 74.231 \\
\hline $1 / 25 / 088: 00$ & 126.958 & 125.041 & & 101.223 & 104.778 & 96.537 & 111.243 & & 80.712 & 74.26 \\
\hline 1/25/08 8:30 & 127.39 & 125.122 & & 101.43 & 105.083 & 96.848 & 111.531 & & & 74.465 \\
\hline $1 / 25 / 089: 00$ & 127.537 & 125.168 & & 101.53 & 105.21 & 96.98 & 111.662 & & & 74.591 \\
\hline 1/25/08 9:30 & 127.617 & 125.199 & & 101.595 & 105.294 & 97.063 & 111.739 & & 80.758 & 74.678 \\
\hline 1/25/08 10:00 & 127.669 & 125.213 & & 101.631 & 105.338 & 97.107 & 111.792 & & & 74.729 \\
\hline $1 / 25 / 0810: 30$ & 127.7 & 125.222 & & 101.652 & 105.372 & 97.14 & 111.825 & & & 74.762 \\
\hline $1 / 25 / 0811: 00$ & 127.719 & 125.233 & & 101.664 & 105.389 & 97.159 & 111.852 & & 80.79 & 74.781 \\
\hline 1/25/08 11:30 & 127.726 & 125.229 & & 101.674 & 105.397 & 97.169 & 111.873 & & & 74.793 \\
\hline $1 / 25 / 0812: 00$ & 127.738 & 125.231 & & 101.681 & 105.407 & 97.178 & 111.887 & & & 74.806 \\
\hline 1/25/08 12:30 & 127.728 & 125.22 & & 101.673 & 105.403 & 97.173 & 111.901 & & 80.804 & 74.804 \\
\hline 1/25/08 13:00 & 127.728 & 125.217 & & 101.683 & 105.403 & 97.173 & 111.912 & & & 74.806 \\
\hline $1 / 25 / 08$ 13:30 & 127.733 & 125.206 & & 101.668 & 105.404 & 97.176 & 111.923 & & & 74.812 \\
\hline 1/25/08 14:00 & 127.716 & 125.204 & & 101.661 & 105.395 & 97.166 & 111.931 & & 80.817 & 74.802 \\
\hline $1 / 25 / 0814: 30$ & 127.709 & 123.315 & & 101.649 & 105.386 & 97.154 & 111.94 & & & 74.797 \\
\hline $1 / 25 / 08$ 15:00 & 127.705 & 123.084 & & 101.232 & 105.387 & 97.157 & 111.946 & & & 74.8 \\
\hline 1/25/08 15:30 & 125.825 & 122.961 & & 100.847 & 104.852 & 96.494 & 111.292 & & 80.804 & 74.69 \\
\hline $1 / 25 / 0816: 00$ & 125.537 & 122.904 & & 100.684 & 104.63 & 96.268 & 111.091 & & & 74.523 \\
\hline $1 / 25 / 0816: 30$ & 125.405 & 122.861 & & 100.608 & 104.515 & 96.155 & 110.992 & & & 74.405 \\
\hline 1/25/08 17:00 & 125.343 & 122.834 & & 100.563 & 104.462 & 96.103 & 110.93 & & 80.758 & 74.349 \\
\hline $1 / 25 / 0817: 30$ & 125.305 & 122.816 & & 100.532 & 104.424 & 96.061 & 110.89 & & & 74.303 \\
\hline $1 / 25 / 0818: 00$ & 125.277 & 122.805 & & 100.506 & 104.401 & 96.037 & 110.862 & & & 74.276 \\
\hline $1 / 25 / 0818: 30$ & 125.251 & 122.805 & & 100.499 & 104.384 & 96.021 & 110.842 & & 80.726 & 74.252 \\
\hline 1/25/08 19:00 & 125.239 & 122.793 & & 100.496 & 104.374 & 96.011 & 110.825 & & & 74.239 \\
\hline $1 / 25 / 0819: 30$ & 125.239 & 122.784 & & 100.489 & 104.377 & 96.014 & 110.815 & & & 74.239 \\
\hline
\end{tabular}


TABLE S1.1 (Cont.)

\begin{tabular}{|c|c|c|c|c|c|c|c|c|c|c|}
\hline \multirow[b]{2}{*}{ Date and Time } & \multicolumn{10}{|c|}{ Depth from Top of Casing (ft) } \\
\hline & MW1D & MW2D & MW3D & MW4D & MW7 & MW9 & Oentrich & MW14D & MW15D & MW16D \\
\hline 1/25/08 20:00 & 125.234 & 122.78 & & 100.484 & 104.367 & 96.004 & 110.806 & & 80.709 & 74.231 \\
\hline $1 / 25 / 0820: 30$ & 125.223 & 122.782 & & 100.475 & 104.356 & 95.995 & 110.804 & & & 74.214 \\
\hline 1/25/08 21:00 & 125.22 & 122.782 & & 100.472 & 104.355 & 95.992 & 110.8 & & & 74.212 \\
\hline $1 / 25 / 0821: 30$ & 125.216 & 122.777 & & 100.475 & 104.356 & 95.995 & 110.797 & & 80.69 & 74.21 \\
\hline $1 / 25 / 0822: 00$ & 125.213 & 122.773 & & 100.477 & 104.354 & 95.99 & 110.793 & & & 74.208 \\
\hline 1/25/08 22:30 & 125.213 & 122.766 & & 100.472 & 104.352 & 95.99 & 110.793 & & & 74.208 \\
\hline 1/25/08 23:00 & 125.211 & 122.762 & & 100.463 & 104.346 & 95.988 & 110.791 & & 80.67 & 74.202 \\
\hline $1 / 25 / 0823: 30$ & 125.199 & 122.773 & & 100.463 & 104.344 & 95.981 & 110.783 & & & 74.194 \\
\hline 1/26/08 0:00 & 125.19 & 122.762 & & 100.468 & 104.335 & 95.971 & 110.781 & & & 74.181 \\
\hline $1 / 26 / 080: 30$ & 125.211 & 122.757 & & 100.47 & 104.352 & 95.99 & 110.778 & & 80.668 & 74.204 \\
\hline 1/26/08 1:00 & 125.206 & 122.748 & & 100.46 & 104.34 & 95.981 & 110.78 & & & 74.194 \\
\hline 1/26/08 1:30 & 125.197 & 122.746 & & 100.451 & 104.334 & 95.973 & 110.778 & & & 74.186 \\
\hline $1 / 26 / 082: 00$ & 125.185 & 122.743 & & 100.449 & 104.327 & 95.964 & 110.776 & & 80.648 & 74.175 \\
\hline $1 / 26 / 082: 30$ & 125.182 & 122.746 & & 100.446 & 104.323 & 95.959 & 110.776 & & & 74.171 \\
\hline 1/26/08 3:00 & 125.18 & 122.741 & & 100.448 & 104.323 & 95.962 & 110.774 & & & 74.173 \\
\hline $1 / 26 / 083: 30$ & 125.182 & 122.737 & & 100.449 & 104.325 & 95.964 & 110.772 & & 80.638 & 74.177 \\
\hline $1 / 26 / 084: 00$ & 125.182 & 122.732 & & 100.448 & 104.323 & 95.964 & 110.772 & & & 74.173 \\
\hline 1/26/08 4:30 & 125.175 & 122.728 & & 100.439 & 104.316 & 95.955 & 110.772 & & & 74.163 \\
\hline $1 / 26 / 085: 00$ & 125.171 & 122.739 & & 100.434 & 104.312 & 95.952 & 110.77 & & 80.621 & 74.165 \\
\hline 1/26/08 5:30 & 125.173 & 122.746 & & 100.429 & 104.314 & 95.957 & 110.77 & & & 74.169 \\
\hline $1 / 26 / 086: 00$ & 125.168 & 122.746 & & 100.451 & 104.321 & 95.959 & 110.765 & & & 74.167 \\
\hline $1 / 26 / 086: 30$ & 125.185 & 122.746 & & 100.453 & 104.329 & 95.971 & 110.765 & & 80.614 & 74.177 \\
\hline $1 / 26 / 087: 00$ & 125.18 & 122.753 & & 100.453 & 104.331 & 95.971 & 110.763 & & & 74.175 \\
\hline 1/26/08 7:30 & 125.182 & 124.351 & & 100.456 & 104.329 & 95.966 & 110.761 & & & 74.173 \\
\hline $1 / 26 / 088: 00$ & 125.192 & 124.823 & & 100.461 & 104.335 & 95.976 & 110.761 & & 80.606 & 74.181 \\
\hline 1/26/08 8:30 & 126.596 & 125.004 & & 101.159 & 104.597 & 96.346 & 111.148 & & & 74.188 \\
\hline 1/26/08 9:00 & 127.305 & 125.115 & & 101.437 & 105.047 & 96.824 & 111.572 & & & 74.39 \\
\hline 1/26/08 9:30 & 127.518 & 125.193 & & 101.578 & 105.225 & 97.008 & 111.736 & & 80.668 & 74.56 \\
\hline 1/26/08 10:00 & 127.634 & 125.251 & & 101.669 & 105.34 & 97.121 & 111.828 & & & 74.671 \\
\hline $1 / 26 / 08 \quad 10: 30$ & 127.716 & 125.295 & & 101.733 & 105.414 & 97.197 & 111.886 & & & 74.75 \\
\hline $1 / 26 / 0811: 00$ & 127.773 & 125.324 & & 101.783 & 105.471 & 97.256 & 111.923 & & 80.734 & 74.81 \\
\hline 1/26/08 11:30 & 127.813 & 125.344 & & 101.814 & 105.513 & 97.298 & 111.953 & & & 74.849 \\
\hline $1 / 26 / 08$ 12:00 & 127.846 & 125.351 & & 101.842 & 105.544 & 97.329 & 111.98 & & & 74.882 \\
\hline $1 / 26 / 0812: 30$ & 127.865 & 125.363 & & 101.857 & 105.563 & 97.345 & 111.998 & & 80.787 & 74.899 \\
\hline 1/26/08 13:00 & 127.88 & 125.358 & & 101.866 & 105.568 & 97.352 & 112.017 & & & 74.907 \\
\hline $1 / 26 / 08$ 13:30 & 127.891 & 125.363 & & 101.873 & 105.58 & 97.367 & 112.03 & & & 74.924 \\
\hline $1 / 26 / 0814: 00$ & 127.887 & 124.27 & & 101.867 & 105.58 & 97.362 & 112.043 & & 80.822 & 74.924 \\
\hline 1/26/08 14:30 & 127.889 & 123.444 & & 101.874 & 105.582 & 97.367 & 112.053 & & & 74.928 \\
\hline $1 / 26 / 0815: 00$ & 127.419 & 123.245 & & 101.306 & 105.578 & 97.374 & 112.057 & & & 74.934 \\
\hline $1 / 26 / 08$ 15:30 & 125.929 & 123.156 & & 100.997 & 104.978 & 96.624 & 111.317 & & 80.834 & 74.818 \\
\hline 1/26/08 16:00 & 125.7 & 123.097 & & 100.87 & 104.795 & 96.438 & 111.142 & & & 74.696 \\
\hline $1 / 26 / 0816: 30$ & 125.596 & 123.065 & & 100.803 & 104.713 & 96.353 & 111.05 & & & 74.611 \\
\hline 1/26/08 17:00 & 125.539 & 123.043 & & 100.766 & 104.656 & 96.301 & 110.999 & & 80.812 & 74.551 \\
\hline 1/26/08 17:30 & 125.504 & 123.022 & & 100.741 & 104.628 & 96.27 & 110.965 & & & 74.506 \\
\hline $1 / 26 / 0818: 00$ & 125.483 & 123.009 & & 100.725 & 104.609 & 96.252 & 110.943 & & & 74.471 \\
\hline $1 / 26 / 0818: 30$ & 125.464 & 122.997 & & 100.71 & 104.59 & 96.233 & 110.928 & & 80.804 & 74.444 \\
\hline 1/26/08 19:00 & 125.447 & 122.995 & & 100.696 & 104.578 & 96.223 & 110.919 & & & 74.421 \\
\hline 1/26/08 19:30 & 125.435 & 122.981 & & 100.692 & 104.569 & 96.212 & 110.909 & & & 74.405 \\
\hline 1/26/08 20:00 & 125.431 & 122.975 & & 100.685 & 104.565 & 96.209 & 110.904 & & 80.797 & 74.388 \\
\hline 1/26/08 20:30 & 125.421 & 122.966 & & 100.673 & 104.554 & 96.195 & 110.9 & & & 74.374 \\
\hline $1 / 26 / 0821: 00$ & 125.412 & 122.961 & & 100.668 & 104.548 & 96.19 & 110.896 & & & 74.359 \\
\hline 1/26/08 21:30 & 125.405 & 122.952 & & 100.663 & 104.54 & 96.183 & 110.89 & & 80.785 & 74.347 \\
\hline 1/26/08 22:00 & 125.402 & 122.943 & & 100.656 & 104.538 & 96.179 & 110.887 & & & 74.339 \\
\hline 1/26/08 22:30 & 125.39 & 122.934 & & 100.649 & 104.529 & 96.169 & 110.883 & & & 74.328 \\
\hline 1/26/08 23:00 & 125.386 & 122.938 & & 100.637 & 104.519 & 96.162 & 110.881 & & 80.775 & 74.32 \\
\hline 1/26/08 23:30 & 125.376 & 122.932 & & 100.634 & 104.51 & 96.15 & 110.879 & & & 74.312 \\
\hline $1 / 27 / 080: 00$ & 125.369 & 122.932 & & 100.634 & 104.512 & 96.15 & 110.875 & & & 74.303 \\
\hline 1/27/08 0:30 & 125.369 & 122.918 & & 100.632 & 104.508 & 96.148 & 110.872 & & 80.77 & 74.295 \\
\hline 1/27/08 1:00 & 125.367 & 122.918 & & 100.627 & 104.508 & 96.148 & 110.868 & & & 74.287 \\
\hline 1/27/08 1:30 & 125.36 & 122.918 & & 100.622 & 104.495 & 96.134 & 110.868 & & & 74.283 \\
\hline $1 / 27 / 082: 00$ & 125.355 & 122.911 & & 100.62 & 104.498 & 96.136 & 110.864 & & 80.76 & 74.276 \\
\hline $1 / 27 / 082: 30$ & 125.357 & 122.909 & & 100.616 & 104.496 & 96.139 & 110.862 & & & 74.27 \\
\hline 1/27/08 3:00 & 125.35 & 122.911 & & 100.613 & 104.493 & 96.131 & 110.86 & & & 74.264 \\
\hline $1 / 27 / 083: 30$ & 125.346 & 122.904 & & 100.615 & 104.489 & 96.127 & 110.857 & & 80.758 & 74.258 \\
\hline $1 / 27 / 084: 00$ & 125.348 & 122.909 & & 100.606 & 104.491 & 96.134 & 110.855 & & & 74.25 \\
\hline 1/27/08 4:30 & 125.341 & 122.916 & & 100.605 & 104.487 & 96.124 & 110.853 & & & 74.248 \\
\hline
\end{tabular}


TABLE S1.1 (Cont.)

\begin{tabular}{|c|c|c|c|c|c|c|c|c|c|c|}
\hline \multirow[b]{2}{*}{ Date and Time } & \multicolumn{10}{|c|}{ Depth from Top of Casing (ft) } \\
\hline & MW1D & MW2D & MW3D & MW4D & MW7 & MW9 & Oentrich & MW14D & MW15D & MW16D \\
\hline 1/27/08 5:00 & 125.348 & 122.9 & & 100.616 & 104.491 & 96.131 & 110.851 & & 80.753 & 74.241 \\
\hline 1/27/08 5:30 & 125.35 & 122.891 & & 100.613 & 104.498 & 96.136 & 110.847 & & & 74.235 \\
\hline 1/27/08 6:00 & 125.341 & 122.891 & & 100.604 & 104.483 & 96.124 & 110.847 & & & 74.233 \\
\hline $1 / 27 / 086: 30$ & 125.334 & 122.884 & & 100.594 & 104.472 & 96.11 & 110.845 & & 80.743 & 74.231 \\
\hline $1 / 27 / 08$ 7:00 & 125.327 & 122.88 & & 100.594 & 104.472 & 96.11 & 110.843 & & & 74.227 \\
\hline $1 / 27 / 087: 30$ & 125.327 & 122.875 & & 100.591 & 104.47 & 96.11 & 110.843 & & & 74.221 \\
\hline $1 / 27 / 088: 00$ & 125.322 & 124.002 & & 100.579 & 104.462 & 96.101 & 110.842 & & 80.734 & 74.217 \\
\hline 1/27/08 8:30 & 125.312 & 124.864 & & 100.575 & 104.455 & 96.096 & 110.84 & & & 74.212 \\
\hline 1/27/08 9:00 & 125.558 & 125.05 & & 101.113 & 104.439 & 96.082 & 110.84 & & & 74.21 \\
\hline 1/27/08 9:30 & 127.322 & 125.158 & & 101.413 & 105.039 & 96.81 & 111.561 & & 80.741 & 74.341 \\
\hline $1 / 27 / 0810: 00$ & 127.542 & 125.229 & & 101.554 & 105.227 & 96.999 & 111.741 & & & 74.483 \\
\hline 1/27/08 10:30 & 127.653 & 125.27 & & 101.65 & 105.34 & 97.107 & 111.839 & & & 74.576 \\
\hline $1 / 27 / 0811: 00$ & 127.731 & 125.295 & & 101.702 & 105.412 & 97.183 & 111.901 & & 80.802 & 74.64 \\
\hline $1 / 27 / 0811: 30$ & 127.771 & 125.299 & & 101.738 & 105.448 & 97.22 & 111.944 & & & 74.688 \\
\hline 1/27/08 12:00 & 127.799 & 125.292 & & 101.743 & 105.475 & 97.246 & 111.972 & & & 74.729 \\
\hline $1 / 27 / 0812: 30$ & 127.799 & 125.283 & & 101.754 & 105.473 & 97.246 & 111.98 & & 80.836 & 74.771 \\
\hline $1 / 27 / 0813: 00$ & 127.804 & 125.265 & & 101.738 & 105.469 & 97.242 & 112.004 & & & 74.805 \\
\hline 1/27/08 13:30 & 127.787 & 125.245 & & 101.728 & 105.46 & 97.232 & 112.022 & & & 74.839 \\
\hline $1 / 27 / 0814: 00$ & 127.785 & 125.213 & & 101.712 & 105.447 & 97.218 & 112.032 & & 80.846 & 74.868 \\
\hline $1 / 27 / 0814: 30$ & 127.792 & 125.195 & & 101.688 & 105.426 & 97.202 & 112.046 & & & 74.891 \\
\hline $1 / 27 / 0815: 00$ & 127.802 & 125.186 & & 101.652 & 105.395 & 97.166 & 112.057 & & & 74.924 \\
\hline $1 / 27 / 0815: 30$ & 127.809 & 125.188 & & 101.644 & 105.374 & 97.145 & 112.064 & & 80.841 & 74.944 \\
\hline 1/27/08 16:00 & 127.811 & 123.886 & & 101.642 & 105.368 & 97.138 & 112.07 & & & 74.957 \\
\hline $1 / 27 / 0816: 30$ & 127.811 & 123.236 & & 101.645 & 105.374 & 97.14 & 112.074 & & & 74.959 \\
\hline $1 / 27 / 0817: 00$ & 126.884 & 123.027 & & 101.082 & 105.302 & 97.039 & 111.923 & & 80.851 & 74.953 \\
\hline 1/27/08 17:30 & 126.189 & 122.92 & & 100.784 & 104.776 & 96.417 & 111.364 & & & 74.828 \\
\hline $1 / 27 / 0818: 00$ & 126.036 & 122.855 & & 100.644 & 104.578 & 96.216 & 111.2 & & & 74.707 \\
\hline 1/27/08 18:30 & 125.948 & 122.802 & & 100.565 & 104.476 & 96.115 & 111.108 & & 80.785 & 74.611 \\
\hline 1/27/08 19:00 & 125.894 & 122.759 & & 100.508 & 104.413 & 96.054 & 111.052 & & & 74.545 \\
\hline $1 / 27 / 0819: 30$ & 125.861 & 122.728 & & 100.468 & 104.365 & 96.004 & 111.016 & & & 74.498 \\
\hline 1/27/08 20:00 & 125.837 & 122.694 & & 100.43 & 104.321 & 95.959 & 110.99 & & 80.736 & 74.467 \\
\hline $1 / 27 / 0820: 30$ & 125.821 & 122.669 & & 100.393 & 104.293 & 95.929 & 110.971 & & & 74.442 \\
\hline $1 / 27 / 0821: 00$ & 125.806 & 122.644 & & 100.372 & 104.258 & 95.896 & 110.958 & & & 74.432 \\
\hline 1/27/08 21:30 & 125.795 & 122.635 & & 100.346 & 104.239 & 95.877 & 110.949 & & 80.69 & 74.417 \\
\hline 1/27/08 22:00 & 125.788 & 122.628 & & 100.327 & 104.213 & 95.853 & 110.939 & & & 74.407 \\
\hline 1/27/08 22:30 & 125.778 & 122.607 & & 100.327 & 104.209 & 95.846 & 110.93 & & & 74.394 \\
\hline 1/27/08 23:00 & 125.769 & 122.58 & & 100.313 & 104.199 & 95.837 & 110.926 & & 80.658 & 74.382 \\
\hline 1/27/08 23:30 & 125.769 & 122.562 & & 100.286 & 104.178 & 95.82 & 110.922 & & & 74.372 \\
\hline $1 / 28 / 080: 00$ & 125.766 & 122.542 & & 100.267 & 104.155 & 95.792 & 110.92 & & & 74.37 \\
\hline 1/28/08 0:30 & 125.764 & 122.521 & & 100.244 & 104.135 & 95.771 & 110.917 & & 80.611 & 74.367 \\
\hline 1/28/08 1:00 & 125.665 & 122.494 & & 100.222 & 104.116 & 95.752 & 110.915 & & & 74.372 \\
\hline 1/28/08 $1: 30$ & 125.634 & 122.462 & & 100.198 & 104.093 & 95.731 & 110.904 & & & 74.372 \\
\hline 1/28/08 2:00 & 125.617 & 122.451 & & 100.174 & 104.066 & 95.702 & 110.89 & & 80.567 & 74.274 \\
\hline 1/28/08 2:30 & 125.544 & 122.442 & & 100.15 & 104.036 & 95.674 & 110.875 & & & 74.179 \\
\hline 1/28/08 3:00 & 125.542 & 122.412 & & 100.137 & 104.028 & 95.662 & 110.858 & & & 74.097 \\
\hline 1/28/08 3:30 & 125.52 & 122.399 & & 100.124 & 104.015 & 95.651 & 110.847 & & 80.521 & 74.028 \\
\hline 1/28/08 4:00 & 125.523 & 122.372 & & 100.107 & 103.992 & 95.627 & 110.838 & & & 73.977 \\
\hline 1/28/08 4:30 & 125.523 & 122.356 & & 100.081 & 103.971 & 95.611 & 110.823 & & & 73.933 \\
\hline 1/28/08 5:00 & 125.523 & 122.342 & & 100.062 & 103.95 & 95.589 & 110.806 & & 80.472 & 73.896 \\
\hline 1/28/08 5:30 & 125.442 & 122.322 & & 100.046 & 103.935 & 95.568 & 110.791 & & & 73.865 \\
\hline $1 / 28 / 086: 00$ & 125.386 & 122.308 & & 100.031 & 103.921 & 95.556 & 110.778 & & & 73.838 \\
\hline 1/28/08 6:30 & 125.388 & 122.294 & & 100.008 & 103.899 & 95.537 & 110.765 & & 80.428 & 73.818 \\
\hline 1/28/08 7:00 & 125.388 & 124.031 & & 99.993 & 103.883 & 95.521 & 110.75 & & & 73.797 \\
\hline $1 / 28 / 087: 30$ & 125.353 & 124.356 & & 99.979 & 103.872 & 95.507 & 110.736 & & & 73.776 \\
\hline 1/28/08 8:00 & 126.818 & 124.503 & & 100.727 & 104.264 & 96.025 & 111.264 & & 80.396 & 73.787 \\
\hline 1/28/08 8:30 & 127.116 & 124.58 & & 100.944 & 104.58 & 96.355 & 111.568 & & & 73.948 \\
\hline 1/28/08 9:00 & 127.246 & 124.626 & & 101.049 & 104.733 & 96.506 & 111.703 & & & 74.072 \\
\hline 1/28/08 9:30 & 127.324 & 124.662 & & 101.113 & 104.805 & 96.582 & 111.781 & & 80.435 & 74.163 \\
\hline $1 / 28 / 0810: 00$ & 127.371 & 124.666 & & 101.158 & 104.856 & 96.631 & 111.828 & & & 74.223 \\
\hline $1 / 28 / 0810: 30$ & 127.409 & 124.682 & & 101.176 & 104.89 & 96.667 & 111.859 & & & 74.268 \\
\hline 1/28/08 11:00 & 127.447 & 124.689 & & 101.18 & 104.892 & 96.669 & 111.88 & & 80.43 & 74.301 \\
\hline 1/28/08 11:30 & 127.468 & 124.68 & & 101.201 & 104.91 & 96.685 & 111.891 & & & 74.308 \\
\hline $1 / 28 / 0812: 00$ & 127.485 & 124.682 & & 101.195 & 104.915 & 96.695 & 111.903 & & & 74.31 \\
\hline $1 / 28 / 0812: 30$ & 127.501 & 124.666 & & 101.192 & 104.907 & 96.685 & 111.903 & & 80.43 & 74.305 \\
\hline 1/28/08 13:00 & 127.516 & 122.966 & & 101.185 & 104.902 & 96.678 & 111.901 & & & 74.293 \\
\hline $1 / 28 / 0813: 30$ & 127.525 & 122.603 & & 101.175 & 104.889 & 96.669 & 111.897 & & & 74.276 \\
\hline
\end{tabular}


TABLE S1.1 (Cont.)

\begin{tabular}{|c|c|c|c|c|c|c|c|c|c|c|}
\hline \multirow[b]{2}{*}{ Date and Time } & \multicolumn{10}{|c|}{ Depth from Top of Casing (ft) } \\
\hline & MW1D & MW2D & MW3D & MW4D & MW7 & MW9 & Oentrich & MW14D & MW15D & MW16D \\
\hline 1/28/08 14:00 & 126.052 & 122.44 & & 100.41 & 104.489 & 96.139 & 111.354 & & 80.426 & 74.237 \\
\hline $1 / 28 / 0814: 30$ & 125.499 & 122.331 & & 100.179 & 104.154 & 95.785 & 111.037 & & & 74.033 \\
\hline 1/28/08 15:00 & 125.135 & 122.292 & & 100.05 & 103.99 & 95.62 & 110.885 & & & 73.882 \\
\hline $1 / 28 / 0815: 30$ & 124.84 & 122.247 & & 99.974 & 103.887 & 95.521 & 110.793 & & 80.345 & 73.772 \\
\hline $1 / 28 / 0816: 00$ & 124.719 & 122.231 & & 99.943 & 103.851 & 95.481 & 110.736 & & & 73.729 \\
\hline $1 / 28 / 0816: 30$ & 124.681 & 122.224 & & 99.916 & 103.811 & 95.448 & 110.701 & & & 73.687 \\
\hline $1 / 28 / 08$ 17:00 & 124.658 & 122.222 & & 99.902 & 103.796 & 95.427 & 110.674 & & 80.289 & 73.667 \\
\hline $1 / 28 / 0817: 30$ & 124.648 & 122.24 & & 99.909 & 103.794 & 95.427 & 110.656 & & & 73.658 \\
\hline $1 / 28 / 0818: 00$ & 124.651 & 122.285 & & 99.912 & 103.796 & 95.429 & 110.644 & & & 73.656 \\
\hline $1 / 28 / 0818: 30$ & 124.662 & 122.31 & & 99.955 & 103.818 & 95.45 & 110.629 & & 80.267 & 73.671 \\
\hline 1/28/08 19:00 & 124.712 & 122.297 & & 100.003 & 103.864 & 95.497 & 110.62 & & & 73.716 \\
\hline 1/28/08 19:30 & 124.748 & 122.295 & & 100.002 & 103.895 & 95.53 & 110.616 & & & 73.741 \\
\hline $1 / 28 / 0820: 00$ & 124.738 & 122.279 & & 99.994 & 103.878 & 95.514 & 110.614 & & 80.264 & 73.722 \\
\hline $1 / 28 / 0820: 30$ & 124.733 & 122.27 & & 99.982 & 103.876 & 95.516 & 110.614 & & & 73.718 \\
\hline 1/28/08 21:00 & 124.717 & 122.258 & & 99.971 & 103.861 & 95.493 & 110.614 & & & 73.7 \\
\hline $1 / 28 / 0821: 30$ & 124.703 & 122.258 & & 99.96 & 103.851 & 95.486 & 110.614 & & 80.23 & 73.689 \\
\hline $1 / 28 / 0822: 00$ & 124.693 & 122.256 & & 99.96 & 103.845 & 95.481 & 110.613 & & & 73.679 \\
\hline 1/28/08 22:30 & 124.696 & 122.251 & & 99.96 & 103.843 & 95.481 & 110.611 & & & 73.681 \\
\hline 1/28/08 23:00 & 124.693 & 122.242 & & 99.959 & 103.843 & 95.476 & 110.613 & & 80.208 & 73.679 \\
\hline 1/28/08 23:30 & 124.691 & 122.224 & & 99.946 & 103.836 & 95.471 & 110.611 & & & 73.671 \\
\hline $1 / 29 / 080: 00$ & 124.681 & 122.22 & & 99.936 & 103.83 & 95.464 & 110.611 & & & 73.663 \\
\hline 1/29/08 0:30 & 124.665 & 122.211 & & 99.924 & 103.811 & 95.446 & 110.614 & & 80.179 & 73.646 \\
\hline 1/29/08 1:00 & 124.658 & 122.204 & & 99.921 & 103.803 & 95.436 & 110.614 & & & 73.638 \\
\hline 1/29/08 1:30 & 124.648 & 122.199 & & 99.914 & 103.798 & 95.431 & 110.614 & & & 73.627 \\
\hline $1 / 29 / 082: 00$ & 124.644 & 122.22 & & 99.903 & 103.792 & 95.427 & 110.615 & & 80.149 & 73.623 \\
\hline 1/29/08 2:30 & 124.634 & 122.24 & & 99.91 & 103.784 & 95.417 & 110.614 & & & 73.613 \\
\hline 1/29/08 3:00 & 124.662 & 122.27 & & 99.943 & 103.82 & 95.457 & 110.609 & & & 73.652 \\
\hline 1/29/08 3:30 & 124.674 & 122.306 & & 99.952 & 103.834 & 95.471 & 110.611 & & 80.144 & 73.656 \\
\hline 1/29/08 4:00 & 124.698 & 122.319 & & 99.989 & 103.862 & 95.495 & 110.607 & & & 73.681 \\
\hline $1 / 29 / 084: 30$ & 124.733 & 122.333 & & 100.017 & 103.898 & 95.535 & 110.601 & & & 73.714 \\
\hline $1 / 29 / 085: 00$ & 124.755 & 122.385 & & 100.04 & 103.917 & 95.556 & 110.601 & & 80.159 & 73.733 \\
\hline 1/29/08 5:30 & 124.776 & 122.419 & & 100.057 & 103.929 & 95.568 & 110.605 & & & 73.745 \\
\hline $1 / 29 / 086: 00$ & 124.809 & 122.453 & & 100.11 & 103.981 & 95.62 & 110.596 & & & 73.789 \\
\hline 1/29/08 6:30 & 124.844 & 122.492 & & 100.148 & 104.017 & 95.653 & 110.598 & & 80.184 & 73.82 \\
\hline 1/29/08 7:00 & 124.892 & 124.167 & & 100.188 & 104.053 & 95.693 & 110.598 & & & 73.859 \\
\hline 1/29/08 7:30 & 124.925 & 124.605 & & 100.222 & 104.091 & 95.726 & 110.594 & & & 73.89 \\
\hline 1/29/08 8:00 & 126.416 & 124.789 & & 100.911 & 104.396 & 96.136 & 110.983 & & 80.23 & 73.929 \\
\hline 1/29/08 8:30 & 127.069 & 124.911 & & 101.168 & 104.805 & 96.57 & 111.366 & & & 74.136 \\
\hline 1/29/08 9:00 & 127.281 & 124.979 & & 101.309 & 104.987 & 96.756 & 111.514 & & & 74.308 \\
\hline 1/29/08 9:30 & 127.395 & 125.038 & & 101.421 & 105.111 & 96.879 & 111.598 & & 80.325 & 74.423 \\
\hline 1/29/08 10:00 & 127.487 & 125.109 & & 101.476 & 105.188 & 96.959 & 111.657 & & & 74.51 \\
\hline $1 / 29 / 08 \quad 10: 30$ & 127.539 & 125.152 & & 101.542 & 105.244 & 97.018 & 111.694 & & & 74.568 \\
\hline 1/29/08 11:00 & 127.589 & 125.197 & & 101.609 & 105.315 & 97.079 & 111.719 & & 80.413 & 74.624 \\
\hline 1/29/08 11:30 & 127.648 & 125.215 & & 101.654 & 105.365 & 97.131 & 111.745 & & & 74.675 \\
\hline $1 / 29 / 0812: 00$ & 127.698 & 125.242 & & 101.69 & 105.408 & 97.178 & 111.779 & & & 74.721 \\
\hline $1 / 29 / 0812: 30$ & 127.724 & 123.777 & & 101.705 & 105.426 & 97.197 & 111.801 & & 80.506 & 74.74 \\
\hline 1/29/08 13:00 & 127.745 & 123.301 & & 101.733 & 105.454 & 97.22 & 111.813 & & & 74.766 \\
\hline 1/29/08 13:30 & 126.506 & 123.138 & & 101.128 & 105.271 & 96.954 & 111.529 & & & 74.783 \\
\hline $1 / 29 / 0814: 00$ & 125.764 & 123.054 & & 100.896 & 104.86 & 96.504 & 111.101 & & 80.528 & 74.632 \\
\hline 1/29/08 14:30 & 125.589 & 123.027 & & 100.78 & 104.706 & 96.346 & 110.956 & & & 74.506 \\
\hline $1 / 29 / 0815: 00$ & 125.502 & 123.004 & & 100.73 & 104.632 & 96.273 & 110.872 & & & 74.429 \\
\hline 1/29/08 15:30 & 125.461 & 122.991 & & 100.715 & 104.599 & 96.24 & 110.819 & & 80.538 & 74.39 \\
\hline 1/29/08 16:00 & 125.442 & 122.986 & & 100.698 & 104.58 & 96.223 & 110.787 & & & 74.37 \\
\hline $1 / 29 / 0816: 30$ & 125.428 & 122.988 & & 100.692 & 104.571 & 96.214 & 110.774 & & & 74.353 \\
\hline 1/29/08 17:00 & 125.424 & 122.991 & & 100.696 & 104.569 & 96.209 & 110.765 & & 80.558 & 74.349 \\
\hline 1/29/08 17:30 & 125.428 & 122.988 & & 100.697 & 104.573 & 96.214 & 110.754 & & & 74.351 \\
\hline $1 / 29 / 0818: 00$ & 125.426 & 122.986 & & 100.703 & 104.578 & 96.219 & 110.744 & & & 74.353 \\
\hline 1/29/08 18:30 & 125.428 & 122.986 & & 100.703 & 104.575 & 96.216 & 110.737 & & 80.575 & 74.351 \\
\hline 1/29/08 19:00 & 125.426 & 122.982 & & 100.697 & 104.571 & 96.214 & 110.729 & & & 74.349 \\
\hline 1/29/08 19:30 & 125.426 & 122.977 & & 100.696 & 104.571 & 96.214 & 110.725 & & & 74.349 \\
\hline $1 / 29 / 0820: 00$ & 125.419 & 122.975 & & 100.694 & 104.567 & 96.209 & 110.72 & & 80.589 & 74.343 \\
\hline $1 / 29 / 0820: 30$ & 125.416 & 122.968 & & 100.689 & 104.565 & 96.207 & 110.716 & & & 74.343 \\
\hline 1/29/08 21:00 & 125.414 & 122.968 & & 100.687 & 104.565 & 96.204 & 110.71 & & & 74.343 \\
\hline $1 / 29 / 0821: 30$ & 125.412 & 122.963 & & 100.686 & 104.555 & 96.197 & 110.709 & & 80.597 & 74.336 \\
\hline 1/29/08 22:00 & 125.405 & 122.97 & & 100.681 & 104.557 & 96.197 & 110.705 & & & 74.332 \\
\hline $1 / 29 / 0822: 30$ & 125.405 & 122.975 & & 100.682 & 104.555 & 96.197 & 110.701 & & & 74.334 \\
\hline
\end{tabular}


TABLE S1.1 (Cont.)

\begin{tabular}{|c|c|c|c|c|c|c|c|c|c|c|}
\hline \multirow[b]{2}{*}{ Date and Time } & \multicolumn{10}{|c|}{ Depth from Top of Casing (ft) } \\
\hline & MW1D & MW2D & MW3D & MW4D & MW7 & MW9 & Oentrich & MW14D & MW15D & MW16D \\
\hline 1/29/08 23:00 & 125.409 & 122.968 & & 100.684 & 104.563 & 96.207 & 110.695 & & 80.611 & 74.343 \\
\hline $1 / 29 / 0823: 30$ & 125.412 & 122.972 & & 100.689 & 104.569 & 96.209 & 110.692 & & & 74.345 \\
\hline 1/30/08 0:00 & 125.409 & 122.963 & & 100.684 & 104.555 & 96.2 & 110.69 & & & 74.341 \\
\hline 1/30/08 0:30 & 125.414 & 122.959 & & 100.677 & 104.561 & 96.204 & 110.688 & & 80.621 & 74.347 \\
\hline 1/30/08 1:00 & 125.402 & 122.945 & & 100.674 & 104.554 & 96.195 & 110.684 & & & 74.336 \\
\hline 1/30/08 1:30 & 125.4 & 122.945 & & 100.665 & 104.552 & 96.193 & 110.68 & & & 74.334 \\
\hline 1/30/08 2:00 & 125.386 & 122.948 & & 100.668 & 104.533 & 96.176 & 110.678 & & 80.624 & 74.318 \\
\hline $1 / 30 / 082: 30$ & 125.39 & 122.945 & & 100.658 & 104.54 & 96.183 & 110.673 & & & 74.328 \\
\hline 1/30/08 3:00 & 125.388 & 122.929 & & 100.665 & 104.54 & 96.181 & 110.669 & & & 74.328 \\
\hline 1/30/08 3:30 & 125.386 & 122.92 & & 100.656 & 104.538 & 96.179 & 110.667 & & 80.631 & 74.324 \\
\hline 1/30/08 4:00 & 125.374 & 122.918 & & 100.641 & 104.521 & 96.162 & 110.665 & & & 74.314 \\
\hline 1/30/08 4:30 & 125.362 & 122.914 & & 100.639 & 104.515 & 96.155 & 110.663 & & & 74.305 \\
\hline 1/30/08 5:00 & 125.362 & 122.914 & & 100.63 & 104.514 & 96.153 & 110.658 & & 80.628 & 74.303 \\
\hline 1/30/08 5:30 & 125.353 & 122.911 & & 100.625 & 104.504 & 96.143 & 110.656 & & & 74.295 \\
\hline 1/30/08 6:00 & 125.348 & 122.909 & & 100.625 & 104.502 & 96.143 & 110.652 & & & 74.295 \\
\hline 1/30/08 6:30 & 125.355 & 122.902 & & 100.632 & 104.504 & 96.148 & 110.648 & & 80.633 & 74.301 \\
\hline 1/30/08 7:00 & 125.355 & 124.694 & & 100.62 & 104.5 & 96.143 & 110.645 & & & 74.299 \\
\hline 1/30/08 7:30 & 125.343 & 125.018 & & 100.618 & 104.498 & 96.139 & 110.643 & & & 74.293 \\
\hline 1/30/08 8:00 & 127.088 & 125.177 & & 101.4 & 104.934 & 96.707 & 111.21 & & 80.648 & 74.336 \\
\hline 1/30/08 8:30 & 127.518 & 125.249 & & 101.619 & 105.247 & 97.03 & 111.503 & & & 74.537 \\
\hline 1/30/08 9:00 & 127.688 & 125.301 & & 101.728 & 105.41 & 97.197 & 111.636 & & & 74.692 \\
\hline 1/30/08 9:30 & 127.771 & 125.329 & & 101.797 & 105.481 & 97.265 & 111.717 & & 80.726 & 74.771 \\
\hline 1/30/08 10:00 & 127.823 & 125.351 & & 101.838 & 105.534 & 97.319 & 111.767 & & & 74.828 \\
\hline $1 / 30 / 0810: 30$ & 127.856 & 125.349 & & 101.864 & 105.561 & 97.348 & 111.801 & & & 74.862 \\
\hline 1/30/08 11:00 & 127.88 & 125.34 & & 101.874 & 105.584 & 97.371 & 111.829 & & 80.77 & 74.891 \\
\hline 1/30/08 11:30 & 127.887 & 125.342 & & 101.871 & 105.584 & 97.376 & 111.852 & & & 74.893 \\
\hline $1 / 30 / 0812: 00$ & 127.882 & 125.342 & & 101.862 & 105.572 & 97.36 & 111.869 & & & 74.886 \\
\hline $1 / 30 / 0812: 30$ & 127.88 & 125.329 & & 101.862 & 105.574 & 97.36 & 111.882 & & 80.792 & 74.888 \\
\hline 1/30/08 13:00 & 127.88 & 123.444 & & 101.857 & 105.566 & 97.352 & 111.891 & & & 74.882 \\
\hline $1 / 30 / 08$ 13:30 & 127.877 & 123.206 & & 101.402 & 105.563 & 97.345 & 111.899 & & & 74.882 \\
\hline $1 / 30 / 0814: 00$ & 126.166 & 123.075 & & 100.985 & 104.986 & 96.638 & 111.221 & & 80.787 & 74.764 \\
\hline 1/30/08 14:30 & 125.816 & 122.993 & & 100.816 & 104.765 & 96.407 & 111.009 & & & 74.605 \\
\hline $1 / 30 / 08$ 15:00 & 125.617 & 122.943 & & 100.72 & 104.641 & 96.282 & 110.902 & & & 74.485 \\
\hline 1/30/08 15:30 & 125.492 & 122.916 & & 100.655 & 104.559 & 96.202 & 110.838 & & 80.746 & 74.401 \\
\hline 1/30/08 16:00 & 125.414 & 122.891 & & 100.625 & 104.512 & 96.15 & 110.795 & & & 74.347 \\
\hline $1 / 30 / 0816: 30$ & 125.367 & 122.88 & & 100.603 & 104.491 & 96.134 & 110.765 & & & 74.324 \\
\hline 1/30/08 17:00 & 125.338 & 122.882 & & 100.582 & 104.466 & 96.11 & 110.74 & & 80.724 & 74.297 \\
\hline 1/30/08 17:30 & 125.32 & 122.886 & & 100.577 & 104.458 & 96.098 & 110.725 & & & 74.285 \\
\hline $1 / 30 / 0818: 00$ & 125.312 & 122.891 & & 100.586 & 104.462 & 96.101 & 110.71 & & & 74.285 \\
\hline $1 / 30 / 0818: 30$ & 125.315 & 122.889 & & 100.591 & 104.472 & 96.11 & 110.697 & & 80.721 & 74.293 \\
\hline 1/30/08 19:00 & 125.32 & 122.9 & & 100.596 & 104.474 & 96.113 & 110.689 & & & 74.295 \\
\hline $1 / 30 / 0819: 30$ & 125.324 & 122.902 & & 100.598 & 104.472 & 96.11 & 110.682 & & & 74.291 \\
\hline 1/30/08 20:00 & 125.329 & 122.914 & & 100.608 & 104.481 & 96.122 & 110.674 & & 80.726 & 74.301 \\
\hline 1/30/08 20:30 & 125.336 & 122.923 & & 100.616 & 104.491 & 96.131 & 110.669 & & & 74.308 \\
\hline $1 / 30 / 0821: 00$ & 125.343 & 122.927 & & 100.627 & 104.5 & 96.143 & 110.661 & & & 74.316 \\
\hline 1/30/08 21:30 & 125.353 & 122.932 & & 100.634 & 104.514 & 96.148 & 110.658 & & 80.731 & 74.322 \\
\hline 1/30/08 22:00 & 125.36 & 122.941 & & 100.637 & 104.519 & 96.155 & 110.652 & & & 74.33 \\
\hline 1/30/08 22:30 & 125.364 & 122.948 & & 100.646 & 104.521 & 96.162 & 110.648 & & & 74.332 \\
\hline 1/30/08 23:00 & 125.372 & 122.943 & & 100.658 & 104.531 & 96.172 & 110.643 & & 80.734 & 74.341 \\
\hline 1/30/08 23:30 & 125.379 & 122.948 & & 100.66 & 104.538 & 96.181 & 110.639 & & & 74.347 \\
\hline $1 / 31 / 080: 00$ & 125.383 & 122.952 & & 100.653 & 104.533 & 96.176 & 110.639 & & & 74.341 \\
\hline 1/31/08 0:30 & 125.379 & 122.959 & & 100.658 & 104.538 & 96.179 & 110.633 & & 80.734 & 74.345 \\
\hline 1/31/08 1:00 & 125.386 & 122.959 & & 100.666 & 104.542 & 96.183 & 110.632 & & & 74.347 \\
\hline $1 / 31 / 081: 30$ & 125.39 & 122.952 & & 100.668 & 104.552 & 96.193 & 110.628 & & & 74.359 \\
\hline 1/31/08 2:00 & 125.393 & 122.961 & & 100.667 & 104.552 & 96.193 & 110.624 & & 80.738 & 74.357 \\
\hline 1/31/08 2:30 & 125.393 & 122.961 & & 100.665 & 104.546 & 96.183 & 110.622 & & & 74.353 \\
\hline 1/31/08 3:00 & 125.39 & 122.966 & & 100.675 & 104.55 & 96.19 & 110.618 & & & 74.355 \\
\hline 1/31/08 3:30 & 125.4 & 122.966 & & 100.674 & 104.554 & 96.195 & 110.615 & & 80.738 & 74.361 \\
\hline $1 / 31 / 084: 00$ & 125.4 & 122.975 & & 100.679 & 104.559 & 96.195 & 110.611 & & & 74.359 \\
\hline $1 / 31 / 084: 30$ & 125.405 & 122.979 & & 100.682 & 104.557 & 96.202 & 110.609 & & & 74.365 \\
\hline 1/31/08 5:00 & 125.409 & 122.988 & & 100.686 & 104.569 & 96.212 & 110.605 & & 80.746 & 74.376 \\
\hline $1 / 31 / 085: 30$ & 125.414 & 122.993 & & 100.694 & 104.571 & 96.209 & 110.601 & & & 74.376 \\
\hline $1 / 31 / 086: 00$ & 125.421 & 123.009 & & 100.706 & 104.582 & 96.226 & 110.598 & & & 74.39 \\
\hline 1/31/08 6:30 & 125.428 & 123.022 & & 100.713 & 104.584 & 96.226 & 110.596 & & 80.758 & 74.388 \\
\hline $1 / 31 / 087: 00$ & 125.442 & 124.873 & & 100.73 & 104.605 & 96.249 & 110.59 & & & 74.409 \\
\hline 1/31/08 7:30 & 125.457 & 125.154 & & 100.794 & 104.616 & 96.261 & 110.588 & & & 74.417 \\
\hline
\end{tabular}


TABLE S1.1 (Cont.)

\begin{tabular}{|c|c|c|c|c|c|c|c|c|c|c|}
\hline \multirow[b]{2}{*}{ Date and Time } & \multicolumn{10}{|c|}{ Depth from Top of Casing (ft) } \\
\hline & MW1D & MW2D & MW3D & MW4D & MW7 & MW9 & Oentrich & MW14D & MW15D & MW16D \\
\hline 1/31/08 8:00 & 127.201 & 125.285 & & 101.501 & 105.077 & 96.841 & 111.178 & & 80.79 & 74.483 \\
\hline $1 / 31 / 088: 30$ & 127.62 & 125.369 & & 101.701 & 105.359 & 97.126 & 111.445 & & & 74.68 \\
\hline 1/31/08 9:00 & 127.778 & 125.419 & & 101.8 & 105.488 & 97.258 & 111.572 & & & 74.812 \\
\hline 1/31/08 9:30 & 127.865 & 125.456 & & 101.869 & 105.574 & 97.348 & 111.655 & & 80.866 & 74.899 \\
\hline 1/31/08 10:00 & 127.917 & 123.977 & & 101.914 & 105.624 & 97.395 & 111.709 & & & 74.955 \\
\hline $1 / 31 / 0810: 30$ & 127.958 & 123.524 & & 101.947 & 105.658 & 97.43 & 111.743 & & & 74.994 \\
\hline 1/31/08 11:00 & 126.705 & 123.376 & & 101.349 & 105.481 & 97.164 & 111.458 & & 80.914 & 75.021 \\
\hline $1 / 31 / 0811: 30$ & 126.012 & 123.292 & & 101.13 & 105.086 & 96.73 & 111.036 & & & 74.886 \\
\hline 1/31/08 12:00 & 125.832 & 123.238 & & 101.025 & 104.948 & 96.593 & 110.908 & & & 74.775 \\
\hline $1 / 31 / 0812: 30$ & 125.74 & 123.183 & & 100.956 & 104.864 & 96.506 & 110.838 & & 80.902 & 74.688 \\
\hline 1/31/08 13:00 & 125.684 & 123.14 & & 100.908 & 104.811 & 96.452 & 110.796 & & & 74.628 \\
\hline 1/31/08 13:30 & 125.632 & 123.097 & & 100.861 & 104.761 & 96.405 & 110.763 & & & 74.578 \\
\hline $1 / 31 / 0814: 00$ & 125.591 & 123.07 & & 100.822 & 104.719 & 96.362 & 110.744 & & 80.885 & 74.537 \\
\hline 1/31/08 14:30 & 125.554 & 123.045 & & 100.791 & 104.675 & 96.32 & 110.731 & & & 74.496 \\
\hline 1/31/08 15:00 & 125.532 & 123.034 & & 100.756 & 104.653 & 96.294 & 110.718 & & & 74.469 \\
\hline 1/31/08 15:30 & 125.506 & 123.031 & & 100.737 & 104.628 & 96.27 & 110.709 & & 80.861 & 74.446 \\
\hline 1/31/08 16:00 & 125.48 & 123.022 & & 100.734 & 104.614 & 96.256 & 110.699 & & & 74.434 \\
\hline 1/31/08 16:30 & 125.471 & 123.011 & & 100.727 & 104.616 & 96.256 & 110.69 & & & 74.434 \\
\hline 1/31/08 17:00 & 125.461 & 123.011 & & 100.717 & 104.605 & 96.245 & 110.684 & & 80.853 & 74.419 \\
\hline 1/31/08 17:30 & 125.452 & 123.004 & & 100.712 & 104.594 & 96.233 & 110.68 & & & 74.413 \\
\hline $1 / 31 / 0818: 00$ & 125.445 & 123.009 & & 100.713 & 104.595 & 96.235 & 110.673 & & & 74.413 \\
\hline 1/31/08 18:30 & 125.442 & 123.007 & & 100.715 & 104.59 & 96.228 & 110.669 & & 80.848 & 74.407 \\
\hline 1/31/08 19:00 & 125.442 & 123.004 & & 100.716 & 104.595 & 96.233 & 110.665 & & & 74.413 \\
\hline 1/31/08 19:30 & 125.442 & 122.995 & & 100.71 & 104.594 & 96.233 & 110.661 & & & 74.411 \\
\hline 1/31/08 20:00 & 125.44 & 122.995 & & 100.708 & 104.59 & 96.23 & 110.656 & & 80.846 & 74.409 \\
\hline 1/31/08 20:30 & 125.438 & 122.993 & & 100.703 & 104.582 & 96.221 & 110.654 & & & 74.401 \\
\hline $1 / 31 / 0821: 00$ & 125.431 & 122.993 & & 100.699 & 104.582 & 96.221 & 110.648 & & & 74.401 \\
\hline $1 / 31 / 0821: 30$ & 125.428 & 122.984 & & 100.701 & 104.578 & 96.216 & 110.646 & & 80.839 & 74.398 \\
\hline 1/31/08 22:00 & 125.428 & 122.979 & & 100.698 & 104.58 & 96.216 & 110.643 & & & 74.398 \\
\hline 1/31/08 22:30 & 125.426 & 122.975 & & 100.689 & 104.573 & 96.209 & 110.641 & & & 74.39 \\
\hline 1/31/08 23:00 & 125.416 & 122.957 & & 100.686 & 104.569 & 96.207 & 110.637 & & 80.831 & 74.386 \\
\hline 1/31/08 23:30 & 125.412 & 122.959 & & 100.672 & 104.567 & 96.204 & 110.633 & & & 74.382 \\
\hline $2 / 1 / 080: 00$ & 125.405 & 122.952 & & 100.675 & 104.544 & 96.183 & 110.633 & & & 74.363 \\
\hline 2/1/08 0:30 & 125.398 & 122.943 & & 100.665 & 104.552 & 96.19 & 110.629 & & 80.824 & 74.367 \\
\hline 2/1/08 1:00 & 125.395 & 122.929 & & 100.66 & 104.542 & 96.179 & 110.628 & & & 74.359 \\
\hline 2/1/08 1:30 & 125.386 & 122.914 & & 100.648 & 104.531 & 96.169 & 110.626 & & & 74.351 \\
\hline 2/1/08 2:00 & 125.376 & 122.9 & & 100.636 & 104.517 & 96.155 & 110.624 & & 80.807 & 74.341 \\
\hline 2/1/08 2:30 & 125.364 & 122.891 & & 100.61 & 104.5 & 96.141 & 110.622 & & & 74.326 \\
\hline 2/1/08 3:00 & 125.346 & 122.884 & & 100.606 & 104.489 & 96.124 & 110.618 & & & 74.312 \\
\hline 2/1/08 3:30 & 125.336 & 122.875 & & 100.599 & 104.481 & 96.115 & 110.616 & & 80.785 & 74.301 \\
\hline 2/1/08 4:00 & 125.329 & 122.866 & & 100.587 & 104.476 & 96.113 & 110.614 & & & 74.301 \\
\hline $2 / 1 / 084: 30$ & 125.315 & 122.859 & & 100.586 & 104.466 & 96.103 & 110.613 & & & 74.291 \\
\hline 2/1/08 5:00 & 125.312 & 122.846 & & 100.572 & 104.458 & 96.094 & 110.611 & & 80.77 & 74.283 \\
\hline 2/1/08 5:30 & 125.305 & 122.841 & & 100.567 & 104.453 & 96.089 & 110.609 & & & 74.276 \\
\hline $2 / 1 / 086: 00$ & 125.296 & 122.841 & & 100.55 & 104.436 & 96.072 & 110.609 & & & 74.264 \\
\hline $2 / 1 / 086: 30$ & 125.279 & 122.83 & & 100.546 & 104.434 & 96.065 & 110.607 & & 80.753 & 74.254 \\
\hline 2/1/08 7:00 & 125.277 & 124.619 & & 100.538 & 104.432 & 96.068 & 110.603 & & & 74.26 \\
\hline 2/1/08 7:30 & 125.27 & 124.934 & & 100.538 & 104.42 & 96.056 & 110.603 & & & 74.245 \\
\hline $2 / 1 / 088: 00$ & 126.884 & 125.066 & & 101.326 & 104.864 & 96.629 & 111.174 & & 80.756 & 74.287 \\
\hline 2/1/08 8:30 & 127.338 & 125.138 & & 101.525 & 105.168 & 96.945 & 111.469 & & & 74.492 \\
\hline 2/1/08 9:00 & 127.549 & 125.188 & & 101.623 & 105.298 & 97.077 & 111.604 & & & 74.613 \\
\hline 2/1/08 9:30 & 127.65 & 125.217 & & 101.683 & 105.374 & 97.152 & 111.685 & & 80.804 & 74.694 \\
\hline 2/1/08 10:00 & 127.712 & 125.247 & & 101.721 & 105.424 & 97.204 & 111.737 & & & 74.75 \\
\hline $2 / 1 / 08 \quad 10: 30$ & 127.747 & 125.274 & & 101.756 & 105.45 & 97.232 & 111.773 & & & 74.779 \\
\hline 2/1/08 11:00 & 127.776 & 123.465 & & 101.773 & 105.485 & 97.263 & 111.797 & & 80.844 & 74.812 \\
\hline 2/1/08 11:30 & 127.797 & 123.192 & & 101.512 & 105.506 & 97.286 & 111.818 & & & 74.835 \\
\hline $2 / 1 / 0812: 00$ & 126.282 & 123.05 & & 100.985 & 105.011 & 96.655 & 111.195 & & & 74.769 \\
\hline 2/1/08 12:30 & 125.944 & 122.948 & & 100.797 & 104.753 & 96.393 & 110.96 & & 80.829 & 74.599 \\
\hline $2 / 1 / 08$ 13:00 & 125.705 & 122.873 & & 100.684 & 104.616 & 96.254 & 110.849 & & & 74.471 \\
\hline $2 / 1 / 08$ 13:30 & 125.542 & 122.807 & & 100.6 & 104.521 & 96.162 & 110.787 & & & 74.37 \\
\hline 2/1/08 14:00 & 125.421 & 122.757 & & 100.536 & 104.451 & 96.087 & 110.748 & & 80.77 & 74.297 \\
\hline $2 / 1 / 08$ 14:30 & 125.331 & 122.734 & & 100.475 & 104.386 & 96.023 & 110.718 & & & 74.231 \\
\hline 2/1/08 15:00 & 125.253 & 122.712 & & 100.442 & 104.333 & 95.969 & 110.693 & & & 74.179 \\
\hline 2/1/08 15:30 & 125.201 & 122.696 & & 100.419 & 104.312 & 95.95 & 110.674 & & 80.724 & 74.157 \\
\hline $2 / 1 / 08$ 16:00 & 125.171 & 122.707 & & 100.403 & 104.293 & 95.929 & 110.661 & & & 74.134 \\
\hline 2/1/08 16:30 & 125.149 & 122.698 & & 100.401 & 104.278 & 95.912 & 110.652 & & & 74.115 \\
\hline
\end{tabular}


TABLE S1.1 (Cont.)

\begin{tabular}{|c|c|c|c|c|c|c|c|c|c|c|}
\hline \multirow[b]{2}{*}{ Date and Time } & \multicolumn{10}{|c|}{ Depth from Top of Casing (ft) } \\
\hline & MW1D & MW2D & MW3D & MW4D & MW7 & MW9 & Oentrich & MW14D & MW15D & MW16D \\
\hline 2/1/08 17:00 & 125.135 & 122.698 & & 100.405 & 104.287 & 95.922 & 110.643 & & 80.699 & 74.121 \\
\hline $2 / 1 / 08 \quad 17: 30$ & 125.135 & 122.705 & & 100.403 & 104.283 & 95.919 & 110.637 & & & 74.119 \\
\hline 2/1/08 18:00 & 125.138 & 122.707 & & 100.405 & 104.285 & 95.919 & 110.633 & & & 74.119 \\
\hline $2 / 1 / 08$ 18:30 & 125.14 & 122.719 & & 100.406 & 104.291 & 95.924 & 110.629 & & 80.682 & 74.119 \\
\hline $2 / 1 / 08$ 19:00 & 125.142 & 122.719 & & 100.417 & 104.295 & 95.931 & 110.624 & & & 74.126 \\
\hline $2 / 1 / 08$ 19:30 & 125.149 & 122.719 & & 100.419 & 104.308 & 95.943 & 110.618 & & & 74.134 \\
\hline $2 / 1 / 0820: 00$ & 125.152 & 122.709 & & 100.424 & 104.304 & 95.938 & 110.618 & & 80.677 & 74.132 \\
\hline $2 / 1 / 0820: 30$ & 125.154 & 122.707 & & 100.42 & 104.306 & 95.94 & 110.616 & & & 74.132 \\
\hline 2/1/08 21:00 & 125.152 & 122.709 & & 100.413 & 104.3 & 95.933 & 110.616 & & & 74.126 \\
\hline $2 / 1 / 0821: 30$ & 125.145 & 122.705 & & 100.413 & 104.296 & 95.931 & 110.613 & & 80.66 & 74.119 \\
\hline 2/1/08 22:00 & 125.147 & 122.703 & & 100.411 & 104.298 & 95.936 & 110.611 & & & 74.126 \\
\hline 2/1/08 22:30 & 125.145 & 122.703 & & 100.405 & 104.297 & 95.931 & 110.611 & & & 74.117 \\
\hline 2/1/08 23:00 & 125.14 & 122.696 & & 100.403 & 104.295 & 95.929 & 110.611 & & 80.646 & 74.117 \\
\hline 2/1/08 23:30 & 125.138 & 122.698 & & 100.4 & 104.289 & 95.926 & 110.609 & & & 74.111 \\
\hline 2/2/08 0:00 & 125.133 & 122.696 & & 100.408 & 104.287 & 95.922 & 110.607 & & & 74.109 \\
\hline $2 / 2 / 080: 30$ & 125.138 & 122.703 & & 100.406 & 104.293 & 95.926 & 110.605 & & 80.636 & 74.111 \\
\hline $2 / 2 / 081: 00$ & 125.138 & 122.696 & & 100.408 & 104.287 & 95.922 & 110.603 & & & 74.107 \\
\hline 2/2/08 1:30 & 125.14 & 122.694 & & 100.41 & 104.295 & 95.931 & 110.603 & & & 74.115 \\
\hline 2/2/08 2:00 & 125.14 & 122.684 & & 100.41 & 104.291 & 95.926 & 110.603 & & 80.624 & 74.109 \\
\hline 2/2/08 2:30 & 125.14 & 122.689 & & 100.401 & 104.289 & 95.924 & 110.601 & & & 74.107 \\
\hline 2/2/08 3:00 & 125.128 & 122.696 & & 100.405 & 104.281 & 95.915 & 110.601 & & & 74.097 \\
\hline 2/2/08 3:30 & 125.133 & 122.703 & & 100.401 & 104.285 & 95.919 & 110.599 & & 80.604 & 74.101 \\
\hline $2 / 2 / 084: 00$ & 125.133 & 122.709 & & 100.413 & 104.293 & 95.926 & 110.597 & & & 74.105 \\
\hline 2/2/08 4:30 & 125.14 & 122.707 & & 100.417 & 104.297 & 95.931 & 110.596 & & & 74.107 \\
\hline $2 / 2 / 085: 00$ & 125.14 & 122.721 & & 100.42 & 104.304 & 95.936 & 110.596 & & 80.602 & 74.113 \\
\hline $2 / 2 / 085: 30$ & 125.149 & 122.73 & & 100.425 & 104.3 & 95.936 & 110.594 & & & 74.115 \\
\hline 2/2/08 6:00 & 125.154 & 122.759 & & 100.441 & 104.319 & 95.955 & 110.592 & & & 74.13 \\
\hline 2/2/08 6:30 & 125.171 & 122.762 & & 100.451 & 104.325 & 95.962 & 110.59 & & 80.599 & 74.138 \\
\hline 2/2/08 7:00 & 125.178 & 122.775 & & 100.475 & 104.356 & 95.99 & 110.586 & & & 74.159 \\
\hline $2 / 2 / 087: 30$ & 125.197 & 124.476 & & 100.48 & 104.356 & 95.992 & 110.586 & & & 74.163 \\
\hline 2/2/08 8:00 & 125.206 & 124.861 & & 100.493 & 104.369 & 96.004 & 110.584 & & 80.616 & 74.173 \\
\hline 2/2/08 8:30 & 126.745 & 125.034 & & 101.23 & 104.727 & 96.485 & 111.069 & & & 74.198 \\
\hline 2/2/08 9:00 & 127.322 & 125.143 & & 101.476 & 105.1 & 96.879 & 111.42 & & & 74.405 \\
\hline 2/2/08 9:30 & 127.539 & 125.213 & & 101.616 & 105.271 & 97.053 & 111.568 & & 80.687 & 74.566 \\
\hline $2 / 2 / 08 \quad 10: 00$ & 127.657 & 125.279 & & 101.705 & 105.386 & 97.164 & 111.653 & & & 74.678 \\
\hline $2 / 2 / 08 \quad 10: 30$ & 127.728 & 125.324 & & 101.769 & 105.456 & 97.235 & 111.705 & & & 74.75 \\
\hline $2 / 2 / 0811: 00$ & 127.79 & 124.204 & & 101.826 & 105.523 & 97.301 & 111.741 & & 80.763 & 74.816 \\
\hline $2 / 2 / 0811: 30$ & 127.832 & 123.433 & & 101.867 & 105.563 & 97.345 & 111.767 & & & 74.859 \\
\hline $2 / 2 / 08 \quad 12: 00$ & 127.22 & 123.238 & & 101.308 & 105.572 & 97.378 & 111.766 & & & 74.893 \\
\hline 2/2/08 12:30 & 126.095 & 123.138 & & 101.006 & 104.991 & 96.636 & 111.054 & & 80.792 & 74.769 \\
\hline 2/2/08 13:00 & 125.811 & 123.081 & & 100.868 & 104.807 & 96.447 & 110.883 & & & 74.62 \\
\hline $2 / 2 / 0813: 30$ & 125.653 & 123.043 & & 100.801 & 104.712 & 96.351 & 110.795 & & & 74.529 \\
\hline $2 / 2 / 08$ 14:00 & 125.532 & 123.016 & & 100.759 & 104.658 & 96.296 & 110.74 & & 80.778 & 74.469 \\
\hline 2/2/08 14:30 & 125.49 & 122.984 & & 100.729 & 104.624 & 96.266 & 110.706 & & & 74.432 \\
\hline $2 / 2 / 08 \quad 15: 00$ & 125.459 & 122.966 & & 100.703 & 104.594 & 96.235 & 110.682 & & & 74.401 \\
\hline 2/2/08 15:30 & 125.431 & 122.952 & & 100.675 & 104.567 & 96.207 & 110.667 & & 80.768 & 74.372 \\
\hline $2 / 2 / 08$ 16:00 & 125.407 & 122.927 & & 100.66 & 104.548 & 96.188 & 110.652 & & & 74.353 \\
\hline 2/2/08 16:30 & 125.393 & 122.927 & & 100.651 & 104.536 & 96.176 & 110.643 & & & 74.341 \\
\hline $2 / 2 / 08 \quad 17: 00$ & 125.374 & 122.927 & & 100.627 & 104.51 & 96.15 & 110.637 & & 80.756 & 74.314 \\
\hline $2 / 2 / 0817: 30$ & 125.362 & 122.925 & & 100.634 & 104.514 & 96.15 & 110.628 & & & 74.314 \\
\hline $2 / 2 / 08 \quad 18: 00$ & 125.364 & 122.934 & & 100.631 & 104.515 & 96.155 & 110.622 & & & 74.318 \\
\hline 2/2/08 18:30 & 125.362 & 122.938 & & 100.634 & 104.512 & 96.15 & 110.616 & & 80.758 & 74.314 \\
\hline 2/2/08 19:00 & 125.364 & 122.952 & & 100.646 & 104.521 & 96.157 & 110.609 & & & 74.32 \\
\hline $2 / 2 / 08 \quad 19: 30$ & 125.372 & 122.961 & & 100.649 & 104.527 & 96.164 & 110.605 & & & 74.326 \\
\hline 2/2/08 20:00 & 125.381 & 122.963 & & 100.667 & 104.538 & 96.179 & 110.599 & & 80.77 & 74.339 \\
\hline 2/2/08 20:30 & 125.398 & 122.957 & & 100.672 & 104.554 & 96.19 & 110.596 & & & 74.351 \\
\hline $2 / 2 / 0821: 00$ & 125.4 & 122.952 & & 100.672 & 104.552 & 96.19 & 110.594 & & & 74.351 \\
\hline 2/2/08 21:30 & 125.398 & 122.948 & & 100.666 & 104.546 & 96.186 & 110.592 & & 80.775 & 74.345 \\
\hline 2/2/08 22:00 & 125.395 & 122.945 & & 100.658 & 104.546 & 96.183 & 110.59 & & & 74.343 \\
\hline 2/2/08 22:30 & 125.386 & 122.936 & & 100.655 & 104.536 & 96.176 & 110.586 & & & 74.336 \\
\hline 2/2/08 23:00 & 125.383 & 122.941 & & 100.651 & 104.536 & 96.176 & 110.584 & & 80.778 & 74.334 \\
\hline 2/2/08 23:30 & 125.379 & 122.934 & & 100.646 & 104.529 & 96.169 & 110.581 & & & 74.33 \\
\hline $2 / 3 / 080: 00$ & 125.379 & 122.918 & & 100.649 & 104.533 & 96.169 & 110.575 & & & 74.334 \\
\hline $2 / 3 / 080: 30$ & 125.376 & 122.904 & & 100.637 & 104.525 & 96.162 & 110.575 & & 80.775 & 74.328 \\
\hline 2/3/08 1:00 & 125.364 & 122.893 & & 100.625 & 104.51 & 96.15 & 110.573 & & & 74.314 \\
\hline 2/3/08 1:30 & 125.35 & 122.88 & & 100.608 & 104.5 & 96.134 & 110.571 & & & 74.301 \\
\hline
\end{tabular}


TABLE S1.1 (Cont.)

Depth from Top of Casing (ft)

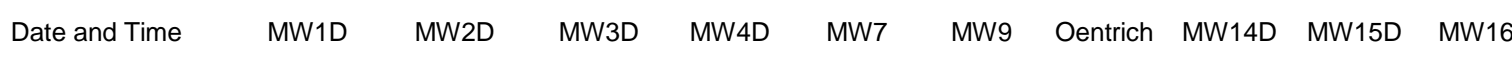

\begin{tabular}{|c|c|c|c|c|c|c|c|c|}
\hline 2/3/08 2:00 & 125.334 & 122.88 & 100.596 & 104.485 & 96.122 & 110.569 & 80.758 & 74.289 \\
\hline $2 / 3 / 082: 30$ & 125.322 & 122.88 & 100.594 & 104.474 & 96.11 & 110.567 & & 74.279 \\
\hline 2/3/08 3:00 & 125.32 & 122.873 & 100.596 & 104.472 & 96.11 & 110.564 & & 74.279 \\
\hline 2/3/08 3:30 & 125.32 & 122.857 & 100.584 & 104.476 & 96.11 & 110.56 & 80.751 & 74.279 \\
\hline 2/3/08 4:00 & 125.31 & 122.855 & 100.58 & 104.462 & 96.103 & 110.558 & & 74.268 \\
\hline $2 / 3 / 084: 30$ & 125.303 & 122.843 & 100.572 & 104.451 & 96.091 & 110.558 & & 74.262 \\
\hline 2/3/08 5:00 & 125.298 & 122.825 & 100.56 & 104.447 & 96.087 & 110.556 & 80.734 & 74.258 \\
\hline $2 / 3 / 085: 30$ & 125.284 & 122.8 & 100.549 & 104.436 & 96.07 & 110.554 & & 74.241 \\
\hline 2/3/08 6:00 & 125.272 & 122.796 & 100.527 & 104.415 & 96.054 & 110.556 & & 74.227 \\
\hline 2/3/08 6:30 & 125.251 & 122.793 & 100.508 & 104.394 & 96.03 & 110.555 & 80.709 & 74.204 \\
\hline 2/3/08 7:00 & 125.239 & 122.786 & 100.506 & 104.388 & 96.023 & 110.552 & & 74.2 \\
\hline $2 / 3 / 087: 30$ & 125.237 & 122.786 & 100.498 & 104.386 & 96.025 & 110.551 & & 74.198 \\
\hline 2/3/08 8:00 & 125.227 & 122.786 & 100.496 & 104.382 & 96.018 & 110.549 & 80.702 & 74.192 \\
\hline 2/3/08 8:30 & 125.22 & 124.616 & 100.504 & 104.375 & 96.011 & 110.547 & & 74.188 \\
\hline 2/3/08 9:00 & 125.23 & 124.886 & 100.572 & 104.382 & 96.021 & 110.543 & & 74.196 \\
\hline 2/3/08 9:30 & 126.972 & 125.007 & 101.238 & 104.824 & 96.579 & 111.12 & 80.709 & 74.239 \\
\hline $2 / 3 / 08$ 10:00 & 127.36 & 125.066 & 101.426 & 105.091 & 96.853 & 111.377 & & 74.425 \\
\hline $2 / 3 / 08$ 10:30 & 127.501 & 125.097 & 101.512 & 105.213 & 96.975 & 111.501 & & 74.547 \\
\hline $2 / 3 / 0811: 00$ & 127.57 & 125.124 & 101.559 & 105.273 & 97.039 & 111.576 & 80.753 & 74.616 \\
\hline 2/3/08 11:30 & 127.608 & 125.131 & 101.588 & 105.304 & 97.07 & 111.625 & & 74.651 \\
\hline $2 / 3 / 08$ 12:00 & 127.631 & 123.306 & 101.612 & 105.336 & 97.103 & 111.657 & & 74.686 \\
\hline $2 / 3 / 0812: 30$ & 127.648 & 123.036 & 101.347 & 105.344 & 97.114 & 111.681 & 80.778 & 74.7 \\
\hline 2/3/08 13:00 & 125.946 & 122.893 & 100.827 & 104.858 & 96.504 & 111.106 & & 74.618 \\
\hline 2/3/08 13:30 & 125.539 & 122.802 & 100.646 & 104.601 & 96.24 & 110.885 & & 74.442 \\
\hline $2 / 3 / 0814: 00$ & 125.355 & 122.741 & 100.53 & 104.466 & 96.103 & 110.78 & 80.721 & 74.312 \\
\hline $2 / 3 / 08$ 14:30 & 125.256 & 122.707 & 100.461 & 104.378 & 96.018 & 110.721 & & 74.223 \\
\hline $2 / 3 / 08$ 15:00 & 125.187 & 122.689 & 100.42 & 104.321 & 95.957 & 110.678 & & 74.161 \\
\hline $2 / 3 / 08$ 15:30 & 125.149 & 122.673 & 100.389 & 104.291 & 95.922 & 110.65 & 80.677 & 74.124 \\
\hline $2 / 3 / 0816: 00$ & 125.123 & 122.641 & 100.386 & 104.276 & 95.91 & 110.629 & & 74.103 \\
\hline 2/3/08 16:30 & 125.119 & 122.623 & 100.36 & 104.262 & 95.898 & 110.616 & & 74.095 \\
\hline $2 / 3 / 08$ 17:00 & 125.088 & 122.623 & 100.329 & 104.228 & 95.86 & 110.609 & 80.646 & 74.057 \\
\hline $2 / 3 / 08$ 17:30 & 125.064 & 122.63 & 100.325 & 104.213 & 95.844 & 110.599 & & 74.041 \\
\hline $2 / 3 / 08$ 18:00 & 125.06 & 122.637 & 100.327 & 104.209 & 95.842 & 110.592 & & 74.039 \\
\hline $2 / 3 / 0818: 30$ & 125.06 & 122.644 & 100.337 & 104.218 & 95.853 & 110.584 & 80.626 & 74.043 \\
\hline 2/3/08 19:00 & 125.067 & 122.641 & 100.345 & 104.226 & 95.86 & 110.581 & & 74.047 \\
\hline 2/3/08 19:30 & 125.076 & 122.617 & 100.353 & 104.234 & 95.867 & 110.575 & & 74.053 \\
\hline $2 / 3 / 0820: 00$ & 125.083 & 122.619 & 100.339 & 104.232 & 95.867 & 110.575 & 80.621 & 74.053 \\
\hline 2/3/08 20:30 & 125.06 & 122.626 & 100.322 & 104.207 & 95.842 & 110.577 & & 74.024 \\
\hline 2/3/08 21:00 & 125.057 & 122.612 & 100.332 & 104.213 & 95.844 & 110.575 & & 74.03 \\
\hline $2 / 3 / 0821: 30$ & 125.062 & 122.619 & 100.325 & 104.217 & 95.849 & 110.573 & 80.604 & 74.035 \\
\hline $2 / 3 / 0822: 00$ & 125.05 & 122.603 & 100.319 & 104.203 & 95.839 & 110.571 & & 74.018 \\
\hline 2/3/08 22:30 & 125.05 & 122.56 & 100.312 & 104.211 & 95.844 & 110.569 & & 74.026 \\
\hline 2/3/08 23:00 & 125.041 & 122.546 & 100.289 & 104.198 & 95.83 & 110.569 & 80.582 & 74.012 \\
\hline 2/3/08 23:30 & 125.005 & 122.517 & 100.272 & 104.146 & 95.778 & 110.571 & & 73.964 \\
\hline $2 / 4 / 080: 00$ & 124.986 & 122.483 & 100.224 & 104.142 & 95.776 & 110.567 & & 73.96 \\
\hline $2 / 4 / 080: 30$ & 124.956 & 122.469 & 100.2 & 104.11 & 95.743 & 110.569 & 80.538 & 73.929 \\
\hline $2 / 4 / 081: 00$ & 124.93 & 122.453 & 100.179 & 104.076 & 95.712 & 110.569 & & 73.9 \\
\hline $2 / 4 / 081: 30$ & 124.908 & 122.424 & 100.175 & 104.062 & 95.7 & 110.566 & & 73.884 \\
\hline $2 / 4 / 082: 00$ & 124.896 & 122.406 & 100.152 & 104.045 & 95.684 & 110.564 & 80.501 & 73.871 \\
\hline $2 / 4 / 082: 30$ & 124.873 & 122.385 & 100.126 & 104.017 & 95.653 & 110.564 & & 73.842 \\
\hline 2/4/08 3:00 & 124.852 & 122.392 & 100.1 & 104 & 95.636 & 110.564 & & 73.832 \\
\hline 2/4/08 3:30 & 124.823 & 122.381 & 100.088 & 103.977 & 95.606 & 110.56 & 80.45 & 73.799 \\
\hline $2 / 4 / 084: 00$ & 124.821 & 122.381 & 100.106 & 103.986 & 95.618 & 110.558 & & 73.807 \\
\hline $2 / 4 / 084: 30$ & 124.826 & 122.365 & 100.086 & 103.971 & 95.603 & 110.558 & & 73.795 \\
\hline $2 / 4 / 085: 00$ & 124.816 & 122.358 & 100.082 & 103.975 & 95.608 & 110.554 & 80.423 & 73.797 \\
\hline 2/4/08 5:30 & 124.814 & 122.351 & 100.07 & 103.963 & 95.596 & 110.554 & & 73.782 \\
\hline $2 / 4 / 086: 00$ & 124.807 & 122.351 & 100.07 & 103.958 & 95.587 & 110.554 & & 73.774 \\
\hline $2 / 4 / 086: 30$ & 124.804 & 122.358 & 100.062 & 103.95 & 95.582 & 110.553 & 80.391 & 73.772 \\
\hline 2/4/08 7:00 & 124.802 & 124.14 & 100.07 & 103.954 & 95.585 & 110.551 & & 73.772 \\
\hline 2/4/08 7:30 & 124.8 & 124.471 & 100.072 & 103.96 & 95.587 & 110.551 & & 73.776 \\
\hline 2/4/08 8:00 & 126.293 & 124.623 & 100.863 & 104.39 & 96.15 & 111.11 & 80.394 & 73.818 \\
\hline $2 / 4 / 088: 30$ & 126.587 & 124.719 & 101.073 & 104.715 & 96.487 & 111.413 & & 74.024 \\
\hline 2/4/08 9:00 & 126.719 & 124.798 & 101.204 & 104.866 & 96.641 & 111.552 & & 74.171 \\
\hline 2/4/08 9:30 & 126.792 & 123.007 & 101.282 & 104.969 & 96.742 & 111.629 & 80.455 & 74.272 \\
\hline $2 / 4 / 08$ 10:00 & 126.835 & 122.784 & 100.961 & 105.045 & 96.82 & 111.679 & & 74.349 \\
\hline $2 / 4 / 08$ 10:30 & 125.301 & 122.68 & 100.561 & 104.573 & 96.212 & 111.048 & & 74.32 \\
\hline
\end{tabular}


TABLE S1.1 (Cont.)

\begin{tabular}{|c|c|c|c|c|c|c|c|c|c|c|}
\hline \multirow[b]{2}{*}{ Date and Time } & \multicolumn{10}{|c|}{ Depth from Top of Casing (ft) } \\
\hline & MW1D & MW2D & MW3D & MW4D & MW7 & MW9 & Oentrich & MW14D & MW15D & MW16D \\
\hline 2/4/08 11:00 & 125.109 & 122.632 & & 100.43 & 104.363 & 95.992 & 110.843 & & 80.465 & 74.179 \\
\hline $2 / 4 / 08$ 11:30 & 125.024 & 122.619 & & 100.353 & 104.262 & 95.893 & 110.751 & & & 74.082 \\
\hline $2 / 4 / 08 \quad 12: 00$ & 124.972 & 122.592 & & 100.32 & 104.222 & 95.856 & 110.695 & & & 74.039 \\
\hline $2 / 4 / 0812: 30$ & 124.939 & 122.562 & & 100.307 & 104.209 & 95.842 & 110.66 & & 80.457 & 74.012 \\
\hline $2 / 4 / 08$ 13:00 & 124.925 & 122.564 & & 100.279 & 104.184 & 95.816 & 110.637 & & & 73.985 \\
\hline 2/4/08 13:30 & 124.934 & 122.558 & & 100.262 & 104.152 & 95.785 & 110.626 & & & 73.954 \\
\hline 2/4/08 14:00 & 124.96 & 122.548 & & 100.267 & 104.156 & 95.787 & 110.613 & & 80.445 & 73.952 \\
\hline $2 / 4 / 08 \quad 14: 30$ & 124.977 & 122.548 & & 100.263 & 104.152 & 95.783 & 110.605 & & & 73.95 \\
\hline $2 / 4 / 08 \quad 15: 00$ & 124.989 & 122.562 & & 100.253 & 104.144 & 95.78 & 110.601 & & & 73.942 \\
\hline $2 / 4 / 08$ 15:30 & 124.986 & 122.578 & & 100.263 & 104.144 & 95.778 & 110.596 & & 80.43 & 73.94 \\
\hline $2 / 4 / 08$ 16:00 & 124.996 & 122.592 & & 100.284 & 104.159 & 95.794 & 110.592 & & & 73.954 \\
\hline $2 / 4 / 0816: 30$ & 125.012 & 122.61 & & 100.299 & 104.18 & 95.811 & 110.584 & & & 73.968 \\
\hline $2 / 4 / 08 \quad 17: 00$ & 125.024 & 122.623 & & 100.317 & 104.19 & 95.825 & 110.581 & & 80.443 & 73.979 \\
\hline $2 / 4 / 08$ 17:30 & 125.043 & 122.655 & & 100.33 & 104.211 & 95.844 & 110.579 & & & 73.995 \\
\hline $2 / 4 / 08$ 18:00 & 125.055 & 122.669 & & 100.353 & 104.222 & 95.858 & 110.579 & & & 74.006 \\
\hline $2 / 4 / 08$ 18:30 & 125.083 & 122.685 & & 100.375 & 104.255 & 95.891 & 110.573 & & 80.467 & 74.037 \\
\hline 2/4/08 19:00 & 125.104 & 122.709 & & 100.395 & 104.27 & 95.905 & 110.573 & & & 74.049 \\
\hline 2/4/08 19:30 & 125.119 & 122.721 & & 100.413 & 104.285 & 95.917 & 110.573 & & & 74.062 \\
\hline $2 / 4 / 0820: 00$ & 125.14 & 122.732 & & 100.432 & 104.31 & 95.945 & 110.573 & & 80.492 & 74.084 \\
\hline $2 / 4 / 0820: 30$ & 125.159 & 122.734 & & 100.446 & 104.321 & 95.957 & 110.575 & & & 74.097 \\
\hline $2 / 4 / 0821: 00$ & 125.171 & 122.75 & & 100.451 & 104.333 & 95.966 & 110.575 & & & 74.107 \\
\hline 2/4/08 21:30 & 125.171 & 122.759 & & 100.463 & 104.333 & 95.966 & 110.577 & & 80.509 & 74.103 \\
\hline $2 / 4 / 0822: 00$ & 125.187 & 122.771 & & 100.472 & 104.354 & 95.988 & 110.573 & & & 74.126 \\
\hline 2/4/08 22:30 & 125.199 & 122.775 & & 100.484 & 104.36 & 95.995 & 110.575 & & & 74.132 \\
\hline 2/4/08 23:00 & 125.206 & 122.78 & & 100.492 & 104.373 & 96.007 & 110.573 & & 80.531 & 74.142 \\
\hline 2/4/08 23:30 & 125.213 & 122.768 & & 100.496 & 104.375 & 96.009 & 110.575 & & & 74.146 \\
\hline 2/5/08 0:00 & 125.216 & 122.773 & & 100.489 & 104.382 & 96.014 & 110.571 & & & 74.148 \\
\hline $2 / 5 / 080: 30$ & 125.213 & 122.775 & & 100.487 & 104.371 & 96.009 & 110.573 & & 80.54 & 74.146 \\
\hline $2 / 5 / 081: 00$ & 125.211 & 122.771 & & 100.498 & 104.375 & 96.011 & 110.569 & & & 74.146 \\
\hline $2 / 5 / 081: 30$ & 125.223 & 122.791 & & 100.496 & 104.378 & 96.016 & 110.569 & & & 74.155 \\
\hline 2/5/08 2:00 & 125.213 & 122.791 & & 100.503 & 104.373 & 96.009 & 110.57 & & 80.545 & 74.148 \\
\hline $2 / 5 / 082: 30$ & 125.22 & 122.807 & & 100.506 & 104.388 & 96.021 & 110.562 & & & 74.157 \\
\hline 2/5/08 3:00 & 125.232 & 122.787 & & 100.513 & 104.396 & 96.035 & 110.562 & & & 74.171 \\
\hline 2/5/08 3:30 & 125.242 & 122.798 & & 100.52 & 104.417 & 96.051 & 110.558 & & 80.565 & 74.188 \\
\hline 2/5/08 4:00 & 125.232 & 122.796 & & 100.506 & 104.39 & 96.025 & 110.56 & & & 74.161 \\
\hline $2 / 5 / 084: 30$ & 125.232 & 122.793 & & 100.525 & 104.403 & 96.039 & 110.556 & & & 74.175 \\
\hline $2 / 5 / 085: 00$ & 125.239 & 122.748 & & 100.522 & 104.398 & 96.035 & 110.556 & & 80.565 & 74.171 \\
\hline $2 / 5 / 085: 30$ & 125.242 & 122.764 & & 100.498 & 104.396 & 96.035 & 110.556 & & & 74.171 \\
\hline 2/5/08 6:00 & 125.204 & 122.789 & & 100.499 & 104.348 & 95.985 & 110.56 & & & 74.126 \\
\hline $2 / 5 / 086: 30$ & 125.208 & 122.8 & & 100.498 & 104.371 & 96.011 & 110.556 & & 80.558 & 74.146 \\
\hline $2 / 5 / 087: 00$ & 125.225 & 124.573 & & 100.501 & 104.392 & 96.03 & 110.551 & & & 74.169 \\
\hline $2 / 5 / 087: 30$ & 125.227 & 124.9 & & 100.522 & 104.403 & 96.039 & 110.547 & & & 74.175 \\
\hline 2/5/08 8:00 & 126.88 & 125.056 & & 101.235 & 104.778 & 96.53 & 111.039 & & 80.592 & 74.204 \\
\hline $2 / 5 / 088: 30$ & 127.364 & 125.124 & & 101.456 & 105.096 & 96.857 & 111.352 & & & 74.39 \\
\hline 2/5/08 9:00 & 127.544 & 125.168 & & 101.564 & 105.256 & 97.02 & 111.49 & & & 74.547 \\
\hline 2/5/08 9:30 & 127.627 & 125.199 & & 101.626 & 105.327 & 97.093 & 111.557 & & 80.655 & 74.624 \\
\hline $2 / 5 / 08$ 10:00 & 127.669 & 125.208 & & 101.654 & 105.372 & 97.138 & 111.61 & & & 74.667 \\
\hline $2 / 5 / 08$ 10:30 & 127.7 & 125.242 & & 101.676 & 105.403 & 97.166 & 111.647 & & & 74.702 \\
\hline 2/5/08 11:00 & 127.712 & 125.247 & & 101.705 & 105.418 & 97.18 & 111.676 & & 80.709 & 74.717 \\
\hline 2/5/08 11:30 & 127.742 & 125.261 & & 101.712 & 105.448 & 97.213 & 111.693 & & & 74.752 \\
\hline 2/5/08 12:00 & 127.75 & 125.265 & & 101.724 & 105.458 & 97.225 & 111.711 & & & 74.766 \\
\hline 2/5/08 12:30 & 127.757 & 125.251 & & 101.736 & 105.468 & 97.23 & 111.723 & & 80.758 & 74.775 \\
\hline $2 / 5 / 08$ 13:00 & 127.766 & 125.238 & & 101.719 & 105.469 & 97.235 & 111.734 & & & 74.781 \\
\hline $2 / 5 / 08$ 13:30 & 127.752 & 125.233 & & 101.717 & 105.462 & 97.225 & 111.743 & & & 74.775 \\
\hline 2/5/08 14:00 & 127.74 & 125.238 & & 101.711 & 105.45 & 97.209 & 111.758 & & 80.78 & 74.764 \\
\hline $2 / 5 / 08 \quad 14: 30$ & 127.74 & 123.442 & & 101.719 & 105.452 & 97.218 & 111.758 & & & 74.773 \\
\hline $2 / 5 / 08 \quad 15: 00$ & 127.742 & 123.186 & & 101.442 & 105.456 & 97.22 & 111.762 & & & 74.781 \\
\hline 2/5/08 15:30 & 125.984 & 123.054 & & 100.978 & 104.994 & 96.634 & 111.17 & & 80.797 & 74.717 \\
\hline $2 / 5 / 08 \quad 16: 00$ & 125.65 & 123.009 & & 100.791 & 104.752 & 96.388 & 110.939 & & & 74.56 \\
\hline $2 / 5 / 08$ 16:30 & 125.504 & 122.943 & & 100.716 & 104.63 & 96.261 & 110.829 & & & 74.438 \\
\hline $2 / 5 / 08$ 17:00 & 125.445 & 122.918 & & 100.673 & 104.588 & 96.221 & 110.763 & & 80.77 & 74.392 \\
\hline $2 / 5 / 08$ 17:30 & 125.398 & 122.9 & & 100.627 & 104.523 & 96.162 & 110.721 & & & 74.33 \\
\hline 2/5/08 18:00 & 125.36 & 122.893 & & 100.61 & 104.502 & 96.141 & 110.693 & & & 74.303 \\
\hline $2 / 5 / 08$ 18:30 & 125.338 & 122.886 & & 100.598 & 104.485 & 96.122 & 110.669 & & 80.753 & 74.281 \\
\hline 2/5/08 19:00 & 125.329 & 122.884 & & 100.591 & 104.478 & 96.115 & 110.652 & & & 74.272 \\
\hline 2/5/08 19:30 & 125.327 & 122.864 & & 100.589 & 104.476 & 96.113 & 110.641 & & & 74.268 \\
\hline
\end{tabular}


TABLE S1.1 (Cont.)

\begin{tabular}{|c|c|c|c|c|c|c|c|c|c|c|}
\hline \multirow[b]{2}{*}{ Date and Time } & \multicolumn{10}{|c|}{ Depth from Top of Casing (ft) } \\
\hline & MW1D & MW2D & MW3D & MW4D & MW7 & MW9 & Oentrich & MW14D & MW15D & MW16D \\
\hline 2/5/08 20:00 & 125.32 & 122.861 & & 100.573 & 104.472 & 96.11 & 110.63 & & 80.751 & 74.258 \\
\hline 2/5/08 20:30 & 125.312 & 122.855 & & 100.572 & 104.455 & 96.094 & 110.624 & & & 74.248 \\
\hline 2/5/08 21:00 & 125.301 & 122.843 & & 100.556 & 104.449 & 96.084 & 110.616 & & & 74.239 \\
\hline $2 / 5 / 0821: 30$ & 125.296 & 122.834 & & 100.551 & 104.447 & 96.082 & 110.611 & & 80.741 & 74.235 \\
\hline 2/5/08 22:00 & 125.282 & 122.832 & & 100.546 & 104.434 & 96.07 & 110.605 & & & 74.223 \\
\hline 2/5/08 22:30 & 125.272 & 122.825 & & 100.541 & 104.428 & 96.063 & 110.603 & & & 74.212 \\
\hline 2/5/08 23:00 & 125.272 & 122.809 & & 100.537 & 104.422 & 96.061 & 110.601 & & 80.734 & 74.214 \\
\hline 2/5/08 23:30 & 125.265 & 122.802 & & 100.523 & 104.418 & 96.056 & 110.596 & & & 74.208 \\
\hline 2/6/08 0:00 & 125.251 & 122.796 & & 100.515 & 104.401 & 96.039 & 110.594 & & & 74.192 \\
\hline $2 / 6 / 080: 30$ & 125.244 & 122.793 & & 100.508 & 104.399 & 96.03 & 110.59 & & 80.724 & 74.188 \\
\hline $2 / 6 / 081: 00$ & 125.237 & 122.784 & & 100.501 & 104.39 & 96.028 & 110.586 & & & 74.181 \\
\hline 2/6/08 1:30 & 125.232 & 122.775 & & 100.494 & 104.384 & 96.021 & 110.582 & & & 74.175 \\
\hline $2 / 6 / 082: 00$ & 125.223 & 122.773 & & 100.491 & 104.378 & 96.014 & 110.581 & & 80.712 & 74.171 \\
\hline $2 / 6 / 082: 30$ & 125.216 & 122.773 & & 100.482 & 104.371 & 96.007 & 110.577 & & & 74.165 \\
\hline 2/6/08 3:00 & 125.211 & 122.764 & & 100.482 & 104.369 & 96.007 & 110.575 & & & 74.159 \\
\hline 2/6/08 3:30 & 125.208 & 122.757 & & 100.481 & 104.369 & 96.004 & 110.569 & & 80.699 & 74.159 \\
\hline $2 / 6 / 084: 00$ & 125.204 & 122.753 & & 100.47 & 104.363 & 95.997 & 110.567 & & & 74.155 \\
\hline 2/6/08 4:30 & 125.194 & 122.753 & & 100.465 & 104.354 & 95.99 & 110.564 & & & 74.144 \\
\hline 2/6/08 5:00 & 125.19 & 122.753 & & 100.463 & 104.35 & 95.988 & 110.564 & & 80.68 & 74.142 \\
\hline $2 / 6 / 085: 30$ & 125.187 & 122.755 & & 100.465 & 104.35 & 95.985 & 110.56 & & & 74.142 \\
\hline 2/6/08 6:00 & 125.19 & 122.759 & & 100.465 & 104.352 & 95.985 & 110.556 & & & 74.14 \\
\hline 2/6/08 6:30 & 125.19 & 122.762 & & 100.468 & 104.352 & 95.985 & 110.554 & & 80.677 & 74.142 \\
\hline $2 / 6 / 08$ 7:00 & 125.194 & 124.573 & & 100.473 & 104.357 & 95.995 & 110.552 & & & 74.146 \\
\hline 2/6/08 7:30 & 125.199 & 124.875 & & 100.477 & 104.36 & 95.997 & 110.551 & & & 74.146 \\
\hline 2/6/08 8:00 & 126.951 & 125.022 & & 101.28 & 104.828 & 96.593 & 111.14 & & 80.69 & 74.204 \\
\hline 2/6/08 8:30 & 127.374 & 125.111 & & 101.485 & 105.121 & 96.9 & 111.42 & & & 74.405 \\
\hline 2/6/08 9:00 & 127.534 & 123.526 & & 101.602 & 105.272 & 97.048 & 111.55 & & & 74.547 \\
\hline 2/6/08 9:30 & 127.631 & 123.19 & & 101.676 & 105.361 & 97.136 & 111.626 & & 80.763 & 74.64 \\
\hline 2/6/08 10:00 & 126.154 & 123.061 & & 100.994 & 105.068 & 96.723 & 111.189 & & & 74.678 \\
\hline $2 / 6 / 08 \quad 10: 30$ & 125.653 & 122.988 & & 100.809 & 104.767 & 96.403 & 110.879 & & & 74.549 \\
\hline $2 / 6 / 08$ 11:00 & 125.509 & 122.943 & & 100.722 & 104.643 & 96.278 & 110.759 & & 80.768 & 74.446 \\
\hline $2 / 6 / 08$ 11:30 & 125.433 & 122.914 & & 100.663 & 104.573 & 96.207 & 110.693 & & & 74.372 \\
\hline $2 / 6 / 08 \quad 12: 00$ & 125.383 & 122.88 & & 100.634 & 104.531 & 96.164 & 110.656 & & & 74.326 \\
\hline $2 / 6 / 08$ 12:30 & 125.355 & 122.866 & & 100.603 & 104.502 & 96.136 & 110.628 & & 80.756 & 74.295 \\
\hline $2 / 6 / 08$ 13:00 & 125.322 & 122.848 & & 100.579 & 104.47 & 96.103 & 110.611 & & & 74.26 \\
\hline $2 / 6 / 08$ 13:30 & 125.303 & 122.823 & & 100.567 & 104.457 & 96.091 & 110.597 & & & 74.248 \\
\hline $2 / 6 / 08 \quad 14: 00$ & 125.289 & 122.807 & & 100.542 & 104.437 & 96.07 & 110.59 & & 80.743 & 74.229 \\
\hline 2/6/08 14:30 & 125.268 & 122.8 & & 100.523 & 104.415 & 96.049 & 110.582 & & & 74.206 \\
\hline $2 / 6 / 08 \quad 15: 00$ & 125.249 & 122.791 & & 100.515 & 104.401 & 96.037 & 110.577 & & & 74.192 \\
\hline $2 / 6 / 08$ 15:30 & 125.242 & 122.793 & & 100.506 & 104.398 & 96.03 & 110.569 & & 80.729 & 74.188 \\
\hline 2/6/08 16:00 & 125.23 & 122.796 & & 100.508 & 104.388 & 96.021 & 110.566 & & & 74.175 \\
\hline $2 / 6 / 08 \quad 16: 30$ & 125.232 & 122.789 & & 100.508 & 104.392 & 96.023 & 110.56 & & & 74.181 \\
\hline $2 / 6 / 08 \quad 17: 00$ & 125.232 & 122.789 & & 100.503 & 104.392 & 96.025 & 110.556 & & 80.724 & 74.179 \\
\hline 2/6/08 17:30 & 125.23 & 122.796 & & 100.504 & 104.388 & 96.021 & 110.554 & & & 74.177 \\
\hline $2 / 6 / 08 \quad 18: 00$ & 125.225 & 122.796 & & 100.51 & 104.386 & 96.021 & 110.552 & & & 74.175 \\
\hline $2 / 6 / 08$ 18:30 & 125.234 & 122.805 & & 100.508 & 104.394 & 96.025 & 110.547 & & 80.714 & 74.183 \\
\hline $2 / 6 / 08 \quad 19: 00$ & 125.232 & 122.809 & & 100.513 & 104.394 & 96.025 & 110.545 & & & 74.179 \\
\hline $2 / 6 / 08$ 19:30 & 125.242 & 122.802 & & 100.518 & 104.405 & 96.037 & 110.541 & & & 74.188 \\
\hline $2 / 6 / 0820: 00$ & 125.246 & 122.812 & & 100.523 & 104.409 & 96.042 & 110.541 & & 80.716 & 74.194 \\
\hline $2 / 6 / 0820: 30$ & 125.244 & 122.807 & & 100.518 & 104.401 & 96.032 & 110.539 & & & 74.188 \\
\hline $2 / 6 / 0821: 00$ & 125.249 & 122.814 & & 100.523 & 104.409 & 96.042 & 110.536 & & & 74.196 \\
\hline $2 / 6 / 0821: 30$ & 125.249 & 122.805 & & 100.525 & 104.409 & 96.039 & 110.536 & & 80.724 & 74.194 \\
\hline 2/6/08 22:00 & 125.251 & 122.805 & & 100.522 & 104.411 & 96.042 & 110.532 & & & 74.198 \\
\hline 2/6/08 22:30 & 125.242 & 122.812 & & 100.52 & 104.398 & 96.03 & 110.534 & & & 74.186 \\
\hline 2/6/08 23:00 & 125.242 & 122.807 & & 100.523 & 104.401 & 96.032 & 110.534 & & 80.721 & 74.188 \\
\hline 2/6/08 23:30 & 125.246 & 122.809 & & 100.522 & 104.409 & 96.039 & 110.53 & & & 74.192 \\
\hline $2 / 7 / 080: 00$ & 125.244 & 122.796 & & 100.523 & 104.403 & 96.035 & 110.528 & & & 74.19 \\
\hline 2/7/08 0:30 & 125.244 & 122.793 & & 100.522 & 104.405 & 96.035 & 110.526 & & 80.729 & 74.19 \\
\hline 2/7/08 1:00 & 125.237 & 122.789 & & 100.506 & 104.394 & 96.025 & 110.526 & & & 74.181 \\
\hline 2/7/08 1:30 & 125.232 & 122.777 & & 100.504 & 104.392 & 96.023 & 110.524 & & & 74.179 \\
\hline 2/7/08 2:00 & 125.225 & 122.762 & & 100.503 & 104.388 & 96.018 & 110.523 & & 80.724 & 74.175 \\
\hline 2/7/08 2:30 & 125.22 & 122.746 & & 100.482 & 104.375 & 96.007 & 110.521 & & & 74.165 \\
\hline 2/7/08 3:00 & 125.201 & 122.737 & & 100.47 & 104.363 & 95.995 & 110.521 & & & 74.152 \\
\hline 2/7/08 3:30 & 125.187 & 122.728 & & 100.46 & 104.346 & 95.976 & 110.521 & & 80.707 & 74.136 \\
\hline 2/7/08 4:00 & 125.178 & 122.719 & & 100.453 & 104.337 & 95.966 & 110.519 & & & 74.13 \\
\hline 2/7/08 4:30 & 125.171 & 122.712 & & 100.439 & 104.329 & 95.962 & 110.517 & & & 74.124 \\
\hline
\end{tabular}


TABLE S1.1 (Cont.)

\begin{tabular}{|c|c|c|c|c|c|c|c|c|c|c|}
\hline \multirow[b]{2}{*}{ Date and Time } & \multicolumn{10}{|c|}{ Depth from Top of Casing (ft) } \\
\hline & MW1D & MW2D & MW3D & MW4D & MW7 & MW9 & Oentrich & MW14D & MW15D & MW16D \\
\hline 2/7/08 5:00 & 125.156 & 122.705 & & 100.43 & 104.318 & 95.95 & 110.515 & & 80.69 & 74.111 \\
\hline 2/7/08 5:30 & 125.149 & 122.703 & & 100.427 & 104.314 & 95.943 & 110.515 & & & 74.105 \\
\hline 2/7/08 6:00 & 125.145 & 122.703 & & 100.42 & 104.308 & 95.936 & 110.513 & & & 74.101 \\
\hline 2/7/08 6:30 & 125.14 & 122.7 & & 100.422 & 104.302 & 95.933 & 110.511 & & 80.672 & 74.095 \\
\hline $2 / 7 / 08$ 7:00 & 125.142 & 124.589 & & 100.417 & 104.304 & 95.933 & 110.509 & & & 74.097 \\
\hline 2/7/08 7:30 & 125.14 & 124.848 & & 100.713 & 104.304 & 95.933 & 110.507 & & & 74.093 \\
\hline 2/7/08 8:00 & 126.986 & 124.979 & & 101.211 & 104.803 & 96.556 & 111.114 & & 80.69 & 74.177 \\
\hline 2/7/08 8:30 & 127.326 & 125.059 & & 101.399 & 105.056 & 96.817 & 111.351 & & & 74.37 \\
\hline 2/7/08 9:00 & 127.466 & 125.109 & & 101.497 & 105.192 & 96.952 & 111.467 & & & 74.498 \\
\hline 2/7/08 9:30 & 127.556 & 125.152 & & 101.568 & 105.27 & 97.03 & 111.54 & & 80.756 & 74.58 \\
\hline $2 / 7 / 08$ 10:00 & 127.608 & 123.571 & & 101.607 & 105.323 & 97.086 & 111.585 & & & 74.638 \\
\hline 2/7/08 10:30 & 127.646 & 123.19 & & 101.64 & 105.367 & 97.124 & 111.617 & & & 74.678 \\
\hline 2/7/08 11:00 & 126.222 & 123.034 & & 101.008 & 105.106 & 96.761 & 111.232 & & 80.797 & 74.694 \\
\hline 2/7/08 11:30 & 125.653 & 122.95 & & 100.794 & 104.763 & 96.395 & 110.89 & & & 74.549 \\
\hline 2/7/08 12:00 & 125.478 & 122.893 & & 100.68 & 104.609 & 96.24 & 110.761 & & & 74.413 \\
\hline $2 / 7 / 0812: 30$ & 125.39 & 122.846 & & 100.623 & 104.535 & 96.167 & 110.686 & & 80.773 & 74.334 \\
\hline $2 / 7 / 0813: 00$ & 125.341 & 122.809 & & 100.573 & 104.476 & 96.108 & 110.644 & & & 74.281 \\
\hline 2/7/08 13:30 & 125.294 & 122.78 & & 100.523 & 104.434 & 96.065 & 110.616 & & & 74.235 \\
\hline 2/7/08 14:00 & 125.249 & 122.746 & & 100.499 & 104.396 & 96.028 & 110.596 & & 80.746 & 74.192 \\
\hline $2 / 7 / 08$ 14:30 & 125.225 & 122.73 & & 100.468 & 104.371 & 96.002 & 110.582 & & & 74.169 \\
\hline $2 / 7 / 08$ 15:00 & 125.187 & 122.714 & & 100.442 & 104.333 & 95.966 & 110.573 & & & 74.136 \\
\hline 2/7/08 15:30 & 125.164 & 122.696 & & 100.434 & 104.32 & 95.952 & 110.564 & & 80.734 & 74.115 \\
\hline $2 / 7 / 08$ 16:00 & 125.159 & 122.696 & & 100.41 & 104.308 & 95.943 & 110.558 & & & 74.109 \\
\hline 2/7/08 16:30 & 125.138 & 122.698 & & 100.399 & 104.291 & 95.924 & 110.552 & & & 74.088 \\
\hline $2 / 7 / 08 \quad 17: 00$ & 125.13 & 122.687 & & 100.406 & 104.291 & 95.922 & 110.547 & & 80.699 & 74.082 \\
\hline $2 / 7 / 08 \quad 17: 30$ & 125.135 & 122.689 & & 100.401 & 104.295 & 95.924 & 110.541 & & & 74.082 \\
\hline $2 / 7 / 08$ 18:00 & 125.128 & 122.685 & & 100.403 & 104.285 & 95.917 & 110.539 & & & 74.078 \\
\hline $2 / 7 / 08$ 18:30 & 125.123 & 122.673 & & 100.399 & 104.285 & 95.917 & 110.536 & & 80.69 & 74.074 \\
\hline 2/7/08 19:00 & 125.121 & 122.678 & & 100.387 & 104.28 & 95.91 & 110.532 & & & 74.072 \\
\hline 2/7/08 19:30 & 125.114 & 122.666 & & 100.389 & 104.27 & 95.9 & 110.532 & & & 74.068 \\
\hline 2/7/08 20:00 & 125.116 & 122.664 & & 100.386 & 104.274 & 95.905 & 110.528 & & 80.675 & 74.062 \\
\hline $2 / 7 / 0820: 30$ & 125.104 & 122.66 & & 100.375 & 104.264 & 95.893 & 110.526 & & & 74.062 \\
\hline 2/7/08 21:00 & 125.097 & 122.653 & & 100.372 & 104.26 & 95.889 & 110.526 & & & 74.053 \\
\hline 2/7/08 21:30 & 125.095 & 122.637 & & 100.37 & 104.257 & 95.889 & 110.526 & & 80.663 & 74.049 \\
\hline 2/7/08 22:00 & 125.093 & 122.619 & & 100.356 & 104.249 & 95.879 & 110.526 & & & 74.047 \\
\hline 2/7/08 22:30 & 125.078 & 122.607 & & 100.337 & 104.234 & 95.865 & 110.528 & & & 74.039 \\
\hline 2/7/08 23:00 & 125.062 & 122.601 & & 100.318 & 104.215 & 95.849 & 110.528 & & 80.643 & 74.028 \\
\hline 2/7/08 23:30 & 125.043 & 122.594 & & 100.318 & 104.202 & 95.832 & 110.526 & & & 74.016 \\
\hline 2/8/08 0:00 & 125.038 & 122.585 & & 100.3 & 104.198 & 95.827 & 110.524 & & & 74.01 \\
\hline 2/8/08 0:30 & 125.031 & 122.573 & & 100.308 & 104.19 & 95.82 & 110.522 & & 80.624 & 73.997 \\
\hline 2/8/08 1:00 & 125.029 & 122.567 & & 100.282 & 104.184 & 95.816 & 110.522 & & & 73.993 \\
\hline $2 / 8 / 081: 30$ & 125.005 & 122.546 & & 100.284 & 104.169 & 95.799 & 110.521 & & & 73.987 \\
\hline 2/8/08 2:00 & 125.005 & 122.535 & & 100.265 & 104.163 & 95.794 & 110.519 & & 80.604 & 73.983 \\
\hline 2/8/08 2:30 & 124.986 & 122.53 & & 100.251 & 104.144 & 95.776 & 110.521 & & & 73.979 \\
\hline 2/8/08 3:00 & 124.97 & 122.517 & & 100.246 & 104.133 & 95.764 & 110.519 & & & 73.975 \\
\hline 2/8/08 3:30 & 124.97 & 122.499 & & 100.236 & 104.131 & 95.761 & 110.517 & & 80.582 & 73.966 \\
\hline $2 / 8 / 084: 00$ & 124.958 & 122.485 & & 100.218 & 104.12 & 95.747 & 110.515 & & & 73.962 \\
\hline 2/8/08 4:30 & 124.939 & 122.478 & & 100.208 & 104.099 & 95.731 & 110.513 & & & 73.956 \\
\hline 2/8/08 5:00 & 124.925 & 122.476 & & 100.194 & 104.083 & 95.712 & 110.515 & & 80.553 & 73.95 \\
\hline 2/8/08 5:30 & 124.913 & 122.485 & & 100.194 & 104.08 & 95.705 & 110.511 & & & 73.944 \\
\hline 2/8/08 6:00 & 124.915 & 122.478 & & 100.189 & 104.08 & 95.71 & 110.509 & & & 73.935 \\
\hline 2/8/08 6:30 & 124.918 & 122.483 & & 100.203 & 104.091 & 95.719 & 110.505 & & 80.531 & 73.927 \\
\hline 2/8/08 7:00 & 124.92 & 124.075 & & 100.191 & 104.085 & 95.71 & 110.505 & & & 73.923 \\
\hline $2 / 8 / 087: 30$ & 124.915 & 124.558 & & 100.206 & 104.085 & 95.712 & 110.504 & & & 73.917 \\
\hline 2/8/08 8:00 & 126.326 & 124.746 & & 100.916 & 104.344 & 96.08 & 110.889 & & 80.528 & 73.913 \\
\hline 2/8/08 8:30 & 127.038 & 124.857 & & 101.18 & 104.799 & 96.57 & 111.315 & & & 74.064 \\
\hline 2/8/08 9:00 & 127.251 & 124.939 & & 101.335 & 104.99 & 96.766 & 111.476 & & & 74.19 \\
\hline 2/8/08 9:30 & 127.371 & 124.997 & & 101.43 & 105.106 & 96.881 & 111.566 & & 80.602 & 74.272 \\
\hline 2/8/08 10:00 & 127.456 & 125.034 & & 101.493 & 105.186 & 96.959 & 111.629 & & & 74.326 \\
\hline $2 / 8 / 08 \quad 10: 30$ & 127.516 & 125.066 & & 101.538 & 105.243 & 97.018 & 111.668 & & & 74.365 \\
\hline 2/8/08 11:00 & 127.551 & 125.095 & & 101.578 & 105.279 & 97.053 & 111.698 & & 80.665 & 74.394 \\
\hline 2/8/08 11:30 & 127.591 & 125.127 & & 101.605 & 105.312 & 97.091 & 111.722 & & & 74.417 \\
\hline 2/8/08 $12: 00$ & 127.617 & 125.138 & & 101.635 & 105.342 & 97.117 & 111.741 & & & 74.438 \\
\hline 2/8/08 12:30 & 127.648 & 125.004 & & 101.661 & 105.369 & 97.143 & 111.754 & & 80.724 & 74.456 \\
\hline 2/8/08 13:00 & 127.664 & 123.249 & & 101.669 & 105.384 & 97.159 & 111.767 & & & 74.471 \\
\hline 2/8/08 13:30 & 127.672 & 123.041 & & 101.135 & 105.391 & 97.166 & 111.781 & & & 74.487 \\
\hline
\end{tabular}


TABLE S1.1 (Cont.)

\begin{tabular}{|c|c|c|c|c|c|c|c|c|c|c|}
\hline \multirow[b]{2}{*}{ Date and Time } & \multicolumn{10}{|c|}{ Depth from Top of Casing (ft) } \\
\hline & MW1D & MW2D & MW3D & MW4D & MW7 & MW9 & Oentrich & MW14D & MW15D & MW16D \\
\hline 2/8/08 14:00 & 125.92 & 122.929 & & 100.809 & 104.809 & 96.447 & 111.063 & & 80.738 & 74.392 \\
\hline $2 / 8 / 08 \quad 14: 30$ & 125.636 & 122.87 & & 100.665 & 104.615 & 96.247 & 110.879 & & & 74.272 \\
\hline 2/8/08 15:00 & 125.471 & 122.83 & & 100.587 & 104.506 & 96.139 & 110.784 & & & 74.192 \\
\hline $2 / 8 / 08 \quad 15: 30$ & 125.369 & 122.809 & & 100.548 & 104.451 & 96.084 & 110.727 & & 80.716 & 74.138 \\
\hline $2 / 8 / 08$ 16:00 & 125.312 & 122.791 & & 100.518 & 104.415 & 96.047 & 110.692 & & & 74.103 \\
\hline 2/8/08 16:30 & 125.27 & 122.791 & & 100.499 & 104.394 & 96.028 & 110.667 & & & 74.078 \\
\hline 2/8/08 17:00 & 125.249 & 122.784 & & 100.496 & 104.38 & 96.014 & 110.656 & & 80.702 & 74.059 \\
\hline $2 / 8 / 08$ 17:30 & 125.23 & 122.782 & & 100.494 & 104.384 & 96.018 & 110.648 & & & 74.045 \\
\hline 2/8/08 18:00 & 125.22 & 122.78 & & 100.492 & 104.377 & 96.011 & 110.645 & & & 74.037 \\
\hline 2/8/08 18:30 & 125.213 & 122.777 & & 100.491 & 104.375 & 96.011 & 110.643 & & 80.699 & 74.028 \\
\hline 2/8/08 19:00 & 125.204 & 122.777 & & 100.487 & 104.375 & 96.009 & 110.641 & & & 74.024 \\
\hline 2/8/08 19:30 & 125.199 & 122.777 & & 100.489 & 104.371 & 96.007 & 110.637 & & & 74.02 \\
\hline 2/8/08 20:00 & 125.194 & 122.768 & & 100.486 & 104.371 & 96.004 & 110.633 & & 80.702 & 74.016 \\
\hline 2/8/08 20:30 & 125.19 & 122.764 & & 100.484 & 104.373 & 96.004 & 110.632 & & & 74.014 \\
\hline 2/8/08 21:00 & 125.19 & 122.764 & & 100.477 & 104.363 & 95.997 & 110.63 & & & 74.014 \\
\hline 2/8/08 21:30 & 125.185 & 122.764 & & 100.477 & 104.36 & 95.992 & 110.628 & & 80.697 & 74.014 \\
\hline 2/8/08 22:00 & 125.185 & 122.762 & & 100.477 & 104.359 & 95.99 & 110.626 & & & 74.014 \\
\hline 2/8/08 22:30 & 125.19 & 122.757 & & 100.473 & 104.359 & 95.992 & 110.624 & & & 74.014 \\
\hline 2/8/08 23:00 & 125.19 & 122.753 & & 100.472 & 104.358 & 95.99 & 110.622 & & 80.694 & 74.012 \\
\hline 2/8/08 23:30 & 125.19 & 122.748 & & 100.467 & 104.356 & 95.985 & 110.622 & & & 74.014 \\
\hline 2/9/08 0:00 & 125.187 & 122.743 & & 100.465 & 104.352 & 95.983 & 110.62 & & & 74.014 \\
\hline 2/9/08 0:30 & 125.185 & 122.741 & & 100.461 & 104.346 & 95.978 & 110.618 & & 80.694 & 74.014 \\
\hline $2 / 9 / 081: 00$ & 125.182 & 122.741 & & 100.458 & 104.342 & 95.973 & 110.618 & & & 74.014 \\
\hline 2/9/08 1:30 & 125.18 & 122.739 & & 100.455 & 104.338 & 95.969 & 110.617 & & & 74.014 \\
\hline 2/9/08 2:00 & 125.175 & 122.739 & & 100.456 & 104.339 & 95.969 & 110.615 & & 80.692 & 74.014 \\
\hline 2/9/08 2:30 & 125.173 & 122.741 & & 100.453 & 104.339 & 95.969 & 110.613 & & & 74.012 \\
\hline 2/9/08 3:00 & 125.173 & 122.741 & & 100.453 & 104.339 & 95.969 & 110.611 & & & 74.012 \\
\hline 2/9/08 3:30 & 125.171 & 122.739 & & 100.455 & 104.34 & 95.971 & 110.609 & & 80.692 & 74.01 \\
\hline 2/9/08 4:00 & 125.168 & 122.737 & & 100.456 & 104.34 & 95.971 & 110.607 & & & 74.01 \\
\hline 2/9/08 4:30 & 125.168 & 122.741 & & 100.455 & 104.338 & 95.969 & 110.605 & & & 74.01 \\
\hline 2/9/08 5:00 & 125.168 & 122.746 & & 100.453 & 104.339 & 95.971 & 110.605 & & 80.685 & 74.01 \\
\hline $2 / 9 / 085: 30$ & 125.166 & 122.753 & & 100.46 & 104.342 & 95.976 & 110.6 & & & 74.008 \\
\hline 2/9/08 6:00 & 125.168 & 122.762 & & 100.465 & 104.348 & 95.981 & 110.6 & & & 74.006 \\
\hline 2/9/08 6:30 & 125.168 & 122.771 & & 100.477 & 104.356 & 95.988 & 110.598 & & 80.685 & 74.006 \\
\hline 2/9/08 7:00 & 125.175 & 122.787 & & 100.482 & 104.363 & 95.997 & 110.596 & & & 74.004 \\
\hline 2/9/08 7:30 & 125.18 & 122.8 & & 100.496 & 104.373 & 96.009 & 110.6 & & & 74.002 \\
\hline 2/9/08 8:00 & 125.187 & 124.478 & & 100.511 & 104.386 & 96.018 & 110.599 & & 80.697 & 74.002 \\
\hline 2/9/08 8:30 & 125.197 & 124.88 & & 100.527 & 104.403 & 96.037 & 110.599 & & & 73.999 \\
\hline 2/9/08 9:00 & 126.596 & 125.05 & & 101.213 & 104.712 & 96.45 & 111.011 & & & 74.008 \\
\hline 2/9/08 9:30 & 127.066 & 125.161 & & 101.459 & 105.1 & 96.857 & 111.369 & & 80.751 & 74.165 \\
\hline 2/9/08 10:00 & 127.317 & 125.231 & & 101.595 & 105.272 & 97.03 & 111.516 & & & 74.285 \\
\hline $2 / 9 / 08 \quad 10: 30$ & 127.492 & 123.9 & & 101.688 & 105.382 & 97.145 & 111.597 & & & 74.361 \\
\hline $2 / 9 / 0811: 00$ & 127.617 & 123.374 & & 101.759 & 105.458 & 97.216 & 111.651 & & 80.848 & 74.415 \\
\hline 2/9/08 11:30 & 126.624 & 123.226 & & 101.211 & 105.382 & 97.081 & 111.45 & & & 74.448 \\
\hline $2 / 9 / 08 \quad 12: 00$ & 125.981 & 123.152 & & 100.983 & 104.948 & 96.586 & 110.984 & & & 74.347 \\
\hline 2/9/08 12:30 & 125.844 & 123.104 & & 100.887 & 104.811 & 96.447 & 110.84 & & 80.875 & 74.252 \\
\hline $2 / 9 / 08$ 13:00 & 125.773 & 123.075 & & 100.83 & 104.742 & 96.377 & 110.765 & & & 74.19 \\
\hline 2/9/08 13:30 & 125.731 & 123.059 & & 100.789 & 104.693 & 96.329 & 110.718 & & & 74.155 \\
\hline 2/9/08 14:00 & 125.702 & 123.047 & & 100.772 & 104.662 & 96.299 & 110.688 & & 80.88 & 74.13 \\
\hline 2/9/08 14:30 & 125.684 & 123.05 & & 100.754 & 104.649 & 96.285 & 110.667 & & & 74.115 \\
\hline $2 / 9 / 08 \quad 15: 00$ & 125.667 & 123.068 & & 100.758 & 104.639 & 96.273 & 110.652 & & & 74.107 \\
\hline 2/9/08 15:30 & 125.653 & 123.065 & & 100.772 & 104.645 & 96.282 & 110.637 & & 80.88 & 74.099 \\
\hline 2/9/08 16:00 & 125.641 & 123.079 & & 100.784 & 104.662 & 96.299 & 110.626 & & & 74.095 \\
\hline 2/9/08 16:30 & 125.636 & 123.081 & & 100.785 & 104.66 & 96.296 & 110.618 & & & 74.095 \\
\hline 2/9/08 17:00 & 125.624 & 123.104 & & 100.791 & 104.676 & 96.311 & 110.609 & & 80.9 & 74.093 \\
\hline 2/9/08 17:30 & 125.617 & 123.106 & & 100.804 & 104.679 & 96.313 & 110.605 & & & 74.088 \\
\hline $2 / 9 / 08$ 18:00 & 125.608 & 123.095 & & 100.82 & 104.702 & 96.339 & 110.643 & & & 74.082 \\
\hline 2/9/08 18:30 & 125.601 & 123.113 & & 100.818 & 104.706 & 96.341 & 110.654 & & 80.919 & 74.078 \\
\hline 2/9/08 19:00 & 125.594 & 123.12 & & 100.822 & 104.697 & 96.334 & 110.653 & & & 74.08 \\
\hline 2/9/08 19:30 & 125.584 & 123.129 & & 100.839 & 104.714 & 96.351 & 110.668 & & & 74.076 \\
\hline 2/9/08 20:00 & 125.58 & 123.143 & & 100.849 & 104.719 & 96.358 & 110.682 & & 80.936 & 74.076 \\
\hline 2/9/08 20:30 & 125.572 & 123.161 & & 100.856 & 104.729 & 96.365 & 110.692 & & & 74.074 \\
\hline 2/9/08 21:00 & 125.568 & 123.163 & & 100.865 & 104.744 & 96.379 & 110.712 & & & 74.074 \\
\hline 2/9/08 21:30 & 125.561 & 123.206 & & 100.882 & 104.759 & 96.395 & 110.72 & & 80.963 & 74.074 \\
\hline 2/9/08 22:00 & 125.561 & 123.195 & & 100.909 & 104.765 & 96.403 & 110.74 & & & 74.074 \\
\hline 2/9/08 22:30 & 125.546 & 123.217 & & 100.937 & 104.803 & 96.438 & 110.782 & & & 74.072 \\
\hline
\end{tabular}


TABLE S1.1 (Cont.)

\begin{tabular}{|c|c|c|c|c|c|c|c|c|c|c|}
\hline \multirow[b]{2}{*}{ Date and Time } & \multicolumn{10}{|c|}{ Depth from Top of Casing (ft) } \\
\hline & MW1D & MW2D & MW3D & MW4D & MW7 & MW9 & Oentrich & MW14D & MW15D & MW16D \\
\hline 2/9/08 23:00 & 125.554 & 123.226 & & 100.909 & 104.79 & 96.428 & 110.797 & & 80.988 & 74.076 \\
\hline 2/9/08 23:30 & 125.546 & 123.245 & & 100.942 & 104.816 & 96.452 & 110.791 & & & 74.076 \\
\hline 2/10/08 0:00 & 125.544 & 123.236 & & 100.935 & 104.824 & 96.457 & 110.804 & & & 74.078 \\
\hline $2 / 10 / 08$ 0:30 & 125.539 & 123.254 & & 100.97 & 104.841 & 96.476 & 110.819 & & 81.024 & 74.08 \\
\hline $2 / 10 / 08$ 1:00 & 125.542 & 123.26 & & 100.97 & 104.839 & 96.478 & 110.831 & & & 74.082 \\
\hline 2/10/08 1:30 & 125.537 & 123.26 & & 100.977 & 104.853 & 96.487 & 110.832 & & & 74.084 \\
\hline 2/10/08 2:00 & 125.539 & 123.254 & & 100.978 & 104.862 & 96.502 & 110.846 & & 81.046 & 74.088 \\
\hline $2 / 10 / 082: 30$ & 125.539 & 123.281 & & 100.987 & 104.862 & 96.502 & 110.851 & & & 74.09 \\
\hline 2/10/08 3:00 & 125.544 & 123.285 & & 100.98 & 104.853 & 96.49 & 110.849 & & & 74.097 \\
\hline $2 / 10 / 083: 30$ & 125.535 & 123.283 & & 100.999 & 104.883 & 96.518 & 110.858 & & 81.071 & 74.093 \\
\hline 2/10/08 4:00 & 125.535 & 123.294 & & 101.009 & 104.889 & 96.523 & 110.864 & & & 74.093 \\
\hline 2/10/08 4:30 & 125.537 & 123.306 & & 101.006 & 104.885 & 96.52 & 110.87 & & & 74.097 \\
\hline $2 / 10 / 085: 00$ & 125.535 & 123.326 & & 101.021 & 104.895 & 96.53 & 110.873 & & 81.095 & 74.095 \\
\hline 2/10/08 5:30 & 125.535 & 123.331 & & 101.035 & 104.908 & 96.544 & 110.885 & & & 74.09 \\
\hline 2/10/08 6:00 & 125.53 & 123.338 & & 101.049 & 104.929 & 96.565 & 110.904 & & & 74.084 \\
\hline $2 / 10 / 086: 30$ & 125.532 & 123.342 & & 101.056 & 104.933 & 96.57 & 110.911 & & 81.117 & 74.084 \\
\hline $2 / 10 / 08$ 7:00 & 125.535 & 123.344 & & 101.059 & 104.938 & 96.577 & 110.917 & & & 74.08 \\
\hline 2/10/08 7:30 & 125.537 & 123.363 & & 101.061 & 104.94 & 96.575 & 110.919 & & & 74.078 \\
\hline 2/10/08 8:00 & 125.539 & 123.372 & & 101.073 & 104.948 & 96.584 & 110.928 & & 81.139 & 74.076 \\
\hline 2/10/08 8:30 & 125.539 & 124.846 & & 101.085 & 104.965 & 96.601 & 110.941 & & & 74.072 \\
\hline 2/10/08 9:00 & 125.542 & 125.408 & & 101.095 & 104.975 & 96.612 & 110.949 & & & 74.072 \\
\hline 2/10/08 9:30 & 126.7 & 125.594 & & 101.759 & 105.138 & 96.855 & 111.236 & & 81.176 & 74.072 \\
\hline $2 / 10 / 0810: 00$ & 127.281 & 125.703 & & 102.04 & 105.647 & 97.426 & 111.747 & & & 74.217 \\
\hline $2 / 10 / 0810: 30$ & 127.464 & 125.775 & & 102.181 & 105.835 & 97.617 & 111.929 & & & 74.349 \\
\hline $2 / 10 / 0811: 00$ & 127.572 & 125.818 & & 102.267 & 105.944 & 97.725 & 112.032 & & 81.266 & 74.438 \\
\hline $2 / 10 / 0811: 30$ & 127.65 & 124.719 & & 102.326 & 106.012 & 97.796 & 112.107 & & & 74.496 \\
\hline 2/10/08 12:00 & 127.728 & 123.904 & & 102.36 & 106.058 & 97.84 & 112.143 & & & 74.539 \\
\hline $2 / 10 / 0812: 30$ & 127.251 & 123.694 & & 101.786 & 106.067 & 97.866 & 112.162 & & 81.332 & 74.574 \\
\hline $2 / 10 / 0813: 00$ & 126.22 & 123.58 & & 101.469 & 105.471 & 97.114 & 111.435 & & & 74.483 \\
\hline $2 / 10 / 08$ 13:30 & 126.062 & 123.499 & & 101.326 & 105.268 & 96.909 & 111.262 & & & 74.382 \\
\hline $2 / 10 / 08$ 14:00 & 125.979 & 123.44 & & 101.235 & 105.161 & 96.799 & 111.172 & & 81.313 & 74.316 \\
\hline $2 / 10 / 0814: 30$ & 125.927 & 123.387 & & 101.171 & 105.081 & 96.721 & 111.116 & & & 74.276 \\
\hline $2 / 10 / 08$ 15:00 & 125.887 & 123.363 & & 101.118 & 105.024 & 96.662 & 111.08 & & & 74.25 \\
\hline $2 / 10 / 0815: 30$ & 125.861 & 123.331 & & 101.077 & 104.976 & 96.612 & 111.054 & & 81.288 & 74.235 \\
\hline $2 / 10 / 08$ 16:00 & 125.832 & 123.31 & & 101.054 & 104.952 & 96.586 & 111.033 & & & 74.225 \\
\hline $2 / 10 / 0816: 30$ & 125.816 & 123.29 & & 101.028 & 104.923 & 96.556 & 111.018 & & & 74.214 \\
\hline $2 / 10 / 08$ 17:00 & 125.792 & 123.26 & & 101.009 & 104.904 & 96.539 & 111.003 & & 81.279 & 74.206 \\
\hline $2 / 10 / 0817: 30$ & 125.773 & 123.238 & & 100.989 & 104.883 & 96.516 & 110.994 & & & 74.2 \\
\hline $2 / 10 / 08$ 18:00 & 125.754 & 123.22 & & 100.961 & 104.853 & 96.487 & 110.986 & & & 74.196 \\
\hline $2 / 10 / 0818: 30$ & 125.724 & 123.213 & & 100.939 & 104.832 & 96.464 & 110.979 & & 81.254 & 74.192 \\
\hline $2 / 10 / 08$ 19:00 & 125.693 & 123.199 & & 100.93 & 104.815 & 96.447 & 110.969 & & & 74.186 \\
\hline 2/10/08 19:30 & 125.669 & 123.179 & & 100.925 & 104.807 & 96.44 & 110.962 & & & 74.181 \\
\hline $2 / 10 / 0820: 00$ & 125.653 & 123.163 & & 100.899 & 104.796 & 96.428 & 110.956 & & 81.235 & 74.175 \\
\hline $2 / 10 / 0820: 30$ & 125.636 & 123.143 & & 100.882 & 104.773 & 96.403 & 110.952 & & & 74.177 \\
\hline $2 / 10 / 0821: 00$ & 125.615 & 123.14 & & 100.865 & 104.759 & 96.391 & 110.947 & & & 74.173 \\
\hline 2/10/08 21:30 & 125.596 & 123.129 & & 100.853 & 104.74 & 96.372 & 110.943 & & 81.215 & 74.173 \\
\hline $2 / 10 / 0822: 00$ & 125.58 & 123.104 & & 100.844 & 104.737 & 96.365 & 110.935 & & & 74.171 \\
\hline 2/10/08 22:30 & 125.572 & 123.09 & & 100.834 & 104.725 & 96.358 & 110.934 & & & 74.167 \\
\hline 2/10/08 23:00 & 125.563 & 123.09 & & 100.801 & 104.698 & 96.329 & 110.932 & & 81.198 & 74.165 \\
\hline $2 / 10 / 0823: 30$ & 125.542 & 123.065 & & 100.803 & 104.685 & 96.315 & 110.928 & & & 74.163 \\
\hline $2 / 11 / 080: 00$ & 125.53 & 123.056 & & 100.785 & 104.685 & 96.315 & 110.923 & & & 74.157 \\
\hline $2 / 11 / 080: 30$ & 125.52 & 123.052 & & 100.761 & 104.666 & 96.296 & 110.92 & & 81.176 & 74.157 \\
\hline 2/11/08 1:00 & 125.502 & 123.031 & & 100.765 & 104.651 & 96.282 & 110.917 & & & 74.152 \\
\hline $2 / 11 / 081: 30$ & 125.492 & 123.013 & & 100.751 & 104.649 & 96.28 & 110.911 & & & 74.15 \\
\hline $2 / 11 / 082: 00$ & 125.48 & 122.986 & & 100.73 & 104.628 & 96.259 & 110.907 & & 81.156 & 74.148 \\
\hline $2 / 11 / 082: 30$ & 125.466 & 122.977 & & 100.713 & 104.611 & 96.24 & 110.904 & & & 74.148 \\
\hline 2/11/08 3:00 & 125.45 & 122.954 & & 100.697 & 104.584 & 96.214 & 110.9 & & & 74.148 \\
\hline 2/11/08 3:30 & 125.431 & 122.929 & & 100.684 & 104.571 & 96.204 & 110.896 & & 81.142 & 74.148 \\
\hline $2 / 11 / 084: 00$ & 125.414 & 122.909 & & 100.649 & 104.55 & 96.183 & 110.892 & & & 74.148 \\
\hline 2/11/08 4:30 & 125.39 & 122.893 & & 100.625 & 104.527 & 96.16 & 110.889 & & & 74.144 \\
\hline $2 / 11 / 085: 00$ & 125.369 & 122.879 & & 100.613 & 104.508 & 96.139 & 110.885 & & 81.09 & 74.14 \\
\hline $2 / 11 / 085: 30$ & 125.35 & 122.868 & & 100.582 & 104.493 & 96.124 & 110.881 & & & 74.138 \\
\hline $2 / 11 / 086: 00$ & 125.329 & 122.85 & & 100.584 & 104.48 & 96.108 & 110.875 & & & 74.132 \\
\hline $2 / 11 / 086: 30$ & 125.32 & 122.845 & & 100.572 & 104.472 & 96.101 & 110.872 & & 81.064 & 74.128 \\
\hline $2 / 11 / 087: 00$ & 125.308 & 124.705 & & 100.567 & 104.447 & 96.077 & 110.872 & & & 74.124 \\
\hline $2 / 11 / 087: 30$ & 125.296 & 124.991 & & 100.835 & 104.449 & 96.077 & 110.868 & & & 74.117 \\
\hline
\end{tabular}


TABLE S1.1 (Cont.)

Depth from Top of Casing (ft)

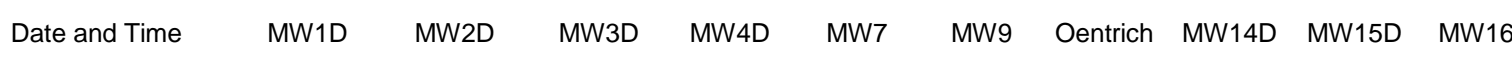

\begin{tabular}{|c|c|c|c|c|c|c|c|c|}
\hline 2/11/08 8:00 & 126.972 & 125.109 & 101.311 & 104.908 & 96.659 & 111.48 & 81.044 & 74.183 \\
\hline 2/11/08 8:30 & 127.315 & 125.161 & 101.514 & 105.195 & 96.952 & 111.713 & & 74.328 \\
\hline $2 / 11 / 089: 00$ & 127.518 & 125.222 & 101.599 & 105.304 & 97.063 & 111.835 & & 74.427 \\
\hline 2/11/08 9:30 & 127.631 & 125.251 & 101.664 & 105.369 & 97.129 & 111.91 & 81.09 & 74.494 \\
\hline 2/11/08 10:00 & 127.695 & 125.251 & 101.705 & 105.431 & 97.19 & 111.951 & & 74.535 \\
\hline $2 / 11 / 08 \quad 10: 30$ & 127.75 & 125.249 & 101.724 & 105.458 & 97.223 & 111.987 & & 74.564 \\
\hline $2 / 11 / 0811: 00$ & 127.773 & 125.265 & 101.717 & 105.466 & 97.228 & 112.011 & 81.117 & 74.591 \\
\hline $2 / 11 / 0811: 30$ & 127.773 & 124.356 & 101.73 & 105.454 & 97.213 & 112.03 & & 74.618 \\
\hline $2 / 11 / 08$ 12:00 & 127.78 & 123.34 & 101.75 & 105.475 & 97.235 & 112.043 & & 74.634 \\
\hline $2 / 11 / 08$ 12:30 & 127.792 & 123.118 & 101.206 & 105.481 & 97.244 & 112.054 & 81.137 & 74.655 \\
\hline 2/11/08 13:00 & 126.18 & 122.984 & 100.892 & 104.902 & 96.539 & 111.367 & & 74.556 \\
\hline $2 / 11 / 08$ 13:30 & 126.01 & 122.914 & 100.725 & 104.689 & 96.322 & 111.195 & & 74.444 \\
\hline $2 / 11 / 0814: 00$ & 125.818 & 122.857 & 100.637 & 104.558 & 96.188 & 111.106 & 81.068 & 74.378 \\
\hline $2 / 11 / 08 \quad 14: 30$ & 125.594 & 122.821 & 100.568 & 104.495 & 96.127 & 111.048 & & 74.332 \\
\hline $2 / 11 / 08$ 15:00 & 125.426 & 122.8 & 100.534 & 104.434 & 96.065 & 111.014 & & 74.301 \\
\hline $2 / 11 / 08$ 15:30 & 125.324 & 122.786 & 100.499 & 104.405 & 96.039 & 110.986 & 81.024 & 74.274 \\
\hline $2 / 11 / 08$ 16:00 & 125.26 & 122.784 & 100.491 & 104.388 & 96.016 & 110.965 & & 74.258 \\
\hline $2 / 11 / 0816: 30$ & 125.237 & 122.782 & 100.486 & 104.375 & 96.004 & 110.952 & & 74.245 \\
\hline $2 / 11 / 08$ 17:00 & 125.22 & 122.78 & 100.486 & 104.379 & 96.007 & 110.939 & 81 & 74.233 \\
\hline $2 / 11 / 08$ 17:30 & 125.218 & 122.784 & 100.489 & 104.377 & 96.004 & 110.93 & & 74.221 \\
\hline $2 / 11 / 08$ 18:00 & 125.213 & 122.777 & 100.48 & 104.373 & 96.002 & 110.922 & & 74.214 \\
\hline $2 / 11 / 0818: 30$ & 125.211 & 122.768 & 100.486 & 104.373 & 96.004 & 110.919 & 80.98 & 74.204 \\
\hline $2 / 11 / 08$ 19:00 & 125.211 & 122.777 & 100.484 & 104.375 & 96.004 & 110.917 & & 74.196 \\
\hline $2 / 11 / 08$ 19:30 & 125.208 & 122.786 & 100.487 & 104.367 & 95.997 & 110.911 & & 74.19 \\
\hline $2 / 11 / 0820: 00$ & 125.213 & 122.796 & 100.492 & 104.373 & 96.002 & 110.905 & 80.956 & 74.179 \\
\hline $2 / 11 / 0820: 30$ & 125.22 & 122.8 & 100.503 & 104.388 & 96.021 & 110.9 & & 74.171 \\
\hline $2 / 11 / 0821: 00$ & 125.223 & 122.793 & 100.508 & 104.394 & 96.023 & 110.896 & & 74.163 \\
\hline 2/11/08 21:30 & 125.239 & 122.805 & 100.506 & 104.4 & 96.03 & 110.894 & 80.949 & 74.157 \\
\hline $2 / 11 / 0822: 00$ & 125.234 & 122.811 & 100.511 & 104.396 & 96.023 & 110.892 & & 74.152 \\
\hline $2 / 11 / 0822: 30$ & 125.237 & 122.8 & 100.527 & 104.405 & 96.035 & 110.89 & & 74.144 \\
\hline $2 / 11 / 0823: 00$ & 125.251 & 122.809 & 100.52 & 104.413 & 96.044 & 110.889 & 80.939 & 74.14 \\
\hline 2/11/08 23:30 & 125.249 & 122.83 & 100.517 & 104.394 & 96.025 & 110.89 & & 74.138 \\
\hline $2 / 12 / 080: 00$ & 125.249 & 122.812 & 100.529 & 104.411 & 96.039 & 110.887 & & 74.132 \\
\hline 2/12/08 0:30 & 125.258 & 122.816 & 100.537 & 104.43 & 96.061 & 110.883 & 80.934 & 74.124 \\
\hline 2/12/08 1:00 & 125.26 & 122.814 & 100.525 & 104.409 & 96.039 & 110.887 & & 74.124 \\
\hline $2 / 12 / 081: 30$ & 125.251 & 122.809 & 100.525 & 104.413 & 96.044 & 110.883 & & 74.119 \\
\hline $2 / 12 / 082: 00$ & 125.253 & 122.809 & 100.527 & 104.411 & 96.042 & 110.883 & 80.922 & 74.115 \\
\hline 2/12/08 2:30 & 125.251 & 122.811 & 100.517 & 104.407 & 96.039 & 110.881 & & 74.111 \\
\hline 2/12/08 3:00 & 125.246 & 122.807 & 100.52 & 104.409 & 96.042 & 110.879 & & 74.107 \\
\hline 2/12/08 3:30 & 125.244 & 122.8 & 100.518 & 104.411 & 96.042 & 110.875 & 80.912 & 74.103 \\
\hline $2 / 12 / 084: 00$ & 125.246 & 122.807 & 100.518 & 104.413 & 96.039 & 110.875 & & 74.099 \\
\hline 2/12/08 4:30 & 125.246 & 122.807 & 100.52 & 104.4 & 96.03 & 110.875 & & 74.097 \\
\hline 2/12/08 5:00 & 125.246 & 122.814 & 100.52 & 104.409 & 96.039 & 110.873 & 80.905 & 74.09 \\
\hline $2 / 12 / 085: 30$ & 125.244 & 122.823 & 100.525 & 104.411 & 96.039 & 110.872 & & 74.084 \\
\hline $2 / 12 / 086: 00$ & 125.251 & 122.823 & 100.537 & 104.419 & 96.049 & 110.87 & & 74.078 \\
\hline $2 / 12 / 086: 30$ & 125.258 & 122.827 & 100.537 & 104.428 & 96.058 & 110.866 & 80.905 & 74.072 \\
\hline $2 / 12 / 087: 00$ & 125.26 & 124.739 & 100.537 & 104.424 & 96.054 & 110.866 & & 74.068 \\
\hline $2 / 12 / 087: 30$ & 125.263 & 124.988 & 100.982 & 104.43 & 96.061 & 110.864 & & 74.057 \\
\hline 2/12/08 8:00 & 127.076 & 125.118 & 101.407 & 104.988 & 96.756 & 111.553 & 80.922 & 74.136 \\
\hline $2 / 12 / 088: 30$ & 127.44 & 125.206 & 101.59 & 105.233 & 97.004 & 111.778 & & 74.283 \\
\hline 2/12/08 9:00 & 127.62 & 123.39 & 101.688 & 105.365 & 97.138 & 111.897 & & 74.378 \\
\hline 2/12/08 9:30 & 127.716 & 123.179 & 101.33 & 105.452 & 97.228 & 111.966 & 80.985 & 74.44 \\
\hline $2 / 12 / 08 \quad 10: 00$ & 125.998 & 123.079 & 100.954 & 104.959 & 96.598 & 111.33 & & 74.394 \\
\hline $2 / 12 / 08 \quad 10: 30$ & 125.676 & 123.029 & 100.82 & 104.769 & 96.396 & 111.133 & & 74.283 \\
\hline 2/12/08 11:00 & 125.544 & 122.988 & 100.754 & 104.666 & 96.299 & 111.042 & 80.973 & 74.21 \\
\hline $2 / 12 / 0811: 30$ & 125.476 & 122.959 & 100.716 & 104.62 & 96.252 & 110.99 & & 74.165 \\
\hline 2/12/08 12:00 & 125.442 & 122.929 & 100.682 & 104.582 & 96.216 & 110.96 & & 74.138 \\
\hline 2/12/08 12:30 & 125.424 & 122.895 & 100.649 & 104.552 & 96.186 & 110.937 & 80.963 & 74.121 \\
\hline $2 / 12 / 08$ 13:00 & 125.419 & 122.873 & 100.618 & 104.518 & 96.153 & 110.928 & & 74.113 \\
\hline 2/12/08 13:30 & 125.407 & 122.85 & 100.592 & 104.489 & 96.122 & 110.917 & & 74.109 \\
\hline $2 / 12 / 0814: 00$ & 125.364 & 122.827 & 100.572 & 104.47 & 96.098 & 110.909 & 80.941 & 74.105 \\
\hline $2 / 12 / 08 \quad 14: 30$ & 125.336 & 122.816 & 100.549 & 104.445 & 96.077 & 110.902 & & 74.103 \\
\hline 2/12/08 15:00 & 125.305 & 122.816 & 100.534 & 104.426 & 96.056 & 110.896 & & 74.101 \\
\hline $2 / 12 / 08$ 15:30 & 125.286 & 122.816 & 100.523 & 104.415 & 96.047 & 110.892 & 80.919 & 74.097 \\
\hline $2 / 12 / 08 \quad 16: 00$ & 125.268 & 122.811 & 100.523 & 104.415 & 96.047 & 110.887 & & 74.093 \\
\hline 2/12/08 16:30 & 125.26 & 122.802 & 100.525 & 104.413 & 96.044 & 110.883 & & 74.088 \\
\hline
\end{tabular}


TABLE S1.1 (Cont.)

Depth from Top of Casing (ft)

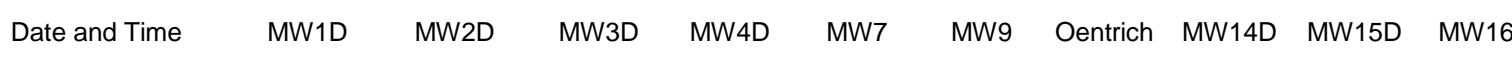

\begin{tabular}{|c|c|c|c|c|c|c|c|c|}
\hline 2/12/08 17:00 & 125.253 & 122.805 & 100.518 & 104.409 & 96.042 & 110.879 & 80.91 & 74.082 \\
\hline 2/12/08 17:30 & 125.249 & 122.805 & 100.513 & 104.4 & 96.032 & 110.877 & & 74.078 \\
\hline $2 / 12 / 08$ 18:00 & 125.242 & 122.805 & 100.522 & 104.405 & 96.035 & 110.873 & & 74.07 \\
\hline $2 / 12 / 0818: 30$ & 125.242 & 122.807 & 100.523 & 104.407 & 96.037 & 110.87 & 80.905 & 74.064 \\
\hline $2 / 12 / 08$ 19:00 & 125.239 & 122.809 & 100.52 & 104.407 & 96.037 & 110.847 & & 74.055 \\
\hline 2/12/08 19:30 & 125.239 & 122.811 & 100.52 & 104.407 & 96.035 & 110.843 & & 74.049 \\
\hline $2 / 12 / 0820: 00$ & 125.242 & 122.798 & 100.525 & 104.409 & 96.039 & 110.845 & 80.897 & 74.039 \\
\hline $2 / 12 / 0820: 30$ & 125.244 & 122.793 & 100.52 & 104.413 & 96.044 & 110.846 & & 74.03 \\
\hline $2 / 12 / 0821: 00$ & 125.246 & 122.786 & 100.51 & 104.4 & 96.032 & 110.85 & & 74.026 \\
\hline $2 / 12 / 0821: 30$ & 125.237 & 122.777 & 100.506 & 104.392 & 96.025 & 110.85 & 80.88 & 74.02 \\
\hline 2/12/08 22:00 & 125.232 & 122.777 & 100.498 & 104.39 & 96.018 & 110.849 & & 74.014 \\
\hline $2 / 12 / 0822: 30$ & 125.227 & 122.775 & 100.496 & 104.382 & 96.014 & 110.849 & & 74.014 \\
\hline $2 / 12 / 0823: 00$ & 125.22 & 122.762 & 100.492 & 104.382 & 96.014 & 110.847 & 80.87 & 74.014 \\
\hline 2/12/08 23:30 & 125.218 & 122.768 & 100.487 & 104.379 & 96.009 & 110.846 & & 74.01 \\
\hline $2 / 13 / 080: 00$ & 125.211 & 122.764 & 100.48 & 104.367 & 96.002 & 110.846 & & 74.008 \\
\hline $2 / 13 / 080: 30$ & 125.208 & 122.762 & 100.482 & 104.373 & 95.999 & 110.845 & 80.856 & 74.002 \\
\hline $2 / 13 / 081: 00$ & 125.206 & 122.753 & 100.48 & 104.369 & 95.999 & 110.844 & & 73.997 \\
\hline $2 / 13 / 081: 30$ & 125.206 & 122.746 & 100.473 & 104.365 & 95.997 & 110.844 & & 73.993 \\
\hline $2 / 13 / 082: 00$ & 125.199 & 122.734 & 100.465 & 104.354 & 95.988 & 110.844 & 80.846 & 73.991 \\
\hline $2 / 13 / 082: 30$ & 125.192 & 122.732 & 100.456 & 104.352 & 95.978 & 110.843 & & 73.987 \\
\hline 2/13/08 3:00 & 125.182 & 122.723 & 100.449 & 104.337 & 95.966 & 110.843 & & 73.981 \\
\hline 2/13/08 3:30 & 125.175 & 122.714 & 100.449 & 104.335 & 95.964 & 110.84 & 80.831 & 73.975 \\
\hline $2 / 13 / 084: 00$ & 125.173 & 122.7 & 100.434 & 104.327 & 95.959 & 110.84 & & 73.968 \\
\hline $2 / 13 / 084: 30$ & 125.161 & 122.696 & 100.422 & 104.32 & 95.95 & 110.84 & & 73.962 \\
\hline $2 / 13 / 085: 00$ & 125.149 & 122.682 & 100.418 & 104.302 & 95.933 & 110.84 & 80.812 & 73.96 \\
\hline 2/13/08 5:30 & 125.145 & 122.675 & 100.406 & 104.299 & 95.929 & 110.84 & & 73.956 \\
\hline $2 / 13 / 086: 00$ & 125.133 & 122.671 & 100.396 & 104.287 & 95.915 & 110.84 & & 73.952 \\
\hline $2 / 13 / 086: 30$ & 125.123 & 122.671 & 100.392 & 104.283 & 95.915 & 110.838 & 80.797 & 73.948 \\
\hline $2 / 13 / 087: 00$ & 125.116 & 124.487 & 100.387 & 104.278 & 95.907 & 110.836 & & 73.946 \\
\hline $2 / 13 / 087: 30$ & 125.112 & 124.791 & 100.387 & 104.274 & 95.903 & 110.836 & & 73.94 \\
\hline $2 / 13 / 088: 00$ & 126.724 & 124.932 & 101.144 & 104.698 & 96.443 & 111.371 & 80.797 & 73.971 \\
\hline $2 / 13 / 088: 30$ & 127.18 & 125.013 & 101.344 & 104.997 & 96.754 & 111.658 & & 74.128 \\
\hline 2/13/08 9:00 & 127.388 & 125.059 & 101.449 & 105.14 & 96.895 & 111.79 & & 74.235 \\
\hline 2/13/08 9:30 & 127.497 & 125.084 & 101.507 & 105.222 & 96.978 & 111.867 & 80.858 & 74.305 \\
\hline $2 / 13 / 0810: 00$ & 127.56 & 125.104 & 101.547 & 105.272 & 97.027 & 111.918 & & 74.353 \\
\hline $2 / 13 / 08 \quad 10: 30$ & 127.594 & 125.118 & 101.571 & 105.298 & 97.058 & 111.953 & & 74.388 \\
\hline $2 / 13 / 08$ 11:00 & 127.622 & 125.118 & 101.588 & 105.315 & 97.074 & 111.98 & 80.89 & 74.417 \\
\hline $2 / 13 / 0811: 30$ & 127.643 & 123.68 & 101.593 & 105.332 & 97.093 & 111.998 & & 74.444 \\
\hline $2 / 13 / 08$ 12:00 & 127.667 & 123.131 & 101.592 & 105.334 & 97.096 & 112.015 & & 74.467 \\
\hline $2 / 13 / 08$ 12:30 & 126.57 & 122.936 & 100.98 & 105.167 & 96.853 & 111.765 & 80.907 & 74.487 \\
\hline $2 / 13 / 08$ 13:00 & 125.946 & 122.816 & 100.703 & 104.7 & 96.337 & 111.303 & & 74.37 \\
\hline $2 / 13 / 08$ 13:30 & 125.66 & 122.737 & 100.558 & 104.512 & 96.146 & 111.153 & & 74.266 \\
\hline $2 / 13 / 0814: 00$ & 125.438 & 122.673 & 100.463 & 104.394 & 96.026 & 111.074 & 80.844 & 74.198 \\
\hline $2 / 13 / 08 \quad 14: 30$ & 125.268 & 122.621 & 100.399 & 104.32 & 95.95 & 111.024 & & 74.152 \\
\hline $2 / 13 / 08$ 15:00 & 125.161 & 122.587 & 100.346 & 104.257 & 95.886 & 110.992 & & 74.121 \\
\hline $2 / 13 / 08$ 15:30 & 125.095 & 122.564 & 100.299 & 104.209 & 95.842 & 110.962 & 80.79 & 74.101 \\
\hline $2 / 13 / 0816: 00$ & 125.043 & 122.537 & 100.274 & 104.173 & 95.804 & 110.93 & & 74.086 \\
\hline $2 / 13 / 0816: 30$ & 125.017 & 122.521 & 100.253 & 104.156 & 95.785 & 110.909 & & 74.088 \\
\hline $2 / 13 / 08$ 17:00 & 124.989 & 122.508 & 100.231 & 104.127 & 95.757 & 110.892 & 80.753 & 74.095 \\
\hline $2 / 13 / 08$ 17:30 & 124.965 & 122.496 & 100.218 & 104.116 & 95.743 & 110.875 & & 74.101 \\
\hline $2 / 13 / 0818: 00$ & 124.951 & 122.485 & 100.206 & 104.099 & 95.731 & 110.864 & & 74.107 \\
\hline $2 / 13 / 08$ 18:30 & 124.939 & 122.476 & 100.198 & 104.093 & 95.721 & 110.855 & 80.721 & 74.113 \\
\hline $2 / 13 / 08$ 19:00 & 124.927 & 122.467 & 100.191 & 104.08 & 95.707 & 110.845 & & 74.117 \\
\hline $2 / 13 / 08$ 19:30 & 124.92 & 122.458 & 100.182 & 104.074 & 95.702 & 110.838 & & 74.117 \\
\hline $2 / 13 / 0820: 00$ & 124.908 & 122.444 & 100.172 & 104.068 & 95.693 & 110.831 & 80.694 & 74.117 \\
\hline $2 / 13 / 0820: 30$ & 124.899 & 122.433 & 100.158 & 104.059 & 95.684 & 110.823 & & 74.119 \\
\hline $2 / 13 / 0821: 00$ & 124.885 & 122.437 & 100.15 & 104.044 & 95.669 & 110.813 & & 74.121 \\
\hline $2 / 13 / 0821: 30$ & 124.875 & 122.431 & 100.148 & 104.034 & 95.66 & 110.804 & 80.66 & 74.121 \\
\hline 2/13/08 22:00 & 124.875 & 122.424 & 100.143 & 104.036 & 95.662 & 110.8 & & 74.121 \\
\hline $2 / 13 / 0822: 30$ & 124.87 & 122.417 & 100.137 & 104.034 & 95.658 & 110.798 & & 74.119 \\
\hline 2/13/08 23:00 & 124.866 & 122.41 & 100.132 & 104.024 & 95.653 & 110.793 & 80.636 & 74.124 \\
\hline 2/13/08 23:30 & 124.859 & 122.403 & 100.125 & 104.017 & 95.644 & 110.789 & & 74.126 \\
\hline $2 / 14 / 080: 00$ & 124.849 & 122.397 & 100.12 & 104.013 & 95.636 & 110.783 & & 74.13 \\
\hline $2 / 14 / 080: 30$ & 124.847 & 122.381 & 100.119 & 104.007 & 95.636 & 110.78 & 80.609 & 74.132 \\
\hline $2 / 14 / 081: 00$ & 124.844 & 122.36 & 100.1 & 104.002 & 95.629 & 110.778 & & 74.136 \\
\hline $2 / 14 / 081: 30$ & 124.828 & 122.36 & 100.086 & 103.986 & 95.613 & 110.77 & & 74.14 \\
\hline
\end{tabular}


TABLE S1.1 (Cont.)

\begin{tabular}{|c|c|c|c|c|c|c|c|c|c|c|}
\hline \multirow[b]{2}{*}{ Date and Time } & \multicolumn{10}{|c|}{ Depth from Top of Casing (ft) } \\
\hline & MW1D & MW2D & MW3D & MW4D & MW7 & MW9 & Oentrich & MW14D & MW15D & MW16D \\
\hline 2/14/08 2:00 & 124.809 & 122.362 & & 100.07 & 103.967 & 95.594 & 110.759 & & 80.572 & 74.144 \\
\hline 2/14/08 2:30 & 124.797 & 122.367 & & 100.075 & 103.962 & 95.587 & 110.75 & & & 74.146 \\
\hline 2/14/08 3:00 & 124.8 & 122.358 & & 100.082 & 103.965 & 95.592 & 110.746 & & & 74.148 \\
\hline $2 / 14 / 083: 30$ & 124.804 & 122.362 & & 100.081 & 103.969 & 95.599 & 110.744 & & 80.548 & 74.148 \\
\hline 2/14/08 4:00 & 124.807 & 122.385 & & 100.069 & 103.964 & 95.592 & 110.746 & & & 74.148 \\
\hline $2 / 14 / 084: 30$ & 124.8 & 122.406 & & 100.088 & 103.964 & 95.592 & 110.742 & & & 74.15 \\
\hline 2/14/08 5:00 & 124.814 & 122.419 & & 100.117 & 103.992 & 95.618 & 110.736 & & 80.538 & 74.146 \\
\hline 2/14/08 5:30 & 124.84 & 122.421 & & 100.124 & 104.015 & 95.641 & 110.735 & & & 74.142 \\
\hline 2/14/08 6:00 & 124.852 & 122.433 & & 100.144 & 104.026 & 95.653 & 110.735 & & & 74.136 \\
\hline $2 / 14 / 086: 30$ & 124.866 & 122.435 & & 100.143 & 104.032 & 95.66 & 110.739 & & 80.536 & 74.136 \\
\hline $2 / 14 / 08$ 7:00 & 124.87 & 124.269 & & 100.158 & 104.047 & 95.672 & 110.735 & & & 74.134 \\
\hline 2/14/08 7:30 & 124.88 & 124.571 & & 100.268 & 104.047 & 95.672 & 110.738 & & & 74.134 \\
\hline $2 / 14 / 088: 00$ & 126.613 & 124.73 & & 100.973 & 104.527 & 96.285 & 111.349 & & 80.548 & 74.183 \\
\hline 2/14/08 8:30 & 127.045 & 124.83 & & 101.194 & 104.822 & 96.589 & 111.619 & & & 74.345 \\
\hline 2/14/08 9:00 & 127.239 & 124.916 & & 101.316 & 104.982 & 96.751 & 111.749 & & & 74.45 \\
\hline 2/14/08 9:30 & 127.348 & 123.247 & & 101.406 & 105.085 & 96.855 & 111.826 & & 80.626 & 74.518 \\
\hline $2 / 14 / 0810: 00$ & 127.435 & 122.959 & & 101.478 & 105.171 & 96.942 & 111.874 & & & 74.564 \\
\hline $2 / 14 / 08 \quad 10: 30$ & 125.87 & 122.857 & & 100.751 & 104.807 & 96.45 & 111.354 & & & 74.558 \\
\hline $2 / 14 / 0811: 00$ & 125.433 & 122.816 & & 100.599 & 104.546 & 96.176 & 111.078 & & 80.66 & 74.438 \\
\hline $2 / 14 / 0811: 30$ & 125.305 & 122.8 & & 100.532 & 104.449 & 96.08 & 110.96 & & & 74.349 \\
\hline 2/14/08 12:00 & 125.253 & 122.8 & & 100.513 & 104.411 & 96.044 & 110.896 & & & 74.287 \\
\hline $2 / 14 / 0812: 30$ & 125.239 & 122.809 & & 100.51 & 104.4 & 96.028 & 110.855 & & 80.68 & 74.243 \\
\hline $2 / 14 / 08$ 13:00 & 125.242 & 122.816 & & 100.52 & 104.401 & 96.035 & 110.83 & & & 74.217 \\
\hline $2 / 14 / 08$ 13:30 & 125.249 & 122.834 & & 100.527 & 104.411 & 96.044 & 110.81 & & & 74.196 \\
\hline $2 / 14 / 0814: 00$ & 125.258 & 122.852 & & 100.542 & 104.42 & 96.054 & 110.802 & & 80.707 & 74.183 \\
\hline $2 / 14 / 08 \quad 14: 30$ & 125.275 & 122.868 & & 100.558 & 104.436 & 96.072 & 110.789 & & & 74.169 \\
\hline $2 / 14 / 08$ 15:00 & 125.291 & 122.889 & & 100.577 & 104.455 & 96.087 & 110.784 & & & 74.161 \\
\hline $2 / 14 / 0815: 30$ & 125.305 & 122.902 & & 100.598 & 104.472 & 96.106 & 110.78 & & 80.736 & 74.15 \\
\hline 2/14/08 16:00 & 125.327 & 122.929 & & 100.61 & 104.493 & 96.124 & 110.776 & & & 74.142 \\
\hline $2 / 14 / 0816: 30$ & 125.341 & 122.95 & & 100.637 & 104.51 & 96.143 & 110.774 & & & 74.136 \\
\hline $2 / 14 / 08$ 17:00 & 125.364 & 122.966 & & 100.658 & 104.54 & 96.174 & 110.776 & & 80.792 & 74.13 \\
\hline $2 / 14 / 0817: 30$ & 125.383 & 122.982 & & 100.675 & 104.558 & 96.19 & 110.774 & & & 74.124 \\
\hline $2 / 14 / 0818: 00$ & 125.398 & 123.002 & & 100.696 & 104.571 & 96.204 & 110.774 & & & 74.115 \\
\hline $2 / 14 / 0818: 30$ & 125.419 & 123.018 & & 100.715 & 104.59 & 96.223 & 110.772 & & 80.829 & 74.105 \\
\hline 2/14/08 19:00 & 125.435 & 123.025 & & 100.741 & 104.607 & 96.245 & 110.77 & & & 74.095 \\
\hline 2/14/08 19:30 & 125.461 & 123.034 & & 100.751 & 104.628 & 96.263 & 110.767 & & & 74.084 \\
\hline $2 / 14 / 0820: 00$ & 125.468 & 123.056 & & 100.754 & 104.634 & 96.268 & 110.767 & & 80.846 & 74.076 \\
\hline 2/14/08 20:30 & 125.473 & 123.07 & & 100.768 & 104.641 & 96.278 & 110.763 & & & 74.068 \\
\hline $2 / 14 / 0821: 00$ & 125.487 & 123.074 & & 100.787 & 104.664 & 96.296 & 110.757 & & & 74.064 \\
\hline $2 / 14 / 0821: 30$ & 125.506 & 123.093 & & 100.806 & 104.678 & 96.313 & 110.755 & & 80.88 & 74.062 \\
\hline 2/14/08 22:00 & 125.516 & 123.104 & & 100.808 & 104.683 & 96.318 & 110.751 & & & 74.057 \\
\hline 2/14/08 22:30 & 125.525 & 123.108 & & 100.825 & 104.698 & 96.332 & 110.748 & & & 74.053 \\
\hline 2/14/08 23:00 & 125.539 & 123.099 & & 100.828 & 104.712 & 96.346 & 110.744 & & 80.912 & 74.049 \\
\hline 2/14/08 23:30 & 125.546 & 123.099 & & 100.825 & 104.717 & 96.353 & 110.742 & & & 74.045 \\
\hline $2 / 15 / 080: 00$ & 125.537 & 123.113 & & 100.827 & 104.708 & 96.341 & 110.74 & & & 74.037 \\
\hline 2/15/08 0:30 & 125.539 & 123.124 & & 100.832 & 104.71 & 96.341 & 110.739 & & 80.927 & 74.033 \\
\hline $2 / 15 / 08$ 1:00 & 125.551 & 123.122 & & 100.846 & 104.721 & 96.355 & 110.733 & & & 74.035 \\
\hline $2 / 15 / 081: 30$ & 125.563 & 123.127 & & 100.853 & 104.737 & 96.367 & 110.727 & & & 74.033 \\
\hline 2/15/08 2:00 & 125.563 & 123.133 & & 100.858 & 104.731 & 96.362 & 110.723 & & 80.954 & 74.028 \\
\hline 2/15/08 2:30 & 125.572 & 123.149 & & 100.859 & 104.737 & 96.37 & 110.724 & & & 74.026 \\
\hline 2/15/08 3:00 & 125.575 & 123.136 & & 100.87 & 104.742 & 96.374 & 110.718 & & & 74.02 \\
\hline 2/15/08 3:30 & 125.584 & 123.142 & & 100.863 & 104.757 & 96.388 & 110.714 & & 80.973 & 74.022 \\
\hline 2/15/08 4:00 & 125.575 & 123.156 & & 100.873 & 104.744 & 96.374 & 110.714 & & & 74.016 \\
\hline $2 / 15 / 084: 30$ & 125.587 & 123.161 & & 100.875 & 104.752 & 96.384 & 110.71 & & & 74.024 \\
\hline 2/15/08 5:00 & 125.584 & 123.149 & & 100.884 & 104.763 & 96.395 & 110.733 & & 80.99 & 74.028 \\
\hline 2/15/08 5:30 & 125.598 & 123.149 & & 100.889 & 104.769 & 96.403 & 110.742 & & & 74.039 \\
\hline $2 / 15 / 086: 00$ & 125.598 & 123.17 & & 100.871 & 104.759 & 96.395 & 110.748 & & & 74.035 \\
\hline 2/15/08 6:30 & 125.582 & 123.167 & & 100.88 & 104.756 & 96.386 & 110.744 & & 81.007 & 74.03 \\
\hline $2 / 15 / 087: 00$ & 125.601 & 124.873 & & 100.899 & 104.778 & 96.414 & 110.755 & & & 74.051 \\
\hline $2 / 15 / 087: 30$ & 125.613 & 125.263 & & 100.892 & 104.775 & 96.41 & 110.763 & & & 74.055 \\
\hline 2/15/08 8:00 & 127.149 & 125.428 & & 101.593 & 105.093 & 96.836 & 111.198 & & 81.039 & 74.066 \\
\hline $2 / 15 / 088: 30$ & 127.724 & 125.517 & & 101.831 & 105.471 & 97.232 & 111.561 & & & 74.274 \\
\hline 2/15/08 9:00 & 127.913 & 125.578 & & 101.959 & 105.641 & 97.404 & 111.715 & & & 74.432 \\
\hline 2/15/08 9:30 & 128.017 & 125.614 & & 102.029 & 105.725 & 97.489 & 111.809 & & 81.13 & 74.523 \\
\hline 2/15/08 10:00 & 128.078 & 125.648 & & 102.083 & 105.791 & 97.555 & 111.865 & & & 74.587 \\
\hline $2 / 15 / 08$ 10:30 & 128.118 & 125.666 & & 102.117 & 105.827 & 97.588 & 111.903 & & & 74.624 \\
\hline
\end{tabular}


TABLE S1.1 (Cont.)

Depth from Top of Casing (ft)

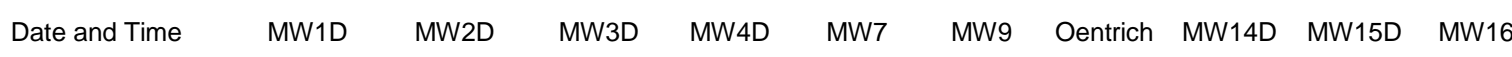

\begin{tabular}{|c|c|c|c|c|c|c|c|c|}
\hline 2/15/08 11:00 & 128.151 & 123.811 & 102.138 & 105.866 & 97.628 & 111.935 & 81.2 & 74.665 \\
\hline 2/15/08 11:30 & 128.17 & 123.569 & 101.754 & 105.883 & 97.647 & 111.951 & & 74.692 \\
\hline $2 / 15 / 08$ 12:00 & 126.563 & 123.449 & 101.356 & 105.374 & 97.013 & 111.33 & & 74.624 \\
\hline $2 / 15 / 08$ 12:30 & 126.241 & 123.362 & 101.197 & 105.144 & 96.78 & 111.125 & 81.205 & 74.489 \\
\hline $2 / 15 / 08$ 13:00 & 126.033 & 123.299 & 101.099 & 105.032 & 96.664 & 111.024 & & 74.405 \\
\hline $2 / 15 / 08$ 13:30 & 125.899 & 123.251 & 101.033 & 104.946 & 96.579 & 110.965 & & 74.345 \\
\hline $2 / 15 / 0814: 00$ & 125.785 & 123.22 & 100.982 & 104.891 & 96.525 & 110.926 & 81.188 & 74.308 \\
\hline $2 / 15 / 08 \quad 14: 30$ & 125.714 & 123.199 & 100.942 & 104.841 & 96.478 & 110.9 & & 74.283 \\
\hline $2 / 15 / 08$ 15:00 & 125.667 & 123.179 & 100.918 & 104.809 & 96.447 & 110.879 & & 74.268 \\
\hline $2 / 15 / 08$ 15:30 & 125.646 & 123.154 & 100.899 & 104.794 & 96.428 & 110.862 & 81.176 & 74.258 \\
\hline 2/15/08 16:00 & 125.624 & 123.136 & 100.878 & 104.771 & 96.405 & 110.849 & & 74.25 \\
\hline $2 / 15 / 0816: 30$ & 125.606 & 123.115 & 100.858 & 104.752 & 96.384 & 110.84 & & 74.243 \\
\hline $2 / 15 / 08$ 17:00 & 125.584 & 123.095 & 100.835 & 104.731 & 96.367 & 110.832 & 81.161 & 74.235 \\
\hline $2 / 15 / 08 \quad 17: 30$ & 125.561 & 123.079 & 100.815 & 104.71 & 96.344 & 110.825 & & 74.225 \\
\hline $2 / 15 / 08$ 18:00 & 125.542 & 123.068 & 100.799 & 104.693 & 96.325 & 110.819 & & 74.212 \\
\hline $2 / 15 / 08$ 18:30 & 125.525 & 123.065 & 100.789 & 104.678 & 96.308 & 110.815 & 81.147 & 74.198 \\
\hline 2/15/08 19:00 & 125.511 & 123.07 & 100.773 & 104.662 & 96.297 & 110.81 & & 74.186 \\
\hline $2 / 15 / 08$ 19:30 & 125.502 & 123.065 & 100.778 & 104.662 & 96.292 & 110.802 & & 74.177 \\
\hline $2 / 15 / 0820: 00$ & 125.502 & 123.059 & 100.782 & 104.668 & 96.301 & 110.795 & 81.147 & 74.171 \\
\hline $2 / 15 / 0820: 30$ & 125.504 & 123.047 & 100.775 & 104.664 & 96.296 & 110.791 & & 74.163 \\
\hline $2 / 15 / 0821: 00$ & 125.502 & 123.043 & 100.763 & 104.658 & 96.292 & 110.789 & & 74.157 \\
\hline $2 / 15 / 0821: 30$ & 125.49 & 123.036 & 100.758 & 104.645 & 96.278 & 110.785 & 81.137 & 74.15 \\
\hline 2/15/08 22:00 & 125.483 & 123.027 & 100.756 & 104.641 & 96.273 & 110.78 & & 74.142 \\
\hline $2 / 15 / 0822: 30$ & 125.478 & 123.022 & 100.744 & 104.634 & 96.266 & 110.778 & & 74.134 \\
\hline 2/15/08 23:00 & 125.468 & 123.011 & 100.737 & 104.628 & 96.259 & 110.774 & 81.13 & 74.126 \\
\hline 2/15/08 23:30 & 125.461 & 123 & 100.728 & 104.624 & 96.254 & 110.77 & & 74.117 \\
\hline $2 / 16 / 080: 00$ & 125.454 & 122.997 & 100.72 & 104.613 & 96.245 & 110.768 & & 74.109 \\
\hline $2 / 16 / 080: 30$ & 125.442 & 122.986 & 100.715 & 104.599 & 96.23 & 110.765 & 81.115 & 74.103 \\
\hline $2 / 16 / 081: 00$ & 125.44 & 122.968 & 100.703 & 104.601 & 96.233 & 110.761 & & 74.097 \\
\hline $2 / 16 / 08$ 1:30 & 125.428 & 122.945 & 100.696 & 104.588 & 96.216 & 110.759 & & 74.09 \\
\hline $2 / 16 / 082: 00$ & 125.416 & 122.923 & 100.673 & 104.571 & 96.2 & 110.759 & 81.098 & 74.082 \\
\hline 2/16/08 2:30 & 125.395 & 122.898 & 100.649 & 104.548 & 96.176 & 110.757 & & 74.078 \\
\hline 2/16/08 3:00 & 125.372 & 122.873 & 100.629 & 104.523 & 96.155 & 110.757 & & 74.07 \\
\hline $2 / 16 / 083: 30$ & 125.353 & 122.855 & 100.601 & 104.5 & 96.131 & 110.755 & 81.066 & 74.066 \\
\hline 2/16/08 4:00 & 125.324 & 122.843 & 100.579 & 104.474 & 96.106 & 110.755 & & 74.059 \\
\hline $2 / 16 / 084: 30$ & 125.305 & 122.841 & 100.567 & 104.459 & 96.087 & 110.751 & & 74.053 \\
\hline 2/16/08 5:00 & 125.286 & 122.834 & 100.554 & 104.445 & 96.072 & 110.748 & 81.034 & 74.047 \\
\hline 2/16/08 5:30 & 125.282 & 122.848 & 100.553 & 104.447 & 96.072 & 110.746 & & 74.041 \\
\hline $2 / 16 / 086: 00$ & 125.277 & 122.82 & 100.541 & 104.438 & 96.068 & 110.742 & & 74.039 \\
\hline 2/16/08 6:30 & 125.268 & 122.811 & 100.553 & 104.453 & 96.08 & 110.736 & 81.024 & 74.026 \\
\hline $2 / 16 / 087: 00$ & 125.268 & 122.798 & 100.541 & 104.424 & 96.054 & 110.738 & & 74.02 \\
\hline $2 / 16 / 087: 30$ & 125.258 & 122.789 & 100.522 & 104.415 & 96.042 & 110.738 & & 74.016 \\
\hline 2/16/08 8:00 & 125.239 & 122.78 & 100.508 & 104.4 & 96.028 & 110.736 & 80.995 & 74.01 \\
\hline $2 / 16 / 088: 30$ & 125.23 & 124.401 & 100.494 & 104.392 & 96.021 & 110.735 & & 74.002 \\
\hline 2/16/08 9:00 & 125.22 & 124.807 & 100.491 & 104.385 & 96.011 & 110.733 & & 73.999 \\
\hline 2/16/08 9:30 & 126.643 & 124.959 & 101.194 & 104.666 & 96.41 & 111.163 & 80.98 & 74.008 \\
\hline 2/16/08 10:00 & 127.265 & 125.022 & 101.423 & 105.053 & 96.822 & 111.561 & & 74.173 \\
\hline $2 / 16 / 08 \quad 10: 30$ & 127.473 & 125.065 & 101.521 & 105.203 & 96.975 & 111.719 & & 74.31 \\
\hline 2/16/08 11:00 & 127.568 & 125.097 & 101.576 & 105.262 & 97.034 & 111.811 & 81.015 & 74.407 \\
\hline $2 / 16 / 0811: 30$ & 127.624 & 123.197 & 101.602 & 105.312 & 97.081 & 111.867 & & 74.467 \\
\hline $2 / 16 / 08$ 12:00 & 127.664 & 122.977 & 101.094 & 105.34 & 97.114 & 111.906 & & 74.508 \\
\hline 2/16/08 12:30 & 125.948 & 122.855 & 100.759 & 104.767 & 96.4 & 111.21 & 81.007 & 74.438 \\
\hline $2 / 16 / 08$ 13:00 & 125.634 & 122.777 & 100.596 & 104.558 & 96.188 & 111.027 & & 74.322 \\
\hline 2/16/08 13:30 & 125.433 & 122.712 & 100.513 & 104.438 & 96.068 & 110.937 & & 74.25 \\
\hline $2 / 16 / 08$ 14:00 & 125.298 & 122.655 & 100.444 & 104.367 & 95.995 & 110.883 & 80.951 & 74.2 \\
\hline $2 / 16 / 08$ 14:30 & 125.208 & 122.61 & 100.382 & 104.302 & 95.931 & 110.828 & & 74.171 \\
\hline 2/16/08 15:00 & 125.14 & 122.585 & 100.332 & 104.247 & 95.875 & 110.807 & & 74.15 \\
\hline $2 / 16 / 08$ 15:30 & 125.083 & 122.562 & 100.299 & 104.203 & 95.83 & 110.796 & 80.89 & 74.142 \\
\hline $2 / 16 / 08$ 16:00 & 125.041 & 122.539 & 100.282 & 104.184 & 95.806 & 110.786 & & 74.136 \\
\hline 2/16/08 16:30 & 125.012 & 122.521 & 100.255 & 104.162 & 95.785 & 110.78 & & 74.128 \\
\hline $2 / 16 / 08 \quad 17: 00$ & 124.989 & 122.508 & 100.232 & 104.137 & 95.766 & 110.776 & 80.851 & 74.124 \\
\hline $2 / 16 / 08 \quad 17: 30$ & 124.967 & 122.478 & 100.225 & 104.118 & 95.745 & 110.774 & & 74.117 \\
\hline 2/16/08 18:00 & 124.953 & 122.458 & 100.201 & 104.106 & 95.733 & 110.77 & & 74.109 \\
\hline $2 / 16 / 08$ 18:30 & 124.934 & 122.433 & 100.17 & 104.076 & 95.705 & 110.772 & 80.807 & 74.103 \\
\hline $2 / 16 / 08$ 19:00 & 124.908 & 122.433 & 100.156 & 104.059 & 95.684 & 110.769 & & 74.097 \\
\hline 2/16/08 19:30 & 124.885 & 122.417 & 100.143 & 104.032 & 95.655 & 110.77 & & 74.093 \\
\hline
\end{tabular}


TABLE S1.1 (Cont.)

\begin{tabular}{|c|c|c|c|c|c|c|c|c|c|c|}
\hline \multirow[b]{2}{*}{ Date and Time } & \multicolumn{10}{|c|}{ Depth from Top of Casing (ft) } \\
\hline & MW1D & MW2D & MW3D & MW4D & MW7 & MW9 & Oentrich & MW14D & MW15D & MW16D \\
\hline 2/16/08 20:00 & 124.873 & 122.417 & & 100.136 & 104.036 & 95.662 & 110.766 & & 80.77 & 74.084 \\
\hline $2 / 16 / 0820: 30$ & 124.866 & 122.412 & & 100.125 & 104.02 & 95.646 & 110.768 & & & 74.08 \\
\hline 2/16/08 21:00 & 124.859 & 122.392 & & 100.129 & 104.019 & 95.646 & 110.765 & & & 74.074 \\
\hline $2 / 16 / 0821: 30$ & 124.856 & 122.372 & & 100.11 & 104.017 & 95.644 & 110.763 & & 80.736 & 74.068 \\
\hline 2/16/08 22:00 & 124.859 & 122.335 & & 100.091 & 103.996 & 95.62 & 110.755 & & & 74.066 \\
\hline 2/16/08 22:30 & 124.859 & 122.328 & & 100.067 & 103.971 & 95.599 & 110.744 & & & 74.062 \\
\hline 2/16/08 23:00 & 124.861 & 122.338 & & 100.046 & 103.939 & 95.561 & 110.723 & & 80.675 & 74.059 \\
\hline 2/16/08 23:30 & 124.859 & 122.297 & & 100.034 & 103.939 & 95.563 & 110.716 & & & 74.055 \\
\hline 2/17/08 0:00 & 124.852 & 122.272 & & 100.053 & 103.945 & 95.571 & 110.703 & & & 74.047 \\
\hline $2 / 17 / 080: 30$ & 124.863 & 122.251 & & 99.993 & 103.903 & 95.53 & 110.705 & & 80.626 & 74.047 \\
\hline $2 / 17 / 081: 00$ & 124.863 & 122.236 & & 99.977 & 103.876 & 95.497 & 110.673 & & & 74.045 \\
\hline 2/17/08 1:30 & 124.863 & 122.199 & & 99.979 & 103.861 & 95.486 & 110.663 & & & 74.043 \\
\hline $2 / 17 / 082: 00$ & 124.866 & 122.192 & & 99.92 & 103.844 & 95.471 & 110.656 & & 80.57 & 74.041 \\
\hline $2 / 17 / 082: 30$ & 124.866 & 122.179 & & 99.91 & 103.804 & 95.427 & 110.618 & & & 74.041 \\
\hline 2/17/08 3:00 & 124.866 & 122.161 & & 99.889 & 103.804 & 95.424 & 110.609 & & & 74.035 \\
\hline $2 / 17 / 083: 30$ & 124.866 & 122.158 & & 99.874 & 103.788 & 95.41 & 110.594 & & 80.521 & 74.033 \\
\hline $2 / 17 / 084: 00$ & 124.868 & 122.168 & & 99.879 & 103.769 & 95.396 & 110.581 & & & 74.03 \\
\hline $2 / 17 / 084: 30$ & 124.868 & 122.163 & & 99.872 & 103.769 & 95.391 & 110.575 & & & 74.024 \\
\hline 2/17/08 5:00 & 124.863 & 122.156 & & 99.881 & 103.777 & 95.403 & 110.568 & & 80.484 & 74.02 \\
\hline 2/17/08 5:30 & 124.868 & 122.149 & & 99.874 & 103.771 & 95.398 & 110.573 & & & 74.016 \\
\hline $2 / 17 / 086: 00$ & 124.868 & 122.158 & & 99.865 & 103.764 & 95.389 & 110.569 & & & 74.014 \\
\hline 2/17/08 6:30 & 124.87 & 122.158 & & 99.869 & 103.76 & 95.384 & 110.564 & & 80.445 & 74.012 \\
\hline $2 / 17 / 08$ 7:00 & 124.87 & 122.177 & & 99.872 & 103.769 & 95.394 & 110.566 & & & 74.008 \\
\hline $2 / 17 / 087: 30$ & 124.87 & 122.197 & & 99.883 & 103.769 & 95.396 & 110.567 & & & 74.006 \\
\hline 2/17/08 8:00 & 124.87 & 122.226 & & 99.901 & 103.788 & 95.415 & 110.566 & & 80.43 & 74.002 \\
\hline $2 / 17 / 088: 30$ & 124.868 & 122.263 & & 99.929 & 103.813 & 95.436 & 110.564 & & & 73.995 \\
\hline 2/17/08 9:00 & 124.868 & 124.102 & & 99.962 & 103.838 & 95.462 & 110.562 & & & 73.989 \\
\hline 2/17/08 9:30 & 124.866 & 124.442 & & 99.998 & 103.882 & 95.504 & 110.56 & & 80.438 & 73.983 \\
\hline 2/17/08 10:00 & 126.369 & 124.614 & & 100.775 & 104.327 & 96.07 & 111.097 & & & 74.018 \\
\hline $2 / 17 / 0810: 30$ & 126.646 & 124.723 & & 101.006 & 104.655 & 96.407 & 111.384 & & & 74.175 \\
\hline $2 / 17 / 08$ 11:00 & 126.771 & 124.791 & & 101.142 & 104.824 & 96.582 & 111.516 & & 80.518 & 74.285 \\
\hline $2 / 17 / 0811: 30$ & 126.844 & 124.85 & & 101.235 & 104.936 & 96.69 & 111.597 & & & 74.359 \\
\hline 2/17/08 12:00 & 126.894 & 124.891 & & 101.297 & 105.011 & 96.766 & 111.647 & & & 74.409 \\
\hline $2 / 17 / 0812: 30$ & 126.936 & 124.923 & & 101.342 & 105.066 & 96.824 & 111.685 & & 80.589 & 74.448 \\
\hline $2 / 17 / 0813: 00$ & 126.977 & 124.95 & & 101.38 & 105.112 & 96.867 & 111.713 & & & 74.479 \\
\hline 2/17/08 13:30 & 127.01 & 124.977 & & 101.418 & 105.142 & 96.902 & 111.734 & & & 74.516 \\
\hline $2 / 17 / 0814: 00$ & 127.073 & 125 & & 101.445 & 105.176 & 96.935 & 111.751 & & 80.646 & 74.636 \\
\hline 2/17/08 14:30 & 127.173 & 125.025 & & 101.464 & 105.201 & 96.959 & 111.764 & & & 74.793 \\
\hline $2 / 17 / 08$ 15:00 & 127.272 & 125.05 & & 101.495 & 105.222 & 96.982 & 111.775 & & & 74.957 \\
\hline $2 / 17 / 0815: 30$ & 127.374 & 123.449 & & 101.519 & 105.252 & 97.011 & 111.782 & & 80.702 & 75.103 \\
\hline 2/17/08 16:00 & 127.459 & 123.093 & & 101.556 & 105.279 & 97.037 & 111.79 & & & 75.24 \\
\hline $2 / 17 / 0816: 30$ & 126.121 & 122.961 & & 100.894 & 104.99 & 96.643 & 111.364 & & & 75.341 \\
\hline $2 / 17 / 08$ 17:00 & 125.615 & 122.891 & & 100.708 & 104.672 & 96.301 & 111.035 & & 80.729 & 74.87 \\
\hline 2/17/08 17:30 & 125.416 & 122.855 & & 100.622 & 104.544 & 96.174 & 110.874 & & & 74.758 \\
\hline $2 / 17 / 0818: 00$ & 125.334 & 122.841 & & 100.572 & 104.48 & 96.11 & 110.806 & & & 74.69 \\
\hline $2 / 17 / 0818: 30$ & 125.291 & 122.823 & & 100.548 & 104.447 & 96.077 & 110.765 & & 80.724 & 74.651 \\
\hline $2 / 17 / 08$ 19:00 & 125.272 & 122.82 & & 100.544 & 104.432 & 96.065 & 110.736 & & & 74.626 \\
\hline 2/17/08 19:30 & 125.263 & 122.809 & & 100.53 & 104.42 & 96.051 & 110.718 & & & 74.605 \\
\hline 2/17/08 20:00 & 125.256 & 122.8 & & 100.525 & 104.42 & 96.051 & 110.703 & & 80.738 & 74.599 \\
\hline 2/17/08 20:30 & 125.249 & 122.793 & & 100.518 & 104.409 & 96.042 & 110.693 & & & 74.585 \\
\hline $2 / 17 / 0821: 00$ & 125.242 & 122.789 & & 100.511 & 104.4 & 96.03 & 110.686 & & & 74.57 \\
\hline 2/17/08 21:30 & 125.234 & 122.789 & & 100.506 & 104.394 & 96.023 & 110.68 & & 80.736 & 74.558 \\
\hline 2/17/08 22:00 & 125.23 & 122.784 & & 100.504 & 104.392 & 96.021 & 110.675 & & & 74.549 \\
\hline 2/17/08 22:30 & 125.225 & 122.777 & & 100.506 & 104.39 & 96.021 & 110.669 & & & 74.543 \\
\hline 2/17/08 23:00 & 125.225 & 122.78 & & 100.499 & 104.39 & 96.021 & 110.665 & & 80.746 & 74.417 \\
\hline 2/17/08 23:30 & 125.218 & 122.787 & & 100.498 & 104.382 & 96.009 & 110.662 & & & 74.398 \\
\hline $2 / 18 / 080: 00$ & 125.218 & 122.789 & & 100.504 & 104.384 & 96.016 & 110.658 & & & 74.469 \\
\hline 2/18/08 0:30 & 125.223 & 122.789 & & 100.504 & 104.39 & 96.021 & 110.654 & & 80.746 & 74.494 \\
\hline 2/18/08 1:00 & 125.225 & 122.805 & & 100.511 & 104.394 & 96.023 & 110.652 & & & 74.576 \\
\hline 2/18/08 1:30 & 125.23 & 122.809 & & 100.53 & 104.396 & 96.025 & 110.65 & & & 74.649 \\
\hline 2/18/08 2:00 & 125.242 & 122.807 & & 100.527 & 104.415 & 96.044 & 110.647 & & 80.758 & 74.709 \\
\hline 2/18/08 2:30 & 125.246 & 122.812 & & 100.525 & 104.417 & 96.049 & 110.643 & & & 74.76 \\
\hline 2/18/08 3:00 & 125.244 & 122.809 & & 100.532 & 104.417 & 96.047 & 110.639 & & & 74.802 \\
\hline $2 / 18 / 083: 30$ & 125.249 & 122.802 & & 100.53 & 104.42 & 96.049 & 110.637 & & 80.756 & 74.831 \\
\hline 2/18/08 4:00 & 125.251 & 122.798 & & 100.53 & 104.42 & 96.051 & 110.635 & & & 74.853 \\
\hline 2/18/08 4:30 & 125.244 & 122.796 & & 100.522 & 104.413 & 96.042 & 110.633 & & & 74.876 \\
\hline
\end{tabular}


TABLE S1.1 (Cont.)

\begin{tabular}{|c|c|c|c|c|c|c|c|c|c|c|}
\hline \multirow[b]{2}{*}{ Date and Time } & \multicolumn{10}{|c|}{ Depth from Top of Casing (ft) } \\
\hline & MW1D & MW2D & MW3D & MW4D & MW7 & MW9 & Oentrich & MW14D & MW15D & MW16D \\
\hline 2/18/08 5:00 & 125.242 & 122.782 & & 100.52 & 104.407 & 96.037 & 110.631 & & 80.753 & 74.897 \\
\hline 2/18/08 5:30 & 125.237 & 122.78 & & 100.51 & 104.403 & 96.035 & 110.628 & & & 74.905 \\
\hline 2/18/08 6:00 & 125.227 & 122.777 & & 100.504 & 104.39 & 96.023 & 110.63 & & & 74.913 \\
\hline 2/18/08 6:30 & 125.223 & 124.065 & & 100.503 & 104.388 & 96.018 & 110.626 & & 80.743 & 74.913 \\
\hline $2 / 18 / 08$ 7:00 & 125.218 & 124.759 & & 100.494 & 104.386 & 96.014 & 110.624 & & & 74.913 \\
\hline 2/18/08 7:30 & 126.078 & 124.954 & & 101.116 & 104.432 & 96.054 & 110.755 & & & 74.917 \\
\hline 2/18/08 8:00 & 127.159 & 123.265 & & 101.411 & 105.018 & 96.787 & 111.392 & & 80.773 & 75.039 \\
\hline 2/18/08 8:30 & 127.442 & 123.047 & & 101.314 & 105.211 & 96.982 & 111.57 & & & 75.17 \\
\hline 2/18/08 9:00 & 125.868 & 122.959 & & 100.835 & 104.841 & 96.48 & 111.046 & & & 75.199 \\
\hline 2/18/08 9:30 & 125.532 & 122.913 & & 100.704 & 104.643 & 96.273 & 110.845 & & 80.8 & 75.114 \\
\hline $2 / 18 / 0810: 00$ & 125.409 & 122.898 & & 100.644 & 104.558 & 96.188 & 110.763 & & & 75.06 \\
\hline 2/18/08 10:30 & 125.357 & 122.895 & & 100.615 & 104.516 & 96.143 & 110.716 & & & 75.017 \\
\hline $2 / 18 / 0811: 00$ & 125.329 & 122.882 & & 100.61 & 104.497 & 96.124 & 110.684 & & 80.8 & 74.988 \\
\hline $2 / 18 / 0811: 30$ & 125.312 & 122.891 & & 100.615 & 104.5 & 96.127 & 110.663 & & & 74.965 \\
\hline 2/18/08 12:00 & 125.308 & 122.884 & & 100.608 & 104.483 & 96.113 & 110.654 & & & 74.562 \\
\hline $2 / 18 / 08$ 12:30 & 125.31 & 122.884 & & 100.61 & 104.497 & 96.127 & 110.643 & & 80.809 & 74.52 \\
\hline $2 / 18 / 08$ 13:00 & 125.32 & 122.873 & & 100.604 & 104.489 & 96.12 & 110.637 & & & 74.533 \\
\hline 2/18/08 13:30 & 125.32 & 122.87 & & 100.601 & 104.489 & 96.12 & 110.631 & & & 74.558 \\
\hline $2 / 18 / 0814: 00$ & 125.317 & 122.866 & & 100.591 & 104.48 & 96.113 & 110.63 & & 80.807 & 74.599 \\
\hline $2 / 18 / 0814: 30$ & 125.308 & 122.873 & & 100.587 & 104.476 & 96.106 & 110.624 & & & 74.628 \\
\hline 2/18/08 15:00 & 125.308 & 122.88 & & 100.589 & 104.474 & 96.101 & 110.622 & & & 74.626 \\
\hline $2 / 18 / 08$ 15:30 & 125.31 & 122.882 & & 100.598 & 104.48 & 96.11 & 110.618 & & 80.814 & 74.628 \\
\hline $2 / 18 / 08$ 16:00 & 125.315 & 122.879 & & 100.606 & 104.487 & 96.117 & 110.613 & & & 74.632 \\
\hline 2/18/08 16:30 & 125.322 & 122.884 & & 100.601 & 104.489 & 96.12 & 110.611 & & & 74.63 \\
\hline $2 / 18 / 08$ 17:00 & 125.317 & 122.889 & & 100.603 & 104.487 & 96.117 & 110.609 & & 80.814 & 74.624 \\
\hline $2 / 18 / 08$ 17:30 & 125.322 & 122.891 & & 100.61 & 104.491 & 96.122 & 110.607 & & & 74.622 \\
\hline 2/18/08 18:00 & 125.327 & 122.902 & & 100.613 & 104.497 & 96.127 & 110.607 & & & 74.62 \\
\hline 2/18/08 18:30 & 125.331 & 122.911 & & 100.618 & 104.499 & 96.129 & 110.605 & & 80.819 & 74.62 \\
\hline 2/18/08 19:00 & 125.336 & 122.925 & & 100.63 & 104.51 & 96.139 & 110.599 & & & 74.518 \\
\hline 2/18/08 19:30 & 125.346 & 122.932 & & 100.644 & 104.519 & 96.15 & 110.597 & & & 74.514 \\
\hline 2/18/08 20:00 & 125.36 & 122.936 & & 100.653 & 104.533 & 96.162 & 110.596 & & 80.834 & 74.514 \\
\hline $2 / 18 / 0820: 30$ & 125.364 & 122.945 & & 100.658 & 104.54 & 96.172 & 110.594 & & & 74.512 \\
\hline 2/18/08 21:00 & 125.372 & 122.954 & & 100.663 & 104.546 & 96.174 & 110.592 & & & 74.514 \\
\hline $2 / 18 / 0821: 30$ & 125.379 & 122.966 & & 100.672 & 104.554 & 96.186 & 110.588 & & 80.851 & 74.514 \\
\hline 2/18/08 22:00 & 125.388 & 122.966 & & 100.687 & 104.563 & 96.195 & 110.586 & & & 74.512 \\
\hline 2/18/08 22:30 & 125.398 & 122.963 & & 100.689 & 104.573 & 96.204 & 110.584 & & & 74.512 \\
\hline 2/18/08 23:00 & 125.402 & 122.968 & & 100.691 & 104.573 & 96.204 & 110.581 & & 80.866 & 74.51 \\
\hline 2/18/08 23:30 & 125.4 & 122.97 & & 100.692 & 104.577 & 96.204 & 110.579 & & & 74.51 \\
\hline $2 / 19 / 080: 00$ & 125.405 & 122.972 & & 100.692 & 104.579 & 96.209 & 110.577 & & & 74.508 \\
\hline 2/19/08 0:30 & 125.405 & 122.975 & & 100.697 & 104.579 & 96.209 & 110.571 & & 80.878 & 74.51 \\
\hline 2/19/08 1:00 & 125.412 & 122.975 & & 100.697 & 104.582 & 96.214 & 110.571 & & & 74.506 \\
\hline $2 / 19 / 08 \quad 1: 30$ & 125.409 & 122.977 & & 100.704 & 104.584 & 96.214 & 110.568 & & & 74.506 \\
\hline 2/19/08 2:00 & 125.412 & 122.975 & & 100.704 & 104.584 & 96.219 & 110.566 & & 80.885 & 74.502 \\
\hline 2/19/08 2:30 & 125.414 & 122.97 & & 100.701 & 104.59 & 96.221 & 110.562 & & & 74.498 \\
\hline 2/19/08 3:00 & 125.414 & 122.954 & & 100.694 & 104.584 & 96.216 & 110.56 & & & 74.496 \\
\hline 2/19/08 3:30 & 125.407 & 122.952 & & 100.694 & 104.581 & 96.212 & 110.556 & & 80.88 & 74.492 \\
\hline 2/19/08 4:00 & 125.405 & 122.934 & & 100.666 & 104.567 & 96.2 & 110.556 & & & 74.492 \\
\hline 2/19/08 4:30 & 125.386 & 122.927 & & 100.668 & 104.563 & 96.193 & 110.551 & & & 74.492 \\
\hline 2/19/08 5:00 & 125.379 & 122.923 & & 100.66 & 104.544 & 96.172 & 110.551 & & 80.868 & 74.489 \\
\hline 2/19/08 5:30 & 125.369 & 122.909 & & 100.647 & 104.541 & 96.169 & 110.547 & & & 74.489 \\
\hline 2/19/08 6:00 & 125.364 & 122.9 & & 100.641 & 104.533 & 96.164 & 110.545 & & & 74.487 \\
\hline 2/19/08 6:30 & 125.353 & 122.886 & & 100.63 & 104.522 & 96.153 & 110.543 & & 80.858 & 74.489 \\
\hline 2/19/08 7:00 & 125.341 & 124.73 & & 100.62 & 104.508 & 96.141 & 110.541 & & & 74.492 \\
\hline $2 / 19 / 087: 30$ & 125.331 & 124.995 & & 100.765 & 104.495 & 96.127 & 110.541 & & & 74.481 \\
\hline 2/19/08 8:00 & 127.059 & 125.12 & & 101.364 & 104.944 & 96.695 & 111.12 & & 80.861 & 74.52 \\
\hline 2/19/08 8:30 & 127.456 & 125.19 & & 101.54 & 105.205 & 96.961 & 111.381 & & & 74.669 \\
\hline 2/19/08 9:00 & 127.605 & 125.229 & & 101.638 & 105.329 & 97.088 & 111.501 & & & 74.771 \\
\hline 2/19/08 9:30 & 127.683 & 125.263 & & 101.692 & 105.403 & 97.164 & 111.576 & & 80.919 & 74.837 \\
\hline 2/19/08 10:00 & 127.733 & 125.267 & & 101.724 & 105.441 & 97.202 & 111.625 & & & 74.886 \\
\hline $2 / 19 / 08 \quad 10: 30$ & 127.764 & 125.261 & & 101.742 & 105.475 & 97.237 & 111.659 & & & 74.94 \\
\hline $2 / 19 / 0811: 00$ & 127.776 & 123.832 & & 101.743 & 105.479 & 97.242 & 111.681 & & 80.956 & 74.975 \\
\hline 2/19/08 11:30 & 127.776 & 123.256 & & 101.721 & 105.481 & 97.244 & 111.702 & & & 74.977 \\
\hline $2 / 19 / 08$ 12:00 & 126.698 & 123.079 & & 101.104 & 105.308 & 97.004 & 111.476 & & & 74.955 \\
\hline $2 / 19 / 08$ 12:30 & 126.114 & 122.97 & & 100.842 & 104.83 & 96.464 & 110.994 & & 80.934 & 74.704 \\
\hline 2/19/08 13:00 & 125.875 & 122.891 & & 100.716 & 104.657 & 96.289 & 110.836 & & & 74.529 \\
\hline 2/19/08 13:30 & 125.717 & 122.834 & & 100.622 & 104.556 & 96.186 & 110.754 & & & 74.432 \\
\hline
\end{tabular}


TABLE S1.1 (Cont.)

\begin{tabular}{|c|c|c|c|c|c|c|c|c|c|c|}
\hline \multirow[b]{2}{*}{ Date and Time } & \multicolumn{10}{|c|}{ Depth from Top of Casing (ft) } \\
\hline & MW1D & MW2D & MW3D & MW4D & MW7 & MW9 & Oentrich & MW14D & MW15D & MW16D \\
\hline 2/19/08 14:00 & 125.584 & 122.796 & & 100.56 & 104.48 & 96.108 & 110.703 & & 80.885 & 74.403 \\
\hline $2 / 19 / 0814: 30$ & 125.466 & 122.757 & & 100.518 & 104.424 & 96.056 & 110.669 & & & 74.392 \\
\hline 2/19/08 15:00 & 125.369 & 122.737 & & 100.482 & 104.388 & 96.016 & 110.645 & & & 74.39 \\
\hline $2 / 19 / 08$ 15:30 & 125.296 & 122.714 & & 100.453 & 104.354 & 95.981 & 110.628 & & 80.841 & 74.396 \\
\hline $2 / 19 / 0816: 00$ & 125.232 & 122.7 & & 100.432 & 104.335 & 95.964 & 110.615 & & & 74.411 \\
\hline 2/19/08 16:30 & 125.192 & 122.691 & & 100.417 & 104.314 & 95.94 & 110.605 & & & 74.419 \\
\hline $2 / 19 / 08$ 17:00 & 125.159 & 122.684 & & 100.406 & 104.301 & 95.926 & 110.594 & & 80.812 & 74.423 \\
\hline $2 / 19 / 08$ 17:30 & 125.14 & 122.687 & & 100.396 & 104.291 & 95.919 & 110.586 & & & 74.423 \\
\hline 2/19/08 18:00 & 125.123 & 122.696 & & 100.396 & 104.287 & 95.912 & 110.582 & & & 74.421 \\
\hline $2 / 19 / 08$ 18:30 & 125.121 & 122.696 & & 100.405 & 104.291 & 95.919 & 110.575 & & 80.787 & 74.413 \\
\hline 2/19/08 19:00 & 125.126 & 122.707 & & 100.405 & 104.297 & 95.926 & 110.573 & & & 74.405 \\
\hline 2/19/08 19:30 & 125.128 & 122.725 & & 100.417 & 104.299 & 95.926 & 110.57 & & & 74.396 \\
\hline $2 / 19 / 0820: 00$ & 125.138 & 122.755 & & 100.432 & 104.316 & 95.94 & 110.564 & & 80.785 & 74.386 \\
\hline $2 / 19 / 0820: 30$ & 125.152 & 122.78 & & 100.455 & 104.329 & 95.955 & 110.562 & & & 74.386 \\
\hline 2/19/08 21:00 & 125.175 & 122.805 & & 100.482 & 104.361 & 95.99 & 110.556 & & & 74.386 \\
\hline $2 / 19 / 0821: 30$ & 125.204 & 122.816 & & 100.513 & 104.386 & 96.014 & 110.553 & & 80.802 & 74.186 \\
\hline 2/19/08 22:00 & 125.23 & 122.83 & & 100.532 & 104.411 & 96.039 & 110.549 & & & 74.167 \\
\hline 2/19/08 22:30 & 125.251 & 122.85 & & 100.541 & 104.424 & 96.051 & 110.549 & & & 74.183 \\
\hline 2/19/08 23:00 & 125.258 & 122.868 & & 100.56 & 104.438 & 96.065 & 110.547 & & 80.812 & 74.192 \\
\hline 2/19/08 23:30 & 125.277 & 122.889 & & 100.579 & 104.46 & 96.087 & 110.543 & & & 74.21 \\
\hline $2 / 20 / 080: 00$ & 125.294 & 122.898 & & 100.599 & 104.476 & 96.106 & 110.543 & & & 74.225 \\
\hline 2/20/08 0:30 & 125.312 & 122.913 & & 100.613 & 104.497 & 96.127 & 110.54 & & 80.834 & 74.241 \\
\hline 2/20/08 1:00 & 125.327 & 122.929 & & 100.622 & 104.508 & 96.139 & 110.538 & & & 74.25 \\
\hline 2/20/08 1:30 & 125.336 & 122.943 & & 100.642 & 104.523 & 96.153 & 110.536 & & & 74.26 \\
\hline 2/20/08 2:00 & 125.353 & 122.95 & & 100.66 & 104.539 & 96.172 & 110.532 & & 80.851 & 74.276 \\
\hline 2/20/08 2:30 & 125.372 & 122.968 & & 100.672 & 104.558 & 96.19 & 110.53 & & & 74.291 \\
\hline 2/20/08 3:00 & 125.386 & 122.977 & & 100.684 & 104.565 & 96.2 & 110.526 & & & 74.299 \\
\hline 2/20/08 3:30 & 125.395 & 122.988 & & 100.699 & 104.58 & 96.212 & 110.555 & & 80.866 & 74.31 \\
\hline 2/20/08 4:00 & 125.407 & 122.993 & & 100.701 & 104.59 & 96.221 & 110.568 & & & 74.32 \\
\hline $2 / 20 / 084: 30$ & 125.416 & 123.002 & & 100.716 & 104.603 & 96.23 & 110.571 & & & 74.326 \\
\hline 2/20/08 5:00 & 125.426 & 123.013 & & 100.72 & 104.603 & 96.235 & 110.579 & & 80.875 & 74.339 \\
\hline $\begin{array}{l}2 / 20 / 085: 30 \\
2\end{array}$ & 125.431 & 123.029 & & 100.728 & 104.617 & 96.249 & 110.594 & & & 74.341 \\
\hline 2/20/08 6:00 & 125.442 & 123.043 & & 100.753 & 104.628 & 96.261 & 110.605 & & & 74.355 \\
\hline 2/20/08 6:30 & 125.454 & 123.05 & & 100.763 & 104.645 & 96.275 & 110.622 & & 80.89 & 74.37 \\
\hline 2/20/08 7:00 & 125.468 & 124.671 & & 100.778 & 104.659 & 96.287 & 110.633 & & & 74.38 \\
\hline 2/20/08 7:30 & 125.485 & 125.14 & & 100.782 & 104.664 & 96.296 & 110.647 & & & 74.394 \\
\hline 2/20/08 8:00 & 126.865 & 125.322 & & 101.514 & 104.95 & 96.692 & 111.061 & & 80.919 & 74.409 \\
\hline 2/20/08 8:30 & 127.551 & 125.435 & & 101.781 & 105.395 & 97.169 & 111.499 & & & 74.603 \\
\hline 2/20/08 9:00 & 127.816 & 125.508 & & 101.916 & 105.57 & 97.345 & 111.666 & & & 74.773 \\
\hline 2/20/08 9:30 & 127.936 & 125.567 & & 102.014 & 105.687 & 97.461 & 111.771 & & 81.02 & 74.884 \\
\hline 2/20/08 10:00 & 128.024 & 125.61 & & 102.072 & 105.763 & 97.541 & 111.848 & & & 74.967 \\
\hline $2 / 20 / 0810: 30$ & 128.081 & 123.718 & & 102.126 & 105.822 & 97.598 & 111.903 & & & 75.025 \\
\hline 2/20/08 11:00 & 128.13 & 123.499 & & 101.637 & 105.866 & 97.64 & 111.944 & & 81.098 & 75.075 \\
\hline 2/20/08 11:30 & 126.308 & 123.383 & & 101.282 & 105.289 & 96.928 & 111.255 & & & 74.988 \\
\hline $2 / 20 / 0812: 00$ & 125.977 & 123.308 & & 101.142 & 105.081 & 96.711 & 111.06 & & & 74.833 \\
\hline $2 / 20 / 08$ 12:30 & 125.842 & 123.251 & & 101.051 & 104.975 & 96.605 & 110.964 & & 81.081 & 74.731 \\
\hline 2/20/08 13:00 & 125.783 & 123.204 & & 100.992 & 104.902 & 96.537 & 110.906 & & & 74.651 \\
\hline $2 / 20 / 08$ 13:30 & 125.752 & 123.165 & & 100.942 & 104.849 & 96.48 & 110.868 & & & 74.597 \\
\hline $2 / 20 / 0814: 00$ & 125.726 & 123.131 & & 100.897 & 104.801 & 96.433 & 110.842 & & 81.066 & 74.551 \\
\hline 2/20/08 14:30 & 125.7 & 123.113 & & 100.866 & 104.769 & 96.398 & 110.821 & & & 74.516 \\
\hline $2 / 20 / 08$ 15:00 & 125.679 & 123.097 & & 100.837 & 104.731 & 96.367 & 110.808 & & & 74.483 \\
\hline $2 / 20 / 08$ 15:30 & 125.667 & 123.084 & & 100.822 & 104.716 & 96.346 & 110.795 & & 81.046 & 74.465 \\
\hline 2/20/08 16:00 & 125.648 & 123.068 & & 100.809 & 104.698 & 96.329 & 110.785 & & & 74.452 \\
\hline $2 / 20 / 0816: 30$ & 125.639 & 123.065 & & 100.792 & 104.683 & 96.315 & 110.776 & & & 74.438 \\
\hline 2/20/08 17:00 & 125.617 & 123.052 & & 100.782 & 104.67 & 96.299 & 110.767 & & 81.029 & 74.423 \\
\hline 2/20/08 17:30 & 125.589 & 123.038 & & 100.775 & 104.67 & 96.301 & 110.761 & & & 74.423 \\
\hline $2 / 20 / 0818: 00$ & 125.565 & 123.041 & & 100.763 & 104.653 & 96.282 & 110.754 & & & 74.409 \\
\hline 2/20/08 18:30 & 125.542 & 123.043 & & 100.756 & 104.645 & 96.275 & 110.752 & & 81.024 & 74.401 \\
\hline 2/20/08 19:00 & 125.516 & 123.036 & & 100.761 & 104.642 & 96.27 & 110.744 & & & 74.396 \\
\hline $2 / 20 / 08$ 19:30 & 125.502 & 123.013 & & 100.763 & 104.651 & 96.28 & 110.738 & & & 74.407 \\
\hline $2 / 20 / 0820: 00$ & 125.492 & 123.009 & & 100.741 & 104.642 & 96.27 & 110.736 & & 81.015 & 74.401 \\
\hline 2/20/08 20:30 & 125.48 & 123 & & 100.727 & 104.622 & 96.252 & 110.735 & & & 74.38 \\
\hline 2/20/08 21:00 & 125.466 & 122.988 & & 100.722 & 104.613 & 96.245 & 110.729 & & & 74.37 \\
\hline $2 / 20 / 0821: 30$ & 125.457 & 122.97 & & 100.718 & 104.605 & 96.235 & 110.727 & & 81.002 & 74.363 \\
\hline 2/20/08 22:00 & 125.447 & 122.968 & & 100.697 & 104.596 & 96.226 & 110.725 & & & 74.357 \\
\hline 2/20/08 22:30 & 125.438 & 122.975 & & 100.689 & 104.575 & 96.204 & 110.725 & & & 74.336 \\
\hline
\end{tabular}


TABLE S1.1 (Cont.)

\begin{tabular}{|c|c|c|c|c|c|c|c|c|c|c|}
\hline \multirow[b]{2}{*}{ Date and Time } & \multicolumn{10}{|c|}{ Depth from Top of Casing (ft) } \\
\hline & MW1D & MW2D & MW3D & MW4D & MW7 & MW9 & Oentrich & MW14D & MW15D & MW16D \\
\hline 2/20/08 23:00 & 125.424 & 122.975 & & 100.687 & 104.575 & 96.207 & 110.72 & & 81.005 & 74.339 \\
\hline 2/20/08 23:30 & 125.416 & 122.968 & & 100.697 & 104.581 & 96.209 & 110.716 & & & 74.343 \\
\hline 2/21/08 0:00 & 125.414 & 122.947 & & 100.689 & 104.581 & 96.209 & 110.714 & & & 74.343 \\
\hline $2 / 21 / 080: 30$ & 125.412 & 122.934 & & 100.675 & 104.577 & 96.204 & 110.71 & & 81.005 & 74.336 \\
\hline $2 / 21 / 081: 00$ & 125.407 & 122.927 & & 100.663 & 104.554 & 96.186 & 110.71 & & & 74.316 \\
\hline $2 / 21 / 081: 30$ & 125.398 & 122.904 & & 100.649 & 104.541 & 96.172 & 110.709 & & & 74.305 \\
\hline $2 / 21 / 082: 00$ & 125.386 & 122.904 & & 100.639 & 104.533 & 96.162 & 110.705 & & 80.983 & 74.297 \\
\hline $2 / 21 / 082: 30$ & 125.376 & 122.893 & & 100.615 & 104.512 & 96.141 & 110.706 & & & 74.279 \\
\hline 2/21/08 3:00 & 125.357 & 122.866 & & 100.62 & 104.51 & 96.139 & 110.699 & & & 74.274 \\
\hline $2 / 21 / 083: 30$ & 125.353 & 122.855 & & 100.601 & 104.5 & 96.127 & 110.697 & & 80.951 & 74.268 \\
\hline 2/21/08 4:00 & 125.341 & 122.836 & & 100.587 & 104.472 & 96.098 & 110.697 & & & 74.241 \\
\hline 2/21/08 4:30 & 125.327 & 122.82 & & 100.567 & 104.468 & 96.094 & 110.695 & & & 74.237 \\
\hline $2 / 21 / 085: 00$ & 125.312 & 122.793 & & 100.548 & 104.445 & 96.07 & 110.693 & & 80.912 & 74.214 \\
\hline 2/21/08 5:30 & 125.298 & 122.784 & & 100.527 & 104.43 & 96.056 & 110.692 & & & 74.202 \\
\hline 2/21/08 6:00 & 125.282 & 122.782 & & 100.508 & 104.404 & 96.028 & 110.69 & & & 74.175 \\
\hline $2 / 21 / 086: 30$ & 125.26 & 122.777 & & 100.506 & 104.394 & 96.021 & 110.686 & & 80.885 & 74.167 \\
\hline $2 / 21 / 08$ 7:00 & 125.249 & 124.644 & & 100.506 & 104.39 & 96.014 & 110.686 & & & 74.163 \\
\hline $2 / 21 / 087: 30$ & 125.239 & 124.913 & & 100.727 & 104.385 & 96.014 & 110.682 & & & 74.159 \\
\hline 2/21/08 8:00 & 126.839 & 125.047 & & 101.276 & 104.856 & 96.603 & 111.26 & & 80.885 & 74.221 \\
\hline $2 / 21 / 088: 30$ & 127.163 & 125.124 & & 101.464 & 105.121 & 96.874 & 111.509 & & & 74.407 \\
\hline 2/21/08 9:00 & 127.348 & 125.174 & & 101.569 & 105.26 & 97.015 & 111.633 & & & 74.541 \\
\hline 2/21/08 9:30 & 127.475 & 125.211 & & 101.628 & 105.338 & 97.091 & 111.71 & & 80.936 & 74.622 \\
\hline $2 / 21 / 0810: 00$ & 127.56 & 125.222 & & 101.671 & 105.39 & 97.145 & 111.758 & & & 74.675 \\
\hline $2 / 21 / 0810: 30$ & 127.627 & 125.233 & & 101.695 & 105.426 & 97.183 & 111.792 & & & 74.715 \\
\hline $2 / 21 / 0811: 00$ & 127.674 & 125.242 & & 101.707 & 105.439 & 97.195 & 111.82 & & 80.973 & 74.729 \\
\hline $2 / 21 / 0811: 30$ & 127.705 & 125.245 & & 101.723 & 105.452 & 97.206 & 111.837 & & & 74.746 \\
\hline 2/21/08 12:00 & 127.726 & 123.925 & & 101.726 & 105.464 & 97.22 & 111.852 & & & 74.76 \\
\hline $2 / 21 / 08$ 12:30 & 127.74 & 123.283 & & 101.73 & 105.47 & 97.225 & 111.863 & & 80.998 & 74.769 \\
\hline 2/21/08 13:00 & 126.78 & 123.086 & & 101.135 & 105.372 & 97.096 & 111.7 & & & 74.766 \\
\hline $2 / 21 / 08$ 13:30 & 126.033 & 122.975 & & 100.854 & 104.855 & 96.485 & 111.161 & & & 74.618 \\
\hline $2 / 21 / 0814: 00$ & 125.795 & 122.9 & & 100.713 & 104.666 & 96.292 & 111 & & 80.961 & 74.46 \\
\hline $2 / 21 / 0814: 30$ & 125.638 & 122.845 & & 100.625 & 104.558 & 96.183 & 110.913 & & & 74.353 \\
\hline $2 / 21 / 08$ 15:00 & 125.525 & 122.8 & & 100.573 & 104.489 & 96.115 & 110.859 & & & 74.281 \\
\hline $2 / 21 / 0815: 30$ & 125.44 & 122.777 & & 100.517 & 104.436 & 96.063 & 110.825 & & 80.91 & 74.229 \\
\hline 2/21/08 16:00 & 125.372 & 122.762 & & 100.494 & 104.394 & 96.018 & 110.801 & & & 74.183 \\
\hline $2 / 21 / 0816: 30$ & 125.317 & 122.755 & & 100.477 & 104.371 & 95.997 & 110.78 & & & 74.161 \\
\hline $2 / 21 / 08$ 17:00 & 125.277 & 122.746 & & 100.467 & 104.36 & 95.988 & 110.763 & & 80.873 & 74.146 \\
\hline 2/21/08 17:30 & 125.244 & 122.732 & & 100.46 & 104.356 & 95.983 & 110.752 & & & 74.138 \\
\hline $2 / 21 / 0818: 00$ & 125.22 & 122.723 & & 100.451 & 104.346 & 95.973 & 110.742 & & & 74.128 \\
\hline $2 / 21 / 08$ 18:30 & 125.206 & 122.718 & & 100.437 & 104.337 & 95.962 & 110.737 & & 80.858 & 74.113 \\
\hline 2/21/08 19:00 & 125.19 & 122.719 & & 100.432 & 104.325 & 95.952 & 110.731 & & & 74.105 \\
\hline $2 / 21 / 0819: 30$ & 125.175 & 122.716 & & 100.43 & 104.325 & 95.95 & 110.725 & & & 74.101 \\
\hline 2/21/08 20:00 & 125.168 & 122.709 & & 100.429 & 104.321 & 95.95 & 110.72 & & 80.824 & 74.101 \\
\hline 2/21/08 20:30 & 125.161 & 122.705 & & 100.425 & 104.32 & 95.945 & 110.718 & & & 74.095 \\
\hline $2 / 21 / 0821: 00$ & 125.159 & 122.705 & & 100.424 & 104.316 & 95.94 & 110.714 & & & 74.088 \\
\hline 2/21/08 21:30 & 125.154 & 122.712 & & 100.42 & 104.31 & 95.938 & 110.712 & & 80.819 & 74.084 \\
\hline $2 / 21 / 0822: 00$ & 125.147 & 122.721 & & 100.422 & 104.31 & 95.938 & 110.71 & & & 74.084 \\
\hline 2/21/08 22:30 & 125.145 & 122.721 & & 100.432 & 104.32 & 95.945 & 110.707 & & & 74.09 \\
\hline 2/21/08 23:00 & 125.147 & 122.723 & & 100.434 & 104.325 & 95.952 & 110.703 & & 80.812 & 74.097 \\
\hline 2/21/08 23:30 & 125.149 & 122.716 & & 100.437 & 104.324 & 95.952 & 110.703 & & & 74.093 \\
\hline $2 / 22 / 080: 00$ & 125.149 & 122.707 & & 100.436 & 104.329 & 95.955 & 110.701 & & & 74.095 \\
\hline 2/22/08 0:30 & 125.154 & 122.696 & & 100.427 & 104.322 & 95.948 & 110.701 & & 80.802 & 74.088 \\
\hline 2/22/08 1:00 & 125.152 & 122.689 & & 100.418 & 104.312 & 95.94 & 110.701 & & & 74.08 \\
\hline $2 / 22 / 081: 30$ & 125.149 & 122.687 & & 100.41 & 104.305 & 95.929 & 110.699 & & & 74.07 \\
\hline 2/22/08 2:00 & 125.145 & 122.685 & & 100.408 & 104.295 & 95.924 & 110.697 & & 80.785 & 74.064 \\
\hline 2/22/08 2:30 & 125.138 & 122.682 & & 100.403 & 104.295 & 95.922 & 110.695 & & & 74.062 \\
\hline 2/22/08 3:00 & 125.133 & 122.675 & & 100.399 & 104.291 & 95.919 & 110.693 & & & 74.059 \\
\hline 2/22/08 3:30 & 125.128 & 122.673 & & 100.398 & 104.289 & 95.917 & 110.691 & & 80.753 & 74.057 \\
\hline 2/22/08 4:00 & 125.123 & 122.669 & & 100.394 & 104.285 & 95.91 & 110.69 & & & 74.051 \\
\hline 2/22/08 4:30 & 125.119 & 122.673 & & 100.389 & 104.283 & 95.91 & 110.69 & & & 74.049 \\
\hline $2 / 22 / 085: 00$ & 125.116 & 122.671 & & 100.392 & 104.28 & 95.905 & 110.688 & & 80.736 & 74.047 \\
\hline 2/22/08 5:30 & 125.114 & 122.669 & & 100.391 & 104.283 & 95.907 & 110.684 & & & 74.047 \\
\hline 2/22/08 6:00 & 125.112 & 122.671 & & 100.389 & 104.281 & 95.905 & 110.684 & & & 74.043 \\
\hline $2 / 22 / 086: 30$ & 125.109 & 122.671 & & 100.387 & 104.281 & 95.905 & 110.682 & & 80.734 & 74.041 \\
\hline 2/22/08 7:00 & 125.109 & 124.619 & & 100.387 & 104.281 & 95.905 & 110.678 & & & 74.045 \\
\hline $2 / 22 / 08$ 7:30 & 125.109 & 124.845 & & 100.909 & 104.28 & 95.907 & 110.678 & & & 74.043 \\
\hline
\end{tabular}


TABLE S1.1 (Cont.)

Depth from Top of Casing (ft)

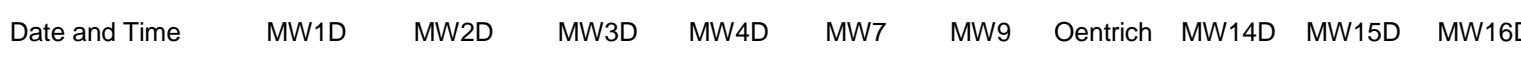

\begin{tabular}{|c|c|c|c|c|c|c|c|c|}
\hline 2/22/08 8:00 & 126.823 & 124.97 & 101.273 & 104.881 & 96.643 & 111.394 & 80.756 & 74.171 \\
\hline 2/22/08 8:30 & 127.109 & 125.052 & 101.43 & 105.106 & 96.872 & 111.604 & & 74.351 \\
\hline 2/22/08 9:00 & 127.281 & 123.397 & 101.519 & 105.232 & 96.997 & 111.713 & & 74.475 \\
\hline 2/22/08 9:30 & 127.404 & 123.088 & 101.578 & 105.31 & 97.077 & 111.784 & 80.817 & 74.556 \\
\hline 2/22/08 10:00 & 126.026 & 122.961 & 100.889 & 104.961 & 96.601 & 111.289 & & 74.572 \\
\hline 2/22/08 10:30 & 125.7 & 122.895 & 100.713 & 104.676 & 96.301 & 111.009 & & 74.429 \\
\hline $2 / 22 / 0811: 00$ & 125.551 & 122.852 & 100.629 & 104.556 & 96.181 & 110.893 & 80.802 & 74.324 \\
\hline $2 / 22 / 08 \quad 11: 30$ & 125.471 & 122.827 & 100.579 & 104.495 & 96.12 & 110.831 & & 74.258 \\
\hline $2 / 22 / 0812: 00$ & 125.44 & 122.8 & 100.551 & 104.453 & 96.08 & 110.791 & & 74.217 \\
\hline $2 / 22 / 08$ 12:30 & 125.424 & 122.773 & 100.525 & 104.426 & 96.054 & 110.769 & 80.78 & 74.19 \\
\hline 2/22/08 13:00 & 125.412 & 122.748 & 100.499 & 104.401 & 96.03 & 110.752 & & 74.165 \\
\hline $2 / 22 / 08$ 13:30 & 125.402 & 122.725 & 100.475 & 104.377 & 96.004 & 110.74 & & 74.136 \\
\hline $2 / 22 / 08$ 14:00 & 125.395 & 122.707 & 100.453 & 104.352 & 95.983 & 110.733 & 80.758 & 74.113 \\
\hline 2/22/08 14:30 & 125.379 & 122.696 & 100.43 & 104.331 & 95.957 & 110.725 & & 74.09 \\
\hline $2 / 22 / 08$ 15:00 & 125.362 & 122.684 & 100.415 & 104.312 & 95.94 & 110.72 & & 74.072 \\
\hline $2 / 22 / 08$ 15:30 & 125.353 & 122.678 & 100.405 & 104.301 & 95.929 & 110.714 & 80.731 & 74.062 \\
\hline 2/22/08 16:00 & 125.338 & 122.669 & 100.396 & 104.293 & 95.919 & 110.709 & & 74.051 \\
\hline 2/22/08 16:30 & 125.329 & 122.666 & 100.391 & 104.285 & 95.912 & 110.705 & & 74.045 \\
\hline $2 / 22 / 08$ 17:00 & 125.315 & 122.664 & 100.387 & 104.278 & 95.905 & 110.703 & 80.712 & 74.037 \\
\hline $2 / 22 / 08$ 17:30 & 125.305 & 122.66 & 100.384 & 104.274 & 95.9 & 110.699 & & 74.033 \\
\hline 2/22/08 18:00 & 125.291 & 122.657 & 100.38 & 104.274 & 95.901 & 110.695 & & 74.033 \\
\hline 2/22/08 18:30 & 125.282 & 122.66 & 100.377 & 104.27 & 95.896 & 110.692 & 80.694 & 74.026 \\
\hline 2/22/08 19:00 & 125.268 & 122.666 & 100.38 & 104.268 & 95.893 & 110.69 & & 74.026 \\
\hline 2/22/08 19:30 & 125.26 & 122.669 & 100.382 & 104.27 & 95.896 & 110.688 & & 74.028 \\
\hline $2 / 22 / 0820: 00$ & 125.249 & 122.671 & 100.389 & 104.276 & 95.9 & 110.684 & 80.685 & 74.03 \\
\hline 2/22/08 20:30 & 125.239 & 122.675 & 100.391 & 104.28 & 95.905 & 110.684 & & 74.035 \\
\hline 2/22/08 21:00 & 125.234 & 122.678 & 100.394 & 104.281 & 95.907 & 110.682 & & 74.035 \\
\hline 2/22/08 21:30 & 125.227 & 122.682 & 100.399 & 104.289 & 95.912 & 110.682 & 80.68 & 74.039 \\
\hline 2/22/08 22:00 & 125.223 & 122.685 & 100.405 & 104.289 & 95.915 & 110.68 & & 74.041 \\
\hline 2/22/08 22:30 & 125.216 & 122.685 & 100.405 & 104.295 & 95.922 & 110.68 & & 74.043 \\
\hline 2/22/08 23:00 & 125.211 & 122.682 & 100.405 & 104.295 & 95.922 & 110.682 & 80.682 & 74.047 \\
\hline 2/22/08 23:30 & 125.206 & 122.68 & 100.408 & 104.295 & 95.919 & 110.68 & & 74.045 \\
\hline 2/23/08 0:00 & 125.204 & 122.687 & 100.403 & 104.295 & 95.922 & 110.68 & & 74.045 \\
\hline $2 / 23 / 080: 30$ & 125.199 & 122.689 & 100.406 & 104.291 & 95.917 & 110.678 & 80.675 & 74.041 \\
\hline $2 / 23 / 081: 00$ & 125.194 & 122.689 & 100.413 & 104.297 & 95.924 & 110.678 & & 74.047 \\
\hline $2 / 23 / 081: 30$ & 125.19 & 122.694 & 100.413 & 104.304 & 95.929 & 110.677 & & 74.051 \\
\hline $2 / 23 / 08$ 2:00 & 125.182 & 122.691 & 100.415 & 104.304 & 95.929 & 110.677 & 80.675 & 74.053 \\
\hline $\begin{array}{l}2 / 23 / 082: 30 \\
2\end{array}$ & 125.18 & 122.689 & 100.418 & 104.304 & 95.931 & 110.675 & & 74.055 \\
\hline 2/23/08 3:00 & 125.178 & 122.687 & 100.417 & 104.305 & 95.931 & 110.675 & & 74.055 \\
\hline 2/23/08 3:30 & 125.173 & 122.682 & 100.413 & 104.304 & 95.929 & 110.673 & 80.672 & 74.049 \\
\hline $2 / 23 / 084: 00$ & 125.171 & 122.682 & 100.408 & 104.302 & 95.929 & 110.671 & & 74.051 \\
\hline $2 / 23 / 084: 30$ & 125.168 & 122.673 & 100.406 & 104.297 & 95.924 & 110.671 & & 74.045 \\
\hline $2 / 23 / 08$ 5:00 & 125.164 & 122.678 & 100.403 & 104.295 & 95.922 & 110.669 & 80.663 & 74.045 \\
\hline $2 / 23 / 085: 30$ & 125.164 & 122.68 & 100.399 & 104.289 & 95.915 & 110.669 & & 74.037 \\
\hline $2 / 23 / 086: 00$ & 125.156 & 122.685 & 100.406 & 104.295 & 95.919 & 110.665 & & 74.041 \\
\hline $2 / 23 / 086: 30$ & 125.154 & 122.684 & 100.408 & 104.295 & 95.922 & 110.663 & 80.655 & 74.043 \\
\hline $2 / 23 / 08$ 7:00 & 125.152 & 122.689 & 100.408 & 104.299 & 95.926 & 110.662 & & 74.045 \\
\hline $2 / 23 / 087: 30$ & 125.149 & 122.689 & 100.415 & 104.301 & 95.924 & 110.66 & & 74.045 \\
\hline $2 / 23 / 088: 00$ & 125.149 & 123.884 & 100.411 & 104.303 & 95.929 & 110.662 & 80.653 & 74.049 \\
\hline $2 / 23 / 088: 30$ & 125.147 & 124.698 & 100.425 & 104.306 & 95.931 & 110.659 & & 74.051 \\
\hline $2 / 23 / 089: 00$ & 125.632 & 124.882 & 101.002 & 104.32 & 95.938 & 110.667 & & 74.057 \\
\hline $2 / 23 / 089: 30$ & 126.832 & 124.993 & 101.292 & 104.919 & 96.671 & 111.381 & 80.682 & 74.208 \\
\hline $2 / 23 / 08$ 10:00 & 127.012 & 125.075 & 101.419 & 105.102 & 96.86 & 111.561 & & 74.365 \\
\hline $2 / 23 / 08 \quad 10: 30$ & 127.114 & 125.122 & 101.523 & 105.215 & 96.968 & 111.657 & & 74.473 \\
\hline $2 / 23 / 0811: 00$ & 127.187 & 125.149 & 101.585 & 105.296 & 97.051 & 111.719 & 80.746 & 74.56 \\
\hline $2 / 23 / 08$ 11:30 & 127.241 & 125.183 & 101.623 & 105.348 & 97.1 & 111.76 & & 74.609 \\
\hline $2 / 23 / 08$ 12:00 & 127.289 & 125.19 & 101.657 & 105.376 & 97.133 & 111.79 & & 74.642 \\
\hline $2 / 23 / 08$ 12:30 & 127.326 & 123.562 & 101.678 & 105.409 & 97.164 & 111.811 & 80.802 & 74.675 \\
\hline $2 / 23 / 08$ 13:00 & 127.355 & 123.179 & 101.681 & 105.416 & 97.173 & 111.829 & & 74.686 \\
\hline $2 / 23 / 08$ 13:30 & 126.038 & 123.027 & 100.999 & 105.106 & 96.754 & 111.407 & & 74.669 \\
\hline $2 / 23 / 0814: 00$ & 125.7 & 122.941 & 100.778 & 104.757 & 96.388 & 111.076 & 80.8 & 74.502 \\
\hline 2/23/08 14:30 & 125.575 & 122.882 & 100.68 & 104.615 & 96.242 & 110.943 & & 74.378 \\
\hline $2 / 23 / 08$ 15:00 & 125.485 & 122.843 & 100.613 & 104.531 & 96.157 & 110.868 & & 74.295 \\
\hline $2 / 23 / 08$ 15:30 & 125.419 & 122.814 & 100.573 & 104.478 & 96.106 & 110.821 & 80.775 & 74.237 \\
\hline 2/23/08 16:00 & 125.355 & 122.796 & 100.536 & 104.441 & 96.07 & 110.791 & & 74.2 \\
\hline $2 / 23 / 08$ 16:30 & 125.31 & 122.787 & 100.518 & 104.415 & 96.042 & 110.768 & & 74.171 \\
\hline
\end{tabular}


TABLE S1.1 (Cont.)

Depth from Top of Casing (ft)

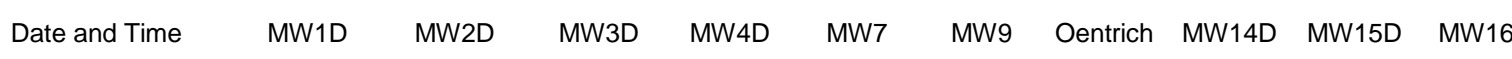

\begin{tabular}{|c|c|c|c|c|c|c|c|c|}
\hline 2/23/08 17:00 & 125.275 & 122.786 & 100.506 & 104.404 & 96.032 & 110.752 & 80.758 & 74.155 \\
\hline 2/23/08 17:30 & 125.246 & 122.793 & 100.499 & 104.388 & 96.018 & 110.739 & & 74.142 \\
\hline $2 / 23 / 08$ 18:00 & 125.227 & 122.802 & 100.508 & 104.39 & 96.018 & 110.729 & & 74.14 \\
\hline $2 / 23 / 08$ 18:30 & 125.218 & 122.807 & 100.517 & 104.404 & 96.03 & 110.72 & 80.758 & 74.15 \\
\hline 2/23/08 19:00 & 125.22 & 122.816 & 100.525 & 104.411 & 96.037 & 110.714 & & 74.159 \\
\hline $2 / 23 / 08$ 19:30 & 125.223 & 122.823 & 100.534 & 104.417 & 96.044 & 110.709 & & 74.163 \\
\hline $2 / 23 / 0820: 00$ & 125.23 & 122.825 & 100.542 & 104.426 & 96.056 & 110.705 & 80.773 & 74.171 \\
\hline $2 / 23 / 0820: 30$ & 125.237 & 122.825 & 100.546 & 104.432 & 96.061 & 110.703 & & 74.177 \\
\hline $2 / 23 / 0821: 00$ & 125.244 & 122.832 & 100.548 & 104.438 & 96.061 & 110.701 & & 74.177 \\
\hline $2 / 23 / 0821: 30$ & 125.251 & 122.834 & 100.553 & 104.438 & 96.063 & 110.699 & 80.76 & 74.177 \\
\hline 2/23/08 22:00 & 125.256 & 122.839 & 100.556 & 104.441 & 96.07 & 110.695 & & 74.181 \\
\hline $2 / 23 / 0822: 30$ & 125.26 & 122.834 & 100.558 & 104.445 & 96.072 & 110.695 & & 74.186 \\
\hline 2/23/08 23:00 & 125.263 & 122.836 & 100.56 & 104.447 & 96.075 & 110.693 & 80.77 & 74.186 \\
\hline 2/23/08 23:30 & 125.265 & 122.843 & 100.561 & 104.445 & 96.075 & 110.692 & & 74.186 \\
\hline $2 / 24 / 080: 00$ & 125.268 & 122.845 & 100.561 & 104.449 & 96.075 & 110.69 & & 74.19 \\
\hline $2 / 24 / 080: 30$ & 125.268 & 122.85 & 100.565 & 104.451 & 96.08 & 110.688 & 80.773 & 74.194 \\
\hline 2/24/08 1:00 & 125.272 & 122.843 & 100.57 & 104.455 & 96.084 & 110.688 & & 74.196 \\
\hline $2 / 24 / 081: 30$ & 125.277 & 122.843 & 100.572 & 104.46 & 96.087 & 110.688 & & 74.2 \\
\hline $2 / 24 / 082: 00$ & 125.279 & 122.843 & 100.567 & 104.455 & 96.082 & 110.686 & 80.778 & 74.196 \\
\hline $2 / 24 / 082: 30$ & 125.282 & 122.841 & 100.567 & 104.457 & 96.084 & 110.684 & & 74.198 \\
\hline $2 / 24 / 083: 00$ & 125.282 & 122.834 & 100.565 & 104.455 & 96.082 & 110.682 & & 74.198 \\
\hline $2 / 24 / 083: 30$ & 125.279 & 122.827 & 100.561 & 104.451 & 96.08 & 110.68 & 80.773 & 74.188 \\
\hline 2/24/08 4:00 & 125.277 & 122.816 & 100.56 & 104.447 & 96.077 & 110.678 & & 74.19 \\
\hline $2 / 24 / 084: 30$ & 125.272 & 122.809 & 100.546 & 104.438 & 96.068 & 110.676 & & 74.183 \\
\hline $2 / 24 / 085: 00$ & 125.265 & 122.809 & 100.536 & 104.428 & 96.056 & 110.673 & 80.768 & 74.171 \\
\hline $2 / 24 / 085: 30$ & 125.263 & 122.818 & 100.53 & 104.419 & 96.044 & 110.673 & & 74.163 \\
\hline $2 / 24 / 086: 00$ & 125.256 & 122.818 & 100.536 & 104.423 & 96.051 & 110.669 & & 74.169 \\
\hline $2 / 24 / 086: 30$ & 125.251 & 122.811 & 100.546 & 104.43 & 96.058 & 110.665 & 80.763 & 74.175 \\
\hline $2 / 24 / 087: 00$ & 125.256 & 122.809 & 100.537 & 104.434 & 96.061 & 110.665 & & 74.177 \\
\hline $2 / 24 / 087: 30$ & 125.253 & 122.812 & 100.532 & 104.424 & 96.049 & 110.663 & & 74.165 \\
\hline $2 / 24 / 088: 00$ & 125.249 & 122.811 & 100.53 & 104.421 & 96.049 & 110.662 & 80.76 & 74.165 \\
\hline $2 / 24 / 088: 30$ & 125.246 & 124.68 & 100.532 & 104.424 & 96.049 & 110.66 & & 74.167 \\
\hline 2/24/08 9:00 & 125.246 & 124.932 & 100.885 & 104.424 & 96.054 & 110.66 & & 74.167 \\
\hline $2 / 24 / 089: 30$ & 126.882 & 125.066 & 101.375 & 104.94 & 96.704 & 111.311 & 80.782 & 74.245 \\
\hline $2 / 24 / 08$ 10:00 & 127.175 & 125.136 & 101.542 & 105.188 & 96.959 & 111.559 & & 74.425 \\
\hline $2 / 24 / 08 \quad 10: 30$ & 127.293 & 125.186 & 101.643 & 105.321 & 97.088 & 111.681 & & 74.549 \\
\hline $2 / 24 / 0811: 00$ & 127.374 & 125.224 & 101.697 & 105.39 & 97.164 & 111.756 & 80.841 & 74.626 \\
\hline $2 / 24 / 0811: 30$ & 127.43 & 125.24 & 101.74 & 105.439 & 97.213 & 111.805 & & 74.675 \\
\hline $2 / 24 / 08$ 12:00 & 127.475 & 123.576 & 101.767 & 105.477 & 97.251 & 111.837 & & 74.719 \\
\hline $2 / 24 / 08 \quad 12: 30$ & 127.513 & 123.21 & 101.778 & 105.49 & 97.265 & 111.865 & 80.888 & 74.733 \\
\hline $2 / 24 / 0813: 00$ & 126.147 & 123.056 & 101.03 & 105.121 & 96.77 & 111.366 & & 74.717 \\
\hline $2 / 24 / 0813: 30$ & 125.802 & 122.959 & 100.806 & 104.792 & 96.424 & 111.054 & & 74.545 \\
\hline $2 / 24 / 0814: 00$ & 125.632 & 122.902 & 100.699 & 104.641 & 96.27 & 110.923 & 80.861 & 74.413 \\
\hline $2 / 24 / 08 \quad 14: 30$ & 125.513 & 122.852 & 100.627 & 104.544 & 96.174 & 110.847 & & 74.318 \\
\hline $2 / 24 / 08$ 15:00 & 125.426 & 122.814 & 100.58 & 104.493 & 96.122 & 110.799 & & 74.264 \\
\hline $2 / 24 / 08 \quad 15: 30$ & 125.367 & 122.796 & 100.536 & 104.445 & 96.075 & 110.77 & 80.826 & 74.214 \\
\hline $2 / 24 / 08$ 16:00 & 125.31 & 122.768 & 100.511 & 104.413 & 96.042 & 110.748 & & 74.181 \\
\hline $2 / 24 / 08 \quad 16: 30$ & 125.268 & 122.743 & 100.492 & 104.394 & 96.021 & 110.731 & & 74.161 \\
\hline $2 / 24 / 08$ 17:00 & 125.239 & 122.73 & 100.465 & 104.371 & 96.002 & 110.72 & 80.8 & 74.138 \\
\hline $2 / 24 / 08 \quad 17: 30$ & 125.211 & 122.723 & 100.453 & 104.348 & 95.976 & 110.71 & & 74.115 \\
\hline $2 / 24 / 08$ 18:00 & 125.19 & 122.709 & 100.439 & 104.333 & 95.964 & 110.703 & & 74.103 \\
\hline $2 / 24 / 08$ 18:30 & 125.168 & 122.698 & 100.427 & 104.327 & 95.957 & 110.693 & 80.775 & 74.093 \\
\hline $2 / 24 / 08$ 19:00 & 125.154 & 122.689 & 100.42 & 104.318 & 95.943 & 110.688 & & 74.082 \\
\hline $2 / 24 / 08$ 19:30 & 125.145 & 122.673 & 100.411 & 104.308 & 95.933 & 110.684 & & 74.074 \\
\hline 2/24/08 20:00 & 125.135 & 122.662 & 100.398 & 104.299 & 95.926 & 110.682 & 80.753 & 74.066 \\
\hline $2 / 24 / 0820: 30$ & 125.126 & 122.657 & 100.38 & 104.278 & 95.905 & 110.678 & & 74.045 \\
\hline $2 / 24 / 0821: 00$ & 125.112 & 122.644 & 100.377 & 104.268 & 95.893 & 110.675 & & 74.035 \\
\hline 2/24/08 21:30 & 125.1 & 122.63 & 100.367 & 104.268 & 95.893 & 110.673 & 80.729 & 74.033 \\
\hline $2 / 24 / 0822: 00$ & 125.095 & 122.625 & 100.356 & 104.251 & 95.879 & 110.671 & & 74.018 \\
\hline $2 / 24 / 0822: 30$ & 125.086 & 122.612 & 100.346 & 104.242 & 95.865 & 110.669 & & 74.006 \\
\hline $2 / 24 / 0823: 00$ & 125.071 & 122.596 & 100.337 & 104.23 & 95.856 & 110.667 & 80.709 & 73.997 \\
\hline $2 / 24 / 08$ 23:30 & 125.064 & 122.576 & 100.324 & 104.221 & 95.846 & 110.665 & & 73.989 \\
\hline $2 / 25 / 080: 00$ & 125.057 & 122.564 & 100.303 & 104.205 & 95.832 & 110.665 & & 73.973 \\
\hline $2 / 25 / 080: 30$ & 125.043 & 122.537 & 100.287 & 104.185 & 95.809 & 110.665 & 80.68 & 73.952 \\
\hline $2 / 25 / 081: 00$ & 125.034 & 122.526 & 100.272 & 104.177 & 95.801 & 110.665 & & 73.946 \\
\hline $2 / 25 / 081: 30$ & 125.022 & 122.505 & 100.232 & 104.15 & 95.776 & 110.665 & & 73.921 \\
\hline
\end{tabular}


TABLE S1.1 (Cont.)

Depth from Top of Casing (ft)

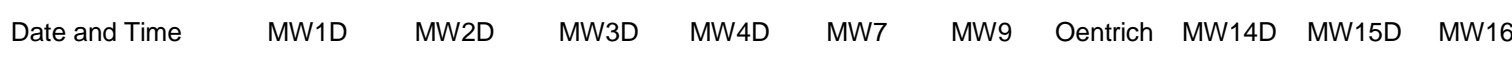

\begin{tabular}{|c|c|c|c|c|c|c|c|c|}
\hline 2/25/08 2:00 & 125 & 122.492 & 100.236 & 104.135 & 95.759 & 110.663 & 80.646 & 73.904 \\
\hline 2/25/08 2:30 & 124.989 & 122.449 & 100.218 & 104.104 & 95.731 & 110.663 & & 73.878 \\
\hline $2 / 25 / 083: 00$ & 124.97 & 122.431 & 100.198 & 104.106 & 95.733 & 110.66 & & 73.88 \\
\hline 2/25/08 3:30 & 124.97 & 122.383 & 100.15 & 104.061 & 95.686 & 110.665 & 80.606 & 73.836 \\
\hline 2/25/08 4:00 & 124.963 & 122.356 & 100.136 & 104.042 & 95.662 & 110.662 & & 73.815 \\
\hline $2 / 25 / 084: 30$ & 124.965 & 122.34 & 100.093 & 103.996 & 95.618 & 110.663 & & 73.774 \\
\hline $2 / 25 / 085: 00$ & 124.96 & 122.313 & 100.069 & 103.966 & 95.592 & 110.667 & 80.55 & 73.745 \\
\hline 2/25/08 5:30 & 124.958 & 122.306 & 100.039 & 103.95 & 95.575 & 110.662 & & 73.733 \\
\hline $2 / 25 / 086: 00$ & 124.951 & 122.299 & 100.022 & 103.924 & 95.547 & 110.663 & & 73.708 \\
\hline $2 / 25 / 086: 30$ & 124.944 & 122.304 & 100.029 & 103.916 & 95.542 & 110.66 & 80.506 & 73.702 \\
\hline 2/25/08 7:00 & 124.937 & 124.079 & 100.017 & 103.914 & 95.535 & 110.658 & & 73.694 \\
\hline $2 / 25 / 087: 30$ & 124.925 & 124.383 & 100.02 & 103.916 & 95.537 & 110.656 & & 73.694 \\
\hline $2 / 25 / 088: 00$ & 126.414 & 124.528 & 100.751 & 104.312 & 96.049 & 111.187 & 80.492 & 73.722 \\
\hline 2/25/08 8:30 & 126.691 & 124.623 & 100.947 & 104.605 & 96.353 & 111.473 & & 73.911 \\
\hline 2/25/08 9:00 & 126.813 & 124.684 & 101.07 & 104.754 & 96.504 & 111.602 & & 74.051 \\
\hline 2/25/08 9:30 & 126.889 & 124.716 & 101.144 & 104.853 & 96.605 & 111.681 & 80.536 & 74.148 \\
\hline 2/25/08 10:00 & 126.941 & 124.757 & 101.192 & 104.914 & 96.664 & 111.732 & & 74.21 \\
\hline $2 / 25 / 08 \quad 10: 30$ & 126.981 & 124.78 & 101.223 & 104.948 & 96.702 & 111.767 & & 74.25 \\
\hline $2 / 25 / 08$ 11:00 & 127.012 & 124.768 & 101.254 & 104.988 & 96.742 & 111.794 & 80.565 & 74.285 \\
\hline $2 / 25 / 0811: 30$ & 127.076 & 123.188 & 101.263 & 105.013 & 96.766 & 111.814 & & 74.312 \\
\hline $2 / 25 / 08 \quad 12: 00$ & 127.147 & 122.841 & 101.252 & 105.003 & 96.761 & 111.835 & & 74.308 \\
\hline $2 / 25 / 08$ 12:30 & 125.823 & 122.716 & 100.642 & 104.742 & 96.388 & 111.413 & 80.587 & 74.328 \\
\hline $2 / 25 / 08$ 13:00 & 125.457 & 122.646 & 100.451 & 104.428 & 96.051 & 111.076 & & 74.19 \\
\hline $2 / 25 / 08$ 13:30 & 125.289 & 122.616 & 100.375 & 104.31 & 95.933 & 110.941 & & 74.088 \\
\hline $2 / 25 / 0814: 00$ & 125.182 & 122.603 & 100.332 & 104.244 & 95.872 & 110.87 & 80.572 & 74.02 \\
\hline $2 / 25 / 08 \quad 14: 30$ & 125.109 & 122.601 & 100.317 & 104.217 & 95.839 & 110.821 & & 73.987 \\
\hline $2 / 25 / 08$ 15:00 & 125.064 & 122.612 & 100.312 & 104.207 & 95.832 & 110.791 & & 73.973 \\
\hline $2 / 25 / 08$ 15:30 & 125.041 & 122.619 & 100.315 & 104.206 & 95.83 & 110.769 & 80.572 & 73.964 \\
\hline $2 / 25 / 0816: 00$ & 125.022 & 122.628 & 100.327 & 104.217 & 95.842 & 110.754 & & 73.975 \\
\hline $2 / 25 / 08 \quad 16: 30$ & 125.01 & 122.657 & 100.337 & 104.228 & 95.856 & 110.735 & & 73.981 \\
\hline $2 / 25 / 08$ 17:00 & 125 & 122.691 & 100.36 & 104.24 & 95.867 & 110.731 & 80.575 & 73.987 \\
\hline $2 / 25 / 08 \quad 17: 30$ & 124.986 & 122.721 & 100.391 & 104.272 & 95.896 & 110.724 & & 74.004 \\
\hline $2 / 25 / 08$ 18:00 & 124.974 & 122.755 & 100.424 & 104.303 & 95.931 & 110.714 & & 74.014 \\
\hline $2 / 25 / 08$ 18:30 & 124.965 & 122.782 & 100.458 & 104.335 & 95.962 & 110.707 & 80.604 & 74.008 \\
\hline 2/25/08 19:00 & 124.956 & 122.802 & 100.496 & 104.371 & 95.995 & 110.705 & & 74.004 \\
\hline $2 / 25 / 08 \quad 19: 30$ & 124.953 & 122.818 & 100.517 & 104.4 & 96.028 & 110.699 & & 74.006 \\
\hline $2 / 25 / 0820: 00$ & 124.953 & 122.834 & 100.536 & 104.419 & 96.049 & 110.699 & 80.631 & 74.012 \\
\hline $2 / 25 / 0820: 30$ & 124.953 & 122.852 & 100.554 & 104.438 & 96.068 & 110.701 & & 74.018 \\
\hline $2 / 25 / 0821: 00$ & 124.958 & 122.877 & 100.572 & 104.453 & 96.082 & 110.703 & & 74.099 \\
\hline 2/25/08 21:30 & 124.96 & 122.891 & 100.592 & 104.474 & 96.101 & 110.705 & 80.658 & 74.192 \\
\hline $2 / 25 / 0822: 00$ & 124.965 & 122.907 & 100.613 & 104.495 & 96.124 & 110.703 & & 74.21 \\
\hline $2 / 25 / 0822: 30$ & 124.97 & 122.918 & 100.623 & 104.508 & 96.141 & 110.707 & & 74.223 \\
\hline 2/25/08 23:00 & 124.977 & 122.934 & 100.646 & 104.522 & 96.153 & 110.707 & 80.685 & 74.237 \\
\hline $2 / 25 / 0823: 30$ & 124.984 & 122.943 & 100.654 & 104.539 & 96.169 & 110.707 & & 74.252 \\
\hline 2/26/08 0:00 & 124.991 & 122.952 & 100.665 & 104.552 & 96.183 & 110.707 & & 74.262 \\
\hline 2/26/08 0:30 & 124.998 & 122.966 & 100.675 & 104.558 & 96.188 & 110.701 & 80.712 & 74.27 \\
\hline 2/26/08 1:00 & 125.005 & 122.968 & 100.691 & 104.573 & 96.204 & 110.699 & & 74.283 \\
\hline 2/26/08 1:30 & 125.012 & 122.975 & 100.692 & 104.581 & 96.214 & 110.697 & & 74.295 \\
\hline 2/26/08 2:00 & 125.022 & 122.986 & 100.696 & 104.581 & 96.214 & 110.701 & 80.731 & 74.295 \\
\hline $2 / 26 / 082: 30$ & 125.026 & 122.995 & 100.704 & 104.592 & 96.221 & 110.699 & & 74.301 \\
\hline 2/26/08 3:00 & 125.029 & 123.004 & 100.722 & 104.602 & 96.233 & 110.695 & & 74.312 \\
\hline 2/26/08 3:30 & 125.038 & 123.004 & 100.727 & 104.613 & 96.24 & 110.693 & 80.758 & 74.322 \\
\hline $2 / 26 / 084: 00$ & 125.045 & 123.011 & 100.735 & 104.622 & 96.252 & 110.69 & & 74.332 \\
\hline 2/26/08 4:30 & 125.052 & 123.018 & 100.737 & 104.622 & 96.252 & 110.69 & & 74.336 \\
\hline 2/26/08 5:00 & 125.06 & 123.027 & 100.746 & 104.626 & 96.256 & 110.682 & 80.775 & 74.339 \\
\hline $2 / 26 / 085: 30$ & 125.069 & 123.038 & 100.753 & 104.636 & 96.268 & 110.682 & & 74.349 \\
\hline 2/26/08 6:00 & 125.076 & 123.045 & 100.765 & 104.643 & 96.273 & 110.677 & & 74.357 \\
\hline 2/26/08 6:30 & 125.081 & 123.056 & 100.773 & 104.655 & 96.285 & 110.675 & 80.797 & 74.367 \\
\hline $2 / 26 / 087: 00$ & 125.088 & 124.821 & 100.784 & 104.661 & 96.289 & 110.671 & & 74.376 \\
\hline 2/26/08 7:30 & 125.097 & 125.17 & 100.797 & 104.676 & 96.306 & 110.665 & & 74.386 \\
\hline $2 / 26 / 088: 00$ & 126.553 & 125.333 & 101.535 & 105.057 & 96.796 & 111.148 & 80.834 & 74.423 \\
\hline 2/26/08 8:30 & 126.87 & 125.428 & 101.752 & 105.397 & 97.15 & 111.459 & & 74.628 \\
\hline 2/26/08 9:00 & 127.017 & 125.49 & 101.878 & 105.559 & 97.315 & 111.595 & & 74.781 \\
\hline 2/26/08 9:30 & 127.114 & 125.537 & 101.954 & 105.656 & 97.414 & 111.702 & 80.934 & 74.884 \\
\hline $2 / 26 / 08$ 10:00 & 127.187 & 125.562 & 102.009 & 105.721 & 97.48 & 111.767 & & 74.946 \\
\hline 2/26/08 10:30 & 127.246 & 125.583 & 102.047 & 105.767 & 97.525 & 111.816 & & 74.998 \\
\hline
\end{tabular}


TABLE S1.1 (Cont.)

Depth from Top of Casing (ft)

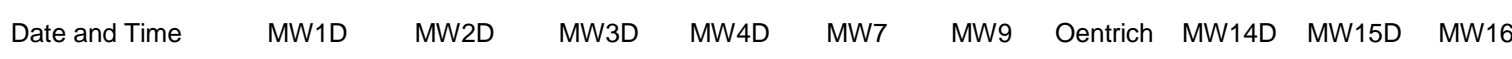

\begin{tabular}{|c|c|c|c|c|c|c|c|c|}
\hline 2/26/08 11:00 & 127.296 & 124.084 & 102.069 & 105.797 & 97.555 & 111.843 & 81.012 & 75.029 \\
\hline 2/26/08 11:30 & 127.334 & 123.628 & 102.091 & 105.818 & 97.576 & 111.861 & & 75.052 \\
\hline $2 / 26 / 08 \quad 12: 00$ & 126.14 & 123.46 & 101.459 & 105.613 & 97.282 & 111.597 & & 75.066 \\
\hline $2 / 26 / 08$ 12:30 & 125.731 & 123.365 & 101.228 & 105.209 & 96.843 & 111.146 & 81.051 & 74.922 \\
\hline 2/26/08 13:00 & 125.606 & 123.301 & 101.109 & 105.047 & 96.681 & 111.001 & & 74.791 \\
\hline $2 / 26 / 08$ 13:30 & 125.542 & 123.251 & 101.037 & 104.956 & 96.586 & 110.921 & & 74.702 \\
\hline $2 / 26 / 0814: 00$ & 125.506 & 123.213 & 100.985 & 104.897 & 96.525 & 110.872 & 81.039 & 74.653 \\
\hline $2 / 26 / 08$ 14:30 & 125.487 & 123.179 & 100.942 & 104.849 & 96.478 & 110.836 & & 74.622 \\
\hline $2 / 26 / 08$ 15:00 & 125.476 & 123.161 & 100.909 & 104.813 & 96.447 & 110.812 & & 74.599 \\
\hline $2 / 26 / 08 \quad 15: 30$ & 125.471 & 123.133 & 100.882 & 104.777 & 96.41 & 110.797 & 81.027 & 74.593 \\
\hline 2/26/08 16:00 & 125.464 & 123.111 & 100.859 & 104.754 & 96.388 & 110.784 & & 74.58 \\
\hline $2 / 26 / 08 \quad 16: 30$ & 125.461 & 123.104 & 100.835 & 104.737 & 96.367 & 110.774 & & 74.58 \\
\hline $2 / 26 / 08$ 17:00 & 125.45 & 123.084 & 100.822 & 104.716 & 96.344 & 110.761 & 81.017 & 74.568 \\
\hline 2/26/08 17:30 & 125.447 & 123.063 & 100.809 & 104.72 & 96.334 & 111.537 & & 74.547 \\
\hline $2 / 26 / 08$ 18:00 & 125.438 & 123.043 & 100.791 & 104.687 & 96.315 & 110.725 & & 74.502 \\
\hline $2 / 26 / 08$ 18:30 & 125.433 & 123.027 & 100.772 & 104.67 & 96.299 & 110.724 & 81.01 & 74.452 \\
\hline 2/26/08 19:00 & 125.426 & 123.02 & 100.756 & 104.649 & 96.28 & 110.721 & & 74.413 \\
\hline $2 / 26 / 08$ 19:30 & 125.419 & 123.009 & 100.744 & 104.636 & 96.263 & 110.718 & & 74.386 \\
\hline $2 / 26 / 0820: 00$ & 125.412 & 123.002 & 100.735 & 104.632 & 96.259 & 110.712 & 80.995 & 74.374 \\
\hline $2 / 26 / 0820: 30$ & 125.407 & 123 & 100.727 & 104.621 & 96.247 & 110.709 & & 74.365 \\
\hline $2 / 26 / 0821: 00$ & 125.402 & 122.991 & 100.722 & 104.611 & 96.237 & 110.705 & & 74.359 \\
\hline $2 / 26 / 0821: 30$ & 125.398 & 122.984 & 100.716 & 104.611 & 96.237 & 110.701 & 80.985 & 74.357 \\
\hline 2/26/08 22:00 & 125.393 & 122.986 & 100.713 & 104.598 & 96.226 & 110.697 & & 74.353 \\
\hline $2 / 26 / 08$ 22:30 & 125.39 & 122.972 & 100.706 & 104.596 & 96.223 & 110.693 & & 74.349 \\
\hline $2 / 26 / 08$ 23:00 & 125.386 & 122.966 & 100.706 & 104.594 & 96.221 & 110.69 & 80.993 & 74.345 \\
\hline 2/26/08 23:30 & 125.383 & 122.959 & 100.692 & 104.583 & 96.209 & 110.688 & & 74.341 \\
\hline $2 / 27 / 080: 00$ & 125.383 & 122.945 & 100.68 & 104.571 & 96.195 & 110.688 & & 74.328 \\
\hline $2 / 27 / 080: 30$ & 125.376 & 122.941 & 100.673 & 104.567 & 96.193 & 110.684 & 80.966 & 74.32 \\
\hline $2 / 27 / 081: 00$ & 125.374 & 122.934 & 100.665 & 104.554 & 96.181 & 110.682 & & 74.312 \\
\hline 2/27/08 1:30 & 125.369 & 122.927 & 100.656 & 104.55 & 96.176 & 110.678 & & 74.305 \\
\hline $2 / 27 / 082: 00$ & 125.367 & 122.923 & 100.653 & 104.544 & 96.169 & 110.677 & 80.956 & 74.301 \\
\hline 2/27/08 2:30 & 125.364 & 122.916 & 100.647 & 104.537 & 96.162 & 110.673 & & 74.297 \\
\hline 2/27/08 3:00 & 125.36 & 122.914 & 100.641 & 104.531 & 96.16 & 110.671 & & 74.291 \\
\hline $2 / 27 / 083: 30$ & 125.355 & 122.904 & 100.639 & 104.527 & 96.153 & 110.667 & 80.946 & 74.287 \\
\hline 2/27/08 4:00 & 125.353 & 122.909 & 100.63 & 104.523 & 96.153 & 110.665 & & 74.283 \\
\hline 2/27/08 4:30 & 125.35 & 122.898 & 100.629 & 104.516 & 96.143 & 110.665 & & 74.276 \\
\hline $2 / 27 / 085: 00$ & 125.346 & 122.891 & 100.625 & 104.52 & 96.146 & 110.662 & 80.936 & 74.276 \\
\hline $2 / 27 / 085: 30$ & 125.346 & 122.891 & 100.618 & 104.51 & 96.139 & 110.66 & & 74.27 \\
\hline $2 / 27 / 086: 00$ & 125.343 & 122.895 & 100.611 & 104.504 & 96.131 & 110.656 & & 74.266 \\
\hline 2/27/08 6:30 & 125.341 & 122.898 & 100.616 & 104.501 & 96.129 & 110.654 & 80.929 & 74.26 \\
\hline $2 / 27 / 087: 00$ & 125.336 & 124.619 & 100.618 & 104.508 & 96.134 & 110.65 & & 74.266 \\
\hline $2 / 27 / 087: 30$ & 125.334 & 124.966 & 100.616 & 104.51 & 96.136 & 110.648 & & 74.27 \\
\hline 2/27/08 8:00 & 126.787 & 125.12 & 101.381 & 104.887 & 96.636 & 111.174 & 80.936 & 74.283 \\
\hline $2 / 27 / 088: 30$ & 127.111 & 125.211 & 101.59 & 105.224 & 96.992 & 111.501 & & 74.475 \\
\hline 2/27/08 9:00 & 127.253 & 123.791 & 101.709 & 105.38 & 97.145 & 111.644 & & 74.62 \\
\hline 2/27/08 9:30 & 127.345 & 123.315 & 101.785 & 105.47 & 97.239 & 111.728 & 81 & 74.715 \\
\hline $2 / 27 / 08$ 10:00 & 126.204 & 123.163 & 101.145 & 105.31 & 96.98 & 111.431 & & 74.779 \\
\hline $2 / 27 / 08 \quad 10: 30$ & 125.757 & 123.079 & 100.927 & 104.9 & 96.53 & 111.016 & & 74.659 \\
\hline 2/27/08 11:00 & 125.632 & 123.022 & 100.823 & 104.76 & 96.384 & 110.877 & 80.993 & 74.537 \\
\hline $2 / 27 / 0811: 30$ & 125.572 & 122.984 & 100.756 & 104.68 & 96.303 & 110.804 & & 74.458 \\
\hline $2 / 27 / 0812: 00$ & 125.539 & 122.952 & 100.713 & 104.621 & 96.249 & 110.761 & & 74.396 \\
\hline 2/27/08 12:30 & 125.518 & 122.927 & 100.68 & 104.586 & 96.212 & 110.733 & 80.973 & 74.361 \\
\hline $2 / 27 / 0813: 00$ & 125.502 & 122.902 & 100.654 & 104.556 & 96.183 & 110.714 & & 74.332 \\
\hline $2 / 27 / 08$ 13:30 & 125.492 & 122.877 & 100.632 & 104.531 & 96.16 & 110.703 & & 74.308 \\
\hline $2 / 27 / 0814: 00$ & 125.466 & 122.857 & 100.601 & 104.506 & 96.136 & 110.693 & 80.954 & 74.283 \\
\hline $2 / 27 / 0814: 30$ & 125.424 & 122.841 & 100.58 & 104.48 & 96.108 & 110.686 & & 74.258 \\
\hline $2 / 27 / 08$ 15:00 & 125.379 & 122.827 & 100.565 & 104.461 & 96.087 & 110.68 & & 74.235 \\
\hline $2 / 27 / 08$ 15:30 & 125.341 & 122.811 & 100.549 & 104.445 & 96.072 & 110.677 & 80.927 & 74.223 \\
\hline $2 / 27 / 0816: 00$ & 125.298 & 122.809 & 100.534 & 104.432 & 96.058 & 110.671 & & 74.206 \\
\hline 2/27/08 16:30 & 125.272 & 122.802 & 100.529 & 104.421 & 96.047 & 110.667 & & 74.196 \\
\hline $2 / 27 / 08$ 17:00 & 125.253 & 122.789 & 100.523 & 104.415 & 96.042 & 110.663 & 80.907 & 74.19 \\
\hline $2 / 27 / 08 \quad 17: 30$ & 125.244 & 122.78 & 100.518 & 104.409 & 96.037 & 110.66 & & 74.183 \\
\hline 2/27/08 18:00 & 125.234 & 122.777 & 100.504 & 104.402 & 96.025 & 110.658 & & 74.175 \\
\hline $2 / 27 / 08$ 18:30 & 125.223 & 122.771 & 100.499 & 104.392 & 96.018 & 110.652 & 80.89 & 74.167 \\
\hline 2/27/08 19:00 & 125.216 & 122.764 & 100.494 & 104.388 & 96.014 & 110.65 & & 74.163 \\
\hline 2/27/08 19:30 & 125.208 & 122.762 & 100.487 & 104.381 & 96.007 & 110.648 & & 74.155 \\
\hline
\end{tabular}


TABLE S1.1 (Cont.)

Depth from Top of Casing (ft)

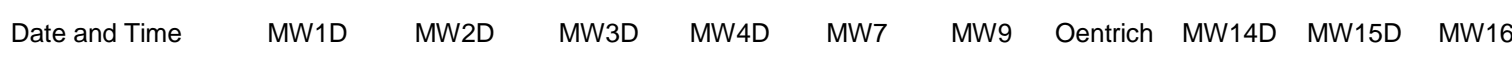

\begin{tabular}{|c|c|c|c|c|c|c|c|c|}
\hline 2/27/08 20:00 & 125.201 & 122.759 & 100.484 & 104.373 & 95.999 & 110.645 & 80.873 & 74.148 \\
\hline 2/27/08 20:30 & 125.194 & 122.752 & 100.48 & 104.371 & 95.997 & 110.643 & & 74.146 \\
\hline $2 / 27 / 0821: 00$ & 125.194 & 122.748 & 100.479 & 104.371 & 95.997 & 110.639 & & 74.146 \\
\hline 2/27/08 21:30 & 125.19 & 122.743 & 100.467 & 104.367 & 95.99 & 110.639 & 80.858 & 74.138 \\
\hline 2/27/08 22:00 & 125.185 & 122.737 & 100.47 & 104.358 & 95.985 & 110.637 & & 74.132 \\
\hline $2 / 27 / 0822: 30$ & 125.18 & 122.734 & 100.463 & 104.356 & 95.983 & 110.635 & & 74.13 \\
\hline $2 / 27 / 0823: 00$ & 125.178 & 122.725 & 100.458 & 104.35 & 95.976 & 110.633 & 80.839 & 74.121 \\
\hline 2/27/08 23:30 & 125.173 & 122.716 & 100.455 & 104.348 & 95.973 & 110.632 & & 74.119 \\
\hline $2 / 28 / 080: 00$ & 125.168 & 122.714 & 100.441 & 104.337 & 95.966 & 110.63 & & 74.113 \\
\hline 2/28/08 0:30 & 125.161 & 122.705 & 100.437 & 104.331 & 95.957 & 110.63 & 80.824 & 74.103 \\
\hline 2/28/08 1:00 & 125.154 & 122.698 & 100.434 & 104.325 & 95.955 & 110.628 & & 74.099 \\
\hline $2 / 28 / 08$ 1:30 & 125.149 & 122.687 & 100.425 & 104.316 & 95.943 & 110.628 & & 74.088 \\
\hline 2/28/08 2:00 & 125.142 & 122.671 & 100.424 & 104.312 & 95.938 & 110.626 & 80.804 & 74.086 \\
\hline 2/28/08 2:30 & 125.135 & 122.66 & 100.399 & 104.299 & 95.926 & 110.626 & & 74.074 \\
\hline 2/28/08 3:00 & 125.121 & 122.653 & 100.386 & 104.284 & 95.912 & 110.626 & & 74.059 \\
\hline 2/28/08 3:30 & 125.107 & 122.641 & 100.377 & 104.27 & 95.898 & 110.624 & 80.778 & 74.047 \\
\hline 2/28/08 4:00 & 125.097 & 122.63 & 100.367 & 104.266 & 95.893 & 110.622 & & 74.043 \\
\hline $2 / 28 / 084: 30$ & 125.086 & 122.623 & 100.361 & 104.255 & 95.879 & 110.622 & & 74.026 \\
\hline $2 / 28 / 085: 00$ & 125.076 & 122.612 & 100.346 & 104.24 & 95.865 & 110.622 & 80.753 & 74.016 \\
\hline 2/28/08 5:30 & 125.064 & 122.605 & 100.334 & 104.236 & 95.863 & 110.62 & & 74.014 \\
\hline $2 / 28 / 086: 00$ & 125.052 & 122.601 & 100.329 & 104.225 & 95.851 & 110.62 & & 73.999 \\
\hline $2 / 28 / 086: 30$ & 125.048 & 122.591 & 100.324 & 104.219 & 95.844 & 110.618 & 80.736 & 73.995 \\
\hline 2/28/08 7:00 & 125.041 & 124.435 & 100.318 & 104.215 & 95.844 & 110.618 & & 73.993 \\
\hline $2 / 28 / 087: 30$ & 125.034 & 124.716 & 100.468 & 104.207 & 95.83 & 110.618 & & 73.981 \\
\hline $2 / 28 / 088: 00$ & 126.679 & 124.861 & 101.082 & 104.657 & 96.403 & 111.185 & 80.734 & 74.039 \\
\hline 2/28/08 8:30 & 127.088 & 124.957 & 101.276 & 104.937 & 96.688 & 111.45 & & 74.229 \\
\hline 2/28/08 9:00 & 127.291 & 124.997 & 101.395 & 105.081 & 96.836 & 111.576 & & 74.37 \\
\hline $2 / 28 / 089: 30$ & 127.402 & 125.031 & 101.462 & 105.178 & 96.933 & 111.649 & 80.797 & 74.467 \\
\hline $2 / 28 / 08 \quad 10: 00$ & 127.478 & 125.066 & 101.495 & 105.224 & 96.98 & 111.7 & & 74.518 \\
\hline $2 / 28 / 08 \quad 10: 30$ & 127.52 & 125.081 & 101.542 & 105.256 & 97.013 & 111.736 & & 74.554 \\
\hline $2 / 28 / 08$ 11:00 & 127.551 & 125.079 & 101.568 & 105.289 & 97.044 & 111.76 & 80.834 & 74.585 \\
\hline $2 / 28 / 0811: 30$ & 127.577 & 125.093 & 101.568 & 105.312 & 97.067 & 111.777 & & 74.611 \\
\hline $2 / 28 / 08 \quad 12: 00$ & 127.589 & 125.081 & 101.576 & 105.308 & 97.065 & 111.796 & & 74.611 \\
\hline $2 / 28 / 08$ 12:30 & 127.591 & 123.265 & 101.58 & 105.323 & 97.081 & 111.807 & 80.866 & 74.628 \\
\hline 2/28/08 13:00 & 127.603 & 123 & 101.257 & 105.317 & 97.077 & 111.818 & & 74.628 \\
\hline $2 / 28 / 08$ 13:30 & 126.01 & 122.868 & 100.784 & 104.838 & 96.476 & 111.234 & & 74.545 \\
\hline $2 / 28 / 0814: 00$ & 125.629 & 122.775 & 100.613 & 104.584 & 96.212 & 111.005 & 80.839 & 74.37 \\
\hline $2 / 28 / 08$ 14:30 & 125.414 & 122.705 & 100.518 & 104.463 & 96.091 & 110.898 & & 74.254 \\
\hline $2 / 28 / 08$ 15:00 & 125.343 & 122.675 & 100.437 & 104.365 & 95.997 & 110.838 & & 74.169 \\
\hline $2 / 28 / 08 \quad 15: 30$ & 125.31 & 122.66 & 100.392 & 104.299 & 95.929 & 110.799 & 80.787 & 74.097 \\
\hline $2 / 28 / 0816: 00$ & 125.275 & 122.65 & 100.363 & 104.27 & 95.898 & 110.765 & & 74.064 \\
\hline $2 / 28 / 08$ 16:30 & 125.213 & 122.655 & 100.361 & 104.261 & 95.886 & 110.746 & & 74.045 \\
\hline 2/28/08 17:00 & 125.149 & 122.66 & 100.348 & 104.255 & 95.882 & 110.729 & 80.77 & 74.039 \\
\hline $2 / 28 / 08 \quad 17: 30$ & 125.112 & 122.66 & 100.339 & 104.263 & 95.889 & 110.716 & & 74.041 \\
\hline $2 / 28 / 08$ 18:00 & 125.1 & 122.664 & 100.377 & 104.266 & 95.891 & 110.708 & & 74.041 \\
\hline $2 / 28 / 08 \quad 18: 30$ & 125.09 & 122.662 & 100.375 & 104.265 & 95.891 & 110.701 & 80.763 & 74.037 \\
\hline 2/28/08 19:00 & 125.088 & 122.666 & 100.38 & 104.27 & 95.898 & 110.695 & & 74.041 \\
\hline $2 / 28 / 08$ 19:30 & 125.09 & 122.678 & 100.384 & 104.27 & 95.898 & 110.692 & & 74.041 \\
\hline $2 / 28 / 0820: 00$ & 125.093 & 122.678 & 100.389 & 104.28 & 95.907 & 110.686 & 80.753 & 74.049 \\
\hline $2 / 28 / 0820: 30$ & 125.093 & 122.673 & 100.399 & 104.289 & 95.917 & 110.682 & & 74.055 \\
\hline $2 / 28 / 0821: 00$ & 125.107 & 122.673 & 100.394 & 104.289 & 95.919 & 110.68 & & 74.062 \\
\hline 2/28/08 21:30 & 125.107 & 122.684 & 100.392 & 104.284 & 95.912 & 110.68 & 80.748 & 74.049 \\
\hline $2 / 28 / 0822: 00$ & 125.102 & 122.682 & 100.405 & 104.285 & 95.912 & 110.675 & & 74.051 \\
\hline $2 / 28 / 08$ 22:30 & 125.109 & 122.685 & 100.406 & 104.297 & 95.924 & 110.673 & & 74.062 \\
\hline 2/28/08 23:00 & 125.114 & 122.685 & 100.405 & 104.299 & 95.924 & 110.671 & 80.743 & 74.062 \\
\hline $2 / 28 / 08$ 23:30 & 125.116 & 122.687 & 100.41 & 104.295 & 95.924 & 110.669 & & 74.059 \\
\hline $2 / 29 / 080: 00$ & 125.119 & 122.689 & 100.41 & 104.299 & 95.926 & 110.669 & & 74.064 \\
\hline 2/29/08 0:30 & 125.119 & 122.696 & 100.413 & 104.303 & 95.929 & 110.665 & 80.734 & 74.064 \\
\hline $2 / 29 / 08$ 1:00 & 125.121 & 122.716 & 100.422 & 104.306 & 95.931 & 110.665 & & 74.064 \\
\hline $2 / 29 / 081: 30$ & 125.126 & 122.728 & 100.429 & 104.312 & 95.94 & 110.663 & & 74.07 \\
\hline 2/29/08 2:00 & 125.13 & 122.739 & 100.451 & 104.331 & 95.957 & 110.66 & 80.738 & 74.086 \\
\hline 2/29/08 2:30 & 125.147 & 122.746 & 100.458 & 104.345 & 95.971 & 110.66 & & 74.101 \\
\hline 2/29/08 3:00 & 125.154 & 122.759 & 100.47 & 104.356 & 95.981 & 110.658 & & 74.107 \\
\hline 2/29/08 3:30 & 125.168 & 122.766 & 100.48 & 104.36 & 95.985 & 110.658 & 80.743 & 74.111 \\
\hline 2/29/08 4:00 & 125.178 & 122.773 & 100.487 & 104.375 & 95.999 & 110.656 & & 74.126 \\
\hline $2 / 29 / 084: 30$ & 125.187 & 122.796 & 100.496 & 104.383 & 96.007 & 110.656 & & 74.132 \\
\hline
\end{tabular}


TABLE S1.1 (Cont.)

\begin{tabular}{|c|c|c|c|c|c|c|c|c|c|c|}
\hline \multirow[b]{2}{*}{ Date and Time } & \multicolumn{10}{|c|}{ Depth from Top of Casing (ft) } \\
\hline & MW1D & MW2D & MW3D & MW4D & MW7 & MW9 & Oentrich & MW14D & MW15D & MW16D \\
\hline 2/29/08 5:00 & 125.197 & 122.821 & & 100.513 & 104.39 & 96.018 & 110.656 & & 80.753 & 74.14 \\
\hline 2/29/08 5:30 & 125.206 & 122.839 & & 100.536 & 104.411 & 96.039 & 110.656 & & & 74.159 \\
\hline 2/29/08 6:00 & 125.225 & 122.861 & & 100.554 & 104.438 & 96.068 & 110.652 & & & 74.186 \\
\hline 2/29/08 6:30 & 125.246 & 122.882 & & 100.58 & 104.457 & 96.084 & 110.652 & & 80.775 & 74.202 \\
\hline $2 / 29 / 08$ 7:00 & 125.268 & 124.694 & & 100.603 & 104.48 & 96.108 & 110.65 & & & 74.223 \\
\hline $2 / 29 / 08$ 7:30 & 125.286 & 125.027 & & 100.642 & 104.499 & 96.129 & 110.648 & & & 74.239 \\
\hline 2/29/08 8:00 & 126.899 & 125.19 & & 101.444 & 104.967 & 96.728 & 111.234 & & 80.817 & 74.301 \\
\hline $2 / 29 / 088: 30$ & 127.338 & 125.29 & & 101.661 & 105.289 & 97.06 & 111.52 & & & 74.52 \\
\hline 2/29/08 9:00 & 127.579 & 123.567 & & 101.786 & 105.455 & 97.225 & 111.651 & & & 74.675 \\
\hline 2/29/08 9:30 & 127.674 & 123.319 & & 101.637 & 105.554 & 97.327 & 111.73 & & 80.914 & 74.775 \\
\hline $2 / 29 / 0810: 00$ & 126.215 & 123.215 & & 101.109 & 105.144 & 96.784 & 111.17 & & & 74.781 \\
\hline $2 / 29 / 08 \quad 10: 30$ & 125.951 & 123.161 & & 100.968 & 104.919 & 96.549 & 110.936 & & & 74.657 \\
\hline $2 / 29 / 0811: 00$ & 125.78 & 123.129 & & 100.897 & 104.819 & 96.447 & 110.832 & & 80.924 & 74.568 \\
\hline $2 / 29 / 0811: 30$ & 125.667 & 123.095 & & 100.858 & 104.765 & 96.395 & 110.774 & & & 74.514 \\
\hline $2 / 29 / 0812: 00$ & 125.596 & 123.077 & & 100.832 & 104.733 & 96.365 & 110.74 & & & 74.481 \\
\hline $2 / 29 / 0812: 30$ & 125.556 & 123.059 & & 100.809 & 104.702 & 96.334 & 110.718 & & 80.932 & 74.45 \\
\hline $2 / 29 / 08$ 13:00 & 125.525 & 123.043 & & 100.789 & 104.685 & 96.315 & 110.701 & & & 74.429 \\
\hline $2 / 29 / 0813: 30$ & 125.506 & 123.029 & & 100.772 & 104.668 & 96.301 & 110.69 & & & 74.413 \\
\hline $2 / 29 / 0814: 00$ & 125.483 & 123.004 & & 100.758 & 104.653 & 96.285 & 110.68 & & 80.936 & 74.396 \\
\hline $2 / 29 / 0814: 30$ & 125.468 & 122.997 & & 100.737 & 104.64 & 96.273 & 110.675 & & & 74.386 \\
\hline $2 / 29 / 08$ 15:00 & 125.45 & 123 & & 100.727 & 104.615 & 96.247 & 110.669 & & & 74.363 \\
\hline $2 / 29 / 0815: 30$ & 125.435 & 122.991 & & 100.722 & 104.607 & 96.242 & 110.663 & & 80.932 & 74.355 \\
\hline $2 / 29 / 08$ 16:00 & 125.428 & 122.981 & & 100.716 & 104.607 & 96.24 & 110.658 & & & 74.355 \\
\hline $2 / 29 / 0816: 30$ & 125.428 & 122.975 & & 100.71 & 104.603 & 96.237 & 110.652 & & & 74.355 \\
\hline $2 / 29 / 08$ 17:00 & 125.419 & 122.966 & & 100.704 & 104.598 & 96.226 & 110.648 & & 80.932 & 74.343 \\
\hline $2 / 29 / 0817: 30$ & 125.414 & 122.961 & & 100.696 & 104.586 & 96.221 & 110.645 & & & 74.339 \\
\hline $2 / 29 / 08$ 18:00 & 125.405 & 122.959 & & 100.687 & 104.577 & 96.212 & 110.643 & & & 74.332 \\
\hline $2 / 29 / 0818: 30$ & 125.395 & 122.961 & & 100.687 & 104.573 & 96.204 & 110.639 & & 80.932 & 74.326 \\
\hline 2/29/08 19:00 & 125.398 & 122.963 & & 100.687 & 104.571 & 96.204 & 110.635 & & & 74.324 \\
\hline $2 / 29 / 08$ 19:30 & 125.398 & 122.959 & & 100.68 & 104.571 & 96.204 & 110.632 & & & 74.326 \\
\hline 2/29/08 20:00 & 125.395 & 122.952 & & 100.687 & 104.575 & 96.204 & 110.626 & & 80.934 & 74.328 \\
\hline $2 / 29 / 0820: 30$ & 125.395 & 122.95 & & 100.68 & 104.571 & 96.204 & 110.626 & & & 74.326 \\
\hline 2/29/08 21:00 & 125.39 & 122.95 & & 100.675 & 104.565 & 96.195 & 110.624 & & & 74.322 \\
\hline 2/29/08 21:30 & 125.386 & 122.941 & & 100.673 & 104.562 & 96.19 & 110.62 & & 80.936 & 74.318 \\
\hline 2/29/08 22:00 & 125.386 & 122.934 & & 100.668 & 104.563 & 96.193 & 110.617 & & & 74.32 \\
\hline 2/29/08 22:30 & 125.379 & 122.923 & & 100.663 & 104.55 & 96.179 & 110.615 & & & 74.31 \\
\hline 2/29/08 23:00 & 125.369 & 122.913 & & 100.653 & 104.544 & 96.174 & 110.613 & & 80.932 & 74.303 \\
\hline $2 / 29 / 0823: 30$ & 125.362 & 122.902 & & 100.642 & 104.535 & 96.167 & 110.611 & & & 74.297 \\
\hline $3 / 1 / 080: 00$ & 125.353 & 122.884 & & 100.637 & 104.525 & 96.157 & 110.609 & & & 74.289 \\
\hline $3 / 1 / 080: 30$ & 125.343 & 122.87 & & 100.615 & 104.516 & 96.148 & 110.607 & & 80.919 & 74.281 \\
\hline $3 / 1 / 081: 00$ & 125.322 & 122.845 & & 100.596 & 104.495 & 96.127 & 110.607 & & & 74.264 \\
\hline $3 / 1 / 081: 30$ & 125.305 & 122.841 & & 100.584 & 104.48 & 96.11 & 110.603 & & & 74.248 \\
\hline $3 / 1 / 082: 00$ & 125.291 & 122.827 & & 100.561 & 104.459 & 96.089 & 110.605 & & 80.9 & 74.229 \\
\hline $3 / 1 / 082: 30$ & 125.275 & 122.807 & & 100.556 & 104.455 & 96.084 & 110.601 & & & 74.225 \\
\hline 3/1/08 3:00 & 125.265 & 122.798 & & 100.541 & 104.438 & 96.068 & 110.6 & & & 74.21 \\
\hline 3/1/08 3:30 & 125.246 & 122.78 & & 100.527 & 104.419 & 96.047 & 110.6 & & 80.878 & 74.192 \\
\hline $3 / 1 / 084: 00$ & 125.234 & 122.771 & & 100.511 & 104.409 & 96.037 & 110.596 & & & 74.186 \\
\hline 3/1/08 4:30 & 125.218 & 122.75 & & 100.499 & 104.394 & 96.021 & 110.596 & & & 74.167 \\
\hline 3/1/08 5:00 & 125.208 & 122.734 & & 100.487 & 104.385 & 96.009 & 110.594 & & 80.856 & 74.157 \\
\hline $3 / 1 / 085: 30$ & 125.194 & 122.721 & & 100.468 & 104.369 & 95.992 & 110.594 & & & 74.144 \\
\hline 3/1/08 6:00 & 125.178 & 122.705 & & 100.451 & 104.346 & 95.971 & 110.594 & & & 74.126 \\
\hline $3 / 1 / 086: 30$ & 125.161 & 122.685 & & 100.434 & 104.335 & 95.962 & 110.59 & & 80.831 & 74.115 \\
\hline $3 / 1 / 087: 00$ & 125.145 & 122.675 & & 100.417 & 104.316 & 95.94 & 110.59 & & & 74.099 \\
\hline 3/1/08 7:30 & 125.128 & 124.449 & & 100.398 & 104.301 & 95.924 & 110.586 & & & 74.082 \\
\hline 3/1/08 8:00 & 125.109 & 124.741 & & 100.391 & 104.287 & 95.915 & 110.585 & & 80.807 & 74.07 \\
\hline $3 / 1 / 088: 30$ & 126.766 & 124.873 & & 101.114 & 104.674 & 96.414 & 111.11 & & & 74.088 \\
\hline 3/1/08 9:00 & 127.199 & 124.929 & & 101.297 & 104.961 & 96.709 & 111.403 & & & 74.27 \\
\hline 3/1/08 9:30 & 127.352 & 124.977 & & 101.39 & 105.091 & 96.843 & 111.533 & & 80.839 & 74.396 \\
\hline $3 / 1 / 08$ 10:00 & 127.428 & 124.997 & & 101.435 & 105.156 & 96.909 & 111.612 & & & 74.467 \\
\hline 3/1/08 10:30 & 127.464 & 125.009 & & 101.469 & 105.194 & 96.945 & 111.66 & & & 74.512 \\
\hline 3/1/08 11:00 & 127.492 & 125.027 & & 101.495 & 105.222 & 96.973 & 111.698 & & 80.87 & 74.537 \\
\hline $3 / 1 / 0811: 30$ & 127.511 & 123.192 & & 101.509 & 105.236 & 96.987 & 111.724 & & & 74.558 \\
\hline 3/1/08 12:00 & 127.518 & 122.941 & & 101.151 & 105.253 & 97.006 & 111.741 & & & 74.574 \\
\hline 3/1/08 12:30 & 125.679 & 122.816 & & 100.725 & 104.761 & 96.393 & 111.144 & & 80.87 & 74.496 \\
\hline 3/1/08 13:00 & 125.39 & 122.725 & & 100.563 & 104.527 & 96.153 & 110.926 & & & 74.334 \\
\hline 3/1/08 13:30 & 125.258 & 122.666 & & 100.47 & 104.409 & 96.035 & 110.823 & & & 74.221 \\
\hline
\end{tabular}


TABLE S1.1 (Cont.)

Depth from Top of Casing (ft)

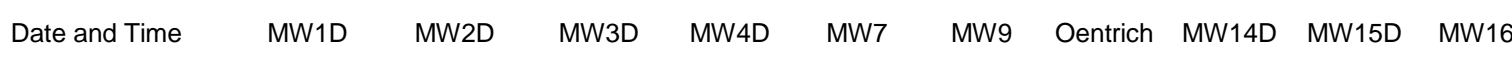

\begin{tabular}{|c|c|c|c|c|c|c|c|c|}
\hline 3/1/08 14:00 & 125.166 & 122.619 & 100.398 & 104.324 & 95.95 & 110.765 & 80.817 & 74.132 \\
\hline 3/1/08 14:30 & 125.102 & 122.582 & 100.346 & 104.265 & 95.889 & 110.729 & & 74.07 \\
\hline 3/1/08 15:00 & 125.055 & 122.553 & 100.305 & 104.215 & 95.842 & 110.705 & & 74.022 \\
\hline 3/1/08 15:30 & 125.017 & 122.533 & 100.277 & 104.183 & 95.806 & 110.684 & 80.785 & 73.985 \\
\hline 3/1/08 16:00 & 124.989 & 122.512 & 100.249 & 104.158 & 95.783 & 110.673 & & 73.958 \\
\hline 3/1/08 16:30 & 124.965 & 122.494 & 100.232 & 104.135 & 95.761 & 110.665 & & 73.937 \\
\hline 3/1/08 17:00 & 124.946 & 122.48 & 100.217 & 104.118 & 95.743 & 110.656 & 80.748 & 73.917 \\
\hline 3/1/08 17:30 & 124.932 & 122.471 & 100.201 & 104.101 & 95.726 & 110.633 & & 73.9 \\
\hline 3/1/08 18:00 & 124.915 & 122.458 & 100.189 & 104.089 & 95.712 & 110.629 & & 73.886 \\
\hline 3/1/08 18:30 & 124.906 & 122.451 & 100.181 & 104.078 & 95.705 & 110.63 & 80.716 & 73.875 \\
\hline 3/1/08 19:00 & 124.896 & 122.442 & 100.174 & 104.07 & 95.695 & 110.63 & & 73.865 \\
\hline 3/1/08 19:30 & 124.889 & 122.435 & 100.163 & 104.061 & 95.684 & 110.63 & & 73.859 \\
\hline 3/1/08 20:00 & 124.88 & 122.426 & 100.16 & 104.055 & 95.679 & 110.628 & 80.687 & 73.851 \\
\hline 3/1/08 20:30 & 124.873 & 122.424 & 100.148 & 104.048 & 95.672 & 110.628 & & 73.842 \\
\hline 3/1/08 21:00 & 124.861 & 122.417 & 100.141 & 104.036 & 95.66 & 110.628 & & 73.83 \\
\hline 3/1/08 21:30 & 124.854 & 122.406 & 100.139 & 104.032 & 95.658 & 110.626 & 80.65 & 73.826 \\
\hline 3/1/08 22:00 & 124.854 & 122.401 & 100.131 & 104.03 & 95.655 & 110.626 & & 73.824 \\
\hline 3/1/08 22:30 & 124.844 & 122.39 & 100.125 & 104.021 & 95.646 & 110.626 & & 73.813 \\
\hline 3/1/08 23:00 & 124.837 & 122.376 & 100.115 & 104.013 & 95.639 & 110.626 & 80.619 & 73.807 \\
\hline 3/1/08 23:30 & 124.826 & 122.36 & 100.105 & 104.002 & 95.627 & 110.624 & & 73.793 \\
\hline 3/2/08 0:00 & 124.814 & 122.344 & 100.089 & 103.992 & 95.615 & 110.626 & & 73.782 \\
\hline 3/2/08 0:30 & 124.797 & 122.322 & 100.074 & 103.975 & 95.596 & 110.63 & 80.587 & 73.766 \\
\hline 3/2/08 1:00 & 124.783 & 122.304 & 100.055 & 103.958 & 95.582 & 110.628 & & 73.749 \\
\hline 3/2/08 1:30 & 124.762 & 122.294 & 100.036 & 103.937 & 95.563 & 110.63 & & 73.731 \\
\hline 3/2/08 2:00 & 124.743 & 122.276 & 100.019 & 103.92 & 95.542 & 110.632 & 80.543 & 73.712 \\
\hline 3/2/08 2:30 & 124.731 & 122.26 & 100.008 & 103.909 & 95.533 & 110.632 & & 73.702 \\
\hline 3/2/08 3:00 & 124.717 & 122.24 & 99.993 & 103.895 & 95.516 & 110.63 & & 73.687 \\
\hline 3/2/08 3:30 & 124.698 & 122.224 & 99.974 & 103.878 & 95.502 & 110.63 & 80.504 & 73.671 \\
\hline 3/2/08 4:00 & 124.681 & 122.215 & 99.953 & 103.855 & 95.481 & 110.62 & & 73.65 \\
\hline 3/2/08 4:30 & 124.66 & 122.204 & 99.938 & 103.84 & 95.462 & 110.607 & & 73.634 \\
\hline 3/2/08 5:00 & 124.648 & 122.202 & 99.934 & 103.832 & 95.45 & 110.596 & 80.465 & 73.623 \\
\hline 3/2/08 5:30 & 124.641 & 122.197 & 99.924 & 103.821 & 95.443 & 110.59 & & 73.613 \\
\hline 3/2/08 6:00 & 124.636 & 122.199 & 99.92 & 103.819 & 95.441 & 110.585 & & 73.611 \\
\hline 3/2/08 6:30 & 124.627 & 122.199 & 99.922 & 103.813 & 95.434 & 110.579 & 80.435 & 73.603 \\
\hline 3/2/08 7:00 & 124.632 & 122.204 & 99.924 & 103.813 & 95.438 & 110.581 & & 73.605 \\
\hline 3/2/08 7:30 & 124.629 & 122.215 & 99.927 & 103.815 & 95.436 & 110.577 & & 73.603 \\
\hline 3/2/08 8:00 & 124.634 & 124.131 & 99.941 & 103.821 & 95.446 & 110.579 & 80.416 & 73.605 \\
\hline 3/2/08 8:30 & 124.646 & 124.369 & 100.372 & 103.834 & 95.455 & 110.579 & & 73.615 \\
\hline 3/2/08 9:00 & 126.457 & 124.508 & 100.766 & 104.365 & 96.11 & 111.225 & & 73.718 \\
\hline 3/2/08 9:30 & 126.733 & 124.594 & 100.944 & 104.598 & 96.351 & 111.445 & 80.45 & 73.904 \\
\hline 3/2/08 10:00 & 126.842 & 124.644 & 101.042 & 104.735 & 96.487 & 111.553 & & 74.039 \\
\hline 3/2/08 10:30 & 126.965 & 123.698 & 101.108 & 104.828 & 96.577 & 111.623 & & 74.13 \\
\hline 3/2/08 11:00 & 127.078 & 122.771 & 101.156 & 104.88 & 96.629 & 111.67 & 80.504 & 74.181 \\
\hline 3/2/08 11:30 & 127.144 & 122.61 & 100.63 & 104.921 & 96.671 & 111.702 & & 74.225 \\
\hline 3/2/08 12:00 & 125.331 & 122.546 & 100.367 & 104.36 & 95.985 & 111.028 & & 74.113 \\
\hline 3/2/08 12:30 & 125.064 & 122.503 & 100.279 & 104.213 & 95.837 & 110.859 & 80.499 & 73.995 \\
\hline $3 / 2 / 08$ 13:00 & 124.984 & 122.48 & 100.232 & 104.156 & 95.78 & 110.778 & & 73.94 \\
\hline 3/2/08 13:30 & 124.946 & 122.517 & 100.21 & 104.11 & 95.738 & 110.733 & & 73.892 \\
\hline 3/2/08 14:00 & 124.92 & 122.512 & 100.229 & 104.093 & 95.719 & 110.701 & 80.496 & 73.865 \\
\hline 3/2/08 14:30 & 124.934 & 122.478 & 100.251 & 104.135 & 95.759 & 110.671 & & 73.892 \\
\hline 3/2/08 15:00 & 124.939 & 122.512 & 100.249 & 104.131 & 95.761 & 110.663 & & 73.896 \\
\hline 3/2/08 15:30 & 124.934 & 122.467 & 100.191 & 104.091 & 95.721 & 110.662 & 80.496 & 73.88 \\
\hline 3/2/08 16:00 & 124.906 & 122.489 & 100.217 & 104.131 & 95.754 & 110.643 & & 73.851 \\
\hline 3/2/08 16:30 & 124.913 & 122.501 & 100.191 & 104.088 & 95.719 & 110.647 & & 73.842 \\
\hline 3/2/08 17:00 & 124.894 & 122.512 & 100.222 & 104.116 & 95.735 & 110.633 & 80.492 & 73.826 \\
\hline 3/2/08 17:30 & 124.892 & 122.533 & 100.229 & 104.124 & 95.752 & 110.632 & & 73.813 \\
\hline 3/2/08 18:00 & 124.885 & 122.56 & 100.567 & 104.135 & 95.761 & 110.63 & & 73.803 \\
\hline 3/2/08 18:30 & 124.873 & 122.569 & 100.672 & 104.154 & 95.783 & 110.624 & 80.499 & 73.793 \\
\hline 3/2/08 19:00 & 124.868 & 122.598 & 100.692 & 104.187 & 95.813 & 110.622 & & 73.782 \\
\hline 3/2/08 19:30 & 124.856 & 122.628 & 100.701 & 104.194 & 95.818 & 110.618 & & 73.772 \\
\hline 3/2/08 20:00 & 124.847 & 122.637 & 100.703 & 104.228 & 95.856 & 110.615 & 81.21 & 73.756 \\
\hline 3/2/08 20:30 & 124.842 & 122.657 & 100.711 & 104.255 & 95.884 & 110.613 & & 73.749 \\
\hline 3/2/08 21:00 & 124.842 & 122.662 & 100.713 & 104.261 & 95.889 & 110.615 & & 73.751 \\
\hline 3/2/08 21:30 & 124.837 & 122.682 & 100.725 & 104.285 & 95.912 & 110.613 & 81.156 & 73.756 \\
\hline 3/2/08 22:00 & 124.837 & 122.716 & 100.728 & 104.291 & 95.919 & 110.613 & & 73.768 \\
\hline 3/2/08 22:30 & 124.833 & 122.718 & 100.741 & 104.308 & 95.938 & 110.613 & & 73.815 \\
\hline
\end{tabular}


TABLE S1.1 (Cont.)

Depth from Top of Casing (ft)

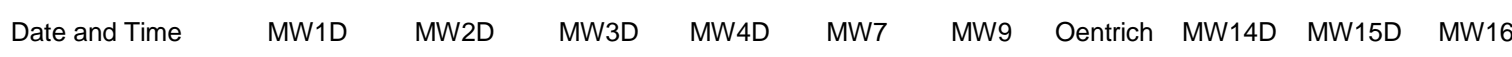

\begin{tabular}{|c|c|c|c|c|c|c|c|c|}
\hline 3/2/08 23:00 & 124.826 & 122.709 & 100.759 & 104.343 & 95.966 & 110.62 & 81.159 & 73.925 \\
\hline 3/2/08 23:30 & 124.826 & 122.728 & 100.763 & 104.345 & 95.976 & 110.632 & & 74.035 \\
\hline 3/3/08 0:00 & 124.828 & 122.725 & 100.758 & 104.329 & 95.959 & 110.635 & & 74.117 \\
\hline 3/3/08 0:30 & 124.821 & 122.739 & 100.766 & 104.354 & 95.983 & 110.637 & 81.171 & 74.198 \\
\hline 3/3/08 1:00 & 124.823 & 122.748 & 100.763 & 104.346 & 95.976 & 110.639 & & 74.285 \\
\hline 3/3/08 1:30 & 124.818 & 122.732 & 100.763 & 104.364 & 95.992 & 110.639 & & 74.384 \\
\hline 3/3/08 2:00 & 124.816 & 122.755 & 100.761 & 104.375 & 96.004 & 110.637 & 81.166 & 74.475 \\
\hline 3/3/08 2:30 & 124.816 & 122.768 & 100.756 & 104.36 & 95.988 & 110.639 & & 74.558 \\
\hline 3/3/08 3:00 & 124.809 & 122.791 & 100.759 & 104.379 & 96.009 & 110.637 & & 74.626 \\
\hline 3/3/08 3:30 & 124.807 & 122.789 & 100.764 & 104.394 & 96.023 & 110.635 & 81.132 & 74.688 \\
\hline 3/3/08 4:00 & 124.802 & 122.811 & 100.766 & 104.424 & 96.049 & 110.635 & & 74.74 \\
\hline 3/3/08 4:30 & 124.804 & 122.802 & 100.761 & 104.411 & 96.039 & 110.637 & & 74.789 \\
\hline 3/3/08 5:00 & 124.802 & 122.811 & 100.589 & 104.438 & 96.068 & 110.635 & 80.809 & 74.828 \\
\hline 3/3/08 5:30 & 124.807 & 122.843 & 100.563 & 104.43 & 96.063 & 110.635 & & 74.868 \\
\hline 3/3/08 6:00 & 124.811 & 122.843 & 100.57 & 104.436 & 96.068 & 110.635 & & 74.895 \\
\hline 3/3/08 6:30 & 124.811 & 122.875 & 100.587 & 104.47 & 96.098 & 110.633 & 80.724 & 74.913 \\
\hline 3/3/08 7:00 & 124.821 & 124.821 & 100.601 & 104.47 & 96.101 & 110.635 & & 74.936 \\
\hline 3/3/08 7:30 & 124.826 & 125.086 & 101.025 & 104.497 & 96.127 & 110.633 & & 74.946 \\
\hline 3/3/08 8:00 & 126.468 & 125.208 & 101.49 & 105.091 & 96.86 & 111.332 & 80.699 & 75.041 \\
\hline 3/3/08 8:30 & 126.698 & 125.301 & 101.691 & 105.346 & 97.121 & 111.559 & & 75.201 \\
\hline 3/3/08 9:00 & 126.828 & 125.349 & 101.797 & 105.477 & 97.256 & 111.674 & & 75.306 \\
\hline 3/3/08 9:30 & 126.913 & 123.535 & 101.867 & 105.563 & 97.336 & 111.747 & 80.782 & 75.389 \\
\hline 3/3/08 10:00 & 126.984 & 123.299 & 101.585 & 105.614 & 97.39 & 111.797 & & 75.457 \\
\hline 3/3/08 10:30 & 125.48 & 123.199 & 101.099 & 105.114 & 96.756 & 111.18 & & 75.436 \\
\hline 3/3/08 11:00 & 125.275 & 123.14 & 100.959 & 104.899 & 96.535 & 110.951 & 80.841 & 75.331 \\
\hline 3/3/08 11:30 & 125.19 & 123.088 & 100.89 & 104.803 & 96.436 & 110.851 & & 75.258 \\
\hline 3/3/08 12:00 & 125.147 & 123.038 & 100.844 & 104.746 & 96.381 & 110.795 & & 75.23 \\
\hline 3/3/08 12:30 & 125.128 & 123.031 & 100.815 & 104.697 & 96.329 & 110.757 & 80.841 & 75.234 \\
\hline 3/3/08 13:00 & 125.114 & 123.011 & 100.791 & 104.649 & 96.282 & 110.737 & & 75.246 \\
\hline 3/3/08 13:30 & 125.09 & 122.97 & 100.785 & 104.647 & 96.278 & 110.716 & & 75.252 \\
\hline 3/3/08 14:00 & 125.081 & 122.961 & 100.772 & 104.626 & 96.256 & 110.705 & 80.853 & 75.252 \\
\hline 3/3/08 14:30 & 125.076 & 122.945 & 100.737 & 104.583 & 96.219 & 110.697 & & 75.106 \\
\hline 3/3/08 15:00 & 125.071 & 122.927 & 100.725 & 104.575 & 96.204 & 110.686 & & 74.841 \\
\hline 3/3/08 15:30 & 125.074 & 122.907 & 100.715 & 104.562 & 96.193 & 110.678 & 80.861 & 74.498 \\
\hline 3/3/08 16:00 & 125.071 & 122.889 & 100.715 & 104.546 & 96.176 & 110.671 & & 74.326 \\
\hline 3/3/08 16:30 & 125.071 & 122.879 & 100.677 & 104.523 & 96.153 & 110.667 & & 74.353 \\
\hline 3/3/08 17:00 & 125.064 & 122.879 & 100.642 & 104.504 & 96.131 & 110.662 & 80.846 & 74.409 \\
\hline 3/3/08 17:30 & 125.06 & 122.882 & 100.63 & 104.497 & 96.127 & 110.658 & & 74.463 \\
\hline 3/3/08 18:00 & 125.052 & 122.886 & 100.675 & 104.501 & 96.127 & 110.652 & & 74.514 \\
\hline 3/3/08 18:30 & 125.045 & 122.882 & 100.692 & 104.501 & 96.129 & 110.648 & 80.809 & 74.554 \\
\hline 3/3/08 19:00 & 125.041 & 122.875 & 100.697 & 104.504 & 96.131 & 110.645 & & 74.593 \\
\hline 3/3/08 19:30 & 125.038 & 122.868 & 100.691 & 104.499 & 96.129 & 110.641 & & 74.634 \\
\hline 3/3/08 20:00 & 125.036 & 122.861 & 100.679 & 104.497 & 96.124 & 110.641 & 80.787 & 74.671 \\
\hline $3 / 3 / 0820: 30$ & 125.029 & 122.859 & 100.665 & 104.489 & 96.117 & 110.637 & & 74.707 \\
\hline $3 / 3 / 0821: 00$ & 125.029 & 122.857 & 100.651 & 104.484 & 96.108 & 110.635 & & 74.742 \\
\hline $3 / 3 / 0821: 30$ & 125.024 & 122.852 & 100.651 & 104.476 & 96.106 & 110.632 & 80.765 & 74.769 \\
\hline 3/3/08 22:00 & 125.022 & 122.836 & 100.642 & 104.476 & 96.103 & 110.63 & & 74.791 \\
\hline $3 / 3 / 08$ 22:30 & 125.017 & 122.823 & 100.641 & 104.47 & 96.101 & 110.626 & & 74.804 \\
\hline 3/3/08 23:00 & 125.017 & 122.807 & 100.641 & 104.455 & 96.082 & 110.626 & 80.741 & 74.814 \\
\hline 3/3/08 23:30 & 125.015 & 122.798 & 100.639 & 104.442 & 96.07 & 110.624 & & 74.816 \\
\hline 3/4/08 0:00 & 125.015 & 122.791 & 100.629 & 104.424 & 96.051 & 110.62 & & 74.818 \\
\hline 3/4/08 0:30 & 125.01 & 122.782 & 100.611 & 104.417 & 96.047 & 110.618 & 80.704 & 74.814 \\
\hline $3 / 4 / 081: 00$ & 125.008 & 122.773 & 100.608 & 104.411 & 96.039 & 110.615 & & 74.812 \\
\hline $3 / 4 / 081: 30$ & 125.008 & 122.762 & 100.592 & 104.4 & 96.028 & 110.611 & & 74.808 \\
\hline 3/4/08 2:00 & 125.005 & 122.755 & 100.587 & 104.396 & 96.021 & 110.609 & 80.663 & 74.804 \\
\hline $3 / 4 / 082: 30$ & 125.005 & 122.739 & 100.582 & 104.381 & 96.009 & 110.605 & & 74.8 \\
\hline 3/4/08 3:00 & 125.003 & 122.721 & 100.57 & 104.377 & 96.004 & 110.603 & & 74.795 \\
\hline 3/4/08 3:30 & 125.003 & 122.712 & 100.549 & 104.36 & 95.988 & 110.601 & 80.628 & 74.791 \\
\hline 3/4/08 4:00 & 125.003 & 122.705 & 100.537 & 104.341 & 95.966 & 110.598 & & 74.787 \\
\hline $3 / 4 / 084: 30$ & 125 & 122.718 & 100.525 & 104.337 & 95.959 & 110.594 & & 74.781 \\
\hline 3/4/08 5:00 & 125 & 122.712 & 100.518 & 104.327 & 95.952 & 110.594 & 80.592 & 74.773 \\
\hline 3/4/08 5:30 & 124.996 & 122.703 & 100.517 & 104.339 & 95.964 & 110.588 & & 74.762 \\
\hline 3/4/08 6:00 & 124.996 & 122.698 & 100.52 & 104.335 & 95.962 & 110.588 & & 74.754 \\
\hline $3 / 4 / 086: 30$ & 124.996 & 122.696 & 100.506 & 104.322 & 95.95 & 110.586 & 80.567 & 74.748 \\
\hline 3/4/08 7:00 & 124.996 & 124.637 & 100.511 & 104.318 & 95.945 & 110.586 & & 74.742 \\
\hline $3 / 4 / 087: 30$ & 124.996 & 124.859 & 100.84 & 104.316 & 95.945 & 110.585 & & 74.735 \\
\hline
\end{tabular}


TABLE S1.1 (Cont.)

\begin{tabular}{|c|c|c|c|c|c|c|c|c|c|c|}
\hline \multirow[b]{2}{*}{ Date and Time } & \multicolumn{10}{|c|}{ Depth from Top of Casing (ft) } \\
\hline & MW1D & MW2D & MW3D & MW4D & MW7 & MW9 & Oentrich & MW14D & MW15D & MW16D \\
\hline 3/4/08 8:00 & 126.629 & 124.975 & & 101.224 & 104.853 & 96.603 & 111.251 & & 80.533 & 74.82 \\
\hline $3 / 4 / 088: 30$ & 126.832 & 125.036 & & 101.397 & 105.076 & 96.832 & 111.463 & & & 74.957 \\
\hline 3/4/08 9:00 & 126.936 & 125.081 & & 101.488 & 105.194 & 96.947 & 111.572 & & & 75.052 \\
\hline 3/4/08 9:30 & 127.534 & 125.104 & & 101.545 & 105.255 & 97.008 & 111.64 & & 80.602 & 75.12 \\
\hline 3/4/08 10:00 & 127.584 & 125.118 & & 101.585 & 105.304 & 97.058 & 111.683 & & & 75.178 \\
\hline $3 / 4 / 08 \quad 10: 30$ & 127.603 & 125.134 & & 101.612 & 105.327 & 97.079 & 111.713 & & & 75.23 \\
\hline 3/4/08 11:00 & & 125.136 & & 101.633 & 105.338 & 97.093 & 111.737 & & 80.704 & 75.248 \\
\hline $3 / 4 / 0811: 30$ & & 125.122 & & 101.65 & 105.359 & 97.117 & 111.754 & & & 75.046 \\
\hline $3 / 4 / 08$ 12:00 & & 125.129 & & 101.666 & 105.361 & 97.119 & 111.767 & & & 74.998 \\
\hline $3 / 4 / 0812: 30$ & & 125.113 & & 101.669 & 105.348 & 97.105 & 111.779 & & 80.787 & 75.017 \\
\hline 3/4/08 13:00 & & 123.301 & & 101.674 & 105.357 & 97.112 & 111.784 & & & 74.806 \\
\hline 3/4/08 13:30 & & 123.015 & & 101.4 & 105.344 & 97.103 & 111.794 & & & 74.649 \\
\hline $3 / 4 / 08$ 14:00 & & 122.868 & & 100.911 & 104.87 & 96.506 & 111.212 & & 80.824 & 74.568 \\
\hline 3/4/08 14:30 & & 122.775 & & 100.696 & 104.603 & 96.23 & 110.969 & & & 74.39 \\
\hline 3/4/08 15:00 & & 122.718 & & 100.568 & 104.461 & 96.087 & 110.847 & & & 74.256 \\
\hline 3/4/08 15:30 & & 122.671 & & 100.506 & 104.371 & 95.997 & 110.765 & & 80.716 & 74.161 \\
\hline 3/4/08 16:00 & & 122.639 & & 100.453 & 104.32 & 95.945 & 110.716 & & & 74.161 \\
\hline $3 / 4 / 08$ 16:30 & & 122.621 & & 100.41 & 104.278 & 95.9 & 110.686 & & & 74.196 \\
\hline $3 / 4 / 08$ 17:00 & & 122.605 & & 100.403 & 104.244 & 95.87 & 110.663 & & 80.648 & 74.239 \\
\hline 3/4/08 17:30 & & 122.585 & & 100.389 & 104.228 & 95.849 & 110.647 & & & 74.274 \\
\hline $3 / 4 / 08$ 18:00 & & 122.582 & & 100.355 & 104.213 & 95.837 & 110.633 & & & 74.312 \\
\hline 3/4/08 18:30 & & 122.598 & & 100.353 & 104.194 & 95.818 & 110.626 & & 80.609 & 74.349 \\
\hline 3/4/08 19:00 & & 122.585 & & 100.343 & 104.194 & 95.816 & 110.617 & & & 74.378 \\
\hline $3 / 4 / 08$ 19:30 & & 122.573 & & 100.337 & 104.213 & 95.839 & 110.609 & & & 74.398 \\
\hline 3/4/08 20:00 & & 122.564 & & 100.334 & 104.198 & 95.823 & 110.605 & & 80.582 & 74.425 \\
\hline 3/4/08 20:30 & 125.03 & 122.571 & & 100.334 & 104.188 & 95.813 & 110.601 & & & 74.372 \\
\hline 3/4/08 21:00 & 125.03 & 122.569 & & 100.33 & 104.181 & 95.801 & 110.6 & & & 74.357 \\
\hline $3 / 4 / 0821: 30$ & 125.031 & 122.544 & & 100.325 & 104.187 & 95.809 & 110.592 & & 80.545 & 74.359 \\
\hline 3/4/08 22:00 & 125.026 & 122.569 & & 100.325 & 104.187 & 95.811 & 110.59 & & & 74.353 \\
\hline 3/4/08 22:30 & 125 & 122.571 & & 100.289 & 104.163 & 95.787 & 110.592 & & & 74.318 \\
\hline $3 / 4 / 0823: 00$ & 125.012 & 122.555 & & 100.294 & 104.183 & 95.806 & 110.583 & & 80.514 & 74.308 \\
\hline 3/4/08 23:30 & 125.019 & 122.544 & & 100.296 & 104.19 & 95.813 & 110.581 & & & 74.332 \\
\hline 3/5/08 0:00 & 125.003 & 122.535 & & 100.296 & 104.173 & 95.799 & 110.581 & & & 74.32 \\
\hline 3/5/08 0:30 & 124.988 & 122.53 & & 100.293 & 104.162 & 95.785 & 110.579 & & 80.504 & 74.291 \\
\hline 3/5/08 1:00 & 124.981 & 122.526 & & 100.294 & 104.16 & 95.783 & 110.577 & & & 74.266 \\
\hline 3/5/08 1:30 & 124.974 & 122.499 & & 100.286 & 104.154 & 95.778 & 110.575 & & & 74.245 \\
\hline 3/5/08 2:00 & 124.965 & 122.514 & & 100.284 & 104.147 & 95.766 & 110.573 & & 80.474 & 74.175 \\
\hline 3/5/08 2:30 & 124.943 & 122.526 & & 100.263 & 104.122 & 95.75 & 110.577 & & & 73.884 \\
\hline 3/5/08 3:00 & 124.955 & 122.544 & & 100.268 & 104.139 & 95.766 & 110.57 & & & 73.904 \\
\hline 3/5/08 3:30 & 124.969 & 122.53 & & 100.258 & 104.154 & 95.78 & 110.566 & & 80.457 & 73.917 \\
\hline 3/5/08 4:00 & 124.979 & 122.535 & & 100.265 & 104.169 & 95.787 & 110.558 & & & 73.909 \\
\hline $3 / 5 / 084: 30$ & 124.967 & 122.546 & & 100.263 & 104.152 & 95.778 & 110.562 & & & 73.911 \\
\hline 3/5/08 5:00 & 124.972 & 122.551 & & 100.262 & 104.158 & 95.78 & 110.558 & & 80.443 & 73.909 \\
\hline 3/5/08 5:30 & 124.984 & 122.569 & & 100.262 & 104.169 & 95.794 & 110.556 & & & 73.919 \\
\hline 3/5/08 6:00 & 124.988 & 122.587 & & 100.262 & 104.173 & 95.799 & 110.555 & & & 73.921 \\
\hline 3/5/08 6:30 & 125.005 & 123.898 & & 100.263 & 104.192 & 95.816 & 110.551 & & 80.423 & 73.937 \\
\hline 3/5/08 7:00 & 125.026 & 124.612 & & 100.282 & 104.213 & 95.834 & 110.549 & & & 73.954 \\
\hline 3/5/08 7:30 & 126.121 & 124.827 & & 100.935 & 104.282 & 95.898 & 110.686 & & & 73.962 \\
\hline 3/5/08 8:00 & 127.12 & 124.961 & & 101.255 & 104.885 & 96.645 & 111.324 & & 80.479 & 74.121 \\
\hline 3/5/08 8:30 & 127.34 & 125.061 & & 101.394 & 105.102 & 96.865 & 111.505 & & & 74.293 \\
\hline 3/5/08 9:00 & 127.48 & 123.304 & & 101.519 & 105.234 & 96.996 & 111.61 & & & 74.415 \\
\hline 3/5/08 9:30 & 127.579 & 123.074 & & 101.242 & 105.335 & 97.098 & 111.683 & & 80.545 & 74.508 \\
\hline 3/5/08 10:00 & 125.762 & 122.988 & & 100.813 & 104.895 & 96.53 & 111.09 & & & 74.52 \\
\hline $3 / 5 / 08 \quad 10: 30$ & 125.513 & 122.932 & & 100.685 & 104.678 & 96.303 & 110.87 & & & 74.448 \\
\hline 3/5/08 11:00 & 125.428 & 122.898 & & 100.627 & 104.602 & 96.226 & 110.774 & & 80.567 & 74.403 \\
\hline 3/5/08 11:30 & 125.371 & & & 100.589 & 104.55 & 96.176 & 110.724 & & & 74.386 \\
\hline 3/5/08 12:00 & 125.334 & & & 100.568 & 104.512 & 96.141 & 110.692 & & & 74.39 \\
\hline 3/5/08 12:30 & 125.322 & & & 100.556 & 104.503 & 96.129 & 110.669 & & 80.572 & 74.405 \\
\hline $3 / 5 / 08$ 13:00 & 125.317 & & & 100.565 & 104.499 & 96.127 & 110.652 & & & 74.429 \\
\hline 3/5/08 13:30 & 125.312 & & & 100.565 & 104.491 & 96.117 & 110.641 & & & 74.456 \\
\hline 3/5/08 14:00 & 125.3 & & & 100.551 & 104.48 & 96.11 & 110.635 & & 80.575 & 74.475 \\
\hline $3 / 5 / 08$ 14:30 & 125.286 & 122.875 & & 100.542 & 104.466 & 96.094 & 110.63 & & & 74.494 \\
\hline 3/5/08 15:00 & 125.286 & 122.859 & & 100.536 & 104.468 & 96.096 & 110.624 & & & 74.318 \\
\hline 3/5/08 15:30 & 125.284 & 122.85 & & 100.53 & 104.466 & 96.096 & 110.617 & & 80.562 & 74.208 \\
\hline 3/5/08 16:00 & 125.279 & 122.852 & & 100.525 & 104.461 & 96.094 & 110.617 & & & 74.194 \\
\hline 3/5/08 16:30 & 125.277 & 122.848 & & 100.534 & 104.461 & 96.091 & 110.611 & & & 74.19 \\
\hline
\end{tabular}


TABLE S1.1 (Cont.)

\begin{tabular}{|c|c|c|c|c|c|c|c|c|c|c|}
\hline \multirow[b]{2}{*}{ Date and Time } & \multicolumn{10}{|c|}{ Depth from Top of Casing (ft) } \\
\hline & MW1D & MW2D & MW3D & MW4D & MW7 & MW9 & Oentrich & MW14D & MW15D & MW16D \\
\hline 3/5/08 17:00 & 125.284 & 122.848 & & 100.541 & 104.474 & 96.098 & 110.609 & & 80.56 & 74.192 \\
\hline $3 / 5 / 08 \quad 17: 30$ & 125.284 & 122.85 & & 100.534 & 104.47 & 96.098 & 110.609 & & & 74.192 \\
\hline 3/5/08 18:00 & 125.286 & 122.857 & & 100.542 & 104.47 & 96.101 & 110.605 & & & 74.194 \\
\hline 3/5/08 18:30 & 125.286 & 122.868 & & 100.553 & 104.474 & 96.103 & 110.605 & & 80.565 & 74.194 \\
\hline 3/5/08 19:00 & 125.296 & 122.877 & & 100.56 & 104.484 & 96.11 & 110.603 & & & 74.2 \\
\hline $3 / 5 / 08$ 19:30 & 125.307 & 122.893 & & 100.565 & 104.493 & 96.12 & 110.6 & & & 74.21 \\
\hline 3/5/08 20:00 & 125.317 & 122.895 & & 100.585 & 104.506 & 96.134 & 110.598 & & 80.575 & 74.223 \\
\hline $3 / 5 / 08$ 20:30 & 125.333 & 122.898 & & 100.589 & 104.523 & 96.15 & 110.6 & & & 74.237 \\
\hline 3/5/08 21:00 & 125.336 & 122.907 & & 100.597 & 104.526 & 96.15 & 110.601 & & & 74.239 \\
\hline 3/5/08 21:30 & 125.341 & 122.916 & & 100.608 & 104.527 & 96.153 & 110.601 & & 80.584 & 74.243 \\
\hline 3/5/08 22:00 & 125.348 & 122.923 & & 100.615 & 104.533 & 96.16 & 110.6 & & & 74.25 \\
\hline 3/5/08 22:30 & 125.357 & 122.923 & & 100.622 & 104.546 & 96.172 & 110.6 & & & 74.262 \\
\hline 3/5/08 23:00 & 125.364 & 122.916 & & 100.634 & 104.55 & 96.179 & 110.6 & & 80.602 & 74.268 \\
\hline 3/5/08 23:30 & 125.364 & 122.913 & & 100.621 & 104.554 & 96.179 & 110.6 & & & 74.268 \\
\hline 3/6/08 0:00 & 125.357 & 122.918 & & 100.618 & 104.543 & 96.172 & 110.6 & & & 74.262 \\
\hline 3/6/08 0:30 & 125.355 & 122.918 & & 100.614 & 104.546 & 96.172 & 110.596 & & 80.606 & 74.262 \\
\hline $3 / 6 / 081: 00$ & 125.359 & 122.907 & & 100.625 & 104.546 & 96.176 & 110.592 & & & 74.264 \\
\hline 3/6/08 1:30 & 125.359 & 122.904 & & 100.618 & 104.548 & 96.176 & 110.592 & & & 74.266 \\
\hline $3 / 6 / 082: 00$ & 125.352 & 122.904 & & 100.615 & 104.537 & 96.167 & 110.59 & & 80.611 & 74.26 \\
\hline 3/6/08 2:30 & 125.348 & 122.898 & & 100.611 & 104.535 & 96.162 & 110.588 & & & 74.254 \\
\hline 3/6/08 3:00 & 125.348 & 122.879 & & 100.608 & 104.537 & 96.164 & 110.586 & & & 74.258 \\
\hline 3/6/08 3:30 & 125.343 & 122.868 & & 100.604 & 104.533 & 96.16 & 110.583 & & 80.621 & 74.254 \\
\hline $3 / 6 / 084: 00$ & 125.324 & 122.861 & & 100.601 & 104.51 & 96.139 & 110.583 & & & 74.235 \\
\hline 3/6/08 4:30 & 125.31 & 122.864 & & 100.597 & 104.501 & 96.127 & 110.581 & & & 74.225 \\
\hline 3/6/08 5:00 & 125.303 & 122.877 & & 100.592 & 104.491 & 96.117 & 110.575 & & 80.594 & 74.217 \\
\hline 3/6/08 5:30 & 125.305 & 122.875 & & 100.591 & 104.495 & 96.122 & 110.573 & & & 74.221 \\
\hline 3/6/08 6:00 & 125.322 & 122.868 & & 100.587 & 104.512 & 96.139 & 110.57 & & & 74.235 \\
\hline 3/6/08 6:30 & 125.317 & 122.87 & & 100.585 & 104.508 & 96.136 & 110.57 & & 80.597 & 74.233 \\
\hline 3/6/08 7:00 & 125.31 & 124.684 & & 100.582 & 104.501 & 96.129 & 110.568 & & & 74.227 \\
\hline $3 / 6 / 087: 30$ & 125.31 & 124.986 & & 100.58 & 104.501 & 96.127 & 110.568 & & & 74.227 \\
\hline 3/6/08 8:00 & 127.156 & 125.133 & & 101.335 & 104.925 & 96.669 & 111.101 & & 80.592 & 74.274 \\
\hline $3 / 6 / 088: 30$ & 127.478 & 125.22 & & 101.523 & 105.218 & 96.97 & 111.388 & & & 74.463 \\
\hline 3/6/08 9:00 & 127.631 & 125.276 & & 101.638 & 105.369 & 97.124 & 111.518 & & & 74.607 \\
\hline 3/6/08 9:30 & 127.721 & 125.313 & & 101.717 & 105.456 & 97.211 & 111.597 & & 80.692 & 74.696 \\
\hline $3 / 6 / 08$ 10:00 & 127.78 & 125.331 & & 101.752 & 105.514 & 97.27 & 111.644 & & & 74.756 \\
\hline $3 / 6 / 08 \quad 10: 30$ & 127.816 & 123.458 & & 101.781 & 105.548 & 97.305 & 111.679 & & & 74.793 \\
\hline $3 / 6 / 08$ 11:00 & 127.839 & 123.46 & & 101.361 & 105.573 & 97.329 & 111.705 & & 80.741 & 74.816 \\
\hline 3/6/08 11:30 & 125.92 & 123.19 & & 101.187 & 105.053 & 96.685 & 111.071 & & & 74.731 \\
\hline $3 / 6 / 08$ 12:00 & 125.955 & 123.09 & & 100.957 & 105.024 & 96.678 & 111.065 & & & 74.613 \\
\hline $3 / 6 / 08$ 12:30 & 125.639 & 123.022 & & 100.833 & 104.796 & 96.424 & 110.834 & & 80.743 & 74.529 \\
\hline 3/6/08 13:00 & 125.537 & 122.966 & & 100.766 & 104.705 & 96.332 & 110.752 & & & 74.448 \\
\hline $3 / 6 / 08$ 13:30 & 125.466 & 122.918 & & 100.725 & 104.638 & 96.266 & 110.705 & & & 74.384 \\
\hline $3 / 6 / 08$ 14:00 & 125.411 & 122.877 & & 100.697 & 104.581 & 96.209 & 110.673 & & 80.716 & 74.328 \\
\hline 3/6/08 14:30 & 125.359 & 122.848 & & 100.675 & 104.535 & 96.162 & 110.65 & & & 74.279 \\
\hline 3/6/08 15:00 & 125.322 & 122.825 & & 100.647 & 104.497 & 96.124 & 110.632 & & & 74.241 \\
\hline $3 / 6 / 08$ 15:30 & 125.289 & 122.807 & & 100.647 & 104.468 & 96.094 & 110.617 & & 80.672 & 74.212 \\
\hline 3/6/08 16:00 & 125.268 & 122.786 & & 100.616 & 104.447 & 96.075 & 110.599 & & & 74.192 \\
\hline 3/6/08 16:30 & 125.248 & 122.768 & & 100.609 & 104.432 & 96.056 & 110.584 & & & 74.175 \\
\hline 3/6/08 17:00 & 125.232 & 122.759 & & 100.594 & 104.411 & 96.037 & 110.578 & & 80.641 & 74.159 \\
\hline 3/6/08 17:30 & 125.213 & 122.752 & & 100.59 & 104.394 & 96.018 & 110.568 & & & 74.138 \\
\hline 3/6/08 18:00 & 125.201 & 122.748 & & 100.585 & 104.388 & 96.011 & 110.563 & & & 74.13 \\
\hline $3 / 6 / 08$ 18:30 & 125.194 & 122.739 & & 100.582 & 104.377 & 96.004 & 110.557 & & 80.604 & 74.121 \\
\hline 3/6/08 19:00 & 125.192 & 122.741 & & 100.577 & 104.375 & 95.999 & 110.551 & & & 74.117 \\
\hline $3 / 6 / 08$ 19:30 & 125.182 & 122.739 & & 100.566 & 104.365 & 95.992 & 110.549 & & & 74.107 \\
\hline 3/6/08 20:00 & 125.182 & 122.734 & & 100.565 & 104.369 & 95.995 & 110.545 & & 80.592 & 74.111 \\
\hline 3/6/08 20:30 & 125.182 & 122.732 & & 100.563 & 104.365 & 95.992 & 110.543 & & & 74.107 \\
\hline 3/6/08 21:00 & 125.175 & 122.734 & & 100.561 & 104.362 & 95.988 & 110.541 & & & 74.103 \\
\hline 3/6/08 21:30 & 125.175 & 122.748 & & 100.558 & 104.36 & 95.983 & 110.538 & & 80.577 & 74.101 \\
\hline 3/6/08 22:00 & 125.18 & 122.746 & & 100.554 & 104.365 & 95.99 & 110.536 & & & 74.105 \\
\hline 3/6/08 22:30 & 125.187 & 122.746 & & 100.554 & 104.375 & 96.002 & 110.532 & & & 74.115 \\
\hline 3/6/08 23:00 & 125.187 & 122.737 & & 100.552 & 104.373 & 95.997 & 110.532 & & 80.575 & 74.115 \\
\hline 3/6/08 23:30 & 125.189 & 122.737 & & 100.552 & 104.373 & 95.995 & 110.53 & & & 74.109 \\
\hline 3/7/08 0:00 & 125.18 & 122.732 & & 100.551 & 104.368 & 95.992 & 110.53 & & & 74.105 \\
\hline 3/7/08 0:30 & 125.177 & 122.741 & & 100.548 & 104.366 & 95.99 & 110.528 & & 80.567 & 74.105 \\
\hline 3/7/08 1:00 & 125.178 & 122.768 & & 100.544 & 104.366 & 95.99 & 110.526 & & & 74.103 \\
\hline 3/7/08 1:30 & 125.185 & 122.752 & & 100.546 & 104.371 & 95.997 & 110.523 & & & 74.109 \\
\hline
\end{tabular}


TABLE S1.1 (Cont.)

\begin{tabular}{|c|c|c|c|c|c|c|c|c|c|c|}
\hline \multirow[b]{2}{*}{ Date and Time } & \multicolumn{10}{|c|}{ Depth from Top of Casing (ft) } \\
\hline & MW1D & MW2D & MW3D & MW4D & MW7 & MW9 & Oentrich & MW14D & MW15D & MW16D \\
\hline 3/7/08 2:00 & 125.211 & 122.752 & & 100.546 & 104.404 & 96.028 & 110.519 & & 80.565 & 74.136 \\
\hline $3 / 7 / 082: 30$ & 125.194 & 122.768 & & 100.541 & 104.385 & 96.009 & 110.521 & & & 74.119 \\
\hline 3/7/08 3:00 & 125.194 & 122.777 & & 100.541 & 104.383 & 96.009 & 110.519 & & & 74.119 \\
\hline 3/7/08 3:30 & 125.213 & 122.791 & & 100.539 & 104.404 & 96.028 & 110.515 & & 80.56 & 74.138 \\
\hline 3/7/08 4:00 & 125.223 & 122.807 & & 100.536 & 104.415 & 96.039 & 110.513 & & & 74.146 \\
\hline 3/7/08 4:30 & 125.234 & 122.825 & & 100.532 & 104.426 & 96.054 & 110.513 & & & 74.159 \\
\hline 3/7/08 5:00 & 125.248 & 122.836 & & 100.529 & 104.442 & 96.07 & 110.509 & & 80.56 & 74.171 \\
\hline 3/7/08 5:30 & 125.267 & 122.843 & & 100.527 & 104.461 & 96.089 & 110.508 & & & 74.188 \\
\hline 3/7/08 6:00 & 125.279 & 122.859 & & 100.525 & 104.47 & 96.096 & 110.509 & & & 74.198 \\
\hline 3/7/08 6:30 & 125.291 & 122.873 & & 100.521 & 104.482 & 96.106 & 110.506 & & 80.565 & 74.206 \\
\hline 3/7/08 7:00 & 125.307 & 124.483 & & 100.613 & 104.497 & 96.122 & 110.502 & & & 74.219 \\
\hline 3/7/08 7:30 & 125.315 & 124.959 & & 100.625 & & 96.136 & 110.502 & & & 74.231 \\
\hline 3/7/08 8:00 & 126.938 & & & 101.357 & & 96.518 & 110.896 & & 80.57 & 74.245 \\
\hline 3/7/08 8:30 & 127.475 & & & 101.619 & & 97.001 & 111.321 & & & 74.452 \\
\hline 3/7/08 9:00 & 127.667 & & & 101.753 & & 97.187 & 111.482 & & & 74.618 \\
\hline 3/7/08 9:30 & 127.787 & 125.4 & & 101.852 & & 97.296 & 111.57 & & 80.653 & 74.729 \\
\hline 3/7/08 10:00 & 127.861 & 125.423 & & 101.907 & & 97.374 & 111.631 & & & 74.808 \\
\hline 3/7/08 10:30 & 127.905 & 125.444 & & 101.945 & & 97.416 & 111.674 & & & 74.851 \\
\hline 3/7/08 11:00 & 127.941 & 125.451 & & 101.972 & & 97.454 & 111.698 & & 80.743 & 74.886 \\
\hline 3/7/08 11:30 & 127.967 & 125.457 & & 101.989 & & 97.475 & 111.724 & & & 74.911 \\
\hline $3 / 7 / 08$ 12:00 & 127.979 & 123.868 & & 102.002 & & 97.489 & 111.741 & & & 74.926 \\
\hline 3/7/08 12:30 & 127.99 & 123.453 & & 102.014 & & 97.503 & 111.751 & & 80.809 & 74.942 \\
\hline 3/7/08 13:00 & 126.374 & 123.274 & & 101.283 & & 97.077 & 111.313 & & & 74.934 \\
\hline 3/7/08 13:30 & 125.913 & 123.172 & & 101.057 & & 96.676 & 110.949 & & & 74.762 \\
\hline 3/7/08 14:00 & 125.731 & 123.106 & & 100.933 & & 96.511 & 110.804 & & 80.822 & \\
\hline 3/7/08 14:30 & 125.622 & 123.058 & & 100.853 & & 96.41 & 110.724 & & & 75.08 \\
\hline 3/7/08 15:00 & 125.558 & 123.02 & & 100.801 & & 96.348 & 110.671 & & & 74.502 \\
\hline 3/7/08 15:30 & 125.506 & 122.993 & & 100.785 & & 96.301 & 110.635 & & 80.822 & 74.326 \\
\hline 3/7/08 16:00 & 125.468 & 122.968 & & 100.733 & & 96.266 & 110.613 & & & 74.281 \\
\hline 3/7/08 16:30 & 125.442 & 122.947 & & 100.725 & & 96.242 & 110.592 & & & 74.25 \\
\hline $3 / 7 / 08$ 17:00 & 125.414 & 122.931 & & 100.69 & & 96.216 & 110.846 & & 80.817 & 74.229 \\
\hline 3/7/08 17:30 & 125.393 & 122.911 & & 100.675 & & 96.197 & 110.55 & & & 74.212 \\
\hline 3/7/08 18:00 & 125.378 & 122.902 & & 100.67 & & 96.183 & 110.543 & & & 74.2 \\
\hline 3/7/08 18:30 & 125.359 & 122.888 & & 100.666 & & 96.164 & 110.536 & & 80.807 & 74.186 \\
\hline 3/7/08 19:00 & 125.35 & 122.877 & & 100.663 & & 96.155 & 110.53 & & & 74.175 \\
\hline 3/7/08 19:30 & 125.333 & 122.861 & & 100.625 & & 96.141 & 110.527 & & & 74.165 \\
\hline 3/7/08 20:00 & 125.324 & 122.85 & & 100.623 & & 96.131 & 110.521 & & 80.804 & 74.155 \\
\hline 3/7/08 20:30 & 125.31 & 122.841 & & 100.622 & & 96.117 & 110.517 & & & 74.148 \\
\hline 3/7/08 21:00 & 125.298 & 122.836 & & 100.591 & & 96.106 & 110.513 & & & 74.136 \\
\hline 3/7/08 21:30 & 125.289 & 122.834 & & 100.589 & & 96.096 & 110.509 & & 80.799 & 74.128 \\
\hline 3/7/08 22:00 & 125.286 & 122.832 & & 100.589 & & 96.094 & 110.504 & & & 74.121 \\
\hline 3/7/08 22:30 & 125.284 & 122.823 & & 100.585 & & 96.089 & 110.5 & & & 74.117 \\
\hline 3/7/08 23:00 & 125.281 & 122.809 & & 100.583 & & 96.089 & 110.498 & & 80.804 & 74.119 \\
\hline 3/7/08 23:30 & 125.27 & 122.8 & & 100.584 & & 96.077 & 110.494 & & & 74.113 \\
\hline 3/8/08 0:00 & 125.255 & 122.786 & & 100.549 & & 96.063 & 110.493 & & & 74.105 \\
\hline 3/8/08 0:30 & 125.248 & 122.761 & & 100.548 & & 96.056 & 110.489 & & 80.802 & 74.093 \\
\hline 3/8/08 1:00 & 125.234 & 122.748 & & 100.516 & & 96.042 & 110.489 & & & 74.082 \\
\hline 3/8/08 1:30 & 125.208 & 122.739 & & 100.496 & & 96.018 & 110.483 & & & 74.068 \\
\hline 3/8/08 2:00 & 125.196 & 122.714 & & 100.485 & & 96.004 & 110.481 & & 80.802 & 74.053 \\
\hline 3/8/08 2:30 & 125.187 & 122.7 & & 100.463 & & 95.995 & 110.476 & & & 74.043 \\
\hline 3/8/08 3:00 & 125.163 & 122.684 & & 100.453 & & 95.971 & 110.472 & & & 74.022 \\
\hline 3/8/08 3:30 & 125.149 & 122.673 & & 100.453 & & 95.959 & 110.47 & & 80.768 & 74.014 \\
\hline 3/8/08 4:00 & 125.133 & 122.653 & & 100.42 & & 95.94 & 110.466 & & & 73.999 \\
\hline 3/8/08 4:30 & 125.121 & 122.641 & & 100.409 & & 95.929 & 110.461 & & & 73.987 \\
\hline 3/8/08 5:00 & 125.102 & 122.623 & & 100.39 & & 95.912 & 110.461 & & 80.738 & 73.977 \\
\hline 3/8/08 5:30 & 125.092 & 122.612 & & 100.373 & & 95.903 & 110.455 & & & 73.958 \\
\hline 3/8/08 6:00 & 125.071 & 122.605 & & 100.361 & & 95.879 & 110.453 & & & 73.942 \\
\hline 3/8/08 6:30 & 125.062 & 122.591 & & 100.361 & & 95.872 & 110.448 & & 80.711 & 73.931 \\
\hline 3/8/08 7:00 & 125.057 & 122.58 & & 100.344 & & 95.867 & 110.446 & & & 73.925 \\
\hline 3/8/08 7:30 & 125.04 & 122.575 & & 100.332 & & 95.851 & 110.442 & & & 73.916 \\
\hline 3/8/08 8:00 & 125.029 & 123.954 & & 100.329 & & 95.837 & 110.442 & & 80.687 & 73.902 \\
\hline 3/8/08 8:30 & 125.026 & 124.573 & & 100.317 & & 95.837 & 110.438 & & & 73.898 \\
\hline 3/8/08 9:00 & 126.293 & 124.743 & & 100.975 & & 95.988 & 110.654 & & & 73.89 \\
\hline 3/8/08 9:30 & 127.094 & 124.836 & & 101.233 & & 96.617 & 111.223 & & 80.685 & 74.041 \\
\hline 3/8/08 10:00 & 127.269 & 124.893 & & 101.348 & & 96.784 & 111.399 & & & 74.181 \\
\hline 3/8/08 10:30 & 127.371 & 124.922 & & 101.426 & & 96.883 & 111.495 & & & 74.266 \\
\hline
\end{tabular}


TABLE S1.1 (Cont.)

\begin{tabular}{|c|c|c|c|c|c|c|c|c|c|c|}
\hline \multirow[b]{2}{*}{ Date and Time } & \multicolumn{10}{|c|}{ Depth from Top of Casing (ft) } \\
\hline & MW1D & MW2D & MW3D & MW4D & MW7 & MW9 & Oentrich & MW14D & MW15D & MW16D \\
\hline 3/8/08 11:00 & 127.43 & 123.12 & & 101.469 & & 96.942 & 111.557 & & 80.743 & 74.328 \\
\hline $3 / 8 / 08 \quad 11: 30$ & 127.459 & 122.857 & & 101.133 & & 96.968 & 111.597 & & & 74.363 \\
\hline 3/8/08 12:00 & 125.591 & 122.73 & & 100.671 & & 96.351 & 110.983 & & & 74.312 \\
\hline 3/8/08 12:30 & 125.31 & 122.657 & & 100.502 & & 96.087 & 110.752 & & 80.753 & 74.161 \\
\hline 3/8/08 13:00 & 125.18 & 122.6 & & 100.408 & & 95.969 & 110.648 & & & 74.055 \\
\hline 3/8/08 13:30 & 125.107 & 122.557 & & 100.354 & & 95.903 & 110.586 & & & 73.977 \\
\hline 3/8/08 14:00 & 125.05 & 122.528 & & 100.306 & & 95.849 & 110.547 & & 80.709 & 73.931 \\
\hline $3 / 8 / 08 \quad 14: 30$ & 125.007 & 122.503 & & 100.273 & & 95.809 & 110.521 & & & 73.887 \\
\hline 3/8/08 15:00 & 124.977 & 122.489 & & 100.249 & & 95.78 & 110.502 & & & 73.859 \\
\hline 3/8/08 15:30 & 124.953 & 122.485 & & 100.234 & & 95.757 & 110.483 & & 80.665 & 73.834 \\
\hline 3/8/08 16:00 & 124.936 & 122.478 & & 100.225 & & 95.743 & 110.47 & & & 73.815 \\
\hline 3/8/08 16:30 & 124.932 & 122.471 & & 100.22 & & 95.74 & 110.442 & & & 73.805 \\
\hline $3 / 8 / 08 \quad 17: 00$ & 124.927 & 122.464 & & 100.218 & & 95.735 & 110.43 & & 80.633 & 73.799 \\
\hline $3 / 8 / 08 \quad 17: 30$ & 124.922 & 122.464 & & 100.21 & & 95.728 & 110.426 & & & 73.795 \\
\hline 3/8/08 18:00 & 124.913 & 122.476 & & 100.204 & & 95.724 & 110.424 & & & 73.787 \\
\hline 3/8/08 18:30 & 124.913 & 122.489 & & 100.216 & & 95.724 & 110.42 & & & 73.78 \\
\hline 3/8/08 19:00 & 124.927 & 122.494 & & 100.23 & & 95.74 & 110.416 & & & 73.787 \\
\hline 3/8/08 19:30 & 124.939 & 122.498 & & 100.239 & & 95.752 & 110.414 & & & 73.797 \\
\hline 3/8/08 20:00 & 124.943 & 122.505 & & 100.241 & & 95.759 & 110.414 & & 80.636 & 73.805 \\
\hline 3/8/08 20:30 & 124.948 & 122.519 & & 100.247 & & 95.761 & 110.412 & & & 73.805 \\
\hline 3/8/08 21:00 & 124.955 & 122.521 & & 100.261 & & 95.768 & 110.412 & & & 73.809 \\
\hline 3/8/08 21:30 & 124.967 & 122.535 & & 100.267 & & 95.783 & 110.41 & & 80.623 & 73.82 \\
\hline 3/8/08 22:00 & 124.972 & 122.546 & & 100.273 & & 95.787 & 110.41 & & & 73.826 \\
\hline 3/8/08 22:30 & 124.981 & 122.555 & & 100.289 & & 95.797 & 110.41 & & & 73.83 \\
\hline 3/8/08 23:00 & 124.991 & 122.557 & & 100.294 & & 95.806 & 110.41 & & 80.631 & 73.84 \\
\hline 3/8/08 23:30 & 125.003 & 122.564 & & 100.303 & & 95.818 & 110.41 & & & 73.847 \\
\hline 3/9/08 0:00 & 125.007 & 122.569 & & 100.308 & & 95.823 & 110.412 & & & 73.853 \\
\hline 3/9/08 0:30 & 125.012 & 122.566 & & 100.318 & & 95.832 & 110.412 & & 80.633 & 73.857 \\
\hline $3 / 9 / 081: 00$ & 125.017 & 122.564 & & 100.318 & & 95.834 & 110.412 & & & 73.863 \\
\hline $3 / 9 / 081: 30$ & 125.017 & 122.564 & & 100.315 & & 95.832 & 110.412 & & & 73.865 \\
\hline 3/9/08 2:00 & 125.012 & 122.569 & & 100.315 & & 95.83 & 110.414 & & 80.631 & 73.863 \\
\hline $3 / 9 / 082: 30$ & 125.015 & 122.575 & & 100.317 & & 95.832 & 110.412 & & & 73.861 \\
\hline 3/9/08 3:00 & 125.019 & 122.575 & & 100.322 & & 95.837 & 110.41 & & & 73.863 \\
\hline 3/9/08 3:30 & 125.024 & 122.591 & & 100.325 & & 95.842 & 110.41 & & 80.628 & 73.867 \\
\hline 3/9/08 4:00 & 125.026 & 122.603 & & 100.339 & & 95.842 & 110.408 & & & 73.869 \\
\hline 3/9/08 4:30 & 125.04 & 122.607 & & 100.353 & & 95.86 & 110.408 & & & 73.878 \\
\hline 3/9/08 5:00 & 125.055 & 122.607 & & 100.363 & & 95.875 & 110.406 & & 80.636 & 73.89 \\
\hline 3/9/08 5:30 & 125.059 & 122.616 & & 100.358 & & 95.877 & 110.406 & & & 73.898 \\
\hline 3/9/08 6:00 & 125.055 & 122.628 & & 100.37 & & 95.875 & 110.406 & & & 73.896 \\
\hline 3/9/08 6:30 & 125.066 & 122.65 & & 100.378 & & 95.886 & 110.408 & & 80.631 & 73.904 \\
\hline 3/9/08 7:00 & 125.076 & 122.659 & & 100.389 & & 95.898 & 110.408 & & & 73.913 \\
\hline 3/9/08 7:30 & 125.097 & 122.668 & & 100.408 & & 95.915 & 110.406 & & & 73.921 \\
\hline 3/9/08 8:00 & 125.111 & 124.358 & & 100.422 & & 95.931 & 110.41 & & 80.645 & 73.935 \\
\hline $3 / 9 / 088: 30$ & 125.118 & 124.764 & & 100.425 & & 95.936 & 110.412 & & & 73.948 \\
\hline 3/9/08 9:00 & 126.844 & 124.941 & & 101.19 & & 96.405 & 110.883 & & & 73.966 \\
\hline 3/9/08 9:30 & 127.291 & 125.043 & & 101.433 & & 96.815 & 111.251 & & 80.697 & 74.163 \\
\hline $3 / 9 / 08$ 10:00 & 127.473 & 125.115 & & 101.562 & & 96.997 & 111.405 & & & 74.318 \\
\hline 3/9/08 10:30 & 127.577 & 125.16 & & 101.645 & & 97.098 & 111.493 & & & 74.419 \\
\hline 3/9/08 11:00 & 127.65 & 125.199 & & & & 97.169 & 111.55 & & 80.785 & 74.492 \\
\hline 3/9/08 11:30 & 127.7 & 125.231 & & & & 97.213 & 111.591 & & & 74.541 \\
\hline 3/9/08 12:00 & 127.737 & 125.256 & & 101.78 & & 97.256 & 111.617 & & & 74.58 \\
\hline $3 / 9 / 08$ 12:30 & 127.773 & 125.269 & & 101.805 & & 97.284 & 111.638 & & 80.858 & 74.611 \\
\hline $3 / 9 / 08$ 13:00 & 127.799 & 125.274 & & 101.822 & & 97.31 & 111.655 & & & 74.642 \\
\hline 3/9/08 13:30 & 127.808 & 125.281 & & 101.827 & & 97.322 & 111.672 & & & 74.663 \\
\hline 3/9/08 14:00 & 127.816 & 123.501 & & 101.836 & & 97.327 & 111.683 & & 80.912 & 74.673 \\
\hline $3 / 9 / 08 \quad 14: 30$ & 127.82 & 123.206 & & 101.567 & & 97.336 & 111.692 & & & 74.682 \\
\hline 3/9/08 15:00 & 125.981 & 123.072 & & 101.019 & & 96.725 & 111.09 & & & 74.624 \\
\hline 3/9/08 15:30 & 125.669 & 122.995 & & 100.844 & & 96.445 & 110.829 & & 80.909 & 74.467 \\
\hline $3 / 9 / 08 \quad 16: 00$ & 125.53 & 122.954 & & 100.752 & & 96.313 & 110.707 & & & 74.355 \\
\hline 3/9/08 16:30 & 125.452 & 122.918 & & 100.701 & & 96.247 & 110.637 & & & 74.283 \\
\hline $3 / 9 / 08$ 17:00 & 125.404 & 122.893 & & 100.664 & & & 110.59 & & 80.892 & 74.235 \\
\hline 3/9/08 17:30 & 125.371 & 122.882 & & 100.64 & & & 110.558 & & & 74.204 \\
\hline 3/9/08 18:00 & 125.348 & 122.87 & & 100.623 & & & 110.536 & & & 74.183 \\
\hline 3/9/08 18:30 & 125.331 & 122.866 & & 100.616 & & 96.141 & 110.517 & & 80.878 & 74.167 \\
\hline 3/9/08 19:00 & 125.324 & 122.863 & & 100.609 & & 96.134 & 110.504 & & & 74.157 \\
\hline 3/9/08 19:30 & 125.317 & 122.87 & & 100.606 & & 96.131 & 110.493 & & & 74.152 \\
\hline
\end{tabular}


TABLE S1.1 (Cont.)

\begin{tabular}{|c|c|c|c|c|c|c|c|c|c|c|}
\hline \multirow[b]{2}{*}{ Date and Time } & \multicolumn{10}{|c|}{ Depth from Top of Casing (ft) } \\
\hline & MW1D & MW2D & MW3D & MW4D & MW7 & MW9 & Oentrich & MW14D & MW15D & MW16D \\
\hline 3/9/08 20:00 & 125.315 & 122.877 & & 100.611 & & 96.129 & 110.483 & & 80.87 & 74.148 \\
\hline 3/9/08 20:30 & 125.322 & 122.882 & & 100.62 & & 96.138 & 110.476 & & & 74.152 \\
\hline 3/9/08 21:00 & 125.327 & 122.888 & & 100.623 & & 96.145 & 110.47 & & & 74.159 \\
\hline 3/9/08 21:30 & 125.334 & 122.893 & & 100.633 & & 96.155 & 110.464 & & 80.875 & 74.161 \\
\hline 3/9/08 22:00 & 125.336 & 122.9 & & 100.637 & & 96.155 & 110.461 & & & 74.169 \\
\hline 3/9/08 22:30 & 125.341 & 122.906 & & 100.642 & & 96.162 & 110.455 & & & 74.173 \\
\hline 3/9/08 23:00 & 125.348 & 122.913 & & 100.651 & & 96.167 & 110.451 & & 80.88 & 74.177 \\
\hline 3/9/08 23:30 & 125.355 & 122.922 & & 100.656 & & 96.178 & 110.451 & & & 74.186 \\
\hline 3/10/08 0:00 & 125.362 & 122.934 & & 100.666 & & 96.181 & 110.448 & & & 74.19 \\
\hline 3/10/08 0:30 & 125.374 & 122.94 & & 100.675 & & 96.19 & 110.444 & & 80.895 & 74.198 \\
\hline 3/10/08 1:00 & 125.383 & 122.945 & & 100.685 & & 96.202 & 110.44 & & & 74.206 \\
\hline 3/10/08 1:30 & 125.39 & 122.95 & & 100.692 & & 96.209 & 110.436 & & & 74.217 \\
\hline 3/10/08 2:00 & 125.395 & 122.95 & & 100.695 & & 96.214 & 110.433 & & 80.909 & 74.223 \\
\hline 3/10/08 2:30 & 125.402 & 122.94 & & 100.697 & & 96.219 & 110.431 & & & 74.227 \\
\hline 3/10/08 3:00 & 125.402 & 122.929 & & 100.689 & & 96.221 & 110.427 & & & 74.229 \\
\hline 3/10/08 3:30 & 125.393 & 122.922 & & 100.68 & & 96.209 & 110.423 & & 80.922 & 74.227 \\
\hline 3/10/08 4:00 & 125.379 & 122.92 & & 100.673 & & 96.195 & 110.421 & & & 74.219 \\
\hline 3/10/08 4:30 & 125.374 & 122.927 & & 100.668 & & 96.193 & 110.417 & & & 74.21 \\
\hline 3/10/08 5:00 & 125.372 & 122.936 & & 100.672 & & 96.19 & 110.414 & & 80.905 & 74.208 \\
\hline 3/10/08 5:30 & 125.376 & 122.95 & & 100.684 & & 96.197 & 110.408 & & & 74.208 \\
\hline 3/10/08 6:00 & 125.383 & 122.972 & & 100.691 & & 96.207 & 110.402 & & & 74.217 \\
\hline 3/10/08 6:30 & 125.398 & 122.968 & & 100.747 & & 96.221 & 110.397 & & 80.917 & 74.225 \\
\hline 3/10/08 7:00 & 125.421 & 122.975 & & 100.716 & & 96.24 & 110.402 & & & 74.241 \\
\hline 3/10/08 7:30 & 125.419 & 122.981 & & 100.722 & & 96.242 & 110.395 & & & 74.245 \\
\hline 3/10/08 8:00 & 125.426 & 122.988 & & 100.727 & & 96.247 & 110.387 & & 80.924 & 74.252 \\
\hline 3/10/08 8:30 & 125.43 & 122.99 & & 100.737 & & 96.252 & 110.386 & & & 74.26 \\
\hline 3/10/08 9:00 & 125.438 & 122.999 & & 100.741 & & 96.259 & 110.38 & & & 74.266 \\
\hline 3/10/08 9:30 & 125.442 & 122.999 & & 100.746 & & 96.263 & 110.376 & & 80.936 & 74.272 \\
\hline 3/10/08 10:00 & 125.449 & 122.999 & & 100.751 & & 96.27 & 110.374 & & & 74.276 \\
\hline 3/10/08 10:30 & 125.449 & 122.997 & & 100.749 & & 96.268 & 110.372 & & & 74.283 \\
\hline 3/10/08 11:00 & 125.447 & 122.984 & & 100.749 & & 96.268 & 110.369 & & 80.946 & 74.281 \\
\hline 3/10/08 11:30 & 125.447 & 122.972 & & 100.74 & & 96.268 & 110.367 & & & 74.281 \\
\hline 3/10/08 12:00 & 125.433 & 122.961 & & 100.725 & & 96.256 & 110.367 & & & 74.279 \\
\hline 3/10/08 12:30 & 125.423 & 122.945 & & 100.714 & & 96.242 & 110.365 & & 80.944 & 74.266 \\
\hline 3/10/08 13:00 & 125.412 & 122.927 & & 100.706 & & 96.228 & 110.363 & & & 74.256 \\
\hline 3/10/08 13:30 & 125.397 & 122.906 & & 100.682 & & 96.216 & 110.359 & & & 74.248 \\
\hline $3 / 10 / 08$ 14:00 & 125.376 & 122.886 & & 100.664 & & 96.197 & 110.359 & & 80.936 & 74.229 \\
\hline 3/10/08 14:30 & 125.359 & 122.87 & & 100.642 & & 96.179 & 110.356 & & & 74.214 \\
\hline 3/10/08 15:00 & 125.338 & 122.848 & & 100.623 & & 96.157 & 110.354 & & & 74.196 \\
\hline 3/10/08 15:30 & 125.319 & 122.834 & & 100.604 & & 96.141 & 110.35 & & 80.912 & 74.179 \\
\hline 3/10/08 16:00 & 125.301 & 122.818 & & 100.587 & & 96.122 & 110.348 & & & 74.165 \\
\hline 3/10/08 16:30 & 125.286 & 122.8 & & 100.57 & & 96.101 & 110.348 & & & 74.148 \\
\hline 3/10/08 17:00 & 125.268 & 122.791 & & 100.554 & & 96.084 & 110.342 & & 80.89 & 74.132 \\
\hline 3/10/08 17:30 & 125.251 & 122.784 & & 100.539 & & 96.07 & 110.339 & & & 74.117 \\
\hline 3/10/08 18:00 & 125.241 & 122.777 & & 100.534 & & 96.061 & 110.337 & & & 74.103 \\
\hline 3/10/08 18:30 & 125.237 & 122.77 & & 100.527 & & 96.058 & 110.329 & & 80.873 & 74.099 \\
\hline 3/10/08 19:00 & 125.227 & 122.764 & & 100.52 & & 96.047 & 110.327 & & & 74.09 \\
\hline 3/10/08 19:30 & 125.222 & 122.77 & & 100.513 & & 96.042 & 110.324 & & & 74.086 \\
\hline 3/10/08 20:00 & 125.216 & 122.766 & & 100.516 & & 96.035 & 110.322 & & 80.848 & 74.08 \\
\hline 3/10/08 20:30 & 125.22 & 122.761 & & 100.517 & & 96.042 & 110.318 & & & 74.078 \\
\hline 3/10/08 21:00 & 125.218 & 122.752 & & 100.513 & & 96.039 & 110.316 & & & 74.08 \\
\hline 3/10/08 21:30 & 125.208 & 122.741 & & 100.504 & & 96.032 & 110.314 & & 80.834 & 74.074 \\
\hline 3/10/08 22:00 & 125.203 & 122.734 & & 100.496 & & 96.023 & 110.314 & & & 74.07 \\
\hline 3/10/08 22:30 & 125.192 & 122.73 & & 100.487 & & 96.011 & 110.312 & & & 74.062 \\
\hline 3/10/08 23:00 & 125.182 & 122.723 & & 100.478 & & 96.004 & 110.31 & & 80.817 & 74.051 \\
\hline 3/10/08 23:30 & 125.177 & 122.711 & & 100.473 & & 95.997 & 110.309 & & & 74.045 \\
\hline 3/11/08 0:00 & 125.173 & 122.702 & & 100.465 & & 95.992 & 110.309 & & & 74.039 \\
\hline 3/11/08 0:30 & 125.166 & 122.687 & & 100.454 & & 95.983 & 110.309 & & 80.804 & 74.033 \\
\hline 3/11/08 1:00 & 125.149 & 122.677 & & 100.44 & & 95.969 & 110.309 & & & 74.022 \\
\hline 3/11/08 1:30 & 125.14 & 122.666 & & 100.428 & & 95.955 & 110.309 & & & 74.01 \\
\hline 3/11/08 2:00 & 125.128 & 122.662 & & 100.422 & & 95.948 & 110.307 & & 80.78 & 73.997 \\
\hline 3/11/08 2:30 & 125.121 & 122.648 & & 100.415 & & 95.938 & 110.305 & & & 73.991 \\
\hline 3/11/08 3:00 & 125.111 & 122.625 & & 100.404 & & 95.929 & 110.303 & & & 73.985 \\
\hline 3/11/08 3:30 & 125.099 & 122.612 & & 100.384 & & 95.917 & 110.301 & & 80.76 & 73.977 \\
\hline 3/11/08 4:00 & 125.078 & 122.6 & & 100.366 & & 95.893 & 110.303 & & & 73.96 \\
\hline 3/11/08 4:30 & 125.064 & 122.587 & & 100.354 & & 95.884 & 110.299 & & & 73.942 \\
\hline
\end{tabular}


TABLE S1.1 (Cont.)

\begin{tabular}{|c|c|c|c|c|c|c|c|c|c|c|}
\hline \multirow[b]{2}{*}{ Date and Time } & \multicolumn{10}{|c|}{ Depth from Top of Casing (ft) } \\
\hline & MW1D & MW2D & MW3D & MW4D & MW7 & MW9 & Oentrich & MW14D & MW15D & MW16D \\
\hline 3/11/08 5:00 & 125.052 & 122.571 & & 100.346 & & 95.875 & 110.299 & & 80.736 & 73.929 \\
\hline 3/11/08 5:30 & 125.038 & 123.707 & & 100.329 & & 95.856 & 110.297 & & & 73.923 \\
\hline 3/11/08 6:00 & 125.021 & 124.575 & & 100.32 & & 95.839 & 110.297 & & & 73.906 \\
\hline 3/11/08 6:30 & 125.431 & 124.761 & & 100.902 & & 95.837 & 110.294 & & 80.709 & 73.896 \\
\hline $3 / 11 / 08$ 7:00 & 127.073 & 124.854 & & 101.187 & & 96.57 & 111.016 & & & 74.033 \\
\hline 3/11/08 7:30 & 127.272 & 124.92 & & 101.312 & & 96.754 & 111.199 & & & 74.183 \\
\hline 3/11/08 8:00 & 127.367 & 124.954 & & 101.392 & & 96.85 & 111.294 & & 80.758 & 74.276 \\
\hline 3/11/08 8:30 & 127.43 & 124.979 & & 101.442 & & 96.919 & 111.354 & & & 74.339 \\
\hline 3/11/08 9:00 & 127.468 & 125.002 & & 101.469 & & 96.956 & 111.394 & & & 74.384 \\
\hline 3/11/08 9:30 & 127.492 & 125.011 & & 101.495 & & 96.985 & 111.414 & & 80.797 & 74.411 \\
\hline $3 / 11 / 0810: 00$ & 127.511 & 125.018 & & 101.51 & & 97.004 & 111.439 & & & 74.432 \\
\hline $3 / 11 / 08$ 10:30 & 127.525 & 125.013 & & 101.516 & & 97.015 & 111.456 & & & 74.452 \\
\hline $3 / 11 / 0811: 00$ & 127.53 & 125.002 & & 101.517 & & 97.02 & 111.469 & & 80.826 & 74.456 \\
\hline $3 / 11 / 0811: 30$ & 127.525 & 123.605 & & 101.512 & & 97.02 & 111.478 & & & 74.463 \\
\hline 3/11/08 12:00 & 127.513 & 123.018 & & 101.51 & & 97.008 & 111.491 & & & 74.463 \\
\hline $3 / 11 / 0812: 30$ & 126.192 & 122.82 & & 100.876 & & 96.806 & 111.259 & & 80.834 & 74.46 \\
\hline $3 / 11 / 0813: 00$ & 125.48 & 122.705 & & 100.606 & & 96.247 & 110.77 & & & 74.303 \\
\hline 3/11/08 13:30 & 125.272 & 122.621 & & 100.465 & & 96.056 & 110.613 & & & 74.152 \\
\hline $3 / 11 / 0814: 00$ & 125.154 & 122.562 & & 100.377 & & 95.945 & 110.526 & & 80.77 & 74.045 \\
\hline $3 / 11 / 0814: 30$ & 125.074 & 122.519 & & 100.311 & & 95.867 & 110.472 & & & 73.971 \\
\hline $3 / 11 / 08$ 15:00 & 125.015 & 122.482 & & 100.266 & & 95.811 & 110.436 & & & 73.911 \\
\hline $3 / 11 / 0815: 30$ & 124.972 & 122.453 & & 100.23 & & 95.768 & 110.402 & & 80.711 & 73.867 \\
\hline $3 / 11 / 0816: 00$ & 124.932 & 122.426 & & 100.198 & & 95.733 & 110.382 & & & 73.832 \\
\hline $3 / 11 / 0816: 30$ & 124.904 & 122.408 & & 100.17 & & 95.707 & 110.369 & & & 73.801 \\
\hline $3 / 11 / 08$ 17:00 & 124.878 & 122.39 & & 100.148 & & 95.684 & 110.356 & & 80.663 & 73.774 \\
\hline 3/11/08 17:30 & 124.856 & 122.378 & & 100.135 & & 95.665 & 110.346 & & & 73.753 \\
\hline $3 / 11 / 08$ 18:00 & 124.84 & 122.365 & & 100.12 & & 95.648 & 110.339 & & & 73.737 \\
\hline 3/11/08 18:30 & 124.828 & 122.355 & & 100.108 & & 95.636 & 110.333 & & 80.616 & 73.72 \\
\hline 3/11/08 19:00 & 124.816 & 122.346 & & 100.099 & & 95.627 & 110.327 & & & 73.71 \\
\hline $3 / 11 / 08$ 19:30 & 124.807 & 122.342 & & 100.089 & & 95.615 & 110.322 & & & 73.698 \\
\hline 3/11/08 20:00 & 124.797 & 122.337 & & 100.086 & & 95.608 & 110.318 & & 80.58 & 73.687 \\
\hline $3 / 11 / 0820: 30$ & 124.792 & 122.333 & & 100.08 & & 95.608 & 110.316 & & & 73.679 \\
\hline $3 / 11 / 0821: 00$ & 124.788 & 122.324 & & 100.077 & & 95.601 & 110.312 & & & 73.675 \\
\hline $3 / 11 / 0821: 30$ & 124.78 & 122.319 & & 100.07 & & 95.596 & 110.31 & & 80.545 & 73.669 \\
\hline 3/11/08 22:00 & 124.776 & 122.317 & & 100.061 & & 95.589 & 110.307 & & & 73.663 \\
\hline 3/11/08 22:30 & 124.769 & 122.315 & & 100.065 & & 95.585 & 110.301 & & & 73.652 \\
\hline 3/11/08 23:00 & 124.769 & 122.31 & & 100.06 & & 95.587 & 110.301 & & 80.516 & 73.65 \\
\hline 3/11/08 23:30 & 124.766 & 122.312 & & 100.058 & & 95.582 & 110.297 & & & 73.644 \\
\hline $3 / 12 / 080: 00$ & 124.762 & 122.303 & & 100.056 & & 95.58 & 110.297 & & & 73.642 \\
\hline 3/12/08 0:30 & 124.762 & 122.299 & & 100.053 & & 95.58 & 110.297 & & 80.492 & 73.638 \\
\hline 3/12/08 1:00 & 124.757 & 122.292 & & 100.049 & & 95.573 & 110.294 & & & 73.636 \\
\hline 3/12/08 1:30 & 124.752 & 122.29 & & 100.041 & & 95.566 & 110.29 & & & 73.629 \\
\hline 3/12/08 2:00 & 124.745 & 122.278 & & 100.036 & & 95.563 & 110.286 & & 80.465 & 73.623 \\
\hline 3/12/08 2:30 & 124.74 & 122.263 & & 100.03 & & 95.556 & 110.282 & & & 73.615 \\
\hline 3/12/08 3:00 & 124.731 & 122.251 & & 100.017 & & 95.549 & 110.275 & & & 73.609 \\
\hline 3/12/08 3:30 & 124.714 & 122.24 & & 99.999 & & 95.528 & 110.264 & & 80.433 & 73.598 \\
\hline 3/12/08 4:00 & 124.702 & 122.229 & & 99.992 & & 95.519 & 110.247 & & & 73.582 \\
\hline 3/12/08 4:30 & 124.691 & 122.224 & & 99.982 & & 95.507 & 110.239 & & & 73.572 \\
\hline 3/12/08 5:00 & 124.679 & 122.222 & & 99.972 & & 95.495 & 110.235 & & 80.413 & 73.561 \\
\hline 3/12/08 5:30 & 124.674 & 122.906 & & 99.972 & & 95.493 & 110.23 & & & 73.549 \\
\hline 3/12/08 6:00 & 124.674 & 124.19 & & 99.968 & & 95.49 & 110.228 & & & 73.547 \\
\hline 3/12/08 6:30 & 124.67 & 124.399 & & 100.573 & & 95.486 & 110.226 & & 80.379 & 73.545 \\
\hline 3/12/08 7:00 & 126.712 & 124.514 & & 100.88 & & 96.247 & 110.979 & & & 73.679 \\
\hline 3/12/08 7:30 & 126.929 & 122.723 & & 101.028 & & 96.452 & 111.17 & & & 73.836 \\
\hline 3/12/08 8:00 & 127.052 & 122.51 & & 100.685 & & 96.572 & 111.274 & & 80.43 & 73.946 \\
\hline 3/12/08 8:30 & 125.197 & 122.408 & & 100.308 & & 95.969 & 110.671 & & & 73.931 \\
\hline 3/12/08 9:00 & 124.967 & 122.353 & & 100.182 & & 95.761 & 110.479 & & & 73.807 \\
\hline 3/12/08 9:30 & 124.861 & 122.317 & & 100.108 & & 95.665 & 110.393 & & 80.401 & 73.725 \\
\hline 3/12/08 10:00 & 124.804 & 122.297 & & 100.075 & & 95.611 & 110.344 & & & 73.667 \\
\hline $3 / 12 / 0810: 30$ & 124.771 & 122.278 & & 100.051 & & 95.58 & 110.316 & & & 73.636 \\
\hline 3/12/08 11:00 & 124.747 & 122.265 & & 100.03 & & 95.563 & 110.295 & & 80.377 & 73.613 \\
\hline 3/12/08 11:30 & 124.731 & 122.251 & & 100.018 & & 95.545 & 110.284 & & & 73.596 \\
\hline $3 / 12 / 08$ 12:00 & 124.717 & 122.24 & & 100.003 & & 95.53 & 110.279 & & & 73.584 \\
\hline 3/12/08 12:30 & 124.702 & 122.226 & & 99.992 & & 95.521 & 110.269 & & 80.352 & 73.567 \\
\hline 3/12/08 13:00 & 124.691 & 122.222 & & 99.984 & & 95.512 & 110.265 & & & 73.559 \\
\hline 3/12/08 13:30 & 124.676 & 122.213 & & 99.97 & & 95.495 & 110.265 & & & 73.547 \\
\hline
\end{tabular}


TABLE S1.1 (Cont.)

Depth from Top of Casing (ft)

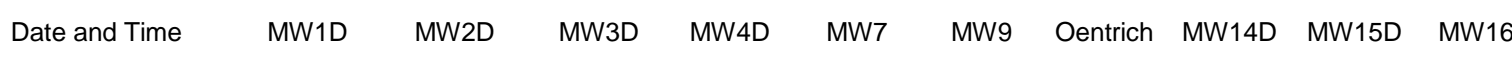

\begin{tabular}{|c|c|c|c|c|c|c|c|}
\hline 3/12/08 14:00 & 124.674 & 122.201 & 99.96 & 95.49 & 110.264 & 80.328 & 73.534 \\
\hline $3 / 12 / 08$ 14:30 & 124.662 & 122.21 & 99.951 & 95.481 & 110.262 & & 73.526 \\
\hline $3 / 12 / 08$ 15:00 & 124.653 & 122.219 & 99.949 & 95.471 & 110.26 & & 73.518 \\
\hline $3 / 12 / 08$ 15:30 & 124.66 & 122.229 & 99.961 & 95.481 & 110.256 & 80.315 & 73.516 \\
\hline $3 / 12 / 08$ 16:00 & 124.672 & 122.235 & 99.972 & 95.493 & 110.252 & & 73.522 \\
\hline $3 / 12 / 08$ 16:30 & 124.679 & 122.242 & 99.98 & 95.502 & 110.25 & & 73.528 \\
\hline $3 / 12 / 08$ 17:00 & 124.686 & 122.249 & 99.991 & 95.512 & 110.249 & 80.308 & 73.534 \\
\hline $3 / 12 / 08$ 17:30 & 124.693 & 122.251 & 99.999 & 95.519 & 110.247 & & 73.543 \\
\hline $3 / 12 / 08$ 18:00 & 124.7 & 122.256 & 100.001 & 95.523 & 110.247 & & 73.549 \\
\hline $3 / 12 / 08$ 18:30 & 124.702 & 122.265 & 100.01 & 95.528 & 110.247 & 80.298 & 73.551 \\
\hline 3/12/08 19:00 & 124.707 & 122.276 & 100.01 & 95.53 & 110.247 & & 73.553 \\
\hline $3 / 12 / 08 \quad 19: 30$ & 124.714 & 122.283 & 100.027 & 95.542 & 110.245 & & 73.553 \\
\hline $3 / 12 / 0820: 00$ & 124.726 & 122.29 & 100.037 & 95.554 & 110.243 & 80.294 & 73.563 \\
\hline $3 / 12 / 0820: 30$ & 124.733 & 122.299 & 100.043 & 95.559 & 110.245 & & 73.574 \\
\hline $3 / 12 / 0821: 00$ & 124.74 & 122.303 & 100.053 & 95.566 & 110.245 & & 73.58 \\
\hline $3 / 12 / 0821: 30$ & 124.747 & 122.31 & 100.055 & 95.575 & 110.245 & 80.291 & 73.586 \\
\hline 3/12/08 22:00 & 124.752 & 122.31 & 100.061 & 95.58 & 110.247 & & 73.588 \\
\hline $3 / 12 / 0822: 30$ & 124.759 & 122.312 & 100.065 & 95.585 & 110.247 & & 73.594 \\
\hline $3 / 12 / 08$ 23:00 & 124.759 & 122.324 & 100.067 & 95.585 & 110.249 & 80.289 & 73.596 \\
\hline 3/12/08 23:30 & 124.761 & 122.315 & 100.072 & 95.589 & 110.25 & & 73.596 \\
\hline 3/13/08 0:00 & 124.773 & 122.312 & 100.077 & 95.599 & 110.25 & & 73.601 \\
\hline 3/13/08 0:30 & 124.766 & 122.324 & 100.07 & 95.589 & 110.254 & 80.286 & 73.607 \\
\hline $3 / 13 / 081: 00$ & 124.764 & 122.326 & 100.075 & 95.589 & 110.256 & & 73.598 \\
\hline $3 / 13 / 081: 30$ & 124.773 & 122.328 & 100.082 & 95.599 & 110.256 & & 73.603 \\
\hline 3/13/08 2:00 & 124.778 & 122.331 & 100.084 & 95.603 & 110.258 & 80.286 & 73.607 \\
\hline $3 / 13 / 082: 30$ & 124.783 & 122.328 & 100.089 & 95.608 & 110.26 & & 73.611 \\
\hline 3/13/08 3:00 & 124.78 & 122.317 & 100.087 & 95.608 & 110.26 & & 73.613 \\
\hline 3/13/08 3:30 & 124.778 & 122.31 & 100.079 & 95.606 & 110.26 & 80.281 & 73.613 \\
\hline 3/13/08 4:00 & 124.769 & 122.308 & 100.07 & 95.596 & 110.26 & & 73.607 \\
\hline 3/13/08 4:30 & 124.764 & 122.308 & 100.07 & 95.589 & 110.256 & & 73.598 \\
\hline 3/13/08 5:00 & 124.759 & 122.308 & 100.065 & 95.587 & 110.256 & 80.274 & 73.596 \\
\hline 3/13/08 5:30 & 124.759 & 122.303 & 100.065 & 95.587 & 110.254 & & 73.594 \\
\hline 3/13/08 6:00 & 124.761 & 123.959 & 100.063 & 95.589 & 110.254 & & 73.592 \\
\hline 3/13/08 6:30 & 124.757 & 124.376 & 100.063 & 95.587 & 110.252 & 80.269 & 73.59 \\
\hline $3 / 13 / 08$ 7:00 & 126.418 & 124.541 & 100.751 & 95.969 & 110.658 & & 73.601 \\
\hline $3 / 13 / 087: 30$ & 126.877 & 124.634 & 100.98 & 96.374 & 111.033 & & 73.774 \\
\hline 3/13/08 8:00 & 127.054 & 124.698 & 101.102 & 96.546 & 111.184 & 80.323 & 73.917 \\
\hline 3/13/08 8:30 & 127.147 & 124.743 & 101.178 & 96.641 & 111.272 & & 74.012 \\
\hline 3/13/08 9:00 & 127.21 & 124.775 & 101.231 & 96.704 & 111.326 & & 74.078 \\
\hline 3/13/08 9:30 & 127.258 & 124.8 & 101.269 & 96.749 & 111.364 & 80.384 & 74.126 \\
\hline $3 / 13 / 08 \quad 10: 00$ & 127.289 & 124.816 & 101.3 & 96.784 & 111.392 & & 74.161 \\
\hline $3 / 13 / 08 \quad 10: 30$ & 127.315 & 124.825 & 101.321 & 96.81 & 111.414 & & 74.19 \\
\hline $3 / 13 / 0811: 00$ & 127.331 & 124.829 & 101.333 & 96.829 & 111.431 & 80.433 & 74.21 \\
\hline 3/13/08 11:30 & 127.345 & 124.832 & 101.342 & 96.843 & 111.445 & & 74.227 \\
\hline $3 / 13 / 08$ 12:00 & 127.343 & 123.02 & 101.347 & 96.846 & 111.456 & & 74.235 \\
\hline $3 / 13 / 08$ 12:30 & 127.348 & 122.75 & 101.026 & 96.85 & 111.463 & 80.474 & 74.245 \\
\hline $3 / 13 / 08$ 13:00 & 125.494 & 122.609 & 100.561 & 96.259 & 110.879 & & 74.179 \\
\hline 3/13/08 13:30 & 125.211 & 122.521 & 100.39 & 95.997 & 110.645 & & 74.02 \\
\hline $3 / 13 / 0814: 00$ & 125.066 & 122.46 & 100.287 & 95.863 & 110.534 & 80.438 & 73.904 \\
\hline $3 / 13 / 08$ 14:30 & 124.977 & 122.408 & 100.218 & 95.778 & 110.468 & & 73.822 \\
\hline $3 / 13 / 08$ 15:00 & 124.913 & 122.369 & 100.167 & 95.717 & 110.425 & & 73.762 \\
\hline $3 / 13 / 08$ 15:30 & 124.861 & 122.344 & 100.125 & 95.672 & 110.395 & 80.396 & 73.714 \\
\hline $3 / 13 / 08$ 16:00 & 124.823 & 122.333 & 100.098 & 95.634 & 110.376 & & 73.679 \\
\hline $3 / 13 / 08$ 16:30 & 124.797 & 122.315 & 100.08 & 95.611 & 110.359 & & 73.648 \\
\hline $3 / 13 / 08$ 17:00 & 124.785 & 122.299 & 100.067 & 95.596 & 110.346 & 80.364 & 73.632 \\
\hline 3/13/08 17:30 & 124.769 & 122.297 & 100.051 & 95.585 & 110.339 & & 73.619 \\
\hline $3 / 13 / 08$ 18:00 & 124.75 & 122.299 & 100.048 & 95.563 & 110.329 & & 73.605 \\
\hline $3 / 13 / 08$ 18:30 & 124.748 & 122.297 & 100.046 & 95.566 & 110.322 & 80.342 & 73.596 \\
\hline 3/13/08 19:00 & 124.75 & 122.294 & 100.049 & 95.573 & 110.316 & & 73.592 \\
\hline $3 / 13 / 08$ 19:30 & 124.747 & 122.283 & 100.044 & 95.566 & 110.31 & & 73.594 \\
\hline $3 / 13 / 0820: 00$ & 124.743 & 122.281 & 100.039 & 95.563 & 110.307 & 80.325 & 73.59 \\
\hline $3 / 13 / 0820: 30$ & 124.738 & 122.272 & 100.036 & 95.559 & 110.305 & & 73.586 \\
\hline $3 / 13 / 0821: 00$ & 124.733 & 122.267 & 100.029 & 95.554 & 110.301 & & 73.582 \\
\hline $3 / 13 / 0821: 30$ & 124.724 & 122.26 & 100.022 & 95.547 & 110.297 & 80.306 & 73.574 \\
\hline 3/13/08 22:00 & 124.717 & 122.256 & 100.018 & 95.54 & 110.295 & & 73.569 \\
\hline $3 / 13 / 0822: 30$ & 124.712 & 122.244 & 100.015 & 95.533 & 110.292 & & 73.563 \\
\hline
\end{tabular}


TABLE S1.1 (Cont.)

\begin{tabular}{|c|c|c|c|c|c|c|c|c|c|c|}
\hline \multirow[b]{2}{*}{ Date and Time } & \multicolumn{10}{|c|}{ Depth from Top of Casing (ft) } \\
\hline & MW1D & MW2D & MW3D & MW4D & MW7 & MW9 & Oentrich & MW14D & MW15D & MW16D \\
\hline 3/13/08 23:00 & 124.707 & 122.247 & & 100.003 & & 95.53 & 110.29 & & 80.284 & 73.559 \\
\hline 3/13/08 23:30 & 124.696 & 122.238 & & 99.999 & & 95.521 & 110.29 & & & 73.549 \\
\hline 3/14/08 0:00 & 124.7 & 122.231 & & 99.998 & & 95.521 & 110.288 & & & 73.545 \\
\hline 3/14/08 0:30 & 124.691 & 122.219 & & 99.989 & & 95.509 & 110.286 & & 80.264 & 73.543 \\
\hline 3/14/08 1:00 & 124.681 & 122.213 & & 99.979 & & 95.504 & 110.286 & & & 73.534 \\
\hline $3 / 14 / 08$ 1:30 & 124.67 & 122.222 & & 99.967 & & 95.493 & 110.284 & & & 73.524 \\
\hline 3/14/08 2:00 & 124.665 & 122.217 & & 99.974 & & 95.488 & 110.282 & & 80.245 & 73.514 \\
\hline 3/14/08 2:30 & 124.672 & 122.213 & & 99.977 & & 95.495 & 110.279 & & & 73.516 \\
\hline 3/14/08 3:00 & 124.67 & 122.206 & & 99.97 & & 95.495 & 110.277 & & & 73.518 \\
\hline 3/14/08 3:30 & 124.662 & 122.206 & & 99.963 & & 95.488 & 110.277 & & 80.228 & 73.512 \\
\hline 3/14/08 4:00 & 124.658 & 122.204 & & 99.963 & & 95.486 & 110.277 & & & 73.505 \\
\hline 3/14/08 4:30 & 124.655 & 122.21 & & 99.965 & & 95.481 & 110.273 & & & 73.501 \\
\hline 3/14/08 5:00 & 124.655 & 122.208 & & 99.961 & & 95.479 & 110.273 & & 80.215 & 73.503 \\
\hline 3/14/08 5:30 & 124.662 & 123.834 & & 99.965 & & 95.486 & 110.271 & & & 73.499 \\
\hline 3/14/08 6:00 & 124.66 & 124.256 & & 99.968 & & 95.486 & 110.269 & & & 73.501 \\
\hline 3/14/08 6:30 & 126.312 & 123.213 & & 100.704 & & 95.9 & 110.703 & & 80.218 & 73.514 \\
\hline 3/14/08 7:00 & 126.783 & 122.571 & & 100.937 & & 96.32 & 111.095 & & & 73.696 \\
\hline 3/14/08 7:30 & 126.033 & 122.43 & & 100.432 & & 96.471 & 111.142 & & & 73.84 \\
\hline 3/14/08 8:00 & 125.036 & 122.355 & & 100.222 & & 95.83 & 110.564 & & 80.257 & 73.799 \\
\hline 3/14/08 8:30 & 124.887 & 122.331 & & 100.137 & & 95.698 & 110.442 & & & 73.71 \\
\hline 3/14/08 9:00 & 124.809 & 122.312 & & 100.086 & & 95.622 & 110.386 & & & 73.656 \\
\hline 3/14/08 9:30 & 124.781 & 122.299 & & 100.068 & & 95.599 & 110.346 & & 80.242 & 73.609 \\
\hline 3/14/08 10:00 & 124.766 & 122.285 & & 100.063 & & 95.587 & 110.325 & & & 73.596 \\
\hline $3 / 14 / 0810: 30$ & 124.752 & 122.258 & & 100.048 & & 95.575 & 110.312 & & & 73.588 \\
\hline 3/14/08 11:00 & 124.738 & 122.258 & & 100.025 & & 95.559 & 110.303 & & 80.232 & 73.574 \\
\hline 3/14/08 11:30 & 124.71 & 122.242 & & 100.013 & & 95.533 & 110.299 & & & 73.559 \\
\hline $3 / 14 / 08$ 12:00 & 124.71 & 122.233 & & 99.999 & & 95.53 & 110.294 & & & 73.545 \\
\hline $3 / 14 / 08$ 12:30 & 124.691 & 122.226 & & 99.991 & & 95.514 & 110.29 & & 80.21 & 73.532 \\
\hline 3/14/08 13:00 & 124.684 & 122.217 & & 99.996 & & 95.509 & 110.29 & & & 73.524 \\
\hline 3/14/08 13:30 & 124.679 & 122.206 & & 99.977 & & 95.5 & 110.286 & & & 73.522 \\
\hline $3 / 14 / 0814: 00$ & 124.672 & 122.199 & & 99.96 & & 95.497 & 110.284 & & 80.196 & 73.512 \\
\hline $3 / 14 / 08$ 14:30 & 124.655 & 122.204 & & 99.956 & & 95.483 & 110.282 & & & 73.497 \\
\hline $3 / 14 / 08$ 15:00 & 124.653 & 122.206 & & 99.955 & & 95.476 & 110.28 & & & 73.491 \\
\hline $3 / 14 / 08$ 15:30 & 124.651 & 122.213 & & 99.965 & & 95.479 & 110.279 & & 80.186 & 73.487 \\
\hline 3/14/08 16:00 & 124.658 & 122.219 & & 99.965 & & 95.486 & 110.275 & & & 73.493 \\
\hline $3 / 14 / 0816: 30$ & 124.665 & 122.219 & & 99.974 & & 95.49 & 110.275 & & & 73.495 \\
\hline 3/14/08 17:00 & 124.674 & 122.219 & & 99.979 & & 95.5 & 110.273 & & 80.183 & 73.505 \\
\hline $3 / 14 / 08$ 17:30 & 124.67 & 122.231 & & 99.98 & & 95.5 & 110.273 & & & 73.507 \\
\hline $3 / 14 / 08$ 18:00 & 124.672 & 122.247 & & 99.982 & & 95.504 & 110.271 & & & 73.507 \\
\hline $3 / 14 / 08$ 18:30 & 124.681 & 122.265 & & 99.998 & & 95.514 & 110.267 & & 80.184 & 73.51 \\
\hline 3/14/08 19:00 & 124.7 & 122.276 & & 100.018 & & 95.528 & 110.265 & & & 73.522 \\
\hline $3 / 14 / 08$ 19:30 & 124.714 & 122.29 & & 100.032 & & 95.547 & 110.264 & & & 73.536 \\
\hline 3/14/08 20:00 & 124.717 & 122.299 & & 100.046 & & 95.559 & 110.262 & & 80.193 & 73.545 \\
\hline 3/14/08 20:30 & 124.719 & 122.312 & & 100.058 & & 95.573 & 110.26 & & & 73.543 \\
\hline $3 / 14 / 0821: 00$ & 124.726 & 122.328 & & 100.067 & & 95.585 & 110.26 & & & 73.534 \\
\hline $3 / 14 / 0821: 30$ & 124.736 & 122.344 & & 100.08 & & 95.599 & 110.258 & & 80.21 & 73.526 \\
\hline 3/14/08 22:00 & 124.736 & 122.351 & & 100.096 & & 95.611 & 110.258 & & & 73.518 \\
\hline 3/14/08 22:30 & 124.736 & 122.367 & & 100.11 & & 95.627 & 110.26 & & & 73.51 \\
\hline 3/14/08 23:00 & 124.74 & 122.378 & & 100.12 & & 95.636 & 110.265 & & 80.208 & 73.51 \\
\hline 3/14/08 23:30 & 124.736 & 122.38 & & 100.135 & & 95.648 & 110.271 & & & 73.514 \\
\hline $3 / 15 / 080: 00$ & 124.736 & 122.378 & & 100.146 & & 95.665 & 110.273 & & & 73.522 \\
\hline 3/15/08 0:30 & 124.797 & 122.374 & & 100.144 & & 95.665 & 110.275 & & 80.215 & 73.522 \\
\hline 3/15/08 1:00 & 124.795 & 122.383 & & 100.141 & & 95.665 & 110.277 & & & 73.534 \\
\hline 3/15/08 1:30 & 124.792 & 122.392 & & 100.141 & & 95.658 & 110.275 & & & 73.559 \\
\hline 3/15/08 2:00 & 124.797 & 122.401 & & 100.151 & & 95.667 & 110.275 & & 80.218 & 73.582 \\
\hline 3/15/08 2:30 & 124.8 & 122.403 & & 100.163 & & 95.679 & 110.275 & & & 73.607 \\
\hline 3/15/08 3:00 & 124.8 & 122.408 & & 100.167 & & 95.688 & 110.277 & & & 73.636 \\
\hline 3/15/08 3:30 & 124.802 & 122.408 & & 100.168 & & 95.691 & 110.275 & & 80.227 & 73.667 \\
\hline 3/15/08 4:00 & 124.8 & 122.417 & & 100.172 & & 95.695 & 110.275 & & & 73.689 \\
\hline 3/15/08 4:30 & 124.823 & 122.433 & & 100.175 & & 95.695 & 110.273 & & & 73.71 \\
\hline 3/15/08 5:00 & 124.821 & 122.435 & & 100.189 & & 95.705 & 110.271 & & 80.237 & 73.725 \\
\hline 3/15/08 5:30 & 124.842 & 122.448 & & 100.199 & & 95.719 & 110.271 & & & 73.735 \\
\hline 3/15/08 6:00 & 124.842 & 122.453 & & 100.203 & & 95.721 & 110.271 & & & 73.753 \\
\hline 3/15/08 6:30 & 124.868 & 122.467 & & 100.218 & & 95.733 & 110.271 & & 80.269 & 73.77 \\
\hline 3/15/08 7:00 & 124.873 & 122.473 & & 100.227 & & 95.745 & 110.26 & & & 73.787 \\
\hline $3 / 15 / 087: 30$ & 124.918 & 124.317 & & 100.235 & & 95.754 & 110.259 & & & 73.797 \\
\hline
\end{tabular}


TABLE S1.1 (Cont.)

\begin{tabular}{|c|c|c|c|c|c|c|c|c|c|c|}
\hline \multirow[b]{2}{*}{ Date and Time } & \multicolumn{10}{|c|}{ Depth from Top of Casing (ft) } \\
\hline & MW1D & MW2D & MW3D & MW4D & MW7 & MW9 & Oentrich & MW14D & MW15D & MW16D \\
\hline 3/15/08 8:00 & 124.925 & 124.634 & & 100.282 & & 95.761 & 110.264 & & 80.284 & 73.793 \\
\hline 3/15/08 8:30 & 126.783 & 124.782 & & 101.037 & & 96.327 & 110.808 & & & 73.811 \\
\hline 3/15/08 9:00 & 127.088 & 124.884 & & 101.238 & & 96.645 & 111.099 & & & 73.983 \\
\hline 3/15/08 9:30 & 127.234 & 124.943 & & 101.355 & & 96.794 & 111.233 & & 80.374 & 74.119 \\
\hline $3 / 15 / 0810: 00$ & 127.362 & 124.979 & & 101.433 & & 96.893 & 111.311 & & & 74.217 \\
\hline $3 / 15 / 08$ 10:30 & 127.419 & 125.009 & & 101.476 & & 96.954 & 111.366 & & & 74.293 \\
\hline $3 / 15 / 0811: 00$ & 127.497 & 125.049 & & 101.507 & & 96.997 & 111.399 & & 80.44 & 74.343 \\
\hline $3 / 15 / 0811: 30$ & 127.523 & 125.065 & & 101.545 & & 97.025 & 111.426 & & & 74.372 \\
\hline 3/15/08 12:00 & 127.563 & 125.074 & & 101.572 & & 97.067 & 111.443 & & & 74.398 \\
\hline $3 / 15 / 08$ 12:30 & 127.584 & 125.074 & & 101.585 & & 97.084 & 111.46 & & 80.509 & 74.429 \\
\hline $3 / 15 / 08$ 13:00 & 127.591 & 125.074 & & 101.586 & & 97.093 & 111.473 & & & 74.448 \\
\hline 3/15/08 13:30 & 127.589 & 125.068 & & 101.591 & & 97.096 & 111.486 & & & 74.454 \\
\hline $3 / 15 / 0814: 00$ & 127.591 & 125.058 & & 101.581 & & 97.093 & 111.493 & & 80.553 & 74.46 \\
\hline $3 / 15 / 0814: 30$ & 127.582 & 123.208 & & 101.571 & & 97.088 & 111.501 & & & 74.458 \\
\hline 3/15/08 15:00 & 127.57 & 122.972 & & 101.147 & & 97.077 & 111.506 & & & 74.454 \\
\hline $3 / 15 / 08$ 15:30 & 125.679 & 122.857 & & 100.775 & & 96.452 & 110.877 & & 80.575 & 74.37 \\
\hline $3 / 15 / 0816: 00$ & 125.428 & 122.789 & & 100.633 & & 96.221 & 110.665 & & & 74.225 \\
\hline 3/15/08 16:30 & 125.312 & 122.741 & & 100.554 & & 96.113 & 110.562 & & & 74.126 \\
\hline $3 / 15 / 08$ 17:00 & 125.244 & 122.714 & & 100.502 & & 96.049 & 110.5 & & 80.55 & 74.064 \\
\hline $3 / 15 / 0817: 30$ & 125.197 & 122.696 & & 100.468 & & 96.007 & 110.459 & & & 74.018 \\
\hline 3/15/08 18:00 & 125.166 & 122.682 & & 100.447 & & 95.978 & 110.429 & & & 73.987 \\
\hline $3 / 15 / 0818: 30$ & 125.147 & 122.684 & & 100.434 & & 95.964 & 110.408 & & 80.558 & 73.968 \\
\hline $3 / 15 / 08$ 19:00 & 125.133 & 122.68 & & 100.432 & & 95.952 & 110.391 & & & 73.954 \\
\hline 3/15/08 19:30 & 125.137 & 122.682 & & 100.432 & & 95.955 & 110.378 & & & 73.95 \\
\hline $3 / 15 / 0820: 00$ & 125.133 & 122.696 & & 100.432 & & 95.955 & 110.369 & & 80.543 & 73.952 \\
\hline $3 / 15 / 0820: 30$ & 125.13 & 122.698 & & 100.442 & & 95.952 & 110.357 & & & 73.95 \\
\hline 3/15/08 21:00 & 125.147 & 122.711 & & 100.451 & & 95.966 & 110.352 & & & 73.956 \\
\hline 3/15/08 21:30 & 125.149 & 122.716 & & 100.459 & & 95.976 & 110.346 & & 80.555 & 73.966 \\
\hline 3/15/08 22:00 & 125.161 & 122.723 & & 100.473 & & 95.988 & 110.341 & & & 73.975 \\
\hline 3/15/08 22:30 & 125.168 & 122.721 & & 100.478 & & 95.995 & 110.335 & & & 73.983 \\
\hline 3/15/08 23:00 & 125.175 & 122.721 & & 100.478 & & 96.002 & 110.331 & & 80.56 & 73.991 \\
\hline 3/15/08 23:30 & 125.173 & 122.718 & & 100.478 & & 96.002 & 110.327 & & & 73.991 \\
\hline $3 / 16 / 080: 00$ & 125.171 & 122.711 & & 100.482 & & 95.999 & 110.325 & & & 73.993 \\
\hline 3/16/08 0:30 & 125.171 & 122.7 & & 100.477 & & 95.997 & 110.322 & & 80.565 & 73.993 \\
\hline 3/16/08 1:00 & 125.166 & 122.711 & & 100.461 & & 95.992 & 110.318 & & & 73.989 \\
\hline 3/16/08 1:30 & 125.151 & 122.707 & & 100.471 & & 95.981 & 110.316 & & & 73.981 \\
\hline 3/16/08 2:00 & 125.164 & 122.698 & & 100.458 & & 95.995 & 110.31 & & 80.565 & 73.983 \\
\hline 3/16/08 2:30 & 125.154 & 122.709 & & 100.466 & & 95.985 & 110.305 & & & 73.975 \\
\hline 3/16/08 3:00 & 125.147 & 122.689 & & 100.466 & & 95.976 & 110.305 & & & 73.981 \\
\hline 3/16/08 3:30 & 125.161 & 122.7 & & 100.465 & & 95.992 & 110.299 & & 80.562 & 73.979 \\
\hline 3/16/08 4:00 & 125.142 & 122.718 & & 100.458 & & 95.969 & 110.301 & & & 73.979 \\
\hline 3/16/08 4:30 & 125.152 & 122.721 & & 100.463 & & 95.983 & 110.294 & & & 73.973 \\
\hline 3/16/08 5:00 & 125.166 & 122.725 & & 100.482 & & 95.999 & 110.288 & & 80.562 & 73.979 \\
\hline 3/16/08 5:30 & 125.168 & 122.727 & & 100.484 & & 95.999 & 110.286 & & & 73.995 \\
\hline 3/16/08 6:00 & 125.173 & 122.721 & & 100.489 & & 96.002 & 110.286 & & & 74.004 \\
\hline 3/16/08 6:30 & 125.178 & 122.723 & & 100.484 & & 96.009 & 110.282 & & 80.575 & 74.002 \\
\hline 3/16/08 7:00 & 125.171 & 122.718 & & 100.487 & & 95.999 & 110.28 & & & 74.002 \\
\hline 3/16/08 7:30 & 125.173 & 124.507 & & 100.484 & & 96.004 & 110.277 & & & 73.997 \\
\hline 3/16/08 8:00 & 125.171 & 124.832 & & 100.48 & & 95.999 & 110.277 & & 80.582 & 74.002 \\
\hline 3/16/08 8:30 & 126.984 & 124.984 & & 101.247 & & 96.518 & 110.791 & & & 74.03 \\
\hline 3/16/08 9:00 & 127.336 & 125.079 & & 101.455 & & 96.855 & 111.078 & & & 74.229 \\
\hline 3/16/08 9:30 & 127.494 & 123.501 & & 101.566 & & 97.006 & 111.208 & & 80.653 & 74.372 \\
\hline 3/16/08 10:00 & 127.589 & 123.154 & & 101.641 & & 97.105 & 111.283 & & & 74.465 \\
\hline $3 / 16 / 08$ 10:30 & 126 & 123.027 & & 100.987 & & 96.747 & 110.9 & & & 74.508 \\
\hline 3/16/08 11:00 & 125.615 & 122.947 & & 100.816 & & 96.417 & 110.583 & & 80.687 & 74.388 \\
\hline 3/16/08 11:30 & 125.483 & 122.9 & & 100.726 & & 96.292 & 110.463 & & & 74.299 \\
\hline $3 / 16 / 08$ 12:00 & 125.405 & 122.872 & & 100.663 & & 96.219 & 110.399 & & & 74.233 \\
\hline 3/16/08 12:30 & 125.353 & 122.829 & & 100.633 & & 96.172 & 110.357 & & 80.68 & 74.181 \\
\hline 3/16/08 13:00 & 125.324 & 122.777 & & 100.606 & & 96.146 & 110.329 & & & 74.152 \\
\hline $3 / 16 / 08$ 13:30 & 125.284 & 122.75 & & 100.561 & & 96.103 & 110.316 & & & 74.13 \\
\hline $3 / 16 / 0814: 00$ & 125.232 & 122.739 & & 100.497 & & 96.051 & 110.307 & & 80.653 & 74.09 \\
\hline $3 / 16 / 08$ 14:30 & 125.204 & 122.718 & & 100.521 & & 96.023 & 110.294 & & & 74.047 \\
\hline $3 / 16 / 08$ 15:00 & 125.187 & 122.698 & & 100.48 & & 96.007 & 110.284 & & & 74.039 \\
\hline $3 / 16 / 08$ 15:30 & 125.168 & 122.682 & & 100.463 & & 95.995 & 110.279 & & 80.631 & 74.02 \\
\hline $3 / 16 / 08$ 16:00 & 125.152 & 122.682 & & 100.446 & & 95.976 & 110.273 & & & 74.006 \\
\hline $3 / 16 / 0816: 30$ & 125.135 & 122.657 & & 100.44 & & 95.959 & 110.267 & & & 73.989 \\
\hline
\end{tabular}


TABLE S1.1 (Cont.)

Depth from Top of Casing (ft)

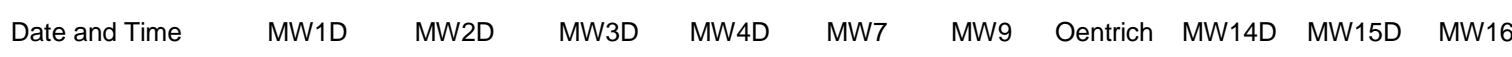

\begin{tabular}{|c|c|c|c|c|c|c|c|}
\hline $3 / 16 / 08$ 17:00 & 125.13 & 122.648 & 100.425 & 95.959 & 110.264 & 80.614 & 73.983 \\
\hline 3/16/08 17:30 & 125.109 & 122.628 & 100.403 & 95.938 & 110.26 & & 73.971 \\
\hline $3 / 16 / 08$ 18:00 & 125.1 & 122.616 & 100.396 & 95.926 & 110.254 & & 73.952 \\
\hline $3 / 16 / 08$ 18:30 & 125.081 & 122.603 & 100.375 & 95.905 & 110.252 & 80.594 & 73.944 \\
\hline $3 / 16 / 08$ 19:00 & 125.067 & 122.589 & 100.361 & 95.893 & 110.249 & & 73.927 \\
\hline $3 / 16 / 08$ 19:30 & 125.055 & 122.582 & 100.356 & 95.882 & 110.247 & & 73.913 \\
\hline $3 / 16 / 0820: 00$ & 125.038 & 122.578 & 100.344 & 95.863 & 110.243 & 80.582 & 73.909 \\
\hline $3 / 16 / 0820: 30$ & 125.031 & 122.58 & 100.335 & 95.86 & 110.237 & & 73.896 \\
\hline $3 / 16 / 0821: 00$ & 125.031 & 122.582 & 100.337 & 95.858 & 110.237 & & 73.89 \\
\hline $3 / 16 / 0821: 30$ & 125.029 & 122.578 & 100.337 & 95.86 & 110.232 & 80.56 & 73.89 \\
\hline $3 / 16 / 0822: 00$ & 125.031 & 122.614 & 100.344 & 95.865 & 110.228 & & 73.888 \\
\hline $3 / 16 / 08$ 22:30 & 125.029 & 122.625 & 100.339 & 95.86 & 110.228 & & 73.894 \\
\hline $3 / 16 / 0823: 00$ & 125.064 & 122.623 & 100.38 & 95.896 & 110.218 & 80.565 & 73.888 \\
\hline $3 / 16 / 0823: 30$ & 125.074 & 122.605 & 100.389 & 95.905 & 110.217 & & 73.919 \\
\hline $3 / 17 / 080: 00$ & 125.074 & 122.591 & 100.37 & 95.903 & 110.217 & & 73.929 \\
\hline 3/17/08 0:30 & 125.057 & 122.585 & 100.353 & 95.884 & 110.217 & 80.553 & 73.919 \\
\hline 3/17/08 1:00 & 125.043 & 122.571 & 100.344 & 95.87 & 110.215 & & 73.9 \\
\hline 3/17/08 1:30 & 125.034 & 122.56 & 100.335 & 95.86 & 110.213 & & 73.89 \\
\hline 3/17/08 2:00 & 125.022 & 122.541 & 100.325 & 95.849 & 110.211 & 80.531 & 73.884 \\
\hline $3 / 17 / 082: 30$ & 125.01 & 122.521 & 100.311 & 95.839 & 110.209 & & 73.873 \\
\hline 3/17/08 3:00 & 124.996 & 122.498 & 100.294 & 95.823 & 110.207 & & 73.863 \\
\hline 3/17/08 3:30 & 124.97 & 122.471 & 100.26 & 95.799 & 110.207 & 80.501 & 73.847 \\
\hline 3/17/08 4:00 & 124.948 & 122.462 & 100.241 & 95.778 & 110.202 & & 73.82 \\
\hline 3/17/08 4:30 & 124.922 & 122.428 & 100.225 & 95.747 & 110.2 & & 73.799 \\
\hline 3/17/08 5:00 & 124.915 & 122.424 & 100.208 & 95.745 & 110.198 & 80.472 & 73.784 \\
\hline 3/17/08 5:30 & 124.878 & 122.421 & 100.175 & 95.705 & 110.2 & & 73.77 \\
\hline 3/17/08 6:00 & 124.873 & 124.276 & 100.182 & 95.702 & 110.19 & & 73.739 \\
\hline 3/17/08 6:30 & 124.873 & 124.537 & 100.456 & 95.7 & 110.19 & 80.438 & 73.743 \\
\hline 3/17/08 7:00 & 126.766 & 124.709 & 100.968 & 96.284 & 110.77 & & 73.803 \\
\hline $3 / 17 / 087: 30$ & 127.036 & 124.798 & 101.15 & 96.537 & 111.03 & & 73.981 \\
\hline 3/17/08 8:00 & 127.189 & 124.859 & 101.281 & 96.718 & 111.142 & 80.494 & 74.068 \\
\hline 3/17/08 8:30 & 127.265 & 124.85 & 101.373 & 96.808 & 111.219 & & 74.13 \\
\hline 3/17/08 9:00 & 127.334 & 124.859 & 101.478 & 96.881 & 111.266 & & 74.175 \\
\hline 3/17/08 9:30 & 127.388 & 124.906 & 101.838 & 96.869 & 111.306 & 80.729 & 74.243 \\
\hline $3 / 17 / 0810: 00$ & 127.414 & 124.922 & 101.898 & 96.874 & 111.328 & & 74.322 \\
\hline $3 / 17 / 08$ 10:30 & 127.43 & 124.92 & 101.865 & 96.919 & 111.341 & & 74.403 \\
\hline $3 / 17 / 0811: 00$ & 127.447 & 124.888 & 101.788 & 96.942 & 111.356 & 80.711 & 74.485 \\
\hline $3 / 17 / 0811: 30$ & 127.461 & 123.052 & 101.707 & 96.94 & 111.371 & & 74.564 \\
\hline $3 / 17 / 08$ 12:00 & 127.473 & 122.8 & 101.367 & 96.912 & 111.383 & & 74.636 \\
\hline $3 / 17 / 08$ 12:30 & 125.598 & 122.673 & 100.832 & 96.292 & 110.77 & 80.633 & 74.618 \\
\hline $3 / 17 / 08$ 13:00 & 125.341 & 122.582 & 100.539 & 96.054 & 110.549 & & 74.541 \\
\hline $3 / 17 / 0813: 30$ & 125.211 & 122.516 & 100.39 & 95.929 & 110.442 & & 74.494 \\
\hline $3 / 17 / 0814: 00$ & 125.119 & 122.469 & 100.32 & 95.839 & 110.38 & 80.548 & 74.469 \\
\hline $3 / 17 / 08$ 14:30 & 125.057 & 122.442 & 100.266 & 95.778 & 110.337 & & 74.458 \\
\hline $3 / 17 / 08$ 15:00 & 125.005 & 122.426 & 100.216 & 95.731 & 110.309 & & 74.452 \\
\hline $3 / 17 / 08$ 15:30 & 124.982 & 122.419 & 100.179 & 95.707 & 110.288 & 80.467 & 74.442 \\
\hline $3 / 17 / 0816: 00$ & 124.967 & 122.408 & 100.173 & 95.693 & 110.273 & & 74.429 \\
\hline $3 / 17 / 0816: 30$ & 124.953 & 122.403 & 100.163 & 95.69 & 110.258 & & 74.413 \\
\hline $3 / 17 / 08$ 17:00 & 124.944 & 122.39 & 100.153 & 95.679 & 110.25 & 80.438 & 74.401 \\
\hline $3 / 17 / 08$ 17:30 & 124.939 & 122.38 & 100.142 & 95.672 & 110.243 & & 74.392 \\
\hline $3 / 17 / 0818: 00$ & 124.937 & 122.38 & 100.139 & 95.658 & 110.239 & & 74.386 \\
\hline $3 / 17 / 08$ 18:30 & 124.934 & 122.38 & 100.13 & 95.653 & 110.235 & 80.413 & 74.38 \\
\hline $3 / 17 / 08$ 19:00 & 124.93 & 122.374 & 100.13 & 95.653 & 110.23 & & 74.374 \\
\hline $3 / 17 / 08$ 19:30 & 124.922 & 122.383 & 100.123 & 95.653 & 110.226 & & 74.365 \\
\hline $3 / 17 / 0820: 00$ & 124.92 & 122.376 & 100.135 & 95.646 & 110.222 & 80.386 & 74.359 \\
\hline $3 / 17 / 0820: 30$ & 124.918 & 122.383 & 100.134 & 95.653 & 110.22 & & 74.349 \\
\hline $3 / 17 / 0821: 00$ & 124.913 & 122.38 & 100.141 & 95.648 & 110.218 & & 74.345 \\
\hline $3 / 17 / 0821: 30$ & 124.911 & 122.387 & 100.129 & 95.657 & 110.215 & 80.377 & 74.336 \\
\hline $3 / 17 / 08$ 22:00 & 124.908 & 122.408 & 100.137 & 95.653 & 110.213 & & 74.332 \\
\hline $3 / 17 / 0822: 30$ & 124.903 & 122.394 & 100.154 & 95.657 & 110.209 & & 74.326 \\
\hline 3/17/08 23:00 & 124.901 & 122.376 & 100.156 & 95.683 & 110.205 & 80.362 & 74.318 \\
\hline $3 / 17 / 0823: 30$ & 124.904 & 122.385 & 100.141 & 95.669 & 110.207 & & 74.32 \\
\hline $3 / 18 / 080: 00$ & 124.904 & 122.365 & 100.134 & 95.65 & 110.207 & & 74.32 \\
\hline 3/18/08 0:30 & 124.899 & 122.34 & 100.141 & 95.665 & 110.202 & 80.347 & 74.314 \\
\hline 3/18/08 1:00 & 124.901 & 122.335 & 100.106 & 95.648 & 110.202 & & 74.314 \\
\hline 3/18/08 1:30 & 124.88 & 122.335 & 100.092 & 95.615 & 110.205 & & 74.314 \\
\hline
\end{tabular}


TABLE S1.1 (Cont.)

\begin{tabular}{|c|c|c|c|c|c|c|c|c|c|c|}
\hline \multirow[b]{2}{*}{ Date and Time } & \multicolumn{10}{|c|}{ Depth from Top of Casing (ft) } \\
\hline & MW1D & MW2D & MW3D & MW4D & MW7 & MW9 & Oentrich & MW14D & MW15D & MW16D \\
\hline 3/18/08 2:00 & 124.87 & 122.331 & & 100.103 & & 95.613 & 110.202 & & 80.318 & 74.297 \\
\hline $3 / 18 / 082: 30$ & 124.866 & 122.342 & & 100.096 & & 95.613 & 110.198 & & & 74.281 \\
\hline 3/18/08 3:00 & 124.863 & 122.333 & & 100.093 & & 95.61 & 110.196 & & & 74.274 \\
\hline 3/18/08 3:30 & 124.856 & 122.333 & & 100.099 & & 95.622 & 110.192 & & 80.303 & 74.27 \\
\hline 3/18/08 4:00 & 124.859 & 122.324 & & 100.087 & & 95.611 & 110.194 & & & 74.272 \\
\hline 3/18/08 4:30 & 124.859 & 122.333 & & 100.091 & & 95.613 & 110.19 & & & 74.27 \\
\hline 3/18/08 5:00 & 124.859 & 122.337 & & 100.087 & & 95.603 & 110.192 & & 80.274 & 74.27 \\
\hline 3/18/08 5:30 & 124.854 & 122.351 & & 100.087 & & 95.611 & 110.187 & & & 74.266 \\
\hline 3/18/08 6:00 & 124.852 & 124.258 & & 100.111 & & 95.618 & 110.188 & & & 74.262 \\
\hline 3/18/08 6:30 & 124.849 & 124.498 & & 100.556 & & 95.627 & 110.185 & & 80.269 & 74.256 \\
\hline 3/18/08 7:00 & 126.714 & 124.618 & & 100.935 & & 96.275 & 110.829 & & & 74.334 \\
\hline 3/18/08 7:30 & 126.986 & 124.693 & & 101.09 & & 96.511 & 111.048 & & & 74.46 \\
\hline 3/18/08 8:00 & 127.121 & 122.906 & & 101.18 & & 96.636 & 111.161 & & 80.328 & 74.541 \\
\hline 3/18/08 8:30 & 127.182 & 122.687 & & 100.873 & & 96.709 & 111.229 & & & 74.599 \\
\hline 3/18/08 9:00 & 125.376 & 122.603 & & 100.496 & & 96.16 & 110.663 & & & 74.572 \\
\hline 3/18/08 9:30 & 125.145 & 122.541 & & 100.373 & & 95.95 & 110.459 & & 80.355 & 74.479 \\
\hline 3/18/08 10:00 & 125.055 & 122.501 & & 100.316 & & 95.867 & 110.363 & & & 74.423 \\
\hline $3 / 18 / 08$ 10:30 & 124.996 & 122.471 & & 100.261 & & 95.811 & 110.314 & & & 74.398 \\
\hline 3/18/08 11:00 & 124.953 & 122.451 & & 100.232 & & 95.771 & 110.282 & & 80.34 & 74.384 \\
\hline 3/18/08 11:30 & 124.92 & 122.442 & & 100.213 & & 95.743 & 110.26 & & & 74.326 \\
\hline 3/18/08 12:00 & 124.906 & 122.426 & & 100.199 & & 95.728 & 110.247 & & & 74.043 \\
\hline 3/18/08 12:30 & 124.894 & 122.401 & & 100.189 & & 95.721 & 110.235 & & 80.32 & 73.813 \\
\hline 3/18/08 13:00 & 124.875 & 122.38 & & 100.165 & & 95.702 & 110.228 & & & 73.749 \\
\hline 3/18/08 13:30 & 124.856 & 122.383 & & 100.154 & & 95.684 & 110.224 & & & 73.722 \\
\hline $3 / 18 / 08$ 14:00 & 124.833 & 122.365 & & 100.148 & & 95.658 & 110.22 & & 80.296 & 73.708 \\
\hline $3 / 18 / 08$ 14:30 & 124.835 & 122.349 & & 100.115 & & 95.665 & 110.215 & & & 73.694 \\
\hline 3/18/08 15:00 & 124.816 & 122.344 & & 100.113 & & 95.639 & 110.213 & & & 73.675 \\
\hline 3/18/08 15:30 & 124.804 & 122.358 & & 100.111 & & 95.632 & 110.207 & & 80.274 & 73.663 \\
\hline 3/18/08 16:00 & 124.8 & 122.349 & & 100.101 & & 95.625 & 110.203 & & & 73.66 \\
\hline $3 / 18 / 08$ 16:30 & 124.807 & 122.362 & & 100.104 & & 95.639 & 110.2 & & & 73.65 \\
\hline 3/18/08 17:00 & 124.799 & 122.369 & & 100.111 & & 95.632 & 110.196 & & 80.262 & 73.652 \\
\hline 3/18/08 17:30 & 124.816 & 122.374 & & 100.123 & & 95.646 & 110.194 & & & 73.658 \\
\hline 3/18/08 18:00 & 124.821 & 122.369 & & 100.135 & & 95.651 & 110.192 & & & 73.667 \\
\hline 3/18/08 18:30 & 124.823 & 122.38 & & 100.12 & & 95.653 & 110.192 & & 80.254 & 73.671 \\
\hline 3/18/08 19:00 & 124.818 & 122.417 & & 100.139 & & 95.646 & 110.19 & & & 73.663 \\
\hline 3/18/08 19:30 & 124.833 & 122.419 & & 100.163 & & 95.662 & 110.188 & & & 73.673 \\
\hline 3/18/08 20:00 & 124.865 & 122.426 & & 100.185 & & 95.698 & 110.183 & & 80.267 & 73.687 \\
\hline 3/18/08 20:30 & 124.868 & 122.439 & & 100.194 & & 95.7 & 110.185 & & & 73.708 \\
\hline 3/18/08 21:00 & 124.878 & 122.448 & & 100.201 & & 95.71 & 110.185 & & & 73.72 \\
\hline 3/18/08 21:30 & 124.889 & 122.462 & & 100.213 & & 95.721 & 110.181 & & 80.269 & 73.727 \\
\hline 3/18/08 22:00 & 124.901 & 122.455 & & 100.22 & & 95.735 & 110.181 & & & 73.733 \\
\hline 3/18/08 22:30 & 124.915 & 122.444 & & 100.222 & & 95.75 & 110.181 & & & 73.741 \\
\hline 3/18/08 23:00 & 124.906 & 122.424 & & 100.21 & & 95.738 & 110.181 & & 80.276 & 73.747 \\
\hline 3/18/08 23:30 & 124.894 & 122.437 & & 100.185 & & 95.726 & 110.181 & & & 73.737 \\
\hline $3 / 19 / 080: 00$ & 124.875 & 122.444 & & 100.191 & & 95.705 & 110.181 & & & 73.718 \\
\hline 3/19/08 0:30 & 124.885 & 122.444 & & 100.201 & & 95.716 & 110.179 & & 80.269 & 73.72 \\
\hline 3/19/08 1:00 & 124.896 & 122.435 & & 100.211 & & 95.733 & 110.173 & & & 73.727 \\
\hline 3/19/08 1:30 & 124.894 & 122.435 & & 100.204 & & 95.728 & 110.173 & & & 73.735 \\
\hline 3/19/08 2:00 & 124.885 & 122.433 & & 100.201 & & 95.719 & 110.173 & & 80.264 & 73.731 \\
\hline 3/19/08 2:30 & 124.885 & 122.424 & & 100.198 & & 95.719 & 110.172 & & & 73.725 \\
\hline 3/19/08 3:00 & 124.885 & 122.408 & & 100.194 & & 95.719 & 110.17 & & & 73.722 \\
\hline 3/19/08 3:30 & 124.875 & 122.403 & & 100.179 & & 95.71 & 110.17 & & 80.257 & 73.72 \\
\hline 3/19/08 4:00 & 124.863 & 122.392 & & 100.167 & & 95.695 & 110.17 & & & 73.71 \\
\hline 3/19/08 4:30 & 124.854 & 122.392 & & 100.161 & & 95.688 & 110.168 & & & 73.698 \\
\hline 3/19/08 5:00 & 124.842 & 122.401 & & 100.156 & & 95.677 & 110.168 & & 80.232 & 73.691 \\
\hline 3/19/08 5:30 & 124.842 & 122.419 & & 100.161 & & 95.677 & 110.164 & & & 73.685 \\
\hline 3/19/08 6:00 & 124.852 & 124.317 & & 100.175 & & 95.686 & 110.16 & & & 73.689 \\
\hline 3/19/08 6:30 & 124.868 & 124.598 & & 100.578 & & 95.705 & 110.158 & & 80.24 & 73.698 \\
\hline 3/19/08 7:00 & 126.83 & 124.739 & & 101.068 & & 96.377 & 110.808 & & & 73.789 \\
\hline 3/19/08 7:30 & 127.128 & 124.829 & & 101.25 & & 96.659 & 111.067 & & & 73.987 \\
\hline 3/19/08 8:00 & 127.272 & 124.891 & & 101.366 & & 96.801 & 111.191 & & 80.32 & 74.115 \\
\hline 3/19/08 8:30 & 127.364 & 124.929 & & 101.435 & & 96.89 & 111.268 & & & 74.208 \\
\hline 3/19/08 9:00 & 127.426 & 124.965 & & 101.479 & & 96.949 & 111.319 & & & 74.27 \\
\hline 3/19/08 9:30 & 127.468 & 124.988 & & 101.517 & & 96.992 & 111.356 & & 80.386 & 74.314 \\
\hline 3/19/08 10:00 & 127.508 & 125.011 & & 101.547 & & 97.032 & 111.384 & & & 74.347 \\
\hline 3/19/08 10:30 & 127.527 & 125.024 & & 101.567 & & 97.051 & 111.405 & & & 74.374 \\
\hline
\end{tabular}


TABLE S1.1 (Cont.)

\begin{tabular}{|c|c|c|c|c|c|c|c|c|c|c|}
\hline \multirow[b]{2}{*}{ Date and Time } & \multicolumn{10}{|c|}{ Depth from Top of Casing (ft) } \\
\hline & MW1D & MW2D & MW3D & MW4D & MW7 & MW9 & Oentrich & MW14D & MW15D & MW16D \\
\hline 3/19/08 11:00 & 127.551 & 125.04 & & 101.585 & & 97.072 & 111.422 & & 80.447 & 74.396 \\
\hline 3/19/08 11:30 & 127.563 & 125.043 & & 101.6 & & 97.088 & 111.433 & & & 74.413 \\
\hline 3/19/08 12:00 & 127.579 & 125.038 & & 101.609 & & 97.102 & 111.448 & & & 74.429 \\
\hline 3/19/08 12:30 & 127.582 & 123.623 & & 101.605 & & 97.107 & 111.458 & & 80.494 & 74.442 \\
\hline 3/19/08 13:00 & 127.577 & 123.045 & & 101.602 & & 97.105 & 111.467 & & & 74.446 \\
\hline 3/19/08 13:30 & 126.201 & 122.854 & & 100.907 & & 96.841 & 111.182 & & & 74.444 \\
\hline 3/19/08 14:00 & 125.511 & 122.741 & & 100.649 & & 96.292 & 110.692 & & 80.487 & 74.283 \\
\hline $3 / 19 / 08$ 14:30 & 125.312 & 122.68 & & 100.518 & & 96.108 & 110.526 & & & 74.142 \\
\hline 3/19/08 15:00 & 125.197 & 122.628 & & 100.435 & & 95.997 & 110.436 & & & 74.041 \\
\hline 3/19/08 15:30 & 125.133 & 122.575 & & 100.389 & & 95.936 & 110.384 & & 80.447 & 73.968 \\
\hline 3/19/08 16:00 & 125.078 & 122.544 & & 100.341 & & 95.891 & 110.346 & & & 73.927 \\
\hline 3/19/08 16:30 & 125.029 & 122.523 & & 100.303 & & 95.839 & 110.318 & & & 73.884 \\
\hline 3/19/08 17:00 & 124.993 & 122.498 & & 100.284 & & 95.809 & 110.299 & & 80.406 & 73.849 \\
\hline 3/19/08 17:30 & 124.974 & 122.476 & & 100.258 & & 95.792 & 110.286 & & & 73.828 \\
\hline 3/19/08 18:00 & 124.948 & 122.464 & & 100.234 & & 95.768 & 110.273 & & & 73.805 \\
\hline 3/19/08 18:30 & 124.925 & 122.453 & & 100.218 & & 95.745 & 110.264 & & 80.374 & 73.782 \\
\hline 3/19/08 19:00 & 124.915 & 122.455 & & 100.208 & & 95.735 & 110.254 & & & 73.768 \\
\hline 3/19/08 19:30 & 124.901 & 122.462 & & 100.208 & & 95.726 & 110.247 & & & 73.758 \\
\hline 3/19/08 20:00 & 124.904 & 122.471 & & 100.216 & & 95.728 & 110.239 & & 80.357 & 73.753 \\
\hline 3/19/08 20:30 & 124.913 & 122.51 & & 100.222 & & 95.738 & 110.234 & & & 73.758 \\
\hline 3/19/08 21:00 & 124.92 & 122.507 & & 100.242 & & 95.745 & 110.228 & & & 73.764 \\
\hline 3/19/08 21:30 & 124.956 & 122.51 & & 100.268 & & 95.783 & 110.22 & & 80.362 & 73.776 \\
\hline 3/19/08 22:00 & 124.958 & 122.507 & & 100.265 & & 95.785 & 110.222 & & & 73.801 \\
\hline 3/19/08 22:30 & 124.96 & 122.51 & & 100.268 & & 95.787 & 110.218 & & & 73.801 \\
\hline 3/19/08 23:00 & 124.958 & 122.51 & & 100.268 & & 95.785 & 110.217 & & 80.359 & 73.801 \\
\hline 3/19/08 23:30 & 124.958 & 122.514 & & 100.272 & & 95.785 & 110.213 & & & 73.803 \\
\hline 3/20/08 0:00 & 124.963 & 122.505 & & 100.275 & & 95.792 & 110.211 & & & 73.803 \\
\hline 3/20/08 0:30 & 124.962 & 122.51 & & 100.272 & & 95.792 & 110.207 & & 80.357 & 73.805 \\
\hline 3/20/08 1:00 & 124.956 & 122.51 & & 100.268 & & 95.785 & 110.207 & & & 73.805 \\
\hline 3/20/08 1:30 & 124.96 & 122.51 & & 100.27 & & 95.792 & 110.202 & & & 73.801 \\
\hline 3/20/08 2:00 & 124.958 & 122.503 & & 100.273 & & 95.787 & 110.2 & & 80.347 & 73.801 \\
\hline 3/20/08 2:30 & 124.958 & 122.485 & & 100.268 & & 95.79 & 110.196 & & & 73.803 \\
\hline 3/20/08 3:00 & 124.953 & 122.478 & & 100.254 & & 95.785 & 110.194 & & & 73.801 \\
\hline 3/20/08 3:30 & 124.934 & 122.473 & & 100.246 & & 95.766 & 110.194 & & 80.328 & 73.791 \\
\hline 3/20/08 4:00 & 124.927 & 122.471 & & 100.235 & & 95.761 & 110.19 & & & 73.782 \\
\hline 3/20/08 4:30 & 124.922 & 122.487 & & 100.232 & & 95.757 & 110.188 & & & 73.774 \\
\hline 3/20/08 5:00 & 124.92 & 122.492 & & 100.246 & & 95.752 & 110.185 & & 80.315 & 73.766 \\
\hline 3/20/08 5:30 & 124.937 & 122.492 & & 100.26 & & 95.771 & 110.181 & & & 73.776 \\
\hline 3/20/08 6:00 & 124.939 & 124.292 & & 100.254 & & 95.776 & 110.177 & & & 73.787 \\
\hline 3/20/08 6:30 & 124.941 & 124.582 & & 100.39 & & 95.773 & 110.177 & & 80.316 & 73.787 \\
\hline 3/20/08 7:00 & 126.768 & 124.73 & & 101.014 & & 96.313 & 110.722 & & & 73.824 \\
\hline 3/20/08 7:30 & 127.088 & 124.804 & & 101.199 & & 96.607 & 110.996 & & & 74.01 \\
\hline 3/20/08 8:00 & 127.237 & 124.848 & & 101.307 & & 96.751 & 111.122 & & 80.369 & 74.134 \\
\hline 3/20/08 8:30 & 127.319 & 122.961 & & 101.359 & & 96.836 & 111.197 & & & 74.223 \\
\hline 3/20/08 9:00 & 127.36 & 122.768 & & 100.854 & & 96.879 & 111.247 & & & 74.272 \\
\hline 3/20/08 9:30 & 125.426 & 122.671 & & 100.575 & & 96.216 & 110.594 & & 80.399 & 74.186 \\
\hline 3/20/08 10:00 & 125.223 & 122.603 & & 100.453 & & 96.03 & 110.429 & & & 74.057 \\
\hline 3/20/08 10:30 & 125.123 & 122.56 & & 100.373 & & 95.936 & 110.346 & & & 73.971 \\
\hline 3/20/08 11:00 & 125.052 & 122.519 & & 100.33 & & 95.872 & 110.299 & & 80.369 & 73.906 \\
\hline 3/20/08 11:30 & 125.015 & 122.482 & & 100.287 & & 95.832 & 110.269 & & & 73.867 \\
\hline 3/20/08 12:00 & 124.97 & 122.446 & & 100.256 & & 95.789 & 110.247 & & & 73.832 \\
\hline 3/20/08 12:30 & 124.934 & 122.396 & & 100.215 & & 95.759 & 110.234 & & 80.342 & 73.799 \\
\hline 3/20/08 13:00 & 124.896 & 122.362 & & 100.179 & & 95.721 & 110.22 & & & 73.766 \\
\hline 3/20/08 13:30 & 124.844 & 122.337 & & 100.129 & & 95.669 & 110.215 & & & 73.733 \\
\hline 3/20/08 14:00 & 124.811 & 122.312 & & 100.104 & & 95.639 & 110.202 & & 80.296 & 73.691 \\
\hline $3 / 20 / 08$ 14:30 & 124.79 & 122.294 & & 100.084 & & 95.617 & 110.192 & & & 73.667 \\
\hline 3/20/08 15:00 & 124.762 & 122.278 & & 100.055 & & 95.589 & 110.181 & & & 73.644 \\
\hline $3 / 20 / 08$ 15:30 & 124.745 & 122.267 & & 100.042 & & 95.573 & 110.172 & & 80.249 & 73.619 \\
\hline 3/20/08 16:00 & 124.726 & 122.242 & & 100.034 & & 95.556 & 110.166 & & & 73.605 \\
\hline 3/20/08 16:30 & 124.717 & 122.224 & & 100.011 & & 95.549 & 110.162 & & & 73.594 \\
\hline 3/20/08 17:00 & 124.691 & 122.204 & & 99.987 & & 95.526 & 110.16 & & 80.22 & 73.578 \\
\hline 3/20/08 17:30 & 124.67 & 122.185 & & 99.968 & & 95.5 & 110.155 & & & 73.555 \\
\hline 3/20/08 18:00 & 124.653 & 122.181 & & 99.951 & & 95.485 & 110.153 & & & 73.536 \\
\hline 3/20/08 18:30 & 124.634 & 122.179 & & 99.941 & & 95.464 & 110.147 & & 80.161 & 73.518 \\
\hline 3/20/08 19:00 & 124.629 & 122.176 & & 99.939 & & 95.462 & 110.136 & & & 73.505 \\
\hline 3/20/08 19:30 & 124.629 & 122.179 & & 99.936 & & 95.46 & 110.134 & & & 73.501 \\
\hline
\end{tabular}


TABLE S1.1 (Cont.)

\begin{tabular}{|c|c|c|c|c|c|c|c|c|c|c|}
\hline \multirow[b]{2}{*}{ Date and Time } & \multicolumn{10}{|c|}{ Depth from Top of Casing (ft) } \\
\hline & MW1D & MW2D & MW3D & MW4D & MW7 & MW9 & Oentrich & MW14D & MW15D & MW16D \\
\hline 3/20/08 20:00 & 124.629 & 122.181 & & 99.937 & & 95.457 & 110.13 & & 80.139 & 73.497 \\
\hline 3/20/08 20:30 & 124.627 & 122.174 & & 99.939 & & 95.462 & 110.128 & & & 73.497 \\
\hline 3/20/08 21:00 & 124.629 & 122.167 & & 99.939 & & 95.462 & 110.125 & & & 73.497 \\
\hline 3/20/08 21:30 & 124.622 & 122.172 & & 99.927 & & 95.457 & 110.126 & & 80.12 & 73.497 \\
\hline 3/20/08 22:00 & 124.613 & 122.172 & & 99.927 & & 95.445 & 110.126 & & & 73.487 \\
\hline 3/20/08 22:30 & 124.617 & 122.172 & & 99.93 & & 95.45 & 110.121 & & & 73.483 \\
\hline 3/20/08 23:00 & 124.62 & 122.176 & & 99.93 & & 95.452 & 110.121 & & 80.103 & 73.485 \\
\hline 3/20/08 23:30 & 124.62 & 122.185 & & 99.936 & & 95.455 & 110.117 & & & 73.487 \\
\hline $3 / 21 / 080: 00$ & 124.62 & 122.199 & & 99.939 & & 95.453 & 110.117 & & & 73.489 \\
\hline 3/21/08 0:30 & 124.632 & 122.204 & & 99.955 & & 95.464 & 110.117 & & 80.091 & 73.491 \\
\hline 3/21/08 1:00 & 124.646 & 122.217 & & 99.961 & & 95.481 & 110.117 & & & 73.503 \\
\hline 3/21/08 1:30 & 124.653 & 122.219 & & 99.968 & & 95.488 & 110.117 & & & 73.507 \\
\hline 3/21/08 2:00 & 124.665 & 122.215 & & 99.991 & & 95.502 & 110.113 & & 80.103 & 73.512 \\
\hline 3/21/08 2:30 & 124.672 & 122.206 & & 99.989 & & 95.507 & 110.115 & & & 73.53 \\
\hline 3/21/08 3:00 & 124.665 & 122.197 & & 99.972 & & 95.5 & 110.115 & & & 73.526 \\
\hline 3/21/08 3:30 & 124.655 & 122.219 & & 99.961 & & 95.49 & 110.113 & & 80.069 & 73.512 \\
\hline 3/21/08 4:00 & 124.644 & 122.247 & & 99.972 & & 95.474 & 110.115 & & & 73.507 \\
\hline 3/21/08 4:30 & 124.667 & 122.274 & & 100.001 & & 95.497 & 110.11 & & & 73.505 \\
\hline 3/21/08 5:00 & 124.691 & 122.287 & & 100.032 & & 95.526 & 110.106 & & 80.066 & 73.522 \\
\hline 3/21/08 5:30 & 124.724 & 122.292 & & 100.048 & & 95.563 & 110.106 & & & 73.555 \\
\hline 3/21/08 6:00 & 124.733 & 124.233 & & 100.053 & & 95.573 & 110.117 & & & 73.572 \\
\hline 3/21/08 6:30 & 124.745 & 124.505 & & 100.559 & & 95.582 & 110.123 & & 80.076 & 73.582 \\
\hline 3/21/08 7:00 & 126.747 & 124.65 & & 100.978 & & 96.296 & 110.819 & & & 73.685 \\
\hline 3/21/08 7:30 & 127.033 & 124.743 & & 101.162 & & 96.567 & 111.061 & & & 73.886 \\
\hline 3/21/08 8:00 & 127.182 & 124.807 & & 101.278 & & 96.714 & 111.18 & & 80.166 & 74.024 \\
\hline 3/21/08 8:30 & 127.277 & 123.75 & & 101.345 & & 96.813 & 111.253 & & & 74.119 \\
\hline 3/21/08 9:00 & 127.341 & 122.947 & & 101.412 & & 96.874 & 111.302 & & & 74.183 \\
\hline 3/21/08 9:30 & 126.676 & 122.775 & & 100.825 & & 96.937 & 111.294 & & 80.249 & 74.237 \\
\hline 3/21/08 10:00 & 125.414 & 122.702 & & 100.577 & & 96.209 & 110.592 & & & 74.146 \\
\hline 3/21/08 10:30 & 125.234 & 122.662 & & 100.471 & & 96.042 & 110.425 & & & 74.035 \\
\hline 3/21/08 11:00 & 125.154 & 122.623 & & 100.427 & & 95.973 & 110.342 & & 80.25 & 73.96 \\
\hline 3/21/08 11:30 & 125.116 & 122.596 & & 100.385 & & 95.936 & 110.299 & & & 73.923 \\
\hline 3/21/08 12:00 & 125.076 & 122.58 & & 100.361 & & 95.903 & 110.267 & & & 73.894 \\
\hline 3/21/08 12:30 & 125.05 & 122.569 & & 100.344 & & 95.877 & 110.247 & & 80.252 & 73.869 \\
\hline 3/21/08 13:00 & 125.031 & 122.557 & & 100.332 & & 95.863 & 110.228 & & & 73.853 \\
\hline 3/21/08 13:30 & 125.022 & 122.544 & & 100.322 & & 95.853 & 110.217 & & & 73.842 \\
\hline $3 / 21 / 08$ 14:00 & 125.012 & 122.537 & & 100.316 & & 95.841 & 110.207 & & 80.252 & 73.836 \\
\hline 3/21/08 14:30 & 124.998 & 122.526 & & 100.301 & & 95.834 & 110.202 & & & 73.826 \\
\hline 3/21/08 15:00 & 124.989 & 122.521 & & 100.296 & & 95.823 & 110.194 & & & 73.815 \\
\hline $3 / 21 / 08$ 15:30 & 124.979 & 122.519 & & 100.287 & & 95.816 & 110.19 & & 80.242 & 73.809 \\
\hline 3/21/08 16:00 & 124.972 & 122.512 & & 100.28 & & 95.809 & 110.185 & & & 73.799 \\
\hline 3/21/08 16:30 & 124.97 & 122.512 & & 100.28 & & 95.806 & 110.181 & & & 73.797 \\
\hline 3/21/08 17:00 & 124.967 & 122.519 & & 100.28 & & 95.801 & 110.179 & & 80.237 & 73.795 \\
\hline 3/21/08 17:30 & 124.965 & 122.532 & & 100.28 & & 95.801 & 110.173 & & & 73.793 \\
\hline 3/21/08 18:00 & 124.972 & 122.541 & & 100.291 & & 95.809 & 110.168 & & & 73.793 \\
\hline $3 / 21 / 08$ 18:30 & 124.982 & 122.546 & & 100.304 & & 95.823 & 110.164 & & 80.237 & 73.801 \\
\hline 3/21/08 19:00 & 124.993 & 122.562 & & 100.313 & & 95.834 & 110.16 & & & 73.813 \\
\hline 3/21/08 19:30 & 124.996 & 122.569 & & 100.323 & & 95.837 & 110.158 & & & 73.82 \\
\hline 3/21/08 20:00 & 125.012 & 122.562 & & 100.337 & & 95.853 & 110.155 & & 80.254 & 73.828 \\
\hline 3/21/08 20:30 & 125.022 & 122.587 & & 100.335 & & 95.86 & 110.153 & & & 73.84 \\
\hline 3/21/08 21:00 & 125.012 & 122.598 & & 100.342 & & 95.853 & 110.155 & & & 73.842 \\
\hline 3/21/08 21:30 & 125.036 & 122.589 & & 100.363 & & 95.879 & 110.149 & & 80.267 & 73.847 \\
\hline 3/21/08 22:00 & 125.047 & 122.605 & & 100.363 & & 95.886 & 110.147 & & & 73.863 \\
\hline 3/21/08 22:30 & 125.041 & 122.609 & & 100.366 & & 95.882 & 110.147 & & & 73.869 \\
\hline 3/21/08 23:00 & 125.057 & 122.612 & & 100.375 & & 95.896 & 110.145 & & 80.279 & 73.871 \\
\hline 3/21/08 23:30 & 125.06 & 122.614 & & 100.378 & & 95.898 & 110.145 & & & 73.878 \\
\hline 3/22/08 0:00 & 125.067 & 122.623 & & 100.384 & & 95.905 & 110.142 & & & 73.882 \\
\hline 3/22/08 0:30 & 125.064 & 122.628 & & 100.387 & & 95.903 & 110.142 & & 80.291 & 73.888 \\
\hline 3/22/08 1:00 & 125.074 & 122.619 & & 100.403 & & 95.915 & 110.138 & & & 73.888 \\
\hline 3/22/08 1:30 & 125.081 & 122.614 & & 100.392 & & 95.922 & 110.138 & & & 73.904 \\
\hline 3/22/08 2:00 & 125.069 & 122.603 & & 100.382 & & 95.91 & 110.138 & & 80.298 & 73.9 \\
\hline 3/22/08 2:30 & 125.064 & 122.594 & & 100.373 & & 95.907 & 110.136 & & & 73.89 \\
\hline 3/22/08 3:00 & 125.052 & 122.598 & & 100.368 & & 95.893 & 110.136 & & & 73.886 \\
\hline 3/22/08 3:30 & 125.045 & 122.598 & & 100.368 & & 95.886 & 110.134 & & 80.296 & 73.882 \\
\hline 3/22/08 4:00 & 125.048 & 122.605 & & 100.372 & & 95.884 & 110.128 & & & 73.875 \\
\hline 3/22/08 4:30 & 125.048 & 122.612 & & 100.372 & & 95.886 & 110.126 & & & 73.882 \\
\hline
\end{tabular}


TABLE S1.1 (Cont.)

Depth from Top of Casing (ft)

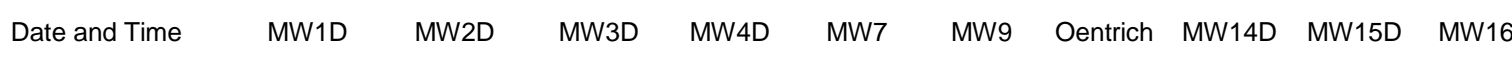

\begin{tabular}{|c|c|c|c|c|c|c|c|}
\hline 3/22/08 5:00 & 125.055 & 122.614 & 100.378 & 95.896 & 110.123 & 80.298 & 73.882 \\
\hline 3/22/08 5:30 & 125.064 & 122.623 & 100.384 & 95.905 & 110.119 & & 73.892 \\
\hline 3/22/08 6:00 & 125.064 & 122.634 & 100.394 & 95.905 & 110.117 & & 73.896 \\
\hline 3/22/08 6:30 & 125.071 & 122.641 & 100.394 & 95.915 & 110.115 & 80.306 & 73.902 \\
\hline 3/22/08 7:00 & 125.083 & 124.494 & 100.409 & 95.926 & 110.111 & & 73.904 \\
\hline 3/22/08 7:30 & 125.088 & 124.8 & 100.611 & 95.929 & 110.11 & & 73.917 \\
\hline 3/22/08 8:00 & 126.977 & 124.943 & 101.212 & 96.508 & 110.671 & 80.338 & 73.979 \\
\hline 3/22/08 8:30 & 127.298 & 125.031 & 101.407 & 96.813 & 110.941 & & 74.169 \\
\hline 3/22/08 9:00 & 127.449 & 125.097 & 101.512 & 96.959 & 111.212 & & 74.31 \\
\hline 3/22/08 9:30 & 127.542 & 125.138 & 101.597 & 97.051 & 111.302 & 80.428 & 74.398 \\
\hline $3 / 22 / 08 \quad 10: 00$ & 127.61 & 125.17 & 101.645 & 97.121 & 111.373 & & 74.467 \\
\hline $3 / 22 / 08 \quad 10: 30$ & 127.648 & 125.192 & 101.676 & 97.159 & 111.411 & & 74.514 \\
\hline $3 / 22 / 08$ 11:00 & 127.681 & 125.204 & 101.7 & 97.185 & 111.441 & 80.496 & 74.545 \\
\hline $3 / 22 / 08 \quad 11: 30$ & 127.702 & 125.222 & 101.719 & 97.211 & 111.465 & & 74.57 \\
\hline $3 / 22 / 08$ 12:00 & 127.714 & 125.233 & 101.734 & 97.225 & 111.48 & & 74.593 \\
\hline $3 / 22 / 08$ 12:30 & 127.733 & 125.238 & 101.746 & 97.244 & 111.493 & 80.557 & 74.607 \\
\hline $3 / 22 / 08$ 13:00 & 127.75 & 123.73 & 101.757 & 97.256 & 111.505 & & 74.624 \\
\hline $3 / 22 / 08$ 13:30 & 127.752 & 123.247 & 101.762 & 97.265 & 111.514 & & 74.636 \\
\hline $3 / 22 / 08$ 14:00 & 126.26 & 123.074 & 101.102 & 96.956 & 111.185 & 80.597 & 74.638 \\
\hline $3 / 22 / 08 \quad 14: 30$ & 125.707 & 122.979 & 100.868 & 96.502 & 110.772 & & 74.487 \\
\hline $3 / 22 / 08$ 15:00 & 125.53 & 122.918 & 100.754 & 96.334 & 110.62 & & 74.353 \\
\hline $3 / 22 / 08$ 15:30 & 125.433 & 122.868 & 100.687 & 96.247 & 110.536 & 80.57 & 74.266 \\
\hline $3 / 22 / 08$ 16:00 & 125.369 & 122.836 & 100.635 & 96.186 & 110.487 & & 74.21 \\
\hline $3 / 22 / 08$ 16:30 & 125.317 & 122.811 & 100.596 & 96.139 & 110.449 & & 74.167 \\
\hline $3 / 22 / 08$ 17:00 & 125.286 & 122.809 & 100.571 & 96.108 & 110.425 & 80.543 & 74.132 \\
\hline $3 / 22 / 08 \quad 17: 30$ & 125.263 & 122.809 & 100.561 & 96.089 & 110.404 & & 74.107 \\
\hline $3 / 22 / 08$ 18:00 & 125.26 & 122.814 & 100.561 & 96.084 & 110.386 & & 74.095 \\
\hline $3 / 22 / 08$ 18:30 & 125.263 & 122.82 & 100.566 & 96.087 & 110.374 & 80.535 & 74.095 \\
\hline $3 / 22 / 08$ 19:00 & 125.263 & 122.829 & 100.575 & 96.091 & 110.363 & & 74.099 \\
\hline $3 / 22 / 08$ 19:30 & 125.27 & 122.836 & 100.583 & 96.103 & 110.384 & & 74.105 \\
\hline $3 / 22 / 0820: 00$ & 125.279 & 122.845 & 100.594 & 96.113 & 110.397 & 80.54 & 74.111 \\
\hline $3 / 22 / 0820: 30$ & 125.286 & 122.859 & 100.601 & 96.12 & 110.402 & & 74.117 \\
\hline $3 / 22 / 0821: 00$ & 125.296 & 122.859 & 100.613 & 96.129 & 110.414 & & 74.126 \\
\hline $3 / 22 / 0821: 30$ & 125.31 & 122.859 & 100.623 & 96.141 & 110.429 & 80.557 & 74.136 \\
\hline $3 / 22 / 08$ 22:00 & 125.312 & 122.857 & 100.623 & 96.143 & 110.433 & & 74.146 \\
\hline $3 / 22 / 08$ 22:30 & 125.31 & 122.852 & 100.62 & 96.146 & 110.433 & & 74.146 \\
\hline $3 / 22 / 08$ 23:00 & 125.308 & 122.848 & 100.616 & 96.143 & 110.427 & 80.557 & 74.144 \\
\hline $3 / 22 / 08$ 23:30 & 125.303 & 122.845 & 100.611 & 96.141 & 110.427 & & 74.142 \\
\hline $3 / 23 / 080: 00$ & 125.298 & 122.843 & 100.608 & 96.134 & 110.423 & & 74.138 \\
\hline 3/23/08 0:30 & 125.296 & 122.836 & 100.606 & 96.131 & 110.419 & 80.557 & 74.138 \\
\hline $3 / 23 / 081: 00$ & 125.294 & 122.829 & 100.601 & 96.129 & 110.417 & & 74.136 \\
\hline $3 / 23 / 08$ 1:30 & 125.289 & 122.829 & 100.596 & 96.124 & 110.412 & & 74.132 \\
\hline $3 / 23 / 08$ 2:00 & 125.277 & 122.823 & 100.594 & 96.117 & 110.408 & 80.557 & 74.128 \\
\hline $3 / 23 / 082: 30$ & 125.279 & 122.807 & 100.589 & 96.12 & 110.404 & & 74.126 \\
\hline 3/23/08 3:00 & 125.275 & 122.795 & 100.578 & 96.11 & 110.401 & & 74.124 \\
\hline 3/23/08 3:30 & 125.255 & 122.786 & 100.566 & 96.094 & 110.399 & 80.553 & 74.113 \\
\hline 3/23/08 4:00 & 125.246 & 122.789 & 100.554 & 96.082 & 110.395 & & 74.103 \\
\hline 3/23/08 4:30 & 125.239 & 122.784 & 100.552 & 96.077 & 110.389 & & 74.09 \\
\hline 3/23/08 5:00 & 125.239 & 122.786 & 100.551 & 96.075 & 110.384 & 80.543 & 74.088 \\
\hline $3 / 23 / 085: 30$ & 125.234 & 122.784 & 100.549 & 96.072 & 110.382 & & 74.09 \\
\hline 3/23/08 6:00 & 125.234 & 122.786 & 100.551 & 96.072 & 110.378 & & 74.086 \\
\hline 3/23/08 6:30 & 125.232 & 122.791 & 100.549 & 96.07 & 110.372 & 80.535 & 74.086 \\
\hline $3 / 23 / 08$ 7:00 & 125.234 & 122.793 & 100.551 & 96.072 & 110.371 & & 74.086 \\
\hline $3 / 23 / 08$ 7:30 & 125.239 & 124.544 & 100.558 & 96.077 & 110.365 & & 74.088 \\
\hline 3/23/08 8:00 & 125.241 & 124.893 & 100.563 & 96.079 & 110.361 & 80.533 & 74.093 \\
\hline $3 / 23 / 088: 30$ & 127.04 & 125.052 & 101.359 & 96.605 & 110.894 & & 74.124 \\
\hline 3/23/08 9:00 & 127.419 & 125.136 & 101.564 & 96.961 & 111.238 & & 74.318 \\
\hline $3 / 23 / 08$ 9:30 & 127.582 & 124.174 & 101.676 & 97.117 & 111.381 & 80.604 & 74.454 \\
\hline $3 / 23 / 0810: 00$ & 127.672 & 123.267 & 101.743 & 97.206 & 111.469 & & 74.545 \\
\hline $3 / 23 / 08 \quad 10: 30$ & 127.449 & 123.079 & 101.152 & 97.263 & 111.521 & & 74.601 \\
\hline $3 / 23 / 08$ 11:00 & 125.728 & 122.977 & 100.882 & 96.525 & 110.795 & 80.631 & 74.502 \\
\hline $3 / 23 / 0811: 30$ & 125.535 & 122.911 & 100.759 & 96.344 & 110.632 & & 74.376 \\
\hline $3 / 23 / 08$ 12:00 & 125.428 & 122.861 & 100.683 & 96.247 & 110.549 & & 74.289 \\
\hline $3 / 23 / 08 \quad 12: 30$ & 125.362 & 122.823 & 100.63 & 96.183 & 110.5 & 80.609 & 74.227 \\
\hline $3 / 23 / 08$ 13:00 & 125.312 & 122.793 & 100.59 & 96.136 & 110.468 & & 74.179 \\
\hline 3/23/08 13:30 & 125.275 & 122.77 & 100.561 & 96.101 & 110.448 & & 74.142 \\
\hline
\end{tabular}


TABLE S1.1 (Cont.)

\begin{tabular}{|c|c|c|c|c|c|c|c|c|c|c|}
\hline \multirow[b]{2}{*}{ Date and Time } & \multicolumn{10}{|c|}{ Depth from Top of Casing (ft) } \\
\hline & MW1D & MW2D & MW3D & MW4D & MW7 & MW9 & Oentrich & MW14D & MW15D & MW16D \\
\hline 3/23/08 14:00 & 125.246 & 122.743 & & 100.537 & & 96.075 & 110.431 & & 80.584 & 74.117 \\
\hline $3 / 23 / 08$ 14:30 & 125.22 & 122.716 & & 100.511 & & 96.054 & 110.421 & & & 74.095 \\
\hline 3/23/08 15:00 & 125.194 & 122.707 & & 100.487 & & 96.023 & 110.41 & & & 74.072 \\
\hline $3 / 23 / 08$ 15:30 & 125.171 & 122.696 & & 100.473 & & 96.004 & 110.402 & & 80.56 & 74.053 \\
\hline $3 / 23 / 08$ 16:00 & 125.159 & 122.68 & & 100.458 & & 95.99 & 110.399 & & & 74.035 \\
\hline 3/23/08 16:30 & 125.142 & 122.664 & & 100.444 & & 95.981 & 110.389 & & & 74.022 \\
\hline $3 / 23 / 08$ 17:00 & 125.13 & 122.653 & & 100.43 & & 95.962 & 110.384 & & 80.531 & 74.01 \\
\hline $3 / 23 / 08 \quad 17: 30$ & 125.116 & 122.648 & & 100.42 & & 95.948 & 110.382 & & & 73.997 \\
\hline $3 / 23 / 08$ 18:00 & 125.104 & 122.639 & & 100.409 & & 95.938 & 110.38 & & & 73.985 \\
\hline 3/23/08 18:30 & 125.097 & 122.634 & & 100.404 & & 95.931 & 110.374 & & 80.504 & 73.975 \\
\hline 3/23/08 19:00 & 125.09 & 122.628 & & 100.397 & & 95.924 & 110.369 & & & 73.968 \\
\hline 3/23/08 19:30 & 125.083 & 122.63 & & 100.39 & & 95.919 & 110.365 & & & 73.962 \\
\hline 3/23/08 20:00 & 125.074 & 122.63 & & 100.387 & & 95.912 & 110.367 & & 80.482 & 73.956 \\
\hline $3 / 23 / 0820: 30$ & 125.078 & 122.632 & & 100.392 & & 95.915 & 110.361 & & & 73.952 \\
\hline $3 / 23 / 0821: 00$ & 125.078 & 122.63 & & 100.394 & & 95.912 & 110.356 & & & 73.954 \\
\hline 3/23/08 21:30 & 125.081 & 122.625 & & 100.392 & & 95.919 & 110.352 & & 80.484 & 73.954 \\
\hline $3 / 23 / 0822: 00$ & 125.078 & 122.623 & & 100.39 & & 95.915 & 110.35 & & & 73.954 \\
\hline 3/23/08 22:30 & 125.076 & 122.614 & & 100.389 & & 95.912 & 110.35 & & & 73.952 \\
\hline 3/23/08 23:00 & 125.069 & 122.603 & & 100.382 & & 95.91 & 110.348 & & 80.474 & 73.95 \\
\hline $3 / 23 / 0823: 30$ & 125.062 & 122.598 & & 100.372 & & 95.9 & 110.344 & & & 73.944 \\
\hline 3/24/08 0:00 & 125.05 & 122.596 & & 100.363 & & 95.889 & 110.342 & & & 73.933 \\
\hline 3/24/08 0:30 & 125.045 & 122.589 & & 100.356 & & 95.884 & 110.341 & & 80.457 & 73.925 \\
\hline 3/24/08 1:00 & 125.045 & 122.58 & & 100.351 & & 95.884 & 110.337 & & & 73.921 \\
\hline 3/24/08 1:30 & 125.036 & 122.575 & & 100.346 & & 95.875 & 110.337 & & & 73.917 \\
\hline $3 / 24 / 082: 00$ & 125.029 & 122.566 & & 100.337 & & 95.865 & 110.335 & & 80.445 & 73.913 \\
\hline $3 / 24 / 082: 30$ & 125.022 & 122.555 & & 100.332 & & 95.86 & 110.333 & & & 73.904 \\
\hline 3/24/08 3:00 & 125.015 & 122.532 & & 100.327 & & 95.856 & 110.329 & & & 73.896 \\
\hline 3/24/08 3:30 & 125.003 & 122.523 & & 100.306 & & 95.844 & 110.331 & & 80.411 & 73.892 \\
\hline 3/24/08 4:00 & 124.984 & 122.51 & & 100.289 & & 95.82 & 110.331 & & & 73.875 \\
\hline 3/24/08 4:30 & 124.97 & 122.498 & & 100.28 & & 95.809 & 110.331 & & & 73.861 \\
\hline 3/24/08 5:00 & 124.958 & 122.487 & & 100.27 & & 95.799 & 110.329 & & 80.382 & 73.849 \\
\hline $3 / 24 / 085: 30$ & 124.944 & 122.471 & & 100.253 & & 95.783 & 110.327 & & & 73.838 \\
\hline 3/24/08 6:00 & 124.932 & 124.14 & & 100.242 & & 95.771 & 110.325 & & & 73.826 \\
\hline 3/24/08 6:30 & 124.918 & 124.526 & & 100.227 & & 95.759 & 110.325 & & 80.352 & 73.813 \\
\hline $3 / 24 / 08$ 7:00 & 126.598 & 124.671 & & 100.923 & & 96.155 & 110.755 & & & 73.813 \\
\hline 3/24/08 7:30 & 127.024 & 124.748 & & 101.13 & & 96.537 & 111.118 & & & 73.983 \\
\hline $3 / 24 / 088: 00$ & 127.173 & 124.793 & & 101.235 & & 96.685 & 111.264 & & 80.379 & 74.111 \\
\hline $3 / 24 / 088: 30$ & 127.253 & 124.818 & & 101.292 & & 96.763 & 111.351 & & & 74.192 \\
\hline 3/24/08 9:00 & 127.3 & 124.825 & & 101.319 & & 96.808 & 111.405 & & & 74.241 \\
\hline 3/24/08 9:30 & 127.322 & 123.24 & & 101.338 & & 96.832 & 111.443 & & 80.411 & 74.27 \\
\hline $3 / 24 / 08 \quad 10: 00$ & 127.336 & 122.814 & & 101.347 & & 96.841 & 111.465 & & & 74.285 \\
\hline 3/24/08 10:30 & 125.75 & 122.643 & & 100.658 & & 96.478 & 111.092 & & & 74.281 \\
\hline $3 / 24 / 0811: 00$ & 125.27 & 122.537 & & 100.44 & & 96.07 & 110.718 & & 80.396 & 74.119 \\
\hline 3/24/08 11:30 & 125.093 & 122.464 & & 100.318 & & 95.903 & 110.579 & & & 73.987 \\
\hline $3 / 24 / 08$ 12:00 & 124.986 & 122.405 & & 100.237 & & 95.801 & 110.493 & & & 73.894 \\
\hline $3 / 24 / 08$ 12:30 & 124.911 & 122.36 & & 100.175 & & 95.733 & 110.449 & & 80.34 & 73.82 \\
\hline $3 / 24 / 08$ 13:00 & 124.849 & 122.317 & & 100.12 & & 95.674 & 110.402 & & & 73.766 \\
\hline 3/24/08 13:30 & 124.8 & 122.274 & & 100.077 & & 95.627 & 110.365 & & & 73.716 \\
\hline $3 / 24 / 08 \quad 14: 00$ & 124.757 & 122.238 & & 100.041 & & 95.582 & 110.331 & & 80.276 & 73.677 \\
\hline $3 / 24 / 08 \quad 14: 30$ & 124.719 & 122.206 & & 99.999 & & 95.545 & 110.314 & & & 73.642 \\
\hline $3 / 24 / 08$ 15:00 & 124.681 & 122.179 & & 99.974 & & 95.512 & 110.29 & & & 73.607 \\
\hline $3 / 24 / 08$ 15:30 & 124.651 & 122.161 & & 99.948 & & 95.481 & 110.275 & & 80.215 & 73.576 \\
\hline $3 / 24 / 08$ 16:00 & 124.622 & 122.151 & & 99.918 & & 95.455 & 110.262 & & & 73.551 \\
\hline 3/24/08 16:30 & 124.601 & 122.133 & & 99.908 & & 95.436 & 110.256 & & & 73.526 \\
\hline $3 / 24 / 08$ 17:00 & 124.594 & 122.117 & & 99.894 & & 95.427 & 110.249 & & 80.169 & 73.512 \\
\hline $3 / 24 / 08 \quad 17: 30$ & 124.575 & 122.097 & & 99.884 & & 95.413 & 110.243 & & & 73.497 \\
\hline $3 / 24 / 08$ 18:00 & 124.563 & 122.081 & & 99.862 & & 95.394 & 110.235 & & & 73.485 \\
\hline $3 / 24 / 08$ 18:30 & 124.54 & 122.063 & & 99.841 & & 95.372 & 110.232 & & 80.115 & 73.464 \\
\hline $3 / 24 / 08$ 19:00 & 124.525 & 122.052 & & 99.831 & & 95.363 & 110.228 & & & 73.443 \\
\hline 3/24/08 19:30 & 124.507 & 122.043 & & 99.813 & & 95.344 & 110.226 & & & 73.427 \\
\hline $3 / 24 / 0820: 00$ & 124.497 & 122.043 & & 99.801 & & 95.332 & 110.224 & & 80.066 & 73.412 \\
\hline $3 / 24 / 0820: 30$ & 124.488 & 122.038 & & 99.8 & & 95.323 & 110.222 & & & 73.402 \\
\hline 3/24/08 21:00 & 124.485 & 122.027 & & 99.798 & & 95.325 & 110.218 & & & 73.396 \\
\hline $3 / 24 / 0821: 30$ & 124.483 & 122.022 & & 99.787 & & 95.318 & 110.218 & & 80.029 & 73.392 \\
\hline $3 / 24 / 08$ 22:00 & 124.469 & 122.011 & & 99.777 & & 95.306 & 110.22 & & & 73.381 \\
\hline 3/24/08 22:30 & 124.464 & 122.006 & & 99.774 & & 95.302 & 110.218 & & & 73.375 \\
\hline
\end{tabular}


TABLE S1.1 (Cont.)

\begin{tabular}{|c|c|c|c|c|c|c|c|c|c|c|}
\hline \multirow[b]{2}{*}{ Date and Time } & \multicolumn{10}{|c|}{ Depth from Top of Casing (ft) } \\
\hline & MW1D & MW2D & MW3D & MW4D & MW7 & MW9 & Oentrich & MW14D & MW15D & MW16D \\
\hline 3/24/08 23:00 & 124.457 & 122.009 & & 99.765 & & 95.297 & 110.217 & & 79.993 & 73.365 \\
\hline 3/24/08 23:30 & 124.447 & 122.013 & & 99.765 & & 95.29 & 110.22 & & & 73.359 \\
\hline 3/25/08 0:00 & 124.45 & 122.006 & & 99.767 & & 95.29 & 110.218 & & & 73.352 \\
\hline 3/25/08 0:30 & 124.455 & 122.02 & & 99.774 & & 95.297 & 110.213 & & 79.961 & 73.354 \\
\hline 3/25/08 1:00 & 124.45 & 122.011 & & 99.774 & & 95.29 & 110.218 & & & 73.357 \\
\hline 3/25/08 1:30 & 124.461 & 122.015 & & 99.784 & & 95.302 & 110.218 & & & 73.357 \\
\hline 3/25/08 2:00 & 124.454 & 122.015 & & 99.777 & & 95.295 & 110.22 & & 79.937 & 73.361 \\
\hline 3/25/08 2:30 & 124.457 & 122.013 & & 99.775 & & 95.299 & 110.217 & & & 73.354 \\
\hline 3/25/08 3:00 & 124.457 & 122.013 & & 99.774 & & 95.295 & 110.218 & & & 73.352 \\
\hline 3/25/08 3:30 & 124.459 & 122.018 & & 99.775 & & 95.297 & 110.218 & & 79.917 & 73.35 \\
\hline 3/25/08 4:00 & 124.457 & 122.027 & & 99.777 & & 95.297 & 110.217 & & & 73.348 \\
\hline 3/25/08 4:30 & 124.459 & 122.047 & & 99.784 & & 95.302 & 110.213 & & & 73.348 \\
\hline 3/25/08 5:00 & 124.471 & 122.061 & & 99.808 & & 95.311 & 110.215 & & 79.9 & 73.354 \\
\hline 3/25/08 5:30 & 124.495 & 122.065 & & 99.822 & & 95.339 & 110.211 & & & 73.369 \\
\hline 3/25/08 6:00 & 124.507 & 123.904 & & 99.832 & & 95.347 & 110.213 & & & 73.383 \\
\hline 3/25/08 6:30 & 124.516 & 124.228 & & 100.055 & & 95.356 & 110.211 & & 79.9 & 73.39 \\
\hline 3/25/08 7:00 & 126.407 & 124.399 & & 100.692 & & 95.971 & 110.808 & & & 73.448 \\
\hline 3/25/08 7:30 & 126.75 & 124.501 & & 100.902 & & 96.289 & 111.097 & & & 73.654 \\
\hline 3/25/08 8:00 & 126.927 & 124.571 & & 101.033 & & 96.461 & 111.229 & & 79.971 & 73.801 \\
\hline 3/25/08 8:30 & 127.031 & 124.634 & & 101.106 & & 96.568 & 111.311 & & & 73.909 \\
\hline 3/25/08 9:00 & 127.104 & 124.666 & & 101.185 & & 96.638 & 111.362 & & & 73.975 \\
\hline 3/25/08 9:30 & 127.17 & 124.705 & & 101.224 & & 96.704 & 111.399 & & 80.059 & 74.037 \\
\hline 3/25/08 10:00 & 127.201 & 122.843 & & 101.255 & & 96.737 & 111.428 & & & 74.078 \\
\hline 3/25/08 10:30 & 127.244 & 122.619 & & 100.744 & & 96.78 & 111.448 & & & 74.109 \\
\hline $3 / 25 / 08$ 11:00 & 125.308 & 122.501 & & 100.425 & & 96.098 & 110.75 & & 80.086 & 74.028 \\
\hline 3/25/08 11:30 & 125.078 & 122.473 & & 100.294 & & 95.891 & 110.555 & & & 73.9 \\
\hline 3/25/08 12:00 & 124.96 & 122.424 & & 100.225 & & 95.78 & 110.459 & & & 73.807 \\
\hline $3 / 25 / 08$ 12:30 & 124.922 & 122.401 & & 100.194 & & 95.75 & 110.399 & & 80.076 & 73.741 \\
\hline 3/25/08 13:00 & 124.875 & 122.38 & & 100.163 & & 95.702 & 110.367 & & & 73.722 \\
\hline 3/25/08 13:30 & 124.849 & 122.362 & & 100.137 & & 95.677 & 110.341 & & & 73.696 \\
\hline $3 / 25 / 08$ 14:00 & 124.833 & 122.342 & & 100.127 & & 95.665 & 110.324 & & 80.061 & 73.675 \\
\hline 3/25/08 14:30 & 124.811 & 122.335 & & 100.111 & & 95.646 & 110.314 & & & 73.658 \\
\hline 3/25/08 15:00 & 124.792 & 122.324 & & 100.103 & & 95.629 & 110.307 & & & 73.648 \\
\hline 3/25/08 15:30 & 124.783 & 122.321 & & 100.089 & & 95.622 & 110.299 & & 80.049 & 73.638 \\
\hline 3/25/08 16:00 & 124.774 & 122.317 & & 100.086 & & 95.611 & 110.294 & & & 73.625 \\
\hline 3/25/08 16:30 & 124.774 & 122.315 & & 100.086 & & 95.613 & 110.286 & & & 73.621 \\
\hline $3 / 25 / 08$ 17:00 & 124.769 & 122.315 & & 100.082 & & 95.606 & 110.282 & & 80.044 & 73.619 \\
\hline 3/25/08 17:30 & 124.766 & 122.321 & & 100.08 & & 95.606 & 110.277 & & & 73.613 \\
\hline 3/25/08 18:00 & 124.764 & 122.333 & & 100.082 & & 95.603 & 110.273 & & & 73.611 \\
\hline 3/25/08 18:30 & 124.769 & 122.333 & & 100.091 & & 95.608 & 110.271 & & 80.037 & 73.613 \\
\hline 3/25/08 19:00 & 124.785 & 122.335 & & 100.099 & & 95.629 & 110.314 & & & 73.623 \\
\hline 3/25/08 19:30 & 124.781 & 122.342 & & 100.103 & & 95.625 & 110.27 & & & 73.623 \\
\hline 3/25/08 20:00 & 124.785 & 122.349 & & 100.108 & & 95.629 & 110.264 & & 80.037 & 73.632 \\
\hline 3/25/08 20:30 & 124.79 & 122.358 & & 100.117 & & 95.632 & 110.265 & & & 73.634 \\
\hline 3/25/08 21:00 & 124.797 & 122.365 & & 100.125 & & 95.641 & 110.268 & & & 73.642 \\
\hline $3 / 25 / 0821: 30$ & 124.809 & 122.365 & & 100.134 & & 95.651 & 110.283 & & 80.039 & 73.648 \\
\hline 3/25/08 22:00 & 124.811 & 122.362 & & 100.135 & & 95.655 & 110.288 & & & 73.656 \\
\hline 3/25/08 22:30 & 124.814 & 122.355 & & 100.135 & & 95.658 & 110.284 & & & 73.656 \\
\hline 3/25/08 23:00 & 124.811 & 122.353 & & 100.129 & & 95.655 & 110.288 & & 80.039 & 73.658 \\
\hline 3/25/08 23:30 & 124.807 & 122.344 & & 100.127 & & 95.651 & 110.288 & & & 73.652 \\
\hline 3/26/08 0:00 & 124.802 & 122.34 & & 100.12 & & 95.644 & 110.286 & & & 73.65 \\
\hline 3/26/08 0:30 & 124.795 & 122.333 & & 100.111 & & 95.639 & 110.286 & & 80.029 & 73.646 \\
\hline 3/26/08 1:00 & 124.788 & 122.333 & & 100.108 & & 95.632 & 110.284 & & & 73.638 \\
\hline 3/26/08 1:30 & 124.781 & 122.34 & & 100.099 & & 95.627 & 110.284 & & & 73.636 \\
\hline 3/26/08 2:00 & 124.781 & 122.337 & & 100.108 & & 95.625 & 110.282 & & 80.029 & 73.627 \\
\hline 3/26/08 2:30 & 124.788 & 122.328 & & 100.113 & & 95.632 & 110.279 & & & 73.634 \\
\hline 3/26/08 3:00 & 124.785 & 122.317 & & 100.103 & & 95.629 & 110.277 & & & 73.638 \\
\hline 3/26/08 3:30 & 124.776 & 122.321 & & 100.089 & & 95.62 & 110.277 & & 80.027 & 73.632 \\
\hline 3/26/08 4:00 & 124.769 & 122.321 & & 100.089 & & 95.613 & 110.277 & & & 73.623 \\
\hline 3/26/08 4:30 & 124.769 & 122.312 & & 100.098 & & 95.613 & 110.273 & & & 73.615 \\
\hline 3/26/08 5:00 & 124.769 & 122.315 & & 100.092 & & 95.615 & 110.271 & & 80.022 & 73.625 \\
\hline 3/26/08 5:30 & 124.764 & 122.319 & & 100.084 & & 95.611 & 110.271 & & & 73.623 \\
\hline 3/26/08 6:00 & 124.764 & 124.14 & & 100.087 & & 95.611 & 110.267 & & & 73.615 \\
\hline 3/26/08 6:30 & 124.766 & 124.444 & & 100.287 & & 95.613 & 110.267 & & 80.015 & 73.617 \\
\hline $3 / 26 / 087: 00$ & 126.622 & 124.58 & & 100.873 & & 96.167 & 110.823 & & & 73.667 \\
\hline 3/26/08 7:30 & 126.943 & 124.659 & & 101.057 & & 96.466 & 111.093 & & & 73.859 \\
\hline
\end{tabular}


TABLE S1.1 (Cont.)

\begin{tabular}{|c|c|c|c|c|c|c|c|c|c|c|}
\hline \multirow[b]{2}{*}{ Date and Time } & \multicolumn{10}{|c|}{ Depth from Top of Casing (ft) } \\
\hline & MW1D & MW2D & MW3D & MW4D & MW7 & MW9 & Oentrich & MW14D & MW15D & MW16D \\
\hline 3/26/08 8:00 & 127.085 & 124.705 & & 101.154 & & 96.608 & 111.221 & & 80.076 & 73.989 \\
\hline 3/26/08 8:30 & 127.166 & 124.741 & & 101.218 & & 96.688 & 111.296 & & & 74.066 \\
\hline 3/26/08 9:00 & 127.213 & 123.165 & & 101.255 & & 96.735 & 111.347 & & & 74.124 \\
\hline 3/26/08 9:30 & 127.253 & 122.745 & & 101.276 & & 96.775 & 111.379 & & 80.135 & 74.159 \\
\hline $3 / 26 / 08$ 10:00 & 125.674 & 122.582 & & 100.604 & & 96.417 & 111.016 & & & 74.167 \\
\hline 3/26/08 10:30 & 125.199 & 122.496 & & 100.382 & & 96.007 & 110.654 & & & 74.016 \\
\hline 3/26/08 11:00 & 125.033 & 122.437 & & 100.277 & & 95.853 & 110.523 & & 80.11 & 73.89 \\
\hline $3 / 26 / 0811: 30$ & 124.946 & 122.387 & & 100.211 & & 95.771 & 110.444 & & & 73.803 \\
\hline $3 / 26 / 08$ 12:00 & 124.885 & 122.351 & & 100.168 & & 95.712 & 110.399 & & & 73.745 \\
\hline 3/26/08 12:30 & 124.837 & 122.319 & & 100.12 & & 95.669 & 110.371 & & 80.086 & 73.708 \\
\hline 3/26/08 13:00 & 124.802 & 122.299 & & 100.098 & & 95.636 & 110.342 & & & 73.669 \\
\hline 3/26/08 13:30 & 124.766 & 122.272 & & 100.072 & & 95.603 & 110.329 & & & 73.642 \\
\hline 3/26/08 14:00 & 124.75 & 122.242 & & 100.048 & & 95.589 & 110.314 & & 80.059 & 73.619 \\
\hline $3 / 26 / 08$ 14:30 & 124.722 & 122.222 & & 100.018 & & 95.561 & 110.303 & & & 73.598 \\
\hline 3/26/08 15:00 & 124.693 & 122.206 & & 99.992 & & 95.53 & 110.29 & & & 73.574 \\
\hline 3/26/08 15:30 & 124.672 & 122.192 & & 99.979 & & 95.509 & 110.279 & & 80.027 & 73.551 \\
\hline 3/26/08 16:00 & 124.655 & 122.185 & & 99.965 & & 95.495 & 110.271 & & & 73.534 \\
\hline 3/26/08 16:30 & 124.641 & 122.179 & & 99.953 & & 95.483 & 110.265 & & & 73.522 \\
\hline 3/26/08 17:00 & 124.636 & 122.167 & & 99.949 & & 95.479 & 110.258 & & 80 & 73.512 \\
\hline 3/26/08 17:30 & 124.629 & 122.154 & & 99.943 & & 95.471 & 110.254 & & & 73.503 \\
\hline 3/26/08 18:00 & 124.615 & 122.147 & & 99.932 & & 95.457 & 110.25 & & & 73.497 \\
\hline 3/26/08 18:30 & 124.606 & 122.129 & & 99.918 & & 95.446 & 110.247 & & 79.973 & 73.487 \\
\hline 3/26/08 19:00 & 124.594 & 122.133 & & 99.905 & & 95.438 & 110.241 & & & 73.474 \\
\hline 3/26/08 19:30 & 124.577 & 122.138 & & 99.899 & & 95.42 & 110.239 & & & 73.464 \\
\hline 3/26/08 20:00 & 124.582 & 122.12 & & 99.905 & & 95.427 & 110.234 & & 79.951 & 73.456 \\
\hline 3/26/08 20:30 & 124.587 & 122.115 & & 99.901 & & 95.431 & 110.23 & & & 73.456 \\
\hline 3/26/08 21:00 & 124.568 & 122.115 & & 99.884 & & 95.415 & 110.234 & & & 73.458 \\
\hline 3/26/08 21:30 & 124.565 & 122.104 & & 99.889 & & 95.413 & 110.23 & & 79.927 & 73.441 \\
\hline 3/26/08 22:00 & 124.565 & 122.088 & & 99.879 & & 95.415 & 110.228 & & & 73.443 \\
\hline 3/26/08 22:30 & 124.554 & 122.077 & & 99.868 & & 95.398 & 110.226 & & & 73.433 \\
\hline 3/26/08 23:00 & 124.54 & 122.061 & & 99.851 & & 95.384 & 110.23 & & 79.897 & 73.427 \\
\hline 3/26/08 23:30 & 124.525 & 122.047 & & 99.836 & & 95.37 & 110.226 & & & 73.41 \\
\hline 3/27/08 0:00 & 124.511 & 122.036 & & 99.831 & & 95.356 & 110.226 & & & 73.394 \\
\hline 3/27/08 0:30 & 124.502 & 122.031 & & 99.81 & & 95.344 & 110.224 & & 79.873 & 73.386 \\
\hline 3/27/08 1:00 & 124.488 & 122.011 & & 99.8 & & 95.33 & 110.224 & & & 73.371 \\
\hline 3/27/08 1:30 & 124.481 & 121.979 & & 99.803 & & 95.323 & 110.22 & & & 73.359 \\
\hline 3/27/08 2:00 & 124.466 & 121.99 & & 99.765 & & 95.311 & 110.222 & & 79.841 & 73.357 \\
\hline 3/27/08 2:30 & 124.431 & 121.975 & & 99.741 & & 95.273 & 110.224 & & & 73.328 \\
\hline 3/27/08 3:00 & 124.44 & 121.979 & & 99.75 & & 95.285 & 110.217 & & & 73.311 \\
\hline 3/27/08 3:30 & 124.424 & 121.975 & & 99.748 & & 95.269 & 110.218 & & 79.812 & 73.309 \\
\hline 3/27/08 4:00 & 124.429 & 121.963 & & 99.75 & & 95.271 & 110.217 & & & 73.305 \\
\hline 3/27/08 4:30 & 124.424 & 121.956 & & 99.734 & & 95.269 & 110.215 & & & 73.307 \\
\hline 3/27/08 5:00 & 124.412 & 121.956 & & 99.725 & & 95.255 & 110.215 & & 79.79 & 73.295 \\
\hline 3/27/08 5:30 & 124.407 & 121.956 & & 99.725 & & 95.25 & 110.215 & & & 73.284 \\
\hline 3/27/08 6:00 & 124.407 & 123.748 & & 99.725 & & 95.252 & 110.213 & & & 73.286 \\
\hline 3/27/08 6:30 & 124.405 & 124.083 & & 99.867 & & 95.25 & 110.211 & & 79.77 & 73.28 \\
\hline 3/27/08 7:00 & 126.256 & 124.244 & & 100.552 & & 95.825 & 110.797 & & & 73.33 \\
\hline 3/27/08 7:30 & 126.61 & 124.331 & & 100.757 & & 96.155 & 111.086 & & & 73.522 \\
\hline 3/27/08 8:00 & 126.776 & 124.385 & & 100.876 & & 96.32 & 111.221 & & 79.827 & 73.663 \\
\hline 3/27/08 8:30 & 126.868 & 124.435 & & 100.937 & & 96.409 & 111.304 & & & 73.762 \\
\hline 3/27/08 9:00 & 126.92 & 122.922 & & 100.987 & & 96.459 & 111.358 & & & 73.811 \\
\hline 3/27/08 9:30 & 126.972 & 122.473 & & 101.038 & & 96.513 & 111.392 & & 79.895 & 73.857 \\
\hline 3/27/08 10:00 & 125.442 & 122.326 & & 100.332 & & 96.179 & 111.02 & & & 73.89 \\
\hline 3/27/08 10:30 & 124.937 & 122.24 & & 100.123 & & 95.743 & 110.624 & & & 73.745 \\
\hline 3/27/08 11:00 & 124.781 & 122.204 & & 100.017 & & 95.601 & 110.479 & & 79.88 & 73.627 \\
\hline 3/27/08 11:30 & 124.688 & 122.183 & & 99.97 & & 95.516 & 110.402 & & & 73.549 \\
\hline 3/27/08 12:00 & 124.653 & 122.174 & & 99.939 & & 95.488 & 110.356 & & & 73.503 \\
\hline $3 / 27 / 08$ 12:30 & 124.632 & 122.167 & & 99.943 & & 95.471 & 110.325 & & 79.873 & 73.481 \\
\hline 3/27/08 13:00 & 124.625 & 122.165 & & 99.937 & & 95.462 & 110.305 & & & 73.476 \\
\hline 3/27/08 13:30 & 124.618 & 122.165 & & 99.929 & & 95.46 & 110.292 & & & 73.47 \\
\hline $3 / 27 / 0814: 00$ & 124.615 & 122.163 & & 99.936 & & 95.455 & 110.28 & & 79.861 & 73.464 \\
\hline 3/27/08 14:30 & 124.618 & 122.156 & & 99.925 & & 95.46 & 110.271 & & & 73.47 \\
\hline 3/27/08 15:00 & 124.611 & 122.158 & & 99.927 & & 95.453 & 110.269 & & & 73.464 \\
\hline 3/27/08 15:30 & 124.606 & 122.167 & & 99.929 & & 95.448 & 110.265 & & 79.853 & 73.462 \\
\hline 3/27/08 16:00 & 124.608 & 122.179 & & 99.934 & & 95.453 & 110.26 & & & 73.462 \\
\hline 3/27/08 16:30 & 124.618 & 122.19 & & 99.949 & & 95.462 & 110.254 & & & 73.464 \\
\hline
\end{tabular}


TABLE S1.1 (Cont.)

\begin{tabular}{|c|c|c|c|c|c|c|c|c|c|c|}
\hline \multirow[b]{2}{*}{ Date and Time } & \multicolumn{10}{|c|}{ Depth from Top of Casing (ft) } \\
\hline & MW1D & MW2D & MW3D & MW4D & MW7 & MW9 & Oentrich & MW14D & MW15D & MW16D \\
\hline 3/27/08 17:00 & 124.627 & 122.188 & & 99.955 & & 95.474 & 110.25 & & 79.858 & 73.474 \\
\hline $3 / 27 / 0817: 30$ & 124.639 & 122.192 & & 99.963 & & 95.486 & 110.25 & & & 73.483 \\
\hline $3 / 27 / 0818: 00$ & 124.641 & 122.197 & & 99.963 & & 95.488 & 110.245 & & & 73.491 \\
\hline $3 / 27 / 0818: 30$ & 124.644 & 122.208 & & 99.972 & & 95.49 & 110.243 & & 79.861 & 73.487 \\
\hline $3 / 27 / 08$ 19:00 & 124.65 & 122.222 & & 99.977 & & 95.5 & 110.243 & & & 73.495 \\
\hline $3 / 27 / 08$ 19:30 & 124.658 & 122.247 & & 99.991 & & 95.504 & 110.239 & & & 73.501 \\
\hline 3/27/08 20:00 & 124.665 & 122.269 & & 100.01 & & 95.518 & 110.234 & & 79.871 & 73.507 \\
\hline $3 / 27 / 0820: 30$ & 124.698 & 122.29 & & 100.032 & & 95.545 & 110.235 & & & 73.524 \\
\hline $3 / 27 / 0821: 00$ & 124.719 & 122.294 & & 100.058 & & 95.566 & 110.235 & & & 73.545 \\
\hline $3 / 27 / 0821: 30$ & 124.738 & 122.306 & & 100.068 & & 95.589 & 110.243 & & 79.897 & 73.567 \\
\hline 3/27/08 22:00 & 124.743 & 122.312 & & 100.08 & & 95.592 & 110.243 & & & 73.58 \\
\hline $3 / 27 / 0822: 30$ & 124.754 & 122.328 & & 100.087 & & 95.608 & 110.243 & & & 73.588 \\
\hline 3/27/08 23:00 & 124.761 & 122.331 & & 100.101 & & 95.613 & 110.243 & & 79.91 & 73.598 \\
\hline 3/27/08 23:30 & 124.776 & 122.335 & & 100.11 & & 95.629 & 110.245 & & & 73.607 \\
\hline $3 / 28 / 080: 00$ & 124.781 & 122.349 & & 100.101 & & 95.634 & 110.247 & & & 73.615 \\
\hline 3/28/08 0:30 & 124.783 & 122.358 & & 100.12 & & 95.634 & 110.247 & & 79.922 & 73.615 \\
\hline 3/28/08 1:00 & 124.795 & 122.355 & & 100.129 & & 95.646 & 110.249 & & & 73.627 \\
\hline 3/28/08 1:30 & 124.807 & 122.365 & & 100.129 & & 95.66 & 110.249 & & & 73.634 \\
\hline 3/28/08 2:00 & 124.807 & 122.374 & & 100.137 & & 95.655 & 110.249 & & 79.932 & 73.64 \\
\hline 3/28/08 2:30 & 124.813 & 122.38 & & 100.149 & & 95.665 & 110.245 & & & 73.644 \\
\hline 3/28/08 3:00 & 124.825 & 122.383 & & 100.154 & & 95.674 & 110.245 & & & 73.652 \\
\hline 3/28/08 3:30 & 124.83 & 122.39 & & 100.161 & & 95.684 & 110.243 & & 79.951 & 73.66 \\
\hline 3/28/08 4:00 & 124.83 & 122.392 & & 100.175 & & 95.683 & 110.243 & & & 73.665 \\
\hline 3/28/08 4:30 & 124.837 & 122.401 & & 100.172 & & 95.688 & 110.241 & & & 73.675 \\
\hline 3/28/08 5:00 & 124.842 & 122.41 & & 100.177 & & 95.695 & 110.237 & & 79.963 & 73.675 \\
\hline 3/28/08 5:30 & 124.852 & 122.417 & & 100.189 & & 95.705 & 110.235 & & & 73.681 \\
\hline 3/28/08 6:00 & 124.859 & 124.213 & & 100.191 & & 95.712 & 110.234 & & & 73.694 \\
\hline 3/28/08 6:30 & 124.863 & 124.564 & & 100.204 & & 95.719 & 110.23 & & 79.983 & 73.696 \\
\hline 3/28/08 7:00 & 126.688 & 124.721 & & 100.978 & & 96.242 & 110.746 & & & 73.743 \\
\hline $3 / 28 / 08$ 7:30 & 127.062 & 124.814 & & 101.185 & & 96.586 & 111.045 & & & 73.942 \\
\hline 3/28/08 8:00 & 127.222 & 124.879 & & 101.305 & & 96.744 & 111.178 & & 80.069 & 74.086 \\
\hline 3/28/08 8:30 & 127.322 & 124.916 & & 101.378 & & 96.841 & 111.255 & & & 74.183 \\
\hline 3/28/08 9:00 & 127.388 & 124.952 & & 101.428 & & 96.909 & 111.306 & & & 74.248 \\
\hline 3/28/08 9:30 & 127.428 & 124.981 & & 101.473 & & 96.947 & 111.339 & & 80.147 & 74.297 \\
\hline $3 / 28 / 0810: 00$ & 127.464 & 124.999 & & 101.5 & & 96.985 & 111.362 & & & 74.334 \\
\hline $3 / 28 / 0810: 30$ & 127.492 & 123.122 & & 101.521 & & 97.015 & 111.381 & & & 74.363 \\
\hline $3 / 28 / 08$ 11:00 & 127.508 & 122.904 & & 101.044 & & 97.032 & 111.394 & & 80.223 & 74.386 \\
\hline $3 / 28 / 0811: 30$ & 125.586 & 122.791 & & 100.721 & & 96.386 & 110.744 & & & 74.303 \\
\hline $3 / 28 / 08$ 12:00 & 125.357 & 122.711 & & 100.58 & & 96.174 & 110.547 & & & 74.169 \\
\hline 3/28/08 12:30 & 125.239 & 122.657 & & 100.496 & & 96.065 & 110.451 & & 80.208 & 74.07 \\
\hline 3/28/08 13:00 & 125.163 & 122.614 & & 100.437 & & 95.995 & 110.395 & & & 73.999 \\
\hline $3 / 28 / 08$ 13:30 & 125.107 & 122.582 & & 100.392 & & 95.938 & 110.357 & & & 73.948 \\
\hline $3 / 28 / 0814: 00$ & 125.067 & 122.555 & & 100.356 & & 95.898 & 110.335 & & 80.193 & 73.911 \\
\hline $3 / 28 / 0814: 30$ & 125.031 & 122.53 & & 100.328 & & 95.865 & 110.318 & & & 73.88 \\
\hline $3 / 28 / 08$ 15:00 & 125.005 & 122.51 & & 100.306 & & 95.842 & 110.305 & & & 73.855 \\
\hline $3 / 28 / 08$ 15:30 & 124.979 & 122.494 & & 100.284 & & 95.818 & 110.294 & & 80.179 & 73.832 \\
\hline $3 / 28 / 0816: 00$ & 124.96 & 122.482 & & 100.266 & & 95.799 & 110.284 & & & 73.813 \\
\hline $3 / 28 / 0816: 30$ & 124.944 & 122.471 & & 100.251 & & 95.785 & 110.277 & & & 73.797 \\
\hline 3/28/08 17:00 & 124.93 & 122.46 & & 100.244 & & 95.771 & 110.271 & & 80.164 & 73.782 \\
\hline $3 / 28 / 08$ 17:30 & 124.92 & 122.451 & & 100.234 & & 95.761 & 110.264 & & & 73.776 \\
\hline $3 / 28 / 08$ 18:00 & 124.908 & 122.444 & & 100.223 & & 95.75 & 110.258 & & & 73.768 \\
\hline 3/28/08 18:30 & 124.899 & 122.437 & & 100.216 & & 95.74 & 110.254 & & 80.157 & 73.758 \\
\hline 3/28/08 19:00 & 124.892 & 122.435 & & 100.208 & & 95.733 & 110.25 & & & 73.751 \\
\hline $3 / 28 / 08$ 19:30 & 124.885 & 122.435 & & 100.206 & & 95.728 & 110.247 & & & 73.743 \\
\hline 3/28/08 20:00 & 124.885 & 122.435 & & 100.206 & & 95.728 & 110.241 & & 80.147 & 73.741 \\
\hline $3 / 28 / 0820: 30$ & 124.885 & 122.439 & & 100.206 & & 95.726 & 110.235 & & & 73.741 \\
\hline $3 / 28 / 0821: 00$ & 124.882 & 122.442 & & 100.206 & & 95.728 & 110.232 & & & 73.741 \\
\hline $3 / 28 / 0821: 30$ & 124.887 & 122.451 & & 100.215 & & 95.731 & 110.228 & & 80.147 & 73.739 \\
\hline $3 / 28 / 0822: 00$ & 124.889 & 122.453 & & 100.22 & & 95.735 & 110.224 & & & 73.747 \\
\hline $3 / 28 / 0822: 30$ & 124.899 & 122.453 & & 100.229 & & 95.747 & 110.22 & & & 73.751 \\
\hline 3/28/08 23:00 & 124.903 & 122.453 & & 100.229 & & 95.75 & 110.217 & & 80.147 & 73.76 \\
\hline 3/28/08 23:30 & 124.901 & 122.446 & & 100.225 & & 95.75 & 110.215 & & & 73.76 \\
\hline $3 / 29 / 080: 00$ & 124.901 & 122.442 & & 100.222 & & 95.75 & 110.213 & & & 73.76 \\
\hline 3/29/08 0:30 & 124.894 & 122.437 & & 100.216 & & 95.738 & 110.211 & & 80.142 & 73.756 \\
\hline 3/29/08 1:00 & 124.889 & 122.428 & & 100.211 & & 95.735 & 110.209 & & & 73.751 \\
\hline $3 / 29 / 08$ 1:30 & 124.887 & 122.424 & & 100.208 & & 95.731 & 110.207 & & & 73.749 \\
\hline
\end{tabular}


TABLE S1.1 (Cont.)

\begin{tabular}{|c|c|c|c|c|c|c|c|c|c|c|}
\hline \multirow[b]{2}{*}{ Date and Time } & \multicolumn{10}{|c|}{ Depth from Top of Casing (ft) } \\
\hline & MW1D & MW2D & MW3D & MW4D & MW7 & MW9 & Oentrich & MW14D & MW15D & MW16D \\
\hline 3/29/08 2:00 & 124.878 & 122.417 & & 100.201 & & 95.724 & 110.205 & & 80.135 & 73.743 \\
\hline 3/29/08 2:30 & 124.873 & 122.408 & & 100.198 & & 95.719 & 110.203 & & & 73.739 \\
\hline 3/29/08 3:00 & 124.866 & 122.399 & & 100.185 & & 95.712 & 110.203 & & & 73.733 \\
\hline 3/29/08 3:30 & 124.856 & 122.385 & & 100.177 & & 95.702 & 110.202 & & 80.125 & 73.727 \\
\hline 3/29/08 4:00 & 124.847 & 122.376 & & 100.168 & & 95.693 & 110.202 & & & 73.716 \\
\hline 3/29/08 4:30 & 124.835 & 122.369 & & 100.156 & & 95.681 & 110.2 & & & 73.71 \\
\hline 3/29/08 5:00 & 124.828 & 122.358 & & 100.151 & & 95.674 & 110.2 & & 80.113 & 73.698 \\
\hline 3/29/08 5:30 & 124.816 & 122.349 & & 100.135 & & 95.667 & 110.196 & & & 73.694 \\
\hline 3/29/08 6:00 & 124.807 & 122.342 & & 100.132 & & 95.655 & 110.196 & & & 73.683 \\
\hline 3/29/08 6:30 & 124.797 & 122.344 & & 100.122 & & 95.646 & 110.194 & & 80.098 & 73.679 \\
\hline 3/29/08 7:00 & 124.792 & 124.065 & & 100.117 & & 95.639 & 110.192 & & & 73.669 \\
\hline 3/29/08 7:30 & 124.79 & 124.435 & & 100.122 & & 95.636 & 110.19 & & & 73.665 \\
\hline 3/29/08 8:00 & 126.558 & 124.582 & & 100.906 & & 96.139 & 110.688 & & 80.11 & 73.685 \\
\hline 3/29/08 8:30 & 126.962 & 124.657 & & 101.109 & & 96.508 & 111.039 & & & 73.884 \\
\hline 3/29/08 9:00 & 127.111 & 124.709 & & 101.209 & & 96.655 & 111.189 & & & 74.018 \\
\hline 3/29/08 9:30 & 127.189 & 124.745 & & 101.266 & & 96.733 & 111.276 & & 80.164 & 74.097 \\
\hline $3 / 29 / 0810: 00$ & 127.244 & 123.147 & & 101.316 & & 96.787 & 111.33 & & & 74.146 \\
\hline 3/29/08 10:30 & 127.284 & 122.739 & & 101.336 & & 96.82 & 111.368 & & & 74.186 \\
\hline $3 / 29 / 0811: 00$ & 125.65 & 122.569 & & 100.589 & & 96.402 & 110.939 & & 80.196 & 74.192 \\
\hline $3 / 29 / 0811: 30$ & 125.197 & 122.462 & & 100.375 & & 96.004 & 110.583 & & & 74.03 \\
\hline 3/29/08 12:00 & 125.022 & 122.394 & & 100.251 & & 95.842 & 110.446 & & & 73.9 \\
\hline $3 / 29 / 08$ 12:30 & 124.91 & 122.335 & & 100.175 & & 95.735 & 110.376 & & 80.147 & 73.805 \\
\hline 3/29/08 13:00 & 124.842 & 122.292 & & 100.113 & & 95.669 & 110.329 & & & 73.735 \\
\hline 3/29/08 13:30 & 124.785 & 122.265 & & 100.073 & & 95.615 & 110.299 & & & 73.685 \\
\hline $3 / 29 / 0814: 00$ & 124.743 & 122.24 & & 100.039 & & 95.575 & 110.277 & & 80.105 & 73.642 \\
\hline 3/29/08 14:30 & 124.714 & 122.217 & & 100.011 & & 95.549 & 110.258 & & & 73.611 \\
\hline 3/29/08 15:00 & 124.686 & 122.199 & & 99.992 & & 95.523 & 110.243 & & & 73.582 \\
\hline $3 / 29 / 08$ 15:30 & 124.669 & 122.181 & & 99.975 & & 95.507 & 110.232 & & 80.073 & 73.565 \\
\hline 3/29/08 16:00 & 124.648 & 122.172 & & 99.955 & & 95.486 & 110.222 & & & 73.547 \\
\hline $3 / 29 / 0816: 30$ & 124.629 & 122.163 & & 99.941 & & 95.471 & 110.211 & & & 73.528 \\
\hline $3 / 29 / 08$ 17:00 & 124.618 & 122.156 & & 99.932 & & 95.46 & 110.207 & & 80.039 & 73.516 \\
\hline $3 / 29 / 08$ 17:30 & 124.61 & 122.154 & & 99.93 & & 95.453 & 110.202 & & & 73.507 \\
\hline 3/29/08 18:00 & 124.606 & 122.154 & & 99.92 & & 95.45 & 110.196 & & & 73.501 \\
\hline $3 / 29 / 0818: 30$ & 124.603 & 122.158 & & 99.92 & & 95.45 & 110.188 & & 80.02 & 73.495 \\
\hline 3/29/08 19:00 & 124.599 & 122.165 & & 99.93 & & 95.446 & 110.187 & & & 73.491 \\
\hline 3/29/08 19:30 & 124.606 & 122.167 & & 99.936 & & 95.45 & 110.183 & & & 73.495 \\
\hline $3 / 29 / 0820: 00$ & 124.613 & 122.167 & & 99.943 & & 95.46 & 110.181 & & 80.003 & 73.499 \\
\hline 3/29/08 20:30 & 124.618 & 122.161 & & 99.943 & & 95.467 & 110.181 & & & 73.505 \\
\hline 3/29/08 21:00 & 124.618 & 122.161 & & 99.932 & & 95.464 & 110.177 & & & 73.505 \\
\hline 3/29/08 21:30 & 124.611 & 122.167 & & 99.936 & & 95.455 & 110.177 & & 79.99 & 73.497 \\
\hline 3/29/08 22:00 & 124.608 & 122.165 & & 99.936 & & 95.455 & 110.175 & & & 73.499 \\
\hline 3/29/08 22:30 & 124.615 & 122.163 & & 99.941 & & 95.467 & 110.172 & & & 73.497 \\
\hline 3/29/08 23:00 & 124.615 & 122.165 & & 99.932 & & 95.464 & 110.172 & & 79.976 & 73.501 \\
\hline 3/29/08 23:30 & 124.613 & 122.158 & & 99.943 & & 95.46 & 110.172 & & & 73.495 \\
\hline $3 / 30 / 080: 00$ & 124.613 & 122.158 & & 99.943 & & 95.464 & 110.168 & & & 73.499 \\
\hline 3/30/08 0:30 & 124.611 & 122.154 & & 99.932 & & 95.46 & 110.17 & & 79.963 & 73.501 \\
\hline 3/30/08 1:00 & 124.608 & 122.154 & & 99.927 & & 95.457 & 110.168 & & & 73.493 \\
\hline 3/30/08 1:30 & 124.599 & 122.154 & & 99.932 & & 95.45 & 110.168 & & & 73.487 \\
\hline 3/30/08 2:00 & 124.603 & 122.149 & & 99.927 & & 95.453 & 110.168 & & 79.946 & 73.487 \\
\hline 3/30/08 2:30 & 124.601 & 122.14 & & 99.927 & & 95.45 & 110.164 & & & 73.485 \\
\hline 3/30/08 3:00 & 124.598 & 122.154 & & 99.922 & & 95.45 & 110.164 & & & 73.483 \\
\hline 3/30/08 3:30 & 124.589 & 122.158 & & 99.924 & & 95.441 & 110.164 & & 79.932 & 73.479 \\
\hline 3/30/08 4:00 & 124.601 & 122.165 & & 99.934 & & 95.453 & 110.162 & & & 73.476 \\
\hline 3/30/08 4:30 & 124.608 & 122.176 & & 99.937 & & 95.457 & 110.16 & & & 73.485 \\
\hline 3/30/08 5:00 & 124.613 & 122.176 & & 99.953 & & 95.462 & 110.158 & & 79.932 & 73.486 \\
\hline 3/30/08 5:30 & 124.624 & 122.19 & & 99.958 & & 95.476 & 110.157 & & & 73.499 \\
\hline 3/30/08 6:00 & 124.625 & 122.181 & & 99.965 & & 95.474 & 110.158 & & & 73.505 \\
\hline 3/30/08 6:30 & 124.639 & 122.185 & & 99.958 & & 95.49 & 110.157 & & 79.934 & 73.51 \\
\hline 3/30/08 7:00 & 124.632 & 124.013 & & 99.961 & & 95.481 & 110.158 & & & 73.51 \\
\hline 3/30/08 7:30 & 124.632 & 124.317 & & 100.142 & & 95.483 & 110.155 & & & 73.505 \\
\hline 3/30/08 8:00 & 126.497 & 124.464 & & 100.761 & & 96.054 & 110.705 & & 79.949 & 73.565 \\
\hline 3/30/08 8:30 & 126.818 & 124.55 & & 100.952 & & 96.355 & 110.988 & & & 73.762 \\
\hline 3/30/08 9:00 & 126.972 & 124.605 & & 101.057 & & 96.502 & 111.116 & & & 73.898 \\
\hline 3/30/08 9:30 & 127.059 & 124.653 & & 101.124 & & 96.586 & 111.195 & & 80.017 & 73.983 \\
\hline $3 / 30 / 08$ 10:00 & 127.116 & 124.675 & & 101.173 & & 96.643 & 111.244 & & & 74.041 \\
\hline $3 / 30 / 0810: 30$ & 127.161 & 124.691 & & 101.209 & & 96.69 & 111.281 & & & 74.084 \\
\hline
\end{tabular}


TABLE S1.1 (Cont.)

\begin{tabular}{|c|c|c|c|c|c|c|c|c|c|c|}
\hline \multirow[b]{2}{*}{ Date and Time } & \multicolumn{10}{|c|}{ Depth from Top of Casing (ft) } \\
\hline & MW1D & MW2D & MW3D & MW4D & MW7 & MW9 & Oentrich & MW14D & MW15D & MW16D \\
\hline 3/30/08 11:00 & 127.189 & 124.689 & & 101.23 & & 96.718 & 111.307 & & 80.073 & 74.121 \\
\hline $3 / 30 / 0811: 30$ & 127.201 & 124.693 & & 101.231 & & 96.735 & 111.326 & & & 74.14 \\
\hline 3/30/08 12:00 & 127.203 & 124.687 & & 101.235 & & 96.735 & 111.343 & & & 74.152 \\
\hline $3 / 30 / 08$ 12:30 & 127.203 & 122.934 & & 101.233 & & 96.739 & 111.356 & & 80.108 & 74.159 \\
\hline $3 / 30 / 08$ 13:00 & 127.199 & 122.65 & & 100.995 & & 96.73 & 111.368 & & & 74.163 \\
\hline 3/30/08 13:30 & 125.447 & 122.516 & & 100.478 & & 96.19 & 110.819 & & & 74.103 \\
\hline $3 / 30 / 0814: 00$ & 125.178 & 122.437 & & 100.311 & & 95.919 & 110.564 & & 80.098 & 73.954 \\
\hline $3 / 30 / 0814: 30$ & 125.041 & 122.387 & & 100.22 & & 95.792 & 110.449 & & & 73.844 \\
\hline 3/30/08 15:00 & 124.96 & 122.344 & & 100.165 & & 95.717 & 110.382 & & & 73.766 \\
\hline $3 / 30 / 08$ 15:30 & 124.906 & 122.31 & & 100.12 & & 95.669 & 110.339 & & 80.071 & 73.716 \\
\hline $3 / 30 / 08$ 16:00 & 124.868 & 122.287 & & 100.084 & & 95.632 & 110.31 & & & 73.679 \\
\hline 3/30/08 16:30 & 124.835 & 122.269 & & 100.06 & & 95.599 & 110.288 & & & 73.646 \\
\hline 3/30/08 17:00 & 124.813 & 122.251 & & 100.041 & & 95.578 & 110.273 & & 80.047 & 73.621 \\
\hline $3 / 30 / 08$ 17:30 & 124.795 & 122.244 & & 100.023 & & 95.561 & 110.256 & & & 73.601 \\
\hline $3 / 30 / 08$ 18:00 & 124.776 & 122.247 & & 100.017 & & 95.545 & 110.247 & & & 73.584 \\
\hline $3 / 30 / 0818: 30$ & 124.766 & 122.24 & & 100.015 & & 95.533 & 110.237 & & 80.029 & 73.574 \\
\hline $3 / 30 / 08$ 19:00 & 124.757 & 122.235 & & 100.015 & & 95.537 & 110.23 & & & 73.567 \\
\hline 3/30/08 19:30 & 124.752 & 122.233 & & 100.005 & & 95.535 & 110.226 & & & 73.563 \\
\hline 3/30/08 20:00 & 124.748 & 122.233 & & 100.008 & & 95.53 & 110.22 & & 80.012 & 73.553 \\
\hline $3 / 30 / 0820: 30$ & 124.745 & 122.222 & & 100.005 & & 95.528 & 110.218 & & & 73.545 \\
\hline 3/30/08 21:00 & 124.738 & 122.224 & & 100.001 & & 95.528 & 110.213 & & & 73.534 \\
\hline $3 / 30 / 0821: 30$ & 124.735 & 122.21 & & 99.998 & & 95.519 & 110.211 & & 79.998 & 73.528 \\
\hline $3 / 30 / 0822: 00$ & 124.731 & 122.183 & & 99.991 & & 95.521 & 110.207 & & & 73.518 \\
\hline 3/30/08 22:30 & 124.731 & 122.174 & & 99.967 & & 95.512 & 110.205 & & & 73.512 \\
\hline 3/30/08 23:00 & 124.731 & 122.151 & & 99.953 & & 95.483 & 110.202 & & 79.976 & 73.507 \\
\hline 3/30/08 23:30 & 124.726 & 122.127 & & 99.932 & & 95.474 & 110.194 & & & 73.501 \\
\hline $3 / 31 / 080: 00$ & 124.703 & 122.104 & & 99.917 & & 95.443 & 110.192 & & & 73.497 \\
\hline 3/31/08 0:30 & 124.684 & 122.095 & & 99.887 & & 95.422 & 110.187 & & 79.944 & 73.493 \\
\hline 3/31/08 1:00 & 124.655 & 122.052 & & 99.868 & & 95.398 & 110.175 & & & 73.489 \\
\hline 3/31/08 1:30 & 124.639 & 121.977 & & 99.851 & & 95.389 & 110.166 & & & 73.479 \\
\hline 3/31/08 2:00 & 124.618 & 121.99 & & 99.787 & & 95.354 & 110.168 & & 79.902 & 73.474 \\
\hline $3 / 31 / 082: 30$ & 124.533 & 121.945 & & 99.77 & & 95.285 & 110.166 & & & 73.474 \\
\hline 3/31/08 3:00 & 124.497 & 121.913 & & 99.731 & & 95.276 & 110.142 & & & 73.456 \\
\hline 3/31/08 3:30 & 124.506 & 121.886 & & 99.725 & & 95.231 & 110.147 & & 79.824 & 73.503 \\
\hline 3/31/08 4:00 & 124.497 & 121.875 & & 99.705 & & 95.215 & 110.147 & & & 73.565 \\
\hline 3/31/08 4:30 & 124.464 & 121.859 & & 99.662 & & 95.182 & 110.149 & & & 73.629 \\
\hline 3/31/08 5:00 & 124.438 & 121.868 & & 99.636 & & 95.167 & 110.145 & & 79.78 & 73.677 \\
\hline 3/31/08 5:30 & 124.424 & 121.857 & & 99.627 & & 95.153 & 110.147 & & & 73.716 \\
\hline 3/31/08 6:00 & 124.419 & 123.555 & & 99.631 & & 95.163 & 110.14 & & & 73.743 \\
\hline 3/31/08 6:30 & 124.421 & 123.95 & & 99.641 & & 95.153 & 110.142 & & 79.748 & 73.768 \\
\hline 3/31/08 7:00 & 126.014 & 124.12 & & 100.415 & & 95.639 & 110.622 & & & 73.801 \\
\hline 3/31/08 7:30 & 126.435 & 124.188 & & 100.639 & & 96.028 & 110.979 & & & 73.987 \\
\hline 3/31/08 8:00 & 126.624 & 122.526 & & 100.739 & & 96.197 & 111.127 & & 79.795 & 74.115 \\
\hline 3/31/08 8:30 & 126.709 & 122.208 & & 100.795 & & 96.268 & 111.219 & & & 74.208 \\
\hline 3/31/08 9:00 & 125.067 & 122.088 & & 100.042 & & 95.785 & 110.716 & & & 74.229 \\
\hline 3/31/08 9:30 & 124.743 & 122.029 & & 99.884 & & 95.486 & 110.451 & & 79.787 & 74.128 \\
\hline $3 / 31 / 0810: 00$ & 124.618 & 122.004 & & 99.801 & & 95.372 & 110.339 & & & 74.062 \\
\hline $3 / 31 / 08$ 10:30 & 124.554 & 121.997 & & 99.775 & & 95.314 & 110.275 & & & 74.02 \\
\hline $3 / 31 / 0811: 00$ & 124.518 & 121.986 & & 99.758 & & 95.292 & 110.237 & & 79.763 & 73.991 \\
\hline 3/31/08 11:30 & 124.495 & 121.988 & & 99.758 & & 95.292 & 110.211 & & & 73.973 \\
\hline $3 / 31 / 08$ 12:00 & 124.485 & 121.981 & & 99.758 & & 95.281 & 110.196 & & & 73.96 \\
\hline 3/31/08 12:30 & 124.471 & 121.981 & & 99.762 & & 95.281 & 110.185 & & 79.768 & 73.952 \\
\hline 3/31/08 13:00 & 124.464 & 121.986 & & 99.753 & & 95.281 & 110.177 & & & 73.942 \\
\hline 3/31/08 13:30 & 124.459 & 121.975 & & 99.756 & & 95.283 & 110.172 & & & 73.822 \\
\hline 3/31/08 14:00 & 124.452 & 121.984 & & 99.756 & & 95.285 & 110.166 & & 79.746 & 73.572 \\
\hline $3 / 31 / 08$ 14:30 & 124.452 & 122.002 & & 99.75 & & 95.276 & 110.166 & & & 73.373 \\
\hline $3 / 31 / 08$ 15:00 & 124.445 & 122.013 & & 99.77 & & 95.283 & 110.16 & & & 73.323 \\
\hline $3 / 31 / 08$ 15:30 & 124.443 & 122.013 & & 99.781 & & 95.299 & 110.157 & & 79.741 & 73.33 \\
\hline $3 / 31 / 08$ 16:00 & 124.455 & 122.031 & & 99.787 & & 95.311 & 110.153 & & & 73.338 \\
\hline $3 / 31 / 0816: 30$ & 124.464 & 122.052 & & 99.796 & & 95.316 & 110.155 & & & 73.342 \\
\hline 3/31/08 17:00 & 124.478 & 122.072 & & 99.822 & & 95.328 & 110.151 & & 79.743 & 73.346 \\
\hline 3/31/08 17:30 & 124.499 & 122.086 & & 99.844 & & 95.354 & 110.147 & & & 73.365 \\
\hline $3 / 31 / 08$ 18:00 & 124.523 & 122.111 & & 99.86 & & 95.375 & 110.143 & & & 73.383 \\
\hline $3 / 31 / 08$ 18:30 & 124.535 & 122.12 & & 99.879 & & 95.387 & 110.145 & & 79.758 & 73.398 \\
\hline $3 / 31 / 08$ 19:00 & 124.559 & 122.154 & & 99.893 & & 95.413 & 110.143 & & & 73.412 \\
\hline $3 / 31 / 0819: 30$ & 124.57 & 122.174 & & 99.913 & & 95.422 & 110.142 & & & 73.427 \\
\hline
\end{tabular}


TABLE S1.1 (Cont.)

\begin{tabular}{|c|c|c|c|c|c|c|c|c|c|c|}
\hline \multirow[b]{2}{*}{ Date and Time } & \multicolumn{10}{|c|}{ Depth from Top of Casing (ft) } \\
\hline & MW1D & MW2D & MW3D & MW4D & MW7 & MW9 & Oentrich & MW14D & MW15D & MW16D \\
\hline 3/31/08 20:00 & 124.601 & 122.188 & & 99.953 & & 95.455 & 110.138 & & 79.795 & 73.443 \\
\hline 3/31/08 20:30 & 124.625 & 122.215 & & 99.963 & & 95.479 & 110.142 & & & 73.474 \\
\hline 3/31/08 21:00 & 124.639 & 122.229 & & 99.996 & & 95.493 & 110.142 & & & 73.487 \\
\hline 3/31/08 21:30 & 124.665 & 122.253 & & 99.999 & & 95.521 & 110.16 & & 79.805 & 73.507 \\
\hline 3/31/08 22:00 & 124.679 & 122.263 & & 100.032 & & 95.535 & 110.177 & & & 73.52 \\
\hline 3/31/08 22:30 & 124.705 & 122.285 & & 100.039 & & 95.561 & 110.183 & & & 73.545 \\
\hline 3/31/08 23:00 & 124.714 & 122.317 & & 100.051 & & 95.566 & 110.19 & & 79.831 & 73.559 \\
\hline 3/31/08 23:30 & 124.738 & 122.321 & & 100.086 & & 95.596 & 110.2 & & & 73.567 \\
\hline 4/1/08 0:00 & 124.761 & 122.342 & & 100.101 & & 95.618 & 110.207 & & & 73.588 \\
\hline 4/1/08 0:30 & 124.766 & 122.337 & & 100.12 & & 95.622 & 110.209 & & 79.861 & 73.609 \\
\hline 4/1/08 1:00 & 124.792 & 122.346 & & 100.125 & & 95.65 & 110.213 & & & 73.621 \\
\hline 4/1/08 1:30 & 124.788 & 122.349 & & 100.122 & & 95.646 & 110.215 & & & 73.634 \\
\hline 4/1/08 2:00 & 124.797 & 122.351 & & 100.127 & & 95.655 & 110.217 & & 79.873 & 73.629 \\
\hline 4/1/08 2:30 & 124.797 & 122.362 & & 100.137 & & 95.655 & 110.215 & & & 73.638 \\
\hline 4/1/08 3:00 & 124.8 & 122.38 & & 100.144 & & 95.658 & 110.215 & & & 73.644 \\
\hline 4/1/08 3:30 & 124.814 & 122.401 & & 100.158 & & 95.672 & 110.215 & & 79.888 & 73.65 \\
\hline 4/1/08 4:00 & 124.832 & 122.417 & & 100.177 & & 95.688 & 110.213 & & & 73.663 \\
\hline 4/1/08 4:30 & 124.849 & 122.424 & & 100.196 & & 95.705 & 110.215 & & & 73.681 \\
\hline 4/1/08 5:00 & 124.866 & 122.442 & & 100.203 & & 95.724 & 110.218 & & 79.919 & 73.696 \\
\hline 4/1/08 5:30 & 124.87 & 122.458 & & 100.222 & & 95.731 & 110.222 & & & 73.704 \\
\hline 4/1/08 6:00 & 124.889 & 124.26 & & 100.234 & & 95.747 & 110.224 & & & 73.718 \\
\hline 4/1/08 6:30 & 124.906 & 124.598 & & 100.303 & & 95.764 & 110.228 & & 79.946 & 73.731 \\
\hline 4/1/08 7:00 & 126.735 & 124.755 & & 101.031 & & 96.294 & 110.757 & & & 73.784 \\
\hline 4/1/08 7:30 & 127.097 & 124.861 & & 101.233 & & 96.633 & 111.052 & & & 73.987 \\
\hline 4/1/08 8:00 & 127.263 & 124.927 & & 101.357 & & 96.796 & 111.187 & & 80.042 & 74.132 \\
\hline 4/1/08 8:30 & 127.371 & 124.975 & & 101.442 & & 96.902 & 111.272 & & & 74.233 \\
\hline 4/1/08 9:00 & 127.438 & 124.999 & & 101.495 & & 96.966 & 111.328 & & & 74.308 \\
\hline 4/1/08 9:30 & 127.49 & 125.031 & & 101.528 & & 97.013 & 111.362 & & 80.135 & 74.359 \\
\hline 4/1/08 10:00 & 127.513 & 125.054 & & 101.562 & & 97.044 & 111.386 & & & 74.392 \\
\hline 4/1/08 10:30 & 127.546 & 123.494 & & 101.588 & & 97.074 & 111.407 & & & 74.423 \\
\hline 4/1/08 11:00 & 127.572 & 123.086 & & 101.607 & & 97.098 & 111.422 & & 80.213 & 74.45 \\
\hline 4/1/08 11:30 & 126.005 & 122.934 & & 100.938 & & 96.751 & 111.045 & & & 74.458 \\
\hline 4/1/08 12:00 & 125.546 & 122.845 & & 100.739 & & 96.365 & 110.673 & & & 74.32 \\
\hline 4/1/08 12:30 & 125.39 & 122.786 & & 100.639 & & 96.216 & 110.534 & & 80.223 & 74.208 \\
\hline 4/1/08 13:00 & 125.298 & 122.745 & & 100.57 & & 96.134 & 110.457 & & & 74.134 \\
\hline 4/1/08 13:30 & 125.239 & 122.709 & & 100.525 & & 96.075 & 110.412 & & & 74.076 \\
\hline 4/1/08 14:00 & 125.197 & 122.689 & & 100.492 & & 96.039 & 110.378 & & 80.218 & 74.035 \\
\hline 4/1/08 14:30 & 125.161 & 122.673 & & 100.468 & & 96.004 & 110.357 & & & 74.006 \\
\hline 4/1/08 15:00 & 125.14 & 122.659 & & 100.453 & & 95.983 & 110.342 & & & 73.983 \\
\hline 4/1/08 15:30 & 125.123 & 122.646 & & 100.44 & & 95.969 & 110.329 & & 80.21 & 73.968 \\
\hline 4/1/08 16:00 & 125.112 & 122.637 & & 100.425 & & 95.959 & 110.32 & & & 73.956 \\
\hline 4/1/08 16:30 & 125.097 & 122.625 & & 100.415 & & 95.943 & 110.31 & & & 73.946 \\
\hline 4/1/08 17:00 & 125.086 & 122.614 & & 100.408 & & 95.933 & 110.305 & & 80.208 & 73.935 \\
\hline 4/1/08 17:30 & 125.076 & 122.603 & & 100.396 & & 95.922 & 110.299 & & & 73.927 \\
\hline 4/1/08 18:00 & 125.064 & 122.607 & & 100.382 & & 95.91 & 110.292 & & & 73.917 \\
\hline $4 / 1 / 08$ 18:30 & 125.055 & 122.6 & & 100.387 & & 95.9 & 110.288 & & 80.22 & 73.909 \\
\hline 4/1/08 19:00 & 125.057 & 122.596 & & 100.38 & & 95.903 & 110.28 & & & 73.909 \\
\hline 4/1/08 19:30 & 125.048 & 122.589 & & 100.368 & & 95.898 & 110.277 & & & 73.906 \\
\hline 4/1/08 20:00 & 125.043 & 122.591 & & 100.366 & & 95.896 & 110.273 & & 80.21 & 73.896 \\
\hline 4/1/08 20:30 & 125.038 & 122.596 & & 100.365 & & 95.884 & 110.269 & & & 73.894 \\
\hline 4/1/08 21:00 & 125.043 & 122.596 & & 100.372 & & 95.891 & 110.262 & & & 73.892 \\
\hline 4/1/08 21:30 & 125.045 & 122.6 & & 100.373 & & 95.893 & 110.258 & & 80.218 & 73.898 \\
\hline 4/1/08 22:00 & 125.045 & 122.603 & & 100.38 & & 95.891 & 110.254 & & & 73.9 \\
\hline 4/1/08 22:30 & 125.05 & 122.6 & & 100.38 & & 95.898 & 110.249 & & & 73.904 \\
\hline 4/1/08 23:00 & 125.05 & 122.596 & & 100.384 & & 95.903 & 110.245 & & 80.22 & 73.909 \\
\hline 4/1/08 23:30 & 125.05 & 122.596 & & 100.38 & & 95.903 & 110.241 & & & 73.909 \\
\hline 4/2/08 0:00 & 125.047 & 122.598 & & 100.375 & & 95.896 & 110.239 & & & 73.911 \\
\hline 4/2/08 0:30 & 125.048 & 122.591 & & 100.368 & & 95.898 & 110.234 & & 80.223 & 73.906 \\
\hline 4/2/08 1:00 & 125.048 & 122.58 & & 100.373 & & 95.9 & 110.23 & & & 73.904 \\
\hline 4/2/08 1:30 & 125.041 & 122.566 & & 100.361 & & 95.893 & 110.226 & & & 73.904 \\
\hline 4/2/08 2:00 & 125.029 & 122.55 & & 100.354 & & 95.877 & 110.224 & & 80.218 & 73.898 \\
\hline 4/2/08 2:30 & 125.015 & 122.535 & & 100.342 & & 95.865 & 110.222 & & & 73.89 \\
\hline 4/2/08 3:00 & 125 & 122.526 & & 100.322 & & 95.853 & 110.22 & & & 73.882 \\
\hline 4/2/08 3:30 & 124.986 & 122.526 & & 100.31 & & 95.837 & 110.217 & & 80.203 & 73.865 \\
\hline 4/2/08 4:00 & 124.974 & 122.519 & & 100.299 & & 95.825 & 110.215 & & & 73.853 \\
\hline 4/2/08 4:30 & 124.974 & 122.51 & & 100.299 & & 95.825 & 110.209 & & & 73.842 \\
\hline
\end{tabular}


TABLE S1.1 (Cont.)

\begin{tabular}{|c|c|c|c|c|c|c|c|c|c|c|}
\hline \multirow[b]{2}{*}{ Date and Time } & \multicolumn{10}{|c|}{ Depth from Top of Casing (ft) } \\
\hline & MW1D & MW2D & MW3D & MW4D & MW7 & MW9 & Oentrich & MW14D & MW15D & MW16D \\
\hline 4/2/08 5:00 & 124.97 & 122.498 & & 100.294 & & 95.818 & 110.205 & & 80.191 & 73.842 \\
\hline 4/2/08 5:30 & 124.96 & 122.498 & & 100.284 & & 95.809 & 110.205 & & & 73.838 \\
\hline 4/2/08 6:00 & 124.948 & 124.292 & & 100.28 & & 95.799 & 110.202 & & & 73.83 \\
\hline 4/2/08 6:30 & 124.944 & 124.612 & & 100.403 & & 95.794 & 110.198 & & 80.183 & 73.824 \\
\hline 4/2/08 7:00 & 126.797 & 124.755 & & 101.093 & & 96.365 & 110.778 & & & 73.861 \\
\hline 4/2/08 7:30 & 127.137 & 124.825 & & 101.288 & & 96.69 & 111.075 & & & 74.053 \\
\hline 4/2/08 8:00 & 127.286 & 123.38 & & 101.383 & & 96.834 & 111.21 & & 80.245 & 74.188 \\
\hline 4/2/08 8:30 & 127.362 & 122.9 & & 101.443 & & 96.907 & 111.292 & & & 74.264 \\
\hline 4/2/08 9:00 & 125.918 & 122.743 & & 100.764 & & 96.631 & 110.984 & & & 74.31 \\
\hline 4/2/08 9:30 & 125.36 & 122.655 & & 100.547 & & 96.174 & 110.562 & & 80.264 & 74.179 \\
\hline 4/2/08 10:00 & 125.194 & 122.598 & & 100.449 & & 96.021 & 110.423 & & & 74.064 \\
\hline 4/2/08 10:30 & 125.104 & 122.555 & & 100.385 & & 95.933 & 110.35 & & & 73.987 \\
\hline 4/2/08 11:00 & 125.048 & 122.514 & & 100.339 & & 95.886 & 110.303 & & 80.24 & 73.933 \\
\hline 4/2/08 11:30 & 125.003 & 122.478 & & 100.297 & & 95.844 & 110.275 & & & 73.894 \\
\hline 4/2/08 12:00 & 124.965 & 122.448 & & 100.266 & & 95.806 & 110.256 & & & 73.861 \\
\hline 4/2/08 12:30 & 124.932 & 122.414 & & 100.235 & & 95.775 & 110.245 & & 80.21 & 73.832 \\
\hline 4/2/08 13:00 & 124.899 & 122.39 & & 100.201 & & 95.742 & 110.232 & & & 73.803 \\
\hline 4/2/08 13:30 & 124.866 & 122.362 & & 100.173 & & 95.712 & 110.226 & & & 73.774 \\
\hline 4/2/08 14:00 & 124.835 & 122.335 & & 100.144 & & 95.684 & 110.22 & & 80.171 & 73.747 \\
\hline 4/2/08 14:30 & 124.811 & 122.315 & & 100.122 & & 95.658 & 110.215 & & & 73.72 \\
\hline 4/2/08 15:00 & 124.788 & 122.285 & & 100.096 & & 95.632 & 110.211 & & & 73.7 \\
\hline 4/2/08 15:30 & 124.764 & 122.251 & & 100.073 & & 95.603 & 110.209 & & 80.127 & 73.677 \\
\hline 4/2/08 16:00 & 124.736 & 122.226 & & 100.037 & & 95.58 & 110.209 & & & 73.654 \\
\hline 4/2/08 16:30 & 124.703 & 122.215 & & 100.01 & & 95.547 & 110.203 & & & 73.623 \\
\hline 4/2/08 17:00 & 124.677 & 122.204 & & 99.996 & & 95.526 & 110.196 & & 80.083 & 73.596 \\
\hline 4/2/08 17:30 & 124.665 & 122.197 & & 99.98 & & 95.514 & 110.188 & & & 73.578 \\
\hline 4/2/08 18:00 & 124.651 & 122.192 & & 99.974 & & 95.497 & 110.185 & & & 73.565 \\
\hline 4/2/08 18:30 & 124.646 & 122.19 & & 99.972 & & 95.495 & 110.181 & & 80.051 & 73.559 \\
\hline 4/2/08 19:00 & 124.641 & 122.188 & & 99.967 & & 95.49 & 110.179 & & & 73.553 \\
\hline 4/2/08 19:30 & 124.641 & 122.185 & & 99.963 & & 95.488 & 110.173 & & & 73.547 \\
\hline 4/2/08 20:00 & 124.636 & 122.188 & & 99.961 & & 95.486 & 110.172 & & 80.032 & 73.543 \\
\hline 4/2/08 20:30 & 124.634 & 122.195 & & 99.963 & & 95.481 & 110.17 & & & 73.541 \\
\hline 4/2/08 21:00 & 124.636 & 122.192 & & 99.967 & & 95.486 & 110.168 & & & 73.541 \\
\hline 4/2/08 21:30 & 124.639 & 122.192 & & 99.968 & & 95.49 & 110.166 & & 80.02 & 73.541 \\
\hline 4/2/08 22:00 & 124.641 & 122.188 & & 99.967 & & 95.488 & 110.164 & & & 73.543 \\
\hline 4/2/08 22:30 & 124.641 & 122.176 & & 99.967 & & 95.493 & 110.164 & & & 73.543 \\
\hline 4/2/08 23:00 & 124.634 & 122.165 & & 99.958 & & 95.485 & 110.164 & & 80 & 73.543 \\
\hline 4/2/08 23:30 & 124.624 & 122.156 & & 99.949 & & 95.476 & 110.164 & & & 73.534 \\
\hline 4/3/08 0:00 & 124.618 & 122.149 & & 99.936 & & 95.469 & 110.16 & & & 73.524 \\
\hline 4/3/08 0:30 & 124.603 & 122.14 & & 99.927 & & 95.455 & 110.157 & & 79.976 & 73.514 \\
\hline 4/3/08 1:00 & 124.596 & 122.124 & & 99.924 & & 95.448 & 110.153 & & & 73.503 \\
\hline 4/3/08 1:30 & 124.589 & 122.108 & & 99.91 & & 95.441 & 110.149 & & & 73.499 \\
\hline 4/3/08 2:00 & 124.573 & 122.095 & & 99.889 & & 95.427 & 110.142 & & 79.946 & 73.489 \\
\hline 4/3/08 2:30 & 124.556 & 122.072 & & 99.88 & & 95.41 & 110.136 & & & 73.472 \\
\hline 4/3/08 3:00 & 124.542 & 122.049 & & 99.863 & & 95.394 & 110.126 & & & 73.458 \\
\hline 4/3/08 3:30 & 124.525 & 122.038 & & 99.832 & & 95.377 & 110.119 & & 79.907 & 73.443 \\
\hline 4/3/08 4:00 & 124.499 & 122.034 & & 99.817 & & 95.351 & 110.102 & & & 73.419 \\
\hline 4/3/08 4:30 & 124.488 & 122.034 & & 99.817 & & 95.337 & 110.093 & & & 73.4 \\
\hline 4/3/08 5:00 & 124.483 & 122.009 & & 99.815 & & 95.337 & 110.089 & & 79.878 & 73.396 \\
\hline 4/3/08 5:30 & 124.483 & 121.995 & & 99.803 & & 95.335 & 110.087 & & & 73.394 \\
\hline 4/3/08 6:00 & 124.457 & 123.947 & & 99.748 & & 95.309 & 110.087 & & & 73.383 \\
\hline 4/3/08 6:30 & 124.438 & 124.16 & & 100.23 & & 95.288 & 110.072 & & 79.844 & 73.348 \\
\hline 4/3/08 7:00 & 126.43 & 124.26 & & 100.611 & & 95.966 & 110.714 & & & 73.441 \\
\hline 4/3/08 7:30 & 126.672 & 124.351 & & 100.749 & & 96.183 & 110.943 & & & 73.636 \\
\hline 4/3/08 8:00 & 126.778 & 124.383 & & 100.83 & & 96.282 & 111.058 & & 79.878 & 73.733 \\
\hline 4/3/08 8:30 & 126.832 & 124.401 & & 100.878 & & 96.367 & 111.12 & & & 73.784 \\
\hline 4/3/08 9:00 & 126.896 & 124.426 & & 100.932 & & 96.414 & 111.167 & & & 73.824 \\
\hline 4/3/08 9:30 & 126.941 & 122.857 & & 100.942 & & 96.426 & 111.2 & & 79.905 & 73.869 \\
\hline 4/3/08 10:00 & 126.977 & 122.464 & & 101.066 & & 96.459 & 111.219 & & & 73.906 \\
\hline 4/3/08 10:30 & 125.502 & 122.315 & & 100.919 & & 96.117 & 110.825 & & & 73.921 \\
\hline $4 / 3 / 0811: 00$ & 125.06 & 122.215 & & 100.751 & & 95.74 & 110.466 & & 79.932 & 73.838 \\
\hline 4/3/08 11:30 & 124.903 & 122.163 & & 100.647 & & 95.596 & 110.329 & & & 73.807 \\
\hline 4/3/08 12:00 & 124.795 & 122.095 & & 100.551 & & 95.5 & 110.256 & & & 73.811 \\
\hline 4/3/08 12:30 & 124.743 & 122.097 & & 100.447 & & 95.457 & 110.209 & & 80.017 & 73.836 \\
\hline 4/3/08 13:00 & 124.603 & 122.083 & & 100.358 & & 95.384 & 110.185 & & & 73.865 \\
\hline 4/3/08 13:30 & 124.58 & 122.065 & & 100.27 & & 95.389 & 110.157 & & & 73.882 \\
\hline
\end{tabular}


TABLE S1.1 (Cont.)

\begin{tabular}{|c|c|c|c|c|c|c|c|c|c|c|}
\hline \multirow[b]{2}{*}{ Date and Time } & \multicolumn{10}{|c|}{ Depth from Top of Casing (ft) } \\
\hline & MW1D & MW2D & MW3D & MW4D & MW7 & MW9 & Oentrich & MW14D & MW15D & MW16D \\
\hline 4/3/08 14:00 & 124.566 & 122.047 & & 100.163 & & 95.38 & 110.142 & & 79.983 & 73.9 \\
\hline 4/3/08 14:30 & 124.556 & 122.034 & & 100.023 & & 95.365 & 110.132 & & & 73.919 \\
\hline 4/3/08 15:00 & 124.549 & 122.031 & & 99.906 & & 95.346 & 110.126 & & & 73.937 \\
\hline $4 / 3 / 08$ 15:30 & 124.542 & 122.031 & & 99.808 & & 95.335 & 110.119 & & 79.88 & 73.954 \\
\hline 4/3/08 16:00 & 124.535 & 122.027 & & 99.808 & & 95.33 & 110.113 & & & 73.966 \\
\hline $4 / 3 / 08 \quad 16: 30$ & 124.525 & 122.036 & & 99.805 & & 95.335 & 110.11 & & & 73.973 \\
\hline $4 / 3 / 08$ 17:00 & 124.523 & 122.056 & & 99.808 & & 95.325 & 110.106 & & 79.8 & 73.983 \\
\hline 4/3/08 17:30 & 124.516 & 122.079 & & 99.827 & & 95.337 & 110.1 & & & 73.991 \\
\hline 4/3/08 18:00 & 124.511 & 122.09 & & 99.849 & & 95.358 & 110.095 & & & 73.993 \\
\hline $4 / 3 / 0818: 30$ & 124.507 & 122.111 & & 99.863 & & 95.377 & 110.093 & & 79.787 & 73.989 \\
\hline $4 / 3 / 08$ 19:00 & 124.504 & 122.122 & & 99.884 & & 95.396 & 110.095 & & & 73.989 \\
\hline 4/3/08 19:30 & 124.525 & 122.131 & & 99.896 & & 95.415 & 110.096 & & & 73.989 \\
\hline $4 / 3 / 0820: 00$ & 124.544 & 122.138 & & 99.906 & & 95.424 & 110.098 & & 79.778 & 73.993 \\
\hline $4 / 3 / 08$ 20:30 & 124.58 & 122.149 & & 99.915 & & 95.434 & 110.1 & & & 73.995 \\
\hline 4/3/08 21:00 & 124.589 & 122.161 & & 99.925 & & 95.443 & 110.1 & & & 73.997 \\
\hline $4 / 3 / 0821: 30$ & 124.599 & 122.161 & & 99.937 & & 95.45 & 110.1 & & 79.787 & 74.002 \\
\hline $4 / 3 / 0822: 00$ & 124.611 & 122.154 & & 99.941 & & 95.464 & 110.106 & & & 74.008 \\
\hline $4 / 3 / 0822: 30$ & 124.61 & 122.151 & & 99.944 & & 95.464 & 110.108 & & & 74.014 \\
\hline $4 / 3 / 0823: 00$ & 124.603 & 122.156 & & 99.936 & & 95.457 & 110.108 & & 79.792 & 73.985 \\
\hline 4/3/08 23:30 & 124.603 & 122.161 & & 99.934 & & 95.455 & 110.106 & & & 73.942 \\
\hline 4/4/08 0:00 & 124.603 & 122.151 & & 99.941 & & 95.457 & 110.104 & & & 73.89 \\
\hline 4/4/08 0:30 & 124.611 & 122.154 & & 99.937 & & 95.467 & 110.106 & & 79.792 & 73.842 \\
\hline 4/4/08 1:00 & 124.601 & 122.149 & & 99.937 & & 95.452 & 110.104 & & & 73.805 \\
\hline 4/4/08 1:30 & 124.601 & 122.149 & & 99.936 & & 95.457 & 110.102 & & & 73.753 \\
\hline $4 / 4 / 082: 00$ & 124.599 & 122.145 & & 99.932 & & 95.453 & 110.102 & & 79.78 & 73.714 \\
\hline 4/4/08 2:30 & 124.599 & 122.145 & & 99.925 & & 95.453 & 110.098 & & & 73.675 \\
\hline 4/4/08 3:00 & 124.596 & 122.14 & & 99.929 & & 95.45 & 110.096 & & & 73.646 \\
\hline 4/4/08 3:30 & 124.594 & 122.145 & & 99.925 & & 95.448 & 110.095 & & 79.773 & 73.625 \\
\hline 4/4/08 4:00 & 124.589 & 122.151 & & 99.93 & & 95.443 & 110.095 & & & 73.615 \\
\hline 4/4/08 4:30 & 124.594 & 122.163 & & 99.932 & & 95.45 & 110.093 & & & 73.609 \\
\hline 4/4/08 5:00 & 124.601 & 122.172 & & 99.948 & & 95.457 & 110.093 & & 79.756 & 73.603 \\
\hline 4/4/08 5:30 & 124.613 & 123.126 & & 99.958 & & 95.471 & 110.096 & & & 73.594 \\
\hline $4 / 4 / 086: 00$ & 124.62 & 124.19 & & 99.967 & & 95.476 & 110.098 & & & 73.592 \\
\hline 4/4/08 6:30 & 124.627 & 124.401 & & 100.616 & & 95.486 & 110.1 & & 79.756 & 73.594 \\
\hline 4/4/08 7:00 & 126.712 & 124.514 & & 100.918 & & 96.266 & 110.872 & & & 73.729 \\
\hline 4/4/08 7:30 & 126.932 & 124.589 & & 101.057 & & 96.48 & 111.063 & & & 73.869 \\
\hline 4/4/08 8:00 & 127.047 & 124.637 & & 101.142 & & 96.593 & 111.168 & & 79.824 & 73.971 \\
\hline 4/4/08 8:30 & 127.121 & 124.675 & & 101.199 & & 96.666 & 111.234 & & & 74.047 \\
\hline 4/4/08 9:00 & 127.17 & 124.696 & & 101.245 & & 96.714 & 111.279 & & & 74.107 \\
\hline 4/4/08 9:30 & 127.211 & 123.056 & & 101.267 & & 96.754 & 111.311 & & 79.895 & 74.152 \\
\hline 4/4/08 10:00 & 127.232 & 122.68 & & 101.288 & & 96.773 & 111.336 & & & 74.188 \\
\hline $4 / 4 / 08 \quad 10: 30$ & 125.549 & 122.519 & & 100.528 & & 96.315 & 110.862 & & & 74.175 \\
\hline $4 / 4 / 0811: 00$ & 125.138 & 122.43 & & 100.325 & & 95.952 & 110.525 & & 79.924 & 73.979 \\
\hline 4/4/08 11:30 & 124.972 & 122.365 & & 100.22 & & 95.799 & 110.389 & & & 73.84 \\
\hline $4 / 4 / 08 \quad 12: 00$ & 124.88 & 122.315 & & 100.156 & & 95.714 & 110.312 & & & 73.749 \\
\hline $4 / 4 / 08$ 12:30 & 124.814 & 122.276 & & 100.101 & & 95.653 & 110.267 & & 79.895 & 73.691 \\
\hline 4/4/08 13:00 & 124.766 & 122.242 & & 100.061 & & 95.606 & 110.239 & & & 73.644 \\
\hline 4/4/08 13:30 & 124.724 & 122.222 & & 100.027 & & 95.57 & 110.217 & & & 73.605 \\
\hline $4 / 4 / 0814: 00$ & 124.693 & 122.195 & & 100.001 & & 95.537 & 110.202 & & 79.861 & 73.574 \\
\hline 4/4/08 14:30 & 124.67 & 122.174 & & 99.982 & & 95.521 & 110.188 & & & 73.549 \\
\hline $4 / 4 / 08 \quad 15: 00$ & 124.643 & 122.161 & & 99.961 & & 95.495 & 110.179 & & & 73.53 \\
\hline 4/4/08 15:30 & 124.627 & 122.142 & & 99.944 & & 95.476 & 110.17 & & 79.846 & 73.512 \\
\hline $4 / 4 / 08$ 16:00 & 124.608 & 122.127 & & 99.924 & & 95.457 & 110.164 & & & 73.495 \\
\hline $4 / 4 / 0816: 30$ & 124.587 & 122.115 & & 99.91 & & 95.438 & 110.158 & & & 73.476 \\
\hline 4/4/08 17:00 & 124.575 & 122.108 & & 99.898 & & 95.424 & 110.155 & & 79.817 & 73.462 \\
\hline 4/4/08 17:30 & 124.565 & 122.104 & & 99.889 & & 95.413 & 110.149 & & & 73.45 \\
\hline $4 / 4 / 08 \quad 18: 00$ & 124.554 & 122.099 & & 99.88 & & 95.41 & 110.145 & & & 73.441 \\
\hline 4/4/08 18:30 & 124.551 & 122.095 & & 99.88 & & 95.405 & 110.142 & & 79.775 & 73.431 \\
\hline $4 / 4 / 08$ 19:00 & 124.547 & 122.086 & & 99.877 & & 95.398 & 110.138 & & & 73.429 \\
\hline $4 / 4 / 08 \quad 19: 30$ & 124.542 & 122.081 & & 99.868 & & 95.396 & 110.138 & & & 73.425 \\
\hline 4/4/08 20:00 & 124.535 & 122.086 & & 99.863 & & 95.387 & 110.136 & & 79.758 & 73.417 \\
\hline $4 / 4 / 0820: 30$ & 124.533 & 122.088 & & 99.863 & & 95.387 & 110.132 & & & 73.41 \\
\hline $4 / 4 / 0821: 00$ & 124.533 & 122.09 & & 99.867 & & 95.387 & 110.13 & & & 73.41 \\
\hline $4 / 4 / 0821: 30$ & 124.535 & 122.088 & & 99.87 & & 95.387 & 110.126 & & 79.746 & 73.412 \\
\hline 4/4/08 22:00 & 124.535 & 122.083 & & 99.868 & & 95.391 & 110.125 & & & 73.414 \\
\hline $4 / 4 / 0822: 30$ & 124.535 & 122.081 & & 99.867 & & 95.391 & 110.123 & & & 73.414 \\
\hline
\end{tabular}


TABLE S1.1 (Cont.)

\begin{tabular}{|c|c|c|c|c|c|c|c|c|c|c|}
\hline \multirow[b]{2}{*}{ Date and Time } & \multicolumn{10}{|c|}{ Depth from Top of Casing (ft) } \\
\hline & MW1D & MW2D & MW3D & MW4D & MW7 & MW9 & Oentrich & MW14D & MW15D & MW16D \\
\hline 4/4/08 23:00 & 124.53 & 122.074 & & 99.865 & & 95.387 & 110.123 & & 79.734 & 73.412 \\
\hline 4/4/08 23:30 & 124.53 & 122.074 & & 99.858 & & 95.384 & 110.121 & & & 73.408 \\
\hline 4/5/08 0:00 & 124.523 & 122.063 & & 99.855 & & 95.377 & 110.121 & & & 73.404 \\
\hline 4/5/08 0:30 & 124.521 & 122.052 & & 99.849 & & 95.377 & 110.117 & & 79.724 & 73.4 \\
\hline 4/5/08 1:00 & 124.511 & 122.049 & & 99.837 & & 95.365 & 110.108 & & & 73.396 \\
\hline 4/5/08 1:30 & 124.502 & 122.045 & & 99.832 & & 95.356 & 110.1 & & & 73.386 \\
\hline 4/5/08 2:00 & 124.497 & 122.031 & & 99.831 & & 95.351 & 110.095 & & 79.699 & 73.379 \\
\hline 4/5/08 2:30 & 124.495 & 122.029 & & 99.824 & & 95.349 & 110.093 & & & 73.377 \\
\hline 4/5/08 3:00 & 124.483 & 122.027 & & 99.813 & & 95.339 & 110.091 & & & 73.371 \\
\hline 4/5/08 3:30 & 124.476 & 122.022 & & 99.81 & & 95.335 & 110.085 & & 79.68 & 73.361 \\
\hline 4/5/08 4:00 & 124.473 & 122.022 & & 99.808 & & 95.33 & 110.081 & & & 73.357 \\
\hline 4/5/08 4:30 & 124.471 & 122.015 & & 99.806 & & 95.332 & 110.08 & & & 73.354 \\
\hline 4/5/08 5:00 & 124.469 & 122.018 & & 99.803 & & 95.328 & 110.08 & & 79.663 & 73.354 \\
\hline 4/5/08 5:30 & 124.462 & 122.018 & & 99.8 & & 95.323 & 110.076 & & & 73.346 \\
\hline 4/5/08 6:00 & 124.464 & 122.018 & & 99.803 & & 95.323 & 110.072 & & & 73.344 \\
\hline 4/5/08 6:30 & 124.464 & 122.024 & & 99.8 & & 95.323 & 110.072 & & 79.646 & 73.346 \\
\hline 4/5/08 7:00 & 124.466 & 122.02 & & 99.808 & & 95.323 & 110.07 & & & 73.342 \\
\hline 4/5/08 7:30 & 124.471 & 123.832 & & 99.803 & & 95.328 & 110.068 & & & 73.346 \\
\hline 4/5/08 8:00 & 124.466 & 124.135 & & 100.003 & & 95.323 & 110.07 & & 79.633 & 73.346 \\
\hline 4/5/08 8:30 & 126.312 & 124.276 & & 100.575 & & 95.865 & 110.622 & & & 73.39 \\
\hline 4/5/08 9:00 & 126.634 & 124.344 & & 100.757 & & 96.164 & 110.898 & & & 73.574 \\
\hline 4/5/08 9:30 & 126.778 & 124.396 & & 100.852 & & 96.306 & 111.03 & & 79.69 & 73.702 \\
\hline 4/5/08 10:00 & 126.851 & 124.419 & & 100.904 & & 96.379 & 111.105 & & & 73.78 \\
\hline 4/5/08 10:30 & 126.901 & 124.435 & & 100.937 & & 96.426 & 111.152 & & & 73.824 \\
\hline 4/5/08 11:00 & 126.925 & 122.63 & & 100.961 & & 96.452 & 111.184 & & 79.729 & 73.857 \\
\hline 4/5/08 11:30 & 126.941 & 122.346 & & 100.658 & & 96.471 & 111.206 & & & 73.88 \\
\hline 4/5/08 12:00 & 125.1 & 122.204 & & 100.182 & & 95.896 & 110.648 & & & 73.822 \\
\hline $4 / 5 / 08 \quad 12: 30$ & 124.8 & 122.115 & & 100.008 & & 95.625 & 110.406 & & 79.717 & 73.663 \\
\hline 4/5/08 13:00 & 124.651 & 122.054 & & 99.906 & & 95.486 & 110.294 & & & 73.543 \\
\hline $4 / 5 / 0813: 30$ & 124.563 & 121.993 & & 99.841 & & 95.401 & 110.232 & & & 73.458 \\
\hline 4/5/08 14:00 & 124.502 & 121.954 & & 99.786 & & 95.342 & 110.19 & & 79.665 & 73.396 \\
\hline 4/5/08 14:30 & 124.44 & 121.92 & & 99.739 & & 95.285 & 110.162 & & & 73.346 \\
\hline $4 / 5 / 08 \quad 15: 00$ & 124.4 & 121.884 & & 99.7 & & 95.243 & 110.145 & & & 73.305 \\
\hline $4 / 5 / 08$ 15:30 & 124.365 & 121.866 & & 99.669 & & 95.215 & 110.128 & & 79.624 & 73.268 \\
\hline 4/5/08 16:00 & 124.332 & 121.852 & & 99.646 & & 95.184 & 110.115 & & & 73.239 \\
\hline 4/5/08 16:30 & 124.313 & 121.839 & & 99.631 & & 95.165 & 110.104 & & & 73.216 \\
\hline 4/5/08 17:00 & 124.301 & 121.839 & & 99.622 & & 95.151 & 110.096 & & 79.57 & 73.197 \\
\hline 4/5/08 17:30 & 124.289 & 121.834 & & 99.615 & & 95.141 & 110.089 & & & 73.187 \\
\hline $4 / 5 / 08 \quad 18: 00$ & 124.284 & 121.829 & & 99.613 & & 95.139 & 110.083 & & & 73.177 \\
\hline $4 / 5 / 08$ 18:30 & 124.282 & 121.827 & & 99.612 & & 95.134 & 110.08 & & 79.541 & 73.175 \\
\hline 4/5/08 19:00 & 124.275 & 121.825 & & 99.607 & & 95.13 & 110.076 & & & 73.171 \\
\hline $4 / 5 / 08 \quad 19: 30$ & 124.275 & 121.829 & & 99.605 & & 95.132 & 110.074 & & & 73.164 \\
\hline $4 / 5 / 0820: 00$ & 124.273 & 121.832 & & 99.605 & & 95.13 & 110.07 & & 79.531 & 73.162 \\
\hline 4/5/08 20:30 & 124.275 & 121.836 & & 99.61 & & 95.132 & 110.068 & & & 73.16 \\
\hline $4 / 5 / 0821: 00$ & 124.28 & 121.848 & & 99.619 & & 95.137 & 110.066 & & & 73.164 \\
\hline $4 / 5 / 0821: 30$ & 124.282 & 121.845 & & 99.617 & & 95.139 & 110.065 & & 79.519 & 73.168 \\
\hline 4/5/08 22:00 & 124.289 & 121.827 & & 99.638 & & 95.146 & 110.063 & & & 73.171 \\
\hline 4/5/08 22:30 & 124.291 & 121.82 & & 99.625 & & 95.148 & 110.063 & & & 73.181 \\
\hline 4/5/08 23:00 & 124.277 & 121.811 & & 99.61 & & 95.132 & 110.065 & & 79.487 & 73.173 \\
\hline 4/5/08 23:30 & 124.268 & 121.784 & & 99.6 & & 95.13 & 110.065 & & & 73.162 \\
\hline $4 / 6 / 08 \quad 0: 00$ & 124.261 & 121.789 & & 99.579 & & 95.12 & 110.065 & & & 73.15 \\
\hline 4/6/08 0:30 & 124.228 & 121.771 & & 99.569 & & 95.087 & 110.066 & & 79.455 & 73.133 \\
\hline 4/6/08 1:00 & 124.235 & 121.761 & & 99.555 & & 95.092 & 110.063 & & & 73.119 \\
\hline 4/6/08 1:30 & 124.221 & 121.752 & & 99.557 & & 95.078 & 110.061 & & & 73.115 \\
\hline 4/6/08 2:00 & 124.209 & 121.748 & & 99.546 & & 95.068 & 110.059 & & 79.448 & 73.106 \\
\hline 4/6/08 2:30 & 124.204 & 121.743 & & 99.531 & & 95.064 & 110.059 & & & 73.096 \\
\hline 4/6/08 3:00 & 124.197 & 121.734 & & 99.529 & & 95.056 & 110.057 & & & 73.084 \\
\hline 4/6/08 3:30 & 124.192 & 121.734 & & 99.52 & & 95.052 & 110.055 & & 79.406 & 73.08 \\
\hline 4/6/08 4:00 & 124.185 & 121.75 & & 99.519 & & 95.042 & 110.055 & & & 73.071 \\
\hline 4/6/08 4:30 & 124.183 & 121.75 & & 99.538 & & 95.042 & 110.053 & & & 73.069 \\
\hline 4/6/08 5:00 & 124.195 & 121.755 & & 99.532 & & 95.054 & 110.05 & & 79.384 & 73.077 \\
\hline 4/6/08 5:30 & 124.199 & 121.759 & & 99.538 & & 95.057 & 110.048 & & & 73.077 \\
\hline 4/6/08 6:00 & 124.202 & 121.768 & & 99.545 & & 95.061 & 110.046 & & & 73.082 \\
\hline 4/6/08 6:30 & 124.209 & 121.773 & & 99.551 & & 95.068 & 110.046 & & 79.372 & 73.084 \\
\hline 4/6/08 7:00 & 124.216 & 121.782 & & 99.557 & & 95.075 & 110.044 & & & 73.09 \\
\hline 4/6/08 7:30 & 124.221 & 123.408 & & 99.563 & & 95.083 & 110.044 & & & 73.094 \\
\hline
\end{tabular}


TABLE S1.1 (Cont.)

\begin{tabular}{|c|c|c|c|c|c|c|c|c|c|c|}
\hline \multirow[b]{2}{*}{ Date and Time } & \multicolumn{10}{|c|}{ Depth from Top of Casing (ft) } \\
\hline & MW1D & MW2D & MW3D & MW4D & MW7 & MW9 & Oentrich & MW14D & MW15D & MW16D \\
\hline 4/6/08 8:00 & 124.23 & 123.861 & & 99.572 & & 95.09 & 110.044 & & 79.367 & 73.098 \\
\hline 4/6/08 8:30 & 125.854 & 124.036 & & 100.27 & & 95.438 & 110.408 & & & 73.111 \\
\hline 4/6/08 9:00 & 126.355 & 124.124 & & 100.508 & & 95.891 & 110.814 & & & 73.299 \\
\hline 4/6/08 9:30 & 126.535 & 124.199 & & 100.63 & & 96.065 & 110.969 & & 79.423 & 73.452 \\
\hline $4 / 6 / 08$ 10:00 & 126.636 & 124.265 & & 100.708 & & 96.164 & 111.06 & & & 73.551 \\
\hline $4 / 6 / 08 \quad 10: 30$ & 126.707 & 124.312 & & 100.773 & & 96.24 & 111.114 & & & 73.615 \\
\hline $4 / 6 / 0811: 00$ & 126.776 & 124.335 & & 100.83 & & 96.303 & 111.15 & & 79.494 & 73.671 \\
\hline $4 / 6 / 08 \quad 11: 30$ & 126.823 & 122.823 & & 100.866 & & 96.355 & 111.176 & & & 73.722 \\
\hline 4/6/08 12:00 & 126.849 & 122.38 & & 100.906 & & 96.383 & 111.197 & & & 73.76 \\
\hline $4 / 6 / 0812: 30$ & 125.343 & 122.226 & & 100.239 & & 96.089 & 110.861 & & 79.541 & 73.784 \\
\hline $4 / 6 / 08$ 13:00 & 124.842 & 122.147 & & 100.037 & & 95.66 & 110.468 & & & 73.642 \\
\hline $4 / 6 / 08$ 13:30 & 124.681 & 122.102 & & 99.939 & & 95.516 & 110.325 & & & 73.524 \\
\hline $4 / 6 / 08 \quad 14: 00$ & 124.603 & 122.065 & & 99.886 & & 95.441 & 110.247 & & 79.523 & 73.448 \\
\hline $4 / 6 / 08 \quad 14: 30$ & 124.554 & 122.043 & & 99.851 & & 95.398 & 110.2 & & & 73.402 \\
\hline $4 / 6 / 08 \quad 15: 00$ & 124.516 & 122.027 & & 99.827 & & 95.365 & 110.168 & & & 73.371 \\
\hline $4 / 6 / 08 \quad 15: 30$ & 124.492 & 122.009 & & 99.812 & & 95.344 & 110.147 & & 79.506 & 73.346 \\
\hline $4 / 6 / 08$ 16:00 & 124.473 & 121.995 & & 99.796 & & 95.33 & 110.13 & & & 73.332 \\
\hline $4 / 6 / 08$ 16:30 & 124.462 & 121.993 & & 99.779 & & 95.314 & 110.119 & & & 73.317 \\
\hline $4 / 6 / 08 \quad 17: 00$ & 124.445 & 121.993 & & 99.777 & & 95.304 & 110.11 & & 79.489 & 73.301 \\
\hline 4/6/08 17:30 & 124.443 & 122.013 & & 99.774 & & 95.302 & 110.1 & & & 73.292 \\
\hline $4 / 6 / 08$ 18:00 & 124.443 & 122.022 & & 99.787 & & 95.299 & 110.095 & & & 73.292 \\
\hline $4 / 6 / 08$ 18:30 & 124.459 & 122.045 & & 99.81 & & 95.318 & 110.087 & & 79.489 & 73.299 \\
\hline 4/6/08 19:00 & 124.471 & 122.058 & & 99.822 & & 95.33 & 110.077 & & & 73.313 \\
\hline $4 / 6 / 08 \quad 19: 30$ & 124.497 & 122.074 & & 99.839 & & 95.356 & 110.062 & & & 73.328 \\
\hline $4 / 6 / 0820: 00$ & 124.507 & 122.09 & & 99.856 & & 95.37 & 110.058 & & 79.501 & 73.342 \\
\hline $4 / 6 / 0820: 30$ & 124.525 & 122.104 & & 99.868 & & 95.384 & 110.058 & & & 73.357 \\
\hline $4 / 6 / 0821: 00$ & 124.537 & 122.113 & & 99.884 & & 95.398 & 110.056 & & & 73.369 \\
\hline $4 / 6 / 0821: 30$ & 124.554 & 122.14 & & 99.898 & & 95.415 & 110.055 & & 79.514 & 73.381 \\
\hline $4 / 6 / 0822: 00$ & 124.559 & 122.163 & & 99.906 & & 95.42 & 110.061 & & & 73.394 \\
\hline $4 / 6 / 0822: 30$ & 124.587 & 122.176 & & 99.943 & & 95.448 & 110.063 & & & 73.402 \\
\hline $4 / 6 / 08$ 23:00 & 124.613 & 122.181 & & 99.958 & & 95.476 & 110.061 & & 79.538 & 73.431 \\
\hline 4/6/08 23:30 & 124.625 & 122.195 & & 99.967 & & 95.486 & 110.063 & & & 73.445 \\
\hline 4/7/08 0:00 & 124.632 & 122.199 & & 99.975 & & 95.493 & 110.063 & & & 73.458 \\
\hline 4/7/08 0:30 & 124.646 & 122.213 & & 99.987 & & 95.507 & 110.063 & & 79.555 & 73.464 \\
\hline 4/7/08 1:00 & 124.651 & 122.215 & & 99.998 & & 95.511 & 110.061 & & & 73.474 \\
\hline 4/7/08 1:30 & 124.66 & 122.206 & & 100.011 & & 95.518 & 110.061 & & & 73.483 \\
\hline 4/7/08 2:00 & 124.665 & 122.183 & & 100.003 & & 95.526 & 110.063 & & 79.572 & 73.497 \\
\hline 4/7/08 2:30 & 124.655 & 122.192 & & 99.98 & & 95.518 & 110.063 & & & 73.493 \\
\hline 4/7/08 3:00 & 124.636 & 122.199 & & 99.97 & & 95.495 & 110.063 & & & 73.476 \\
\hline 4/7/08 3:30 & 124.644 & 122.195 & & 99.989 & & 95.507 & 110.057 & & 79.572 & 73.468 \\
\hline 4/7/08 4:00 & 124.651 & 122.192 & & 99.975 & & 95.514 & 110.055 & & & 73.483 \\
\hline 4/7/08 4:30 & 124.644 & 122.188 & & 99.992 & & 95.509 & 110.051 & & & 73.479 \\
\hline 4/7/08 5:00 & 124.639 & 122.179 & & 99.98 & & 95.502 & 110.05 & & 79.589 & 73.481 \\
\hline $4 / 7 / 085: 30$ & 124.636 & 122.192 & & 99.968 & & 95.504 & 110.048 & & & 73.481 \\
\hline 4/7/08 6:00 & 124.627 & 124.029 & & 99.977 & & 95.493 & 110.044 & & & 73.466 \\
\hline 4/7/08 6:30 & 124.636 & 124.326 & & 100.216 & & 95.502 & 110.04 & & 79.589 & 73.47 \\
\hline 4/7/08 7:00 & 126.509 & 124.476 & & 100.776 & & 96.063 & 110.6 & & & 73.528 \\
\hline 4/7/08 7:30 & 126.825 & 124.548 & & 100.95 & & 96.365 & 110.872 & & & 73.722 \\
\hline 4/7/08 8:00 & 126.979 & 123.135 & & 101.057 & & 96.511 & 111 & & 79.648 & 73.849 \\
\hline 4/7/08 8:30 & 127.057 & 122.63 & & 101.118 & & 96.589 & 111.076 & & & 73.935 \\
\hline 4/7/08 9:00 & 125.707 & 122.476 & & 100.496 & & 96.412 & 110.857 & & & 73.987 \\
\hline 4/7/08 9:30 & 125.088 & 122.426 & & 100.278 & & 95.914 & 110.406 & & 79.68 & 73.863 \\
\hline $4 / 7 / 08 \quad 10: 00$ & 124.93 & 122.374 & & 100.223 & & 95.773 & 110.265 & & & 73.747 \\
\hline 4/7/08 10:30 & 124.875 & 122.342 & & 100.172 & & 95.721 & 110.192 & & & 73.698 \\
\hline 4/7/08 11:00 & 124.826 & 122.319 & & 100.13 & & 95.674 & 110.153 & & 79.68 & 73.663 \\
\hline 4/7/08 11:30 & 124.792 & 122.31 & & 100.113 & & 95.641 & 110.125 & & & 73.625 \\
\hline 4/7/08 12:00 & 124.769 & 124.133 & & 100.104 & & 95.622 & 110.108 & & & 73.611 \\
\hline 4/7/08 12:30 & 124.759 & 124.387 & & 100.366 & & 95.613 & 110.091 & & 79.685 & 73.601 \\
\hline 4/7/08 13:00 & 126.65 & 122.906 & & 100.902 & & 96.223 & 110.712 & & & 73.646 \\
\hline 4/7/08 13:30 & 126.922 & 122.496 & & 101.049 & & 96.475 & 110.975 & & & 73.809 \\
\hline 4/7/08 14:00 & 125.416 & 122.374 & & 100.373 & & 96.178 & 110.682 & & 79.714 & 73.898 \\
\hline 4/7/08 14:30 & 124.956 & 122.285 & & 100.199 & & 95.789 & 110.346 & & & 73.78 \\
\hline 4/7/08 15:00 & 124.828 & 122.238 & & 100.113 & & 95.672 & 110.228 & & & 73.675 \\
\hline 4/7/08 15:30 & 124.743 & 122.192 & & 100.005 & & 95.589 & 110.173 & & 79.685 & 73.619 \\
\hline 4/7/08 16:00 & 124.686 & 122.158 & & 99.984 & & 95.537 & 110.132 & & & 73.536 \\
\hline 4/7/08 16:30 & 124.644 & 122.165 & & 99.939 & & 95.493 & 110.111 & & & 73.512 \\
\hline
\end{tabular}


TABLE S1.1 (Cont.)

\begin{tabular}{|c|c|c|c|c|c|c|c|c|c|c|}
\hline \multirow[b]{2}{*}{ Date and Time } & \multicolumn{10}{|c|}{ Depth from Top of Casing (ft) } \\
\hline & MW1D & MW2D & MW3D & MW4D & MW7 & MW9 & Oentrich & MW14D & MW15D & MW16D \\
\hline 4/7/08 17:00 & 124.608 & 122.14 & & 99.951 & & 95.457 & 110.096 & & 79.653 & 73.468 \\
\hline 4/7/08 17:30 & 124.613 & 122.151 & & 99.934 & & 95.471 & 110.081 & & & 73.468 \\
\hline 4/7/08 18:00 & 124.596 & 122.161 & & 99.948 & & 95.453 & 110.072 & & & 73.456 \\
\hline 4/7/08 18:30 & 124.599 & 122.158 & & 99.934 & & 95.453 & 110.063 & & 79.646 & 73.46 \\
\hline 4/7/08 19:00 & 124.611 & 122.163 & & 99.939 & & 95.471 & 110.051 & & & 73.452 \\
\hline 4/7/08 19:30 & 124.615 & 122.163 & & 99.949 & & 95.464 & 110.046 & & & 73.464 \\
\hline 4/7/08 20:00 & 124.611 & 122.161 & & 99.956 & & 95.474 & 110.042 & & 79.633 & 73.466 \\
\hline $4 / 7 / 0820: 30$ & 124.603 & 122.163 & & 99.948 & & 95.474 & 110.038 & & & 73.464 \\
\hline 4/7/08 21:00 & 124.599 & 122.156 & & 99.948 & & 95.471 & 110.033 & & & 73.462 \\
\hline 4/7/08 21:30 & 124.594 & 122.129 & & 99.949 & & 95.476 & 110.033 & & 79.631 & 73.464 \\
\hline 4/7/08 22:00 & 124.592 & 122.133 & & 99.929 & & 95.471 & 110.031 & & & 73.466 \\
\hline 4/7/08 22:30 & 124.594 & 122.133 & & 99.913 & & 95.443 & 110.033 & & & 73.454 \\
\hline 4/7/08 23:00 & 124.582 & 122.108 & & 99.939 & & 95.441 & 110.025 & & 79.619 & 73.433 \\
\hline 4/7/08 23:30 & 124.585 & 122.104 & & 99.898 & & 95.45 & 110.023 & & & 73.45 \\
\hline 4/8/08 0:00 & 124.582 & 122.09 & & 99.894 & & 95.42 & 110.025 & & & 73.425 \\
\hline 4/8/08 0:30 & 124.577 & 122.086 & & 99.882 & & 95.417 & 110.019 & & 79.604 & 73.417 \\
\hline 4/8/08 1:00 & 124.575 & 122.111 & & 99.87 & & 95.401 & 110.019 & & & 73.41 \\
\hline 4/8/08 1:30 & 124.568 & 122.115 & & 99.887 & & 95.398 & 110.016 & & & 73.396 \\
\hline 4/8/08 2:00 & 124.563 & 122.086 & & 99.901 & & 95.422 & 110.006 & & 79.607 & 73.388 \\
\hline 4/8/08 2:30 & 124.57 & 122.049 & & 99.899 & & 95.427 & 110.004 & & & 73.383 \\
\hline 4/8/08 3:00 & 124.587 & 121.981 & & 99.87 & & 95.398 & 110.006 & & & 73.386 \\
\hline 4/8/08 3:30 & 124.587 & 122.006 & & 99.855 & & 95.363 & 110.008 & & 79.565 & 73.39 \\
\hline 4/8/08 4:00 & 124.556 & 122.038 & & 99.805 & & 95.297 & 110.01 & & & 73.402 \\
\hline 4/8/08 4:30 & 124.514 & 122.015 & & 99.817 & & 95.313 & 109.999 & & & 73.394 \\
\hline 4/8/08 5:00 & 124.511 & 122.015 & & 99.832 & & 95.351 & 109.991 & & 79.543 & 73.4 \\
\hline 4/8/08 5:30 & 124.518 & 122.031 & & 99.8 & & 95.328 & 109.993 & & & 73.421 \\
\hline 4/8/08 6:00 & 124.507 & 123.789 & & 99.832 & & 95.321 & 109.988 & & & 73.433 \\
\hline 4/8/08 6:30 & 124.509 & 124.104 & & 100.101 & & 95.351 & 109.984 & & 79.528 & 73.441 \\
\hline 4/8/08 7:00 & 126.246 & 124.294 & & 100.606 & & 95.865 & 110.617 & & & 73.53 \\
\hline 4/8/08 7:30 & 126.596 & 124.41 & & 100.794 & & 96.185 & 110.887 & & & 73.691 \\
\hline 4/8/08 8:00 & 126.795 & 124.421 & & 100.971 & & 96.369 & 111.007 & & 79.575 & 73.789 \\
\hline 4/8/08 8:30 & 126.92 & 124.473 & & 100.985 & & 96.499 & 111.082 & & & 73.853 \\
\hline 4/8/08 9:00 & 126.969 & 124.51 & & 101.038 & & 96.504 & 111.139 & & & 73.917 \\
\hline 4/8/08 9:30 & 127.003 & 122.646 & & 101.08 & & 96.556 & 111.172 & & 79.626 & 73.954 \\
\hline 4/8/08 10:00 & 127.029 & 122.43 & & 100.666 & & 96.591 & 111.197 & & & 73.989 \\
\hline 4/8/08 10:30 & 125.199 & 122.328 & & 100.403 & & 95.922 & 110.517 & & & 73.929 \\
\hline 4/8/08 11:00 & 124.986 & 122.263 & & 100.303 & & 95.714 & 110.305 & & 79.633 & 73.83 \\
\hline 4/8/08 11:30 & 124.889 & 122.224 & & 100.458 & & 95.62 & 110.202 & & & 73.774 \\
\hline 4/8/08 12:00 & 124.835 & 122.197 & & 100.471 & & 95.559 & 110.143 & & & 73.756 \\
\hline 4/8/08 12:30 & 124.8 & 122.17 & & 100.453 & & 95.526 & 110.104 & & 79.861 & 73.751 \\
\hline 4/8/08 13:00 & 124.774 & 122.147 & & 100.446 & & 95.495 & 110.08 & & & 73.751 \\
\hline 4/8/08 13:30 & 124.743 & 122.133 & & 100.423 & & 95.471 & 110.063 & & & 73.768 \\
\hline 4/8/08 14:00 & 124.717 & 122.113 & & 100.394 & & 95.45 & 110.048 & & 79.748 & 73.793 \\
\hline 4/8/08 14:30 & 124.7 & 122.104 & & 100.341 & & 95.438 & 110.038 & & & 73.811 \\
\hline 4/8/08 15:00 & 124.672 & 122.102 & & 100.253 & & 95.417 & 110.031 & & & 73.836 \\
\hline $4 / 8 / 08 \quad 15: 30$ & 124.648 & 122.09 & & 100.142 & & 95.413 & 110.021 & & 79.643 & 73.863 \\
\hline 4/8/08 16:00 & 124.644 & 122.077 & & 100.027 & & 95.413 & 110.016 & & & 73.882 \\
\hline 4/8/08 16:30 & 124.625 & 122.081 & & 99.918 & & 95.398 & 110.008 & & & 73.822 \\
\hline 4/8/08 17:00 & 124.611 & 122.081 & & 99.86 & & 95.387 & 110.006 & & 79.572 & 73.468 \\
\hline 4/8/08 17:30 & 124.601 & 122.086 & & 99.865 & & 95.389 & 109.999 & & & 73.4 \\
\hline 4/8/08 18:00 & 124.592 & 122.09 & & 99.87 & & 95.389 & 109.993 & & & 73.394 \\
\hline 4/8/08 18:30 & 124.585 & 122.09 & & 99.872 & & 95.398 & 109.989 & & 79.536 & 73.394 \\
\hline 4/8/08 19:00 & 124.58 & 122.088 & & 99.879 & & 95.401 & 109.986 & & & 73.394 \\
\hline 4/8/08 19:30 & 124.573 & 122.102 & & 99.874 & & 95.401 & 109.984 & & & 73.4 \\
\hline 4/8/08 20:00 & 124.563 & 122.108 & & 99.882 & & 95.398 & 109.98 & & 79.519 & 73.396 \\
\hline 4/8/08 20:30 & 124.551 & 122.115 & & 99.894 & & 95.412 & 109.975 & & & 73.4 \\
\hline 4/8/08 21:00 & 124.547 & 122.124 & & 99.903 & & 95.417 & 109.973 & & & 73.408 \\
\hline $4 / 8 / 0821: 30$ & 124.54 & 122.136 & & 99.91 & & 95.424 & 109.969 & & 79.514 & 73.414 \\
\hline 4/8/08 22:00 & 124.535 & 122.138 & & 99.918 & & 95.436 & 109.967 & & & 73.421 \\
\hline 4/8/08 22:30 & 124.561 & 122.138 & & 99.924 & & 95.445 & 109.963 & & & 73.431 \\
\hline 4/8/08 23:00 & 124.561 & 122.147 & & 99.93 & & 95.446 & 109.963 & & 79.514 & 73.435 \\
\hline 4/8/08 23:30 & 124.559 & 122.145 & & 99.932 & & 95.45 & 109.963 & & & 73.439 \\
\hline 4/9/08 0:00 & 124.596 & 122.136 & & 99.937 & & 95.46 & 109.963 & & & 73.439 \\
\hline 4/9/08 0:30 & 124.594 & 122.129 & & 99.927 & & 95.455 & 109.963 & & 79.521 & 73.448 \\
\hline 4/9/08 1:00 & 124.585 & 122.117 & & 99.918 & & 95.45 & 109.963 & & & 73.441 \\
\hline 4/9/08 1:30 & 124.577 & 122.12 & & 99.908 & & 95.438 & 109.961 & & & 73.433 \\
\hline
\end{tabular}


TABLE S1.1 (Cont.)

\begin{tabular}{|c|c|c|c|c|c|c|c|c|c|c|}
\hline \multirow[b]{2}{*}{ Date and Time } & \multicolumn{10}{|c|}{ Depth from Top of Casing (ft) } \\
\hline & MW1D & MW2D & MW3D & MW4D & MW7 & MW9 & Oentrich & MW14D & MW15D & MW16D \\
\hline 4/9/08 2:00 & 124.566 & 122.124 & & 99.899 & & 95.427 & 109.963 & & 79.509 & 73.423 \\
\hline 4/9/08 2:30 & 124.566 & 122.106 & & 99.912 & & 95.429 & 109.958 & & & 73.414 \\
\hline 4/9/08 3:00 & 124.573 & 122.111 & & 99.906 & & 95.438 & 109.954 & & & 73.421 \\
\hline 4/9/08 3:30 & 124.566 & 122.102 & & 99.889 & & 95.42 & 109.954 & & 79.509 & 73.419 \\
\hline 4/9/08 4:00 & 124.561 & 122.092 & & 99.894 & & 95.417 & 109.95 & & & 73.404 \\
\hline 4/9/08 4:30 & 124.556 & 122.09 & & 99.88 & & 95.415 & 109.948 & & & 73.408 \\
\hline 4/9/08 5:00 & 124.549 & 122.108 & & 99.877 & & 95.408 & 109.944 & & 79.489 & 73.396 \\
\hline 4/9/08 5:30 & 124.547 & 122.097 & & 99.889 & & 95.403 & 109.942 & & & 73.394 \\
\hline 4/9/08 6:00 & 124.535 & 123.92 & & 99.898 & & 95.424 & 109.937 & & & 73.4 \\
\hline 4/9/08 6:30 & 124.537 & 124.228 & & 100.135 & & 95.41 & 109.937 & & 79.504 & 73.41 \\
\hline 4/9/08 7:00 & 126.386 & 124.392 & & 100.723 & & 96.002 & 110.534 & & & 73.45 \\
\hline 4/9/08 7:30 & 126.733 & 124.485 & & 100.919 & & 96.313 & 110.821 & & & 73.642 \\
\hline 4/9/08 8:00 & 126.899 & 124.532 & & 101.037 & & 96.475 & 110.953 & & 79.533 & 73.774 \\
\hline 4/9/08 8:30 & 126.995 & 124.575 & & 101.1 & & 96.565 & 111.032 & & & 73.873 \\
\hline 4/9/08 9:00 & 127.052 & 124.603 & & 101.135 & & 96.617 & 111.084 & & & 73.933 \\
\hline 4/9/08 9:30 & 127.111 & 123.099 & & 101.181 & & 96.659 & 111.118 & & 79.602 & 73.964 \\
\hline 4/9/08 10:00 & 127.14 & 122.6 & & 101.192 & & 96.683 & 111.146 & & & 74.002 \\
\hline 4/9/08 10:30 & 125.636 & 122.417 & & 100.466 & & 96.365 & 110.803 & & & 74.012 \\
\hline 4/9/08 11:00 & 125.057 & 122.303 & & 100.241 & & 95.877 & 110.367 & & 79.629 & 73.851 \\
\hline 4/9/08 11:30 & 124.87 & 122.244 & & 100.12 & & 95.705 & 110.218 & & & 73.718 \\
\hline 4/9/08 12:00 & 124.757 & 122.181 & & 100.046 & & 95.599 & 110.138 & & & 73.625 \\
\hline 4/9/08 12:30 & 124.696 & 122.142 & & 99.982 & & 95.545 & 110.089 & & 79.592 & 73.559 \\
\hline 4/9/08 13:00 & 124.634 & 122.145 & & 99.932 & & 95.483 & 110.059 & & & 73.503 \\
\hline 4/9/08 13:30 & 124.589 & 122.122 & & 99.927 & & 95.438 & 110.033 & & & 73.458 \\
\hline 4/9/08 14:00 & 124.594 & 122.097 & & 99.908 & & 95.448 & 110.012 & & 79.57 & 73.441 \\
\hline 4/9/08 14:30 & 124.57 & 122.047 & & 99.889 & & 95.424 & 110.001 & & & 73.433 \\
\hline 4/9/08 15:00 & 124.547 & 122.018 & & 99.848 & & 95.403 & 109.991 & & & 73.412 \\
\hline 4/9/08 15:30 & 124.497 & 121.99 & & 99.813 & & 95.354 & 109.99 & & 79.531 & 73.383 \\
\hline 4/9/08 16:00 & 124.469 & 121.97 & & 99.793 & & 95.323 & 109.98 & & & 73.348 \\
\hline 4/9/08 16:30 & 124.443 & 121.95 & & 99.762 & & 95.302 & 109.975 & & & 73.326 \\
\hline 4/9/08 17:00 & 124.421 & 121.938 & & 99.741 & & 95.281 & 109.969 & & 79.492 & 73.301 \\
\hline 4/9/08 17:30 & 124.4 & 121.929 & & 99.731 & & 95.259 & 109.967 & & & 73.28 \\
\hline 4/9/08 18:00 & 124.388 & 121.922 & & 99.717 & & 95.25 & 109.959 & & & 73.268 \\
\hline 4/9/08 18:30 & 124.381 & 121.9 & & 99.708 & & 95.245 & 109.952 & & 79.46 & 73.255 \\
\hline 4/9/08 19:00 & 124.372 & 121.886 & & 99.708 & & 95.236 & 109.95 & & & 73.245 \\
\hline 4/9/08 19:30 & 124.351 & 121.87 & & 99.674 & & 95.212 & 109.948 & & & 73.241 \\
\hline 4/9/08 20:00 & 124.336 & 121.85 & & 99.66 & & 95.2 & 109.946 & & 79.421 & 73.216 \\
\hline 4/9/08 20:30 & 124.322 & 121.85 & & 99.648 & & 95.186 & 109.942 & & & 73.204 \\
\hline 4/9/08 21:00 & 124.303 & 121.845 & & 99.643 & & 95.165 & 109.941 & & & 73.191 \\
\hline 4/9/08 21:30 & 124.299 & 121.848 & & 99.636 & & 95.163 & 109.931 & & 79.387 & 73.181 \\
\hline 4/9/08 22:00 & 124.296 & 121.834 & & 99.636 & & 95.156 & 109.926 & & & 73.175 \\
\hline 4/9/08 22:30 & 124.299 & 121.805 & & 99.627 & & 95.163 & 109.92 & & & 73.171 \\
\hline 4/9/08 23:00 & 124.282 & 121.786 & & 99.608 & & 95.149 & 109.918 & & 79.355 & 73.166 \\
\hline 4/9/08 23:30 & 124.256 & 121.773 & & 99.584 & & 95.118 & 109.916 & & & 73.154 \\
\hline 4/10/08 0:00 & 124.242 & 121.746 & & 99.563 & & 95.097 & 109.915 & & & 73.14 \\
\hline 4/10/08 0:30 & 124.242 & 121.698 & & 99.55 & & 95.082 & 109.909 & & 79.321 & 73.135 \\
\hline 4/10/08 1:00 & 124.242 & 121.678 & & 99.501 & & 95.059 & 109.907 & & & 73.135 \\
\hline 4/10/08 1:30 & 124.247 & 121.689 & & 99.467 & & 95.012 & 109.907 & & & 73.137 \\
\hline 4/10/08 2:00 & 124.242 & 121.673 & & 99.474 & & 94.995 & 109.896 & & 79.286 & 73.133 \\
\hline 4/10/08 2:30 & 124.232 & 121.648 & & 99.476 & & 95.005 & 109.882 & & & 73.125 \\
\hline 4/10/08 3:00 & 124.232 & 121.623 & & 99.45 & & 94.991 & 109.879 & & & 73.125 \\
\hline 4/10/08 3:30 & 124.213 & 121.582 & & 99.424 & & 94.958 & 109.879 & & 79.242 & 73.125 \\
\hline 4/10/08 4:00 & 124.19 & 121.548 & & 99.386 & & 94.939 & 109.879 & & & 73.125 \\
\hline $4 / 10 / 084: 30$ & 124.126 & 121.514 & & 99.355 & & 94.894 & 109.881 & & & 73.127 \\
\hline 4/10/08 5:00 & 124.098 & 121.494 & & 99.312 & & 94.863 & 109.881 & & 79.198 & 73.125 \\
\hline 4/10/08 5:30 & 124.062 & 121.492 & & 99.29 & & 94.828 & 109.883 & & & 73.123 \\
\hline 4/10/08 6:00 & 124.046 & 123.301 & & 99.284 & & 94.809 & 109.879 & & & 73.117 \\
\hline 4/10/08 6:30 & 124.039 & 123.582 & & 99.517 & & 94.809 & 109.875 & & 79.159 & 73.106 \\
\hline $4 / 10 / 087: 00$ & 125.745 & 123.696 & & 100.039 & & 95.342 & 110.429 & & & 73.154 \\
\hline $4 / 10 / 08$ 7:30 & 126.057 & 123.764 & & 100.192 & & 95.62 & 110.701 & & & 73.305 \\
\hline 4/10/08 8:00 & 126.175 & 123.786 & & 100.275 & & 95.733 & 110.829 & & 79.154 & 73.396 \\
\hline 4/10/08 8:30 & 126.248 & 123.786 & & 100.855 & & 95.804 & 110.906 & & & 73.458 \\
\hline 4/10/08 9:00 & 126.331 & 121.977 & & 100.995 & & 95.825 & 110.957 & & & 73.512 \\
\hline 4/10/08 9:30 & 126.367 & 121.675 & & 100.785 & & 95.825 & 110.99 & & 79.286 & 73.561 \\
\hline 4/10/08 10:00 & 124.54 & 121.532 & & 100.196 & & 95.255 & 110.433 & & & 73.538 \\
\hline $4 / 10 / 08 \quad 10: 30$ & 124.244 & 121.442 & & 100.08 & & 94.962 & 110.175 & & & 73.437 \\
\hline
\end{tabular}


TABLE S1.1 (Cont.)

\begin{tabular}{|c|c|c|c|c|c|c|c|c|c|c|}
\hline \multirow[b]{2}{*}{ Date and Time } & \multicolumn{10}{|c|}{ Depth from Top of Casing (ft) } \\
\hline & MW1D & MW2D & MW3D & MW4D & MW7 & MW9 & Oentrich & MW14D & MW15D & MW16D \\
\hline 4/10/08 11:00 & 124.086 & 121.381 & & 99.946 & & 94.826 & 110.05 & & 79.201 & 73.375 \\
\hline 4/10/08 11:30 & 123.996 & 121.324 & & 99.753 & & 94.734 & 109.974 & & & 73.34 \\
\hline 4/10/08 12:00 & 123.942 & 121.281 & & 99.694 & & 94.677 & 109.918 & & & 73.311 \\
\hline 4/10/08 12:30 & 123.906 & 121.272 & & 99.75 & & 94.623 & 109.847 & & 79.142 & 73.295 \\
\hline 4/10/08 13:00 & 123.868 & 121.226 & & 99.734 & & 94.583 & 109.772 & & & 73.28 \\
\hline 4/10/08 13:30 & 123.828 & 121.204 & & 99.725 & & 94.576 & 109.738 & & & 73.261 \\
\hline $4 / 10 / 0814: 00$ & 123.826 & 121.201 & & 99.703 & & 94.538 & 109.728 & & 79.052 & 73.259 \\
\hline $4 / 10 / 0814: 30$ & 123.72 & 121.208 & & 99.693 & & 94.505 & 109.71 & & & 73.266 \\
\hline 4/10/08 15:00 & 123.727 & 121.192 & & 99.631 & & 94.507 & 109.704 & & & 73.286 \\
\hline $4 / 10 / 08$ 15:30 & 123.729 & 121.183 & & 99.5 & & 94.51 & 109.693 & & 78.934 & 73.311 \\
\hline 4/10/08 16:00 & 123.727 & 121.181 & & 99.384 & & 94.498 & 109.689 & & & 73.313 \\
\hline 4/10/08 16:30 & 123.724 & 121.19 & & 99.298 & & 94.488 & 109.689 & & & 73.301 \\
\hline 4/10/08 17:00 & 123.722 & 121.199 & & 99.305 & & 94.481 & 109.682 & & 78.812 & 73.286 \\
\hline 4/10/08 17:30 & 123.717 & 121.211 & & 99.26 & & 94.493 & 109.678 & & & 73.272 \\
\hline 4/10/08 18:00 & 123.715 & 121.211 & & 99.203 & & 94.507 & 109.674 & & & 73.255 \\
\hline $4 / 10 / 08$ 18:30 & 123.712 & 121.204 & & 99.181 & & 94.522 & 109.672 & & 78.756 & 73.239 \\
\hline 4/10/08 19:00 & 123.705 & 121.213 & & 99.147 & & 94.522 & 109.672 & & & 73.222 \\
\hline 4/10/08 19:30 & 123.705 & 121.226 & & 99.105 & & 94.519 & 109.668 & & & 73.208 \\
\hline 4/10/08 20:00 & 123.698 & 121.24 & & 99.059 & & 94.526 & 109.667 & & 78.729 & 73.189 \\
\hline $4 / 10 / 0820: 30$ & 123.696 & 121.269 & & 99.022 & & 94.54 & 109.665 & & & 73.171 \\
\hline 4/10/08 21:00 & 123.691 & 121.285 & & 98.993 & & 94.552 & 109.661 & & & 73.158 \\
\hline 4/10/08 21:30 & 123.689 & 121.294 & & 99.074 & & 94.58 & 109.663 & & 78.719 & 73.142 \\
\hline 4/10/08 22:00 & 123.696 & 121.297 & & 99.088 & & 94.604 & 109.659 & & & 73.125 \\
\hline 4/10/08 22:30 & 123.717 & 121.301 & & 99.091 & & 94.613 & 109.657 & & & 73.113 \\
\hline $4 / 10 / 0823: 00$ & 123.715 & 121.308 & & 99.093 & & 94.613 & 109.657 & & 78.722 & 73.102 \\
\hline 4/10/08 23:30 & 123.712 & 121.317 & & 99.098 & & 94.621 & 109.657 & & & 73.094 \\
\hline $4 / 11 / 080: 00$ & 123.734 & 121.331 & & 99.107 & & 94.625 & 109.655 & & & 73.086 \\
\hline 4/11/08 0:30 & 123.734 & 121.342 & & 99.121 & & 94.637 & 109.655 & & 78.705 & 73.08 \\
\hline 4/11/08 1:00 & 123.755 & 121.353 & & 99.134 & & 94.649 & 109.654 & & & 73.075 \\
\hline 4/11/08 1:30 & 123.755 & 121.365 & & 99.147 & & 94.663 & 109.654 & & & 73.071 \\
\hline $4 / 11 / 082: 00$ & 123.776 & 121.374 & & 99.157 & & 94.672 & 109.652 & & 78.695 & 73.067 \\
\hline 4/11/08 2:30 & 123.8 & 121.387 & & 99.164 & & 94.684 & 109.65 & & & 73.065 \\
\hline 4/11/08 3:00 & 123.797 & 121.399 & & 99.178 & & 94.693 & 109.65 & & & 73.063 \\
\hline 4/11/08 3:30 & 123.833 & 121.41 & & 99.191 & & 94.705 & 109.65 & & 78.688 & 73.061 \\
\hline 4/11/08 4:00 & 123.845 & 121.424 & & 99.202 & & 94.719 & 109.648 & & & 73.059 \\
\hline 4/11/08 4:30 & 123.859 & 121.433 & & 99.215 & & 94.731 & 109.642 & & & 73.057 \\
\hline 4/11/08 5:00 & 123.871 & 121.449 & & 99.224 & & 94.743 & 109.646 & & 78.688 & 73.057 \\
\hline 4/11/08 5:30 & 123.878 & 121.464 & & 99.234 & & 94.755 & 109.644 & & & 73.057 \\
\hline 4/11/08 6:00 & 123.894 & 123.283 & & 99.257 & & 94.767 & 109.642 & & & 73.057 \\
\hline 4/11/08 6:30 & 123.916 & 123.628 & & 99.403 & & 94.785 & 109.64 & & 78.683 & 73.057 \\
\hline 4/11/08 7:00 & 125.792 & 123.795 & & 100.115 & & 95.375 & 110.209 & & & 73.094 \\
\hline 4/11/08 7:30 & 126.154 & 123.902 & & 100.328 & & 95.716 & 110.508 & & & 73.259 \\
\hline 4/11/08 8:00 & 126.326 & 123.972 & & 100.451 & & 95.884 & 110.645 & & 78.802 & 73.359 \\
\hline 4/11/08 8:30 & 126.435 & 124.029 & & 100.535 & & 95.987 & 110.726 & & & 73.425 \\
\hline 4/11/08 9:00 & 126.509 & 122.684 & & 100.599 & & 96.063 & 110.78 & & & 73.476 \\
\hline 4/11/08 9:30 & 126.57 & 122.117 & & 100.645 & & 96.119 & 110.82 & & 78.839 & 73.52 \\
\hline 4/11/08 10:00 & 125.279 & 121.961 & & 99.992 & & 95.964 & 110.587 & & & 73.557 \\
\hline $4 / 11 / 0810: 30$ & 124.577 & 121.886 & & 99.775 & & 95.408 & 110.065 & & & 73.448 \\
\hline 4/11/08 11:00 & 124.414 & 121.845 & & 99.689 & & 95.257 & 109.907 & & 78.895 & 73.361 \\
\hline 4/11/08 11:30 & 124.336 & 121.82 & & 99.643 & & 95.189 & 109.826 & & & 73.311 \\
\hline $4 / 11 / 08$ 12:00 & 124.294 & 121.809 & & 99.615 & & 95.151 & 109.772 & & & 73.276 \\
\hline 4/11/08 12:30 & 124.27 & 121.8 & & 99.601 & & 95.132 & 109.744 & & 78.903 & 73.158 \\
\hline 4/11/08 13:00 & 124.261 & 121.802 & & 99.594 & & 95.122 & 109.717 & & & 73.077 \\
\hline 4/11/08 13:30 & 124.251 & 121.802 & & 99.593 & & 95.116 & 109.706 & & & 73.059 \\
\hline 4/11/08 14:00 & 124.251 & 121.809 & & 99.598 & & 95.118 & 109.695 & & 78.91 & 73.051 \\
\hline 4/11/08 14:30 & 124.254 & 121.811 & & 99.596 & & 95.118 & 109.691 & & & 73.059 \\
\hline 4/11/08 15:00 & 124.256 & 121.816 & & 99.605 & & 95.125 & 109.686 & & & 73.057 \\
\hline 4/11/08 15:30 & 124.265 & 121.82 & & 99.61 & & 95.13 & 109.684 & & 78.922 & 73.063 \\
\hline 4/11/08 16:00 & 124.265 & 121.823 & & 99.61 & & 95.137 & 109.682 & & & 73.069 \\
\hline $4 / 11 / 0816: 30$ & 124.265 & 121.829 & & 99.617 & & 95.137 & 109.678 & & & 73.071 \\
\hline 4/11/08 17:00 & 124.275 & 121.839 & & 99.622 & & 95.144 & 109.674 & & 78.949 & 73.075 \\
\hline 4/11/08 17:30 & 124.277 & 121.845 & & 99.632 & & 95.151 & 109.672 & & & 73.075 \\
\hline $4 / 11 / 08$ 18:00 & 124.289 & 121.854 & & 99.638 & & 95.158 & 109.672 & & & 73.086 \\
\hline 4/11/08 18:30 & 124.296 & 121.861 & & 99.648 & & 95.17 & 109.674 & & 78.959 & 73.092 \\
\hline 4/11/08 19:00 & 124.301 & 121.873 & & 99.658 & & 95.179 & 109.672 & & & 73.1 \\
\hline 4/11/08 19:30 & 124.31 & 121.884 & & 99.669 & & 95.184 & 109.669 & & & 73.109 \\
\hline
\end{tabular}


TABLE S1.1 (Cont.)

\begin{tabular}{|c|c|c|c|c|c|c|c|c|c|c|}
\hline \multirow[b]{2}{*}{ Date and Time } & \multicolumn{10}{|c|}{ Depth from Top of Casing (ft) } \\
\hline & MW1D & MW2D & MW3D & MW4D & MW7 & MW9 & Oentrich & MW14D & MW15D & MW16D \\
\hline 4/11/08 20:00 & 124.325 & 121.888 & & 99.679 & & 95.196 & 109.665 & & 78.956 & 73.117 \\
\hline $4 / 11 / 0820: 30$ & 124.332 & 121.9 & & 99.684 & & 95.207 & 109.665 & & & 73.109 \\
\hline 4/11/08 21:00 & 124.339 & 121.904 & & 99.696 & & 95.212 & 109.663 & & & 73.133 \\
\hline $4 / 11 / 0821: 30$ & 124.348 & 121.907 & & 99.705 & & 95.224 & 109.657 & & 78.966 & 73.142 \\
\hline $4 / 11 / 0822: 00$ & 124.355 & 121.898 & & 99.708 & & 95.229 & 109.657 & & & 73.15 \\
\hline $4 / 11 / 0822: 30$ & 124.353 & 121.898 & & 99.701 & & 95.229 & 109.657 & & & 73.156 \\
\hline 4/11/08 23:00 & 124.348 & 121.891 & & 99.698 & & 95.222 & 109.654 & & 78.971 & 73.152 \\
\hline 4/11/08 23:30 & 124.346 & 121.891 & & 99.696 & & 95.219 & 109.65 & & & 73.148 \\
\hline $4 / 12 / 080: 00$ & 124.343 & 121.898 & & 99.691 & & 95.217 & 109.65 & & & 73.14 \\
\hline 4/12/08 0:30 & 124.343 & 121.898 & & 99.696 & & 95.219 & 109.644 & & 78.974 & 73.142 \\
\hline 4/12/08 1:00 & 124.343 & 121.895 & & 99.698 & & 95.219 & 109.64 & & & 73.146 \\
\hline 4/12/08 1:30 & 124.346 & 121.891 & & 99.696 & & 95.224 & 109.637 & & & 73.15 \\
\hline $4 / 12 / 082: 00$ & 124.343 & 121.895 & & 99.692 & & 95.221 & 109.635 & & 78.976 & 73.148 \\
\hline 4/12/08 2:30 & 124.339 & 121.898 & & 99.693 & & 95.217 & 109.633 & & & 73.144 \\
\hline 4/12/08 3:00 & 124.343 & 121.895 & & 99.696 & & 95.219 & 109.627 & & & 73.144 \\
\hline 4/12/08 3:30 & 124.346 & 121.898 & & 99.696 & & 95.221 & 109.624 & & 78.978 & 73.148 \\
\hline 4/12/08 4:00 & 124.343 & 121.895 & & 99.696 & & 95.219 & 109.622 & & & 73.148 \\
\hline 4/12/08 4:30 & 124.346 & 121.898 & & 99.696 & & 95.221 & 109.618 & & & 73.148 \\
\hline 4/12/08 5:00 & 124.346 & 121.898 & & 99.698 & & 95.224 & 109.616 & & 78.978 & 73.15 \\
\hline 4/12/08 5:30 & 124.346 & 121.902 & & 99.696 & & 95.224 & 109.612 & & & 73.15 \\
\hline 4/12/08 6:00 & 124.343 & 121.907 & & 99.698 & & 95.224 & 109.61 & & & 73.152 \\
\hline 4/12/08 6:30 & 124.348 & 121.913 & & 99.703 & & 95.224 & 109.605 & & 78.978 & 73.152 \\
\hline 4/12/08 7:00 & 124.353 & 122.478 & & 99.71 & & 95.231 & 109.601 & & & 73.154 \\
\hline 4/12/08 7:30 & 124.36 & 123.936 & & 99.72 & & 95.238 & 109.599 & & & 73.162 \\
\hline 4/12/08 8:00 & 124.372 & 124.149 & & 100.323 & & 95.25 & 109.595 & & 78.993 & 73.171 \\
\hline 4/12/08 8:30 & 126.43 & 124.274 & & 100.623 & & 95.978 & 110.303 & & & 73.313 \\
\hline 4/12/08 9:00 & 126.65 & 124.346 & & 100.769 & & 96.19 & 110.493 & & & 73.487 \\
\hline 4/12/08 9:30 & 126.783 & 124.399 & & 100.857 & & 96.317 & 110.589 & & 79.076 & 73.603 \\
\hline $4 / 12 / 0810: 00$ & 126.854 & 124.437 & & 100.918 & & 96.391 & 110.654 & & & 73.681 \\
\hline 4/12/08 10:30 & 126.91 & 124.453 & & 100.959 & & 96.44 & 110.697 & & & 73.735 \\
\hline 4/12/08 11:00 & 126.943 & 124.467 & & 100.979 & & 96.48 & 110.72 & & 79.154 & 73.772 \\
\hline 4/12/08 11:30 & 126.965 & 124.478 & & 101.004 & & 96.499 & 110.746 & & & 73.795 \\
\hline 4/12/08 12:00 & 126.981 & 124.485 & & 101.012 & & 96.511 & 110.759 & & & 73.815 \\
\hline $4 / 12 / 0812: 30$ & 126.991 & 124.48 & & 101.025 & & 96.52 & 110.769 & & 79.208 & 73.83 \\
\hline 4/12/08 13:00 & 127 & 122.596 & & 101.024 & & 96.53 & 110.776 & & & 73.84 \\
\hline 4/12/08 13:30 & 126.998 & 122.358 & & 100.537 & & 96.535 & 110.788 & & & 73.849 \\
\hline 4/12/08 14:00 & 125.062 & 122.238 & & 100.184 & & 95.879 & 110.146 & & 79.225 & 73.753 \\
\hline $4 / 12 / 0814: 30$ & 124.811 & 122.165 & & 100.041 & & 95.646 & 109.931 & & & 73.598 \\
\hline 4/12/08 15:00 & 124.686 & 122.12 & & 99.963 & & 95.533 & 109.83 & & & 73.493 \\
\hline 4/12/08 15:30 & 124.615 & 122.09 & & 99.915 & & 95.464 & 109.766 & & 79.181 & 73.425 \\
\hline $4 / 12 / 0816: 00$ & 124.573 & 122.074 & & 99.88 & & 95.427 & 109.725 & & & 73.377 \\
\hline 4/12/08 16:30 & 124.542 & 122.068 & & 99.86 & & 95.398 & 109.693 & & & 73.348 \\
\hline 4/12/08 17:00 & 124.525 & 122.063 & & 99.856 & & 95.382 & 109.672 & & 79.169 & 73.328 \\
\hline $4 / 12 / 0817: 30$ & 124.518 & 122.063 & & 99.851 & & 95.379 & 109.652 & & & 73.321 \\
\hline $4 / 12 / 0818: 00$ & 124.514 & 122.068 & & 99.851 & & 95.375 & 109.639 & & & 73.317 \\
\hline 4/12/08 18:30 & 124.516 & 122.068 & & 99.856 & & 95.379 & 109.627 & & 79.162 & 73.317 \\
\hline 4/12/08 19:00 & 124.516 & 122.063 & & 99.86 & & 95.382 & 109.616 & & & 73.319 \\
\hline 4/12/08 19:30 & 124.518 & 122.061 & & 99.86 & & 95.384 & 109.605 & & & 73.321 \\
\hline $4 / 12 / 0820: 00$ & 124.514 & 122.063 & & 99.858 & & 95.382 & 109.599 & & 79.157 & 73.321 \\
\hline $4 / 12 / 0820: 30$ & 124.509 & 122.068 & & 99.858 & & 95.375 & 109.594 & & & 73.317 \\
\hline 4/12/08 21:00 & 124.514 & 122.068 & & 99.861 & & 95.384 & 109.586 & & & 73.317 \\
\hline 4/12/08 21:30 & 124.516 & 122.065 & & 99.865 & & 95.386 & 109.58 & & 79.164 & 73.319 \\
\hline $4 / 12 / 0822: 00$ & 124.516 & 122.063 & & 99.863 & & 95.386 & 109.573 & & & 73.323 \\
\hline 4/12/08 22:30 & 124.514 & 122.056 & & 99.858 & & 95.386 & 109.567 & & & 73.323 \\
\hline 4/12/08 23:00 & 124.511 & 122.049 & & 99.856 & & 95.382 & 109.562 & & 79.152 & 73.319 \\
\hline 4/12/08 23:30 & 124.509 & 122.045 & & 99.853 & & 95.377 & 109.556 & & & 73.317 \\
\hline $4 / 13 / 080: 00$ & 124.502 & 122.04 & & 99.846 & & 95.372 & 109.552 & & & 73.313 \\
\hline 4/13/08 0:30 & 124.495 & 122.04 & & 99.841 & & 95.368 & 109.547 & & 79.14 & 73.309 \\
\hline 4/13/08 1:00 & 124.49 & 122.038 & & 99.837 & & 95.363 & 109.541 & & & 73.305 \\
\hline 4/13/08 1:30 & 124.49 & 122.034 & & 99.837 & & 95.361 & 109.535 & & & 73.301 \\
\hline 4/13/08 2:00 & 124.49 & 122.031 & & 99.836 & & 95.358 & 109.53 & & 79.132 & 73.301 \\
\hline 4/13/08 2:30 & 124.485 & 122.031 & & 99.834 & & 95.358 & 109.526 & & & 73.299 \\
\hline 4/13/08 3:00 & 124.483 & 122.034 & & 99.829 & & 95.356 & 109.522 & & & 73.299 \\
\hline 4/13/08 3:30 & 124.481 & 122.034 & & 99.829 & & 95.353 & 109.515 & & 79.123 & 73.295 \\
\hline 4/13/08 4:00 & 124.481 & 122.036 & & 99.831 & & 95.353 & 109.511 & & & 73.292 \\
\hline 4/13/08 4:30 & 124.483 & 122.043 & & 99.831 & & 95.356 & 109.505 & & & 73.295 \\
\hline
\end{tabular}


TABLE S1.1 (Cont.)

\begin{tabular}{|c|c|c|c|c|c|c|c|c|c|c|}
\hline \multirow[b]{2}{*}{ Date and Time } & \multicolumn{10}{|c|}{ Depth from Top of Casing (ft) } \\
\hline & MW1D & MW2D & MW3D & MW4D & MW7 & MW9 & Oentrich & MW14D & MW15D & MW16D \\
\hline 4/13/08 5:00 & 124.483 & 122.052 & & 99.839 & & 95.358 & 109.503 & & 79.12 & 73.297 \\
\hline 4/13/08 5:30 & 124.495 & 122.063 & & 99.848 & & 95.37 & 109.496 & & & 73.301 \\
\hline 4/13/08 6:00 & 124.502 & 122.07 & & 99.858 & & 95.375 & 109.488 & & & 73.307 \\
\hline 4/13/08 6:30 & 124.511 & 122.077 & & 99.868 & & 95.386 & 109.485 & & 79.128 & 73.317 \\
\hline 4/13/08 7:00 & 124.518 & 122.083 & & 99.875 & & 95.391 & 109.481 & & & 73.323 \\
\hline 4/13/08 7:30 & 124.525 & 122.088 & & 99.882 & & 95.401 & 109.473 & & & 73.332 \\
\hline 4/13/08 8:00 & 124.533 & 124.086 & & 99.887 & & 95.405 & 109.469 & & 79.132 & 73.338 \\
\hline 4/13/08 8:30 & 124.54 & 124.308 & & 100.519 & & 95.412 & 109.464 & & & 73.344 \\
\hline 4/13/08 9:00 & 126.608 & 124.426 & & 100.832 & & 96.178 & 110.217 & & & 73.483 \\
\hline 4/13/08 9:30 & 126.839 & 124.503 & & 100.976 & & 96.4 & 110.408 & & 79.191 & 73.66 \\
\hline 4/13/08 10:00 & 126.96 & 122.664 & & 101.068 & & 96.52 & 110.513 & & & 73.77 \\
\hline 4/13/08 10:30 & 127.04 & 122.43 & & 100.625 & & 96.596 & 110.575 & & & 73.849 \\
\hline 4/13/08 11:00 & 125.133 & 122.321 & & 100.27 & & 95.957 & 109.93 & & 79.24 & 73.805 \\
\hline 4/13/08 11:30 & 124.887 & 122.26 & & 100.135 & & 95.733 & 109.721 & & & 73.673 \\
\hline 4/13/08 12:00 & 124.778 & 122.217 & & 100.065 & & 95.632 & 109.62 & & & 73.58 \\
\hline 4/13/08 12:30 & 124.714 & 122.188 & & 100.017 & & 95.575 & 109.564 & & 79.225 & 73.522 \\
\hline $4 / 13 / 0813: 00$ & 124.67 & 122.161 & & 99.989 & & 95.533 & 109.53 & & & 73.483 \\
\hline 4/13/08 13:30 & 124.639 & 122.133 & & 99.958 & & 95.504 & 109.503 & & & 73.454 \\
\hline 4/13/08 14:00 & 124.611 & 122.117 & & 99.934 & & 95.476 & 109.488 & & 79.203 & 73.429 \\
\hline $4 / 13 / 08 \quad 14: 30$ & 124.585 & 122.104 & & 99.92 & & 95.452 & 109.475 & & & 73.406 \\
\hline 4/13/08 15:00 & 124.566 & 122.09 & & 99.903 & & 95.436 & 109.464 & & & 73.39 \\
\hline 4/13/08 15:30 & 124.554 & 122.079 & & 99.892 & & 95.424 & 109.453 & & 79.186 & 73.375 \\
\hline $4 / 13 / 0816: 00$ & 124.54 & 122.065 & & 99.878 & & 95.41 & 109.443 & & & 73.365 \\
\hline 4/13/08 16:30 & 124.53 & 122.058 & & 99.866 & & 95.401 & 109.438 & & & 73.354 \\
\hline 4/13/08 17:00 & 124.516 & 122.047 & & 99.858 & & 95.389 & 109.432 & & 79.172 & 73.342 \\
\hline 4/13/08 17:30 & 124.507 & 122.04 & & 99.846 & & 95.38 & 109.426 & & & 73.334 \\
\hline 4/13/08 18:00 & 124.495 & 122.034 & & 99.839 & & 95.368 & 109.419 & & & 73.323 \\
\hline 4/13/08 18:30 & 124.49 & 122.029 & & 99.832 & & 95.361 & 109.415 & & 79.159 & 73.315 \\
\hline 4/13/08 19:00 & 124.483 & 122.022 & & 99.829 & & 95.356 & 109.407 & & & 73.311 \\
\hline 4/13/08 19:30 & 124.478 & 122.02 & & 99.82 & & 95.351 & 109.402 & & & 73.305 \\
\hline 4/13/08 20:00 & 124.469 & 122.015 & & 99.818 & & 95.344 & 109.396 & & 79.145 & 73.299 \\
\hline $4 / 13 / 0820: 30$ & 124.464 & 122.018 & & 99.815 & & 95.342 & 109.391 & & & 73.295 \\
\hline 4/13/08 21:00 & 124.464 & 122.013 & & 99.813 & & 95.339 & 109.387 & & & 73.292 \\
\hline 4/13/08 21:30 & 124.462 & 122.013 & & 99.813 & & 95.335 & 109.38 & & 79.14 & 73.292 \\
\hline $4 / 13 / 0822: 00$ & 124.462 & 122.011 & & 99.812 & & 95.335 & 109.374 & & & 73.29 \\
\hline 4/13/08 22:30 & 124.461 & 122.004 & & 99.811 & & 95.337 & 109.37 & & & 73.288 \\
\hline 4/13/08 23:00 & 124.457 & 122.002 & & 99.806 & & 95.335 & 109.365 & & 79.14 & 73.288 \\
\hline 4/13/08 23:30 & 124.452 & 121.995 & & 99.803 & & 95.328 & 109.361 & & & 73.284 \\
\hline 4/14/08 0:00 & 124.45 & 121.99 & & 99.799 & & 95.325 & 109.355 & & & 73.282 \\
\hline 4/14/08 0:30 & 124.443 & 121.984 & & 99.792 & & 95.321 & 109.351 & & 79.113 & 73.278 \\
\hline 4/14/08 1:00 & 124.438 & 121.975 & & 99.787 & & 95.314 & 109.346 & & & 73.272 \\
\hline 4/14/08 1:30 & 124.431 & 121.97 & & 99.779 & & 95.309 & 109.342 & & & 73.266 \\
\hline 4/14/08 2:00 & 124.424 & 121.966 & & 99.772 & & 95.299 & 109.336 & & 79.093 & 73.261 \\
\hline 4/14/08 2:30 & 124.414 & 121.963 & & 99.765 & & 95.295 & 109.333 & & & 73.251 \\
\hline 4/14/08 3:00 & 124.412 & 121.952 & & 99.762 & & 95.287 & 109.327 & & & 73.247 \\
\hline 4/14/08 3:30 & 124.41 & 121.95 & & 99.76 & & 95.29 & 109.323 & & 79.079 & 73.243 \\
\hline 4/14/08 4:00 & 124.4 & 121.943 & & 99.75 & & 95.28 & 109.321 & & & 73.241 \\
\hline 4/14/08 4:30 & 124.393 & 121.947 & & 99.746 & & 95.273 & 109.316 & & & 73.23 \\
\hline 4/14/08 5:00 & 124.391 & 121.952 & & 99.746 & & 95.271 & 109.312 & & 79.062 & 73.228 \\
\hline 4/14/08 5:30 & 124.393 & 121.952 & & 99.751 & & 95.273 & 109.308 & & & 73.226 \\
\hline 4/14/08 6:00 & 124.398 & 123.757 & & 99.754 & & 95.278 & 109.3 & & & 73.228 \\
\hline 4/14/08 6:30 & 124.398 & 124.101 & & 99.901 & & 95.278 & 109.299 & & 79.059 & 73.233 \\
\hline 4/14/08 7:00 & 126.26 & 124.256 & & 100.597 & & 95.851 & 109.873 & & & 73.272 \\
\hline 4/14/08 7:30 & 126.624 & 124.342 & & 100.792 & & 96.19 & 110.173 & & & 73.474 \\
\hline 4/14/08 8:00 & 126.785 & 124.399 & & 100.902 & & 96.341 & 110.329 & & 79.12 & 73.615 \\
\hline 4/14/08 8:30 & 126.875 & 124.435 & & 100.964 & & 96.431 & 110.406 & & & 73.704 \\
\hline 4/14/08 9:00 & 126.932 & 124.453 & & 101.01 & & 96.487 & 110.457 & & & 73.76 \\
\hline 4/14/08 9:30 & 126.967 & 124.471 & & 101.033 & & 96.52 & 110.493 & & 79.174 & 73.799 \\
\hline 4/14/08 10:00 & 126.991 & 124.485 & & 101.052 & & 96.544 & 110.517 & & & 73.822 \\
\hline 4/14/08 10:30 & 127.007 & 122.548 & & 101.068 & & 96.558 & 110.534 & & & 73.84 \\
\hline 4/14/08 11:00 & 127.024 & 122.317 & & 100.471 & & 96.575 & 110.547 & & 79.216 & 73.855 \\
\hline 4/14/08 11:30 & 125.012 & 122.19 & & 100.151 & & 95.832 & 109.819 & & & 73.743 \\
\hline 4/14/08 12:00 & 124.771 & 122.104 & & 100.008 & & 95.613 & 109.62 & & & 73.588 \\
\hline 4/14/08 12:30 & 124.641 & 122.045 & & 99.913 & & 95.493 & 109.518 & & 79.169 & 73.481 \\
\hline 4/14/08 13:00 & 124.556 & 122.002 & & 99.848 & & 95.413 & 109.462 & & & 73.404 \\
\hline 4/14/08 13:30 & 124.492 & 121.966 & & 99.799 & & 95.351 & 109.421 & & & 73.342 \\
\hline
\end{tabular}


TABLE S1.1 (Cont.)

\begin{tabular}{|c|c|c|c|c|c|c|c|c|c|c|}
\hline \multirow[b]{2}{*}{ Date and Time } & \multicolumn{10}{|c|}{ Depth from Top of Casing (ft) } \\
\hline & MW1D & MW2D & MW3D & MW4D & MW7 & MW9 & Oentrich & MW14D & MW15D & MW16D \\
\hline 4/14/08 14:00 & 124.45 & 121.929 & & 99.763 & & 95.311 & 109.393 & & 79.128 & 73.297 \\
\hline $4 / 14 / 0814: 30$ & 124.412 & 121.902 & & 99.732 & & 95.278 & 109.372 & & & 73.264 \\
\hline 4/14/08 15:00 & 124.377 & 121.873 & & 99.699 & & 95.243 & 109.359 & & & 73.235 \\
\hline $4 / 14 / 08$ 15:30 & 124.346 & 121.841 & & 99.672 & & 95.215 & 109.346 & & 79.084 & 73.206 \\
\hline $4 / 14 / 0816: 00$ & 124.32 & 121.818 & & 99.643 & & 95.188 & 109.334 & & & 73.179 \\
\hline 4/14/08 16:30 & 124.287 & 121.8 & & 99.619 & & 95.155 & 109.327 & & & 73.152 \\
\hline $4 / 14 / 08$ 17:00 & 124.265 & 121.784 & & 99.599 & & 95.134 & 109.319 & & 79.04 & 73.127 \\
\hline $4 / 14 / 08$ 17:30 & 124.244 & 121.768 & & 99.579 & & 95.118 & 109.312 & & & 73.109 \\
\hline 4/14/08 18:00 & 124.23 & 121.752 & & 99.565 & & 95.101 & 109.304 & & & 73.092 \\
\hline 4/14/08 18:30 & 124.216 & 121.737 & & 99.551 & & 95.087 & 109.299 & & 79.003 & 73.075 \\
\hline 4/14/08 19:00 & 124.199 & 121.716 & & 99.537 & & 95.068 & 109.293 & & & 73.063 \\
\hline 4/14/08 19:30 & 124.18 & 121.714 & & 99.517 & & 95.052 & 109.289 & & & 73.049 \\
\hline $4 / 14 / 0820: 00$ & 124.164 & 121.703 & & 99.508 & & 95.038 & 109.284 & & 78.966 & 73.03 \\
\hline $4 / 14 / 0820: 30$ & 124.159 & 121.705 & & 99.503 & & 95.035 & 109.278 & & & 73.02 \\
\hline 4/14/08 21:00 & 124.15 & 121.705 & & 99.498 & & 95.028 & 109.273 & & & 73.013 \\
\hline $4 / 14 / 0821: 30$ & 124.15 & 121.7 & & 99.501 & & 95.028 & 109.265 & & 78.944 & 73.009 \\
\hline $4 / 14 / 0822: 00$ & 124.152 & 121.696 & & 99.501 & & 95.028 & 109.261 & & & 73.009 \\
\hline 4/14/08 22:30 & 124.145 & 121.684 & & 99.496 & & 95.024 & 109.256 & & & 73.009 \\
\hline 4/14/08 23:00 & 124.143 & 121.673 & & 99.486 & & 95.019 & 109.252 & & 78.922 & 73.003 \\
\hline 4/14/08 23:30 & 124.128 & 121.666 & & 99.472 & & 95.009 & 109.248 & & & 72.997 \\
\hline 4/15/08 0:00 & 124.119 & 121.662 & & 99.467 & & 94.995 & 109.244 & & & 72.984 \\
\hline 4/15/08 0:30 & 124.112 & 121.653 & & 99.46 & & 94.986 & 109.239 & & 78.888 & 72.976 \\
\hline 4/15/08 1:00 & 124.105 & 121.639 & & 99.455 & & 94.983 & 109.237 & & & 72.968 \\
\hline 4/15/08 1:30 & 124.098 & 121.621 & & 99.444 & & 94.976 & 109.231 & & & 72.962 \\
\hline 4/15/08 2:00 & 124.086 & 121.598 & & 99.427 & & 94.965 & 109.229 & & 78.856 & 72.953 \\
\hline 4/15/08 2:30 & 124.065 & 121.582 & & 99.406 & & 94.943 & 109.226 & & & 72.939 \\
\hline 4/15/08 3:00 & 124.046 & 121.569 & & 99.388 & & 94.924 & 109.224 & & & 72.92 \\
\hline 4/15/08 3:30 & 124.029 & 121.555 & & 99.376 & & 94.908 & 109.222 & & 78.822 & 72.902 \\
\hline 4/15/08 4:00 & 124.013 & 121.539 & & 99.36 & & 94.891 & 109.218 & & & 72.889 \\
\hline 4/15/08 4:30 & 123.998 & 121.528 & & 99.343 & & 94.877 & 109.214 & & & 72.873 \\
\hline 4/15/08 5:00 & 123.984 & 121.517 & & 99.329 & & 94.863 & 109.212 & & 78.78 & 72.858 \\
\hline 4/15/08 5:30 & 123.972 & 123.106 & & 99.322 & & 94.849 & 109.21 & & & 72.846 \\
\hline 4/15/08 6:00 & 123.963 & 123.566 & & 99.31 & & 94.84 & 109.207 & & & 72.836 \\
\hline 4/15/08 6:30 & 125.542 & 123.73 & & 99.996 & & 95.151 & 109.554 & & 78.758 & 72.829 \\
\hline 4/15/08 7:00 & 126.062 & 123.816 & & 100.213 & & 95.608 & 109.974 & & & 72.999 \\
\hline 4/15/08 7:30 & 126.234 & 123.859 & & 100.325 & & 95.771 & 110.132 & & & 73.137 \\
\hline 4/15/08 8:00 & 126.319 & 123.875 & & 100.382 & & 95.855 & 110.22 & & 78.795 & 73.224 \\
\hline 4/15/08 8:30 & 126.364 & 121.979 & & 100.407 & & 95.898 & 110.275 & & & 73.272 \\
\hline 4/15/08 9:00 & 126.383 & 121.737 & & 99.932 & & 95.917 & 110.314 & & & 73.299 \\
\hline 4/15/08 9:30 & 124.438 & 121.616 & & 99.574 & & 95.269 & 109.686 & & 78.79 & 73.21 \\
\hline 4/15/08 10:00 & 124.178 & 121.539 & & 99.424 & & 95.028 & 109.479 & & & 73.051 \\
\hline 4/15/08 10:30 & 124.057 & 121.483 & & 99.348 & & 94.915 & 109.376 & & & 72.937 \\
\hline 4/15/08 11:00 & 123.979 & 121.435 & & 99.284 & & 94.847 & 109.318 & & 78.734 & 72.871 \\
\hline 4/15/08 11:30 & 123.925 & 121.399 & & 99.24 & & 94.792 & 109.284 & & & 72.815 \\
\hline 4/15/08 12:00 & 123.878 & 121.369 & & 99.196 & & 94.748 & 109.259 & & & 72.772 \\
\hline $4 / 15 / 08$ 12:30 & 123.842 & 121.335 & & 99.169 & & 94.712 & 109.241 & & 78.68 & 72.73 \\
\hline 4/15/08 13:00 & 123.814 & 121.303 & & 99.147 & & 94.686 & 109.229 & & & 72.705 \\
\hline 4/15/08 13:30 & 123.786 & 121.283 & & 99.105 & & 94.656 & 109.218 & & & 72.676 \\
\hline 4/15/08 14:00 & 123.743 & 121.26 & & 99.074 & & 94.618 & 109.212 & & 78.622 & 72.645 \\
\hline 4/15/08 14:30 & 123.717 & 121.231 & & 99.062 & & 94.595 & 109.201 & & & 72.614 \\
\hline 4/15/08 15:00 & 123.701 & 121.197 & & 99.034 & & 94.58 & 109.199 & & & 72.598 \\
\hline 4/15/08 15:30 & 123.675 & 121.17 & & 99.002 & & 94.547 & 109.194 & & 78.568 & 72.575 \\
\hline 4/15/08 16:00 & 123.639 & 121.158 & & 98.974 & & 94.515 & 109.186 & & & 72.546 \\
\hline 4/15/08 16:30 & 123.611 & 121.143 & & 98.953 & & 94.491 & 109.182 & & & 72.519 \\
\hline 4/15/08 17:00 & 123.599 & 121.131 & & 98.94 & & 94.477 & 109.173 & & 78.514 & 72.497 \\
\hline 4/15/08 17:30 & 123.58 & 121.122 & & 98.921 & & 94.458 & 109.167 & & & 72.482 \\
\hline 4/15/08 18:00 & 123.566 & 121.118 & & 98.915 & & 94.439 & 109.162 & & & 72.464 \\
\hline 4/15/08 18:30 & 123.559 & 121.109 & & 98.915 & & 94.439 & 109.156 & & 78.475 & 72.455 \\
\hline 4/15/08 19:00 & 123.556 & 121.109 & & 98.907 & & 94.439 & 109.152 & & & 72.451 \\
\hline 4/15/08 19:30 & 123.554 & 121.102 & & 98.903 & & 94.432 & 109.148 & & & 72.443 \\
\hline 4/15/08 20:00 & 123.549 & 121.106 & & 98.896 & & 94.425 & 109.139 & & 78.433 & 72.437 \\
\hline 4/15/08 20:30 & 123.542 & 121.104 & & 98.895 & & 94.422 & 109.141 & & & 72.433 \\
\hline 4/15/08 21:00 & 123.549 & 121.104 & & 98.9 & & 94.43 & 109.135 & & & 72.43 \\
\hline 4/15/08 21:30 & 123.547 & 121.097 & & 98.902 & & 94.427 & 109.132 & & 78.409 & 72.428 \\
\hline 4/15/08 22:00 & 123.542 & 121.081 & & 98.897 & & 94.42 & 109.13 & & & 72.428 \\
\hline 4/15/08 22:30 & 123.54 & 121.07 & & 98.883 & & 94.418 & 109.128 & & & 72.424 \\
\hline
\end{tabular}


TABLE S1.1 (Cont.)

\begin{tabular}{|c|c|c|c|c|c|c|c|c|c|c|}
\hline \multirow[b]{2}{*}{ Date and Time } & \multicolumn{10}{|c|}{ Depth from Top of Casing (ft) } \\
\hline & MW1D & MW2D & MW3D & MW4D & MW7 & MW9 & Oentrich & MW14D & MW15D & MW16D \\
\hline 4/15/08 23:00 & 123.523 & 121.054 & & 98.864 & & 94.404 & 109.128 & & 78.375 & 72.41 \\
\hline 4/15/08 23:30 & 123.507 & 121.052 & & 98.855 & & 94.389 & 109.124 & & & 72.397 \\
\hline 4/16/08 0:00 & 123.495 & 121.045 & & 98.848 & & 94.378 & 109.12 & & & 72.387 \\
\hline 4/16/08 0:30 & 123.486 & 121.043 & & 98.846 & & 94.371 & 109.117 & & 78.336 & 72.375 \\
\hline 4/16/08 1:00 & 123.485 & 121.027 & & 98.84 & & 94.368 & 109.113 & & & 72.373 \\
\hline 4/16/08 1:30 & 123.478 & 121.006 & & 98.829 & & 94.361 & 109.109 & & & 72.366 \\
\hline 4/16/08 2:00 & 123.464 & 120.993 & & 98.807 & & 94.347 & 109.109 & & 78.299 & 72.356 \\
\hline $4 / 16 / 082: 30$ & 123.445 & 120.986 & & 98.795 & & 94.328 & 109.105 & & & 72.34 \\
\hline 4/16/08 3:00 & 123.431 & 120.979 & & 98.786 & & 94.319 & 109.104 & & & 72.323 \\
\hline 4/16/08 3:30 & 123.424 & 121 & & 98.779 & & 94.305 & 109.102 & & 78.262 & 72.313 \\
\hline 4/16/08 4:00 & 123.422 & 120.986 & & 98.79 & & 94.3 & 109.098 & & & 72.302 \\
\hline 4/16/08 4:30 & 123.438 & 120.975 & & 98.784 & & 94.326 & 109.09 & & & 72.306 \\
\hline 4/16/08 5:00 & 123.426 & 120.961 & & 98.774 & & 94.312 & 109.09 & & 78.23 & 72.306 \\
\hline 4/16/08 5:30 & 123.419 & 120.988 & & 98.774 & & 94.305 & 109.09 & & & 72.3 \\
\hline 4/16/08 6:00 & 123.403 & 122.745 & & 98.774 & & 94.286 & 109.09 & & & 72.296 \\
\hline 4/16/08 6:30 & 123.429 & 123.117 & & 98.791 & & 94.314 & 109.081 & & 78.209 & 72.288 \\
\hline 4/16/08 7:00 & 125.23 & 123.278 & & 99.606 & & 94.825 & 109.605 & & & 72.325 \\
\hline 4/16/08 7:30 & 125.639 & 123.385 & & 99.81 & & 95.205 & 109.944 & & & 72.53 \\
\hline 4/16/08 8:00 & 125.804 & 123.457 & & 99.925 & & 95.365 & 110.089 & & 78.262 & 72.672 \\
\hline $4 / 16 / 088: 30$ & 125.906 & 123.501 & & 100.015 & & 95.46 & 110.172 & & & 72.765 \\
\hline 4/16/08 9:00 & 125.986 & 123.557 & & 100.07 & & 95.544 & 110.221 & & & 72.846 \\
\hline
\end{tabular}


Argonne

Environmental Science Division

Argonne National Laboratory

9700 South Cass Avenue, Bldg. 203

Argonne, IL 60439-4843

www.anl.gov

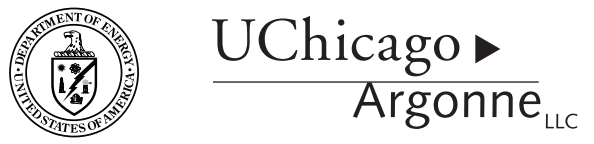

A U.S. Department of Energy laboratory

managed by UChicago Argonne, LLC 